EXECUTIVE SUMMARY

\title{
THE DEVELOPMENT OF A PROCEDURE TO FORECAST TRAFFIC VOLUMES ON URBAN SEGMENTS OF THE STATE AND INTERSTATE HIGHWAY SYSTEMS
}

by

Sunil K. Saha

Graduate Research Assistant

and

Jon D. Fricker

Research Engineer

Joint Highway Research Project

Project No.: HPR-0313-(028)

File No.: $3-3-41$

Prepared as Part of an Investigation

conducted by the

Joint Highway Research Project

Engineering Experiment Station

Purdue University

In Cooperation with the

Indiana Department of Transportation

and the

U.S. Department of Transportation

Federal Highway Administration

The contents of this report reflect the views of the author who is responsible for the facts and accuracy of the data presented herein. The contents do not necessarily reflect the official views or policies of the Federal Highway Administration. This report does not constitute a standard, specifications or regulations.

Department of Civil Engineering

Purdue University

West Lafayette, Indiana 47907

August 1990 
TECHNICAL REPORT STANDARD TITLE PAGE

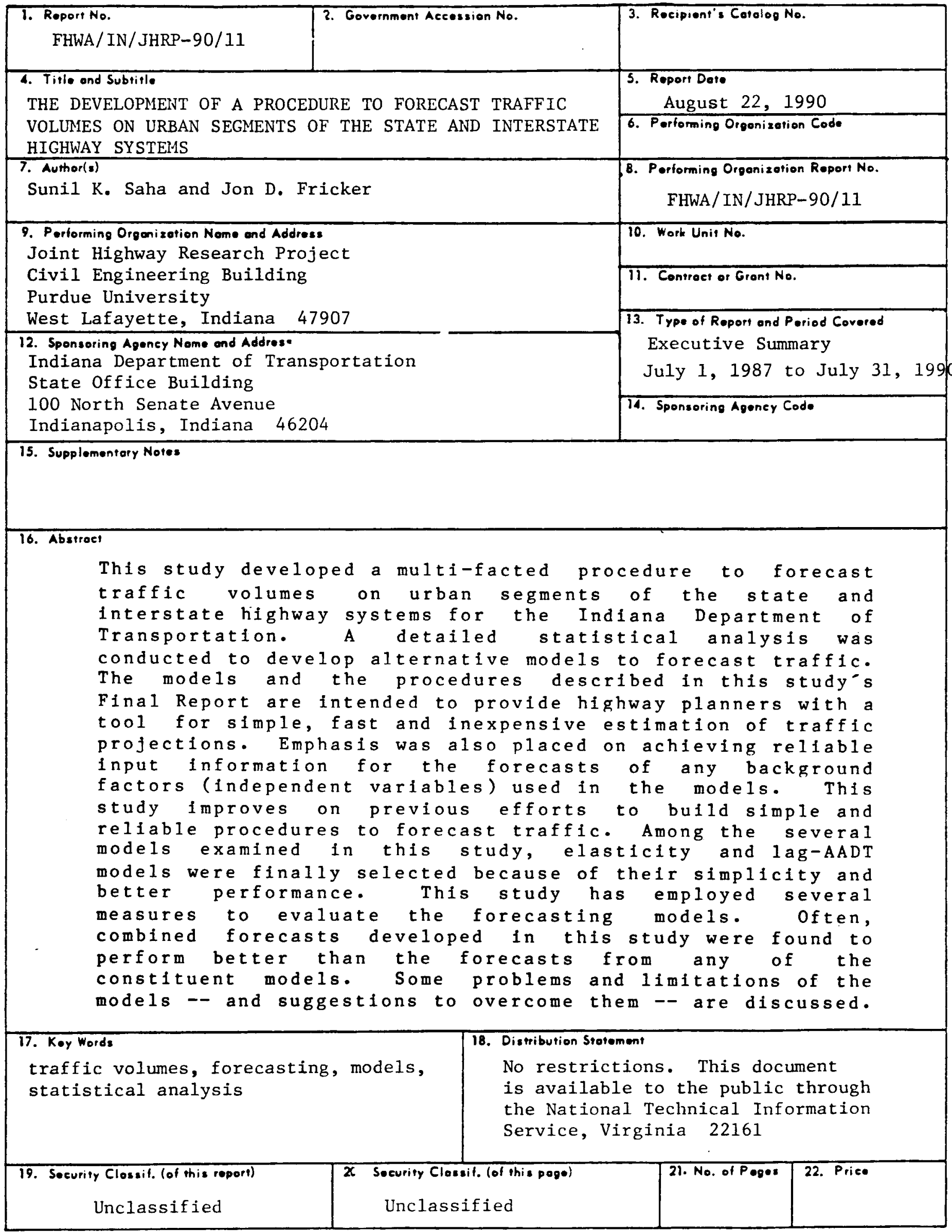


EXECUTIVE SUMMARY

The analyses conducted in this study were performed with traffic data gathered from the paper files of INDOT. Other input data were obtained from a variety of sources, for example, the libraries at Purdue University, Census Publications, IU Business Center, and the firm of Woods \& Poole Economics. A nationwide survey on "Forecasting Traffic on State Highways" by ITE Technical Council Committee 6F-4l showed the lack of any universally accepted method to address the issue of traffic forecasts in smal1- to medium-sized urban areas. This study has developed an efficient, inexpensive and easy-to-use method to forecast the traffic on state highways. The greatest strength of this study is the application and description of different statistics, ranging from simple to sophisticated. This report contains a full description of the entire model building process. The major findings of the research are summarized below, with reference to specific chapters and sections of the Final Report.

\section{Data Evaluation and Candidate Elasticity Model}

The variables and the data bases used in this study have 
been described in Chapter 4. Table 4.7 contains a summary of the quality (cost, avaliability and reliability) of the variables. The details of the analyses and their results for the evaluation of data and for selection of the candidate regression/elasticity models are presented in Chapter 5. The framework of the analyses for interstate and noninterstate highway are presented in Figures 5.1 and 5.2 , respectively. Several variable selection criteria $\left(R_{p}^{2}, R_{a}^{2}, C_{p}, P C, A I C\right)$ have been employed to select the most appropriate model. Despite the merits and demerits of using the $R^{2}\left(R_{p}^{2}\right)$ criterion, it has been adopted by the researchers as the single most useful criterion to gauge the "goodness" of a statistical mode1. However, analyses in this research were aided by other statistics. The data structure of this study includes the two data bases (segment and county/city average) and the four formats (raw, raw E\&L, density, and density E\&L). A sensitivity analysis of the "maximum" $R^{2}$ value among the competing models that are without any inconsistency in the sign of regression coefficients identifies the combination of county/city average data base and raw format as the best combination from all possible combination of the data structure. A significant improvement in model statistics was found in county/city average data bases over segment data bases. Because all the predictor variables used in this study are totals at either the city, county, or state level, it was not surprising to have traffic data on a county/city average basis perform better than traffic data on a more localized basis. 
A similar sensitivity analysis among the data groups revealed the following:

- For Interstate Highways: (1) For separate analysis of rural and urban interstate highways, both statewide and regional (north, central and south regions) models are found to be suitable. (2) A statewide model for the entire interstate network in Indiana was not satisfactory. (3) The improvement of the model statistics is better in case of analysis by geographical region than by the extent of roadway mileage and city group.

- For Urban Principal Arterial: (1) The combination of city group and geographic region performs better than any other possible combination. This second-level stratification by geographical region under each city group has been established as superior to grouping by city group alone. Results improved when the data of city groups $A, B$ and $C$ in the south region were pooled. The alternate was having unstable results with too few observations in each city group.

- For Minor Arterial \& Collector: The second-level stratification by geographic region and extent of highway mileage resulted in poor results and groups with too few observations. However, the analysis at this level of stratification identified areas of weakness in the data. After pool- 
ing data by city group, the quality of model statistics Improved.

The candidate models resulted from narrowing down a list of possible candidate models by comparing the model statistics $\left(R^{2}, R_{a}^{2}\right.$, PC, RMSE, AIC and $C_{p}$ ) and by applying judgment on the quality (availability, cost and reliability) of the data for the predictor variables in the model. The list of those candidate models are shown in Chapter 5 .

\section{Elasticity Based Traffic Mode1}

The final regression equations and the elasticity models developed in this study were shown in Chapter 6. The influential analysis identified the individual outliers and the outlier groups and their effect on model statistics. The diagnostic statistics employed to do influential analysis were shown in section 6.2.1. Modification of the data (if necessary) or deletion of outliers (if justified) was found to improve the quality of model statistics significantly. For example, it improved the statistics for the normality assumption of errors. Validity of the regression assumptions (linearity, constant variance, and normality of errors) were checked through formal tests and graphlcal plots -- residual and normal probability plots. Results of significance tests showed that the model parameters are highly significant. A significance of relation also resulted for all the models. The collinearity test found that no significant 
relationship existed between the variables of each model. The final regression equations of each group, shown in Tables 6.236.25, were produced by comparing the results of the above tests and some model statistics $\left(R^{2}, R_{a}^{2}, R M S E\right.$, and $\left.C V\right)$ of the candidate models, and by applying judgment regarding the quality of predictor variables. The $R^{2}$ values for the regression models of interstate highways, urban principal arterial, and urban minor arterial \& collector have ranges 50.35-88.86, 51.81-94.14 and 62.52-87.18 percent, respectively, with one or two variables in each equation. Each of these models is simple and does not contain more than two predictor variables. The elasticity models developed from the regression models are shown in Tables 6.266.28. These models are statistically sound and simple. They require the base year traffic data and base and future year data for the predictor variable(s).

\section{Principal Component Based Traffic Mode1}

The procedure and results for Principal Component Analysis (PCA) were shown in Chapter 7. The PCA was carried out for each group of filtered data which resulted from the influential analysis of Chapter 6. The PCA used 11 predictor variables, but had eliminated the problem of multicolinearity by computing the artificial variables (principal components) which are independent to each other. Some key results of the Principal component Regression (PSR) model with PCs in the equation are shown in 
Tables 7.2-7.4. Appendix Tables D1-D3 show the relationship between the PCs entered in the equation and the original variables. The PCR equations for interstate highways, urban principal arterial and urban minor arterial \& collector are shown in Tables $7.5,7.6$ and 7.7 , respectively. These tables show a oneto-one comparison of $R^{2}$ values between the PCR models and the regression or elasticity models from Chapter 6 . The $\mathrm{R}^{2}$ values for the PCR models had ranges 61.46-90.26, 52.87-97.57 and 66.87-89.31 percent for the three major highway categories in this study. The $\mathrm{R}^{2}$ ranges for the regression/elasticity models are 50.35-88.86, 51.81-94.14 and 62.52-87.18 percent. This range comparison, plus one-to-one comparisons for each model shown in Tables 7.5-7.7, do not show any significant improvement from the complex PCR model over the simple regression or elasticity model developed in Chapter 6. However, the complex PCR models, which need all variables to compute PCs in the model equation, certified the goodness of the model statistics for the regression or elasticity models that have fewer variables in their equations.

\section{Lagged Traffic (Lag-AADT) Mode 1}

The information of traffic data itself and the rate at which traffic had changed were used to develop the inexpensive and simple lagged traffic model. The segment data base of chapter 4 with a "raw" format (see Chapter 5) was used to build this model. Although Equation ( 8.2 ) had used "rate of AADT change", in its 
rearranged form, Equation ( 8.3 ) needs only one AADT value to forecast traffic on a highway segment. Because the data bases used to build lag-AADT models are different from those used in in Chapters 6 and 7 , the model statistics of the lag models are not directly compared to those of the other models. However, the traffic forecasting performance of the lag-AADT models were compared with the others in Chapter 10. The lag traffic models for Interstate, urban principal arterial and urban minor arterial and collector were presented in Tables $8.1,8.2$ and 8.3 , respectively. These tables show, as expected, a very high $R^{2}$ values (about 99 percent). The parameters of the models and the regression relationships were found highly significant at very low levels. The nonnormality of the error terms were because of the presence of outliers. The forecasting performance of these models was tested by skipping the last year of AADT values from each segment, reformulating the models, and comparing the resulting forecasts against the skipped values. Very high forecasting quality performance measures and model statistics were found for the models (shown in Appendix E) built with partial data.

\section{Time Series and Ratio Method Forecasts}

No forecasts of vehicle registrations and city population are avallable from outside sources. The structure and results of procedures developed to make these forecasts are shown in Chapter 9 and Appendix F. A ratio method of forecasting city population 
was described in section 9.3.1. Its forecasts for the cities of Indiana were shown in Appendix $F$. Forecasts of vehicle registration were made through two time series analysis techniques -- the Box-Jenkins ARIMA model and the autoregressive procedure. Parameters of the models were found to be significant at 5 percent level of significance. Both autoregressive and ARIMA procedures produce high quality forecasts in this study. Use of these two different types of models also increased confidence in the forecasts. A combined forecast of vehicle registrations was made by simple averaging of the forecasts from the Box-Jenkins and the autoregressive procedures. The forecast measures of the combined forecasts and the forecasts from the constituent models for the two selected counties were shown in Table 9.7. The values of the performance criteria verified the reliability of the vehicle registration forecasts.

\section{Traffic Model Evaluation}

Leve1 of accuracy is a primary concern for the users of traffic forecasts. Inaccurate forecasts can lead to under or over-designed facilities. To determine the tolerable errors in forecast, one needs to know how much error a traffic forecast can have without causing major design changes. However, boundary cases must be examined more carefully. In that case, it will be wise to develop forecasts from several methods to arrive at a "consensus range" of the traffic estimate. 
Usually, the error in various travel forecasting methods can be estimated by testing the methods against the actual counts and comparing the results. This study used several forecasting measuring criteria (Section 10.2) to evaluate the performance of the traffic models. The results, shown in Tables 10.1-10.3, show that the mean absolute percentage errors, defined in section 10.2, are usually less than 10 percent. Regression models developed in Chapter 6 were found to be ineffective in forecasting project site traffic. Despite the difference in model statistics and procedures, both elasticity and lag-AADT models were found to be highly efficient models for forecasting traffic. They were found to work equally well. The combined forecasts, formed by simple averaging of the forecasts from the elasticity and lag-AADT models, did better than either the constituent model in many cases. Combined forecasts are recommended to forecast traffic on the state highway segments. Forecasts from several models provides the opportunity to assess the sensitivity of the forecasts to different model forms. Finally, a procedure, shown in Section 10.4, was developed to monitor the forecasts and to see if there will be any need in the future to recalibrate the models and their parameters. 
JOINT HIGHWAY RESEARCH PROJECT FHWA/IN/JHRP-90/11

Draft Final Report

THE DEVELOPMENT OF A PROCEDURE TO FORECAST TRAFFIC VOLUMES ON URBAN SEGMENTS OF THE STATE

AND INTERSTATE HIGHWAY SYSTEMS

Sunil K. Saha

Jon D. Fricker 
Draft

Final Report

The Development Of A Procedure To Forecast Traffic Volumes On Urban Segments Of The State And Interstate Highway Systems

TO:

FROM: Jon D. Fricker

H.L. Michael, Director

Joint Highway Research Project

Associate Professor of Civil Engineering

Purdue University

West Lafayette, Indiana
August 22, 1990

Project No. C-36-5400

File No: $3-3-41$

SUBJ: The Development of a Procedure to Forecast Traffic Volumes on Urban Segments of the State and Interstate Highway Systems

Attached is the final report of the subject report, which describes the work completed under the expanded project having the title "A Method of Establishing Traffic Growth Factors". The project has been performed by Mr. Sunil Saha, Graduate Research Assistant, under my direction.

After extensive data retrieval and preparation, painstaking statistical analysis and engineering judgement have produced a forecasting procedure that combines explanatory power and ease of use. Using a menu-driven computer program developed to implement the models built during this study, INDOT personnel can now quickly generate a welldocumented traffic volume forecast for any urban segment of the state or interstate highway system.

The final report is submitted for review, comment, and acceptance in fulfillment of the referenced study.

cc:
A.G. Altschaeffl
D. Andrewski
J.L. Chameau
W.L. Dolch
A.R. Fendrick
J.D. Fricker
D.W. Halpin
K.R. Hoover
R.H. Lee
C.W. Letts
C.W. Lovell
D.W. Lucas
H.L. Michael
B.K. Partridge
G.J. Rorbakken
C.F. Scholer

Respectfully Submitted

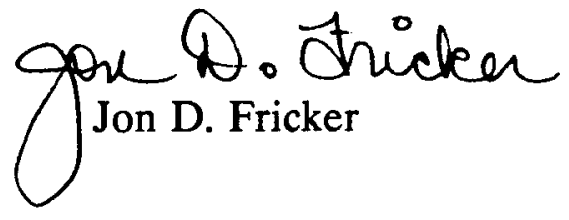




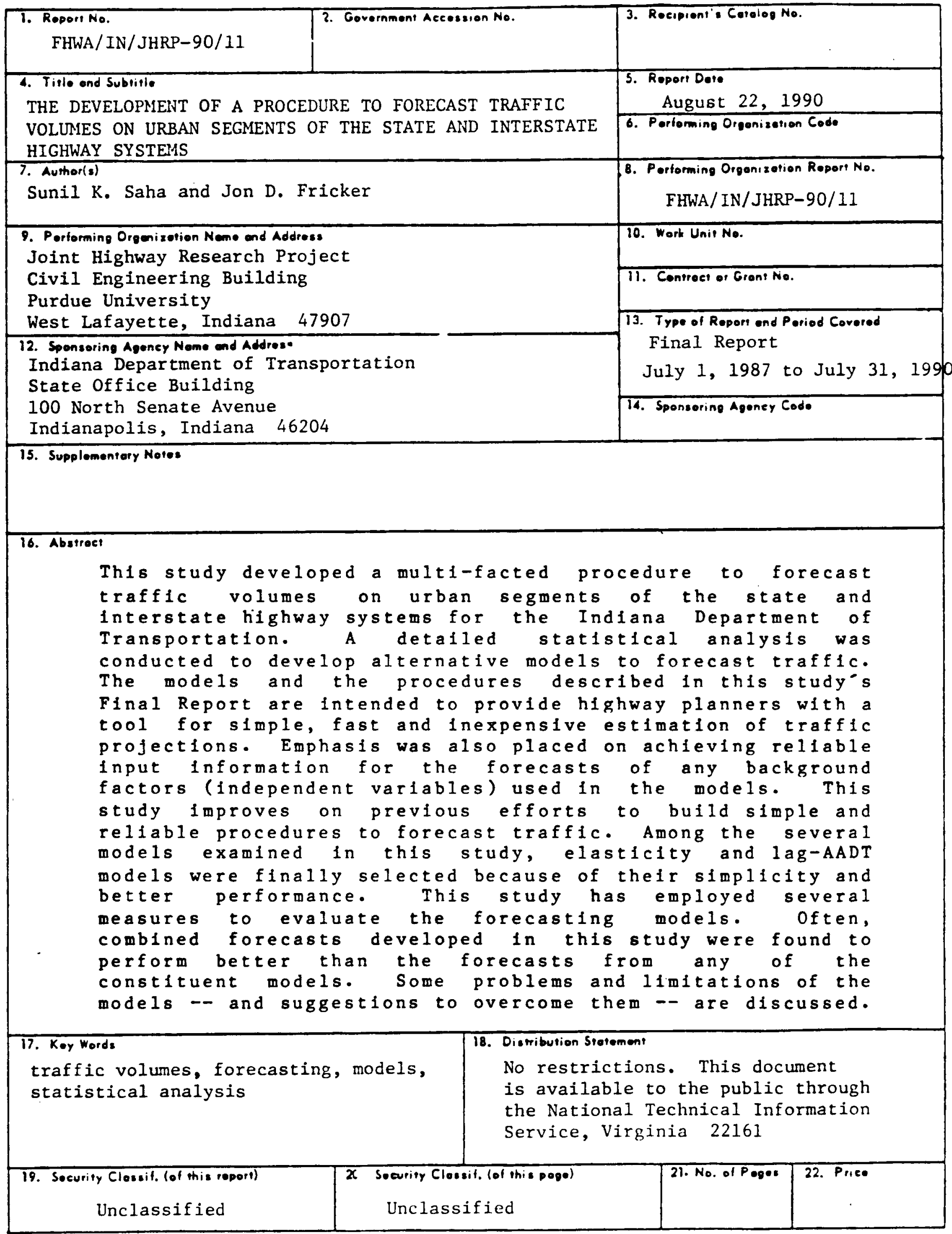


FINAL REPORT

THE DEVELOPMENT OF A PROCEDURE TO FORECAST TRAFFIC VOLUMES ON URBAN SEGMENTS OF THE STATE AND INTERSTATE HIGHWAY SYSTEMS

by

Sunil K. Saha

Graduate Research Assistant

and

Jon D. Fricker

Research Engineer

Joint Highway Research Project

Project No.: HPR-0313-(028)

File No.: $3-3-41$

Prepared as Part of an Investigation

conducted by the

Joint Highway Research Project

Engineering Experiment Station

Purdue University

In Cooperation with the

Indiana Department of Transportation

and the

U.S. Department of Transportation

Federal Highway Administration

The contents of this report reflect the views of the author who is responsible for the facts and accuracy of the data presented herein. The contents do not necessarily reflect the official views or policies of the Federal Highway Administration. This report does not constitute a standard, specifications or regulations.

Department of Civil Engineering

Purdue University

West Lafayette, Indiana 47907

August 1990 


\section{ACKNOWLEDGMENTS}

The authors extend their sincere appreciation to the following persons, who provided valuable technical assistance at various stages of the project: Professor Kumares C. Sinha for his suggestions on the general conduct and orientation of the study; Professor Tom Kuczek for his advice and comments on the extensive statistical analyses in this research; and Professor Arnold L. Sweet for his assistance and comment on the time series analysis in this study.

Mr. John Nagle was the project advisor from the Indiana Department of Transportation (INDOT). The authors offer ther special thanks to him for his comments, suggestions, patience, and support during the course of this study. Sincere appreciation is also extended to Mr. John Breitweiser, the project advisor from the Federal Highway Administration, for his comments and suggestions during this study's quarterly advisory committee meetings. The authors thankfully acknowledge the assistance of Ms. Rebecca Archibald and Mr. Kirk Mangold of INDOT in obtaining the traffic data, and Mr. Maher Al-Kilani and Mr. Stephen Kalanjee of Purdue University for their help in data entry.

The writers are also grateful to the Indiana Department of Transportation, the Federal Highway Administration and Purdue University for the financial support provided through grants administered by the Joint Highway Research Project. 
TABLE OF CONTENTS

Page

LIST OF TABLES $\ldots \ldots \ldots \ldots \ldots \ldots \ldots \ldots \ldots \ldots \ldots \ldots \ldots \ldots \ldots \ldots \ldots \ldots \ldots \ldots \ldots$

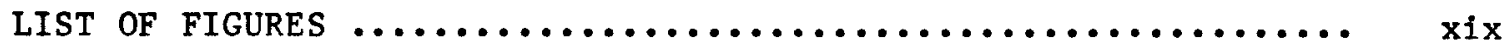

CHAPTER 1 - INTRODUCTION $\ldots \ldots \ldots \ldots \ldots \ldots \ldots \ldots \ldots \ldots \ldots \ldots \ldots \ldots \ldots \ldots$

1.1 Introduction $\ldots \ldots \ldots \ldots \ldots \ldots \ldots \ldots \ldots \ldots \ldots \ldots \ldots \ldots \ldots \ldots \ldots$

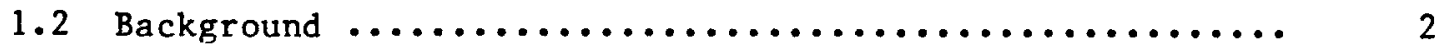

1.3 Purpose and Scope of the Research $\ldots \ldots \ldots \ldots \ldots \ldots \ldots \ldots \ldots$

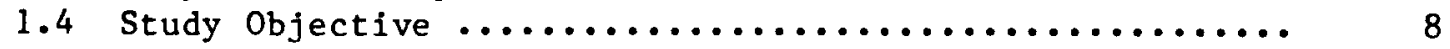

1.5 Study Organization $\ldots \ldots \ldots \ldots \ldots \ldots \ldots \ldots \ldots \ldots \ldots \ldots \ldots \ldots \ldots$

CHAPTER 2 - LITERATURE REVIEW ....................... 12

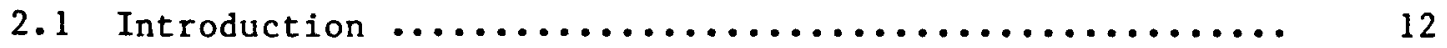

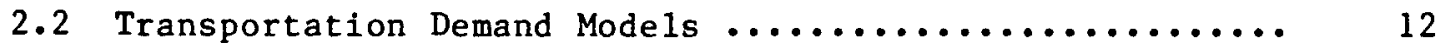

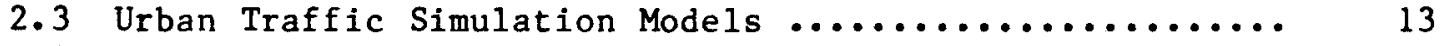

2.4 ITE Survey Results of State Traffic

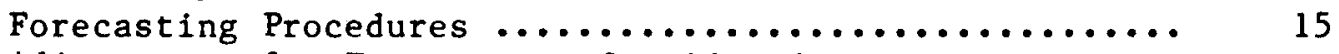

2.5 Adjustments for Factors not Considered

Explicitly in the Model $\ldots \ldots \ldots \ldots \ldots \ldots \ldots \ldots \ldots \ldots \ldots . \ldots \ldots 17$

2.6 Past Research $\ldots \ldots \ldots \ldots \ldots \ldots \ldots \ldots \ldots \ldots \ldots \ldots \ldots \ldots \ldots \ldots . . \ldots \ldots$

2.6.1 Site Traffic Estimate from Volume Ratio

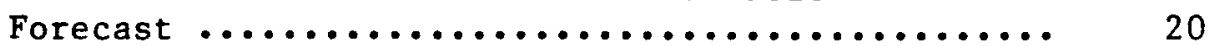

2.6.2 Project-Level Traffic Forecasting ............. 20

2.7 AADT Estimate from Short-Term Counts ............... 22

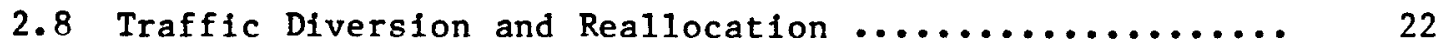

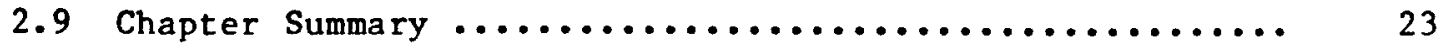

CHAPTER 3 - PROBLEM STATEMENT AND EVALUATION FRAMEWORK ........ 24

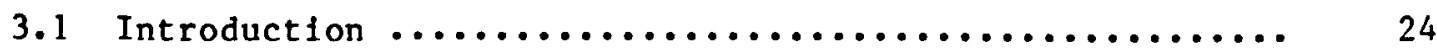

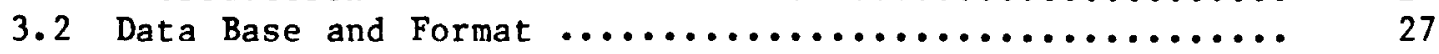

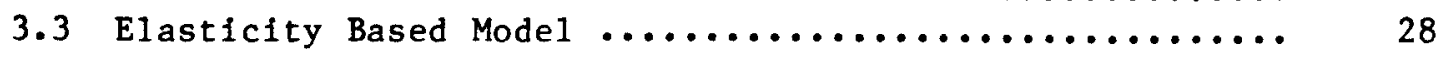


3.4 Principal Component Analysis (PCA) Based Model ........ 31

3.5 Lag-AADT Mode1 ............................... 32

3.6 Time Series and Ratio Method Forecasts ................ 33

3.7 Evaluation of Traffic Mode1 ...................... 34

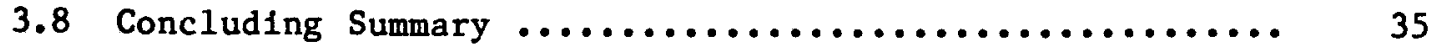

CHAPTER 4 - DATA BASES ........................... 36

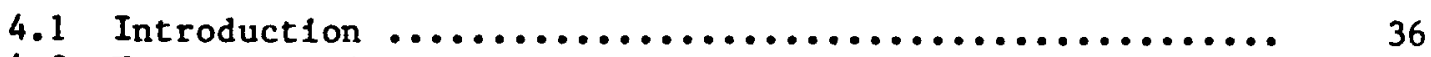

4.2 Forecasts of Demographic and Economic Variables ........ 37

4.3 W\&P's Forecast Methods ....................... 38

4.3.1 The "Export-Base" Economic Models .............. 40

4.3.2 The Demographic Mode1 ...................... 41

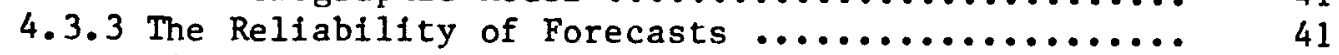

4.4 Methodology for IU's Population Projections ........... 42

4.5 The Data Tables ................................ 44

4.5.1 Data Tables with Segment AADT ................. 49

4.5.2 Data Tables with County and City Average AADT .... 54

4.5 .3 Number of Cases ........................... 54

4.6 Description of Variables $\ldots \ldots \ldots \ldots \ldots \ldots \ldots \ldots \ldots \ldots \ldots \ldots$

4.6.1 Index or Categorical Variables ............... 55

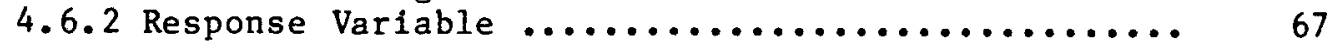

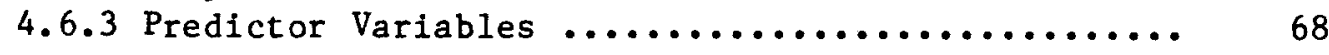

4.7 Chapter Summary $\ldots \ldots \ldots \ldots \ldots \ldots \ldots \ldots \ldots \ldots \ldots \ldots \ldots \ldots \ldots . \ldots 2$

CHAPTER 5 - SELECTION OF DATA FORMAT AND CANDIDATE MODELS ..... 83

5.1 Introduction $\ldots \ldots \ldots \ldots \ldots \ldots \ldots \ldots \ldots \ldots \ldots \ldots \ldots \ldots \ldots . \ldots \ldots$

5.2 Ordinary Least Squares (OLS) $\ldots \ldots \ldots \ldots \ldots \ldots \ldots \ldots \ldots \ldots \ldots . \ldots \ldots$

5.2.1 Correlation and the Regression Coefficient ...... 85

5.3 Some Alternative Variable Selection Rules ............ 87

$5.3 .1 \mathrm{R}_{\mathrm{p}}^{2}$ Criterion $\ldots \ldots \ldots \ldots \ldots \ldots \ldots \ldots \ldots \ldots \ldots \ldots . \ldots \ldots \ldots$

5.3.2 Adjusted $R^{2}\left(R_{a}^{2}\right)$ or $M S E$ Criterion ............. 89

5.3 .3 Mallows ${ }^{-} c_{p}$ Criterion $\ldots \ldots \ldots \ldots \ldots \ldots \ldots \ldots \ldots$ 
Page

5.3.4 Amemiya's Prediction Criterion (PC) .............. 91

5.3.5 Aka1ke's Information Criterion (AIC) ........... 92

5.4 Multiple Linear Regression Analysis ................. 94

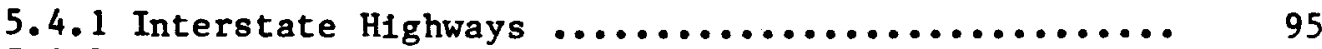

5.4.2 Noninterstate Urban State Highways ............ 97

5.5 Data Format and Group Selection ................... 100

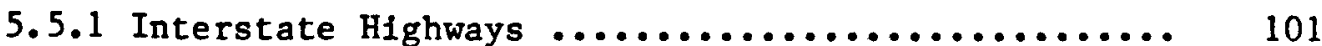

5.5.2 Urban Principal Arterial .................... 116

5.5.3 Urban Minor Arterial and Collector ............ 125

5.6 Candidate Regression Mode1 Selection .............. 135

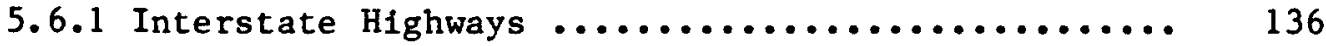

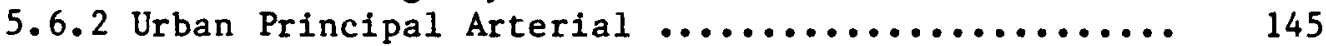

5.6.3 Urban Minor Arterial and Collector ............ 155

5.7 Chapter Summary $\ldots \ldots \ldots \ldots \ldots \ldots \ldots \ldots \ldots \ldots \ldots \ldots \ldots \ldots \ldots$

CHAPTER 6 - DIAGNOSIS AND FINAL SELECTION OF MODEL .......... 160

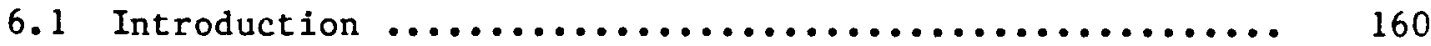

6.2 Detecting Outliers and Influential Observations ....... 161

6.2.1 Diagnostic Techniques of Detecting Outliers ..... 162

6.2.1.1 Partial Regression Residual P1ots ........ 163

6.2.1.2 Studentized Residual (RSTUDENT) .......... 164

6.2.1.3 Change in the Fitted Values (DFFITS) ..... 168

6.2.1.4 Change in the Regression Coefficients (DFBETAS) ................ 172

6.2.1.5 Diagonal Element of Hat Matrix

(HAT DIAG) .......................... 172

6.2.1.6. Residual and Normal Probability Plots .... 173

6.2.2 Pre and Post Analysis of Outliers ............ 181

6.3 Validity of Regression Assumptions and Model Aptness ... 197

6.3.1 Scatterplots: Linear Pattern of the Model ....... 198

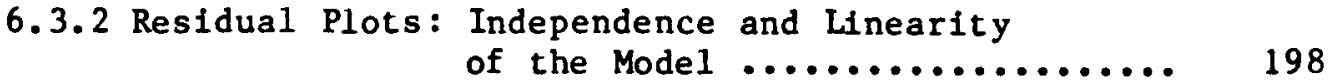

6.3.3 Residual Plots: Homoscedasticity of Variance ..... 203

6.3.4 Normality Tests and Plots: Normality of Errors ... 203 
Page

6.4 Significance and Collinearity of Regression

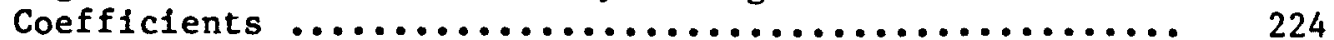

6.4.1 Collinearity Diagnostics of Regression

Coefficients ............................ 224

6.4.2 Testing Hypothesis Concerning Regression

Coefficients .............................. 241

6.4.3 Standardized Regression Coefficients ........... 264

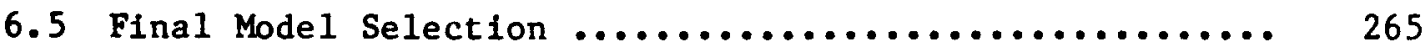

6.6 Chapter Summary ............................. 279

CHAPTER 7 - PRINCIPAL COMPONENT ANALYSIS BASED MODEL ......... 280

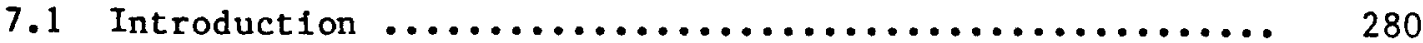

7.2 Principal Component Analys1s ...................... 281

7.3 Principal Component Regression ..................... 288

7.3.1 Final Principal Component Regression Model ...... 289

7.4 Chapter Summary .............................. 303

CHAPTER 8 - LAGGED TRAFFIC MODEL ........................ 304

8.1 Introduction .................................... 304

8.2 Lagged Model Formulation ........................ 305

8.2.1 Modelling Approach ....................... 305

8.2.2 Model Formulation ....................... 306

8.3 Lagged Regression Model ........................ 307

8.3.1 Ful1-Lagged Model ........................ 308

8.3.2 Partial-Lagged Model and Its Performance ....... 326

8.3.2.1 Partial-Lagged Mode1 ................. 326

8.3.2.2 Performance of Partial-Lagged Model ..... 327

8.4 Chapter Summary ................................. 329

CHAPTER 9 - VEHICLE REGISTRATION AND CITY POPULATION

FORECASTS ............................. 331

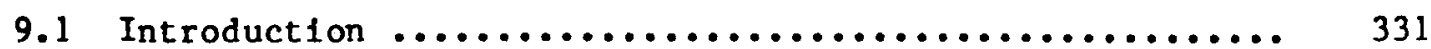

9.2 Time Series Forecasts of Vehicle Registration ......... 332 
Page

9.2.1 Introduction to Forecast Procedures and

Estimation Method ......................... 332

9.2 .2 ARIMA Model $\ldots \ldots \ldots \ldots \ldots \ldots \ldots \ldots \ldots \ldots \ldots \ldots \ldots . \ldots \ldots$

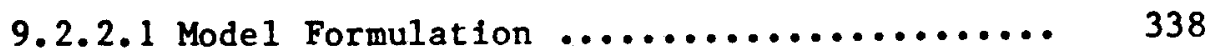

9.2.2.2 Chi-Squared Check of Autocorrelation ..... 342

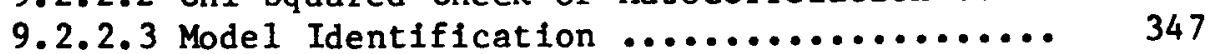

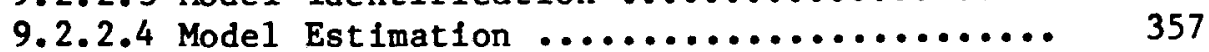

9.2.2.5 Model Forecast and Validation .......... 361

9.2.3 Autoregression Procedure ................. 362

9.2.3.1 First Order Autocorrelation Check of

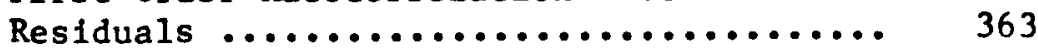

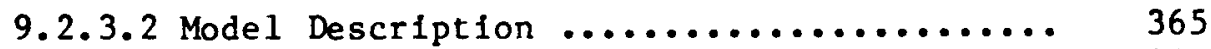

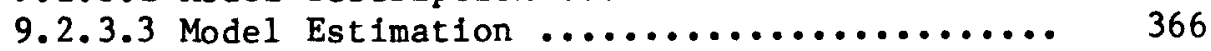

9.2.3.4 Model Forecast and Validation ............ 369

9.2.4 Combination of Forecasts and Comparison of

Models ............................... 370

9.3 City Population Forecasts .................... 374

9.3.1 Ratio Method of City Population Forecasts ....... 374

9.4 Chapter Summary $\ldots \ldots \ldots \ldots \ldots \ldots \ldots \ldots \ldots \ldots \ldots \ldots \ldots \ldots \ldots \ldots$

CHAPTER 10 - MODEL VALIDATION, COMBINATION AND CALIBRATION ..... 378

10.1 Introduction $\ldots \ldots \ldots \ldots \ldots \ldots \ldots \ldots \ldots \ldots \ldots \ldots \ldots \ldots \ldots . \ldots \ldots \ldots$

10.2 Performance Measures Criteria ................... 378

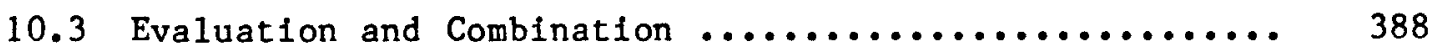

10.4 Calibration and Tracking Signal ................... 400

10.5 Chapter Summary ........................... 403

CHAPTER 11 - SUMMARY AND CONCLUSIONS .................. 405

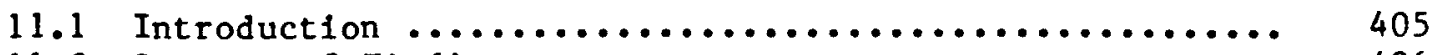

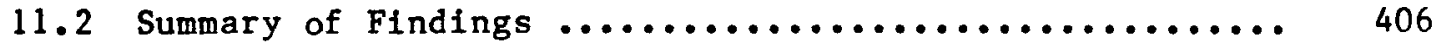

11.2.1 Data Evaluation and Candidate Elasticity Model.. 407 11.2.2 Elasticity Based Traffic Mode1 .............. 409 11.2.3 Principal Component Based Traffic Model ....... 410 11.2 .4 Lagged Traffic (Lag-AADT) Model ............. 411 11.2.5 Time Series and Ratio Method Forecasts ........ 412 11.2.6 Traffic Model Evaluation ................ 413 
11.3 Implementation Guldelines ....................... 414

11.4 Problems, Limitations and Suggestions ................ 424

11.5 Future Research Direction ........................ 426

11.6 Conclusions ................................. 430

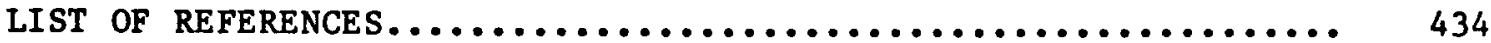

APPENDICES

Appendix A.................................... 441

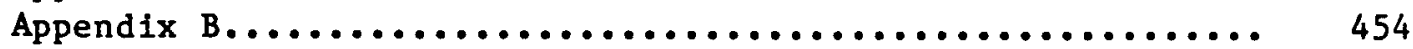

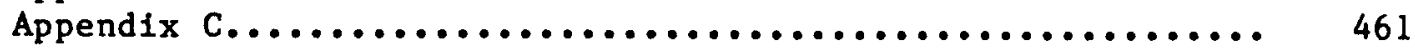

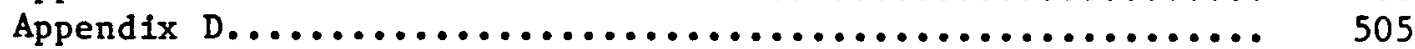

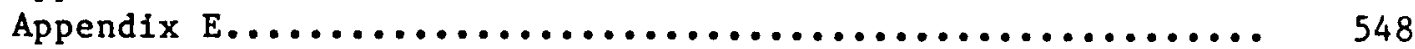

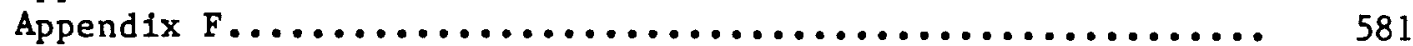

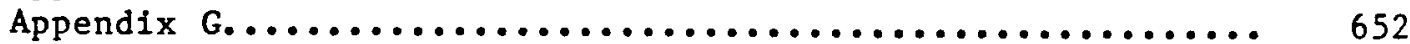




\section{LIST OF TABLES}

Table

Page

4.1 Urban Functional Classification of Highways ............ 45

4.2 Groupings of Roadway Segments $\ldots \ldots \ldots \ldots \ldots \ldots \ldots \ldots \ldots \ldots \ldots . \ldots 4$

4.3 Description of Items in Data-Bases .................. 48

4.4 Number of Cases for Urban Minor Arterial \& Collector ...... 56

4.5 Number of Cases for Urban Principal Arterial ........... 57

4.6 Number of Cases for Interstate Highways .............. 58

4.7 Availability, Cost and Reliability of the Predictor Variables .................................... 70

5.1 Maximum $\mathrm{R}^{2}$ value for Interstate Highways $\ldots \ldots \ldots \ldots \ldots \ldots$

5.2 Number of Variables for Maximum $R^{2}$ value for Interstate Highways .............................. 104

5.3 Marginal Improvement in Maximum $R^{2}$ value of County/City Average AADT over Segment AADT for Interstate Highways ..... 106

5.4 Marginal Improvement in Maximum $R^{2}$ value of Raw E\&L, Density and Density E\&L Formats over Raw Format for Interstate Highways ............................. 109

5.5 Marginal Improvement in Maximum $R^{2}$ value of Earlier \& Later $(E \& L)$ Year Data Format for Interstate Highways ...... 111

5.6 Marginal Improvement in Maximum $R^{2}$ value of Density Format over Raw Format for Interstate Highways ........... 113

5.7 Maximum $\mathrm{R}^{2}$ value for Urban Principal Arterial .......... 117

5.8 Number of Variables for Maximum $R^{2}$ value for Urban Principal Arterial ......................... 118

5.9 Marginal Improvement in Maximum $R^{2}$ value of County/City Average AADT over Segment AADT for Urban Principal Arterial 
Table

5.10 Marginal Improvement in Maximum $R^{2}$ value of Raw E\&L, Density and Density E\&L Formats over Raw Format for

Urban Principal Arterial

5.11 Marginal Improvement in Maximum $R^{2}$ value of Earlier \& Later (E\&L) Year Data Format for Urban Principal Arterial ..

5.12 Marginal Improvement in Maximum $R^{2}$ value of Density Format over Raw Format for Urban Principal Arterial ...... 122

5.13 Maximum $R^{2}$ value for Urban Minor Arterial \& Collector ..... 126

5.14 Number of Variables for Maximum $R^{2}$ value for

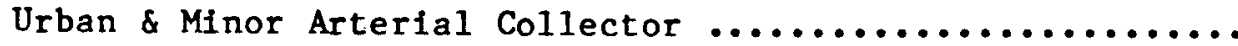

5.15 Marginal Improvement in Maximum $\mathrm{R}^{2}$ value of County/City Average AADT over Segment AADT for Urban Minor

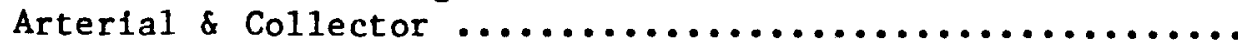

5.16 Marginal Improvement in Maximum $R^{2}$ value of Raw $E \& L$, Density and Density E\&L Formats over Raw Format for

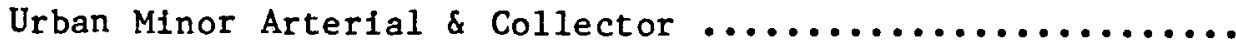

5.17 Marginal Improvement in Maximum $R^{2}$ value of Earlier \& Later (E\&L) Year Data Format for Urban Minor Arterial

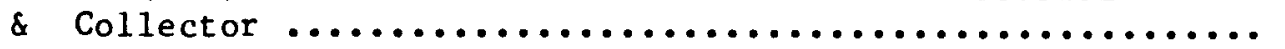

5.18 Marginal Improvement in Maximum $\mathrm{R}^{2}$ value of Density Format over Raw Format for Urban Minor Arterial \&

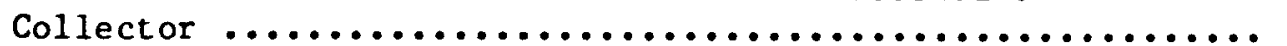

5.19 Possible Candidate Models for Interstate Highways ........ 138

5.20 Possible Candidate Models for Urban Principal Arterial .... 146

5.20.1 Possible Candidate Models for South Region of CTYGR A, B and $C$ of Urban Principal Arterial ................ 154

5.21 Possible Candidate Models for Urban Minor Arterial and Collector

6.1 Some Major Influential Diagnostics of Model Cn 5 [An 1llustration of individual effect before deleting any observation ] $\ldots \ldots \ldots \ldots \ldots \ldots \ldots \ldots \ldots \ldots \ldots \ldots \ldots \ldots \ldots$

6.2 Some Major Influential Diagnostics of Model Un4 [An 1llustration of group effect before deleting any observation] 
6.3 Some Major Influentlal Diagnostics of Model Cn5 [After deleting observation 11 of Table 6.1] ............ 184

6.4 Some Major Influential Diagnostics of Model Un4 [After deleting OBS 48-52 with ID (City \#) 6 of Table 6.2].. 191

6.5 Normality Statistics of Residuals in Pre-Influence Analysis [Interstate Highways] ........................ 209

6.6 Normality Statistics of Residuals in Pre-Influence Analysis [Urban Principal Arteria1] ................... 212

6.7 Normality Statistics of Residuals in Pre-Influence Analysis [Urban Minor Arterial \& Collector] ............. 214

6.8 Normality Statistics of Residuals in Post-Influence Analysis [Interstate Highways] ..................... 215

6.9 Normality Statistics of Residuals in Post-Influence Analysis [Urban Principal Arterial] ................... 218

6.10 Normality Statistics of Residuals in Post-Influence Analysis [Urban Minor Arterial \& Collector] ............. 220

6.11 Collinearity Statistics for $>2$ variables in the Model in Pre-Influence Analysis [Interstate Highways] ...........

6.12 Collinearity Statistics for $>2$ varlables in the Model in Pre-Influence Analysis [Urban Principal Arterial] ...... 230

6.13 Collinearity Statistics for $>2$ variables in the Model in Pre-Influence Analysis [Urban Minor Arterial \& Collector] $\ldots \ldots \ldots \ldots \ldots \ldots \ldots \ldots \ldots \ldots \ldots \ldots \ldots \ldots \ldots \ldots \ldots \ldots \ldots$

6.14 Collinearity Statistics for $>2$ variables in the Model in Post-Influence Analysis [Interstate Highways] ......... 234

6.15 Collinearity Statistics for $>2$ variables in the Model in Post-Influence Analysis [Urban Principal Arterial] ......

6.16 Collinearity Statistics for $>2$ variables in the Mode 1 in Post-Influence Analysis [Urban Minor Arterial \&

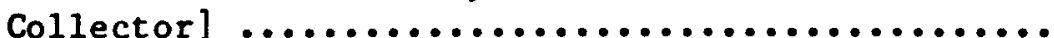

6.17 Significance of Varlable(s) in Model in Pre-Influence Analysis [Interstate Highways] ....................... 242

6.18 Significance of Variable(s) in Model in Pre-Influence Analysis [Urban Principal Arterial] ................... 
Table

6.19 Significance of Variable(s) in Model in Pre-Influence Analysis [Urban Minor Arterial \& Collector] ............. 250

6.20 Significance of Variable(s) in Model in Post-Influence Analysis [Interstate Highways] ....................... 253

6.21 Significance of Variable(s) in Model in Post-Influence Analysis [Urban Principal Arterial] .................. 258

6.22 Significance of Variable(s) in Model in Post-Influence Analysis [Urban Minor Arterial \& Collector] ............. 261

6.23 Final Regression Equations for Interstate Highways ........ 268

6.24 Final Regression Equations for Urban Principal Arterial .... 270

6.25 Final Regression Equations for Urban Minor Arterial \&

6. 26 Elasticity-based Traffic Models for Interstate Highways .... 274

6.27 Elasticity-based Traffic Models for Urban Principal Arterial

6.28 Elasticity-based Traffic Models for Urban Minor Arterial \& Collector .............................. 278

7.1 Eigen Values and Component Variances of Selected Data

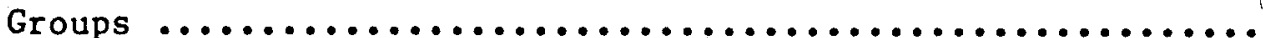

7.2 Some Key Results of Principal Component Regression for Interstate Highways ............................. 291

7.3 Some Key Results of Principal Component Regression for Urban Principal Arterial ......................... 293

7.4 Some Key Results of Principal Component Regression for Urban Minor Arterial \& Collector .....................

7.5 Final Principal Component Regression (PCR) Equations for Interstate Highways .............................

7.6 Final Principal Component Regression (PCR) Equations for Urban Principal Arterial .......................... 300

7.7 Final Principal Component Regression (PCR) Equations for Urban Minor Arterial \& Collector .................... 302 
Table

Page

8.1 Full-Lagged Regression Results of Interstate Highways ..... 309

8.2 Fu11-Lagged Regression Results of Urban Principal

Arterial .....................................

8.3 Full-Lagged Regression Results of Urban Minor Arterial \& Collector

8.4 Lagged Traffic (Lag-AADT) Models for Interstate Highways ..

8.5 Lagged Traffic (Lag-AADT) Models for Urban Principal

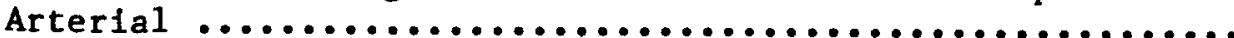

8.6 Lagged Traffic (Lag-AADT) Models for Urban Minor

Arterial \& Collector .............................

9.1 Example of Selected ARIMA Models from General Form

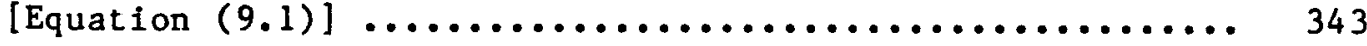

9.2 Steps of ARIMA Model for Nonseasonal Univariate Series .... 345

9.3 Statistics of ARIMA Models for Few Selected Counties ...... 358

9.4 Significance and Correlation of Parameters of ARIMA

Models for Few Selected Counties .................. 359

9.5 Nonsignificance of Overfitted Autoregressive Lags for

Franklin County Vehicle Registrations .............. 367

9.6 Key Statistics of OLS, AUTOREG and ARIMA Model for Few Selected Counties Vehicle Registrations ...............

9.7 Forecast Measures of Time Series Models for Two Selected

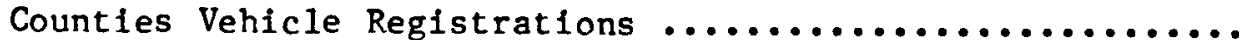

10.1 Forecast Measures from Regression, Elasticity and Lag-AADT

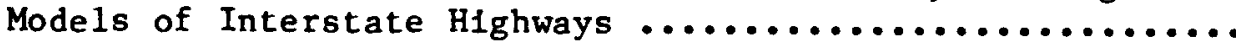

10.2 Forecast Measures from Regression, Elasticlty and Lag-AADT

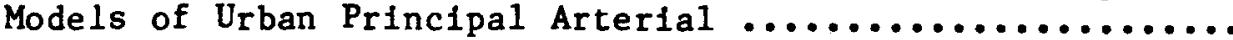

10.3 Forecast Measures from Regression, Elasticity and Lag-AADT Models of Urban Minor Arterial \& Collector ..............

10.4 Ranges of Forecast Measures of Models for Major Highway

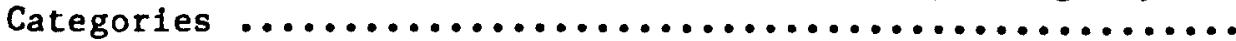


Append1x

Table

Page

Al Segment AADT Raw Data Base for Urban Minor Arterial \&

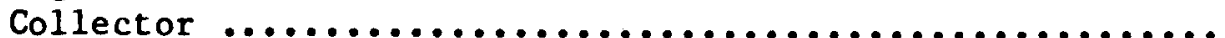

442

A2 Segment AADT Density Data Base for Urban Minor Arterial \&

Collector ....................................

A3 Segment AADT, Earlier \& Later Years Density Data Base for

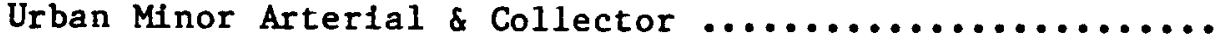

A4 County or City Average Raw Data Base for Urban Minor

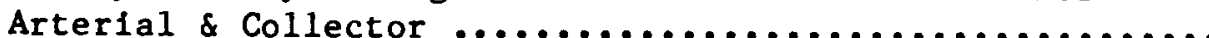

A5 County or City Average Raw Data Base for Urban Principal

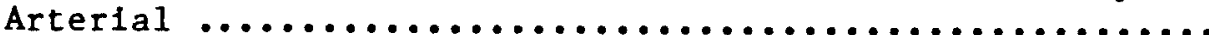

A6 County or City Average Raw Data Base for Interstate

Highways .................................... 452

B1 City Number, Name and Land Area .................... 455

B2 County Number, Name and Land Area .................... 456

B3 1988 County Functional Class Mileage of State of Indiana .. 457

B4 Traffic Data Retrieval Form ....................... 460

$\mathrm{Cl}$ Best Mode1s for Interstate Highways

[Data Format: Raw and County Avg. by Year] ............ 462

C2 Best Models for Interstate Highways

[Data Format: Raw E\&L and County Avg. by Year] ......... 467

C3 Best Models for Interstate Highways

[Data Format: Density and County Avg. by Year] ..........

C4 Best Models for Interstate Highways

[Data Format: Density E\&L and County Avg . by Year] ........

C5 Best Models for Urban Principal Arterial

[Data Format: Raw and City Avg. by Year] .............. 482

C5.1 Best Models for Urban Principal Arterlal by Region

[Data Format: Raw and City Avg. by Year] ............... 485

C6 Best Mode1s for Urban Principal Arterfal

[Data Format: Raw E\&L and C1ty Avg. by Year] .......... 486 
Appendix

Table

C7 Best Models for Urban Principal Arterial

[Data Format: Density and City Avg. by Year] ............ 489

C8 Best Models for Urban Principal Arterial

[Data Format: Density E\&L and City Avg. by Year] ......... 492

C9 Best Mode1s for Urban Minor Arterlal \& Collector

[Data Format: Raw and City Avg. by Year] .............. 495

C10 Best Models for Urban Minor Arterlal \& Collector

[Data Format: Raw E\&L and City Avg. by Year] ........... 498

C11 Best Models for Urban Minor Arterial \& Collector

[Data Format: Density and City Avg. by Year] .............

C12 Best Models for Urban Minor Arterial \& Collector

[Data Format: Density E\&L and City Avg. by Year] .........

D1 Eigenvectors or Coefficients of Principal Components (PCs) of Final Regression Equations for Interstate Highways .....

D2 Eigenvectors or Coefficients of Principal Components (PCs) of Final Regression Equations for Urban Principal

Arterial ..................................

D3 Eigenvectors or Coefficients of Principal Components (PCs) of Final Regression Equations for Urban Minor

Arterial \& Collector

E1.1 Partial-Lagged Regression Results of Interstate Highways ..

E1.2 Partial-Lagged Regression Results of Urban Principal

Arterial ...................................

E1.3 Partial-Lagged Regression Results of Urban Minor Arterial

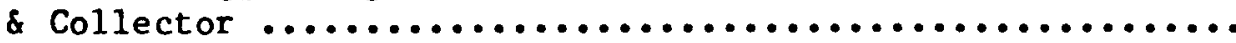

E2.1 Forecast Measures from Partial-Lagged Model of Interstate Highways $\ldots \ldots \ldots \ldots \ldots \ldots \ldots \ldots \ldots \ldots \ldots \ldots \ldots$

E2.2 Forecast Measures from Partial-Lagged Model of Urban Principal Arterial .......................

E2.3 Forecast Measures from Partial-Lagged Model of

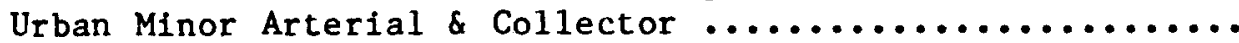


F1 ARIMA Model for Vehicle Registrations ............... 582

F2 Parameter of ARIMA Model for Vehicle Registrations ....... 585

F3 1989-2015 Vehicle Registration Forecasts by ARIMA Model ... 589

F4 Autoregression Model for Vehicle Registrations .......... 598

F5 Parameter of Autoregression Model for Vehicle Registrations .................................. 601

F6 1989-2015 Vehicle Registration Forecasts by Autoregressive Procedure .......................... 604

F7 1989-2015 Vehicle Registration Forecasts (Combined) ...... 613

F8 Surrounding County and Township for Cities of Indiana .... 622

F9 City Population Forecasts by Ratio Method ............. 625

G1 90-Percent Confidence Limits of Forecast Measures for Models of Interstate Highways ..................... 653

G2 95-Percent Confidence Limits of Forecast Measures for

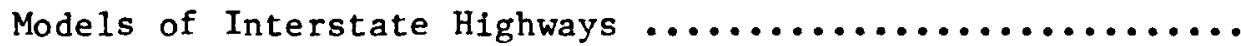

G3 90-Percent Confidence Limits of Forecast Measures for Models of Urban Principal Arterial .................. 657

G4 95-Percent Confidence Limits of Forecast Measures for Models of Urban Principal Arterial .................. 659

G5 90-Percent Confidence Limits of Forecast Measures for Models of Urban Minor Arterial \& Collector .............. 661

G6 95-Percent Confidence Limits of Forecast Measures for Models of Urban Minor Arterial \& Collector .............. 
4.1 Schematic Representation of "SCALE" Problem ............. 51

4.2 Suggested Geographical Boundaries of State of Indiana ..... 63

5.1 Layout of Analysis for Interstate Highways ............ 96

5.2 Layout of Analysis for Non-Interstate Urban State

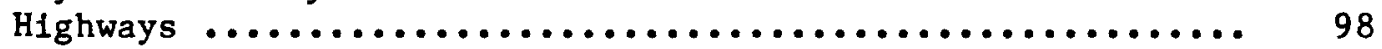

6.1 Partial Regression Residual Plot for Regressor CNTYINC of Model Cn5 [Individual case of Pre-Influence Analysis] .....

6.2 Partial Regression Residual Plot for Regressor CNTYVR of Model Cn5 [Individual case of Pre-Influence Analysis] ..... 166

6.3 Partial Regression Residual Plot for Regressor CNTYHH of Model Un4 [Group case of Pre-Influence Analys1s] .........

6.4 Residual Plot against Predicted value of Model Cn5

[Individual case of Pre-Influence Analysis] ............ 174

6.5 Residual Plot against Regressor CNTYINC of Model Cn5

[Individual case of Pre-Influence Analysis] ........... 175

6.6 Residual P1ot against Regressor CNTYVR of Mode1 Cn5 [Individual case of Pre-Influence Analysis] ............ 176

6.7 Normal Probability Plot of Model Cn5 [Individual case of Pre-Influence Analysis] ............. 177

6.8 Residual Plot against Predicted value of Model Un4 [Group case of Pre-Influence Analysis] ............... 178

6.9 Residual Plot against Regressor CNTYHH of Model Un4 [Group case of Pre-Influence Analysis] ................ 179

6.10 Normal Probability Plot of Model Un4 [Group case of Pre-Influence Analys1s] ................. 180 
Figure

Page

6.11 Partial Regression Residual Plot for Regressor CNTYINC of Model Cn5 [Individual case of Post-Influence Analysis] ....

6.12 Partial Regression Residual Plot for Regressor CNTYVR of Model Cn5 [Individual case of Post-Influence Analysis] ....

6.13 Residual Plot against Predicted value of Model Cn5 [Individual case of Post-Influence Analysis] ............

6.14 Residual Plot against Regressor CNTYINC of Model Cn5

[Individual case of Post-Influence Analysis] .............

6.15 Residual Plot against Regressor CNTYVR of Model Cn5 [Individual case of Post-Influence Analysis] ............

6.16 Normal Probability P1ot of Model Cn5

[Individual case of Post-Influence Analysis] ............ 190

6.17 Partial Regression Residual Plot for Regressor CNTYHH of Model Un4 [Group case of Post-Influence Analysis] ........

6.18 Residual Plot against Predicted value of Model Un4

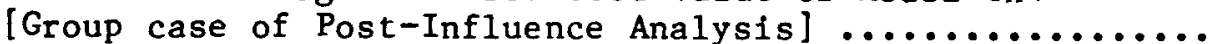

6.19 Residual Plot against Regressor CNTYHH of Model Un4 [Group case of Post-Influence Analysis] ...............

6.20 Normal Probability Plot of Model Un4

[Group case of Post-Influence Analysis] ............... 196

6.21 A Scatterplot for Region "s" of Urban Interstate

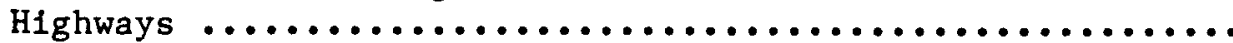

6.22 A Scatterplot for City Group " $C$ " and Region " $n$ " of

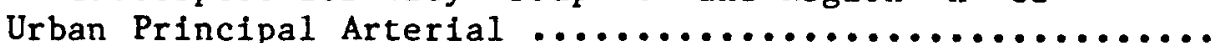

6.23 A Scatterplot for City Group " $D$ " and Region "ctn" of

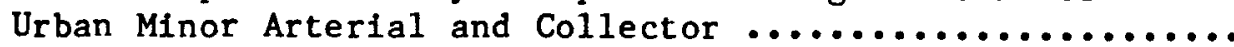

6.24 Residual Plot against CNTYINC of Model Rs 1 [Region "s" of Rural Interstate Highways] .............. 204

6.25 Residual Plot against CNTYINC of Model Ds4 [C1ty Group "D" \& Region "s" of Urban Principal Arteria1]..

6.26 Residual Plot against CNTYINC of Model C4 [City Group " $C$ " of Urban Minor Arterial \& Collector] ...... 
6. 27 Normal Probability Plot of Model Rs 1

[Region "s" of Rural Interstate Highways] ..............

6. 28 Normal Probability Plot of Model Ds 4

[City Group "D" \& Region "s" of Urban Principal Arterial].. 222

6. 29 Normal Probability Plot of Model C4

[City Group "C" of Urban Minor Arteria1 \& Collector] ......

8.1 Scatterplot of AADT against LAADT for City Group "A" and Region " $n$ " of Urban Principal Arterial ................

8.2 Scatterplot of AADT against RAADT for City Group "A" and Region " $n$ " of Urban Principal Arterial ...............

8.3 Residual Plot against RAADT without RAADT in Model for City Group "A" and Region " $n$ " of Urban Principal Arteria1 ....................................

9.1 Observed Series of Franklin County Vehicle Registration ...

9.2 Observed Series of Tippecanoe County Vehicle Registration ................................

9.3 First Difference Series of Franklin County Vehicle

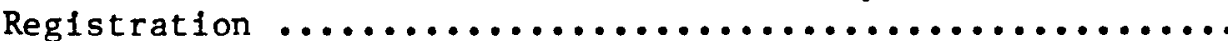

9.4 First Difference Series of Tippecanoe County Vehicle

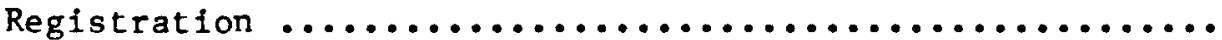

9.5 Autocorrelation of Observed Series for Franklin County Vehicle Registration

9.6 Autocorrelation of Observed Series for Tippecanoe

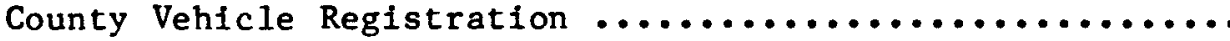

9.7 (a) Q-statistic and (b) Partial Autocorrelation of Observed Series for Franklin County Vehicle Registration ..........

9.8 (a) Q-statistic and (b) Partial Autocorrelation of Observed Series for Tippecanoe County Vehicle Registration ........

9.9 Autocorrelation of First Difference Series for Franklin County Vehicle Registration 
Figure

Page

9.10 Autocorrelation of First Difference Series for Tippecanoe

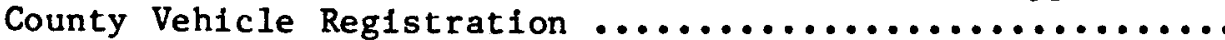

9.11 (a) Q-statistic and (b) Partial Autocorrelation of First Difference Series for Franklin County Vehicle Registration

9.12 (a) Q-statistic and (b) Partial Autocorrelation of First Difference Series for Tippecanoe County Vehfcle Registration

11.1 Process for Implementation of the Mode1 on Interstate

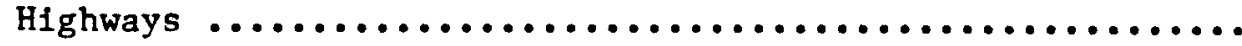

11.2 Process for Implementation of the Model on Urban Principal Arterial

11.3 Process for Implementation of the Model on Urban

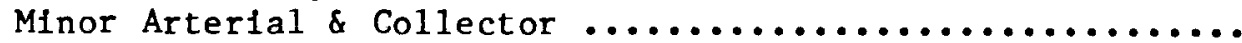

Appendix

Figure

Page

D1.1 Partial Regression Residual P1ot of PC1 for Central Region of Rural Interstate Highways ...............

D1.2 Partial Regression Residual Plot of PC2 for Central Region of Rural Interstate Highways $\ldots \ldots \ldots \ldots \ldots \ldots \ldots \ldots$

D1.3 Partial Regression Residual Plot of PC3 for Central Region of Rural Interstate Highways $\ldots \ldots \ldots \ldots \ldots \ldots \ldots \ldots$

D1.4 Partial Regression Residual P1ot of PC1 for Central Region of Urban Interstate Highways .................

D1.5 Partial Regression Residual P1ot of PC2 for Central Region of Urban Interstate Highways ............... 516

D1.6 Partial Regression Residual P1ot of PC4 for Central Region of Urban Interstate Highways .................

D1.7 Partial Regression Residual Plot of PC1 for City Group "A" and Region "c" of Urban Principal Arterial 
Appendix

Figure

Page

D1.8 Partial Regression Residual Plot of PC3 for C1ty Group "A" and Region "c" of Urban Principal Arterial .............

D1.9 Partial Regression Residual Plot of PC4 for City Group "A" and Region "c" of Urban Principal Arterial .............

D1.10 Partial Regression Residual Plot of PC1 for City Group "D" of Urban Minor Arterial \& Collector ...................

D1.11 Partial Regression Residual P1ot of PC3 for City Group "D" of Urban Minor Arterial \& Collector ..................

D1.12 Partial Regression Residual Plot of PC5 for City Group "D"

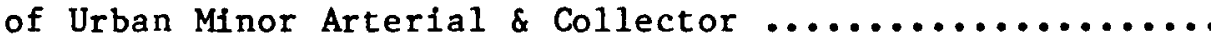

D2.1 Normal Probability P1ot for Central Region of Rural Interstate Highways ......................

D2.2 Normal Probability Plot for Central Region of Urban Interstate Highways .....................

D2.3 Normal Probability P1ot for City Group "A" and Region "c" of Urban Principal Arterial ......................

D2.4 Normal Probability P1ot for City Group "D" of

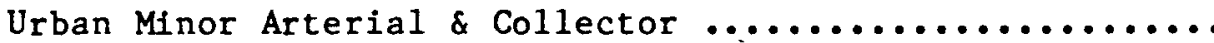

D3.1 Residual Plot against Predicted value for Central Region

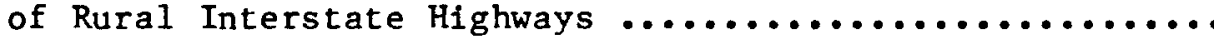

D3.2 Residual P1ot against Predicted value for Central Region

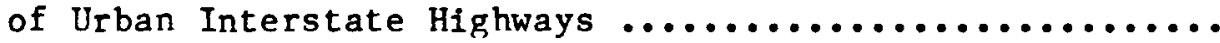

D3.3 Residual Plot against Predicted value for City Group "A" and Region "c" of Urban Principal Arterial ..............

D3.4 Residual P1ot against Predicted value for City Group "D" of Urban Minor Arterial \& Collector ....................

D4.1 Residual P1ot against PCl for Central Region of Rural Interstate Highways .....................

D4.2 Residual Plot against PC2 for Central Region of Rural Interstate Highways ....................

D4.3 Residual Plot against PC3 for Central Region

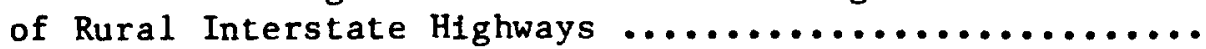


Appendix

Figure

D4.4 Residual Plot against PC1 for Central Region

of Urban Interstate Highways ..................... 535

D4.5 Residual P1ot against PC2 for Central Region

of Urban Interstate Highways .................... 536

D4.6 Residual Plot against PC4 for Central Region

of Urban Interstate Highways .................... 537

D4.7 Residual P1ot against PC1 for City Group "A" and Region "c" of Urban Principal Arterial ............. 538

D4.8 Residual P1ot against PC3 for City Group "A" and Regton "c" of Urban Principal Arterial ............ 539

D4.9 Residual Plot against PC4 for City Group "A" and Region "c" of Urban Principal Arterial ............. 540

D4.10 Residual P1ot against PCl for City Group "D"

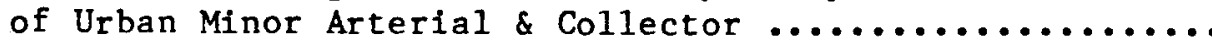

D4.11 Residual P1ot against PC3 for City Group "D" of Urban Minor Arterial \& Collector ..................

D4.12 Residual P1ot against PC5 for City Group "D"

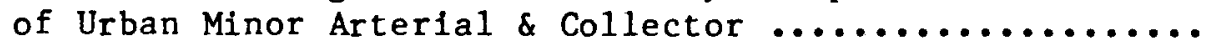

D5.1 Residual P1ot against PC8 (not in mode1) for Central Region of Rural Interstate Highways .................

D5.2 Residual Plot against PC8 (not in mode1) for Central

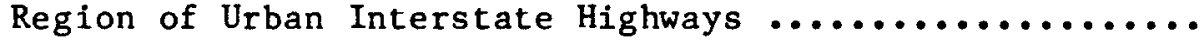

D5.3 Residual P1ot against PC8 (not in mode1) for City Group "A" and Region "c" of Urban Principal Arterial ..........

D5.4 Residual P1ot against PC8 (not in model) for City Group

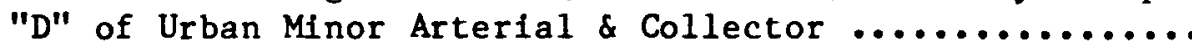

E1.1 Partial Regression Residual Plot of LAADT for North Region of Urban Interstate Highways ................

E1.2 Partial Regression Residual Plot of RAADT for North Region of Urban Interstate Highways 
Appendix

Figure

Page

E1.3 Partial Regression Residual Plot of LAADT for City Group " $A$ " and Region " $n$ " of Urban Principal Arterial ..........

E1.4 Partial Regression Residua1 P1ot of RAADT for City Group "A" and Region " $n$ " of Urban Principal Arterial .......... 560

E1.5 Partial Regression Residual Plot of LAADT for C1ty Group "C" of Urban Minor Arterial \& Collector.................

E1.6 Partial Regression Residual Plot of RAADT for City Group "C" of Urban Minor Arterial \& Collector...................

E2.1 Normal Probability P1ot for North Region of Urban Interstate Highways $\ldots \ldots \ldots \ldots \ldots \ldots \ldots \ldots \ldots \ldots \ldots \ldots$

E2.2 Normal Probability P1ot for City Group " $A$ " and Region "n" of Urban Principal Arterial .......................

E2.3 Normal Probability P1ot for City Group "C" of

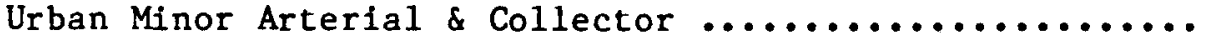

E3.l Residual Plot against Predicted value for North Region of Urban Interstate Highways

E3.2 Residual Plot against Predicted value for City Group "A" and Region "n" of Urban Principal Arterial ..............

E3.3 Residual Plot against Predicted value for City Group "C"

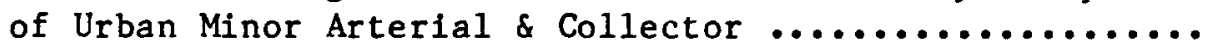

E4.1 Residual Plot against LAADT for North Region of

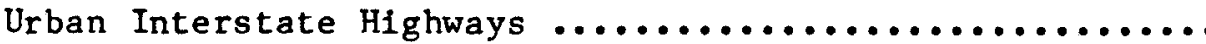

E4.2 Residual P1ot against RAADT for North Region of Urban Interstate Highways

E4.3 Residual P1ot against LAADT for City Group " $A$ " and Region " $n$ " of Urban Principal Arterial ............

E4.4 Residual Plot against RAADT for City Group "A" and Region "n" of Urban Principal Arterial

E4.5 Residual Plot against LAADT for City Group "C" of Urban Minor Arterial \& Collector ..................

E4.6 Residual Plot against RAADT for City Group "C" of Urban Minor Arterial \& Collector 
Appendix

Figure

Page

E5.1 P1ot of Observed against Predicted AADT from Lagged Regression for North Regional Model of Urban Interstate

Highways ..................................

E5.2 Plot of Observed against Predicted AADT from Lagged

Regression for City Group " $A$ " and Region " $n$ " of

Urban Principal Arterial ........................

576

E5.3 P1ot of Observed against Predicted AADT from Lagged

Regression for City Group "C" of Urban Minor Arterial

\& Collector ..................................

E6.1 Scatterplot of Change in Observed vs. Change in Predicted AADT from Lagged Model for North Region of Urban

Interstate Highways ..........................

578

E6.2 Scatterplot of Change in Observed vs. Change in Predicted AADT from Lagged Model for City Group " $A$ " and Region " $n$ "

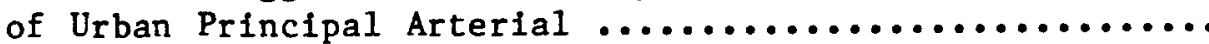

E6.3 Scatterplot of Change in Observed vs. Change in Predicted AADT from Lagged Model for City Group " $\mathrm{C}$ " of Urban Minor

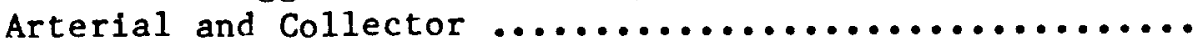

F1.1 Normal Probability Plot of Residuals from $\operatorname{ARIMA}(0,1,0)$

Forecasts of Franklin County Vehicle Registration .......

F1.2 Normal Probability Plot of Residuals from $\operatorname{ARIMA}(1,1,0)$ Forecasts of Tippecanoe County Vehicle Registration ......

F1.3 Normal Probability Plot of Residuals from $\operatorname{ARIMA}(1,1,0)$ Forecasts of White County Vehicle Registration ..........

F1.4 Normal Probability Plot of Residuals from Autoregressive Forecasts of Franklin County Vehicle Registration ........

F1.5 Normal Probability Plot of Residuals from Autoregressive Forecasts of Tippecanoe County Vehicle Registration ......

F1.6 Normal Probability Plot of Residuals from Autoregressive Forecasts of White County Vehicle Registration ..........

F2.1 Residual P1ot against Time from $\operatorname{ARIMA}(0,1,0)$ Forecasts of Franklin County Vehicle Registration .......

F2.2 Residual P1ot against Time from $\operatorname{ARIMA}(1,1,0)$ Forecasts of Tippecanoe County Vehicle Registration ...... 
Appendix

Figure

Page

F2.3 Residual P1ot against Time from $\operatorname{ARIMA}(1,1,0)$

Forecasts of White County Vehicle Registration ..........

F2.4 Residual P1ot against Time from Autoregressive

Forecasts of Franklin County Vehicle Registration ........

F2.5 Residual P1ot against Time from Autoregressive

Forecasts of Tippecanoe County Vehicle Registration ......

F2.6 Residual P1ot against Time from Autoregressive

Forecasts of White County Vehicle Registration ............

F3.1 Plot of Observed values against Autoregressive

Forecasts of Franklin County Vehicle Registration ....... 640

F3.2 P1ot of Observed values against Box-Jenkin

Forecasts of Franklin County Vehicle Registration ........

F3.3 P1ot of Observed values against Combined

Forecasts of Franklin County Vehicle Registration ........

F3.4 Plot of Observed values against Autoregressive

Forecasts of Tippecanoe County Vehicle Registration ......

F3.5 P1ot of Observed values against Box-Jenkin

Forecasts of Tippecanoe County Vehicle Registration ...... 644

F3.6 P1ot of Observed values against Combined

Forecasts of Tippecanoe County Vehicle Registration ...... 645

F4.1 Observed values and Autoregressive Forecasts of

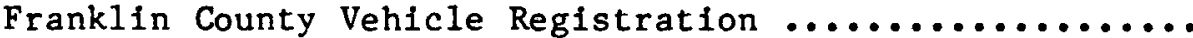

F4.2 Observed values and Autoregressive Forecasts of

Tippecanoe County Vehicle Registration ................

F4.3 Observed values and Box-Jenkin Forecasts of

Franklin County Vehicle Registration .................. 648

F4.4 Observed values and Box-Jenkin Forecasts of

Tippecanoe County Vehicle Registration ................ 649

F4.5 Observed values and Combined Forecasts of

Franklin County Vehicle Registration ................ 650

F4.6 Observed values and Combined Forecasts of

Tippecanoe County Vehicle Registration 
CHAPTER 1

INTRODUCTION

\subsection{Introduction}

Among the most important factors in public investment decisions are the projected demand for an existing or proposed highway factlity. The pattern of traffic growth and projected traffic volumes have been recognized as prime factors in most analyses of highway projects. Developing future traffic estimates is not an exact science, dependent as it is on so many hard-to-predict varlables. The traffic growth factor has a significant effect on highway investment decisions pertainIng to increasing the capacity of existing highways and the construction of new facilities when limited funds are available. Traffic forecasting procedures must be reasonably easy and economical to carry out, be sensitive to a wide range of policy issues and alternatives, and produce information useful to decision-makers in a form that does not require extensive training to understand.

Estimates of future traffic can be arrived at by two very different methods: projections and forecasts. Projections have been used for years and are based on a historfcal record of the desired data 1tem. Trend lines drawn through prior year data observations are 
extrapolated to the target year. In some cases, these extrapolated trends are modified by the analyst based on his experience and knowledge of the route, state or region. Whereas with projections we are dealing only with the traffic data, forecasting techniques are concerned with predicting the future values of economic and other measures or Indicators of person and vehicle travel. In forecasting techniques, a relationship between traffic and assoctated factor(s) is established. The terms prediction and forecasting have been used interchangeably in this dissertation, 1rrespective of their inherent definitions.

A clear distinction should be made about the nature of traffic forecasting methodologies. They are divided into two separate groups: (1) Those that address the forecasting problem as a network analysis, based on the traditional four-step process that requires large amounts of data and sophisticated computer resources, while not guaranteeing forecasts that are appreciably superior to less detalled methods, and (2) the simple, easy-to-use forecasts on a road-by-road basis that fulfill the particular needs of the local highway departments.

\subsection{Background}

Traffic data are essential in nearly every step of the planning process. In highway investment (major maintenance, reconstruction or new construction), a reliable estimate of future trafflc volume is a key element. The design of roads and analysis of alternative highway projects are dependent on these forecasts. 
Traffic forecasts can be prepared with a varlety of approaches, depending on whether the forecast refers to an urban or rural area. In urban areas, forecasts are generally based on the four-step (trip generation model, trip distribution model, modal split model and traffic assignment model) travel-simulation process. In these situations, travel on the road network is an output of the assignment process. Most large metropolitan areas have developed and 1mplemented a fairly sophisticated set of computer-based travel simulation models based on the traditional four-step process.

In rural areas, when assignment-based models do not exist or are not practical to apply, traffic estimates are generally made by expanding present traffic into the future based on projections of population, employment, vehicle registration, land-use data, or other parameters [Hazen 1982, Memmott et al. 1981, Neveu 1982]. A fam11y of models for use in forecasting average annual dafly traffic (AADT) volumes on the following classes of rural highway segments in the Indiana state and Interstate system has been developed [Saha 1986, Fricker \& Saha 1987]:

- Rural Interstate

- Rural Principal Arterial

- Rural Minor Arterial

- Rural Major Collector

These models were designed to capture the relationship between AADT and the factors (economic, demographic, etc.) that influence traffic volumes, using input data that are reasonably avallable and model forms that are flexible and easy to use. While a forecasting 
tool can never be validated until the forecast or horizon year becomes the current year, the models that were developed appear to be (a) on a sounder theoretical and statistical basis than those previously found In the 11terature and (b) in conformity with the goals of the rural study.

It is likely that the rural factors and coefficient values are not applicable to urban segments. Two principal reasons for this probable lack of transferabllity stand out:

1. Traffic on urban highway segments is more a function of local conditions and factors than the typical rural segment.

2. Traffic on urban segments is much more sensitive to changes in other links in the urban road network.

The urban traffic forecast procedures reviewed to date can be grouped under two general categories: trend forecasting and theoretical forecasting. Trend forecasting can be subdivided into two procedural types: mechanical and analytical. The mechanical methods assume that future travel patterns will be a continuation of past experience, allowing simple projections of past trends forward. The analytical method recognizes the fact that traffic growth is a product, not only of time, but of certain varying internal forces and external stimuli that operate to affect the rate of growth of each contributing factor to the composite growth. Theoretical forecasting procedures can fall Into any of the following categories: (a) traditional aggregate 4step traffic simulation models, (b) disaggregate stochastic behavior models, and (c) econometric models.

Traffic forecasting is the backbone of the transportation planning process. Urban areas approaching the 50,000 population level 
face the formidable task of Initiating a comprehensive transportation planning process. The following statement in regards to the results of the investigation was made in the executive summary and introduction of the "Framework for the Transferabllity of Trlp Generation Parameters of Small Urban Areas" [Mahmassan1 \& Sinha 1978]:

"Initiating a continuing, comprehensive and coordinated transportation planning process is a considerable task that faces small metropolitan areas as their population reaches 50,000. These areas usually lack the technical know-how as well as the financial capabilities needed to use the battery of computer-oriented planning models more commonly used in large metropolitian areas. In most cases such sophistication is not needed because the problems faced by smaller areas are different in nature and in complexity from those in larger areas."

In early 1987, Purdue University and the Program Development Division of INDOT recognized the need for simplified procedures for small- and medium-sized urban areas, and therefore, organlzed a research project to develop a procedure to forecast traffic on state highway systems for small- and medium-sized urban areas of Indiana. The planning division of INDOT was aware of the past resources expended in the transportation planning process for many of the urbanized areas in Indiana and therefore realized that the results derived from such a project could be very beneficial.

\subsection{Purpose and Scope of the Research}

The goal of this research study is to develop a method of establishing a procedure to forecast traffic for the Interstate and smal1to mid-size urban state highways that can be used by the Indiana Department of Transportation (INDOT). The research has been carried out by the Joint Highway Research Project (JHRP) at Purdue University 
with the sponsorship of the Federal Highway Administration (FHWA) and INDOT .

The primary purpose of this research is to develop model(s) to forecast traffic for use by personnel responsible in planning, design and decision making. A secondary purpose of the proposed research is to advance the body of knowledge regarding traffic forecasts. The model bullding approach of traffic forecasts has not been confined to the State of Indiana. A related purpose of the proposed research is to establish the groundwork for follow-up studies in other states.

This study has met the ongoing needs of INDOT for a reliable method of estimating future traffic on individual routes, as an aid to the planning process and in implementing the Highway Improvement Program. The proposed method is reliable, with modest data and computational requirements, and is well-documented and flexible. The model developed in this study is simple to apply. A hand calculator is adequate for the application of the model. But, the use of spreadsheet and the Interactive programming will improve the efficiency of model application.

Some common criticisms of the models in transportation planning studies are [Richards \& Ben-akiva 1975]:

1. The models are too time-consuming, complex and expensive to operate.

2. Too much thought is given to the model bullding and too little to the elements that are really important in the practical use or implementation.

3. The users (as opposed to the model bullders) do not always understand the models. 
4. The researchers usually concentrate on bullding the model, but seldom devote enough effort to model implementation and validation.

5. The models are too data-hungry.

One of the purposes of this study is to develop a model less vulnerable to the above criticisms.

The primary focus of this study is the design and testing of the simple, fast methods to forecast traffic volumes. This study detalls the development of such a procedure in order to update the method in future years. This study examines previous efforts almed at forecasting traffic, particularly by using simple and efficient methods, describes the chosen methodology, and evaluates the results of the investigation.

The scope of this study is limited in some 1mportant ways. First, although studies from other states and countries have been reviewed and, when appropriate, their findings have been incorporated Into the present study, the data bases of this study were created for Indiana. The development of growth factor models for urban state highways in Indiana has remained the primary focus of this research. 


\subsection{Study Object1ve}

In the overall investigation, the objective will be to make the model(s) as simple as possible. The overall objective of the proposed study is to provide, for the urban highways in Indiana for which INDOT has responsibility, a means of predicting future traffic volumes that are :

- rellable,

- we11-documented,

- easy-to-use, and

- based on input factors for which clear relationships and usable forecasts will continue to exist.

The goals leading to the above-mentioned general technical objectives are:

a Establish a database of maximum practical accuracy, consistency, and size.

b. Identify the variables/factors that best describe the response variable. Both judgment and statistical analyses will play a role in this selection process.

c. Establish causal relationships between traffic volumes on urban highways and input factors of a demographic, economic, and "structural" nature, using statistical analysis techniques.

d. Test and validate the model(s), and develop guidelines for use of the model(s).

\subsection{Study Organization}

Th1s study consists of eleven chapters and seven appendices. Chapter 2 reviews the literature on forecasting traffic that uses simple and easy-to-use methods and looks at some of the current procedures practiced by some state highway departments. 
Chapter 3 presents the framework of overall analysis for constructing statistical models. This chapter primarily explains only the conceptual ideas behind overall analysis. Chapter 4 describes the variables in the data bases, the data formats and their use in the analyses.

Some statistical analyses of the data gathered in Chapter 4 are provided in Chapter 5. Some useful statistics of the data bases, their formats, and groups are being compared. The candidate models for each group are selected from the best data base and format. Chapter 6 contains all the results of influential analysis, significance, collinearity, and normality statistics of the candidate models identified in Chapter 5. Model statistics are presented as pre and post influence analysis. Sample residual and normality plots are shown for some selected models. Final regression and its variant elasticity model are presented in this chapter.

Chapter 7 contalns the results of of the analysis done for the principal component based regression model. Some key statistics are compared with models of Chapter 6 . Chapter 8 reports another alternative model - the lagged traffic model. The model statistics and the sample graphical plots are also shown in this chapter, as with the models in Chapters 6 and 7.

Chapter 9 discusses and presents the results of forecasts of Input variables for which no forecast is avallable from outside sources. Two efficlent time series analysis techniques to forecast vehicle registration and the "rat1o" method to forecast city popula- 
tion have been used in this chapter.

Chapter 10 presents the results of some performance measuring criterion to evaluate (a) the traffic models developed in earlier chapters and (b) the forecasts found by combining the efficient models. A monitoring device has been proposed to detect the need for calibration in future. Chapter 11 gives the conclusions of the research as well as guidelines to implement the models developed. This chapter also provides probable problems, limitations and recommendations for further research in areas of traffic forecasting that can use less complicated models.

Some sample data tables developed and analyzed in Chapters 5 to 8 are presented in Appendix A. The complete data bases used in this study are saved on several floppy diskettes for updating the data base with recent information and for future modification of the model, if desired. Appendix B presents the land areas of cities and counties and data for functional class mileage to complete the data bases in Appendix A. A form used for manual retrieval of traffic data is shown in Table B4 of Appendix B.

Appendix C presents the best models without any inconsistency in the regression coefficients for different numbers of variables for each data group, format and data base. The statistics of these models are used to do sensitivity analysis with respect to different data bases and formats. Appendix $D$ shows the elgenvectors of the principal components used in the models, standard deviation and mean of the original variables, and sample graphical plots for principal component 
regression. Appendix E contains the results of regression and forecast measures of the lagged model in Chapter 8 with partial data. Sample graphical plots of residual and normality for some selected models are also shown in Appendix E.

Appendix F presents (1) models, forecasts and sample plots of vehicle registration (VR) from the Box-Jenkins and the autoregressive models, (11) combined VR forecasts and sample plots, (111) sample plots of residuals and normality, and (iv) city population forecasts. Appendix G presents the 90- and 95-percent confidence limits of the forecast measures for combined traffic forecasts and for the forecasts from each of the constituent models. 
CHAPTER 2

\section{LITERATURE REVIEW}

\subsection{Introduction}

It is the goal of this research to sort through and select the most relevant literature where an abundance presents itself and to add to the store of knowledge where a scarcity exists. The literature of particular interest for this study is that dealing with traffic volume and the background factors that influence it. A review of the literature on traffic forecasting, with particular emphasis on simplified procedures is presented in this chapter. A review of the literature reveals that limited research has been accomplished on the topic of forecasting traffic for smal1- and medium-sized urban areas. Some Ideas from this review study have been incorporated into the present study.

\subsection{Transportation Demand Models}

The process of relating the demand for transportation to the socioeconomic activities that generate it is known as transportation demand analysis [Kanafani 1983]. The results of this analysis are relationships (often as models) between measures of activity and 
measures of transport demand. Such relationships are often referred to as transportation demand models. Although demand analysis is distinct from traffic forecasting, one can use the results of demand analysis to forecast future traffic volumes. The demand models provide major input into the forecasting process. It should be recognized that there are limitations to demand models as forecasting tools. The analyst might well find it reasonable to use models of demand analysis for short-term forecasting to study the impacts of changes in the demand and supply environments of transportation. But as the time frame of forecasting becomes longer, it is unlikely that the same models will continue to be of as much relevance.

\subsection{Urban Traffic Simulation Models}

The traditional travel simulation model is avallable in many urban areas. The system was originally developed in the early 1960's and has been widely used in many urban areas ever since. The highly sophisticated simulation procedures are based on a set of procedures to predict urban area trip generation, trip distribution and trip assignment. It is not the purpose of this section to give a detailed description of the procedures, which would be lengthy and time consum1ng. The principles involved in the system have been described in many urban transportation planning textbooks, such as Hutchinson [1974], Stopher and Meyburg [1975], and Meyer and Miller [1984]. However, this section is intended to introduce an overview of that sophisticated process. 
The network-wide travel simulation system requires extensive Inputs describing urban area socioeconomic, travel, and highway network characteristics. In general terms, the process consists of developing a trip file describing the rate of trip making in each of the analysis zones of the study area and then distributing those trips between the zones using some form of attraction-production model (for Instance, the gravity model or intervening opportunity mode1). Finally, trip interchanges are assigned to the network based on some preferred 1terative technique - for example, minimum path (al1-ornothing), equilibrium or stochastic assignment. Many software packages based on this modeling concept are avallable on the market.

Prem [1987] had discussed the following travel forecasting tools In terms of their general description, primary task, features and constraints.

1. Urban Transportation Planning Process (UTPS)

2. Manual Quick Response System (QRS)

3. Microcomputer Assignment Models

4. Simplified Project Forecasting (SPF)

5. Traffic Simulation Models

The scope of this study does not allow the researchers to apply these techniques in their forecasts. But, avallable forecasts from any of the travel simulation model can be easily compared with the forecasts produced by this study. 
2.4 ITE Survey Results of State Traff1c Forecasting Procedures

\begin{abstract}
A summary of the state of the art of procedures used to forecast traffic by states and provinces was addressed by ITE Technical Committee 6F-41 [ITE, 1990]. The following results are summarized in that report.
\end{abstract}

- Traffic forecasting remains one of the most important functions of state and provincial planning staffs. Main department offices are primarily responsible for preparing both urban and rural forecasts. Decentralization of the forecasting task to regional groups has not been undertaken, perhaps because such activity requires a specialized set of skills not widely available in regional groups.

- Traffic forecasting remains a combination of art and science requiring the user's judgment and experience. In developing forecasting studies, the following points are stressed [ITE, 1990]:

a. Review of Traffic Count History - To develop an understanding of the pattern of growth around the project site.

b. Focus on Inputs -- GIGO ("Garbage In Garbage Out"). A model is only as valid as the data used to develop the profections.

c. Balance Effort with Project Scale - A complex and expensive forecasting method may be unnecessary for a project of limited scope and 1mpact.

d. Select an Appropriate Level of Accuracy - The purpose of the project will determine the accuracy level desired. 
e. Use Several Methods -- If possible, it may be highly desirable to apply several forecasting methods on the same project. This sensitivity analysis will assist the planner in establishing the range of potential error in a particular forecast.

- Traffic forecasting requires the application of both technical procedures and practical experience. A wide range of procedures have been developed and applied. In general, the methods that have been used are related to the project needs, level of investment, and the range of acceptable error.

- The traditional four-step urban modeling procedure is used in many urban areas. However, a number of states simply extrapolate current trends in traffic counts or use growth factor procedures. In rural areas, simple extrapolation and growth factor procedures are used extensively.

- Certain standard techniques (i.e. extrapolation, time series analysis, elasticity-based, site specific, functional classification based, multivariate statistical analysis, etc.) are 11kely to predominate. But, very few written documents on these techniques have been published to date. Very few studies are known to the researchers in this study about the quality of the results of the methods practiced by different state highway agencies.

- Many states remain actively engaged in 1mproving forecasting procedures and adopting microcomputer techniques. In addition, improvIng truck forecasting procedures appears to be a priority for many states. 
2.5 Adjustments for Factors not Considered Explicitly in the Model

Many background (socioeconomic) factors may seem to influence travel on roadways. A discussion on (1) how the background factors affect traffic and (2) the role of the background factors can be found In Chapter 2 of Indlana's rural study [Fricker \& Saha 1987]. But, most forecasts developed in the past did not include consideration of energy price and supply or improvements in auto fuel economy. Moreover, forecasts do not of ten recognize the transformations that have occurred in travel-related variables such as household size, auto size, inflation, employment, population age, and settlement patterns. But not all of those factors entered in the model equation because of (a) inadequate information in the past, (b) forecasts into the future are either uncertain or not reliable with a scanty data base, and (c) the factors not significant in the model bullding process. Additional research is required in this field to develop methods that will adjust traffic for the factors not considered in model. Chapter 9 of the New York State Traffic Forecasting Guide [Trombly \& Ouderkirk 1986] presented the tabular values to adjust traffic forecasts. The results are based on some empirical relationships between travel and background factors in the past. So, the results should be applied cautlously. In the absence of any other good source on adjustments or further research, users of the this study may adjust the forecasts if they feel the future conditions are warranted to made such adjustments. Again, a word of caution mentioned in the Guide [Trombly \& Ouderkirk 1986] is as follows:

"The adjustment procedures were developed using the best 
Information avallable at the time. These data come primarily from the $1970^{\circ} \mathrm{s}$ and earlier. New factors not accounted for in these procedures may influence travel behavior in the future. Two such issues are the economic conditions in the early $1980^{\circ} \mathrm{s}$, and the decontrol of crude ofl prices in early 1981. Finally, the user should be aware of these shortcomings, and is responsible for altering the procedure to more accurately reflect the particular situation."

In general, forecast can not be done without error. Those errors can be attributed to (1) model specification (1.e., wrong or incomplete model terms), (ii) error in the input varlables (1.e., a change in the assumptions regarding growth of an input variable), or (111) calibration errors (i.e., estimation of model parameters). The adjustments presented in the New York State Traffic Forecasting Guide are intended to offset the errors related to model specification and for projection of model input variables.

\subsection{Past Research}

Traffic forecasting in urban areas has been extensively explored and the forecasting methodologies, mainly based on sophisticated computer modeling programs, are highly advanced. Methods of traffic forecasting were advanced during the mid-sixties when statewide transportation studies were conducted by many states to fulfill the need for developing final statewide transportation plans. Traffic forecasting was a basic input for these studies. However, very little work has been published on developing and testing the simple methods, where sophisticated computer modeling is not possible because of time, cost, technical expertise. Standardized methodologies for nationwide use have not been established for small- to medium-sized urban areas and state authorities develop their own procedures to 
accommodate their needs. A main reason for the development of different procedures by different state authorities might be that, since the development of traffic projections is not an exact science, planners base their methods on different conceptual models and thus use different procedures to reduce the uncertainty associated with their projections.

The varlous state departments of highways developed their own methods to forecast traffic when the 4-step modeling process did not fit the purpose and scope of their study. Very few of these studies are well documented. Some of these studies were summarized in Chapter 1 of Indiana's rural study [Fricker \& Saha 1987]. Independent variables used by the model of Indiana's study were population, households, vehicle registrations, employment, and fuel price. Aggregate models represented four categorles of rural highways -Interstates, Principal Arterial, Minor Arterial, and Major Collector -- and resulted in reasonably accurate estimates of future volumes. Disaggregate models were calibrated for specific continuous traffic counting stations and the accuracy of prediction was very good. The $\mathrm{R}^{2}$-values for Aggregate and Disaggregate models were 65.8-83.7 percent and 51.5-97.8 percent, respectively. Besides, the varlables in the models were statistically significant and no other variables were found to provide additional predictive power to the models. The vartables in the models were selected based on statistical analysis as well as on subjective judgment. 


\subsubsection{Site Traffic Estimate from Volume Ratio Forecast}

The Kentucky study [Deacon et al. 1987] to forecast highway traffic volumes involved a two-step modeling process. In the first step, a linear regression relates average travel to personal income, fuel price, and total miles of streets and highways. In the second step, cross-tabulation models relate site-specific traffic growth to statewide ADT. The cross-tabulation refers to highway functional classification, rural/urban location, county population growth, SMSA/non-SMSA designation, and volume level.

The forecast of future traffic volumes at a specific location requires two types of models, one for forecasting average travel (AADT) on all Kentucky highways and the second for forecasting volume ratio (ratio of project site AADT to statewide AADT) and 1ts growth at locations simflar to the one under investigation. Forecasts of future year statewide AADT do not vary from project to project. Then, the forecast of site AADI is simply the product of the volume ratio and statewide traffic forecast.

\subsubsection{Project-Leve1 Traffic Forecasting}

The Simplified Project Forecasting (SPF) [COMSIS 1985, Ismart and Schoener 1987] procedure was developed by COMSIS as a quickresponse alternative to the traditional 4-step simulation process. The SPF procedure is designed to produce link-specific traffic forecasts, simllar to the 4-step precess, with modest data requirements. It produces growth factors to be applied to a base traffic volume 
estimate, rather than producing the absolute traffic volume as its primary output. A growth factor is produced for any facility in question by running the SPF model system for the base year and target year and calculating the growth factor as the ratio of the target year to base year traffic volume estimates. This growth factor incorporates the effects of changes both in land use and in the transportation network. This approach parallels the traditional modeling process used by most transportation agencies. While it has the Important benefits of familiarity, understandability, and comparabil1ty to these existing processes, the data requirements are modest in comparison and most of the operations are transparent to the user.

A typical application of this procedure is when traffic congestion develops on existing urban arterial streets in response to rapid increases in land development. This type of application is becoming prevalent in the fast-growing suburbs of major metropolitan areas. In such an environment, SPF could be used to obtain a quick, yet technically defensible, estimate of traffic for determining the nature of improvement needed to solve the problem. To apply the microcomputer SPF software in this situation, the user supplies as Input the population and employment characterfstics for the base year and a forecast year for geographical areas surrounding the facility. In addition, coordinates are provided for each geographical area and the facility being evaluated. These coordinates are used by the program to reformat the population and employment data. Default values are also provided in the computer software for link speeds and other factors, some of which may be changed by the user. 


\subsection{AADT Estimate from Short-Term Counts}

The traffic forecasting methods developed in this study need the apply of base year or lag year traffic estimation. Irrespective of these different name (base or lag), one need to obtain or estimate traffic at current or any other chosen year designated as base or lag year. Base or lag year may be different from the current year, but 1t is the year for which most recent data needed by the model are available. If AADT at base or lag year is not avaflable, then the method described in this section or the one practiced by INDOT can be used to estimate AADT.

INDOT has been developed factors to apply to short-term coverage counts to estimate AADT at a site. The factoring procedure (see Section 2.6 [Fricker \& Saha, 1987]) of INDOT follows the recommendation of the Traffic Monitoring Guide of Federal Highway Administration [FHWA 1970]. Those factors are based on the seasonal variation of the traffic, with similar variation patterns grouped according to location (rural/urban) and type of facilities. The data from the continuous count stations located throughout the state are used to develop the factors. These factors can be easily procured by contacting the Division of Program Development, INDOT.

\subsection{Traffic Diversion and Reallocation}

The traffic models developed in this chapter do not address the issue of increase in capacity or decrease in travel time that resulted from some roadway improvements. There will be a need (1) to 
reallocate the traffic in a corridor between the parallel facilities to simulate the actual operating conditions, and (2) to modify traffic flow on a highway segment to reflect the travel time 1mprovements. The above issues are more related to an impact study than a volume forecasting procedure. The scope of this study does not allow the researchers to incorporate such speclalized techniques directly. Network-based simulation models would be more appropriate to consider those 1ssues. The simplified techniques found in the literature [NCHRP 187 and 255, Trombly and Ouderkirk 1986] can be applied to the results of this study, if the user decides new improved roads or major development may affect the highway segment for which a forecast is destred.

\subsection{Chapter Summary}

The objective of this chapter is to provide a brief review of the literature on traffic forecasting, with an emphasis on simplified procedures. Sources to adjust the traffic forecast for the factors not considered explicitly in the models and to refine the forecast for parallel construction and improvement of travel time are presented. Results from a nationwide survey on "Traffic Forecasting on State Highway Segments" by an ITE technical counctl committee show the status of the procedures practiced by states and provinces. The commonly-clted background factors for traffic forecasting have been Identified in this chapter. Some of those factors, for which data are available, are used in this research. 
CHAPTER 3

PROBLEM STATEMENT AND EVALUATION FRAMEWORK

\subsection{Introduction}

Much of the work in forecasting traffic on urban highways was done in the 1960s and 1970s, and was based on the the 4-step sequential modeling process. Few efforts to develop and test simple methods have been found in literature. It is this vold that has received the most attention during this research. In this research, an effort has been made to develop models to predict future traffic on Interstate Highways and on state highways in small- to medium-sized urban areas. A previous study [Fricker \& Saha 1987] developed a procedure to forecast traffic on rural state highways in Indiana.

The current method at the Indiana Department of Transportation (INDOT) to forecast future traffic on urban state highways is based on 20-year growth factors for each of Indiana's 92 counties. Recognizing that the current set of traffic growth factors are outdated, overly simplistic, and lacking the documentation necessary to update them, the proposed method will provide a means of predicting future traffic volumes that is reliable, well-documented, flexible, and based on 
Input factors for which clear relationships and usable forecasts will continue to exist.

A clear distinction should be made about the nature of traffic forecasting methodologies. They are divided into two separate groups: (1) Those that address the forecasting problem as a network analysis, based on the traditional four-step process that requires enormous amounts of data and sophisticated computer resources, while not guaranteelng forecasts that are appreciably superior to less detalled methods, and (2) the simple, easy-to-use forecasts on a road-to-road basis that fulfill the particular needs of the local highway departments.

The proposed method seeks a suitable "middle ground" - a method that provides a reliable forecast with modest data and computational requirements. The models developed should be relatively simple to use and be updated without difficulty. This study will meet the continuous needs of INDOT for a reliable method of estimating future traffic on Individual routes as an aid to the planning process and in implementIng the Highway Improvement Program. In this study, several methods have been examined and developed to determine the sensitivity of the forecasts and to improve the rellability of the forecasts by combining the forecasts.

The study by Morf and Houska [1958] leads to the conclusion that the characteristic "type of service" has a remarkable effect on traffic growth rates. Highways with the greatest percentage of Interurban or interregional service generally had the largest 
Increases in travel. Roads that serve largely urban-to-rural or rural-to-urban travel had the smallest increases. The results of the Morf and Houska study suggest different traffic forecasting models for different functional classes of highway. The functional classification of highway are interstates (representing interurban and interregional service), principal arterials (representing rural-to-urban service), and minor arterials and major collectors (representing ruralto-rural and urban-to-urban service). By using functional class as the determinant, the three major road types - interstate (rural \& urban), urban principal arterial, and urban minor arterial \& collector - were identified in this study.

The principles of the traffic forecasting methods developed in this dissertation can be grouped into the following main phases:

1. Identification and selection of the underlying factors (independent variables) to be taken into account in traffic (dependent varfable) forecasting.

2. Determination of the type of functional relationship existing between the dependent variable and the independent variables.

3. Developing and testing the databases, and filtering the data (1f necessary).

4. Empirical testing of the mathematical expression for the relationship between the dependent and independent variables, including evaluation of coefficients and validation of model assumptions.

5. Forecast of future values of the independent varlables and subsequent derfvation of traffic forecast.

6. Comparative evaluation of the level of accuracy between the alternative models and combination of the efficient models.

Statistical analyses in Chapters 5 to 8 suggest different models for each of the three highway categories. Except for the elasticity 
model, only conceptual descriptions of the models developed and examined in this study and some key point of analysis are presented in the following sections. A detalled description of the models (other than the elasticity model) and the process by which they were developed can be found in later chapters.

\subsection{Data Base and Format}

The success of a forecasting method not only depends on the model specification, but also on the rellability of the data and the way the data are used in the model. Avallability of past data and projections of future values were also important considerations in selecting data for model development. The traffic volume data will be the dependent variable. The several independent variables will reflect condition at both the local and state level, and the type of highways.

A difference between this study and the rural study [Fricker \& Saha 1987] is the primary source of traffic data. The rural study was based on traffic data from continuous count stations. This urban study has used traffic data primarily from coverage count stations, with a few traffic counts from continuous count stations at urban locations. It is expected that 60 telemetering stations, installed in the mid 1980s, w11l provide adequate data in the future. The large number of traffic volume observations from the coverage count stations are expected to provide better statistical results and more stability in model parameters. In addition, having more data offers the opportunity to test different data structures. One unique aspect of this study is the successful use of coverage count station traffic data and 
the evaluation of the alternative data structures.

After some preliminary analysis, the following categorical variables have been chosen to determine the layout of the analysis: (1) city size, (2) geographical region, and (3) extent of roadway mileage. These varlables will be used to stratify or group the data base into more homogeneous data groups so that more stable results w1ll be achieved. The way in which the varlables are entered in the analysis have depicted in Figures 5.1 (for interstate highways) and 5.2 (for noninterstate highways) in Chapter 5. The two data bases and four data formats will be examined to select the best suitable data base and its format based on the sensitivity of the statistical analysis and engineering judgment.

\subsection{Elasticity Based Mode1}

A varfety of forecasting models were examined. The simplest one was AADT (Annual Average Daily Traffic) belng directly proportional to the socioeconomic factors, such as population or number of households. It was felt that the explanatory power of such a model would be too low to provide reasonably accurate forecasts. This procedure also carries with it a problem inherent in all regression models: the problem of forecasting outside the range of predictor variables in which it was callbrated.

An elast1city-based model [Fricker \& Saha 1987] was selected and used to relate future year AADT to present year AADT with a number of background factors. The general form of the model is as follows: 


$$
\operatorname{AADT}_{f}=\operatorname{AADT}_{b}\left[1.0+\sum_{j=1}^{n} e_{j}\left(x_{j, f}-x_{j, b}\right) / x_{j, b}\right]
$$

or, after rearrangement,

$$
\frac{\operatorname{AADT}_{f}-\mathrm{AADT}_{b}}{\mathrm{AADT}_{b}}=\sum_{j=1}^{n} e_{j}\left[\frac{x_{j, f}-x_{j, b}}{x_{j, b}}\right]
$$

where,

$$
\begin{aligned}
& \operatorname{AADT}_{f}=\text { AADT in future year, } \\
& \text { AADT }_{b}=\text { AADT in base year, } \\
& x_{j, f}=\text { value of variable } x_{j} \text { in the future year, } \\
& x_{j, b}=\text { value of variable } x_{j} \text { in the base year, } \\
& e_{j} \quad=\text { elasticity of AADT with respect to } x_{j}, \\
& n \quad=\text { number of associated variables. }
\end{aligned}
$$

The elasticlty-based model was preferred for several reasons. The most important reason was that it was believed that the range of volumes over which the model would be applied would be much greater than that used in developing the model, making a simple linear regression model that relates AADT to the background factors directly inappropriate. A proof for this statement can be found during model evaluation in Chapter 10. Second, the use of present year AADT to estimate future year AADT (as a sort of pivot point) would lessen the problem of nonresident (or non-site traffic). Also, the elasticity portion of the model calculates a growth factor directly. (See the right hand side of Equation 3.2) 
The elasticities and the approprlate background factors are derived from a linear equation that relates AADT to a varlety of the factors in Table 4.3. It can be shown mathematically that, given an equation of the form

$$
Y_{1}=a+\sum_{j=1}^{n} a_{j} X_{1 j}
$$

where,

$$
\begin{aligned}
& Y_{1}=\text { value of dependent variable at } 1 \text { th observation; } \\
& 1=1, \ldots, n_{1} \\
& x_{i j}=\text { value of } j \text { th independent variable at ith observation; } \\
& j=1, \ldots, n, \\
& a=\text { constant term, } \\
& a_{j}=\text { regression coefficient for } j \text { th independent variable, } \\
& \mathrm{n}_{1}=\text { observation number, } \\
& \mathrm{n}=\text { number of independent variables, }
\end{aligned}
$$

elasticity measures can be estimated by:

$$
e_{f}=a_{f}\left[\frac{\bar{x}_{1 j}}{\bar{Y}_{1}}\right]
$$

where,

$$
\begin{aligned}
& e_{j}=\text { elasticity of AADT with respect to independent variable } x_{j}, \\
& \bar{X}_{1 j}=\text { overall mean of the } j \text { th independent variable, } \\
& \bar{Y}_{1}=\text { overall mean value of dependent variable, }
\end{aligned}
$$


$a_{j}$ as defined below Equation (3.3).

Thus, using multiple linear regression, the background factors that best estimate AADT and their respective elasticities can be derived. The data for estimation of the background factors and elasticities came from a variety of sources. Detalls regarding the data are presented in the next chapter. Both the regression model (Equation 3.3) and the "elasticity model (Equation 3.1) are presented in Chapter 6.

\subsection{Principal Component Analysis (PCA) Based Model}

Multicollinearity with a large number of independent varlables in regression is not unusual. This study has nominated twelve predictor variables to explain variability in traffic because (1) the study did not want to overlook any variables that may be found significant and for which usable data are present for model building, and (ii) the forecasts of those variables are either available or can be generated with rellable methods. Although adequate safeguards have been taken to select the best regression equation in Chapter 6 , a complete elimination of collinearity is almost impossible without some advanced techniques. Principal component analysis reduces the dimension of the problem by using some independent factors from the original interrelated Independent variables. The PCA-based regression models are presented in Chapter 7. The main purpose of these models is to compare the statistics of these complex models to the regression and elasticity models developed in Chapter 6 . The use of PCA models in 
forecasting is very $11 \mathrm{mited}$ because forecasts of all independent var1ables used in the analysis w111 be required to permit computation of the principal components in the PCA models. The following two reasons justify the use of PCA models in forecasting:

1. A significant improvement in the model statistics in PCA model about the statistics in regression or elasticity model of Chapter 6.

2. The poor performance of the elasticity and regression models.

Otherwise, PCA models w1Il be used to assess the quality of the models in Chapter 6.

\subsection{Lag-AADT Model}

One of the primary purposes of this research is to develop reliable models for forecasting future traffic volume on state roads and streets of Indiana. Alternative models (for instance, Lag-AADT model) have been developed in this study for use in either urban or rural locations at the project level. The lag-AADT model is simple and Inexpensive because only the information on traffic data will be used to build and to use the model. To evaluate the performance of this model, the model will be fitted through a partial data base, which lacks the last year of AADT for each highway segment. The model with this partial data base will be used to forecast the last year of traffic data for each segment. The last year forecasts will then be compared to the observed AADT. The final model will be formed with the complete data base. The lag-AADT models developed in this study are presented in Chapter 8. The performance of the lag-AADT model, regression and elasticity model in Chapter 6, and PCA-based model in 
Chapter 7 (1f warranted) w111 be compared in Chapter 10.

\subsection{Time Serles and Ratio Method Forecasts}

The accurate and independent forecasts of input variables in models are important to get rellable estimates and forecasts of traffic. Forecasts from various sources will mainly be used as the input variables for the models in the study. In addition, some efficlent and rellable methods have been used to forecast the input variables for which no forecasts are avallable from any sources. Two time series analysis techniques (Box-Jenkins "ARIMA" and Autoregressive procedures) have been used to forecast vehicle registrations at the county and state levels. In summary, the Autoregressive Integrated Moving Average (ARIMA) procedure fits the model that allows an error term generated by an ARIMA process. The autoregressive process does multiple regression, which allows for a serially correlated error term generated by an autoregressive process.

All time series techniques are based upon the fundamental assumption that a past pattern will be repeated in the future. The methodology suggested by Box and Jenkins represents a systematic approach to modeling and forecasting discrete time series. There are two basic reasons why this methodology can lead to better forecasts than other statistical forecasting methods. First, using traditional approaches requires that the analyst use his or her own experience as a basis of model development. In the past, experienced analysts have developed methods for 1dentifying appropriate mathematical models. They generally relied on plots and indices that alded in Identifying the 
presence of trends and seasonal patterns. Based upon this analysis, a spectfic model would be defined. On the other hand, using the BoxJenkins technique, the analyst does not arbitrarily pick a specific model, but instead eliminates inappropriate models until he or she is left with the most suitable one. Second, the spectfic form of a given model which is to be used has traditionally been the result of a trial-and-error procedure. Moreover, a good deal of experience and Intuitive judgment on the part of the forecaster is often involved. Box-Jenkins [1976] procedure, however, presents a rational structured approach to the determination of a specific model.

A detailed description of modeling with time series analysis will be shown in Chapter 9. Only the input data have been used to forecast the series. Vehicle registration forecasts from the Box-Jenkins and autoregressive procedures will be compared, and then combined based on their performance. A simple but reliable method of population projection, the "ratio" method, will be used to forecast the city population. Section 9.3 presents this technique.

\subsection{Evaluation of Traffic Model}

In the absence of any universally accepted performance criteria, several measures w11l be used to compare the different models developed in this study. The description of the performance criterion will be provided in Chapter 10. Forecasts from the efficient models will be combined based on the performance of the models in forecasts. Finally, a monftoring device, also based on performance, will be developed to detect any need for model recalibration in the future. 


\subsection{Concluding Summary}

Travel in urban areas has been increasing due to changing land use, increase in human activity, and growth in vehicle ownership. There are numerous forces that shape the demand for traffic on highways. It is very difficult (to say the least) to accurately represent all interacting forces in a procedure to forecast traffic. The forecasting models and the procedures developed in this study should be used as general guidance. This study restricts its attention to reasonably simple procedures for highway travel demand forecasting.

It is felt that greater sensitivity of forecasts will be obtalned by developing alternative models and using varlables having a more direct interaction with the variables that create demand for highway traffic. However, there is a great deal of multi-collinearity between many potential decision-related variables. Adequate measures have taken in this dissertation to avoid any severe multicollinearity problem.

The procedures developed in this study are belleve to provide an Inexpensive, quick, and transparent technique for forecasting travel in small- and medium-slzed urban areas. The model is also suggested for use in cities in less developed and developing countries because of Its simplicity and low cost. 
CHAPTER 4

DATA BASES

\begin{abstract}
4.1 Introduction
In this chapter, a number of variables that have been used to create tables of input data will be discussed, along with the traffic data for which forecasts are desired. The methodology and quality of forecasts of some demographic and economic variables from our two principal sources will be discussed in this chapter. Advice on how to forecast values of the predictor variables for which forecasts are not available will be offered. The different forms of data tables for the different highway categories will also be discussed. These data tables are the input medium for statistical analysis. The main objective of this chapter is to describe the variables and the evolution of the data tables used in the analysis. These data tables could be modified when new counts and/or new census reports become avallable, in order to recalibrate and modify the developed traffic forecasting models for use in the future traffic.
\end{abstract}




\subsection{Forecasts of Demographic and Economic Variables}

The future-year values for many of the predictor variables used In this study were obtained from

a. Woods \& Poole Economics, Inc. and

b. the Indiana University Business Research Center.

No demographic and economic data at the city level (except population) have been found that could be usable in model formulation. However, there will be a need to forecast city populations, as well as vehicle registrations at the county and state level. The appropriate forecasting techniques (for example, smoothing and time series analysis) and/or some ad hoc methods (for example, the ratio method) will be used to obtain forecasts of those variables for which there is no forecast available from existing sources.

When comparing the population projections published by the IU Business Research Center and Woods \& Poole (W\&P), sometimes the W\&P values are higher than $\mathrm{IU}^{\prime} \mathrm{s}$, and sometimes they are lower. There is no consistent trend. IU used Cohort Analysis [Krueckeberg \& Silver 1974, Boyce 1985] on1y. W\&P began with Cohort Analysis, then modified the results with economic adjustment factors [W\&P Economics 1988], constrained by national, regional, and state forecasted totals (See Section 4.3 for a brief description). The regional model that produces the forecast component of this database is the product of extensive research by Woods \& Poole economists. It is frequently revised and 1mproved to reflect ongoing research by the professional staff, new computational techniques and new sources of regional economic and demographic information. Each year a new forecast is produced with 
the most current data avallable. The sophistication of the W\&P economic and demographic forecasting models, and the fact that they rely on a very detalled database, makes the regional model and database one of the most comprehensive county-level forecasting tools available.

\subsection{W\&P'으 Forecast Methods}

At the most general level, the strengths of the economic and demographic forecasts. [W\&P Economics, Inc.] stem from the comprehensive nature of the county database and the integrated nature of the model. The forecast for each county in the United States is done simultaneously so that changes in one county will affect growth or decline in other counties. This reflects the flow of economic activity around the county as new industries spring up or relocate in growing areas and as people migrate, in part because of job opportunities. The county forecasts are also developed within the framework of the United States forecast that is determined from Woods \& Poole's macro-economic model. This approach of linking the counties together to capture regional flows and constraining the results to a previously determined United States total avoids a common pitfall in regional forecasting. The regional forecasts are sometimes made for a city or county without regard for potential growth in surrounding areas or other areas in the country. Such forecasts may be simple extrapolations of historical trends and may tend to be overly optimistic or pessimistic. If these county forecasts were added together, the total might be considerably different from any concelvable national forecast 
scenario, which is the result of the regional forecasts being generated independently without interactive procedures and not being constrained to a consistent national total.

The primary methods used to generate the complete country forecasts proceed in three stages. First, the country is divided into 183 Economic Areas (EAs) as defined by the U.S. Department of Commerce's Bureau of Economic Analysis. The EAs are aggregates of contiguous counties and attempt to measure cohesive economic regions in the United States. For each EA a forecast is made for employment, using an "export-base" approach (explained below), and the employment forecast results are then constrained to a previously determined national forecast. The employment forecast for each EA is then used to estimate earnings in each EA; the earnings forecasts are similarly constrained to the national forecast total. The employment and earnings forecasts then become the principal explanatory variables used to estimate population and number of households in each EA. The population and household forecasts are also constrained to the national totals.

The second stage of the forecast procedure is to make population by age, sex and race forecasts on the basis of net migration rates profected from employment opportunities. The third stage is a replication of the first two stages, except that it is performed at the county level, using the EAs as the control total for the county forecast. 


\subsubsection{The "Export-Base" Economic Models}

The specific economic forecasting models used by Woods \& Poole that generate the employment, earnings and income forecasts for each county in the United States generally follow a standard economic "export-base" approach. It is a relatively simple approach to regional employment forecasting. Certain industrial sectors at the regional level are considered "basic". This means that these sectors produce output that is not consumed locally, but is produced for "export" out of the region for national or international consumption. Normally the "basic" sectors are mining, agriculture, manufacturing and the Federal Government. In contrast, "non-basic" sectors are those, such as retafl trade, transportation, communication and construction, with output that is usually consumed locally. The growth of the "non-bastc" sectors depends largely on the growth of the "basic" sectors that form the basis of the region's economy. Although the theory is appealing, some shortcomings do exist with the "exportbase" approach [W\&P Economics, Inc.]. For example, some "basic" commodities produced locally are consumed locally. However, despite the shortcomings, the avallability of relatively clean data for subnational geographic areas makes the "export-base" approach very useful, albeit not perfect. The analytical framework for constructing regional models using the "export-base" approach entails estimating demand equations for output by sector. The principal explanatory varfable is the national demand for the output of that sector. A modification of the current "export-base" approach of W\&P is used in the current W\&P forecasting model to account for regional variants to 
normal basic/non-basic industry definitions. In addition to following an "export-base" approach, the regional model uses individual EA and county models as forecasting tools.

\subsubsection{The Demographic Model}

The demographic portion of the reglonal model follows a traditional cohort-component analysis [Krueckeberg \& Silvers 1974] based on net migration rates for the period 1970 to 1982. The migration patterns in the forecast period for population (by age in 5-year cohort groups up to 85 and over), sex, and race (white, black and other) are largely based on employment opportunities. The expected economic growth, or decline, of a city or county therefore has a resulting impact on population growth. This integration of economic and demographic regional analysis is a significant strength of the model.

\subsubsection{The Reliability of Forecasts}

Unlike other sciences, economics cannot rely on experimentation to test theories and verify hypotheses. Rather, historical data are analyzed and theories are developed that explain the historical data and then are used to make a forecast. The Woods \& Poole forecast, like all economic and demographic forecasts, uses this approach. There are, however, some inherent 11mitations.

One limftation is that the future is not usually known with any reasonable degree of certainty. There is always the possibility of an unprecedented shock to the economy - a shock that could not be fore- 
cast based on any analysis of historical data. For instance, a local government may enact a new Industrial pollcy that has an unexpected, beneficial effect on employment growth. Or an abrupt economic change, although anticlpated, may occur with much greater intensity or in a shorter time period than expected. Another limitation is a result of dolng forecasts for small geographic areas with a small number of observations. Statistically, forecasts become more reliable as the area becomes larger. Error can be reduced, although never eliminated, by constraining the small area forecasts to the forecast totals for a larger area. This is the method used by Woods \& Poole. Given these limitations and the inability in economics to experimentally test theorfes, the most acceptable economic models are those that are modeled on not only known facts, i.e., historical data, but also explain those facts well. One way to evaluate how a forecast explains the historical data is to do "relro-forecasts" and measure how well the model predicts the past. The divergence of the retro-forecast from the actual data measures the accuracy of the model. On the average, all endogenous variables in the Voods \& Poole national and regional models have a percent root mean square error of less than three percent.

\subsection{Methodology for IU's Population Profections}

Indiana Unfversity (IU) used demographic models to produce their 1988 population projections at the county and township levels. The demographic approach uses patterns and models of births, deaths, and migration changes to project population. It has been assumed that 
general social, environmental, economic and political features of the base year will continue for the life of the projection. The model can be summarized by the following equation:

new population = old population + births - deaths + net migration

The projections were calculated for each of the 92 counties for fiveyear Intervals from 1985-2020. Within each county projection, there are 18 age cohorts (from $0-4$ to $85+$ ) for male and for female. The fertility component model was calculated using age-specific birth rates for 1980 and 1984 from the Indiana State Board of Health. The mortality component model was calculated using age-specific deaths for Indiana counties for 1979-81, obtained from Indiana state Board of Health. The age-specific mortality rates used in population projections are assumed to remain constant through the life of the projections.

The Indiana township population projections [Business Research Center, IU] are an extension of the Indiana county population projection. Therefore, the demographic model assumes that population changes within a township are the result of births, deaths, and migration into and out of the township. Similar to the county population projections, township population was divided into cohorts classified by race (white and non-white), sex, and age categorles. After the projected population was computed for each township in a county, they were adfusted so that their sum was equal to the profected county population. The assumptions made in computing the township population profections were the same as those for county population profections. 
At the township level, the most difficult component of the basic demographic equation was net migration. Knowledge of local conditions provided an important source of information for adjusting and profecting net migration. Although the adjustment factors were based on the extent and number of changing local conditions, the actual assignment of magnitudes was judgmental.

\subsection{The Data Tables}

This study has been focussed mainly on bullding traffic forecasting model(s) for urban highway segments in the State of Indiana. The general characteristics of each functional class of urban highway are presented in Table 4.1 .

The definition of "urban" highway segments, for purposes of this study, Includes Federal Aid Primary, Federal Aid Urban, and other state highways within an urbanized area, as commonly defined. The researcher and advisory committee members agreed to group the urban area into the following three levels:

1. Highest leve1: Areas with designated Metropolitan Planning Organizations or with population greater than 200,000 .

2. Middle-population level: Cities and towns with population greater than 5,000 and not included in level 1 .

3. Low population: Cities and towns under 5,000 population.

Since level 2 localities are not 11kely to have in-house planning capability, the inftial research focus was on this level.

The functional classes of all the urban state highway segments for which AADT figures were collected have been identifled by examin- 
Table 4.1: Urban Functional Classification of Highways

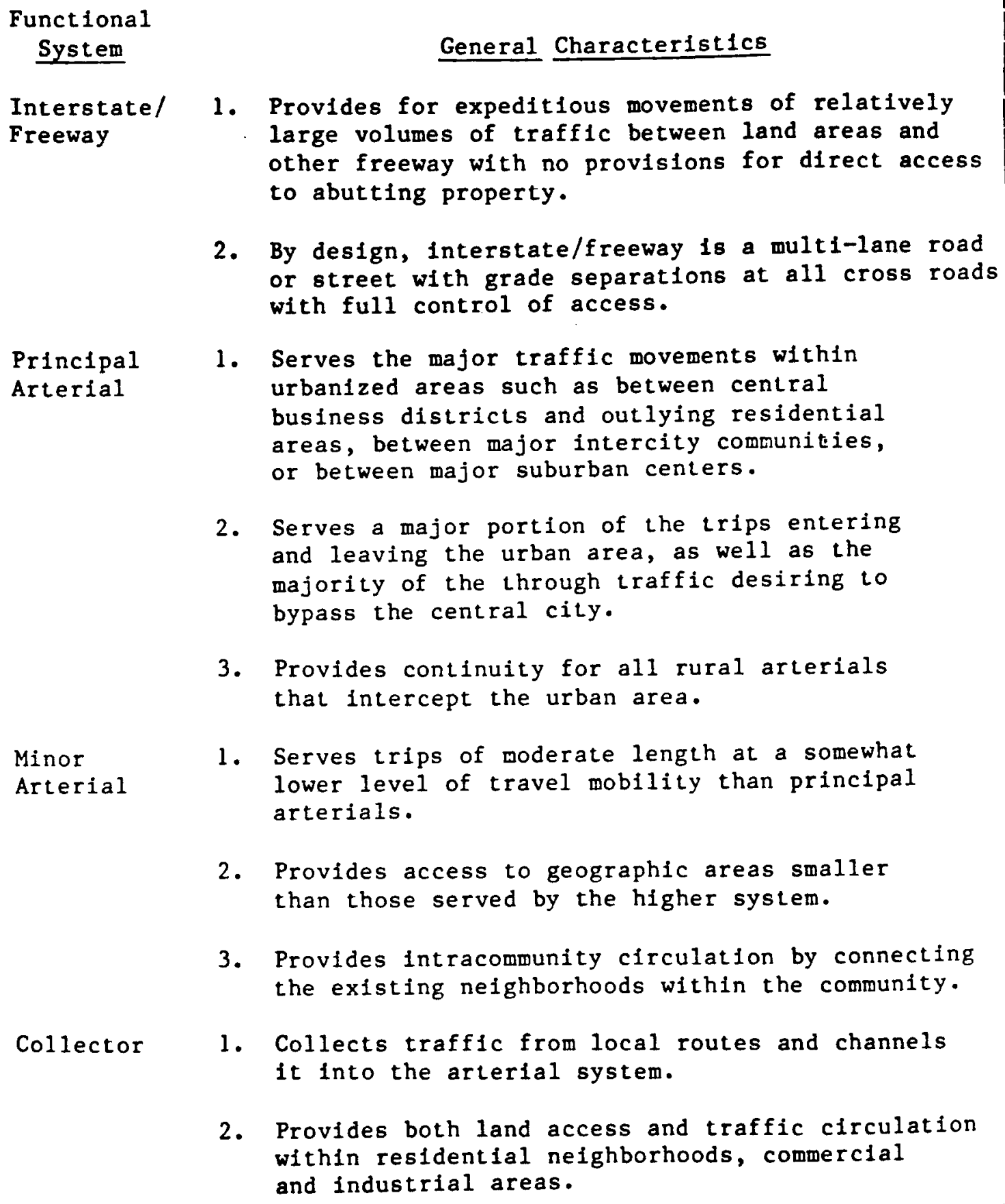

2. By design, interstate/freeway is a multi-lane road or street with grade separations at all cross roads with full control of access.

Principal

1. Serves the major traffic movements within urbanized areas such as between central business districts and outlying residential areas, between major intercity communities, or between major suburban centers.

2. Serves a major portion of the trips entering and leaving the urban area, as well as the majority of the through traffic desiring to bypass the central cily.

3. Provides continuity for all rural arterials that intercept the urban area.

Minor

Arterial

1. Serves trips of moderate length at a somewhat lower level of travel mobility than principal arterials.

2. Provides access to geographic areas smaller than those served by the higher system.

3. Provides intracommunity circulation by connecting the existing neighborhoods within the community.

Collector 1. Collects traffic from local routes and channels it into the arterial system.

2. Provides both land access and traffic circulation within residential neighborhoods, commercial and industrial areas.

Sources: 1. AASHTO [1984].

2. INDOT Road Inventory file.

3. Meyer and Miller [1984]. 
Ing the Road Inventory f1le of INDOT (Indiana Department of Transportation). There were only a few urban segments in the "collector" class, and the number of minor arterial segments is much smaller than that of principal arterlal. Moreover, the traffic levels of these two class of highways (minor arterial and collector) are almost the same. Therefore, the "collector" segments were pooled with "minor arterial" segments for statistical analysis purposes. The 77 citles in level 2 were further divided into four groups, depending on 1980 city population (see Table 4.2), to determine the effect of city size on traffic. Moreover, to reduce the variation in the data, it was necessary to organize the segments in each population group by common variable characteristics. The variables used to define groups, or "families", of segments are functional classification and city size. The cities for noninterstate highways and the sections for interstate highways are sampled randomly from the available number of cities and roadway sections, respectively. All the segments of the sampled cities are entered in the noninterstate data tables. Table 4.2 shows the number of sampled sections for each group of cities for noninterstate highways and for the two groupings of interstate highways (rural \& urban). The growth factor model for rural interstates will be compared with the one developed for urban interstates. Finally, it will be determined if there are advantages to pooling the interstate highway groups Into a single group.

The 11st of 1tems incorporated in data bases is presented in Table 4.3. The description of all the variables is provided in Section 4.6. The column number in the data base for each variable is 
Table 4.2: Groupings of Roadway Segments

A. Noninterstate State Highways (Urban):

\begin{tabular}{|ccc|cc|}
\hline \multirow{2}{*}{$\begin{array}{c}\text { City Group } \\
(\star)\end{array}$} & $\begin{array}{c}\text { Total No. } \\
\text { of Cities }\end{array}$ & $\begin{array}{c}\text { No. of } \\
\text { Cities } \\
\text { Sampled }\end{array}$ & $\begin{array}{c}\text { No. of Road Segments/Sections } \\
\text { in Cities Sampled }\end{array}$ \\
\hline A & 10 & 9 & 142 & 12 \\
Prterial & $\begin{array}{c}\text { Minor Arterial } \\
\text { and Collector }\end{array}$ \\
B & 9 & 8 & 59 & 14 \\
C & 25 & 19 & 136 & 18 \\
\hline D & 33 & 25 & 98 & 42 \\
\hline
\end{tabular}

B. Interstate Highways: (Note: 50 out of the 92 counties have interstate highways)

\begin{tabular}{|lccc|}
\hline Highway Class & $\begin{array}{c}\text { Tolal No. } \\
\text { of Sections }\end{array}$ & $\begin{array}{c}\text { No. of } \\
\text { Sections Sampled }\end{array}$ & $\begin{array}{c}\text { Percent of } \\
\text { Sections Sampled }\end{array}$ \\
\hline Rural Interstate & 220 & 117 & 53.18 \\
Urban Interstate & 79 & 79 & 100.00 \\
\hline
\end{tabular}

(*) See Section 4.6 for City Group Classification. 
Table 4.3: Description of Items in Data-Bases

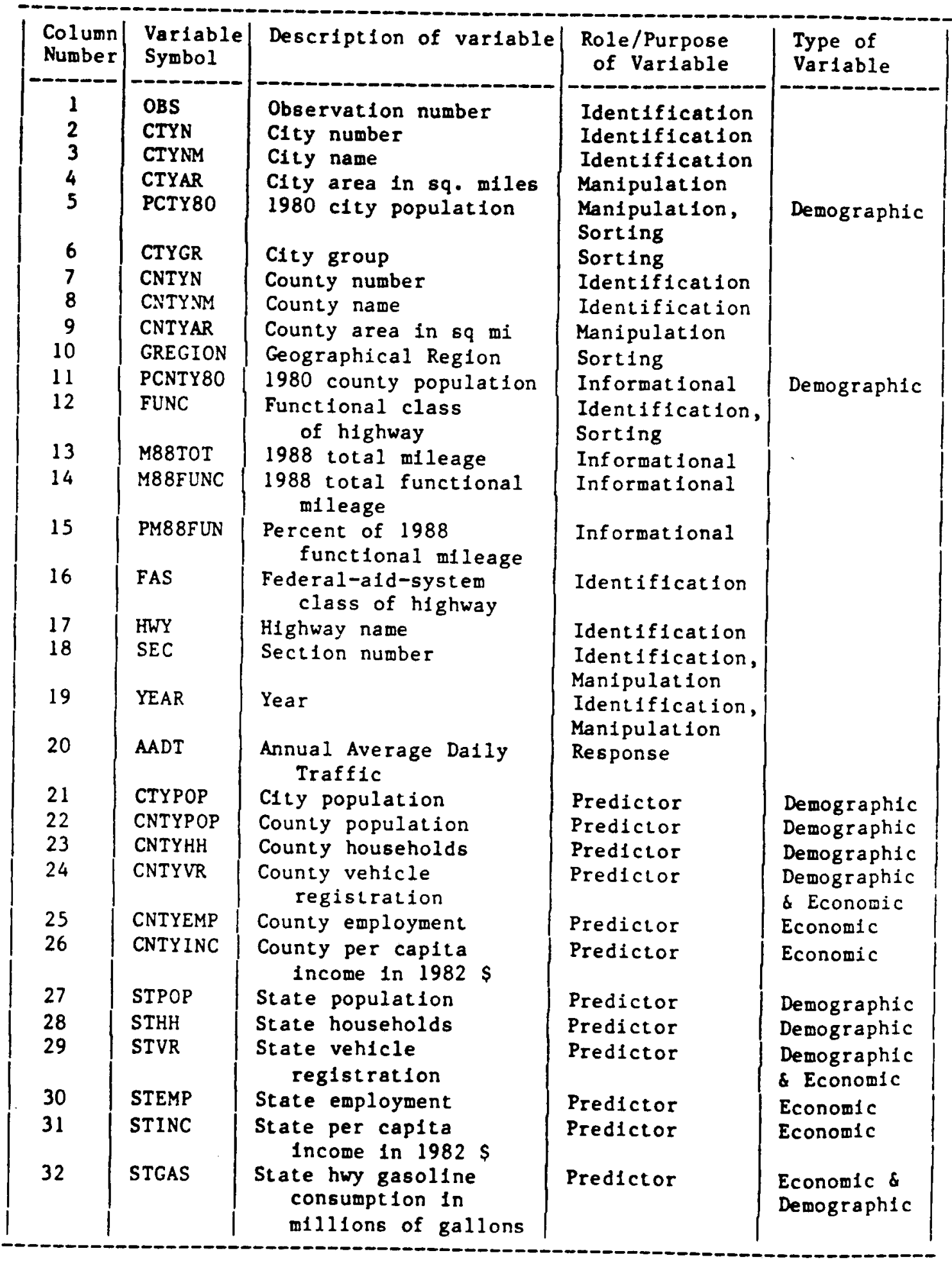


shown in Table 4.3, along with its corresponding variable name and symbol. The data bases with all the varlables of Table 4.3 w1ll be used in statistical analyses to develop the prediction model. There Is a separate data base for each noninterstate state highway. Each of these data bases for noninterstate highways is further divided into the four city groups. Similar groupings were done for interstate highway data bases. The following subsections will present the data bases under the two broad headings - data bases with segment AADT and data bases with county or city average AADT.

\subsubsection{Data Tables with Segment AADT}

All the data bases began with the AADT of each segment. For these segment AADT data bases, traffic data are taken from all the sampled segments of the selected city or county. In cases where AADT and predictor variable values were not avallable for the same years, linear interpolations were carried out for the predictor variables to match the year of AADT. The following four forms of data bases were produced from the data bases with segment AADT.

1. Raw form (RAW)

2. Earlier and later years version of the raw form (RAW E\&L)

3. Density form (DENSITY)

4. Earlier and later years version of the density form (DENSITY E\&L) A brlef description for each of these forms is presented below. 
RAW, Raw form and

DENSITY, Density form

The raw form of each data set was developed by direct entry, through a LOTUS 1-2-3 worksheet, of the values of all the variables in Table 4.3. A portion of the data base for urban minor arterial and collector functional class is shown in Appendix Table Al. The item in column 20 is the response variable and 1tems from columns 21 through 32 will be used as predictor variables. All the predictor variables are numerical in nature. The data bases created with the variables described in Table 4.3 w111 be referred to as "RAW" data bases.

The socioeconomic variables, used as predictor variables in this study, entered in the "RAW" data bases are aggregate over city, county or state. Despite the same level of traffic between two cities or counties, a "scale problem" may arise because of wide variation of the aggregate socloeconomic variables. A schematic representation of the "scale problem" is illustrated in Figure 4.1. In this figure, two cities/counties -- small and large - with almost identical values of AADT on their roadway sections show the effect of the level of demographic and economic data. In part a of Figure 4.1, large city/county data consistently fall above the data of the small clty/county at different years. When these data are employed to predict AADT, they w111 exhibit the scale effect, as shown in part b of Figure 4.1. In part b of Figure 4.1, large and small city/county data points are separated from each other, In spite of almost Identical AADT values. This scale effect will be minimized in the following cases:

1. AADT values are proportional to the level of demographic and economic data of small and large city/county. 


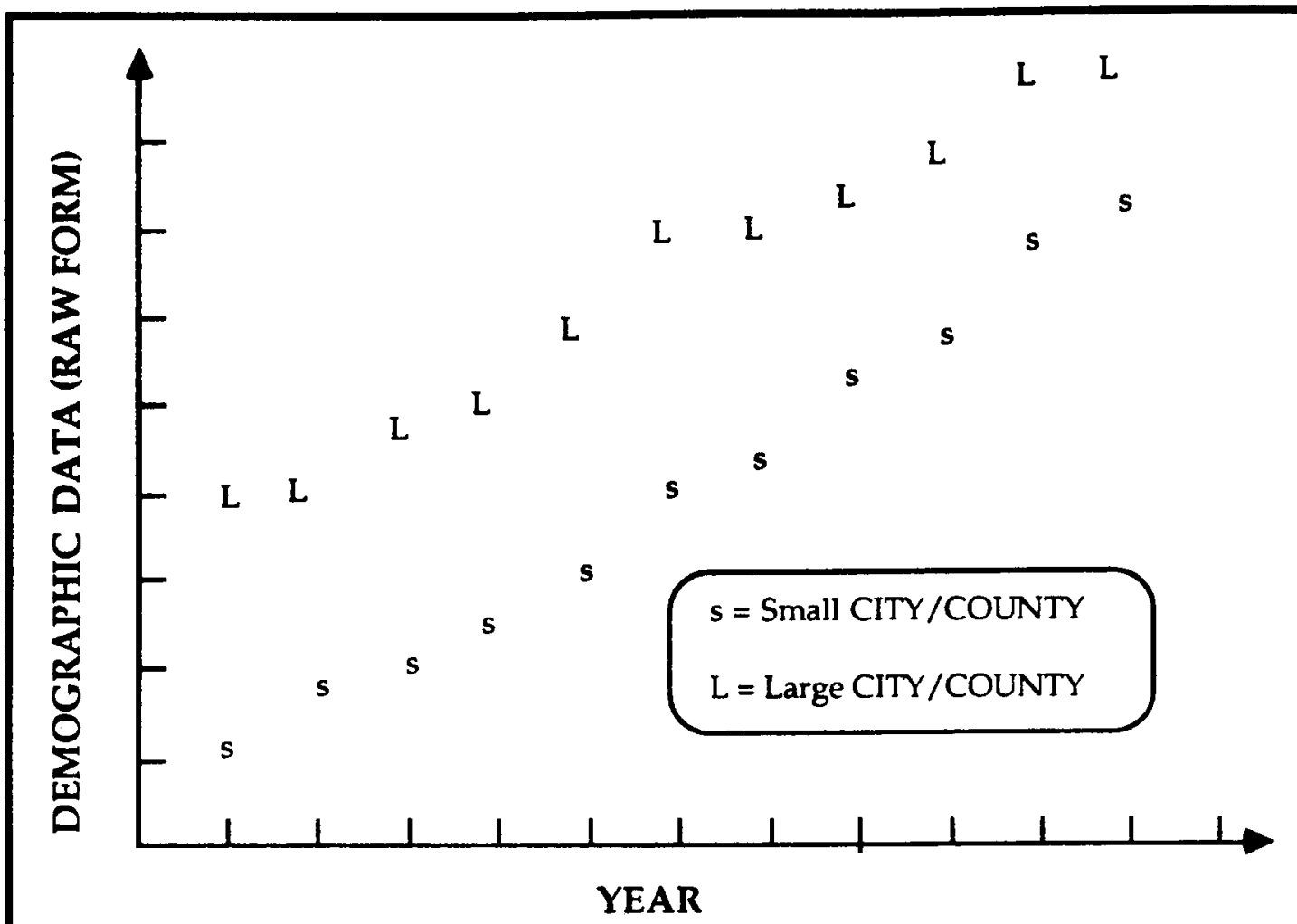

(a) Demographic Data vs. Year

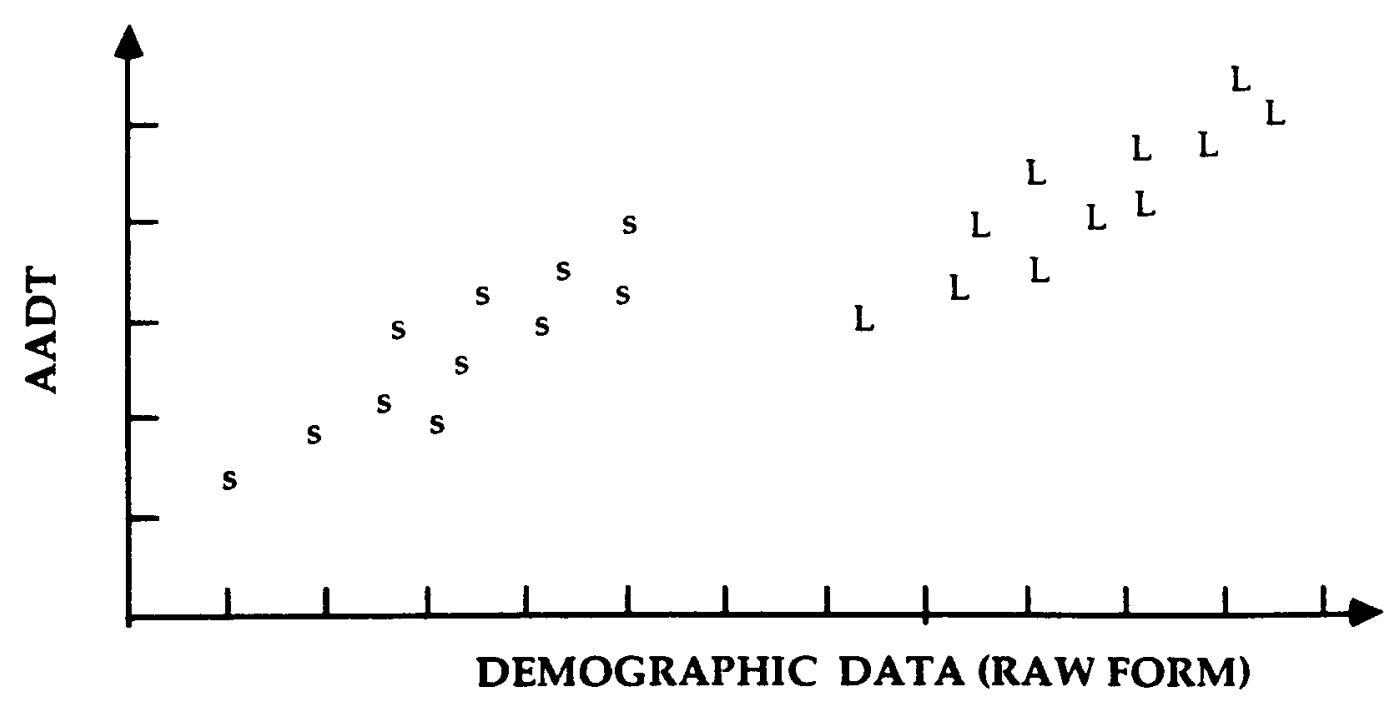

(b) AADT vs Demographic Data

Figure 4.1: Schematic Representation of "SCALE" Problem 
2. Segregat1ng/subsetting data bases according to levels of demographic and economic data. (See Section 4.5)

3. Normalizing the data bases (for example, the density format described next).

To remove/minimize the scale problem caused by big differences among c1ty/county-level demographic and economic variables in a data base, the RAW data bases have been transformed to a density form. It was expected that a normalized form of the demographic and economic variables would reduce the variation in predictor varlables used to explain AADT levels. The DENSITY form of data set was produced by redefining some of the demographic and economic variables in Table 4.3 as follows:

1. CTYPOP: City population per square mile of city area.

2. CNTYPOP: County population per square mile of county area.

3. CNTYHH: County households per square mile of county area.

4. CNTYEMP: County employment per square mile of county area.

5. STPOP: State population per square mile of state area.

6. STHH: State households per square mile of state area.

7. STEMP: State employment per square mile of state area.

8. CNTYVR: County vehicle registrations per 100 county population.

9. STVR: State vehicle registrations per 100 state population.

The variables -- county income, state income and state highway gasoIine consumption - in the DENSITY data set remalned unchanged from "RAW" form. A computer program coded in FORTRAN 77 was written to develop the DENSITY data set from the raw data set. A portion of DENSITY data base for urban minor arterial and collector is shown in 
Appendix Table A2. (Note: Complete raw data bases for each functional class of highway have been saved on floppy diskettes to permit updatIng in the future.)

RAW E\&L, Earlier \& 1ater version of raw form and DENSITY E\&L, Ear11er \& later version of density form

New data bases, represented as earller and later year version of raw and density form, were prepared with average values of earlier and later years of traffic and socio-economic variables. Let " $a$ " and " $b$ " be the earliest and latest year of AADT counts for a highway segment. Then, the middle year $(m)$ is the average of $a$ and $b, m=(a+b) / 2$. The earlier years $\left(x^{-} s\right)$ and later years $\left(y^{-} s\right)$ are defined as follows:

$$
\begin{aligned}
& \text { Earlier years: } a<x<m \text {, and } \\
& \text { Later years: } m<y<b .
\end{aligned}
$$

The data for the middle year, $m$, has been included in both earlier and later year data. A computer program, coded in FORTRAN 77, has been written to develop earlier and later year version of raw and density data sets from raw and density data sets. A portion of the earlier and later (E\&L) year version of the DENSITY data base for urban minor arterial and collector is shown in Appendix Table A3. The E\&L data consist of two data points for each section of highway: the average values of the earlier years data and of the later years ${ }^{-}$of data. The E\&L transformation will provide a longer interval between observations than that provided by the RAW and DENSITY data bases (See column 19 of Appendix Tables Al to A6). This longer interval would presumably reduce/eliminate short term fluctuations in data points. 


\subsubsection{Data Tables with County and C1ty Average AADT}

Considerable variability in the data sets was noticed in the segment AADT data base because of wide variation in AADT among the different highway sections within a city or county. Meanwhlle, the predictor variables for each section within a city are the same. To remove these variations in segment AADT data bases, the earlier four forms of data (raw, density, raw E\&L, and density E\&L) were modified by averaging the Individual segment AADT values to produce a city or county-wide average AADT for each year under the different functional roadway classes. For rural interstates, averaging within a county was done. For other functional classes of urban highways, averaging over a city was done. Appendix Tables A4 to A6 show the portion of the data tables with county or city average AADT for the three main functional classes, identified in earlier sections.

The statistical quality of the eight different forms of the data bases -- four with segment AADTs and four with county or city average AADTs -- will be evaluated in the next chapter.

\subsubsection{Number of Cases}

A case is a row or record in a data table. A filter analysis can be performed to remove data points that are obviously wrong (examples: faulty observations, data entry error, etc.). Outlier analysis can be used to remove points that have a high probability of not belonging to the family of data points. Inftially, all observations in a data base were used in the analysis. If the data items or amount are found to 
be insufficient during the analysis, necessary data will be collected appropriately. Tables 4.4 to 4.6 present the number of cases for each category of highway for all forms of data bases described in earlier sections. These tables also show the number of cases when they are sorted under different variables. All the possibilities w111 be analyzed in the next chapter to select a particular form of data tables or a combination of forms.

\subsection{Description of Variables}

In this section, the variables have been grouped into three categorles -- Index (categorical) variables, Response variables and Predictor variables -- depending on their roles in the analysis. Table 4.3 has presented the list of all variables with their role, symbol, type and column number of data sets. All these variables are described in this section with respect to their definition, avallabilIty, quality and role in analysis.

\subsubsection{Index or Categorical Variables}

The index or categorical variables are used to perform mainly three kinds of operation - sorting, identification and manipulation. The sorting of the data sets is performed through the Index variable(s) to stratify the data sets. These variables are listed in Table 4.3 wth their role as "sorting". Some of the Index variables are used to identify the observation during statistical analysis and can be used to modify the data sets in the future for calibration of model. Their roles have been shown as "identification" in Table 4.3. 
Table 4.4: Number of Cases for Urban Minor Arterial \& Collector

\begin{tabular}{|c|c|c|c|c|c|c|}
\hline $\begin{array}{l}\mathrm{C} \\
\mathrm{T}\end{array}$ & $\begin{array}{l}\mathbf{M} \\
\mathbf{F}\end{array}$ & & Segmen & Database & County/City & Ave. Database \\
\hline $\begin{array}{l}Y \\
G\end{array}$ & $\mathrm{U}$ & $\begin{array}{l}\mathbf{E} \\
\mathbf{G}\end{array}$ & $\begin{array}{l}\text { RAW } \\
\text { or }\end{array}$ & $\begin{array}{l}\text { RAW or } \\
\text { DENSITY }\end{array}$ & $\begin{array}{l}\text { RAW } \\
\text { or }\end{array}$ & $\begin{array}{l}\text { RAW or } \\
\text { DENSITY }\end{array}$ \\
\hline $\mathbf{R}$ & C & ION & DENSITY & E\&L & DENSITY & E\&L \\
\hline & ALL & & 392 & 172 & 119 & 54 \\
\hline $\begin{array}{l}A \\
B \\
C \\
D\end{array}$ & & & $\begin{array}{r}56 \\
64 \\
78 \\
194\end{array}$ & $\begin{array}{l}24 \\
28 \\
36 \\
84\end{array}$ & $\begin{array}{l}18 \\
13 \\
30 \\
58\end{array}$ & $\begin{array}{r}8 \\
6 \\
14 \\
26\end{array}$ \\
\hline $\begin{array}{l}\mathrm{A} \\
\mathrm{A} \\
\mathrm{A} \\
\mathrm{A}\end{array}$ & $\begin{array}{l}\text { F1 } \\
\text { F2 } \\
\text { F3 } \\
\text { F4 }\end{array}$ & & $\begin{array}{l}35 \\
21\end{array}$ & $\begin{array}{l}14 \\
10\end{array}$ & $\begin{array}{r}10 \\
8\end{array}$ & $\begin{array}{l}4 \\
4\end{array}$ \\
\hline $\begin{array}{l}\mathrm{B} \\
\mathrm{B} \\
\mathrm{B} \\
\mathrm{B}\end{array}$ & $\begin{array}{l}\text { F1 } \\
\text { F2 } \\
\text { F3 } \\
\text { F4 }\end{array}$ & & 64 & 28 & 13 & 6 \\
\hline $\begin{array}{l}c \\
c \\
c \\
c\end{array}$ & $\begin{array}{l}\text { F1 } \\
\text { F2 } \\
\text { F3 } \\
\text { F4 }\end{array}$ & 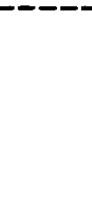 & $\begin{array}{l}23 \\
20 \\
35\end{array}$ & $\begin{array}{r}10 \\
8 \\
18\end{array}$ & $\begin{array}{r}14 \\
5 \\
11\end{array}$ & $\begin{array}{l}6 \\
2 \\
6\end{array}$ \\
\hline $\begin{array}{l}D \\
D \\
D \\
D\end{array}$ & $\begin{array}{l}\text { F1 } \\
\text { F2 } \\
\text { F3 } \\
\text { F4 }\end{array}$ & & $\begin{array}{r}116 \\
14 \\
55 \\
9\end{array}$ & $\begin{array}{r}52 \\
6 \\
22 \\
4\end{array}$ & $\begin{array}{r}30 \\
9 \\
10 \\
9\end{array}$ & $\begin{array}{r}14 \\
4 \\
4 \\
4\end{array}$ \\
\hline $\begin{array}{l}\text { A } \\
\text { A } \\
\text { A }\end{array}$ & & $\begin{array}{l}n \\
c \\
s\end{array}$ & $\begin{array}{l}21 \\
10 \\
25\end{array}$ & $\begin{array}{r}10 \\
4 \\
10\end{array}$ & $\begin{array}{l}8 \\
5 \\
5\end{array}$ & $\begin{array}{l}4 \\
2 \\
2\end{array}$ \\
\hline $\begin{array}{l}\text { B } \\
\text { B } \\
\text { B }\end{array}$ & & $\begin{array}{l}\mathrm{n} \\
\mathrm{c} \\
\mathrm{s}\end{array}$ & $\begin{array}{l}24 \\
40\end{array}$ & $\begin{array}{l}12 \\
16\end{array}$ & $\begin{array}{l}8 \\
5\end{array}$ & $\begin{array}{l}4 \\
2\end{array}$ \\
\hline $\begin{array}{l}\bar{c} \\
C \\
c\end{array}$ & & $\begin{array}{l}\text { n } \\
\text { c } \\
\text { s }\end{array}$ & $\begin{array}{l}52 \\
16 \\
10\end{array}$ & $\begin{array}{r}24 \\
4\end{array}$ & $\begin{array}{r}13 \\
5\end{array}$ & $\begin{array}{l}6 \\
2\end{array}$ \\
\hline $\begin{array}{l}D \\
D \\
D\end{array}$ & & $\begin{array}{l}\mathrm{n} \\
\mathrm{c} \\
\mathrm{s}\end{array}$ & $\begin{array}{r}32 \\
20 \\
142\end{array}$ & $\begin{array}{l}16 \\
10 \\
58\end{array}$ & $\begin{array}{r}16 \\
8 \\
34\end{array}$ & $\begin{array}{r}8 \\
4 \\
14\end{array}$ \\
\hline
\end{tabular}

Note: See section 4.6 .1 for description of CTYGR, MFUNC \& GREGION. 
Table 4.5: Number of Cases for Urban Principal Arterial

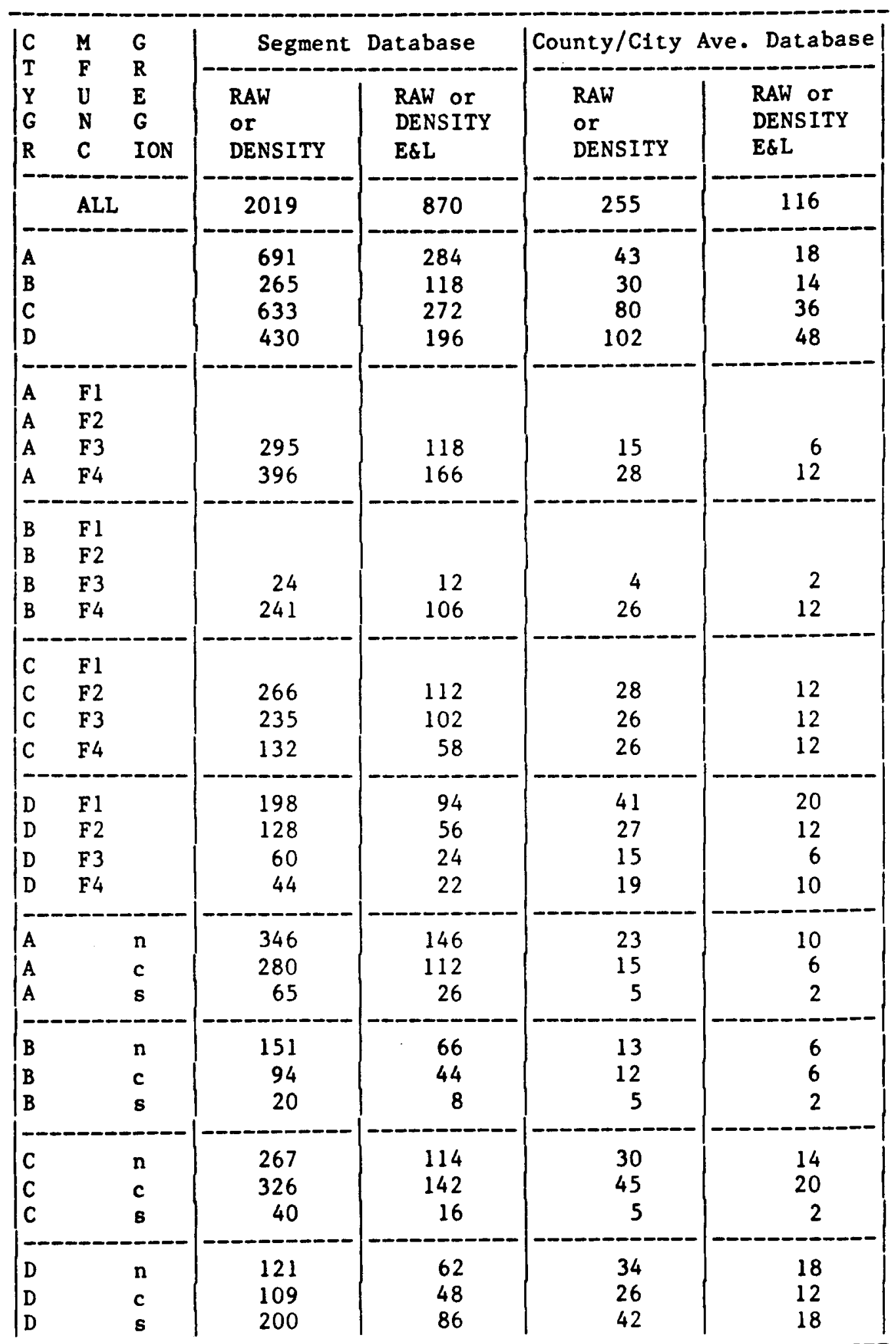

Note: See section 4.6 .1 for description of CTYGR, MFUNC \& GREGION. 
Table 4.6: Number of Cases for Interstate Highways

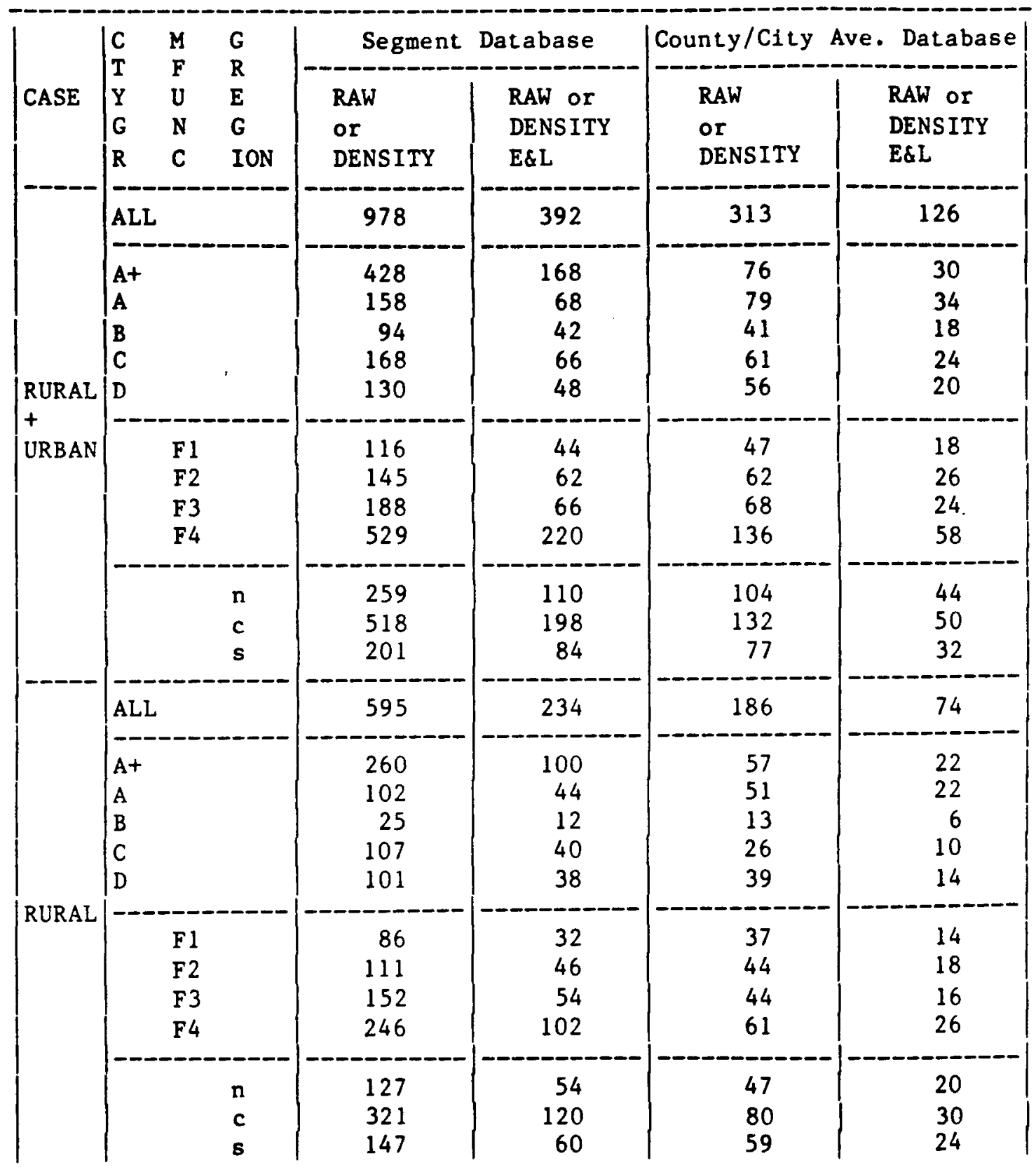


Table 4.6, continued

\begin{tabular}{|c|c|c|c|c|c|c|}
\hline \multirow[b]{2}{*}{ CASE } & $M$ & G & \multicolumn{2}{|c|}{ Segment Database } & County/City & Ave. Database \\
\hline & $\begin{array}{ll}Y & U \\
G & N \\
R & C\end{array}$ & $\begin{array}{l}\text { E } \\
\text { G } \\
\text { ION }\end{array}$ & $\begin{array}{l}\text { RAW } \\
\text { or } \\
\text { DENSITY }\end{array}$ & $\begin{array}{l}\text { RAW or } \\
\text { DENSITY } \\
\text { E\&L }\end{array}$ & $\begin{array}{l}\text { RAW } \\
\text { Or } \\
\text { DENSITY }\end{array}$ & $\begin{array}{l}\text { RAW or } \\
\text { DENSITY } \\
\text { E\&L }\end{array}$ \\
\hline & ALL & & 383 & 158 & 127 & 52 \\
\hline & $\begin{array}{l}\text { At } \\
\text { A } \\
\text { B } \\
\text { C } \\
\text { D }\end{array}$ & & $\begin{array}{r}168 \\
56 \\
69 \\
61 \\
29\end{array}$ & $\begin{array}{l}68 \\
24 \\
30 \\
26 \\
10\end{array}$ & $\begin{array}{l}19 \\
28 \\
28 \\
35 \\
17\end{array}$ & $\begin{array}{r}8 \\
12 \\
12 \\
14 \\
6\end{array}$ \\
\hline URBAN & $\begin{array}{l}\mathrm{F} 1 \\
\mathrm{~F} 2 \\
\mathrm{~F} 3 \\
\mathrm{~F} 4\end{array}$ & $-\infty$ & $\begin{array}{r}30 \\
34 \\
36 \\
283\end{array}$ & $\begin{array}{r}12 \\
16 \\
12 \\
118\end{array}$ & $\begin{array}{l}10 \\
18 \\
24 \\
75\end{array}$ & $\begin{array}{r}4 \\
8 \\
8 \\
32\end{array}$ \\
\hline & & $\begin{array}{l}\mathrm{n} \\
\mathrm{c} \\
\mathrm{s}\end{array}$ & $\begin{array}{r}132 \\
197 \\
54\end{array}$ & $\begin{array}{l}56 \\
78 \\
24\end{array}$ & $\begin{array}{l}57 \\
52 \\
18\end{array}$ & $\begin{array}{r}24 \\
20 \\
8\end{array}$ \\
\hline
\end{tabular}

Note: See section 4.6 .1 for description of CTYGR, MFUNC \& GREGION. 
Also to manipulate the data sets from one form to another, some of the Index variables was used. The roles of these index variables are stated as "manipulation" in Table 4.3. Those index variables not used for any of the above three purposes are designated as "informational" in Table 4.3. Sometimes, the informational index variable was used to designate the sorting variable. Some of the variables play more than one role.

OBS, Observation Number

The variable OBS (observation number) is simply the serial number of each observation or record in the data set. The purpose of this variable is to identify the observation during the statistical analyses.

CTYN, City Number and

CTYNM, City Name

The varlables CTYN and CTYNM were entered in each record of a data set to identify the observation. They are used in data manipulation and will be helpful modifying the data bases in the future. For each urban highway segment, the CTYN and CTYMM identify the city within which the segment is located. For rural interstates, fudgment was applied to Identify the city, by size and closeness that is thought to have the largest impact on the highway section. The variables CTYN and CTYNM are shown in Appendix Table Bl. 
CTYAR, C1ty Area and

CNTYAR, County Area

The 1980 census record was the source of variables CTYAR, C1ty Area in square miles, and CNTYAR, County Area in square miles. The varlable CTYAR was used to convert the clty population to its density form as city population per square mile. The variable CNTYAR was used to get the density of county population, county household and county employment. The role of CTYAR and CNTYAR is manipulation of data sets from their raw format to density format. Appendix Tables B1 and B2 show the areas in square miles for cities and countles of Indiana.

PCTY80, 1980 City Population

CTYGR, City Group

The variable PCTY80 represents the city population in year 1980 . Bureau of Census records (County and City Data Book, Characteristics of the Population) and the STATIS database of Indiana University were used to get the 1980 city population. According to the 1980 census, there were 77 cities in Indiana with a population between 5000 and 50000. For noninterstate highways, the following groupings of the cities were made:

\begin{tabular}{|c|c|}
\hline CTYGR & 1980 City Population (1000) \\
\hline A & $30-50$ \\
B & $20-30$ \\
C & $10-20$ \\
D & $5-10$ \\
\hline
\end{tabular}

For interstate highways, an additional group, A+, was designated for cities with more than 50,000 population in 1980. The variable CTYGR was used to sort the data tables by city group based on the 
informational variable PCTY 80 . This subsetting of the data sets will provide on opportunity to observe any improvement in statistical analysis.

CNTYN, County Number

CNTYNM, County Name

Like city number and city name, county number (CNTYN) and county name (CNTYNM) were entered in data sets according to the county in which a highway segment is located. These variables also have a role In Identifying an observation and will be useful in modifying the data-base(s) in the future. These variables were used to manipulate the data sets. Appendix Table B2 presents the variable CNTYN and CNTYNM.

GREGION, Geographical Region

As with City Group, the data sets were stratifled by geographical region (GREGION) to reduce the variability of the data sets or to improve the quality of results. Figure 4.2 shows the boundaries to demarcate the north, central and south regions of the State of Indiana. A highway section was classifled as north, central or south by its location within a city/county. The variable GREGION was recorded as $n$, $c$ or $s$ in the data sets for north, central and south, respectively.

PCNTY 80, 1980 County Population

The county populations were obtalned from census records. The 


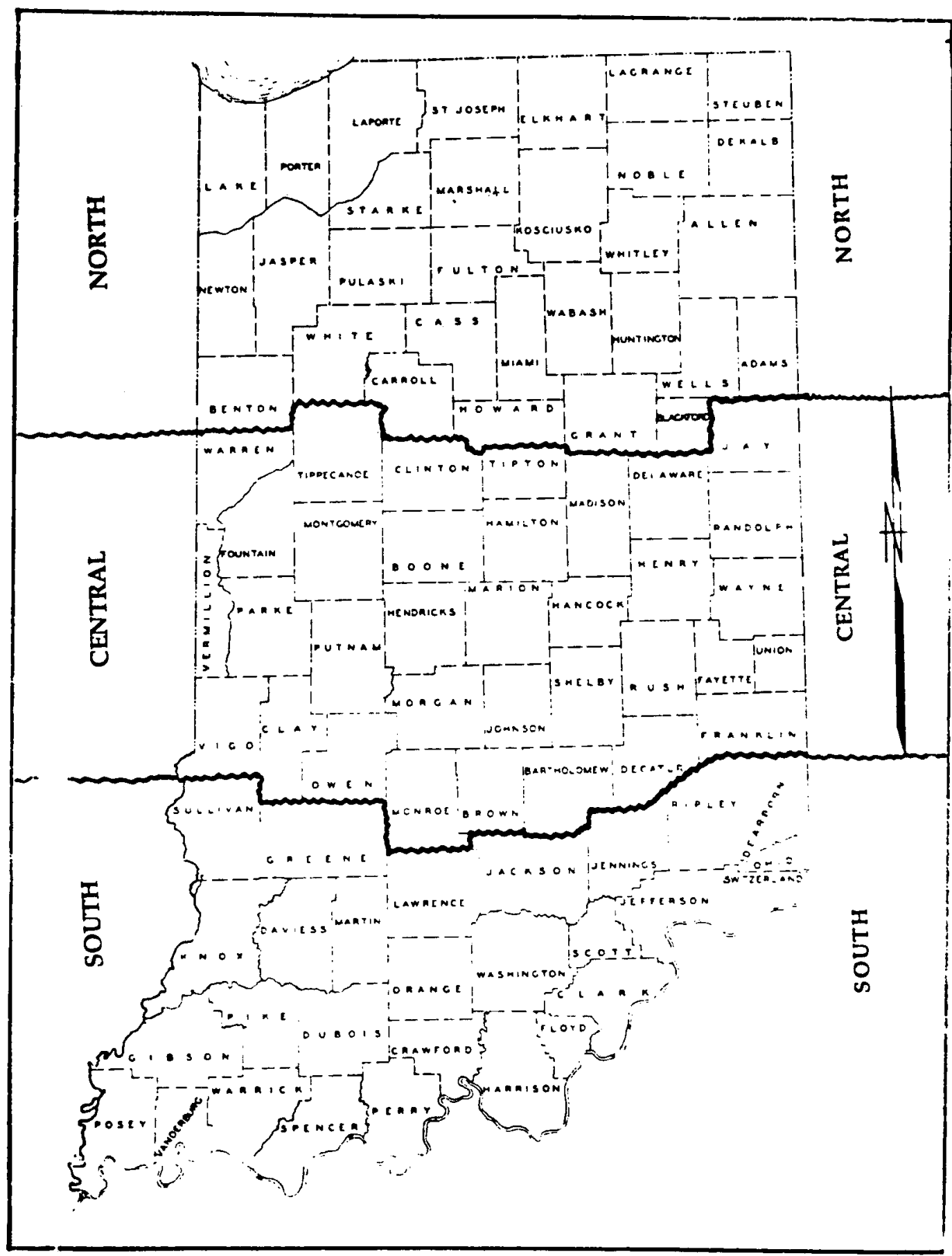

Figure 4.2: Suggested Geographical Boundaries of State of Indiana 
variable PCNTY80 represents the county total population in the 1980 census year. PCNTY 80 was not used to stratify the data sets, but it was kept in the data sets if any stratification needed to be done based on county population.

FUNC, Functional Class

FAS, Federal-A1d System

Classification is the tool by which a complex network of highways can be allocated into groups or systems of routes having simflar characteristics. For transportation planning purposes, as well as for design purposes, highways are most effectively classifled by function. Table 4.1 has shown the general definitions of Urban Functional Class (FUNC) -- Interstate, principal arterial, minor arterial, collector, and local. Federal-aid system (FAS) classifications -- interstate, primary, secondary, urban, non FAS -- categorize highways based on the distribution and administration of federal-aid highway funds. Both the functional and federal-aid classes of all the state highway segments for which AADT figures have been collected were identified by examining the Road Inventory file of INDOT. The data FUNC and FAS for different highway classes were recorded in data sets as follows:

\begin{tabular}{|cl|ll|}
\hline Functional Class & FUNC & Federal-aid Class & FAS \\
\hline Urban Interstate & ui & Federal-Aid Interstate & fai \\
Rural Interstate & r1 & Federal-Aid Primary & fap \\
Urban Principal - & upa & Federal-Aid Secondary & fas \\
Arterlal & & Federal-Aid Urban & fau \\
Urban Minor Arterial & uma & & \\
Urban Collector & uc & & \\
\hline
\end{tabular}

Initially, all the data sets have been created by functional class on 
a LOTUS 1-2-3 worksheet. The variable FUNC has been used for sorting as well as for identification purposes, whereas the varlable FAS has been used only for identification.

M88T0T, 1988 Total Mileage

M88FUNC, 1988 Total Functional Mileage

MFUNC, Mileage Group

PM88FUN, Percent of 1988 Functional Mileage

The varlable M88TOT represents the total county mileage, both rural and urban, for interstate highways. For noninterstate highways, M88TOT represents the total county urban mfleage. For interstates, M88FUNC and PM88FUN represent the miles of interstate highways in a county and the percent of interstate highways in a county with respect to the county's total mileage. For noninterstate highways, M88FUNC represents the miles of state highways within a county other than interstate and PM88FUN is expressed as a percent of M88FUNC with respect to M88TOT for noninterstates.

As with City Group, the data sets were stratified by the extent of miles of road in a city/county based on the values of M88TOT. These groupings were based on quartile ranges. The univariate procedure of SAS [SAS/Basics 1985] was used to find the quartile boundaries. According to the 1988 mileage report of INDOT, there are some counties without any urban mileage. To establish the groupings for non-interstate highways, counties without any urban mileage were dropped from the analysis. The results of the groupings (MFUNC), based on the quartile ranges, are as follows: 
(Case A) Non-1nterstate Urban Highways:

\begin{tabular}{|c|c|}
\hline $\begin{array}{c}\text { M1leage Group } \\
\text { (MFUNC) }\end{array}$ & $\begin{array}{c}\text { County Urban Total Mileage } \\
\text { (M88TOT) }\end{array}$ \\
\hline F1 & $33-57$ \\
F2 & $57-96$ \\
F3 & $96-262$ \\
F4 & $262-3340$ \\
\hline
\end{tabular}

(Case B) Interstate Highways:

\begin{tabular}{|c|c|}
\hline $\begin{array}{c}\text { Mileage Group } \\
\text { (MFUNC) }\end{array}$ & $\begin{array}{c}\text { County Total Mileage } \\
\text { (M88TOT) }\end{array}$ \\
\hline F1 & $170-820$ \\
F2 & $820-940$ \\
F3 & $940-1090$ \\
F4 & $1090-3340$ \\
\hline
\end{tabular}

The variable MFUNC is not recorded in data-sets, but was created durIng several statistical analyses based on the values of M88TOT. The variable MFUNC has been used to stratify or sort the data-sets. The role of M88TOT, M88FUNC and PM88FUN is informational. The values of M88TOT, M88FUNC and PM88FUN for all 92 counties of Indiana are presented in Appendix Table B3.

HWY, H1ghway Name

SEC, $\overline{\text { Section Number }}$

The varfable, HWY, represents the jurisdictional name of each section of highway (for example, SR26, US52 and I65). For the RAW data bases, the section number (SEC) was obtalned from "Traffic Statistics" book of Indiana Department of Transportation. But in county or cfty average data bases, the section number (SEC) represents 
the number of a section within each county/city used to arrive at the average values. These variables were mainly used for identification of each observation and will be helpful to modify the data-base(s) in future.

YEAR

The varlable YEAR represents the year in which all the variables, both dependent and independent, apply for a particular observation. This variable was introduced to reflect the time effect on AADT and the predictor variable(s), and to study patterns of the residuals against time. As a general trend, AADT increases as time passes. It was thought that using YEAR as a predictor variable will lead to high statistical correlation with the other predictor variables. For that reason, YEAR has not used as a predictor variable.

\subsubsection{Response variable}

The only response variable that needs to be predicted in future years is AADT (Annual Average Dally Traffic). In Indiana, five out of 31 continuous traffic count stations are located in urban areas [INDOT-Traffic Statistics Book, Feb. -87]. The rural study [Fricker and Saha 1987] was based on the continuous count AADT. Because of the limited number of continuous count stations at urban locations, this study was carried out with coverage traffic counts, which are generally done at 3- to 4-year intervals. The coverage count is converted to AADT based on the adjustment factors developed from continuous count stations. Some noise in coverage count traffic data is 
expected because of short term counts and the use of adjustment factors developed from continuous count station data. It was felt that averaging over county/city would be useful to filter some of those noises in data.

AADT, Annual Average Dally Traffic (AADT)

AADT is the average 24-hour traffic volume for a given year, for both directions of travel, unless otherwise specified.

There are 77 cities/towns in Indiana with a population between 5,000 and 50,000 (Leve1 2), and about 850 segments of state, US and interstate routes in these cities. The historical traffic counts were retrleved for most of those segments for certain past years during the period of 1970 to 1987 from INDOT files. The form that was used to record those traffic data over different years is shown in Appendix Table B4. For the raw traffic counts, adjustment factors (prepared by INDOT) were used to convert them to AADT.

\subsubsection{Predictor Variables}

There are a number of predictor variables that have been 1dentifled historically as the primary determinants of highway travel, the major ones being population, income, employment, energy, auto ownersh1p, and household. Twelve predictor varlables (see Table 4.3) are used in this study. The main role of these predictor varlables is to explain AADT. These predictor variables can be categorized as either demographic or economic. Some fundamental criterla adopted in select- 
Ing the predictor variables are [Draper \& Smith 1981, Freund \& Minton 1979] :

1. Are the proposed variables fundamental to the problem?

2. Avallability of data (varlables)

a. Are annual data avallable?

b. Are historfcal data available?

c. What is the most. recent year of data?

d. Will data be available in the future?

3. Cost to obtain data

4. How reliable are the data?

In this section, each of the predictor variables is discussed with respect to their availability in the past and in the future. Historical data for each of the predictor variables for all the counties or cities are recorded on floppy diskettes using the LoTUS 1-2-3 worksheet. Based on the discussion of the predictor varlables in sections $4.3,4.4$ and the current section, a summary table (see Table 4.7) has been prepared regarding fundamental selection criterla. The avallability, cost and reliablifty of the variables is used to identify the "quality" of the variables. An equal welght placed on each of the selection criteria result in the following order of importance (1=most preferable varlable):

1. Employment (county and state level)

2. (a). Population, Vehicle Registration and Income (county and state level)

(b). State Highway Gasoline consumption 
Table 4.7: Availability, Cost and Reliability of the Prediclor Variables

\begin{tabular}{|c|c|c|c|c|c|}
\hline $\begin{array}{l}\text { Variable } \\
\text { Symbol }\end{array}$ & $\begin{array}{l}\text { Variable } \\
\text { Description }\end{array}$ & $\begin{array}{l}\text { Past } \\
\text { Data } \\
(*)\end{array}$ & $\begin{array}{l}\text { Fore- } \\
\text { cast } \\
(* *)\end{array}$ & $\begin{array}{l}\text { Cost of } \\
\text { data } \\
(* * \star)\end{array}$ & $\begin{array}{l}\text { Reli- } \\
\text { ability } \\
(\star \star \star \star)\end{array}$ \\
\hline$=x=x==x=x$ & 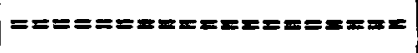 & $== \pm==x=0$ & $=x===x==$ & $=====$ & $==x===x=$ \\
\hline CTYPOP & City Population & 3 & 3 & 2 & 4 \\
\hline CNTYPOP & County Population & 2 & 1 & 1 & 2 \\
\hline CNTYHH & County Households & 4 & 1 & 1 & 2 \\
\hline CNTYVR & County Veh. Regis. & 1 & 2 & 2 & 1 \\
\hline CNTYEMP & County Employment & 1 & 1 & 1 & 2 \\
\hline CNTYINC & $\begin{array}{l}\text { County Percapita } \\
\text { Income in } 82 \$\end{array}$ & 1 & 1 & 1 & 3 \\
\hline STPOP & State Population & 2 & 1 & 1 & 2 \\
\hline STHH & State Households & 4 & 1 & 1 & 2 \\
\hline STVR & State Veh. Regis. & 1 & 2 & 2 & 1 \\
\hline STEMP & State Employment & 1 & 1 & 1 & 2 \\
\hline STINC & $\begin{array}{l}\text { State Percapita } \\
\text { Income in } 82 \$\end{array}$ & 1 & 1 & 1 & 3 \\
\hline STGAS & $\begin{array}{l}\text { State Hwy. Gas. } \\
\text { Consump. in mil- } \\
\text { lions of gallons }\end{array}$ & 1 & 2 & 2 & 1 \\
\hline
\end{tabular}

(*) PAST DATA:

1 = available at all years

2 = available at census years $\delta$ intercensus estimation present

3 - avallable at more than census years \& estimates done for missing year(s)

4 - avallable at census years \& data for missing years are estimated
(**) FORECAST:

1 = forecasts available from W\&P Economics, Inc, and/or IU Business Center

2 = Forecast will be generated in this study

3 = Forecast available but not complete, missing forecast will be made $(\star \star \star)$ COST OF DATA:

1 = easily available from different sources with negligible cost

2 = in-house forecast may make the data comparatively expensive $(* \star * *)$ RELIABILITY:

$1=$ good historical series, so forecasts are expected to be reliable

2 = data sources claim their forecasts are reliable

3 = despite the reliability claimed by data sources, researchers expect uncertainty in future because of nature of the variable

4 = lack of good historical data makes the forecast less reliable 
3. Households (county and state level)

4. City population

This order of preference in addition to the statistical results will be referred to in later chapter(s) to identify the candidate and final model(s). The 11terature [McKay 1925, Morf \& Houska 1958, Hartgen 1981, Memmott 1983] on traffic forecasting shows that all the predictor varfables are relevant to traffic forecasting. The question of relevance will be further examined with respect to the functional class of highway. For example, state level data should have more influence on interstate and principal arterial highways than minor arterial and collector. In addition to the Information in Table 4.7, detalled discussions for each of the predictor variables are presented below with respect to their definition, sources, and evolution in data bases.

CTYPOP, City Population

Decennial Bureau of Census [County and City Data Book, Characteristics of the Population] records on population are only done for some major cities. The database STATIS (Statistical Information System), a component of EDIN (Economic Development Information Network) at Indiana University has records of city population for certain past years of all levels of citles. The STATIS database was accessed through the Purdue University Computer Center to obtain the city population data for the years 1970, 1973, 1975, 1978, 1980, 1982, 1984 and 1986. To use in data tables, linear interpolation was done to find 
c1ty population for missing years 1971, 1972, 1974, 1976, 1977, 1979, 1981,1983 and 1985. The c1ty population for 1987 was estimated by adding annual changes between 1984 and 1986 to 1986 population.

An understanding of what is happening to the population is necessary in determining highway travel demands. The variable, C1ty Population (CTYPOP), is taken as a predictor variable on the assumption that the response variable AADT in a particular year at a place is dependent on the number of people living nearby.

Woods and Poole (W\&P) Economics Inc. provides the forecast on population of cities, classified as Metropolitan Statistical Areas (MSA). The Indiana Business School also projects the township population for every fifth year over 20-30 future years [Indiana University 1986]. The Indiana township population projections are an extension of the Indiana county population projections. To get the projection for city population other than the MSA population, a "ratio" method -the proportion of city population to county/township will be used. Based on this proportionality assumption, unknown city populations can be estimated by using equation 4.1.

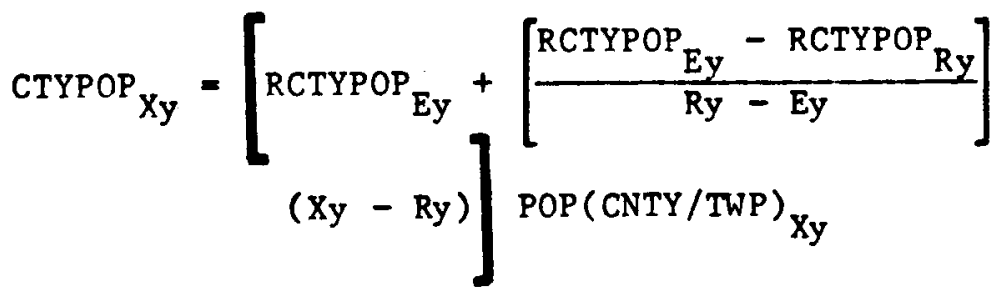

where,

$$
\begin{aligned}
\text { RCTYPOP }_{\text {Ry }}= & \text { Ratio of city population to county or township } \\
& \text { population in a recent known year, Ry, }
\end{aligned}
$$




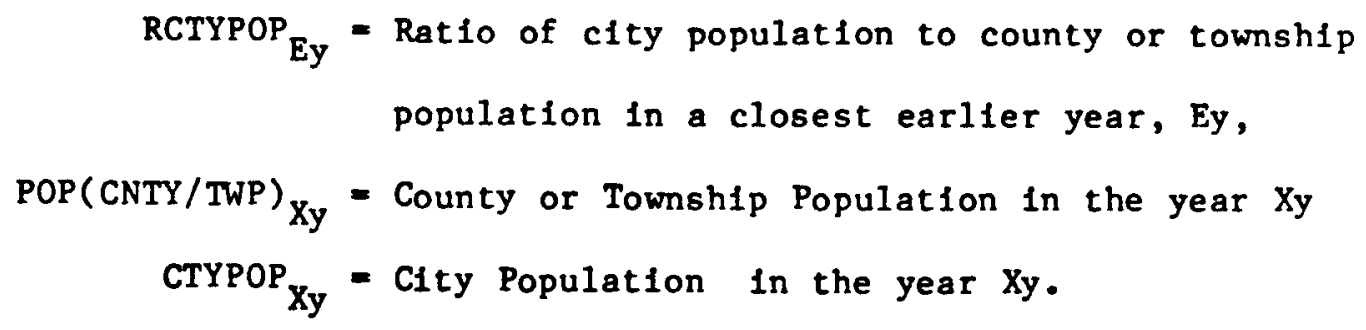

A small city is generally contained within a township. On the other hand, there are some cities that extend over two or more townships. For the bigger city, ratlos can be developed based on multiple townships which enclosed the cities or total county(les) population projections.

CNTYPOP, County Population and

STPOP, State Population

Decennial Bureau of Census [County and City Data Book, Characteristics of the Population] records on population provide state and county values. These variables are taken as predictor variables on the assumption that the response variable AADT in a particular year at a particular place is dependent on the number of people living nearby. Intercensus estimates of CNTYPOP and STPOP from the Indiana School of Business [Indiana University] were used in the data tables for years other than census years.

The Indiana Business School also projects the population for every fifth year over 20-30 future years. Its projections are made at the county level, based on the fertility, mortality, and net migration experiences of the county populations. The state forecasts are the results of the sum of the forecasts of the 92 individual counties. The projections are based on past trends and patterns, but also 
involve judgments, because simple historfcal extrapolation is not always rellable. Wood and Poole Economics, Inc. population projections [W\&P, 1987 Indiana State Proffle] are also based on cohortcomponent analysis with consideration of economic growth/decline and constrained by national, reglonal, and state forecasted totals. Population forecasts from efther IU Business School or W\&P Economics can be used, but the inclusion of economic factors with the cohort analysis and the consideration of smaller area projections constrained to broader area profectlons make W\&P's forecasts preferable to IU's forecasts.

CNTYHH, County Households, and STHH, State Households

A household includes all persons who occupy a housing unit. A housing unft is a house, an apartment, a group of rooms, or a single room occupied as separate living quarters or, if vacant, intended for occupancy as separate living quarters. Data for total households include all occupied housing units. The number of occupled housing units is the same as the number of households. The housing statistics presented here for the years 1970 and 1980 are based on the results of the 1970 and 1980 Census of Population and Housing, conducted by the Bureau of Census as of April 1, 1970 and 1980 [Bureau of the Census, County and City Data Book]. Some of the data collected by the Bureau of Census were collected on a 100 percent, or complete-count, housing Inventory, while other data were obtained from sample estimates. The samples were 5 percent, 15 percent, and 20 percent, depending on the subject covered. The sample data have been "weighted" or "Inflated" 
to reflect the entire population or unfverse. Exact agreement, therefore, is not to be expected between data based on samples and data resulting from complete counts.

A large portion of our society is organized into households. The total number of households in a county in a particular year is CNTYHH for that year. STHH is the statewide value. It was felt that travel demand would very much dependent on this variable. Although the household size and demand are changing, these changes are felt to be somewhat predictable. It was found in a previous study [Neveu 1982] that number of households is a better predictor of AADT than population. The predictor variables - CNTYHH and STHH - are chosen on the assumption that the response varlable, AADT, will be adequately explained by using them in models. The Bureau of Census gives the values of CNTYHH and STHH for each census year. Like population, number of households forecasts for county and state can be obtained from Woods and Poole Economics, Inc. The estimates of intercensus households between 1970 and 1987 were accomplished by the interpolation procedure described below [Fricker \& Saha 1987].

The slopes of population/household between 1970 and 1980 are less than 0.04 per year [See Figure 4.1 of Fricker \& Saha 1987]. With these mild slopes, it has been assumed that the average household size 1s changing uniformly between the census years. To develop the slope of household size between 1980 and 1990, the 1990 forecasts from W\&P forecasts were used with 1980 census data. Based on the above assumptions, households for years 1971 to 1979 are computed by using Equa$\operatorname{tion}(4.2)$. 
${ }_{19 X X}=\left[\mathrm{POP} / \mathrm{HH}_{1970}+\left[\frac{\mathrm{POP} / \mathrm{HH}_{1980}-\mathrm{POP} / \mathrm{HH}_{1970}}{10}\right](19 \mathrm{XX}-1970)\right]_{\ldots(4.2)}^{-1} \mathrm{POP}_{19 X X}$

where,

$$
\begin{aligned}
\mathrm{POP} / \mathrm{HH}_{1970} & =\text { Ratio of population to households in year 1970, } \\
\mathrm{POP} / \mathrm{HH}_{1980} & =\text { Rat1o of population to households in year 1980, } \\
\mathrm{POP}_{19 \mathrm{XX}} & =\text { Population in year } 19 \mathrm{XX}(1971<19 \mathrm{XX}<1979), \\
\mathrm{HH}_{19 \mathrm{XX}} & =\text { Households in year } 19 \mathrm{XX} .
\end{aligned}
$$

Households at years 1981 to 1987 are also computed using Equation (4.2) by replacing (1) POP/HH ratios for 1970 and 1980 by those ratios for 1980 and 1990 respectively, (11) year 1970 by 1980 , and (1i1) the range of years $19 \mathrm{XX}$ as $1981<19 \mathrm{XX}<1987$.

CNTYVR, County Vehicle Registrations and STVR, State Vehicle Registrations

The total number of vehicle registrations in the county where a highway segment is located (CNTYVR) and that for the whole state of Indiana (STVR) is published each year by the Indiana Bureau of Motor Vehicles. Historical data for these variables are available for years of 1940, 1941, and 1950 through 1988. Data for vehicle registrations are not avallable at the city level. The available data are reliable in the sense that they are not estimates, but are counts made at motor vehicle registration offices throughout the state. These variables are proposed to explain AADT on the assumption that AADT in a particular year at a given place is closely related to the number of vehicles registered then and there. The prediction of expected future traffic based on the projection of the trend of motor vehicle registrations is a reasonably accurate indication of future highway traffic. The value 
of vartables CNTYVR and STVR was used in the data tables for all categorles of highways. Because of the good series of historfcal data, time sertes analysis and a combination of regression and time serles methods will be used to predict the future year values of CNTYVR and STVR. The validity of our forecasts will be assessed through retro-forecasting procedures. The reliability of these forecasts can be assessed by comparing the forecasts with the yearly report on motor vehicle registrations of the Bureau of Motor Vehicles, as they become avallable from the Bureau's yearly publication.

CNTYEMP, County Employment and STEMP, State Employment

Employment data [Bureau of Census, Council of Economic Advisers, Indiana Employment Security Division (IESD)] is an economic variable. The County Employment Patterns [IESD] are released each summer and provide "covered employment" data for each month, each county, and each employment category for the previous calendar year. The total "covered employment" [Bureau of Census, IESD],

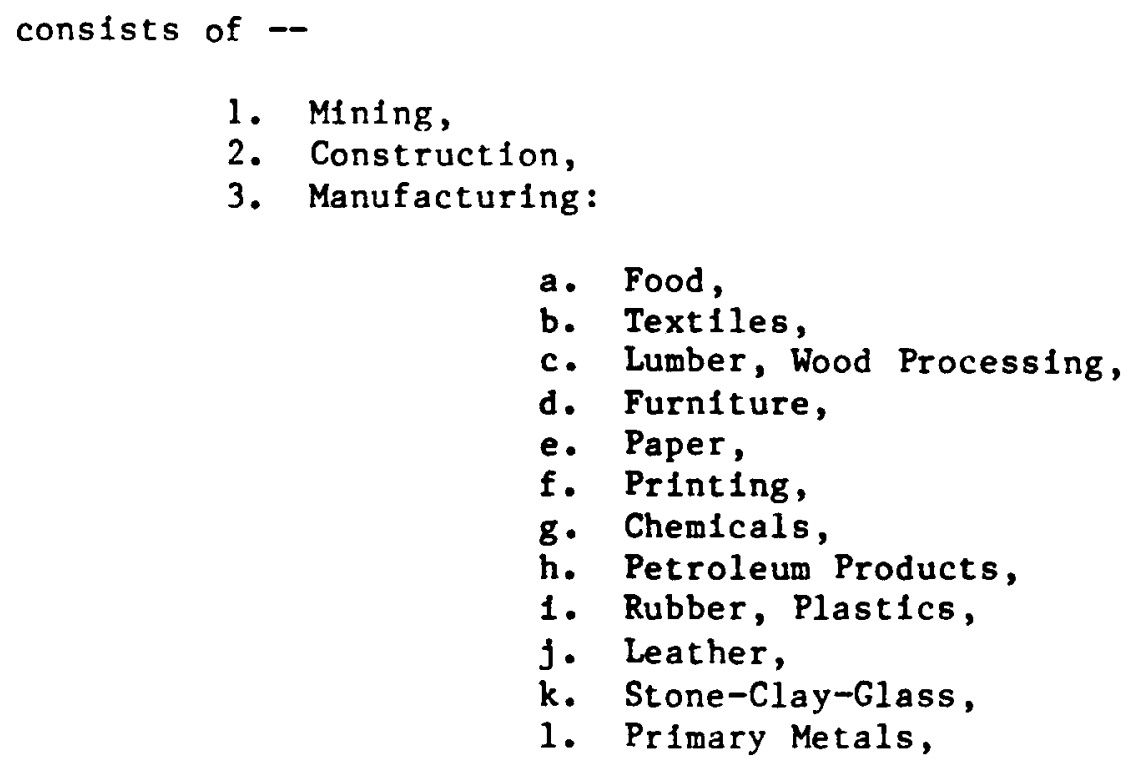


m. Fabricated Materlals,

n. Non-electric Machinery,

o. Electric Machinery,

p. Transportation Equipment,

q. Instruments and

r. Misc. Manufacturing,

4. Transportation, Communication, Public Utilities,

5. Wholesale Trade,

6. Retail Trade,

7. Finance,

8. Agriculture \& Services, and

9. Government.

County Employment Patterns published prior to 1983 do not provide state employment figures. According to the 1976 edition, covered employment represents about 85 percent of nonagricultural wage and salary employment and 78 percent of all employment. Major exceptions to coverage of wage and salary employment are in rallroads, small nonprofit institutions, churches, private households, and most government units. State hospitals, schools of higher education, and local government utilities are covered. In addition to these exceptions, self-employed workers (both farm and non-farm) are excluded from coverage.

"County Business Patterns - Indiana", a publication of the US Bureau of Census, furnishes employment data for each year for the week Including March 12, and provides such data for the county and state levels. This summary of employment excludes government employees, raflroad employees, self-employed persons, etc. Th1s publication also provides Federal Civilian Employment for the mid-March pay period by county and state. The "City and County Data Book" is another publication of the Bureau of Census that presents employment data by county and state in every tenth year. The employment figures in the "City 
and County Data Book" are prepared from household surveys, where workers are counted according to their place of residence; whereas for "County Business Patterns", they are counted according to their place of work. There are varlous reasons for differences in the two serles of data:

a. differences in the reporting systems they use,

b. differences in the time period to which the reports refer,

c. sampling variations in the figures based on the sample survey, and

d. differences in industrial classification.

For the City and County Data Book, the survey information is obtained from respondents in workers' households, while the County Business Patterns industrial classification is based on information either from the employer or administrative sources.

There exists 1ittle difference between the numbers in "County Business Patterns" and "County Employment Patterns". The difference Is mainly due to the reporting systems and the perfods to which the reports refer. The employment numbers from the "County Business Patterns - Indiana" were taken as varlable CNTYEMP, County Employment, for the years 1970 to 1987 . The state employment totals (STEMP) are found by summing the 92 county employment figures in "County Business Patterns - Indiana".

The forecasts of employment data [W\&P,1987] are based on establishment-based employment data, where employment figures are counted according to the place of work. This was one of the main 
reasons to choose County Business Patterns historical employment data -- which are also establishment-based data. The employment forecasts from Wood \& Poole Economics Inc. will be used in traffic forecasting model(s). Those forecasts are based on "Export-Base" economic models (see Section 4.3.1). The superfority of these forecasts was discussed In some earlier $\operatorname{section}(s)$.

CNTYINC, County Per Capita Income ( $-\frac{82}{\$)} \$$ )

STINC, State Per Capita Income ( 82 \$)

Income is a economic indicator. Like employment, it is believed that this county and state income influences the traffic on highways. Income was used earlier In New Mexico's [Albright 1985] and Neveu's [1982] study. The City and County Data Book [Bureau of the Census] publishes the per capita personal income and median family income at the state and county level for the year before the census years. The database STATIS (Statistical Information System), a component of EDIN (Economic Development Information Network) of Indiana University has historical records of income along with other information. The STATIS database was accessed through the Purdue University Computer Center to obtain the per capita income in current dollars at county and state for the years 1970 to 1987. For use in the data tables, the county and state per capita incomes were converted to 1982 dollars by applyIng the consumer price index (CPI) [Bureau of Labor Statistics, Economic Statistics Bureau of Washington] in Equation (4.3).

$$
\mathrm{INC}_{1982}=\left[\frac{\mathrm{CPI}{ }_{1982}}{\mathrm{CPI}_{19 \mathrm{XX}}}\right] \mathrm{INC}_{19 \mathrm{XX}}
$$

where, 


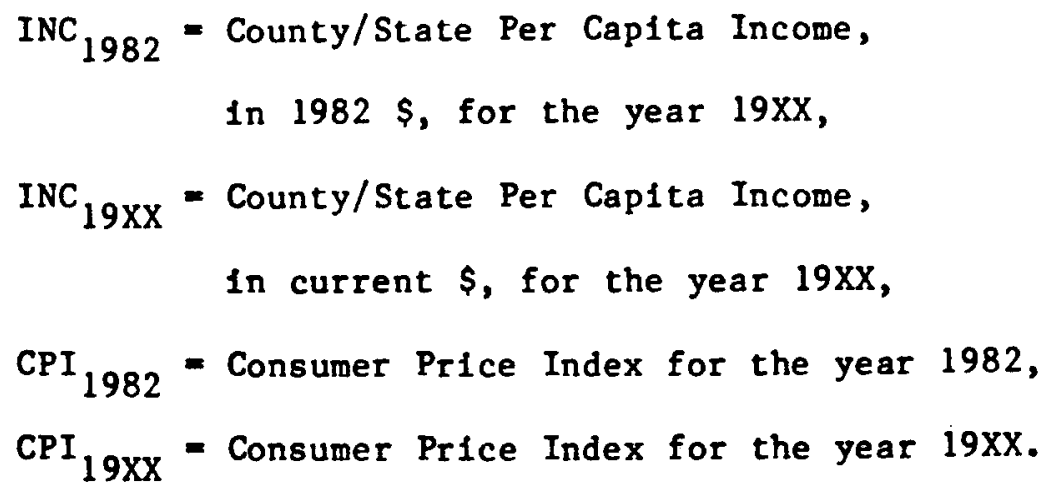

The converted county and state per capita income in 1982 dollars are represented as variables CNTYINC and STINC in the data tables, respectively. These varfables are adopted on the assumption that there exists a significant relationship between income and magnitude of travel. The historical income data are easily avallable. The forecasts of per capita Income at state and county levels are also available from Woods and Poole Economics Inc. Like employment, their income forecasts are also based on their "Export-Base" Economic Models.

STGAS, State Highway Gasoline Consumption in Millions of Gallons

The total amount of yearly gasoline consumption by the vehicles on the roads of Indiana is taken as variable STGAS. The historical data on STGAS are available from Highway Statistics of Federal Highway Administration (FHWA) since 1949. This variable is proposed to explain AADT on the assumption that AADT in a particular year is closely related to amount of highway gasoline used in that year. Because of good historical data, time series analysis and a combination of regression and time serles methods will be used to predict the future year values of STGAS. The validity of our forecasts will be 
made through retro-forecasting. The reliablity of these forecasts can be assessed by comparing the forecasts with data in the yearly publications of FHWA, as they become avallable.

\subsection{Chapter Summary}

The central idea of this chapter is to describe the variables and data bases used in model development. The variables have been discussed and the sources of their numerical values are given. The summary regarding avallability, cost and rellability of the predictor variables is presented (See Table 4.7). Explanations behind the uses of all the predictor variables (or independent variables, $X^{-} s$ ) are given. The role of index or categorical variables is also provided. The methods by which certain data are estimated or interpolated are presented. This chapter is a guide to the data bases created during the study. The chapter also acts as a useful resource for any future modification of the data bases. 
CHAPTER 5

SELECTION OF DATA FORMAT AND CANDIDATE MODELS

\subsection{Introduction}

The statistical quality of the two alternate data bases and their formats/structures (described in Chapter 4) will be evaluated in this chapter. The results of some statistical analyses and judgment will be employed to select the best usable format for each class of highway. The results of these analyses under different groupings will be compared to identify the best grouping of data for further analyses. Some of the frequently used statistical selection criteria will be discussed in this chapter. The candidate models will be identified for the selected group(s) of the data structure.

\subsection{Ordinary Least Squares (OLS)}

The most common method of fitting a line to a set of data is the method of least squares. The method of least squares chooses a Iine/plane that makes the sum of the squares of the residuals as small as possible. Consider a dependent variable, $Y$, and set of independent varlables, $X^{\prime} s$, which are linearly related in the paramelers so that: 


$$
Y_{1}=B_{0}+\sum_{j=1}^{n} B_{j} X_{1 j}+e_{1}
$$

where,

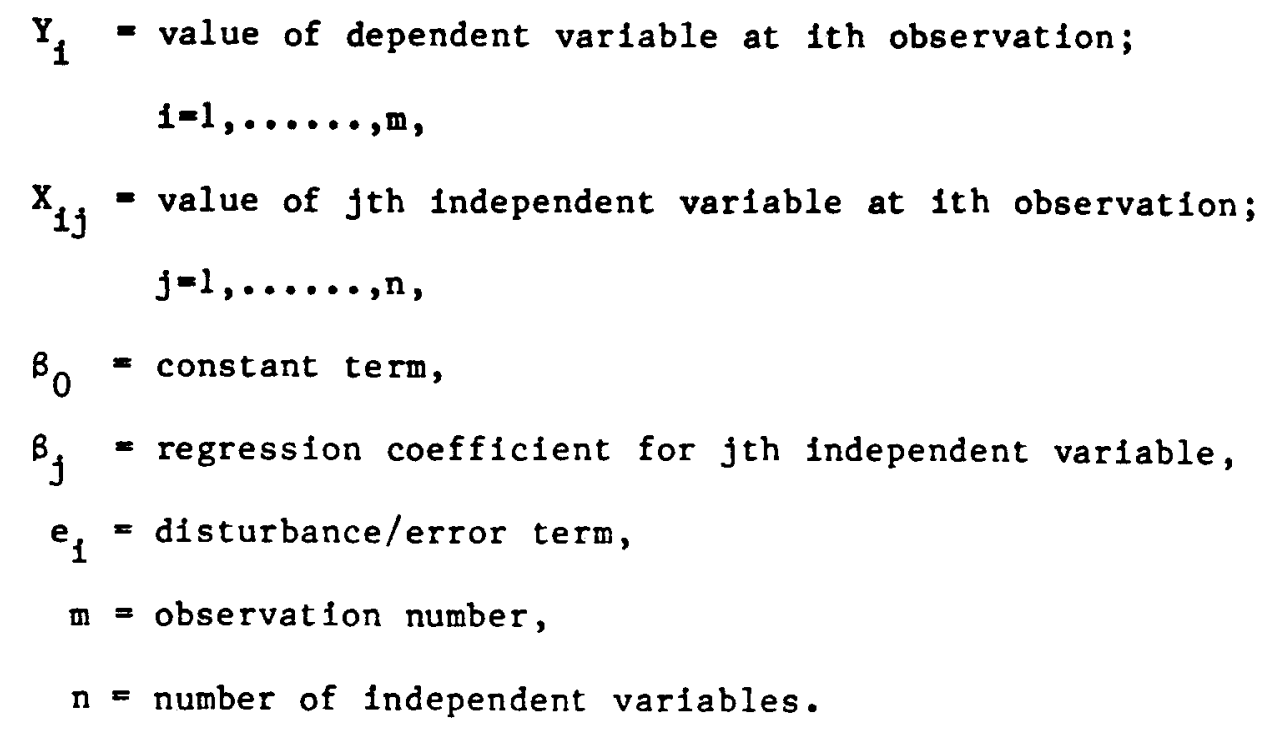

The method of ordinary least squares (OLS) assures that, under certain assumptions, the $B^{-}$s are chosen so that in a given sample, $\Sigma e_{1}^{2}$, the sum of the squared disturbance term, is as small as possible. When these assumptions are satisfied, then $B^{-} s$ have the properties of being the best linear unbiased and efficient estimator among the class of estimators for the given sample observations [Neter et al. 1985]. Some other important properties of OLS are:

1. The estimated regression line/plane will pass through the "point of means", the point $\left(\bar{Y}, \bar{X}_{2}, \ldots, \bar{X}_{n}\right)$.

2. The est 1mated errors have mean zero, $\Sigma e_{1}=0$, where $1=1$ to $\mathrm{m}$.

3. The estimated residuals are orthogonal to each 
explanatory/predictor variable: the sum of their cross products is zero.

\subsubsection{Correlation and the Regression Coefficient}

The correlation coefficient measures the strength of the linear association between two quantitative variables. For m observations on two varlables $x_{1}$ and $x_{2}$, denoted by

$$
\left(x_{11}, x_{21}\right), \cdots,\left(x_{11}, x_{21}\right), \cdots,\left(x_{1 m}, x_{2 m}\right),
$$

the correlation coefficient between $x_{1}$ and $x_{2}, r_{12}$, can be defined as

$$
r_{12}=\frac{1}{n-1} \sum_{i=1}^{m}\left[\frac{x_{11^{-x_{1}}}}{s_{x 1}}\right]\left[\frac{x_{21}-\bar{x}_{2}}{s_{x 2}}\right]
$$

Here $\bar{x}_{1}$ and $s_{x l}$ are the mean and standard deviation of the $x_{1}$ observations alone, and similarly $\bar{x}_{2}$ and $s_{x 2}$ refer to the $x_{2}$ observations.

Multicollinearity is said to exist in a regression analysis when the predictor variables or linear combinations of them are highly correlated to each other. It is well known that collinearity need not harm forecasts, even if it has harmed structural estimation, as long as it continues into the forecast period [Draper \& Smith 1981, Judge et al. 1980]. In the problem we are confronting with the socloeconomic variables, it is likely that collinearity will continue in future. But, we cannot proceed on that assumption. Consequently, adequate care has been taken by dropping varlates thought to be col11near. The dropped varfates, which really do belong to the model, may lead to specification errors with their consequent estimation blases. These estimation biases become forecasting biases when 
projected into situations where collinearfty no longer prevalls. An Important fact regarding the correlation coefficient is that, when 1ndependent variables are highly correlated, the regression coefficlent of any Independent variable depends on which other independent varlables are included in the model. For highly correlated independent variables, a regression coefficlent does not reflect any inherent effect of the particular independent variable on the dependent variable, but only a marginal or partial effect, given whatever other correlated Independent variables are included in the model. [Draper \& Smith 1981, Neter et al. 1985]

In general, when two independent variables are correlated with each other, intercorrelation or multicollinearity among them is said to exist [Neter et al. 1985]. The three important problems that arise when using highly correlated variables are:

1. Adding or deleting an independent varlable changes the regression coefficients.

2. The extra sum of squares of regression associated with an independent variable varies depending upon which independent variables are already in the model.

3. The estimated regression coefficients individually may not be statistically significant, even though a definite statistical relationship exists between the dependent variable and a set of Independent variables. 
The existence of multicollinearity does not invalidate a regression analysis, but neither is the absence of multicollinearity a validation of a particular regression model. Multicollinearity is also not a specification error [Freund \& Minton 1979]. However, in this study, the expected signs and the values of the variables in the regression equations will be carefully examined to avold the problem of multicollinearity in the choice of variables.

\subsection{Some Alternative Variable Selection Rules}

In this section, some of the most common model selection rules will be discussed. Although most of the criterla can be found in many applied statistical texts, their underlying principles are presented in this section to understand their role in model selection. Moreover, many of these criteria are difficult to understand. Therefore, this section is devoted to a simple presentation. In general, most of these rules rely on discrimination criteria, such as maximizing some modified $R^{2}$, and each one measures how well the models fit the data after some adjustment for number of variables in the equation.

\section{$5.3 .1 \mathrm{R}_{\mathrm{p}}^{2}$ Criterion}

The $R_{p}^{2}$ criterion calls for an examination of the coefficient of multiple determination $R^{2}$, to select one or several subsets of $X-$ variables. The subscript " $p$ " of $R_{p}^{2}$ suggests that there are $p$ parameters, or $p-1$ predictor variables. In this section, $R_{p}^{2}$ will be referred as $R^{2}$ - the coefficient of multiple determination. $R^{2}$ measures the proportion of the total variance accounted for regression 
relationship through the linear influence of the explanatory variables. The total sum of squares of $Y$, the dependent varlable, about its mean may be partitioned as follows:

where,

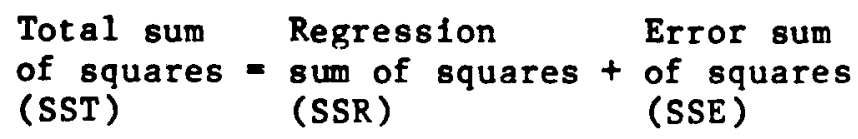

A graphical description of the partitioning of SST can be found in the literature on applied statistics (for example, Figure 3.5 of Ref. Neter, Wasserman \& Kutner [1985]). Given this decomposition of the sample variation of $\mathrm{Y}$ about its mean, the coefficient of determination is represented as

$$
R_{p}^{2}=\frac{S S R}{S S T}=1-\frac{S S E}{S S T}
$$

Since $R_{p}^{2}$ is a ratio of the sum of squares and the denominator is constant for all possible regressions, $R_{p}^{2}$ varies inversely with the error sum of squares, $\mathrm{SSE}_{\mathrm{P}}$. But $\mathrm{SSE}_{\mathrm{p}}$ can never increase as additional independent varlables are included in the model. Thus, $R_{p}^{2}$ will be at a maximum when all $\mathrm{p}-1$ potential $\mathrm{X}$-variables are included in the regression model. The reason for using the $R_{p}^{2}$ criterion with the all possible regressions approach therefore cannot be to $\max 1 m 1 z e R_{p}^{2}$. The goal is to find the point where adding more variables is not worthwhlle because it leads to only a small increase in $R_{p}^{2}$ often, this point is reached when only a limited number of $X$-variables is 
included in the regression model. Clearly, the determination of where diminishing returns occurs is a judgmental one.

\section{3 .2 Adjusted $\mathrm{R}^{2}\left(\mathrm{R}_{\mathrm{a}}^{2}\right)$ or ${ }^{\mathrm{MSE}} \mathrm{P}$ Criterion}

As a basis for model cholce, the $R^{2}$ measure has an obvious fault - It can be increased by increasing the number of explanatory variables. To take this characteristic of the measure into account, Theil [Judge et al. 1980] proposed a corrected coefficient of multiple determination $R_{a}^{2}$. The corrected or adjusted $R_{a}^{2}$ is represented as

$$
R_{a}^{2}=1-\frac{n-1}{n-p}\left(1-R^{2}\right)=1-\frac{M S E}{S S T /(n-1)}
$$

where

$$
\begin{aligned}
\mathrm{R}^{2} & =1-\mathrm{SSE} / \mathrm{SST} \\
\mathrm{SSE} & =\text { Error Sum of Squares } \\
\mathrm{MSE} & =\text { Mean Square Error }[\mathrm{SSE} /(\mathrm{n}-\mathrm{p})] \\
\mathrm{SST} & =\text { Total Sum of Squares } \\
\mathrm{n} & =\text { number of observations } \\
\mathrm{p} & =\text { number of parameters including intercept, if any. }
\end{aligned}
$$

Unlike the $R_{p}^{2}$ criterion, the $R_{a}^{2}$ criterion takes the number of parameters in the model into account through the degrees of freedom. It can be seen from equation (5.4) that $R_{a}^{2}$ decreases if and only if MSE decreases, since $S S T /(n-1)$ is fixed for the given Y-observations. Thus, $R_{a}^{2}$ or MSE are equivalent criteria. To represent the number of parameters ( $p)$ in the equation, $M S E_{p}$ is used. The $M S E_{p}$ criterion 
seeks to find either the subset of $X$ variables that minimizes MSE $p$, or one or several subsets for which $\mathrm{MSE}_{\mathrm{p}}$ is so close to the minimum that adding more varlables is not worthwhile.

The $R_{a}^{2}$ criterion will lead us to the right model "on the average". However, the expectation property alone is no guarantee of success. Also, the $R_{p}^{2}$ criterion need not be the most powerful of the criteria involving a quadratic form of the residuals that have the property: the expected value is minimized by the true model. A Iimitation of both the $R_{p}^{2}$ and $R_{a}^{2}$ measures is that they do not include a consideration of the losses associated with choosing an incorrect model. With the goal of eliminating this deficiency, next two criteria based on the mean square error estimate $-C_{p}$ and $P C--$ are presented. [Judge et al. 1980]

\subsubsection{Mallows ${ }^{-} \mathrm{C}_{\mathrm{P}}$ Criterion}

The $\mathrm{C}_{\mathrm{P}}$-criterion is concerned with the total mean squared error (MSE) of the $n$ fitted values for each of the various subset regression models. The $C_{p}$ statistic is estimated by the following equation

$$
C_{p}=\frac{S S E}{\partial^{2}}+2 p-n
$$

where

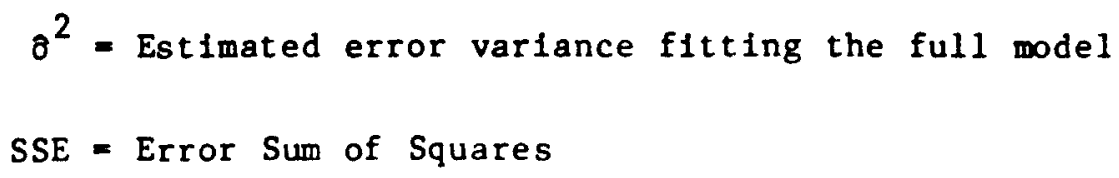




$$
\begin{aligned}
& n=\text { number of observations } \\
& p=\text { number of parameters including intercept, if any. }
\end{aligned}
$$

When the $C_{P}$ values for all possible regression models are plotted agalnst $P$, those models with little bias - measured numerically as the difference between the center of the sampling distribution and the true parameter value - will tend to fall near the line $C_{P}=P$ [Draper \& Smith 1981]. Models with substantial bias will tend to fall considerably above this line.

In using the $C_{P}$-criterion, the subsets of $X$ variables for which (1) $C_{P}$ value is small and (2) the $C_{P}$ value is near $P$, are considered for the model. Sets of $X$ varlables with small $C_{P}$ values have a small total mean squared error and, when the $C_{P}$ value is also near $P$, the bias of the regression model is sma11. It may sometimes occur that the regression model based on the subset of $X$ varlables with the smallest $C_{P}$ value involves substantial bias. In that case, one may at times prefer a regression model on a somewhat larger subset of $X$ variables for which the $C_{P}$ value is slightly larger and that does not involve a substantial blas component. Thus, one should look for a regression with a low $C_{P}$ value about equal to $P$. When the choice is not clear-cut, then it is a matter of personal judgment whether one prefers a blased equation or an equation with more parameters.

\subsubsection{Amemiya's Prediction Criterion (PC)}

To include a consideration of the losses associated with choosing an Incorrect model, Amemfya [1980] developed a criterion based on the 
unconditional mean squared prediction error as the risk function. This results in a modification of $\mathrm{R}^{2}$ that corrects the degrees of freedom to a greater extent than Theil's $R_{a}^{2}$. Amemiya's criterion is known as the Prediction Criterion (PC), which can also be used as a selection criterion in a nonlinear regression model. In terms of $R^{2}$, PC can be represented as [Amemiya 1980]:

$$
P C=\frac{n+P}{n-P}\left(1-R^{2}\right)
$$

where the definitions of $n, P$ and $R^{2}$ are same as under equations 5.4 and 5.5. The PC has a higher penalty for adding variables than Theil's adjusted $R^{2}$. In using the PC criterion, one seeks to identify subsets of $X$ variables for which the $P C$ value is small.

\subsubsection{Akaike's Information Criterion (AIC)}

The information measure or criterion seeks to incorporate in the model selection the divergent conslderations of accuracy of estimation and the "best" approximation to reality. Thus use of this criterion Involves a statistic that incorporates a measure of the precision of the estimate and a measure of the rule of parsimony, to be defined in Section 5.6, in the parameterization of a statistical model.

The classical method of maximum likelihood could be represented to Identify a statistical model by maximizing an estimate of the generalized entropy, or the expected log 11 kelihood, of the model being fitted [Akaike 1981]. The $\log$ maximum-likelihood is a biased estimate of the expected log likelihood of the model with parameters determined 
by the method of maximum-likelihood. The correction of this bias leads to an information criterion, AIC - the Akalke Information Criterion [Akaike 1981] -- defined by

$$
\text { AIC }=(-2) \text { In (maximum likelihood) }
$$

+2 (number of free parameters within the model)

Therefore, many important statistical problems could be solved simply by specifying a set of models and estimating the parameters within each model by the method of maximum likelihood and then selecting a model with the minimum value of AIC. This procedure, called the minimum AIC estimation procedure, produces a new approach to statistical model identification that could provide a unifying view of the problems. AIC has an important advantage, in that it can be used not only in regression analysis, but also to choose among varlous linear constraints in the general parametric model. The AIC can be used in any model where the likelihood function is specified. For the OLS (see equation 5.1 ), equation 5.7 reduces to

$$
A I C=(n) \ln (S S E / n)+2 p
$$

where the definitions of $n$ and $p$ remain the same as equation 5.4 . The above equation shows explicitly the penalty for increasing the number of parameters. In using the AIC criterion, the user needs to identify subsets of $X$ variables for which AIC value is small. 


\subsection{Multiple Linear Regression Analysis}

In this section, the results of some analyses are presented. Each analysis is discussed briefly, together with some interpretations and criteria for selection. The RSQUARE procedure [SAS/Statistics 1985] was used to select the the optimal subsets of predictor variables in a multiple regression analysis. The procedure finds the subsets of predictor variables that best predict a response variable by linear regression in the given sample. The $R^{2}$ statistic is the criterion for selecting subsets. PROC RSQUARE can also efficlently evaluate all possible subset regressions and print the models in decreasing order of $R^{2}$ magnitude within each subset size. Other statistics (for example, $R_{a}^{2}, P C, R M S E, A I C$ and $C_{p}$ ) are also available for comparing subsets of different sizes. The definitions of $R_{p}^{2}, R_{a}^{2}, P C, A I C$ and $C_{P}$ have been provided in earlier sections. RMSE is the square root of Mean Square Error (MSE) and is calculate by the following equation

$$
\text { RMSE }=\sqrt{\frac{S S E}{n-p}}
$$

These statistics, as well as estimated regression coefficients, were computed through RSQUARE procedure of SAS [SAS/Statistics 1985]. While RSQUARE is a useful tool for exploratory model bullding, no statistical method can be relied on to 1dentify the "true" model. Effective model bullding requires background theory to suggest relevant predictors and plausible functional forms for the model. 


\subsubsection{Interstate Highways}

A11 four formats of data bases - Raw, Raw E\&L, Density and Density E\&L (for definitions, see Section 4.5.1) - are analyzed using segment AADT and county \& clty average AADT. The layout of analysis is shown in Figure 5.1. This layout is formulated under the following assumptions: (1) traffic growth is primarily associated with the functional class of highway and (11) CTYGR, MFUNC and GREGION have a secondary effect on traffic growth. These effects are shown as Phases 1 and 2 in Figure 5.1. In Phase 1, the data bases are grouped and analyzed by rural, urban location and both together. Phase 1 will compare the results of analysis for the three groupings of highways. In Phase 2, the data bases are grouped and analyzed by the categorical variables CTYGR, MFUNC and GREGION (for definitions, see Section 4.6.1) under each combination of Phase 1. CTYGR, MFUNC and GREGION have 5,4 and 3 levels, respectively. Phase 2 will compare the results within the groupings of each of the three categorlcal variables and between the groups. Moreover, a comparison between the two main forms of data bases by the1r four formats will be done at each phase. Also, a comparison among the four formats within each of the main data forms can be made. The results of Phase 2 will also identify any misclassified observations.

The best models without any inconsistency in the sign of regression coefficlent of all possible combinations are identified for different number of variables in the equation. For example, a positive sign is expected for population on the assumption of increase of AADT because of population 1ncrease. Appendix Tables $\mathrm{Cl}$ and $\mathrm{C} 3$ present the 


\section{Data Structure}

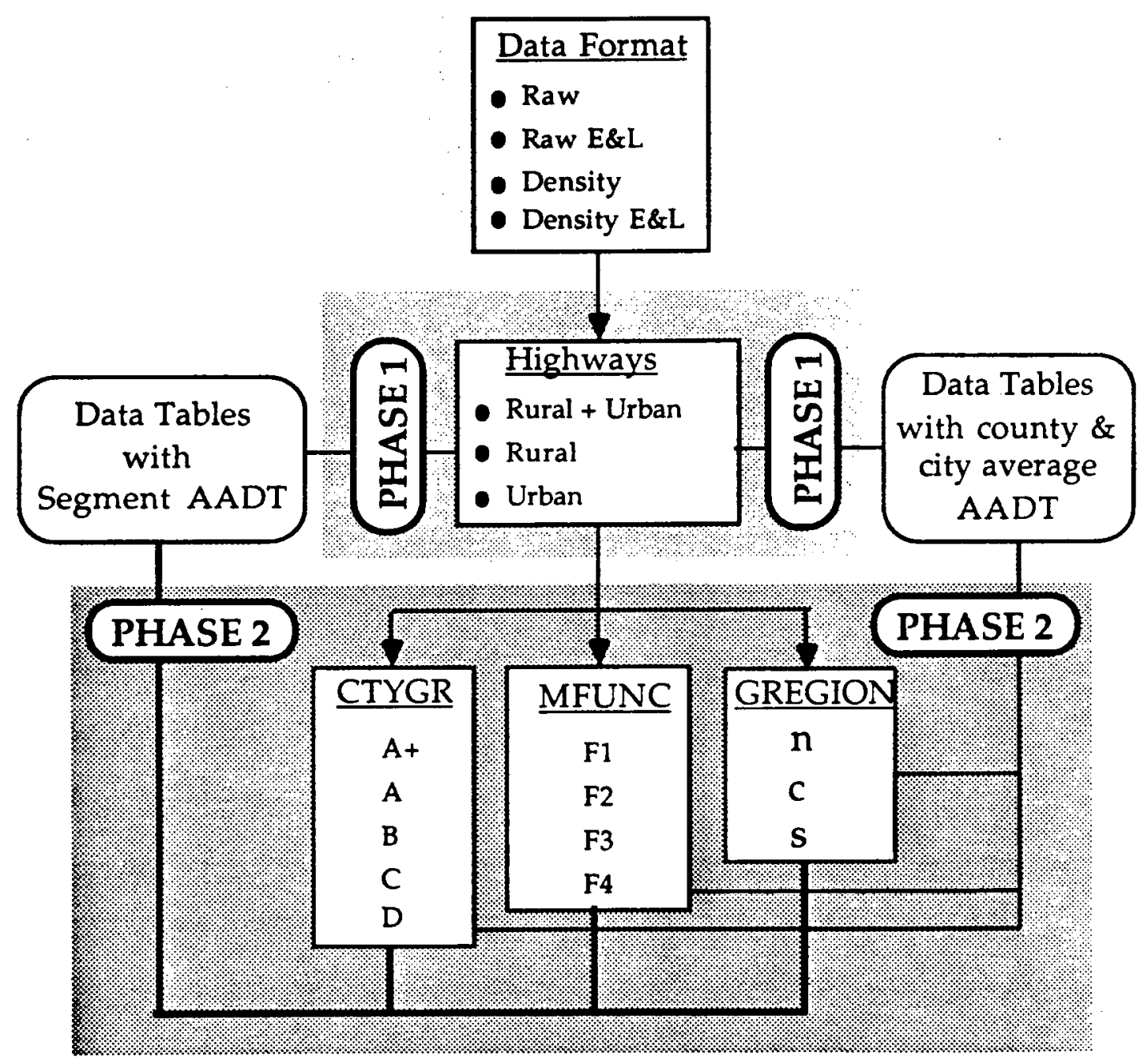

Figure 5.1: Layout of Analysis for Interstate Highways 
results of raw and density format data bases for county or clty average AADT. The results of earlier and later year versions of raw and density data bases for the same are shown in Tables C2 and C4. These results are based on a linear functional form of predictor variables. The first column in these tables presents the combination/case and the number of observations, N. The second and the ninth columns present the number of variables $(p-1)$ and the corresponding variable names in the equation. The second through elghth columns show criterion measures and statistics. The last (tenth) column contains the values of regression coefficients corresponding to the variables in the ninth column. Similar data tables result from using segment AADT, but these tables are not shown.

\subsubsection{Noninterstate Urban State Highways}

The layout of analysis for noninterstate urban state highways is shown in Figure 5.2. This layout is formulated under the following assumptions: (i) the functional class of highway is the most important determinant of traffic growth, (1i) CTYGR, based on city population, has the next greatest impact on traffic growth and (1i1) MFUNC or GREGION has a tertiary effect on traffic growth. With the data bases, these three levels of influences are represented as Phases 1, 2 and 3. In Phase 1, the data bases are grouped and analyzed by the following functional classes:
a. Urban Principal Arterial, and
b. Urban Minor Arterlal and Collector

In Phase 2, the data bases are grouped and analyzed by the categorfcal 


\section{Data Structure}

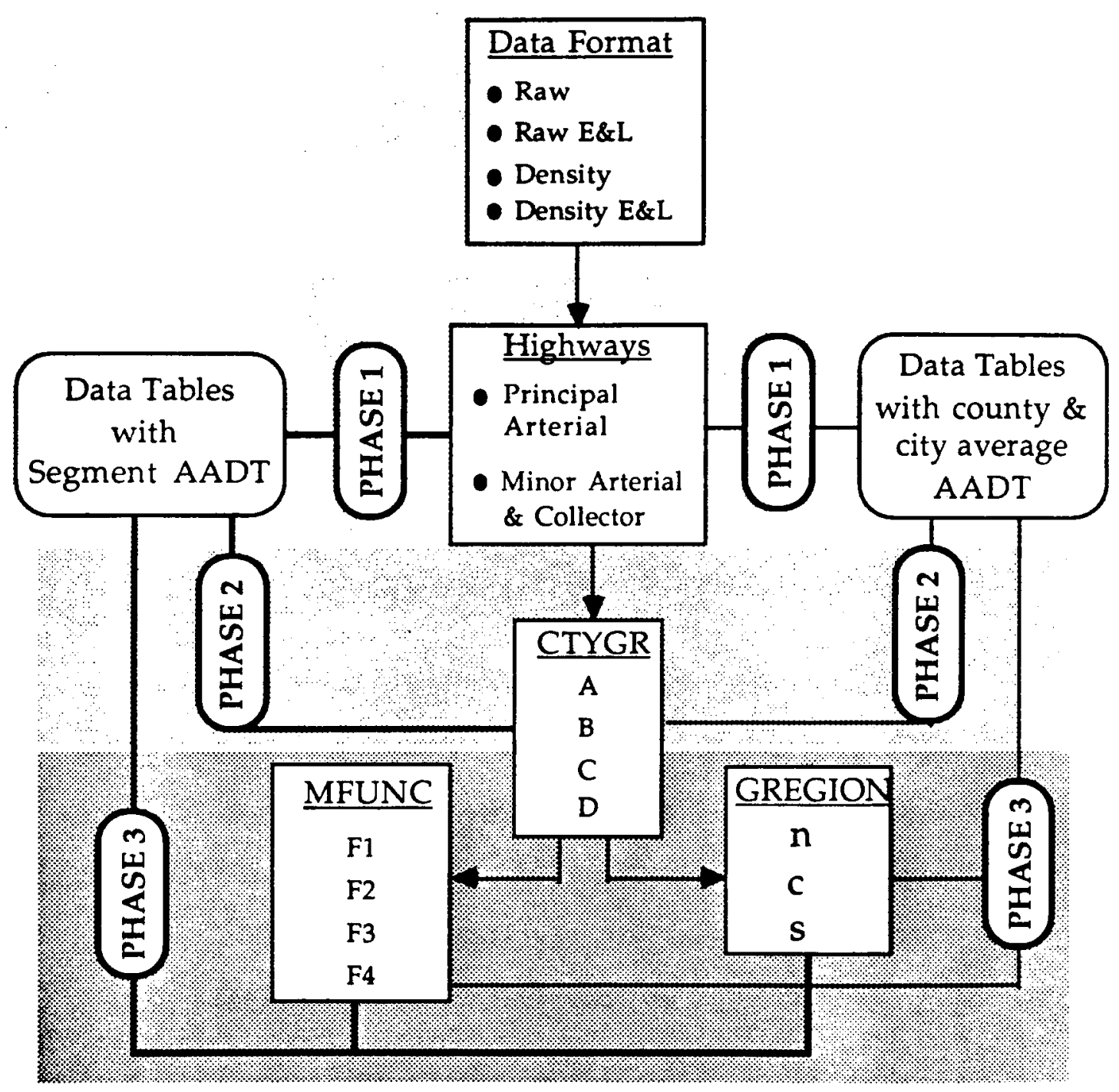

Figure 5.2: Layout of Analysis for Non-Interstate Urban State Highways 
variable CTYGR, with four levels under each level of Phase 1 . In Phase 3, the variables MFUNC and GREGION, with 4 and 3 levels, respectively, are grouped and analyzed with each combination of Phases 1 and 2. For interstate highways, CTYGR, MFUNC and GREGION are all addressed in Phase 2. As for interstate highways, the results at different phases will be used to compare the different format of data bases and to identify the misclassified observations in the data bases.

The best models without any inconsistency in regression coefficient are identified for all possible combinations of variables in the equation for the two main functional classes of highways. Appendix Tables $C 5$ and $C 7$ present the results of raw and density format data bases for county or city average AADT on urban principal arterials. The results of the earlier and later year version of raw and density data bases for the same highway class are shown in Tables $\mathrm{C} 6$ and $\mathrm{C} 8$. An analysis by GREGION is done for city and county average raw data bases. The results of north $(n), \operatorname{central}(c)$ and $\operatorname{south}(s)$ are presented in Appendix Table C5.1. The statistical quality of results by GREGION and CTYGR (see Tables C5 and C5.1) does not provide an edge for one over the other. Table C5.1 also shows the results of pooled CTYGR A, $B$ and $C$ for the south region. One main reason for this pooling is that only five observations resulted in county and city average data base for each of the three city groups in the south region. This pooling has Improved some statistics of the models - for example, $C_{P}$ value in Table C5.1 with respect to that in Table C5. Some other considerations in this pooling will be addressed in Section 5.6.2. 
Tables C9 to C12 (Tables C9\&C11 for raw and density format; Tables $\mathrm{C} 10$ and $\mathrm{C} 12$ for raw E\&L and density E\&L format) present the results of urban minor arterial and collector for county and city average AADT. All these results are based on linear functional form. The column descriptions of the tables follow the descriptions, provided in the latter part of Section 5.4.1.

\subsection{Data Format and Group Selection}

Several alternative selection rules and the values of the relevant statistics have been presented in sections 5.3 and 5.4 .1 . It is not the intention of this study to use any single criterion as the sole basis for selecting a model. It can be argued from this study's results that, in general, most of the selection rules supplement each other in the selection of the regressor variable(s). If one reviews the procedures on variable selection of statistical model buflding, there appears to have been a strong desire by many to designate some single statistic or index to gauge the "goodness" of a statistical model. One natural candidate for this honor would be the coefficient of multiple determination, $\mathrm{R}^{2}$.

In the following subsections, the values of $R^{2}$ for the different data formats w111 be compared to each other to select the most promising and practical data format(s). The values of $R^{2}$ will play a major role in this selection process. In the final selection of data format, the criteria of establishing high $R^{2}$ will not be considered exclusively. Because $R^{2}$ is a relative quantity, it shows how large the Regression Sum of Squares (SSR) is relative to the Total Sum of 
Squares (SST). In some situations, data may be scattered and a large $\mathrm{R}^{2}$ may not show a good $\mathrm{fit}$. In more controlled situations, a small $\mathrm{R}^{2}$ may show a good fit [Freund \& Minton 1979]. A high $\mathrm{R}^{2}$ value does not necessarily mean a good fit. In that respect, the selection process will occasionally be alded by the other statistics $-R_{a}^{2}, P C, R M S E$, AIC \& $C_{p}$. In addition to data format selection, subsets or groups of data will be 1dentified to do further analysis from the selected data format.

\subsubsection{Interstate Highways}

The maximum values of $\mathrm{R}^{2}$ for the "all data" formats and their subsets are 1dentified in Tables $\mathrm{Cl}$ to $\mathrm{C} 4$ for county and city average AADT data tables. Similar Identification was carried out for segment AADT data tables. Table 5.1 shows the maximum $R^{2}$ value, in percent, for all possible comblnations of Interstate highways. These results have evolved without any inconsistency in the regression coefficients. The number of variables that corresponds to each of the $R^{2}$ values in Table 5.1 is shown in Table 5.2. By observing the results of Table 5.1 with an almost equal number of variables in the equation (see Table 5.2), one can observe the superiority of the county/city average data bases over the segment data bases. The marginal improvement in the maximum $R^{2}$ value for all four formats of data tables is shown in Table 5.3. This table shows that there is a significant improvement In most of the cases.

For the "raw" format, there is 12.03 percent increase in $R^{2}$ value, when all data for interstate have been analyzed together. An 
Table 5.1: Maximum $\mathrm{R}^{2}$ value for Interstate Highways

\begin{tabular}{|c|c|c|c|c|c|c|c|c|c|c|}
\hline \multirow{2}{*}{ CASE } & \multirow{2}{*}{$\begin{array}{l}\mathrm{C} \\
\mathrm{T} \\
\mathrm{Y} \\
\mathrm{GR}\end{array}$} & \multirow{2}{*}{$\begin{array}{ll}M & G \\
F & R \\
\text { U } & \text { E } \\
\text { NC } & \text { GION }\end{array}$} & \multicolumn{4}{|c|}{ Segment Data base } & \multicolumn{4}{|c|}{ County/City Average Data base } \\
\hline & & & Raw & $\begin{array}{l}\text { Raw } \\
\text { E\&L }\end{array}$ & Density & $\begin{array}{l}\text { Density } \\
\text { E\&L }\end{array}$ & Raw & $\begin{array}{l}\text { Raw } \\
\text { E\&L }\end{array}$ & Dens1ty & $\begin{array}{c}\text { Density } \\
\text { E\&L }\end{array}$ \\
\hline & ALL & & 40.26 & 41.00 & 50.14 & 51.95 & 52.29 & 51.70 & 49.42 & 49.56 \\
\hline RURAL & $\begin{array}{l}\text { At } \\
\text { A } \\
\text { B } \\
\text { C } \\
\text { D }\end{array}$ & & $\begin{array}{r}26.30 \\
28.70 \\
60.31 \\
35.60 \\
9.28\end{array}$ & $\begin{array}{l}27.58 \\
23.89 \\
55.02 \\
34.05 \\
X\end{array}$ & $\begin{array}{r}49.56 \\
39.19 \\
61.32 \\
37.16 \\
9.35\end{array}$ & $\begin{array}{l}55.25 \\
32.17 \\
63.09 \\
36.39 \\
x\end{array}$ & $\begin{array}{l}40.28 \\
29.98 \\
64.15 \\
67.45 \\
12.90\end{array}$ & $\begin{array}{l}41.59 \\
20.72 \\
63.32 \\
65.84 \\
X\end{array}$ & $\begin{array}{l}33.16 \\
32.74 \\
53.13 \\
63.16 \\
21.32\end{array}$ & $\begin{array}{l}35.46 \\
25.78 \\
49.05 \\
63.04 \\
12.69\end{array}$ \\
\hline URBAN & $\begin{array}{l}\text { F1 } \\
\text { F2 } \\
\text { F3 } \\
\text { P4 }\end{array}$ & & $\begin{array}{l}53.53 \\
72.55 \\
28.62 \\
23.69\end{array}$ & $\begin{array}{l}54.79 \\
72.47 \\
28.42 \\
26.49\end{array}$ & $\begin{array}{l}59.72 \\
70.83 \\
28.60 \\
40.59\end{array}$ & $\begin{array}{l}60.96 \\
72.54 \\
28.54 \\
45.72\end{array}$ & $\begin{array}{l}52.98 \\
75.59 \\
52.55 \\
44.66\end{array}$ & $\begin{array}{l}54.53 \\
74.63 \\
52.14 \\
46.48\end{array}$ & $\begin{array}{l}63.53 \\
71.76 \\
52.59 \\
40.45\end{array}$ & $\begin{array}{l}65.40 \\
73.26 \\
52.13 \\
43.06\end{array}$ \\
\hline & & $\begin{array}{l}n \\
\mathbf{c} \\
\mathbf{s}\end{array}$ & $\begin{array}{l}37.87 \\
54.58 \\
63.42\end{array}$ & $\begin{array}{l}35.83 \\
55.65 \\
66.12\end{array}$ & $\begin{array}{l}37.17 \\
55.86 \\
57.68\end{array}$ & $\begin{array}{l}32.53 \\
55.84 \\
58.51\end{array}$ & $\begin{array}{l}47.31 \\
73.08 \\
61.77\end{array}$ & $\begin{array}{l}45.32 \\
75.91 \\
64.68\end{array}$ & $\begin{array}{l}48.98 \\
73.47 \\
56.67\end{array}$ & $\begin{array}{l}47.19 \\
76.30 \\
57.67\end{array}$ \\
\hline & ALL & & 47.59 & 48.95 & 46.54 & 48.08 & 40.60 & 37.14 & 40.19 & 36.61 \\
\hline & At & & $\begin{array}{l}49.62 \\
51.25\end{array}$ & $\begin{array}{l}52.38 \\
44.91\end{array}$ & $\begin{array}{l}44.88 \\
47.12\end{array}$ & $\begin{array}{l}47.99 \\
41.75\end{array}$ & $\begin{array}{l}66.09 \\
47.06\end{array}$ & $\begin{array}{l}68.62 \\
38.31\end{array}$ & $\begin{array}{l}51.92 \\
42.54\end{array}$ & $\begin{array}{l}53.19 \\
37.65\end{array}$ \\
\hline & $\begin{array}{l}\text { B } \\
\text { C }\end{array}$ & & $\begin{array}{l}30.87 \\
24.77\end{array}$ & $\begin{array}{l}36.14 \\
30.28\end{array}$ & $\begin{array}{l}44.88 \\
15.30\end{array}$ & $\begin{array}{l}57.82 \\
18.63\end{array}$ & $\begin{array}{l}30.79 \\
75.06\end{array}$ & $\begin{array}{l}52.51 \\
81.76\end{array}$ & $\begin{array}{l}70.61 \\
91.69\end{array}$ & $\begin{array}{l}65.86 \\
94.81\end{array}$ \\
\hline & D & & 7.49 & $\mathrm{x}$ & 7.49 & $x$ & 9.67 & $x$ & 16.37 & $x$ \\
\hline RURAL & $\begin{array}{l}F 1 \\
F 2 \\
F 3 \\
F 4\end{array}$ & & $\begin{array}{l}40.38 \\
66.45 \\
27.06 \\
43.18\end{array}$ & $\begin{array}{l}41.42 \\
64.89 \\
28.40 \\
45.78\end{array}$ & $\begin{array}{l}46.12 \\
64.28 \\
27.04 \\
43.61\end{array}$ & $\begin{array}{l}48.91 \\
65.36 \\
28.40 \\
45.84\end{array}$ & $\begin{array}{l}37.26 \\
76.77 \\
50.41 \\
37.75\end{array}$ & $\begin{array}{l}37.81 \\
75.27 \\
54.03 \\
35.41\end{array}$ & $\begin{array}{l}48.42 \\
72.20 \\
54.07 \\
39.00\end{array}$ & $\begin{array}{l}50.85 \\
73.27 \\
53.97 \\
35.36\end{array}$ \\
\hline & & $\begin{array}{l}n \\
c \\
\end{array}$ & $\begin{array}{l}29.75 \\
40.73 \\
42.61\end{array}$ & $\begin{array}{l}25.58 \\
44.76 \\
44.78\end{array}$ & $\begin{array}{l}27.51 \\
40.79 \\
48.98\end{array}$ & $\begin{array}{l}25.58 \\
44.49 \\
49.66\end{array}$ & $\begin{array}{l}30.64 \\
51.12 \\
41.76\end{array}$ & $\begin{array}{l}27.32 \\
56.21 \\
44.69\end{array}$ & $\begin{array}{l}30.60 \\
51.49 \\
55.11\end{array}$ & $\begin{array}{l}27.31 \\
51.21 \\
52.46\end{array}$ \\
\hline
\end{tabular}


Table 5.1, continued

\begin{tabular}{|c|c|c|c|c|c|c|c|c|c|c|}
\hline \multirow{2}{*}{ CASE } & \multirow{2}{*}{\multicolumn{2}{|c|}{$\mid \begin{array}{lll}C & M & G \\
T & F & R \\
Y & U & E \\
G R & \text { NC } & \text { GION }\end{array}$}} & \multicolumn{4}{|c|}{ Segment Dala base } & \multicolumn{4}{|c|}{ County/C1ty Average Data base } \\
\hline & & & Raw & $\begin{array}{l}\text { Raw } \\
\text { E\&L }\end{array}$ & Densily & $\begin{array}{l}\text { Density } \\
\text { E\&L }\end{array}$ & Raw & $\begin{array}{l}\text { Raw } \\
\text { E\&L }\end{array}$ & Densily & $\begin{array}{c}\text { Densily } \\
\text { E\&L }\end{array}$ \\
\hline & ALL & & 32.05 & 32.37 & 51.58 & 54.97 & 55.57 & 53.63 & 50.75 & 50.86 \\
\hline URBAN & $\begin{array}{l}A+ \\
A \\
B \\
C \\
D \\
-\end{array}$ & & $\begin{array}{r}2.80 \\
15.26 \\
75.20 \\
36.68 \\
35.60\end{array}$ & $\begin{array}{c}X \\
X \\
80.00 \\
32.30 \\
38.84\end{array}$ & $\begin{array}{l}68.91 \\
22.43 \\
74.72 \\
35.37 \\
58.64\end{array}$ & $\begin{array}{l}71.12 \\
16.84 \\
80.77 \\
33.38 \\
60.72\end{array}$ & $\begin{array}{r}7.48 \\
20.13 \\
80.83 \\
67.27 \\
34.63\end{array}$ & $\begin{array}{l}6.52 \\
X \\
89.46 \\
65.48 \\
31.18\end{array}$ & $\begin{array}{l}38.41 \\
19.93 \\
80.26 \\
60.72 \\
60.64\end{array}$ & $\begin{array}{l}11.36 \\
12.66 \\
90.74 \\
60.06 \\
58.57\end{array}$ \\
\hline & $\begin{array}{l}\text { F1 } \\
\text { F2 } \\
\text { F3 } \\
\text { F4 }\end{array}$ & & $\begin{array}{l}49.86 \\
70.08 \\
63.76 \\
14.09\end{array}$ & $\begin{array}{l}47.56 \\
70.54 \\
62.91 \\
17.51\end{array}$ & $\begin{array}{l}49.65 \\
70.40 \\
65.21 \\
46.69\end{array}$ & $\begin{array}{l}47.55 \\
71.38 \\
70.15 \\
52.95\end{array}$ & $\begin{array}{l}91.57 \\
79.47 \\
67.77 \\
45.26\end{array}$ & $\begin{array}{l}(\star \star) \\
79.65 \\
72.48 \\
47.07\end{array}$ & $\begin{array}{l}93.28 \\
78.14 \\
67.74 \\
39.09\end{array}$ & $\begin{array}{l}(\star \star) \\
78.07 \\
76.94 \\
41.78\end{array}$ \\
\hline & & $\begin{array}{l}n \\
c \\
s\end{array}$ & $\begin{array}{l}36.05 \\
61.43 \\
62.78\end{array}$ & $\begin{array}{l}32.10 \\
61.01 \\
66.48\end{array}$ & $\begin{array}{l}44.09 \\
66.46 \\
58.60\end{array}$ & $\begin{array}{l}38.11 \\
64.11 \\
63.93\end{array}$ & $\begin{array}{l}50.03 \\
84.52 \\
81.34\end{array}$ & $\begin{array}{l}46.73 \\
88.34 \\
85.23\end{array}$ & $\begin{array}{l}51.63 \\
86.81 \\
77.05\end{array}$ & $\begin{array}{l}48.63 \\
88.52 \\
82.94\end{array}$ \\
\hline
\end{tabular}


Table 5.2: Number of Variables for Maximum $R^{2}$ value for Interstate Highways

\begin{tabular}{|c|c|c|c|c|c|c|c|c|c|c|c|}
\hline \multirow{2}{*}{ CASE } & \multirow{2}{*}{$\begin{array}{ll}\text { C } & \\
\text { T } & 1 \\
Y & 1 \\
\text { GR }\end{array}$} & \multirow{2}{*}{$\begin{array}{l}\text { G } \\
\text { R } \\
\text { E } \\
\text { GION }\end{array}$} & \multicolumn{4}{|c|}{ Segment Data base } & \multicolumn{5}{|c|}{ County/C1Ly Average Data base } \\
\hline & & & Raw & $\begin{array}{l}\text { Raw } \\
\text { E\&L }\end{array}$ & Density & $\begin{array}{c}\text { Density } \\
\text { E\&L }\end{array}$ & Raw & $\begin{array}{l}\text { Raw } \\
\text { E\&L }\end{array}$ & Density & $\begin{array}{c}\text { Density } \\
\text { E\&L }\end{array}$ & \\
\hline & ALL & & 2 & 2 & 4 & 3 & 2 & 2 & & 2 & 2 \\
\hline RURAL & $\begin{array}{l}\mathrm{A}+ \\
\mathrm{A} \\
\mathrm{B} \\
\mathrm{C} \\
\mathrm{D}\end{array}$ & & $\begin{array}{l}2 \\
2 \\
3 \\
2 \\
1\end{array}$ & $\begin{array}{l}2 \\
2 \\
2 \\
2 \\
x\end{array}$ & $\begin{array}{l}4 \\
3 \\
3 \\
2 \\
1\end{array}$ & $\begin{array}{l}3 \\
2 \\
3 \\
2 \\
x\end{array}$ & $\begin{array}{l}2 \\
2 \\
4 \\
3 \\
1\end{array}$ & $\begin{array}{l}2 \\
2 \\
3 \\
2 \\
x\end{array}$ & & $\begin{array}{l}1 \\
2 \\
3 \\
2 \\
2\end{array}$ & $\begin{array}{l}1 \\
2 \\
2 \\
2 \\
1\end{array}$ \\
\hline $\mid+\infty$ & $\begin{array}{l}F 1 \\
F 2 \\
F 3 \\
F 4\end{array}$ & $-\infty-1$ & $\begin{array}{l}2 \\
4 \\
2 \\
2 \\
\end{array}$ & $\begin{array}{l}2 \\
2 \\
2 \\
2\end{array}$ & $\begin{array}{l}2 \\
3 \\
2 \\
3\end{array}$ & $\begin{array}{l}2 \\
2 \\
2 \\
2\end{array}$ & $\begin{array}{l}2 \\
4 \\
2 \\
2\end{array}$ & $\begin{array}{l}2 \\
2 \\
2 \\
2\end{array}$ & & $\begin{array}{l}2 \\
3 \\
2 \\
2\end{array}$ & \begin{tabular}{l|}
2 \\
2 \\
2 \\
2
\end{tabular} \\
\hline & & $\begin{array}{l}n \\
\mathrm{c} \\
\mathrm{s}\end{array}$ & $\begin{array}{l}2 \\
3 \\
2\end{array}$ & $\begin{array}{l}2 \\
3 \\
2\end{array}$ & $\begin{array}{l}3 \\
3 \\
2\end{array}$ & $\begin{array}{l}2 \\
2 \\
2 \\
\end{array}$ & $\begin{array}{l}2 \\
3 \\
2\end{array}$ & $\begin{array}{l}2 \\
3 \\
1\end{array}$ & & $\begin{array}{l}2 \\
3 \\
2\end{array}$ & 2 \\
\hline & $A L L$ & & 4 & 4 & 3 & 3 & 3 & 3 & & 3 & \\
\hline & $\begin{array}{l}\mathrm{A}^{+} \\
\mathrm{A} \\
\mathrm{B} \\
\mathrm{C} \\
\mathrm{D}\end{array}$ & & $\begin{array}{l}4 \\
2 \\
2 \\
3 \\
1\end{array}$ & $\begin{array}{l}4 \\
2 \\
1 \\
3 \\
x\end{array}$ & $\begin{array}{l}2 \\
3 \\
2 \\
3 \\
1\end{array}$ & $\begin{array}{l}2 \\
2 \\
2 \\
2 \\
X\end{array}$ & $\begin{array}{l}4 \\
2 \\
2 \\
2 \\
1\end{array}$ & $\begin{array}{l}3 \\
2 \\
2 \\
2 \\
x\end{array}$ & & $\begin{array}{l}2 \\
2 \\
3 \\
4 \\
2\end{array}$ & $\begin{array}{l}2 \\
2 \\
3 \\
x\end{array}$ \\
\hline KLRAL & $\begin{array}{l}\text { F1 } \\
\text { F2 } \\
\text { F3 } \\
\text { F4 }\end{array}$ & . & $\begin{array}{l}2 \\
4 \\
2 \\
3\end{array}$ & $\begin{array}{l}2 \\
2 \\
2 \\
4\end{array}$ & $\begin{array}{l}2 \\
2 \\
2 \\
3\end{array}$ & $\begin{array}{l}2 \\
2 \\
2 \\
3\end{array}$ & $\begin{array}{l}2 \\
4 \\
2 \\
3\end{array}$ & $\begin{array}{l}2 \\
3 \\
2 \\
3\end{array}$ & $\begin{array}{l}2 \\
2 \\
2 \\
3\end{array}$ & $\begin{array}{l}2 \\
2 \\
2 \\
3\end{array}$ & $\begin{array}{l}2 \\
2 \\
2 \\
2\end{array}$ \\
\hline & & $\begin{array}{l}n \\
c \\
5\end{array}$ & $\begin{array}{l}3 \\
3 \\
2\end{array}$ & $\begin{array}{l}2 \\
4 \\
2\end{array}$ & $\begin{array}{l}2 \\
3 \\
2\end{array}$ & $\begin{array}{l}2 \\
3 \\
2\end{array}$ & $\begin{array}{l}2 \\
3 \\
2\end{array}$ & $\begin{array}{l}2 \\
3 \\
1\end{array}$ & & $\begin{array}{l}2 \\
3 \\
2\end{array}$ & $\begin{array}{l}2 \\
2 \\
1\end{array}$ \\
\hline
\end{tabular}


Table 5.2, continued

\begin{tabular}{|c|c|c|c|c|c|c|c|c|c|c|}
\hline \multirow{2}{*}{ CASE } & \multirow{2}{*}{$\begin{array}{ll}C & H \\
T & F \\
Y & U \\
G R & N\end{array}$} & \multirow{2}{*}{$\begin{array}{l}\text { G } \\
\text { R } \\
\text { E } \\
\text { GION }\end{array}$} & \multicolumn{4}{|c|}{ Segment Data base } & \multicolumn{4}{|c|}{ County/C1ty Average Data base } \\
\hline & & & Raw & $\begin{array}{l}\text { Raw } \\
\text { E\&L }\end{array}$ & Density & $\begin{array}{c}\text { Density } \\
\text { E\&L }\end{array}$ & Raw & $\begin{array}{l}\text { Raw } \\
\text { E\&L }\end{array}$ & Densfiy & $\begin{array}{c}\text { Density } \\
\text { E\&L }\end{array}$ \\
\hline & ALI & & 2 & & 4 & 3 & 2 & 2 & 2 & 2 \\
\hline & $\begin{array}{l}A+ \\
A \\
B \\
C \\
D\end{array}$ & & $\begin{array}{l}1 \\
1 \\
3 \\
2 \\
1\end{array}$ & & $\begin{array}{l}x \\
x \\
3 \\
2 \\
1\end{array}$ & $\begin{array}{l}3 \\
1 \\
4 \\
2 \\
1\end{array}$ & $\begin{array}{l}1 \\
1 \\
3 \\
3 \\
2\end{array}$ & $\begin{array}{l}1 \\
x \\
3 \\
2 \\
1\end{array}$ & $\begin{array}{l}2 \\
1 \\
3 \\
2 \\
2\end{array}$ & $\begin{array}{l}1 \\
1 \\
4 \\
2 \\
1\end{array}$ \\
\hline |URBAN & $\begin{array}{rr} & \\
F 1 \\
F 2 \\
F 3 \\
F 4\end{array}$ & $-\infty$ & $\begin{array}{l}2 \\
2 \\
2 \\
2\end{array}$ & 2 & $\begin{array}{l}2 \\
2 \\
2 \\
3\end{array}$ & $\begin{array}{l}1 \\
2 \\
2 \\
2\end{array}$ & $\begin{array}{l}2 \\
2 \\
2 \\
2\end{array}$ & $\begin{array}{l}X \\
2 \\
2 \\
2\end{array}$ & $\begin{array}{l}2 \\
2 \\
2 \\
2\end{array}$ & $\begin{array}{l}x \\
2 \\
2 \\
2\end{array}$ \\
\hline & & $\begin{array}{l}\mathrm{n} \\
\mathrm{c} \\
\mathrm{g}\end{array}$ & $\begin{array}{l}2 \\
2 \\
2\end{array}$ & 2 & $\begin{array}{l}2 \\
3 \\
2\end{array}$ & $\begin{array}{l}2 \\
3 \\
2\end{array}$ & $\begin{array}{l}2 \\
3 \\
2\end{array}$ & $\begin{array}{l}2 \\
2 \\
2\end{array}$ & $\begin{array}{l}2 \\
3 \\
2\end{array}$ & $\begin{array}{l}2 \\
2 \\
2\end{array}$ \\
\hline
\end{tabular}


Table 5.3: Marginal Improvement in Maximum $R^{2}$ value of County/City
Average AADT over Segment AADT for Interstate Highways

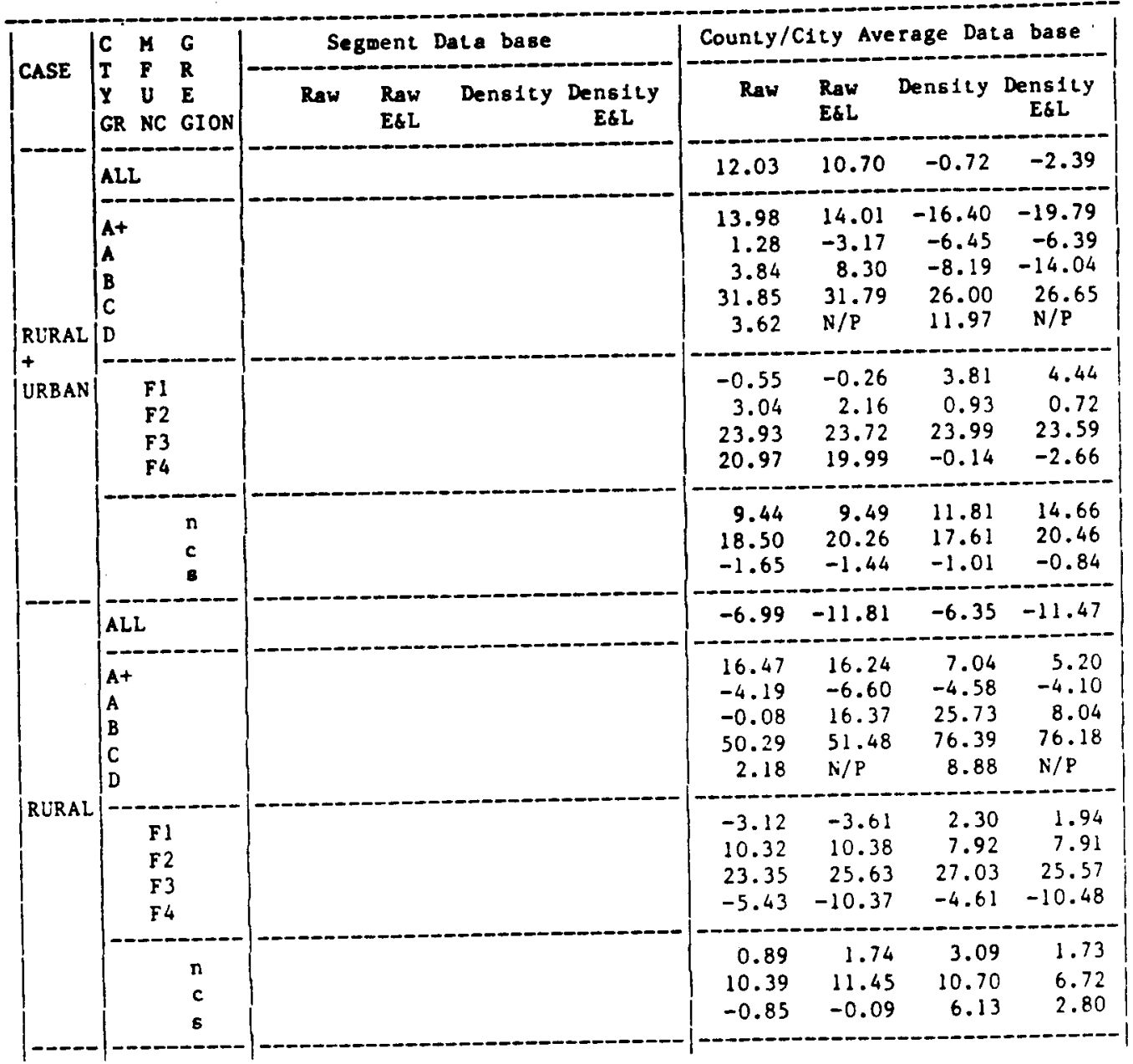


Table 5.3, continued

\begin{tabular}{|c|c|c|c|c|c|c|c|c|c|}
\hline \multirow{2}{*}{ CASE } & \multirow{2}{*}{$\mid \begin{array}{ll}C & M \\
T & F \\
Y & V \\
\text { GR } & \text { NC }\end{array}$} & \multirow{2}{*}{$\begin{array}{l}\text { G } \\
\text { R } \\
\text { E } \\
\text { GION }\end{array}$} & \multicolumn{3}{|c|}{ Segwent Data base } & \multicolumn{4}{|c|}{ County/C1ty Average Data base } \\
\hline & & & $\begin{array}{ll}\text { Raw } & \text { Raw } \\
& \text { E\&L }\end{array}$ & Density & $\begin{array}{c}\text { Dens 1ty } \\
\text { E\& L }\end{array}$ & Raw & $\begin{array}{l}\text { Raw } \\
\text { E\&L }\end{array}$ & Density & $\begin{array}{c}\text { Density } \\
\text { E\&L }\end{array}$ \\
\hline & ALL & & & & & 23.52 & 21.26 & -0.83 & -4.11 \\
\hline & $\begin{array}{l}A+ \\
A \\
B \\
C \\
D\end{array}$ & & & & & $\begin{array}{r}4.68 \\
4.87 \\
5.63 \\
30.59 \\
-0.97\end{array}$ & $\begin{array}{l}N / P \\
N / P \\
9.46 \\
33.18 \\
-7.66\end{array}$ & $\begin{array}{l}\mathrm{N} / \mathrm{P} \\
-2.50 \\
5.54 \\
25.35 \\
2.00\end{array}$ & $\begin{array}{l}N / P \\
-4.18 \\
9.97 \\
26.68 \\
-2.15\end{array}$ \\
\hline URBAN & $\begin{array}{l}\text { F1 } \\
\text { F2 } \\
\text { F3 } \\
\text { F4 }\end{array}$ & --- & $-\infty-\infty,-\infty$ & - & -...- & $\begin{array}{r}41.71 \\
9.39 \\
4.01 \\
31.17\end{array}$ & $\begin{array}{l}\text { N/P } \\
9.11 \\
9.57 \\
29.56\end{array}$ & $\begin{array}{r}43.63 \\
7.74 \\
2.53 \\
-7.60\end{array}$ & $\begin{array}{c}\mathrm{N} / \mathrm{P} \\
6.69 \\
6.79 \\
-11.17\end{array}$ \\
\hline & & $\begin{array}{l}n \\
c \\
8\end{array}$ & & & & $\begin{array}{l}13.98 \\
23.09 \\
18.56\end{array}$ & $\begin{array}{l}14.63 \\
27.33 \\
18.75\end{array}$ & $\begin{array}{r}7.54 \\
20.35 \\
18.45\end{array}$ & $\begin{array}{l}10.52 \\
24.41 \\
19.01\end{array}$ \\
\hline
\end{tabular}

Note: $N / P=$ Not Possible 
Increase of 23.52 percent $R^{2}$ value is observed for the "urban-all" case, but a decrease of 6.99 percent $R^{2}$ value is found for the "rural-all" case. The values of PC, RMSE, AIC and $C_{p}$ for the "ruralall" case with $R^{2}$ of 40.60 percent $\left(R_{a}^{2}=39.62\right.$ percent $)$ and 3 variables in the equation are $0.6201,7344,3315.4$ and 24.73 respectively for county/city average data table (see Appendix Table Cl). On the other hand, the values of PC, RMSE, AIC and $C_{p}$ for the same with $R^{2}$ of 47.59 percent $\left(R_{a}^{2}=47.23\right.$ percent) and 4 variables in the equation are $0.5330,9300,10879$ and 33.90 for segment AADT data table. All these statistics except $P C$ establish that the results from county and city average AADT data table are better for rural interstates in spite of a small decrease in $\mathrm{R}^{2}$ value.

A further examination of Table 5.1 reveals that the results have usually been improved when the data tables have been grouped by CTYGR, MFUNC and GREGION. Table 4.6 has shown the number of observations for different groupings according to the layout analysis presented in Figure 5.1. The analysis by CTYGR shows that data for 'CTYGR D' are not good for the "rural" and "ruralturban" cases (see Table 5.1). The data for 'CTYGR At' are also not successful in producing statistically good results. By observing the results of Table 5.1 and its "parent" (Appendix Tables C.l to C.4), In general, the results by GREGION are better than those by CTYGR and MFUNC.

Tables $5.4,5.5$ and 5.6 are produced from Table 5.1 to answer the following questions:

1. Are the improvements in raw E\&L, density and density E\&L formats about raw format enough to justify their selection? (Table 5.4) 
Table 5.4: Marginal Improvement in Maximum $R^{2}$ value of Raw E\&L, Density and Denstty E\&L Formats over Raw Formal for Interslate Highways

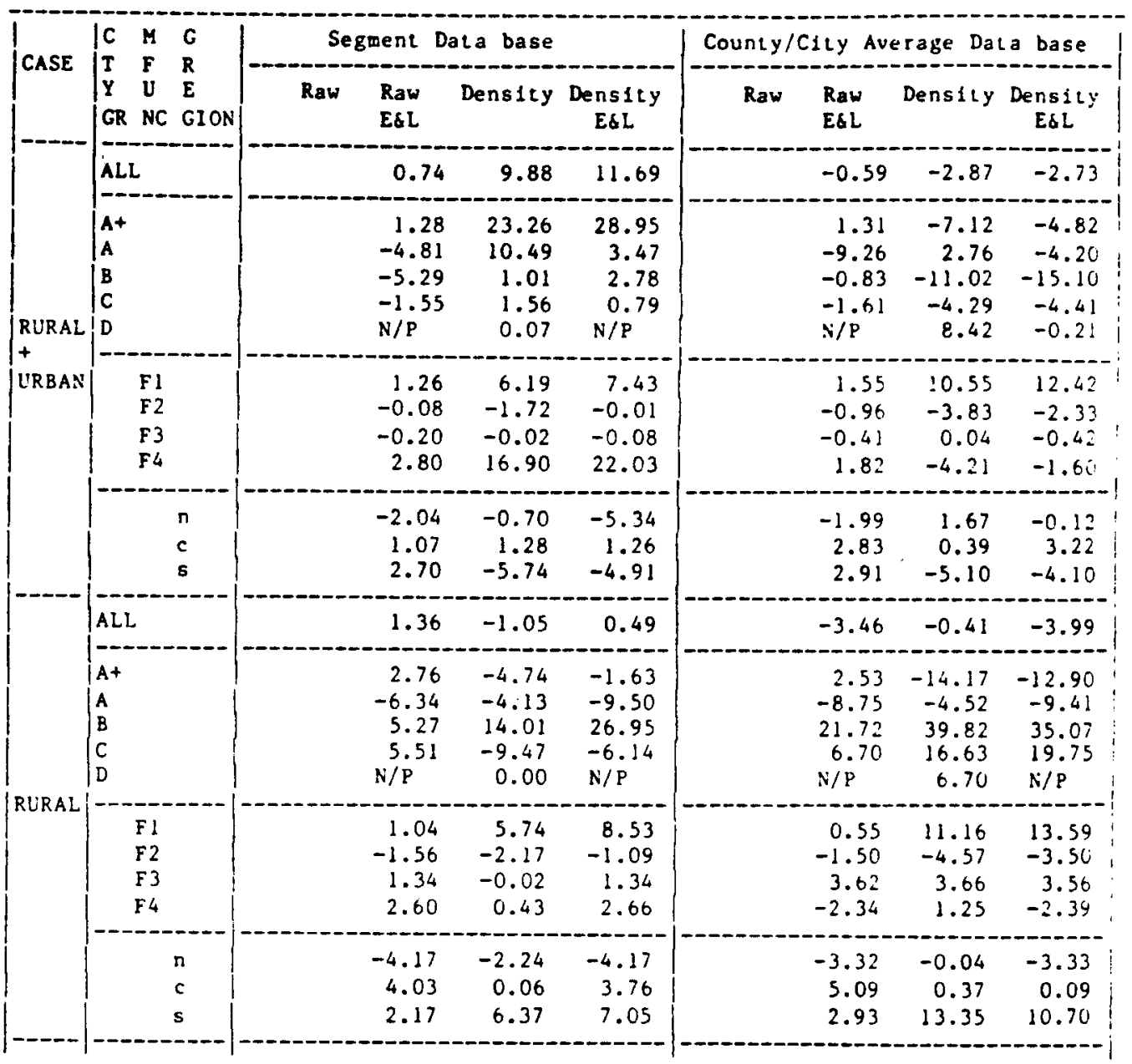


Table 5.4, continued

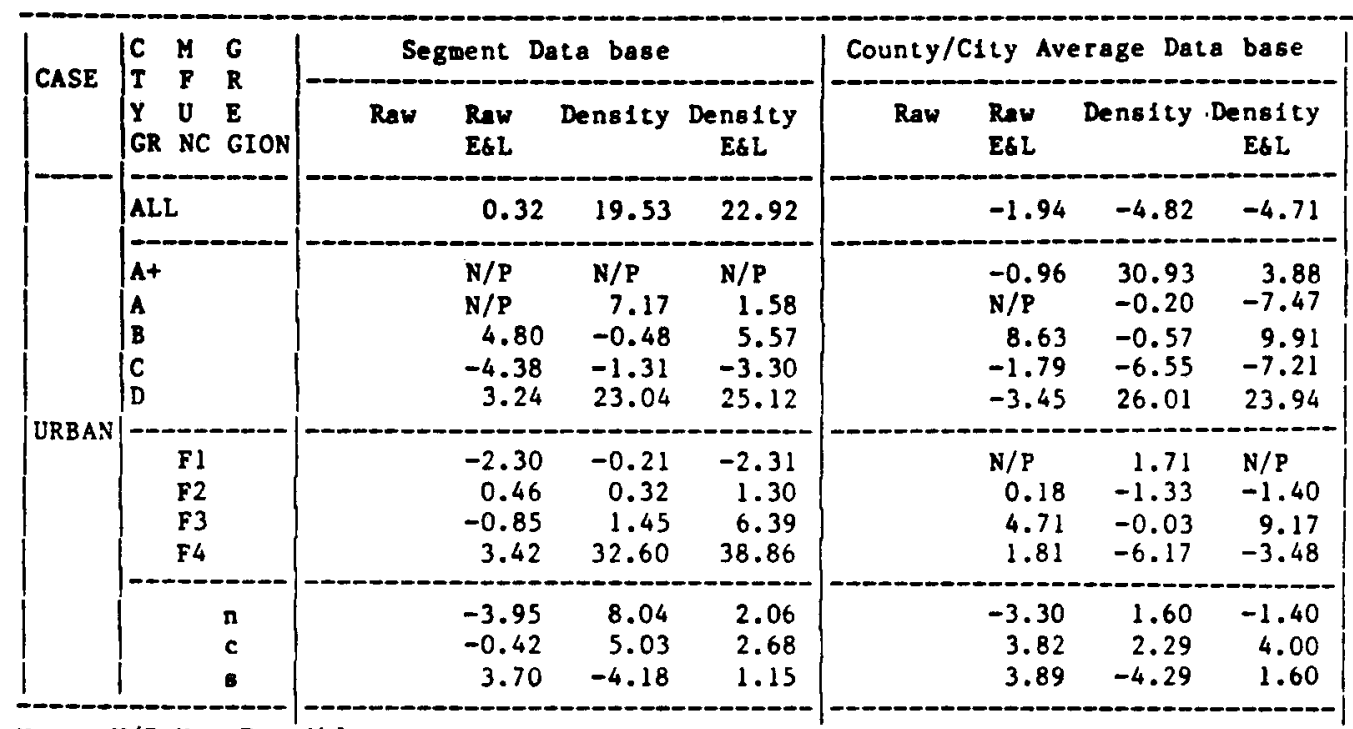

Note: N/P=Not Possible 
Table 5.5: Marginal Improvement in Maximum $R^{2}$ value of Earlier \& Later (E\&L) Year Data Format for Interstate Highways

\begin{tabular}{|c|c|c|c|c|c|c|c|c|c|c|}
\hline \multirow{2}{*}{ CASE } & \multirow{2}{*}{\begin{tabular}{|ll}
$\mathrm{C}$ & $\mathrm{M}$ \\
$\mathrm{T}$ & $\mathrm{F}$ \\
$\mathrm{Y}$ & $\mathrm{U}$ \\
$\mathrm{GR}$ & $\mathrm{NC}$
\end{tabular}} & \multirow{2}{*}{$\begin{array}{l}G \\
R \\
E \\
\text { GION }\end{array}$} & \multicolumn{4}{|c|}{ Segment Data base } & \multicolumn{4}{|c|}{ County/City Average Data base } \\
\hline & & & Raw & $\begin{array}{l}\text { Raw } \\
\text { E\&I }\end{array}$ & Density & $\begin{array}{c}\text { Density } \\
\text { E\&L }\end{array}$ & Raw & $\begin{array}{l}\text { Raw } \\
\text { E\&L }\end{array}$ & Density & $\begin{array}{l}\text { Density } \\
\text { E\&L }\end{array}$ \\
\hline & ALL & & & 0.74 & & 1.81 & & -0.59 & & 0.14 \\
\hline RURAL & $\begin{array}{l}\mathrm{A}+ \\
\mathrm{A} \\
\mathrm{B} \\
\mathrm{C} \\
\mathrm{D}\end{array}$ & & & $\begin{array}{l}1.28 \\
-4.81 \\
-5.29 \\
-1.55 \\
\text { N/P }\end{array}$ & & $\begin{array}{r}5.69 \\
-7.02 \\
1.77 \\
-0.77 \\
\mathrm{~N} / \mathrm{P}\end{array}$ & & $\begin{array}{l}1.31 \\
-9.26 \\
-0.83 \\
-1.61 \\
\mathrm{~N} / \mathrm{P}\end{array}$ & & $\begin{array}{r}2.30 \\
-6.96 \\
-4.08 \\
-0.12 \\
-8.63\end{array}$ \\
\hline URBAN & $\begin{array}{l}\text { F1 } \\
\text { F2 } \\
\text { F3 } \\
\text { F4 }\end{array}$ & & & $\begin{array}{r}1.26 \\
-0.08 \\
-0.20 \\
2.80\end{array}$ & & $\begin{array}{r}1.24 \\
1.71 \\
-0.06 \\
5.13\end{array}$ & & $\begin{array}{r}1.55 \\
-0.96 \\
-0.41 \\
1.82\end{array}$ & & $\begin{array}{r}1.87 \\
1.50 \\
-0.46 \\
2.61\end{array}$ \\
\hline & & $\begin{array}{l}\mathrm{n} \\
\mathrm{c} \\
\mathrm{s}\end{array}$ & & $\begin{array}{r}-2.04 \\
1.07 \\
2.70\end{array}$ & & $\begin{array}{r}-4.64 \\
-0.02 \\
0.83\end{array}$ & & $\begin{array}{r}-1.99 \\
2.83 \\
2.91\end{array}$ & & $\begin{array}{r}-1.79 \\
2.83 \\
1.00\end{array}$ \\
\hline & ALL & & & 1.36 & & 1.54 & & -3.46 & & -3.58 \\
\hline & $\begin{array}{l}\text { At } \\
A \\
\text { B } \\
\text { C } \\
\text { D }\end{array}$ & & & $\begin{array}{r}2.76 \\
-6.34 \\
5.27 \\
5.51 \\
\mathrm{~N} / \mathrm{P}\end{array}$ & & $\begin{array}{c}3.11 \\
-5.37 \\
12.94 \\
3.33 \\
\mathrm{~N} / \mathrm{P}\end{array}$ & & $\begin{array}{r}2.53 \\
-8.75 \\
21.72 \\
6.70 \\
\mathrm{~N} / \mathrm{P}\end{array}$ & & $\begin{array}{r}1.27 \\
-4.89 \\
-4.75 \\
3.12 \\
x / p\end{array}$ \\
\hline RURAL & $\begin{array}{l}--- \\
\text { F1 } \\
\text { F2 } \\
\text { F3 } \\
\text { F4 }\end{array}$ & & & $\begin{array}{r}1.04 \\
-1.56 \\
1.34 \\
2.60 \\
\end{array}$ & & $\begin{array}{l}2.79 \\
1.08 \\
1.36 \\
2.23 \\
\end{array}$ & & $\begin{array}{r}0.55 \\
-1.50 \\
3.62 \\
-2.34\end{array}$ & & $\begin{array}{r}2.43 \\
1.07 \\
-0.10 \\
-3.64 \\
-\end{array}$ \\
\hline & & $\begin{array}{l}\mathrm{n} \\
\mathrm{c} \\
\mathrm{s}\end{array}$ & & $\begin{array}{r}-4.17 \\
4.03 \\
2.17\end{array}$ & & $\begin{array}{r}-1.93 \\
3.70 \\
0.68\end{array}$ & & $\begin{array}{r}-3.32 \\
5.09 \\
2.93\end{array}$ & & $\begin{array}{l}-3.29 \\
-0.28 \\
-2.65\end{array}$ \\
\hline
\end{tabular}


Table 5.5, continued

\begin{tabular}{|c|c|c|c|c|c|c|c|c|c|c|}
\hline \multirow{2}{*}{ CASE } & \multirow{2}{*}{ 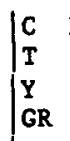 } & \multirow{2}{*}{$\begin{array}{ll}M & G \\
F & R \\
\text { U } & E \\
\text { NC } & \text { GION }\end{array}$} & \multicolumn{4}{|c|}{ Segment Data base } & \multicolumn{4}{|c|}{ County/City Average Data base } \\
\hline & & & Raw & $\begin{array}{l}\text { Raw } \\
\text { E\&L }\end{array}$ & Density & $\begin{array}{c}\text { Density } \\
\text { E\&L }\end{array}$ & Raw & $\begin{array}{l}\text { Raw } \\
\text { E\&L }\end{array}$ & Densily & $\begin{array}{c}\text { Density } \\
\text { E\&L }\end{array}$ \\
\hline & ALL & & & 0.3 & & 3.39 & & -1.94 & & 0.11 \\
\hline & $\begin{array}{l}\text { At } \\
A \\
B \\
C \\
D\end{array}$ & & & $\begin{array}{l}N / P \\
N / P \\
4.8 \\
-4.3 \\
3.2\end{array}$ & & $\begin{array}{r}2.21 \\
-5.59 \\
6.05 \\
-1.99 \\
2.08\end{array}$ & & $\begin{array}{l}-0.96 \\
N / P \\
8.63 \\
-1.79 \\
-3.45\end{array}$ & & $\begin{array}{r}-27.05 \\
-7.27 \\
10.48 \\
-0.66 \\
-2.07\end{array}$ \\
\hline URBAN & $\begin{array}{l}\text { F1 } \\
\text { F2 } \\
\text { F3 } \\
\text { F4 }\end{array}$ & $-m$ & & $\begin{array}{r}-2.3 \\
0.4 \\
-0.8 \\
3.4\end{array}$ & & $\begin{array}{r}-2.10 \\
0.98 \\
4.94 \\
6.26\end{array}$ & & $\begin{array}{l}N / P \\
0.18 \\
4.71 \\
1.81\end{array}$ & & $\begin{array}{l}N / P \\
-0.07 \\
9.20 \\
2.69\end{array}$ \\
\hline & & $\begin{array}{l}\mathrm{n} \\
\mathrm{c} \\
\mathrm{s}\end{array}$ & & $\begin{array}{r}-3.9 \\
-0.4 \\
3.7\end{array}$ & & $\begin{array}{r}-5.98 \\
-2.35 \\
5.33\end{array}$ & & $\begin{array}{r}-3.30 \\
3.82 \\
3.89\end{array}$ & & $\begin{array}{r}-3.00 \\
1.71 \\
5.89\end{array}$ \\
\hline
\end{tabular}

Note: $N / P=$ Not Possible 
Table 5.6: Marginal Improvement in Maximum $R^{2}$ value of Density Format over Raw Format for Interstate Highways

\begin{tabular}{|c|c|c|c|c|c|c|c|c|c|c|}
\hline \multirow{2}{*}{ CASE } & \multirow{2}{*}{$\mid \begin{array}{ll}C & M \\
T & F \\
Y & U \\
G R & N C\end{array}$} & \multirow{2}{*}{$\begin{array}{l}\text { G } \\
\text { R } \\
\text { E } \\
\text { GION }\end{array}$} & \multicolumn{4}{|c|}{ Segment Data base } & \multicolumn{4}{|c|}{ County/City Average Dala base } \\
\hline & & & Raw & $\begin{array}{l}\text { Raw } \\
\text { E\&L }\end{array}$ & Density & $\begin{array}{l}\text { Density } \\
\text { E\&L }\end{array}$ & Raw & $\begin{array}{l}\text { Raw } \\
\text { E\&L }\end{array}$ & Density & $\begin{array}{c}\text { Density } \\
\text { E\&L }\end{array}$ \\
\hline & ALL & & & & 9.88 & 10.95 & & & -2.87 & -2.14 \\
\hline RURAL & $\begin{array}{l}\text { At } \\
\text { A } \\
B \\
\text { C } \\
\text { D }\end{array}$ & & & & $\begin{array}{r}23.26 \\
10.49 \\
1.01 \\
1.56 \\
0.07\end{array}$ & $\begin{array}{r}27.67 \\
8.28 \\
8.07 \\
2.34 \\
N / P\end{array}$ & & & $\begin{array}{r}-7.12 \\
2.76 \\
-11.02 \\
-4.29 \\
8.42\end{array}$ & $\begin{array}{r}-6.13 \\
5.06 \\
-14.27 \\
-2.80 \\
N / P\end{array}$ \\
\hline URBAN & $\begin{array}{l}\text { F1 } \\
\text { F2 } \\
\text { F3 } \\
\text { F4 }\end{array}$ & & & & $\begin{array}{r}6.19 \\
-1.72 \\
-0.02 \\
16.90\end{array}$ & $\begin{array}{r}6.17 \\
0.07 \\
0.12 \\
19.23\end{array}$ & & & $\begin{array}{r}10.55 \\
-3.83 \\
0.04 \\
-4.21\end{array}$ & $\begin{array}{l}10.87 \\
-1.37 \\
-0.01 \\
-3.42\end{array}$ \\
\hline & & $\begin{array}{l}\mathrm{n} \\
\mathrm{c} \\
\mathrm{s}\end{array}$ & & & $\begin{array}{r}-0.70 \\
1.28 \\
-5.74\end{array}$ & $\begin{array}{r}-3.30 \\
0.19 \\
-7.61\end{array}$ & & & $\begin{array}{r}1.67 \\
0.39 \\
-5.10\end{array}$ & $\begin{array}{r}1.87 \\
0.39 \\
-7.01\end{array}$ \\
\hline & ALL & & & & -1.05 & -0.87 & & & -0.41 & -0.53 \\
\hline & $\begin{array}{l}\text { At } \\
\text { A } \\
\text { B } \\
\text { C } \\
\text { D }\end{array}$ & & & & $\begin{array}{r}-4.74 \\
-4.13 \\
14.01 \\
-9.47 \\
0.00\end{array}$ & $\begin{array}{r}-4.39 \\
-3.16 \\
21.68 \\
-11.65 \\
\text { N/P }\end{array}$ & & & $\begin{array}{r}-14.17 \\
-4.52 \\
39.82 \\
16.63 \\
6.70\end{array}$ & $\begin{array}{r}-15.43 \\
-0.66 \\
13.35 \\
13.05 \\
N / P\end{array}$ \\
\hline RURAL & $\begin{array}{l}\text { F1 } \\
\text { F2 } \\
\text { F3 } \\
\text { F4 }\end{array}$ & & & & $\begin{array}{r}5.74 \\
-2.17 \\
-0.02 \\
0.43\end{array}$ & $\begin{array}{l}7.49 \\
0.47 \\
0.00 \\
0.06\end{array}$ & & & $\begin{array}{r}11.16 \\
-4.57 \\
3.66 \\
1.25\end{array}$ & $\begin{array}{l}13.04 \\
-2.00 \\
-0.06 \\
-0.05\end{array}$ \\
\hline & & $\begin{array}{l}n \\
\mathrm{c} \\
\mathrm{s}\end{array}$ & & & $\begin{array}{r}-2.24 \\
0.06 \\
6.37\end{array}$ & $\begin{array}{r}0.00 \\
-0.27 \\
4.88\end{array}$ & & & $\begin{array}{r}-0.04 \\
0.37 \\
13.35\end{array}$ & $\begin{array}{r}-0.01 \\
-5.00 \\
7.77\end{array}$ \\
\hline
\end{tabular}


Table 5.6, continued

\begin{tabular}{|c|c|c|c|c|c|c|c|c|c|c|}
\hline \multirow{2}{*}{ CASE } & \multirow{2}{*}{$\mid \begin{array}{ll}C & M \\
T & F \\
Y & U \\
G R & N C\end{array}$} & \multirow{2}{*}{$\begin{array}{l}\text { G } \\
\text { R } \\
\text { E } \\
\text { GION }\end{array}$} & \multicolumn{4}{|c|}{ Segment Data base } & \multicolumn{4}{|c|}{ County/C1ty Average Data base } \\
\hline & & & Raw & $\begin{array}{l}\text { Raw } \\
\text { E\&L }\end{array}$ & Density & $\begin{array}{c}\text { Density } \\
\text { E\&L }\end{array}$ & Raw & $\begin{array}{l}\text { Raw } \\
\text { E\&L }\end{array}$ & Dens 1Ly & $\begin{array}{c}\text { Dens Ity } \\
\text { E\&L }\end{array}$ \\
\hline 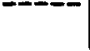 & ALL & & & & 19.53 & 22.60 & & & -4.82 & -2.77 \\
\hline & $\begin{array}{l}\text { At } \\
A \\
B \\
C \\
D\end{array}$ & & & & $\begin{array}{l}N / P \\
7.17 \\
-0.48 \\
-1.31 \\
23.04\end{array}$ & $\begin{array}{l}N / P \\
N / P \\
0.77 \\
1.08 \\
21.88\end{array}$ & & & $\begin{array}{l}30.93 \\
-0.20 \\
-0.57 \\
-6.55 \\
26.01\end{array}$ & $\begin{array}{l}4.84 \\
\mathrm{~N} / \mathrm{P} \\
1.28 \\
-5.42 \\
27.39\end{array}$ \\
\hline URBAN & $\begin{array}{r}F 1 \\
F 2 \\
F 3 \\
F 4\end{array}$ & $\cdots$ & & & $\begin{array}{r}-0.21 \\
0.32 \\
1.45 \\
32.60\end{array}$ & $\begin{array}{r}-0.01 \\
0.84 \\
7.24 \\
35.44\end{array}$ & & & $\begin{array}{r}1.71 \\
-1.33 \\
-0.03 \\
-6.17\end{array}$ & $\begin{array}{l}\text { N/P } \\
-1.58 \\
4.46 \\
-5.29\end{array}$ \\
\hline & & $\begin{array}{l}n \\
c \\
s\end{array}$ & & & $\begin{array}{r}8.04 \\
5.03 \\
-4.18\end{array}$ & $\begin{array}{r}6.01 \\
3.10 \\
-2.55\end{array}$ & & & $\begin{array}{r}1.60 \\
2.29 \\
-4.29\end{array}$ & $\begin{array}{r}1.90 \\
0.18 \\
-2.29\end{array}$ \\
\hline
\end{tabular}


2. Does the Earlier and Later (E\&L) year data format 1mprove the results significantly? (Table 5.5 )

3. Is the density data format better than the raw format? (Table 5.6)

The results of Table 5.4 and the other related statistics of Appendix Tables Cl to C4 do not show any significant change in the quality of the results except the AIC values, which are more directly related to the number of observations (see equation 5.7). Tables C2 and C4 show that AIC values for the "Earlier \& Later" format are much lower than the AIC values of the non-E\&L format. Thus statistics 1ike AIC will be more appropriate to compare the different models under a data format that keeps their observations the same. For "GREGION $c$ " of rural highways under the county/city average data base, there is a 5.09 percent increase in $R^{2}$ value for the "raw E\&L" format (see Table 5.4). The associated statistics from Tables $\mathrm{Cl}$ and $\mathrm{C} 2$ for this case of "raw" and "raw E\&L" formats are as follows:

\begin{tabular}{|c|c|c|c|c|c|c|}
\hline Format & $\mathrm{R}^{2}$ & $R_{a}^{2}$ & PC & AIC & $C_{p}$ & $\begin{array}{c}\text { No of } \\
\text { Vartables }\end{array}$ \\
\hline $\begin{array}{l}\text { Raw } \\
\text { Raw E\&L }\end{array}$ & $\begin{array}{l}51.12 \\
56.21(*)\end{array}$ & $\begin{array}{l}49.19 \\
51.16(*)\end{array}$ & $\begin{array}{l}0.5402(*) \\
0.5726\end{array}$ & $\begin{array}{l}1411.9 \\
528.1(*)\end{array}$ & $\begin{array}{l}10.01 \\
3.83(*)\end{array}$ & $\begin{array}{l}3 \\
3\end{array}$ \\
\hline
\end{tabular}

$(*)=$ the better values.

Despite this small improvement of "raw E\&L" format over "raw" format, the "raw E\&L" format will not be considered because of the simplicity of "raw" format when compared with the "raw E\&L" format. Similar arguments could be made from the results of Tables $\mathrm{Cl}$ to $\mathrm{C} 4$ and Tables 5.5 and 5.6 to justify the raw data format over the others.

By considering the results of Table 5.1 and Table C.1, the interstate highways will be further analyzed with the "raw" format of the 
county/city average data base. The analysis will carried out by Phase 1 (rural interstate, urban interstate and the1r combined form) and by Phase 2 with GREGION.

\subsubsection{Urban Principal Arterial}

The analysis for urban principal arterials follows Figure 5.2 (see Section 5.4.2). Table 5.7 presents the maximum $\mathrm{R}^{2}$ value, in percent, corresponding to the layout of Figure 5.2. The results of county/city average data base are obtained from Appendix Tables C.5 to C.8. The number of variables for each value of $\mathrm{R}^{2}$ in Table 5.7 is shown in Table 5.8. In most of the cases, the number of variables entered in equations for each combination under the different data formats of the segment and county/city average data bases are almost equal. Thus the number of variables do not pose a problem in comparing different data bases. Table 5.7 shows a clear margin of improvement for county/city average data base over its counterpart -- the segment data base. One reason behind this improvement is the elimination of variation in AADT within the sections of urban principal arterials in a city or county for county/city average data base.

Tables 5.9, 5.10, 5.11 and 5.12 result from Table 5.7 and show the extent of improvement in maximum $R^{2}$ value for

1. County/city average data bases over segment data bases (Table 5.9),

2. Raw E\&L, density and density E\&L formats over raw format (Table 5.10),

3. Earlier and Later (E\&L) formats over non-E\&L formats (Table 5.11), and 
Table 5.7: Maximum $\mathrm{R}^{2}$ value for Urban Principal Arterial

\begin{tabular}{|c|c|c|c|c|c|c|c|c|c|c|}
\hline \multirow{2}{*}{$\begin{array}{l}C \\
T \\
Y \\
G R\end{array}$} & \multirow{2}{*}{$\begin{array}{l}M \\
E \\
\text { U } \\
\text { NC }\end{array}$} & \multirow{2}{*}{$\begin{array}{l}\mathbf{G} \\
\mathbf{R} \\
\mathbf{E} \\
\mathbf{G N}\end{array}$} & \multicolumn{4}{|c|}{ Segment Data base } & \multicolumn{4}{|c|}{ County/City Average Data base } \\
\hline & & & Raw & $\begin{array}{l}\text { Raw } \\
\text { E\&L }\end{array}$ & Density & $\begin{array}{l}\text { Density } \\
\text { E\&L }\end{array}$ & Raw & $\begin{array}{l}\text { Raw } \\
\text { E\&L }\end{array}$ & Density & $\begin{array}{l}\text { Density } \\
\text { E\&L }\end{array}$ \\
\hline & ALL & & 14.39 & 16.57 & 14.33 & 16.55 & 37.11 & 39.15 & 34.98 & 35.79 \\
\hline $\begin{array}{l}A \\
B \\
C \\
D\end{array}$ & & & $\begin{array}{r}6.11 \\
18.28 \\
19.23 \\
10.54\end{array}$ & $\begin{array}{r}7.46 \\
24.60 \\
19.31 \\
10.67\end{array}$ & $\begin{array}{r}7.08 \\
23.96 \\
15.99 \\
10.01\end{array}$ & $\begin{array}{r}8.04 \\
29.24 \\
14.76 \\
9.96\end{array}$ & $\begin{array}{l}34.48 \\
36.84 \\
49.89 \\
39.67\end{array}$ & $\begin{array}{l}42.85 \\
50.75 \\
46.73 \\
41.25\end{array}$ & $\begin{array}{l}33.43 \\
50.50 \\
40.63 \\
35.70\end{array}$ & $\begin{array}{l}41.09 \\
60.49 \\
36.27 \\
38.83\end{array}$ \\
\hline A & $\begin{array}{l}\text { F3 } \\
\text { F4 }\end{array}$ & & $\begin{array}{r}17.26 \\
8.95\end{array}$ & $\begin{array}{l}21.58 \\
11.13\end{array}$ & $\begin{array}{r}17.26 \\
4.46\end{array}$ & $\begin{array}{r}21.15 \\
3.76\end{array}$ & $\begin{array}{l}70.37 \\
53.67\end{array}$ & $\begin{array}{l}88.76 \\
70.31\end{array}$ & $\begin{array}{l}70.37 \\
24.40\end{array}$ & $\begin{array}{l}85.91 \\
26.59\end{array}$ \\
\hline B & $\begin{array}{l}\text { F3 } \\
\text { F4 }\end{array}$ & & $\begin{array}{l}x \\
18.75\end{array}$ & $\begin{array}{r}0.03 \\
25.20\end{array}$ & $\begin{array}{r}0.06 \\
26.30\end{array}$ & $\begin{array}{r}0.03 \\
31.52\end{array}$ & $\begin{array}{l}(\star \star) \\
35.31\end{array}$ & $\begin{array}{l}(* *) \\
48.92\end{array}$ & $\begin{array}{l}(* *) \\
57.85\end{array}$ & $\begin{array}{l}(\star *) \\
66.35\end{array}$ \\
\hline $\begin{array}{l}c \\
C \\
C\end{array}$ & $\begin{array}{l}\text { F2 } \\
\text { F3 } \\
\text { F4 }\end{array}$ & & $\begin{array}{r}4.83 \\
30.59 \\
42.25\end{array}$ & $\begin{array}{r}4.20 \\
36.60 \\
41.00\end{array}$ & $\begin{array}{r}4.72 \\
22.44 \\
40.03\end{array}$ & $\begin{array}{r}4.55 \\
28.99 \\
39.72\end{array}$ & $\begin{array}{l}45.79 \\
81.14 \\
66.27\end{array}$ & $\begin{array}{l}51.32 \\
85.27 \\
56.07\end{array}$ & $\begin{array}{l}48.75 \\
59.71 \\
64.64\end{array}$ & $\begin{array}{l}51.73 \\
67.31 \\
56.80\end{array}$ \\
\hline $\begin{array}{l}D \\
D \\
D \\
D\end{array}$ & $\begin{array}{l}\text { F1 } \\
\text { F2 } \\
\text { F3 } \\
\text { F4 }\end{array}$ & & $\begin{array}{r}3.16 \\
28.52 \\
31.45 \\
45.24\end{array}$ & $\begin{array}{c}X \\
29.37 \\
31.92 \\
45.32\end{array}$ & $\begin{array}{r}3.16 \\
12.58 \\
21.72 \\
41.26\end{array}$ & $\begin{array}{r}2.39 \\
20.07 \\
21.81 \\
44.53\end{array}$ & $\begin{array}{r}9.06 \\
86.29 \\
89.71 \\
68.01\end{array}$ & $\begin{array}{c}x \\
92.36 \\
95.34 \\
66.01\end{array}$ & $\begin{array}{r}9.06 \\
42.12 \\
61.96 \\
62.08\end{array}$ & $\begin{array}{c}X \\
57.08 \\
66.20 \\
60.53\end{array}$ \\
\hline $\begin{array}{l}\mathrm{A} \\
\mathrm{A} \\
\mathrm{A}\end{array}$ & & $\begin{array}{l}\mathrm{n} \\
\mathrm{c} \\
\mathrm{s}\end{array}$ & $\begin{array}{r}9.40 \\
16.66 \\
1.26\end{array}$ & $\begin{array}{r}11.76 \\
20.40 \\
1.13\end{array}$ & $\begin{array}{r}4.57 \\
14.50 \\
1.26\end{array}$ & $\begin{array}{r}4.04 \\
20.43 \\
1.13\end{array}$ & $\begin{array}{l}53.03 \\
82.30 \\
89.09\end{array}$ & $\begin{array}{l}X \\
96.38 \\
(* *)\end{array}$ & $\begin{array}{l}26.20 \\
71.31 \\
89.11\end{array}$ & $\begin{array}{l}36.00 \\
97.83 \\
(* *)\end{array}$ \\
\hline $\begin{array}{l}B \\
B \\
B\end{array}$ & & $\begin{array}{l}\mathrm{n} \\
\mathrm{c} \\
\mathrm{s}\end{array}$ & $\begin{array}{r}7.63 \\
41.93 \\
41.07\end{array}$ & $\begin{array}{r}8.44 \\
45.16 \\
42.68\end{array}$ & $\begin{array}{r}9.03 \\
42.18 \\
41.60\end{array}$ & $\begin{array}{r}8.87 \\
45.55 \\
42.68\end{array}$ & $\begin{array}{l}83.95 \\
95.30 \\
99.30\end{array}$ & $\begin{array}{l}88.73 \\
99.43 \\
(* *)\end{array}$ & $\begin{array}{l}92.90 \\
95.85 \\
99.30\end{array}$ & $\begin{array}{l}99.34 \\
99.99 \\
(\star *)\end{array}$ \\
\hline $\begin{array}{l}C \\
C \\
C\end{array}$ & & $\begin{array}{l}n \\
c \\
8\end{array}$ & $\begin{array}{l}23.00 \\
24.24 \\
11.82\end{array}$ & $\begin{array}{r}21.97 \\
27.21 \\
6.34\end{array}$ & $\begin{array}{l}21.12 \\
18.39 \\
11.90\end{array}$ & $\begin{array}{c}18.35 \\
17.83 \\
6.34\end{array}$ & $\begin{array}{l}40.16 \\
62.04 \\
81.68\end{array}$ & $\begin{array}{l}20.53 \\
69.85 \\
(\star \star)\end{array}$ & $\begin{array}{l}36.66 \\
49.24 \\
82.25\end{array}$ & $\begin{array}{l}24.04 \\
48.05 \\
(\star \star)\end{array}$ \\
\hline $\begin{array}{l}D \\
D \\
D\end{array}$ & & $\begin{array}{l}n \\
\text { c } \\
\text { s }\end{array}$ & $\begin{array}{l}18.11 \\
15.20 \\
12.58\end{array}$ & $\begin{array}{l}19.81 \\
17.90 \\
14.50\end{array}$ & $\begin{array}{r}29.27 \\
7.23 \\
12.51\end{array}$ & $\begin{array}{r}32.52 \\
6.64 \\
14.33\end{array}$ & $\begin{array}{l}49.28 \\
65.50 \\
45.20\end{array}$ & $\begin{array}{l}50.37 \\
79.61 \\
51.38\end{array}$ & $\begin{array}{l}66.90 \\
20.53 \\
42.99\end{array}$ & $\begin{array}{l}70.10 \\
22.92 \\
50.48\end{array}$ \\
\hline
\end{tabular}

Note: $X=$ Not Available; $(* *)=N<5$ 
Table 5.8: Number of Variables for Maximum $R^{2}$ value for Urban Principal Arterial

\begin{tabular}{|c|c|c|c|c|c|c|c|c|c|c|}
\hline \multirow{2}{*}{$\begin{array}{l}C \\
T \\
Y \\
G R\end{array}$} & \multirow{2}{*}{$\begin{array}{l}M \\
F \\
U \\
N C\end{array}$} & \multirow{2}{*}{$\begin{array}{l}G \\
R \\
E \\
G N\end{array}$} & \multicolumn{4}{|c|}{ Segment Data base } & \multicolumn{4}{|c|}{ County/City Average Data base } \\
\hline & & & Raw & $\begin{array}{l}\text { Raw } \\
\text { E\&L }\end{array}$ & Density & $\begin{array}{c}\text { y Density } \\
\text { E\&I }\end{array}$ & Raw & $\begin{array}{l}\text { Raw } \\
\text { E\&L }\end{array}$ & Density & $\begin{array}{c}\text { Density } \\
\text { E\&L }\end{array}$ \\
\hline & ALI & & 4 & 4 & 4 & 4 & 4 & 4 & 4 & 3 \\
\hline $\begin{array}{l}\text { A } \\
B \\
C \\
D\end{array}$ & & & $\begin{array}{l}2 \\
2 \\
3 \\
3\end{array}$ & $\begin{array}{l}2 \\
2 \\
3 \\
3\end{array}$ & 3 & $\begin{array}{l}3 \\
3 \\
2 \\
2\end{array}$ & $\begin{array}{l}3 \\
2 \\
3 \\
4\end{array}$ & $\begin{array}{l}3 \\
2 \\
3 \\
3\end{array}$ & $\begin{array}{l}3 \\
3 \\
2 \\
3\end{array}$ & $\begin{array}{l}3 \\
3 \\
2 \\
2\end{array}$ \\
\hline A & $\begin{array}{l}\text { F3 } \\
\text { F4 }\end{array}$ & & $\begin{array}{l}1 \\
2\end{array}$ & $\begin{array}{l}2 \\
2\end{array}$ & & $\begin{array}{l}1 \\
3\end{array}$ & $\begin{array}{l}1 \\
3\end{array}$ & $\begin{array}{l}2 \\
3\end{array}$ & $\begin{array}{l}1 \\
3\end{array}$ & $\frac{1}{3}$ \\
\hline B & $\begin{array}{l}\text { F3 } \\
\text { F4 }\end{array}$ & & $\begin{array}{l}x \\
2\end{array}$ & $\begin{array}{l}1 \\
2\end{array}$ & & $\begin{array}{l}1 \\
3\end{array}$ & $\begin{array}{r}(* \star) \\
2\end{array}$ & $\begin{array}{r}(* *) \\
2\end{array}$ & $\begin{array}{r}(* *) \\
3\end{array}$ & $\begin{array}{r}(* *) \\
3\end{array}$ \\
\hline $\begin{array}{l}c \\
c \\
c\end{array}$ & $\begin{array}{l}\text { F2 } \\
\text { F3 } \\
\text { F4 }\end{array}$ & & $\begin{array}{l}2 \\
4 \\
2\end{array}$ & $\begin{array}{l}2 \\
3 \\
2\end{array}$ & 2 & $\begin{array}{l}2 \\
4 \\
2\end{array}$ & $\begin{array}{l}2 \\
4 \\
2\end{array}$ & $\begin{array}{l}2 \\
3 \\
2\end{array}$ & $\begin{array}{l}3 \\
4 \\
2\end{array}$ & $\begin{array}{l}2 \\
4 \\
2\end{array}$ \\
\hline $\mid \begin{array}{l}D \\
D \\
D \\
D\end{array}$ & $\begin{array}{l}\text { F1 } \\
\text { F2 } \\
\text { F3 } \\
\text { F4 }\end{array}$ & & $\begin{array}{l}1 \\
3 \\
3 \\
3\end{array}$ & $\begin{array}{l}x \\
2 \\
3 \\
3\end{array}$ & 3 & $\begin{array}{l}1 \\
3 \\
3 \\
3\end{array}$ & $\begin{array}{l}1 \\
3 \\
3 \\
3\end{array}$ & $\begin{array}{l}x \\
3 \\
3 \\
3\end{array}$ & $\begin{array}{l}1 \\
3 \\
2 \\
3\end{array}$ & $\begin{array}{l}x \\
3 \\
2 \\
2\end{array}$ \\
\hline $\begin{array}{l}A \\
A \\
A\end{array}$ & & $\begin{array}{l}n \\
c \\
s\end{array}$ & $\begin{array}{l}2 \\
2 \\
1\end{array}$ & $\begin{array}{l}2 \\
2 \\
1\end{array}$ & 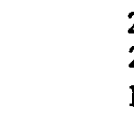 & $\begin{array}{l}2 \\
3 \\
1\end{array}$ & $\begin{array}{l}3 \\
2 \\
1\end{array}$ & $\begin{array}{r}x \\
2 \\
(* *)\end{array}$ & $\begin{array}{l}2 \\
2 \\
1\end{array}$ & $(\star \star *)^{3}$ \\
\hline \begin{tabular}{|l} 
B \\
B \\
B
\end{tabular} & & $\begin{array}{l}\mathrm{n} \\
\mathrm{c} \\
\mathrm{s}\end{array}$ & $\begin{array}{l}2 \\
2 \\
2\end{array}$ & $\begin{array}{l}2 \\
2 \\
1\end{array}$ & 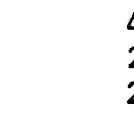 & $\begin{array}{l}3 \\
3 \\
1\end{array}$ & $\begin{array}{l}3 \\
2 \\
2\end{array}$ & $\begin{array}{r}2 \\
2 \\
(* *)^{2}\end{array}$ & $\begin{array}{l}4 \\
2 \\
2\end{array}$ & $(* *)^{3}$ \\
\hline $\begin{array}{l}\mathrm{c} \\
\mathrm{c} \\
\mathrm{c}\end{array}$ & & $\begin{array}{l}n \\
c \\
s\end{array}$ & $\begin{array}{l}2 \\
2 \\
2\end{array}$ & $\begin{array}{l}2 \\
2 \\
1\end{array}$ & & $\begin{array}{l}2 \\
2 \\
1\end{array}$ & $\begin{array}{l}2 \\
3 \\
2\end{array}$ & $(* *)^{2}$ & $\begin{array}{l}2 \\
2 \\
2\end{array}$ & $(\star \star *)$ \\
\hline $\begin{array}{l}D \\
D \\
D\end{array}$ & & $\begin{array}{l}n \\
c \\
s\end{array}$ & $\begin{array}{l}2 \\
3 \\
3\end{array}$ & $\begin{array}{l}2 \\
3 \\
2\end{array}$ & & $\begin{array}{l}3 \\
2 \\
2\end{array}$ & $\begin{array}{l}3 \\
3 \\
3\end{array}$ & $\begin{array}{l}2 \\
4 \\
2\end{array}$ & $\begin{array}{l}4 \\
2 \\
2\end{array}$ & $\begin{array}{l}3 \\
2 \\
1\end{array}$ \\
\hline
\end{tabular}

Note: $X-$ Not Available; $(\star \star)=N<5$ 
Table 5.9: Marginal Improvement in Maximum $R^{2}$ value of County/City Average AADT over Segment AADT for Urban Principal Arterial

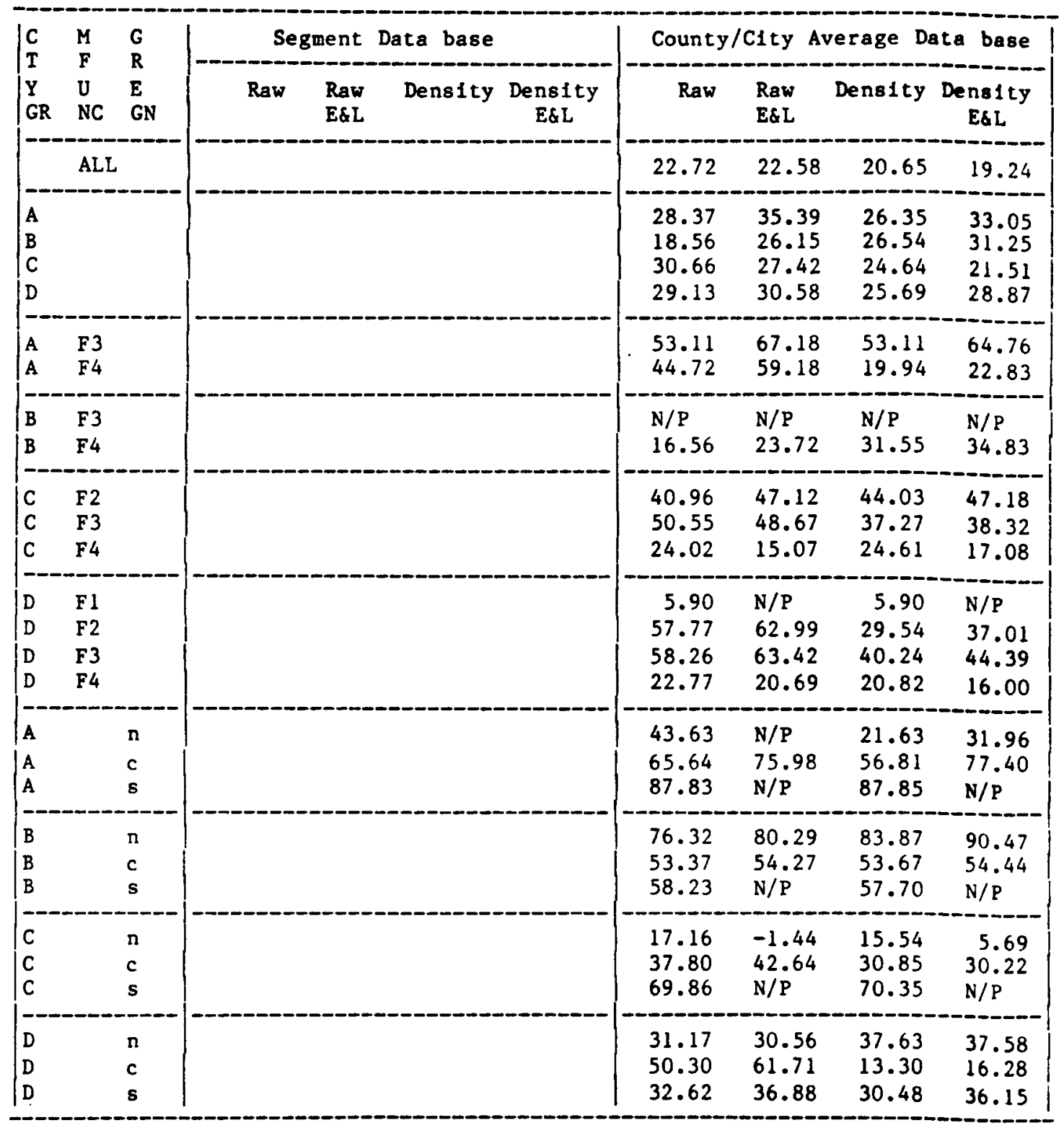

Note: $N / P=$ Not Possible 
Table 5.10: Marginal Improvement in Maximum $\mathrm{R}^{2}$ value of Raw E\&L, Density and Density E\&L Formats over Raw Format for Urban Principal Arterial

\begin{tabular}{|c|c|c|c|c|c|c|c|c|c|c|}
\hline \multirow{2}{*}{$\begin{array}{l}\text { C } \\
T \\
Y \\
\text { GR }\end{array}$} & \multirow{2}{*}{$\begin{array}{l}M \\
F \\
U \\
N C\end{array}$} & \multirow{2}{*}{$\begin{array}{l}\text { G } \\
\text { R } \\
\text { E } \\
\text { GN }\end{array}$} & \multicolumn{4}{|c|}{ Segment Data base } & \multicolumn{4}{|c|}{ County/City Average Data base } \\
\hline & & & Raw & $\begin{array}{l}\text { Raw } \\
\text { E\&L }\end{array}$ & Density & $\begin{array}{c}\text { Density } \\
\text { E\&L }\end{array}$ & Raw & $\begin{array}{l}\text { Raw } \\
\text { E\&L }\end{array}$ & Density & $\begin{array}{c}\text { Density } \\
\text { E\&L }\end{array}$ \\
\hline & ALL & & & 10.46 & 8.22 & 10.44 & & 2.04 & -2.13 & -1.32 \\
\hline $\begin{array}{l}A \\
B \\
C \\
D \\
--\end{array}$ & & & & $\begin{array}{l}1.35 \\
6.32 \\
0.08 \\
0.13\end{array}$ & $\begin{array}{r}0.97 \\
5.68 \\
-3.24 \\
-0.53\end{array}$ & $\begin{array}{r}1.93 \\
10.96 \\
-4.47 \\
-0.58\end{array}$ & & $\begin{array}{r}8.37 \\
13.91 \\
-3.16 \\
1.58\end{array}$ & $\begin{array}{r}-1.05 \\
13.66 \\
-9.26 \\
-3.97\end{array}$ & $\begin{array}{r}6.61 \\
23.65 \\
-13.62 \\
-0.84 \\
\end{array}$ \\
\hline A & $\begin{array}{l}\text { F3 } \\
\text { F4 }\end{array}$ & & & $\begin{array}{l}4.32 \\
2.18\end{array}$ & $\begin{array}{r}0.00 \\
-4.49 \\
\end{array}$ & $\begin{array}{r}3.89 \\
-5.19 \\
\end{array}$ & & $\begin{array}{l}18.39 \\
16.64\end{array}$ & $\begin{array}{r}0.00 \\
-29.27 \\
\end{array}$ & $\begin{array}{r}15.54 \\
-27.08 \\
\end{array}$ \\
\hline $\begin{array}{l}\mathrm{B} \\
\mathrm{B}\end{array}$ & $\begin{array}{l}\text { F3 } \\
\text { F4 }\end{array}$ & & & $\begin{array}{l}N / P \\
6.45\end{array}$ & $\begin{array}{l}\mathrm{N} / \mathrm{P} \\
7.55\end{array}$ & $\begin{array}{l}\text { N/P } \\
12.77\end{array}$ & & $\begin{array}{l}N / P \\
13.61\end{array}$ & $\begin{array}{l}N / P \\
22.54\end{array}$ & $\begin{array}{l}N / P \\
31.04\end{array}$ \\
\hline $\begin{array}{l}c \\
c \\
c\end{array}$ & $\begin{array}{l}\text { F2 } \\
\text { F3 } \\
\text { F4 }\end{array}$ & & & $\begin{array}{r}-0.63 \\
6.01 \\
-1.25 \\
\end{array}$ & $\begin{array}{l}-0.11 \\
-8.15 \\
-2.22 \\
\end{array}$ & $\begin{array}{l}-0.28 \\
-1.60 \\
-2.53\end{array}$ & & $\begin{array}{r}5.53 \\
4.13 \\
-10.20 \\
\end{array}$ & $\begin{array}{r}2.96 \\
-21.43 \\
-1.63 \\
\end{array}$ & $\begin{array}{r}5.94 \\
-13.83 \\
-9.47 \\
\end{array}$ \\
\hline $\begin{array}{l}D \\
D \\
D \\
D\end{array}$ & $\begin{array}{l}\text { F1 } \\
\text { F2 } \\
\text { F3 } \\
\text { F4 }\end{array}$ & & & $\begin{array}{l}\mathrm{N} / \mathrm{P} \\
0.85 \\
0.47 \\
0.08\end{array}$ & $\begin{array}{r}0.00 \\
-15.94 \\
-9.73 \\
-3.98\end{array}$ & $\begin{array}{l}-0.77 \\
-8.45 \\
-9.64 \\
-0.71\end{array}$ & & $\begin{array}{l}N / P \\
6.07 \\
5.63 \\
-2.00\end{array}$ & $\begin{array}{r}0.00 \\
-44.17 \\
-27.75 \\
-5.93\end{array}$ & $\begin{array}{l}N / P \\
-29.21 \\
-23.51 \\
-7.48\end{array}$ \\
\hline $\begin{array}{l}A \\
A \\
A\end{array}$ & & $\begin{array}{l}\mathrm{n} \\
\mathrm{c} \\
\mathrm{s}\end{array}$ & & $\begin{array}{r}2.36 \\
3.74 \\
-0.13\end{array}$ & $\begin{array}{r}-4.83 \\
-2.16 \\
0.00\end{array}$ & $\begin{array}{r}-5.36 \\
3.77 \\
-0.13 \\
\end{array}$ & & $\begin{array}{l}N / P \\
14.08 \\
N / P\end{array}$ & $\begin{array}{r}-26.83 \\
-10.99 \\
0.02\end{array}$ & $\begin{array}{l}-17.03 \\
15.53 \\
N / P\end{array}$ \\
\hline $\begin{array}{l}B \\
B \\
B\end{array}$ & & $\begin{array}{l}n \\
c \\
s\end{array}$ & & $\begin{array}{l}0.81 \\
3.23 \\
1.61\end{array}$ & $\begin{array}{l}1.40 \\
0.25 \\
0.53\end{array}$ & $\begin{array}{l}1.24 \\
3.62 \\
1.61\end{array}$ & & $\begin{array}{r}4.78 \\
4.13 \\
\mathrm{~N} / \mathrm{P}\end{array}$ & $\begin{array}{l}8.95 \\
0.55 \\
0.00\end{array}$ & $\begin{array}{l}15.39 \\
4.69 \\
N / P\end{array}$ \\
\hline $\begin{array}{l}C \\
C \\
C\end{array}$ & & $\begin{array}{l}\mathrm{n} \\
\mathrm{c} \\
\mathrm{s}\end{array}$ & & $\begin{array}{r}-1.03 \\
2.97 \\
-5.48\end{array}$ & $\begin{array}{r}-1.88 \\
-5.85 \\
0.08\end{array}$ & $\begin{array}{l}-4.65 \\
-6.41 \\
-5.48 \\
-\end{array}$ & . & $\begin{array}{c}-19.63 \\
7.81 \\
N / P\end{array}$ & $\begin{array}{r}-3.50 \\
-12.80 \\
0.57\end{array}$ & $\left\{\begin{array}{c}-16.12 \\
-13.99 \\
N / P\end{array}\right.$ \\
\hline $\begin{array}{l}D \\
D\end{array}$ & & $\begin{array}{l}\mathbf{n} \\
\mathrm{c} \\
\mathbf{s}\end{array}$ & & $\begin{array}{l}1.70 \\
2.70 \\
1.92\end{array}$ & $\begin{array}{l}11.16 \\
-7.97 \\
-0.07\end{array}$ & $\begin{array}{r}14.41 \\
-8.56 \\
1.75\end{array}$ & & $\begin{array}{r}1.09 \\
14.11 \\
6.18\end{array}$ & $\begin{array}{rr}17.62 \\
-44.97 \\
-2.21\end{array}$ & $\begin{array}{r}20.82 \\
-42.58 \\
5.28\end{array}$ \\
\hline
\end{tabular}


Table 5.11: Marginal Improvement in Maximum $\mathrm{R}^{2}$ value of Earlier \& Later (E\&L) Year Data Format for Urban Principal Arterial

\begin{tabular}{|c|c|c|c|c|c|c|c|c|c|c|}
\hline \multirow{2}{*}{$\begin{array}{l}\mathrm{C} \\
\mathrm{T} \\
\mathrm{Y} \\
\mathrm{GR}\end{array}$} & \multirow{2}{*}{$\begin{array}{l}M \\
F \\
U \\
N C\end{array}$} & \multirow{2}{*}{$\begin{array}{l}\text { G } \\
R \\
E \\
G N\end{array}$} & \multicolumn{4}{|c|}{ Segment Data base } & \multicolumn{4}{|c|}{ County/City Average Data base } \\
\hline & & & Raw & $\begin{array}{l}\text { Raw } \\
\text { E\&L }\end{array}$ & Density & $\begin{array}{c}\text { Density } \\
\text { E\&L }\end{array}$ & Raw & $\begin{array}{l}\text { Raw } \\
\text { E\&L }\end{array}$ & Density & $\begin{array}{l}\text { Density } \\
\text { E\&L }\end{array}$ \\
\hline & ALL & & & 2.18 & & 2.22 & & 2.04 & & 0.81 \\
\hline $\begin{array}{l}A \\
B \\
C \\
D\end{array}$ & & & & $\begin{array}{l}1.35 \\
6.32 \\
0.08 \\
0.13\end{array}$ & & $\begin{array}{r}0.96 \\
5.28 \\
-1.23 \\
-0.05\end{array}$ & & $\begin{array}{r}8.37 \\
13.91 \\
-3.16 \\
1.58\end{array}$ & & $\begin{array}{r}7.66 \\
9.99 \\
-4.36 \\
3.13\end{array}$ \\
\hline A & $\begin{array}{l}\text { F3 } \\
\text { F4 }\end{array}$ & & & $\begin{array}{l}4.32 \\
2.18\end{array}$ & & $\begin{array}{r}3.89 \\
-0.70\end{array}$ & & $\begin{array}{l}18.39 \\
16.64\end{array}$ & & $\begin{array}{r}15.54 \\
2.19\end{array}$ \\
\hline B & $\begin{array}{l}\text { F3 } \\
\text { F4 }\end{array}$ & & & $\begin{array}{l}\mathrm{N} / \mathrm{P} \\
6.45\end{array}$ & & $\begin{array}{r}-0.03 \\
5.22\end{array}$ & & $\begin{array}{l}N / P \\
13.61\end{array}$ & & $\begin{array}{l}\mathrm{N} / \mathrm{P} \\
8.50\end{array}$ \\
\hline $\begin{array}{l}c \\
c \\
c\end{array}$ & $\begin{array}{l}\text { F2 } \\
\text { F3 } \\
\text { F4 }\end{array}$ & & & $\begin{array}{r}-0.63 \\
6.01 \\
-1.25\end{array}$ & & $\begin{array}{r}-0.17 \\
6.55 \\
-0.31\end{array}$ & & $\begin{array}{r}5.53 \\
4.13 \\
-10.20\end{array}$ & & $\begin{array}{r}2.98 \\
7.60 \\
-7.84\end{array}$ \\
\hline $\begin{array}{l}D \\
D \\
D \\
D\end{array}$ & $\begin{array}{l}\text { F1 } \\
\text { F2 } \\
\text { F3 } \\
\text { F4 }\end{array}$ & & & $\begin{array}{l}\mathrm{N} / \mathrm{P} \\
0.85 \\
0.47 \\
0.08\end{array}$ & & $\begin{array}{r}-0.77 \\
7.49 \\
0.09 \\
3.27\end{array}$ & & $\begin{array}{r}\mathrm{N} / \mathrm{P} \\
6.07 \\
5.63 \\
-2.00\end{array}$ & & $\begin{array}{r}\mathrm{N} / \mathrm{P} \\
14.96 \\
4.24 \\
-1.55\end{array}$ \\
\hline $\mid \begin{array}{l}A \\
A \\
A\end{array}$ & & $\begin{array}{l}n \\
\mathrm{c} \\
\mathrm{s}\end{array}$ & & $\begin{array}{r}2.36 \\
3.74 \\
-0.13\end{array}$ & & $\begin{array}{r}-0.53 \\
5.93 \\
-0.13\end{array}$ & & $\begin{array}{l}\mathrm{N} / \mathrm{P} \\
14.08 \\
\mathrm{~N} / \mathrm{P}\end{array}$ & & $\begin{array}{l}9.80 \\
26.52 \\
\mathrm{~N} / \mathrm{P}\end{array}$ \\
\hline $\begin{array}{l}B \\
B \\
B\end{array}$ & & $\begin{array}{l}n \\
\mathrm{c} \\
\mathrm{s}\end{array}$ & & $\begin{array}{l}0.81 \\
3.23 \\
1.61\end{array}$ & & $\begin{array}{r}-0.16 \\
3.37 \\
1.08\end{array}$ & & $\begin{array}{l}4.78 \\
4.13 \\
\mathrm{~N} / \mathrm{P}\end{array}$ & & $\begin{array}{l}6.44 \\
4.14 \\
\mathrm{~N} / \mathrm{P}\end{array}$ \\
\hline $\mid \begin{array}{l}c \\
c \\
c\end{array}$ & & $\begin{array}{l}n \\
c \\
s\end{array}$ & & $\begin{array}{r}-1.03 \\
2.97 \\
-5.48\end{array}$ & & $\begin{array}{l}-2.77 \\
-0.56 \\
-5.56\end{array}$ & & $\begin{array}{r}-19.63 \\
7.81 \\
N / \mathrm{P}\end{array}$ & & $\begin{array}{r}-12.62 \\
-1.19 \\
N / P\end{array}$ \\
\hline $\mid \begin{array}{l}D \\
D \\
D\end{array}$ & & $\begin{array}{l}n \\
c \\
s\end{array}$ & & $\begin{array}{l}1.70 \\
2.70 \\
1.92\end{array}$ & & $\begin{array}{r}3.25 \\
-0.59 \\
1.82\end{array}$ & & $\begin{array}{r}1.09 \\
14.11 \\
6.18\end{array}$ & & $\begin{array}{l}3.20 \\
2.39 \\
7.49\end{array}$ \\
\hline
\end{tabular}

Note: N/P=NoL Possible 
Table 5.12: Marginal Improvement in Maximum $R^{2}$ value of Density Format over Raw Format for Urban Principal Arterial

\begin{tabular}{|c|c|c|c|c|c|c|c|c|c|c|}
\hline \multirow{2}{*}{$\begin{array}{l}\mathrm{C} \\
\mathrm{T} \\
\mathrm{Y} \\
\mathrm{GR}\end{array}$} & \multirow{2}{*}{$\begin{array}{l}M \\
F \\
\text { U } \\
\text { NC }\end{array}$} & \multirow{2}{*}{$\begin{array}{l}\mathbf{G} \\
\mathbf{R} \\
\mathbf{E} \\
\mathrm{GN}\end{array}$} & \multicolumn{4}{|c|}{ Segment Data base } & \multicolumn{4}{|c|}{ County/City Average Data base } \\
\hline & & & Raw & $\begin{array}{l}\text { Raw } \\
\text { E\&L }\end{array}$ & Density & $\begin{array}{c}\text { Density } \\
\text { E\&L }\end{array}$ & Raw & $\begin{array}{l}\text { Raw } \\
\text { E\&L }\end{array}$ & Density & $\begin{array}{c}\text { Density } \\
\text { E\&L }\end{array}$ \\
\hline & ALL & & & & -0.06 & -0.02 & & & -2.13 & -3.36 \\
\hline $\begin{array}{l}A \\
B \\
C \\
D\end{array}$ & & & & & $\begin{array}{r}0.97 \\
5.68 \\
-3.24 \\
-0.53\end{array}$ & $\begin{array}{r}0.58 \\
4.64 \\
-4.55 \\
-0.71\end{array}$ & & & $\begin{array}{l}-1.05 \\
13.66 \\
-9.26 \\
-3.97\end{array}$ & $\begin{array}{r}-1.76 \\
9.74 \\
-10.46 \\
-2.42\end{array}$ \\
\hline $\begin{array}{l}A \\
A\end{array}$ & $\begin{array}{l}\text { F3 } \\
\text { F4 }\end{array}$ & & & & $\begin{array}{r}0.00 \\
-4.49\end{array}$ & $\begin{array}{l}-0.43 \\
-7.37\end{array}$ & & & $\begin{array}{r}0.00 \\
-29.27\end{array}$ & $\begin{array}{r}-2.85 \\
-43.72\end{array}$ \\
\hline B & $\begin{array}{l}\text { F3 } \\
\text { F4 }\end{array}$ & & & & $\begin{array}{l}\mathrm{N} / \mathrm{P} \\
7.55\end{array}$ & $\begin{array}{l}0.00 \\
6.32\end{array}$ & & & $\begin{array}{l}N / P \\
22.54\end{array}$ & $\begin{array}{l}N / P \\
17.43\end{array}$ \\
\hline $\begin{array}{l}c \\
c \\
c\end{array}$ & $\begin{array}{l}\text { F2 } \\
\text { F3 } \\
\text { F4 }\end{array}$ & & & & $\begin{array}{l}-0.11 \\
-8.15 \\
-2.22\end{array}$ & $\begin{array}{r}0.35 \\
-7.61 \\
-1.28\end{array}$ & & & $\begin{array}{r}2.96 \\
-21.43 \\
-1.63\end{array}$ & $\begin{array}{r}0.41 \\
-17.96 \\
0.73\end{array}$ \\
\hline $\begin{array}{l}D \\
D \\
D \\
D\end{array}$ & $\begin{array}{l}\text { F1 } \\
\text { F2 } \\
\text { F3 } \\
\text { F4 }\end{array}$ & & & & $\begin{array}{r}0.00 \\
-15.94 \\
-9.73 \\
-3.98\end{array}$ & $\begin{array}{l}N / P \\
-9.30 \\
-10.11 \\
-0.79\end{array}$ & & & $\begin{array}{r}0.00 \\
-44.17 \\
-27.75 \\
-5.93\end{array}$ & $\begin{array}{c}N / P \\
-35.28 \\
-29.14 \\
-5.48\end{array}$ \\
\hline $\begin{array}{l}A \\
A \\
A\end{array}$ & & $\begin{array}{l}\mathrm{n} \\
\mathrm{c} \\
\mathrm{s}\end{array}$ & & & $\begin{array}{r}-4.83 \\
-2.16 \\
0.00\end{array}$ & $\begin{array}{r}-7.72 \\
0.03 \\
0.00\end{array}$ & & & $\begin{array}{r}-26.83 \\
-10.99 \\
0.02\end{array}$ & $\begin{array}{l}N / P \\
1.45 \\
N / P\end{array}$ \\
\hline $\begin{array}{l}B \\
B \\
B\end{array}$ & & $\begin{array}{l}\mathrm{n} \\
\mathrm{c} \\
\mathrm{s}\end{array}$ & & & $\begin{array}{l}1.40 \\
0.25 \\
0.53\end{array}$ & $\begin{array}{l}0.43 \\
0.39 \\
0.00\end{array}$ & & & $\begin{array}{l}8.95 \\
0.55 \\
.0 .00\end{array}$ & $\begin{array}{l}10.61 \\
0.56 \\
\mathrm{~N} / \mathrm{P}\end{array}$ \\
\hline $\begin{array}{l}c \\
C \\
c\end{array}$ & & $\begin{array}{l}\mathrm{n} \\
\mathrm{c} \\
\mathrm{s}\end{array}$ & & & $\begin{array}{r}-1.88 \\
-5.85 \\
0.08\end{array}$ & $\begin{array}{r}-3.62 \\
-9.38 \\
0.00\end{array}$ & & & $\begin{array}{r}-3.50 \\
-12.80 \\
0.57\end{array}$ & $\begin{array}{r}3.51 \\
-21.80 \\
\mathrm{~N} / \mathrm{P}\end{array}$ \\
\hline $\mid \begin{array}{l}D \\
D \\
D\end{array}$ & & $\begin{array}{l}n \\
\mathbf{c} \\
\mathbf{s}\end{array}$ & & & $\begin{array}{l}11.16 \\
-7.97 \\
-0.07\end{array}$ & $\begin{array}{r}12.71 \\
-11.26 \\
-0.17\end{array}$ & & & $\begin{array}{r}17.62 \\
-44.97 \\
-2.21\end{array}$ & $\begin{array}{r}19.73 \\
-56.69 \\
-0.90\end{array}$ \\
\hline
\end{tabular}

Note: N/P=Not Possible 
4. Density formats over raw formats (Table 5.12).

Table 5.10 does not show any significant improvement of raw E\&L, density and density E\&L formats over raw format. For example, the different statistics for "CTYGR $D$ " and "GREGION C" of raw E\&L format over raw format with a 14.11 percent increase in $R^{2}$, for county/city average data base are:

\begin{tabular}{|c|c|c|c|c|c|c|}
\hline Format & $\mathrm{R}^{2}$ & $\mathrm{R}_{\mathrm{a}}^{2}$ & PC & AIC & $\mathrm{C}_{\mathrm{p}}$ & $\begin{array}{c}\text { No of } \\
\text { Variable }\end{array}$ \\
\hline $\begin{array}{ll}\text { Raw } & \\
\text { Raw } & E \& L\end{array}$ & $\begin{array}{l}65.50 \\
79.61(*)\end{array}$ & $\begin{array}{l}60.79 \\
67.96(*)\end{array}$ & $\begin{array}{l}0.4705(*) \\
0.4951\end{array}$ & $\begin{array}{l}362.7 \\
164.4(*)\end{array}$ & $\begin{array}{l}2.15(*) \\
N / A\end{array}$ & $\begin{array}{l}3 \\
4\end{array}$ \\
\hline
\end{tabular}

$(*)=$ the better values, N/A = Not Available.

In spite of a 14.11 percent increase of $R^{2}$ for the "raw E\&L" format, there is only a 7.17 percent increase in $R_{a}^{2}$, which is $R^{2}$ with adjustment for the number of variables in the model equation. It has been mentioned in Section 5.5.1 that AIC is suitable for comparing models that use the same data sets and equal observations. Therefore, AIC values will not be considered in the above tables, because of the unequal numbers of observations in the two different data sets (see Table 4.5). The value of PC supports the raw format over the raw E\&L format. The data structure of the raw format is also much simpler than that of raw E\&L format. A similar argument can be made from Tables 5.11 and 5.12 to establish the superfority of the raw format over its counterparts -- raw E\&L, density and density E\&L formats.

The following points are derived from careful examination of the values in Table 5.7 and its parent Table C5: 
1. No significant change in $\mathrm{R}^{2}$ value at Phase 2 (analysis by CTYGR) versus Phase 1 ("al1" case). The definitions of Phase 1, 2 and 3 analysis are provided in Figure 5.2.

2. The data structure of Phase 3 analysis by the CTYGR and MFUNC combination is incomplete. For example, CTYGR "A" and MFUNC "F1" combination are absent in the data sets.

3. At Phase 3, the results for CTYGR \& GREGION combination have improved in CTYGR A and B than those for CTYGR \& MFUNC combination.

4. Some of the $C_{p}$ values are not available at Phase 3 for the CTYGR-GREGION combination, because of error variances too small to compute $\mathrm{C}_{\mathrm{p}}$.

5. The $R^{2}$ values at Phase 3 for the CTYGR-GREGION combination ranges 53-89, 83-99, 40-82 and 49-66 percent for CTYGR A, B, C and D, respectively.

6. The PC values at Phase 3 with CTYGR-GREGION combination ranges $0.25-0.66,0.03-0.30,0.45-0.73$ and $0.47-0.66$ for CTYGR A, B, C and D, respectively. The PC values at Phase 2 by CTYGR are 0.79 , $0.77,0.53$ and 0.67 for CTYGR A, B, C and D, respectively. As for $\mathrm{R}^{2}$ and PC values, the improvement in RMSE and AIC is noticed at the Phase 3 analysis with the CTYGR-GREGION combination, when compared to the Phase 2 analysis. Thus, further analyses for urban principal arterials will be carried out with the raw format of county/city average data base at Phase 3 with the CTYGR-GREGION combination. 


\subsubsection{Urban Minor Arterial and Collector}

The layout of analysis for urban minor arterials and collectors was kept the same as for urban principal arterials (see Figure 5.2). The maximum $\mathrm{R}^{2}$ values for urban minor arterials and collectors are shown in Table 5.13. The corresponding numbers of variables in the equations are presented in Table 5.14. The parent tables of Table 5.13 are Appendix Tables C9 to C12. Table 5.13 shows the improvement in $\mathrm{R}^{2}$ value with county/city average data bases over segment data bases. The data structure for Phase 3 analysis in both of the CTYGRMFUNC and CTYGR-GREGION combinations is incomplete. Many cells in Phase 3 also have few (for example, fewer than five) observations in Earlier and Later (E\&L) data format (see Table 4.4). As with urban principal arterial and interstate highway, Tables 5.15, 5.16, 5.17 and 5.18 are constructed from Table 5.13 to show the marginal 1mprovement in the maximum $R^{2}$ value for

1. County/city average data bases over segment data bases (Table 5.15),

2. Raw E\&L, density and density E\&L formats over raw format (Table 5.16),

3. Earlier and Later (E\&L) formats over non-E\&L formats (Table $5.17)$, and

4. Density formats over raw formats (Table 5.18).

Despite the gaps in the data structure, Table 5.16 shows the superiority of county/city average data bases over its counterpart, the segment data bases. As for interstate highways and urban principal arterials, Table 5.16 does not show any consistent or significant gain for the raw E\&L, density and density E\&L formats over raw format. For example, the CTYGR A at Phase 2 analysis shows a 17.54 percent 
Table 5.13: Maximum $\mathrm{R}^{2}$ value for Urban Minor Arterial \& Collector

\begin{tabular}{|c|c|c|c|c|c|c|c|c|c|c|}
\hline |c & $\mathbf{M}$ & G & & ment $\mathrm{D}$ & ata base & & Count: & City Ay & verage $\mathrm{Da}$ & at a base \\
\hline $\begin{array}{l}T \\
Y \\
G R\end{array}$ & $\begin{array}{l}\mathbf{F} \\
\mathbf{U} \\
\mathrm{NC}\end{array}$ & $\begin{array}{l}\mathbf{R} \\
\mathrm{E} \\
\mathrm{GN}\end{array}$ & Raw & $\begin{array}{l}\text { Raw } \\
\text { E\&L }\end{array}$ & Density & $\begin{array}{c}\text { Density } \\
\text { E\&L }\end{array}$ & Raw & $\begin{array}{l}\text { Raw } \\
\text { E\&L }\end{array}$ & Density & $\begin{array}{c}\text { Density } \\
\text { E\&L }\end{array}$ \\
\hline & ALL & & 14.14 & 18.36 & 11.24 & 14.34 & 33.83 & 40.75 & 21.33 & 25.24 \\
\hline $\begin{array}{l}A \\
B \\
C \\
D\end{array}$ & & & $\begin{array}{r}30.47 \\
36.13 \\
7.06 \\
11.18\end{array}$ & $\begin{array}{c}40.67 \\
42.96 \\
10.69 \\
x\end{array}$ & $\begin{array}{r}36.96 \\
36.06 \\
10.07 \\
8.58\end{array}$ & $\begin{array}{r}41.23 \\
44.04 \\
9.99 \\
5.58\end{array}$ & $\begin{array}{r}65.94 \\
69.50 \\
9.40 \\
21.40\end{array}$ & $\begin{array}{l}83.48 \\
78.06 \\
12.56 \\
17.11\end{array}$ & $\begin{array}{r}78.29 \\
72.55 \\
9.30 \\
15.69\end{array}$ & $\begin{array}{r}87.35 \\
83.12 \\
11.52 \\
9.78\end{array}$ \\
\hline $\begin{array}{l}\text { A } \\
\text { A }\end{array}$ & $\begin{array}{l}\text { F3 } \\
\text { F4 }\end{array}$ & & $\begin{array}{r}16.42 \\
3.98\end{array}$ & $\begin{array}{r}36.44 \\
6.57\end{array}$ & $\begin{array}{r}44.93 \\
6.78\end{array}$ & $\begin{array}{r}50.92 \\
6.57\end{array}$ & $\begin{array}{l}27.90 \\
42.74\end{array}$ & $\begin{array}{l}(\star \star) \\
(\star \star)\end{array}$ & $\begin{array}{l}88.49 \\
75.39\end{array}$ & $\begin{array}{l}(* *) \\
(* *)\end{array}$ \\
\hline B & F4 & & 36.13 & 42.96 & 36.06 & 44.04 & 69.50 & 78.06 & 72.55 & 83.12 \\
\hline $\begin{array}{l}\mathrm{c} \\
\mathrm{C} \\
\mathrm{C}\end{array}$ & $\begin{array}{l}\text { F2 } \\
\text { F3 } \\
\text { F4 }\end{array}$ & & $\begin{array}{r}16.27 \\
0.23 \\
46.62\end{array}$ & $\begin{array}{r}33.36 \\
0.09 \\
47.59\end{array}$ & $\begin{array}{r}16.05 \\
0.23 \\
46.64\end{array}$ & $\begin{array}{r}33.37 \\
0.10 \\
47.30\end{array}$ & $\begin{array}{l}17.45 \\
16.34 \\
83.63\end{array}$ & $\begin{array}{l}46.10 \\
(* *) \\
84.17\end{array}$ & $\begin{array}{l}17.16 \\
16.35 \\
81.09\end{array}$ & $\begin{array}{l}41.59 \\
(\star *) \\
83.59\end{array}$ \\
\hline $\mid \begin{array}{l}D \\
D \\
D \\
D\end{array}$ & $\begin{array}{l}\text { F1 } \\
\text { F2 } \\
\text { F3 } \\
\text { F4 }\end{array}$ & & $\begin{array}{c}X \\
85.99 \\
21.53 \\
98.30\end{array}$ & $\begin{array}{l}43.60 \\
91.84 \\
22.20 \\
(\star \star)\end{array}$ & $\begin{array}{l}47.77 \\
72.00 \\
21.53 \\
98.62\end{array}$ & $\begin{array}{l}43.60 \\
91.84 \\
22.20 \\
(\star \star)\end{array}$ & $\begin{array}{c}x \\
96.78 \\
81.71 \\
98.40\end{array}$ & $\begin{array}{c}X \\
(* *) \\
(* \star) \\
(* *)\end{array}$ & $\begin{array}{l}50.18 \\
84.78 \\
81.68 \\
98.62\end{array}$ & $\begin{array}{l}42.94 \\
(* \star) \\
(\star *) \\
(* *)\end{array}$ \\
\hline A & & $\begin{array}{l}\mathrm{n} \\
\mathrm{c} \\
\mathrm{s}\end{array}$ & $\begin{array}{r}3.98 \\
14.42 \\
27.17\end{array}$ & $\begin{array}{l}6.57 \\
(* \star) \\
25.36\end{array}$ & $\begin{array}{r}6.78 \\
14.65 \\
27.17\end{array}$ & $\begin{array}{l}6.57 \\
(* \star) \\
25.36\end{array}$ & $\begin{array}{l}42.74 \\
51.65 \\
79.65\end{array}$ & $\begin{array}{l}(\star \star) \\
(\star \star) \\
(* \star)\end{array}$ & $\begin{array}{l}75.39 \\
51.03 \\
79.66\end{array}$ & $\begin{array}{l}(* *) \\
(* *) \\
(* *)\end{array}$ \\
\hline $\begin{array}{l}\text { B } \\
\text { B } \\
\text { B }\end{array}$ & & $\begin{array}{l}\mathbf{n} \\
\mathbf{c} \\
\mathbf{s}\end{array}$ & $\begin{array}{l}X \\
0.35\end{array}$ & $\begin{array}{l}x \\
0.42\end{array}$ & $\begin{array}{r}50.84 \\
0.35\end{array}$ & $\begin{array}{r}56.26 \\
0.42\end{array}$ & $\begin{array}{l}33.89 \\
20.99\end{array}$ & $\begin{array}{l}(\star \star) \\
(\star \star)\end{array}$ & $\begin{array}{l}89.72 \\
21.03\end{array}$ & $\begin{array}{l}(* \star) \\
(* *)\end{array}$ \\
\hline $\begin{array}{l}\mathrm{C} \\
\mathrm{C} \\
\mathrm{C}\end{array}$ & & $\begin{array}{l}\mathrm{n} \\
\mathrm{c} \\
\mathrm{s}\end{array}$ & $\begin{array}{c}24.58 \\
\times \\
79.88\end{array}$ & $\begin{array}{l}28.80 \\
26.99 \\
(\star *)\end{array}$ & $\begin{array}{l}29.12 \\
10.25 \\
79.76\end{array}$ & $\begin{array}{l}31.82 \\
26.96 \\
(\star \star)\end{array}$ & $\begin{array}{c}78.69 \\
\times \\
99.40\end{array}$ & $\begin{array}{l}85.97 \\
35.48 \\
(\star \star)\end{array}$ & $\begin{array}{l}93.25 \\
14.25 \\
99.26\end{array}$ & $\begin{array}{l}94.97 \\
35.44 \\
(\star \star)\end{array}$ \\
\hline $\mid \begin{array}{l}D \\
D \\
D\end{array}$ & & $\begin{array}{l}\text { n } \\
\text { c } \\
\text { s }\end{array}$ & $\begin{array}{l}9.67 \\
\times \\
9.11\end{array}$ & $\begin{array}{r}14.90 \\
26.19 \\
7.19\end{array}$ & $\begin{array}{r}14.79 \\
25.62 \\
9.82\end{array}$ & $\begin{array}{r}14.90 \\
26.19 \\
7.19\end{array}$ & $\begin{array}{l}35.88 \\
88.06 \\
18.97\end{array}$ & $\begin{array}{l}35.81 \\
(\star \star) \\
19.06\end{array}$ & $\begin{array}{l}54.92 \\
88.06 \\
20.52\end{array}$ & $\begin{array}{l}55.14 \\
(\star \star) \\
20.11\end{array}$ \\
\hline
\end{tabular}

Note: $X=$ Not Ava1lable; $(* \star)=N<5$ 
Table 5.14: Number of Variables for Maximum $R^{2}$ value for Urban Minor Arterial Collector

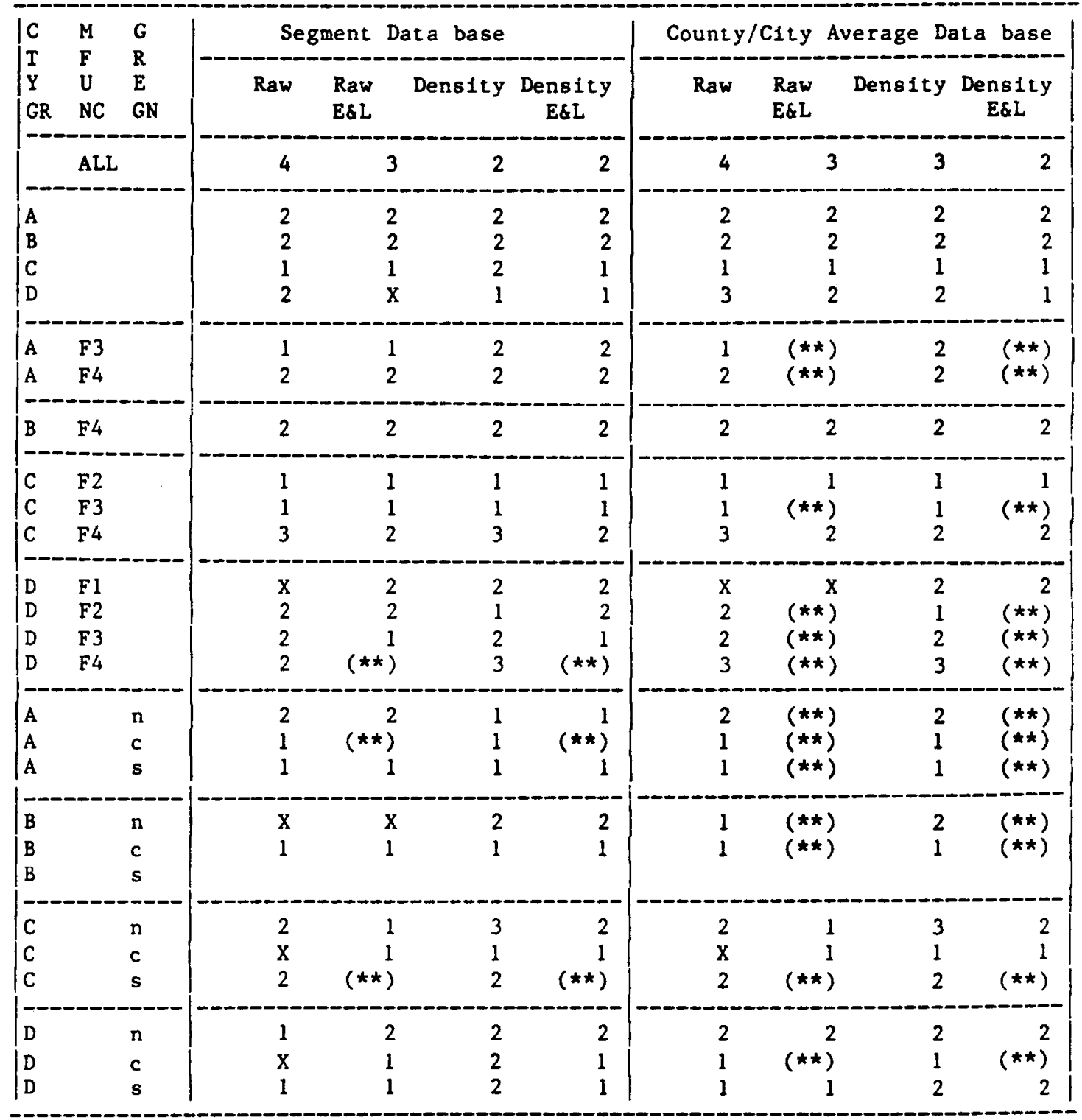

Note: $X=$ Not Available; $(* \star)=N<5$ 
Table 5.15: Marginal Improvement in Maximum $R^{2}$ value of County/City Average AADT over Segment AADT for Urban Minor Arterial \& Collector

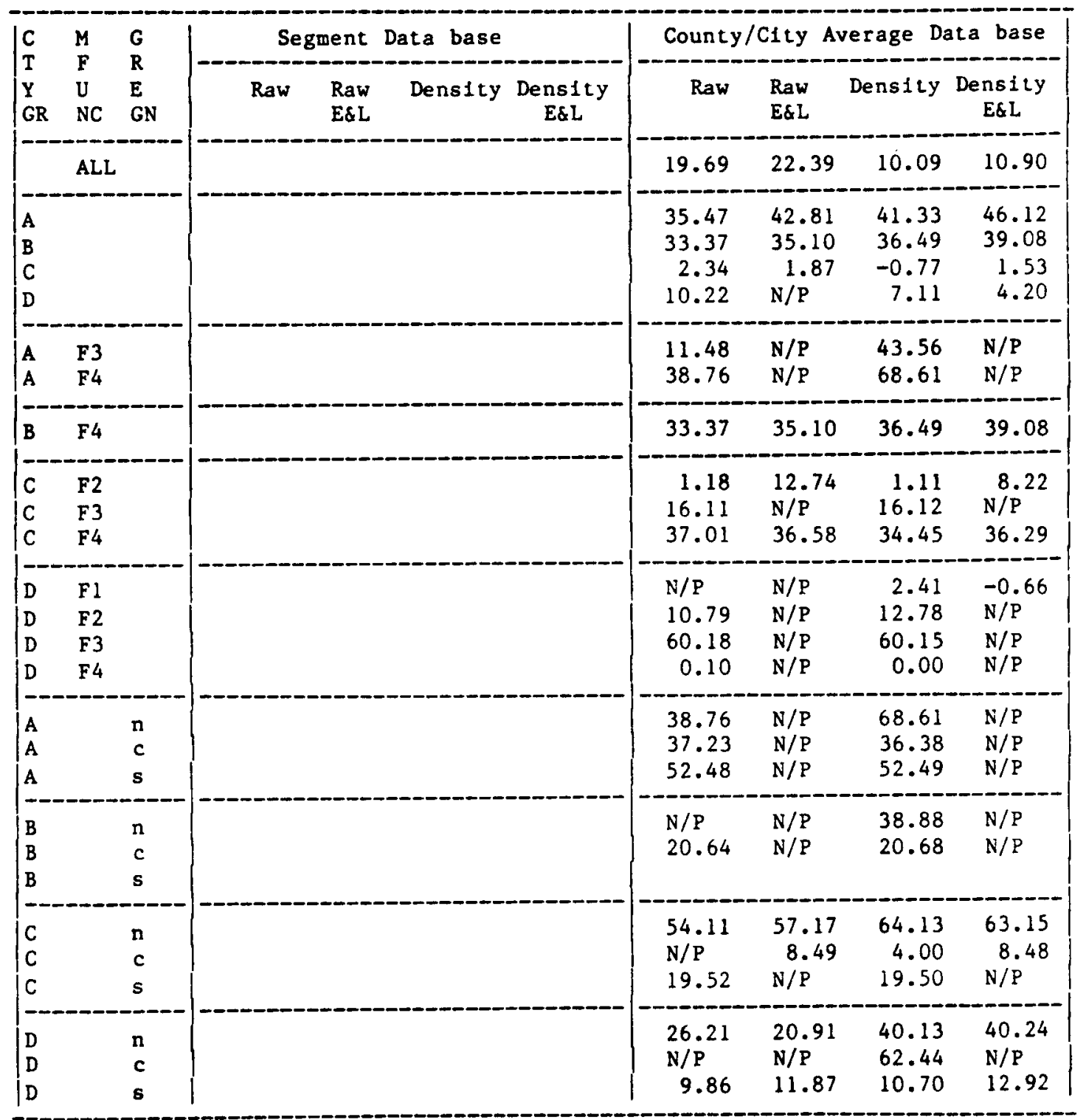

Note: $\mathrm{N} / \mathrm{P}=\mathrm{Not}$ Possible 
Table 5.16: Marginal Improvement in Maximum $R^{2}$ value of Raw E\&L, Density and Density E\&L Formats over Raw Format for Urban Minor Arterial \& Collector

\begin{tabular}{|c|c|c|c|c|c|c|c|c|c|c|}
\hline \multirow{2}{*}{$\mid \begin{array}{l}\mathrm{C} \\
\mathrm{T} \\
\mathrm{Y} \\
\mathrm{GR}\end{array}$} & \multirow{2}{*}{$\begin{array}{l}M \\
F \\
U \\
\text { NC }\end{array}$} & \multirow{2}{*}{$\begin{array}{l}\text { G } \\
\text { R } \\
\text { E } \\
\text { GN }\end{array}$} & \multicolumn{4}{|c|}{ Segment Data base } & \multicolumn{4}{|c|}{ County/City Average Data base } \\
\hline & & & Raw & $\begin{array}{l}\text { Raw } \\
\text { E\&L }\end{array}$ & Density & $\begin{array}{l}\text { Density } \\
\text { E\&L }\end{array}$ & Raw & $\begin{array}{l}\text { Raw } \\
\text { E\&L }\end{array}$ & Density & $\begin{array}{c}\text { Density } \\
\text { E\&L }\end{array}$ \\
\hline & ALL & & & -12.11 & -19.23 & -16.13 & & 6.92 & -12.50 & -8.59 \\
\hline $\begin{array}{l}\text { A } \\
\text { B } \\
\text { C } \\
\text { D }\end{array}$ & & & & $\begin{array}{r}10.20 \\
6.83 \\
3.63 \\
\mathrm{~N} / \mathrm{P}\end{array}$ & $\begin{array}{r}6.49 \\
-0.07 \\
3.01 \\
-2.60\end{array}$ & $\begin{array}{r}10.76 \\
7.91 \\
2.93 \\
-5.60\end{array}$ & & $\begin{array}{r}17.54 \\
8.56 \\
3.16 \\
-4.29\end{array}$ & $\begin{array}{r}12.35 \\
3.05 \\
-0.10 \\
-5.71\end{array}$ & $\begin{array}{r}21.41 \\
13.62 \\
2.12 \\
-11.62\end{array}$ \\
\hline A & $\begin{array}{l}\text { F3 } \\
\text { F4 }\end{array}$ & & & $\begin{array}{r}20.02 \\
2.59\end{array}$ & $\begin{array}{r}28.51 \\
2.80\end{array}$ & $\begin{array}{r}34.50 \\
2.59\end{array}$ & & $\begin{array}{l}N / P \\
N / P\end{array}$ & $\begin{array}{l}60.59 \\
32.65\end{array}$ & $\begin{array}{l}N / P \\
N / P\end{array}$ \\
\hline B & F4 & & & 6.83 & -0.07 & 7.91 & & 8.56 & 3.05 & 13.62 \\
\hline $\begin{array}{l}c \\
c \\
c\end{array}$ & $\begin{array}{l}\text { F2 } \\
\text { F3 } \\
\text { F4 }\end{array}$ & & & $\begin{array}{r}17.09 \\
-0.14 \\
0.97\end{array}$ & $\begin{array}{r}-0.22 \\
0.00 \\
0.02\end{array}$ & $\begin{array}{r}17.10 \\
-0.13 \\
0.68\end{array}$ & & $\begin{array}{l}28.65 \\
\mathrm{~N} / \mathrm{P} \\
0.54\end{array}$ & $\begin{array}{r}-0.29 \\
0.01 \\
-2.54\end{array}$ & $\begin{array}{l}24.14 \\
\mathrm{~N} / \mathrm{P} \\
-0.04\end{array}$ \\
\hline $\begin{array}{l}\text { D } \\
\text { D } \\
\text { D } \\
\text { D }\end{array}$ & $\begin{array}{l}\text { F1 } \\
\text { F2 } \\
\text { F3 } \\
\text { F4 }\end{array}$ & & & $\begin{array}{l}\mathrm{N} / \mathrm{P} \\
5.85 \\
0.67 \\
\mathrm{~N} / \mathrm{P}\end{array}$ & $\begin{array}{r}\mathrm{N} / \mathrm{P} \\
-13.99 \\
0.00 \\
0.32\end{array}$ & $\begin{array}{l}N / P \\
5.85 \\
0.67 \\
N / P\end{array}$ & & $\begin{array}{l}N / P \\
N / P \\
N / P \\
N / P\end{array}$ & $\begin{array}{r}\mathrm{N} / \mathrm{P} \\
-12.00 \\
-0.03 \\
0.22\end{array}$ & $\begin{array}{l}N / P \\
N / P \\
N / P \\
N / P\end{array}$ \\
\hline $\begin{array}{l}A \\
A \\
A\end{array}$ & & $\begin{array}{l}n \\
c \\
s\end{array}$ & & $\begin{array}{l}2.59 \\
N / P \\
-1.81\end{array}$ & $\begin{array}{l}2.80 \\
0.23 \\
0.00\end{array}$ & $\begin{array}{l}2.59 \\
\mathrm{~N} / \mathrm{P} \\
-1.81\end{array}$ & & $\begin{array}{l}N / P \\
N / P \\
N / P\end{array}$ & $\begin{array}{r}32.65 \\
-0.62 \\
0.01\end{array}$ & $\begin{array}{l}N / P \\
N / P \\
N / P\end{array}$ \\
\hline $\begin{array}{l}\text { B } \\
\text { B } \\
\text { B }\end{array}$ & & $\begin{array}{l}n \\
c \\
s\end{array}$ & & $\begin{array}{l}N / P \\
0.07\end{array}$ & $\begin{array}{l}\mathrm{N} / \mathrm{P} \\
0.00\end{array}$ & $\begin{array}{l}\mathrm{N} / \mathrm{P} \\
0.07\end{array}$ & & $\begin{array}{l}\mathrm{N} / \mathrm{P} \\
\mathrm{N} / \mathrm{P}\end{array}$ & $\begin{array}{r}55.83 \\
0.04\end{array}$ & $\begin{array}{l}N / P \\
N / P\end{array}$ \\
\hline $\begin{array}{l}c \\
c \\
c\end{array}$ & & $\begin{array}{l}n \\
c \\
s\end{array}$ & & $\begin{array}{l}4.22 \\
N / P \\
N / P\end{array}$ & $\begin{array}{l}4.54 \\
\mathrm{~N} / \mathrm{P} \\
-0.12\end{array}$ & $\begin{array}{l}7.24 \\
N / P \\
N / P\end{array}$ & & $\begin{array}{l}7.28 \\
35.48 \\
\mathrm{~N} / \mathrm{P}\end{array}$ & $\begin{array}{r}14.56 \\
14.25 \\
-0.14\end{array}$ & $\begin{array}{l}16.28 \\
35.44 \\
\mathrm{~N} / \mathrm{P}\end{array}$ \\
\hline $\begin{array}{l}\text { D } \\
\text { D } \\
\text { D }\end{array}$ & & $\begin{array}{l}n \\
c \\
s\end{array}$ & & $\begin{array}{l}5.23 \\
\mathrm{~N} / \mathrm{P} \\
-1.92\end{array}$ & $\begin{array}{c}5.12 \\
\mathrm{~N} / \mathrm{P} \\
0.71\end{array}$ & $\begin{array}{l}5.23 \\
N / P \\
-1.92\end{array}$ & & $\begin{array}{c}-0.07 \\
\mathrm{~N} / \mathrm{P} \\
0.09\end{array}$ & $\begin{array}{r}19.04 \\
0.00 \\
1.55\end{array}$ & $\begin{array}{c}19.26 \\
\mathrm{~N} / \mathrm{P} \\
1.14\end{array}$ \\
\hline
\end{tabular}

Note: N/P=Not Possible 
Table 5.17: Marginal Improvement in Maximum $R^{2}$ value of Earlier \& Later (E\&L) Year Data Format for Urban Minor Arterial \& Collector

\begin{tabular}{|c|c|c|c|c|c|c|c|c|c|c|}
\hline \multirow{2}{*}{\begin{tabular}{|l}
$C$ \\
$T$ \\
$Y$ \\
$G R$
\end{tabular}} & \multirow{2}{*}{$\begin{array}{l}\text { M } \\
F \\
\text { U } \\
\text { NC }\end{array}$} & \multirow{2}{*}{$\begin{array}{l}\text { G } \\
\text { R } \\
\text { E } \\
\text { GN }\end{array}$} & \multicolumn{4}{|c|}{ Segment Data base } & \multicolumn{4}{|c|}{ County/City Average Data base } \\
\hline & & & Raw & $\begin{array}{l}\text { Raw } \\
\text { E\&L }\end{array}$ & Density & $\begin{array}{c}\text { Density } \\
\text { E\&L }\end{array}$ & Raw & $\begin{array}{l}\text { Raw } \\
\text { E\&L }\end{array}$ & Density & $\begin{array}{c}\text { Density } \\
\text { E\&L }\end{array}$ \\
\hline & ALL & & & 4.22 & & 3.10 & & 6.92 & & 3.91 \\
\hline $\begin{array}{l}A \\
B \\
C \\
D\end{array}$ & & & & $\begin{array}{l}10.20 \\
6.83 \\
3.63 \\
\mathrm{~N} / \mathrm{P}\end{array}$ & & $\begin{array}{r}4.27 \\
7.98 \\
-0.08 \\
-3.00\end{array}$ & & $\begin{array}{r}17.54 \\
8.56 \\
3.16 \\
-4.29\end{array}$ & & $\begin{array}{r}9.06 \\
10.57 \\
2.22 \\
-5.91\end{array}$ \\
\hline A & $\begin{array}{l}\text { F3 } \\
\text { F4 }\end{array}$ & & & $\begin{array}{r}20.02 \\
2.59\end{array}$ & & $\begin{array}{r}5.99 \\
-0.21\end{array}$ & & $\begin{array}{l}N / P \\
N / P\end{array}$ & & $\begin{array}{l}N / P \\
N / P\end{array}$ \\
\hline B & F4 & & & 6.83 & & 7.98 & & 8.56 & & 10.57 \\
\hline $\mid \begin{array}{l}c \\
c \\
c\end{array}$ & $\begin{array}{l}\text { F2 } \\
\text { F3 } \\
\text { F4 }\end{array}$ & & & $\begin{array}{r}17.09 \\
-0.14 \\
0.97\end{array}$ & & $\begin{array}{r}17.32 \\
-0.13 \\
0.66\end{array}$ & & $\begin{array}{c}28.65 \\
\text { N/P } \\
0.54\end{array}$ & & $\begin{array}{l}24.43 \\
\mathrm{~N} / \mathrm{P} \\
2.50\end{array}$ \\
\hline $\mid \begin{array}{l}D \\
D \\
D \\
D\end{array}$ & $\begin{array}{l}\text { F1 } \\
\text { F2 } \\
\text { F3 } \\
\text { F4 }\end{array}$ & & & $\begin{array}{l}\mathrm{N} / \mathrm{P} \\
5.85 \\
0.67 \\
\mathrm{~N} / \mathrm{P}\end{array}$ & & $\begin{array}{c}-4.17 \\
19.84 \\
0.67 \\
\mathrm{~N} / \mathrm{P}\end{array}$ & & $\begin{array}{l}N / P \\
N / P \\
N / P \\
N / P\end{array}$ & & $\begin{array}{l}-7.24 \\
N / P \\
N / P \\
N / P\end{array}$ \\
\hline $\begin{array}{l}\mathrm{A} \\
\mathrm{A} \\
\mathrm{A}\end{array}$ & & $\begin{array}{l}n \\
c \\
\text { s }\end{array}$ & & $\begin{array}{l}2.59 \\
\mathrm{~N} / \mathrm{P} \\
-1.81\end{array}$ & & $\begin{array}{l}-0.21 \\
\mathrm{~N} / \mathrm{P} \\
-1.81\end{array}$ & & $\begin{array}{l}\mathrm{N} / \mathrm{P} \\
\mathrm{N} / \mathrm{P} \\
\mathrm{N} / \mathrm{P}\end{array}$ & & $\begin{array}{l}N / P \\
N / P \\
N / P\end{array}$ \\
\hline $\begin{array}{l}\text { B } \\
\text { B } \\
\text { B }\end{array}$ & & $\begin{array}{l}\mathrm{n} \\
\mathrm{c} \\
\mathrm{s}\end{array}$ & & $\begin{array}{l}\mathrm{N} / \mathrm{P} \\
0.07\end{array}$ & & $\begin{array}{l}5.42 \\
0.07\end{array}$ & & $\begin{array}{l}\mathrm{N} / \mathrm{P} \\
\mathrm{N} / \mathrm{P}\end{array}$ & & $\begin{array}{l}N / P \\
N / P\end{array}$ \\
\hline $\begin{array}{l}c \\
c \\
c\end{array}$ & & $\begin{array}{l}\mathrm{n} \\
\mathrm{c} \\
\mathrm{s}\end{array}$ & & $\begin{array}{l}4.22 \\
\mathrm{~N} / \mathrm{P} \\
\mathrm{N} / \mathrm{P}\end{array}$ & & $\begin{array}{l}2.70 \\
16.71 \\
\mathrm{~N} / \mathrm{P}\end{array}$ & & $\begin{array}{l}7.28 \\
N / P \\
N / P\end{array}$ & & $\begin{array}{l}1.72 \\
21.19 \\
\mathrm{~N} / \mathrm{P}\end{array}$ \\
\hline $\begin{array}{l}D \\
D \\
D\end{array}$ & & $\begin{array}{l}\mathrm{n} \\
\mathrm{c} \\
\mathrm{s}\end{array}$ & & $\begin{array}{l}5.23 \\
\mathrm{~N} / \mathrm{P} \\
-1.92\end{array}$ & & $\begin{array}{r}0.11 \\
0.57 \\
-2.63\end{array}$ & & $\begin{array}{c}-0.07 \\
\mathrm{~N} / \mathrm{P} \\
0.09\end{array}$ & & $\begin{array}{c}0.22 \\
\mathrm{~N} / \mathrm{P} \\
-0.41\end{array}$ \\
\hline
\end{tabular}

Note: $\mathrm{N} / \mathrm{P}=$ Not Possible 
Table 5.18: Marginal Improvement in Maximum $R^{2}$ value of Density Format over Raw Format for Urban Minor Arterial \& Collector

\begin{tabular}{|c|c|c|c|c|c|c|c|c|c|c|}
\hline $\mid \begin{array}{l}C \\
T\end{array}$ & & & & ment & ta base & & County & City & verage $D a$ & ata basse \\
\hline $\begin{array}{l}Y \\
G R\end{array}$ & $\begin{array}{l}\mathrm{U} \\
\mathrm{NC}\end{array}$ & $\begin{array}{l}\mathrm{E} \\
\mathrm{GN}\end{array}$ & Raw & $\begin{array}{l}\text { Raw } \\
\text { E\&L }\end{array}$ & Density & $\begin{array}{c}\text { Density } \\
\text { E\&L }\end{array}$ & Raw & $\begin{array}{l}\text { Raw } \\
\text { E\&L }\end{array}$ & Density & $\begin{array}{c}\text { Density } \\
\text { E\&L }\end{array}$ \\
\hline & ALL & & & & -2.90 & -4.02 & & & -12.50 & -15.51 \\
\hline $\begin{array}{l}A \\
B \\
C \\
D\end{array}$ & & & & & $\begin{array}{r}6.49 \\
-0.07 \\
3.01 \\
-2.60\end{array}$ & $\begin{array}{l}0.56 \\
1.08 \\
-0.70 \\
N / P\end{array}$ & & & $\begin{array}{r}12.35 \\
3.05 \\
-0.10 \\
-5.71\end{array}$ & $\begin{array}{r}3.87 \\
5.06 \\
-1.04 \\
-7.33\end{array}$ \\
\hline A & $\begin{array}{l}\text { F3 } \\
\text { F4 }\end{array}$ & & & & $\begin{array}{r}28.51 \\
2.80\end{array}$ & $\begin{array}{r}14.48 \\
0.00\end{array}$ & & & $\begin{array}{l}60.59 \\
32.65\end{array}$ & $\begin{array}{l}\mathrm{N} / \mathrm{P} \\
\mathrm{N} / \mathrm{P}\end{array}$ \\
\hline B & F4 & & & & -0.07 & 1.08 & & & 3.05 & 5.06 \\
\hline $\begin{array}{l}c \\
c \\
c\end{array}$ & $\begin{array}{l}\text { F2 } \\
\text { F3 } \\
\text { F4 }\end{array}$ & & & & $\begin{array}{r}-0.22 \\
0.00 \\
0.02\end{array}$ & $\begin{array}{r}0.01 \\
0.01 \\
-0.29\end{array}$ & & & $\begin{array}{r}-0.29 \\
0.01 \\
-2.54\end{array}$ & $\begin{array}{l}-4.51 \\
\mathrm{~N} / \mathrm{P} \\
-0.58\end{array}$ \\
\hline $\begin{array}{l}D \\
D \\
D \\
D\end{array}$ & $\begin{array}{l}\text { F1 } \\
\text { F2 } \\
\text { F3 } \\
\text { F4 }\end{array}$ & & & & $\begin{array}{r}\mathrm{N} / \mathrm{P} \\
-13.99 \\
0.00 \\
0.32\end{array}$ & $\begin{array}{l}0.00 \\
0.00 \\
0.00 \\
\text { N/P }\end{array}$ & & $\cdot$ & $\begin{array}{l}\mathrm{N} / \mathrm{P} \\
-12.00 \\
-0.03 \\
0.22\end{array}$ & $\begin{array}{l}N / P \\
N / P \\
N / P \\
N / P\end{array}$ \\
\hline $\begin{array}{l}A \\
A \\
A\end{array}$ & & $\begin{array}{l}n \\
c \\
s\end{array}$ & & & $\begin{array}{l}2.80 \\
0.23 \\
0.00\end{array}$ & $\begin{array}{c}0.00 \\
\mathrm{~N} / \mathrm{P} \\
0.00\end{array}$ & & & $\begin{array}{r}32.65 \\
-0.62 \\
0.01\end{array}$ & $\begin{array}{l}N / P \\
N / P \\
N / P\end{array}$ \\
\hline \begin{tabular}{|l}
$B$ \\
$B$ \\
$B$
\end{tabular} & & $\begin{array}{l}\mathrm{n} \\
\mathrm{c} \\
\mathrm{s}\end{array}$ & & & $\begin{array}{l}\mathrm{N} / \mathrm{P} \\
0.00\end{array}$ & $\begin{array}{l}N / P \\
0.00\end{array}$ & & & $\begin{array}{c}55.83 \\
0.04\end{array}$ & $\begin{array}{l}\mathrm{N} / \mathrm{P} \\
\mathrm{N} / \mathrm{P}\end{array}$ \\
\hline $\begin{array}{l}c \\
c \\
c\end{array}$ & & $\begin{array}{l}n \\
c \\
s\end{array}$ & & & $\begin{array}{r}4.54 \\
10.25 \\
-0.12\end{array}$ & $\begin{array}{l}3.02 \\
-0.03 \\
\mathrm{~N} / \mathrm{P}\end{array}$ & & & $\begin{array}{l}14.56 \\
14.25 \\
-0.14\end{array}$ & $\begin{array}{l}9.00 \\
-0.04 \\
\mathrm{~N} / \mathrm{P}\end{array}$ \\
\hline $\begin{array}{l}\text { D } \\
\text { D } \\
\text { D }\end{array}$ & & $\begin{array}{l}n \\
c \\
\text { s }\end{array}$ & & & $\begin{array}{r}5.12 \\
25.62 \\
0.71\end{array}$ & $\begin{array}{l}0.00 \\
0.00 \\
0.00\end{array}$ & & & $\begin{array}{r}19.04 \\
0.00 \\
1.55\end{array}$ & $\begin{array}{l}19.33 \\
\mathrm{~N} / \mathrm{P} \\
1.05\end{array}$ \\
\hline
\end{tabular}

Note: $N / P=$ Not Possible 
increase in $R^{2}$ value for raw $E \& L$ format over raw format for county/city average data base. The statistics for this case are (see Tables $(9$ and $(10)$ :

\begin{tabular}{|c|c|c|c|c|c|c|}
\hline Format & $\mathrm{R}^{2}$ & $\mathrm{R}_{\mathrm{a}}^{2}$ & PC & AIC & $c_{p}$ & $\begin{array}{c}\text { No of } \\
\text { Variable }\end{array}$ \\
\hline $\begin{array}{l}\text { Raw } \\
\text { Raw E\&L }\end{array}$ & $\begin{array}{l}65.94 \\
83.48(*)\end{array}$ & $\begin{array}{l}61.39 \\
76.87(*)\end{array}$ & $\begin{array}{l}0.4769 \\
0.3638(*)\end{array}$ & $\begin{array}{l}280.6 \\
121.9(*)\end{array}$ & $\begin{array}{c}62.54(*) \\
\text { N/A }\end{array}$ & $\begin{array}{l}2 \\
2\end{array}$ \\
\hline
\end{tabular}

$(*)=$ the better values, N/A $=$ Not Avallable.

In spite of the minor improvement in some of these statistics for the E\&L format over the raw format; one will prefer raw format because of 1ts simplicity in data structure. The "raw" data is simple, that is only averaged values over county/city for each highway class. But additional work is involved to build the "raw E\&L" data structure (See Chapter 4). Usually, the improvement in $\mathrm{R}^{2}$ value is not enough (see Table 5.16). The rest of this section's discussion will be concerned with the raw format of the county/city average data base.

Because of incompleteness of data structure and the fewer observations at Phase 3 , the quality of Phase 3 analysis will not be judged against phase 2 or 1 . But the results of Phase 3 will be used to Identify the way to improve the Phase 2 results. The Phase 2 results show the clear margin of improvement for CTYGR A and $B$ by comparing results with Phase 1 ("a11" case). The rest of the analysis for urban minor arterials and collectors for CTYGR A and B will be done at the Phase 2 level. For CTYGR C, the value of $\mathrm{R}^{2}$ is only 9.40 percent (see Table 5.13), with PC of 1.0355 (A PC value greater than unity suggests a poor result). Some statistics for possible $\mathrm{R}^{2}$ cases after phase 3 analysis with both MFUNC and GREGION are (from Table C5): 


\begin{tabular}{|cc|ccccc|}
\hline MFUNC & GREGION & $\mathrm{R}^{2}$ & $\mathrm{R}_{\mathrm{a}}^{2}$ & $\mathrm{PC}$ & $\begin{array}{c}\text { No of } \\
\text { Variables }\end{array}$ & $\begin{array}{c}\text { No of } \\
\text { Observations }\end{array}$ \\
\hline F2 & & 17.45 & 10.57 & 1.1007 & 1 & 14 \\
F3 & & 16.34 & 11.55 & 1.9521 & 1 & 5 \\
\hline & 83.63 & 76.62 & 0.3507 & 3 & 11 \\
\hline $\mathbf{C} 4$ & N/A & N/A & N/A & N/A & 12 \\
& $\mathbf{n}(*)$ & 78.69 & 74.43 & 0.3409 & 2 & 13 \\
& 99.40 & 98.80 & 0.0239 & 2 & 5 \\
\hline
\end{tabular}

$(\star)=$ the case shows better statistical results, N/A = Not Available.

The results for the combination MFUNC "F2", "F3" and GREGION "c" are not promising. For example, MFUNC "F2" and "F3" have PC values more than unity - unacceptable PC values - and low $\mathrm{R}^{2}$ values. Regression analysis with the RSQUARE procedure of SAS [SAS/Statistics 1985] was performed on the modified data set of "CTYGR C" - deleting the GREGION "c" observations and then combining the rest of the observations at phase 3. The analysis on this modified data set shows only one variable in the equation without inconsistency in the regression coefficients. The maximum $R^{2}$ values for this one-variable case are as follows:

$$
\begin{aligned}
& -\mathrm{R}^{2}=0.2692, \mathrm{R}_{\mathrm{a}}^{2}=0.2235, \mathrm{RMSE}=2269 \\
& \text { - } \mathrm{PC}=0.9136, \mathrm{AIC}=281.6 \text { and } \mathrm{C}_{\mathrm{P}}=11.03 .
\end{aligned}
$$

These statistics show the significant change in $R^{2}$ value for analysis of GREGION " $n$ " and "s" separately. The GREGION "s" has few observations in the current data set. Considering all these facts, the rest of the analysis of "CTYGR C" will be followed with the following groups of raw format of county/city average data base: 
Later, the deleted observations could be used to evaluate the performance of the final model(s).

The value 21.40 percent for $R^{2}$ and $P C$ equal to 0.9025 for CTYGR $D$ at Phase 2 are not good enough to pursue the rest of its analysis. The maximum $R^{2}$ values for CTYGR " $D$ " at the Phase 3 analysis with MFUNC and GREGION are (from Table C5):

\begin{tabular}{|lc|ccccc|}
\hline MFUNC & GREGION & $\mathrm{R}^{2}$ & $\mathrm{R}_{\mathrm{a}}^{2}$ & $\mathrm{PC}$ & $\begin{array}{c}\text { No of } \\
\text { Variable }\end{array}$ & $\begin{array}{c}\text { No of } \\
\text { Observation }\end{array}$ \\
\hline F1 & N/A & N/A & N/A & N/A & 30 \\
F2 & & 96.78 & 95.70 & 0.0645 & 2 & 9 \\
F3 & & 81.71 & 76.49 & 0.3395 & 2 & 10 \\
F4 & & 98.40 & 97.44 & 0.4160 & 3 & 9 \\
\hline & $\mathrm{C}$ & 88.06 & 86.06 & 0.1991 & 1 & 8 \\
& $\mathrm{n}$ & 35.88 & 26.01 & 0.9372 & 2 & 16 \\
& $\mathrm{~S}$ & 18.97 & 16.44 & 0.9115 & 1 & 34 \\
\hline
\end{tabular}

This table suggests the following alternatives:

1. Delete MFUNC "Fl" observations and experiment with Phase 2 analysis by combining other groups of MFUNC.

2. Delete GREGION " $n$ or $s$ " observations and do Phase 2 analysis by combining " $c$ and $s$ or $n$ " groups of GREGION.

Regression analysis with the RSQUARE procedure is conducted for these cases. The resulting maximum $R^{2}$ values without any inconsistency in the regression coefficients are as follows: 


\begin{tabular}{|c|c|c|c|c|c|c|}
\hline Case & Varlable in model & $\mathrm{R}^{2}$ & $\mathrm{R}_{\mathrm{a}}^{2}$ & PC & AIC & $c_{p}$ \\
\hline $\begin{array}{c}\text { MFUNC }= \\
F 2+F 3+F 4\end{array}$ & $\begin{array}{l}\text { CNTYINC } \\
\text { CNTYINC CTYPOP }\end{array}$ & $\begin{array}{l}.3998 \\
.5437\end{array}$ & $\begin{array}{l}.3767 \\
.5072\end{array}$ & $\begin{array}{l}.6925 \\
.5658\end{array}$ & $\begin{array}{l}444.1 \\
438.4\end{array}$ & $\begin{array}{l}146.74 \\
107.82\end{array}$ \\
\hline $\begin{array}{l}\text { GREGION= } \\
\quad \mathrm{c}+\mathrm{n}\end{array}$ & $\begin{array}{l}\text { CNTYINC } \\
\text { CNTYINC STEMP }\end{array}$ & $\begin{array}{l}.5398 \\
.5426\end{array}$ & $\begin{array}{l}.5189 \\
.4991\end{array}$ & $\begin{array}{l}.5439 \\
.5881\end{array}$ & $\begin{array}{l}340.9 \\
342.7\end{array}$ & $\begin{array}{l}-2.34 \\
-0.35\end{array}$ \\
\hline $\begin{array}{c}\text { GREGION= } \\
c+8\end{array}$ & $\begin{array}{l}\text { CNTYINC } \\
\text { CNTYINC STEMP }\end{array}$ & $\begin{array}{l}.1712 \\
.1944\end{array}$ & $\begin{array}{l}.1505 \\
.1531\end{array}$ & $\begin{array}{l}.9117 \\
.9295\end{array}$ & $\begin{array}{l}681.4 \\
682.2\end{array}$ & $\begin{array}{l}16.94 \\
17.40\end{array}$ \\
\hline
\end{tabular}

This table shows that "MFUNC=F2+F3+F4" and "GREGION=c+n" w11l be two good choices to do further analyses for CTYGR D under the raw format of the county/city average data base. The deleted observations may be used to evaluate the performance of the final model(s).

\subsection{Candidate Regression Mode1 Selection}

Sections 5.5.1 to 5.5.3 have identifled the most promising data group and data format for the three classes of highways, primarily to achieve high $R^{2}$ values. The "raw" format under the city/county average data base has been chosen in all cases. In this section, candidate models from those identified groups will be selected from a pool of possible candidate models. The possible candidate models will be those models that have good statistics and are free from any inconsistency in regression coefficients. The statistics $-R^{2}, R_{a}^{2}, P C$, RMSE, AIC and $C_{p}$ - have been used to compare the possible candidate models and narrow down the number of candidate regression models. In addition, the quality of the predictor variables (i.e., avallability, cost and reliability) has been considered to identify the candidate regression models. Based on these considerations, the following order of importance $(1=$ most preferable) of the predictor variables was 
determined (See Chapter 4):

1. Employment (county and state level)

2. (a) Population, Vehicle Registration and Income (county \& state level)

(b) State Highway Gasoline consumption

3. Households (county and state level)

4. City population.

The choices will lead to parsimony - a decrease in the number of variables in the equation with a minor loss in statistical quality. In addition, the variables will be judged by whether they are fundamental to the problem. For example, a state-level variable is preferable to a local-level variable for interstate highways and vice versa for local highways. Thus, both statistical results and judgment will be employed to select the candidate regression models.

The final selection of the model variables will be aided by residual analyses. Information gained by these analyses, together with the investigator's knowledge about the phenomenon under study, will be helpful in choosing the final regression model to be employed [Neter et a1. 1985].

\subsubsection{Interstate Highways}

The analysis of Section 5.5.1 determined the following twelve groups, comprising Phase 1 and GREGION combination of Phase 2, to select candidate models from each of these groups for interstate high- 
ways :

$$
\begin{aligned}
& \text { 1. Interstate Highways (rural and urban) } \\
& \text { 11. Rural Interstate Highways } \\
& \text { 111. Urban Interstate Highways } \\
& \text { 1v. Region ' } c \text { ' of Interstate Highways } \\
& \text { v. Region ' } n \text { ' of Interstate Highways } \\
& \text { vi. Region ' } s \text { ' of Interstate Highways } \\
& \text { vi1. Region ' } c \text { ' of Rural Interstate Highways } \\
& \text { vi1i. Region ' } n \text { ' of Rural Interstate Highways } \\
& \text { 1x. Region ' } s \text { ' of Rural Interstate Highways } \\
& x \text {. Region ' } c \text { ' of Urban Interstate Highways } \\
& x i \text {. Region ' } n \text { ' of Urban Interstate Highways } \\
& x 11 \text {. Region ' } s \text { ' of Urban Interstate Highways }
\end{aligned}
$$

These groups are not mutually exclusive. For example, groups (iv) through (vi) are subsets of group (i). But the groups (iv) to (vi) are mutually exclusive. Thus four alternate models are possible from the above twelve groups. For example, the models from groups ( 1 ), (ii), (iv) and (vii) are applicable for a rural interstate section in GREGION ${ }^{\prime} c^{\prime}$. Table 5.19 shows all the possible candidate models under each group, without any inconsistency in the sign of regression coefficlents. From this table, the following remarks can be drawn about the associated statistics for the models of each group:

- Group 1: No significant changes in the statistics among the 13 possible candidate models. UR 4 and UR 13 are the two best models for the 1 variable and 2 variable equations. 
Table 5.19: Possible Candidate Models for Interstate Highways

1. Interstate Highways [DATA - "ALL"]

\begin{tabular}{|c|c|c|c|c|c|c|c|c|c|}
\hline del & $\mathbf{R}^{2}$ & $R_{a}^{2}$ & PC & RMSE & AIC & $\mathrm{C}$ & Var & sbles in Model & $b$-coefficients in ord \\
\hline UR 1 & 41.90 & 41.72 & 0.5884 & 14827.2 & 6014.24 & 124.25 & CNTYEMP & & 0.12650 \\
\hline UR2 & 48.94 & 48.78 & 0.5172 & 13900.2 & 5973.82 & 71.77 & CNTYVR & & 0.08801 \\
\hline UR3 & 49.39 & 49.22 & 0.5126 & 13839.2 & 5971.06 & 68.43 & CNTYHH & & 0.17075 \\
\hline UR4 & 50.30 & 50.14 & 0.5034 & 13714.0 & 5965.38 & 61.64 & CNTYPOP & & 0.06291 \\
\hline UR5 & 51.02 & 50.70 & 0.4993 & 13636.0 & 5962.80 & 58.25 & CNTYHH & STEMP & $\begin{array}{lll}0.17067 & 0.02192\end{array}$ \\
\hline UR6 & 51.23 & 50.91 & 0.4972 & 13607.0 & 5961.47 & 56.70 & CNTYHH & STINC & $0.16966 \quad 4.64730$ \\
\hline UR7 & 51.24 & 50.93 & 0.4970 & 13605.2 & 5961.38 & 56.61 & CNTYHH & CNTYINC & $0.15836 \quad 2.03630$ \\
\hline UR8 & 51.28 & 50.97 & 0.4966 & 13599.3 & 5961.11 & 56.29 & CNTYPOP & STPOP & $0.06261 \quad 0.02499$ \\
\hline UR9 & 51.38 & 51.07 & 0.4956 & 13585.9 & 3960.49 & 55.57 & CNTYPOP & STVR & $0.06231 \quad 0.00633$ \\
\hline UR 10 & 51.45 & 51.14 & 0.4948 & 13575.4 & 5960.01 & 55.02 & CNTYPOP & STHH & 0.062540 .02041 \\
\hline UR 11 & 52.12 & 51.81 & 0.4881 & 13482.3 & 5955.70 & 50.07 & CNTYPOP & CNTYINC & $0.05845 \quad 2.01590$ \\
\hline UR 12 & 52.27 & 51.96 & 0.4866 & 13461.3 & 5954.73 & 48.95 & |CNTY POP & STINC & $10.06259 \quad 4.80470$ \\
\hline |UR 13 & 52.29 & 51.99 & 0.4863 & 13457.5 & 5954.55 & 48.75 & | CNTYPOP & STEMP & 10.063110 .02423 \\
\hline
\end{tabular}

11. Rural Interstate Highways [DATA - "RURAL-ALL"]

\begin{tabular}{|c|c|c|c|c|c|c|c|c|c|c|c|c|}
\hline $\mid R \mathbf{R}$ & 31.01 & 30.64 & 0. & 7871.8 & 3339.22 & 53.45 & |CNTYINC & & & 3.54721 & & \\
\hline R2 & 35.68 & 34.98 & 0.6642 & 7621.3 & 3328.17 & 39.50 & CNTYINC & CTYPOP & & .95107 & 0.00916 & \\
\hline R3 & 36.28 & 35.58 & 0.6581 & 7586.0 & 3326.44 & 37.47 & CNTYINC & CNTYEMP & & .09435 & 0.03361 & \\
\hline $\mathrm{R} 4$ & 37.49 & 36.81 & 0.6456 & 7513.6 & 3322.88 & 33.34 & CNTYINC & CNTYPOP & & 3.01986 & 0.01746 & \\
\hline R5 & 37.63 & 36.95 & 0.6441 & 7505.1 & 3322.46 & 32.86 & CNTYINC & CNTYHH & & 02135 & 0.04812 & \\
\hline R6 & 37.82 & 37.14 & & 7493.6 & 3321.89 & 32.21 & CNTYINC & CNTYVR & & 4719 & 0.02519 & \\
\hline R7 & 38.31 & 37.29 & 0.6440 & 7484.6 & 3322.42 & 32.54 & CNTYINC & CNTYVR & STINC & 72424 & 0.02598 & 1.28150 \\
\hline R8 & 38.32 & 37 & 39 & .7 & & 49 & NC & CNTYHH & STHH & 2675 & 0.04831 & 0.00772 \\
\hline R9 & 38.4 & 37.47 & & 74 & 33 & 31.95 & CNTYINC & CNTYVR & STHH & 5593 & 0.02523 & 0.00754 \\
\hline R 10 & 3 & 3 & & & & 4 & CNI & CNTYPOP & STEMP & & & 940 \\
\hline R11 & 38.73 & 37.72 & 0.6 & 7459.2 & 3321.15 & 31.11 & CNTYINC & CNTYHH & STEMP & 8521 & 0.04992 & 0.00909 \\
\hline R12 & 38. & 37.90 & & & & & $\mathrm{CN}$ & CNTYVR & STEMP & & 307 & 0.00903 \\
\hline RI3 & 39.38 & 38.38 & 0.6 & 741 & 331 & 28.88 & CNTYINC & CNTYEMP & CTYPOP & 6636 & 0.02867 & 0.00760 \\
\hline R14 & 40.47 & 39.49 & & 735 & & 25 & CNTYINC & CNTYPOP & CTYPOP & & 0.01527 & 0.00744 \\
\hline R15 & 40.49 & 39.50 & 0.6213 & 7351.4 & 3315.74 & 25.12 & CNTYINC & CNTYHH & CTYPOP & 1528 & 0.04179 & 0.00730 \\
\hline 1016 & $10<0$ & 39.62 & 0.6201 & 7344.5 & & & CNTYINC & CNTYVR & CTYPOP & 2.55744 & 0.02185 & 0.00721 \\
\hline
\end{tabular}


Table 5.19, continued

111. Urban Interstate M1ghways [DATA - "URBAN-ALL"]

\begin{tabular}{|c|c|c|c|c|c|c|c|c|}
\hline Model & $R^{2}$ & $R_{a}^{2}$ & PC & RMSF. & AIC. & $c_{p}-a-g h$ & Vartables in Model & b-coefficlents in order \\
\hline ul & 43.25 & 42.80 & 0.5856 & 18430.7 & 2496.71 & 45.12 & CNTYEMP & 0.13161 \\
\hline U2 & 51.20 & 50.81 & 0.5036 & 17091.9 & 2477.56 & 21.58 & CNTYVR & 0.09331 \\
\hline u3 & 52.28 & 51.90 & 0.4924 & 16900.6 & 12474.70 & 18.37 & CNTYHH & 0.18115 \\
\hline U4 & 53.38 & 53.01 & 0.4811 & 16704.4 & 2471.73 & 15.10 & CNTYPOP & 0.06708 \\
\hline us & 53.88 & 53.13 & 0.4836 & 16683.0 & 2472.39 & 15.65 & |CNTYHH STEMP & 10.180690 .02769 \\
\hline U6 & 54.07 & 53.33 & 0.4815 & 15647.4 & 2471.85 & 15.06 & CNTYHH STINC & $0.17935 \quad 5.77150$ \\
\hline U7 & 54.82 & 54.09 & 0.4737 & 16511.8 & 2469.77 & 12.86 & CNTYPOP STPOP & $0.06628 \quad 0.03756$ \\
\hline u8 & 55.13 & 54.41 & 0.4704 & 16453.9 & 2468.88 & 11.92 & CNTYPOP STHH & $0.06613 \quad 0.03200$ \\
\hline U9 & 55.25 & 54.53 & 0.4691 & 16432.3 & 2468.54 & 11.57 & CNTYPOP STVR & 0.065770 .01081 \\
\hline U10 & 55.37 & 54.65 & 0.4679 & 16411.0 & 2468.21 & 11.23 & CNTYPOP STINC & $0.06651 \quad 6.07240$ \\
\hline ju11 & 55.57 & 54.85 & 10.4658 & 16373.8 & 2467.64 & 10.63 & |CNTYPOP STEMP & $\mid 0.06728 \quad 0.03243$ \\
\hline
\end{tabular}

1v. Region " $c$ " of Interstate Highways [DATA = "ALL \& GREGION-c"]

\begin{tabular}{|c|c|c|c|c|c|c|c|c|c|c|c|c|}
\hline |URel & 64.83 & 4.56 & 0.3625 & 9560.0 & 2421.63 & 47.15 & CNTYP & & & 0.04996 & & \\
\hline URc2 & 65.41 & 65.14 & 0.3566 & 9481.2 & 2419.45 & 44.27 & CNTYEMP & & & 0.09792 & & \\
\hline URe3 & 65.72 & 65.45 & 0.3534 & 9438.8 & 2418.27 & 42.74 & CNTYHH & & & 0.13260 & & \\
\hline URe4 & 66.29 & 66.03 & 0.3475 & 9359.3 & 2416.03 & 39.87 & CNTYVR & & & 0.06850 & & \\
\hline URe5 & 68.91 & 68.43 & 0.3254 & 9023.4 & 2407.37 & 28.84 & CNTYHн & STINC & & 0.13135 & 4.98860 & \\
\hline URe6 & 69.04 & 68.56 & 0.3240 & 9003.6 & 2406.79 & 28.16 & CNTYVR & STHH & & 0.06642 & 0.02454 & \\
\hline URe? & 69.11 & 68.63 & 0.3232 & 8993.8 & 2406.50 & 27.82 & CNTYEMP & STEMP & & 0.09640 & 0.02599 & \\
\hline URc8 & 69.12 & 68.64 & 0.3232 & 8992.8 & 2406.47 & 27.79 & CNTY POP & STEMP & & 0.04936 & 0.02794 & \\
\hline URc9 & 69.26 & 68.79 & 0.3216 & 8971.5 & 2405.84 & 27.06 & CNTYVR & STINC & & 0.06777 & 4.81640 & \\
\hline URe IO & 69.71 & 69.24 & 0.3170 & 8906.6 & 240 & 24.86 & CNTYHH & STEMP & & 13078 & 0.02697 & \\
\hline URc11 & 70.00 & 69.53 & 10.3140 & 8863.8 & 2402.65 & 23.41 & CNTYVR & STEMP & & 0.06746 & 0.02600 & \\
\hline URc12 & 71.32 & 70.87 & 10.3002 & 8666.5 & 239 & 16.83 & CNTYPOP & CNTYINC & & 0.04418 & 3.26150 & \\
\hline & 71.66 & 71.22 & n.29hh & Rń 14.4 & 2395.12 & 15.12 & CNTYEMP & CNTYINC & & In.08668 & 3.20780 & \\
\hline 'Re 14 & 71.98 & 71.55 & 0.2932 & 8565.8 & 2393.52 & 13.53 & |CNTYVR & CNTYINC & & 6075 & 3.07490 & \\
\hline URe15 & 72.02 & 71.59 & 0.2928 & 8559.8 & 239 & 13.34 & CNTYHH & CNTYINC & & 10.11747 & 3.21770 & \\
\hline URc16 & 72.43 & 71.78 & 0.2929 & 8529.9 & 49 & 13.29 & ICNTYHH & CNTYINC & STVR & 729 & 2.8959n & .00318 \\
\hline URe17 & 72.50 & 71.86 & 0.2922 & .1 & & 12.94 & CNTYPOP & CNTYINC & STEMP & 0.04500 & 2.60420 & 0.01622 \\
\hline URCis & 72.50 & 71.86 & 0.2921 & 8518.5 & 2393.14 & 12.93 & CNTYEMP & CNTYINC & STHH & 8655 & 2.76990 & 0.01446 \\
\hline URc19! & 72.58 & 71.93 & 0.2914 & & & 12.57 & CNTYVR & CNTYINC & STPOP & 0.06061 & 2.68320 & 0.01637 \\
\hline URe 20 & 72.59 & 71.94 & 0.2913 & 8506.0 & 2392.75 & 12.52 & CNTYEMP & CNTY INC & STEMP & 0.08785 & 2.63570 & 0.01430 \\
\hline URe 21 & 72.67 & 72.03 & 0.2904 & 8493.3 & 2392.35 & 12.12 & CNTYHH & CNTYINC & STPOP & 0.11727 & 2.80680 & 0.01709 \\
\hline URc 22 & 72.77 & 72.13 & & 8476.9 & 2391.85 & 11.59 & CNTYVR & CNTYINC & STHH & 0.06063 & 2.65250 & 0.01401 \\
\hline URc23 & 72.86 & 72.23 & 0.2883 & 8462.5 & 2391.40 & 11.13 & CNTYHH & CNTYINC & STHH & 0.11729 & 2.77910 & 0.01448 \\
\hline URc 24 & 73.01 & 72.37 & 0.2868 & 8440.5 & 2390.71 & 10.43 & CNTYVR & CNTYINC & STEMP & 0.06168 & 2.46660 & 0.01508 \\
\hline URc2s & 73.08 & 72.45 & 0.2860 & 8428.6 & 2390.34 & 10.05 & CNTYHH & CNTYINC & STEMP & 0.11934 & 2.59940 & 0.01535 \\
\hline
\end{tabular}


Table 5.19, continued

v. Region " $n$ - of Interstate H1ghways [DATA = "ALL \& GREGION-n"]

\begin{tabular}{|c|c|c|c|c|c|c|c|c|}
\hline Mode 1 & $R^{2}$ & $\mathrm{R}_{\mathrm{a}}^{2}$ & PC & RMSE & AIC & $c_{p}$ & Varlables In Model & $\mid b$-coefficients in \\
\hline URn! & 37.64 & 37.03 & 0.6481 & 19365.8 & 2055.20 & 35.79 & CNTYEMP & 0.20 \\
\hline URn2 & 43.77 & 43.22 & 0.5844 & 18389.0 & 2044.44 & 22.44 & CNTYVR & 0.11874 \\
\hline URn3 & 45.08 & 44.54 & 0.5707 & 18173.2 & 2041.98 & 19.58 & CNTYPOP & 0.07776 \\
\hline URn4 & 45.33 & 44.79 & 0.5681 & 18132.0 & 2041.51 & 19.04 & CNTYHH & 0.22742 \\
\hline URn5 & 46.16 & 45.09 & 0.5704 & 18082.6 & 2041.92 & 19.23 & CNTYPOP STHH & $0.07833 \quad 0.02566$ \\
\hline URn6 & 46.20 & 45.13 & 0.5700 & 18076.0 & 2041.84 & 19.15 & CNTYPOP STVR & $0.07762 \quad 0.00859$ \\
\hline URn? & 46.30 & 45.24 & 0.5689 & 18058.8 & 2041.64 & 18.93 & CNTYPOP STPOP & 0.078990 .03740 \\
\hline URn8 & 46.33 & 45.27 & 0.5685 & 18053.5 & 2041.58 & 18.86 & CNTYHH CNTYINC & $0.21994 \quad 2.21680$ \\
\hline URn9 & 46.63 & 45.57 & 0.5654 & 18003.8 & 2041.01 & 18.21 & CNTYHH STEMP & $0.22956 \quad 0.02588$ \\
\hline URn10 & 47.02 & 45.97 & 0.5613 & 17938.0 & 2040.25 & 17.37 & CNTYPOP STEMP & $0.07926 \quad 0.03170$ \\
\hline URn 1 & 47.24 & 46.19 & 0.5590 & 17900.8 & 2039.82 & 16.89 & CNTYHH STINC & $0.22531 \quad 6.25960$ \\
\hline $\ln$ & 47.31 & 46.26 & 0.5582 & 17889.0 & 2039.68 & 16.74 & CNTYPOP STINC & $0.07726 \quad 6.75860$ \\
\hline
\end{tabular}

vi. Region ' $s$ ' of Interstate Highways [DATA = "ALL \& GREGION-s"]

\begin{tabular}{|c|c|c|c|c|c|c|c|c|c|}
\hline $\begin{array}{l}\text { |URs1 } \\
\text { URs2 } \\
\text { URs3 } \\
\text { URs4 } \\
\text { URR5 }\end{array}$ & $\begin{array}{l}58.60 \\
59.82 \\
60.19 \\
61.42 \\
61.77\end{array}$ & $\begin{array}{l}58.05 \\
59.28 \\
59.66 \\
60.90 \\
60.74\end{array}$ & $\begin{array}{l}10.4361 \\
0.4232 \\
0.4193 \\
0.4064 \\
0.4133\end{array}$ & $\begin{array}{l}8117.5 \\
7997.2 \\
7959.9 \\
7836.7 \\
7853.2\end{array}$ & $\mid \begin{array}{l}1388.25 \\
1385.95 \\
1385.23 \\
1382.83 \\
1384.12\end{array}$ & $\begin{array}{l}47.19 \\
43.66 \\
42.57 \\
39.02 \\
39.99\end{array}$ & $\begin{array}{l}\text { | CNTYEMP } \\
\text { |CNTYPOP } \\
\text { ICNTYVR } \\
\text { | CNTYHH } \\
\text { CNTYHH }\end{array}$ & STEMP & 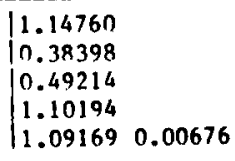 \\
\hline
\end{tabular}

v11. Region " $c^{-}$of Rural Interstate H1ghways [DATA = "RURAL-ALL \& GRGION-c"]

\begin{tabular}{|c|c|c|c|c|c|c|c|c|c|c|c|c|}
\hline 1 & & & & 7351.6 & 142 & 27.40 & ONTY INC & & & $\begin{array}{l}3.89030 \\
3.29554\end{array}$ & & \\
\hline$R \in 2$ & 4. & 42.8 & & 7035. & & $\begin{array}{l}19.48 \\
14.08\end{array}$ & $\begin{array}{l}\text { CNTYINC } \\
\text { CNTY INC }\end{array}$ & $\begin{array}{l}\text { CTYPOP } \\
\text { CNTYEMP }\end{array}$ & & 3.43086 & $\begin{array}{l}0.00181 \\
0.03198\end{array}$ & \\
\hline Da & 47.51 & 46.14 & & 6829.2 & 38 & 14.08 & CNTYINC & CNTYPOP & & 3.37292 & 0.01724 & \\
\hline $\operatorname{Re} 4$ & 48.45 & 47.11 & & 6767.7 & 4.13 & $\begin{array}{l}12.50 \\
12.43\end{array}$ & CNTY INC & CNTYHH & & 863 & 0.04462 & \\
\hline & & $\begin{array}{l}47 \\
47\end{array}$ & & $\begin{array}{l}6764 . \\
6749 .\end{array}$ & $\begin{array}{l}1414.06 \\
1413.70\end{array}$ & 12.04 & CNTYINC & CNTYVR & & 3.30847 & 0.02388 & \\
\hline 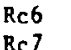 & & $\begin{array}{l}47 \\
48\end{array}$ & & $\begin{array}{l}6749.4 \\
6671.4\end{array}$ & $\begin{array}{l}1413.70 \\
1412.79\end{array}$ & 10.97 & CNTYINC & CNTYEMP & CTYPOP & 3.05733 & 0.02732 & 0.00578 \\
\hline Rc7 & & 48 & & 666 & 1412.6 & 10.85 & CNTYINC & CNTYHH & STHH & 3.03607 & 0.04534 & 0.01286 \\
\hline Rc9 & 67 & 48. & In & $66 \theta$ & 30 & 10. & CNTY INC & CNTYPOP & STH & 3.02088 & 0.01722 & 313 \\
\hline Rel & 50 & 48.8 & 0. & & & 10. & CNTYINC & CNTYHH & STI & 2.95025 & 0.04675 & 237 \\
\hline Rc & & 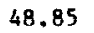 & & 66 & & 10 & CNTY INC & CNTYPOP & STI & 80 & 73 & 0.0 \\
\hline $\operatorname{Re} 12$ & 50 & 48.8 & 0. & & 40 & 10.57 & CNTYINC & CNTYVR & STHH & 314 & 367 & 267 \\
\hline $\mathrm{Rel} 3$ & & 49. & & & & & CNTYINC & CNTYVR & STE & 330 & 0. & 0.01226 \\
\hline $\mathrm{Re}$ & & 49 & & 66 & & 10.24 & CNTYINC & CNTYPOP & CTY & 313 & 0.0 & 0.00534 \\
\hline Rc & & 49. & & 6638. & 1411.99 & 10.14 & CNTYINC & CNTYHH & CTY & 6997 & 0.03886 & 535 \\
\hline Rc16 & 51.12 & 49.19 & 10.5402 & 6632.9 & 1411.86 & 10.01 & CNTYINC, & CNTYYR & CTYPOP & 2.99880 & 0.02032 & 0.00521 \\
\hline
\end{tabular}


Table 5.19, continued

v111. Region " $n$ " of Rural Interstate Highways [DATA - "RURAL-ALL \& GREGION-n"]

\begin{tabular}{|c|c|c|c|c|c|c|c|c|}
\hline Model & $\mathrm{R}^{2}$ & $\mathrm{R}_{\mathrm{a}}^{\mathrm{L}}$ & PC & RMSE & AIC & $c_{p}$ & Variables in Model & b-coefficlents in order \\
\hline $\begin{array}{l}R_{n 1} \\
R_{n} 2 \\
R_{n} 3 \\
R_{n 4} \\
R_{n} 5 \\
R_{n 6}\end{array}$ & $\begin{array}{l}27.90 \\
29.96 \\
30.24 \\
30.34 \\
30.37 \\
30.64\end{array}$ & $\begin{array}{l}26.19 \\
26.77 \\
27.07 \\
27.17 \\
27.20 \\
27.49\end{array}$ & $\begin{array}{l}0.7863 \\
0.7959 \\
0.7927 \\
0.7916 \\
0.7913 \\
0.7882\end{array}$ & $\begin{array}{l}7504.4 \\
7474.4 \\
7459.4 \\
7454.1 \\
7452.7 \\
7437.9\end{array}$ & $\begin{array}{l}840.74 \\
841.31 \\
841.12 \\
841.05 \\
841.03 \\
840.85\end{array}$ & $\begin{array}{l}67.66 \\
66.34 \\
65.91 \\
65.75 \\
65.71 \\
65.29\end{array}$ & $\begin{array}{l}\text { CNTYINC } \\
\text { CNTYINC STUR } \\
\text { CNTYINC STINC } \\
\text { CNTYINC STPOP } \\
\text { CNTYINC STEMP } \\
\text { ICNTYINC STHY }\end{array}$ & \begin{tabular}{|ll}
3.62066 & \\
3.47581 & 0.00390 \\
2.91200 & 2.96540 \\
3.45135 & 0.01861 \\
3.12513 & 0.01362 \\
3.47227 & 0.01405
\end{tabular} \\
\hline
\end{tabular}

ix. Region ' $s$ ' of Rural Interstate H1ghways IDATA = "RURAL-ALL \& GREGION-s"!

\begin{tabular}{|c|c|c|c|c|c|c|c|c|}
\hline $\mid \begin{array}{l}\text { Rs } 1 \\
\text { Rs2 } \\
\text { Rs 3 } \\
\text { Rs4 }\end{array}$ & $\begin{array}{l}40.46 \\
40.88 \\
41.73 \\
41.76\end{array}$ & $\begin{array}{l}39.42 \\
39.84 \\
39.65 \\
39.68\end{array}$ & $\left|\begin{array}{l}0.6371 \\
0.6327 \\
0.6451 \\
0.6448\end{array}\right|$ & $\begin{array}{l}4999.4 \\
4981.8 \\
4989.7 \\
4988.7\end{array}$ & $\mid \begin{array}{l}1006.98 \\
1006.56 \\
1007.71 \\
1007.68\end{array}$ & $\begin{array}{l}43.62 \\
42.93 \\
43.51 \\
43.47\end{array}$ & \begin{tabular}{|l} 
CNTYPOP \\
CNTYHH \\
CNTYPOP STEMP \\
CNTYHH STEMP
\end{tabular} & $\mid \begin{array}{ll}0.20608 & \\
0.58700 & \\
0.20333 & 0.00698 \\
0.57794 & 0.00582\end{array}$ \\
\hline
\end{tabular}

$x$. Region ' $\mathrm{c}$ ' of Utban Interstate Highways [DATA = "URBAN-ALL \& GREGION-c"]

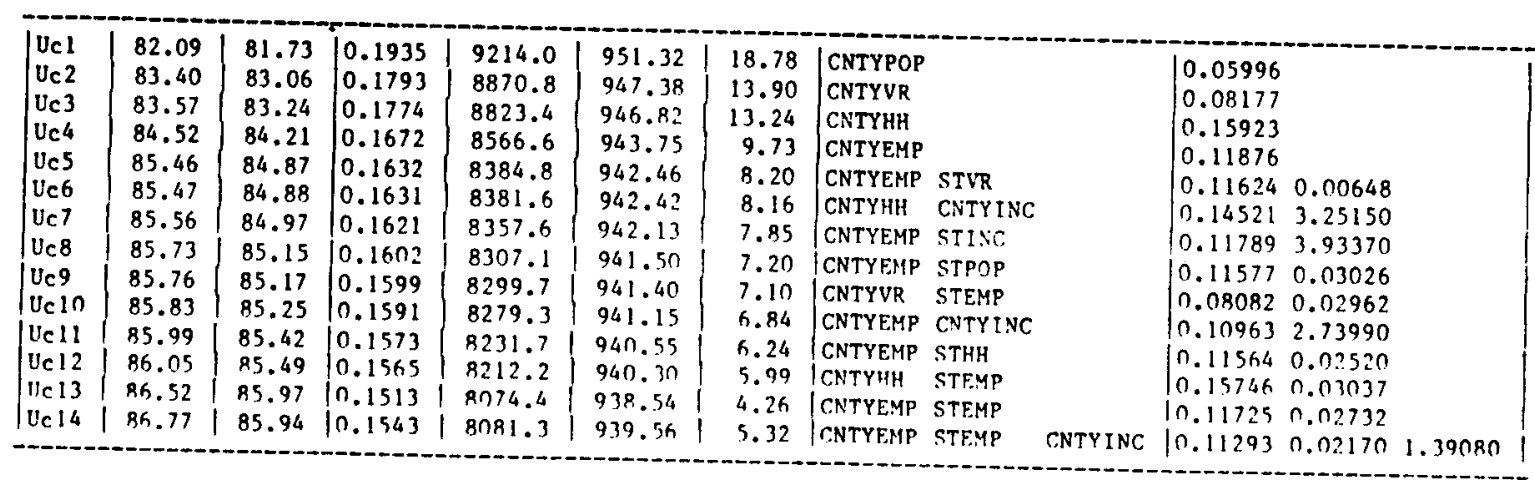


Table 5.19, continued

$x 1$. Region " $n$ ' of Orban Interstate H1ghways [DATA = "URBAN-ALL \& GREGION- $n$ "]

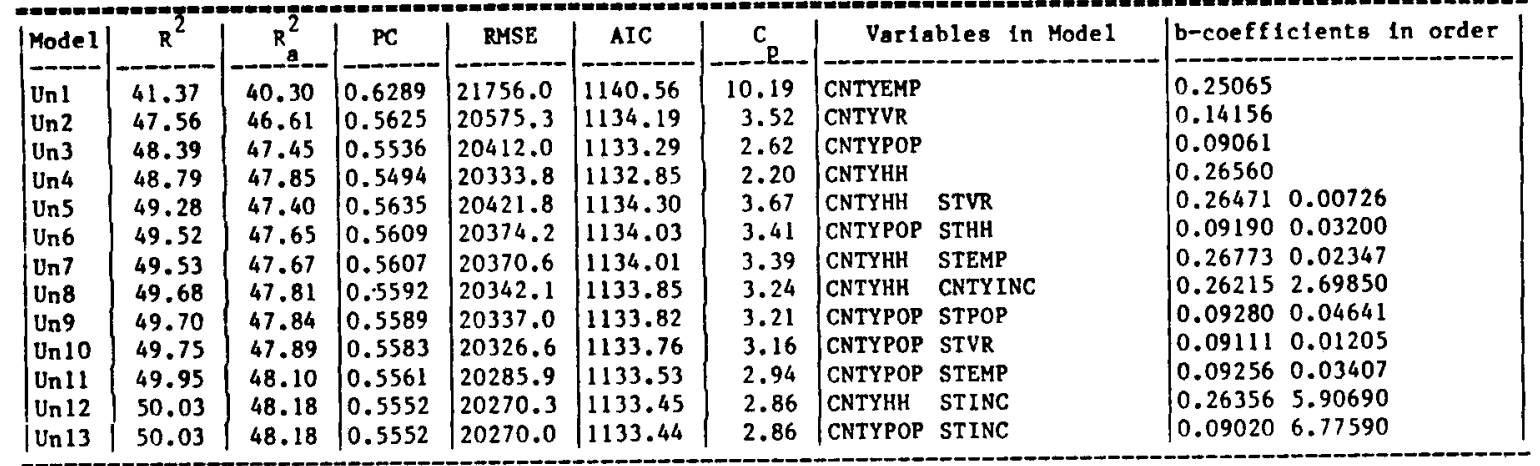

xi1. Region " $s$ " of Urban Interstate Highways [DATA = "URBAN-ALL \& GREGION-s"]

\begin{tabular}{|c|c|c|c|c|c|c|c|c|c|}
\hline Usi & 62.88 & 60.56 & 0.4640 & 9070.2 & 329.94 & 110.62 & CNTYPOP & & 0.39544 \\
\hline Us 2 & 64.42 & 62.19 & 0.4448 & 8880.3 & 329.18 & 105.45 & CNTYEMP & & 1.10581 \\
\hline Us 3 & 68.73 & 66.77 & 0.3909 & 8324.8 & 326.85 & 90.98 & CNTYHH & & 1.16050 \\
\hline log 4 & 73.11 & 71.43. & 0.3361 & 7719.7 & 324.13 & 76.27 & CNTYVR & & 0.52365 \\
\hline U. 5 & 76.01 & 72.81 & 0.3359 & 7530.8 & 324.08 & 68.54 & | CNTYVR & STEMP & $0.48955 \quad 0.01921$ \\
\hline Us 6 & 76.23 & 73.06 & 10.3328 & 7496.7 & 323.92 & 67.81 & ICNTYHH & STVR & $1.0 n 7530.01153$ \\
\hline Us 7 & 76.90 & 73.82 & 0.3234 & 7389.2 & 323.40 & 65.54 & CNTYEMP & STPOP & $1.02348 \quad 0.05393$ \\
\hline Us 8 & 77.68 & 74.70 & 0.3125 & 7263.5 & 322.78 & 62.92 & CNTYUR & STPOP & $0.47823 \quad 0.03405$ \\
\hline Us 9 & 77.98 & 75.04 & 0.3083 & 7214.7 & 322.54 & 61.92 & CNTYHH & STHH & $1.03188 \quad 0.04050$ \\
\hline Us 10 & 78.52 & 75.66 & 0.3007 & 7125.3 & 322.09 & 60.10 & CNTYEMP & STVR & 0.974390 .01515 \\
\hline Us 11 & 78.78 & 75.95 & 0.2971 & 7083.1 & 321.88 & 59.25 & CNTYUR & STVR & 0.459670 .01017 \\
\hline IU⿴ 12 & 80.48 & 77.88 & 0.2732 & 6792.0 & 320.36 & 53.51 & CNTYVR & STHH & $0.46748 \quad 0.03654$ \\
\hline |Us 13 & 81.34 & 78.86 & 0.2612 & 6640.8 & 319.55 & 50.63 & CNTYEMP & STHH & $\mid \begin{array}{lll}1.01685 & 0.05309\end{array}$ \\
\hline
\end{tabular}


- Groups ii and vii: The improvement in the statistics of R6 (Group ii) and Rc6 (Group vii), the best model with 2 variables in equation in the respective groups, establishes their superiority over R1 (Group $i i$ ) and Rcl (Group vii), the best models with 1 variable in equation, respectively.

- Groups iii and v: Among the l-variable cases, Ul of Group $i i i$ and Urnl of Group $v$ are inferior to others in their respective groups. Only a slight difference in statistics is noticed among the 2-variable cases.

- Group iv: Virtually no difference in statistics is noticed among, the candidate models with either 1 or 2 or 3 variables in the equation.

- Groups vi and $x$ : For Group vi, all the statistics show the superiority of URs 4 , the best model with 1 variable in the equation, over Urs5 - the only possible model with 2 variables in the equation. For Group $x$, Uc13, the best model with 2 variables in the equation, supercedes the choice Uc14, the only possible model with 3 variables. There is almost no change in the statistics among the choices with 1 or 2 variables in the equation of Group $x$.

- Groups vili and $1 x$ : Despite low values in $R^{2}$ and $R_{a}^{2}$, the $P C$ and AIC statistics suggest that Rnl (the only possible model with 1 variable in equation of Group vili) and Rs 1 of Group ix are better choices than others in their respective groups. 
- Group xi: The models Un12 and Un13 have almost Identical statistics. But the preference of population over household cancels the cholce Un12 in favor of Un13. For the 1-variable case, Unl is inferior to others. The results of the 2-variable cases show that the choices are almost identical.

- Group xif: The latter choices of either 1 or 2 varlables in the equation are better than the former of those, respectively.

The foregoing comparative evaluations among the models of each group with respect to their statistics, parsimony, and the preferences of variables concerning their quality (see Chapter 4) have been used to Identify the following candidate models for each group of Interstate highways.

\begin{tabular}{|c|c|c|c|c|c|c|}
\hline Group & \multicolumn{6}{|c|}{ Model Number } \\
\hline 1 & UR2 & UR4 & UR 12 & UR 13 & & \\
\hline 11 & R3 & $\mathrm{R} 4$ & R6 & $\mathrm{R} 16$ & & \\
\hline 111 & $\mathrm{U} 2$ & U4 & U9 & U11 & & \\
\hline Iv & URe 3 & URc 4 & URc 14 & URcl & 5 URc2 & 4 URc 25 \\
\hline $\mathrm{v}$ & URn 2 & URn 3 & URn 4 & URn 1 & 0 URn 1 & \\
\hline vi & URs 1 & URs 2 & URs 3 & URs 4 & & \\
\hline vii & $\mathrm{Rc4}$ & $\operatorname{Rc} 5$ & $\operatorname{Rc} 6$ & $\operatorname{Rcl} 14$ & $\mathrm{Rc} 15$ & $\mathrm{Rc} 16$ \\
\hline viil & $\mathrm{Rn} 1$ & $\operatorname{Rn} 4$ & $\operatorname{Rn} 5$ & Rn6 & & \\
\hline $1 x$ & Rs 1 & Rs 2 & Rs 3 & $\operatorname{Rs} 4$ & & \\
\hline $\mathbf{x}$ & $\mathrm{Uc} 1$ & Uc2 & Uc3 & Uc4 & $\mathrm{Uc} 12$ & $\mathrm{Uc13}$ \\
\hline$x 1$ & Un2 & Un3 & Un4 & Un 11 & Un 13 & \\
\hline$x 11$ & Us 3 & Us 4 & Us 10 & Us 11 & Us 12 & Us 13 \\
\hline
\end{tabular}

Further analyses in later chapter(s) will determine the final model from these candidate models in each group. 


\subsubsection{Urban Principal Arterial}

Based on the analysis of Section 5.5.2, the following twelve groups, which are mutually exclusive, are determined to find the model(s) from each of these groups:

$$
\begin{aligned}
\text { i. City Group }=A \text { and Region }=c \\
\text { ii. City Group }=A \text { and Region }=n \\
\text { iii. City Group }=A \text { and Region }=s \\
\text { iv. City Group }=B \text { and Region }=c \\
\text { v. City Group }=B \text { and Region }=n \\
\text { vi. City Group }=B \text { and Region }=s \\
\text { vii. City Group }=C \text { and Region }=c \\
\text { viii. City Group }=C \text { and Region }=n \\
\text { ix. City Group }=C \text { and Region }=s \\
x \cdot \text { City Group }=D \text { and Region }=c \\
\text { xi. City Group }=D \text { and Region }=n \\
\text { xii. City Group }=D \text { and Region }=s
\end{aligned}
$$

As the groups are mutually exclusive, model(s) from a particular group is(are) applicable for each highway section. The applicable group will be determined through the definition of CTYGR and GREGION from the location of the highway section. Table 5.20 shows all the possible candidate models under each group that have no inconsistency in their regression coefficlents. From this table, the following remarks could be made about the statistics associated with the models of each group :

- Group 1: The possible candidate models show a clear margin of improvement in 2-variable cases over 1-variable cases. 
Table 5.20: Possible Candidate Models for Urban Principal Arterial

1. City Group $=A \&$ Region $=c[$ DATA $=\operatorname{CTYGR}(A) \& \operatorname{GREGION}(c)]$

\begin{tabular}{|c|c|c|c|c|c|c|c|c|c|}
\hline Mode 1 & $\mathrm{R}^{2}$ & $\mathbf{R}_{\mathrm{a}}^{2}$ & PC & RMSE & AIC & $c_{p}$ & Vari & ables In Model & b-coefficients in order \\
\hline Acl 1 & 45.71 & 41.53 & 0.7099 & 1993.6 & 229.78 & 317.28 & CNTYVR & & 0.12476 \\
\hline Ac2 2 & 57.71 & 54.46 & 0.5530 & 1759.5 & 226.04 & 244.72 & CNTYEMP & & 0.37527 \\
\hline Ac 3 & 64.52 & 58.61 & 0.5322 & 1677.4 & 225.40 & 205.52 & CNTYEMP & STHн & $\begin{array}{lll}0.33280 & 0.00520\end{array}$ \\
\hline Ae4 & 76.19 & 72.22 & 0.3571 & 1374.1 & 219.42 & 134.96 & | CNTYHH & CNTYINC & 0.290432 .00873 \\
\hline Ac5 5 & 77.13 & 73.32 & 0.3430 & 1346.7 & $21^{R} .81$ & 129.28 & | CNTYVR & CNTY INC & 10.143541 .40742 \\
\hline Ac6 & 78.18 & 74.54 & 0.3274 & 1315.6 & 218.12 & 122.97 & CNTYEMP & CNTYINC & 0.381591 .11802 \\
\hline Ac? & 82.30 & 79.35 & 10.2655 & 11184.8 & |214.97 & 98.03 & | CNTYPOP & CNTYINC & $10.09702 \quad 2.26556$ \\
\hline
\end{tabular}

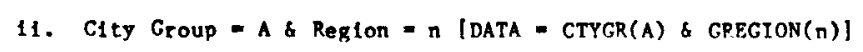

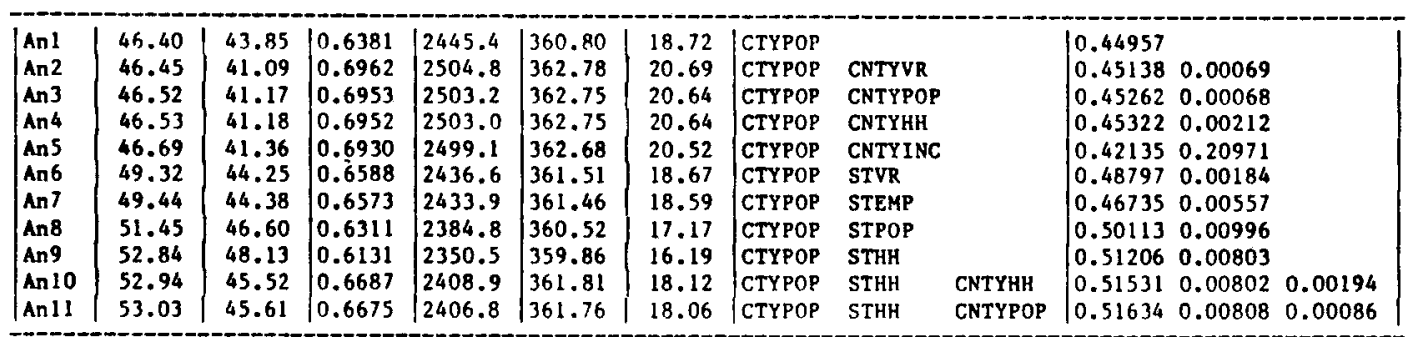

111. City Group $=A \&$ Region $=s[$ DATA $-\operatorname{CTYGR(A)\& ~} \operatorname{GrEGION}(s)]$

\begin{tabular}{|c|c|c|c|c|c|c|c|c|}
\hline A\& 1 & 73.56 & 64.75 & 10.6169 & 231.7 & 55.90 & $(*)$ & STVR & 10.00059 \\
\hline$A_{82}$ & 75.79 & 67.73 & 0.5648 & 221.7 & 55.46 & $(*)$ & |STPOP & 0.00251 \\
\hline A 3 & 82.32 & 76.43 & 0.4125 & 189.5 & 53.89 & $(\star)$ & CNTYVR & 0.04496 \\
\hline A 84 & 83.14 & 77.52 & 0.3934 & 185.0 & 53.65 & $(\star)$ & STHH & 0.00226 \\
\hline As 5 & 88.43 & 84.58 & n.2699 & 153.3 & 51.77 & $(\star)$ & CNTYHH & 10.17139 \\
\hline IAs 6 & 89.09 & 85.45 & $\ln .2546$ & 148.9 & 51.48 & $(\star)$ & |CNTYPOP & 0.11112 \\
\hline
\end{tabular}


Table 5.20, continued

1v. City Group - B Reglon - c [DATA - CTYGR(B) \& $\operatorname{CREGLON(C)]}$

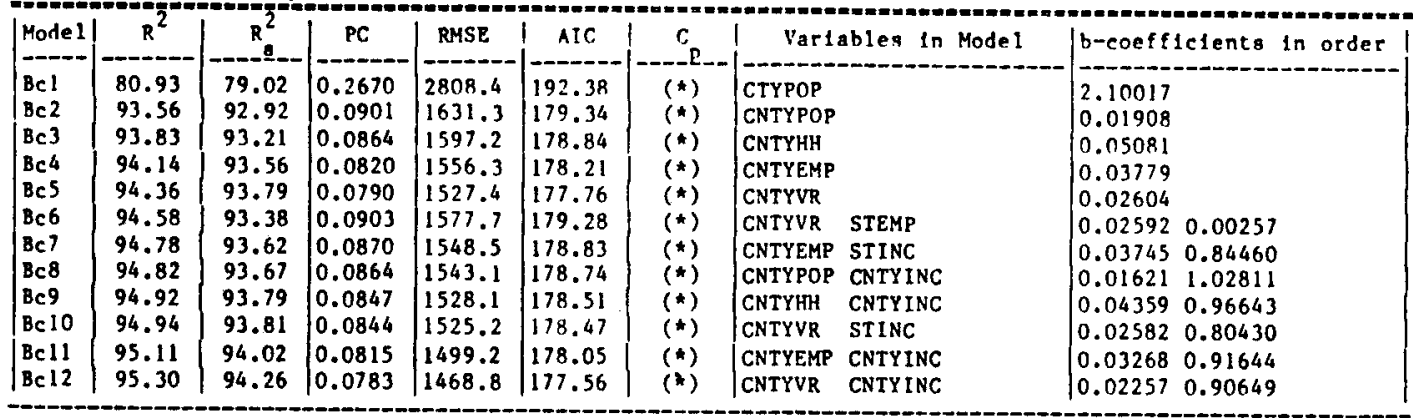

v. City Group - B \& Region - n [DATA = $\operatorname{CTYGR(B)\& ~} \operatorname{GREGION(n)]}$

\begin{tabular}{|c|c|c|c|c|c|c|c|c|c|c|c|c|}
\hline Bn! & 41.61 & 36.30 & 0.7963 & 1450.9 & 191.11 & 15.25 & STPOP & & & 0.01581 & & \\
\hline Bn2 & 48.08 & 43.36 & 0.7080 & 1368.1 & 189.58 & 12.57 & STINC & & & 2.08902 & & \\
\hline & 49.38 & 44.78 & 0.6902 & 1350.8 & 189.25 & 12.02 & STVR & & & 0.00365 & & \\
\hline Bn4 & 56.55 & 52.61 & 0.5924 & 1251.5 & 187.26 & 9.05 & STEMP & & & 0.01250 & & \\
\hline Bn5 & 59.08 & 55.36 & 0.5580 & 1214.6 & 186.49 & 8.00 & CNTYVR & & & 0.11499 & & \\
\hline Bn6 & 67.08 & 60.50 & 0.5267 & 1142.5 & 185.66 & 6.67 & STEMP & CNTY INC & & 0.00992 & 0.58040 & \\
\hline Bn? & 70.91 & 65.10 & 0.4654 & 1074.0 & 184.05 & 5.08 & CNTYVR & STGAS & & 10.12776 & 3.26653 & \\
\hline $\operatorname{BnB} 8$ & 71.19 & 65.43 & 0.4610 & 1068.9 & 183.92 & 4.97 & CNTYING, & CNTYPOP & & 1.04606 & 0.10919 & \\
\hline & 73.24 & 67.89 & 0.4281 & 1030.1 & 182.96 & 4.11 & ICNTYVR & STEMP & & 0.07498 & 0.00768 & \\
\hline Bn 10 & 75.16 & 70.19 & 0.3975 & 992.5 & 182.00 & 3.31 & I CNTYVR & STINC & & 10.08630 & 1.33923 & \\
\hline$B n 11$ & 76.16 & 71.39 & $\mid 0.3814$ & 972.3 & 181.46 & 2.90 & STPOP & CNTYINC & & 0.01513 & 0.94934 & \\
\hline Bn12 & 76.74 & 72.09 & 0.3721 & $96 n .4$ & 181.14 & 2.6h & STVR & CNTYINC & & 0326 & 0.85245 & \\
\hline Bn13 & 76.75 & 72.10 & 0.3720 & 960.2 & 181.13 & 2.66 & CNTYINC & CNTYHH & & 1.22230 & 0.27179 & \\
\hline Bn14 & 78.67 & 74.40 & 0.3413 & 919.8 & 180.02 & 1.86 & CNTYVR & CNTYINC & & 0.09824 & 0.73647 & \\
\hline & 78.80 & 74.55 & 0.3393 & 917.0 & 179.94 & 1.81 & CNTYINC & STHH & & 1.05470 & 0.01052 & \\
\hline Bn16 & 81.21 & 74.95 & 0.3549 & 909.8 & 180.36 & 2.80 & CNTYUR & CNTYINC & SIPOP & 0.06187 & 0.81100 & 0.00698 \\
\hline Bn 17 & 81.89 & 75.85 & 0.3422 & 893.4 & 179.89 & 2.52 & CNTYYR & CNTYINC & TTH & 0.05244 & 0.88939 & 0.00572 \\
\hline $\operatorname{Bn} 18$ & 82.12 & 76.17 & 0.3376 & 887.5 & 179.72 & 2.42 & CNTYVR & CNTYINC & STINC & 0.08694 & 0.52885 & 0.7 \\
\hline Bn 19 & 82.13 & 76.18 & & 887.3 & & 2.42 & CNTYINC & STHH & CNTYPOP & 1.06051 & 0.00760 & 0.04831 \\
\hline $\begin{array}{l}\mathrm{Bn} 20 \\
\mathrm{Bn} 21\end{array}$ & $\begin{array}{l}82.68 \\
83.95\end{array}$ & $\begin{array}{l}76.90 \\
78.61\end{array}$ & 0.3272 & $\begin{array}{l}873.6 \\
840.8\end{array}$ & 179.31 & 2.20 & STEMP & CNTYINC & CNTYPOP & 0.00680 & 0.74845 & 0.08160 \\
\hline & & & 0.3031 & 840.8 & 178.31 & 1.66 & CNTYUR & CNTYINC & STEMP & 0.07539 & 0.58548 & 0.00504 \\
\hline
\end{tabular}


Table 5.20, continued

v1. City Group - B \& Region - \& [DATA = CTYGR(B) \& GREGION(s)]

\begin{tabular}{|c|c|c|c|c|c|c|c|c|}
\hline Mode1 & $R^{2}$ & $R_{a}^{2}$ & PC & RMSE & AIC & $c_{p}$ & Variables In Model & $b$-coeffictents in order \\
\hline B. 1 & 95.76 & 94.34 & 0.0990 & 758.8 & 67.76 & $(*)$ & STHн & 0.01985 \\
\hline B8 2 & 96.08 & 94.77 & 0.0916 & 729.7 & 67.37 & $(*)$ & STVR & 0.00549 \\
\hline $\mathrm{Bs} 3$ & 97.09 & 96.12 & 0.0679 & 628.3 & 65.88 & (*) & CNTYVR & 0.25577 \\
\hline$B 84$ & 97.53 & 96.71 & 0.0575 & 578.5 & 65.05 & $(*)$ & СNTYHН & 0.84968 \\
\hline B85 & 98.00 & 97.34 & 0.0466 & 520.3 & 63.99 & (*) & CNTYPOP & 0.54790 \\
\hline Bs 6 & 98.97 & 98.63 & 0.0241 & 374.1 & 60.69 & (*) & STPOP & 0.02344 \\
\hline B87 & 99.30 & 98.61 & 0.0279 & 376.7 & 60.73 & $(\star)$ & CNTYPOP CNTYEMP & $0.57032 \quad 0.22379$ \\
\hline
\end{tabular}

v11. City Group - C \& Region - c [DATA - CTYGR(C)\& GREGION(C)]

\begin{tabular}{|c|c|c|c|c|c|c|c|c|c|c|c|}
\hline Cel & .48 & 29.89 & 10.7489 & 13762.9 & 742.92 & 99.81 & | СTYPOP & & 0.73585 & & \\
\hline Cc2 2 & 43.95 & 42.64 & 0.6127 & 3403.5 & 733.88 & 74.19 & I CNTYINC & & 1.87331 & & \\
\hline $\mid c c_{3}$ & 44.49 & 41.85 & 0.6344 & 3426.9 & 735.44 & 75.07 & | CNTYINC CNTYHH & & 1.82311 & 0.03172 & \\
\hline Ce4 & 45.05 & 42.43 & 0.6280 & 3409.7 & 734.99 & 73.93 & ICNTYINC CNTYVR & & 1.75708 & 0.01953 & \\
\hline & 45.17 & 42.56 & 0.6267 & 3406.0 & 734.89 & 73.68 & CNTYINC CNTYPOP & & 1.79287 & 0.01645 & \\
\hline ce6 & 61.65 & 59.82 & 0.4383 & 2848.5 & 718.80 & 39.81 & CNTYINC CTYPOP & & 1.59431 & 0.56681 & \\
\hline Ce7 & 61.96 & 59.18 & 0.4546 & 2871.2 & 720.43 & 41.16 & CNTYINC CTYPOP & CNTYVR & 1.53609 & 0.55790 & 0.01052 \\
\hline juer & 62.04 & 59.27 & 0.4536 & 2868.2 & 720.34 & 41.00 & CNTYINC CTYPOP & CNTYPOP & 1.55306 & 0.55707 & 0.00942 \\
\hline
\end{tabular}

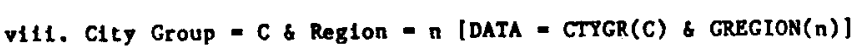

\begin{tabular}{|l|l|l|l|l|l|l|l|l|l|}
\hline$C_{n 1}$ & 31.79 & 29.36 & 0.7795 & 2259.7 & 465.31 & 7.56 & CNTYINC & 1.38579 \\
$C_{n 2}$ & 32.93 & 27.96 & 0.8198 & 2281.9 & 466.81 & 9.00 & CNTYINC STHH & 1.28106 & 0.00254 \\
$C_{n} 3$ & 33.11 & 28.16 & 0.8175 & 2278.8 & 466.72 & 8.90 & CNTYINC CNTYPOP & 1.30983 & 0.00160 \\
$C_{n} 4$ & 33.26 & 28.32 & 0.8157 & 2276.2 & 466.86 & 8.83 & CNTYINC CNTYHH & 1.30868 & 0.00498 \\
$C_{n} 5$ & 33.55 & 28.63 & 0.8122 & 2271.4 & 466.53 & 8.69 & CNTYINC CNTYVR & 1.28313 & 0.00290 \\
$C_{n} 6$ & 34.57 & 29.73 & 0.7997 & 2253.8 & 466.06 & 8.19 & CNTYINC CNTYEMP & 1.22026 & 0.00713 \\
$C_{n} 7$ & 40.16 & 35.72 & 0.7314 & 2155.5 & 463.38 & 5.44 & CNIYINC CTYPOP & 1.18695 & 0.25756 \\
\hline
\end{tabular}

1x. City Group $=C$ \& Region $=8$ [DATA $=$ CTYGR(C) \& GREGION(s)]

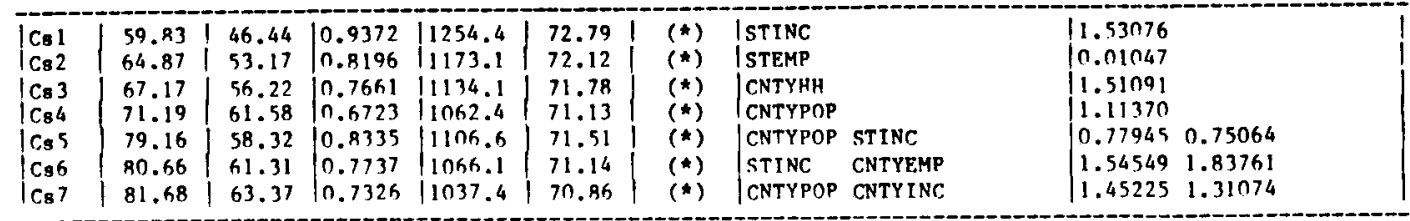


Table 5.20, continued

x. City Group - D \& Region - c [DATA = CTYGR(D) \& GREGLON(C)]

\begin{tabular}{|c|c|c|c|c|c|c|c|c|c|c|c|c|}
\hline Yode & $R^{2}$ & $R^{2}$ & PC & RMSF, & AIC & sp & & & & & & \\
\hline Del & & 34.12 & $n .7$ & $1293^{\circ}$ & & 17. & & & & 0.6 & & \\
\hline $\mathrm{De} 2$ & & 34.34 & 10.7616 & 11291.0 & 375.30 & 15.27 & CTYPOP & CNTYPOP & & 0.69688 & 0.00644 & \\
\hline$c^{3}$ & 39.70 & 34.45 & 0.7603 & 1290.0 & | 375.25 & 15.21 & |CTYPOP & CNTYEMP & & 0.70634 & 0.01910 & \\
\hline & & .73 & 0.7571 & 1287.2 & 375.14 & 15.06 & CTYPOP & CNTYHH & & 0.70246 & & \\
\hline Dc5 & 40.96 & 35.82 & 0.7443 & 1276.4 & 374.70 & 14.47 & CTYPOP & CNTYVR & & 0.70908 & 0.00989 & \\
\hline$D c 6$ & 53.38 & 49.33 & 0.5878 & 1134.2 & 368.56 & 7.22 & CTYPOP & CNTYINC & & 0.98844 & 0.66616 & \\
\hline 7 & 53.97 & 49.97 & 0.5804 & 1127.0 & 23 & 6.88 & CTYPOP & STPOP & & 713 & 0.00741 & \\
\hline $0<8$ & 54 & 50.43 & 0. & 112 & & 6.63 & CTYPOP & STINC & & 541 & 1.10903 & \\
\hline De9 & 56.10 & 52.29 & 0.5535 & 1100.6 & 367.00 & 5.63 & CTYPOP & STHH & & 0.70696 & 81 & \\
\hline 10 & 56.98 & 53.24 & 0.5424 & 1089.5 & 366.47 & 5.12 & |CTYPOP & STVR & & 0.73143 & 0.00175 & \\
\hline 11 & 58.88 & 55.30 & 35 & 1065.2 & & 4.01 & CTYPOP & STEMP & & 274 & 0.00725 & \\
\hline$D_{c} 12$ & 59.99 & 54.54 & 0.5456 & 1074.3 & 366.59 & 5.36 & CTYPOP & STVR & ST & 0.76234 & 0.00120 & 0.59380 \\
\hline & 60.06 & 54.62 & 0.5446 & 107 & & 5.32 & CTYPOP & STEMP & $\mathrm{CN}^{\prime}$ & 0.74883 & 0.00699 & 0.01197 \\
\hline 10 & 60.24 & 54.82 & 0.5421 & 1070.9 & 366.42 & 5.21 & CTYPOP & STEMP & CNT & & 690 & 0.00574 \\
\hline De1s & 61.07 & 55.76 & 0.5308 & 1059.7 & 365.88 & 4.73 & СTYPOP & STHH & STING & 0.7 & 0.00400 & 59 \\
\hline De16 & 62.45 & 57.33 & 0.5121 & 1040.8 & 364. & 3.93 & CTYPOP & STPOP & CNTYINC & 0.94436 & 0.00567 & 0.50163 \\
\hline & 64.23 & 59.35 & 0.4878 & 1015.9 & 363.68 & 2.89 & CTYPOP & STVR & CNTYINC & 0.94046 & 0.00137 & \\
\hline & 64.32 & 59.46 & 0.4865 & 1014.5 & 363 & 2.83 & CTYPOP & STEMP & CNTY INC & 0.91773 & 0.00561 & 0.41917 \\
\hline & 6 & 60.79 & 0.4705 & 997.7 & 362.74 & 2.15 & CTYPOP & STHH & CNTYINC, & 0.94592 & 0.00475 & 0.51758 \\
\hline
\end{tabular}

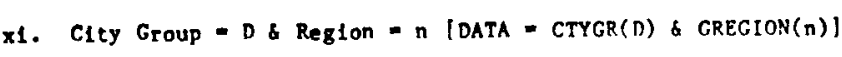

\begin{tabular}{|c|c|c|c|c|c|c|c|c|c|c|c|c|}
\hline $\mid \begin{array}{l}D n 1 \\
\text { Dn2 }\end{array}$ & 42.03 & 40.22 & |n.6522 & 2447.8 & 532.54 & 0.30 & CNTYEMP & & & 0.03529 & & \\
\hline $\begin{array}{l}\text { On2 } \\
\text { On3 }\end{array}$ & 42.19 & 40.38 & 0.6504 & 2444.5 & 532.45 & 0.21 & CNTYPOP & & & 10.01324 & & \\
\hline Dn3 & 42.78 & 40.99 & 10.6438 & 2432.0 & 1532.10 & -0.09 & CNTYHH & & & n.03865 & & \\
\hline $\mid \begin{array}{l}D_{n} 4 \\
D_{n} 5\end{array}$ & 43.82 & 42.05 & 0.6321 & $24 n 9.8$ & 531.48 & $-n .54$ & I CNTYUR & & & 0.01941 & & \\
\hline $\mid \begin{array}{l}\text { Dn5 } \\
\text { Dnn6 }\end{array}$ & 45.90 & 42.41 & 0.6457 & 12402.5 & 1532.19 & 0.27 & CNTYVR & STEMP & & $\ln .01857$ & 0.00455 & \\
\hline $\mid \begin{array}{l}\text { Dn6 } \\
D_{n} 7\end{array}$ & 45.94 & 42.45 & 0.6453 & 12401.7 & 1532.17 & 0.25 & |CNTYEMP & STPOP & & 0.03310 & $n .0 n 754$ & \\
\hline $\mid \begin{array}{l}D_{n} 7 \\
D_{n} 8\end{array}$ & 46.01 & 42.53 & 0.6444 & 2400.1 & 1532.12 & 0.22 & |CNTYHH & STPOP & & $10.0362 n$ & n.0nk90 & \\
\hline $\begin{array}{l}\text { DnR } \\
\text { Dng }\end{array}$ & 46.08 & 42.60 & 0.6436 & 2398.6 & 532.08 & 0.18 & CNTYPOP & sTHII & & $\ln .01246$ & 0.00548 & \\
\hline Dn9 & 46.15 & 42.68 & 0.6427 & 2397.0 & 532.03 & 0.14 & CNTYHH & STHH & & 10.03634 & 0.00512 & \\
\hline Dn 10 & 46.28 & 42.81 & 0.6412 & 2394.2 & 531.95 & 0.08 & CNTYEMP & STHH & & 0.03329 & 0.00571 & \\
\hline DnII & 46.33 & 42.86 & 0.6406 & 2393.0 & 531.92 & 0.05 & CNTYVR & STVR & & 0.01872 & 0.07134 & \\
\hline $10 n 12$ & 46.57 & 43.12 & 0.6377 & 2387.6 & 1531.71 & -0.07 & CNTYPOP & CNIYINC & & 0.00964 & 1.00114 & \\
\hline Dn 13 & 46.90 & 43.47 & 0.6338 & 2380.3 & 531.56 & -0.25 & CNTYVH & CNTYINC. & & 0.01464 & 0.87511 & \\
\hline DnI4 & 46.90 & 43.47 & 0.6338 & 2380.2 & 531.56 & -0.25 & CNTYVR & STPOP & & 1820 & 0.00674 & \\
\hline Dn 15 & 47.11 & 43.70 & 0.6312 & 2375.4 & 531.42 & -0.36 & | CNTYVR & STHH & & |0.01827 & 0.00507 & \\
\hline Dn 16 & 47.25 & 43.85 & 0.6296 & 2372.3 & 531.33 & -0.43 & CNTYMH & CNTYINC, & & 0.02839 & 1.00117 & \\
\hline Dn17 & 48.86 & 43.75 & $0.64,77$ & 2374.4 & 512.27 & 0.73 & CNTYUR & STPOP & CNTYINC. & 10.011551 & 0.0050 & 0.71667 \\
\hline Dn18 & 49.94 & 43.83 & 0.6468 & 2372.7 & 532.23 & 0.69 & |CNTYPOP & CNTYINC & STHH & 0.00963 & 0.83035 & 0.00439 \\
\hline $\operatorname{Dn} 19$ & 49.08 & 43.99 & 0.6449 & 2369.3 & 532.13 & 0.61 & CNTYHH & CNTYTAC & STPOP & 0.02801 & 0.85218 & \\
\hline Dn 20 & 49.10 & 44.01 & 0.6447 & 2368.9 & 532.12 & 0.60 & CNTYVR & STHH & CNTYINC. & 0.01455 & 0.00423 & 312 \\
\hline n.1 & 49.28 & 144.21 & .6425 & $276,4.7$ & חוס. יד3, & 0.51 & |CNTYIIII & CNTYINC. & stril & $\mid 0.0128017$ & $0.456,3$ & $n .0 n 4 n t$ \\
\hline
\end{tabular}


Table 5.20, continued

xi1. City Group = D \& Region - \& [DATA $=\operatorname{CTYGR(D)\& ~} \operatorname{GREGION(s)]}$

\begin{tabular}{|c|c|c|c|c|c|c|c|c|c|c|c|c|}
\hline Mode 1 & $R^{2}$ & $\mathbf{R}^{2}$ & PC & RMSE & AIC & \multicolumn{4}{|c|}{ Variables In Model } & \multicolumn{3}{|c|}{ b-coefficients in order } \\
\hline & 35.40 & & 0.7106 & 1790.2 & 631.12 & 2.28 & STVR & & & 0.00352 & & \\
\hline Ds 2 & 37.43 & 35.87 & 0.6882 & 1761.8 & 629.77 & 1.01 & STEMP & & & 0.01286 & & \\
\hline DE 3 & 37.48 & 35.91 & 0.6878 & 1761.2 & 629.75 & 0.99 & STHH & & & 0.01153 & & \\
\hline D\& 4 & 39.85 & 38.34 & 0.6617 & 1727.5 & 628.12 & -0.49 & STPOP & & & 0.01546 & & \\
\hline Do 5 & 40.46 & 37.41 & 0.6870 & 1740.5 & 629.69 & 1.13 & STEMP & CTYPOP & & 0.01228 & 0.22443 & \\
\hline D86 6 & 40.48 & 37.43 & 0.6868 & 1740.2 & 629.68 & 1.11 & STEMP & STVR & & 0.00793 & 0.00173 & \\
\hline De7 & 40.59 & 37.54 & 0.6855 & 1738.7 & 629.60 & 1.05 & STPOP & STGAS & & 0.01574 & 1.17000 & \\
\hline $\mathrm{D}_{8} 8$ & 41.03 & 38.01 & 0.6804 & 1732.1 & 629.28 & 0.77 & STPOP & CNTY INC & & 0.01450 & 0.19043 & \\
\hline Das & 42.21 & 39.25 & 0.6668 & 1714.7 & 628.43 & 0.03 & STHH & STEMP & & 0.00651 & 0.00723 & \\
\hline Ds 10 & 42.34 & 39.38 & 0.6653 & 1712.8 & 628.34 & -0.05 & STPOP & CTYPOP & & 0.01477 & 0.20442 & \\
\hline$D_{8} 11$ & 42.16 & 39.46 & 0.6644 & 1711.7 & 628.29 & -0.09 & STPOP & STEMP & & 0.00971 & 0.00598 & \\
\hline Ds 12 & 42.84 & 39.91 & 0.6595 & 1705.3 & 627.97 & -0.36 & STHK & STINC & & 0.00842 & 1.08856 & \\
\hline Ds 13 & 43.03 & 40.10 & 0.6574 & 1702.6 & 627.84 & -0.47 & STPOP & STINC & & 0.01177 & 0.89367 & \\
\hline D8: 14 & 44.67 & 40.30 & 0.6698 & 1699.8 & 628.61 & 0.50 & STHH & STEMP & CTYPOP & 0.00613 & 0.00704 & 0.20273 \\
\hline De 15 & 44.82 & 40.47 & 0.6679 & 1697.5 & 628.50 & 0.41 & STPOP & STEMP & CTYPOP & 10.00912 & 0.00588 & 0.20086 \\
\hline Ds16 & 45.02 & 40.68 & 0.6655 & 1694.4 & |628.34 & 0.28 & STHH & STINC & CTYPOP & 0.00809 & 1.03582 & 0.19128 \\
\hline |Ds 17 & 45.20 & 40.87 & 10.6634 & |1691.7 & |628.21 & 0.17 & STPOP & STINC & CTYPOP & 0.01130 & 0.84841 & 0.19102 \\
\hline
\end{tabular}


- Group ii: Despite large $R^{2}$ values, the $R_{a}^{2}, P C, R M S E, A I C$ and $C_{p}$ statistics make An10 and Anl1 -- models with 3 variables in their equations -- inferior to the best model with 2 variables in its equation (An9). There is practically no difference in associated statistics among models An2 to An5. For 2-variable cases, a slight improvement in statistics of An6 to An 9 is noticed in comparison to the statistics of An2 to An9.

- Group iii: The associated statistics of As 3 to As6 make them preferable to As 1 and As 2 .

- Group iv: The improvement in the statistics of $\mathrm{BC} 2$ to $\mathrm{BC} 12$, when compared to the statistics of $\mathrm{BCl}$, makes $\mathrm{BCl}$ inferior to other choices with 1 variable. Only slight improvement in statistics is noticed for the best models with 2 variables over those with 1 variable. $P C$ and AIC criteria invalidate the choices Bc6-Bcll versus $\mathrm{BC} 5$.

- Group v: The statistics of 2-variable cases make them preferable to l-variable cases. Despite a higher $\mathrm{R}^{2}$ for $B n 16$ than for $\mathrm{Bn} 15$, the PC and AIC criteria reject $B n 16$ in favor of $B n 15$.

- Group vi: Despite a small improvement in $R^{2}$ value for $B s 7$ over Bs6, the associated statistics make Bs6 more worthwhile to pursue than Bs7.

- Group vii: The statistics show a significant improvement in Cc2 over Ccl. Despite high values in $\mathrm{R}^{2}$, the other associated statistics suggest that $\mathrm{Cc} 7$ and $\mathrm{Cc} 8$ are inferior to $\mathrm{Cc}_{\mathrm{c}}$. 
- Groups vili and 1x: Each of these groups shows some improvement in statistics of latter choices $(\operatorname{Cn} 5-\operatorname{Cn} 7, \operatorname{Cs} 4-\operatorname{Cs} 7)$ in comparison to earlier cholces $(\operatorname{Cn} 1-\operatorname{Cn} 4, \operatorname{Cs} 1-\operatorname{Cs} 3)$ in the lists.

- Group x: For 2-variable cases, the latter models (Dc6-D11) are better than the earlier choices (Dc2-Dc5). There is only slight improvement in statistics of the better models (Dc18-Dc19) with 3 variables over those with 2 variables.

- Groups $x 1$ and $x i 1:$ An increase in the number of variables has not changed the associated statistics significantly. For group $x 1$, the PC and AIC criteria make Dn5-Dn14 inferior to Dn4. For group $x i 1$, the PC and AIC criterion establish the superiority of Ds 4 and Ds 12-Ds 13 over Ds 5-Ds 11 and Ds 14-Ds 17, respectively. These comparative evaluations, parsimony, and the quality of the variables have been used to identify the following candidate models for each group of urban principal arterials.

\begin{tabular}{|l|llll|}
\hline Group & \multicolumn{3}{|c|}{ Mode1 } & Number \\
\hline 1 & Ac4 & Ac5 & Ac6 & Ac7 \\
11 & An6 & An7 & An8 & An9 \\
111 & As3 & As4 & As5 & As6 \\
\hline $1 v$ & Bc3 & Bc4 & Bc5 & Bc12 \\
$v$ & Bn12 & Bn14 & Bn15 & Bn21 \\
vi & Bs3 & Bs4 & Bs5 & Bs6 \\
\hline vi1 & Cc5 & Cc6 & & \\
v111 & Cn5 & Cn6 & Cn7 & \\
$1 x$ & Cs4 & Cs5 & Cs6 & Cs7 \\
\hline$x$ & Dc10 & Dc11 & Dc18 & Dc19 \\
x1 & Dn3 & Dn4 & Dn15 & Dn16 \\
x11 & Ds3 & Ds4 & Ds 12 & Ds 13 \\
\hline
\end{tabular}


In Section 5.4.2, an analysis of pooled data for CTYGR A, B and C has been undertaken because of the low number of observations for each of the city groups. This pooling has given the opportunity to produce statistically more significant results by incorporating a larger data set $(n=15)$ than the data set $(n=5)$ for each group separately. Table C5.1 has some of the statistical measures for the best models with 1 or 2 variables in the equation for this pooled data set. The separation of the city group ' $D$ ' from the pooled data set has resulted in good statistical measures. Moreover, the resulting observations $(n=42)$ can be considered large enough to do the further statistical analyses and to produce significant statistical results. Thus, a common final model for the south region of CTYGR $A, B$ and $C$ will be selected from the pooled data set -- Group xili. And the models for the groups iii, vi and ix - the individual groups of CTYGR A, B and C in the south region -- will not be evaluated. The nine possible candidate models for the pooled group are shown in Table 5.20.1. Of those, the five models ( $A B C s 3-A B C s 4$ and $A B C s 7-A B C s 9$ ) have been selected as the candidate models based on their associated statistics and quality of variables in the models. Further analyses in later chapter(s) will determine the final model from these candidate models under each of the selected groups. 

Table 5.20.1: Possible Candidate Models for South Region of
CTYGR A, B and C of Urban Principal Arterial

x11. City Group $=A+B+C \& \operatorname{Region}=s \quad[\operatorname{DATA}=\operatorname{CTYGR}(A+B+C) \& \operatorname{GREGION}(s)]$

\begin{tabular}{|c|c|c|c|c|c|c|c|c|}
\hline Mode1 & $\mathrm{R}^{2}$ & $\mathrm{R}^{2}$ & PC & RMSE & AIC & C & Varlables in Model & b-coefficlents in order \\
\hline ABCs $1 \mid$ & 67.38 & 64.87 & 0.4266 & 3889.2 & 249.83 & 158.50 & CNTYPOP & 0.12476 \\
\hline$A B C s 2$ & 69.03 & 66.65 & 0.4049 & 3789.0 & 249.05 & 149.88 & CNTYHH & 0.37527 \\
\hline $\begin{array}{l}A B C s 3 \\
A B C s 4\end{array}$ & $\begin{array}{l}72.43 \\
81.08\end{array}$ & $\begin{array}{l}70.31 \\
79.63\end{array}$ & $\begin{array}{l}0.3605 \\
0.2474\end{array}$ & $\begin{array}{l}3575.2 \\
2961.5\end{array}$ & $\begin{array}{l}247.31 \\
241.66\end{array}$ & $\begin{array}{r}132.23 \\
87.28\end{array}$ & $\begin{array}{l}\text { CNTYVR } \\
\text { CNTYEMP }\end{array}$ & $\mid \begin{array}{ll}0.33280 & 0.00520 \\
0.29043 & 2.00873\end{array}$ \\
\hline$A B C s 5$ & 83.50 & 80.74 & 0.2476 & 2879.2 & 241.61 & 76.75 & CNTYEMP STEMP & $0.14354 \quad 1.40742$ \\
\hline$A B C 56$ & 84.19 & 81.55 & 0.2372 & 2818.2 & 240.97 & 73.15 & CNTYEMP STINC & $0.38159 \quad 1.11802$ \\
\hline$A B C s 7$ & 85.14 & 82.66 & 0.2229 & 2732.1 & 240.04 & 68.21 & CNTYEMP STVR & $0.09702 \quad 2.26556$ \\
\hline $\mathrm{ABCs} 8$ & 85.44 & 83.01 & 0.2184 & 2704.2 & 239.73 & 66.64 & CNTYEMP STHH & $0.38159 \quad 1.11802$ \\
\hline$|A B C s 9|$ & 85.53 & 83.12 & 0.2170 & 2695.6 & 239.63 & 66.16 & CNTYEMP STPOP & $0.09702 \quad 2.26556$ \\
\hline
\end{tabular}




\subsubsection{Urban Minor Arterial and Collector}

The data groups determined in Section 5.5 .3 for minor arterials and collectors are as follows:

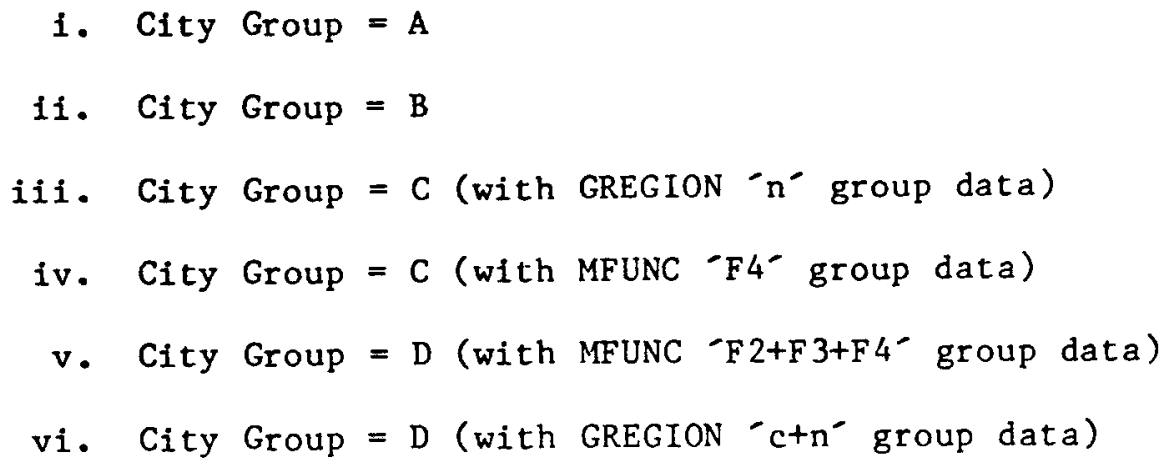
sistency in regression coefficients, are presented in Table 5.21. The following remarks apply to the associated statistics for the models in each group:

- Group 1 : The possible candidate models show a clear margin of improvement for $A 4-A 7$ with 2 variables versus $A 2$ with 1 variable. For the two 1-variable cases, $A 2$ is preferable to A1.

- Group 11: For 1-variable cases, the statistics of B2 invalidate the choice of $\mathrm{B} 1$. Despite a minor improvement in the $\mathrm{R}^{2}$ value of 2-variable cases over B2 with 1 variable, the associated statistics make 2-variable choices inferior to B2.

- Group 1i1: A significant improvement is noticed in the statistics of $\mathrm{C} 2, \mathrm{C} 3$ and $\mathrm{C} 4$ over $\mathrm{Cl}$, all with 1 variable in their equations. The associated statistics make the choices with 2 variables 
Table 5.21: Possible Candidate Models for Urban Minor Arterial and Collector

1. City Group $=$ A [DATA $=\operatorname{CTYGR}(A)]$

\begin{tabular}{|c|c|c|c|c|c|c|c|c|}
\hline Mode1 & $\mathbf{R}^{2}$ & $R_{a}$ & PC & RMSE & AIC & $C_{p}$ & Variables in Model & b-coefficlents in order \\
\hline Al & 43.40 & 39.86 & 0.7075 & 2805.5 & 287.70 & 109.86 & CNTYEMP & 0.04083 \\
\hline A2 & 53.57 & 50.67 & 0.5804 & 2540.9 & 284.13 & 87.60 & CNTYINC & 2.09310 \\
\hline A3 & 56.02 & 50.16 & 0.6157 & 2554.0 & 285.15 & 84.23 & CNTYINC STHH & 1.876790 .00497 \\
\hline A4 & 62.07 & 57.01 & 0.5310 & 2372.0 & 282.49 & 71.00 & CNTYINC CNTYHH & $1.84437 \quad 0.01934$ \\
\hline A5 & 62.07 & 57.02 & 0.5310 & 2371.8 & 282.49 & 70.99 & CNTYINC CNTYPOP & 1.833700 .00668 \\
\hline A6 & 64.04 & & 0.5034 & 2309.5 & 281.53 & 66.69 & CNTYINC CNTYVR & $1.73620 \quad 0.01145$ \\
\hline A7 & 65.94 & 61.39 & 0.4769 & 2247.8 & 280.56 & 62.54 & CNTYINC CNTYEMP & 1.545690 .02481 \\
\hline
\end{tabular}

11. City Group $=$ B $[$ DATA $=\operatorname{CTYGR}(B)]$

\begin{tabular}{|c|c|c|c|c|c|c|c|c|}
\hline $\begin{array}{l}\text { B1 } \\
\text { B2 } \\
\text { B3 } \\
\text { B4 } \\
\text { B5 } \\
\text { B6 } \\
\text { B7 } \\
\text { B8 }\end{array}$ & $\begin{array}{l}40.76 \\
66.94 \\
66.96 \\
66.97 \\
67.00 \\
67.15 \\
67.23 \\
67.58\end{array}$ & $\begin{array}{l}35.40 \\
63.94 \\
60.35 \\
60.37 \\
60.41 \\
60.68 \\
61.17 \\
63.40\end{array}$ & $\mid \begin{array}{l}0.8079 \\
0.4507 \\
0.5287 \\
0.5284 \\
0.5279 \\
0.5243 \\
0.5177 \\
0.4880\end{array}$ & $\mid \begin{array}{l}3096.8 \\
2313.1 \\
2425.6 \\
2425.1 \\
2423.9 \\
2415.5 \\
2400.4 \\
2330.4\end{array}$ & \begin{tabular}{|l}
210.82 \\
203.23 \\
205.23 \\
205.22 \\
205.21 \\
205.12 \\
204.96 \\
204.19
\end{tabular} & $\begin{array}{l}(*) \\
(*) \\
(*) \\
(*) \\
(*) \\
(*) \\
(*) \\
(*)\end{array}$ & 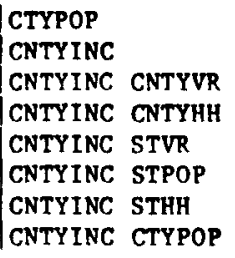 & $\mid \begin{array}{ll}1.91741 & \\
2.40789 & \\
2.40330 & 0.00034 \\
2.40411 & 0.00094 \\
2.38507 & 0.00025 \\
2.36248 & 0.00240 \\
2.36762 & 0.00284 \\
2.02801 & 0.61712\end{array}$ \\
\hline
\end{tabular}

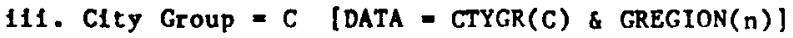

\begin{tabular}{|c|c|c|c|c|c|c|c|c|}
\hline Model & $\mathbf{R}^{2}$ & $\mathbf{R}_{\mathbf{a}}$ & PC & RMSE & AIC & $\mathrm{C}_{\mathrm{r}}$ & Variables in Model & b-coefficients in order \\
\hline $\mathrm{Cl}$ & 61.45 & 57.95 & 0.5257 & 1229.4 & 186.80 & $(*)$ & CNTYEMP & 0.02291 \\
\hline $\mathrm{C} 2$ & 71.58 & 68.99 & 0.3876 & 1055.7 & 182.84 & (*) & CNTYVR & 0.01283 \\
\hline C3 & 73.88 & 71.50 & 0.3562 & 1012.0 & 181.74 & (*) & CNTYPOP & 0.00849 \\
\hline C4 & 75.74 & 73.53 & 0.3309 & 975.4 & 180.78 & (*) & CNTYHH & 0.02504 \\
\hline C5 & 77.04 & 72.44 & 0.3674 & 995.2 & 182.06 & $(*)$ & CNTYHH STPOP & 0.023990 .00226 \\
\hline C6 & 77.79 & 73.35 & 0.3554 & 978.7 & 181.63 & (*) & CNTYPOP STHH & $0.00797 \quad 0.00310$ \\
\hline C7 & 77.94 & 73.52 & 0.3530 & 975.5 & 181.54 & (*) & CNTYHH STEMP & $0.02430 \quad 0.00234$ \\
\hline C8 & 78.69 & 74.43 & 0.3409 & 958.7 & 181.09 & $(\star)$ & CNTYHH STHH & $\mid 0.02358 \quad 0.00271$ \\
\hline
\end{tabular}


Table 5.21, continued

1v. City Group = C [DATA = CTYGR(C) \& MFUNC(F4)]

\begin{tabular}{|c|c|c|c|c|c|c|c|c|c|c|c|c|}
\hline Mode1 & $R^{2}$ & $\mathbf{R}_{a}^{2}$ & PC & RMSE & AIC & $c_{p}$ & Varla & bles in & Mode 1 & $b-c o e f f 1$ & lclents & In order \\
\hline C9 & 74.85 & 72.05 & 0.3633 & 1267.9 & 158.99 & (*) & CNTYEMP & & & 0.03364 & & \\
\hline $\mathrm{ClO}$ & 76.60 & 74.00 & 0.3381 & 1223.0 & 158.19 & (*) & CNTYPOP & & & 0.01123 & & \\
\hline C11 & $78.08^{\circ}$ & 75.64 & 0.3166 & 1183.6 & 157.47 & (*) & CNTYVR & & & 0.01828 & & \\
\hline C12 & 78.30 & 75.88 & 0.3135 & 1177.8 & 157.36 & (*) & CNTYHH & & & 10.03289 & & \\
\hline C13 & 80.39 & 75.49 & 10.3431 & 1187.3 & 158.24 & (*) & CNTYPOP & STHH & & 10.01093 & 0.00532 & \\
\hline C14 & 80.69 & 75.86 & 0.3379 & 1178.3 & 158.08 & (*) & | CNTYVR & STEMP & & 0.01830 & 0.00548 & \\
\hline C1s & 80.74 & 75.93 & 0.3370 & $117 \mathrm{~h} .7$ & 158.05 & $(\star)$ & CNTYEMP & STHH & & $n .033 n 3$ & $0.0 \cap 660$ & \\
\hline cla & 80.76 & 75.95 & 10.3367 & 1176.2 & 1158.04 & $(\star)$ & CNTYFMP & STVR & & 0.03305 & 0.00245 & \\
\hline C17 & 80.92 & 76.15 & 0.3339 & 1171.3 & 157.94 & (*) & CNTYVR & STHH & & $\ln .01775$ & 0.00462 & \\
\hline $\mathrm{Cl} 18$ & 80.94 & 76.17 & 0.3335 & 1170.6 & 157.93 & $(*)$ & CNTYHH & STHH & & 0.03193 & 0.00447 & \\
\hline C19 & 81.06 & 76.32 & 0.3315 & 1167.0 & 157.87 & $(\star)$ & CNTYHH & STEMP & & 0.03297 & 0.00564 & \\
\hline C2O & 81.09 & 76.37 & 0.3309 & 1166.0 & 157.85 & (*) & |CNTYPOP & STPOP & & 0.01091 & 0.00901 & \\
\hline C21 & 81.55 & 76.94 & 0.3229 & 1151.8 & 157.58 & (*) & CNTYVR & STPOP & & 0.01772 & 0.00795 & \\
\hline C22 & 81.71 & 77.13 & 0.3201 & 1146.8 & 157.48 & (*) & CNTYHH & STPOP & & 0.03187 & 0.00788 & \\
\hline C23 & 81.80 & 77.25 & 0.3185 & 1143.9 & 157.43 & (*) & CNTYEMP & STPOP & & 0.03306 & 0.01115 & \\
\hline C24 & 83.14 & 75.92 & 0.3612 & 1176.8 & 158.58 & (*) & CNTYPOP & STPOP & CNTYEMP & 0.00501 & 0.01865 & 0.01012 \\
\hline C25 & 83.63 & 76.62 & 0.3507 & 1159.7 & 158.26 & (*) & СNTYнH & STPOP & CNTYEMP & 0.01616 & 0.00944 & 0.01716 \\
\hline
\end{tabular}

v. City Group $=D$ [DATA $=\operatorname{CTYGR}(D) \& \operatorname{MFUNC}(F 2+F 3+F 4)]$

\begin{tabular}{|c|c|c|c|c|c|c|c|c|}
\hline$\left|\begin{array}{l}D 1 \\
D 2 \\
D 3 \\
D 4 \\
D 5 \\
D 6\end{array}\right|$ & $\begin{array}{l}39.98 \\
39.98 \\
39.98 \\
39.99 \\
41.18 \\
54.37\end{array}$ & $\begin{array}{l}37.67 \\
35.18 \\
35.18 \\
35.19 \\
36.48 \\
50.72\end{array}$ & $\mid \begin{array}{l}0.6925 \\
0.7442 \\
0.7442 \\
0.7441 \\
0.7293 \\
0.5658\end{array}$ & $\begin{array}{l}2686.4 \\
2739.6 \\
2739.5 \\
2739.3 \\
2712.1 \\
2388.8\end{array}$ & $\mid \begin{array}{l}446.10 \\
446.10 \\
446.09 \\
445.53 \\
438.43 \\
181.63\end{array}$ & $\begin{array}{l}146.74 \\
148.74 \\
148.73 \\
148.71 \\
145.33 \\
107.82\end{array}$ & 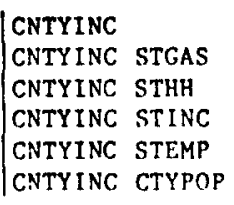 & $\mid \begin{array}{ll}2.04400 & \\
2.03917 & 0.09900 \\
2.03185 & 0.00021 \\
2.01787 & 0.07403 \\
1.84212 & 0.00424 \\
1.68745 & 0.88901\end{array}$ \\
\hline
\end{tabular}

v1. City Croup $=$ D IDATA $=$ CTYGR(D) \& GRFGION $(C+n) \mid$

\begin{tabular}{|c|c|c|c|c|c|c|c|c|}
\hline D7 & 53.98 & 51.89 & 10.5439 & 11167.0 & 340.89 & 1146.74 & I CNTYING & 11.16659 \\
\hline D8 & 54.12 & 49.75 & 10.5898 & 11102.5 & 342.82 & 148.74 & | CNTYING STHH & 1.154320 .00060 \\
\hline 109 & 54.13 & 49.76 & 10.5898 & 11192.5 & 342.82 & 148.73 & CNTYINC STPOP & $1.15390 \quad 0.00087$ \\
\hline D10 & 54.22 & 49.86 & $\ln .5887$ & 1191.3 & 342.77 & 148.71 & CNTYINC STVR & $1.15009 \quad 0.00024$ \\
\hline D11 & 54.26 & 49.91 & 10.5881 & 1190.7 & 1342.75 & 145.33 & CNTYINC STEMP & 1.148980 .00103 \\
\hline
\end{tabular}

(*) Error-Vartance to ton small to compute $c_{p}$ 
inferior to $\mathrm{C} 4$, which is the best model with one variable.

- Group iv: A minor amount of change in the statistics is noticed among the possible candidate models. The PC and AIC criteria establish that $C 12$ with 1 varlable is slightly better than the chotces with 2 and 3 variables.

- Group v: The statistics of D6 establish that it is the best selection among all cholces.

- Group vi: Despite a small improvement in $\mathrm{R}^{2}$ value for D8-D11 with 2 variables over D7 with 1 variable, the associated statistics qualify $D 7$ over D8-D11.

These comparative evaluations, parsimony and the quality of variables have been used to identify the following candidate model(s) for each group of urban minor arterials and collectors.

\begin{tabular}{|l|llll|}
\hline Group & \multicolumn{3}{|c|}{ Model } & Number \\
\hline i & A2 & A5 & A6 & A7 \\
i1 & B2 & & & \\
iii & C2 & C3 & C4 & \\
iv & C9 & C10 & C11 & C12 \\
v & D6 & & & \\
vi & D7 & & & \\
\hline
\end{tabular}

Further analyses in the next chapter(s) will determine the final model from these candidate models under each group. 


\subsection{Chapter Summary}

This chapter has described all the statistics that were employed to compare the data bases, data formats and groups. A sumnary of all important results has been provided. Among the four alternative data formats, the "RAW" data format of county/city average data base is found to be the best. The evaluation of alternative data structures has avoided the risk of working with a weak data structure and thus avoided accepting poor statistical results. In addition to data format and structure selection, groupings of data have been made to produce statistically sound results. Finally, the combination of statistical evaluation and judgment based on quality of the predictor variables is employed to identify candidate regression models for further analysis in the following chapter and in earlier in chapter. 
CHAPTER 6

DIAGNOSIS AND FINAL SELECTION OF MODEL

\subsection{Introduction}

The aim of regression diagnostics is to exhibit the degree of appropriateness of the assumptions made in fitting a regression model to a specific set of data. Answers are sought to such questions as

1. Are there outliers?

11. Should the response variable be transformed?

111. Is the variance constant?

iv. Are all the explanatory variables in the model necessary?

v. Is the systematic part of the model adequate? If not, should variables be added or should the form of the model be altered?

v1. How do the answers to these questions depend on individual observations?

In this chapter, influential analysis will be carried out to Identify the outliers and influential observations (if present) for all the candidate models selected in Chapter 5. The statistical background and mechanism for doing this influential analysis will be 
described in this chapter. The statistical results of pre and post influence analysis have shown significant improvements in some of the statistics in later. Thus, the influence analysis has avoided many unexpected transformation to satisfy some assumptions of regression analysis. All important assumptions regarding regression analysis and model aptness are examined for the candidate models. Significance and collinearity of regression coefficients will be evaluated to find the most useful variables and to avoid the problem of selection with multicollinearity. The selection of the final model, both regression and elasticity-based, for each group of different highways under consideration will be based primarily on the quality of statistical results of the candidate model.

\subsection{Detecting Outliers and Influential Observations}

Outliers are extreme observations. In general, an observation is considered influential if removing it would markedly change the position of the regression 1ine. But, not all influential observations are outliers. After the multiple regression has been performed, most detection procedures focus on the residuals, the fitted(predicted) values, and the explanatory variables.

Outliers can create great difficulty. A major reason for discarding outlier(s) is that, under the least squares method, a fitted line may be pulled disproportionately toward an outlier because the sum of the squared deviations is minimized. The three main problems with an outlier are as follows: 
1. An outlier can pull down the value of regression coefficient(s) toward zero so that the value of the t-statistic for testing $\mathrm{H}_{0}$ : $B_{f}=0$ is also going to be smaller. Thus, it would cause the null hypothesis to be unnecessarily accepted even though the dependent and the independent variables do have a significant regression relation.

2. Outliers could also pull up the value of the slope of the fitted line and also the t-statistic for testing for significance of regression. Thus, a significant regression relation may be concluded when there is no such relationship.

3. The outliers are unlikely to affect the analysis if regarded one at a time. But a cluster of outliers can affect the analysis as a group. Thus, studying the effect of influential observations, it is not enough to consider them one at a time, but it is also necessary to study them as a group.

In this study, some techniques [SAS/STATISTICS 1985; Belsley, Kuh \& Welsch 1980] are used for diagnosing influential data points that avold some of the problems described in above paragraphs. Two representative candidate models, $\mathrm{Cn} 5$ and Un4 (see Chapter 5), are selected to show the individual and group effect of outliers, respectively.

\subsubsection{Diagnostic Techniques of Detecting Outliers}

A brief description from SAS/STATISTICS [1985] and Belsley, Kuh, and Welsch [1980] regarding the technical background for diagnosing 
influential data points is provided in this section. In interpreting the results of each of the diagnostic techniques, judgment and intuition, guided by statistical theory, are used to determine the reasonable cutoffs beyond which a particular measure of leverage or influence is large enough to be worthy of further examination. An outlier or a group of outliers are considered influential when several of the diagnostic techniques mentioned in the next sections determine the fact.

\subsubsection{Partial Regression Residual Plots}

Simple 2-variable regression scatterplots contain much diagnostic information about residuals and provide much guidance about influential subsets of data that might escape detection with the formal diagnostic techniques. The partial "regression leverage/residual plot" is the plot of the response variable and the regressor after they have been made orthogonal to the other regressors in the model. These plots (see Figures $6.1-6.3$ ) are generated by plotting the residuals of the response variable omitting the selected regressor against the residuals of the selected regressor on all the other regressor(s). A line fit to the points has a slope equal to the parameter estimate in the full model.

The partial regression residual plots are an important part of regression diagnostics. They supplant the traditional plots of residuals against explanatory/independent variables. However, partialregression leverage plots cannot tell us everything. Certain types of influential data can be overlooked and the influence of the leverage 
points detected in the plot will sometimes be difficult to quantify. [Belsley, Kuh and Welsch 1980]

The partial regression plots of model $\mathrm{Cn} 5$ are shown in Figures 6.1 and 6.2. Both of these plots show that one observation $(1 d=35$, where 35 represents the variable CTYN) is a possible candidate for an outlier. The "*' $s$ " In these plots are representation of overlapping of observations. The mild slope of the plot in Figure 6.2 suggests that the variable CNTYVR is not useful in the presence of variable CNTYINC in the model. Figure 6.3 presents the partial regression plot of model Un4. Although the outlier(s) is(are) not clear in this plot, some other diagnostic measures of the following subsections have proved that a group of observations, with $1 d=6$ in the plot, is a potential candidate as a group of outliers. The mild slope and more scattered observation indicate a lower value of $R^{2}$.

\subsubsection{Studentized Residual (RSTUDENT)}

One way to try to detect outliers is to produce the standardized residual at the ith observation $\left(d_{f}\right)$ as follows:

$$
d_{1}=\frac{e_{i}}{\sqrt{M S E}}
$$

where, $e_{1}$ is the residual corresponding to i-th observation and MSE stands for the Mean Square Error, taken as an estimate of standard deviation. A rough rule-of-thumb - if the true errors are $11 d$ and $\mathrm{N}\left(0, \sigma^{2}\right)$, then 95 percent and 99.7 percent of standardized residuals 
AADT

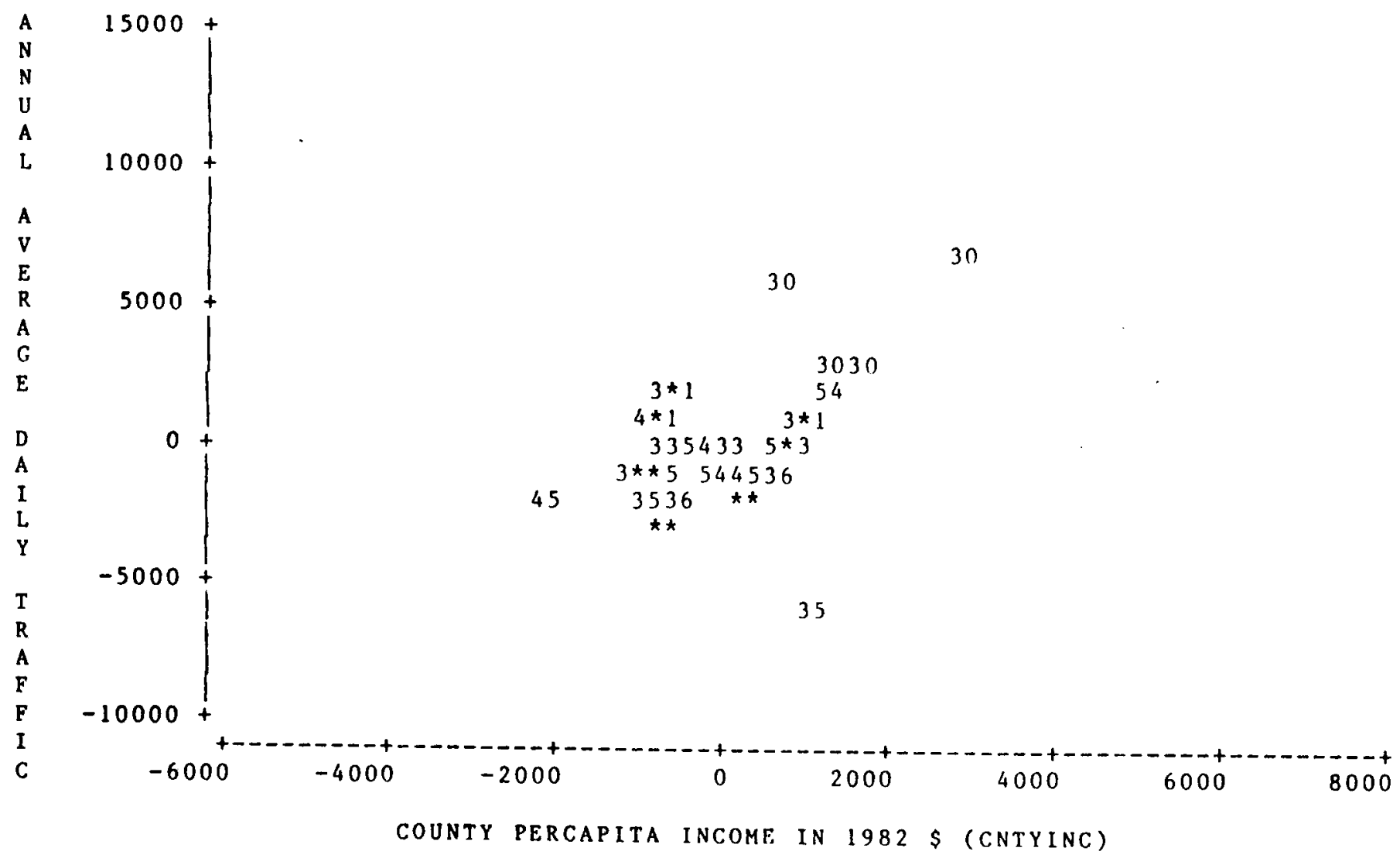

Figure 6.1: Partial Regression Residual Plot for Regressor CNTYINC of Model Cn5 [Individual case of Pre-Influence Analysis] 
Slope of the plot: 0.0029

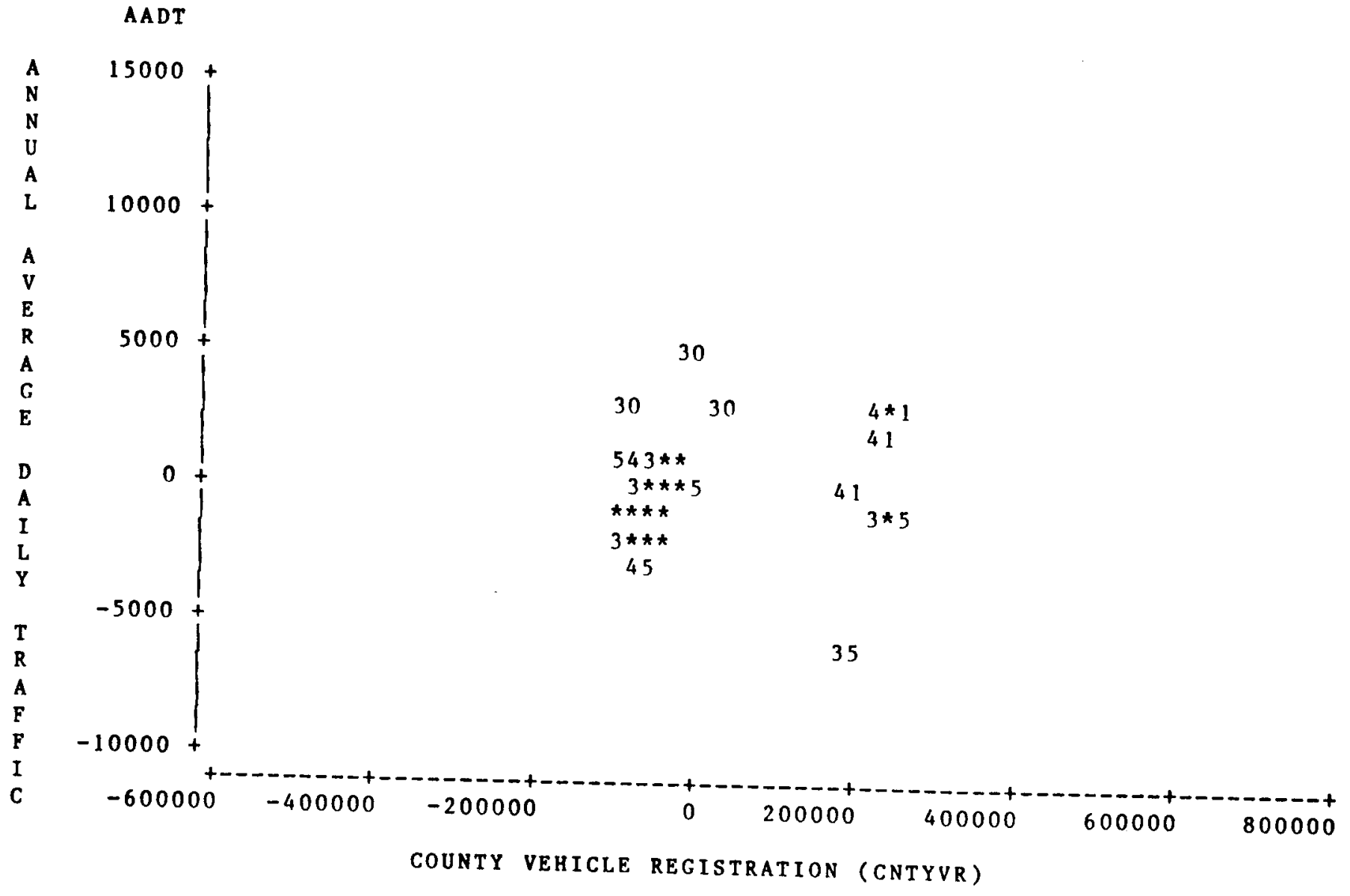

Figure 6.2: Partial Regression Residual Plot for Regressor CNTYVR of Model Cn5 [Individual case of Pre-Influence Analysis] 
Slope of the p1ot: 0.2656

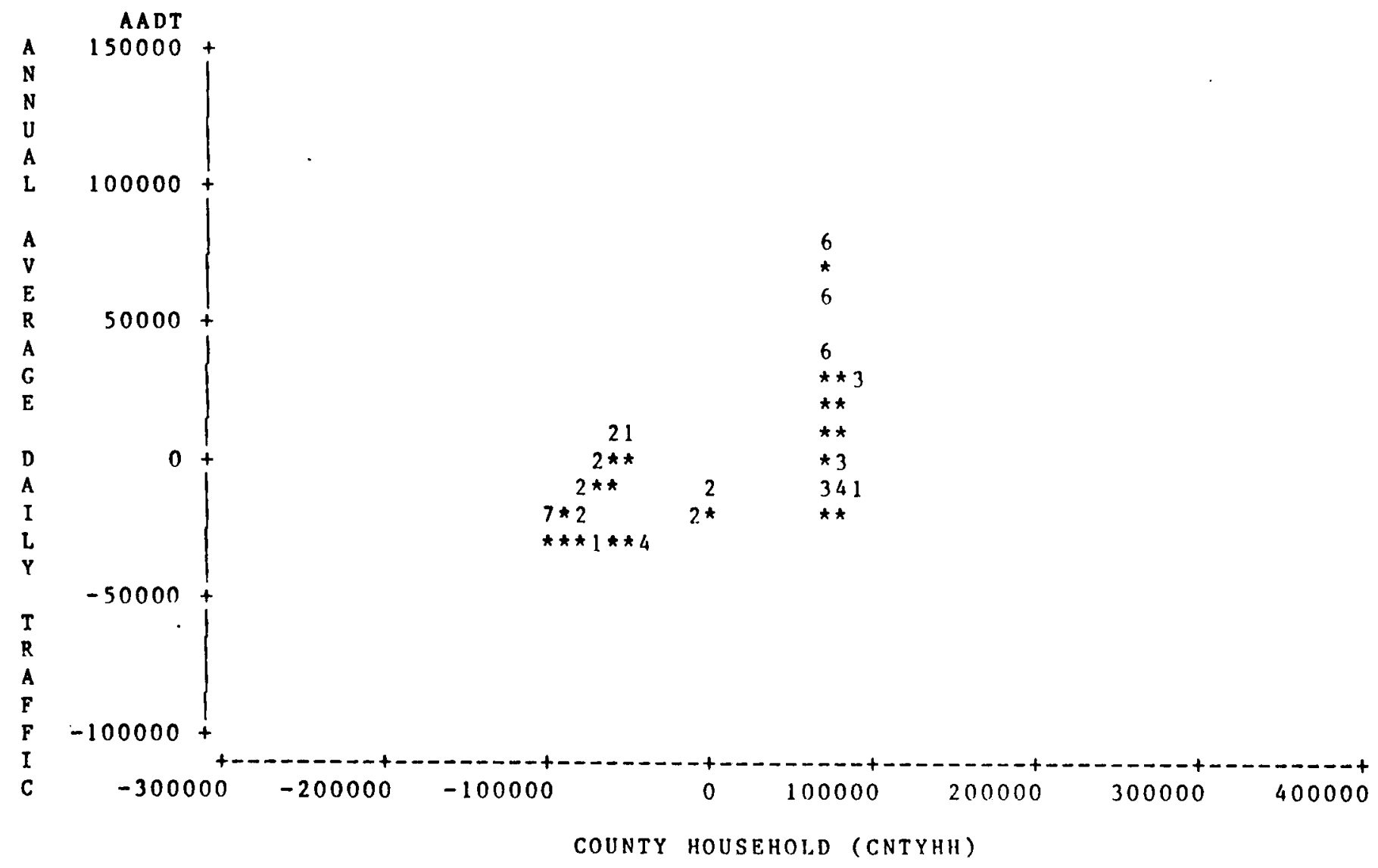

Figure 6.3: Partial Regression Residual Plot for Regressor CNTYHH of Model In 4 [Group case of Pre-Influence Analysis] 
should fall between \pm 2 and \pm 3 , respectively. Thus, if a standardized residual is bigger than 3 , then it is a potential outlier with much skewness and/or kurtosis.

The studentized residual (RSTUDENT) differs slightly from the $d_{i}$ because RSTUDENT is calculated with the current observation deleted. The cutoff value for RSTUDENT is usually taken as 2.0. This absolute cut off value is independent on the sample size.

The third column of Tables 6.1 and 6.2 shows the values of RSTUDENT in models $\mathrm{Cn} 5$ and Un4, respectively. The observations with (*) beside the statistics have exceeded the suggested cutoff values. The group effect of City Number (CTYN) 6 is evident from the results of Table 6.2.

\subsubsection{Change in the Fitted Values (DFFITS)}

A way to summarize coefficient changes and, at the same time, to gain insight into the forecasting effects when an observation is deleted, is by the change in fit. The DFFITS statistic is a scaled measure of the change in the predicted value for the th observation and is calculated by deleting the ith observation. The observation i is considered to be influential if

$$
\left|\operatorname{DFFITS}_{1}\right|>2 \sqrt{\frac{q+1}{n}}
$$

where $q$ and $n$ are the number of predictor variables and total number of observations, respectively. The right hand side of Equation (6.2) 


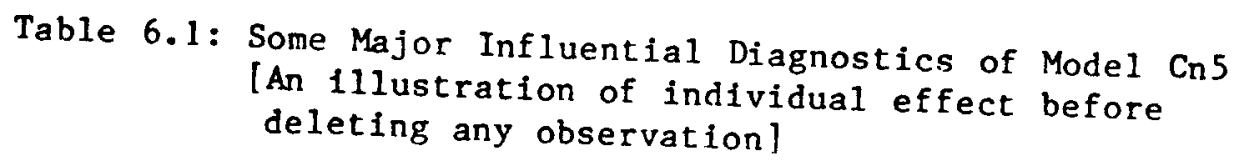

\begin{tabular}{|c|c|c|c|c|c|c|c|}
\hline OBS & $\begin{array}{c}\text { ID } \\
\text { (City }\end{array}$ & \#) RSTUDENT & $\begin{array}{r}\text { HAT DIAG } \\
\mathrm{H}\end{array}$ & DFFITS & $\begin{array}{r}\text { INTERCEP } \\
\text { DFBETAS }\end{array}$ & $\begin{array}{c}\text { CNTYINC } \\
\text { DFEETAS }\end{array}$ & $\begin{array}{c}\text { CNTYVR } \\
\text { DFEETAS }\end{array}$ \\
\hline 1 & 30 & 0.2908 & 0.1061 & 0.1002 & -0.0758 & 0.0826 & -0.0325 \\
\hline 2 & 30 & 0.5997 & 0.0768 & 0.1729 & -0.1170 & 0.1298 & -0.0474 \\
\hline 3 & 30 & 1.1604 & 0.0507 & 0.2680 & 0.1766 & -0.1566 & 0.0414 \\
\hline 4 & 30 & $2.3459(*)$ & 0.0429 & 0.4967 & -0.1915 & 0.2342 & -0.0578 \\
\hline 5 & 30 & 1.8285 & $0.2995(*)$ & $1.1956(*)$ & $-1.0810(\star)$ & $1.1269(*)$ & -0.3171 \\
\hline 6 & 33 & 0.4459 & 0.1041 & 0.1520 & 0.1213 & -0.1084 & -0.0274 \\
\hline 7 & 33 & 0.0277 & 0.0497 & 0.0063 & 0.0011 & -0.0003 & -0.0034 \\
\hline 8 & 33 & -0.3251 & 0.0677 & -0.0876 & 0.0368 & -0.0469 & 0.0534 \\
\hline 9 & 33 & 0.6679 & 0.0737 & 0.1884 & 0.1290 & -0.1101 & -0.0486 \\
\hline 10 & 33 & -0.1163 & 0.0697 & -0.0318 & 0.0139 & -0.0175 & 0.0195 \\
\hline 11 & 35 & $-4.3482(\star)$ & 0.1849 & $-2.0712(*)$ & $0.9574(*)$ & $-0.9005(t)$ & $-1.2981(*)$ \\
\hline 12 & 35 & -0.6100 & 0.1661 & -0.2723 & -0.0989 & 0.1079 & -0.2406 \\
\hline 13 & 35 & -0.8647 & 0.1445 & -0.3554 & -0.1143 & 0.1240 & -0.3099 \\
\hline 14 & 36 & -1.0096 & 0.0722 & -0.2817 & -0.1787 & 0.1487 & 0.0923 \\
\hline 15 & 36 & -1.0029 & 0.0514 & -0.2336 & -0.0063 & -0.0242 & 0.1374 \\
\hline 16 & 36 & -0.6248 & 0.0591 & -0.1566 & -0.0790 & 0.0607 & 0.0617 \\
\hline 17 & 36 & -0.7057 & 0.0578 & -0.1747 & 0.0432 & -0.0649 & 0.1085 \\
\hline 18 & 41 & 1.3302 & 0.1364 & 0.5287 & 0.1534 & -0.1685 & $0.4584(\star)$ \\
\hline 19 & 41 & -0.0104 & 0.1849 & -0.0050 & 0.0023 & -0.0022 & -0.0031 \\
\hline 20 & 41 & 1.0379 & 0.1661 & 0.4633 & 0.1682 & -0.1835 & $0.4093(*)$ \\
\hline 21 & 41 & 0.8621 & 0.1445 & 0.3543 & 0.1140 & -0.1245 & 0.3090 \\
\hline 22 & 45 & 0.2272 & 0.1927 & 0.1110 & 0.1014 & -0.0946 & -0.0053 \\
\hline 23 & 45 & -0.1565 & 0.0748 & -0.0445 & -0.0295 & 0.0249 & 0.0133 \\
\hline 24 & 45 & -1.0733 & 0.0516 & -0.2505 & 0.0142 & -0.0467 & 0.1491 \\
\hline 25 & 45 & -0.0988 & 0.0804 & -0.0292 & -0.0208 & 0.0179 & 0.0073 \\
\hline 26 & 45 & -0.4946 & 0.0506 & -0.1142 & 0.0018 & -0.0166 & 0.0666 \\
\hline 27 & 54 & -0.2471 & 0.0468 & -0.0547 & -0.0143 & 0.0074 & 0.0248 \\
\hline 28 & 54 & -0.4545 & 0.0577 & -0.1124 & 0.0430 & -0.0559 & 0.0617 \\
\hline 29 & 54 & 0.3494 & 0.0475 & 0.0780 & 0.0337 & -0.0246 & -0.0258 \\
\hline 30 & 54 & 0.3881 & 0.0891 & 0.1214 & -0.0777 & 0.0888 & -0.0615 \\
\hline
\end{tabular}

(*) Exceeds cutoff values: RSTUDENT $=2.0$; HAT DIAG $=0.20 ;$ DFFITS $=0.6325 ;$ DFBETAS $=0.3651$. 
Table 6.2: Some Major Influential Diagnostics of Model Un4 [An illustration of group effect before deleting any observation]

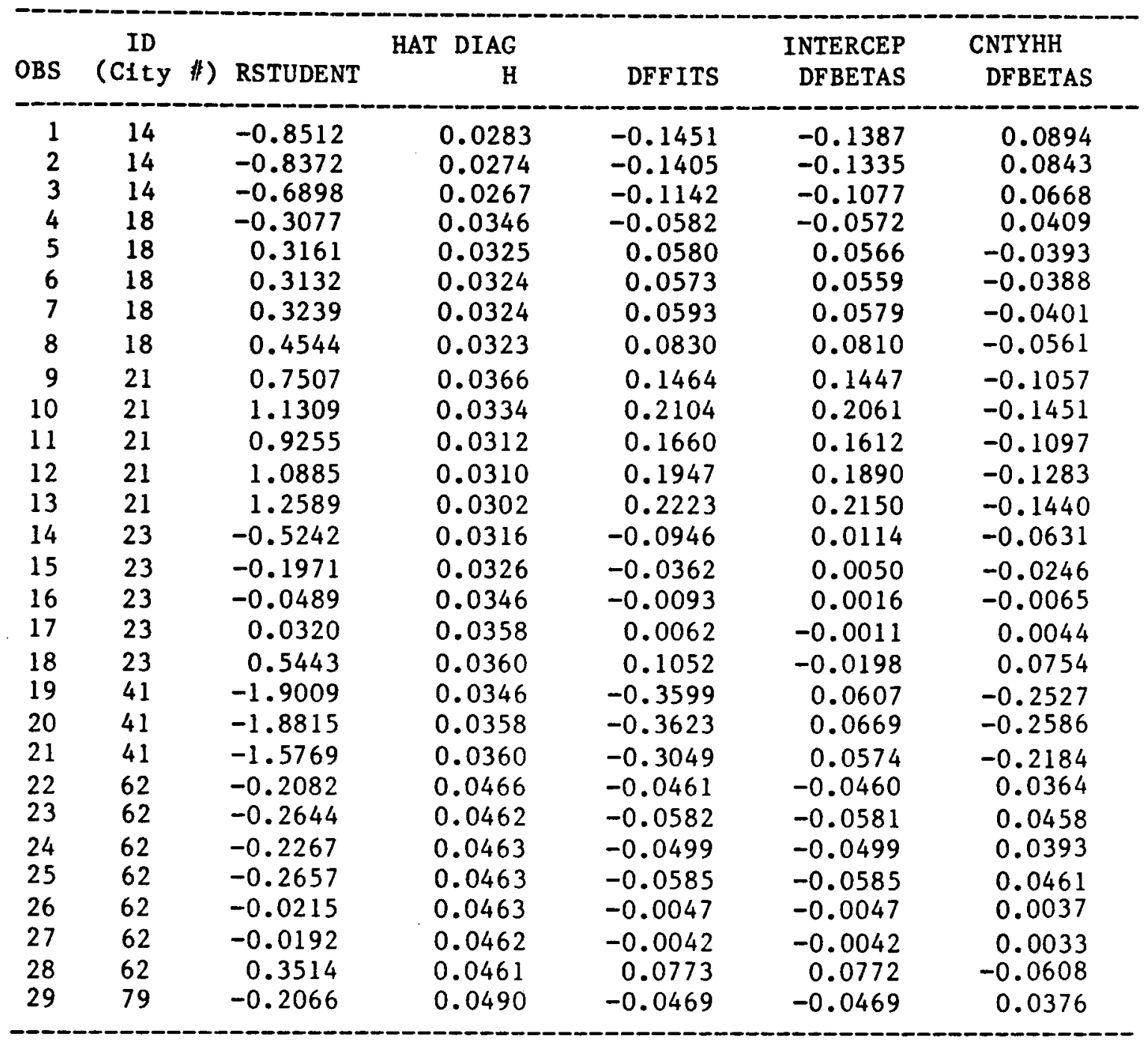


Table 6.2, continued

\begin{tabular}{|c|c|c|c|c|c|c|}
\hline & ID & & DIAG & & INTERCEP & CNTYHH \\
\hline OBS & (City \#) & RSTUDENT & $\mathrm{H}$ & DFFITS & DFBETAS & DFBETAS \\
\hline 30 & 79 & -0.3525 & 0.0479 & -0.0790 & -0.0790 & 0.0629 \\
\hline 31 & 79 & -0.4232 & 0.0479 & -0.0949 & -0.0948 & 0.0755 \\
\hline 32 & 79 & -0.2386 & 0.0477 & -0.0534 & -0.0534 & 0.0425 \\
\hline 33 & 79 & 0.0264 & 0.0475 & 0.0059 & 0.0059 & -0.0047 \\
\hline 34 & 2 & -0.9912 & 0.0180 & -0.1341 & -0.0928 & 0.0208 \\
\hline 35 & 2 & -1.1132 & 0.0175 & -0.1488 & -0.0863 & 0.0015 \\
\hline 36 & 2 & -0.8797 & 0.0175 & -0.1176 & -0.0671 & -0.0001 \\
\hline 37 & 2 & -0.2671 & 0.0176 & -0.0357 & -0.0198 & -0.0008 \\
\hline 38 & 23 & -0.6433 & 0.0316 & -0.1161 & 0.0140 & -0.0774 \\
\hline 39 & 23 & -0.4059 & 0.0326 & -0.0745 & 0.0103 & -0.0506 \\
\hline 40 & 23 & -0.5878 & 0.0346 & -0.1113 & 0.0188 & -0.0781 \\
\hline 41 & 23 & -0.6092 & 0.0353 & -0.1166 & 0.0209 & -0.0828 \\
\hline 42 & 23 & -0.4113 & 0.0358 & -0.0792 & 0.0146 & -0.0565 \\
\hline 43 & 3 & -1.2241 & 0.0316 & -0.2210 & 0.0266 & -0.1473 \\
\hline 44 & 3 & -0.7281 & 0.0326 & -0.1337 & 0.0184 & -0.0908 \\
\hline 45 & 3 & -0.7914 & 0.0346 & -0.1498 & 0.0253 & -0.1052 \\
\hline 46 & 3 & -0.6854 & 0.0353 & -0.1312 & 0.0235 & -0.0931 \\
\hline 47 & 3 & -0.5720 & 0.0358 & -0.1102 & 0.0203 & -0.0786 \\
\hline 48 & 6 & 1.2954 & 0.0316 & 0.2339 & -0.0281 & 0.1558 \\
\hline 49 & 6 & $2.3499(*)$ & 0.0326 & $0.4314(*)$ & -0.0594 & $0.2931(*)$ \\
\hline 50 & 6 & $2.7940(*)$ & 0.0346 & $0.5289(*)$ & -0.0892 & $0.3714(*)$ \\
\hline 51 & 6 & $2.8883(*)$ & 0.0353 & $0.5529(*)$ & -0.0990 & $0.3923(*)$ \\
\hline 52 & 6 & $3.0217(*)$ & 0.0358 & $0.5819(*)$ & -0.1075 & $0.4153(*)$ \\
\hline 53 & 41 & -0.3867 & 0.0316 & -0.0698 & 0.0084 & -0.0465 \\
\hline 54 & 41 & 0.4664 & 0.0326 & 0.0856 & -0.0118 & 0.0582 \\
\hline 55 & 41 & 0.6321 & 0.0346 & 0.1197 & -0.0202 & 0.0840 \\
\hline 56 & 41 & 0.5102 & 0.0353 & 0.0977 & -0.0175 & 0.0693 \\
\hline 57 & 41 & 0.4485 & 0.0358 & 0.0864 & -0.0160 & 0.0616 \\
\hline
\end{tabular}

(*) Exceeds cutoff values: RSTUDENT=2.0; HAT DIAG=0.0702;

DFFITS $=0.3746$; DFBETAS $=0.2649$. 
represents the cutoff value for DFFITS. The values of DFFITS statistics (column 5) and their cutoff values for models Cn5 and Un4 are shown in Table 6.1 and Table 6.2 , respectively.

\subsubsection{Change in the Regression Coefficients (DFBETAS)}

The estimated regression coefficients are primarily of interest to the users of regression mode1s. DFBETAS are the scaled measures of the change in each parameter/coefficlent estimate and are calculated by deleting the ith observation. The cutoff for DFBETAS is calculated as $\frac{2}{\sqrt{n}}$, where $n$ is the total number of observations. If the absolute value of DFBETAS is at least equal to this cutoff value, then the observation is a potential candidate to be an outlier. The last columns of Tables 6.1 and 6.2 show the values of DFBETAS of the parameter of the model.

\subsubsection{Diagonal Element of Hat Matrix (HAT DIAG)}

HAT DIAG $\left(h_{1}\right)$ is the ith diagonal element of the hat matrix [SAS/STATISTICS 1985; Belsley, Kuh and Welsch 1980]. The $h_{1}$ can be viewed as the coefficient of dependent variable $\left(y_{1}\right)$ in its predicted value $\left(\hat{y}_{1}\right)$. Thus, if one $h_{1}$ 's ls larger compared to the rest, then that element has more influence on the analysis than the rest. The observation 1 is considered to be influential if

$$
h_{1}>2 \frac{q+1}{n}
$$

where the definitions of $q$ and $n$ remain the same as in Equation 6.2 . 
The fourth column of Tables 6.1 and 6.2 show the values of $h_{1}$ ("HAT DIAG").

\subsubsection{Residual and Normal Probability P1ots}

Oftentimes, the residual plots convey more information about outllers and are generally used to detect them. The normal probability plot is not generally taken as a suggestive measure to detect the outlier(s). However, the effect of an outlier is often evident in the normal probability plot. A detailed discussion of the use of these plots is presented in Section 6.3 and its subsections. In using the residual plots, the observations with more than 2-3 times the standard deviation of the residuals are taken as possible candidates for the outlier. The residual plots and RSTUDENT measure in Section 6.2.1.2 are very close to each other. The residual plots of Figures 6.4 to 6.6 against predicted value and the regressor variables suggest that one observation (represented by the right hand bottom point of these plots) is a potential candidate to be an influential observation. Despite a higher P-value for normality, the normal probability plot of mode1 Cn5 in Figure 6.7 also concludes that the observation corresponding to the lower left hand corner of Figure 6.7 is a suspected outlier - the same suspected point as in Figures 6.4 to 6.6 .

The residual plots against the predicted values, regressor in the model, and the normal probability plot model Un4 are shown in Figures $6.8,6.9$, and 6.10 , respectively. The four observations (represented by four right hand upper points in these plots) are suspected as a 
LEGEND: $A=1$ OBS, B $=2$ OBS, ETC.

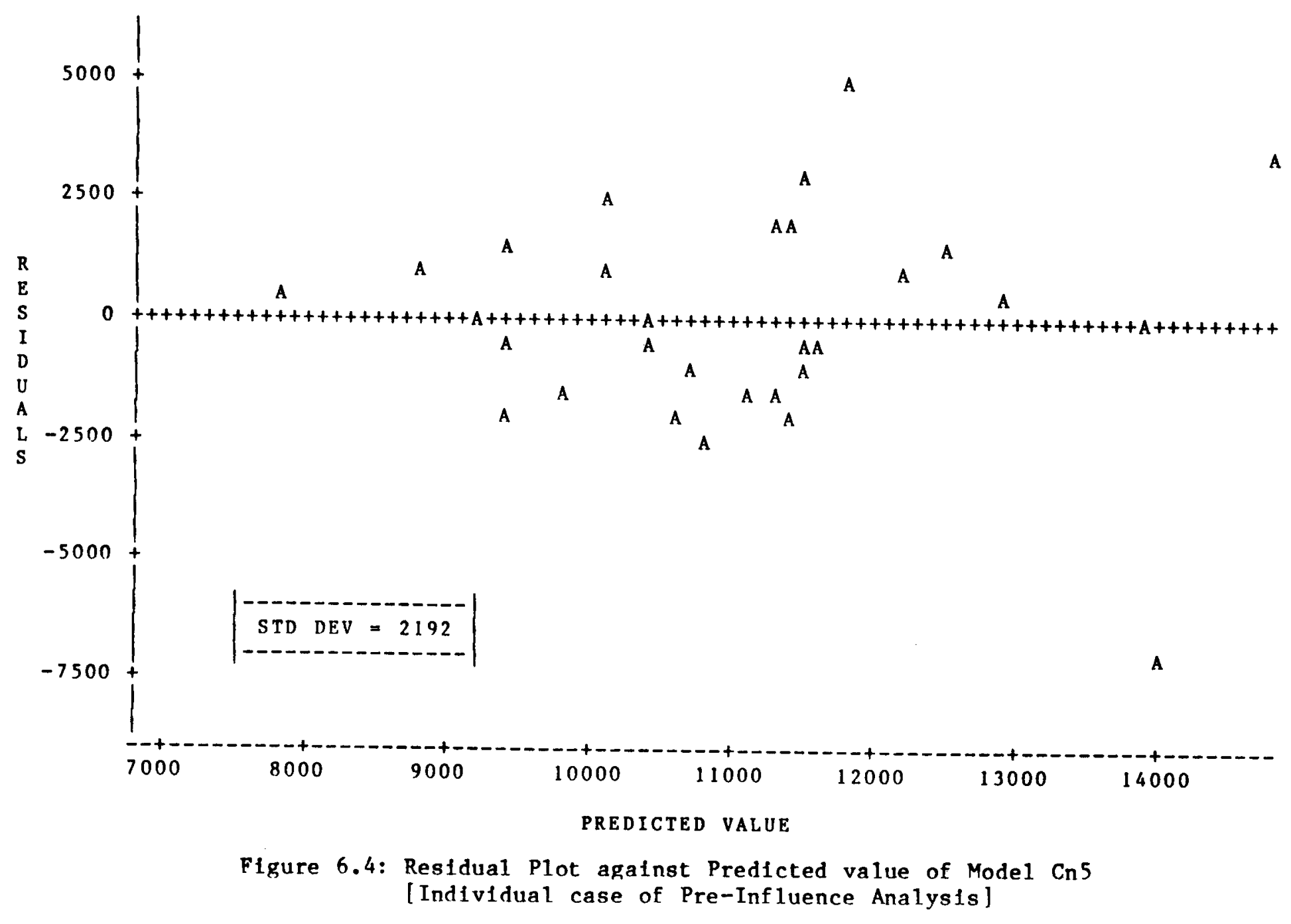


LEGEND: $A=1$ OBS, $B=2$ OBS, ETC.

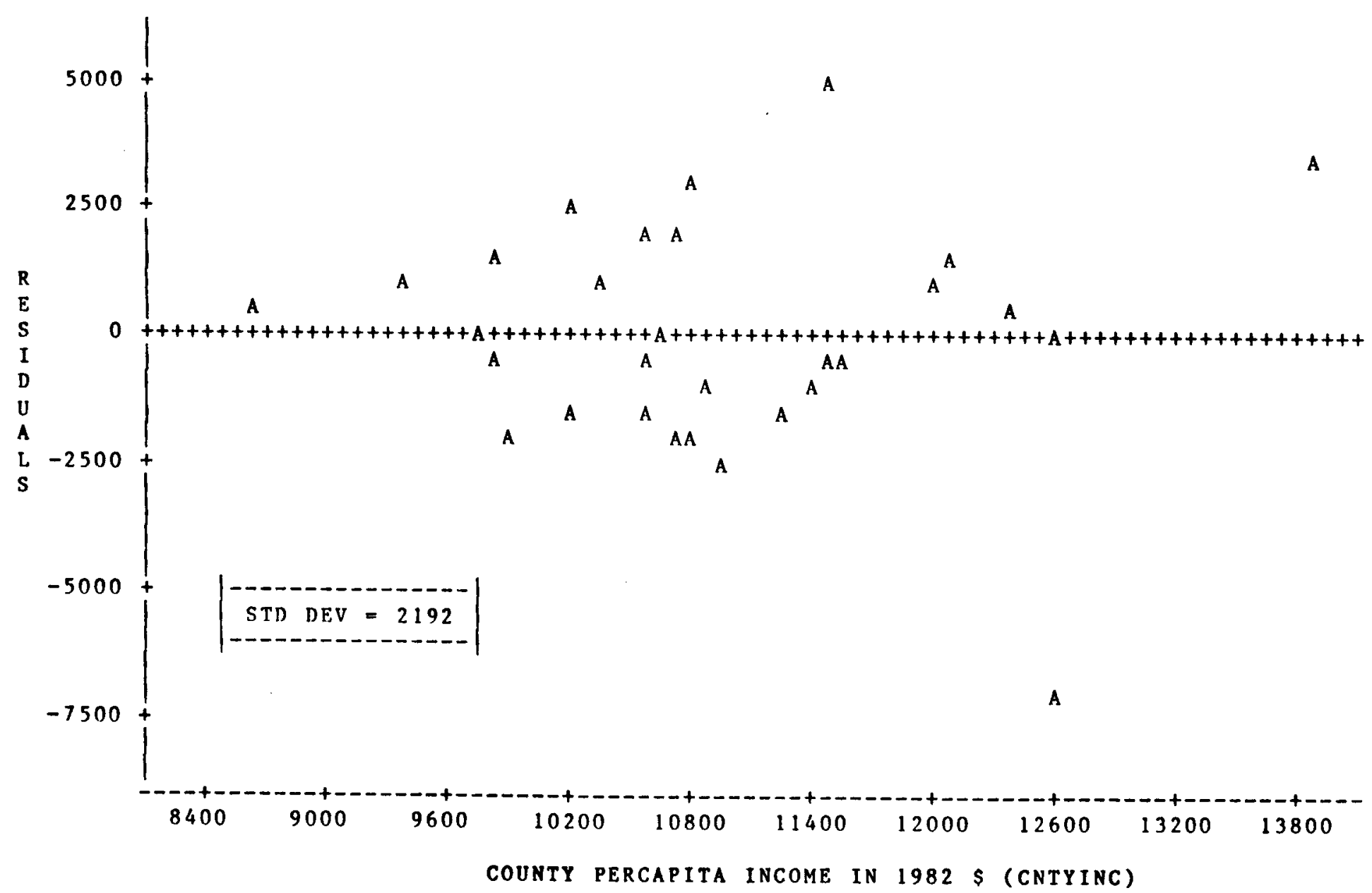

Figure 6.5: Residual Plot against Regressor CNTYINC of Mode1 Cn5

[Individual case of Pre-Influence Analysis] 
LEGEND: $A=1$ OBS, B $=2$ OBS, ETC.

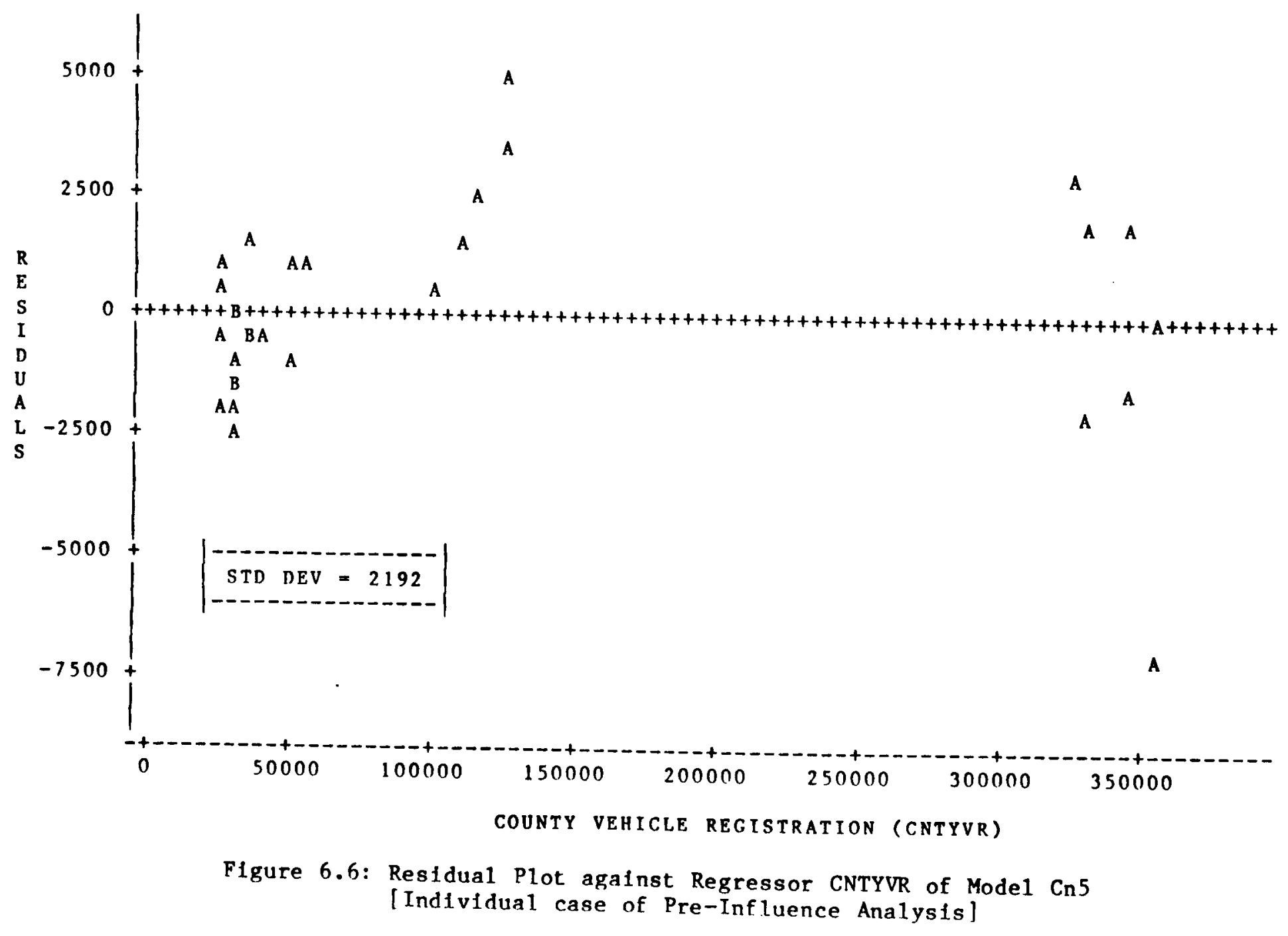


LEGEND: $A=1$ OBS, $B=20 B S$, ETC.

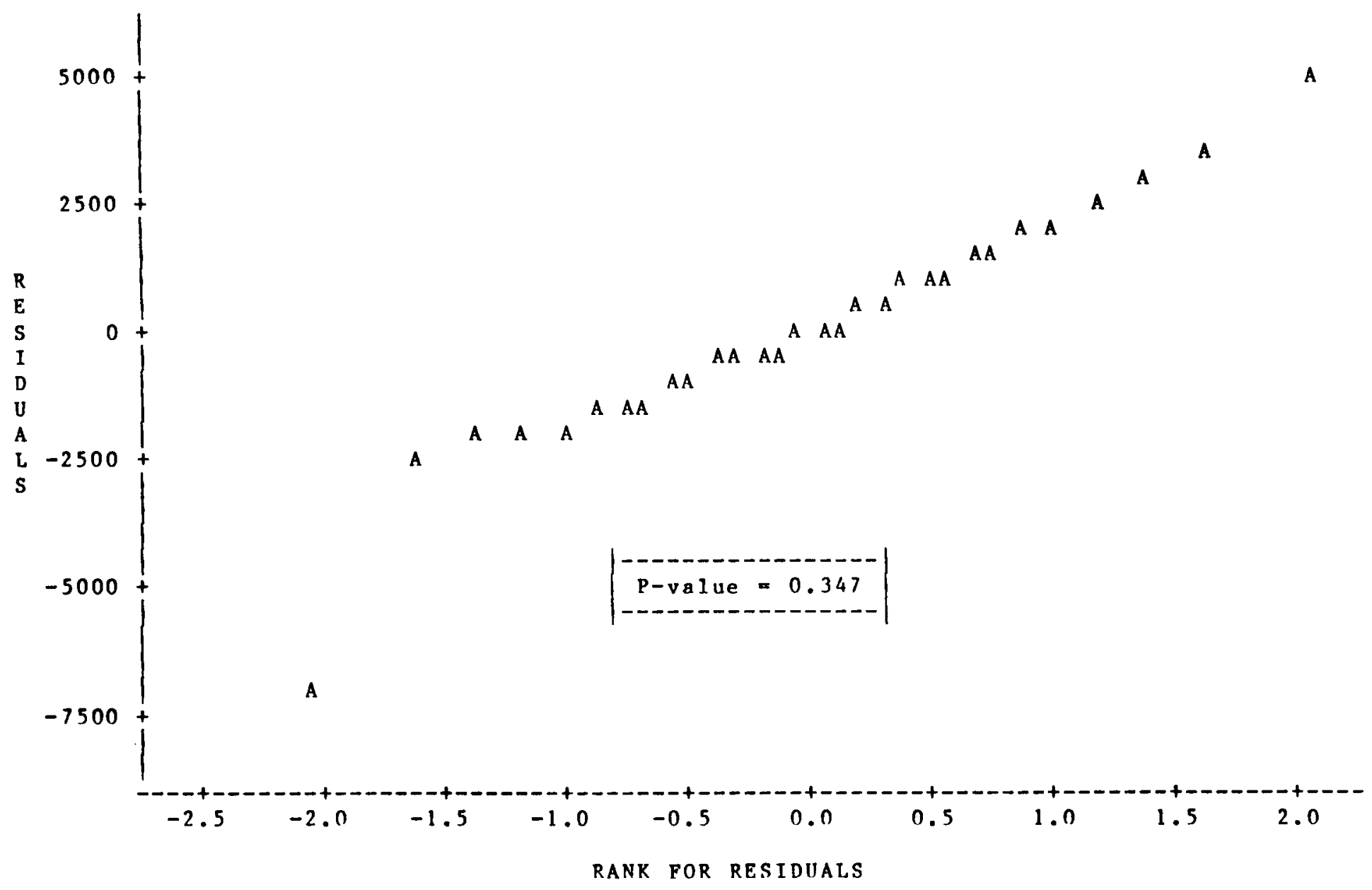

Figure 6.7: Norma1 Probability P1ot of Model Cn5

[Individual case of Pre-Influence Analysis] 
LEGEND: $A=1$ OBS, $B=2$ OBS, ETC.

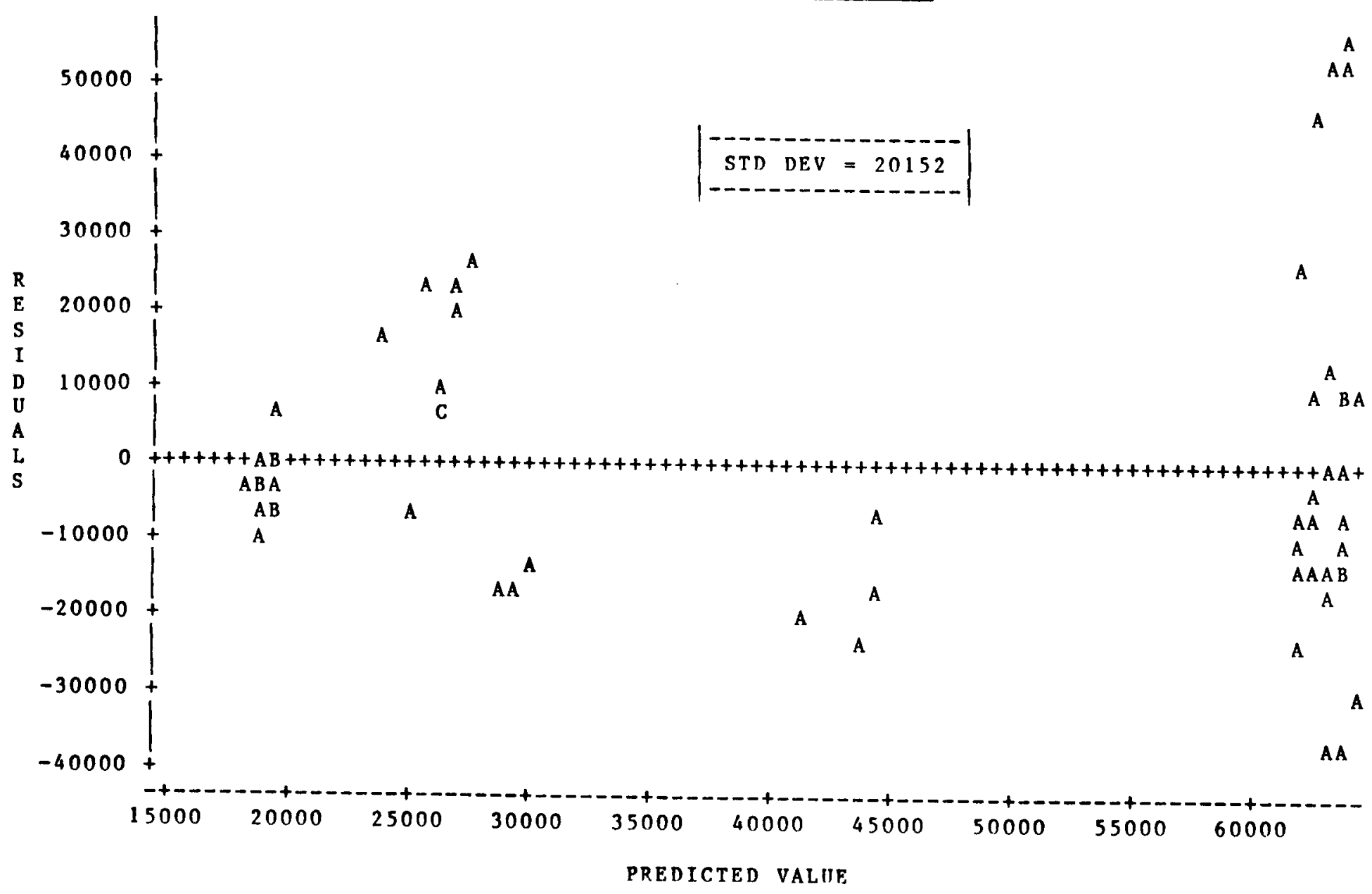

Figure 6.8: Residual Plot against Predicted value of Model Un4 [Group case of Pre-Influence Analysis] 
LEGEND: $A=1$ OBS, $B=2$ OBS, ETC.

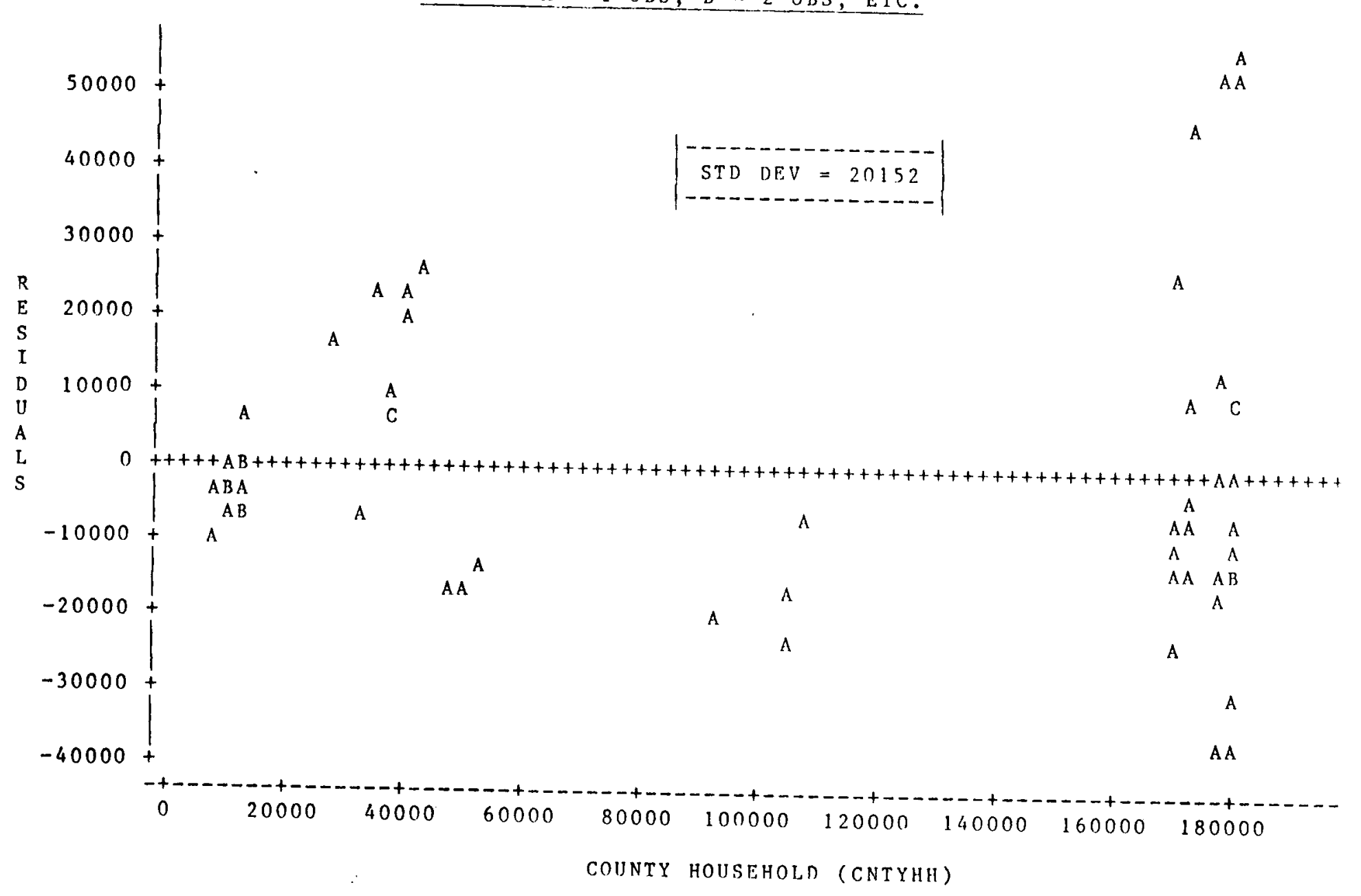

Figure 6.9: Residual Plot against Regressor CNTYHH of Model Un't [Group case of Pre-Influence Analysis] 
LEGEND: $A=1$ OBS, $B=2$ OBS, ETC.

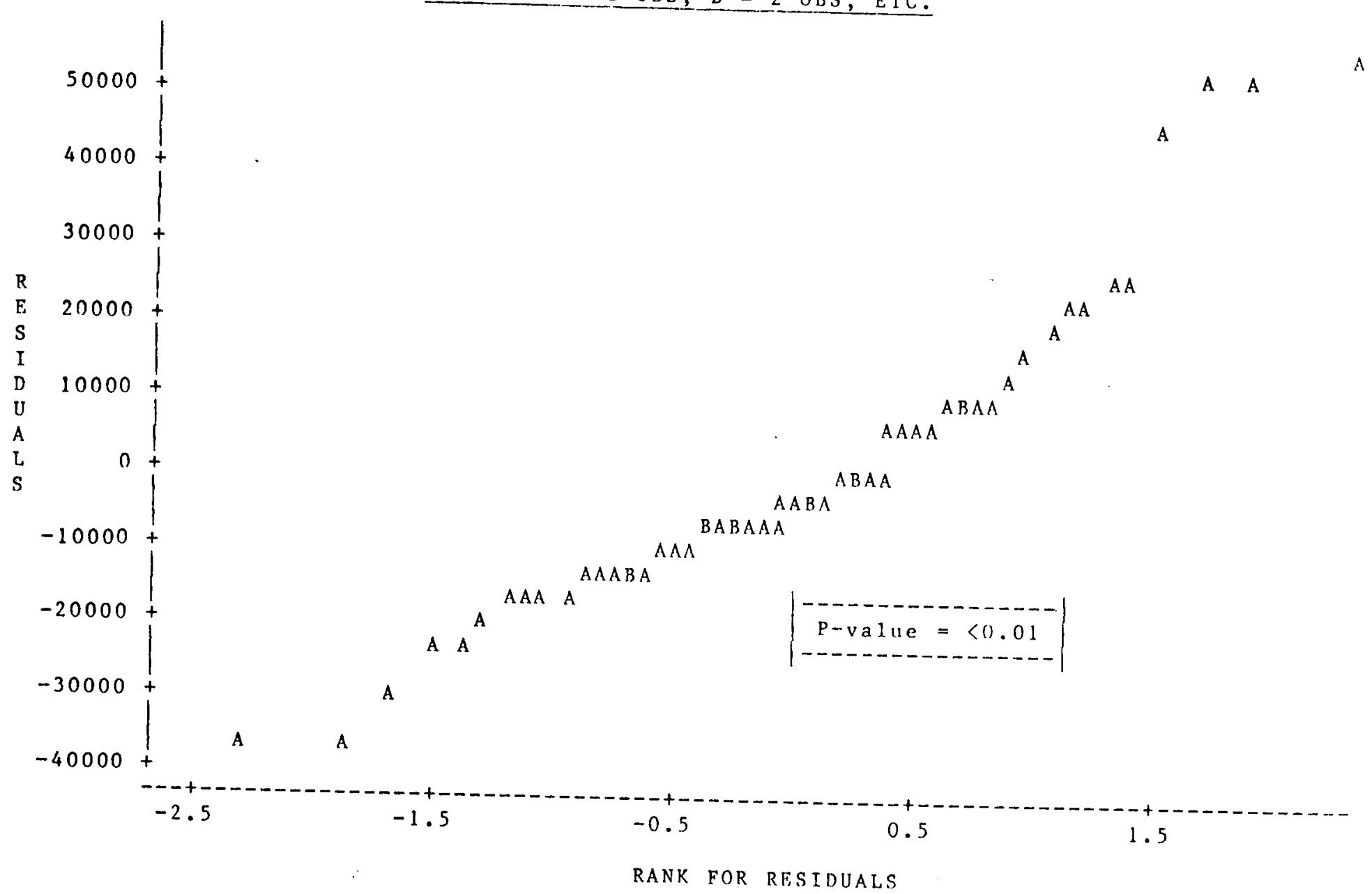

Figure 6.10: Normal Probability P1ot of Model Un4 [Group case of Pre-Influence Analysis] 
possible group to be considered influential.

\subsubsection{Pre and Post Analysis of Outliers}

Outlier observations, if present, have an unusually high influence on parameter estimates, the ANOVA table, etc. A safe rule frequently suggested is to discard an outlier only if there is direct evidence that it represents an error in recording, processing, or a similar type of circumstance. Once an influential unit has been identified, efforts should be made to find out if any error was made in data collection or data manipulation associated with that unit. In the absence of such errors, the units that exceed at least three of the diagnostic cutoff values referred to Section 6.2 .1 can be deleted from the data set. For example, the influential observations identified by the diagnostic measures -- RSTUDENT, DFFITS, HAT DIAG and DFBETAS -- have been examined in terms of partial regression plots, the normality plot, and residual plots against the predicted values and regressors to see whether they are posing any problem in getting statistically good results. Once an outlier or group of outliers has been identified, the observation(s) corresponding to the outlier(s) is(are) carefully examined to see if any of the following is the cause of being influential.

i. Is there any error in data entry?

1i. Is there any error in processing the data sets? (For example, computing county \& city average data from the segment data.) 
iii. Did the county \& city average AADT data get influenced by one or more segment's data? In that case, the influential segment(s) is(are) dropped from the segment data base and the county \& city average data are recomputed from the remaining segments in the segment data base.

iv. If some years of AADT values in any data base are thought to be due to faulty recording and/or an abnormal increase/decrease in AADT for some reasons (for example, construction, parallel/alternate road closure/opening, etc.), then those years of data were omitted in further analysis.

v. Finally, if the outliers are not errors or mistakes, they are legitimate data values that just don't fit our simple linear model. Our theoretical model is not good enough to classify those outliers, or the prescribed model may not be correct.

After deletion of any influential observations, all the influential diagnostic measures and plots of residual and normal probability plots are reevaluated on the modified data sets. Such analysis after deletion of outliers is referred to as "Post-Influence Analysis", whereas the analysis before deletion of observations has been referred to as "Pre-Influence Analysis". The diagnostics and plots of Pre-Influence analysis confirmed that one observation (OBS 11 of Table 6.1) at year 1978 of city number 35 (Crown Point) is an outlier for model Cn5. A very low county or city average AADT for observation 11 causes it to be an outlier. For model Un4, the group of observations (OBS 49-52 of Table 6.2) with "city number $=6 "$ (Hammond) appeared to be influen- 
tial. Although observations 49-52 (see $6^{-} \mathrm{s}$ in the partial regression plot of Figure 6.3) have predictor variable values within the range of values associated with the other observations, these four observations have AADT values outside the range of the other observations. Perhaps factors other than those included in our analysis could explain this anomaly. However, our only recourse here is to remove the outlying observations from the otherwise consistent data set.

At this stage, the model parameters are recalculated by deleting the outlier(s) confirmed through the influence analysis. The diagnostic statistics and the associated plots of this post-influence analysis for model $\mathrm{Cn} 5$ are shown in Table 6.3 and Figures 6.11 to 6.16. At least three of the four tests have been required as evidence of an outlier. Some judgment was used to verify outliers by reviewing the diagnostic measures and plots. The post-analysis results for model Un4 are shown in Table 6.4 and Figures 6.17 to 6.20. The results of the post-influence analysis are examined carefully to detect any additional possible outliers in the data set. If necessary, post-influence analysis is carried out further until the data set appears to be reasonably good about any apparent outlier. .

Comparing the results of pre- and post-influence analysis (see table below), one can easily see the improvement in the model statistics. For example, the results of the P-value for normality, ST. DEV. of residuals, $R^{2}, R_{a}^{2}$, RMSE and Coefficient of Variation (C.V.) for the models $\mathrm{Cn} 5$ and Un4 are as follows: 
Table 6.3: Some Major Influential Diagnostics of Model Cn5 [After deleting observation 11 of Table 6.11

\begin{tabular}{|c|c|c|c|c|c|c|c|}
\hline OBS & ID & RSTUDENT & AT DIAG & DFFITS & $\begin{array}{r}\text { INTERCEP } \\
\text { DFBETAS }\end{array}$ & $\begin{array}{l}\text { CNTYINC } \\
\text { DFBETAS }\end{array}$ & $\begin{array}{l}\text { CNTYVP. } \\
\text { DFBET }\end{array}$ \\
\hline & & & & & & & \\
\hline 1 & 30 & 0.0013 & 0.1129 & 0.0005 & -0.0004 & 0.0004 & -0.non! \\
\hline 2 & 30 & 0.4392 & 0.0823 & 0.1316 & -0.0913 & 0.1003 & -0.0236 \\
\hline 3 & 30 & 1.4855 & 0.0507 & 0.3433 & 0.2182 & -0.1939 & 0.0542 \\
\hline 4 & 30 & $2.8758(*)$ & 0.0464 & 0.6344 & -0.2671 & 0.3170 & -0.0182 \\
\hline 5 & 30 & 1.5598 & $0.3213(*)$ & $1.0732(*)$ & $-0.9751(*)$ & $1.0130(*)$ & -0.1833 \\
\hline 6 & 33 & 0.8626 & 0.1078 & 0.2998 & 0.2417 & -0.2170 & -0.0560 \\
\hline 7 & 33 & 0.0948 & 0.0499 & 0.0217 & 0.0041 & -0.0013 & -0.0114 \\
\hline 8 & 33 & -0.5068 & 0.0680 & -0.1369 & 0.0583 & -0.0736 & 0.0768 \\
\hline 9 & 33 & 1.0667 & 0.0755 & 0.3049 & 0.2115 & -0.1819 & -0.0879 \\
\hline 10 & 33 & -0.2398 & 0.0701 & -0.0658 & 0.0291 & -0.0364 & 0.0370 \\
\hline 11 & 35 & -1.4441 & 0.1823 & $-0.6818(*)$ & -0.1872 & 0.2113 & $-0.6091(*)$ \\
\hline 12 & 35 & -1.7951 & 0.1601 & $-0.7836(*)$ & -0.1813 & 0.2066 & $-0.6921(*)$ \\
\hline 13 & 36 & -1.1076 & 0.0742 & -0.3135 & -0.2026 & 0.1702 & 0.1117 \\
\hline 14 & 36 & -1.2709 & 0.0515 & -0.2962 & -0.0102 & -0.0278 & 0.1700 \\
\hline 15 & 36 & -0.6655 & 0.0601 & -0.1684 & -0.0869 & 0.0678 & 0.0692 \\
\hline 16 & 36 & -0.9477 & 0.0578 & -0.2347 & 0.0581 & -0.0867 & 0.1377 \\
\hline 17 & 41 & 1.1199 & 0.1522 & 0.4744 & 0.0945 & -0.1093 & $0.4169(*)$ \\
\hline 18 & 41 & -1.0283 & $0.2269(*)$ & -0.5571 & 0.2785 & -0.2627 & -0.3705 \\
\hline 19 & 41 & 0.7322 & 0.1823 & 0.3457 & 0.0949 & -0.1072 & 0.3088 \\
\hline 20 & 41 & 0.5278 & 0.1601 & 0.2304 & 0.0533 & -0.0608 & 0.2035 \\
\hline 21 & 45 & 0.7479 & 0.2014 & 0.3756 & 0.3444 & -0.3224 & -0.0391 \\
\hline 22 & 45 & 0.0003 & 0.0768 & 0.0001 & 0.0000 & -0.0000 & -0.0000 \\
\hline 23 & 45 & -1.3917 & 0.0517 & -0.3248 & 0.0171 & -0.0584 & 0.1865 \\
\hline 24 & 45 & 0.0870 & 0.0828 & 0.0261 & 0.0188 & -0.0164 & -0.0075 \\
\hline 25 & 45 & -0.6197 & 0.0506 & -0.1431 & 0.0015 & -0.0198 & 0.0807 \\
\hline 26 & 54 & -0.2681 & 0.0469 & -0.0595 & -0.0158 & 0.0085 & 0.0267 \\
\hline 27 & 54 & -0.6940 & 0.0582 & -0.1726 & 0.0677 & -0.0870 & 0.0854 \\
\hline 28 & 54 & 0.5134 & 0.0477 & 0.1149 & 0.0500 & -0.0368 & -0.0385 \\
\hline 29 & 54 & 0.2841 & 0.0914 & 0.0901 & -0.0587 & 0.0667 & -0.0391 \\
\hline
\end{tabular}

(*) Exceeds cutoff values: RSTUDENT $=2.0$; HAT DIAG $=0.2069$; DFFITS $=0.6433 ;$ DFBETAS $=0.3714$. 
Slope of the plot: 1.5654

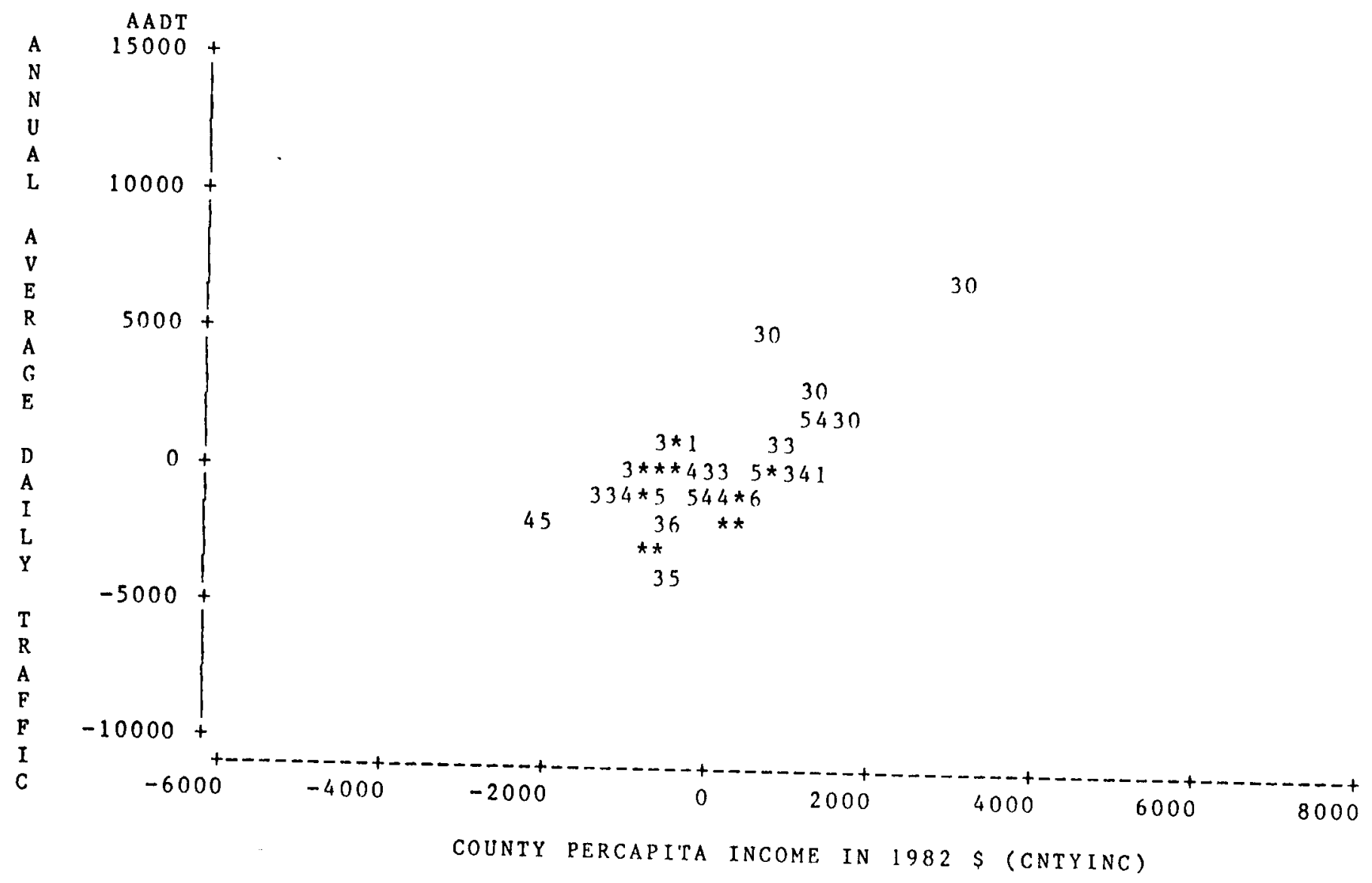

Figure 6.11: Partial Regression Residual Plot for Regressor CNTYINC of Model Cn5 [Individual case of Post-Influence Analysis] 
Slope of the plot: 0.0064

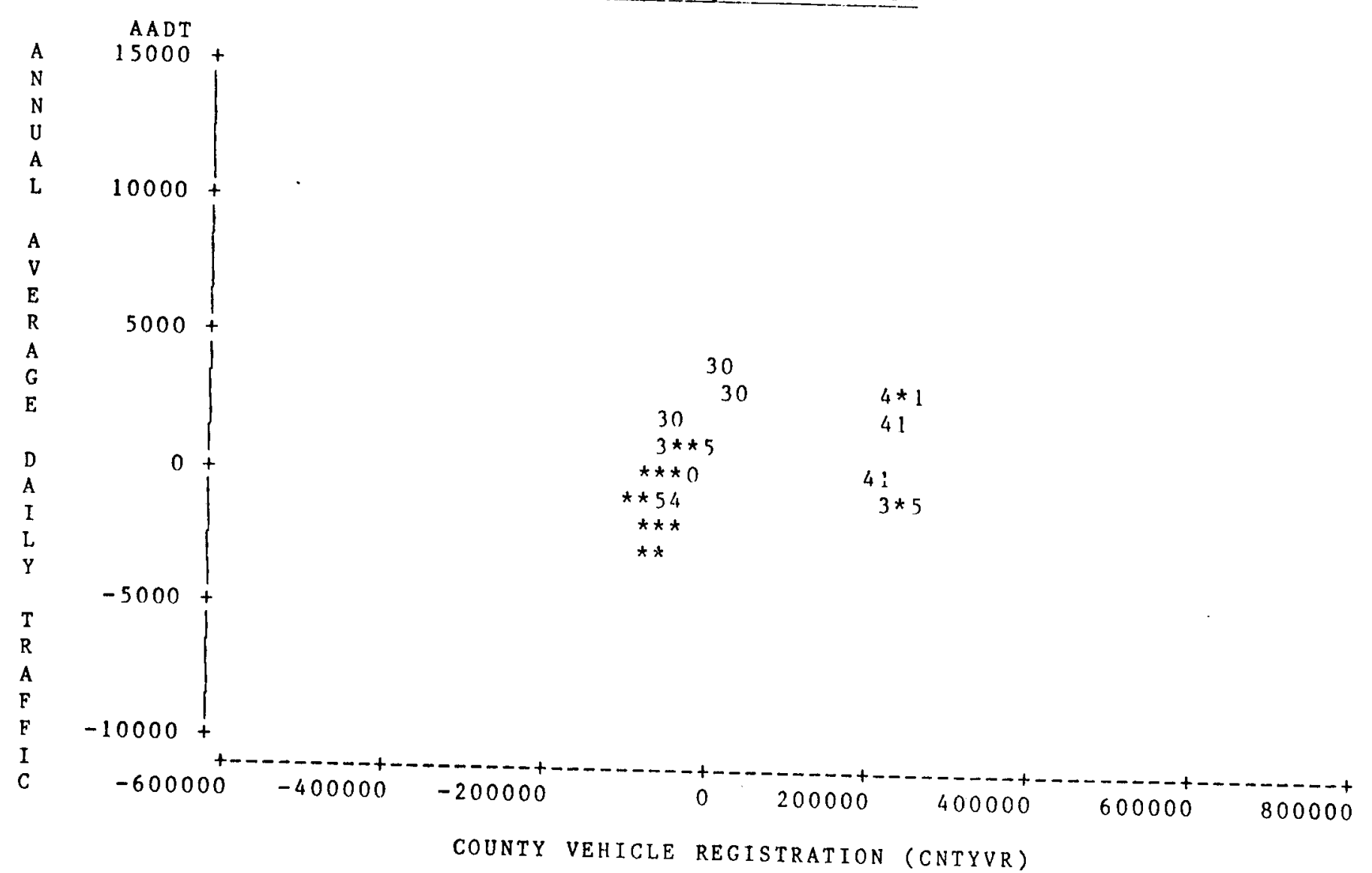

Figure 6.12: Partia1 Regression Residual Plot for Regressor CNTYVR of Model Cn 5 [Individual case of Post-Influence Analysisl 
LEGEND: $A=1$ OBS, $B=2$ OBS, ETC.

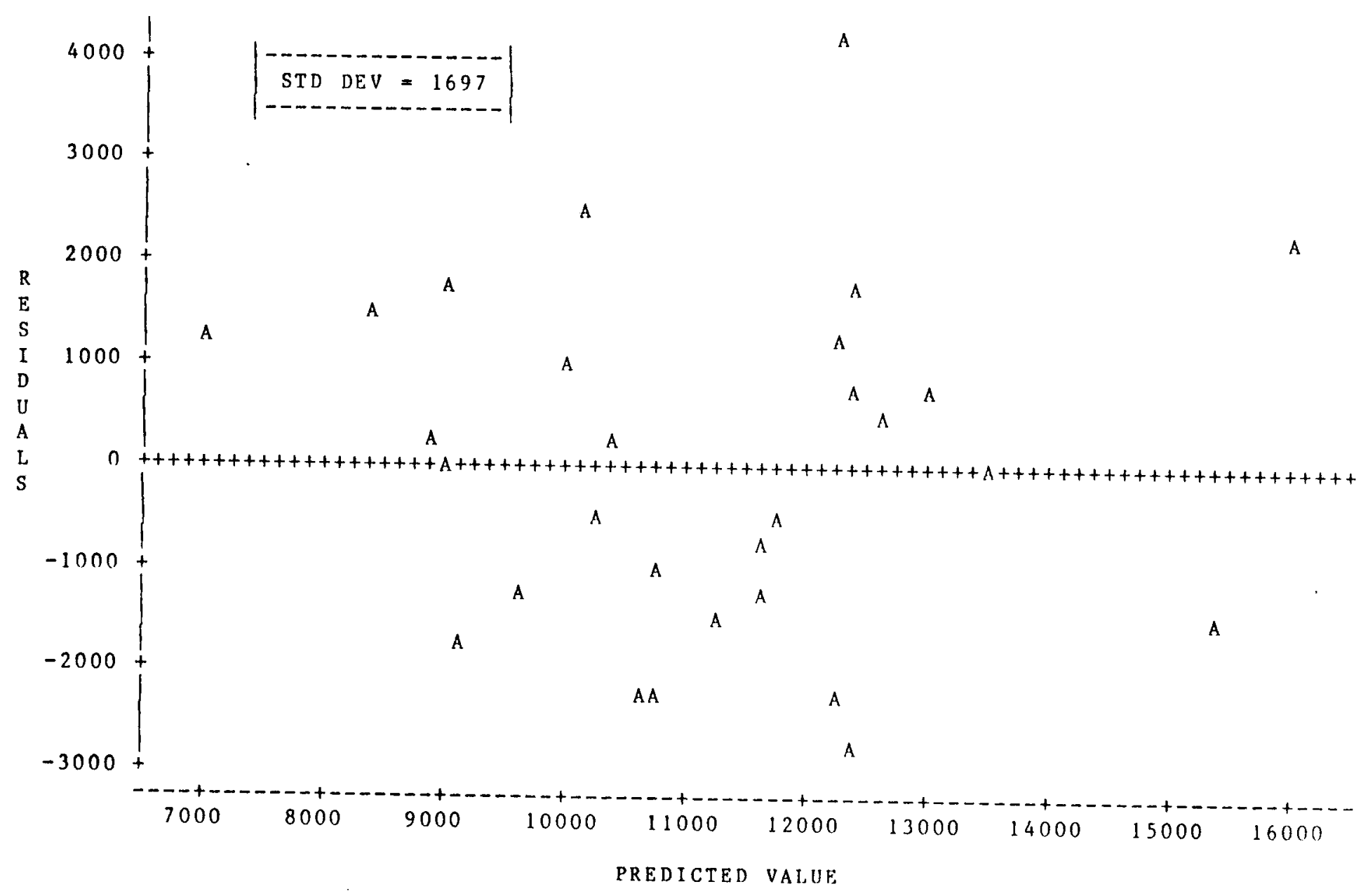

Figure 6.13: Residual Plot against Predicted value of Model Cn5

[Individual case of Post-Influence Analysis] 
LEGEND: $A=1$ OBS, $B=2$ OBS, FTC.

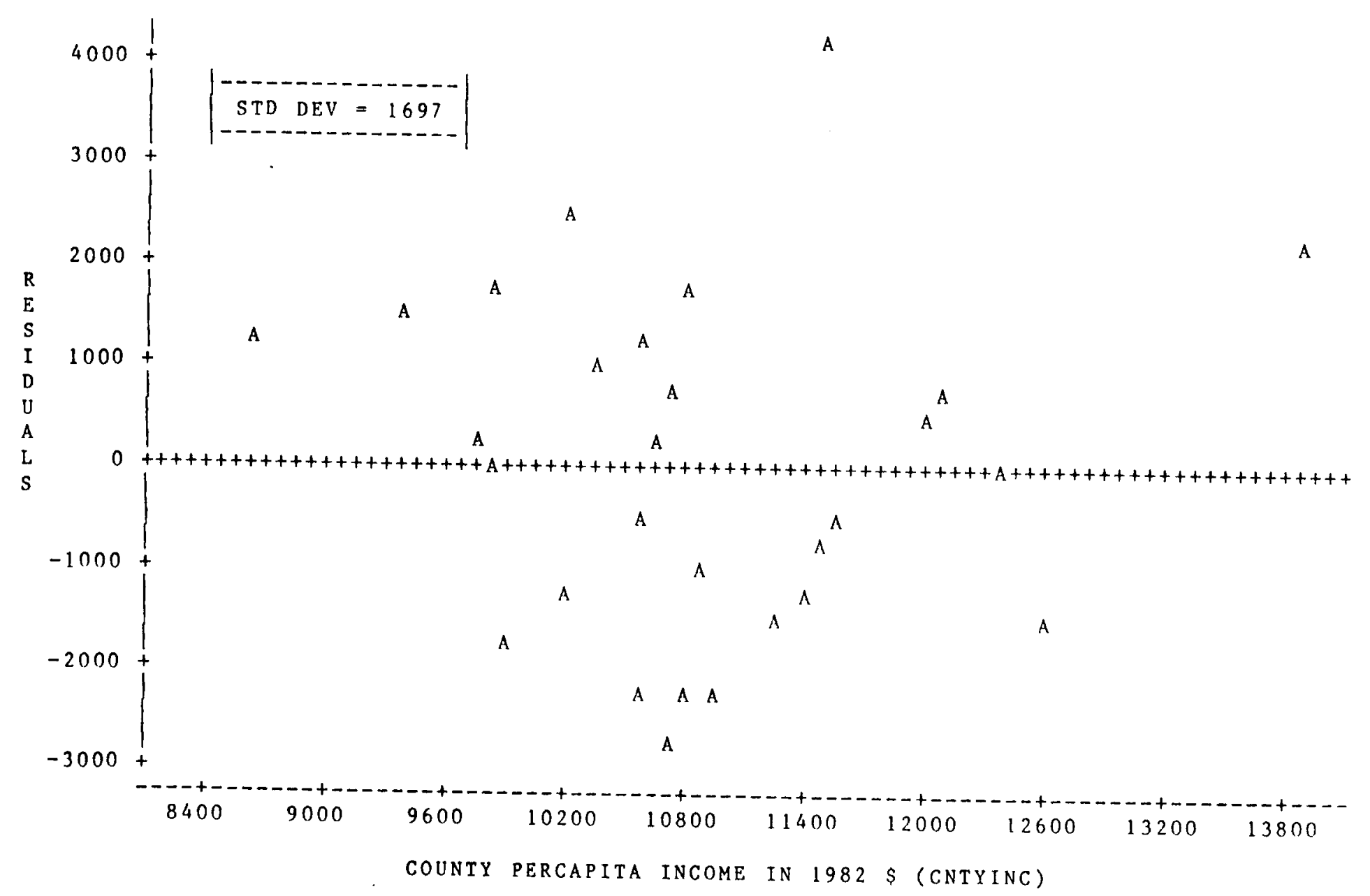

Figure 6.14: Residual Plot against Regressor CNTYINC of Model Cn 5 IIntividual case of Post-Influence Analysis I 
LEGEND: $A=1$ OBS, $B=2$ OBS, ETC.

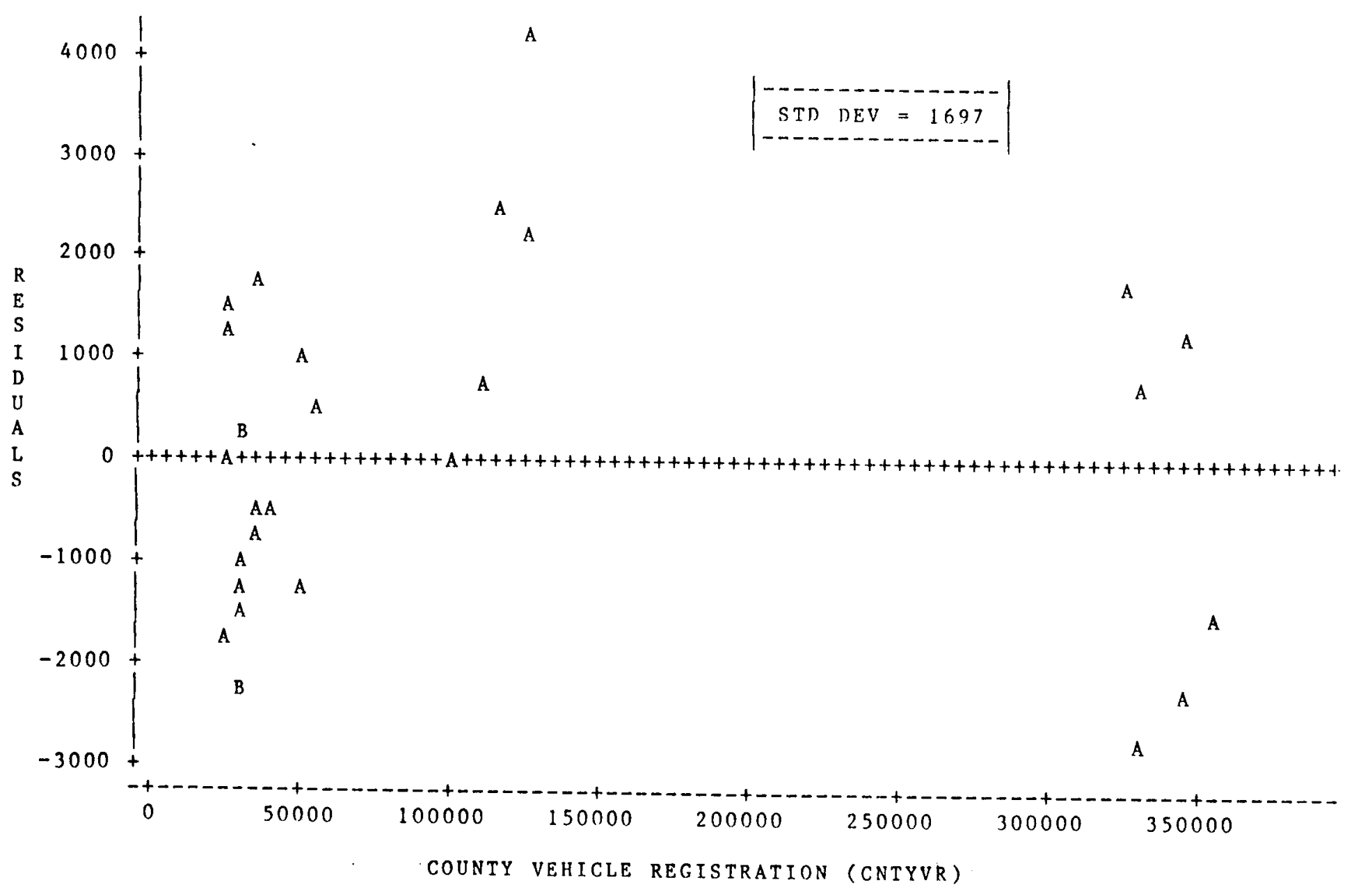

Figure 6.15: Residual Plot against Regressor CNTYVR of Mode1 Cn5

[Individual case of Post-Influence Analysis] 
LEGEND: $A=1$ OBS, $B=2$ OBS, ETC.

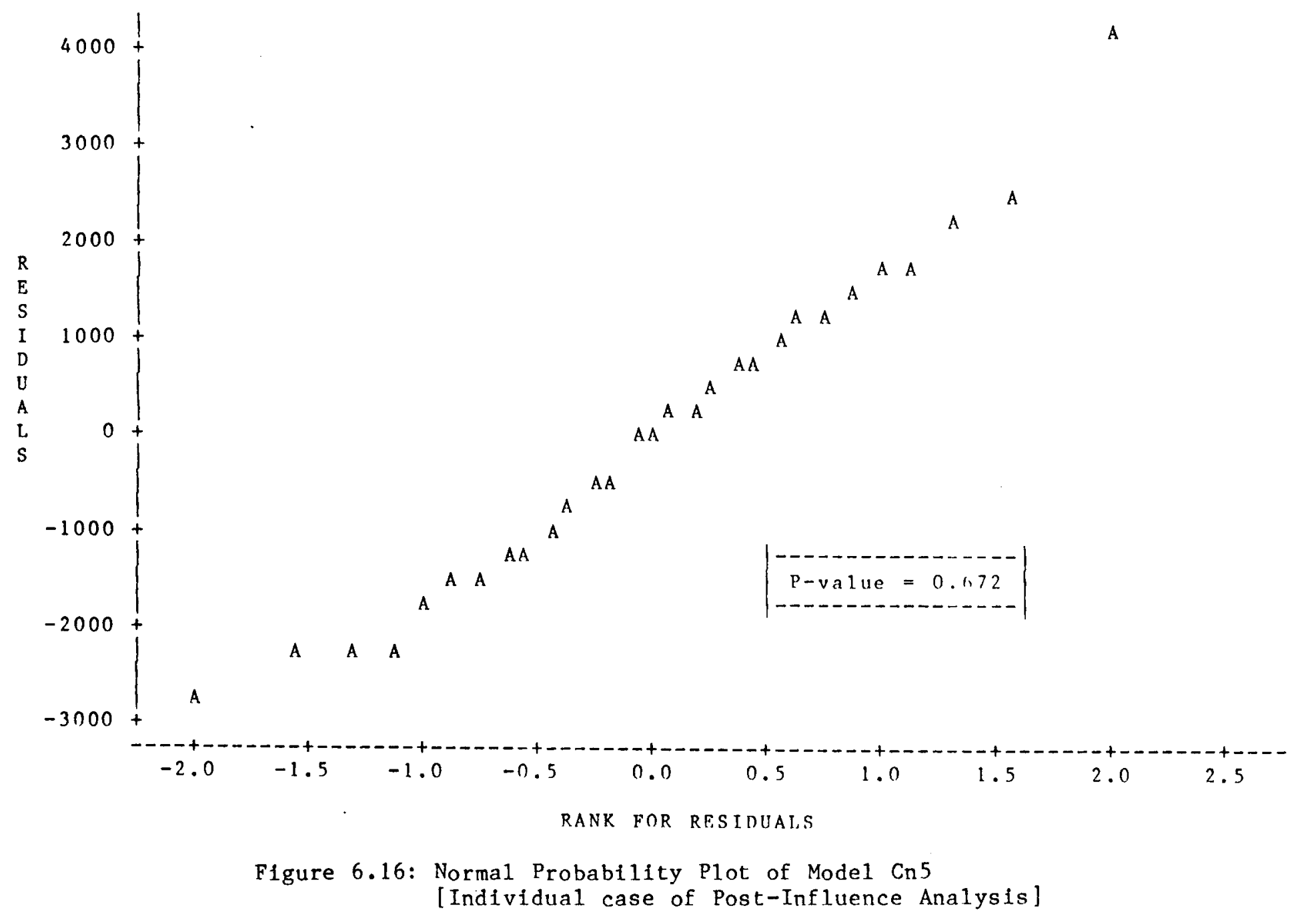


Table 6.4: Some Major Influential Diagnoslics of Model Un4 [After deleting OBS 48-52 with ID (City \#) 6 of Table 6.2]

\begin{tabular}{|c|c|c|c|c|c|c|}
\hline OBS & $\begin{array}{c}\text { ID } \\
(\text { City } \#)\end{array}$ & RSTUDENT & $\begin{array}{r}\text { DIAG } \\
\mathrm{H}\end{array}$ & DFFITS & $\begin{array}{r}\text { INTERCEP } \\
\text { DFBETAS }\end{array}$ & $\begin{array}{l}\text { CNTYHH } \\
\text { DFBETAS }\end{array}$ \\
\hline 1 & 14 & -1.2175 & 0.0284 & -0.2080 & -0.1970 & 0.1181 \\
\hline 2 & 14 & -1.1837 & 0.0276 & -0.1992 & -0.1872 & 0.1095 \\
\hline 3 & 14 & -0.9443 & 0.0268 & -0.1568 & -0.1462 & 0.0835 \\
\hline 4 & 18 & -0.4547 & 0.0346 & -0.0861 & -0.0843 & 0.0573 \\
\hline 5 & 18 & 0.5193 & 0.0326 & 0.0953 & 0.0926 & -0.0609 \\
\hline 6 & 18 & 0.5166 & 0.0324 & 0.0945 & 0.0918 & -0.0602 \\
\hline 7 & 18 & 0.5326 & 0.0324 & 0.0975 & 0.0947 & -0.0622 \\
\hline 8 & 18 & 0.7342 & 0.0323 & 0.1342 & 0.1303 & -0.0854 \\
\hline 9 & 21 & 1.1460 & 0.0367 & 0.2235 & 0.2203 & -0.1541 \\
\hline 10 & 21 & 1.7845 & 0.0335 & 0.3321 & $0.3240(*)$ & $-0: 2166$ \\
\hline 11 & 21 & 1.4835 & 0.0312 & 0.2662 & 0.2571 & -0.1649 \\
\hline 12 & 21 & 1.7466 & 0.0310 & 0.3126 & $0.3016(*)$ & -0.1929 \\
\hline 13 & 21 & $2.0347(*)$ & 0.0303 & 0.3596 & $0.3454(*)$ & -0.2173 \\
\hline 14 & 23 & -0.0977 & 0.0380 & -0.0194 & 0.0027 & -0.0137 \\
\hline 15 & 23 & 0.4133 & 0.0393 & 0.0836 & -0.0129 & 0.0597 \\
\hline 16 & 23 & 0.6647 & 0.0417 & 0.1386 & -0.0253 & 0.1017 \\
\hline 17 & 23 & 0.8033 & 0.0431 & 0.1704 & -0.0337 & 0.1268 \\
\hline 18 & 23 & 1.6240 & 0.0434 & 0.3459 & -0.0694 & 0.2581 \\
\hline 19 & 41 & $-2.1752(*)$ & 0.0417 & $-0.4536(*)$ & 0.0829 & $-0.3329(*)$ \\
\hline 20 & 41 & $-2.1303(*)$ & 0.0431 & $-0.4519(*)$ & 0.0892 & $-0.3362(*)$ \\
\hline 21 & 41 & -1.6497 & 0.0434 & -0.3513 & 0.0705 & -0.2622 \\
\hline 22 & 62 & -0.4143 & 0.0468 & -0.0917 & -0.0916 & 0.0704 \\
\hline 23 & 62 & -0.4967 & 0.0463 & -0.1094 & -0.1093 & 0.0837 \\
\hline 24 & 62 & -0.4395 & 0.0464 & -0.0969 & -0.0968 & 0.0742 \\
\hline 25 & 62 & -0.4994 & 0.0464 & -0.1102 & -0.1100 & 0.0843 \\
\hline 26 & 62 & -0.1262 & 0.0464 & -0.0278 & -0.0278 & 0.0213 \\
\hline 27 & 62 & -0.1221 & 0.0463 & -0.0269 & -0.0269 & 0.0206 \\
\hline 28 & 62 & 0.4434 & 0.0462 & 0.0976 & 0.0974 & -0.0746 \\
\hline
\end{tabular}


Table 6.4 , continued

\begin{tabular}{|c|c|c|c|c|c|c|}
\hline OBS & $\begin{array}{c}\text { ID } \\
(\text { City \#) }\end{array}$ & RSTUDENT & $\begin{array}{r}\text { HAT DIAG } \\
\mathrm{H}\end{array}$ & DFFITS & $\begin{array}{r}\text { INTERCEP } \\
\text { DFBETAS }\end{array}$ & $\begin{array}{l}\text { CNTYHH } \\
\text { DFBETAS }\end{array}$ \\
\hline 29 & 79 & -0.4313 & 0.0492 & -0.0981 & -0.0981 & 0.0766 \\
\hline 30 & 79 & -0.6456 & 0.0480 & -0.1450 & -0.1448 & 0.1122 \\
\hline 31 & 79 & -0.7545 & 0.0480 & -0.1694 & -0.1693 & 0.1312 \\
\hline 32 & 79 & -0.4697 & 0.0479 & -0.1053 & -0.1052 & 0.0815 \\
\hline 33 & 79 & -0.0634 & 0.0477 & -0.0142 & -0.0142 & 0.0110 \\
\hline 34 & 2 & -1.2004 & 0.0193 & -0.1684 & -0.1090 & 0.0108 \\
\hline 35 & 2 & -1.3342 & 0.0194 & -0.1875 & -0.0991 & -0.0156 \\
\hline 36 & 2 & -0.9695 & 0.0194 & -0.1364 & -0.0708 & $-n .0128$ \\
\hline 37 & 2 & -0.0279 & 0.0195 & -0.0039 & -0.0020 & -0.0004 \\
\hline 38 & 23 & -0.2784 & 0.0380 & -0.0553 & 0.0077 & -0.0389 \\
\hline 39 & 23 & 0.0943 & 0.0393 & 0.0191 & -0.0029 & 0.0136 \\
\hline 40 & 23 & -0.1595 & 0.0417 & -0.0333 & 0.0061 & -0.0244 \\
\hline 41 & 23 & -0.1837 & 0.0426 & -0.0387 & 0.0075 & -0.0287 \\
\hline 42 & 23 & 0.1212 & 0.0431 & 0.0257 & -0.0051 & 0.0191 \\
\hline 43 & 3 & -1.1597 & 0.0380 & -0.2305 & 0.0320 & -0.1620 \\
\hline 44 & 3 & -0.3945 & 0.0393 & -0.0797 & 0.0123 & -0.0570 \\
\hline 45 & 3 & -0.4676 & 0.0417 & -0.0975 & 0.0178 & -0.0716 \\
\hline 46 & 3 & -0.2991 & 0.0426 & -0.0631 & 0.0121 & -0.0467 \\
\hline 47 & 3 & -0.1230 & 0.0431 & -0.0261 & 0.0052 & -0.0194 \\
\hline 48 & 41 & 0.1113 & 0.0380 & 0.0221 & -0.0031 & 0.0155 \\
\hline 49 & 41 & 1.4557 & 0.0393 & 0.2943 & -0.0456 & 0.2102 \\
\hline 50 & 41 & 1.7517 & 0.0417 & 0.3653 & -0.0668 & 0.2681 \\
\hline 51 & 41 & 1.5603 & 0.0426 & 0.3290 & -0.0633 & 0.2436 \\
\hline 52 & 41 & 1.4646 & 0.0431 & 0.3107 & -0.0614 & 0.2311 \\
\hline
\end{tabular}

(*) Exceeds cutoff values: RSTUDENT=2.0; HAT DIAG=0.0769;

DFFITS $=0.3992 ;$ DFBETAS $=0.2774$. 
Slope of the plot: 0.1998

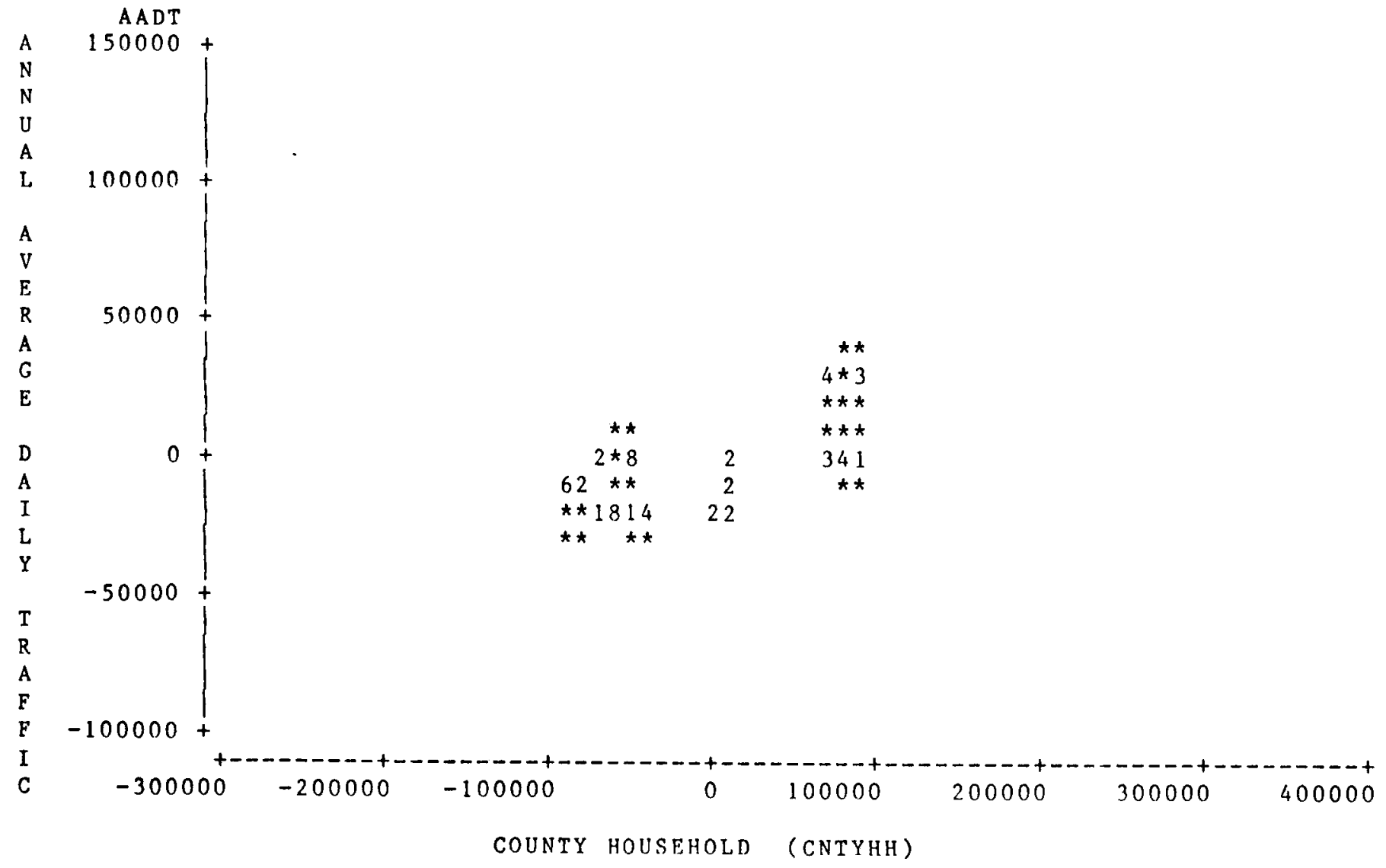

Figure 6.17: Partial Regression Residual Plot for Regressor CNTYHH of Model Un4 [Group case of Post-Influence Analysis] 


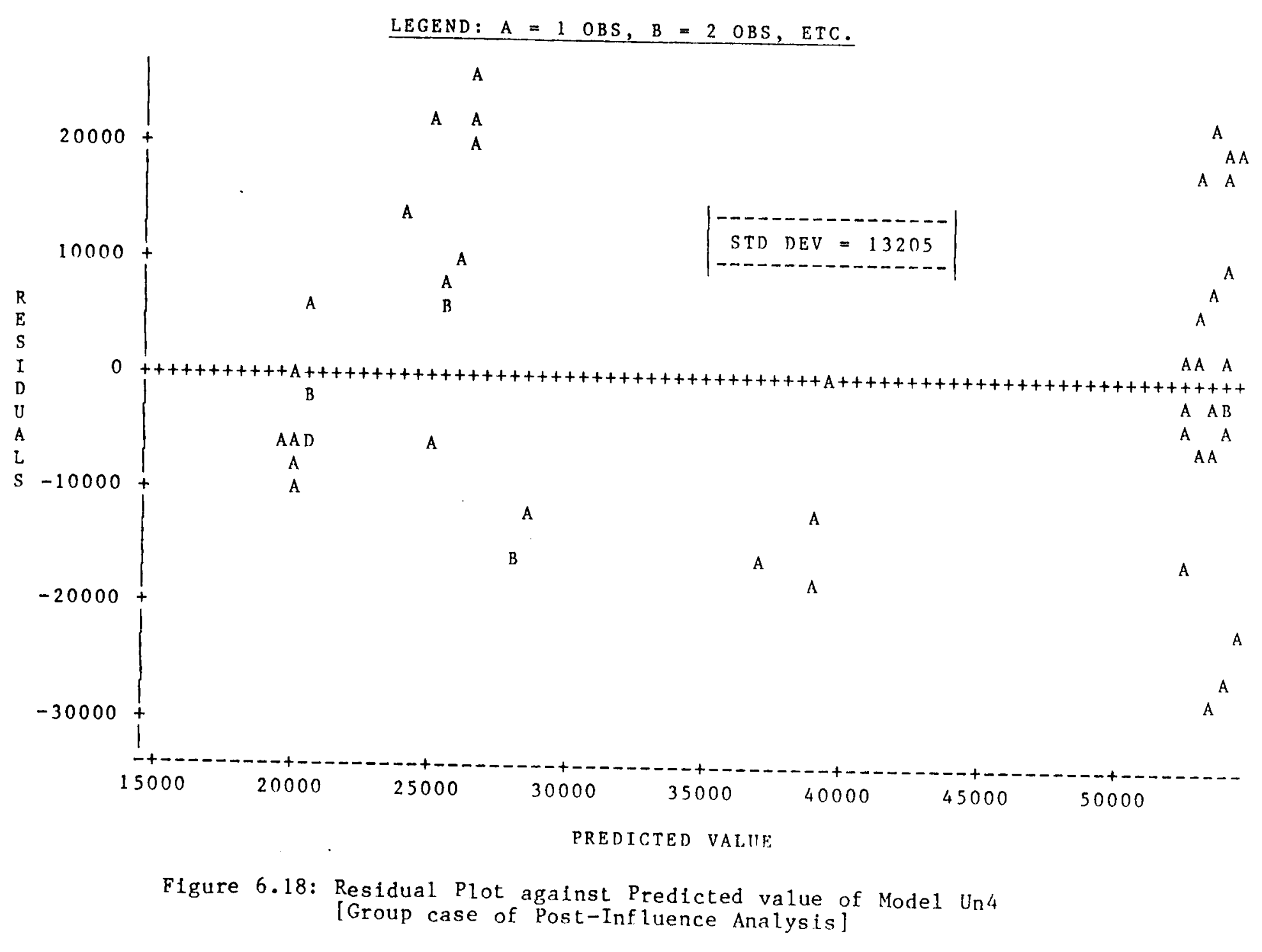


LEGEND: $A=1$ OBS, $B=20 B S, E T C$.

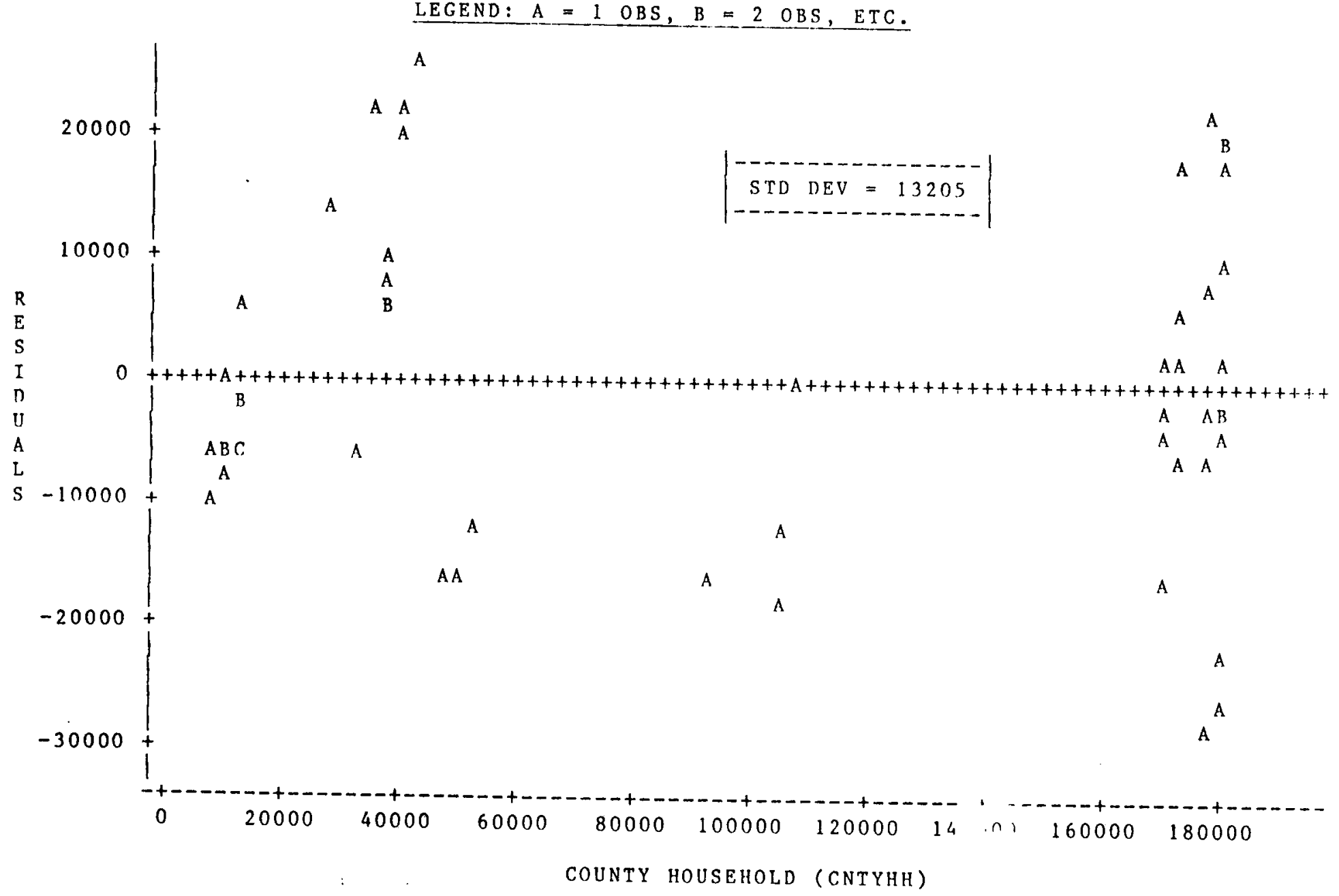

Figure 6.19: Residual P1ot agalnst Regressor CNTYHH of Model Un4 [Group case of Post-Influence Analysis] 
LEGEND: $A=1$ OBS, $B=2$ OBS, ETC.

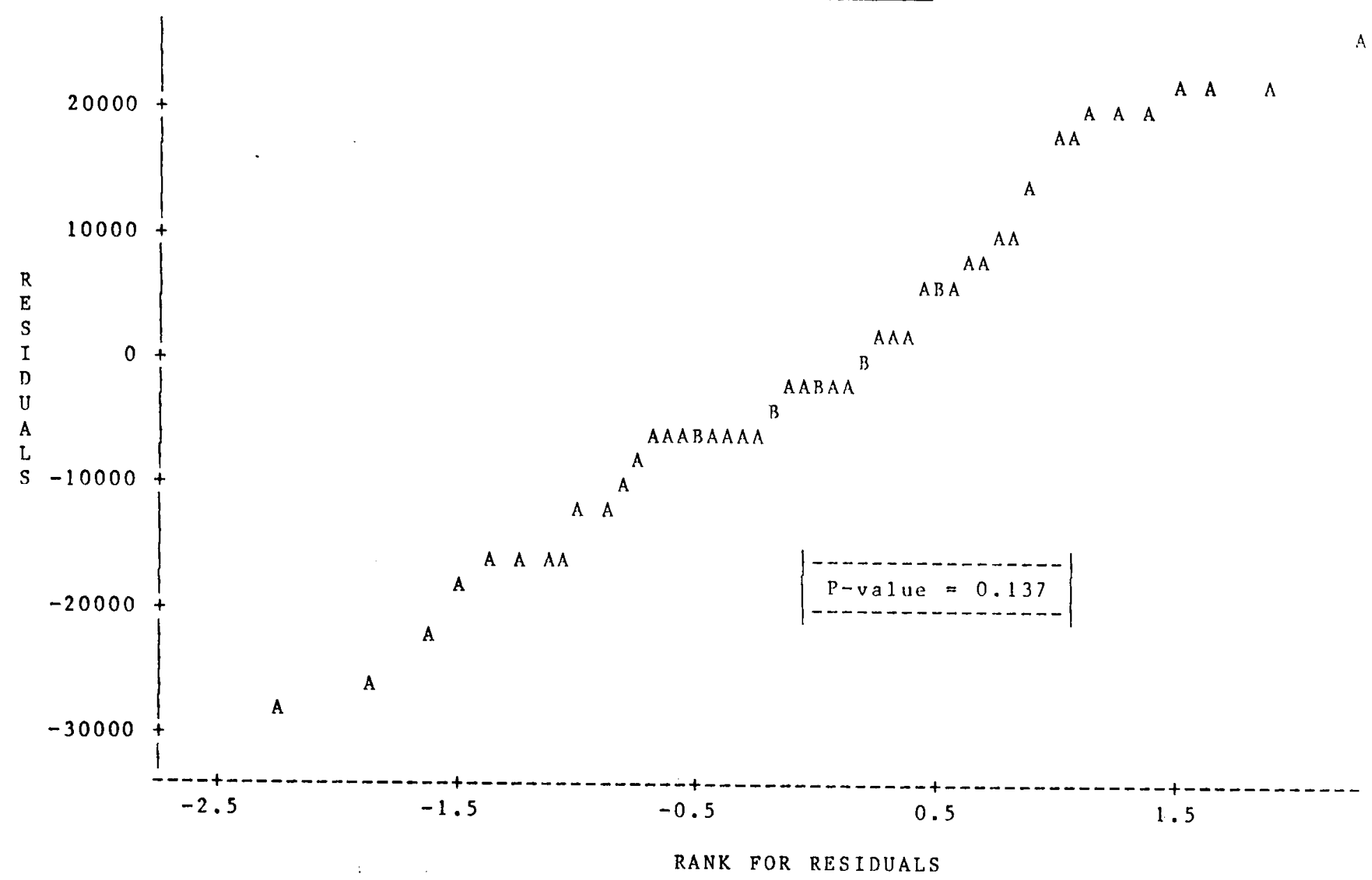

Figure 6.20: Norma1 Probability P1ot of Model Un.

[Group case of Post-Influence Analys 1s] 


\begin{tabular}{|c|c|c|c|c|c|c|c|}
\hline $\begin{array}{l}\text { Mode1 } \\
(* * *)\end{array}$ & $\begin{array}{l}\text { Ana- } \\
\text { lysis }\end{array}$ & $\begin{array}{l}\text { ST DEV } \\
(*)\end{array}$ & $\begin{array}{c}P \text {-value } \\
\text { of } W \text {-test } \\
(*)\end{array}$ & $\begin{array}{c}\mathrm{R}^{2} \\
(* *)\end{array}$ & $\begin{array}{c}\mathrm{R}_{\mathrm{a}}^{2} \\
(* *)\end{array}$ & $\begin{array}{l}\text { RMSE } \\
(* *)\end{array}$ & $\begin{array}{l}\text { C.V. } \\
(* *)\end{array}$ \\
\hline $\operatorname{Cn} 5$ & $\begin{array}{l}\text { Pre } \\
\text { Post }\end{array}$ & $\begin{array}{l}2192 \\
1697\end{array}$ & $\begin{array}{l}0.347 \\
0.672\end{array}$ & $\begin{array}{l}33.55 \\
58.04\end{array}$ & $\begin{array}{l}28.63 \\
54.81\end{array}$ & $\begin{array}{l}2271 \\
1761\end{array}$ & $\begin{array}{l}20.57 \\
15.74\end{array}$ \\
\hline Un4 & $\begin{array}{l}\text { Pre } \\
\text { Post }\end{array}$ & $\begin{array}{l}20152 \\
13205\end{array}$ & $\begin{array}{c}<0.01 \\
0.137\end{array}$ & $\begin{array}{l}48.79 \\
55.64\end{array}$ & $\begin{array}{l}47.85 \\
54.76\end{array}$ & $\begin{array}{l}20333 \\
13336\end{array}$ & $\begin{array}{l}46.04 \\
35.24\end{array}$ \\
\hline
\end{tabular}

$(* *)$ See Figures $6.4-6.10,6.13-6.16,6.18-6.20$.

$(* * *)$ Model Cn5: 1 of 30 original observations deleted as outlier. Model Un 4: 4 of 56 original observations deleted as outliers.

Tables in Section 6.3 and 6.4 show the improvement in statistics for all the candidate models owing to this influence analysis.

\subsection{Validity of Regression Assumptions and Model Aptness}

Due to the fact that the estimation of model regression parameters depends on assumptions concerning the statistical characteristics of the error components in the model, these assumptions are crucial in determining the specific estimation procedure. Three most important assumptions regarding regression are

1. Linearity of the model,

2. Homoscedasticity of variance, and

3. Normality of the errors.

In this section, some formal tests and a commonly used graphic residual analysis are used to verify the above assumptions and to check the aptness of the models. The validity of some of the tests (for example, the significance test of the parameter - see Section 6.4.2) depends on one or some of these assumptions (for example, normality assumption is crucial for significance test). 
The residual plots shown in this section are generated by the SAS package [SAS/Basics 1985]. The plots were done to check the aptness of each model. The ith residual, denoted by $e_{i}$, is the difference between the observed value $Y_{i}$ and the corresponding fitted value $\hat{Y}_{i}$ (i.e., $e_{i}=Y_{i}-\hat{Y}_{i}$ ).

\subsubsection{Scatterplots: Linear Pattern of the Model}

Scatterplots display the relationships between two quantitative variables graphically. It is a good plot if the general pattern shows the direction, form, and strength of the relationship between the variables. The strength of a scatterplot is that it provides a complete picture of the relationship between two variables - at least as far as that relationship is reflected in the available data. A complete picture is often too detailed for easy interpretation. But one can easily see a general pattern by smoothing a scatterplot. The smoothing is done by averaging the $y$ values separately for each $x$ value. Figures $6.21-6.23$ show the three representative plots, one from each highway category. A linear pattern is plausible in each of these plots.

\subsubsection{Residual Plots: Independence and Linearity of the Model}

The independence assumption is all but impossible to check (or correct) after the experiment is over. The plot of residuals against the time in which data were collected, help to detect correlations or dependence in the residuals. But runs of positive and negative residuals is usually an expected result from the cross-classified (data 
Scatterplot of AADT vS CNTYEMP

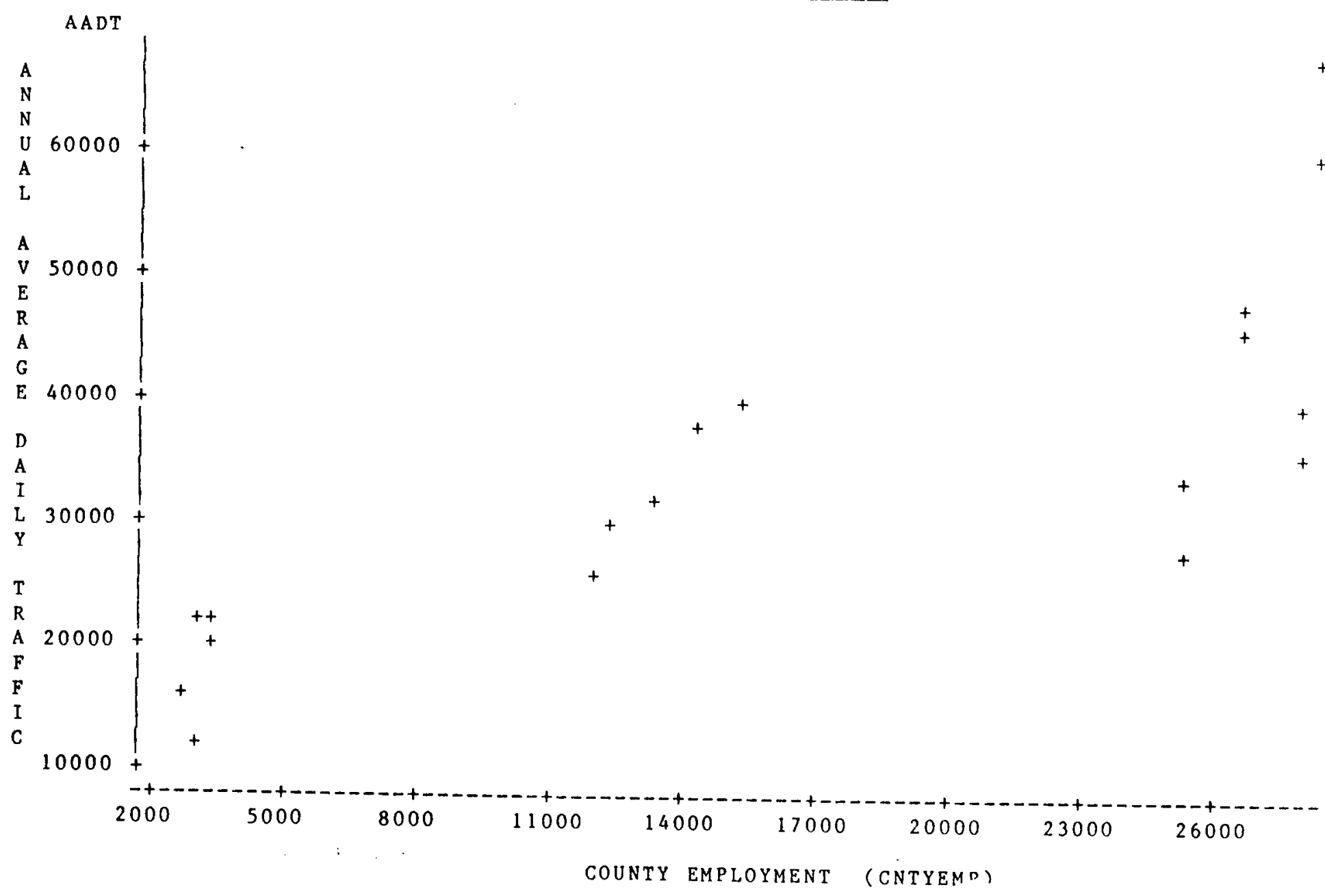

Figure 6.21: A Scatterplot for Reglon "s" of Urban Inte $r$ Highways 
Scatterplot of AADT vs CNTYINC

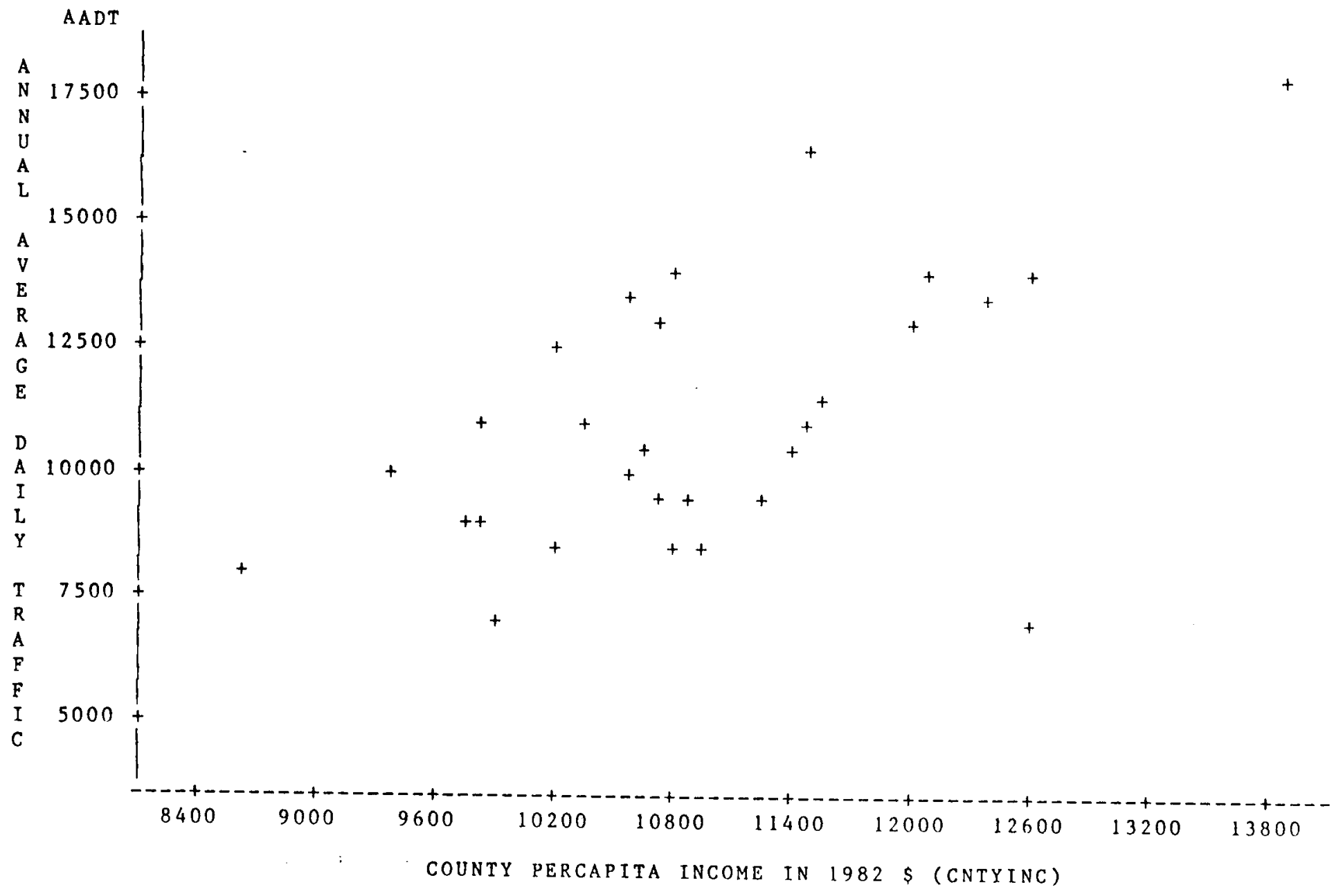

Figure 6.22: A Scatterplot for City Group " $C$ " and Region " $n$ " of Urban Principal Arterial. 
Scatterplot of AADT US CNTYINC

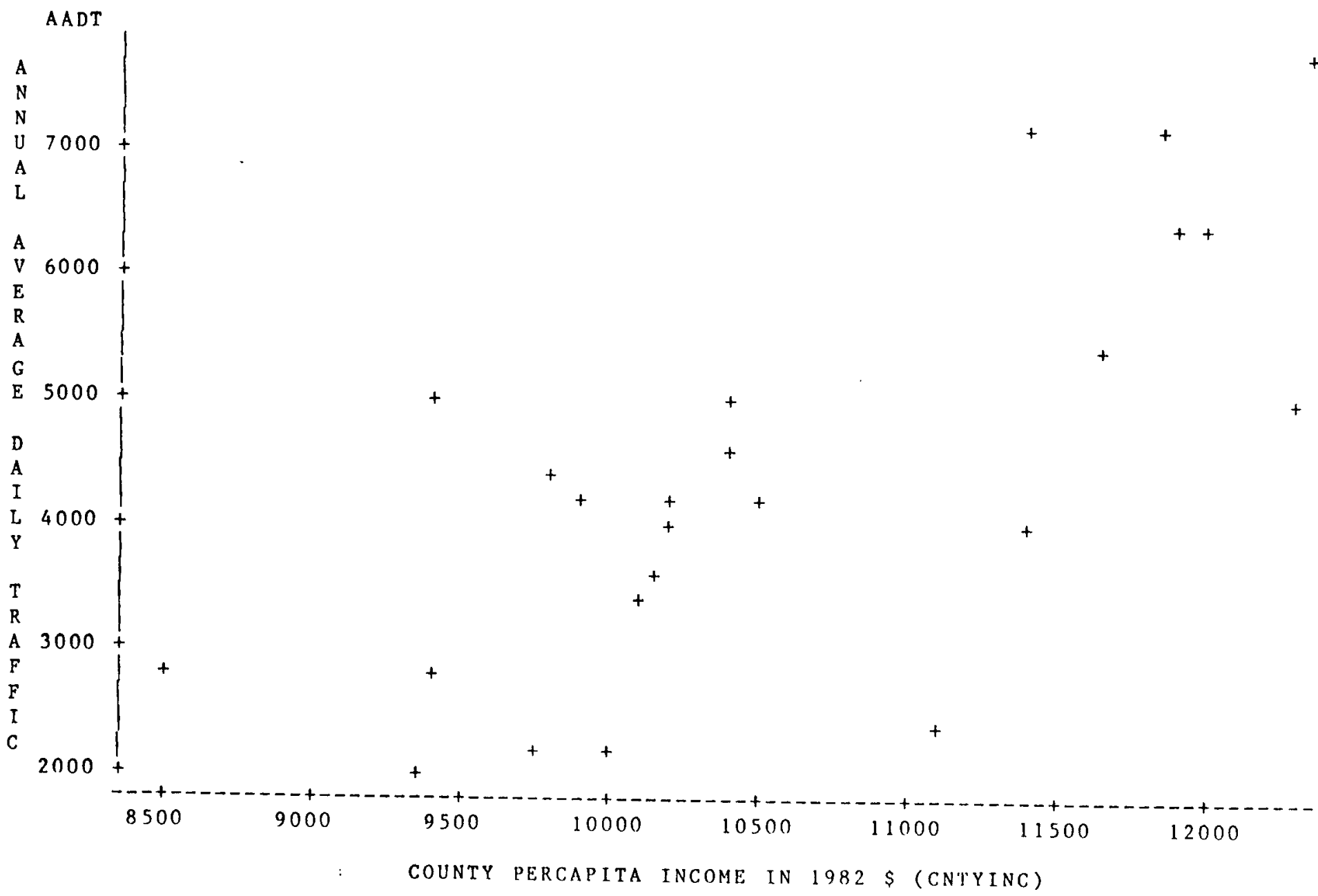

Ftgure 6.23: A Scatterplot for City Group "D" and Region "ctn" of Urban Minor Artertal and Collector 
from different counties/cities) data we have. If the residuals tend to spread more or less over time, then there is a problem of nonconstant error variance. For a linear model, the scatter plots of $Y$ against $X^{-} s$ should depict no nonlinear trend. Also, a parallel horizontal band of the plots of residuals against each predictor variable suggests linearity of the model.

The plots of residuals against the fitted response variable and predictor variables (see the representative Figures 6.13-6.15, 6.18 and 6.19) suggest no grounds for questioning the appropriateness of the linearity of the regression function or the constancy of the error variance. There are no suggestions in any of these plots that systematic deviation from the fitted response plane (for more than one variable in the equation) or line (for one variable in the equation) is present. The error variance varies in some of these plots with the level of $\hat{Y}$ and $X^{-} s$, but this variation does not exhibit any gross departure. After correction through influence analysis, the residual plots against the $\hat{Y}$ (predicted values) and the $X^{\prime} s$ (regressors in the mode1) do not suggest the presence of any outlier. In a residual plot, outliers are the points that lie far beyond the scatter of the remaining residuals, perhaps 3 or more standard deviations from zero [Neter et al. 1985].

Residual plots were also generated against variables not included in the model, to check whether some key independent or predictor variables could provide important additional descriptive and predictive power to the model. Three representative plots (Figures 6.24-6.26) of residuals against CNTYINC for models Rs 1 ( $\mathrm{Gr}-\mathrm{ix}), \mathrm{Ds} 4(\mathrm{Gr}-\mathrm{xi} i)$ and $\mathrm{C} 4$ 
(Gr-iii) (one from each highway category) do not show any correlation between the error ierms and the variable, CNTYINC, which is not included in codels. This is because the residuals are random around the zer.e. Thus, it is confirmed that the appropriate variables are included in the model and no additional variable will provide significant additional power to the model.

\subsubsection{Res $\quad$ ots: Homoscedasticity of Variance}

If the model is correct, and three assumptions -- normality, independence and linearity - hold, the residuals should be without pattern or structure. To see if there is a pattern or structure, we plot the residuals against: (1) the dependent variable, (2) the predicted values and (3) independent variables. This type of test to see the non-constant variance is an informal, but most widely accepted, procedure for testing the homogeneity of residuals. A horizontal random parallel band of these plots (see the representative plots $6.13-6.15,6.18-6.19,6.24-6.26)$ suggests the homoscedasticity of variances. In other words, absence of heteroscedasticity is visually indicated by a scatter of points uniformly distributed across the graph. As with the normality assumption (see Section 6.3.4), the assumption of constant variance is important for consistent and efficient estimates of the regression coefficients.

\subsubsection{Normality Tests and Plots: Normality of Errors}

The assumption of normality is often made that data come from a suitable normal distribution. Since the validity of many results 
LEGEND: $A=1$ OBS, $B=2$ OBS, ETC.

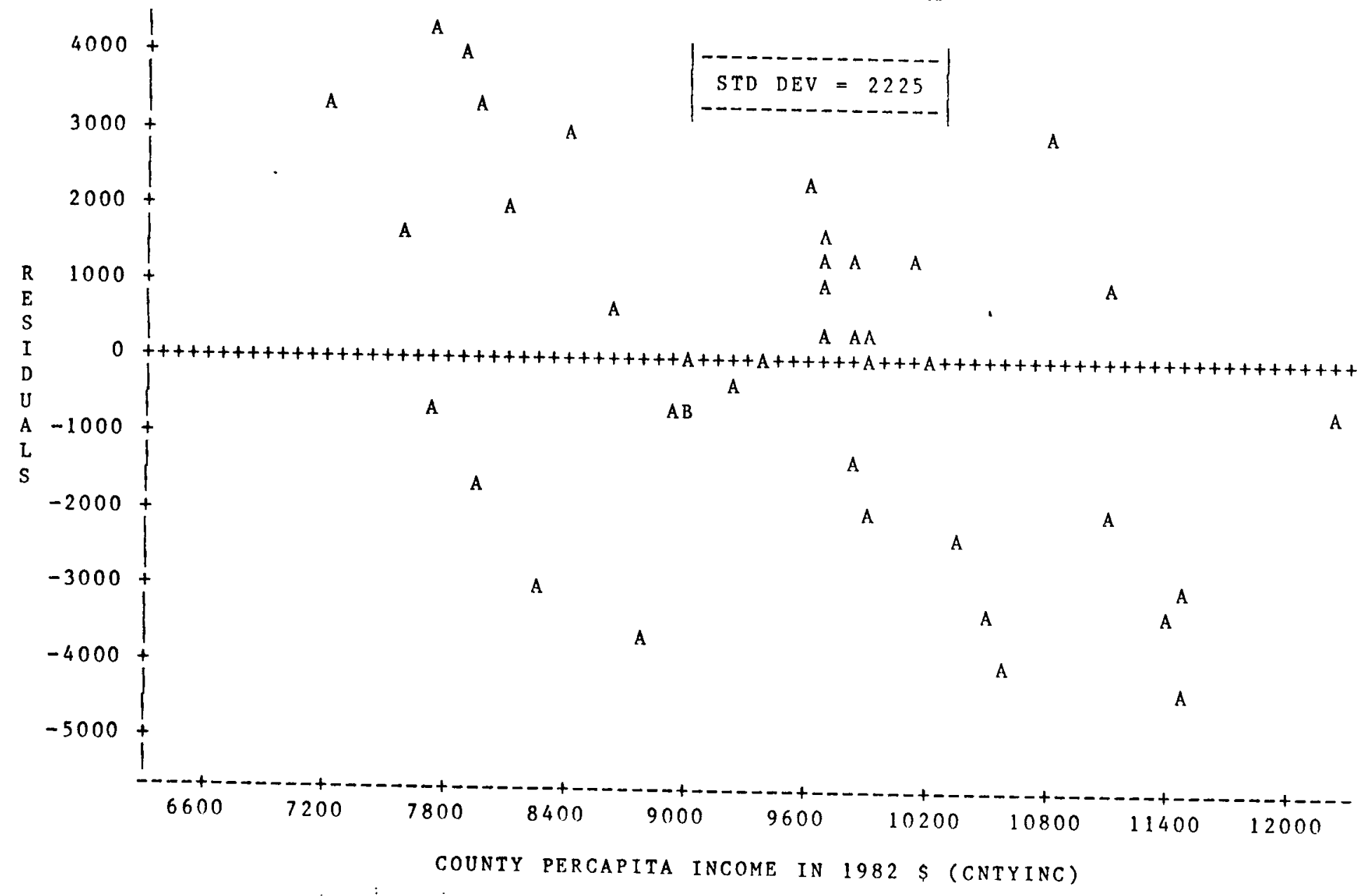

Figure 6.24: Residual Plot against CNTYINC of Model Rs 1 [Region "s" of Rural Interstate Highways] 
LEGEND: $A=1$ OBS,$B=2 O B S$,

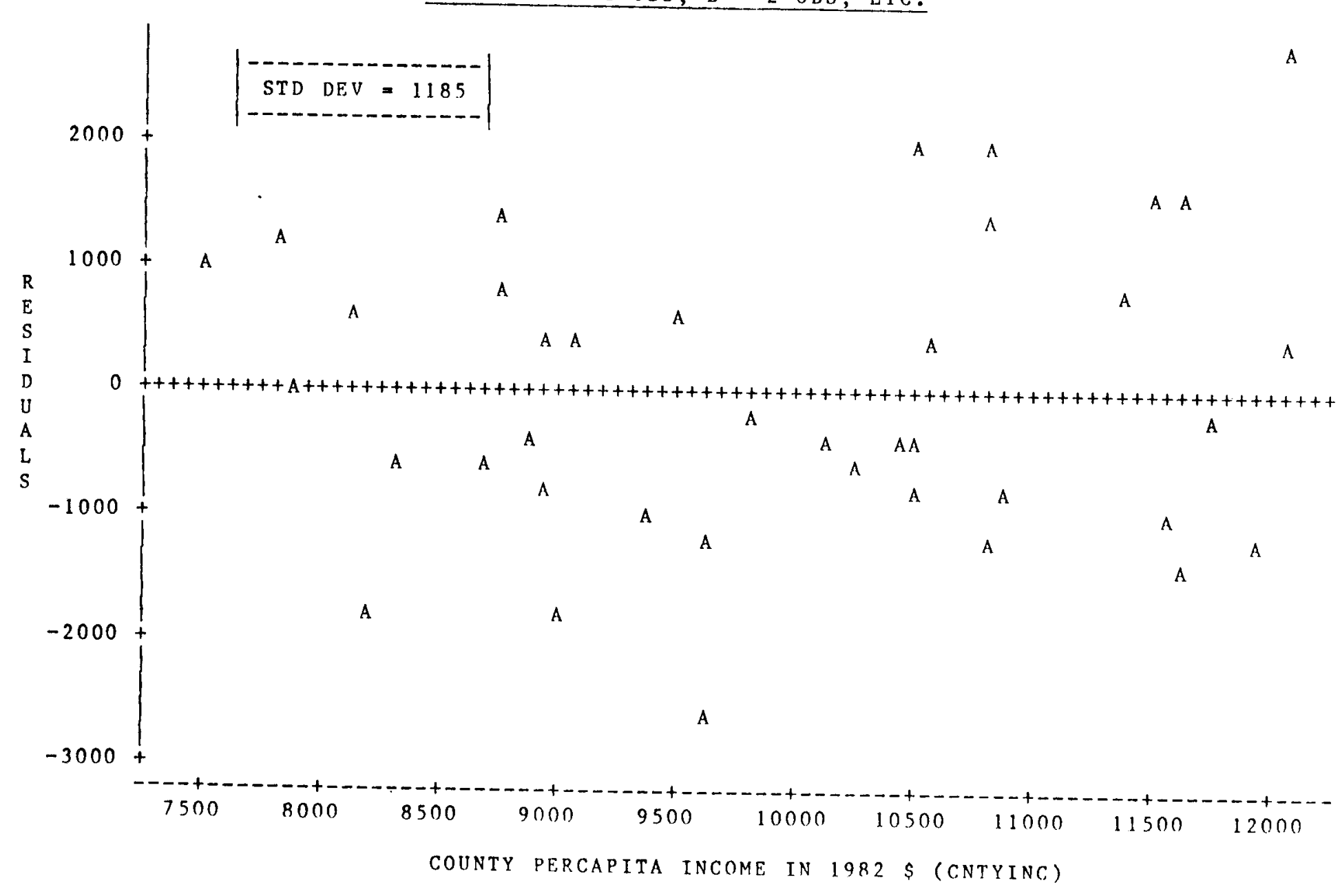

Figure 6.25: Residual Plot against CNTYINC of Model Ds4

[City Group "D" \& Regiton "s" of Urban Principal Arterial.] 
LEGEND: $A=1$ OBS, $B=2$ OBS, ETC.

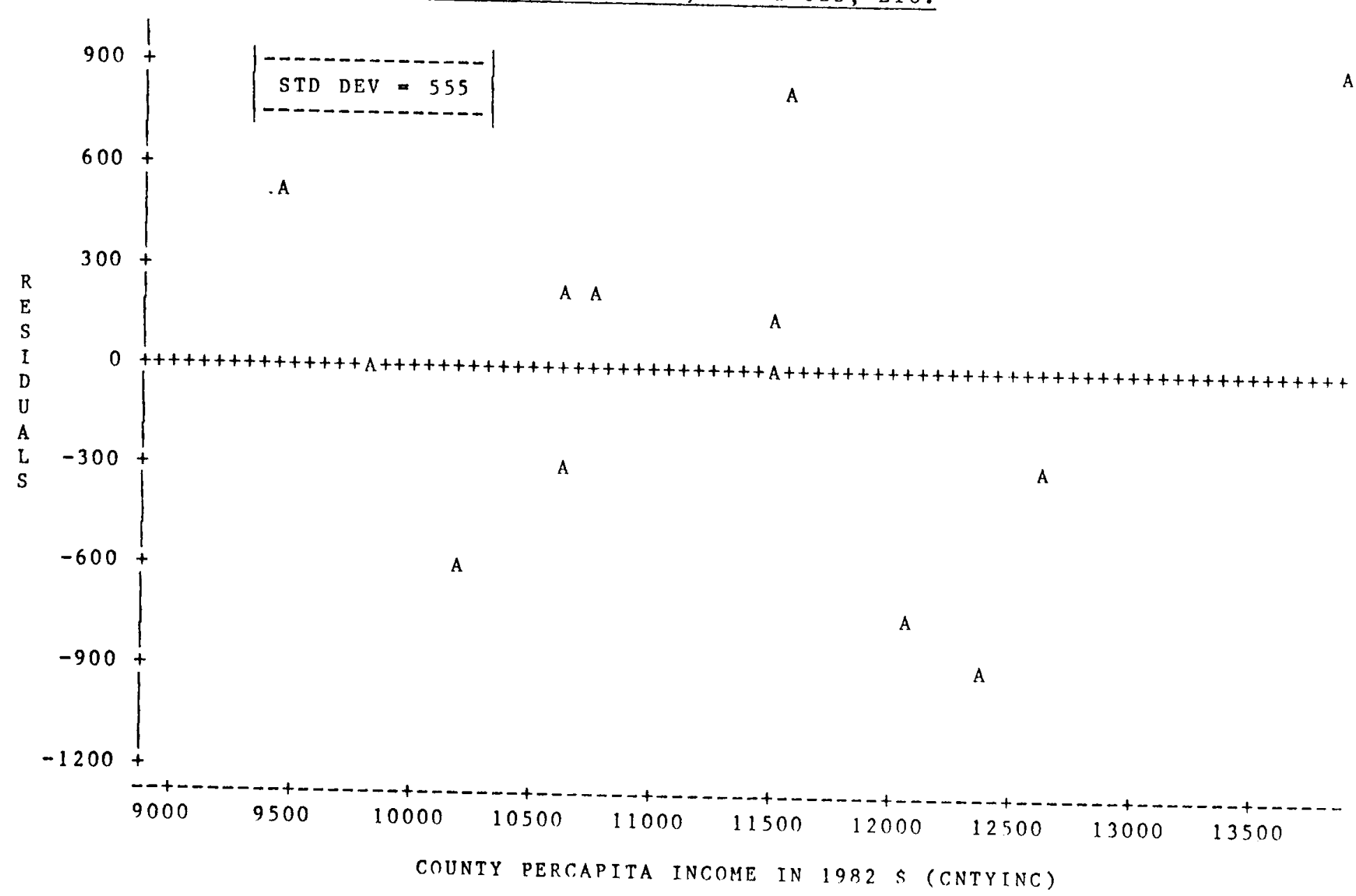

Figure 6.26: Residual Plot against CNTYINC of Model C4

[City Group "C" of Urban Minor Arterial \& Collector] 
often depend on the validity of the normal assumption, it is important to test for $n c: i$ ity. If the true errors are iid and $N\left(0, \sigma^{2}\right)$, then the normal prol ty plot should be (close to) a straight line. In general, the ir the amount of non-normality, the lower the power of the ANOVA F-test to detect the significance of the variable(s).

A heurist: test of normality is to draw the histogram to see if the dist:- is reasonably symmetric. Two informal tests of normality are is done through the coefficient of skewness and the coefficient of kurcusis. The coefficient of skewness indicates whether the distribution is symmetric or not. For a normal distribution, the expected value of this coefficient is zero. Since normal distributions are not the only symmetric distributions, a skewness test is not by itself a sufficient check for normality. The coefficient of kurtosis measures the peakedness of the data set. For a normal distribution, the expected value of this coefficient is 3 .

The standard and widely used tests for normality are the WilkShapiro $W$ test and normal probability plots. SAS procedure UNIVARIATE [SAS/BASICS 1985] was used to test normality. For fewer than 51 observations, the $W$ statistic is calculated, whereas for 51 or more observations, the Kolmogorov $D$ statistic is computed by the SAS UNIVARIATE procedure. Small values of $W$ leads to the rejection of the null hypothesis of normality. Probabilities, PROB $>W$ (for $N<51$ ) and $P R O B<D$ (for $N \geqslant 51$ ) are used for testing that the data come from a normal distribution. Values of these probabilities higher than the specified $\alpha$-level (usually, 5 percent) show that data are drawn from a normal distribution. In pre-influence analysis, the results of these 
normality tests along with standard deviation, skewness and kurtosis of residuals of all the ... idate models are shown in Table 6.5 to 6.7 for different groups ighway classes, identified in Chapter 5. Tables 6.8 to 6.10 shor normality test results in post-influence analysis. Comparing the results of normality tests in pre- and postinfluence analysis, one an see the improvement in results in postinfluence analysis. all data groups in Tables 6.8 to 6.10 except Group $i$ of Interst ays, the $P$-value ( $P R O B \angle D$ or $P R O B>W$ ) of the normality test is $\ldots$ it. .05 . With these general improvements in the results of normality tests in post-influence analysis (compared to pre-influence analysis), a significant improvement - from non-normal distributions to normal distributions-- is noticed in Groups iii, v, vi, $i x, x i$ of Interstate Highways (see Tables 6.5 and 6.8 ). These results also prove the usefulness of the influence analysis.

Normal quantile plots (often called normal probability plots) are recommended for assessing normality. There are several variations on the normal quantile plot. Approximately normal data will produce plotted points that lie close to a line. Real data will almost always show some departure from the theoretical normal model, so it is. important to confine the examination of a normal quantile plot to searching for shapes that show clear departures from normality. In other words, minor wiggles are not a major concern. [Moore \& McCabe 1989]. Three representative normal probability plots after the influence analysis, one from each highway category, are presented in Figures 6.27 to 6.29 . Figures $6.7,6.10,6.16$ and 6.20 also have illustrated the normal probability plots. All these normal probability plots fall reasonably 
Table 6.5: Normality Statistics of Residuals in Pre-Influence Analysis [Interstate Highways]

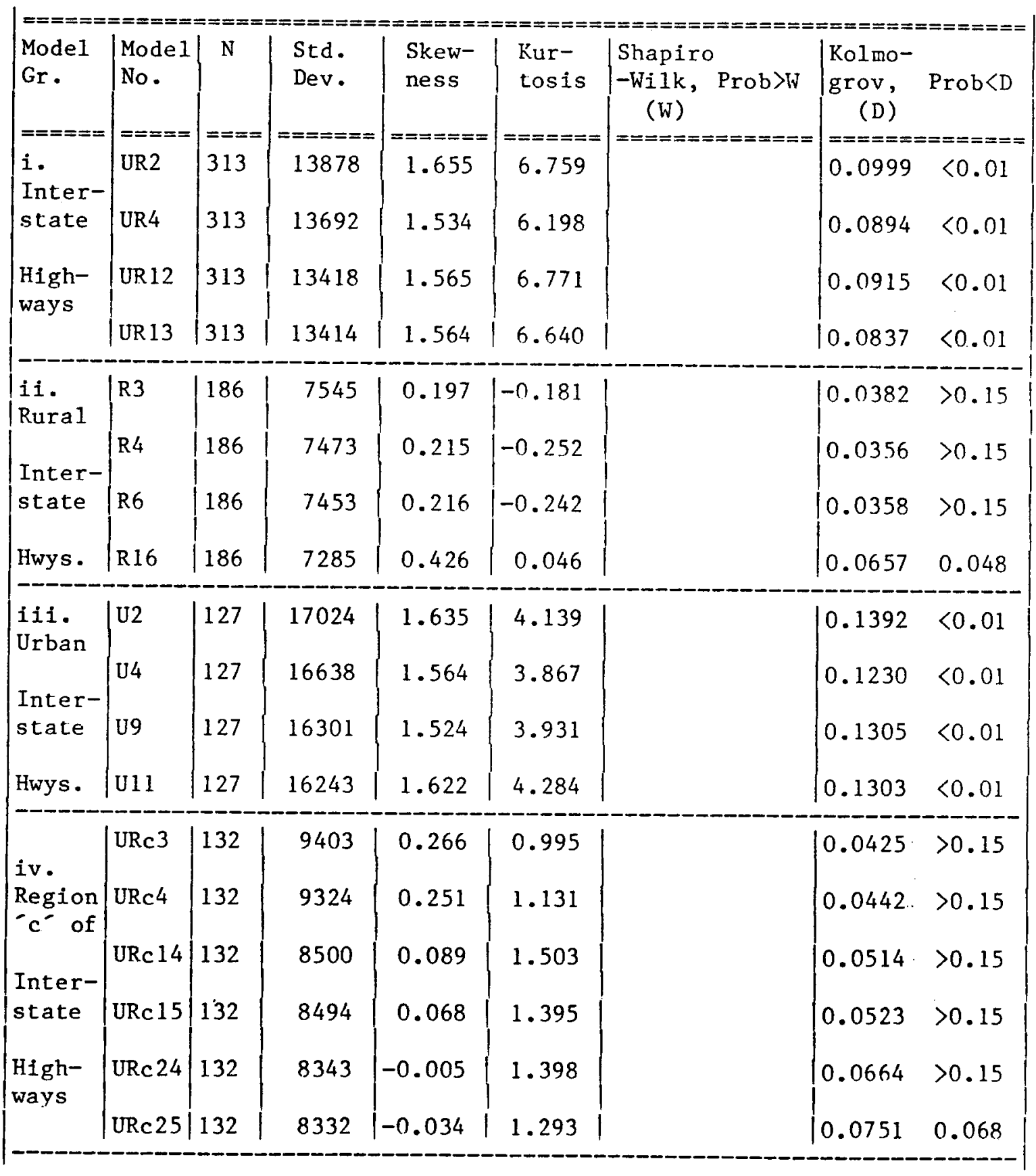


Table 6.5, continued

\begin{tabular}{|c|c|c|c|c|c|c|c|c|c|}
\hline \multirow{4}{*}{$\begin{array}{l}\text { Mode1 } \\
\text { Gr. } \\
====== \\
\mathrm{v} \cdot \\
\text { Region } \\
-n^{-} \text {of }\end{array}$} & $\begin{array}{l}\mid \text { Model } \\
\text { No. }\end{array}$ & $N$ & $\begin{array}{l}\text { Std. } \\
\text { Dev. }\end{array}$ & $\begin{array}{l}\text { Skew- } \\
\text { ness }\end{array}$ & $\begin{array}{l}\text { Kur- } \\
\text { tosis }\end{array}$ & \multirow{2}{*}{\multicolumn{2}{|c|}{$\mid \begin{array}{l}\text { Shapiro } \\
- \text { Wilk, Prob }>\text { W } \\
\text { (W) } \\
==============\end{array}$}} & \multicolumn{2}{|c|}{$\begin{array}{l}\text { Kolmo- } \\
\text { grov, Prob<D } \\
\text { (D) } \\
=============\end{array}$} \\
\hline & $=====$ & $====$ & $=======$ & $=======$ & $======\pi$ & & & & \\
\hline & URn2 & 104 & 18300 & 1.108 & 2.985 & & & 0.1189 & $<0.01$ \\
\hline & URn 3 & 104 & 18085 & 1.123 & 3.146 & & & 0.1170 & $<0.01$ \\
\hline $\begin{array}{l}\text { Inter- } \\
\text { state }\end{array}$ & URn 4 & 104 & 18044 & 1.080 & 2.921 & & & 0.1187 & $<0.01$ \\
\hline High- & URn 10 & 104 & 17763 & 1.124 & 3.318 & & & 0.1309 & $<0.01$ \\
\hline ways & URn 12 & 104 & 17715 & 1.175 & 3.616 & & & 0.1368 & $<0.01$ \\
\hline vi. & URs 1 & 77 & 8064 & 0.799 & 0.948 & & & 0.1253 & $<0.01$ \\
\hline 's' of & URs 2 & 77 & 7944 & 1.049 & 2.156 & & & 0.1141 & 0.014 \\
\hline $\begin{array}{l}\text { Inter- } \\
\text { state }\end{array}$ & URs 3 & 77 & 7907 & 0.848 & 1.214 & & & 0.1108 & 0.020 \\
\hline Hwys. & URs 4 & 77 & 7785 & 0.979 & 1.748 & & & 0.1180 & $<0.01$ \\
\hline $\begin{array}{l}\text { vii. } \\
\text { Region }\end{array}$ & Rc4 & 80 & 6681 & 0.060 & -0.149 & & & 0.0528 & $>0.15$ \\
\hline$c^{\prime}$ of & $\operatorname{Rc} 5$ & 80 & 6678 & 0.061 & -0.128 & & & 0.0555 & $>0.15$ \\
\hline Rural & $R c 6$ & 80 & 6663 & 0.064 & -0.159 & & & 0.0536 & $>0.15$ \\
\hline $\begin{array}{l}\text { Inter- } \\
\text { state }\end{array}$ & Rel 14 & 80 & 6515 & 0.312 & 0.068 & & & 0.0763 & $>0.15$ \\
\hline High- & Re 15 & 80 & 6511 & 0.320 & 0.086 & & & 0.0821 & $>0.15$ \\
\hline ways & Re 16 & 80 & 6506 & 0.311 & 0.047 & & & 0.0840 & $>0.15$ \\
\hline $\begin{array}{l}\text { viii. } \\
\text { Region }\end{array}$ & Rn 1 & 47 & 7422 & 0.419 & 0.658 & 0.9814 & 0.769 & & \\
\hline $\begin{array}{l}\text { 'n' of } \\
\text { Rural }\end{array}$ & $\operatorname{Rn} 4$ & 47 & 7290 & 0.273 & 0.671 & 0.9815 & 0.774 & & \\
\hline $\begin{array}{l}\text { Int. } \\
\text { Hwys. }\end{array}$ & Rn5 & 47 & 7289 & 0.310 & 0.522 & 0.9801 & 0.722 & & \\
\hline & Rn6 & 47 & 7274 & 0.249 & 0.703 & 0.9808 & 0.746 & & \\
\hline
\end{tabular}


Table $f . r$ : continued

\begin{tabular}{|c|c|c|c|c|c|c|c|c|c|}
\hline$====5$ & $=-1$ & $;====$ & $== \pm==$ & $===$ & $\therefore=====$ & $=-2$ & $=-=$ & & \\
\hline Model & |Model| & $\mathrm{N}$ & Std. & Sk & Kur- & Shapiro & & Kolmo- & \\
\hline $\mathrm{Gr}$ & No. & & Dev. & ne & tosis & $\left\{\begin{array}{c}-W i 1 k, \\
(W)\end{array}\right.$ & Prob $>W$ & $\begin{array}{r}\text { grov } \\
\text { (D) }\end{array}$ & Prob $<D$ \\
\hline$== \pm===$ & $=====$ & $== \pm=$ & $=======$ & $==.=-===$ & $1=======$ & $=======$ & $===\approx====$ & |====== & $========$ \\
\hline ix. & Rs 1 & 59 & 4956 & $0 . " 19$ & 1.007 & & & 0.1457 & $<0.01$ \\
\hline $\begin{array}{l}\text { Region } \\
\text { - } \mathrm{s}^{-} \text {of }\end{array}$ & Rs 2 & 59 & 4939 & & 1.080 & & & 0.1623 & $<0.01$ \\
\hline Rural & |Rs & בנה & ענצע & & 1.000 & & & & 10.01 \\
\hline Int. & Rs 3 & 59 & 4903 & & 1.316 & & & 0.1548 & $<0.01$ \\
\hline Hoys. & Rs 4 & 59 & 4902 & & 1.351 & & & 0.1290 & 0.016 \\
\hline$x$. & $\mathrm{U}_{\mathrm{C}} 1$ & 52 & 9123 & 0.571 & U.395 & & & 0.0821 & $>0.15$ \\
\hline $\begin{array}{l}\text { Region } \\
-c^{-} \text {of }\end{array}$ & Uc2 & 52 & 8783 & 0.518 & 0.207 & & & 0.0754 & $>0.15$ \\
\hline Urban & Uc3 & 52 & 8736 & 0.490 & 0.114 & & & 0.0862 & $>0.15$ \\
\hline Inter- & $10 c 4$ & 52 & 8482 & 0.405 & -0.352 & & & 0.0904 & $>0.15$ \\
\hline state & Ucl 12 & 52 & 8050 & 0.330 & -0.270 & & & 0.1038 & $>0.15$ \\
\hline High- & & & & & & & & & \\
\hline ways & Uc13 & 52 & 7915 & 0.312 & $\mid-0.461$ & & & 0.0942 & $>0.15$ \\
\hline xi. & Un2 & 57 & 20391 & 1.013 & 1.451 & & & 0.1170 & 0.050 \\
\hline $\begin{array}{l}\text { Region } \\
\text { 'n' of }\end{array}$ & Un 3 & 57 & 20229 & 1.073 & 1.582 & & & 0.1291 & 0.019 \\
\hline Urban & Un 4 & 57 & 20152 & 1.004 & 1.423 & & & 0.1396 & $<0.01$ \\
\hline Int. & Un 11 & 57 & 19920 & 1.055 & 1.668 & & & 0.1251 & 0.025 \\
\hline Hwys. & Un 13 & 57 & 19905 & 1.124 & 1.836 & & & $0.1223 .$. & 0.033 \\
\hline xii. & Us 3 & 18 & 8076 & 0.948 & 1.374 & 0.9373 & 0.322 & & \\
\hline 's' of & Us4 & 18 & 7489 & 0.873 & 1.179 & 0.9450 & 0.395 & & \\
\hline Urban & Us 10 & 18 & 6693 & 0.876 & 1.232 & 0.9491 & 0.434 & & \\
\hline Inter- & Us 11 & 18 & 6653 & 1.087 & 1.736 & 0.9184 & 0.142 & & \\
\hline & Us 12 & 18 & 6380 & 1.262 & 2.016 & 0.8974 & 0.051 & & \\
\hline $\begin{array}{l}\text { High- } \\
\text { ways }\end{array}$ & Us 13 & 18 & 6238 & 1.117 & 1.613 & 0.9221 & 0.177 & & \\
\hline
\end{tabular}


Table 6.6: Normality Statistics of Res: is in Pre-Influence Analysis [Irrban Principal Arterial]

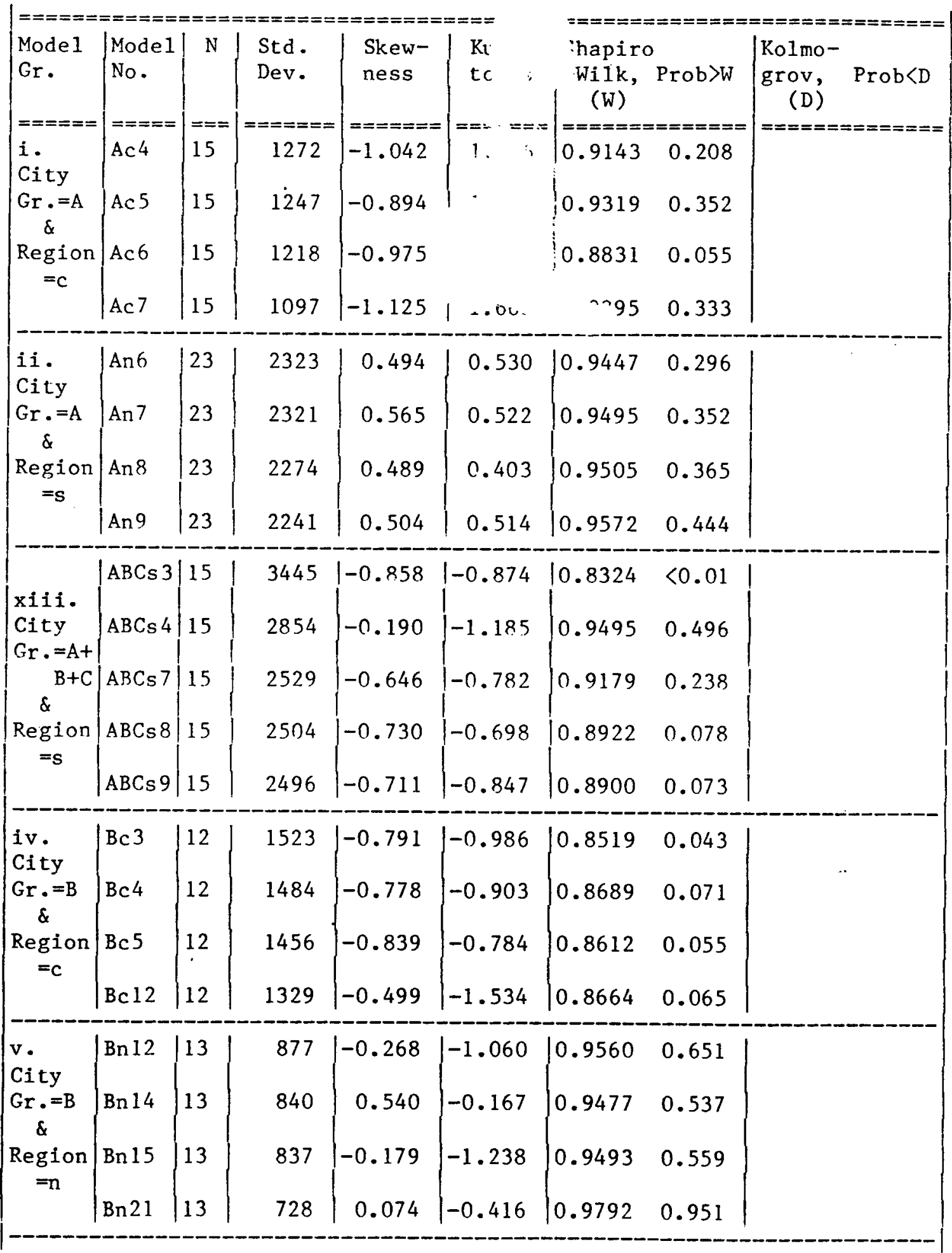


Table 6.6 , continued

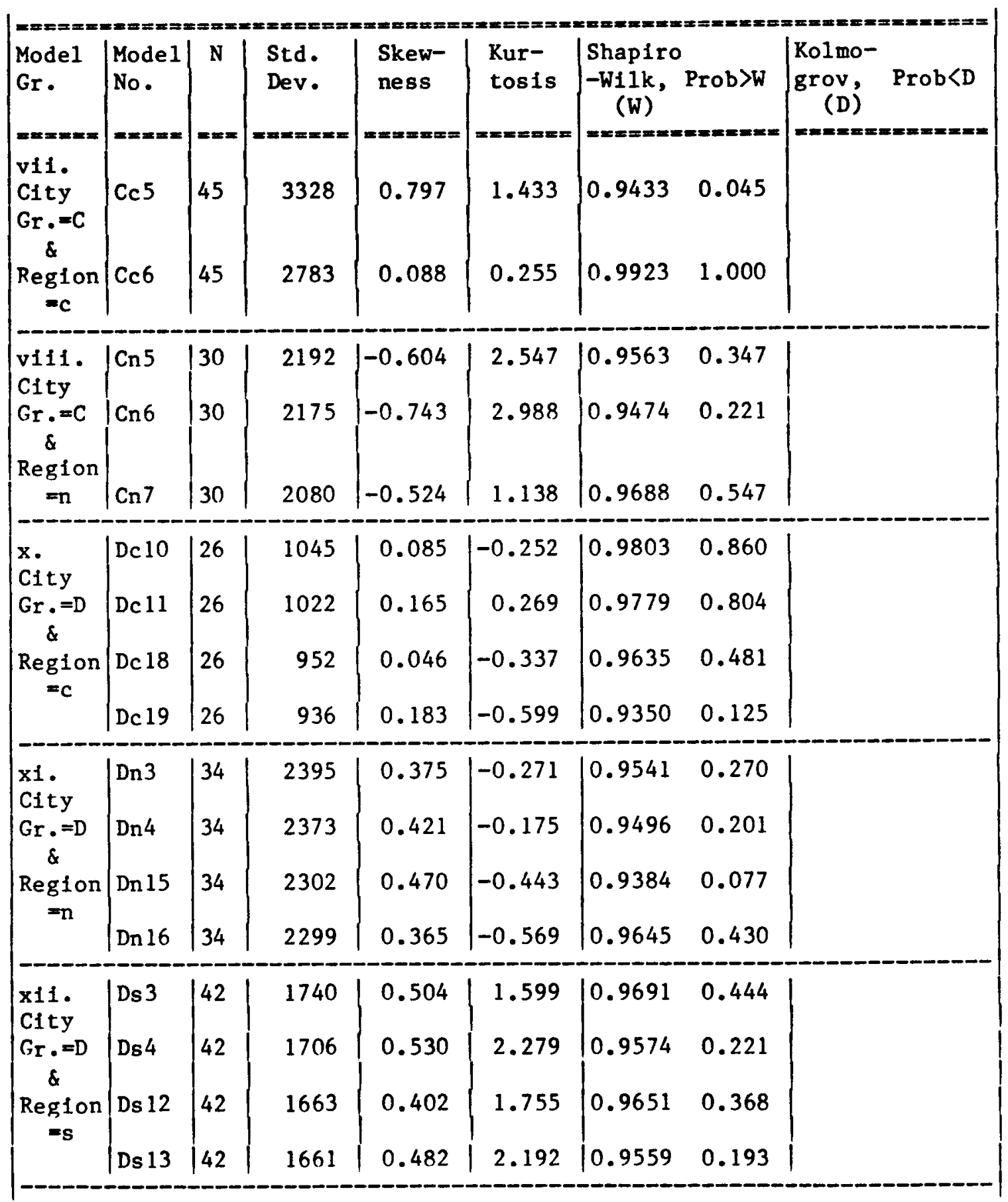


Table 6.7: Normality Statistics of Residuals in Pre-Influence Analysis [Urban Minor Arterial \& Collector]

\begin{tabular}{|c|c|c|c|c|c|c|c|c|}
\hline \multirow[t]{2}{*}{$\begin{array}{l}\text { Mode1 } \\
\text { Gr. } \\
\text { =x=x=m }\end{array}$} & $\left|\begin{array}{l}\text { Mode } 1 \\
\text { No. } \\
== \pm==\end{array}\right|$ & $\left|\begin{array}{c}N \\
==2\end{array}\right|$ & $\mid \begin{array}{l}\text { Std. } \\
\text { Dev. } \\
\\
\end{array}$ & $\begin{array}{l}\text { Skew- } \\
\text { ness } \\
==x=m=x\end{array}$ & \multirow[t]{2}{*}{$\mid \begin{array}{l}\text { Kur- } \\
\text { tosis } \\
==x==== \\
-0,201\end{array}$} & \multicolumn{2}{|c|}{$\begin{array}{l}\text { Shapiro } \\
\text {-Wilk, Prob }>W \\
\text { (W) } \\
==\pi=x===\pi== \pm==\end{array}$} & \multirow[t]{5}{*}{$\mid \begin{array}{l}\text { Kolmo- } \\
\text { grov, Prob }<D \\
\text { (D) } \\
=x=m=x=m== \pm=x\end{array}$} \\
\hline & A2 & 18 & 2465 & 0.592 & & 0.9341 & 0.291 & \\
\hline $\begin{array}{l}\text { City } \\
\text { City }\end{array}$ & A5 & 18 & 2228 & 0.815 & 0.365 & 0.9356 & 0.306 & \\
\hline $\mathrm{Gr} .=\mathrm{A}$ & A6 & 18 & 2169 & 0.748 & 0.214 & 0.9393 & 0.341 & \\
\hline & A7 & 18 & 2111 & 0.651 & 0.989 & 10.9449 & 0.394 & \\
\hline $\begin{array}{l}11 \\
\text { City } \\
\text { Gr.=B }\end{array}$ & B2 & 13 & 2215 & -0.066 & -0.887 & 0.9714 & 0.864 & \\
\hline & $\mathrm{C} 2$ & 13 & 1011 & -0.923 & -0.542 & 0.8452 & 0.028 & \\
\hline City & C3 & 13 & 969 & -0.818 & -0.591 & 0.8752 & 0.070 & \\
\hline & 104 & 13 & 934 & -0.844 & -0.465 & 0.8544 & 0.038 & \\
\hline & C9 & 11 & 1203 & -0.052 & -1.762 & 0.9034 & 0.271 & \\
\hline $\begin{array}{l}\text { iv. } \\
\text { City }\end{array}$ & $\mathrm{C} 10$ & 11 & 1160 & -0.183 & 0.052 & 0.9739 & 0.907 & \\
\hline $\mathrm{Gr} .=\mathrm{C}$ & $C 11$ & 11 & 1123 & 0.304 & -0.049 & 0.9533 & 0.661 & \\
\hline & $\mathrm{C} 12$ & 11 & 1117 & -0.198 & 0.338 & 0.9741 & 0.909 & \\
\hline $\begin{array}{l}\text { v. } \\
\text { City } \\
\text { Gr.=D }\end{array}$ & D6 & 28 & 2299 & 0.878 & -0.055 & 0.9062 & 0.018 & \\
\hline $\begin{array}{l}\text { vi. } \\
\text { City } \\
\text { Gr.=D }\end{array}$ & D7 & 24 & 1141 & -0.311 & -0.185 & 0.9788 & 0.851 & \\
\hline
\end{tabular}


Table 6.8: Normality Statistics of Residuals in Post-Influence Analysis [Interstate Highways]

\begin{tabular}{|c|c|c|c|c|c|c|c|c|}
\hline $\begin{array}{l}\text { Model } \\
\text { Gr. } \\
\text { = }\end{array}$ & $\mid \begin{array}{l}\text { Model } \\
\text { No. } \\
\text { No=e=n }\end{array}$ & | & $\begin{array}{l}\text { Std. } \\
\text { Dev. }\end{array}$ & $\begin{array}{l}\text { Skew- } \\
\text { ness }\end{array}$ & $\left\{\begin{array}{l}\text { Kur- } \\
\text { tosis }\end{array}\right.$ & $\begin{array}{l}\text { Shapiro } \\
- \text { Wilk, Prob }>\text { W } \\
\text { (W) }\end{array}$ & $\mid \begin{array}{l}\text { Kolmo- } \\
\text { grov, } \\
\text { (D) }\end{array}$ & Prob $<D$ \\
\hline & UR2 & 225 & 5899 & 0.214 & -1.037 & & 0.0782 & $<0.01$ \\
\hline $\begin{array}{l}\text { Inter- } \\
\text { state }\end{array}$ & UR4 & 225 & 6109 & 0.241 & -1.025 & & 0.0733 & $<0.01$ \\
\hline High- & UR 12 & 225 & 5989 & 0.195 & -0.962 & & 0.0674 & $<0.01$ \\
\hline & UR 13 & 225 & 5981 & 0.196 & -1.007 & & 0.0720 & $<0.01$ \\
\hline ii. & |R3 & 121 & 3983 & 0.258 & -0.899 & & 0.0632 & $>0.15$ \\
\hline & R4 & 121 & 3809 & 0.403 & -0.753 & & 0.0854 & 0.029 \\
\hline $\begin{array}{l}\text { inter- } \\
\text { state }\end{array}$ & R6 & 121 & 3767 & 0.454 & -0.738 & & 0.0823 & 0.043 \\
\hline Hwys. & $R 16$ & 121 & 3766 & 0.441 & -0.748 & & 0.0844 & 0.034 \\
\hline |iii. & U2 & 103 & 8774 & 0.119 & -0.698 & & 0.0693 & $>0.15$ \\
\hline & U4 & 103 & 8585 & 0.207 & -0.687 & & 0.0800 & 0.102 \\
\hline $\begin{array}{l}\text { Inter- } \\
\text { state }\end{array}$ & U9 & 103 & 8251 & 0.034 & -0.667 & & 0.0716 & $>0.15$ \\
\hline Hwys. & |u11 & 103 & 8105 & 0.133 & -0.488 & & 0.0528 & $>0.15$ \\
\hline & | URc3 & 116 & 6831 & 0.080 & -0.730 & & 0.0468 & $>0.15$ \\
\hline $\begin{array}{l}\text { iv } \\
\text { Region }\end{array}$ & URc4 & 116 & 6745 & 0.090 & -0.737 & & 0.0490 & $>0.15$ \\
\hline & URc 14 & 116 & 6124 & -0.083 & -0.620 & & 0.0559 & $>0.15$ \\
\hline $\begin{array}{l}\text { Inter- } \\
\text { state }\end{array}$ & URc 15 & 116 & 6131 & -0.111 & -0.577 & & 0.0470 & $>0.15$ \\
\hline High- & URc24 & 116 & 6035 & -0.190 & -0.662 & & 0.0683 & $>0.15$ \\
\hline & URc25 & 116 & 6035 & -0.230 & -0.607 & & 0.0593 & $>0.15$ \\
\hline
\end{tabular}


Table 6.8 , continued

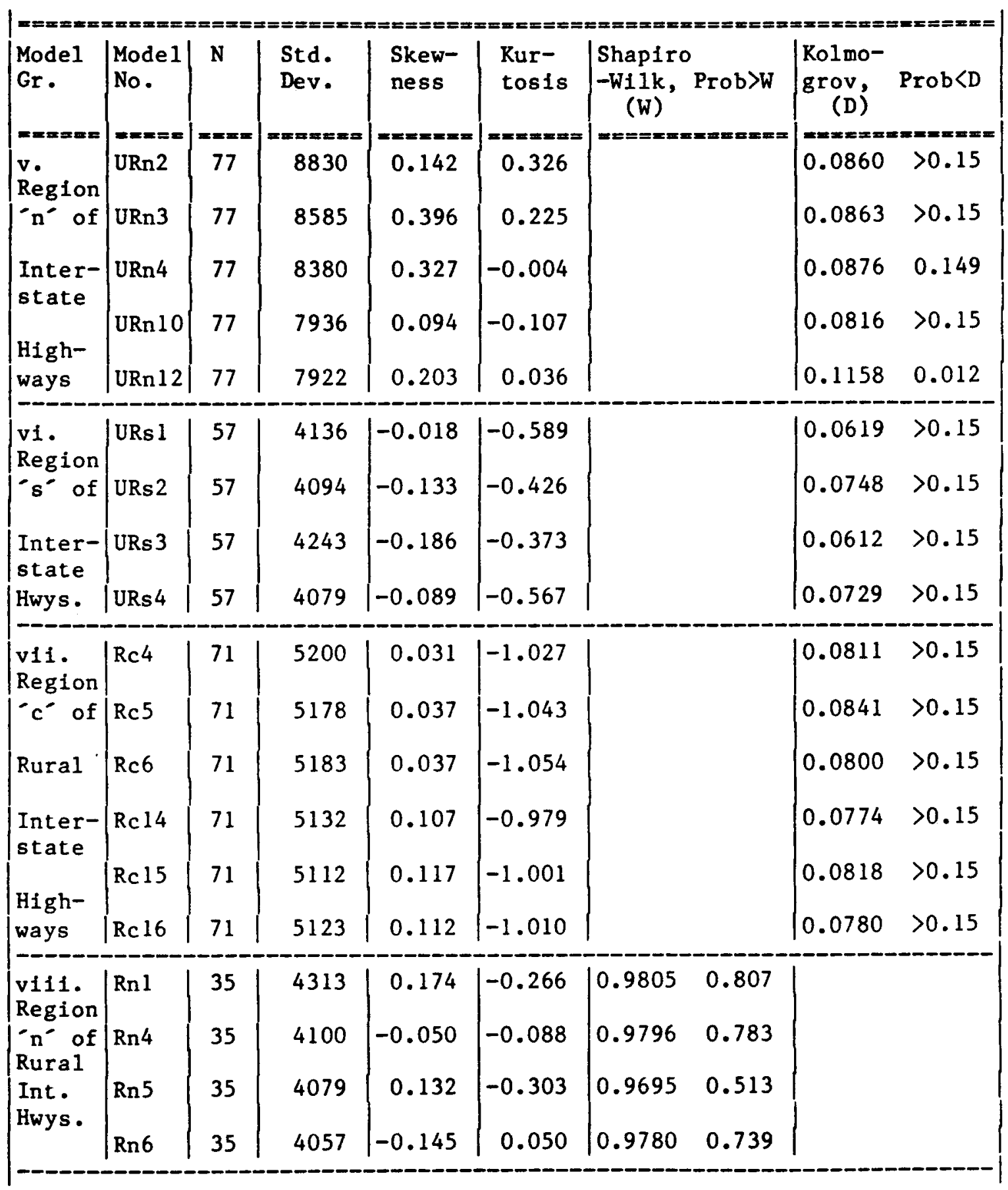


Table 6.8 , continued

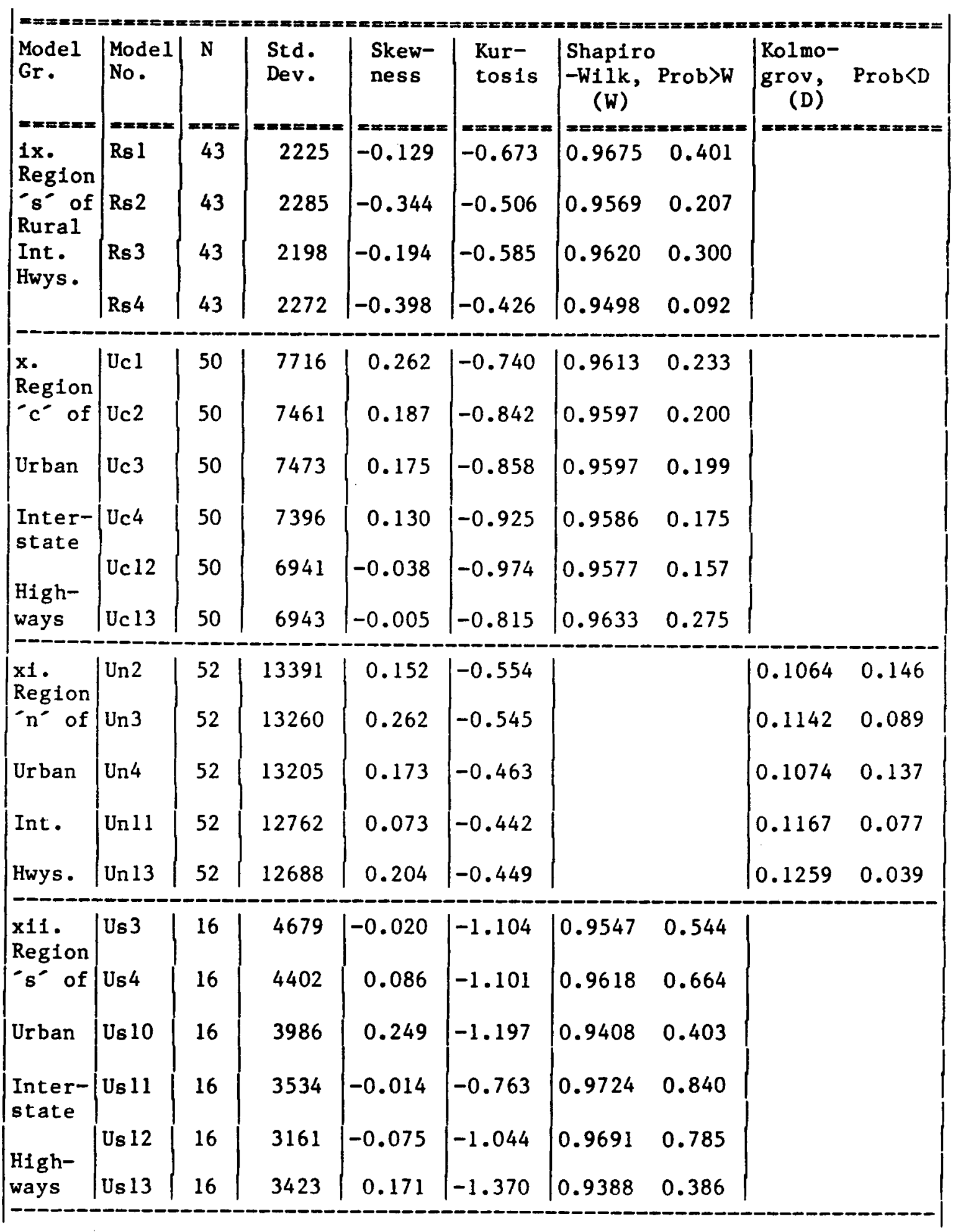


Table 6.9: Normality Statistics of Residuals in Post-Influence Analysis [Urban Principal Arterial]

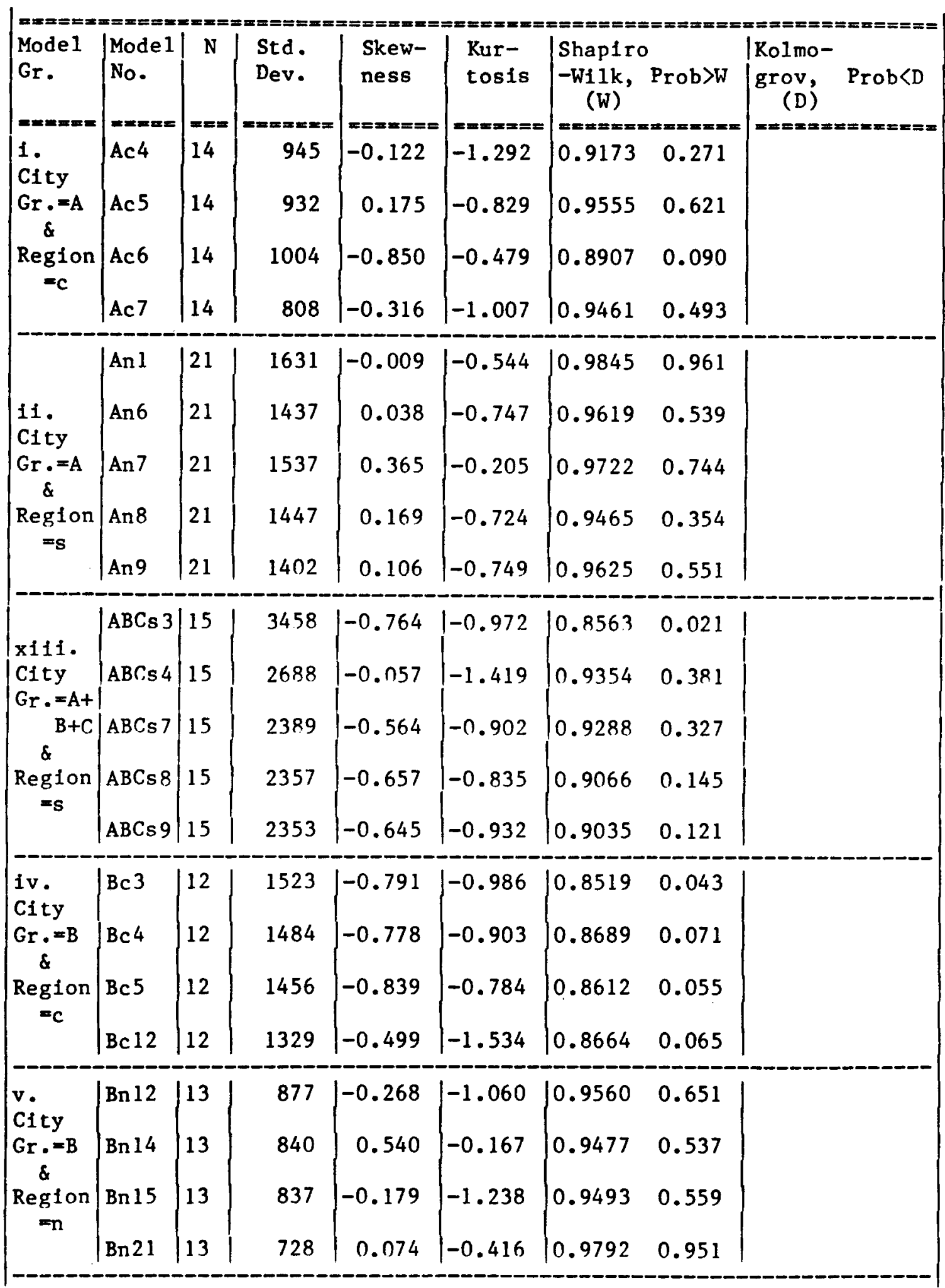


Table 6.9, continued

\begin{tabular}{|c|c|c|c|c|c|c|c|c|}
\hline $\begin{array}{l}\text { Model } \\
\text { Gr. } \\
\text { =m==== } \\
\text { vi1. } \\
\text { City } \\
\text { Gr.=C } \\
\& \\
\text { Region } \\
=c\end{array}$ & $\begin{array}{l}\text { Mode1 } \\
\text { No. } \\
==== \\
\text { Cc5 } \\
\text { Cc6 }\end{array}$ & $\mid \begin{array}{c}N \\
=-= \\
42\end{array}$ & 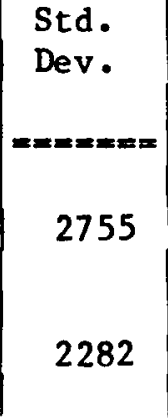 & $\mid \begin{array}{l}\text { Skew- } \\
\text { ness } \\
=m=m=m= \\
0.161 \\
-0.009\end{array}$ & $\left\{\begin{array}{l}\text { Kur- } \\
\text { tosis } \\
==\pi x=== \\
0.672 \\
-0.619\end{array}\right.$ & $\begin{array}{c}\text { Shapir } \\
\text {-Wi1k, } \\
(W) \\
0.9616 \\
0.9786\end{array}$ & $\begin{array}{c}x=x=== \\
0.303 \\
0.704\end{array}$ & $\left\{\begin{array}{l}\text { Kolmo- } \\
\text { grov, Prob }<D \\
\text { (D) } \\
=x==x==x=m=x\end{array}\right.$ \\
\hline $\begin{array}{l}\text { vili. } \\
\text { City } \\
\text { Gr.=C } \\
\& \\
\text { Region } \\
=n\end{array}$ & $\left\{\begin{array}{l}C_{n} 1 \\
\ln 5 \\
\ln 6\end{array}\right.$ & $\left\{\begin{array}{l}29 \\
29 \\
29\end{array}\right.$ & $\begin{array}{l}1860 \\
1697 \\
1641\end{array}$ & $\begin{array}{l}0.546 \\
0.422 \\
0.305 \\
0.309\end{array}$ & $\left\{\begin{array}{l}-0.365 \\
-0.003 \\
-0.057 \\
-0.647\end{array}\right.$ & $\left\{\begin{array}{l}0.9597 \\
0.9729 \\
0.9768 \\
0.9602\end{array}\right.$ & $\begin{array}{l}0.413 \\
0.672 \\
0.771 \\
0.421\end{array}$ & \\
\hline $\begin{array}{l}\text { X. } \\
\text { City } \\
\text { Gr }=D \\
\& \\
\text { Region } \\
=C\end{array}$ & $\left\{\begin{array}{l}D c 10 \\
D c 11 \\
D c 18 \\
D c 19\end{array}\right.$ & $\mid \begin{array}{l}25 \\
25 \\
25 \\
25\end{array}$ & $\begin{array}{l}876 \\
805 \\
774 \\
820\end{array}$ & $\begin{array}{r}0.055 \\
-0.181 \\
0.031 \\
0.445\end{array}$ & $\mid \begin{array}{l}-0.874 \\
-1.071 \\
-1.170 \\
-1.021\end{array}$ & $\mid \begin{array}{l}0.9752 \\
0.9549 \\
0.9426 \\
0.9212\end{array}$ & $\begin{array}{l}0.765 \\
0.390 \\
0.241 \\
0.062\end{array}$ & \\
\hline $\begin{array}{l}x i . \\
\text { City } \\
\text { Gr.=D } \\
\& \\
\text { Region } \\
=n\end{array}$ & $\mid \begin{array}{l}\text { Dn } 3 \\
\text { Dn4 } \\
\text { Dn } 15 \\
\text { Dn } 16\end{array}$ & $\mid \begin{array}{l}33 \\
33 \\
33 \\
33\end{array}$ & $\begin{array}{l}2169 \\
2141 \\
2107 \\
2129\end{array}$ & $\begin{array}{l}0.099 \\
0.137 \\
0.273 \\
0.226\end{array}$ & $\mid \begin{array}{l}-1.043 \\
-0.990 \\
-0.936 \\
-0.851\end{array}$ & $\mid \begin{array}{l}0.9402 \\
0.9371 \\
0.9269 \\
0.9509\end{array}$ & $\begin{array}{l}0.092 \\
0.078 \\
0.041 \\
0.237\end{array}$ & \\
\hline $\begin{array}{l}\text { xi1. } \\
\text { City } \\
\text { Gr.=D } \\
\& \\
\text { Region } \\
=s\end{array}$ & $\mid \begin{array}{l}\text { Ds } 3 \\
D s 4 \\
D s 12 \\
D s 13\end{array}$ & 39 & $\begin{array}{l}1273 \\
1185\end{array}$ & $\left\{\begin{array}{c}0.115 \\
0.223 \\
-0.036 \\
0.120\end{array}\right.$ & $\left\{\begin{array}{r}-0.407 \\
-0.205 \\
0.308 \\
0.270\end{array}\right.$ & $\mid \begin{array}{l}0.9749 \\
0.9825 \\
0.9922 \\
0.9896\end{array}$ & $\begin{array}{l}0.621 \\
0.853 \\
1.000 \\
0.990\end{array}$ & \\
\hline
\end{tabular}


Table 6.10: Normality Statistics of Residuals in Post-Influence Analysis [Urban Minor Arterial \& Collector]

\begin{tabular}{|c|c|c|c|c|c|c|c|c|}
\hline $\begin{array}{l}\text { Mode1 } \\
\text { Gr. } \\
==x==2\end{array}$ & $\left\{\begin{array}{l}\text { Mode } 1 \\
\text { No. } \\
==-==2\end{array}\right.$ & 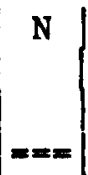 & $\begin{array}{l}\text { Std. } \\
\text { Dev. } \\
=====x==\end{array}$ & $\mid \begin{array}{l}\text { Skew- } \\
\text { ness } \\
\\
=x=x=m=\end{array}$ & \multirow[t]{2}{*}{$\mid \begin{array}{l}\mathrm{Kur}- \\
\text { tosis } \\
\\
=x==x=x\end{array}$} & \multicolumn{2}{|c|}{$\begin{array}{l}\text { Shapiro } \\
\text {-Wilk, Prob }>W \\
\text { (W) }\end{array}$} & \multirow[t]{5}{*}{$\mid \begin{array}{l}\text { Kolmo- } \\
\text { grov, Prob }<D \\
\text { (D) } \\
===x=m==x===x\end{array}$} \\
\hline & A2 & 16 & 1959 & 0.515 & & 0.9605 & 0.642 & \\
\hline $\begin{array}{l}\text { i. } \\
\text { City }\end{array}$ & A5 & 16 & 1543 & 0.020 & -0.959 & 0.9761 & 0.901 & \\
\hline $\mathrm{Gr} \cdot=\mathrm{A}$ & A6 & 16 & 1533 & -0.001 & -0.960 & 0.9777 & 0.917 & \\
\hline & A7 & 16 & 1551 & 0.095 & -0.766 & 0.9768 & 0.908 & \\
\hline $\begin{array}{l}\text { ii. } \\
\text { City } \\
G r=B\end{array}$ & B2 & 12 & 1905 & 0.218 & -1.412 & 0.9362 & 0.455 & \\
\hline \multirow{3}{*}{$\begin{array}{l}\text { iif. } \\
\text { City } \\
\text { Gr.=C }\end{array}$} & $\mathrm{C} 2$ & 13 & 625 & -0.285 & $\mid-1.157$ & 10.9383 & 0.452 & \\
\hline & C3 & 13 & 598 & -0.111 & -1.073 & 0.9601 & 0.709 & \\
\hline & 104 & 13 & 555 & -0.056 & -0.810 & 0.9583 & 0.684 & \\
\hline \multirow{4}{*}{$\begin{array}{l}\text { iv. } \\
\text { City } \\
\text { Gr.=C }\end{array}$} & $\mathrm{Cg}$ & 11 & 1203 & -0.052 & -1.762 & 0.9034 & 0.271 & \\
\hline & 1010 & 11 & 1160 & -0.183 & 0.052 & 0.9739 & 0.907 & \\
\hline & C11 & 11 & 1123 & 0.304 & -0.049 & 0.9533 & 0.661 & \\
\hline & $\mathrm{C} 12$ & 11 & 1117 & -0.198 & 0.338 & 0.9741 & 0.909 & \\
\hline $\begin{array}{l}\text { vity } \\
\text { Gr.=D }\end{array}$ & D6 & 25 & 1649 & 0.614 & -0.703 & 0.9132 & 0.041 & \\
\hline $\begin{array}{l}\text { vi. } \\
\text { City } \\
\text { Gr. }=D\end{array}$ & D7 & 23 & 1018 & -0.019 & -0.659 & 0.9713 & 0.696 & \\
\hline
\end{tabular}


LEGEND: $A=1$ OBS, $B=2$ OBS, ETC.

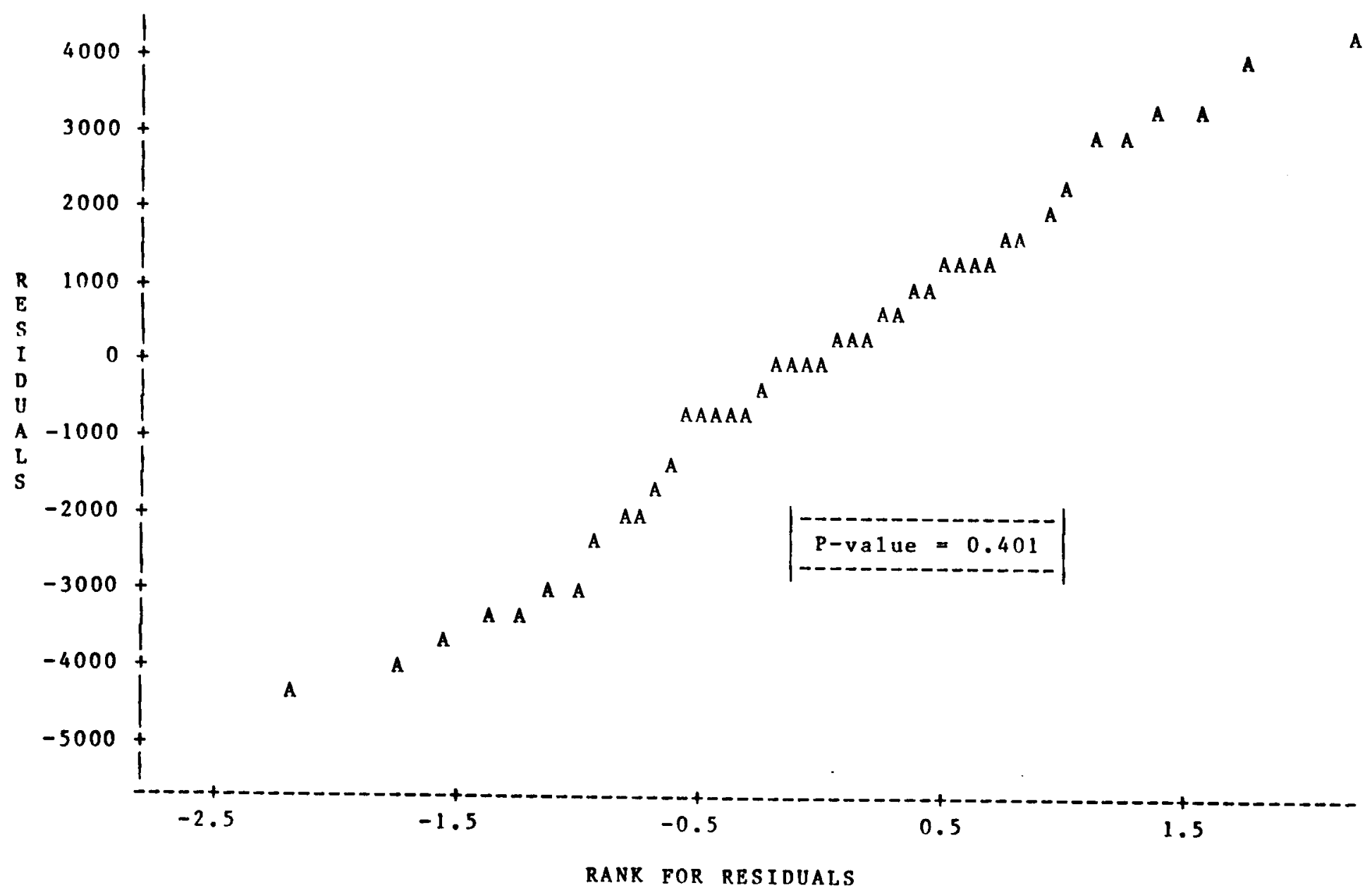

Figure 6.27: Normal Probability Plot of Model Rs 1

[Region "s" of Rural Interstate Highways] 
LEGEND: $A=1$ OBS, B $=2$ OBS, ETC.

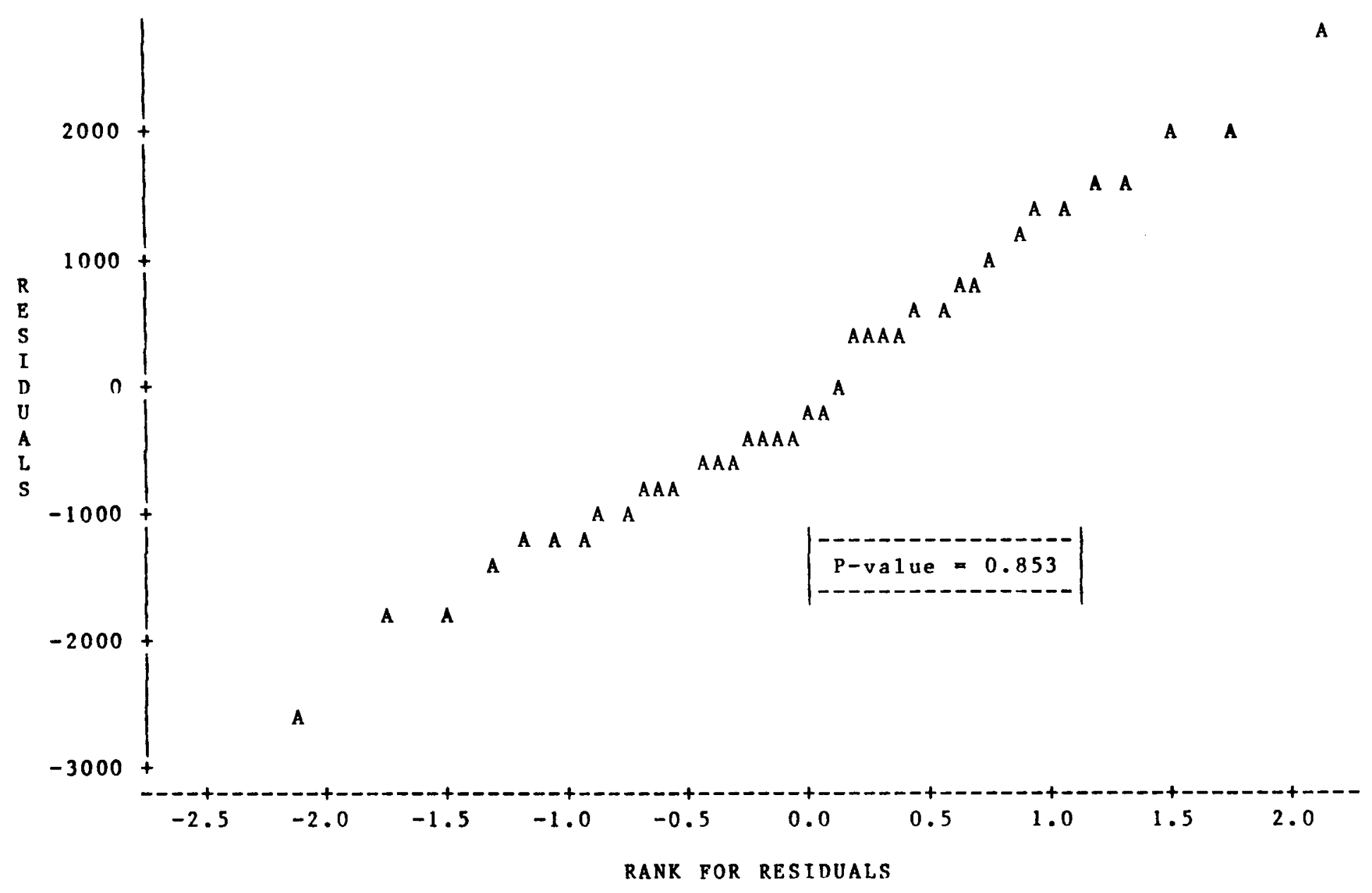

Figure 6.28: Normal Probability Plot of Model Ds4

[City Group "D" \& Region "s" of Urban Principal Arterial] 
LEGEND: $A=1$ OBS, $B=2$ OBS, ETC.

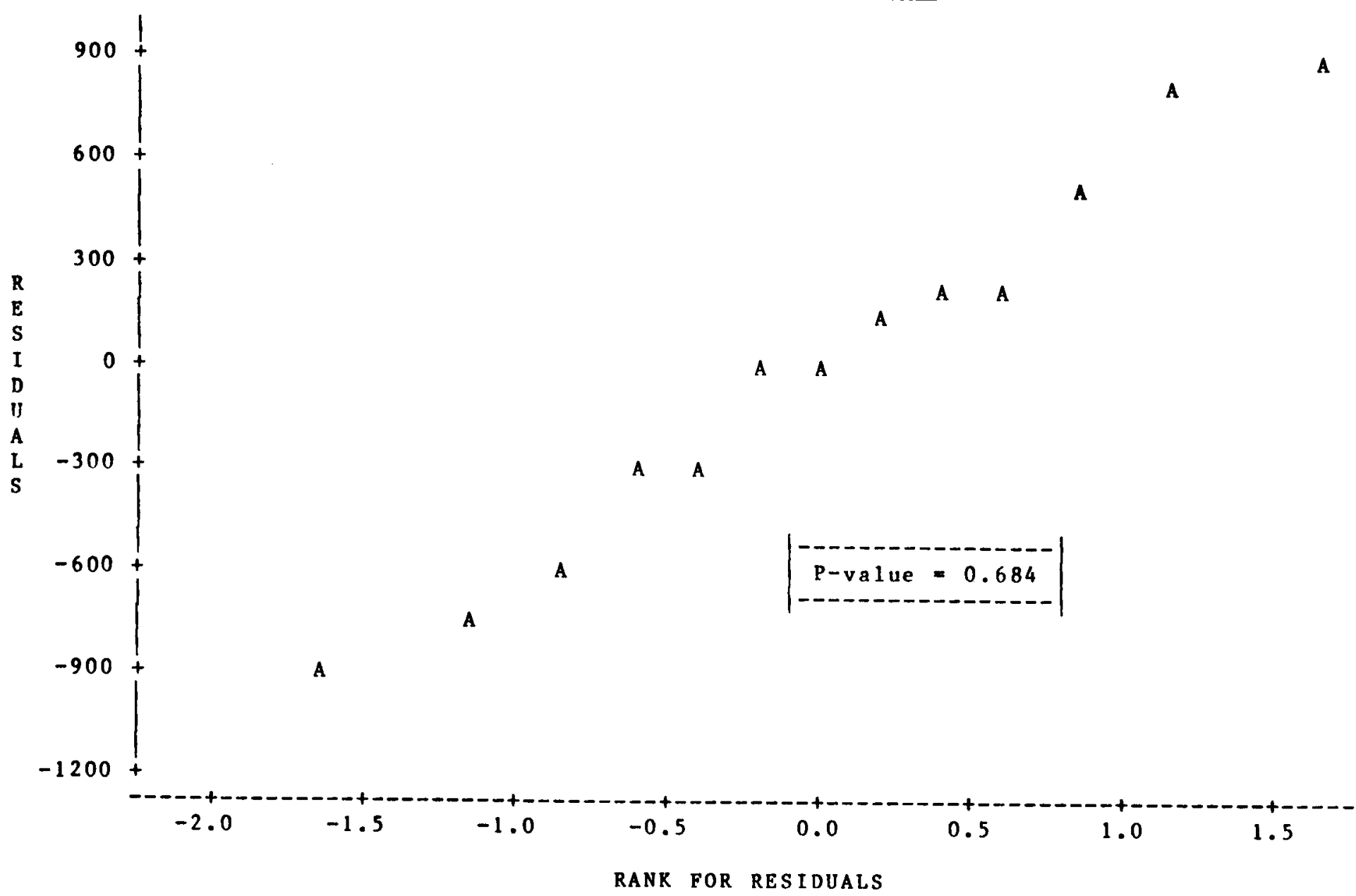

Figure 6.29: Normal Probabllity Plot of Model C4

[City Group "C" of Urban Minor Artertal \& Collector] 
close to straight lines, suggesting that the error terms are approximately normally distributed. A slight departure is noticed for the normal probability plot of some cases. It is belleved [Neter et al. 1985] that this small departure from normality will not create any serious problems.

\subsection{SIgnificance and Collinearity of Regression Coefficients}

During the process of selecting the possible candidate models in Chapter 5, the models with consistency in sign of their regression coefficlents were chosen. The correspondence between the regression coefficients and collinearity problem does not guarantee that our selections are free of collinearity problem. In subsection 6.4.1, some formal tests of collinearity are performed on the selected candidate models.

The test for significance of the parameters in the model and overall significance test for regression relation are carried out in Subsection 6.4.2. The presence of multicollinearity affects the significance of these tests. But the absence of multicollinearity does not guarantee that all the variables in the model are significant.

\subsubsection{Collinearity Diagnostics of Regression Coefficlents}

When a regressor is a nearly linear combination of other regressors In the model, the affected estimates are unstable and have high standard errors. Examining the correlation matrix of the explanatory variables is a commonly employed procedure since this matrix is a 
standard output of most regression packages. A high correlation coefficient between the two explanatory variates can indeed point to a possible collinearity problem. The diagonal elements of the inverse of the correlation matrix are called the Variance Inflation Factors (VIF). The diagnostic value of VIF of the $k$-th independent variable $\left(X_{k}\right)$ is calculated as follows:

$$
\mathrm{VIF}_{k}=\frac{1}{1-\mathrm{R}_{\mathrm{k}}^{2}}
$$

where, $R_{k}^{2}$ is the multiple correlation coefficient of $X_{k}$ regressed on the remaining explanatory variables. Thus, VIF $\mathrm{k}_{\mathrm{k}}$ is equal to 1 when $R_{k}^{2}=0$, 1.e., when $X_{k}$ is not linearly related to the other $X$ variables. on the other hand, a high VIF indicates an $R_{k}^{2}$ near unity, and hence points to collinearity. A maximum $V_{k} F_{k}$ above 10 is often taken as an indication that multicollinearity may be unduly influencing the least squares estimates.

A limitation of the correlation matrix and VIF for detecting multicollinearities is that they cannot distinguish between several simultaneous multicollinearities. It is clearly possible for three or more variates to be collinear while no two of the variates taken alone are highly correlated. The correlation matrix and the VIF are wholly incapable of diagnosing such a situation. Condition numbers/indices and the variance-decomposition proportion [Belsley, Kuh and Welsch 1980] have been used to Identify this collinearity problem. The condition numbers are defined as the square roots of the ratio of the largest eigenvalue to each individual eigenvalue. When this number is 
large, the problem is said to be 111 -conditioned. Weak dependencies are associated with condition indexes around 5 or 10 , whereas moderate to strong relations are associated with condition indices of 30 to 100. A high condition index causes substantial varlance inflation and great potential harm to the regression estimates. For each predictor variable, the SAS regression procedure prints the proportion of the variance of the estimate accounted for by each principal component. The diagnostic procedure: A collinearity problem occurs when a component associated with a high condition index contributes strongly to the variance of two or more variables. Thus high condition index (greater than 30) and the large variance-decomposition proportions (say, greater than .60) associated with each high condition index identifies the involving variates have the problem of multicollinearity. [Belsley, Kuh and Welsch 1980; SAS/Statistics 1985]

The above interpretation of VIF, condition number and variancedecomposition presumes at least two variates in the equation. The results of these collinearity statistics in pre- and post-influence analyses of models with at least 2 variables are shown in Tables 6.11-6.13 and 6.14-6.16, respectively. In general, there is no problem of collinearity statistics for most of the candidate models. The results of the collinearity diagnostics are useful for comparative evaluation of models within a selected group and then for selecting the best model. 
Table 6.11: Collinearity Statistics for $>2$ variables in the Model in Pre-Influence Analysis [Interstate Highways]

\begin{tabular}{|c|c|c|c|c|c|c|c|c|}
\hline \multirow{3}{*}{$\begin{array}{l}\text { Mode1 } \\
\text { Gr. } \\
======\end{array}$} & \multirow{3}{*}{$\begin{array}{l}\text { Mode 1 } \\
\text { No. }\end{array}$} & \multirow{2}{*}{\multicolumn{2}{|c|}{$\mid \begin{array}{l}\text { Varlance Influ- } \\
\text { ence Factor (VIF) }\end{array}$}} & \multirow{3}{*}{$\begin{array}{l}\text { Num- } \\
\text { ber } \\
== \pm=x\end{array}$} & \multirow{3}{*}{$\begin{array}{l}\text { Cond1- } \\
\text { tion } \\
\text { Index } \\
== \pm== \pm=\end{array}$} & \multicolumn{3}{|c|}{ Variance Proportion by } \\
\hline & & & & & & $\operatorname{ar} 1$ & $\operatorname{lar} 2$ & $\operatorname{ar} 3$ \\
\hline & & $\operatorname{Var} \cdot(*)$ & Value & & & $(*)$ & $(*)$ & (*) \\
\hline & UR 12 & CNTYPOP & 1.0007 & 1 & 1.000 & 0.4869 & 0.4869 & \\
\hline $\begin{array}{l}\text { Inter- } \\
\text { state }\end{array}$ & & STINC & 1.0007 & 2 & 1.026 & 0.5131 & 0.5131 & \\
\hline $\begin{array}{l}\text { High- } \\
\text { ways }\end{array}$ & |UR 13 & $\begin{array}{l}\text { CNTYPOP } \\
\text { STEMP }\end{array}$ & $\begin{array}{l}1.0003 \\
1.0003\end{array}$ & $\begin{array}{l}1 \\
2\end{array}$ & $\begin{array}{l}1.000 \\
1.016\end{array}$ & $\mid \begin{array}{l}0.4919 \\
0.5081\end{array}$ & $\mid \begin{array}{l}0.4919 \\
0.5081\end{array}$ & \\
\hline & |R3 & $\begin{array}{l}\text { CNTYINC } \\
\text { CNTYEMP }\end{array}$ & $\begin{array}{l}1.0959 \\
1.0959\end{array}$ & $\begin{array}{l}1 \\
2\end{array}$ & $\begin{array}{l}1.000 \\
1.357\end{array}$ & $\mid \begin{array}{l}0.3521 \\
0.6479\end{array}$ & $\begin{array}{l}0.3521 \\
0.6479\end{array}$ & \\
\hline $\begin{array}{l}\text { ii. } \\
\text { Rural }\end{array}$ & R4 & $\begin{array}{l}\text { CNTYINC } \\
\text { CNTYPOP }\end{array}$ & $\begin{array}{l}1.1058 \\
1.1058\end{array}$ & $\begin{array}{l}1 \\
2\end{array}$ & $\begin{array}{l}1.000 \\
1.377\end{array}$ & $\begin{array}{l}0.3453 \\
0.6547\end{array}$ & $\mid \begin{array}{l}0.3453 \\
0.6547\end{array}$ & \\
\hline $\begin{array}{l}\text { Inter- } \\
\text { state }\end{array}$ & |R6 & $\begin{array}{l}\text { CNTYINC } \\
\text { CNTYVR }\end{array}$ & $\begin{array}{l}1.1303 \\
1.1303\end{array}$ & $\begin{array}{l}1 \\
2\end{array}$ & $\begin{array}{l}1.000 \\
1.424\end{array}$ & $\begin{array}{l}0.3302 \\
0.6698\end{array}$ & $\begin{array}{l}0.3302 \\
0.6698\end{array}$ & \\
\hline $\begin{array}{l}\text { High- } \\
\text { ways }\end{array}$ & R16 & $\begin{array}{l}\text { CNTYINC } \\
\text { CNTYVR } \\
\text { CTYPOP }\end{array}$ & $\begin{array}{l}1.2652 \\
1.1790 \\
1.2386\end{array}$ & $\begin{array}{l}1 \\
2 \\
3\end{array}$ & $\begin{array}{l}1.000 \\
1.557 \\
1.684\end{array}$ & $\mid \begin{array}{l}0.1664 \\
0.0771 \\
0.7564\end{array}$ & $\mid \begin{array}{l}0.1506 \\
0.8176 \\
0.0318\end{array}$ & $\mid \begin{array}{l}0.1618 \\
0.2945 \\
0.5437\end{array}$ \\
\hline $\begin{array}{l}111 . \\
\text { Urban }\end{array}$ & U9 & $\begin{array}{l}\text { | CNTYPOP } \\
\text { |STVR }\end{array}$ & $\begin{array}{l}1.0109 \\
1.0109\end{array}$ & $\begin{array}{l}1 \\
2\end{array}$ & $\begin{array}{l}1.000 \\
1.110\end{array}$ & $\mid \begin{array}{l}0.4481 \\
0.5519\end{array}$ & $\mid \begin{array}{l}0.4481 \\
0.5519\end{array}$ & \\
\hline $\begin{array}{l}\text { Int. } \\
\text { Hwys. }\end{array}$ & U11 & $\begin{array}{l}\text { CNTYPOP } \\
\text { STEMP }\end{array}$ & $\begin{array}{l}1.0002 \\
1.0002\end{array}$ & $\begin{array}{l}1 \\
2\end{array}$ & $\begin{array}{l}1.000 \\
1.015\end{array}$ & $\mid \begin{array}{l}0.4925 \\
0.5075\end{array}$ & $\mid \begin{array}{l}0.4925 \\
0.5075\end{array}$ & \\
\hline & URc 14 & $\begin{array}{l}\text { CNTYVR } \\
\text { CNTYINC }\end{array}$ & $\begin{array}{l}1.1492 \\
1.1492\end{array}$ & $\begin{array}{l}1 \\
2\end{array}$ & $\begin{array}{l}1.000 \\
1.458\end{array}$ & $\mid \begin{array}{l}0.3199 \\
0.6801\end{array}$ & $\mid \begin{array}{l}0.3199 \\
0.6801\end{array}$ & \\
\hline $\mid \begin{array}{l}\text { Iv. } \\
\text { Region } \\
{ }^{-} c^{-} \text {of }\end{array}$ & |URc 15 & $\begin{array}{l}\text { CNTYHH } \\
\text { CNTYINC }\end{array}$ & $\begin{array}{l}1.1357 \\
1.1357\end{array}$ & $\begin{array}{l}1 \\
2\end{array}$ & $\begin{array}{l}1.000 \\
1.434\end{array}$ & $\begin{array}{l}0.3371 \\
0.6729\end{array}$ & $\begin{array}{l}0.3371 \\
0.6729\end{array}$ & \\
\hline $\begin{array}{l}\text { Inter- } \\
\text { state }\end{array}$ & URc 24 & $\begin{array}{l}\text { CNTYVR } \\
\text { CNTYINC } \\
\text { STEMP }\end{array}$ & $\begin{array}{l}1.1610 \\
1.3991 \\
1.2225\end{array}$ & $\begin{array}{l}1 \\
2 \\
3\end{array}$ & $\begin{array}{l}1.000 \\
1.301 \\
1.816\end{array}$ & $\begin{array}{l}0.1272 \\
0.5280 \\
0.3448\end{array}$ & $\begin{array}{l}0.2126 \\
0.0002 \\
0.7872\end{array}$ & $\begin{array}{l}0.1524 \\
0.3719 \\
0.4756\end{array}$ \\
\hline $\begin{array}{l}\text { High- } \\
\text { ways }\end{array}$ & |URc25 & \begin{tabular}{|l} 
CNTYHH \\
CNTYINC \\
STEMP
\end{tabular} & $\begin{array}{l}1.1481 \\
1.3849 \\
1.2232\end{array}$ & $\begin{array}{l}1 \\
2 \\
3\end{array}$ & $\begin{array}{l}1.000 \\
1.289 \\
1.799\end{array}$ & $\begin{array}{l}0.1236 \\
0.5472 \\
0.3292\end{array}$ & $\begin{array}{l}0.2184 \\
0.0003 \\
0.7813\end{array}$ & $\begin{array}{l}0.1576 \\
0.3508 \\
0.4912\end{array}$ \\
\hline
\end{tabular}


Table 6.11 , continued

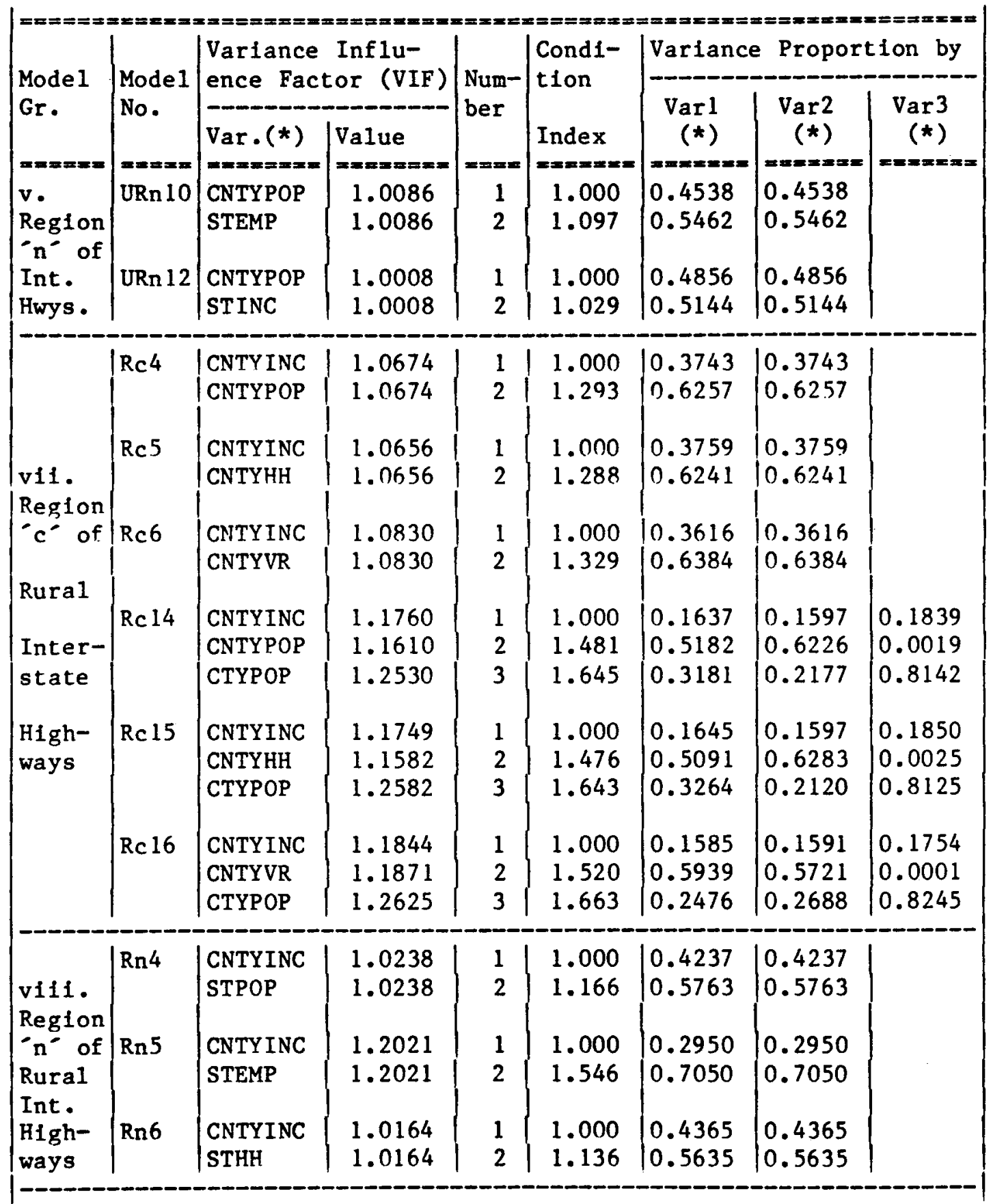


Table 6.11 , continued

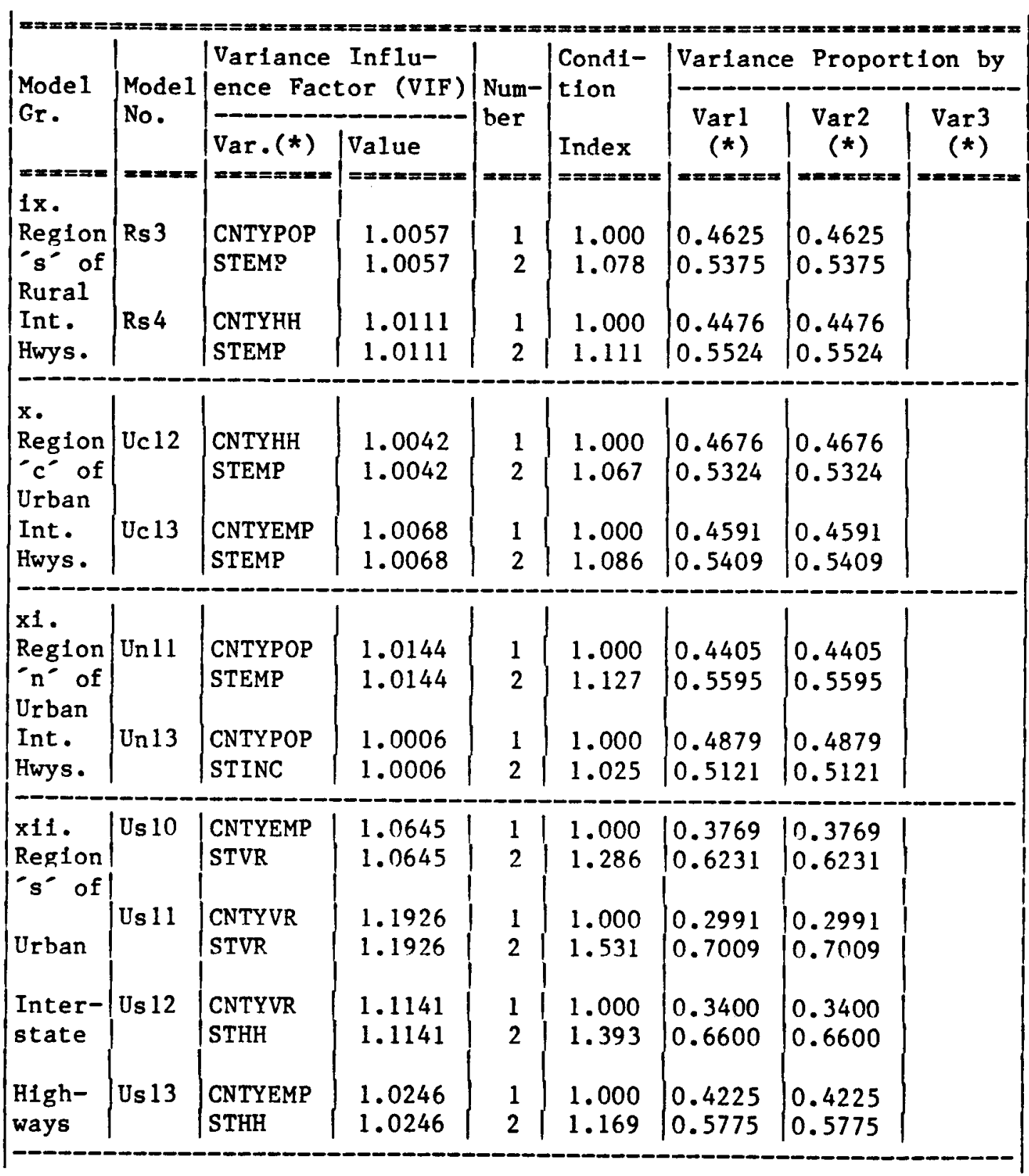

(*) Var1, Var2 and Var3 represent the variables in the order that shown under column 3 (Var.) for each model. 
Table 6.12: Collinearity Statistics for $>2$ variables in the Model in Pre-Influence Analysis [Urban Princlpal Arterial]

\begin{tabular}{|c|c|c|c|c|c|c|c|c|}
\hline \multirow{3}{*}{$\begin{array}{l}\text { Model } \\
\text { Gr. } \\
\text { m=r=m }\end{array}$} & \multirow{3}{*}{$\begin{array}{l}\text { Mode 1 } \\
\text { No. }\end{array}$} & \multirow{2}{*}{\multicolumn{2}{|c|}{$\begin{array}{l}\text { Variance Influ- } \\
\text { ence Factor (VIF) }\end{array}$}} & \multirow{3}{*}{$\begin{array}{l}\text { Num- } \\
\text { ber }\end{array}$} & \multirow{3}{*}{$\left|\begin{array}{l}\text { Condi- } \\
\text { tion } \\
\text { Index } \\
=x= \pm=x=x\end{array}\right|$} & \multicolumn{3}{|c|}{ Variance Proportion by } \\
\hline & & & & & & $\operatorname{Var} 1$ & $\operatorname{Var} 2$ & $\operatorname{Var} 3$ \\
\hline & & Var.(*) & Value & & & $(*)$ & $(*)$ & $(*)$ \\
\hline \multirow{8}{*}{$\begin{array}{l}\text { 1. } \\
\text { City } \\
\text { Gr. }=\mathrm{A} \\
\quad \& \\
\text { Region } \\
\quad=\mathrm{C}\end{array}$} & $==2=x$ & 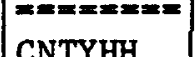 & $\begin{array}{c}3==x=2=0 \\
1,2537\end{array}$ & $==2$ & $\begin{array}{c}=x=x=x= \\
100 n\end{array}$ & $\begin{array}{l}=2=2 x=1 \\
0.2751\end{array}$ & $\begin{array}{l}=12=31 \\
0.2751\end{array}$ & \multirow{8}{*}{$=2 x=2 x=0$} \\
\hline & & CNTYINC & 1.2537 & 2 & 1.623 & 0.7249 & 0.7249 & \\
\hline & Ac5 & CNTYVR & 1.0330 & 1 & 1.000 & 0.4107 & 0.4107 & \\
\hline & & CNTYINC & 1.0330 & 2 & 1.197 & 0.5893 & 0.5893 & \\
\hline & Ac6 & CNTYEMP & 1.0010 & 1 & 1.000 & 0.4859 & 0.4859 & \\
\hline & & CNTYINC & 1.0010 & 2 & 1.029 & 0.5141 & 0.5141 & \\
\hline & \multirow[t]{2}{*}{ Ac7 } & CNTYPOP & 1.3710 & 1 & 1.000 & 0.2399 & 0.2399 & \\
\hline & & CNTYINC & 1.3710 & 2 & 1.780 & 0.7601 & $10.76 n 1$ & \\
\hline \multirow{8}{*}{$\begin{array}{l}11 . \\
\text { City } \\
\text { Gr.=A } \\
\& \\
\begin{array}{c}\text { Region } \\
=s\end{array}\end{array}$} & \multirow[t]{2}{*}{ An6 } & |CTYPOP & 1.1159 & 1 & 1.000 & 0.3388 & 10.3388 & \\
\hline & & STVR & 1.1159 & 2 & 1.397 & 0.6612 & 0.6612 & \\
\hline & \multirow[t]{2}{*}{ An 7} & |CTYPOP & 1.0239 & 1 & 1.000 & 0.4236 & 0.4236 & \\
\hline & & STEMP & 1.0239 & 2 & 1.167 & 0.5764 & 0.5764 & \\
\hline & \multirow[t]{2}{*}{ An 8} & CTYPOP & 1.2080 & 1 & 1.000 & \multirow{2}{*}{$\begin{array}{l}0.3359 \\
0.6641\end{array}$} & \multirow{2}{*}{$\begin{array}{l}0.3359 \\
0.6641\end{array}$} & \\
\hline & & STPOP & 1.2080 & 2 & 1.406 & & & \\
\hline & \multirow[t]{2}{*}{ An 9} & СTYPOP & 1.1392 & 1 & 1.000 & 0.3252 & 0.3252 & \\
\hline & & STHH & 1.1392 & 2 & 1.440 & 0.6748 & 0.6748 & \\
\hline \multirow{6}{*}{$\begin{array}{l}\text { xili. } \\
\text { City } \\
\text { Gr.=A+ } \\
B+C \\
\& \\
\text { Region } \\
=8\end{array}$} & \multirow[t]{2}{*}{$|\mathrm{ABCs} 7|$} & | CNTYEMP & 1.0002 & 1 & 1.000 & 0.4932 & \multirow{2}{*}{$\mid \begin{array}{l}0.4932 \\
0.5068\end{array}$} & \\
\hline & & STVR & 1.0002 & 2 & 1.014 & 0.5068 & & \\
\hline & \multirow[t]{2}{*}{$A B C s 8$} & CNTYEMP & 1.0017 & 1 & 1.000 & 0.4797 & \multirow{2}{*}{$\begin{array}{l}0.4797 \\
0.5203\end{array}$} & \\
\hline & & STHH & 1.0017 & 2 & 1.042 & 0.5203 & & \\
\hline & \multirow[t]{2}{*}{$A B C s 9$} & CNTYEMP & 1.0024 & 1 & 1.000 & 0.4755 & \multirow{2}{*}{$\begin{array}{l}0.4755 \\
0.5245\end{array}$} & \\
\hline & & |STPOP & 1.0024 & 2 & 1.050 & 10.5245 & & \\
\hline
\end{tabular}


Table 6.12, continued

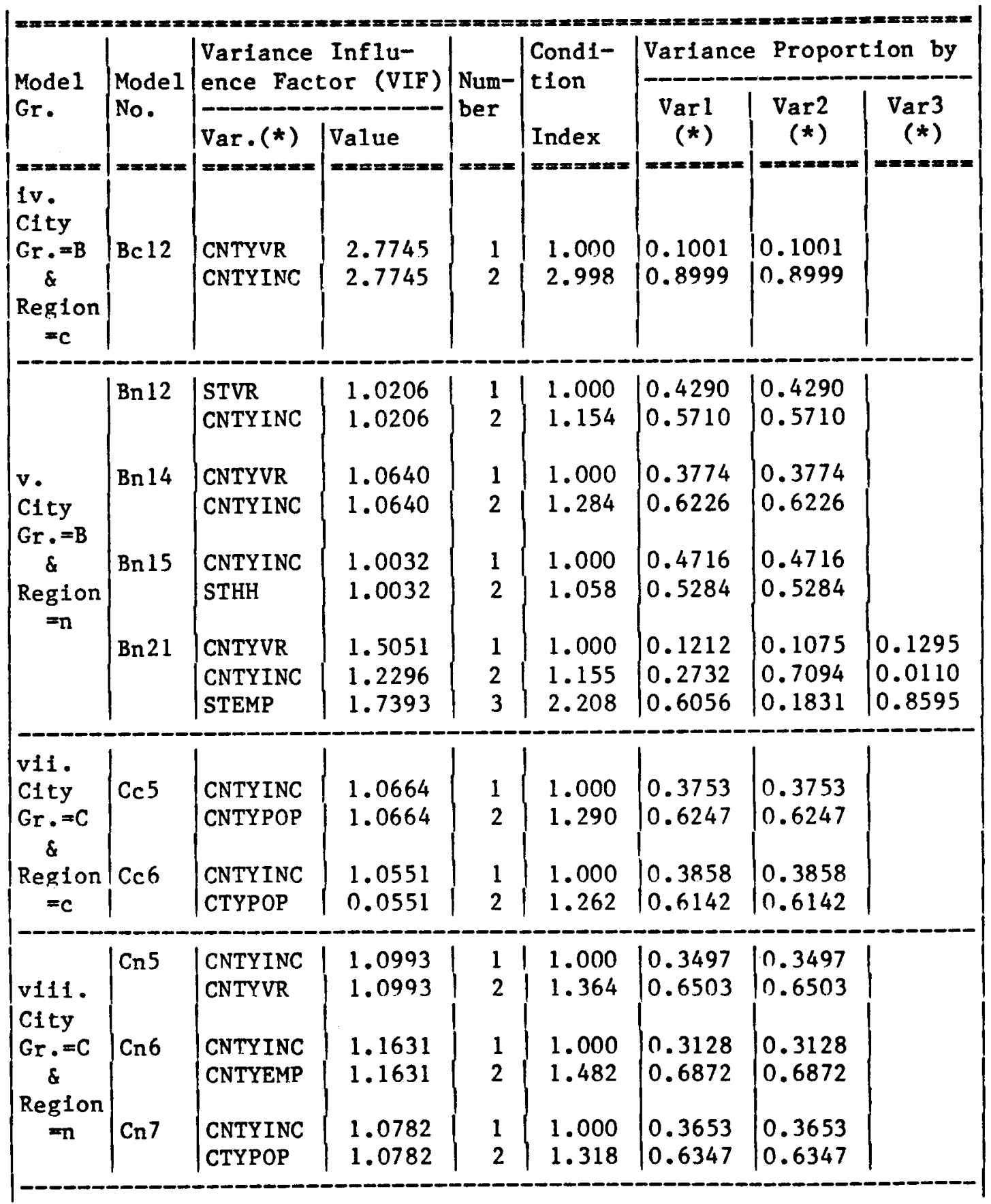


Table 6.12 , continued

\begin{tabular}{|c|c|c|c|c|c|c|c|c|}
\hline \multirow{4}{*}{$\begin{array}{l}\text { Model } \\
\text { Gr. } \\
== \pm=m=\end{array}$} & \multirow{3}{*}{$\begin{array}{l}\text { Mode1 } \\
\text { No. }\end{array}$} & \multirow{2}{*}{\multicolumn{2}{|c|}{$\begin{array}{l}\text { Variance Influ- } \\
\text { ence Factor (VIF) }\end{array}$}} & \multirow{4}{*}{$\begin{array}{l}\text { Num- } \\
\text { ber }\end{array}$} & \multirow{4}{*}{$\begin{array}{l}\text { Condi- } \\
\text { tion } \\
\text { Index } \\
=x=m=x=\end{array}$} & \multicolumn{3}{|c|}{ Variance Proportion by } \\
\hline & & & & & & $\mathbf{V}$ & Var2 & $\operatorname{lar} 3$ \\
\hline & & $\operatorname{Var} \cdot(*)$ & Value & & & (*) & (*) & (*) \\
\hline & $\begin{array}{l}n=x=0 \\
0=10\end{array}$ & $==2=x=2=$ & $=0=2$ & & & $==2 x=\pi$ & $=m=2==$ & \\
\hline \multirow{8}{*}{$\begin{array}{l}\text { x. } \\
\text { City } \\
\text { Gr }=D \\
\& \\
\text { Region } \\
=c\end{array}$} & Dc 10 & $\begin{array}{l}\text { CTYPOP } \\
\text { STVR }\end{array}$ & $\begin{array}{l}1.0137 \\
1.0137\end{array}$ & $\begin{array}{l}1 \\
2\end{array}$ & $\begin{array}{l}1.000 \\
1.124\end{array}$ & $\begin{array}{l}0.4418 \\
0.5582\end{array}$ & $\begin{array}{l}0.4418 \\
0.5582\end{array}$ & \\
\hline & De 11 & CTYPOP & 1.0131 & 1 & 1.000 & 0.4431 & 0.4431 & \\
\hline & & SIEMP & 31 & & 1.121 & y & & \\
\hline & Dc 18 & $\begin{array}{l}\text { CTYPOP } \\
\text { STEMP }\end{array}$ & $\begin{array}{l}1.5233 \\
1.2247\end{array}$ & $\begin{array}{l}1 \\
2\end{array}$ & $\begin{array}{l}1.000 \\
1.403\end{array}$ & $\begin{array}{l}0.1260 \\
0.2378\end{array}$ & $\begin{array}{l}0.0940 \\
0.6156\end{array}$ & $\begin{array}{l}0.1460 \\
0.0020\end{array}$ \\
\hline & & CNTYINC & 1.7966 & 3 & 2.240 & 0.6362 & 0.2904 & 0.8520 \\
\hline & Dc 19 & CTYPOP & 1.4983 & 1 & 1.000 & 0.1672 & 0.0618 & 0.1821 \\
\hline & & STHH & 1.0735 & 2 & 1.318 & 0.1064 & 0.8265 & 0.0040 \\
\hline & & |CNTYINC & 876 & 3 & 2.031 & 265 & 117 & 139 \\
\hline \multirow{5}{*}{$\begin{array}{l}x i . \\
\text { City } \\
\text { Gr.=D } \\
\& \\
\begin{array}{c}\text { Region } \\
=n\end{array}\end{array}$} & & & & & & & & \\
\hline & Dn 15 & CNTYVR. & 1.0460 & 1 & 1.000 & 0.3952 & & \\
\hline & & STHH & 460 & 2 & 1.237 & 0.6048 & & \\
\hline & $\operatorname{Dn} 16$ & CNTYHH & 1.6755 & 1 & 1.000 & 0.1825 & 825 & \\
\hline & & | CNTYINC & 755 & 2 & 2.116 & 0.8175 & 10 & \\
\hline \multirow{5}{*}{$\begin{array}{l}\text { xil. } \\
\text { City } \\
\text { Gr.=D } \\
\quad \& \\
\text { Region } \\
\quad=s\end{array}$} & & & & & & & & \\
\hline & Ds 12 & STHH & 1.5069 & 1 & 1.000 & 0.2100 & 0.2100 & \\
\hline & & STINC & 1.5069 & 2 & 1.940 & 0.7900 & 0.7900 & \\
\hline & Ds 13 & STPOP & 1.7158 & 1 & 1.000 & 0.1770 & 0.1770 & \\
\hline & & STINC & 1.7158 & 2 & 2.156 & 0.8230 & 0.8230 & \\
\hline
\end{tabular}

(*) Var1, Var2 and Var3 represent the variables in the order that shown under column 3 (Var.) for each model. 
Table 6.13: Collinearity Statistics for $\geqslant 2$ variables in the Model in Pre-Influence Analysis [Urban Minor Arterial \& Collector

\begin{tabular}{|c|c|c|c|c|c|c|c|}
\hline \multirow{3}{*}{$\begin{array}{l}\text { Model } \\
\text { Gr. } \\
===x=x\end{array}$} & \multirow{3}{*}{$\begin{array}{l}\text { Mode1 } \\
\text { No. }\end{array}$} & \multirow{2}{*}{\multicolumn{2}{|c|}{$\begin{array}{l}\text { Variance Influ- } \\
\text { ence Factor (VIF) }\end{array}$}} & \multirow{3}{*}{$\begin{array}{l}\text { Num- } \\
\text { ber }\end{array}$} & \multirow{3}{*}{$\begin{array}{l}\text { Condi- } \\
t \text { Ion } \\
\text { Index } \\
==m=x=m\end{array}$} & \multicolumn{2}{|c|}{ Var1. Prop. by } \\
\hline & & & & & & Varl & $\operatorname{Var} 2$ \\
\hline & & Var.(*) & Value & & & $(\star)$ & (*) \\
\hline & A5 & $\begin{array}{l}\text { CNTYINC } \\
\text { CNTYPOP }\end{array}$ & $\begin{array}{l}1.0968 \\
1.0968\end{array}$ & $\begin{array}{l}1 \\
2\end{array}$ & $\begin{array}{l}1.000 \\
1.358\end{array}$ & $\begin{array}{l}0.3515 \\
0.6485\end{array}$ & $\begin{array}{l}0.3515 \\
0.6485\end{array}$ \\
\hline $\begin{array}{l}\text { i. } \\
\text { City } \\
\text { Gr.=A }\end{array}$ & A6 & $\begin{array}{l}\text { CNTYINC } \\
\text { CNTYVR }\end{array}$ & $\begin{array}{l}1.1488 \\
1.1488\end{array}$ & $\begin{array}{l}1 \\
2\end{array}$ & $\begin{array}{l}1.000 \\
1.458\end{array}$ & $\mid \begin{array}{l}0.3201 \\
0.6799\end{array}$ & $\begin{array}{l}0.3201 \\
0.6799\end{array}$ \\
\hline & A7 & $\begin{array}{l}\text { CNTYINC } \\
\text { CNTYEMP }\end{array}$ & $\begin{array}{l}1.2963 \\
1.2963\end{array}$ & $\begin{array}{l}1 \\
2\end{array}$ & $\begin{array}{l}1.000 \\
1.683\end{array}$ & $\begin{array}{l}0.2609 \\
0.7391\end{array}$ & $\mid \begin{array}{l}0.2609 \\
0.7391\end{array}$ \\
\hline $\begin{array}{l}\text { v. } \\
\text { City } \\
\text { Gr.=D }\end{array}$ & D6 & \begin{tabular}{|l} 
CNTYINC \\
CTYPOP
\end{tabular} & $\begin{array}{l}1.0845 \\
1.0845\end{array}$ & $\begin{array}{l}1 \\
2\end{array}$ & $\begin{array}{l}1.000 \\
1.332\end{array}$ & $\begin{array}{l}0.3604 \\
0.6396\end{array}$ & $\mid \begin{array}{l}0.3604 \\
0.6396\end{array}$ \\
\hline
\end{tabular}

(*) Varl and Var2 represent the variables in the order that shown under column 3 (Var.) for each model. 
Table 6.14: Collinearity Statistics for $>2$ variables in the Model in Post-Influence Analysis [Interstate Highways]

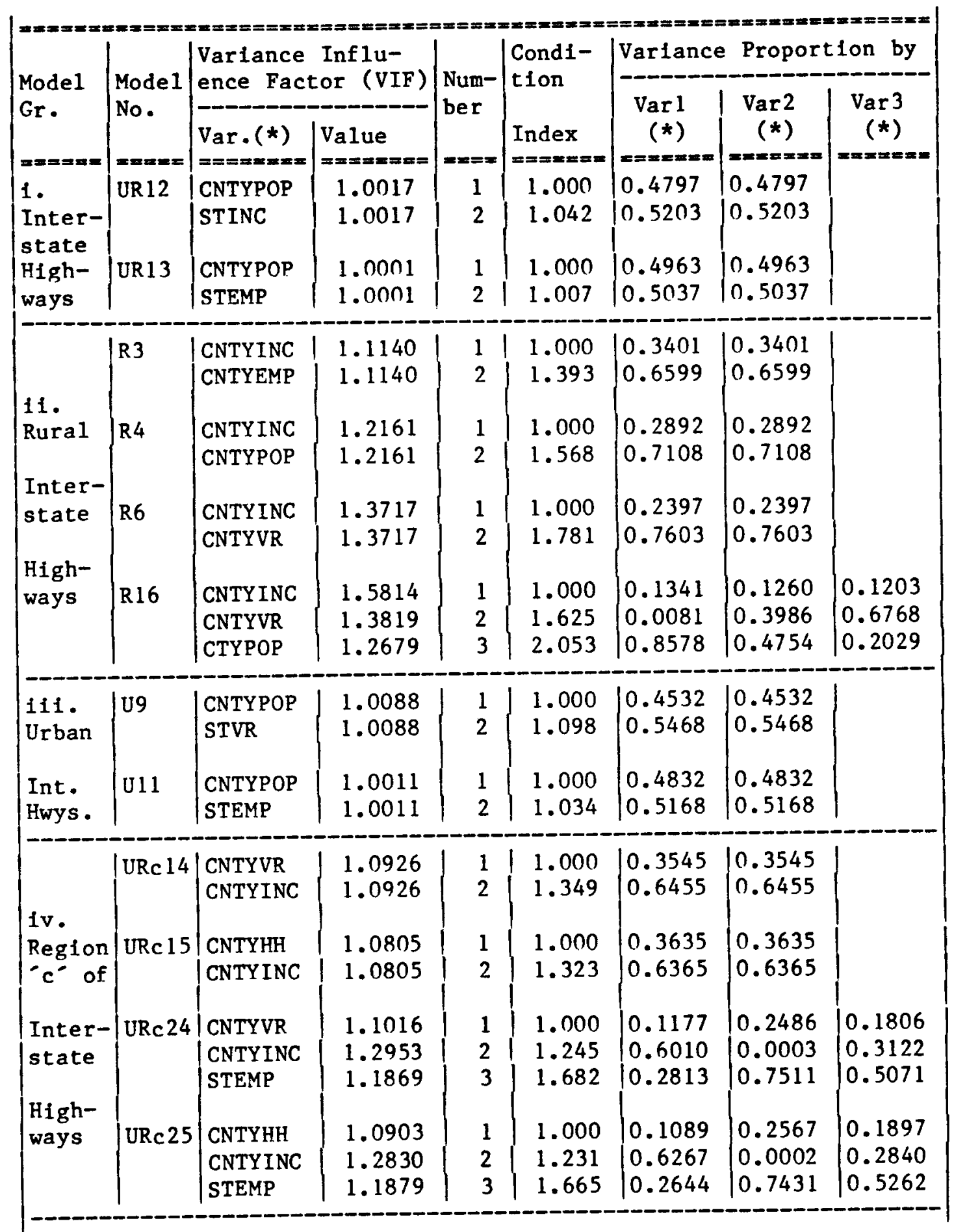


Table 6.14, continued

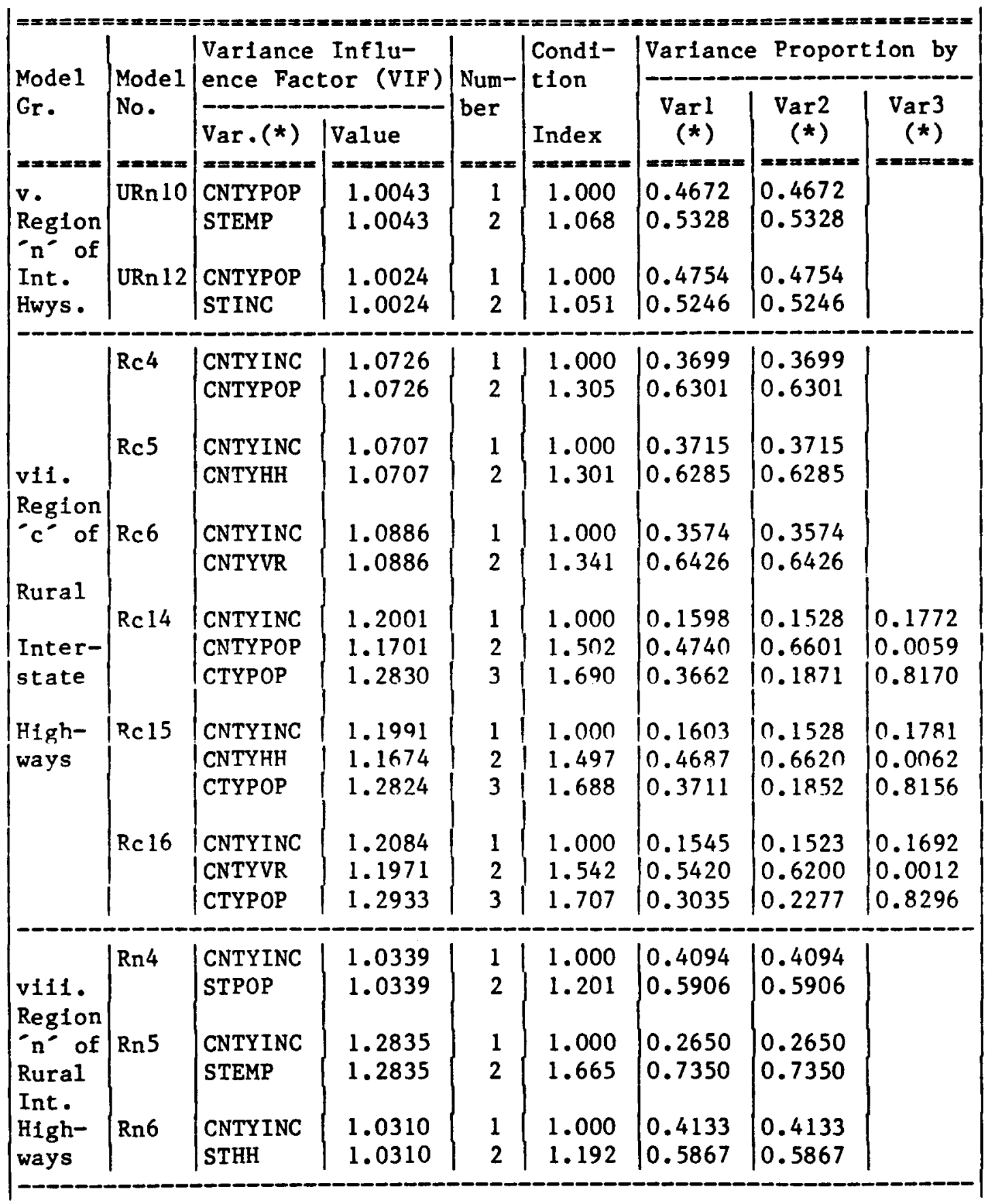


Table 6.14 , continued

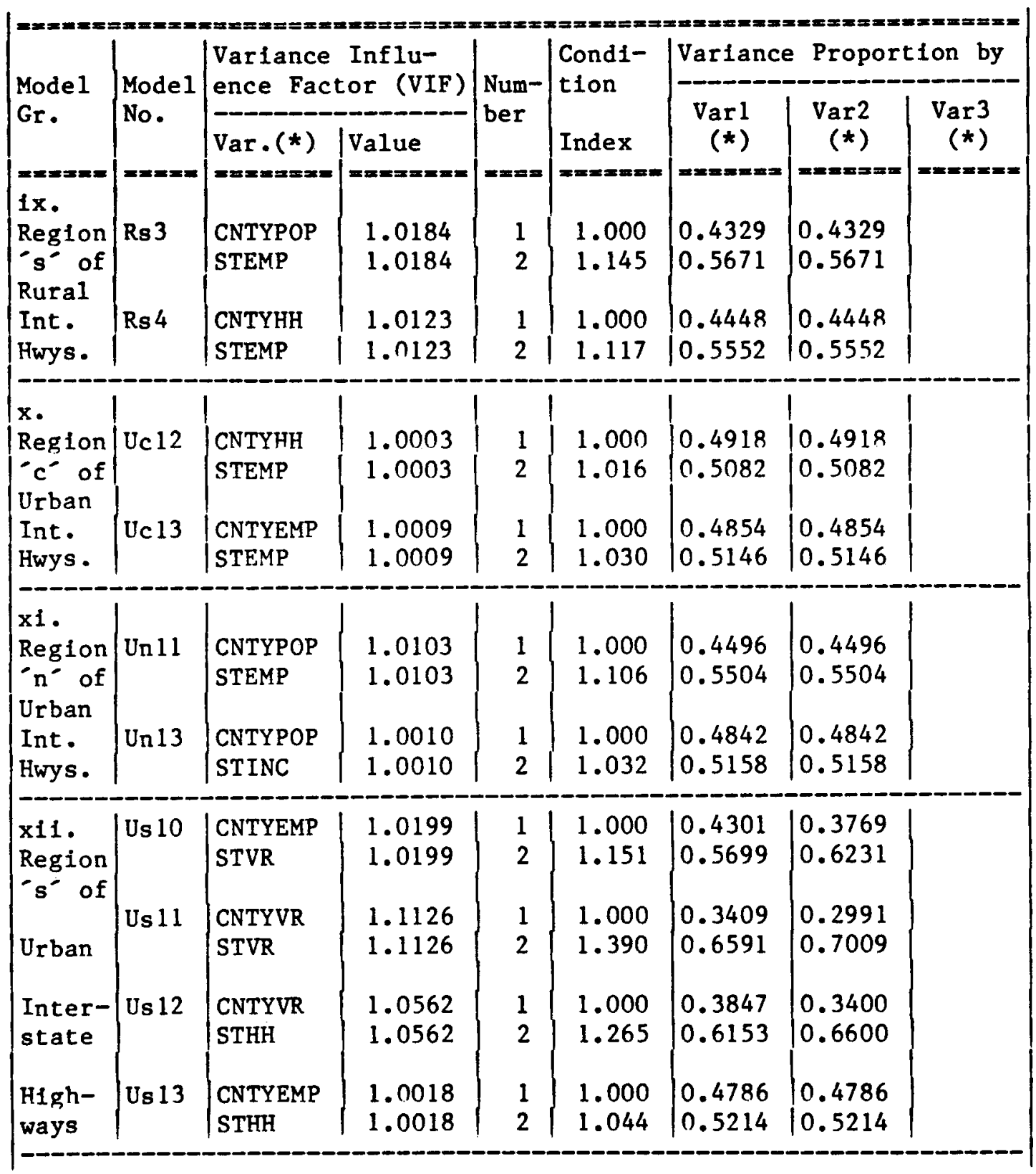

(*) Var1, Var2 and Var3 represent the variables in the order that shown under column 3 (Var.) for each model. 
Table 6.15: Collinearity Statistics for $>2$ variables in the Model in Post-Influence Analysis [Urban Principal arterial]

\begin{tabular}{|c|c|c|c|c|c|c|c|c|}
\hline \multirow{4}{*}{$\begin{array}{l}\text { Model } \\
\text { Gr. } \\
=x== \pm=x\end{array}$} & \multirow{3}{*}{$\begin{array}{l}\text { Mode } 1 \\
\text { No. }\end{array}$} & \multirow{2}{*}{\multicolumn{2}{|c|}{$\begin{array}{l}\text { Variance Influ- } \\
\text { ence Factor (VIF) }\end{array}$}} & \multirow{3}{*}{$\begin{array}{l}\text { Num- } \\
\text { ber }\end{array}$} & \multirow{2}{*}{$\begin{array}{l}\text { Cond1- } \\
\text { tion }\end{array}$} & \multicolumn{3}{|c|}{ Variance Proportion by } \\
\hline & & & & & & $\operatorname{Var} 1$ & Var2 & $\operatorname{Var} 3$ \\
\hline & & $\operatorname{Var} \cdot(*)$ & Value & & Index & $(\star)$ & $(*)$ & $(*)$ \\
\hline & $=2=x=3$ & $=x=2 x=x=2 x$ & |=x=s=mex & $== \pm=x$ & $==2 x=x$ & =x=x=x= & 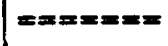 & \multirow[t]{9}{*}{$===\equiv=x$} \\
\hline \multirow{8}{*}{$\begin{array}{l}\text { City } \\
\text { Gr. }=A \\
\& \\
\text { Region } \\
\quad=c\end{array}$} & Ac4 & CNTYHH & 1.2518 & 1 & 1.000 & 0.2758 & 0.2758 & \\
\hline & & CNTYINC & 1.2518 & 2 & 1.621 & 0.7242 & 0.7242 & \\
\hline & Ac5 5 & CNTYVR & 1.0322 & 1 & 1.000 & 0.4117 & 0.4117 & \\
\hline & & CNTYINC & 1.0322 & 2 & 1.195 & 0.5883 & 0.5883 & \\
\hline & Ac6 6 & CNTYEMP & 1.0005 & 1 & 1.000 & 0.4884 & 0.4884 & \\
\hline & & CNTYINC & 1.0005 & 2 & 1.024 & 0.5116 & 0.5116 & \\
\hline & \multirow{2}{*}{ Ac7 } & CNTYPOP & 1.3685 & 1 & 1.000 & 0.2406 & 0.2406 & \\
\hline & & CNTYINC & 1.3685 & 2 & 1.777 & 0.7594 & 0.7594 & \\
\hline \multirow{8}{*}{$\begin{array}{l}\text { ii. } \\
\text { City } \\
\text { Gr.=A } \\
\& \\
\text { Region } \\
\quad=s\end{array}$} & An6 & CTYPOP & 1.3052 & 1 & 1.000 & 0.2582 & 0.2582 & \\
\hline & & STVR & 1.3052 & 2 & 1.695 & 0.7418 & 0.7418 & \\
\hline & \multirow[t]{2}{*}{ An 7} & CTYPOP & 1.1030 & 1 & 1.000 & 0.3472 & 0.3472 & \\
\hline & & STEMP & 1.1030 & 2 & 1.371 & 0.6528 & 0.6528 & \\
\hline & \multirow[t]{2}{*}{ An 8} & CTYPOP & 1.2917 & 1 & 1.000 & 0.2624 & 10.2624 & \\
\hline & & STPOP & 1.2917 & 2 & 1.677 & 0.7376 & 0.7376 & \\
\hline & \multirow[t]{2}{*}{ An 9} & CTYPOP & 1.3271 & 1 & 1.000 & 0.2518 & 10.2518 & \\
\hline & & STHH & 1.3271 & 2 & 1.724 & 10.7482 & 10.7482 & \\
\hline \multirow{3}{*}{$\begin{array}{l}\text { xifi. } \\
\text { City } \\
\text { Gr. }=A+ \\
B+C\end{array}$} & \multirow[t]{2}{*}{$\mathrm{ABCs} 7$} & | CNTYEMP & 1.0002 & 1 & 1.000 & 0.4932 & 0.4932 & \\
\hline & & STVR & 1.0002 & 2 & 1.014 & 0.5068 & 0.5068 & \\
\hline & \multirow[t]{2}{*}{$A B C s 8$} & CNTYEMP & 1.0017 & 1 & 1.000 & 0.4797 & 0.4797 & \\
\hline \multirow{3}{*}{$\begin{array}{c}\& \\
\text { Region } \\
=s\end{array}$} & & STHH & 1.0017 & 2 & 1.042 & 0.5203 & 0.5203 & \\
\hline & \multirow[t]{2}{*}{$\mathrm{ABCs} 9$} & CNTYEMP & 1.0024 & 1 & 1.000 & 0.4755 & 0.4755 & \\
\hline & & STPOP & 1.0024 & 2 & 1.050 & 0.5245 & 0.5245 & \\
\hline
\end{tabular}


Table 6.15 , continued

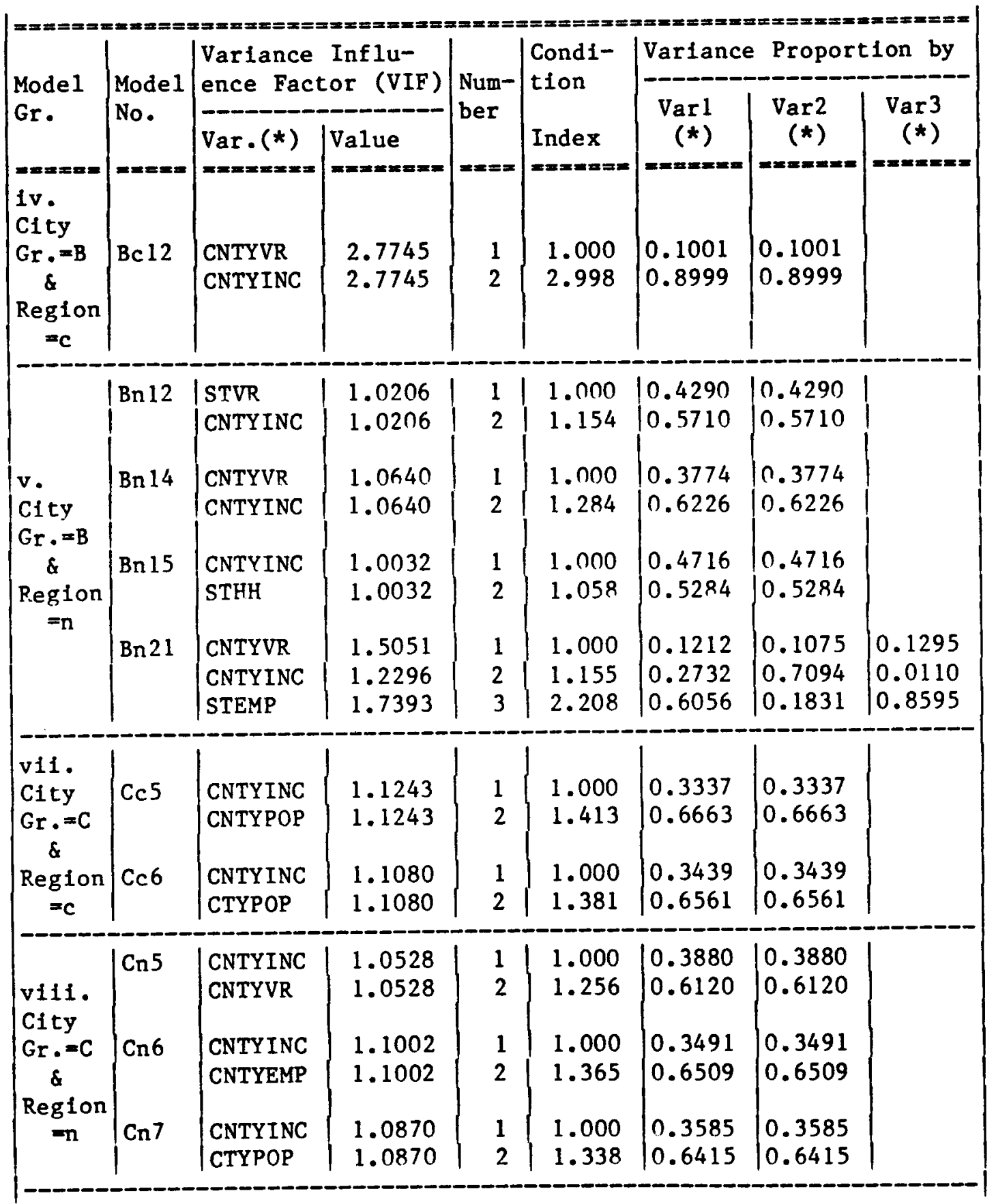


Table 6.15, continued

\begin{tabular}{|c|c|c|c|c|c|c|c|c|}
\hline \multirow{4}{*}{$\begin{array}{l}\text { Mode } 1 \\
\text { Gr. } \\
=x== \pm=\end{array}$} & \multirow{4}{*}{$\begin{array}{l}\text { Model } \\
\text { No. } \\
== \pm==\end{array}$} & \multirow{2}{*}{\multicolumn{2}{|c|}{$\mid \begin{array}{l}\text { Variance Influ- } \\
\text { ence Factor (VIF) }\end{array}$}} & \multirow{3}{*}{$\begin{array}{l}\text { Num- } \\
\text { ber }\end{array}$} & \multirow{4}{*}{$\begin{array}{l}\text { Condi- } \\
\text { tion } \\
\text { Index } \\
=x=x=x=2\end{array}$} & \multicolumn{3}{|c|}{ Variance Proportion by } \\
\hline & & & & & & Varl & Var2 & $\operatorname{Var} 3$ \\
\hline & & Var.(*) & Value & & & $(*)$ & $(*)$ & $(*)$ \\
\hline & & 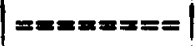 & 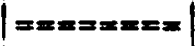 & $==x= \pm$ & & 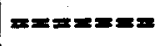 & |z=men=1 & \\
\hline \multirow{9}{*}{$\begin{array}{l}\text { X. } \\
\text { City } \\
\text { Gr. }=D \\
\& \\
\text { Region } \\
\quad=c\end{array}$} & Dc 10 & CTYPOP & 1.0075 & 1 & 1.000 & 0.4569 & 0.4569 & \\
\hline & & STVR & 1.0075 & 2 & 1.090 & 0.5431 & 0.5431 & \\
\hline & De 11 & CTYPOP & 1.0103 & 1 & 1.000 & 0.4496 & 0.4496 & \\
\hline & & STEMP & 1.0103 & 2 & 1.106 & 0.5504 & 0.5504 & \\
\hline & Dc 18 & CTYPOP & 1.4406 & 1 & 1.000 & 0.1296 & 0.1007 & 0.1567 \\
\hline & & STEMP & 1.2312 & 2 & 1.383 & 0.2777 & 0.5731 & 0.0011 \\
\hline & & CNTYINC & 1.7134 & 3 & 2.158 & 0.5927 & 0.3262 & 0.8422 \\
\hline & De 19 & CTYPOP & 1.4180 & 1 & 1.000 & 0.1957 & 0.0422 & 0.2115 \\
\hline & & $\begin{array}{l}\text { STHH } \\
\text { CNTYINC }\end{array}$ & $\begin{array}{l}1.0475 \\
1.4717\end{array}$ & $\begin{array}{l}2 \\
3\end{array}$ & $\begin{array}{l}1.268 \\
1.901\end{array}$ & $\begin{array}{l}0.0813 \\
0.7231\end{array}$ & $\begin{array}{l}0.8601 \\
0.0977\end{array}$ & $\begin{array}{l}0.0009 \\
0.7876\end{array}$ \\
\hline \multirow{5}{*}{$\begin{array}{l}x i . \\
\text { City } \\
\text { Gr.=D } \\
\quad \& \\
\text { Region } \\
\quad=n\end{array}$} & \multirow{5}{*}{$D n 15$} & & & \multirow[b]{2}{*}{1} & \multirow{3}{*}{1.000} & \multirow{3}{*}{0.3855} & \multirow{3}{*}{0.3855} & \\
\hline & & CNTYVR & 1.0554 & & & & & \\
\hline & & STHH & 1.0554 & 2 & & & & \\
\hline & & ICNTYHH & 1.7547 & 1 & 1.000 & 10.1721 & 0.1721 & \\
\hline & & CNTYINC & 1.7547 & 2 & 2.193 & 0.8279 & 0.8279 & \\
\hline xii. & & & & & & & & \\
\hline City & Ds 12 & STHH & 1.4693 & 1 & 1.000 & 0.2174 & 0.2174 & \\
\hline $\mathrm{Gr}_{\&}=\mathrm{D}$ & & STINC & 1.4693 & 2 & 1.897 & 0.7826 & 0.7826 & \\
\hline Region & Ds 13 & STPOP & 1.6820 & 1 & 1.000 & 0.1816 & 0.1816 & \\
\hline$=s$ & & STINC & 1.6820 & 2 & 2.123 & 0.8184 & 0.8184 & \\
\hline
\end{tabular}

(*) Varl, Var 2 and $\operatorname{Var} 3$ represent the variables in the order that shown under column 3 (Var.) for each model. 
Table 6.16: Collinearity Statistics for $>2$ variables in the Model in Post-Influence Analysis [Urban Minor Arterial \& Collector]

\begin{tabular}{|c|c|c|c|c|c|c|c|}
\hline \multirow{4}{*}{$\begin{array}{l}\text { Model } \\
\text { Gr. } \\
======\end{array}$} & \multirow{3}{*}{$\begin{array}{l}\text { Model } \\
\text { No. }\end{array}$} & \multirow{2}{*}{\multicolumn{2}{|c|}{$\begin{array}{l}\text { Variance Influ- } \\
\text { ence Factor (VIF) }\end{array}$}} & \multirow{3}{*}{$\begin{array}{l}\text { Num- } \\
\text { ber }\end{array}$} & \multirow{4}{*}{$\begin{array}{l}\text { Condi- } \\
\text { tion } \\
\text { Index } \\
=x= \pm=x\end{array}$} & \multicolumn{2}{|c|}{ Var1. Prop. by } \\
\hline & & & & & & Var & $r^{2}$ \\
\hline & & $\operatorname{Var} \cdot(*)$ & Value & & & (1) & (1) \\
\hline & $=$ & & & $= \pm=2$ & & $=2 x=2 x=1$ & \\
\hline & A5 & $\begin{array}{l}\text { CNTYINC } \\
\text { CNTYPOP }\end{array}$ & $\begin{array}{l}1.0995 \\
1.0995\end{array}$ & $\begin{array}{l}1 \\
2\end{array}$ & $\begin{array}{l}1.000 \\
1.364\end{array}$ & $\begin{array}{l}0.3596 \\
0.6504\end{array}$ & $\begin{array}{l}0.3596 \\
0.6504\end{array}$ \\
\hline $\begin{array}{l}\text { 1. } \\
\text { City } \\
\text { Gr.=A }\end{array}$ & A6 & $\begin{array}{l}\text { CNTYINC } \\
\text { CNTYVR }\end{array}$ & $\begin{array}{l}1.1524 \\
1.1524\end{array}$ & $\begin{array}{l}1 \\
2\end{array}$ & $\begin{array}{l}1.000 \\
1.464\end{array}$ & $\begin{array}{l}0.3182 \\
0.6818\end{array}$ & $\mid \begin{array}{l}0.3182 \\
0.6818\end{array}$ \\
\hline & A7 & $\begin{array}{l}\text { CNTYINC } \\
\text { CNTYEMP }\end{array}$ & $\begin{array}{l}1.2970 \\
1.2970\end{array}$ & $\begin{array}{l}1 \\
2\end{array}$ & $\begin{array}{l}1.000 \\
1.684\end{array}$ & $\mid \begin{array}{l}0.2607 \\
0.7393\end{array}$ & $\mid \begin{array}{l}0.2607 \\
0.7393\end{array}$ \\
\hline $\begin{array}{l}\text { vity } \\
\text { Gr.=D }\end{array}$ & D6 & $\begin{array}{l}\text { CNTYINC } \\
\text { CTYPOP }\end{array}$ & $\begin{array}{l}1.0358 \\
1.0577\end{array}$ & $\begin{array}{l}1 \\
2\end{array}$ & $\begin{array}{l}1.000 \\
1.207\end{array}$ & $\mid \begin{array}{l}0.4071 \\
0.5929\end{array}$ & $\mid \begin{array}{l}0.4071 \\
0.5929\end{array}$ \\
\hline
\end{tabular}

(*) Varl and Var2 represent the variables in the order that shown under column 3 (Var.) for each model. 


\subsubsection{Testing Hypothesis Concerning Regression Coefficients}

The F-test for the regression relation explains whether the variables in the model have any statistical relation to the dependent variable. The hypotheses are

$$
\begin{aligned}
& H_{0}: B_{1}=B_{2}=\ldots \ldots . .=B_{P-1}=0 \\
& H_{a}: a 11 B_{k}(k=1, \ldots \ldots, P-1) \neq 0
\end{aligned}
$$

The test statistic is given by F-value, the ratio of Mean Square Regression (MSR) to Mean Square Error (MSE).

If the calculated F-value $<F(1-\alpha, P-1, n-P)$ or the $P$-value $(P R O B>F)$ is greater than than the specified -level, then $H_{0}$ holds and proves that the variables in the model do not have any statistical relation to the dependent variable. Larger values of $F$ or smaller P-values lead to the conclusion $\mathrm{H}_{\mathrm{a}}$. The results of this F-test on candidate models of Interstate, Urban Principal Arterial and Urban Minor Arterfal \& Collector for the pre-influence case have been presented in Tables $6.17,6.18$ and 6.19 , respectively. The test results conclude that hypothesis $\mathrm{H}_{\mathrm{a}}$ (i.e., the relationships among the variables in the models exist) cannot be rejected at an a level of 0.01 . A $p$-value $(P R O B>F)$ of 0.0001 in the tables indicates that there is less than one chance in 10,000 of obtaining an $F$ this large or larger by chance alone if $\mathrm{H}_{0}$ holds. 
Table 6.17: Significance of Variable(s) in Model in Pre-Influence Analysis [Interstate Highways]

\begin{tabular}{|c|c|c|c|c|c|c|c|c|c|}
\hline \multirow{2}{*}{$\begin{array}{l}\text { Mode1 } \\
\text { Gr. }\end{array}$} & \multirow{2}{*}{\begin{tabular}{|l|} 
Model 1 \\
No.
\end{tabular}} & \multicolumn{4}{|c|}{ Model Statistics } & \multicolumn{4}{|c|}{ Variables in Model } \\
\hline & & $\begin{array}{l}R^{2} \\
R_{a}^{2} \\
===\end{array}$ & $\mid \begin{array}{c}\text { RMSE } \\
\text { C.V. } \\
\text { C=m=E= }\end{array}$ & $\begin{array}{l}d f_{r} \\
d f_{e}\end{array}$ & $\mid \begin{array}{c}\text { F-Value } \\
\text { Prob }>\text { F } \\
=x=m=m=x\end{array}$ & $\mid \begin{array}{l}\text { Varlable } \\
==2=m==\end{array}$ & $\begin{array}{c}t- \\
\text { value } \\
=x=x=m\end{array}$ & Prob $>t$ & $\begin{array}{l}\text { Standard- } \\
\text { Ized Est. }\end{array}$ \\
\hline & UR2 & $\begin{array}{l}48.94 \\
48.78\end{array}$ & $\begin{array}{l}13900 \\
50.513\end{array}$ & $\begin{array}{r}1 \\
311\end{array}$ & $\begin{array}{c}298.089 \\
0.0001\end{array}$ & CNTYVR & 17.265 & 0.0001 & 0.6996 \\
\hline $\begin{array}{l}\text { Inter- } \\
\text { state }\end{array}$ & UR4 & $\begin{array}{l}50.30 \\
50.14\end{array}$ & $\begin{array}{l}13713 \\
49.837\end{array}$ & $\begin{array}{r}1 \\
311\end{array}$ & $\left\{\begin{array}{r}314.741 \\
0.0001\end{array}\right.$ & CNTYPOP & 17.741 & 0.0001 & 0.7092 \\
\hline $\begin{array}{l}\text { High- } \\
\text { ways }\end{array}$ & UR 12 & $\begin{array}{l}52.27 \\
51.96\end{array}$ & $\begin{array}{l}13461 . \\
48.918\end{array}$ & $\begin{array}{r}2 \\
310\end{array}$ & $\begin{array}{r}169.727 \\
0.0001\end{array}$ & $\begin{array}{l}\text { CNTYYOP } \\
\text { STINC }\end{array}$ & $\begin{array}{r}17.974 \\
3.576\end{array}$ & $\begin{array}{l}0.0001 \\
0.0004\end{array}$ & $\begin{array}{l}0.7055 \\
0.1404\end{array}$ \\
\hline & UR 13 & $\begin{array}{l}52.29 \\
51.99\end{array}$ & $\begin{array}{l}13457 \\
48.904\end{array}$ & $\begin{array}{r}2 \\
310\end{array}$ & $\begin{array}{r}169.912 \\
0.0001\end{array}$ & $\begin{array}{l}\text { CNTYPOP } \\
\text { STEMP }\end{array}$ & $\begin{array}{r}18.135 \\
3.601\end{array}$ & $\begin{array}{l}0.0001 \\
0.0004\end{array}$ & $\begin{array}{l}0.7115 \\
0.1413\end{array}$ \\
\hline & R3 & $\begin{array}{l}36.28 \\
35.58\end{array}$ & $\mid \begin{array}{l}7585.9 \\
38.047\end{array}$ & $\begin{array}{r}2 \\
183\end{array}$ & $\begin{array}{l}52.095 \\
0.0001\end{array}$ & $\begin{array}{l}\text { CNTYINC } \\
\text { CNTYEMP }\end{array}$ & $\begin{array}{l}7.864 \\
3.889\end{array}$ & $\begin{array}{l}0.0001 \\
0.0001\end{array}$ & $\begin{array}{l}0.4858 \\
0.2403\end{array}$ \\
\hline Rural & R4 & $\begin{array}{l}37.49 \\
36.81\end{array}$ & $\begin{array}{l}7513.5 \\
37.684\end{array}$ & $\begin{array}{r}2 \\
183\end{array}$ & $\begin{array}{l}54.875 \\
0.0001\end{array}$ & $\begin{array}{l}\text { CNTYINC } \\
\text { CNTYPOP }\end{array}$ & $\begin{array}{l}7.714 \\
4.355\end{array}$ & $\begin{array}{l}0.0001 \\
0.0001\end{array}$ & $\begin{array}{l}0.4741 \\
0.2676\end{array}$ \\
\hline state & R6 & $\begin{array}{l}37.82 \\
37.14\end{array}$ & $\begin{array}{l}7493.5 \\
37.583\end{array}$ & $\begin{array}{r}2 \\
183\end{array}$ & $\begin{array}{l}55.658 \\
0.0001\end{array}$ & $\begin{array}{l}\text { CNTYINC } \\
\text { CNTYVR }\end{array}$ & $\begin{array}{l}7.466 \\
4.477\end{array}$ & $\begin{array}{l}0.0001 \\
0.0001\end{array}$ & $\begin{array}{l}0.4627 \\
0.2774\end{array}$ \\
\hline $\begin{array}{l}\text { nagn- } \\
\text { ways }\end{array}$ & R16 & $\begin{array}{l}40.60 \\
39.62\end{array}$ & $\begin{array}{l}7344.4 \\
36.836\end{array}$ & $\begin{array}{r}3 \\
182\end{array}$ & $\begin{array}{l}41.462 \\
0.0001\end{array}$ & $\begin{array}{l}\text { CNTYINC } \\
\text { CNTYVR } \\
\text { CTYPOP }\end{array}$ & $\begin{array}{l}6.248 \\
3.880 \\
2.916\end{array}$ & $\begin{array}{l}0.0001 \\
0.0001 \\
0.0040\end{array}$ & $\begin{array}{l}0.4015 \\
0.2407 \\
0.1854 \\
\end{array}$ \\
\hline $\begin{array}{l}\text { il1. } \\
\text { Urban }\end{array}$ & U2 & $\begin{array}{l}51.20 \\
50.81\end{array}$ & $\mid \begin{array}{l}17091 \\
44.258\end{array}$ & $\begin{array}{r}1 \\
125\end{array}$ & $\left|\begin{array}{c}131.132 \\
0.0001\end{array}\right|$ & CNTYVR & 11.451 & 0.0001 & 0.7155 \\
\hline $\begin{array}{l}\text { Inter- } \\
\text { state }\end{array}$ & 04 & $\begin{array}{l}53.38 \\
53.10\end{array}$ & $\begin{array}{l}16704 \\
43.255\end{array}$ & $\begin{array}{r}1 \\
125\end{array}$ & $\begin{array}{r}143.153 \\
0.0001\end{array}$ & CNTYPOP & 11.965 & 0.0001 & 0.7306 \\
\hline $\begin{array}{l}\text { High- } \\
\text { ways }\end{array}$ & |U9 & $\begin{array}{l}55.25 \\
54.53\end{array}$ & $\begin{array}{l}16432 . \\
42.550\end{array}$ & $\begin{array}{r}2 \\
124\end{array}$ & $\begin{array}{l}76.553 \\
0.0001\end{array}$ & \begin{tabular}{|l} 
CNTYPOP \\
STVR
\end{tabular} & $\begin{array}{r}11.861 \\
2.275\end{array}$ & $\begin{array}{l}0.0001 \\
0.0247\end{array}$ & $\begin{array}{l}0.7164 \\
0.1374\end{array}$ \\
\hline & U11 & $\begin{array}{l}55.57 \\
54.85\end{array}$ & $\begin{array}{l}16373 . \\
42.399\end{array}$ & $\begin{array}{r}2 \\
124\end{array}$ & & \begin{tabular}{|l} 
CNTYPOP \\
STEMP
\end{tabular} & $\begin{array}{r}12.242 \\
2.470\end{array}$ & & $\begin{array}{l}0.7329 \\
0.1478\end{array}$ \\
\hline
\end{tabular}


Table 6.17 , continued

\begin{tabular}{|c|c|c|c|c|c|c|c|c|c|}
\hline \multirow{2}{*}{$\begin{array}{l}\text { Model } \\
\text { Gr. }\end{array}$} & \multirow{2}{*}{\begin{tabular}{|l|} 
Mode 1 \\
No.
\end{tabular}} & \multicolumn{4}{|c|}{ Model Statistics } & \multicolumn{4}{|c|}{ Variables in Model } \\
\hline & & $\begin{array}{l}\mathrm{R}^{2} \\
\mathrm{R}^{2}\end{array}$ & $\begin{array}{l}\text { RMSE } \\
\text { C.V. }\end{array}$ & $\begin{array}{l}d f_{r} \\
d f_{e}\end{array}$ & $\mid \begin{array}{r}\text { F-Value } \\
\text { Prob }>F\end{array}$ & Variable & $\begin{array}{l}\text { t- } \\
\text { value }\end{array}$ & Prob $>t$ & $\begin{array}{l}\text { Standard- } \\
\text { ized Est. }\end{array}$ \\
\hline & $\begin{array}{l}\ln =2= \pm \\
\text { URc3 }\end{array}$ & $\begin{array}{l}65.72 \\
65.45\end{array}$ & $\mid \begin{array}{l}===-1= \\
9438.8 \\
33.865\end{array}$ & $\begin{array}{r}=m= \\
1 \\
130\end{array}$ & $\begin{array}{c}0=0= \\
249.175 \\
0.0001\end{array}$ & 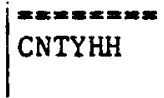 & $\begin{array}{l}==-==2 \\
15.785\end{array}$ & $\begin{array}{c}==x= \pm= \\
0.0001\end{array}$ & $\begin{array}{c}=1=x=5= \\
0.8106\end{array}$ \\
\hline & URc4 & $\begin{array}{l}66.29 \\
66.03\end{array}$ & $\begin{array}{l}9359.2 \\
33.579\end{array}$ & $\begin{array}{r}1 \\
130\end{array}$ & $\begin{array}{r}255.647 \\
0.0001\end{array}$ & CNTYVR & 15.989 & 0.0001 & 0.8142 \\
\hline $\begin{array}{l}\text { Region } \\
\text { Regiof } \\
c^{-} \text {of }\end{array}$ & URe 14 & $\begin{array}{l}71.98 \\
71.45\end{array}$ & $\begin{array}{l}8565.7 \\
30.732\end{array}$ & $\begin{array}{r}2 \\
129\end{array}$ & $\begin{array}{r}165.705 \\
0.0001\end{array}$ & $\begin{array}{l}\text { CNTYVR } \\
\text { CNTYINC }\end{array}$ & $\begin{array}{r}14.453 \\
5.119\end{array}$ & $\begin{array}{l}0.0001 \\
0.0001\end{array}$ & $\begin{array}{l}0.7221 \\
0.2557\end{array}$ \\
\hline $\begin{array}{l}\text { Inter- } \\
\text { state }\end{array}$ & URc15 & $\begin{array}{l}72.02 \\
71.59\end{array}$ & $\begin{array}{l}8559.7 \\
30.711\end{array}$ & $\begin{array}{r}2 \\
129\end{array}$ & $\begin{array}{r}166.026 \\
0.0001\end{array}$ & $\begin{array}{l}\text { CNTYHH } \\
\text { CNTYINC }\end{array}$ & $\begin{array}{r}14.469 \\
5.392\end{array}$ & $\begin{array}{l}0.0001 \\
0.0001\end{array}$ & $\begin{array}{l}0.7181 \\
0.2676\end{array}$ \\
\hline $\begin{array}{l}\text { High- } \\
\text { ways }\end{array}$ & URc24 & $\begin{array}{l}73.01 \\
72.37\end{array}$ & $\begin{array}{l}8440.5 \\
30.283\end{array}$ & $\begin{array}{r}3 \\
128\end{array}$ & $\begin{array}{r}115.392 \\
0.0001\end{array}$ & $\begin{array}{l}\text { CNTYVR } \\
\text { CNTYINC } \\
\text { STEMP }\end{array}$ & $\begin{array}{r}14.815 \\
3.777 \\
2.204\end{array}$ & $\begin{array}{l}0.0001 \\
0.0002 \\
0.0293\end{array}$ & $\begin{array}{l}0.7331 \\
0.2051 \\
0.1119\end{array}$ \\
\hline & URc25 & 73.08 & 8428.6 & 3 & 115.838 & CNTYHH & 14.848 & 0.0001 & 0.7296 \\
\hline & & 72.45 & 30.240 & 128 & 0.0001 & $\mid \begin{array}{l}\text { CNTYINC } \\
\text { STEMP }\end{array}$ & $\begin{array}{l}4.006 \\
2.246\end{array}$ & $\begin{array}{l}0.0001 \\
0.0264\end{array}$ & $\begin{array}{l}0.2162 \\
0.1139\end{array}$ \\
\hline & URn2 & $\begin{array}{l}43.77 \\
43.22\end{array}$ & $\begin{array}{l}18388 \\
54.335\end{array}$ & $\begin{array}{r}1 \\
102\end{array}$ & $\begin{array}{l}79.397 \\
0.0001\end{array}$ & CNTYVR & 8.911 & 0.0001 & 0.6616 \\
\hline $\begin{array}{l}\text { ve } \\
\text { Region } \\
\text { - } n^{-} \text {of }\end{array}$ & |URn 3 & $\begin{array}{l}45.08 \\
44.54\end{array}$ & $\begin{array}{l}18173 . \\
53.698\end{array}$ & $\begin{array}{r}1 \\
102\end{array}$ & $\begin{array}{l}83.729 \\
0.0001\end{array}$ & CNTYPOP & 9.150 & 0.0001 & 0.6714 \\
\hline $\begin{array}{l}\text { Inter- } \\
\text { state }\end{array}$ & URn 4 & $\begin{array}{l}45.33 \\
44.79\end{array}$ & $\begin{array}{l}18131 . \\
53.576\end{array}$ & $\begin{array}{r}1 \\
102\end{array}$ & $\begin{array}{l}84.576 \\
0.0001\end{array}$ & CNTYHH & 9.197 & 0.0001 & 0.6733 \\
\hline $\begin{array}{l}\text { High- } \\
\text { ways }\end{array}$ & URn 10 & $\begin{array}{l}47.02 \\
45.97\end{array}$ & $\begin{array}{l}17937 \\
53.002\end{array}$ & $\begin{array}{r}2 \\
101\end{array}$ & $\begin{array}{l}44.816 \\
0.0001\end{array}$ & $\begin{array}{l}\text { CNTYPOP } \\
\text { STEMP }\end{array}$ & $\begin{array}{l}9.408 \\
1.922\end{array}$ & $\begin{array}{l}0.0001 \\
0.0575\end{array}$ & $\begin{array}{l}0.6843 \\
0.1398\end{array}$ \\
\hline & URn12 & $\begin{array}{l}47.31 \\
46.26\end{array}$ & $\begin{array}{l}17889 . \\
52.858\end{array}$ & $\begin{array}{r}2 \\
101\end{array}$ & $\begin{array}{l}45.339 \\
0.0001\end{array}$ & \begin{tabular}{|l} 
CNTYPOP \\
STINC
\end{tabular} & $\begin{array}{l}9.232 \\
2.066\end{array}$ & $\begin{array}{l}0.0001 \\
0.0414\end{array}$ & $\begin{array}{l}0.6671 \\
0.1493\end{array}$ \\
\hline
\end{tabular}


Table 6.17 , continued

\begin{tabular}{|c|c|c|c|c|c|c|c|c|c|}
\hline \multirow[b]{2}{*}{$\begin{array}{l}\text { Model } \\
\text { Gr. }\end{array}$} & \multirow{2}{*}{$\begin{array}{l}\text { Model } \\
\text { No. }\end{array}$} & \multicolumn{4}{|c|}{ Model Statistics } & \multicolumn{4}{|c|}{ Variables in Model } \\
\hline & & $\mathrm{R}^{2}$ & $\begin{array}{l}\text { RMSE } \\
\text { C.v. }\end{array}$ & $d f_{r}$ & $\mid \begin{array}{r}\text { F-Value } \\
\text { Prob }>F\end{array}$ & Variable & $\begin{array}{c}t- \\
\text { value }\end{array}$ & Prob>t & $\begin{array}{l}\text { Standard- } \\
\text { ized Est. }\end{array}$ \\
\hline $\begin{array}{l}==-==0= \\
v 1 .\end{array}$ & \begin{tabular}{|l} 
=e=-= \\
URs 1
\end{tabular} & $\begin{array}{r}=-8=- \\
58.60 \\
58.05\end{array}$ & $\begin{array}{l}=1 \\
8117.4 \\
44.196\end{array}$ & $\begin{array}{r}1 \\
75\end{array}$ & $\begin{array}{r}106.167 \\
0.0001\end{array}$ & $\mid \begin{array}{l}\text {-me= } \\
\text { CNTYEMP }\end{array}$ & 10.304 & 0.0001 & 0.7655 \\
\hline $\begin{array}{l}\text { Region } \\
\text { - } s^{-} \text {of }\end{array}$ & | URs2 & $\begin{array}{l}59.82 \\
59.28\end{array}$ & $\begin{array}{l}7997.1 \\
43.541\end{array}$ & $\begin{array}{r}1 \\
75\end{array}$ & $\begin{array}{r}111.661 \\
0.0001\end{array}$ & CNTYPOP & 10.567 & 0.0001 & 0.7734 \\
\hline $\begin{array}{l}\text { Inter- } \\
\text { state }\end{array}$ & URs 3 & $\begin{array}{l}60.19 \\
59.66\end{array}$ & $\begin{array}{l}7959.9 \\
43.338\end{array}$ & $\begin{array}{r}1 \\
75\end{array}$ & $\begin{array}{r}113.411 \\
0.0001\end{array}$ & CNTYVR & 10.649 & 0.0001 & 0.7758 \\
\hline $\begin{array}{l}\text { High- } \\
\text { ways }\end{array}$ & |URs4 & $\begin{array}{l}61.42 \\
60.90\end{array}$ & $\begin{array}{l}7836.7 \\
42.668\end{array}$ & $\begin{array}{r}1 \\
75\end{array}$ & $\begin{array}{r}119.381 \\
0.0001\end{array}$ & CNTYHH & 10.926 & 0.0001 & 0.7837 \\
\hline & Rc4 & $\begin{array}{l}48.45 \\
47.11\end{array}$ & $\mid \begin{array}{l}6767.7 \\
28.345\end{array}$ & 77 & $\begin{array}{l}36.182 \\
0.0001\end{array}$ & $\begin{array}{l}\text { CNTYINC } \\
\text { CNTYPOPP }\end{array}$ & $\begin{array}{l}6.354 \\
3.878\end{array}$ & $\begin{array}{l}0.0001 \\
0.0002\end{array}$ & $\begin{array}{l}0.5371 \\
0.3278\end{array}$ \\
\hline vii. & $\operatorname{Rc} 5$ & $\begin{array}{l}48.49 \\
47.16\end{array}$ & $\begin{array}{l}6764.6 \\
28.333\end{array}$ & $\begin{array}{r}2 \\
77\end{array}$ & $\begin{array}{l}36.251 \\
0.0001\end{array}$ & $\begin{array}{l}\text { CNTYINC } \\
\text { CNTYHH }\end{array}$ & $\begin{array}{l}6.373 \\
3.889\end{array}$ & $\begin{array}{l}0.0001 \\
0.0002\end{array}$ & $\begin{array}{l}0.5380 \\
0.3283\end{array}$ \\
\hline$-c^{-}$of & Rc6 & $\begin{array}{l}48.73 \\
47.40\end{array}$ & $\begin{array}{l}6749.3 \\
28.269\end{array}$ & $\begin{array}{r}2 \\
77\end{array}$ & $\begin{array}{l}36.588 \\
0.0001\end{array}$ & $\begin{array}{l}\text { CNTYINC } \\
\text { CNTYVR }\end{array}$ & $\begin{array}{l}6.204 \\
3.942\end{array}$ & $\begin{array}{l}0.0001 \\
0.0002\end{array}$ & $\begin{array}{l}0.5269 \\
0.3348\end{array}$ \\
\hline $\mid \begin{array}{l}\text { Rural } \\
\text { Inter- } \\
\text { state }\end{array}$ & Rcl4 & $\begin{array}{l}50.99 \\
49.06\end{array}$ & $\begin{array}{l}6641.8 \\
27.818\end{array}$ & $\begin{array}{r}3 \\
76\end{array}$ & $\begin{array}{l}26.359 \\
0.0001\end{array}$ & $\begin{array}{l}\text { CNTYINC } \\
\text { CNTYPOP } \\
\text { CTYPOP }\end{array}$ & $\begin{array}{l}5.565 \\
3.223 \\
1.986\end{array}$ & $\begin{array}{l}0.0001 \\
0.0019 \\
0.0506\end{array}$ & $\begin{array}{l}0.4846 \\
0.2790 \\
0.1785\end{array}$ \\
\hline $\begin{array}{l}\text { High- } \\
\text { ways }\end{array}$ & $\operatorname{Rc} 15$ & $\begin{array}{l}51.05 \\
49.11\end{array}$ & $\begin{array}{l}6638.1 \\
27.803\end{array}$ & $\begin{array}{r}3 \\
76\end{array}$ & $\begin{array}{l}26.417 \\
0.0001\end{array}$ & $\begin{array}{l}\text { CNTYINC } \\
\text { CNTYHH } \\
\text { CTYPOP }\end{array}$ & $\begin{array}{l}5.578 \\
3.239 \\
1.991\end{array}$ & $\begin{array}{l}0.0001 \\
0.0018 \\
0.0501\end{array}$ & $\begin{array}{l}0.4852 \\
0.2797 \\
0.1787\end{array}$ \\
\hline & $R c 16$ & $\begin{array}{l}51.12 \\
49.19\end{array}$ & $\begin{array}{l}6632.9 \\
27.781\end{array}$ & $\begin{array}{r}3 \\
76\end{array}$ & $\begin{array}{l}26.499 \\
0.0001\end{array}$ & $\begin{array}{l}\text { CNTYINC } \\
\text { CNTYVR } \\
\text { CTYPOP }\end{array}$ & $\begin{array}{l}5.472 \\
3.259 \\
1.931\end{array}$ & $\begin{array}{l}0.0001 \\
0.0017 \\
0.0573\end{array}$ & $\begin{array}{l}0.4775 \\
0.2848 \\
0.1740\end{array}$ \\
\hline
\end{tabular}


Table 6.17 , continued

\begin{tabular}{|c|c|c|c|c|c|c|c|c|c|}
\hline \multirow{2}{*}{$\begin{array}{l}\text { Model } \\
\text { Gr. }\end{array}$} & \multirow{2}{*}{$\begin{array}{l}\text { Model } \\
\text { No. }\end{array}$} & \multicolumn{4}{|c|}{ Model Statistics } & \multicolumn{4}{|c|}{ Varlables in Model } \\
\hline & & $\begin{array}{c}\mathrm{R}^{2} \\
\mathrm{R}_{\mathrm{a}}^{2} \\
== \pm \mathrm{a}=\mathrm{E}\end{array}$ & $\begin{array}{l}\text { RMSE } \\
\text { C.V. } \\
x= \pm x=x\end{array}$ & $\begin{array}{l}d f_{r} \\
d f_{e} \\
d=x==\end{array}$ & $\left\{\begin{array}{c}\text { F-Value } \\
\text { Prob }>\text { F } \\
== \pm=x=m=\end{array}\right.$ & $\left\{\begin{array}{l}\text { Variable } \\
\text { zx=mex=m }\end{array}\right.$ & $\begin{array}{c}t- \\
\text { value }\end{array}$ & Prob $>t$ & $\begin{array}{l}\text { Standard- } \\
\text { ized Est. }\end{array}$ \\
\hline $\begin{array}{l}\text { vili. } \\
\text { Region } \\
\text { - } n^{-} \text {of }\end{array}$ & $\operatorname{Rn} 1$ & $\begin{array}{l}27.90 \\
26.19\end{array}$ & $\begin{array}{l}7504.4 \\
35.182\end{array}$ & $\frac{1}{45}$ & $\begin{array}{l}17.318 \\
0.0001\end{array}$ & CNTYINC & 4.162 & 0.0001 & 0.5272 \\
\hline Rural & Rn4 & $\begin{array}{l}30.34 \\
27.17\end{array}$ & $\begin{array}{l}7454.0 \\
34.946\end{array}$ & $\begin{array}{r}2 \\
44\end{array}$ & $\begin{array}{r}9.581 \\
0.0004\end{array}$ & $\begin{array}{l}\text { CNTYINC } \\
\text { STPOP }\end{array}$ & $\begin{array}{l}3.947 \\
1.269\end{array}$ & $\begin{array}{l}0.0003 \\
0.2112\end{array}$ & $\begin{array}{l}0.5025 \\
0.1615\end{array}$ \\
\hline $\begin{array}{l}\text { Inter- } \\
\text { state }\end{array}$ & $\operatorname{Rn} 5$ & $\begin{array}{l}30.37 \\
27.20\end{array}$ & $\begin{array}{l}7452.6 \\
34.942\end{array}$ & $\begin{array}{r}2 \\
44\end{array}$ & $\begin{array}{r}9.593 \\
0.0003\end{array}$ & $\begin{array}{l}\text { CNTYINC } \\
\text { STEMP }\end{array}$ & $\begin{array}{l}3.299 \\
1.276\end{array}$ & $\begin{array}{l}0.0019 \\
0.2088\end{array}$ & $\begin{array}{l}0.4550 \\
0.1759\end{array}$ \\
\hline $\begin{array}{l}\text { High- } \\
\text { ways }\end{array}$ & $\operatorname{Rn} 6$ & $\begin{array}{l}30.64 \\
27.49\end{array}$ & \begin{tabular}{|l}
7437.8 \\
34.871
\end{tabular} & $\begin{array}{r}2 \\
44\end{array}$ & $\begin{array}{r}9.719 \\
0.0003\end{array}$ & $\begin{array}{l}\text { CNTYINC } \\
\text { STHH }\end{array}$ & $\begin{array}{l}3.994 \\
1.345\end{array}$ & $\begin{array}{l}0.0002 \\
0.0186\end{array}$ & $\begin{array}{l}0.5056 \\
0.1702\end{array}$ \\
\hline $\begin{array}{l}\text { ix. } \\
\text { Region }\end{array}$ & Rs 1 & 40.46 & 4999.3 & 1 & 38.742 & CNTYPOP & 6.224 & 0.0001 & 0.6361 \\
\hline Rural & Rs 2 & $\begin{array}{l}40.88 \\
39.84\end{array}$ & $\begin{array}{l}4981.8 \\
36.926\end{array}$ & $\begin{array}{r}1 \\
57\end{array}$ & $\begin{array}{l}39.416 \\
0.0001\end{array}$ & CNTYHH & 6.278 & 0.0001 & 0.6394 \\
\hline $\begin{array}{l}\text { Inter- } \\
\text { state }\end{array}$ & Rs 3 & $\begin{array}{l}41.73 \\
39.65\end{array}$ & $\begin{array}{l}4989.7 \\
36.984\end{array}$ & $\begin{array}{r}2 \\
56\end{array}$ & $\begin{array}{l}20.056 \\
0.0001\end{array}$ & $\begin{array}{l}\text { CNTYPOP } \\
\text { STEMP }\end{array}$ & $\begin{array}{l}6.136 \\
1.105\end{array}$ & $\begin{array}{l}0.0001 \\
0.2740\end{array}$ & $\begin{array}{l}0.6276 \\
0.1130\end{array}$ \\
\hline $\begin{array}{l}\text { High- } \\
\text { ways }\end{array}$ & Rs 4 & $\begin{array}{l}41.76 \\
39.68\end{array}$ & $\begin{array}{l}4988.6 \\
36.976\end{array}$ & $\begin{array}{r}2 \\
56\end{array}$ & $\begin{array}{l}20.076 \\
0.0001\end{array}$ & $\begin{array}{l}\text { CNTYHH } \\
\text { STEMP }\end{array}$ & $\begin{array}{l}6.139 \\
0.918\end{array}$ & $\begin{array}{l}0.0001 \\
0.3623\end{array}$ & $\begin{array}{l}0.6295 \\
0.0942\end{array}$ \\
\hline & Uel & $\begin{array}{l}82.09 \\
81.73\end{array}$ & $\begin{array}{l}9213.9 \\
27.083\end{array}$ & $\begin{array}{r}1 \\
50\end{array}$ & $\begin{array}{r}229.126 \\
0.0001\end{array}$ & CNTYPOP & $\mid 15.137$ & 0.0001 & .9060 \\
\hline & Uc2 & $\begin{array}{l}83.40 \\
83.06\end{array}$ & $\begin{array}{l}8870.8 \\
26.075\end{array}$ & $\begin{array}{r}1 \\
50\end{array}$ & $\begin{array}{r}251.137 \\
0.0001\end{array}$ & CNTYVR & 15.847 & 0.0001 & 0.9132 \\
\hline$c^{\prime}$ of & Uc3 & $\begin{array}{l}83.57 \\
83.24\end{array}$ & $\begin{array}{l}8823.3 \\
25.935\end{array}$ & $\begin{array}{r}1 \\
50\end{array}$ & $\begin{array}{r}254.386 \\
0.0001\end{array}$ & CNTYHН & 15.949 & 0.0001 & 0.9142 \\
\hline $\begin{array}{l}\text { Urban } \\
\text { Inter- }\end{array}$ & Uc4 & $\begin{array}{l}84.52 \\
84.21\end{array}$ & $\begin{array}{l}8566.6 \\
25.181\end{array}$ & $\begin{array}{r}1 \\
50\end{array}$ & $\begin{array}{r}272.901 \\
0.0001\end{array}$ & CNTYEMP & 16.520 & 0.0001 & 0.9193 \\
\hline H1gh- & Uc 12 & $\begin{array}{l}86.05 \\
85.49\end{array}$ & $\begin{array}{l}8212.1 \\
24.139\end{array}$ & $\begin{array}{r}2 \\
49\end{array}$ & $\begin{array}{r}151.188 \\
0.0001\end{array}$ & $\begin{array}{l}\text { CNTYHH } \\
\text { STEMP }\end{array}$ & $\begin{array}{r}16.909 \\
2.953\end{array}$ & $\begin{array}{l}0.0001 \\
0.0048\end{array}$ & $\begin{array}{l}0.9040 \\
0.1579\end{array}$ \\
\hline & Uc13 & $\begin{array}{l}86.52 \\
85.97\end{array}$ & $\begin{array}{l}8074.4 \\
23.734\end{array}$ & $\begin{array}{r}2 \\
49\end{array}$ & $\begin{array}{r}157.235 \\
0.0001\end{array}$ & $\begin{array}{l}\text { CNTYEMP } \\
\text { STEMP }\end{array}$ & $\begin{array}{r}17.247 \\
2.699\end{array}$ & $\begin{array}{l}0.0001 \\
0.0095\end{array}$ & $\begin{array}{l}0.9077 \\
0.1420\end{array}$ \\
\hline
\end{tabular}


Table 6.17 , continued

\begin{tabular}{|c|c|c|c|c|c|c|c|c|c|}
\hline \multirow{2}{*}{$\begin{array}{l}\text { Mode } 1 \\
\text { Gr. }\end{array}$} & \multirow{2}{*}{$\begin{array}{l}\text { Mode1 } \\
\text { No. }\end{array}$} & \multicolumn{4}{|c|}{ Model Statistics } & \multicolumn{4}{|c|}{ es in Model } \\
\hline & & $\begin{array}{l}\mathrm{R}^{2} \\
\mathrm{R}^{2}\end{array}$ & $\begin{array}{l}\text { RMSE } \\
\text { C.v. }\end{array}$ & $\mathrm{df}_{\mathrm{r}}$ & $\mid \begin{array}{c}\text { F-Value } \\
\text { Prob }>\text { F }\end{array}$ & Variable & $\begin{array}{c}\text { t- } \\
\text { value }\end{array}$ & Prob>t & $\mid \begin{array}{l}\text { Standard- } \\
\text { ized Est. }\end{array}$ \\
\hline$=m=x=$ & $\mid \begin{array}{l}==== \\
\text { Un2 }\end{array}$ & $\begin{array}{l}4=0= \\
47.56 \\
46.61\end{array}$ & $\begin{array}{l}==1=0= \\
20575 . \\
46.590\end{array}$ & $==x=$ & $\begin{array}{c}=1 \\
49.886 \\
0.0001\end{array}$ & $\begin{array}{l}\Rightarrow=m=m== \\
\text { CNTYVR }\end{array}$ & $\begin{array}{c}x=x=0 \\
7.063\end{array}$ & 0.0001 & $\begin{array}{r}x \\
0.6897\end{array}$ \\
\hline $\begin{array}{l}\text { xi. } \\
\text { Region } \\
{ }_{n}{ }^{-} \text {of }\end{array}$ & Un 3 & $\begin{array}{l}48.39 \\
47.45\end{array}$ & $\begin{array}{l}20412 . \\
46.221\end{array}$ & $\begin{array}{r}1 \\
55\end{array}$ & $\begin{array}{l}51.571 \\
0.0001\end{array}$ & CNTYPOP & 7.181 & 0.0001 & 0.6956 \\
\hline Urban & Un4 & $\begin{array}{l}48.79 \\
47.85\end{array}$ & $\begin{array}{l}20333 \\
46.044\end{array}$ & $\begin{array}{r}1 \\
55\end{array}$ & $\begin{array}{l}52.392 \\
0.0001\end{array}$ & CNTYHH & 7.238 & 0.0001 & 0.6985 \\
\hline $\begin{array}{l}\text { Inter- } \\
\text { state }\end{array}$ & Un 11 & $\begin{array}{l}49.95 \\
48.10\end{array}$ & $\begin{array}{l}20285 \\
45.935\end{array}$ & $\begin{array}{r}2 \\
54\end{array}$ & $\begin{array}{l}26.951 \\
0.0001\end{array}$ & \begin{tabular}{|l} 
CNTYPOP \\
STEMP
\end{tabular} & $\begin{array}{l}7.329 \\
1.298\end{array}$ & $\begin{array}{l}0.0001 \\
0.1996\end{array}$ & $\begin{array}{l}0.7106 \\
0.1259\end{array}$ \\
\hline $\begin{array}{l}\text { High- } \\
\text { ways }\end{array}$ & Un 13 & $\begin{array}{l}50.03 \\
48.18\end{array}$ & $\begin{array}{l}20269 \\
45.899\end{array}$ & $\begin{array}{r}2 \\
54\end{array}$ & $\begin{array}{l}27.035 \\
0.0001\end{array}$ & \begin{tabular}{|l} 
CNTYPOP \\
STINC
\end{tabular} & $\begin{array}{l}7.197 \\
1.332\end{array}$ & $\begin{array}{l}0.0001 \\
0.1885\end{array}$ & $\begin{array}{l}0.6925 \\
0.1281\end{array}$ \\
\hline & |Us 3 & $\begin{array}{l}68.73 \\
66.77\end{array}$ & $\begin{array}{l}8324.8 \\
24.237\end{array}$ & $\begin{array}{r}1 \\
16\end{array}$ & $\begin{array}{l}35.163 \\
0.0001\end{array}$ & |CNTYHH & 5.930 & 0.0001 & 3290 \\
\hline $\begin{array}{l}\text { xiig. } \\
\text { Region }\end{array}$ & Us4 & $\begin{array}{l}73.11 \\
71.43\end{array}$ & $\begin{array}{l}7719.6 \\
22.475\end{array}$ & $\begin{array}{r}1 \\
16\end{array}$ & $\begin{array}{l}43.499 \\
0.0001\end{array}$ & CNTYVR & 6.595 & 0.0001 & 0.8550 \\
\hline & Us 10 & $\begin{array}{l}78.52 \\
75.66\end{array}$ & $\begin{array}{l}7125.3 \\
20.745\end{array}$ & $\begin{array}{r}2 \\
15\end{array}$ & $\begin{array}{l}27.421 \\
0.0001\end{array}$ & $\begin{array}{l}\text { CNTYEMP } \\
\text { STVR }\end{array}$ & $\begin{array}{l}5.728 \\
3.139\end{array}$ & $\begin{array}{l}0.0001 \\
0.0068\end{array}$ & $\begin{array}{l}0.7072 \\
0.3875\end{array}$ \\
\hline $\begin{array}{l}\text { Inter- } \\
\text { state }\end{array}$ & Us 11 & $\begin{array}{l}78.78 \\
75.95\end{array}$ & $\begin{array}{l}7083.0 \\
20.622\end{array}$ & $\begin{array}{r}2 \\
15\end{array}$ & $\begin{array}{l}27.837 \\
0.0001\end{array}$ & $\begin{array}{l}\text { CNTYVR } \\
\text { STVR }\end{array}$ & $\begin{array}{l}5.778 \\
2.001\end{array}$ & $\begin{array}{l}0.0001 \\
0.0638\end{array}$ & $\begin{array}{l}0.7506 \\
0.2600\end{array}$ \\
\hline $\begin{array}{l}\text { High- } \\
\text { ways }\end{array}$ & Us 12 & $\begin{array}{l}80.48 \\
77.88\end{array}$ & $\begin{array}{l}6791.9 \\
19.774\end{array}$ & $\begin{array}{r}2 \\
15\end{array}$ & $\begin{array}{l}30.931 \\
0.0001\end{array}$ & $\begin{array}{l}\text { CNTYVR } \\
\text { STHH }\end{array}$ & $\begin{array}{l}6.340 \\
2.381\end{array}$ & $\begin{array}{l}0.0001 \\
0.0310\end{array}$ & $\begin{array}{l}0.7633 \\
0.2867\end{array}$ \\
\hline & Us 13 & $\begin{array}{l}81.34 \\
78.86\end{array}$ & $\begin{array}{l}6640.8 \\
19.334\end{array}$ & $\begin{array}{r}2 \\
15\end{array}$ & $\begin{array}{l}32.701 \\
0.0001\end{array}$ & $\begin{array}{l}\text { CNTYEMP } \\
\text { STHH }\end{array}$ & $\begin{array}{l}6.538 \\
3.689\end{array}$ & $\begin{array}{l}0.0001 \\
0.0022\end{array}$ & $\begin{array}{l}0.7380 \\
0.4165\end{array}$ \\
\hline
\end{tabular}


Table 6.18: Significance of Variable(s) in Model in Pre-Influence Analysis [Urban Principal Arterial]

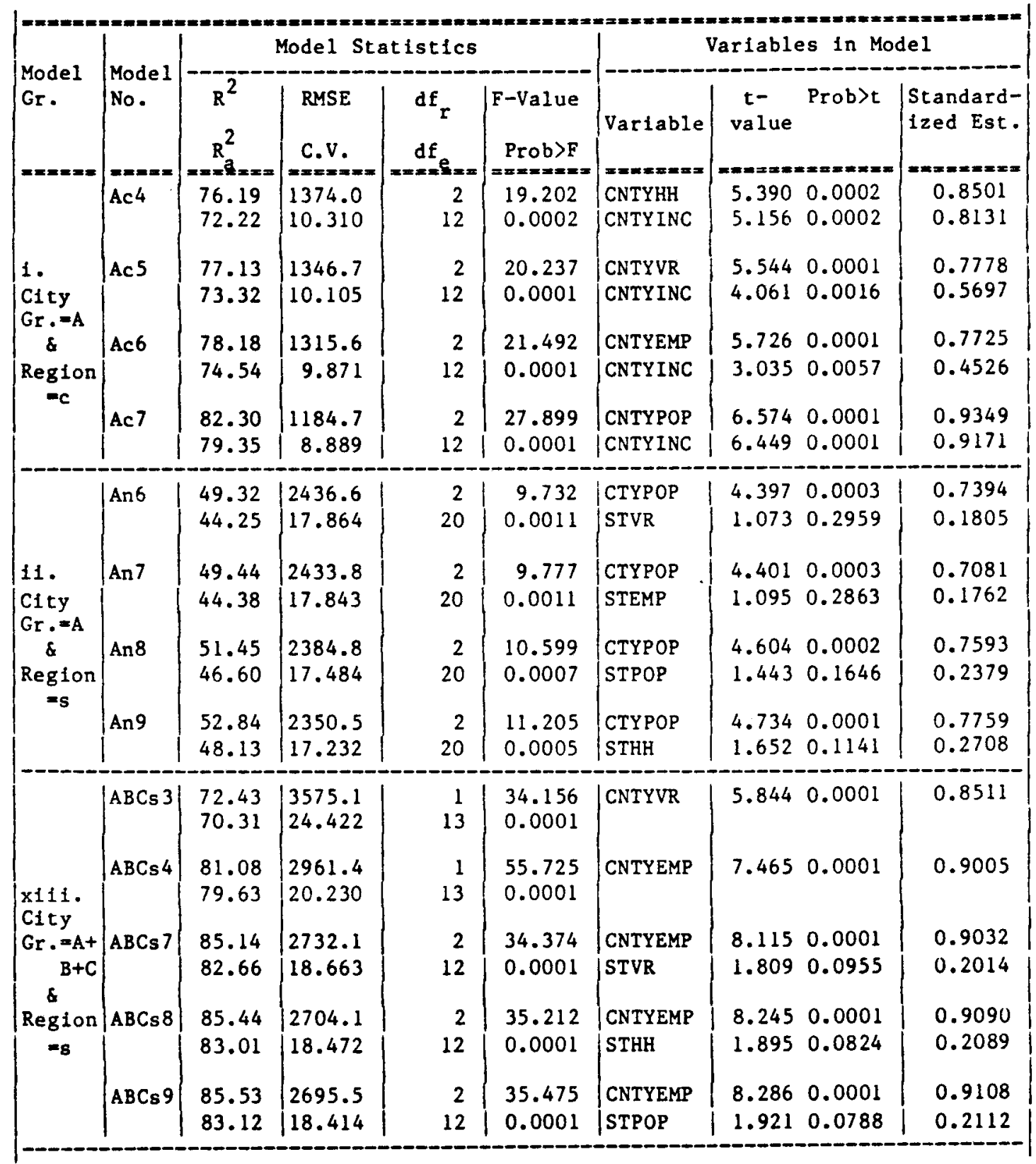


Table 6.18 , continued

\begin{tabular}{|c|c|c|c|c|c|c|c|c|c|}
\hline \multirow{2}{*}{$\begin{array}{l}\text { Model } \\
\text { Gr. } \\
\text { =e=-=e }\end{array}$} & \multirow{2}{*}{$\begin{array}{l}\text { Mode 1 } \\
\text { No. }\end{array}$} & \multicolumn{4}{|c|}{ Model Statistics } & \multicolumn{4}{|c|}{ Variables In Model } \\
\hline & & $\begin{array}{l}\mathrm{R}^{2} \\
\mathrm{R}_{\mathrm{a}}^{2}\end{array}$ & $\begin{array}{l}\text { RMSE } \\
\text { C.v. }\end{array}$ & $\begin{array}{l}d f_{r} \\
d f_{e}\end{array}$ & $\mid \begin{array}{r}\text { F-Value } \\
\text { Prob }>F\end{array}$ & Variable & $\begin{array}{l}\text { t- } \\
\text { value }\end{array}$ & Prob $>t$ & $\begin{array}{l}\text { SLandard- } \\
\text { ized Est. }\end{array}$ \\
\hline$=2= \pm x$ & $\begin{array}{l}==2=\pi \\
\mathrm{Bc} 3\end{array}$ & $\begin{array}{c}=2=0=3 \\
93.83 \\
93.21\end{array}$ & $\mid \begin{array}{l}=x=1 \\
1597.1 \\
10.576\end{array}$ & $\begin{array}{r}-5= \\
1 \\
10\end{array}$ & $\begin{array}{r}152.108 \\
0.0001\end{array}$ & $\begin{array}{l}==\ln =2=x= \\
\text { CNTYHH }\end{array}$ & $\begin{array}{l}=x=x= \\
12.333\end{array}$ & $\begin{array}{c}==x=x= \\
0.0001\end{array}$ & $\begin{array}{c}=x=1=x= \\
0.9687\end{array}$ \\
\hline $\begin{array}{l}\text { iv. } \\
\text { City } \\
\text { Gr.=B }\end{array}$ & Bc4 & $\begin{array}{l}94.14 \\
93.56\end{array}$ & $\mid \begin{array}{l}1556.2 \\
10.306\end{array}$ & $\begin{array}{r}1 \\
10\end{array}$ & $\begin{array}{r}160.736 \\
0.0001\end{array}$ & CNTYEMP & 12.678 & 0.0001 & 0.9703 \\
\hline$\stackrel{\&}{\text { Region }}$ & Bc5 & $\begin{array}{l}94.36 \\
93.79\end{array}$ & $\begin{array}{l}1527.3 \\
10.114\end{array}$ & $\begin{array}{r}1 \\
10\end{array}$ & $\begin{array}{r}167.261 \\
0.0001\end{array}$ & CNTYVR & 12.933 & 0.0001 & 0.9714 \\
\hline & Bcl2 & $\begin{array}{l}95.30 \\
94.26\end{array}$ & $\begin{array}{r}1468.8 \\
9.727\end{array}$ & $\begin{array}{l}2 \\
9\end{array}$ & $\begin{array}{l}91.336 \\
0.0001\end{array}$ & \begin{tabular}{|l} 
CNTYVR \\
CNTYINC
\end{tabular} & $\begin{array}{l}6.997 \\
1.346\end{array}$ & $\begin{array}{l}0.0001 \\
0.2111\end{array}$ & $\begin{array}{l}0.8418 \\
0.1620\end{array}$ \\
\hline & Bn 12 & $\begin{array}{l}76.74 \\
72.09\end{array}$ & $\begin{array}{l}960.4 \\
7.794\end{array}$ & $\begin{array}{r}2 \\
10\end{array}$ & $\begin{array}{l}16.498 \\
0.0007\end{array}$ & $\begin{array}{l}\text { STVR } \\
\text { CNTYINC }\end{array}$ & $\begin{array}{l}4.074 \\
3.430\end{array}$ & $\begin{array}{l}0.0022 \\
0.0064\end{array}$ & $\begin{array}{l}0.6277 \\
0.5284\end{array}$ \\
\hline $\begin{array}{l}\text { v. } \\
\text { City } \\
\text { Gr.=B }\end{array}$ & Bn 14 & $\begin{array}{l}78.67 \\
74.40\end{array}$ & $\begin{array}{l}919.8 \\
7.465\end{array}$ & $\begin{array}{r}2 \\
10\end{array}$ & $\begin{array}{l}18.436 \\
0.0004\end{array}$ & $\begin{array}{l}\text { CNTYVR } \\
\text { CNTYYNC }\end{array}$ & $\begin{array}{l}4.359 \\
3.030\end{array}$ & $\begin{array}{l}0.0014 \\
0.0127\end{array}$ & $\begin{array}{l}0.6567 \\
0.4565\end{array}$ \\
\hline${ }_{\text {Region }}^{\delta}$ & Bn 15 & $\begin{array}{l}78.80 \\
74.55\end{array}$ & $\begin{array}{l}916.9 \\
7.442\end{array}$ & $\begin{array}{r}2 \\
10\end{array}$ & $\begin{array}{l}18.579 \\
0.0004\end{array}$ & \begin{tabular}{|l} 
CNTYINC \\
STHH
\end{tabular} & $\begin{array}{l}4.482 \\
4.379\end{array}$ & $\begin{array}{l}0.0012 \\
0.0014\end{array}$ & $\begin{array}{l}0.6538 \\
0.6387\end{array}$ \\
\hline & Bn21 & $\begin{array}{l}83.95 \\
78.61\end{array}$ & $\begin{array}{l}840.8 \\
6.824\end{array}$ & $\begin{array}{l}3 \\
9\end{array}$ & $\begin{array}{l}15.697 \\
0.0006\end{array}$ & \begin{tabular}{|l} 
CNTYVR \\
CNTYINC \\
STEMP
\end{tabular} & $\begin{array}{l}3.076 \\
2.451 \\
1.722\end{array}$ & $\begin{array}{l}0.0132 \\
0.0367 \\
0.1191\end{array}$ & $\begin{array}{l}0.5039 \\
0.3629 \\
0.3033\end{array}$ \\
\hline $\begin{array}{l}\text { vil. } \\
\text { City } \\
G r=C \\
\&\end{array}$ & Cc5 & $\begin{array}{l}45.17 \\
42.56\end{array}$ & $\begin{array}{l}3406.0 \\
26.870\end{array}$ & $\begin{array}{r}2 \\
42\end{array}$ & $\begin{array}{l}17.298 \\
0.0001\end{array}$ & $\begin{array}{l}\text { CNTYINC } \\
\text { CNTYPOP }\end{array}$ & $\begin{array}{l}5.377 \\
0.967\end{array}$ & $\begin{array}{l}0.0001 \\
0.3390\end{array}$ & $\begin{array}{l}0.6344 \\
0.1141\end{array}$ \\
\hline $\begin{array}{l}\text { Region } \\
=c\end{array}$ & Cc6 & $\begin{array}{r}61.65 \\
59.82\end{array}$ & $\begin{array}{l}2848.5 \\
22.472\end{array}$ & $\begin{array}{r}2 \\
42\end{array}$ & $\begin{array}{l}33.757 \\
0.0001\end{array}$ & $\mid \begin{array}{l}\text { CNTYINC } \\
\text { CTYPOP }\end{array}$ & $\begin{array}{l}5.748 \\
4.403\end{array}$ & $\begin{array}{l}0.0001 \\
0.0001\end{array}$ & $\begin{array}{l}0.5642 \\
0.4322\end{array}$ \\
\hline $\begin{array}{l}\text { vili. } \\
\text { City }\end{array}$ & $\cos 5$ & $\begin{array}{l}33.55 \\
28.63\end{array}$ & $\mid \begin{array}{l}2271.3 \\
20.565\end{array}$ & $\begin{array}{r}2 \\
27\end{array}$ & $\begin{array}{l}6.815 \\
0.004\end{array}$ & $\begin{array}{l}\text { CNTYINC } \\
\text { CNTYVR }\end{array}$ & $\begin{array}{l}3.174 \\
0.845\end{array}$ & $\begin{array}{l}0.0037 \\
0.4056\end{array}$ & $\begin{array}{l}0.5221 \\
0.1390\end{array}$ \\
\hline $\begin{array}{c}\mathrm{Gr}:=\mathrm{C} \\
\& \\
\operatorname{Region}\end{array}$ & Cn6 & $\begin{array}{l}34.57 \\
29.73\end{array}$ & $\begin{array}{l}2253.7 \\
20.406\end{array}$ & $\begin{array}{r}2 \\
27\end{array}$ & $\begin{array}{r}7.133 \\
0.0033\end{array}$ & $\begin{array}{l}\text { CNTYINC } \\
\text { CNTYEMP }\end{array}$ & $\begin{array}{l}2.957 \\
1.071\end{array}$ & $\begin{array}{l}0.0064 \\
0.2936\end{array}$ & $\begin{array}{l}0.4965 \\
0.1798\end{array}$ \\
\hline$-n$ & Cn7 & $\begin{array}{l}40.16 \\
35.72\end{array}$ & $\begin{array}{l}2155.4 \\
19.515\end{array}$ & $\begin{array}{r}2 \\
27\end{array}$ & $\begin{array}{r}9.059 \\
0.0001\end{array}$ & $\begin{array}{l}\text { CNTYINC } \\
\text { CTYPOP }\end{array}$ & $\begin{array}{l}3.124 \\
1.943\end{array}$ & $\begin{array}{l}0.0042 \\
0.0625\end{array}$ & $\begin{array}{l}0.4829 \\
0.3003\end{array}$ \\
\hline
\end{tabular}


Table 6.18 , continued

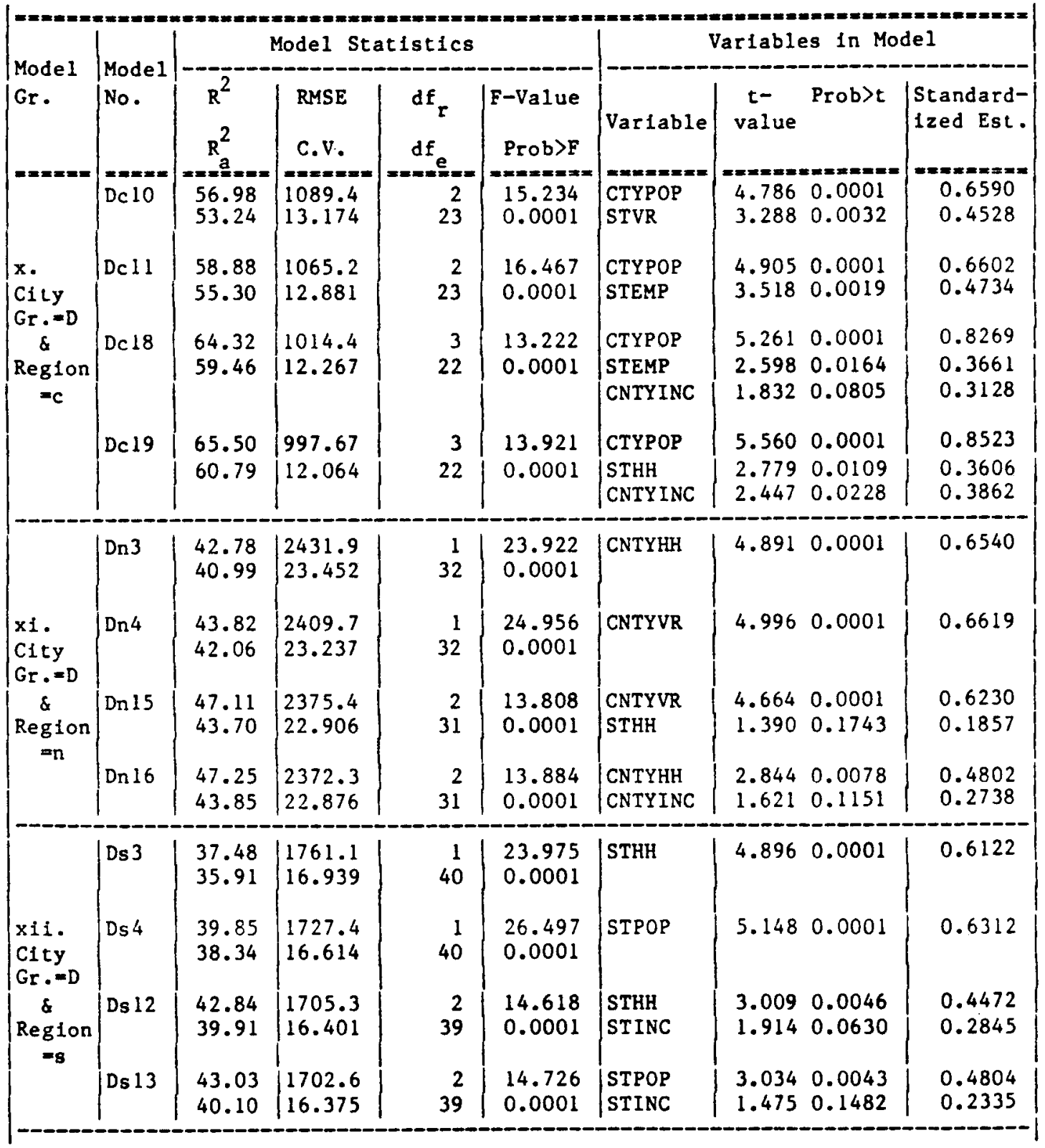


Table 6.19: Significance of Varlable(s) in Model in Pre-Influence Analysis [Urban Minor Arterial \& Collector]

\begin{tabular}{|c|c|c|c|c|c|c|c|c|c|}
\hline \multirow[b]{2}{*}{$\begin{array}{l}\text { Model } \\
\text { Gr. }\end{array}$} & \multirow[b]{2}{*}{$\begin{array}{l}\text { Mode } 1 \\
\text { No. }\end{array}$} & \multicolumn{4}{|c|}{ Model Statistics } & \multicolumn{4}{|c|}{ Variables in Model } \\
\hline & & $\mathrm{R}^{2}$ & $\begin{array}{l}\text { RMSE } \\
\text { C.v. }\end{array}$ & $d f_{r}$ & $\mid \begin{array}{r}\text { F-Value } \\
\text { Prob }>\text { F }\end{array}$ & Variable & $\begin{array}{c}t- \\
\text { value }\end{array}$ & Prob $>t$ & $\mid \begin{array}{l}\text { Standard- } \\
\text { ized Est. }\end{array}$ \\
\hline \multirow{4}{*}{$\begin{array}{l}1 . \\
\text { City } \\
\text { Gr.=A }\end{array}$} & $\begin{array}{l}==x=x \\
A 2\end{array}$ & $\begin{array}{c}=-x=2= \\
53.57 \\
50.67\end{array}$ & $\begin{array}{l}=x=3== \\
2540.9 \\
23.074\end{array}$ & $\begin{array}{r}=x x=x= \\
1 \\
16\end{array}$ & $\begin{array}{c}=3 x=18=0 \\
18.461 \\
0.0006\end{array}$ & $\begin{array}{l}==x=x=x \\
\text { CNTYINC }\end{array}$ & $\begin{array}{c}=x=x=x \\
4.297\end{array}$ & 0.0006 & $\begin{array}{c}==-1=x=x \\
0.7319\end{array}$ \\
\hline & A5 & $\begin{array}{l}62.07 \\
57.02\end{array}$ & $\begin{array}{l}2371.8 \\
21.539\end{array}$ & $\begin{array}{r}2 \\
15\end{array}$ & $\begin{array}{l}12.275 \\
0.0007\end{array}$ & $\begin{array}{l}\text { CNTYINC } \\
\text { CNTYPOP }\end{array}$ & $\begin{array}{l}3.850 \\
1.834\end{array}$ & $\begin{array}{l}0.0016 \\
0.0866\end{array}$ & $\begin{array}{l}0.6412 \\
0.3054\end{array}$ \\
\hline & A6 & $\begin{array}{l}64.04 \\
59.25\end{array}$ & $\begin{array}{l}2309.5 \\
20.973\end{array}$ & $\begin{array}{r}2 \\
15\end{array}$ & $\begin{array}{l}13.357 \\
0.0005\end{array}$ & $\begin{array}{l}\text { CNTYINC } \\
\text { CNTYVR }\end{array}$ & $\begin{array}{l}3.658 \\
2.090\end{array}$ & $\begin{array}{l}0.0023 \\
0.0541\end{array}$ & $\begin{array}{l}0.6071 \\
0.3468\end{array}$ \\
\hline & A7 & $\begin{array}{l}65.94 \\
61.39\end{array}$ & $\mid \begin{array}{l}2247.8 \\
20.412\end{array}$ & $\begin{array}{r}2 \\
15\end{array}$ & $\begin{array}{l}14.517 \\
0.0003\end{array}$ & $\begin{array}{l}\text { CNTYINC } \\
\text { CNTYEMP }\end{array}$ & $\begin{array}{l}3.150 \\
2.333\end{array}$ & $\begin{array}{l}0.0066 \\
0.0340\end{array}$ & $\begin{array}{l}0.5405 \\
0.4004\end{array}$ \\
\hline \multirow[t]{2}{*}{$\begin{array}{l}\text { i1. } \\
\text { City } \\
G r .=B\end{array}$} & | B2 & $\begin{array}{l}66.95 \\
63.94\end{array}$ & $\mid \begin{array}{l}2313.1 \\
27.638\end{array}$ & $\begin{array}{r}1 \\
11\end{array}$ & $\begin{array}{l}22.278 \\
0.0006\end{array}$ & CNTYINC & 4.720 & 0.0006 & 0.8182 \\
\hline & $\mathrm{C} 2$ & $\begin{array}{l}71.58 \\
68.99\end{array}$ & $\mid \begin{array}{l}1055.6 \\
12.641\end{array}$ & $\begin{array}{r}1 \\
11\end{array}$ & $\begin{array}{l}27.701 \\
0.0003\end{array}$ & | CNTYVR & 5.263 & 0.0003 & 0.8460 \\
\hline \multirow{2}{*}{$\begin{array}{l}\text { iii. } \\
\text { City } \\
\text { Gr.=C }\end{array}$} & |c3 & $\begin{array}{l}73.88 \\
71.50\end{array}$ & $\begin{array}{l}1012.0 \\
12.118\end{array}$ & $\begin{array}{r}1 \\
11\end{array}$ & $\begin{array}{l}31.111 \\
0.0002\end{array}$ & CNTYPOP & 5.578 & 0.0002 & 0.8595 \\
\hline & 104 & $\begin{array}{l}75.74 \\
73.53\end{array}$ & $\begin{array}{r}975.4 \\
11.679\end{array}$ & $\begin{array}{r}1 \\
11\end{array}$ & $\begin{array}{l}34.334 \\
0.0001\end{array}$ & CNTYHH & 5.860 & 0.0001 & 0.8703 \\
\hline \multirow{4}{*}{$\begin{array}{l}\text { iv. } \\
\text { CIty } \\
\text { Gr. =C }\end{array}$} & |cg & $\begin{array}{l}74.85 \\
72.05\end{array}$ & $\mid \begin{array}{l}1267.8 \\
12.371\end{array}$ & $\begin{array}{l}1 \\
9\end{array}$ & $\begin{array}{l}26.781 \\
0.0006\end{array}$ & |CNTYEMP & 5.175 & 0.0006 & 0.8651 \\
\hline & $\mathrm{ClO}$ & $\begin{array}{l}76.60 \\
74.00\end{array}$ & $\begin{array}{l}1223.0 \\
15.052\end{array}$ & $\begin{array}{l}1 \\
9\end{array}$ & $\begin{array}{l}29.456 \\
0.0004\end{array}$ & CNTYPOP & 5.427 & 0.0004 & 0.8752 \\
\hline & c11 & $\begin{array}{l}78.08 \\
75.64\end{array}$ & $\begin{array}{l}1183.6 \\
14.567\end{array}$ & $\begin{array}{l}1 \\
9\end{array}$ & $\begin{array}{l}32.058 \\
0.0003\end{array}$ & CNTYVR & 5.662 & 0.0003 & 0.8836 \\
\hline & $C 12$ & $\begin{array}{l}78.30 \\
75.88\end{array}$ & $\begin{array}{l}1177.7 \\
14.495\end{array}$ & $\begin{array}{l}1 \\
9\end{array}$ & $\begin{array}{l}32.467 \\
0.0003\end{array}$ & CNTYHH & 5.698 & 0.0003 & 0.8849 \\
\hline
\end{tabular}


Table 6.19, continued

\begin{tabular}{|c|c|c|c|c|c|c|c|c|c|}
\hline \multirow[b]{2}{*}{$\begin{array}{l}\text { Mode } 1 \\
\text { Gr. } \\
====== \\
\text { v. } \\
\text { City } \\
\text { Gr. }=D\end{array}$} & \multirow[b]{2}{*}{$\begin{array}{l}\text { Mode } 1 \\
\text { No. } \\
\text { D6 }\end{array}$} & \multicolumn{4}{|c|}{ Model Statistics } & \multicolumn{4}{|c|}{ Varlables in Model } \\
\hline & & $\begin{array}{c}R^{2} \\
R_{a}^{2} \\
==x=== \\
54.37 \\
50.72\end{array}$ & $\mid \begin{array}{c}\text { RMSE } \\
\text { C.V. } \\
==x==x= \\
2388.8 \\
38.123\end{array}$ & $\begin{array}{r}\mathrm{df}_{\mathrm{x}} \mathrm{df} \\
2 \\
25\end{array}$ & $\mid \begin{array}{c}\text { F-Value } \\
\text { Prob }>\text { F } \\
==x=x=m \\
14.893 \\
0.0001\end{array}$ & $\left|\begin{array}{l}\text { Variable } \\
======x \neq \\
\text { CNTYINC } \\
\text { CTYPOP }\end{array}\right|$ & $\begin{array}{c}t- \\
\text { value } \\
====x=s \\
3.710 \\
2.807\end{array}$ & $\begin{array}{l}\text { Prob>t } \\
===x=m=x \\
0.0011 \\
0.0095\end{array}$ & $\begin{array}{l}\text { Standard- } \\
\text { 1zed Est. } \\
===m=m=\pi \\
0.5220 \\
0.3950\end{array}$ \\
\hline $\begin{array}{l}\text { vi. } \\
\text { City } \\
\text { Gr. =D }\end{array}$ & D7 & $\begin{array}{l}53.98 \\
51.89\end{array}$ & $\begin{array}{l}1166.9 \\
26.347\end{array}$ & $\begin{array}{r}1 \\
22\end{array}$ & $\begin{array}{l}25.804 \\
0.0001\end{array}$ & CNTYINC & 5.080 & 0.0001 & 0.7347 \\
\hline
\end{tabular}


In addition to the F-value and the corresponding P-value $(P R O B>F)$, the associated degrees of freedom for the residual $\left(\mathrm{df}_{\mathbf{r}}\right)$ and the error ( $\mathrm{df}_{\mathrm{e}}$ ) are shown in Tables 6.17-6.19. The values of $\mathrm{R}^{2}, \mathrm{R}_{\mathrm{a}}^{2}$, RMSE (see Chapter 5 for definition) and coefficient of variation (C.V.) for each of the candidate models are also shown in these tables. The coefficient of variation (C.V.) is expressed as a percentage; it is a measure of the relative variation of the ratio of RMSE to the mean of the dependent variable. The C.V. is sometimes used as a standard to gauge the relative magnitude of error variation. The results of the F-test and other associated model statistics from post-influence analysis for all the candidate models of three highway classes are shown in Tables 6.20-6.22. By comparing the results of pre- and post-influence cases, one can see the significant improvements in model statistics in post-influence analysis. In general, higher values of $R^{2}, R_{a}^{2}$ and F-ratio, and lower values of RMSE and C.V., result from influence analysis.

To test the significance of each variable $\quad \mathrm{H}_{0}: \beta_{k}=0$; $H_{a}: B_{k} \neq 0$ for $\left.1<k<P-1\right)$, the usual t-test is employed. A high value of $t$ or a small $P$-value $(P R O B>t)$ concludes that the parameter involved is significant. The results of this t-test and the associated P-value (PROB $>t$ ) are presented in Tables 6.17-6.19 (pre-1nfluence case) and in Tables 6.20-6.22 (post-influence case). For a 1-variable model, the $t$ test is equivalent to the F-test because $F=t^{2}$. A P-value greater than 0.05 has been taken as an indication that the parameter involved is not significant, where 0.05 represents the user-specified risk or a level. The t-value (P-value) for CNTYVR in model $\mathrm{Cn} 5$ in pre 
Table 6.20: Significance of Varlable(s) in Model in Post-Influence Analysis [Interstate Highways]

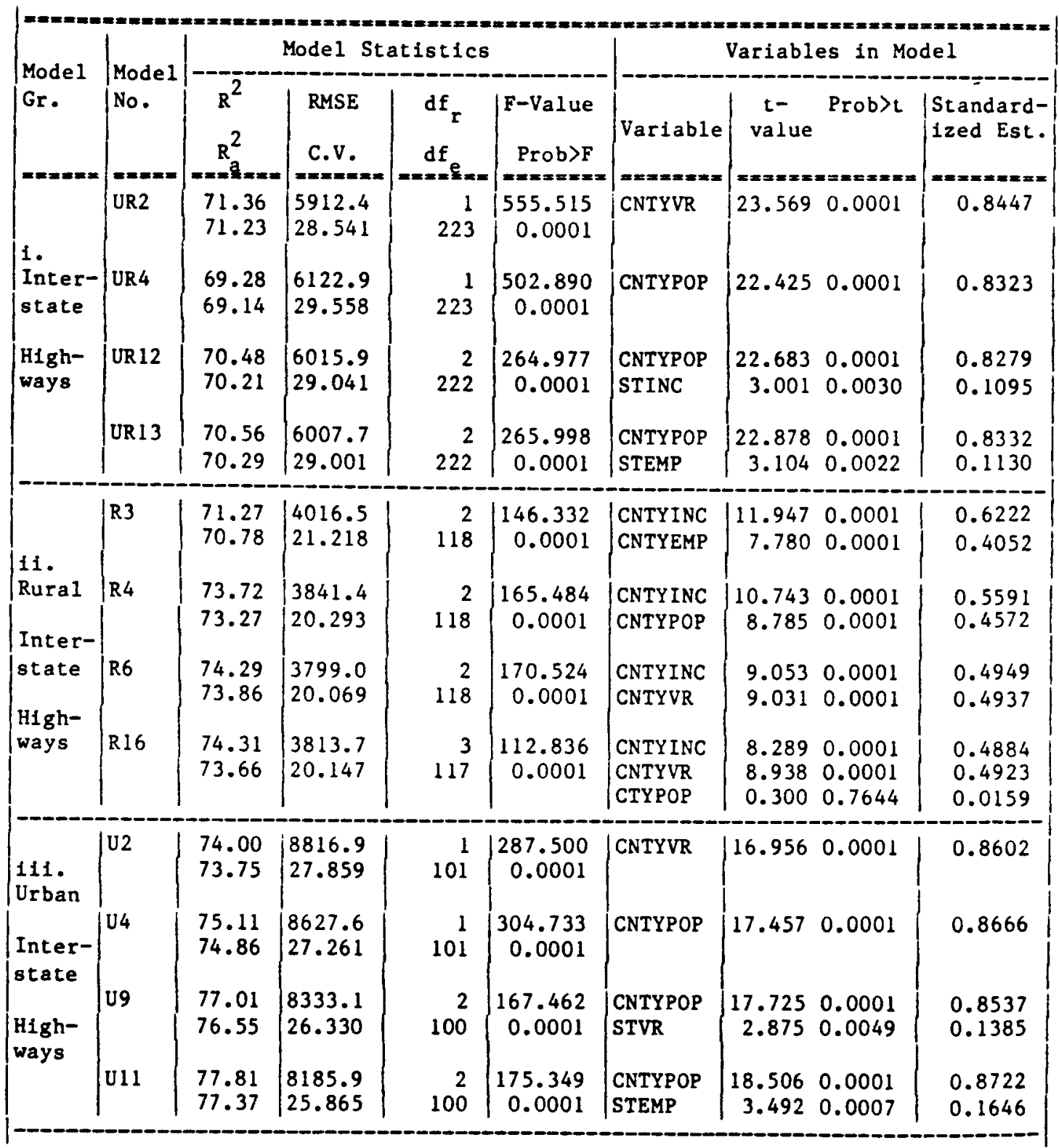


Table 6.20, continued

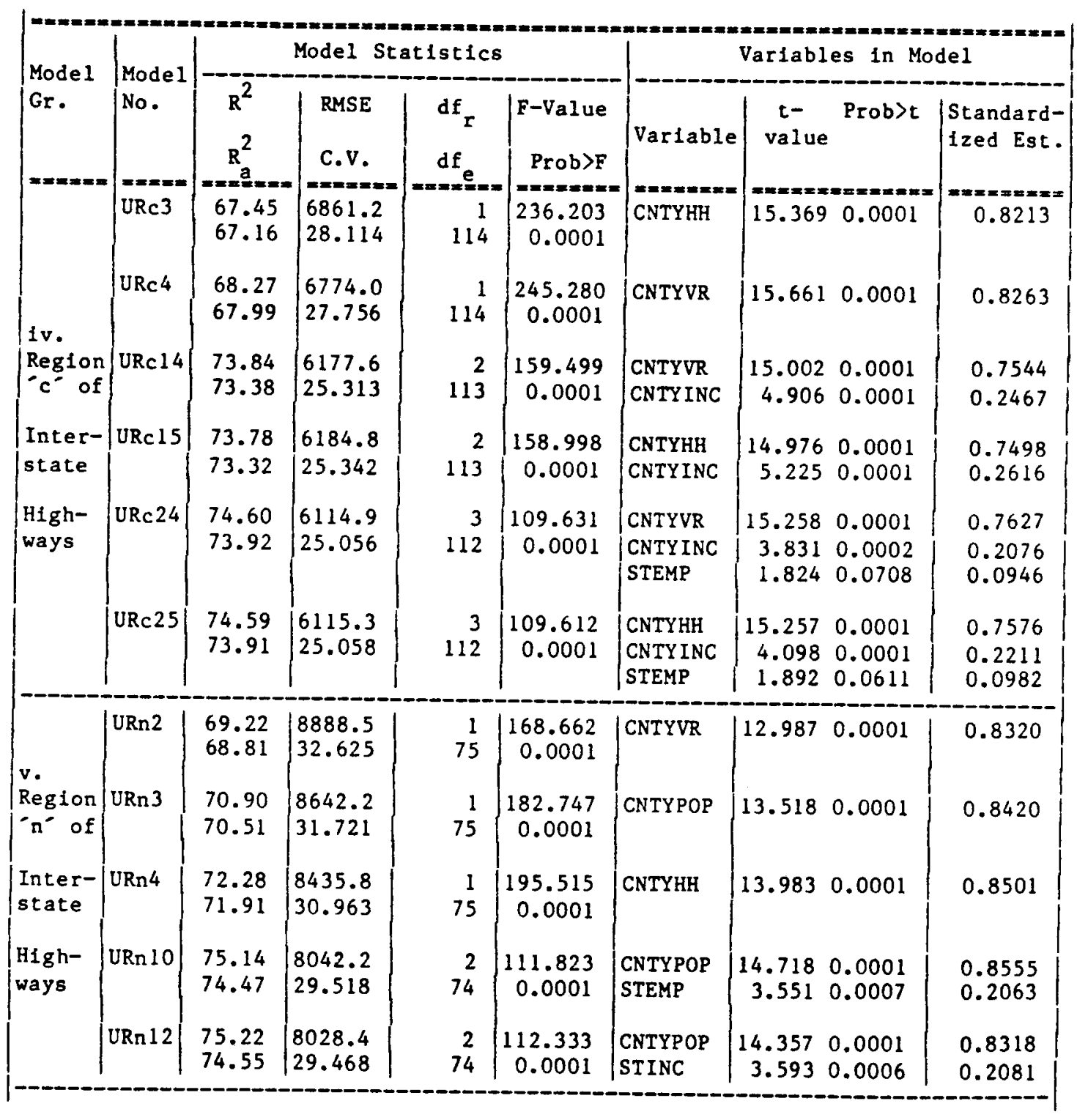


Table 6.20 , continued

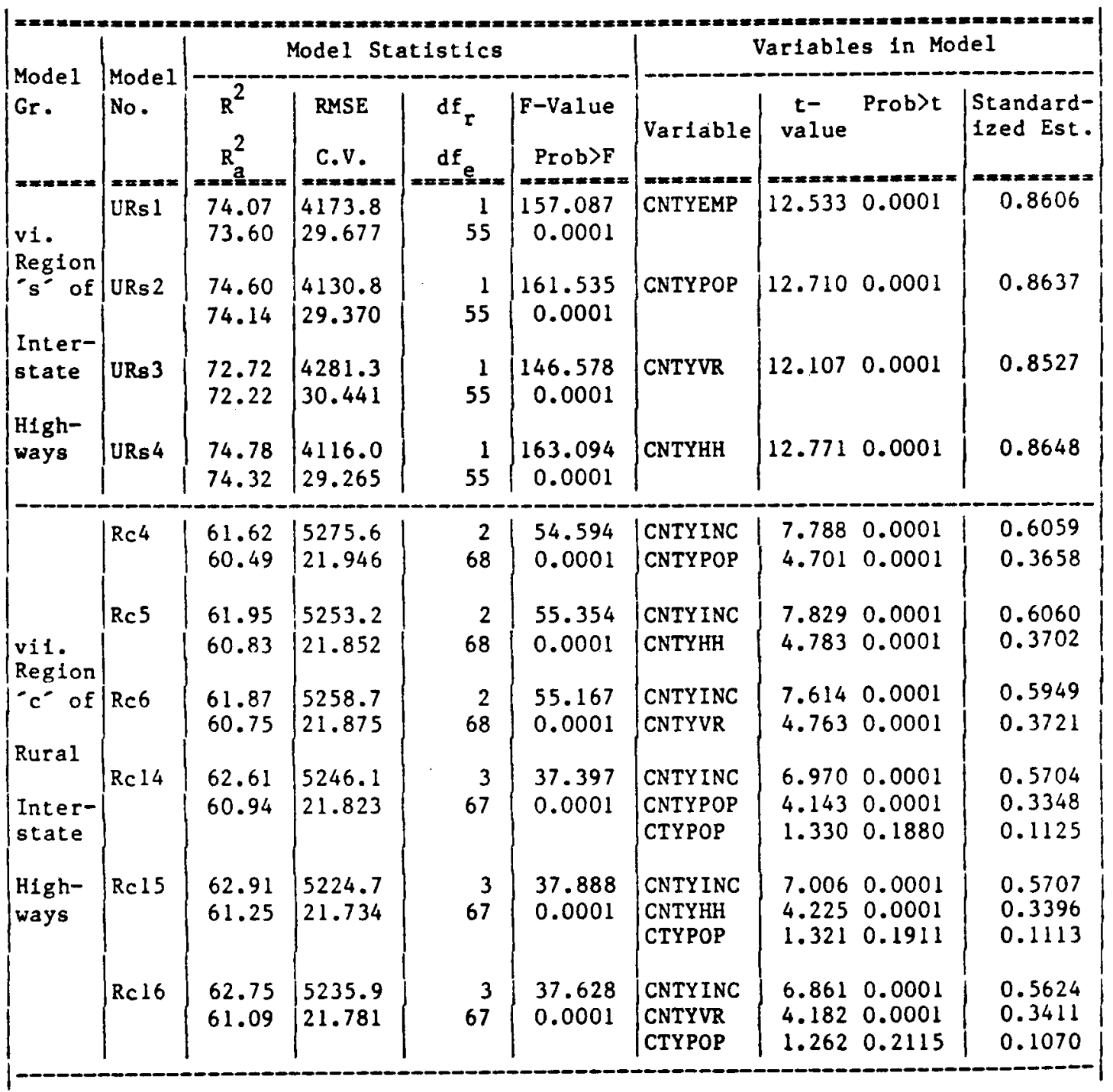


Table 6.20, continued

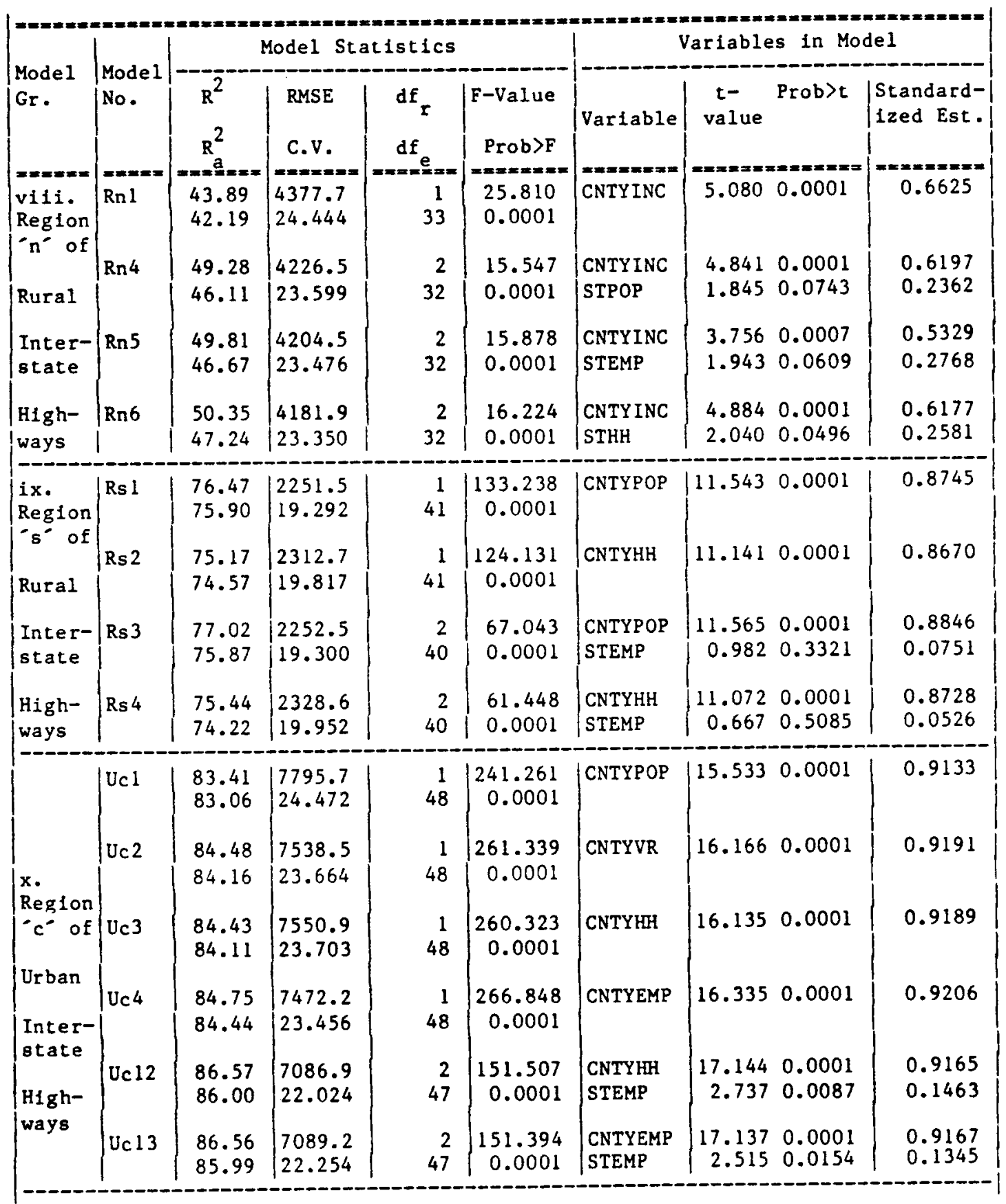


Table 6.20, continued

\begin{tabular}{|c|c|c|c|c|c|c|c|c|c|}
\hline \multirow{2}{*}{$\begin{array}{l}\text { Model } \\
\text { Gr. }\end{array}$} & \multirow{2}{*}{\begin{tabular}{|l|} 
Model \\
No.
\end{tabular} \mid} & \multicolumn{4}{|c|}{ Model Statistics } & \multicolumn{4}{|c|}{ Variables In Model } \\
\hline & & $\begin{array}{c}R^{2} \\
R_{a}^{2} \\
== \pm==\end{array}$ & $\left\{\begin{array}{l}\text { RMSE } \\
\text { C.V. } \\
== \pm=2=0\end{array}\right.$ & $\begin{array}{l}d f_{r} \\
d f_{e} \\
c=x==\end{array}$ & $\mid \begin{array}{c}\text { F-Value } \\
\text { Prob }>\text { F } \\
\text { =0mx=m=x }\end{array}$ & Variable & $\begin{array}{c}t- \\
\text { value } \\
=x= \pm= \pm=\end{array}$ & Prob $>t$ & $\begin{array}{l}\text { Standard- } \\
\text { ized Est. }\end{array}$ \\
\hline & Un2 & $\begin{array}{l}54.38 \\
53.47\end{array}$ & $\begin{array}{l}13523 \\
35.731\end{array}$ & $\begin{array}{l}1 \\
50\end{array}$ & $\begin{array}{l}59.612 \\
0.0001\end{array}$ & CNTYVR & 7.721 & 0.0001 & 0.7375 \\
\hline $\begin{array}{l}\text { Region } \\
n^{-} \text {of }\end{array}$ & $\ln 3$ & $\begin{array}{l}55.27 \\
54.38\end{array}$ & $\begin{array}{l}13391 . \\
35.383\end{array}$ & $\begin{array}{r}1 \\
50\end{array}$ & $\begin{array}{l}61.782 \\
0.0001\end{array}$ & CNTYPOP & 7.860 & 0.0001 & 0.7434 \\
\hline Urban & Un4 & $\begin{array}{l}55.64 \\
54.76\end{array}$ & $\begin{array}{l}13336 . \\
35.235\end{array}$ & $\begin{array}{r}1 \\
50\end{array}$ & $\begin{array}{l}62.721 \\
0.0001\end{array}$ & CNTYHH & 7.920 & 0.0001 & 0.7459 \\
\hline $\begin{array}{l}\text { Inter- } \\
\text { state }\end{array}$ & Un11 & $\begin{array}{l}58.57 \\
56.88\end{array}$ & $\begin{array}{l}13019 \\
34.400\end{array}$ & $\begin{array}{r}2 \\
49\end{array}$ & $\begin{array}{l}34.632 \\
0.0001\end{array}$ & $\begin{array}{l}\text { CNTYPOP } \\
\text { STEMP }\end{array}$ & $\begin{array}{l}8.243 \\
1.975\end{array}$ & $\begin{array}{l}0.0001 \\
0.0540\end{array}$ & $\begin{array}{l}0.7618 \\
0.1825\end{array}$ \\
\hline ways & Un 13 & $\begin{array}{l}59.05 \\
57.37\end{array}$ & $\begin{array}{l}12944 . \\
34.200\end{array}$ & $\begin{array}{r}2 \\
49\end{array}$ & $\begin{array}{l}35.323 \\
0.0001\end{array}$ & $\begin{array}{l}\text { CNTYPOP } \\
\text { STINC }\end{array}$ & $\begin{array}{l}8.061 \\
2.125\end{array}$ & $\begin{array}{l}0.0001 \\
0.0386\end{array}$ & $\begin{array}{l}0.7373 \\
0.1944\end{array}$ \\
\hline & Us 3 & $\begin{array}{l}79.19 \\
77.70\end{array}$ & $\begin{array}{l}4843.1 \\
15.787\end{array}$ & $\begin{array}{r}1 \\
14\end{array}$ & $\begin{array}{l}53.273 \\
0.0001\end{array}$ & CNTYHH & 7.299 & 0.0001 & 0.8899 \\
\hline $\mid \begin{array}{l}x i 1 \\
\text { Region } \\
\text { - }- \text { of }\end{array}$ & Us4 & $\begin{array}{l}81.58 \\
80.27\end{array}$ & $\begin{array}{l}4556.2 \\
14.852\end{array}$ & $\begin{array}{r}1 \\
14\end{array}$ & $\begin{array}{l}62.012 \\
0.0001\end{array}$ & CNTYVR & 7.875 & 0.0001 & 0.9032 \\
\hline Urban & Us 10 & $\begin{array}{l}84.90 \\
82.57\end{array}$ & $\begin{array}{l}4281.8 \\
13.957\end{array}$ & $\begin{array}{r}2 \\
13\end{array}$ & $\begin{array}{l}36.535 \\
0.0001\end{array}$ & $\begin{array}{l}\text { CNTYEMP } \\
\text { STVR }\end{array}$ & $\begin{array}{l}6.993 \\
3.890\end{array}$ & $\begin{array}{l}0.0001 \\
0.0019\end{array}$ & $\begin{array}{l}0.7613 \\
0.4235\end{array}$ \\
\hline $\begin{array}{l}\text { Inter- } \\
\text { state }\end{array}$ & Us 11 & $\begin{array}{l}88.13 \\
86.30\end{array}$ & $\begin{array}{l}3796.3 \\
12.375\end{array}$ & $\begin{array}{r}2 \\
13\end{array}$ & $\begin{array}{l}48.244 \\
0.0001\end{array}$ & $\begin{array}{l}\text { CNTYVR } \\
\text { STVR }\end{array}$ & $\begin{array}{l}8.108 \\
2.677\end{array}$ & $\begin{array}{l}0.0001 \\
0.0190\end{array}$ & $\begin{array}{l}0.8174 \\
0.2698\end{array}$ \\
\hline $\begin{array}{l}\text { High- } \\
\text { ways }\end{array}$ & Us 12 & $\begin{array}{l}90.50 \\
89.04\end{array}$ & $\begin{array}{l}3395.6 \\
11.068\end{array}$ & $\begin{array}{r}2 \\
13\end{array}$ & $\begin{array}{l}61.928 \\
0.0001\end{array}$ & $\begin{array}{l}\text { CNTYVR } \\
\text { STHH }\end{array}$ & $\begin{array}{l}9.476 \\
3.494\end{array}$ & $\begin{array}{l}0.0001 \\
0.0040\end{array}$ & $\begin{array}{l}0.8324 \\
0.3069\end{array}$ \\
\hline & Us 13 & $\begin{array}{l}88.86 \\
87.15\end{array}$ & $\begin{array}{l}3676.7 \\
11.985\end{array}$ & $\begin{array}{r}2 \\
13\end{array}$ & $\begin{array}{l}51.863 \\
0.0001\end{array}$ & $\begin{array}{l}\text { CNTYEMP } \\
\text { STHH }\end{array}$ & $\begin{array}{l}8.364 \\
5.015\end{array}$ & $\begin{array}{l}0.0001 \\
0.0002\end{array}$ & $\begin{array}{l}0.8005 \\
0.4646\end{array}$ \\
\hline
\end{tabular}


Table 6.21: Significance of Varlable(s) in Model in Post-Influence Analysis [Urban Principal Arterial]

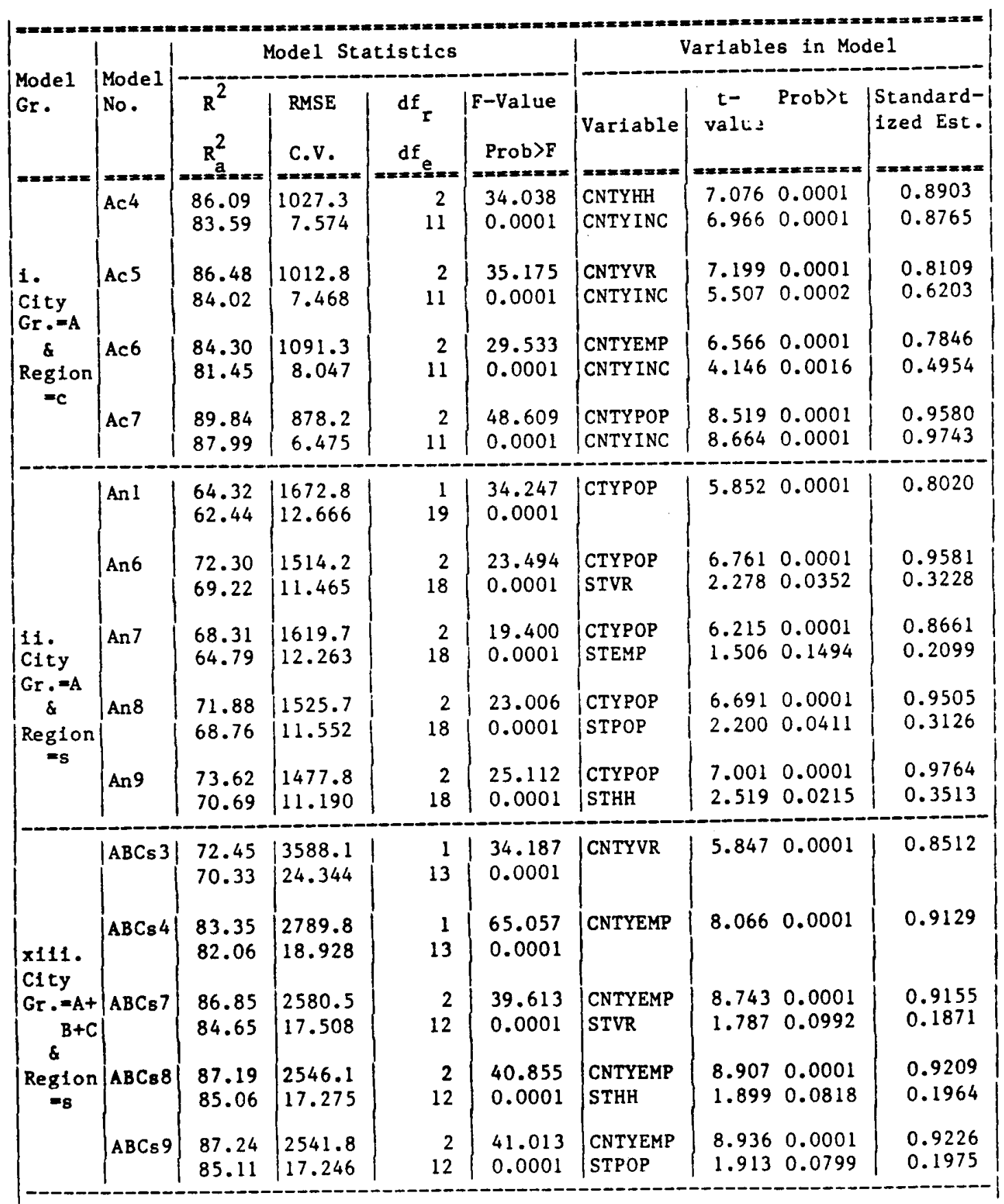


Table 6.21, continued

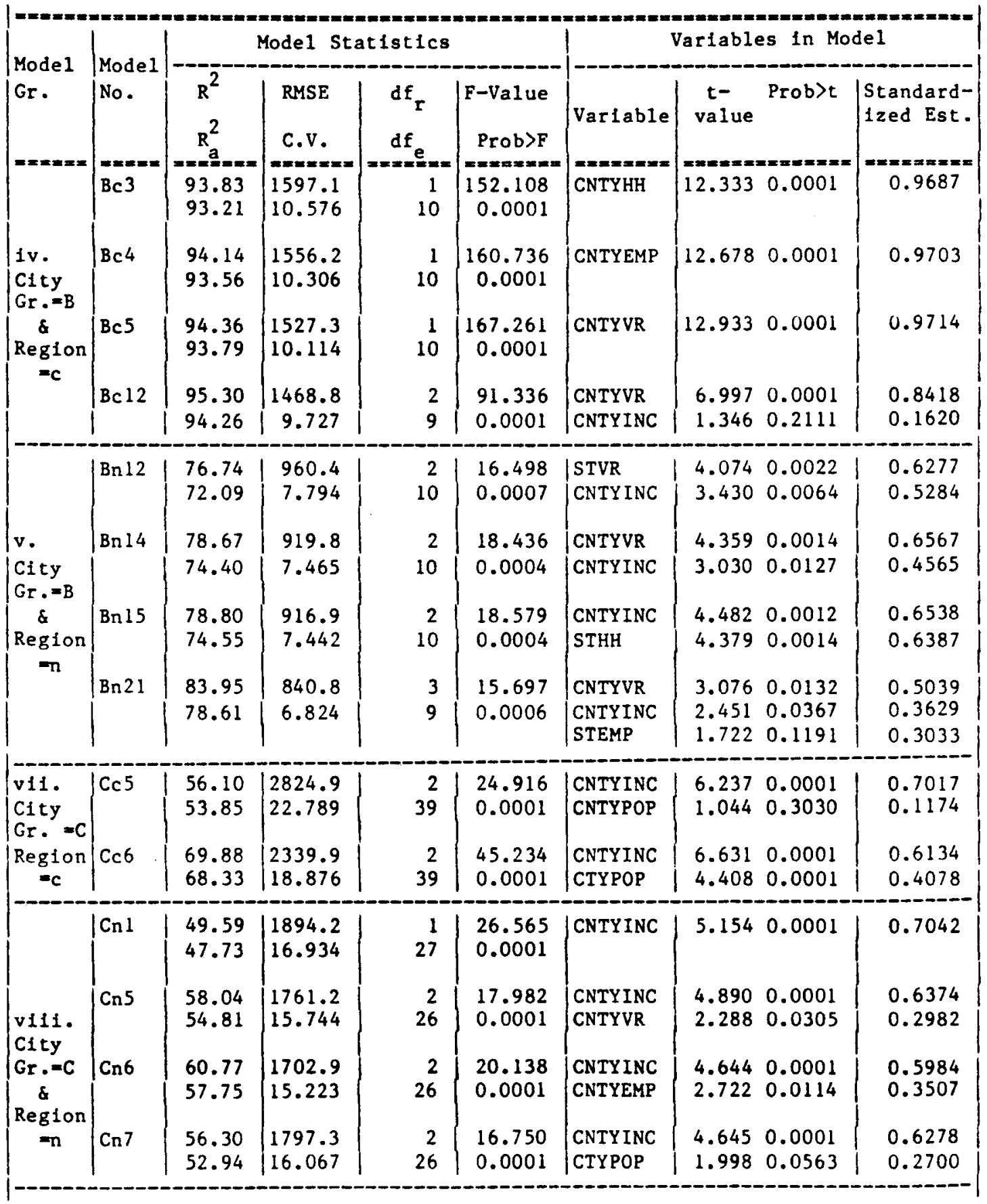


Table 6.21, continued

\begin{tabular}{|c|c|c|c|c|c|c|c|c|c|}
\hline \multirow{3}{*}{$\begin{array}{l}\text { Model } \\
\text { Gr. }\end{array}$} & \multirow{2}{*}{$\begin{array}{l}\text { Mode1 } \\
\text { No. }\end{array}$} & \multicolumn{4}{|c|}{ Model Statistics } & \multicolumn{4}{|c|}{ Variables in Model } \\
\hline & & $\mathrm{R}^{2}$ & RMSE & $\mathrm{df}_{\mathrm{r}}$ & F-Value & Variable & $\begin{array}{c}t- \\
\text { value }\end{array}$ & Prob $>t$ & $\mid \begin{array}{l}\text { Standard- } \\
\text { Ized Est. }\end{array}$ \\
\hline & $==5= \pm$ & $=m=\frac{a}{2}=x=$ & C.V. & $==x=E=$ & 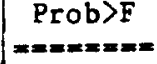 & & & & \\
\hline \multirow{7}{*}{$\begin{array}{l}x . \\
\text { City } \\
G r .=D \\
\& \\
\text { Region } \\
=c\end{array}$} & Dc 10 & $\begin{array}{l}70.44 \\
67.76\end{array}$ & $\begin{array}{r}915.4 \\
11.093\end{array}$ & $\begin{array}{r}2 \\
22\end{array}$ & $\begin{array}{l}26.218 \\
0.0001\end{array}$ & \begin{tabular}{|l} 
CTYPOP \\
STVR
\end{tabular} & $\begin{array}{l}6.486 \\
3.767\end{array}$ & $\begin{array}{l}0.0001 \\
0.0011\end{array}$ & $\begin{array}{l}0.7546 \\
0.4382\end{array}$ \\
\hline & Dc11 & 75.05 & 841.1 & 2 & 33.085 & CTYPOP & 7.157 & 0.0001 & 0.7661 \\
\hline & & 72.78 & 10.193 & 22 & 0.0001 & STEMP & 4.568 & 0.0001 & 0.4890 \\
\hline & De18 & 76.96 & 827.2 & 3 & 23.384 & |CTYPOP & 6.816 & 0.0001 & 0.8568 \\
\hline & & 73.67 & 10.024 & 21 & 0.0001 & $\begin{array}{l}\text { STEMP } \\
\text { CNTYINC }\end{array}$ & $\begin{array}{l}3.648 \\
1.321\end{array}$ & $\begin{array}{l}0.0015 \\
0.2008\end{array}$ & $\begin{array}{l}0.4240 \\
0.1811\end{array}$ \\
\hline & Dc19 & 74.16 & 876.2 & 3 & 20.086 & CTYPOP & 6.739 & 0.0001 & 0.8902 \\
\hline & & 70.46 & 10.617 & 21 & 0.0001 & \begin{tabular}{|l} 
STHH \\
CNTYINC
\end{tabular} & $\begin{array}{l}3.096 \\
2.265\end{array}$ & $\begin{array}{l}0.0055 \\
0.0342\end{array}$ & $\begin{array}{l}0.3515 \\
0.3049\end{array}$ \\
\hline \multirow{4}{*}{$\begin{array}{l} \\
x 1 . \\
\text { City } \\
\text { Gr.=D } \\
\quad \& \\
\operatorname{Region} \\
\quad=n\end{array}$} & Dn3 & $\begin{array}{l}50.52 \\
48.92\end{array}$ & $\mid \begin{array}{l}2204.0 \\
21.573\end{array}$ & $\begin{array}{r}1 \\
31\end{array}$ & $\begin{array}{l}31.650 \\
0.0001\end{array}$ & |CNTYHH & 5.626 & 0.0001 & 0.7108 \\
\hline & Dn 4 & $\begin{array}{l}51.81 \\
50.26\end{array}$ & $\begin{array}{l}2175.0 \\
21.289\end{array}$ & $\begin{array}{l}1 \\
31\end{array}$ & $\begin{array}{l}33.332 \\
0.0001\end{array}$ & CNTYVR & 5.773 & 0.0001 & 0.7198 \\
\hline & Dn15 & $\begin{array}{l}53.32 \\
50.21\end{array}$ & $\begin{array}{l}2176.1 \\
21.300\end{array}$ & $\begin{array}{r}2 \\
30\end{array}$ & $\begin{array}{l}17.133 \\
0.0001\end{array}$ & \begin{tabular}{|l} 
CNTYVR \\
STHH
\end{tabular} & $\begin{array}{l}5.392 \\
0.984\end{array}$ & $\begin{array}{l}0.0001 \\
0.3331\end{array}$ & $\begin{array}{l}0.6909 \\
0.1261\end{array}$ \\
\hline & Dn 16 & $\begin{array}{l}52.35 \\
49.17\end{array}$ & $\begin{array}{l}2198.6 \\
21.519\end{array}$ & $\begin{array}{r}2 \\
30\end{array}$ & $\begin{array}{l}16.481 \\
0.0001\end{array}$ & $\mid \begin{array}{l}\text { CNTYHH } \\
\text { CNTYINC }\end{array}$ & $\begin{array}{l}3.553 \\
1.074\end{array}$ & $\begin{array}{l}0.0013 \\
0.2913\end{array}$ & $\begin{array}{l}0.5932 \\
0.1793\end{array}$ \\
\hline \multirow{5}{*}{$\begin{array}{l}\text { xif. } \\
\text { City } \\
\text { Gr.=D } \\
\& \\
\operatorname{Region} \\
=-8\end{array}$} & Ds 3 & $\begin{array}{l}45.61 \\
44.14\end{array}$ & $\mid \begin{array}{l}1290.0 \\
12.653\end{array}$ & $\begin{array}{r}1 \\
37\end{array}$ & $\begin{array}{l}31.023 \\
0.0001\end{array}$ & STHH & 5.570 & 0.0001 & 0.6753 \\
\hline & Ds 4 & $\begin{array}{l}52.85 \\
51.58\end{array}$ & $\begin{array}{l}1201.0 \\
11.781\end{array}$ & $\begin{array}{r}1 \\
37\end{array}$ & $\begin{array}{l}41.481 \\
0.0001\end{array}$ & STPOP & 6.441 & 0.0001 & 0.7270 \\
\hline & Ds 12 & 50.88 & 1242.9 & 2 & 18.641 & STHH & 3.659 & 0.0008 & 0.5181 \\
\hline & & & & 50 & & J IINC & • & & 0.2102 \\
\hline & Ds 13 & $\begin{array}{l}54.82 \\
52.31\end{array}$ & $\mid \begin{array}{l}1191.9 \\
11.690\end{array}$ & $\begin{array}{r}2 \\
36\end{array}$ & $\begin{array}{l}21.840 \\
0.0001\end{array}$ & \begin{tabular}{|l} 
STPOP \\
STINC
\end{tabular} & $\begin{array}{l}4.207 \\
1.251\end{array}$ & $\begin{array}{l}0.0002 \\
0.2189\end{array}$ & $\begin{array}{l}0.6112 \\
0.1818\end{array}$ \\
\hline
\end{tabular}


Table 6.22: Significance of Vartable(s) in Model in Post-Influence Analysis [Urban Minor Arterial \& Collector]

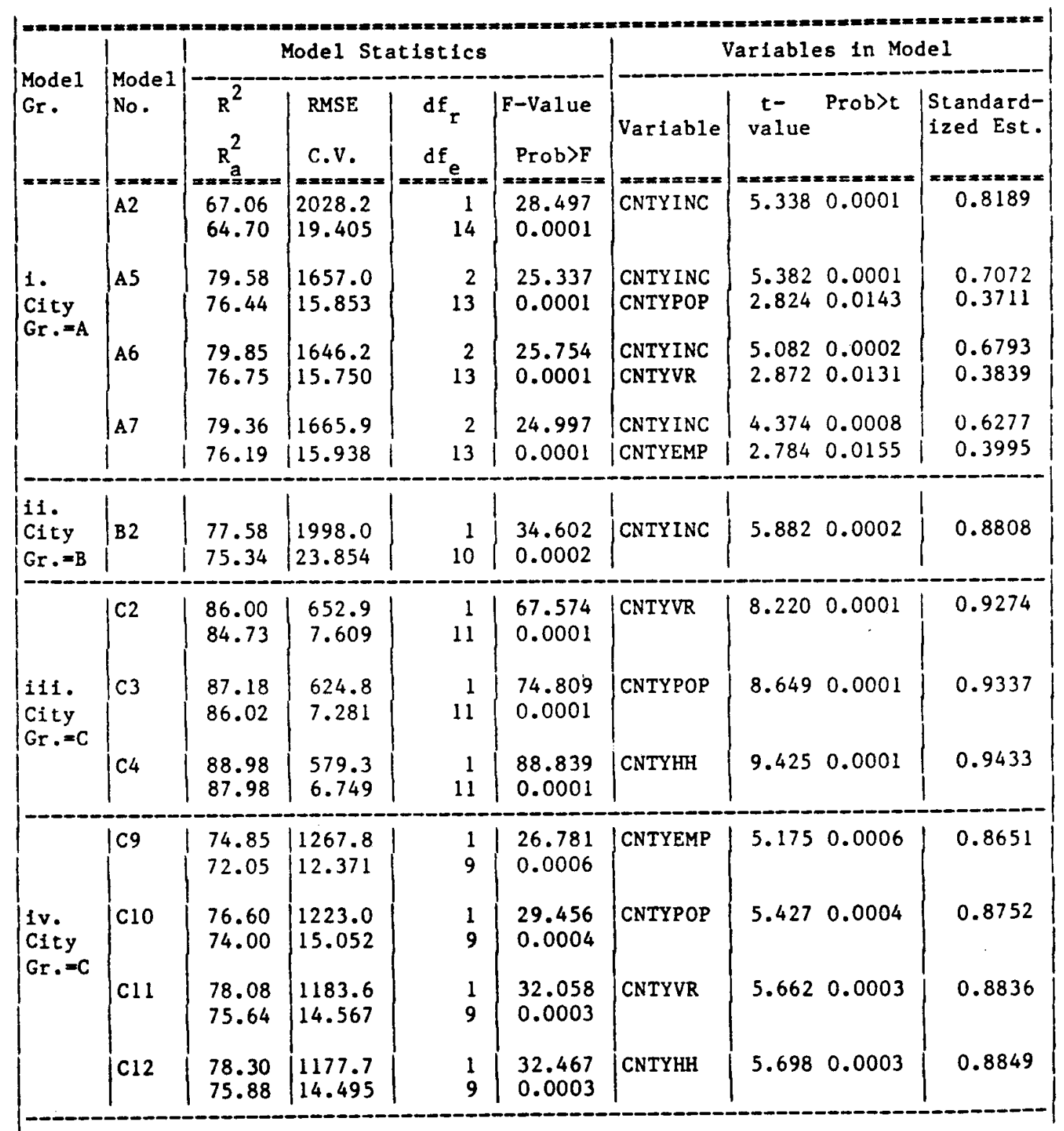


Table 6.22, continued

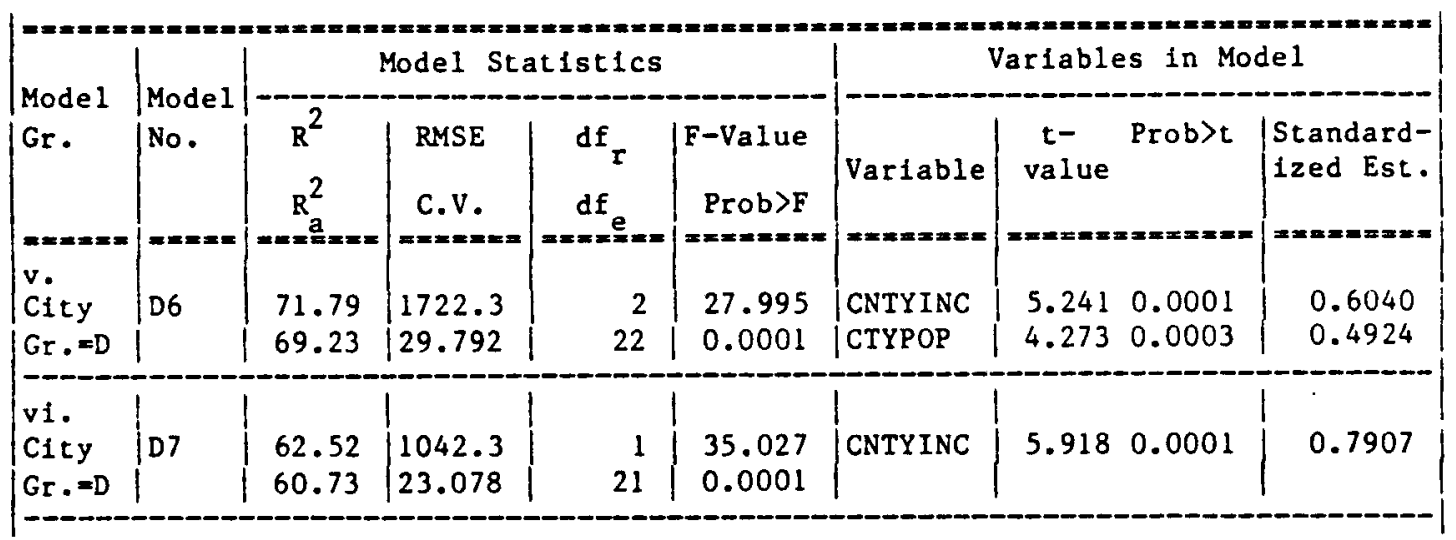


and post influence cases is $0.845(0.4056)$ and $2.288(0.0305)$, respectively. The partial regression residual plot in Figure 6.2 is also an Indication of nonsignificance of the predictor variable in the preinfluence case, because the plot is scattered and because the presence of an outlier causes a mild slope. Removing the outlier resulted in an increased slope and less scatter (see Figure 6.12). Thus, the results for model Ca5 prove that a variable that was found to be nonsignificant in the pre-influence case may become significant in the post-influence case. A decreased slope could also happen, if the outlier(s) had caused the particular predictor variable to be less significant. Additional candidate models are evaluated (if necessary) In the post-influence case by dropping the nonsignificant variable in the pre-influence case. For example, model Cnl (see Table 6.21) is considered in the post-influence case by dropping variable CNTYVR from $\mathrm{Cn} 5$ of the pre-influence case. In section 6.5, only the models with significant variables in their equations will be considered to find the final selection.

To test the significance of each subset with more than one variable $\left(H_{0}: B_{1}=\ldots=\beta_{j}=0 ; H_{a}:\right.$ all $\beta_{j} \neq 0$ for $\left.1<j<P-1\right)$, a general 1inear test [Neter et a1. 1985] was employed. The applicable Fstatistic is shown in equation 6.7.

$$
F^{*}=\frac{\frac{\operatorname{SSE}(R)-\operatorname{SSE}(F)}{d f_{R}-d f}}{\frac{\operatorname{SSE}(F)}{d f}}
$$

where, 


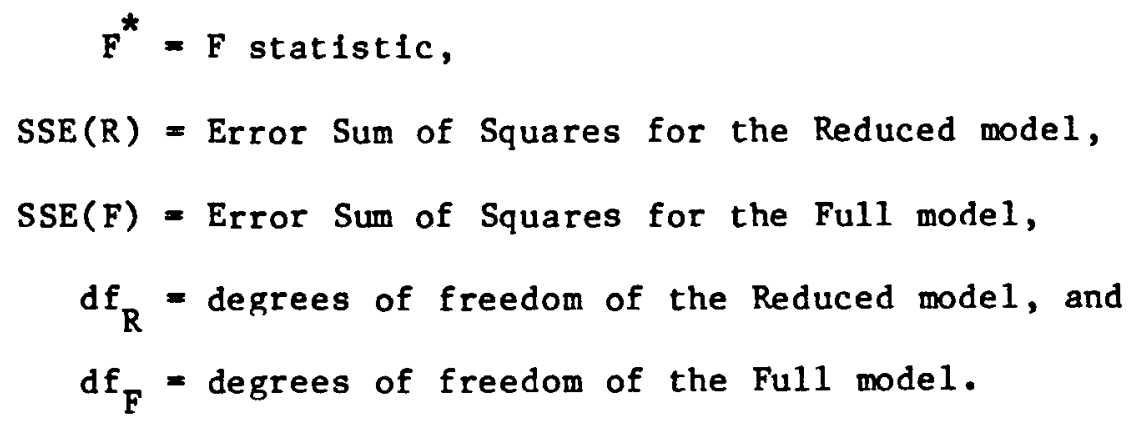

The reduced model was obtained by dropping the element(s) to be tested from the full model under $\mathrm{H}_{0}$. The test results (not presented) show that when variables are dropped from the model, there still exist regression relationships. The hypothesis $\mathrm{H}_{\mathrm{a}}$ cannot be rejected at an $\alpha$ level of 0.01 .

\subsubsection{Standardized Regression Coefficients}

Ordinarily, it is difficult to compare regression coefficients because of differences in the units involved (for example, income in dollars and population in number of persons). Standardized regression coefficients help to compare the regression coefficients. The standardized regression coefficients $\left(B_{f}^{-} s\right)$ are defined as follows:

$$
B_{j}=b_{j}\left[\frac{s_{j}}{s_{Y}}\right]
$$

where $s_{j}$ and $s_{Y}$ are the standard deviation of $X_{j}$ and $Y$ observations, respectively, and $b_{j}$ is the parameter estimate of $x_{j}$ (i.e., $\beta_{j}$ of Equation 5.1). The term in brackets makes $B_{j}$ dimensionless. The coefficient of $B_{j}$ reflects the change in the independent variable $x_{j}$ (In units of standard deviation of $Y$ ) per unit change in the indepen- 
dent variable $x_{j}$ (in units of standard deviation of $x_{j}$ ) when all other independent variables are held constant. [Neter et. a1. 1985]

The standardized estimate for each variable of the model is shown in the last column of Tables 6.19-6.24. Interpretation regarding the importance of independent variables in regression coefficients can be affected by the problem of multicollinearity. So, interpretation is not wise if multicollinearity exists. But, tests in Section 6.4.1 have shown that in most of the selections, the problem of multicollinearity is efther absent or not significant. Thus, the values of standardized estimates provide information about the importance of variables in the models and for comparison of different candidate models.

\subsection{Final Model Selection}

The preceding sections have demonstrated the usefulness of influence analysis through improvement in statistical results. For example, influence analysis has satisfied the normality assumption of regression analysis. It has also avoided the awkward transformations often needed to satisfy the normality assumptions. All the final model selections are based on the results of post-influence analysis. The results (see Tables $6.8-6.10$ ) of normality statistics of residuals in "post-influence" indicate that all the selected groups except Group 1 (Interstate Highways) have models that satisfy the normality assumptions of residuals. Because of the Information described above and the high number of influential observations for overall/combined analysis of Interstate Highways, Group 1 of interstate highways has 
not been considered further to select any model from this group. Instead, regional analysis (Groups iv-vi in Section 5.6.1) of the combined urban-rural mode1, separate rural and urban analysis (Groups 11 and $11 i$ ) and combined regional analysis (Groups vil-xi1) of Interstate Highways have been used to select one final model from each of these groups. Thus, three alternate elasticity models for Interstate Highways are possible. One such model has finally selected from the candidate model(s) for each group of non-interstate highways.

Both statistical results and judgment are employed to decide the final selections. The following criteria are employed to draw the final selection from the candidate models for each group under consideration.

1. Elimination of candidate models having nonsignificant predictor variables. (Tables 6.20-6.22)

2. Comparison of the results of the standard regression assumptions (for example, normality and homogeneity) for the candidate models. (Tables $6.8-6.10)$

3. Overall significance of the candidate models. In other words, how useful is the candidate model. (Tables 6.20-6.22)

4. Comparative evaluation of the collinearity statistics of the candidate models. (Tables $6.14-6.16$ )

5. Comparison of some model statistics $\left(R^{2}, R_{a}^{2}\right.$, RMSE, C.V., standardized regression coefficients etc.) for the candidate models. Elimination of candidate model(s) having more predictor varlables 
without having a significant change in the quality of results in comparison to the models with fewer predictor variable(s). In other words, the final selection should lead to parsimony - a decrease in number of variables with minor cost in statistical quality.

6. Consideration of the quality (avallability, cost and reliabllity - See Chapter 4) of the predictor variables in the model. For almost identical statistical results for candidate models, the order of importance of predictor variables (See Section 5.6) has been considered to evaluate the candidate models and to make the final model selection.

The first four criteria are primarily considered to select the final model. Criteria 5 and 6 have been taken into account in candidate model selection (Chapter 5). In final model selection, the last two criteria are used to break any ties that result from the first four criteria.

The final regression equations, along with the $R^{2}$ values and elasticities, are presented in Tables $6.23,6.24$ and 6.25 for Interstates, Urban Principal Arterial, and Urban Minor Arterial \& Collector, respectively. The elasticities shown in these tables were obtained from the output of Multiple Linear Regression on final selected varlables computed according to Equation (3.4) of Chapter 3. The equations that resulted from the specifled criteria are the best possible, considering all the limftations. The equations in the final selections use variables that are easily available from a variety of sources for both historical and future trends, or else future values 
Table 6.23: Final Regression Equations for Interstate Highways

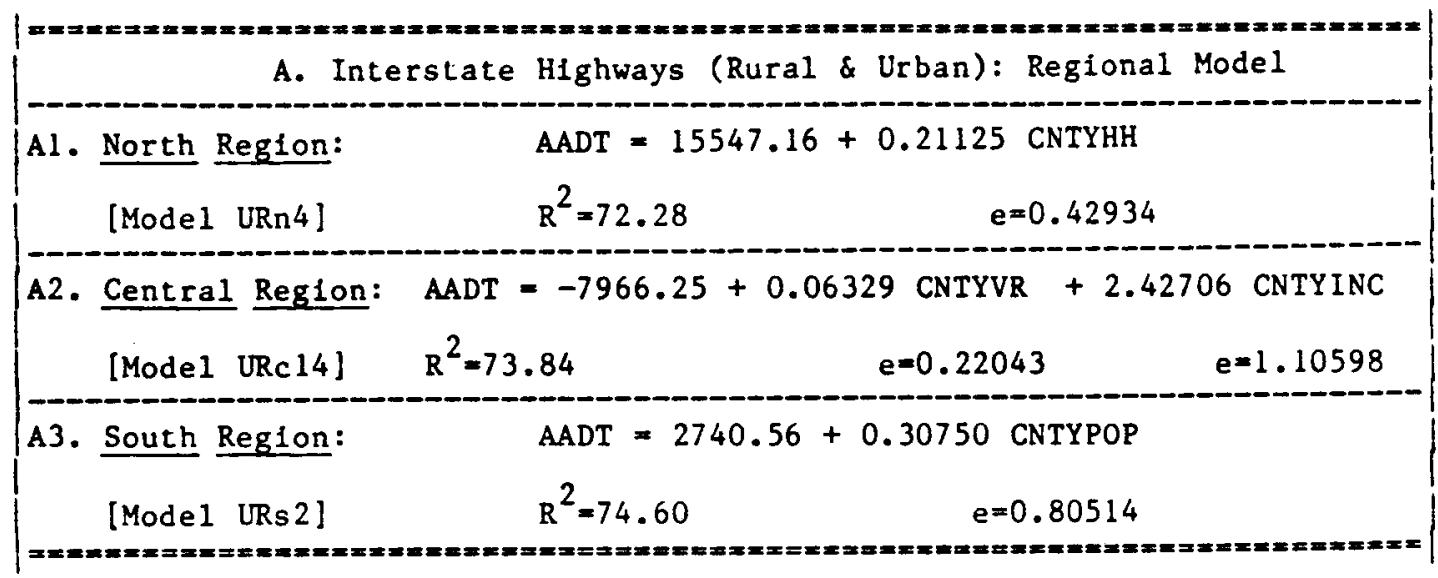

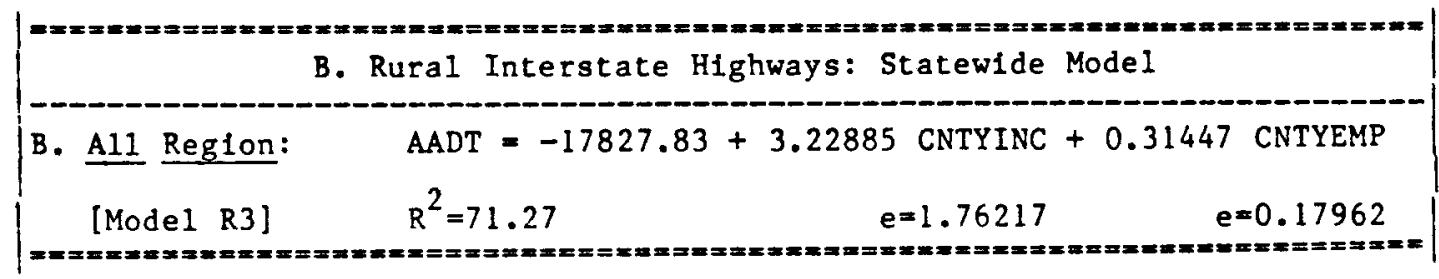

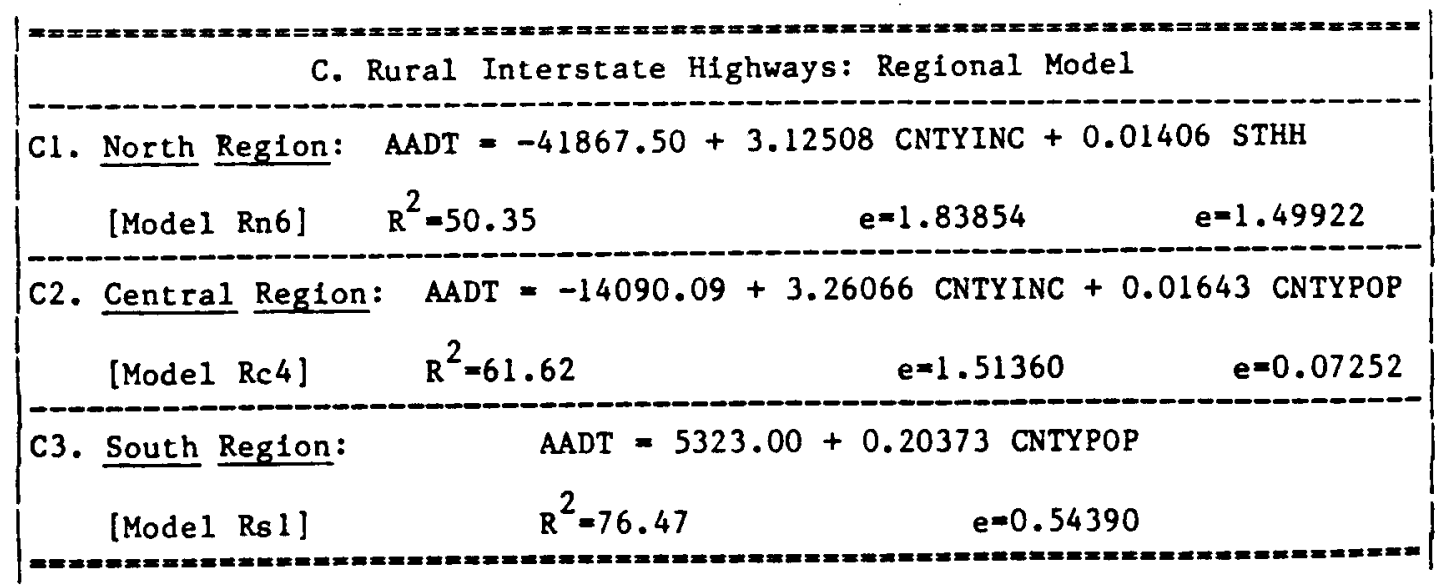


Table 6.23, continued

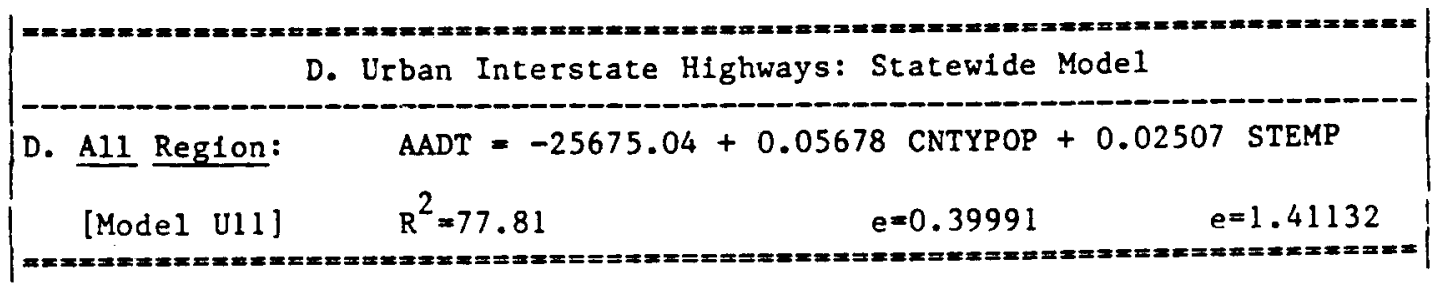

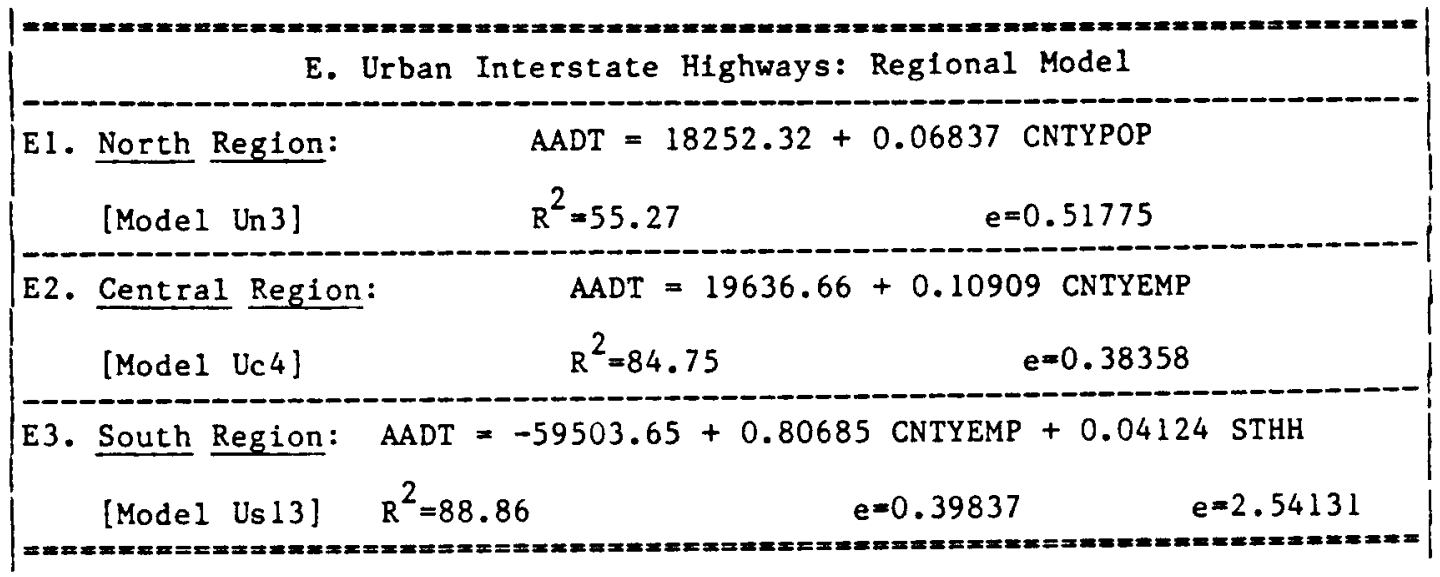

Note: See Table 4.3 for description of variables. 
Table 6.24: Final Regression Equations for Urban Principal Arterial

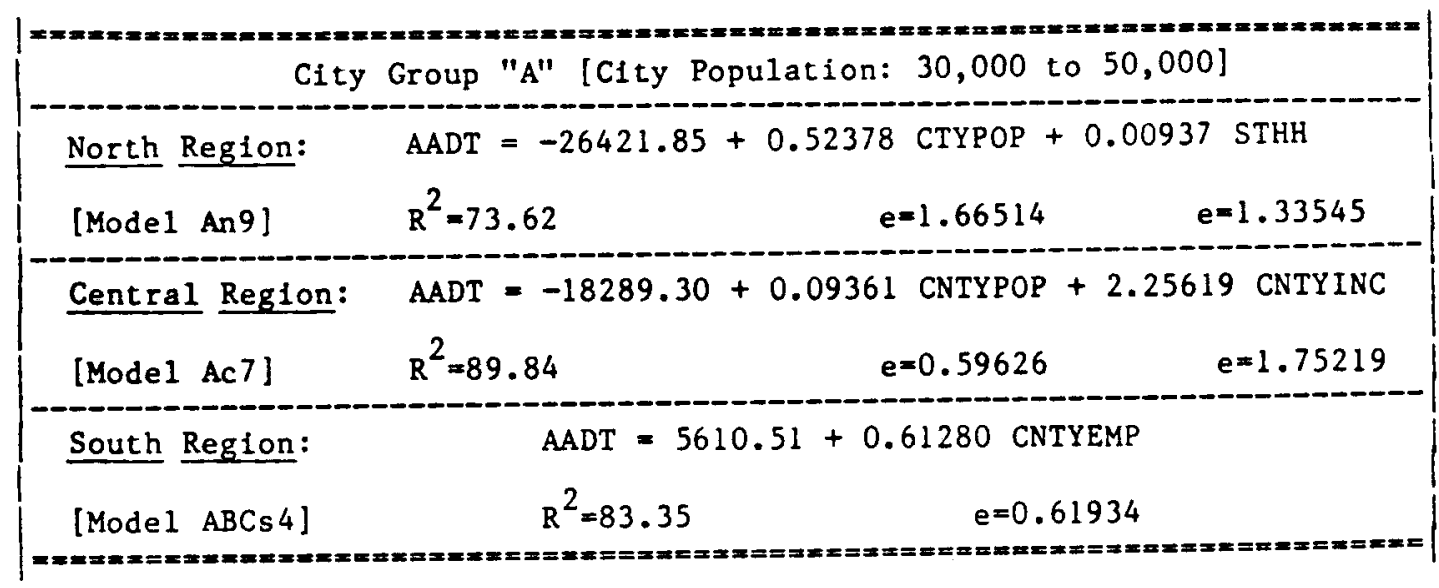

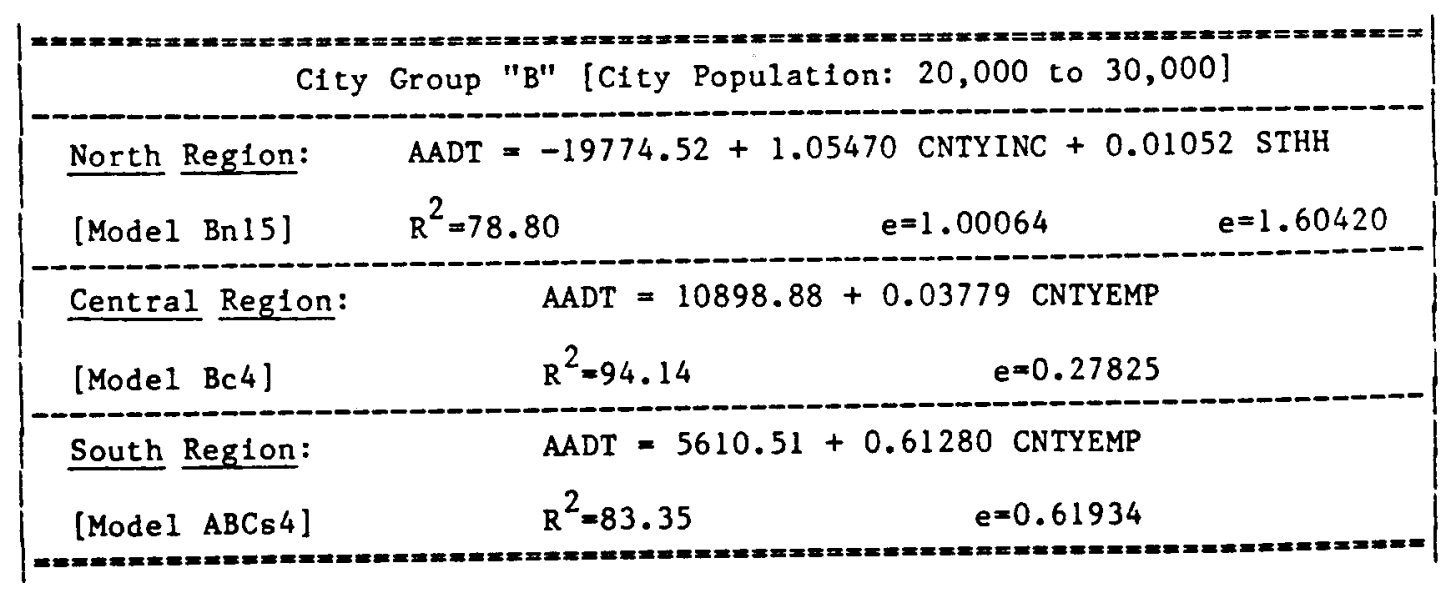


Table 6.24, continued

\begin{tabular}{|c|c|}
\hline Cit & oup "C" [City Population: 10, \\
\hline $\begin{array}{l}\text { North Region: } \\
{[\text { Model } \operatorname{Cn} 6]}\end{array}$ & $\begin{array}{l}\text { AADT }=-5610.46+1.46963 \text { CNTYINC }+0.01433 \text { CNTYEMP } \\
R^{2}=60.77 \quad \mathrm{e}=1.42975 \quad e^{=0.07181}\end{array}$ \\
\hline $\begin{array}{l}\text { Central Region: } \\
\text { [Mode1 Cc6] }\end{array}$ & $\begin{array}{l}\text { AADT }=-13752.45+1.57494 \text { CNTYINC }+0.55774 \text { CTYPOP } \\
R^{2}=69.88 \quad e=1.50307 \quad e=0.60636\end{array}$ \\
\hline South Region: & $\begin{array}{l}\text { AADT }=5610.51+0.61280 \text { CNTYEMP } \\
R^{2}=83.35 \quad e=0.61934\end{array}$ \\
\hline
\end{tabular}

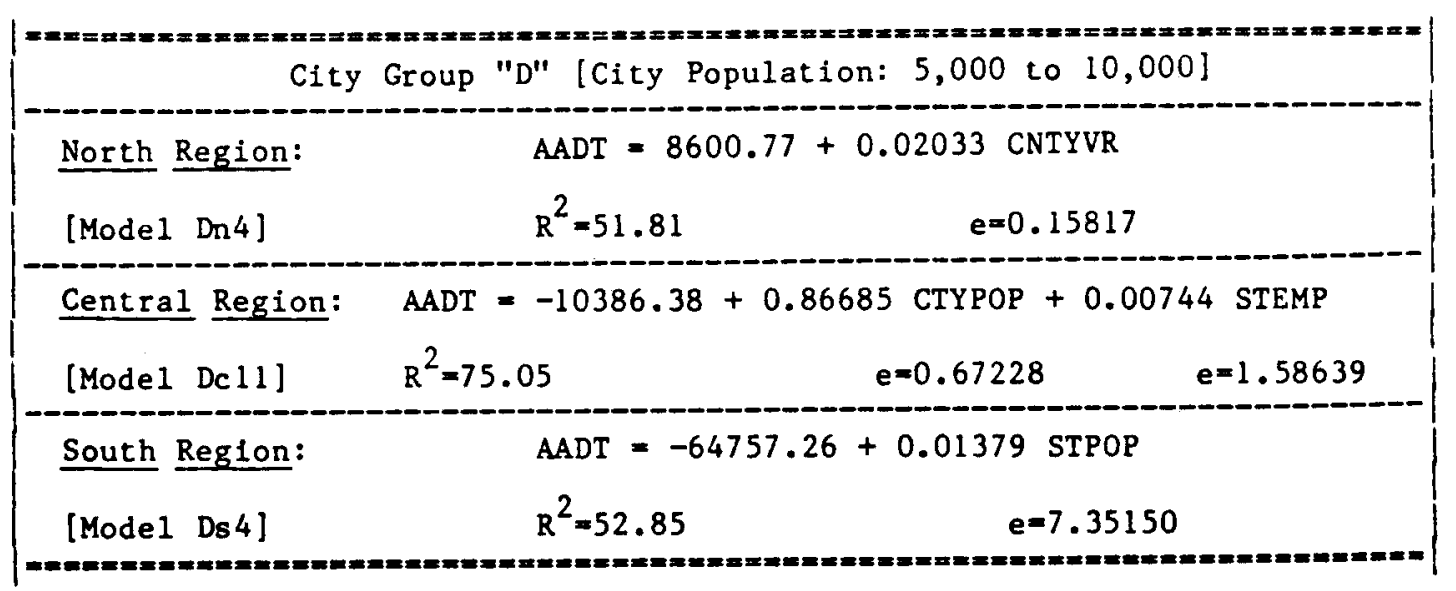

Note: See Table 4.3 for description of variables. 
Table 6.25: Final Regression Equations for Urban Minor Arterial \& Collector

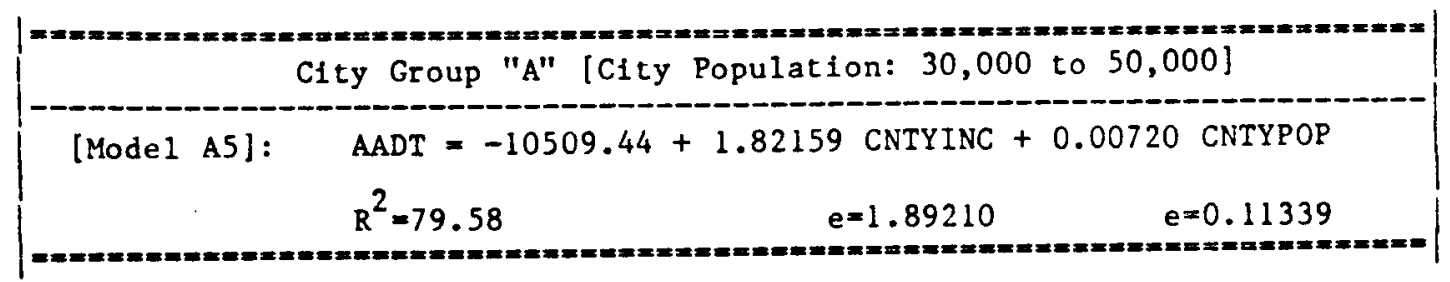

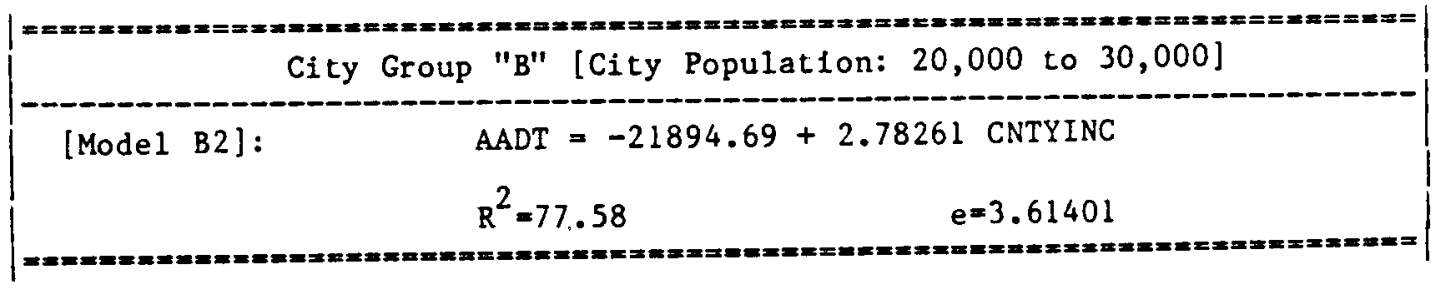

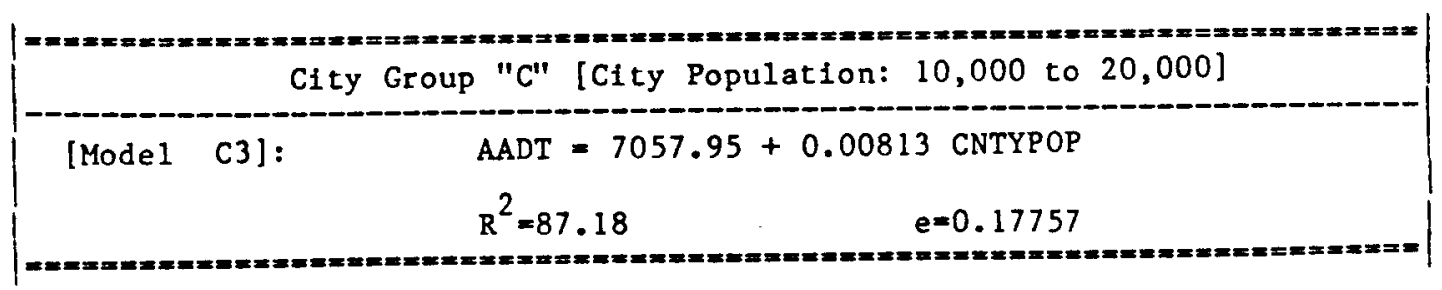

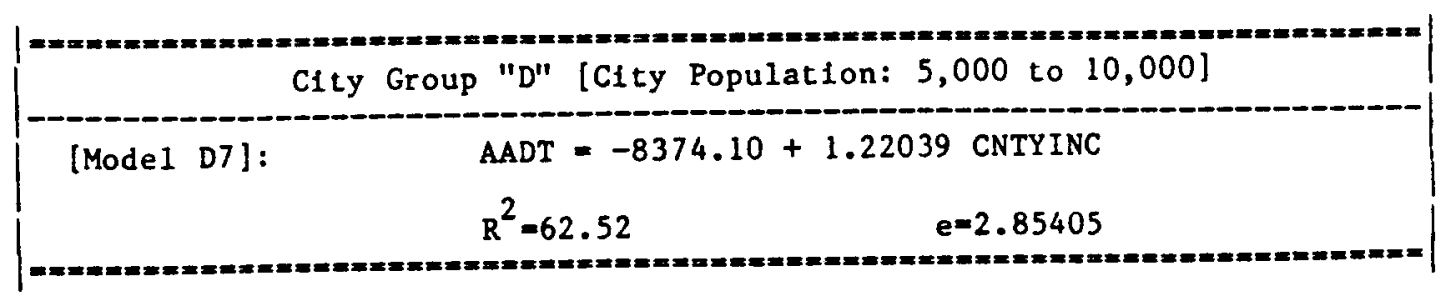

Note: See Table 4.3 for description of variables. 
can be generated from the available information. Each of the variables is significant at the 95 percent confidence level.

Using the elasticities obtalned from the regression analysis, a forecasting model was developed for each selected group under different highway categories by substituting those elasticities into Equation (3.1) of Chapter 3. The elasticity models are presented in Tables $6.26,6.27$ and 6.28 for Interstates, Urban Principal Arterial, and Urban Minor Arterlal \& Collector, respectively. These models generally satisfy all the criteria specified earlier. Each of the models is relatively simple, containing not more than two variables. The use of these models is also straightforward. The input values are the base year AADT and the base and future year value (the year for which the traffic forecast is needed) of the predictor variables.

The elasticity of urban interstate AADT in the north region is 0.51775 (See Table 6.26) with respect to county population, meaning that a 1 percent change in the number of county population results in 0.52 percent increase in urban interstate traffic in the north region. The larger the elasticity of a given model, the greater the variable's effect on AADT. Because of this and the linear relationship of the model, an error of 10 percent in the predicted value of county population w111 change profected traffic by 5.18 percent in the urban interstate north region model. Thus, accurate forecasts for the input variables is a requirement for the success of the elasticity model in traffic forecasting. 
Table 6.26: Elasticity-based Traffic Models for Interstate Highways

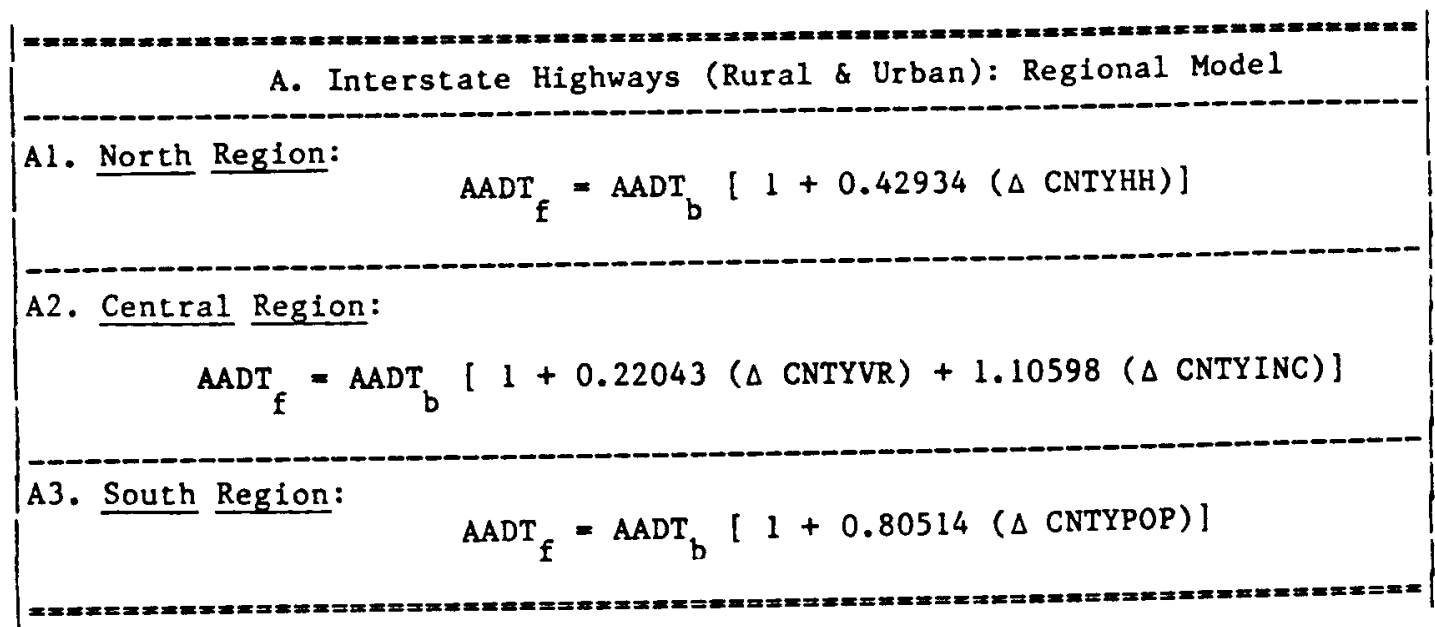

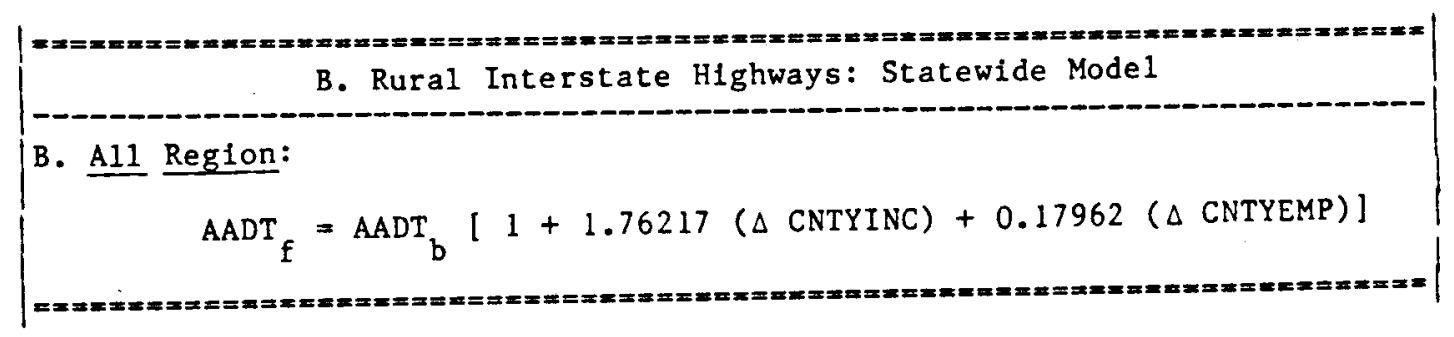

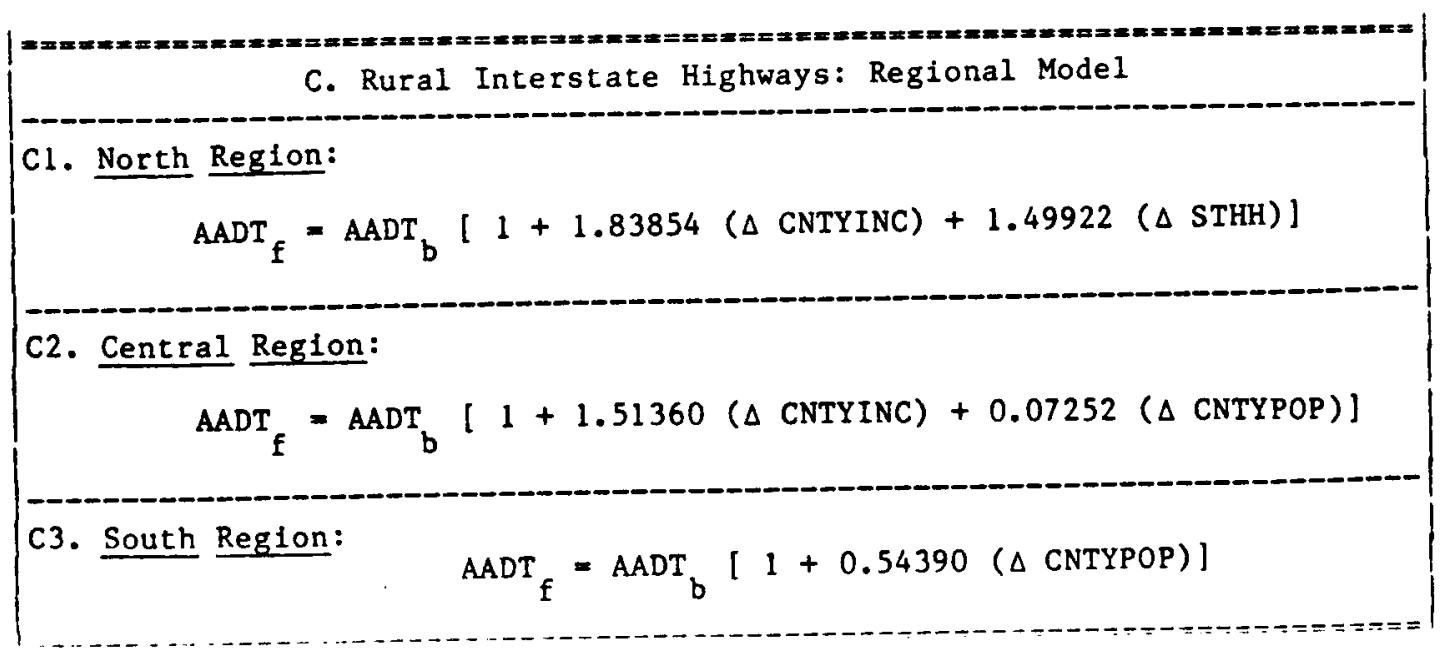


Table 6.26, continued

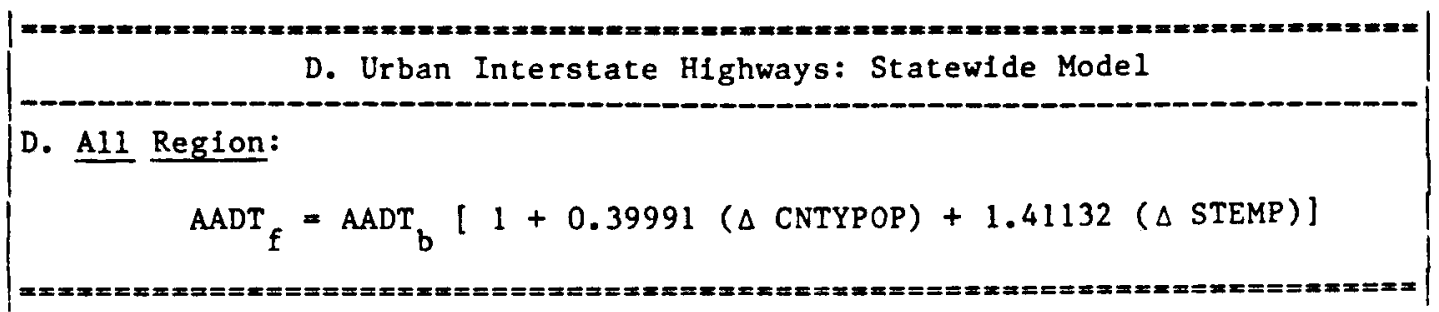

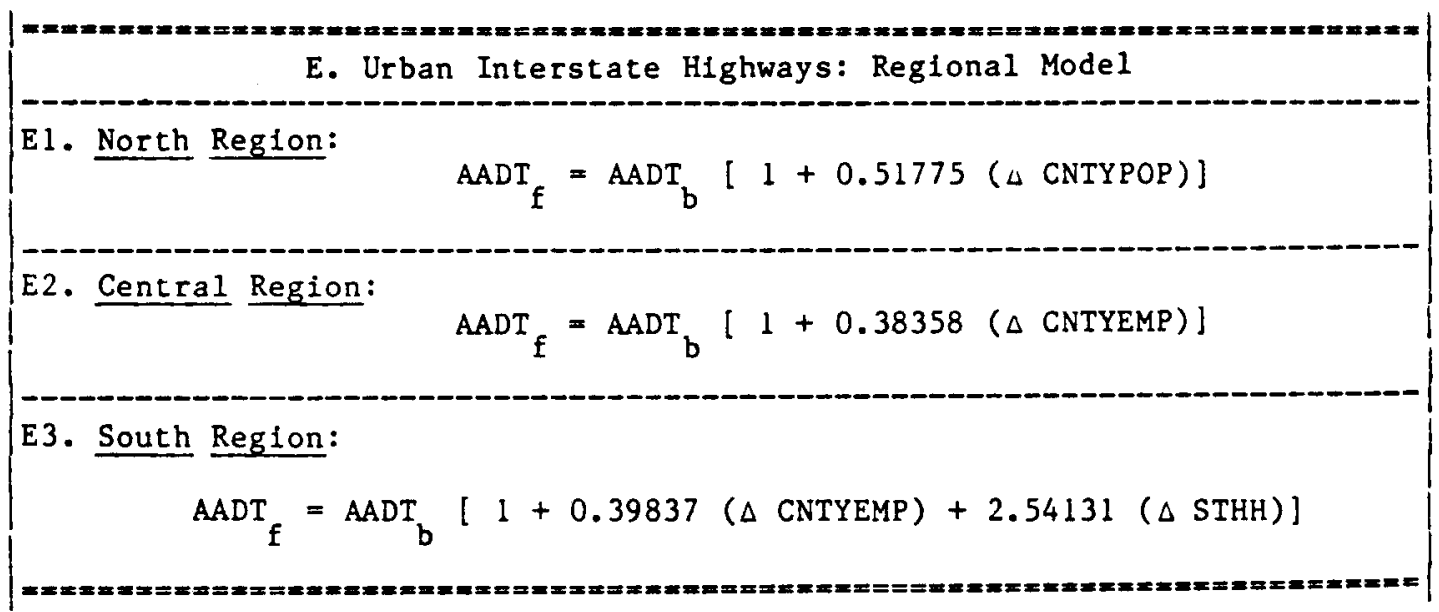

Note: (i) See Table 4.3 for description of variables.

(ii) $\Delta$ represents change in predictor variable with respect to its base value in fraction. For example, $\Delta x=\frac{X_{f}-X_{b}}{X_{b}}$, where $x_{b}$ and $x_{f}$ denote base and future values of $x$. 
Table 6.27: Elasticity-based Traffic Models for Urban Princlpal Arterial

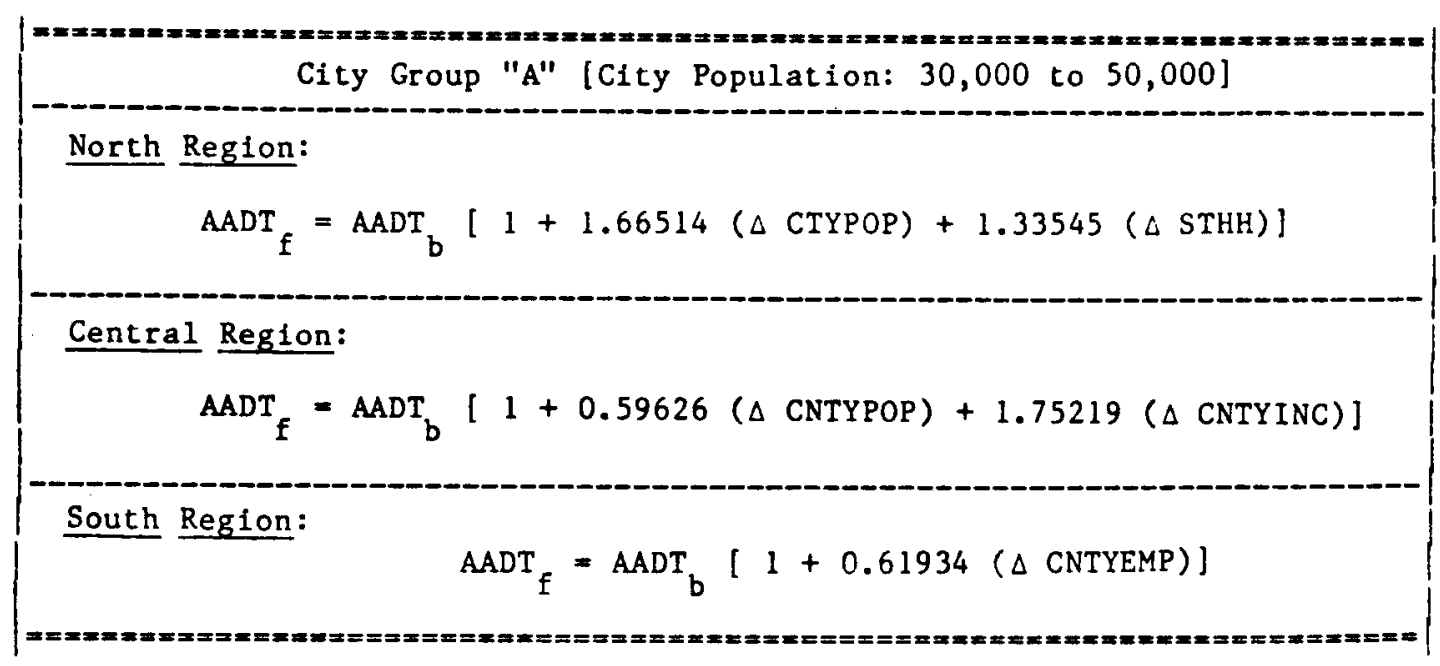

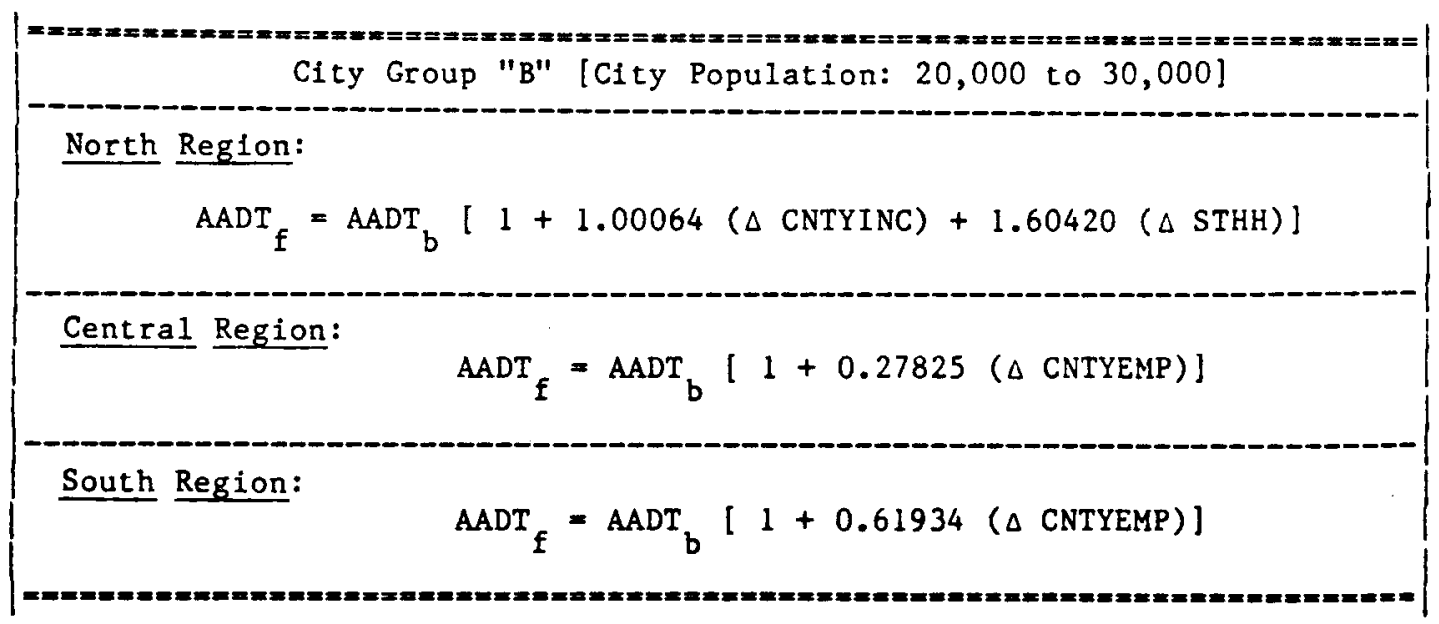


Table 6.27 , continued

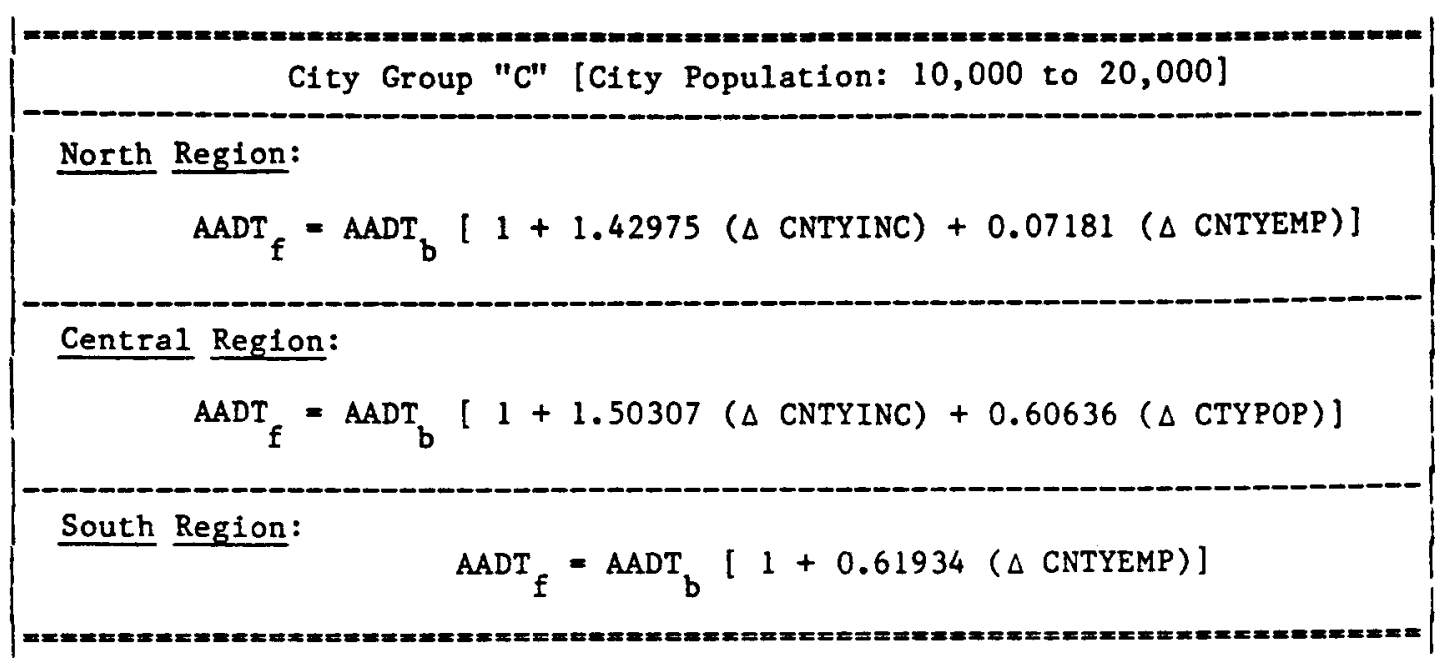

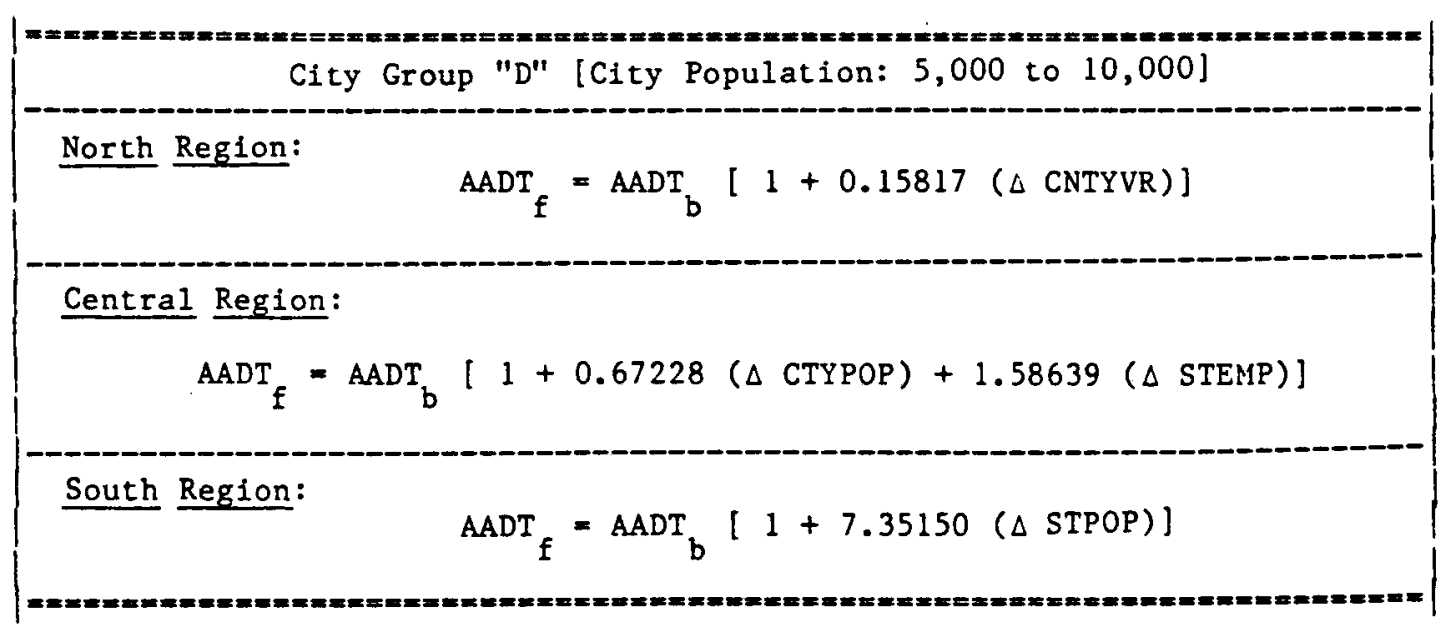

Note: (1) See Table 4.3 for description of variables.

(1i) $\Delta$ represents change in predictor variable with respect to its base value in fraction. For example, $\Delta x=\frac{x_{f}-x_{b}}{x_{b}}$, where $x_{b}$ and $x_{f}$ denote base and future values of $x$. 
Tahle 6.28: Elasticlty-based Traffic Models for Urban Minor Arterial \& Collector

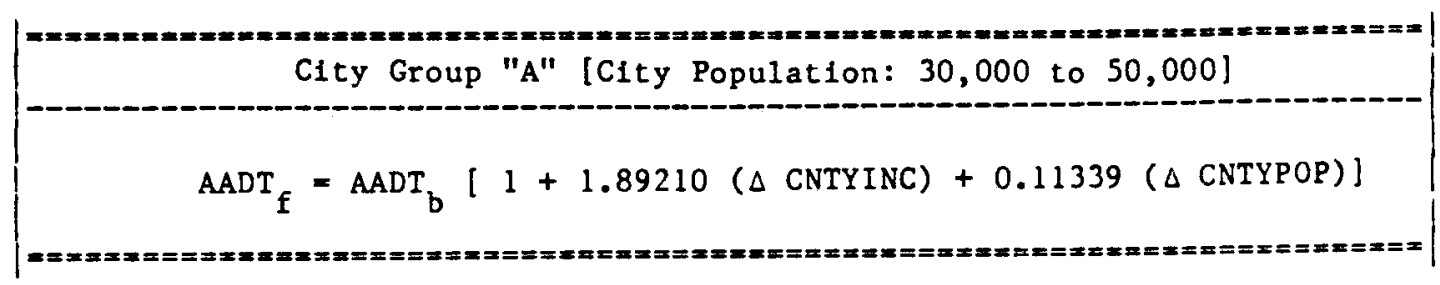

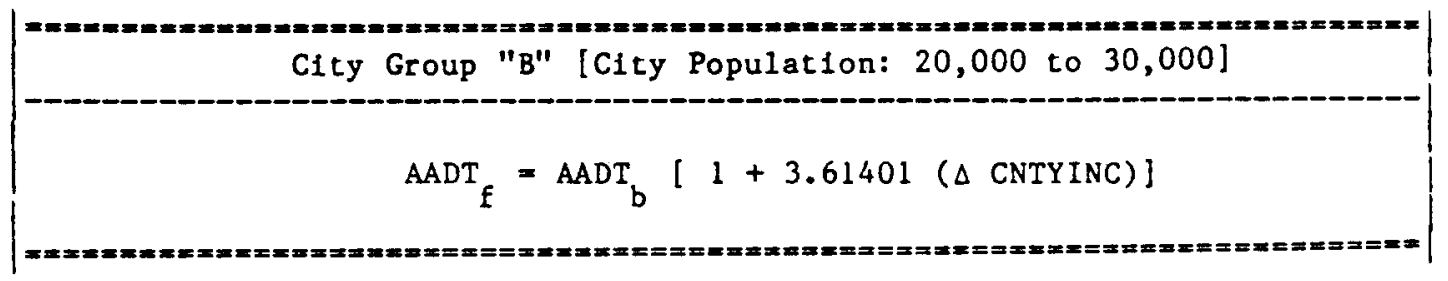

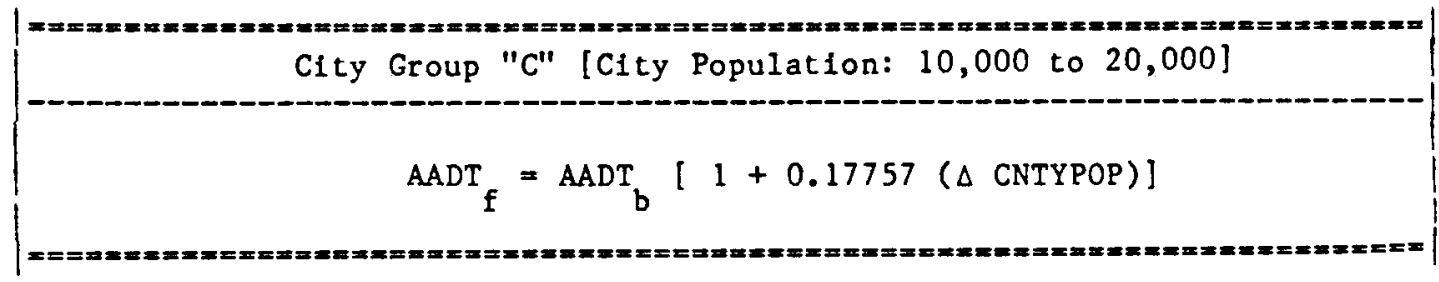

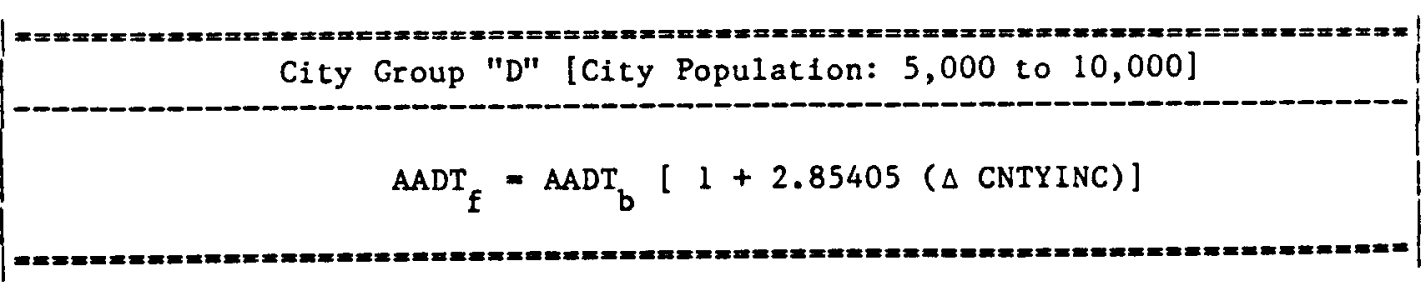

Note: (1) See Table 4.3 for description of variables.

(11) $\Delta$ represents change in predictor varlable with respect to its base value in fraction. For example, $\Delta x=\frac{x_{f}-x_{b}}{x_{b}}$, where $x_{b}$ and
$x_{f}$ denote base and future values of $x$. 


\subsection{Chapter Summary}

All the statistics employed in influential analysis, testing regression assumptions, and significance and collinearity of the regression assumptions have been described in this chapter. A summary of results of all statistical analyses has been provided. Results of pre and post influence analys is have been compared to show the usefulness of detecting outliers and influential observations. Significance tests on variables in the candidate models have found useful predictor variables, whereas the overall significance test has found that strong relationships exist between the response and the selected predictor variables. Collinearity statistics on regression coefficients are used to detect the degree of multicollinearity (if any exists). The collinearity statistics and significance tests have identified useful models with minimized multicolifnearity and fewer variables ( $<2$ ) in each final model selected. The statistical results of post-influence analysis are used to draw a final selection from the group of candidate models. Finally, elasticity models (See Tables 6.26-6.28) are formed for different groups under each highway class. Performance of this model form, in addition to some alternative model structures, will be evaluated in a later chapter. 
CHAPTER 7

PRINCIPAL COMPONENT ANALYSIS BASED MODEL

\subsection{Introduction}

The forecasting of traffic volumes is a complex task. Besides the many measurable variables that influence traffic, there are others that cannot be measured. This study has been based on a large number of variables (twelve) that may explain traffic volumes. In Chapter 6, it has been noted that at most two variables out of twelve useful varlates are retained in our final regression equations. The main reason for this is that we are primarily working with socioeconomic variables, and $i t$ is more likely that these variates are collinear. In Chapters 5 and 6 , variates thought to be collinear were dropped and diagnostic tests for collinearity were carried out. Another strategy that can be applied is replacing subsets of the collinear variates by specific linear combinations (such as princtpal components). Principal Component Analysis (PCA) is a multivariate technique for examining relationships among several quantitative variables, and it can be used to reduce the number of variables in regression. 


\subsection{Principal Component Analysis}

The statistical technique of Principal Component Analysis (PCA) can be related in many aspects to the "principal stress analysis" of structural analysis. For example, the equivalents of the direction cosine vectors in structural analysis are eigenvectors in this statistical technique [Harr 1987]. PCA is a multivariate analysis technique that attempts to describe interrelationships among a set of variables. PCA can be thought as a dimension reduction technique and is useful because problems with many variables are hard to model, to parametrize, to analyze, to interpret. Also, it is difficult to keep track of the correlation structure with a large number of variables. The description of principal component analysis in this section focuses on the concept and uses behind 1t. A complete description of principal component analysis can be found in Johnson and Wichern [1982].

Procedure "PRINCOMP" of SAS package [SAS/Statistics 1985] is used to perform Principal Component Analysis. The procedure is used to calculate a maximum of 11 principal components from 11 original predictor variables. One original variable, STGAS, is dropped in this analysis because it was absent from the final selection in Chapter 6 . The PCA is carried out for each filtered data group - data groups that remained after influential analysis in Chapter 6 . The following four data groups, one from each major highway class, have been selected to 1llustrate some concepts of PCA in this section and principal component regression in subsequent sections. 
a. Central Region of Rural Interstate.

b. Central Region of Urban Interstate.

c. City Group "A" and Central Region of Urban Principal Arterial.

d. City Group "D" of Urban Minor arterlal and Collector.

The important results of PCA for the above selected data groups are shown in Table 7.1 .

In statistical analysis, a model containing a large number of independent variables is not uncommon. In most cases, statistical analyses reveal the appropriate model. Our analyses start with a large list of variables because we do not initially want to overlook the variables that may be explanatory factors in a model. However, the inclusion of a large number of variables in a regression model often results in multicollinearity. In Chapters 5 and 6 , it has been mentioned how multicollinearity may inhibit the usefulness of the results. Principal Component Analysis (PCA) can be used to study the structure of multicollinearity, and the results of such study can be used to improve the results of regression analysis. This method (described below) produces a set of artificial, uncorrelated $(r=0)$ variables that can then be used in a regression model.

Starting with a data set with $p$ numeric variables, which is usually large in number, the PCA tries to find if there exist $k$ linear combinations $\mathrm{PC} 1, \ldots ., \mathrm{PCk}$ of the original variables $\mathrm{X} 1, \ldots, \mathrm{Xp}$ such that the $\mathrm{PCi}^{\prime} \mathrm{s}$ account for most of the variation in the original $\mathrm{X}^{\prime} \mathrm{s}$. For the $\mathrm{PC}^{-} s$ to be useful as a dimension reduction technique, $k$ should 
Table 7.1: Eigen Values and Component Varlances of Selected Data Groups

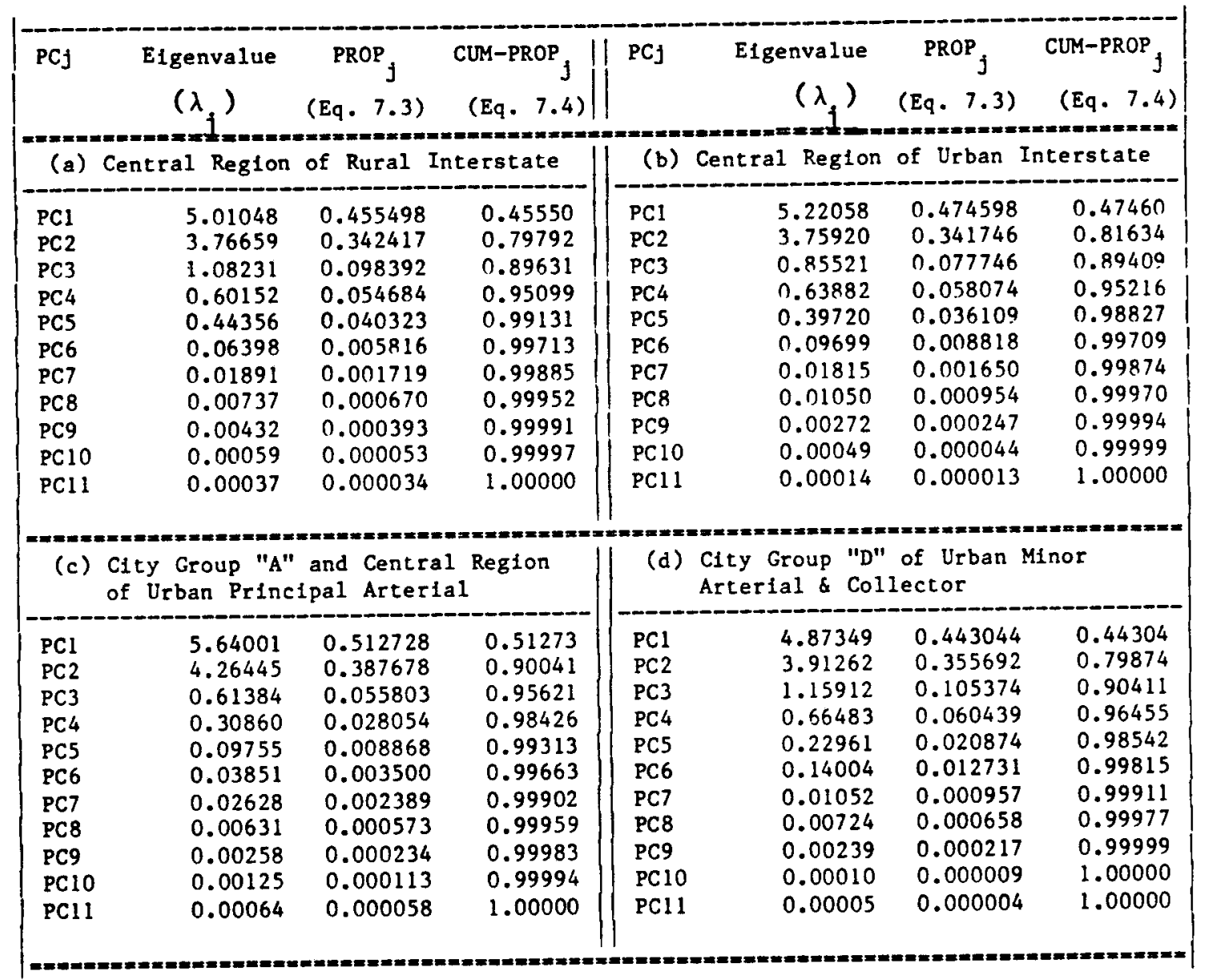


be substantially smaller than p. At the end of this section, it is shown that the value of $k$ in most of the data groups is 2 or 3 , which is small compared to our 11 original variables used to derlve Principal Components (PCs). Each principal component is a linear combination of the original variables, with coefficients equal to the eigenvectors of the correlation or covariance matrix. Let $\lambda_{1}>\lambda_{2}>\lambda_{3}>\ldots .>\lambda_{p}>0$ be the ordered eigenvalues of the variance-covarlance matrix of $X^{-} s$, and $\underline{\alpha}_{1}, \underline{\alpha}_{2}, \underline{\alpha}_{3}, \ldots, \frac{\alpha}{p}$ be the corresponding normalized eigenvector [Johnson \& Wichern 1982]. Then the $j$-th Principal Component $(P C j)$ is defined as

$$
P C_{j}=\sum_{i=1}^{p} \alpha_{1 j} X 1=\underline{a}_{j} X
$$

The PCs selected in the regression model (see Section 7.3.1) are presented in Appendix Tables D1, D2 and D3 for the data groups Interstate, Urban Principal Arterial, and Urban Minor Arterial and Collector, respectively. These tables show the elements of the eigenvectors, or the coefficients of the PCs. Means and standard deviations of the original variables are also shown in these tables. The first Principal Component $(\mathrm{PC} 1)$ of $\mathrm{X} 1, \mathrm{X} 2, \ldots \ldots, \mathrm{X}_{\mathrm{p}}$ (the original variables) is defined as the Iinear combination $\underline{\alpha}_{1}^{-} X$ of $\times 1, \times 2, \ldots, X_{p}$ that has the maximum variance among all possible linear combinations $\underline{\alpha} \underline{x}$, subject to the constraint $\underline{\alpha} \underline{-}<1$. The second Principal Component (PC2) is defined as the linear combination, $\underline{\alpha}_{2}^{-} \underline{x}$, that has the maximum variance among all possible linear combinations $\underline{\alpha} \underline{x}$, is uncorrelated with $\mathrm{PCl}$, and satisfies the constraint $\underline{a} \underline{\alpha}<1$. At each 
stage $j, P C j$ is uncorrelated with $P C 1, P C 2, \ldots . . ., P C(j-1)$. Thus, the principal components have the following properties:

- The principal component variables are jointly uncorrelated.

- The first principal component has the largest variance of any linear function of the original variables. The second component has the second largest variance, and so forth.

The zero correlation condition allows dimension reduction through the independent factors. As a result, the regression coefficients for $\mathrm{PCs}^{-}$are independent on the number of $\mathrm{PCs}^{-}$in the regression equation.

Principal components can be obtained by computing the eigenvalues and eigenvectors of the correlation or covariance matrix. In this study, the correlation matrix has been used so that the components are not affected by the scales of measurement of the original variables. Our original variables are qualitatively different variables and are measured/expressed in different units. In such cases, it is very difficult to construct a linear combination of original variables. The correlation option of the SAS PRINCOMP procedure has eliminated this problem by standardizing each original variable as

$$
x_{i}^{s t d}=\frac{x_{1}-\mu_{1}}{\sigma_{1}}
$$

where, $x_{1}^{\text {std }}$ is the standardized form of original variable $x_{1}$, $\mu_{1}$ and $\sigma_{1}$ are the mean and standard deviation of $x_{1}$. The values of the means and standard deviations of all the original 
variables for the data groups are presented in Appendix Tables Dl to D3. The variables $x_{1}^{s t d}$ are now unitless. The variable $X i$ in Equation (7.1) is the standardized form of Equation (7.2). The above standardization carries out PCA on the correlation matrix of the original variables. Since all the diagonal elements in a correlation matrix are 1.0 , the total variation is equal to number of variables ( $p)$. This Implies that average variance of the Principal Component is $\mathrm{p} / \mathrm{p}=$ 1. Because the average variance is 1 , sometimes the following rule of thumb is used to determine the number of components to be retained:

$$
\text { Retain } P C j, \text { if Variance }(P C j)>1 .
$$

The principal components in Table 7.1 are sorted in descending order of the eigenvalues, $\lambda_{j}$, which are equal to the variances of the components. The above rule of thumb suggests that only 2 or 3 PCs in Table 7.1 with $\lambda_{j}$ greater than 1 are to be retained in our four selected data groups. A similar result has been found for the rest of our data groups (results not shown). Because the correlation matrix is used, the sum of the variances of both the original and the principal component variables is equal to the number of variables. A set of elgenvalues of almost equal magnitudes suggests that there is 11ttle multicollinearity, while a wide variation of magnitudes indicates severe multicollinearity. The elgenvalues in Table 7.1 suggest that our data sets have severe multicollinearity. The number of large (usually greater than unity) elgenvalues may be taken as a crude indication of the factors needed to describe the behavior of the full set of variables. The efgenvectors are the coefficlents for the linear transformation of the standardized variables with mean zero and 
variance one. These coefficients are used to create observed values of the new component variables and can be used to interpret the structure of these variables.

The fraction of the total varlation accounted for by the $j$-th Principal Component is

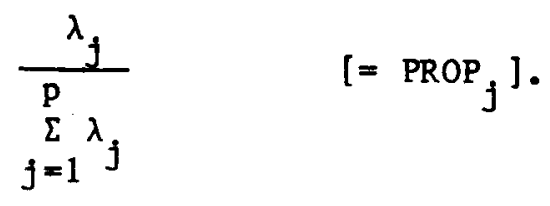

The values of PROP ${ }_{j}$ for the four selected data groups in Table 7.1 suggest that the first 2 or 3 PCs account for most of the variation. The fraction of the total variation accounted for by the first $k(k<p)$ Principal Components is

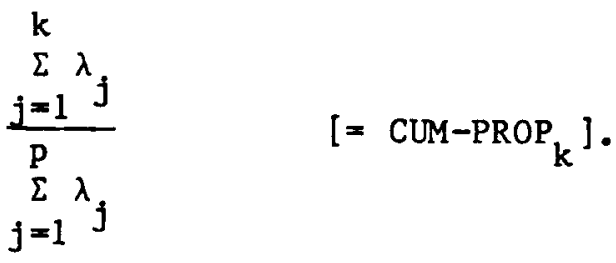

These fractions are calculated for each $j$. The first $k$ components are retained if CUM-PROP $k$ is large (generally in the 90 percent neighborhood) and if the fraction PROP $j$ for $j>k$ does not provide enough contribution to CUM-PROP $_{k}$. Table 7.1 has presented the values of PROP $j$ and CUM-PROP $f$ for the four representative data groups selected at the start of this section. The stopping rule based on CUM-PROP $j$ of Equation (7.4) suggests that 2 or $3 \mathrm{PC}^{-s}$ are enough to describe the data sets. While trying to decide upon a stopping point, the jumps in the 
ratios of Equation (7.4) are also noticed. The results are same as those concluded by the rule of thumb for retaining PCs. The retained PCs of each data group are used in the following section to check their significance in regression analysis.

\subsection{Principal Component Regression}

The combination of principal component analysis and regression analysis presents the analyst with the means of reducing the number of significant variables and of expressing the remaining variables in a linear composite relationship [Harr 1987]. In principal component regression, the set of principal component varlables (PCs), Instead of the original variables, is used in a regression analysis. Since the principal components are uncorrelated, there is no multicollinearity in the regression. Thus, it is possible to determine the important coeficients without attempting any variable selection techniques. Regression analysis with the retained PCs of Section 7.2 is performed to determine the significance of the PCs. In addition, the different variable selection rules (see Section 5.3 and 1 ts subsections) are used to find the candidate models with PCs for each data group. The models with retained PCs in Section 7.2 are examined to see if they belong to the set of candidate models and if they can be selected finally after further diagnosis. In a forecast, users need the future values of all the original variables. Use of the results of principal component regression may be costly due to the forecasts of a large number of original variables. However, the principal component regression analysis provides a direct check on the standard regression 
model and its derfvative "elasticity model" to see how good the models in Chapter 6 compare to collinearity-free principal component regression. Because the princlpal component regression starts with data sets after influence analysis (Chapter 6), further influentlal diagnosis is not necessary. The residual plots in Section 7.3 .1 have shown that there is no need for influence analysis for the filtered data, resulted after influence analysis in Chapter 6. At the same time, collinearity analysis can be skipped because, by definition, PCs are 1ndependent of each other and no problem of multicollinearity will arise. But the other tests of Chapter 6 (for example, significance test, normality and homoscedasticity, and model aptness) are used to find the best model from the group of candidate models.

\subsubsection{Final Principal Component Regression Mode1}

The PCA in Section 7.2 has resulted in the most useful PCs, based on the original predictor variables. But it has not been shown whether the retained components are significant in describing the response variable AADT. The "REG" procedure of SAS [SAS/Statistics] is used to perform the regression with the $\mathrm{PC}^{-} \mathrm{s}$. The validity of the regression assumptions and model aptness (see Section 6.3) are checked for all the candidate models of each data group. In addition, the significance tests of Section 6.4 are performed to determine the overall significance and the significance of each PC in the regression equation. The comparative evaluation (as was done in Chapter 6) of all these tests and the information on variance explained by the PCs in the PCA of Section 7.2 are used to select the final model for each 
group of data under consideration. Some important model statistics -$R^{2}$ (Coefficient of Multiple Determination), $R_{a}^{2}$ (Adjusted Coefficient of Multiple Determination), RMSE (Root Mean Square Error) and C.V. (Coefficient of Variation) - for the final selected models are shown In Tables $7.2,7.3$, and 7.4 for Interstate, Urban Principal arterial and Urban Minor arterial and Collector, respectively. The models in these tables will provide three alternative options for Interstate Highways and only one option for non-interstate urban highways. The ranges of the varfous model statistics in Tables 7.2 to 7.4 are as follows:

\begin{tabular}{|c|c|c|c|c|}
\hline $\begin{array}{l}\text { Highway } \\
\text { Category }\end{array}$ & {$\left[R^{2}\right](*)$} & $\mathrm{R}_{\mathrm{a}}^{2}$ & RMSE & C.V. \\
\hline $\begin{array}{l}\text { Interstate } \\
\text { Highways }\end{array}$ & $\begin{array}{c}61.46-90.26 \\
{[50.35-88.86]}\end{array}$ & $59.51-89.23$ & $1505-12616$ & $12.33-33.33$ \\
\hline $\begin{array}{l}\text { Urban Prin- } \\
\text { cipal Art. }\end{array}$ & $\begin{array}{c}52.87-97.57 \\
{[51.81-94.14]}\end{array}$ & $49.72-96.91$ & $762-2187$ & $5.62-21.04$ \\
\hline $\begin{array}{l}\text { Urban Minor } \\
\text { Arterial \& } \\
\text { Collector }\end{array}$ & $\begin{array}{c}66.87-89.31 \\
{[62.52-87.18]}\end{array}$ & $59.51-86.64$ & $695-2560$ & $8.10-30.56$ \\
\hline
\end{tabular}

(*) $R^{2}$ ranges of the final selections in Chapter 6 (See Tables 6.23-6.25).

The above table also compares the ranges of $R^{2}$ values to the final selections of Chapter 6.

The P-values (PROB $>F$ ) of the significance statistics of Tables 7.2-7.4 show that the minimum $\alpha-1$ evels at which a significant relation exists between PCs and AADT are $0.0001,0.0003$ and 0.0069 for Interstate, Urban Principal Arterial and Urban Minor Arterial \& Collector, respectively. The significance at an $\alpha$-level of 0.0001 indicates that 
Table 7.2: Some Key Results of Princlpal Component Regression for Interstate Highways

\begin{tabular}{|c|c|c|c|c|c|c|c|c|}
\hline \multirow{2}{*}{ Region } & \multicolumn{2}{|c|}{ Model Statistics } & \multicolumn{4}{|c|}{ Significance Statistics } & \multicolumn{2}{|c|}{ Normality Stat. } \\
\hline & $\mathrm{R}^{2} \mathrm{R}_{\mathrm{a}}^{2}$ & $\begin{array}{l}\text { RMSE } \\
\text { C.V. }\end{array}$ & $\begin{array}{r}\text { F-Value } \\
\text { Prob }>\text { F }\end{array}$ & $\left|\begin{array}{l}\text { Mode1 } \\
\text { PC (*) }\end{array}\right|$ & $\begin{array}{c}t- \\
\text { value }\end{array}$ & Prob>t & $\mathbf{N}$ & $\begin{array}{l}W / D(* *) \\
\text { (P-value) }\end{array}$ \\
\hline & A. Int & ate $\mathrm{Hi}$ & ways ( $\mathrm{Ru}$ & al \& & ban): & $\operatorname{Reg} 1$ & Mode 1 & \\
\hline North & $\begin{array}{l}74.32 \\
73.26\end{array}$ & $\begin{array}{l}8229.5 \\
30.206\end{array}$ & $\begin{array}{l}70.418 \\
0.0001\end{array}$ & $\begin{array}{l}\mathrm{PC} 1 \\
\mathrm{PC} 2 \\
\text { PC4 }\end{array}$ & $\begin{array}{r}9.691 \\
9.870 \\
-4.463\end{array}$ & $\begin{array}{l}0.0001 \\
0.0001 \\
0.0001\end{array}$ & 77 & $\begin{array}{r}0.0841 \\
(>0.15)\end{array}$ \\
\hline Central & $\begin{array}{l}76.08 \\
75.44\end{array}$ & $\begin{array}{l}5934.1 \\
24.315\end{array}$ & $\begin{array}{r}118.729 \\
0.0001\end{array}$ & $\begin{array}{l}\text { PC1 } \\
\text { PC } 2 \\
\text { PC3 }\end{array}$ & $\begin{array}{r}14.134 \\
12.100 \\
3.167\end{array}$ & $\begin{array}{l}0.0001 \\
0.0001 \\
0.0020\end{array}$ & 14 & $\begin{array}{r}0.0623 \\
(>0.15)\end{array}$ \\
\hline South & $\begin{array}{l}88.12 \\
87.21\end{array}$ & $\begin{array}{l}2905.4 \\
20.658\end{array}$ & $\begin{array}{l}96.430 \\
0 . n 001\end{array}$ & $\begin{array}{l}\text { PC1 } \\
\text { PC } 2 \\
\text { PC3 } \\
\text { PC } 5\end{array}$ & $\begin{array}{r}13.730 \\
11.186 \\
-6.413 \\
5.563\end{array}$ & $\begin{array}{l}0.0001 \\
0.0001 \\
0.0001 \\
0.0001\end{array}$ & 57 & $\begin{array}{r}0.0789 \\
(>0.15)\end{array}$ \\
\hline$=x==$ & $\begin{array}{l}=\times=1 \\
\text { B. Rura1 }\end{array}$ & $\begin{array}{l}=\mathbf{x}=\mathbf{x}= \\
\text { ntersta }\end{array}$ & $\begin{array}{l}=====- \\
\text { e Highw }\end{array}$ & $\begin{array}{l}==== \\
s: S\end{array}$ & $\begin{array}{l}c===== \\
\text { ewide }\end{array}$ & $\begin{array}{l}\text { Mode } 1 \\
\text { Mode }\end{array}$ & 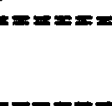 & \\
\hline ALL & $\begin{array}{l}71.44 \\
70.71\end{array}$ & $\begin{array}{l}4021.7 \\
21.245\end{array}$ & $\begin{array}{l}97.545 \\
0.0001\end{array}$ & $\begin{array}{l}\mathrm{PC} 1 \\
\mathrm{PC} 2 \\
\mathrm{PC} 3\end{array}$ & $\begin{array}{r}12.276 \\
10.135 \\
6.262\end{array}$ & $\begin{array}{l}0.0001 \\
0.0001 \\
0.0001\end{array}$ & 121 & $\begin{array}{r}0.0817 \\
(0.046)\end{array}$ \\
\hline$=$ & $\begin{array}{l}====x== \\
\text { C. Rura1 }\end{array}$ & $\begin{array}{l}===x= \\
\text { Interst }\end{array}$ & $\begin{array}{l}==== \\
\text { Highw }\end{array}$ & $\begin{array}{l}\text { s=s=s } \\
s: \mathrm{R} e\end{array}$ & $\begin{array}{l}x=== \pm \\
\text { onal } M\end{array}$ & lode & & \\
\hline North & $\begin{array}{l}69.84 \\
66.92\end{array}$ & $\begin{array}{l}3311.3 \\
18.489\end{array}$ & $\begin{array}{l}23.930 \\
0.0001\end{array}$ & $\begin{array}{l}\mathrm{PC} 1 \\
\mathrm{PC} 3 \\
\mathrm{PC} 7\end{array}$ & $\begin{array}{l}6.869 \\
3.127 \\
3.851\end{array}$ & $\begin{array}{l}0.0001 \\
0.0038 \\
0.0006\end{array}$ & 35 & $\begin{array}{r}0.9481 \\
(0.165)\end{array}$ \\
\hline Central & $\begin{array}{l}61.46 \\
59.73\end{array}$ & $\begin{array}{l}5326.1 \\
22.156\end{array}$ & $\begin{array}{l}35.616 \\
0.0001\end{array}$ & $\begin{array}{l}\text { PC 1 } \\
\text { PC2 } \\
\text { PC } 3\end{array}$ & $\begin{array}{l}8.844 \\
2.373 \\
4.796\end{array}$ & $\begin{array}{l}0.0001 \\
0.0205 \\
0.0001\end{array}$ & 71 & $\begin{array}{r}0.0914 \\
(0.147)\end{array}$ \\
\hline South & $\begin{array}{l}90.26 \\
89.23\end{array}$ & $\begin{array}{l}1504.7 \\
12.893\end{array}$ & $\begin{array}{l}88.034 \\
0.0001\end{array}$ & $\begin{array}{l}\text { PC } 1 \\
\text { PC2 } \\
\text { PC } 3 \\
\text { PC4 }\end{array}$ & $\begin{array}{r}14.008 \\
7.658 \\
-6.154 \\
-7.707\end{array}$ & $\begin{array}{l}0.0001 \\
0.0001 \\
0.0001 \\
0.0001\end{array}$ & 43 & $\begin{array}{r}0.9603 \\
(0.269)\end{array}$ \\
\hline
\end{tabular}


Table 7.2 , continued

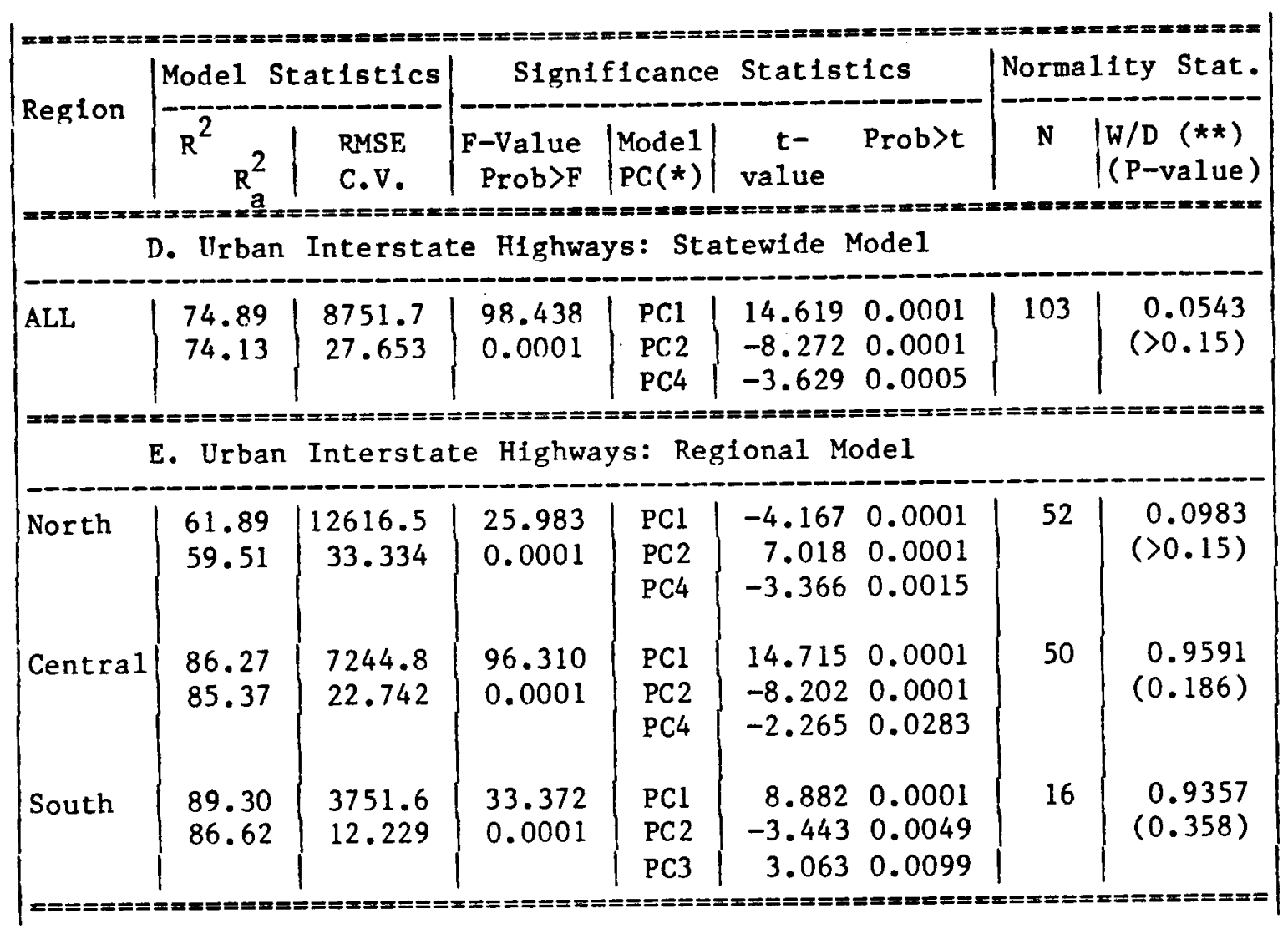

(*) See Table Dl for description of PCs' (Principal Components).

(**) N11k-Shapiro ' $W$ ' for $N<51$ or Kolmogorov ${ }^{\prime} D^{\prime}$ for $N \geqslant 51$, and $\mathrm{P}$-value $=\mathrm{PROB}>\mathrm{D}$ or $\mathrm{PROB} \angle \mathrm{W}$. 
Table 7.3: Some Key Results of Principal Component Regression for Urban Principal Arterial

\begin{tabular}{|c|c|c|c|c|c|c|c|c|}
\hline \multirow{2}{*}{ Region } & \multicolumn{2}{|c|}{ Model Statistics } & \multicolumn{4}{|c|}{ Significance Statistics } & \multicolumn{2}{|c|}{ Normality Stat. } \\
\hline & $\mathrm{R}^{2}$ & $\begin{array}{l}\text { RMSE } \\
\text { C.V. }\end{array}$ & $\mid \begin{array}{c}\text { F-Value } \\
\text { Prob }>\text { F }\end{array}$ & $\begin{array}{l}\text { Mode1 } \\
\text { PC }(*)\end{array}$ & $\mid \begin{array}{c}t- \\
\text { value }\end{array}$ & Prob $>t$ & $\mathbf{N}$ & $\mid \begin{array}{l}\text { W/D ( }(* *) \\
\text { (P-value) }\end{array}$ \\
\hline \multicolumn{9}{|c|}{ City Group "A" [City Population: 30,000 to 50,000 ] } \\
\hline North & $\begin{array}{l}59.05 \\
54.50\end{array}$ & $\begin{array}{l}1841.1 \\
13.941\end{array}$ & $\begin{array}{l}12.980 \\
0.0003\end{array}$ & $\begin{array}{l}\mathrm{PC} 3 \\
\mathrm{PC} 4\end{array}$ & $\begin{array}{l}3.075 \\
4.063\end{array}$ & 0.0065 & 21 & $\begin{array}{r}0.9360 \\
(0.240)\end{array}$ \\
\hline Central & $\begin{array}{l}93.05 \\
90.97\end{array}$ & $\begin{array}{l}761.5 \\
5.615\end{array}$ & $\begin{array}{l}44.634 \\
0.0001\end{array}$ & $\begin{array}{l}\text { PC 1 } \\
\text { PC3 } \\
\text { PC4 }\end{array}$ & $\begin{array}{r}8.983 \\
6.585 \\
-3.138\end{array}$ & $\begin{array}{l}0.0001 \\
0.0001 \\
0.0106\end{array}$ & 14 & $\begin{array}{l}0.9243 \\
(0.326)\end{array}$ \\
\hline South & $\begin{array}{l}97.57 \\
96.91\end{array}$ & $\begin{array}{r}1157.4 \\
7.853\end{array}$ & $\begin{array}{c}147.500 \\
0.0001\end{array}$ & $\begin{array}{l}\text { PC 1 } \\
\text { PC2 } \\
\text { PC } 3\end{array}$ & $\left\{\begin{array}{r}11.384 \\
11.501 \\
-13.440\end{array}\right.$ & $\begin{array}{l}0.0001 \\
0.0001 \\
0.0001\end{array}$ & 15 & $\begin{array}{r}0.9587 \\
(0.640)\end{array}$ \\
\hline \multicolumn{9}{|c|}{ 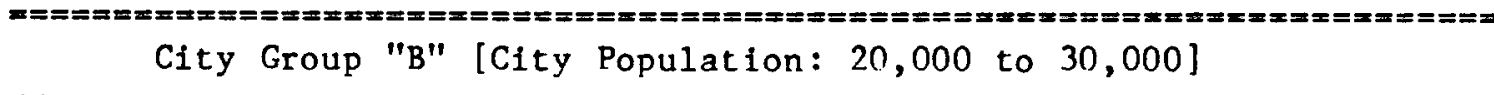 } \\
\hline North & $\begin{array}{l}74.24 \\
69.08\end{array}$ & $\begin{array}{r}1010.8 \\
8.203\end{array}$ & $\begin{array}{l}14.407 \\
0.0011\end{array}$ & $\begin{array}{l}\text { PC } 1 \\
\text { PC2 }\end{array}$ & $\begin{array}{l}4.474 \\
2.966\end{array}$ & $\begin{array}{l}0.0012 \\
0.0141\end{array}$ & 13 & $\begin{array}{r}0.9589 \\
(0.692)\end{array}$ \\
\hline Central & $\begin{array}{l}93.61 \\
92.19\end{array}$ & $\begin{array}{l}1713.1 \\
11.344\end{array}$ & $\begin{array}{l}65.959 \\
0.0001\end{array}$ & $\begin{array}{l}\text { PC1 } \\
\text { PC2 }\end{array}$ & $\begin{array}{l}10.646 \\
-4.310\end{array}$ & 0.0001 & 12 & $\begin{array}{r}0.8842 \\
(0.108)\end{array}$ \\
\hline South & $\begin{array}{l}97.57 \\
96.91\end{array}$ & $\begin{array}{r}1157.4 \\
7.853\end{array}$ & $\begin{array}{r}147.500 \\
0.0001\end{array}$ & $\begin{array}{l}\text { PC1 } \\
\text { PC2 } \\
\text { PC3 }\end{array}$ & $\begin{array}{r}11.384 \\
11.501 \\
-13.440\end{array}$ & $\begin{array}{l}0.0001 \\
0.0001 \\
0.0001\end{array}$ & 15 & $\begin{array}{r}0.9587 \\
(0.640)\end{array}$ \\
\hline \multicolumn{9}{|c|}{ 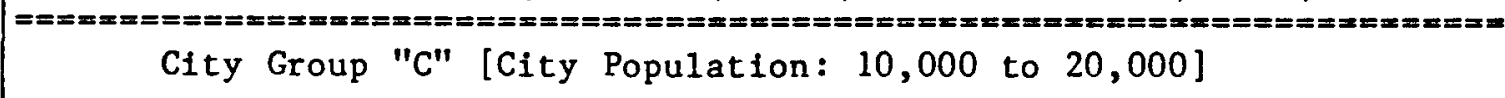 } \\
\hline North & $\begin{array}{l}70.26 \\
65.30\end{array}$ & $\begin{array}{l}1543.4 \\
13.797\end{array}$ & $\begin{array}{l}14.173 \\
0.0001\end{array}$ & $\begin{array}{l}\text { PC 1 } \\
\text { PC3 } \\
\text { PC5 } \\
\text { PC6 }\end{array}$ & $\begin{array}{r}4.901 \\
4.578 \\
-2.335 \\
-2.503\end{array}$ & $\begin{array}{l}0.0001 \\
0.0001 \\
0.0282 \\
0.0195\end{array}$ & 29 & $\begin{array}{r}0.9750 \\
(0.725)\end{array}$ \\
\hline Centra1 & $\begin{array}{l}77.26 \\
75.47\end{array}$ & $\begin{array}{l}2059.4 \\
16.614\end{array}$ & $\begin{array}{l}43.047 \\
0.0001\end{array}$ & $\begin{array}{l}\mathrm{PCl} \\
\mathrm{PC} 3 \\
\text { PC5 }\end{array}$ & $\begin{array}{r}5.526 \\
9.490 \\
-2.924\end{array}$ & $\begin{array}{l}0.0001 \\
0.0001 \\
0.0058\end{array}$ & 42 & $\begin{array}{r}0.9543 \\
(0.163)\end{array}$ \\
\hline South & $\begin{array}{l}97.57 \\
96.91\end{array}$ & $\begin{array}{r}1157.4 \\
7.853\end{array}$ & $\begin{array}{r}147.500 \\
0.0001\end{array}$ & $\begin{array}{l}\text { PC1 } \\
\text { PC } 2 \\
\text { PC3 }\end{array}$ & $\begin{array}{r}11.384 \\
11.501 \\
-13.440\end{array}$ & $\begin{array}{l}0.0001 \\
0.0001 \\
0.0001\end{array}$ & 15 & $\begin{array}{r}0.9587 \\
(0.640)\end{array}$ \\
\hline
\end{tabular}


Table 7.3 , continued

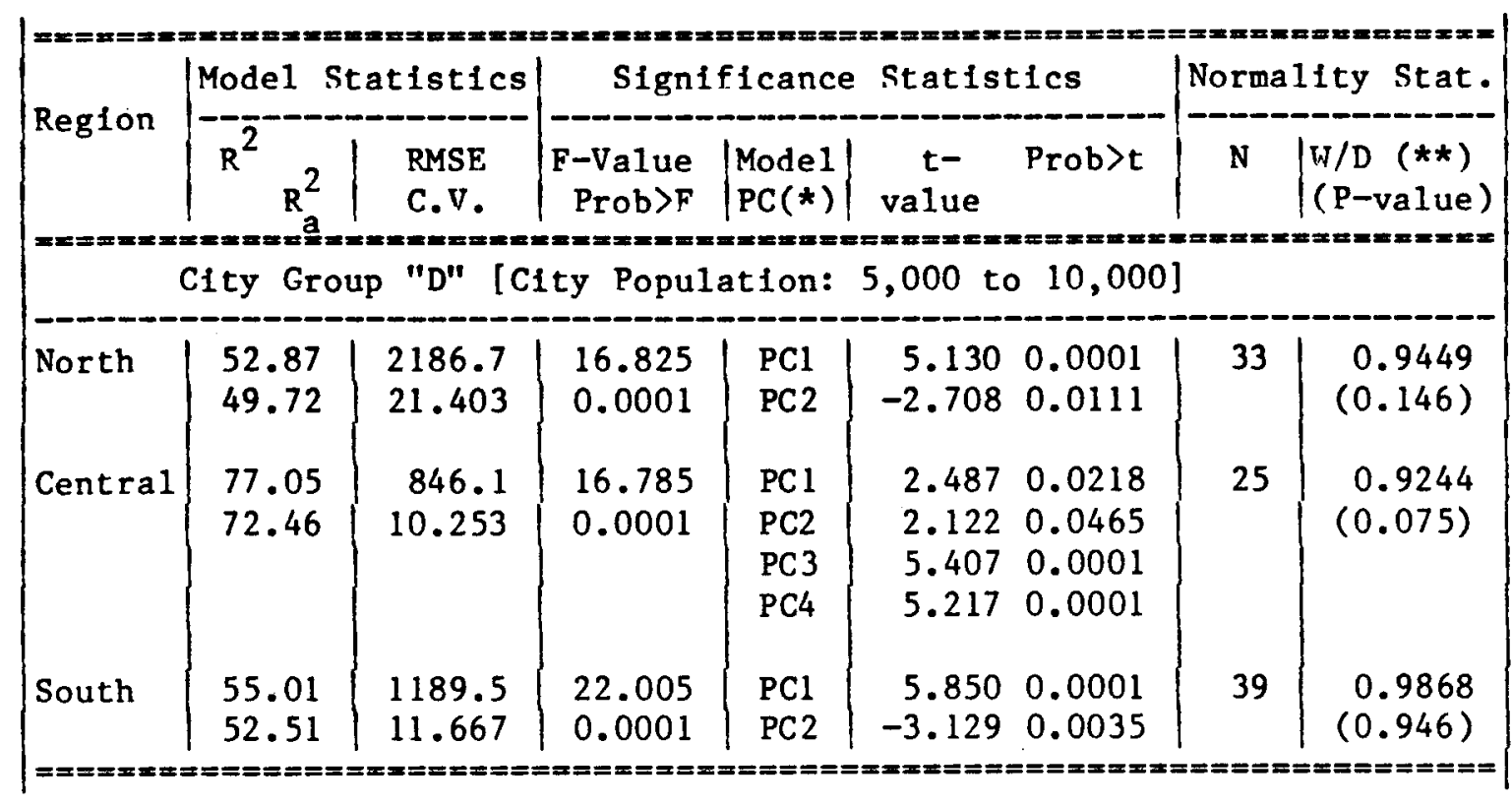

(*) See Table D2 for description of PCs' (Principal Components).

$(* *)$ Wilk-Shapiro ${ }^{\prime} W^{\prime}$ for $N<51$ or Kolmogorov ${ }^{\prime} D^{\prime}$ for $N>51$, and $\mathrm{P}-\mathrm{value}=\mathrm{PROB}>\mathrm{D}$ or $\mathrm{PROB} \angle \mathrm{W}$. 
Table 7.4: Some Key Results of Principal Component Regression for Urban Minor Arterial \& Collector

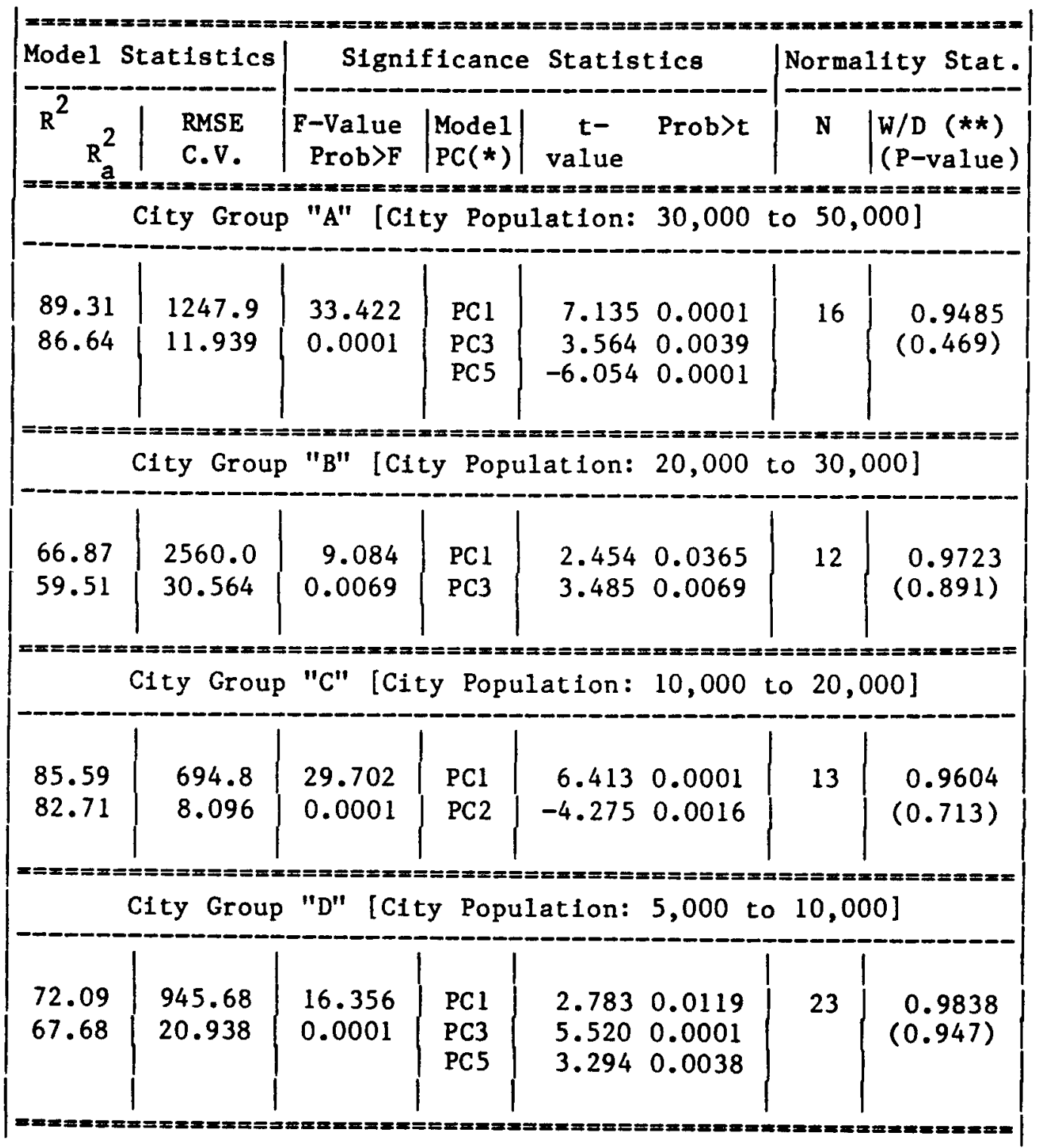

(*) See Table D3 for description of PCs- (Principal Components).

$(* *)$ Wilk-Shapiro ${ }^{\prime} W^{-}$for $N<51$ or Kolmogorov ${ }^{\prime} D^{-}$for $N 51$, and $\mathrm{P}$-value $=\mathrm{PROB}>\mathrm{D}$ or $\mathrm{PROB}<\mathrm{W}$. 
9,999 out of 10,000 times a significant relationship exists between the selected PCs and AADT. The significance statistics for each individual $P C$ in the models of Tables 7.2-7.4 state that the minimum Pvalues $(P R O B>t)$, for which the $P C s$ are significant are $0.028,0.0465$ and 0.0365 for for Interstate, Urban Principal Arterial and Urban Minor Arterial \& Collector, respectively. So the PCs are significant at least at $95 \%$ level of confidence. Usually, the P-value for the PC is as low as 0.0001 (see Tables $7.2-7.4$ ). Partial regression residual plots of PCs for the selected model, one from each major highway class, are presented in Appendix Figures Dl.1-Dl.12. These sample plots also suggest the PCs in the equation are significant and the plots do not suggest any observation to be outlier.

By definition, the $\mathrm{PC}^{\prime}$ s are uncorrelated, so there is no problem of multicollinearity. Since the principal components are linear combinations of the original variables, it is not unreasonable to expect them to be nearly normal [Johnson \& Wichern 1982]. The P-values of normality statistics in Tables $7.2-7.4$ show that the normality assumption is satisfied for $\alpha-1$ evel as low as 0.05 and the results are much better in most of the cases. Appendix Figures D2.1 to D2.4 show the normality plots for the four selected models, one from each major highway class. These sample normality plots, as well as the normality statistics in Tables $7.2-7.4$, suggest that the normality assumption of the regression models has been satisfled.

The residual plots for each of the selected model groups (one from each major highway class) are drawn against 
1. the predicted values of AADT (see Appendix Figures D3.1D3.4),

2. the PCs in the model (see Appendix Figures D4.1-D4.12), and

3. the PC not in the model (see Appendix Figures D5.1-D5.4).

These plots do not suggest any gross departure from non-constancy of error variance and non-linearlty of the regression relation. These residual plots also do not suggest any observation to be outlier. This is an expected result because PCA has performed with the filtered data sets after influential analysis in Chapter 6 . The plots of residuals in Appendix Figures D3.1-D3.4 and D4.1-D4.2 also justify that the most appropriate PCs are entered into the model. The sample plots in Figures D5.1-D5.4 indicate that no additional PCs - rather than those already entered into the model - will provide any significant additional strength in the model. All these sample plots and some key regression statistics in Tables $7.2-7.4$ show that the final selections are statistically significant and most appropriate. The regression equations of these final selected models for each data group are shown In Tables $7.5,7.6$ and 7.7 for Interstate, Urban Principal Arterial and Urban Minor Arterial \& Collector, respectively. These tables show the $R^{2}$ and $R_{a}^{2}$ values for Principal Component based Regression (PSR) model and, for comparison, the $R^{2}$ values for the final models selected in Chapter 6. The comparison of these $R^{2}$ values for the two alternative model forms does not support one form over the other. According to the principle behind developing PCR models (see discussion in Section 3.4), further evaluations of these relatively complex PCR models 
Table 7.5: Final Principal Component Regression (PCR) Equations for Interstate Highways

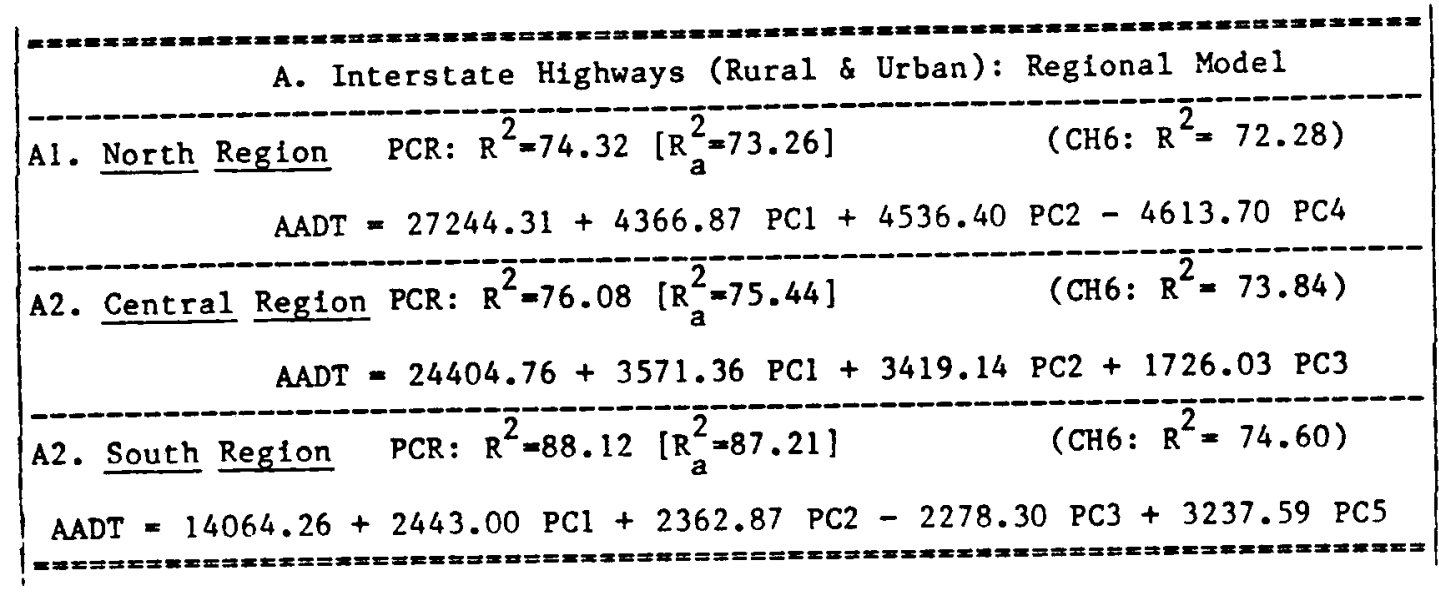

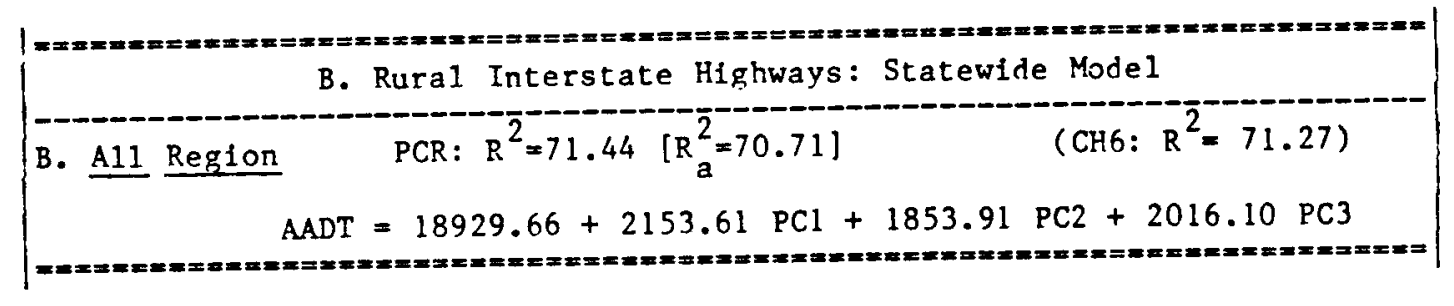

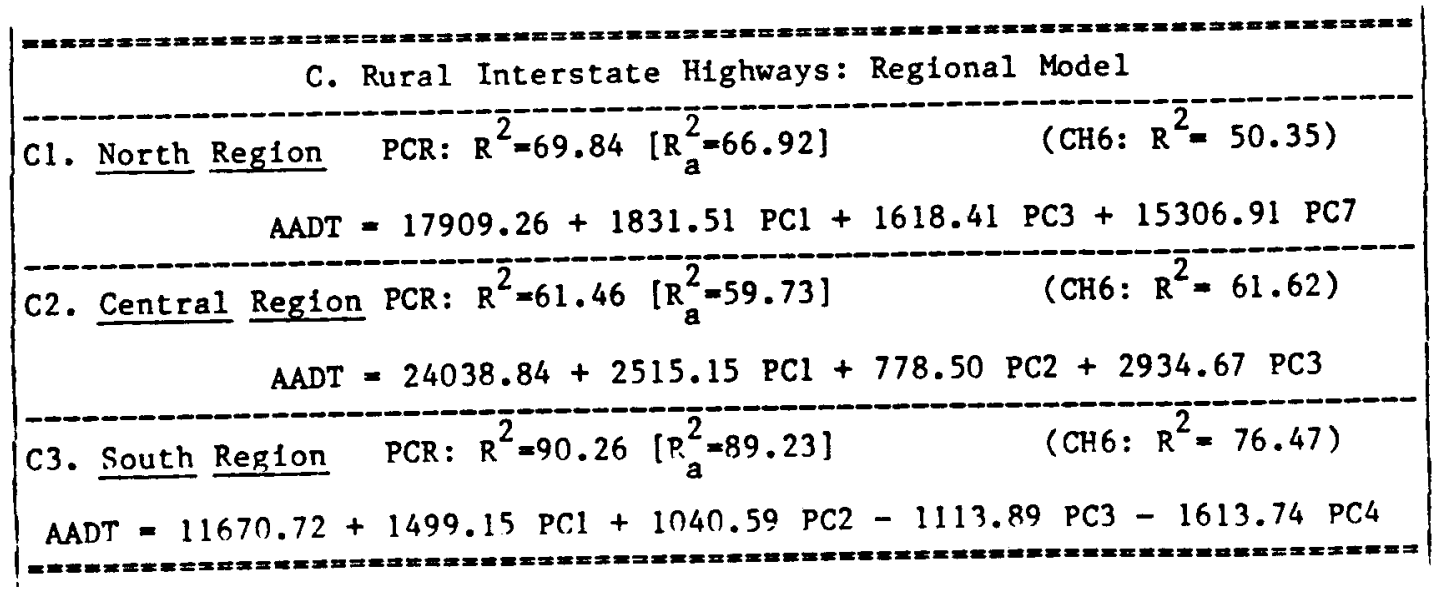


Table 7.5, continued

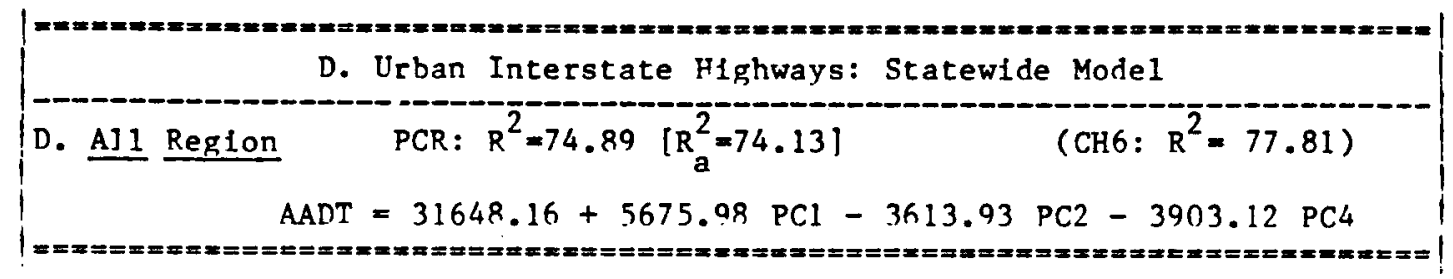

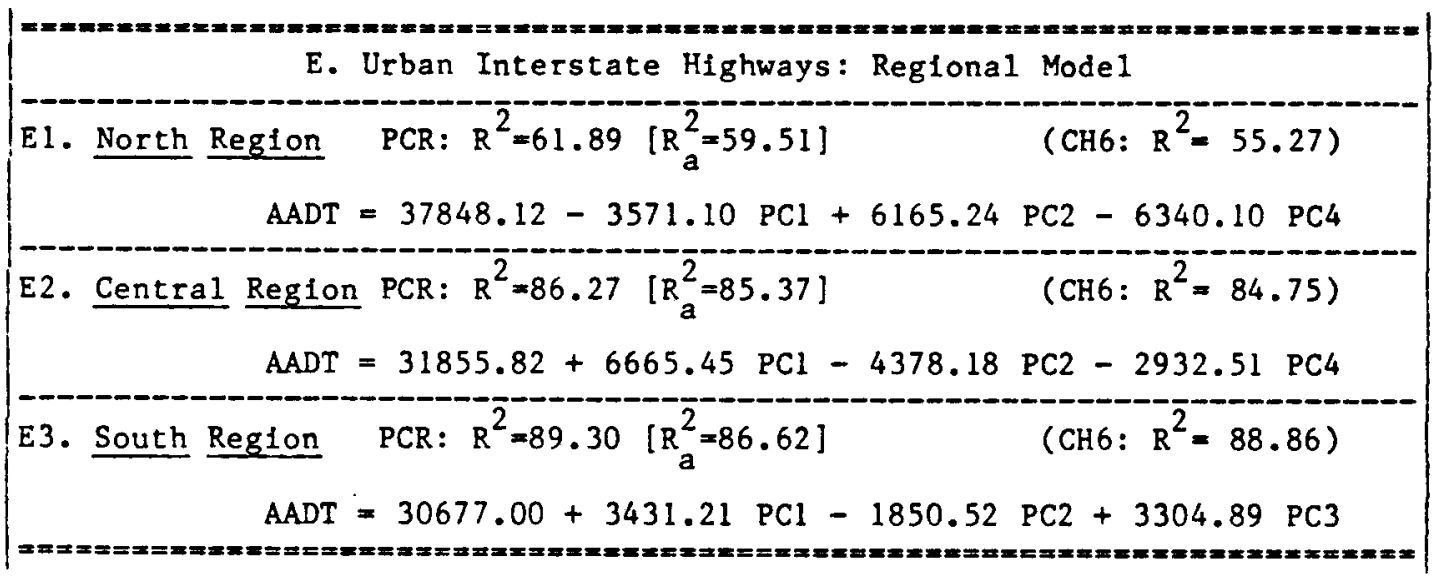

Note: See Table Dl for description of PCs' (Principal Components). 
Table 7.6: Final Principal Component Regression (PCR) Equations for Urban Principal Arterial

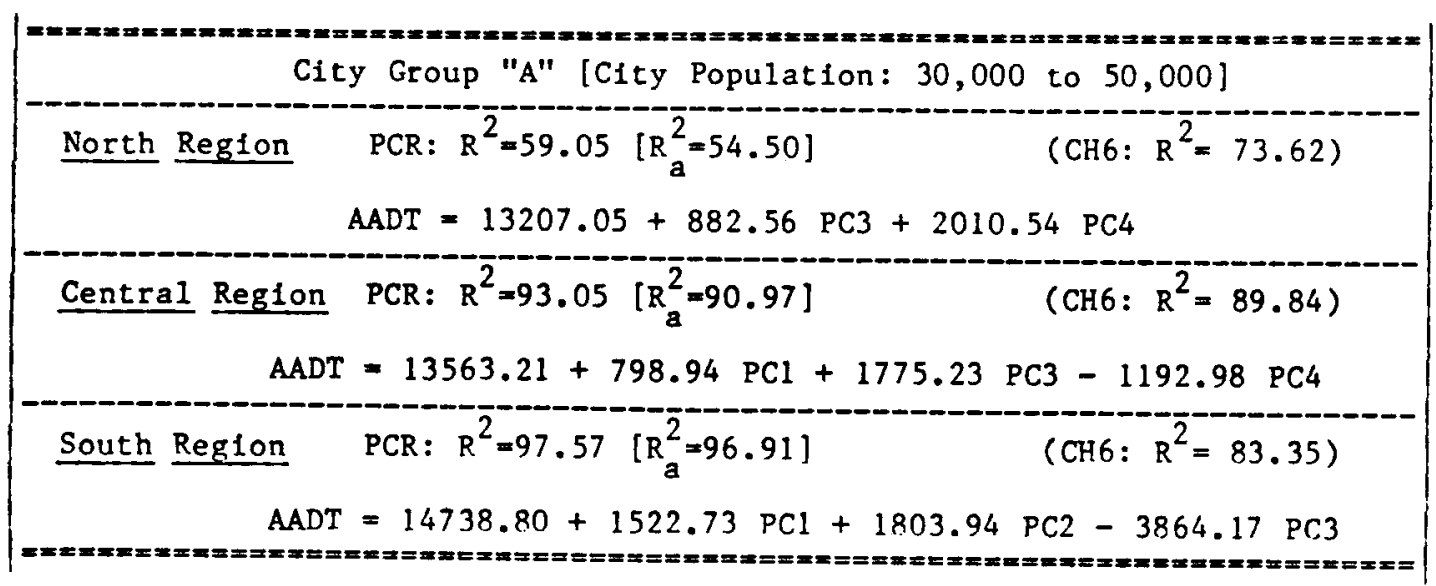

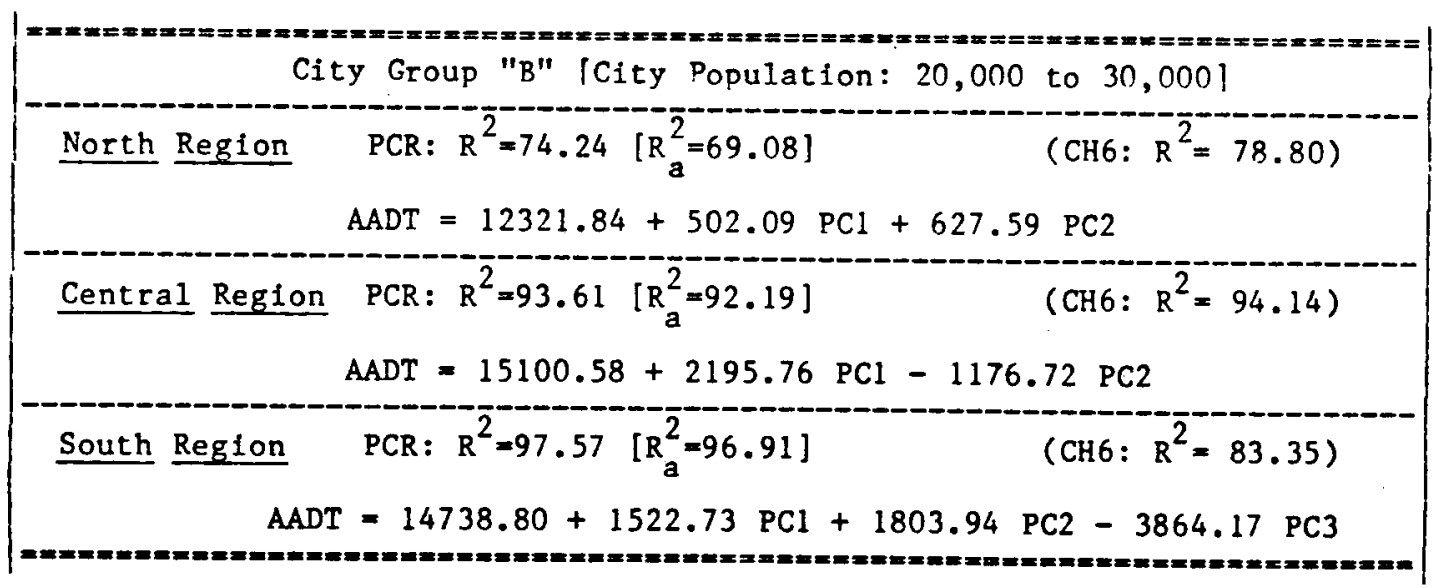


Table 7.6, continued

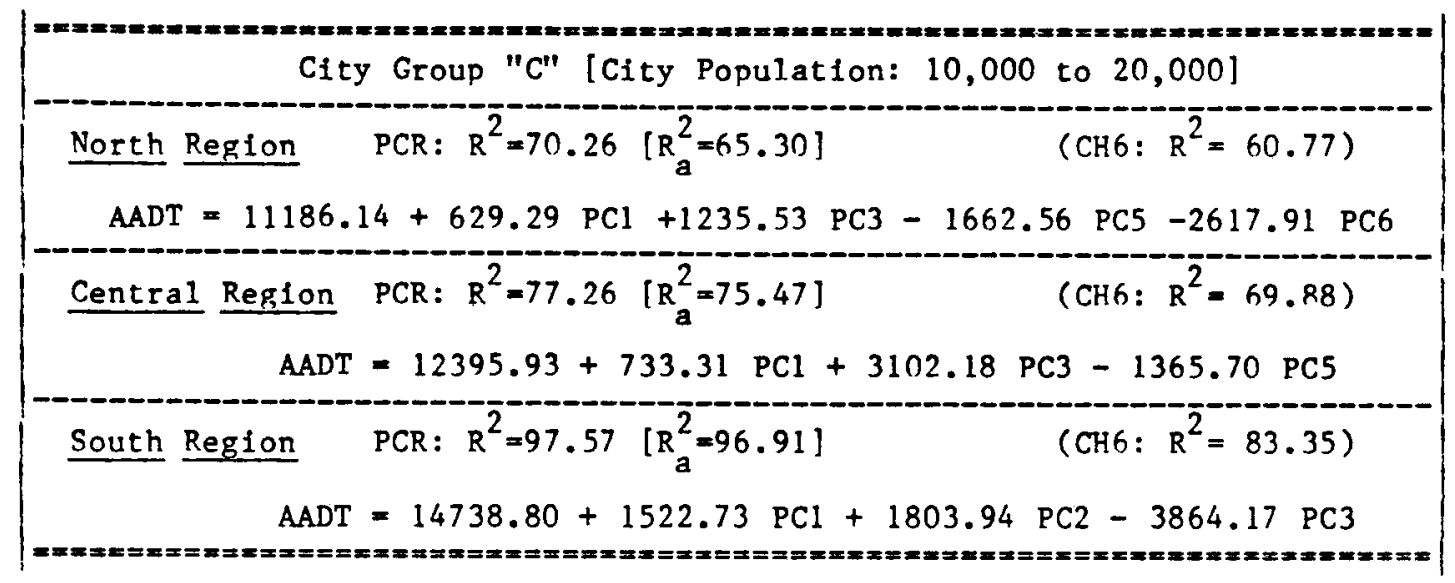

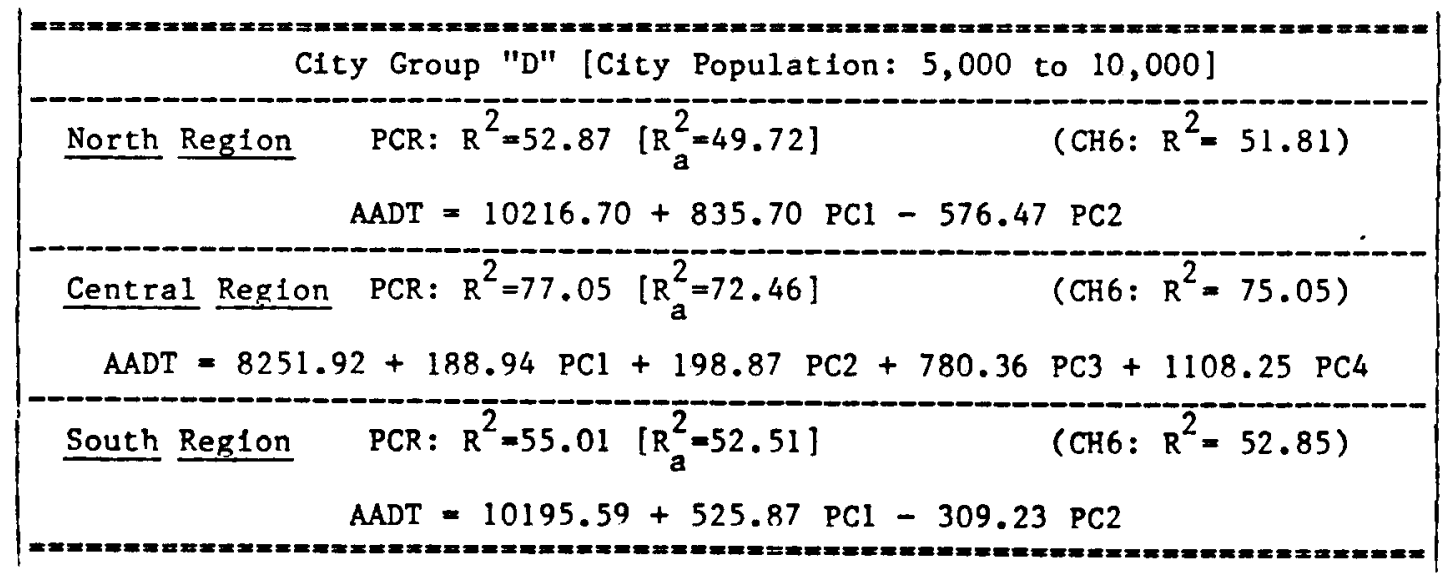

Note: See Table D2 for description of PCs' (Principal Components). 
Table 7.7: Final Princlpal Component Regression (PCR) Equations for Urban Minor Arterial \& Collector

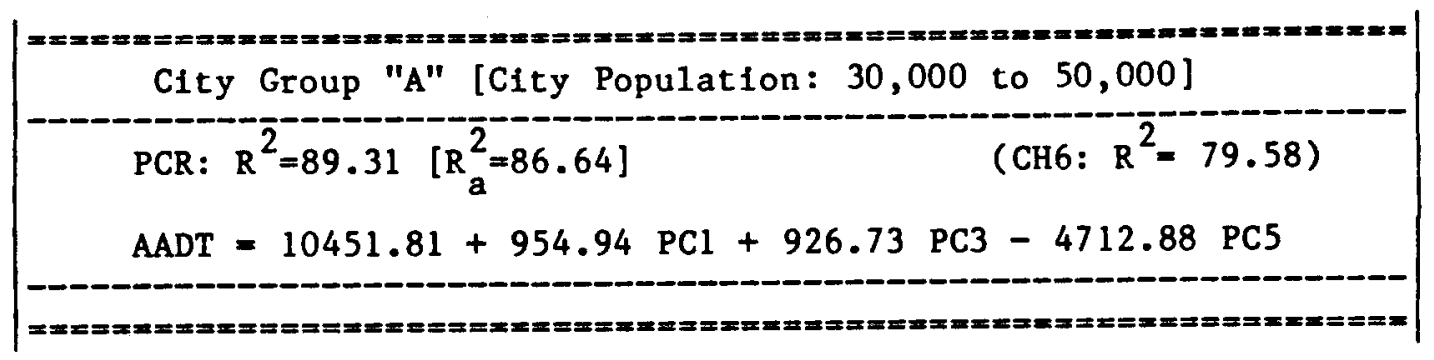

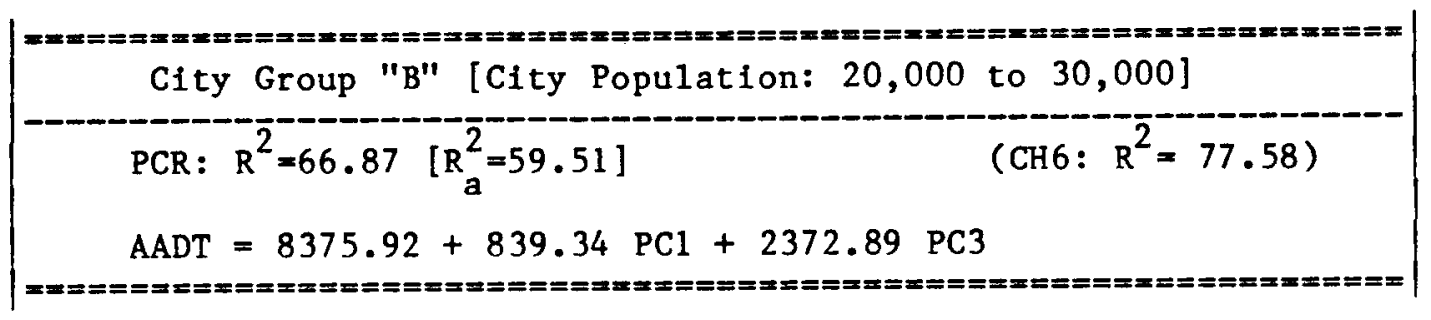

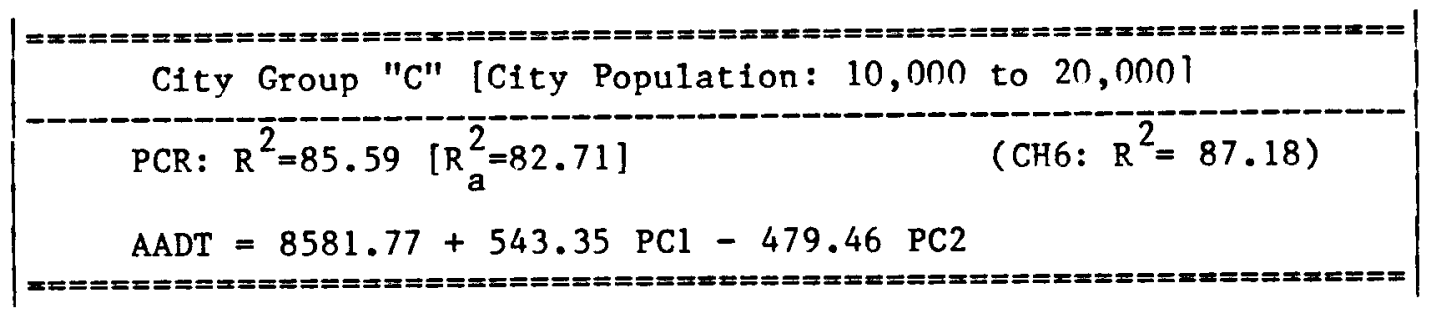

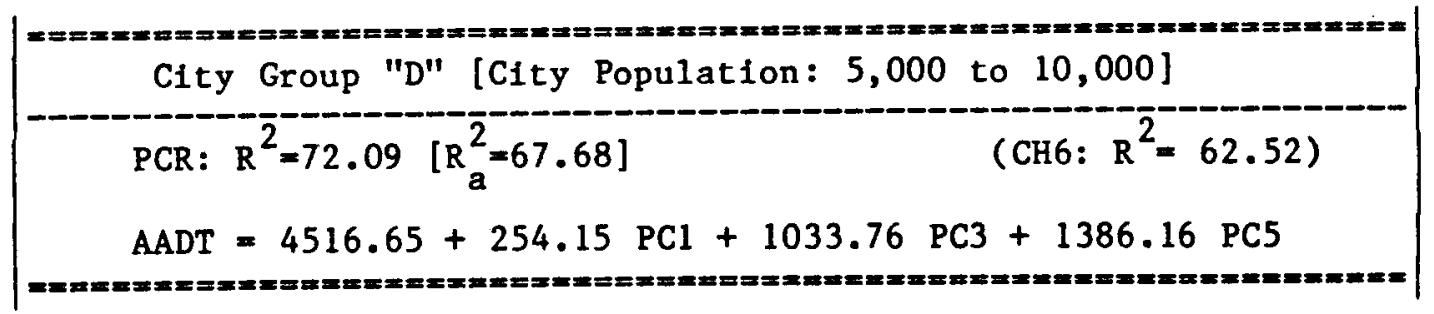

Note: See Table D3 for description of PCs' (Principal Components). 
are not warranted. The constant term of the models in Tables 7.5 to 7.7 represents the mean of the response variable, AADT. The PC values entered in each of these models in Tables $7.5-7.7$ can be computed from their relation to the eigenvectors presented in Appendix Tables D1-D3. The absolute values of the coefficients of the PCs in Tables D1-D3 represent the importance of each original variable in defining the PCs.

\subsection{Chapter Summary}

Principal Component Regression (PCR) models have been developed for each data group, shown in Chapters 5 and 6 . These alternative models are based on Principal Component Analysis (PCA). The PCA has reduced the dimension of the problem significantly. For example, it has formed only 2 or 3 useful uncorrelated variables (see the PCs with eigenvalues greater than one in Table 7.1) from 11 original variables. Each of the final PCR models is found to be statistically significant, with $\mathrm{R}^{2}$ values similar to the final selections in Chapter 6 . The concepts and uses of the PCA have been provided in this chapter. The complete model bullding approach of Chapter 6 has been used to find the PCR models. The key results are presented in the figures and tables. Since by definition, each of the principal components is Independent of each other, the problem of multicollinearity - a problem that often arises with socioeconomic variables - has been completely eliminated. The alternative PCR models have also proved the suitability of models in Chapter 6 - regression and its derivative elasticity model -- with fewer original variables. 
CHAPTER 8

LAGGED TRAFFIC MODEL

\subsection{Introduction}

In this chapter, an inexpensive and simple model to forecast traffic will be developed, based on the traffic data and the rate at which traffic has changed over the years. The lagged traffic model developed in this chapter can be considered as a substitute for hundreds of "trend projections" employed often to forecast traffic at the project sites. The segment data bases (See Chapter 5) have been used to build the models. The lagged regression models have been developed with a full set of data. Statistical analyses and tests have been performed to find the quality of the models and to identify the strength of relation of the models. The partial lagged models are developed with the data set by omitting the last year of AADT for each highway segment in the data bases. The performance of the models has been evaluated by comparing the predicted last year AADT using the partial models with the observed AADT value of each highway segment. 


\subsection{Lagged Model Formulation}

The statistical analysis of lagged regression is not uncommon in transportation planning, economics and forecasting. For example, Chan [1985] used this technique to estimate highway travel and fuel consumption. In his formulation, both lagged endogeneous (dependent) variable and current exogeneous (independent) variables were used.

\subsubsection{Mode1ing Approach}

Often in statistical and econometric models involving time, a change in an explanatory variable has an affect that is distributed over time. A part of the impact may occur immediately, but the additional effects may persist through time. Sometimes, there may be no immediate effect and a lag is required before a response occurs. The general form of the lag model with equal time intervals between observations can be represented as

$$
Y_{t}=k_{0}+\sum_{i=1}^{P} k_{y i} Y_{t-1}+\sum_{j=1}^{Q} k_{x j} X_{t-j}+\varepsilon_{t}
$$

Both explanatory (Independent, X) and response (dependent, $Y$ ) var1ables can enter into the lag structure. In Equation (8.1), the $k^{\prime} s$ are constant and $\varepsilon_{t}$ is the random error term. In general, more than one lag variable $(P>1$ and/or $Q>1$ in Equation (8.1)) will tend to be highly correlated and will not be able to obtain reliable estimates. At the same time, models with more than one lagged variable lead to more complicated models to estimate. On the other hand, no guidelines 
are avallable as to an acceptable length of the lag. With the objective of building simple model, the analysis in this chapter has shown that the simple model - $\mathrm{P}=1$, no explanatory lag variable and a modification to account for unequal time interval between observations -is enough to describe most of the variation in traffic data.

\subsubsection{Model Formulation}

The formulation of lagged regression is simple when the data are equally spaced (for example, data are available for each year). The origina1 highway segment data bases (See Chapter 5 and the tables in Appendix A) are used in the analysis. Time intervals between AADT observations in these data bases are not the same. A modified formulation of the lagged regression of Equation (8.1) is warranted to account for these unequal time intervals. To make the model simple, only the first lagged value - P $=1$ in Equation (8.1) - of AADT is used in this model. The models with exogeneous variables are developed in earlier chapters.

It has been postulated that the amount of traffic would follow this time-series pattern:

$$
\text { AADT }_{t}=k_{0}+k_{m} \text { LAADT }_{t-m}+k_{r} \text { RAADT }_{t m}+\varepsilon_{t}
$$

where,

$$
\begin{aligned}
\operatorname{AADT}_{t} & =\text { AADT in year } t \\
\text { LAADT }_{t \rightarrow m} & =\text { Lagged AADT in year } t-m,
\end{aligned}
$$




$$
\begin{aligned}
& \text { RAADT }_{t m}=\text { Rate of AADT change between years } t \text { and } t-m, \\
&=\left[\text { AADT }_{t}-\text { AADT }_{t-m}\right] / m \text {, and } \\
& k_{0}, k_{m} \text { and } k_{r} \text { are the coefficients. }
\end{aligned}
$$

Linear regression of SAS [SAS/STATISTICS 1985] has been used to determine the coefficients of the Equation (8.2). The term " $k_{0}$ " in Equation (8.2) represents the average effect of all excluded (not explicitly considered) factors in the model. The $\varepsilon_{t}$ is the part of the model that is "unexplained" and is caused by the factors about which analysts remain ignorant. The expected value of $\varepsilon_{t}$ is zero. Then on rearrangement, Equation $(8.2)$ can be transformed to:

$$
\operatorname{AADT}_{t}=\frac{k_{0}^{m}}{m-k_{r}}+\frac{k_{m}^{m}-k_{r}}{m-k_{r}} \text { LAADT }_{t-m}
$$

The terms in Equation (8.3) are defined under Equation (8.2). Equation (8.3) will be used to predict AADT at any year by knowing the lag year AADT (LAADT) and $m$ - the difference of year between lag year $(t-m)$ and the year $(t)$ at which forecast is desired. The term "m" w111 be referred as "forecast period".

\subsection{Lagged Regression Mode1}

For comparison, the groupings of data sets are unchanged with respect to those used in earlier chapters. The "REG" procedure of SAS [SAS/Statistics 1985] is used to perform the regression in Equation (8.2). The model using a full data set has been referred to as the 
"full-lagged model" or simply as the "lagged model". To evaluate the performance of the models, models are formed by deleting the last year AADT observation for each roadway segment in the segment data bases. The model with this partial data sets is referred to as "Partial Lagged Model" in this chapter.

To depict the graphical plots associated with residual analyses, the following three models - one from each major highway class -have been selected:

\author{
Model 1: North Region of Urban Interstate, \\ Model 2: City Group "A" and North Region of \\ Urban Principal Arterial; and \\ Model 3: City Group "C" of Urban Minor \\ Arterial \& Collector.
}

\title{
8.3.1 Full-Lagged Model
}

The estimates of the parameters and the other key regression results of the lagged regression model (Equation 8.2) for interstate, urban minor arterial \& collector are presented in Tables $8.1,8.2$ and 8.3, respectively. The ranges of the varlous model statistics in these tables are as follows: 
Table 8.1: Fu11-Lagged Regression Results of Interstate Highways

\begin{tabular}{|c|c|c|c|c|c|c|c|c|c|}
\hline \multirow{3}{*}{ Region } & \multicolumn{2}{|c|}{ Model Statistics } & \multicolumn{5}{|c|}{ Significance Statistics \& Parameter Values } & \multicolumn{2}{|c|}{ Normality Stat. } \\
\hline & $R^{2} R^{2}$ & $\begin{array}{l}\text { RMSE } \\
\text { C.V. }\end{array}$ & $\left\{\begin{array}{r}\text { F-Value } \\
\text { Prob }>\text { F }\end{array}\right.$ & Para & $\begin{array}{l}\text { ameter } \\
\text { Estimate }\end{array}$ & $\mid \begin{array}{c}t- \\
\text { value }\end{array}$ & Prob>t & $\mathrm{N}$ & $\mid \begin{array}{c}W / D(\star) \\
(P \text {-value })\end{array}$ \\
\hline & \multicolumn{9}{|c|}{ A. Interstate Highways (Rural \& Urban): Regional Model } \\
\hline North & $\begin{array}{l}99.46 \\
99.45\end{array}$ & $\begin{array}{r}2526.6 \\
6.289\end{array}$ & $\begin{array}{r}18488.8 \\
0.0001\end{array}$ & $\begin{array}{l}\text { CONST } \\
\text { LAADT } \\
\text { RAADT }\end{array}$ & $\begin{array}{r}250.05261 \\
1.00029 \\
2.36768\end{array}$ & $\begin{array}{r}0.906 \\
177.514 \\
28.105\end{array}$ & $\begin{array}{l}0.3663 \\
0.0001 \\
0.0001\end{array}$ & 204 & $\begin{array}{r}0.1288 \\
(<0.01)\end{array}$ \\
\hline Central & $\begin{array}{l}98.42 \\
98.41\end{array}$ & $\begin{array}{r}2770.5 \\
7.224\end{array}$ & $\begin{array}{r}12962.6 \\
0.0001\end{array}$ & $\begin{array}{l}\text { CONST } \\
\text { LAADT } \\
\text { RAADT }\end{array}$ & $\begin{array}{r}62.02860 \\
1.03957 \\
1.54560\end{array}$ & $\begin{array}{r}0.226 \\
146.955 \\
31.521\end{array}$ & $\begin{array}{l}0.8211 \\
0.0001 \\
0.0001\end{array}$ & 419 & $\begin{array}{r}0.1126 \\
(<0.01)\end{array}$ \\
\hline South & $\begin{array}{l}98.10 \\
98.07\end{array}$ & $\begin{array}{l}2021.4 \\
10.222\end{array}$ & $\begin{array}{l}4021.5 \\
0.0001\end{array}$ & $\begin{array}{l}\text { CONST } \\
\text { LAADT } \\
\text { RAADT }\end{array}$ & $\begin{array}{r}-846.55778 \\
1.10673 \\
1.85193\end{array}$ & $\begin{array}{l}-2.965 \\
74.320 \\
17.914\end{array}$ & $\begin{array}{l}0.0035 \\
0.0001 \\
0.0001\end{array}$ & 159 & $\begin{array}{r}0.1557 \\
(<0.01)\end{array}$ \\
\hline & B. Rural & Interst & te Highwa & ys: Sta & atewide Model & & & & \\
\hline ALL & $\begin{array}{l}97.53 \\
97.52\end{array}$ & $\begin{array}{r}2088.0 \\
8.925\end{array}$ & $\begin{array}{l}9371.6 \\
0.0001\end{array}$ & $\begin{array}{l}\text { CONST } \\
\text { LAADT } \\
\text { RAADT } \\
\text { RAA }\end{array}$ & $\begin{array}{r}177.88057 \\
1.02221 \\
1.79409\end{array}$ & $\left\{\begin{array}{r}0.895 \\
115.594 \\
32.858 \\
===x==m\end{array}\right.$ & $\begin{array}{l}0.3711 \\
0.0001 \\
0.0001 \\
== \pm==1=1\end{array}$ & 478 & $\begin{array}{r}0.1462 \\
(<0.01)\end{array}$ \\
\hline & C. Rural & Interst & te Highr & ys: $\operatorname{Reg}$ & gional Model & & & & \\
\hline North & $\left|\begin{array}{l}94.61 \\
94.50\end{array}\right|$ & $\begin{array}{r}1859.1 \\
8.815\end{array}$ & $\begin{array}{r}851.6 \\
0.0001\end{array}$ & $\begin{array}{l}\text { I CONST } \\
\text { ILAADT } \\
\text { IRAADT }\end{array}$ & $\begin{array}{r}1097.59856 \\
0.96235 \\
2.14349\end{array}$ & $\begin{array}{r}1.998 \\
34.289 \\
19.105\end{array}$ & $\begin{array}{l}0.0485 \\
0.0001 \\
0.0001\end{array}$ & 100 & $\begin{array}{r}0.1432 \\
(<0.01)\end{array}$ \\
\hline Central & $\begin{array}{l}97.16 \\
97.14\end{array}$ & $\begin{array}{r}2398.6 \\
8.307\end{array}$ & $\begin{array}{l}4411.3 \\
0.0001\end{array}$ & $\begin{array}{l}\text { CONST } \\
\text { LAADT } \\
\text { RAADT }\end{array}$ & $\begin{array}{r}-118.49997 \\
1.03520 \\
1.70124\end{array}$ & $\begin{array}{l}-0.338 \\
79.899 \\
22.975\end{array}$ & $\begin{array}{l}0.7356 \\
0.0001 \\
0.0001\end{array}$ & 261 & $\begin{array}{l}0.1190 \\
(<0.01)\end{array}$ \\
\hline South & $\begin{array}{l}96.04 \\
95.97\end{array}$ & $\begin{array}{r}1271.5 \\
9.675\end{array}$ & $\begin{array}{l}1382.3 \\
0.0001\end{array}$ & $\begin{array}{l}\text { CONST } \\
\text { LAADT } \\
\text { RAADT }\end{array}$ & $\begin{array}{r}-207.38543 \\
1.05773 \\
1.80581\end{array}$ & $\begin{array}{l}-0.736 \\
49.623 \\
15.789\end{array}$ & $\begin{array}{l}0.4630 \\
0.0001 \\
0.0001\end{array}$ & 117 & $\begin{array}{r}0.1761 \\
(<0.01)\end{array}$ \\
\hline
\end{tabular}


Table 8.1 , continued

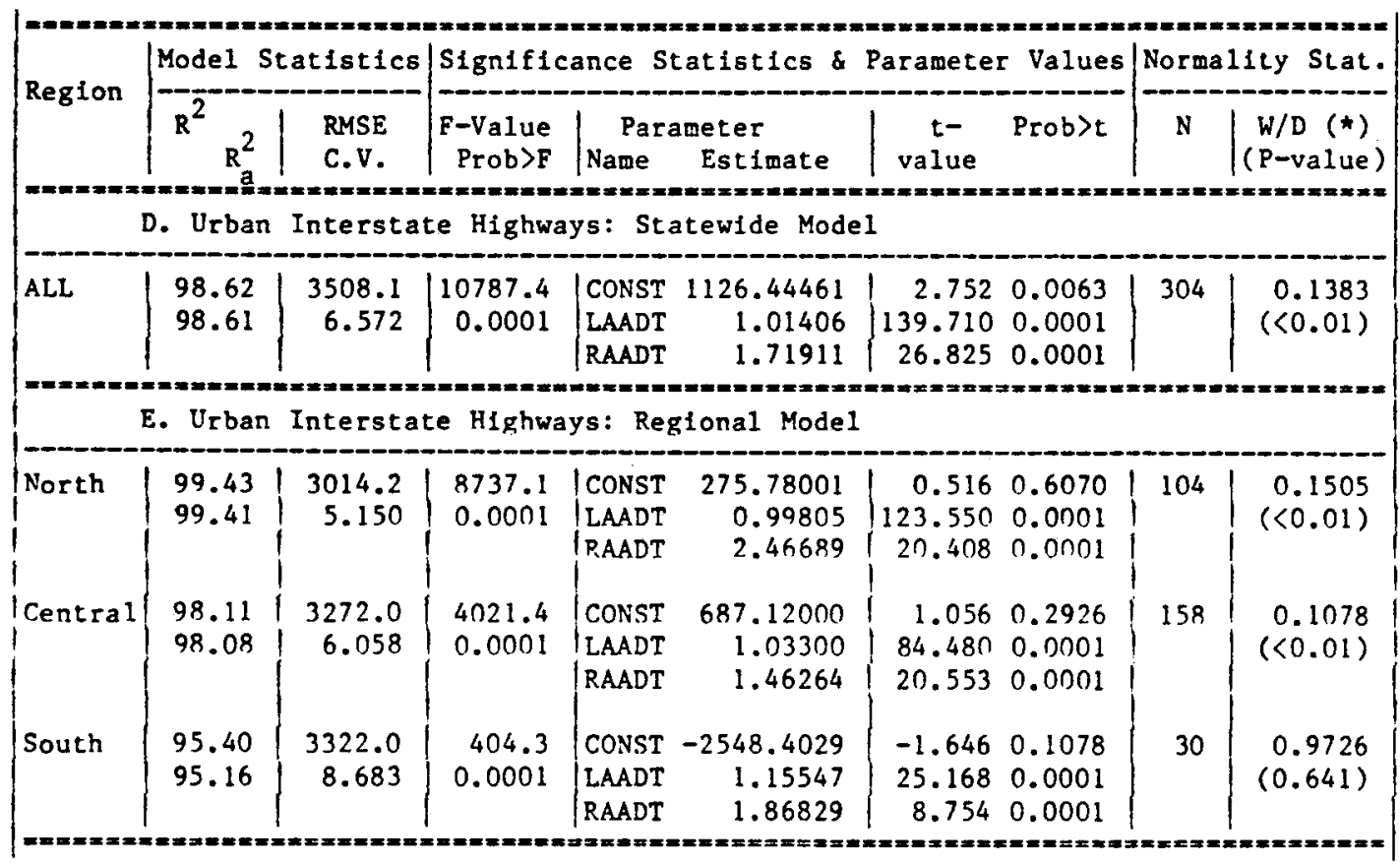

(*) Wilk-Shapiro ' $W$ ' for $N<51$ or Kolmogorov ' $D$ ' for $N>51$, and $P$-value $=P R O B>D$ or $P R O B<W$. 
Table 8.2: Ful1-Lagged Regression Results of Urban Principal
Arterial

\begin{tabular}{|c|c|c|c|c|c|c|c|c|c|}
\hline \multirow{3}{*}{ Region } & \multicolumn{2}{|c|}{ Moriel Statistics } & \multicolumn{5}{|c|}{ Significance Statistics \& Parameter Values } & \multicolumn{2}{|c|}{ Normality Stat. } \\
\hline & 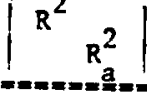 & $\begin{array}{l}\text { RMSE } \\
\text { C.V. }\end{array}$ & 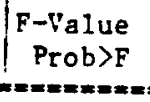 & $\prod_{\substack{\text { Name } \\
\text { Nanas }}}^{\text {Para }}$ & 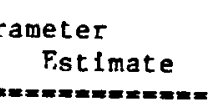 & 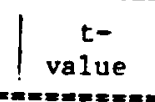 & Prob $>t$ & $\mathrm{~N}$ & $\mid \begin{array}{c}W / D(*) \\
\text { ( } P \text {-value })\end{array}$ \\
\hline & City Gr & "A" [C & ity Popul & lation: & 30,000 to 5 & 50,0001 & & & \\
\hline North & $\begin{array}{l}99.46 \\
99.45\end{array}$ & $\begin{array}{l}439.9 \\
3.399\end{array}$ & $\begin{array}{r}24800.5 \\
0.0001\end{array}$ & $\begin{array}{l}\text { CONST } \\
\text { LAADT } \\
\text { RAADT }\end{array}$ & $\begin{array}{r}27.83083 \\
0.98818 \\
3.61995\end{array}$ & $\begin{array}{r}0.422 \\
212.149 \\
124.378\end{array}$ & $\begin{array}{l}0.6737 \\
0.0001 \\
0.0001\end{array}$ & 273 & $\begin{array}{r}0.1229 \\
(<0.01)\end{array}$ \\
\hline Central & $\begin{array}{r}99.18 \\
99.18\end{array}$ & $\begin{array}{l}511.8 \\
3.889\end{array}$ & $\begin{array}{r}13406.7 \\
0.0001\end{array}$ & $\begin{array}{l}\text { CONST } \\
\text { LAADT } \\
\text { RAADT }\end{array}$ & $\begin{array}{r}46.81155 \\
0.99679 \\
3.77805\end{array}$ & $\begin{array}{r}0.526 \\
151.545 \\
61.846\end{array}$ & $\begin{array}{l}0.5992 \\
0.0001 \\
0.0001\end{array}$ & 224 & $\begin{array}{r}0.1161 \\
(<0.01)\end{array}$ \\
\hline South & $\begin{array}{l}99.35 \\
99.32\end{array}$ & $\begin{array}{l}247.7 \\
2.379\end{array}$ & $\begin{array}{l}3729.5 \\
0.0001\end{array}$ & $\begin{array}{l}\text { CONST } \\
\text { LAADT } \\
\text { RAADT }\end{array}$ & $\begin{array}{r}214.42029 \\
0.97593 \\
3.93023\end{array}$ & $\begin{array}{r}1.650 \\
80.058 \\
41.882\end{array}$ & $\begin{array}{l}0.1053 \\
0.0001 \\
0.0001\end{array}$ & 52 & $\begin{array}{r}0.1631 \\
(<0.01)\end{array}$ \\
\hline & City Gro & "B" [C & ty Popula & Lation: & 20,000 to 30 & 30,0001 & & & \\
\hline North & $\begin{array}{l}99.98 \\
99.98\end{array}$ & $\begin{array}{r}86.5 \\
0.706\end{array}$ & $\begin{array}{l}259068 \\
0.0001\end{array}$ & $\begin{array}{l}\text { CONST } \\
\text { LAADT } \\
\text { RAADT }\end{array}$ & $\begin{array}{r}-6.30353 \\
1.00180 \\
3.96802\end{array}$ & $\left\{\begin{array}{r}-0.332 \\
675.741 \\
266.187\end{array}\right.$ & $\begin{array}{l}0.7405 \\
0.0001 \\
0.0001\end{array}$ & 118 & $\begin{array}{r}0.2710 \\
(<0.01)\end{array}$ \\
\hline Central & $\begin{array}{l}99.61 \\
99.60\end{array}$ & $\begin{array}{l}458.0 \\
3.435\end{array}$ & $\begin{array}{l}8886.8 \\
0.0001\end{array}$ & $\begin{array}{l}\text { CONST } \\
\text { LAADT } \\
\text { RAADT }\end{array}$ & $\begin{array}{r}-98.00552 \\
1.00303 \\
3.45706\end{array}$ & $\begin{array}{r}-0.855 \\
132.321 \\
52.871\end{array}$ & $\begin{array}{l}0.3952 \\
0.0001 \\
0.0001\end{array}$ & 72 & $\begin{array}{r}0.0769 \\
(<0.01)\end{array}$ \\
\hline South & $\begin{array}{l}99.22 \\
99.10\end{array}$ & $\begin{array}{l}378.5 \\
1.552\end{array}$ & $\begin{array}{r}823.6 \\
0.0001\end{array}$ & $\begin{array}{l}\text { CONST } \\
\text { LAADT } \\
\text { RAADT }\end{array}$ & $\begin{array}{r}1757.29224 \\
0.93057 \\
3.76437\end{array}$ & $\begin{array}{r}3.106 \\
40.464 \\
20.326\end{array}$ & $\begin{array}{l}0.0084 \\
0.0001 \\
0.0001\end{array}$ & 16 & $\begin{array}{r}0.9188 \\
(0.212)\end{array}$ \\
\hline
\end{tabular}


Table 8.2 , continued

\begin{tabular}{|c|c|c|c|c|c|c|c|c|c|}
\hline \multirow{3}{*}{ Region } & \multicolumn{2}{|c|}{ Model Statistics } & \multicolumn{5}{|c|}{ Significance Statistics \& Parameter Values } & \multicolumn{2}{|c|}{ Normality Stat. } \\
\hline & $\mathrm{R}^{2} \mathrm{R}^{2}$ & $\begin{array}{l}\text { RMSE } \\
\text { C.V. }\end{array}$ & $\left\{\begin{array}{r}\text { F-Value } \\
\text { Prob }>\text { F }\end{array}\right.$ & $\begin{array}{l}\text { Para } \\
\text { Name }\end{array}$ & $\begin{array}{l}\text { meter } \\
\text { Estimate }\end{array}$ & $\begin{array}{c}t- \\
\text { value }\end{array}$ & Prob $>t$ & $\mathbf{N}$ & $\begin{array}{c}W / D(*) \\
(P \text {-value })\end{array}$ \\
\hline & \multicolumn{9}{|c|}{ City Group "C" [City Population: 10,000 to 20,000 ] } \\
\hline North & $\begin{array}{l}99.05 \\
99.04\end{array}$ & $\begin{array}{l}331.9 \\
3.632\end{array}$ & $\begin{array}{r}10838.3 \\
0.0001\end{array}$ & $\begin{array}{l}\text { CONST } \\
\text { LAADT } \\
\text { RAADT }\end{array}$ & $\begin{array}{r}331.91553 \\
0.96816 \\
3.63161\end{array}$ & $\begin{array}{r}3.880 \\
128.524 \\
76.250\end{array}$ & $\begin{array}{l}0.0001 \\
0.0001 \\
0.0001\end{array}$ & 210 & $\begin{array}{r}0.1270 \\
(<0.01)\end{array}$ \\
\hline Central & $\begin{array}{l}99.07 \\
99.07\end{array}$ & $\begin{array}{l}530.0 \\
4.487\end{array}$ & $\begin{array}{r}13484.1 \\
0.0001\end{array}$ & $\begin{array}{l}\text { CONST } \\
\text { LAADT } \\
\text { RAADT }\end{array}$ & $\begin{array}{r}-3.43535 \\
1.00199 \\
3.63089\end{array}$ & $\left\{\begin{array}{r}-0.042 \\
147.624 \\
66.623\end{array}\right.$ & $\begin{array}{l}0.9664 \\
0.0001 \\
0.0001\end{array}$ & 255 & $\begin{array}{r}0.1389 \\
(<0.01)\end{array}$ \\
\hline South & $\begin{array}{l}98.12 \\
97.99\end{array}$ & $\begin{array}{l}619.2 \\
5.692\end{array}$ & $\begin{array}{r}756.9 \\
0.0001\end{array}$ & $\begin{array}{l}\text { CONST } \\
\text { I LAADT } \\
\text { RAADT }\end{array}$ & $\begin{array}{r}378.46475 \\
0.97113 \\
3.23398\end{array}$ & $\begin{array}{r}1.100 \\
28.834 \\
18.683\end{array}$ & $\begin{array}{l}0.2802 \\
0.0001 \\
0.0001\end{array}$ & 32 & $\begin{array}{r}0.7370 \\
(<0.01)\end{array}$ \\
\hline & City Gro & "D" IC & -y Popt & ation: & 5,000 to 10 &, 0001 & & & \\
\hline North & $\begin{array}{l}98.83 \\
98.80\end{array}$ & $\begin{array}{l}429.8 \\
4.432\end{array}$ & $\begin{array}{l}3679.6 \\
0.0001\end{array}$ & $\begin{array}{l}\text { CONST } \\
\text { LAADT } \\
\text { RAADT }\end{array}$ & $\begin{array}{r}106.51264 \\
0.99136 \\
3.95393\end{array}$ & $\begin{array}{r}0.973 \\
81.650 \\
39.398\end{array}$ & $\begin{array}{l}0.3848 \\
0.0001 \\
0.0001\end{array}$ & 90 & $\begin{array}{r}0.2553 \\
(<0.01)\end{array}$ \\
\hline Central & $\begin{array}{l}99.57 \\
99.56\end{array}$ & $\begin{array}{l}217.4 \\
2.405\end{array}$ & $\begin{array}{l}9406.2 \\
0.0001\end{array}$ & $\begin{array}{l}\text { CONST } \\
\text { LAADT } \\
\text { RAADT }\end{array}$ & $\begin{array}{r}82.77866 \\
0.99630 \\
3.72608\end{array}$ & $\begin{array}{r}1.121 \\
119.990 \\
58.926\end{array}$ & $\begin{array}{l}0.2655 \\
0.0001 \\
0.0001\end{array}$ & 85 & $\begin{array}{r}0.1648 \\
(<0.01)\end{array}$ \\
\hline South & $\begin{array}{l}99.13 \\
99.12\end{array}$ & $\begin{array}{l}359.1 \\
3.344\end{array}$ & $\begin{array}{l}8761.5 \\
0.0001\end{array}$ & $\begin{array}{l}\text { CONST } \\
\text { LAADT } \\
\text { RAADT }\end{array}$ & $\begin{array}{r}200.54730 \\
0.99216 \\
3.15041\end{array}$ & $\begin{array}{r}2.270 \\
116.576 \\
53.639\end{array}$ & $\begin{array}{l}0.0246 \\
0.0001 \\
0.0001\end{array}$ & 157 & $\begin{array}{c}0.2548 \\
(<0.01)\end{array}$ \\
\hline
\end{tabular}

(*) Wilk-Shapiro ' $W$ ' for $N<51$ or Kolmogorov ' $D$ - for $N>51$, and $P$-value $=P R O B>D$ or $P R O B<W$. 

Table 8.3: Ful1-Lagged Regression Results of Urban Minor
Arterial \& Collector

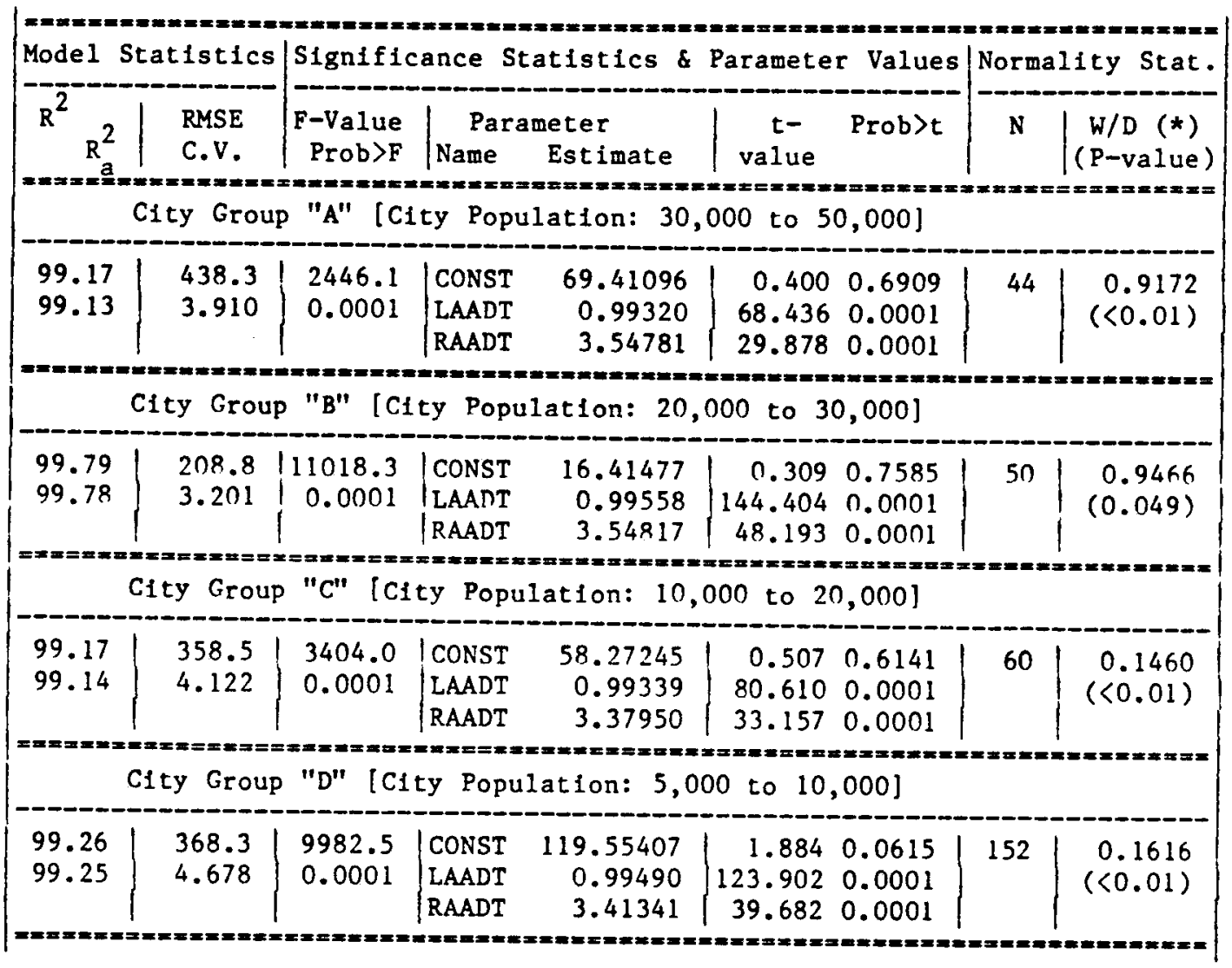

(*) Wilk-Shapiro ' $W$ ' for $N<51$ or Kolmogorov ' $D$ ' for $N>51$, and $\mathrm{P}$-value $=\mathrm{PROB}>\mathrm{D}$ or $\mathrm{PROB}<\mathrm{W}$. 


\begin{tabular}{|l|cccc|}
\hline $\begin{array}{l}\text { Highway } \\
\text { Class }\end{array}$ & $\mathrm{R}^{2}$ & $\mathrm{R}_{\mathrm{a}}{ }^{2}$ & $\mathrm{RMSE}$ & $\mathrm{C} . \mathrm{V}$. \\
\hline $\begin{array}{l}\text { Interstate } \\
\text { Highways }\end{array}$ & $94.61-99.46$ & $94.50-99.45$ & $1272-3508$ & $5.15-10.22$ \\
$\begin{array}{l}\text { Urban Prin- } \\
\text { cipal Art. }\end{array}$ & $98.12-99.98$ & $97.99-99.98$ & $87-619$ & $0.71-5.69$ \\
$\begin{array}{l}\text { Urban Minor } \\
\text { Arterial \& } \\
\text { Collector }\end{array}$ & $99.17-99.79$ & $99.13-99.78$ & $209-438$ & $3.20-4.68$ \\
\hline
\end{tabular}

The high $R^{2}$ (Coefficient of Multiple Determination) and $R_{a}^{2}$ (Adjusted Coefficient of Multiple Determination) statistics of the lagged models imply that no additional variables is necessary to reduce the variation of traffic. The RMSE (Root Mean Square Error) and the C.V. (Coefficient of Variation) values of the models in Tables $8.1-8.3$ also have been reduced significantly as compared to the models in the Chapters 6 and 7 .

The parameter estimates, which correspond to parameter names CONST, LAADT and RAADT in Tables 8.1-8.3, represent the values of $k_{0}$, $k_{m}$ and $k_{r}$ of Equations 8.2 and 8.3. In addition, the significance tests of Section 6.4 are performed to see the significance of overall regression relation and the significance of each term in Equation (8.1). The significance statistics (F-value, PROB $>F, t-v a l u e$ and $\mathrm{PROB}>\mathrm{t}$ ) show that a strong overall relationship exists and the parameters (LAADT and RAADT) are highly significant in explaining traffic volumes (AADT). The P-values in most of these tests show that the overall regression relation and the variables in Equation (8.2) are significant at an a level of 0.0001 . 
Partial regression residual plots of LAADT and RAADT for the selected models, one from each major highway class, are presented in Appendix Figures El.1-E1.6. These sample plots also show that the variables in the equation are significant. The "CONST" (Constant or Intercept) term has been found insignificant in most of the cases for an $\alpha$ level of 0.05 . The "CONST" term in the model represents the average effect of all factors not explicitly considered in the model. The term has a meaning when the explicitly considered factors in the model can take a value of zero. Thus, nonsignificance of the "CONST" term is not taken as evidence to drop the term from the model.

Nonnormality of the error terms is found for most of the models of Tables 8.1-8.3 for an a level of 0.01 . Three sample normal probability plots of the selected models, one from each major highway class, are presented in Appendix Figures E2.1-E2.3. The curvature of these plots also indicates that the errors are not normally distributed. Much of the curvature in these plots are due to the presence of outlier(s) in the data set. The normality of error terms is important to support the validity of the significance test. In Chapter 6 , normality statistics have been found to be improved in the postInfluence case. For segment data bases, influential analysis is done (results not shown) to identify the outlier(s), but the outliers are not deleted from the data sets because they are thought to be valid our simple model has simply falled to model the outlier(s). Moreover, the high degree of significance of the variables in the model equation and the highly significant regression relation mean that the results of the significance test are not expected to be altered, whether or 
not the error terms have a normal distribution or not.

The collinearity statistics of the models in Tables 8.1-8.3, introduced in Chapter 6, are examined. Although, all the results of collinearity are not shown, the expected sign of the parameters (LAADT and RAADT) and their high probability of significance are enough to convince us that there is no problem of multicollinearity.

The residual plots for each of the selected model group (one from each major highway class) are drawn against --

1. predicted values (see Appendix Figures E3.1-E3.3) and

2. variables in the model - LAADT and RAADT (see Appendix Figures E4.1-E4.6).

These plots do not suggest any gross departure from non-constancy of error variance and non-linearity of the regression relation. But, evidence of outlier(s) is present in these graphical plots. For example, an observation in these plots at a distance of more than 3 times the standard deviation (STD DEV) can be considered to be an outlier. In Chapter 6, outliers are removed, if possible, to eliminate their influence on the average county and city average AADT. But for segment AADT data base, the outllers are considered valid data and no modification except a check for coding error is done for the observation(s) suspected to be outlier(s). A trend of increasing the band width of residuals from near-zero values of RAADT to the higher absolute values of RAADT in the plots is an expected result, because at low rate of AADT change errors are expected to be small and viceversa. Moreover, the usual transformations (for example, log, 
square-root, $\log -\log$, etc.) to remove this trend will complicate the model structure and create a problem for some negative values of RAADT. Also, any transformations are not warranted from the sample scatterplots in Figures 8.1 and 8.2 of AADT against LAADT and RAADT, because a linear trend is evident in these plots. A sample residual plot against RAADT when the variable RAADT is not in model is shown in Figure 8.3. This sample residual plot also does not suggest any form but linear. It also shows that RAADT is an important variable. The model statistics of the three selected representative models, one from each major highway class, with and without RAADT, in the presence of LAADT in the models, are

\begin{tabular}{|c|l|rrrr|}
\hline $\begin{array}{c}\text { Selected } \\
\text { Mode1 }\end{array}$ & $\begin{array}{l}\text { RAADT } \\
\text { in Mode } 1\end{array}$ & $\mathrm{R}^{2}$ & $\mathrm{R}_{\mathrm{a}}^{2}$ & $\mathrm{RMSE}$ & $\mathrm{C.V}$. \\
\hline Mode1 1 & Absent & 97.06 & 97.03 & 6789 & 11.60 \\
& Present & 99.43 & 99.41 & 3014 & 5.15 \\
\hline Mode1 22 & Absent & 68.44 & 68.32 & 3353 & 25.90 \\
& Present & 99.46 & 99.45 & 440 & 3.40 \\
\hline Mode1 3 & Absent & 83.16 & 82.87 & 1601 & 18.40 \\
& Present & 99.17 & 99.14 & 358 & 4.12 \\
\hline
\end{tabular}

Thus, RAADT in the model has significantly improved all the model statistics and consequently will improve the predictive power of the model. This improvement with RAADT is applicable for all the lagged models.

The Prediction models of Equation (8.3) of the full-lagged regression models are shown in Tables $8.4,8.5$ and 8.6 for Interstate, Urban Principal Arterial and Urban Minor Arterial \& Collector, respectively. These models are derived by using the values of parameter 
LEGEND: $A=1$ OBS, $B=2$ OBS, ETC.

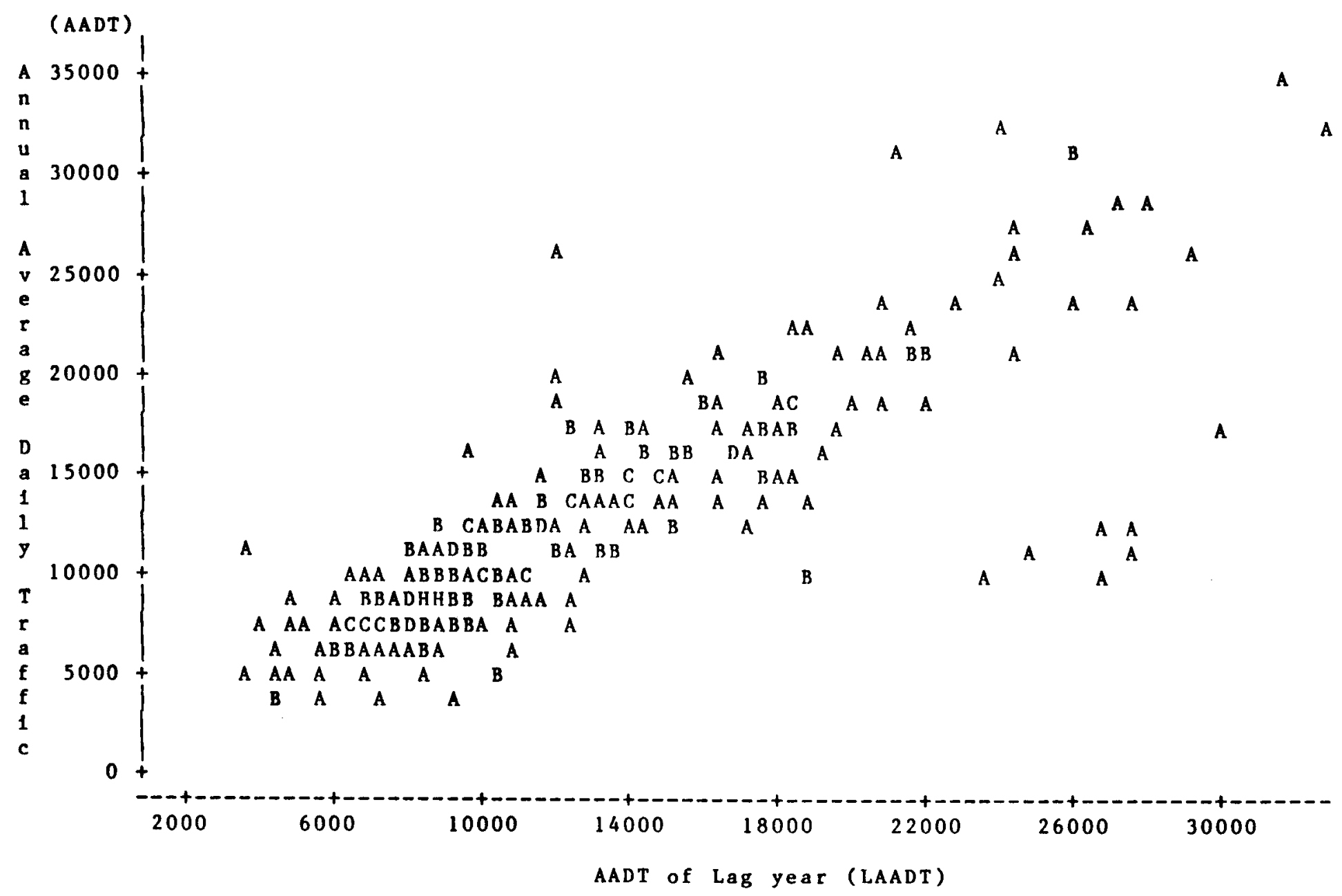

Figure 8.1: Scatterplot of AADT against LAADT for City Group "A" and Region " $n$ " of Urban Principal Arterial 


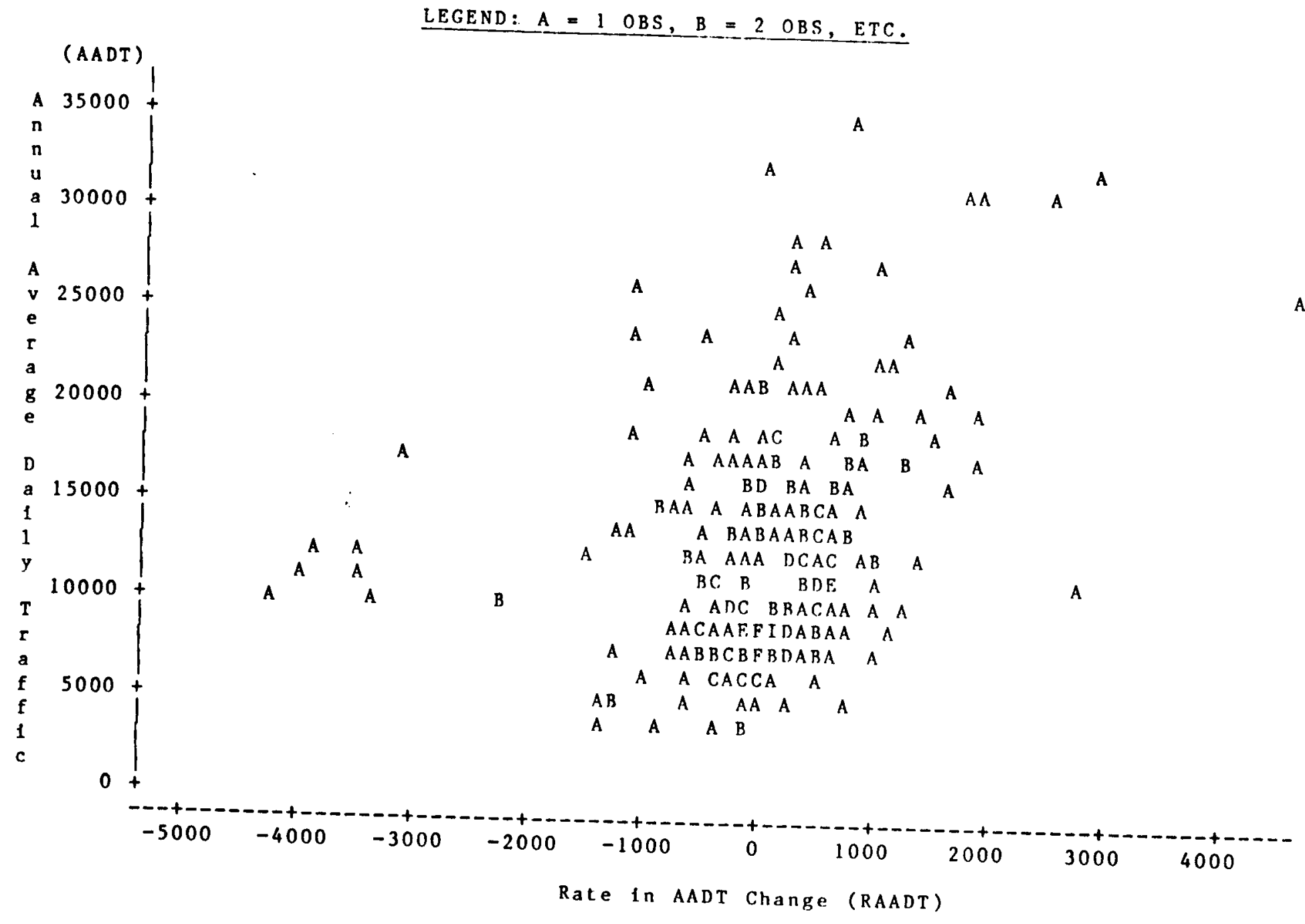

Figure 8.2: Scatterplot of AADT agatnst RAADT for city Croup "A" and Region " $n$ " of Urban Princtpal Arterlal 
Table 8.4: Lagged Traffic (Lag-AADT) Models for Interstate Highways

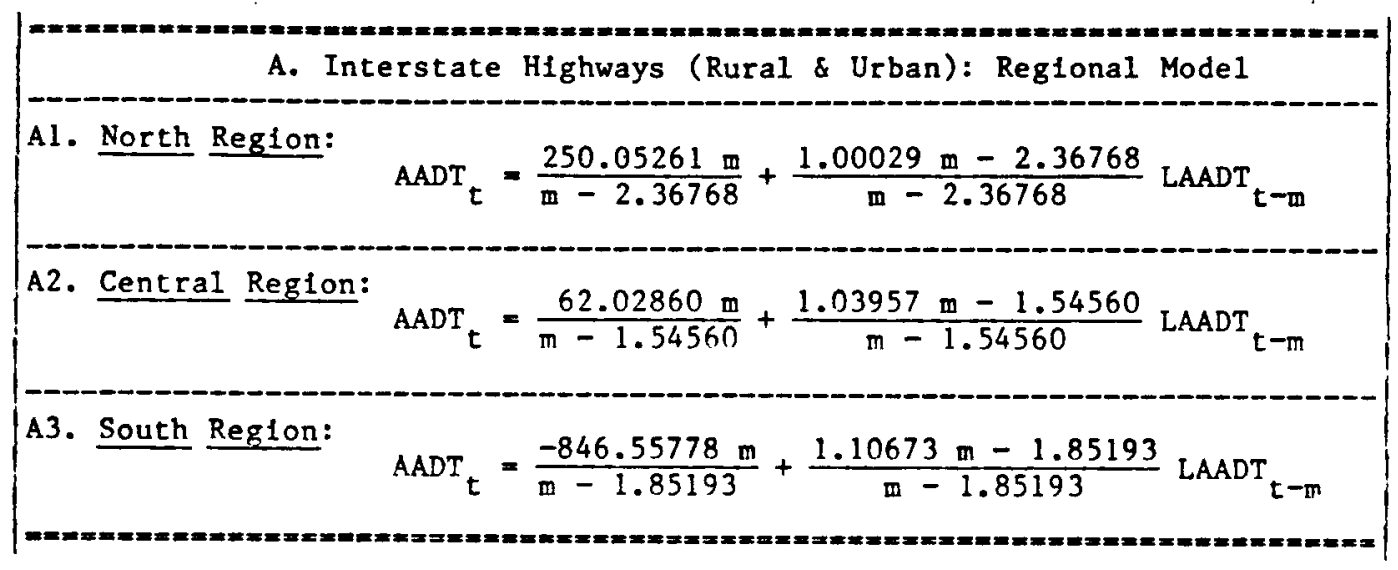

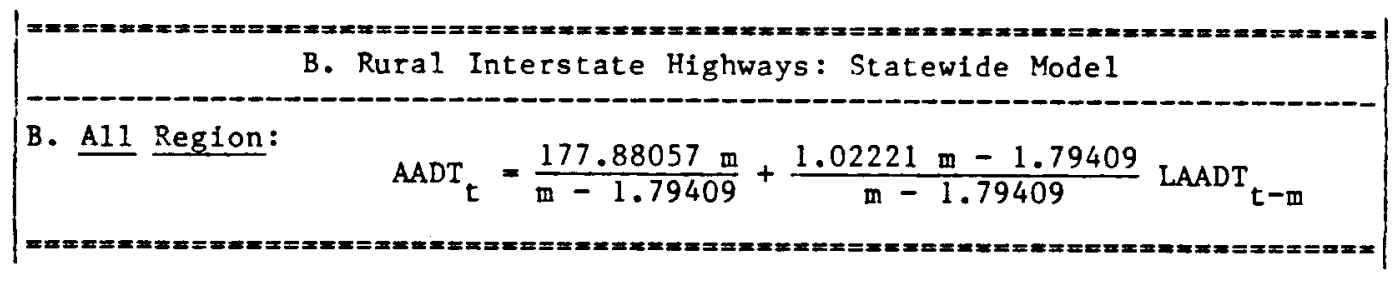

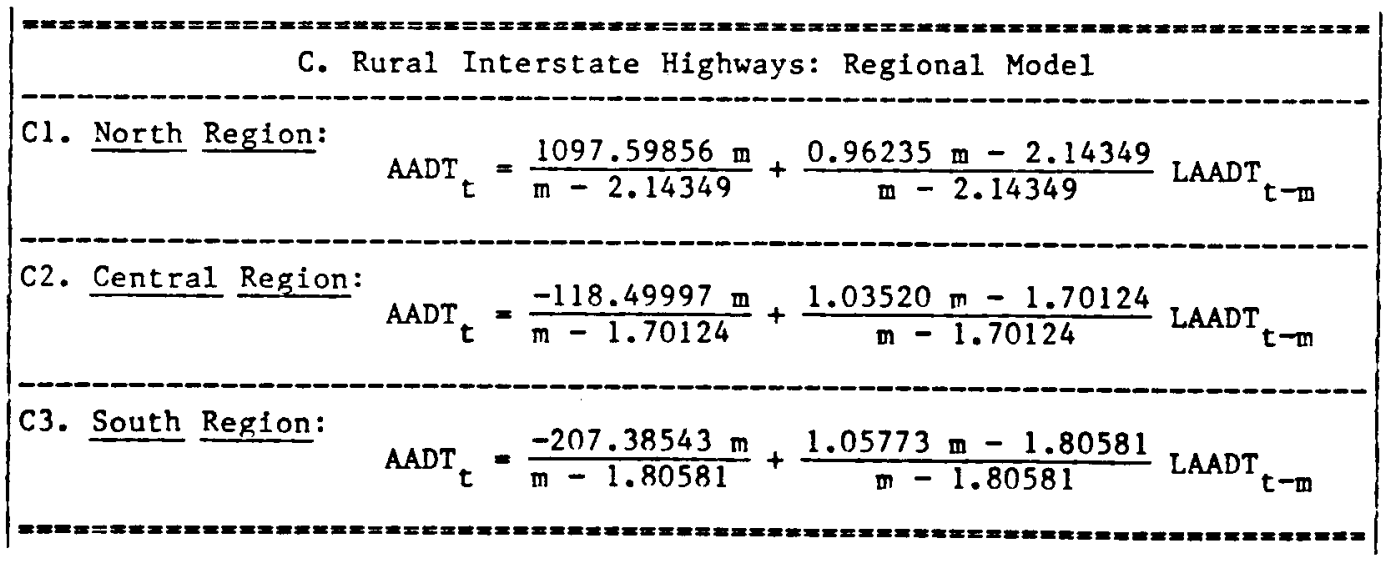


Table 8.4 , continued

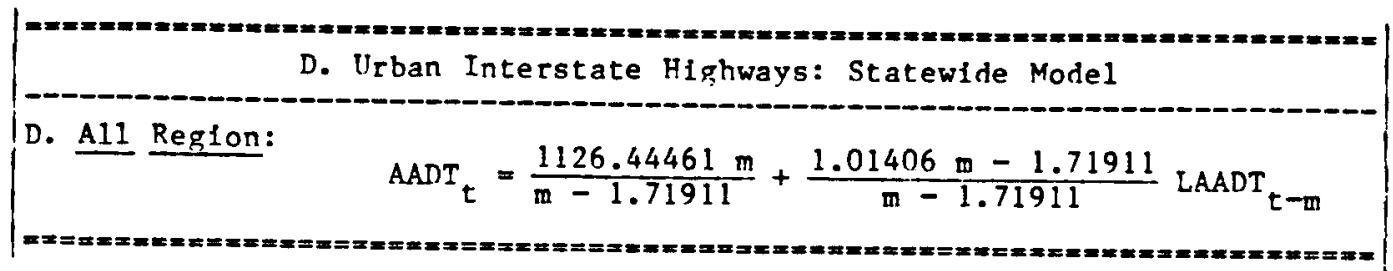

\begin{tabular}{|c|c|c|}
\hline E1. North Region: & $\mathrm{AADT}_{t}=\frac{275.78001 \mathrm{~m}}{\mathrm{~m}-2.46689}+\frac{0.99805 \mathrm{~m}-2.46689}{\mathrm{~m}-2.46689}$ & LAADT $_{t-m}$ \\
\hline E2. Central Reglon: & $\mathrm{AADT}_{t}=\frac{687.12000 \mathrm{~m}}{\mathrm{~m}-1.46264}+\frac{1.03300 \mathrm{~m}-1.46264}{\mathrm{~m}-1.46264}$ & LAADT $_{t-m}$ \\
\hline E3. South Region: & $\mathrm{AADT}_{t}=\frac{-2548.4029 m}{\mathrm{~m}-1.86829}+\frac{1.15547 \mathrm{~m}-1.86829}{\mathrm{~m}-1.86829}$ & LAADT $_{t-m}$ \\
\hline
\end{tabular}


Table 8.5: Lagged Traffic (Lag-AADT) Models for Urban Principal Arterial

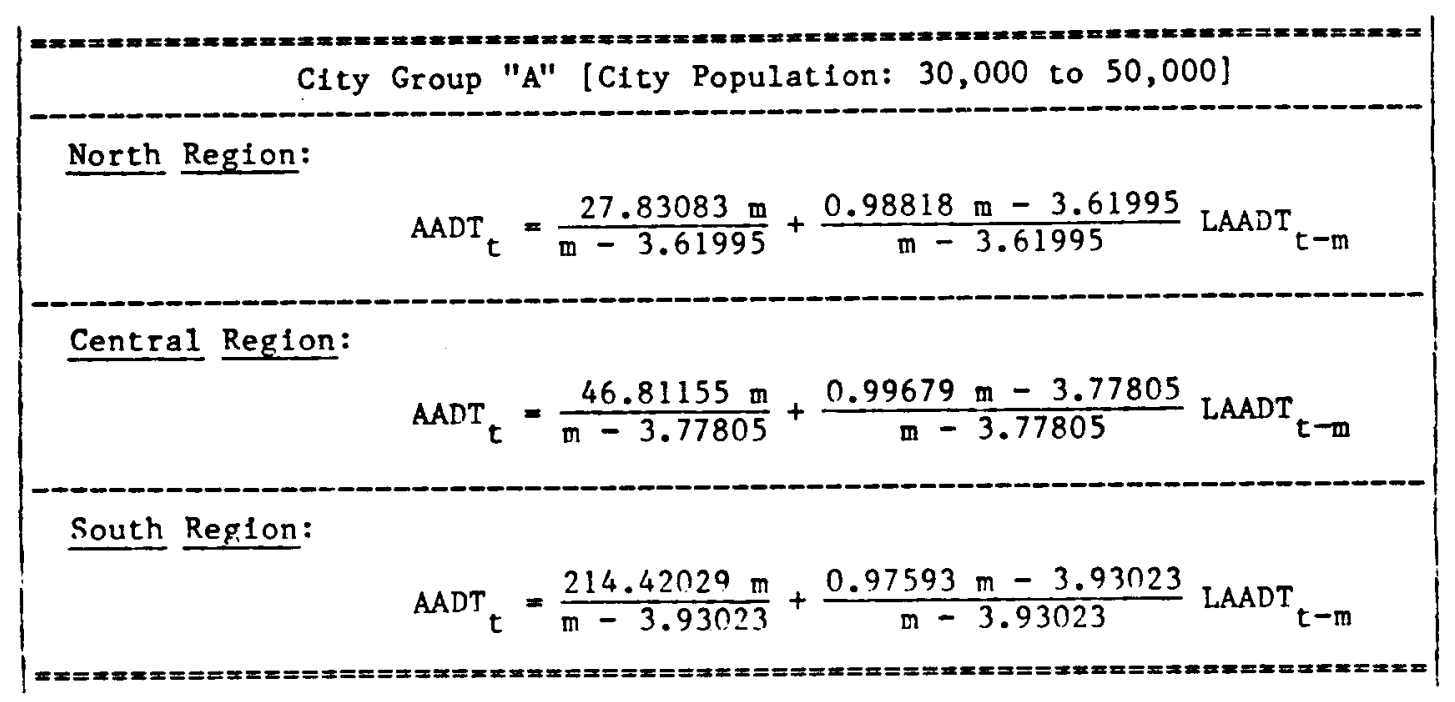

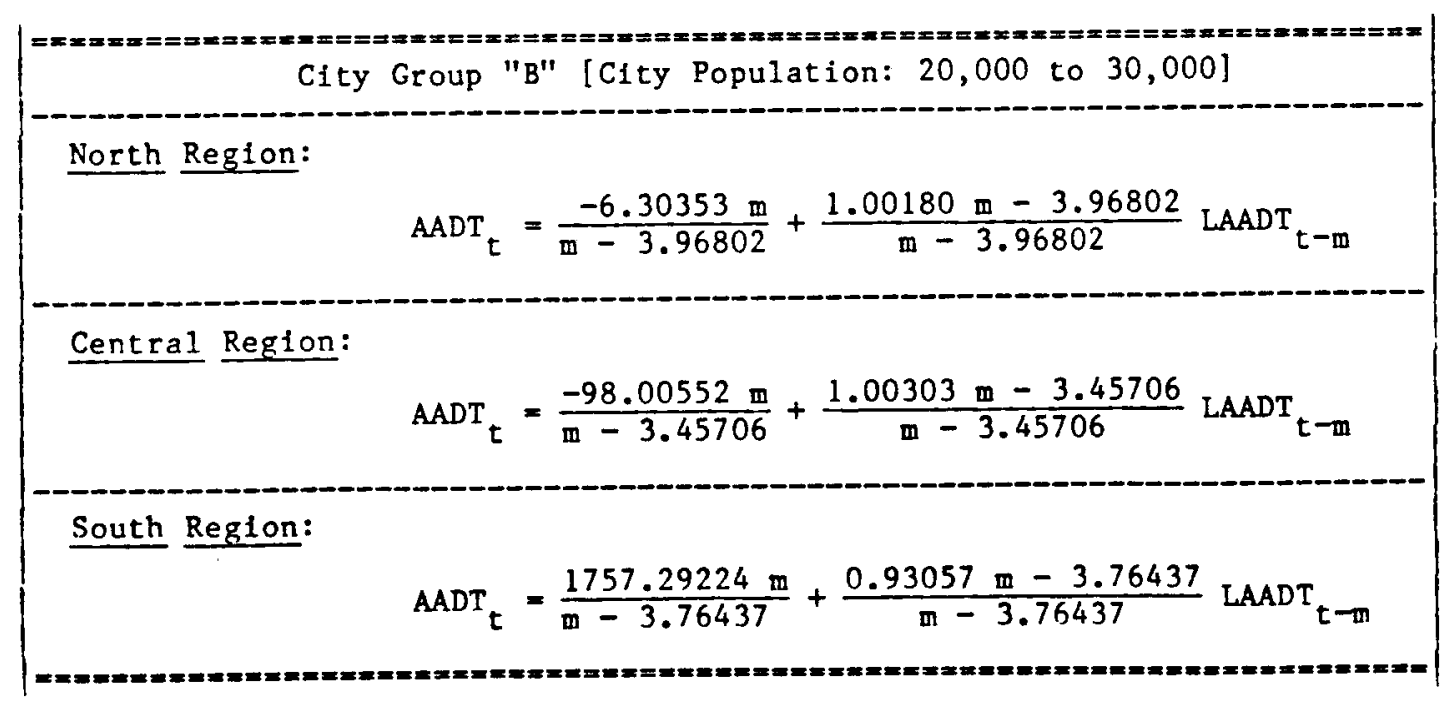


Table 8.5, continued

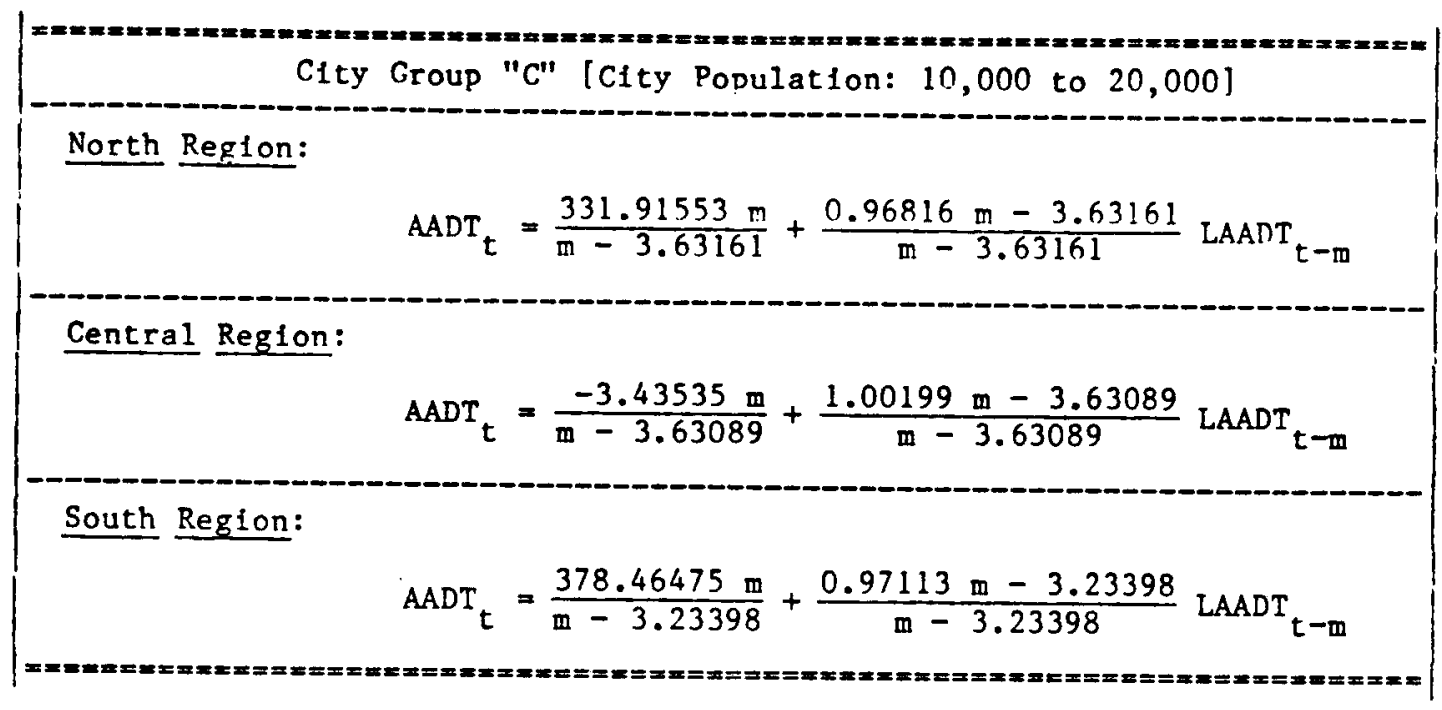

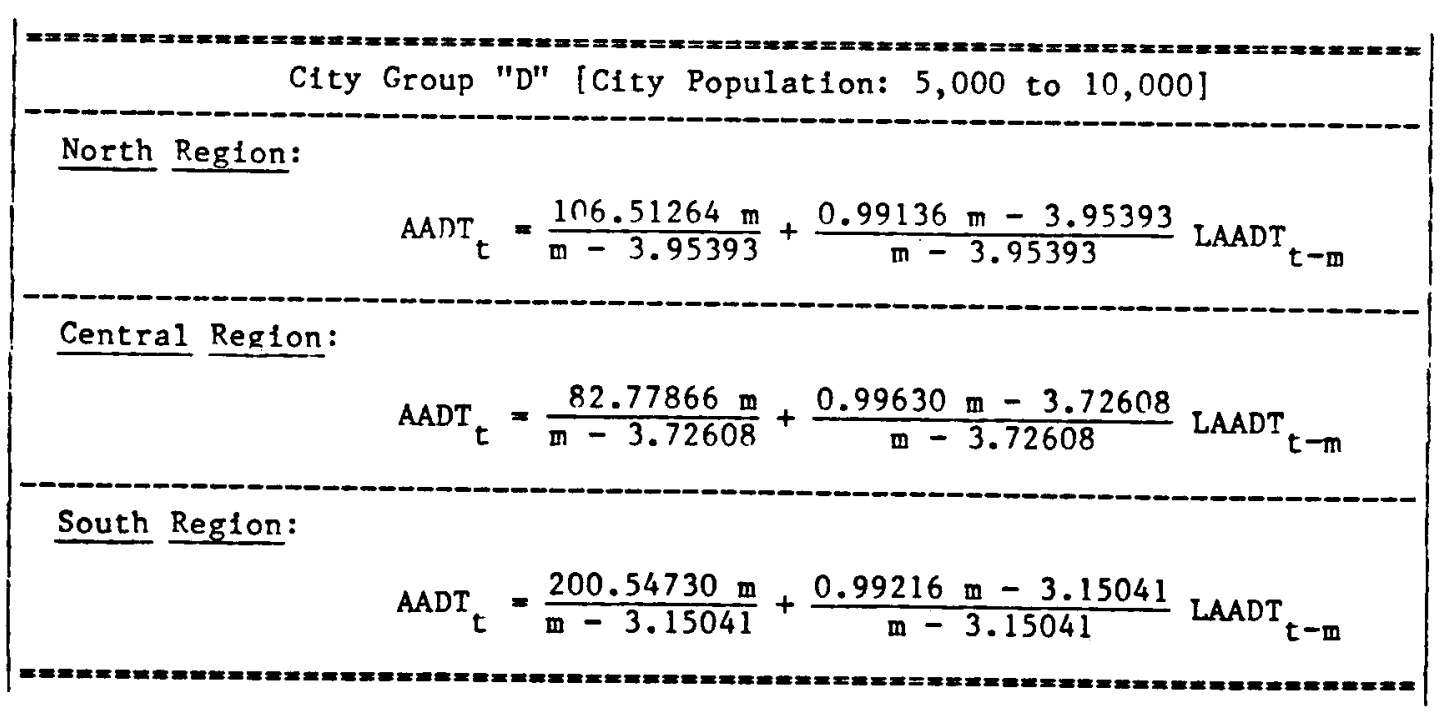


Table 8.6: Lagged Traffic (Lag-AADT) Models for Urban Minor Arterial \& Collector

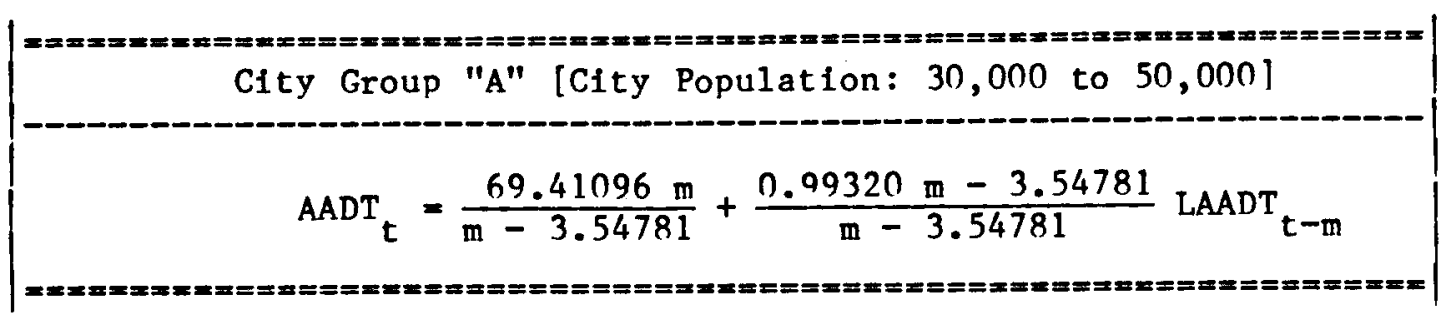

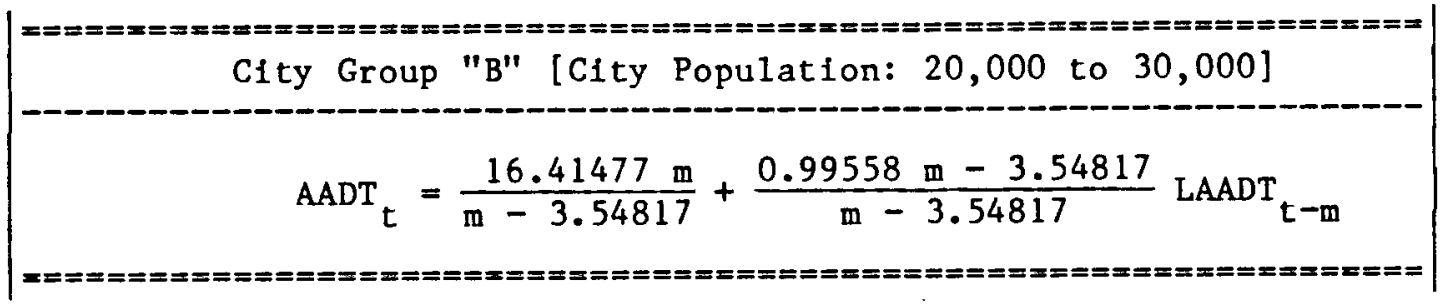

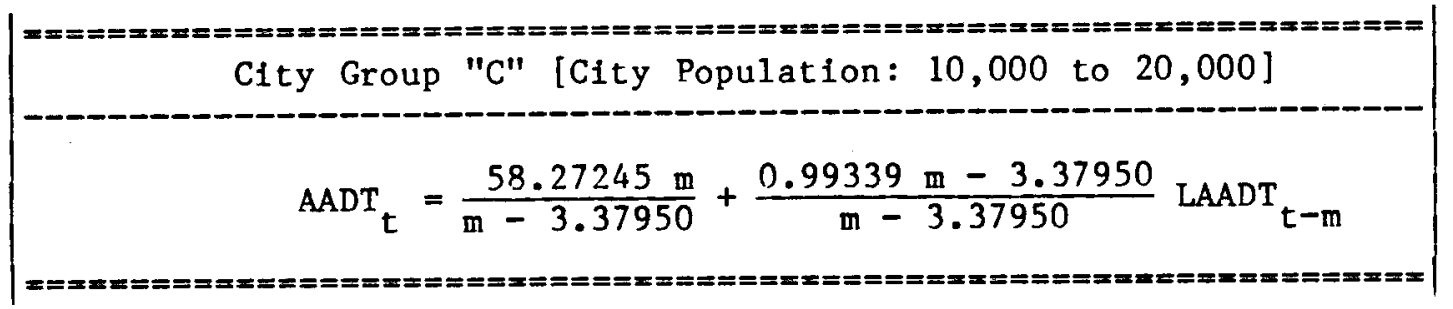

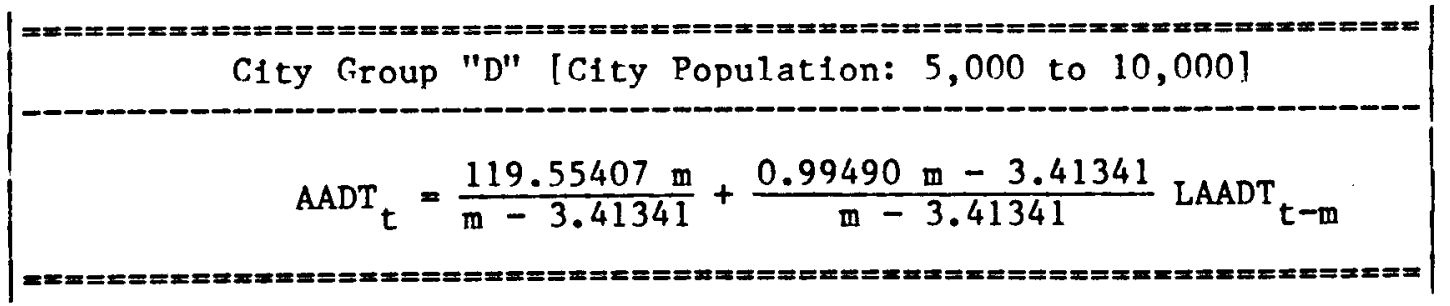


from Tables 8.1 to 8.3 in Equation (8.3). Like elasticity models in Chapter 6, three options are present for interstate highways and for one option on non-interstate highways. The use of these models is simple and the only information needed is a one lag year AADT value and the forecast period. Further evaluations of these differently approached models will be carried out in Chapter 10. It is expected that lagged model will perform as well as the models developed in Chapter 6.

\subsubsection{Partial-Lagged Mode1 and Its Performance}

The partial-lagged regression model of Equation (8.2) is formed by omitting the last year observation from each highway section of the segment data base. The major purpose of forming this partial model is to evaluate the performance of the lagged regression model. The partial models also verify the stablity of the model parameter estimates and of their significance of relation.

\subsubsection{Partial-Lagged Model}

The results of partial lagged regression analysis are presented In Appendix Tables E1.1 to E1.3 for interstate, urban principal arterial and urban minor arterial \& collector, respectively. The ranges of the various partial model statistics from Appendix Tables E1.1- E1.3 and their comparison with the full lagged model from Tables 8.1-8.3 are as follows: 


\begin{tabular}{|l|cccc|}
\hline $\begin{array}{l}\text { Highway } \\
\text { Class }[(*)]\end{array}$ & $\mathrm{R}^{2}$ & $\mathrm{R}_{\mathrm{a}}^{2}$ & $\mathrm{RMSE}$ & $\mathrm{C.V}$ \\
\hline $\begin{array}{l}\text { Interstate } \\
\text { Highways }\end{array}$ & $95.49-99.82$ & $95.36-99.81$ & $1051-3285$ & $2.99-8.56$ \\
& {$[94.61-99.46]$} & {$[94.50-99.45]$} & {$[1272-3508]$} & {$[5.15-10.22]$} \\
$\begin{array}{l}\text { Urban Prin- } \\
\text { pal Arterial }\end{array}$ & $96.53-99.97$ & $96.20-99.97$ & $101-568$ & $0.86-6.96$ \\
& {$[98.12-99.98]$} & {$[97.99-99.98]$} & {$[87-619]$} & {$[0.71-5.69]$} \\
$\begin{array}{l}\text { Urban Minor } \\
\text { Arteria1 \& } \\
\text { Collector }\end{array}$ & $98.97-99.82$ & $98.91-99.81$ & $193-444$ & $3.12-5.07$ \\
\hline
\end{tabular}

(*) Ranges of the full-lagged models statistics are shown in [ ].

No major change in the statistics is noticed for omitting the last observation from each segment. Thus, the stability of the lagged model is verified. The qualities of the statistics in Tables E1.1E 1.3 are almost Identical to those for the full-lagged regression presented in Tables 8.1 to 8.3 . The discussions about the statistics presented in Section 8.3 .1 are also valid for these partial regression models.

\subsubsection{Performance of Partial-Lagged Model}

To evaluate the partial-lagged model, the forecast model form of Equation (8.3) has been used to forecast the last year of traffic data, which have been deleted during formulation of partial-lagged model. In using Equation (8.3) to forecast AADT, the users need only the information about one lag year traffic data (LAADT) -- which could be the present year AADT - and the "forecast period" from the lagged year traffic data to the year for which the forecast is desired. A wide range of possible values of this "forecast period" has been used to capture the both short-term and long-term performance of the model. 
To evaluate the efficiency of model forecasts, the following five forecast-measures have been used:

a. Mean Error (ME)

b. Mean Percentage Error (MPE)

c. Mean Absolute Error (MAE)

d. Mean Absolute Percentage Error (MAPE)

e. Pearson Correlation Coefficients $(r)$ between the observed and predicted values.

The formal definitions of these measures, as well as their comparison for use in forecast evaluation will be provided in Chapter 10 . The forecast measures, the number of forecasts and ranges of "forecast period" of the partial-lagged prediction models for Interstate, Urban Principal Arterial, and Urban Minor Arterial \& Collector are presented in Appendix Tables E2.1 to E2.3, respectively. The range of results from Appendix Tables E2.1 to E2.3 can he summarized as follows:

\begin{tabular}{|c|c|c|c|c|c|}
\hline Highway Class & ME & MPE & MAE & MAPE & $\mathbf{r}$ \\
\hline $\begin{array}{l}\text { Interstate } \\
\text { Highways }\end{array}$ & $\begin{array}{l}-1066 \\
-5424\end{array}$ & $\begin{array}{l}-3.96 \\
-10.31\end{array}$ & $\begin{array}{l}1311 \\
5472\end{array}$ & $\begin{array}{r}7.30 \\
12.43\end{array}$ & $\begin{array}{l}0.952, \\
0.995\end{array}$ \\
\hline $\begin{array}{l}\text { Urban Prin- } \\
\text { pal Arterial }\end{array}$ & $\begin{array}{l}-114 \\
-1588\end{array}$ & $\begin{array}{c}-14.69 \\
0.41\end{array}$ & $\begin{array}{l}656 \\
1588\end{array}$ & $\begin{array}{r}5.32 \\
14.69\end{array}$ & $\begin{array}{l}0.904 \\
0.986\end{array}$ \\
\hline $\begin{array}{l}\text { Urban Minor } \\
\text { Arterial \& } \\
\text { Collector }\end{array}$ & $\begin{array}{c}-558 \\
-4\end{array}$ & $\begin{array}{c}-4.40 \\
0.37\end{array}$ & $\begin{array}{l}663 \\
1309\end{array}$ & $\begin{array}{r}7.95 \\
11.20\end{array}$ & $\begin{array}{l}0.941, \\
0.986\end{array}$ \\
\hline
\end{tabular}

It is evident from the range of values of ME and MPE that the lagged models in general are underpredicting traffic. The positive and negative prediction errors are cancelled each other in ME and MPE. The MAE measure shows high values and ranges for interstate highways compared to those in noninterstate highways. But MAPE (Mean Absolute Percentage Error) shows that the values and thelr ranges have almost remained same for all highway classes. Pearson correlation coeffi- 
cient ( $r$ ) shows that a high degree of correlation exists between predicted and observed AADT values. In addition to these forecast measures, three selected sample plots - one from each major highway category - of Observed AADT and Predicted AADT are presented in Appendix Figures E5.1-E5.3. These plots and the forecast measures in Tables E2.1-E2.3 establish that model has performed well to predict the last year of each segment traffic data for a short to long term forecast perlod.

The sample plots, one from each major highway category, of change in predicted and observed AADT are presented in Appendix Figures E6.1-E6.3 to see if the variable, change in observed AADT, will be helpful to forecast the change in predicted AADT. The random nature of these plots of change in observed and predicted AADT, and their low correlation coefficients, suggest that the model with change in AADT will not be useful to capture the change in predicted AADT. It will not be as effective as with the lagged model with a change to account for unequal time intervals between observations.

\subsection{Chapter Summary}

Alternative time-series models, based on past AADT data and the rate at which $\mathrm{AADT}$ has been changing in past years to account for the unequal time spacing between observations, have been developed for each data group produced in Chapters 5 and 6 . These alternative models are based on lagged regression analysis. Each of the models is found statistically significant with high $\mathrm{R}^{2}$ values. The complete model buflding approach of Chapter 6 has been used to confirm the 
suitability of the models. The performance evaluation of the models is tested by forming models with partial data. The partial lagged models show the stability of the model parameters. The comparison of the models developed in this chapter with those in earlier chapters will be done in Chapter 10. 
CHAPTER 9

VEHICLE REGISTRATION AND CITY POPULATION FORECASTS

\subsection{Introduction}

Forecasts of predictor variables entered in the models in this study, other than vehicle registrations and city population, are available from outside sources (see Chapter 4). This chapter explores the application of Time Series Analysis to produce the forecasts of vehicle registrations at the state and county level. Following a brief introduction to Time Series Analysis, model development and fitting are discussed in some detail. This chapter addresses the development of computationally efficient models for forecasting of vehicle registrations based on the time series analysis framework. The combined forecasts of vehicle registration are based on two components: (1) forecasts produced by an Autoregressive procedure and (2) those produced using the Box-Jenkins ARIMA technique. The performance of the forecasting models produced in this study is evaluated. To forecast city population, a simple but widely accepted "ratio" method has been used. The terms prediction and forecasting have been used interchangeably in this chapter and throughout the report, although philosophical distinctions can be made. 


\subsection{Time Series Forecasts of Vehicle Registration}

A time series is a sequence of observations arranged in order of some other variable, usually a time variable. Yearly vehicle registration data for the years 1950 to 1988 for the state of Indiana and each of its 92 counties are used to generate the independent forecasts of vehicle registration for the state and each county. Final results of the forecasts and models are presented for all 93 series of data. To illustrate model identification, estimation and validation, two counties (24-Franklin and 79-Tippecanoe) are highlighted in this chapter. In addition, 91-White County is used to show certain results of the time series analysis. The yearly total number of vehicle registrations have been used in this study, so each series of data is non-seasonal. The representative plots of series for 24-Franklin and 79-Tippecanoe are shown in Figures 9.1 and 9.2 , respectively. Despite an occasional "shock" in the series, these plots show that there is an increasing trend in vehicle registrations and a lack of affinity towards the mean value of the series.

\subsubsection{Introduction to Forecast Procedures and Estimation Method}

Using other predictor variables in this study (for example, income, employment, population, and household) as a predictor variable(s) to forecast of Vehicle Registrations is possible. But, to obtain accurate forecasts and confidence limits, the input series must be independent of one another. Also, the past values of the response series cannot contain additional information about future values of the input series beyond that contained in the past values of the input 


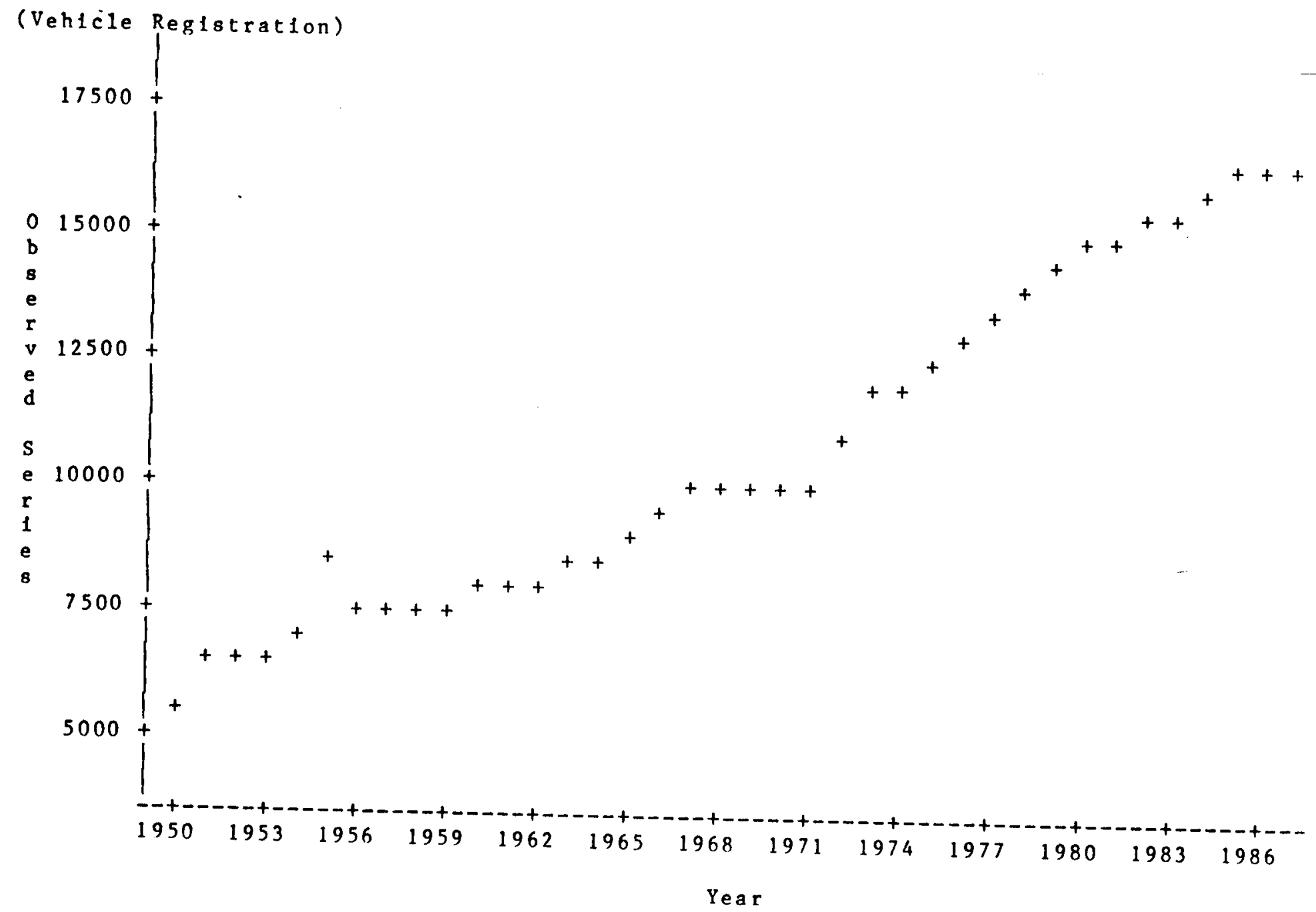

Figure 9.1: Observed Series of Franklin County Vehicle Registration 


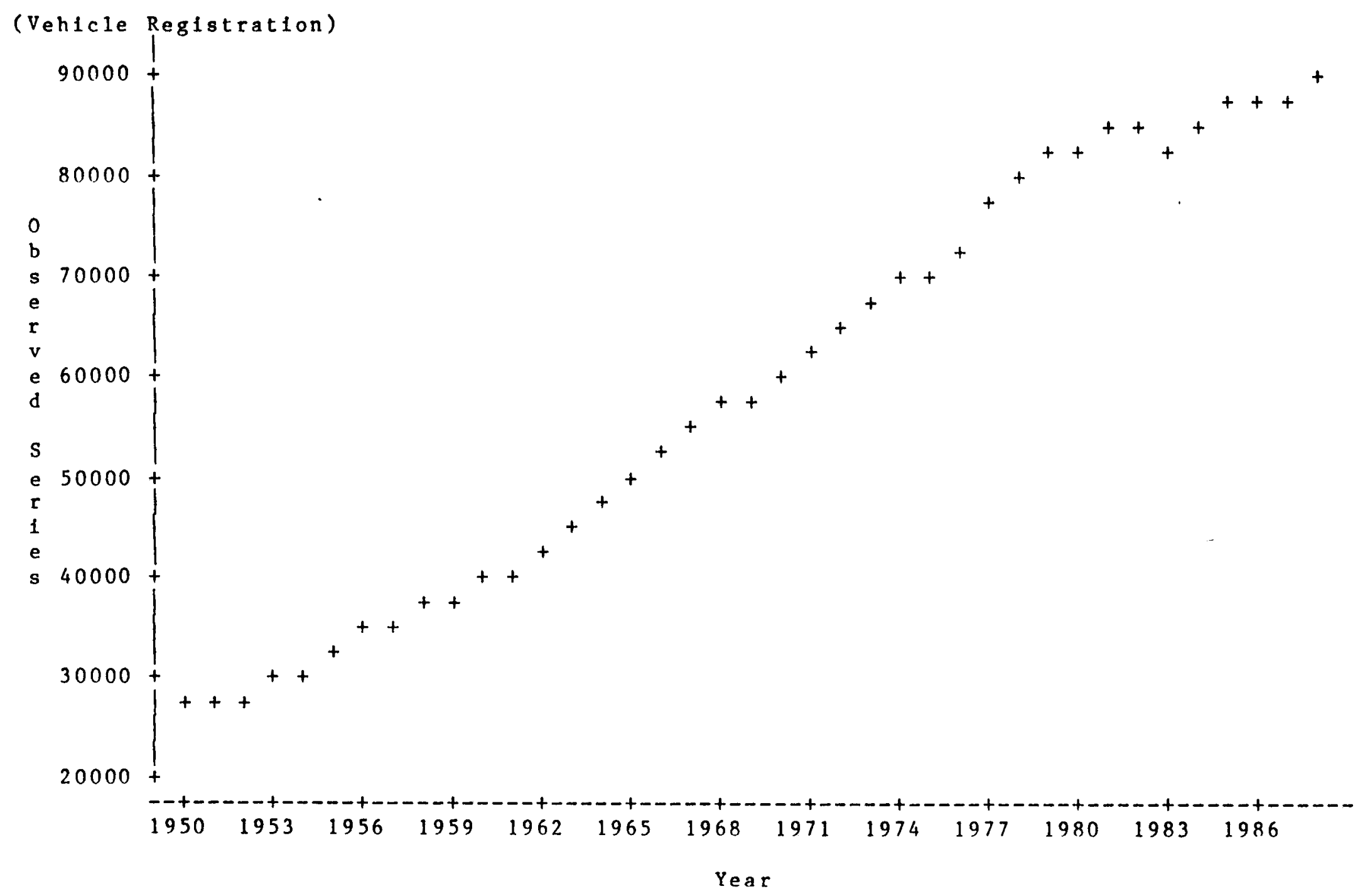

Figure 9.2: Observed Series of Tippecanoe County Vehicle Registration 
series. That is, there should be no feedback loops between the response series and the input series. Thus, the time series analysis employed in this chapter, which depends on only on time, seems to be a more appropriate method to forecast vehicle registrations at county and state level. The following time series procedures appear to be appropriate for this study:

a. ARIMA - fits models that allow for an error term generated by an AutoRegressive Integrated Moving Average (ARIMA) process. A brief description of this method is presented in Section 9.2.2.

b. AUTOREG - does multiple regression allowing for a serially correlated error term that is generated by an AUTOREGressive (AUTOREG) process. The procedure has been addressed concisely in Section 9.2.3.

The estimation techniques [SAS/ETS 1984, Brocklebank \& Dickey 1986] that can be used in the above procedures are as follows:

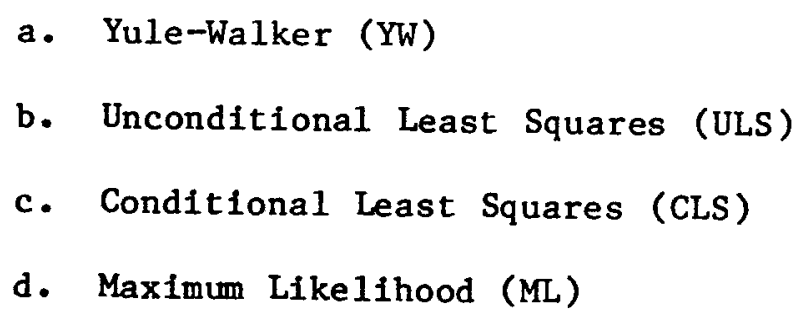

The ULS estimates are also referred to as Nonlinear Least Squares (NLS) estimates or Exact Least Squares (ELS) estimates. A detailed description of these methods is beyond the scope of this study, but the following comparative evaluations from the 1iterature [Brocklebank \& Dickey 1986] are made to select the most accurate method. 
i. Computationally, YW estimation is the fastest.

i1. The ULS technique more accurately computes prediction error variances and finite sample prediction than the CLS technique.

11i. The CLS method assumes a constant variance and the same linear combination of past values as the optimum prediction.

iv. In the ML method, the quantity to be minimized is not the sum of squares; Instead, it is the negative $\log$ of the likelihood function.

The Ordinary Least Squares (OLS) estimates and YW estimates are used as starting values for the ML and ULS methods. Although the CLS, ULS, and ML methods should give similar results for reasonably large data sets, studies [SAS/ETS 1984] comparing the three methods suggest that ML is the most accurate method. Based on the literature and some pilot runs (results not shown) with different methods, this study has used the ML method in model estimation.

\subsubsection{ARIMA Mode1}

ARIMA is an acronym for AutoRegressive Integrated Moving-Average mode1s, usually denoted by the notation $\operatorname{ARIMA}(p, d, q)$, where

$p$ is the degree of the autoregressive part,

$d$ is the degree of the differencing, and

$q$ is the degree of the moving-average process.

The ARIMA procedure analyzes and forecasts univariate time series data using the autoregressive integrated moving average model developed by Box and Jenkins [1976]. 
ARIMA is an iterative method for modeling the dependence among the observations in a time series. It is a model building process rather than model fitting process, because the model is determined from the data rather than by assumption. The model determination stage, called identification, is followed by parameter estimation, and then diagnostic checking to determine if the model provides an adequate description of the data. If the checking stage shows that the model is deficient in some way, then it is necessary to go back to the identification stage and go through the process again. It is also desirable to develop a model that uses parameters parsimoniously, i.e., contains a small number of autoregressive and moving average terms.

The ARIMA procedure assumes that the time series is either stationary or can be made into a stationary time series after suitable differencing operations. Intuitively, the series needs to have a constant mean and to fluctuate about that mean with a constant variance. In other words, the covariance between two elements in the series depends only on the time difference between the two elements.

For the autocorrelated error terms, the usual ordinary least squares (OLS), estimates of regression parameters may not be enough, and the usual estimates of standard errors may be biased. Further, because of serial autocorrelation, past and present observations can be used to improve predictions in the near future. So, in the ARIMA model, as opposed to the normal assumption of stochastic independence of observations, we specifically assume that these observations are correlated. Fitting complicated models with the SAS ARIMA procedure 
[SAS/ETS 1984] usually requires the user to provide input and judgment. The SAS ARIMA procedure can handle time series of moderate size. The procedure needs more than thirty observations and fewer than 2000. With fewer than thirty, the parameters are not estimated very well. With thousands of observations, the method requires excessive computer time and memory.

\subsubsection{Model Formulation}

Let $\left\{z_{t}\right\}$ be observations on the state variable taken at equally spaced intervals. The observation at time $t$ can be thought of as the sum of a deterministic component $\left(f_{t}\right)$ plus a stochastic noise component $\left(\varepsilon_{t}\right)$, i.e., $z_{t}=f_{t}+\varepsilon_{t}$. If the $\left\{z_{t}\right\}$ series is stationary, then $f_{t}$ can be represented by a mean level $\mu$. The time series of vehicle registrations (see the representative plots in Figures 9.1 and 9.2) exhibits some degrees of non-stationarity. For simplicity, the differencing technique [Box-Jenkins 1976] has been used to overcome the problem of non-stationarity. Figures 9.3 and 9.4 show the plots of the first differences of the series presented in Figures 9.1 and 9.2, respectively. The increasing trend in the observed series has been eliminated in the first difference series.

The noise components $\left\{\varepsilon_{t}\right\}$ in vehicle registration data are assumed to be serially correlated and they induce correlation in the $\left\{z_{t}\right\}$ series. Therefore, the ARIMA process represents $\varepsilon_{t}$ by a 1 inear combination of a white nolse sequence $\left\{a_{t}\right\}$, where the $a^{\prime} s$ are independent variables with zero mean and variance $\sigma_{a}^{2}$. 


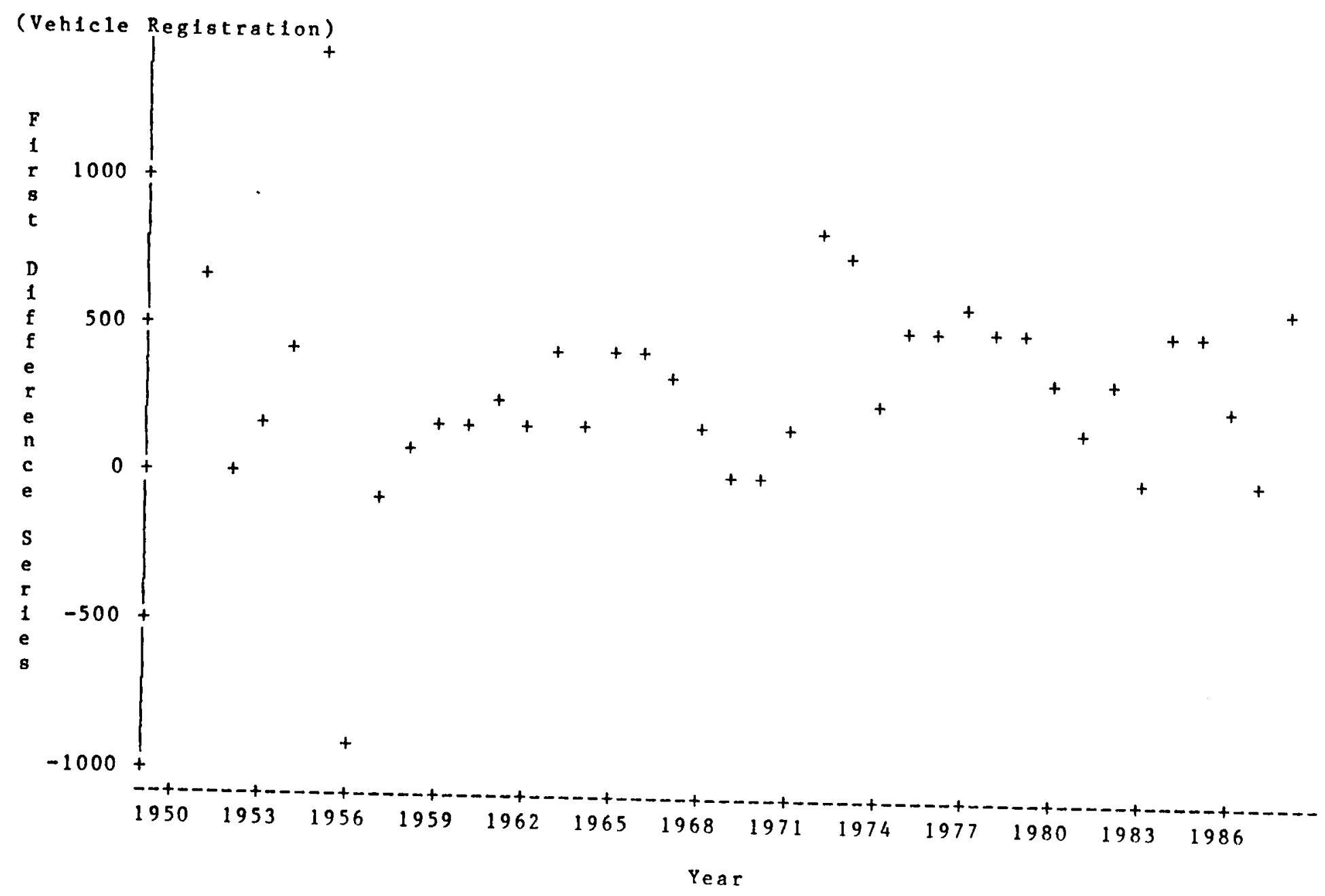

Figure 9.3: First Difference Series of Franklin County Vehicle Registration 


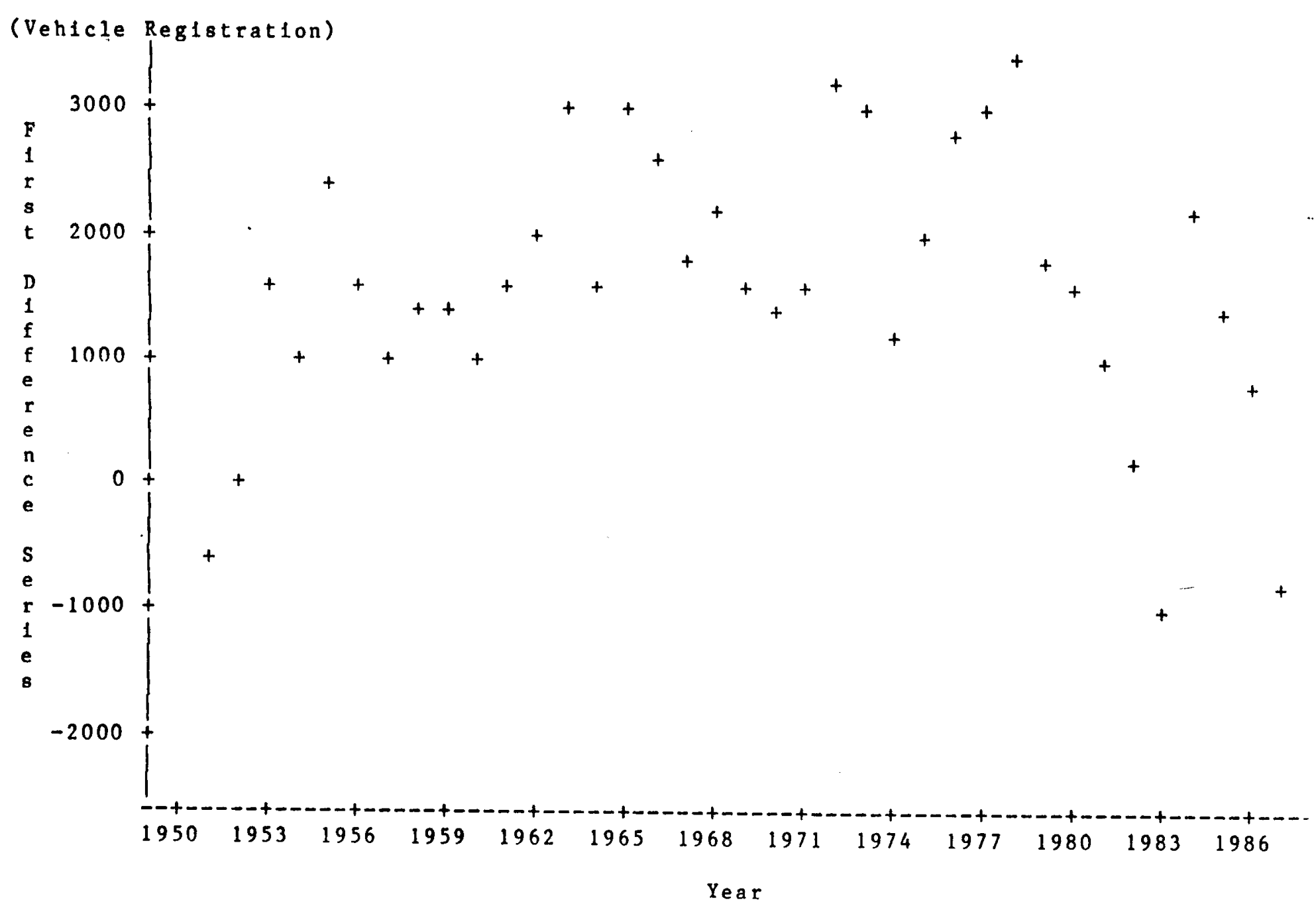

Figure 9.4: First Difference Series of Tippecanoe County Vehicle Registration 
The mathematical representation of ARIMA $(p, d, q)$, originally formulated by Box and Jenkins [1976], of the non-seasonal data, $Z_{t}$, is as follows:

$$
\Phi_{p}(B) \nabla_{z_{t}}=\theta_{0}+\theta_{q}(B) a_{t}
$$

where,

$$
\begin{aligned}
{ }_{p}(B) & =1-\phi_{1} B-\ldots-\phi_{p}{ }^{P} \\
\theta_{q}(B) & =1-\theta_{1} B-\ldots-\theta_{p}{ }^{q} \\
B_{z_{t}}^{m} & =z_{t-m} \\
\nabla_{z_{t}}^{m_{z}} & =(1-B){ }^{m_{z}} \\
z_{t} & =z_{t}-E\left(z_{t}\right) \\
E\left(z_{t}\right) & - \text { Mean of the Process } z_{t} \\
a_{t} & - \text { White noise with zero means and variance } \\
\phi & - \text { An Autoregressive (AR) parameter } \\
\theta_{0} & - \text { Constant Term (MU) } \\
\theta & - \text { A Moving Average (MA) parameter } \\
p & - \text { The order of the differenced or AR series } \\
q & - \text { The order of the white noise or MA series } \\
d & - \text { The order of differencing }
\end{aligned}
$$

Here, $\Phi_{p}(B)$ and $\theta_{q}(B)$ are polynomials of degree $p$ and $q$, respectively, in the backshift operator B. The models of Equation (9.1) have been formulated by Box and Jenkins (1976), and they are usually referred to as autoregressive integrated moving average (ARIMA) models of order $(p, d, q)$, generally represented as $\operatorname{ARIMA}(p, d, q)$. The general form of 
Equation (9.1) has been elaborated in Table 9.1 for a few representative ARIMA models being produced in this study (see Appendix Tables Fl and F2). An example for each of these representative models is also shown in Table 9.1.

The analysis to develop ARIMA models is divided into three stages (identification, estimation and forecast), corresponding to the stages described by Box and Jenkins [1976]. These stages to prescribe a ARIMA model are described in Sections 9.2.2.3 to 9.2.2.6. The overall steps involved in modeling the nonseasonal time serles are outlined in Table 9.2.

\subsubsection{Chi-Squared Check of Autocorrelation}

One way of viewing the process of modeling time series is an attempt to find a transformation that reduces the observed data to random noise. To do that, one needs to examine the sample autocorrelations of the residuals as a diagnostic check. Autocorrelation, if evident in the residuals, may help to suggest the direction in which the model should be modified. Box and Pierce [1970] have developed a test (known as the Box-Pierce Q-statistic) that is capable of determining whether several autocorrelation coefficients are significantly different from zero. This test is based on the $x^{2}$ (chi-square) distribution of the autocorrelation coefficients. If the computed value of the test is less than that from the table of values of the $x^{2}$ statistic, the autocorrelations used to calculate the test are not significantly different from zero. This suggests that the data generating the autocorrelations are random. If the computed $x^{2}$-test 
Table 9.1: Example of Selected ARIMA Models from General Form [Equation (9.1)]

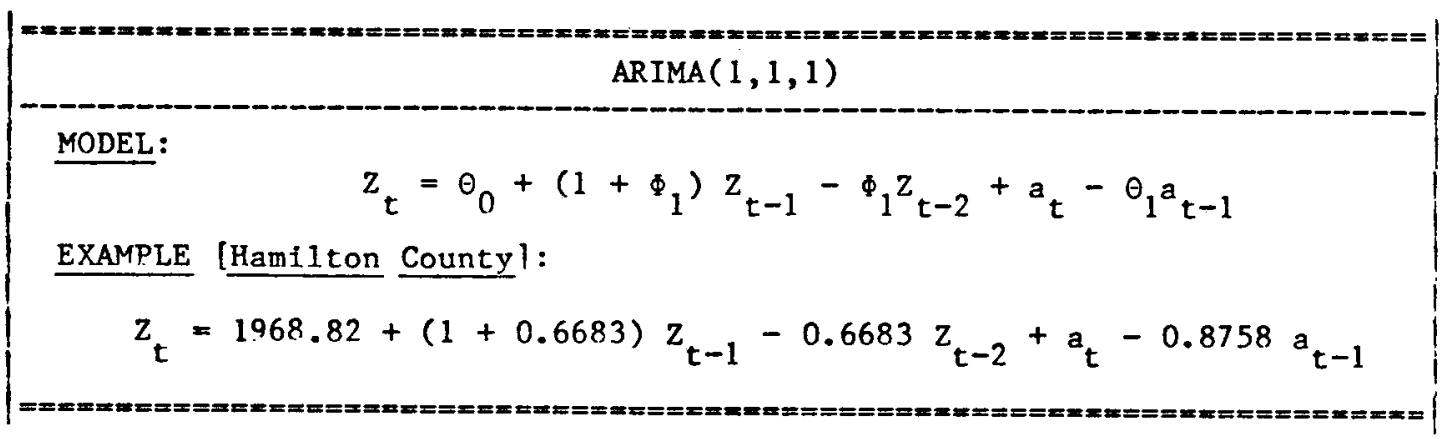

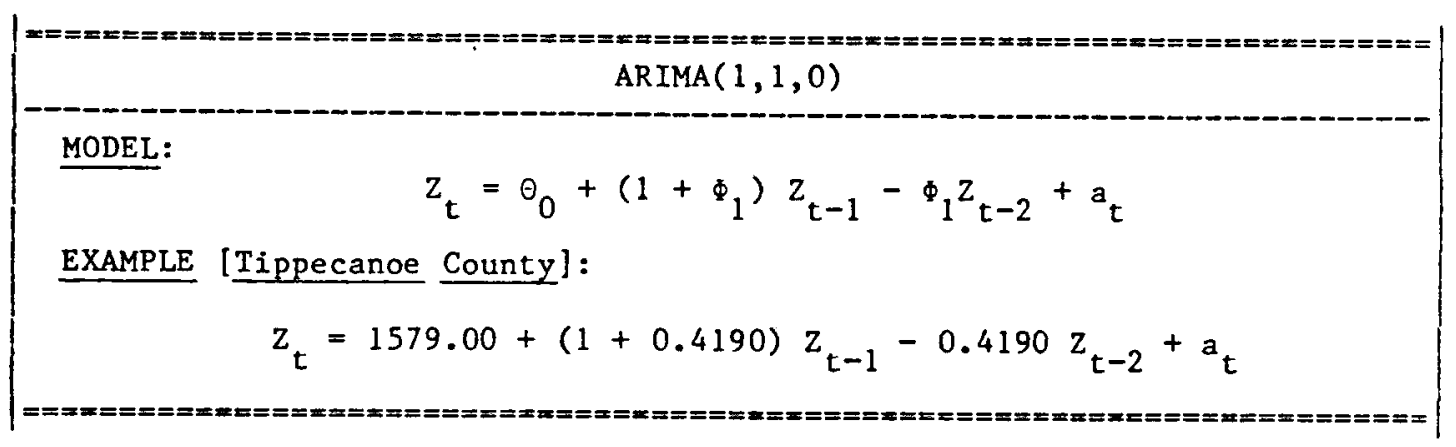

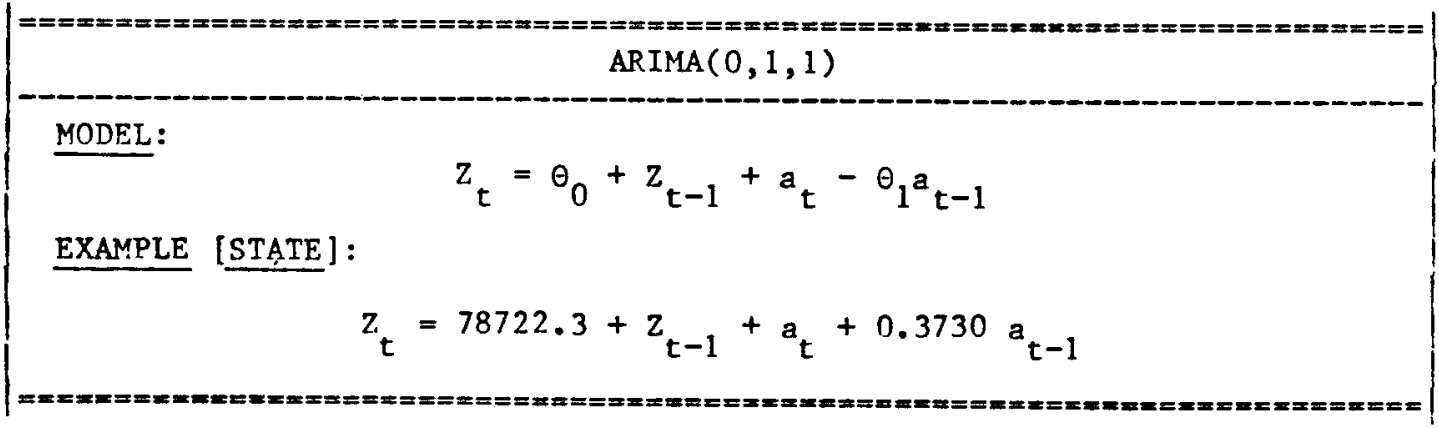


Table 9.1, continued

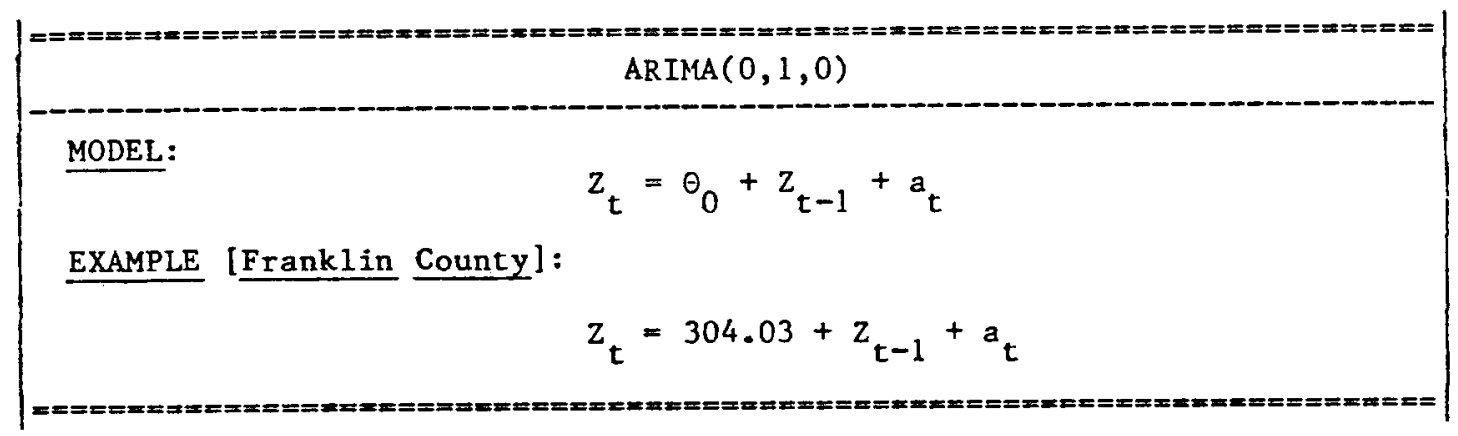

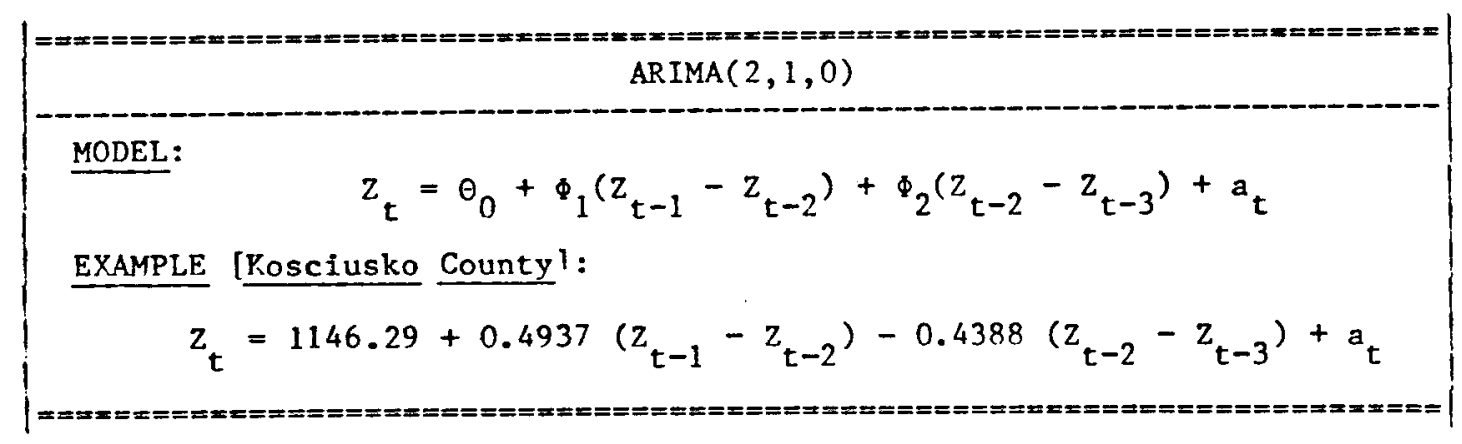

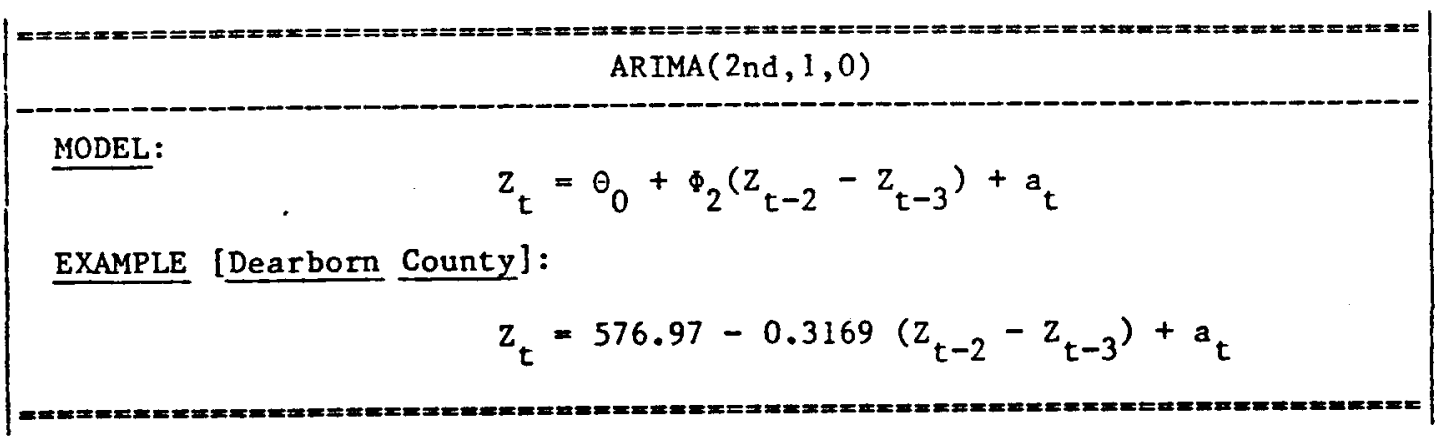


Table 9.2: Steps of ARIMA Model for Nonseasonal Univariate Series

1. Check for nonstationarity using
data plot to monitor slow level shifts in the data
ACF to monitor very slow decay
If any of these tests indicate nonstationarity, difference the series
and repeat the steps above. If necessary, second differencing will be

2. Check the $Q$ statistic (CHI SQUARE). If $Q$ is small (in other words, PROB is fairly large) and if the first few autocorrelations are small, then one may assume that the original (possibly differenced) series is just
white noise.

3. Check the ACF and PACF [Box and Jenkins 1976, Abraham and Leodolter 1983] to identify a model. If the ACF drops to zero after $q$ lags, this indicates an MA(q) model. If the PACF drops after $p$ lags, this
indicates an $\operatorname{AR}(\mathrm{p})$ model.

4. Estimate the model (or several candidate models) of step 3 .

5. Check the $Q$ statistic (CHI-SQUARE) after estimation. If it is insignificant, the model fits reasonably well according to this criterion. Otherwise, return to the original ACF and PACF of the original (possibly differenced) data to determine if something has been missed. This is generally more advisable than plotting the ACF of the residuals from the misspecified model.

6. Forecast the series from the fitted model of step 5. It is a good idea to start the forecast a few steps before the end of series to compare the last forecasts to the data values at the end of the series. In case of a large discrepancy, the user may want to adjust the forecasts. In the final forecast, omit the start the forecast from few steps back. It is used only as a diagnostic tool.

7. Examine plots of residuals and possibly use PROC UNIVARIATE [SAS/Bas1cs, 1985] to examine the distribution to test the white noise assumption further.

Sources: 1. SAS/ETS [1984] and SAS [1986].

2. Box and Jenkins [1976], and Abraham and Leodolter [1983]. 
statistics (i.e., Q-statistic) is larger than the value from the table, the autocorrelations are significantly different from zero, indicating the existence of some pattern. The Q-statistic is computed as

$$
\chi^{2} \text {-test }=n \sum_{j=1}^{k} r_{j}^{2},
$$

where $k$ is the largest time lag included. The smaller the autocorrelations $\left(r_{j}\right)$, the smaller the value of $x^{2}$.

In the identification stage, the SAS ARIMA procedure uses autocorrelations to form a statistic whose approximate distribution is chi-squared under the null hypothesis that the series is white noise. The test is the Ljung modification of the Box-Pierce Q statistic. The formula for this statistic is

$$
n(n+2) \sum_{j=1}^{k} \frac{r^{2}(j)}{(n-j)}
$$

where $r(j)$ is the estimated autocorrelation at lag $j$ and $k$ is any positive integer. SAS ARIMA procedure uses several values of $k$ to compute the $x^{2}$ statistic. In the modeling stage, the SAS ARIMA procedure calculates the same statistic on the residuals to test the hypothesis that they are white noise. The statistic is compared to critical values from a chi-squared distribution. If the model is correct, the residuals should be white noise and the chi-squared statistic should be small (the probability value should be large). A 
significant chi-square statistic concludes that the model does not fit we11.

\subsubsection{Model Identification}

The IDENTIFY statement of the SAS ARIMA procedure [SAS/ETS 1984] has been used to identify the mode1. PROC ARIMA reads in the time series, differencing them if a trend is detected, and computes autocorrelations and partial autocorrelations. These results usually suggest one or more models that could be fit to the data. At the identification stage, Autocorrelated Function (ACF) and Partial Autocorrelated Function (PACF) [Box \& Jenkins 1976] are the keys to model identification. The behavior of functions for different processes is summarized below:

\begin{tabular}{|c|c|c|c|c|}
\hline & $\operatorname{MA}(q)$ & $\operatorname{AR}(p)$ & $\operatorname{ARMA}(p, q)$ & White Noise \\
\hline $\begin{array}{l}\text { ACF } \\
\text { PACF }\end{array}$ & $\begin{array}{c}D(q) \\
T\end{array}$ & T & $T$ & zero \\
\hline
\end{tabular}

where,

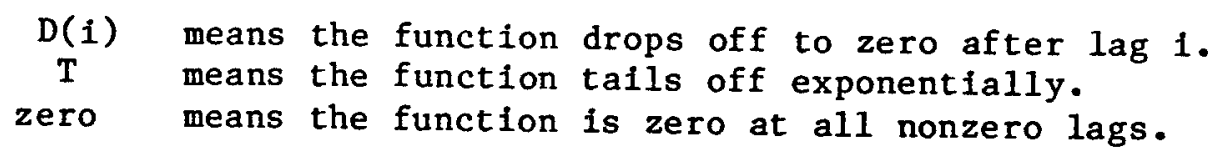

ACFs for the observed series of 24-Franklin and 79-Tippecanoe counties are shown in Figures 9.5 and 9.6, respectively. A slow decay of ACF and an increasing trend in the plots in Figures 9.1 and 9.2 suggest that the observed series of vehicle registrations are nonstationary. The PACFs of this observed series are presented in Figures 9.7 and 9.8. One may want to analyze $A R(1)$ - an autoregressive model with lag 1 - based on identification rules. The chi-square statistic and 


\section{Franklin County Vehicle Registration Series: Observed}

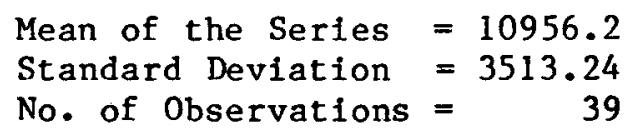

AUTOCORRELATIONS

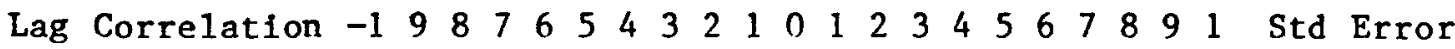

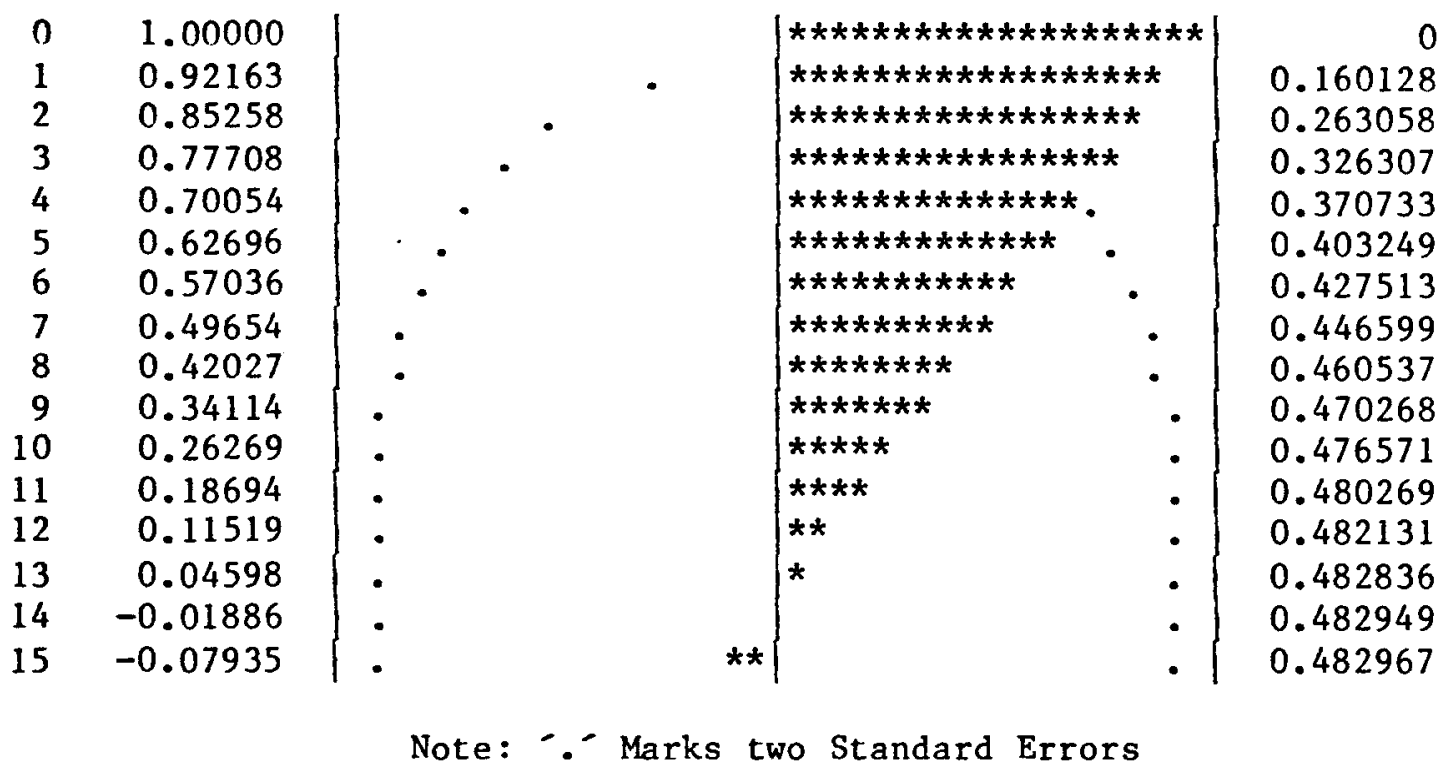

Figure 9.5: Autocorrelation of Observed Series for Frank1in County Vehicle Registration 


\section{Tippecanoe County Vehicle Registration Series: Observed}

Mean of the Series $=58565.3$

Standard Deviation $=21081.3$

No. of Observations $=39$

\section{AUTOCORRELATIONS}

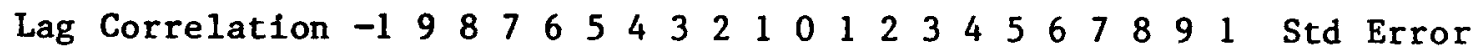
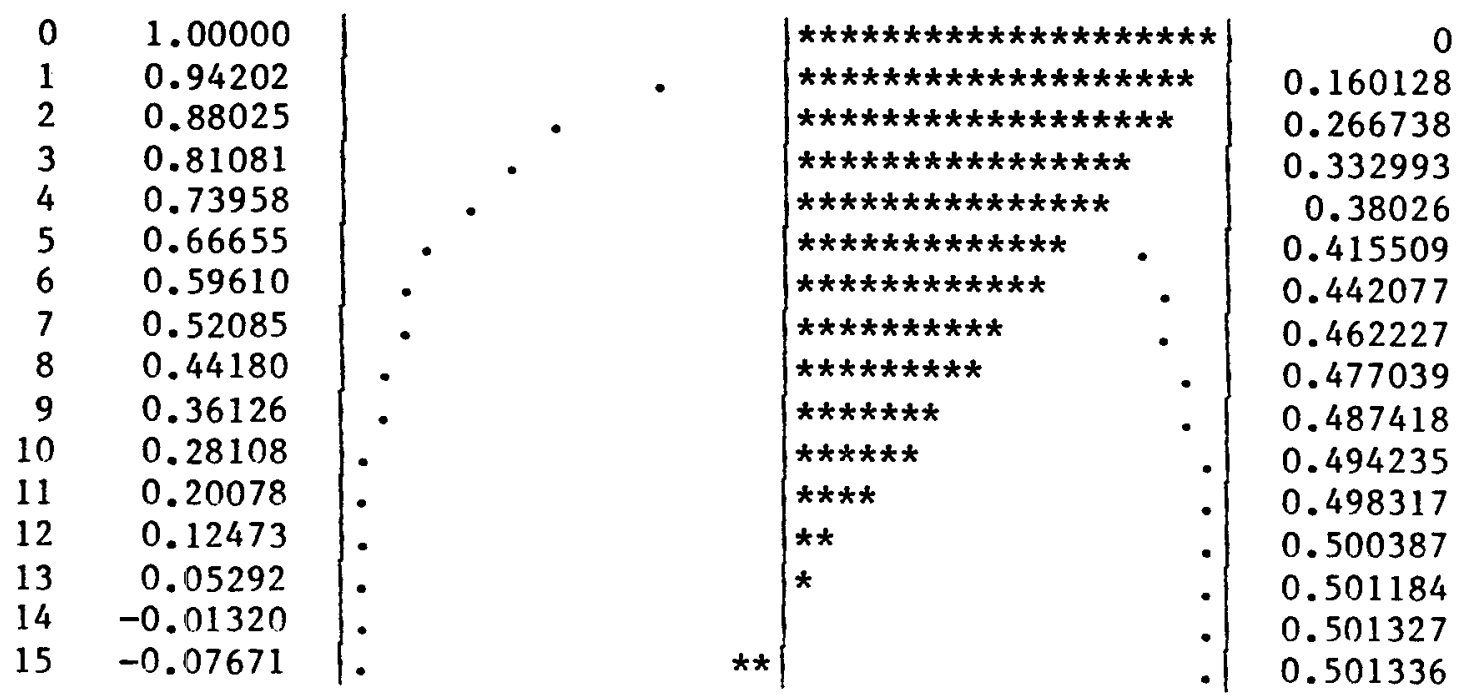

Note: "- Marks two Standard Errors

Figure 9.6: Autocorrelation of Observed Series for Tippecanoe County Vehicle Registration 
Franklin County Vehicle Registration

Series: Observed

(a) Box-Pierce Q-statistic:

AUTOCORRELATION CHECK FOR WHITE NOISE

\begin{tabular}{|c|c|c|c|c|c|c|c|c|c|}
\hline $\begin{array}{r}\text { To } \\
\text { Lag }\end{array}$ & $\begin{array}{l}\text { Chi- } \\
\text { Square }\end{array}$ & $\mathrm{df}$ & $\begin{array}{c}\mathrm{P} \text { (White- } \\
\text { Noise) }\end{array}$ & & Autoco & crelati & ons & & \\
\hline $\begin{array}{r}6 \\
12\end{array}$ & $\begin{array}{l}150.65 \\
184.87\end{array}$ & $\begin{array}{r}6 \\
12\end{array}$ & $\begin{array}{l}0.000 \\
0.000\end{array}$ & $\begin{array}{l}0.922 \\
0.497\end{array}$ & $\begin{array}{l}0.853 \\
0.420\end{array}$ & $\begin{array}{l}0.777 \\
0.341\end{array}$ & $\begin{array}{l}0.701 \\
0.263\end{array}$ & $\begin{array}{l}0.627 \\
0.187\end{array}$ & $\begin{array}{l}0.570 \\
0.115\end{array}$ \\
\hline
\end{tabular}

(b) PARTIAL AUTOCORRELATIONS

Partial

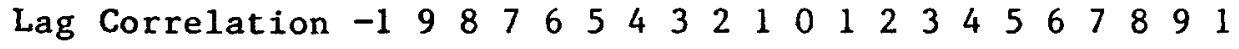

\begin{tabular}{rr|}
1 & 0.92163 \\
2 & 0.02116 \\
3 & -0.07680 \\
4 & -0.05395 \\
5 & -0.02474 \\
6 & 0.07156 \\
7 & -0.14165 \\
8 & -0.08749 \\
9 & -0.06955 \\
10 & -0.04604 \\
11 & -0.03287 \\
12 & -0.05851 \\
13 & -0.04384 \\
14 & -0.03370 \\
15 & -0.02814
\end{tabular}

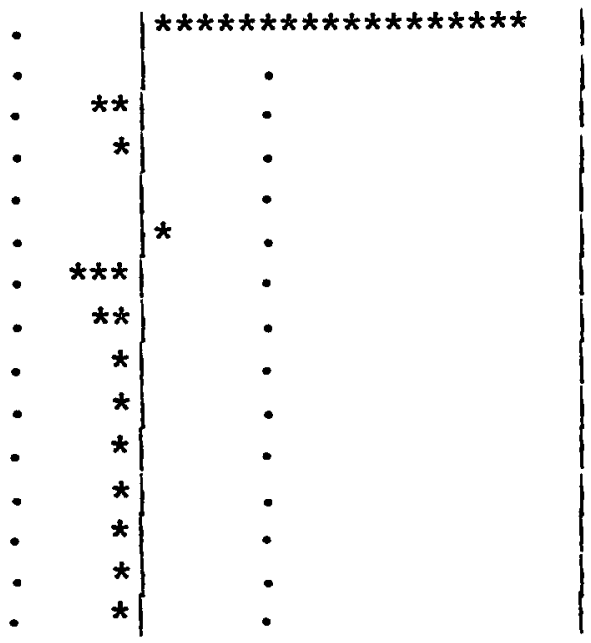

Note: - Marks of Approx. two Standard Errors

Figure 9.7: (a) Q-statistic and (b) Partial Autocorrelation of Observed Series for Franklin County Vehicle Registration 


\section{Tippecanoe County Vehicle Registration Series: Observed}

(a) Box-Pierce Q-statistic:

AUTOCORRELATION CHECK FOR WHITE NOISE

\begin{tabular}{|c|c|c|c|c|c|c|c|c|c|}
\hline $\begin{array}{r}\text { To } \\
\text { Lag }\end{array}$ & $\begin{array}{l}\text { Chi- } \\
\text { Square }\end{array}$ & $\mathrm{df}$ & $\begin{array}{l}\text { P(White } \\
\text { Noise) }\end{array}$ & & Autoce & rrelat & ons & & \\
\hline $\begin{array}{r}6 \\
12\end{array}$ & $\begin{array}{l}163.13 \\
2.01 .29\end{array}$ & $\begin{array}{r}6 \\
12\end{array}$ & $\begin{array}{l}0.000 \\
0.000\end{array}$ & $\begin{array}{l}0.942 \\
0.521\end{array}$ & $\begin{array}{l}0.880 \\
0.442\end{array}$ & $\begin{array}{l}0.811 \\
0.361\end{array}$ & $\begin{array}{l}0.740 \\
0.281\end{array}$ & $\begin{array}{l}0.667 \\
0.201\end{array}$ & $\begin{array}{l}0.596 \\
0.125\end{array}$ \\
\hline
\end{tabular}

(b) PARTIAL AUTOCORRELATIONS

Partial

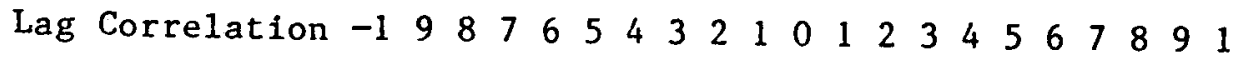

$\begin{array}{|rr|}1 & 0.94202 \\ 2 & -0.06354 \\ 3 & -0.10024 \\ 4 & -0.05072 \\ 5 & -0.05235 \\ 6 & -0.01839 \\ 7 & -0.08693 \\ 8 & -0.08502 \\ 9 & -0.06316 \\ 10 & -0.05164 \\ 11 & -0.06116 \\ 12 & -0.02995 \\ 13 & -0.03022 \\ 14 & -0.01891 \\ 15 & -0.04739\end{array}$

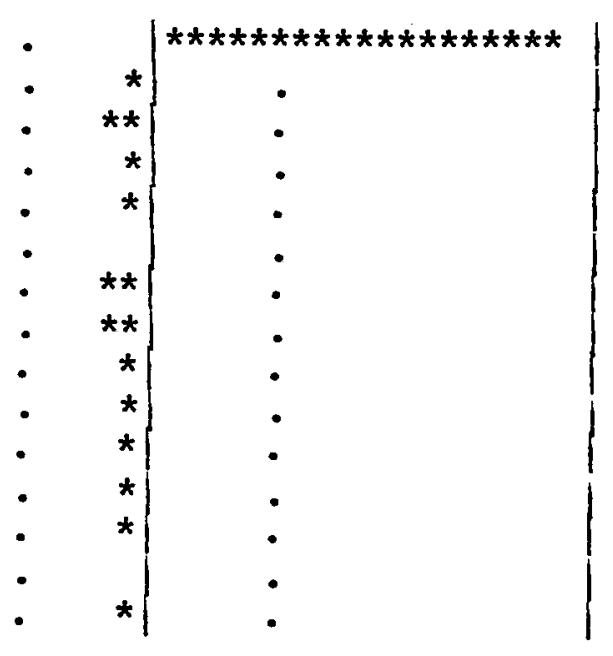

Note: - Marks of Approx. two Standard Errors

Figure 9.8: (a) Q-statistic and (b) Partial Autocorrelation of Observed Series for Tippecanoe County Vehicle Registration 
the associated Prob(White Noise) presented in part (a) of PACFs of Figures 9.7 and 9.8 show that chi-square statistic are significant. The first difference series is tried because of nonstationarity of the observed series and a significant chi-square statistic. ACFs and PACFs for the first difference series (see Figures 9.3 and 9.4) are presented in Figures 9.9-9.10 and 9.11-9.12, respectively. ACFs in Figure 9.9 and 9.10 and plots in Figures 9.3 and 9.4 suggest that the first difference series is stationary. Further, the low chi-square statistics and the high probability of white noise in part (a) of Figures 9.11 and 9.12 suggest that the first difference series is appropriate. According to our identification rules from $A C F$ and PACF, the following models are identified:

1. $\operatorname{ARIMA}(0,1,0)$ seems to be the most appropriate model for 24Franklin County because of no significant lags in ACF and PACF in Figures 9.9 and 9.11. Although not warranted, two other models - $\operatorname{Arima}(1,1,0)$ and $\operatorname{ARIMA}(0,1,1)-$ have been examined to make the most appropriate selection.

2. From the plots of ACF and PACF in Figures 9.10 and 9.12 , it appears that $\operatorname{ARIMA}(1,1,0)$ is a good model for Tippecanoe County. But the other possibilities -- $\operatorname{ARIMA}(0,1,1), \operatorname{ARIMA}(1,1,1)$ and $\operatorname{ARIMA}(0,1,0)$ - also need to be investigated to make a good final selection. $\operatorname{ARIMA}(0,1,0)$ is not warranted, based on the $\operatorname{ACF}$ and PACF plots, but it will be the appropriate model when there are no significant autoregressive or moving average parameters in other ARIMA mode1s. 
Franklin County Vehicle Registration Series: First Difference

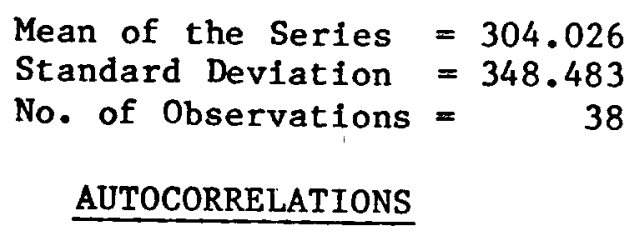

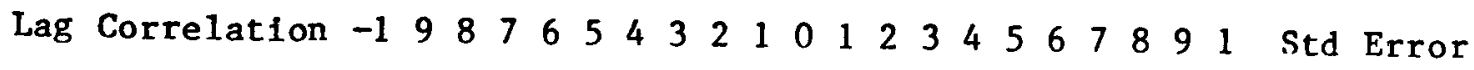

\begin{tabular}{rr|}
0 & 1.00000 \\
1 & -0.07353 \\
2 & -0.09935 \\
3 & -0.07115 \\
4 & 0.19828 \\
5 & -0.07131 \\
6 & 0.03567 \\
7 & -0.05452 \\
8 & -0.01340 \\
9 & -0.07698 \\
10 & -0.02184 \\
11 & -0.00350 \\
12 & 0.14593 \\
13 & 0.09804 \\
14 & -0.06982 \\
15 & -0.14890
\end{tabular}

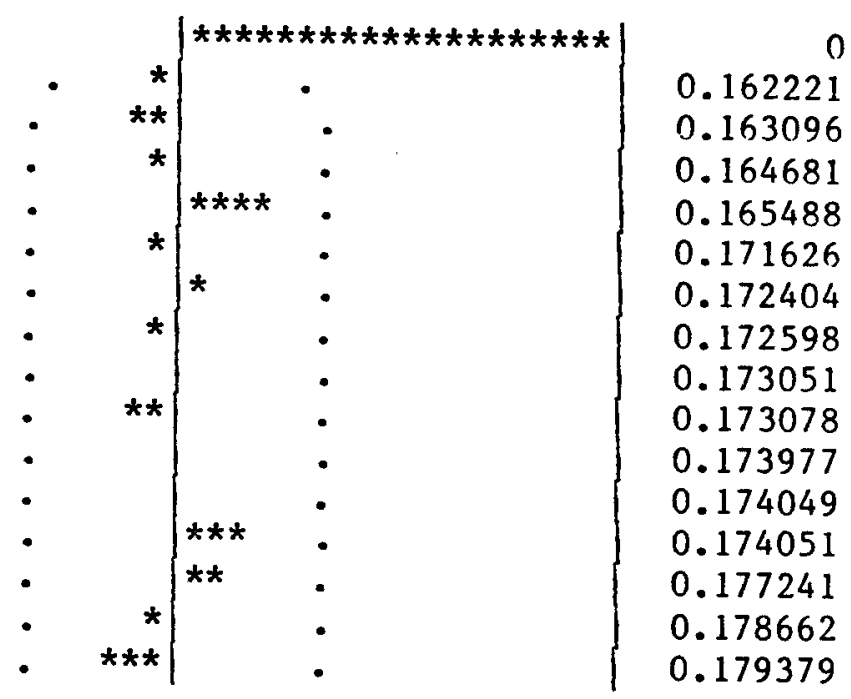

Note: - Marks two Standard Errors Figure 9.9: Autocorrelation of First Difference Series for Franklin
County Vehicle Registration 


\section{Tippecanoe County Vehicle Registration Series: First Difference}

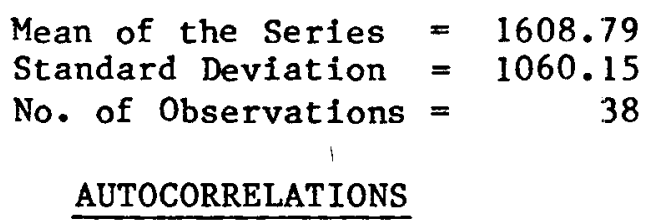

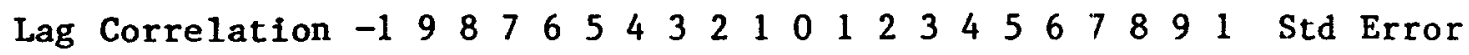

\begin{tabular}{rr|}
0 & 1.00000 \\
1 & 0.38190 \\
2 & 0.14201 \\
3 & 0.07239 \\
4 & 0.16620 \\
5 & 0.02764 \\
6 & 0.03347 \\
7 & 0.00868 \\
8 & 0.00145 \\
9 & -0.12615 \\
10 & -0.10449 \\
11 & -0.12317 \\
12 & 0.02922 \\
13 & 0.02926 \\
14 & -0.20256 \\
15 & -0.15564
\end{tabular}

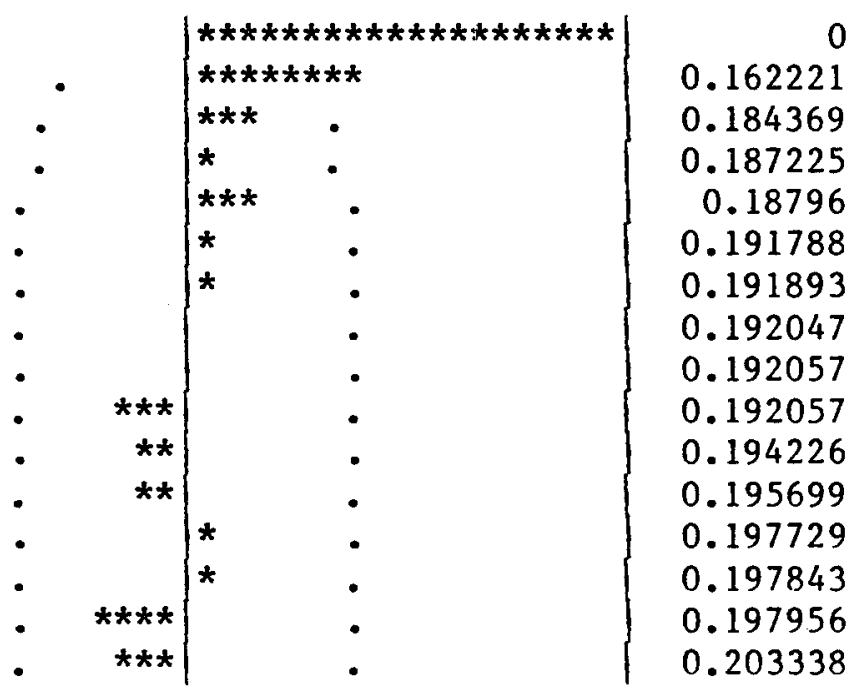

Note: -" Marks two Standard Errors

Figure 9.10: Autocorrelation of First Difference Series for Tippecanoe County Vehicle Registration 
Franklin County Vehicle Registration Series: First Difference

(a) Box-Pierce Q-statistic:

AUTOCORRELATION CHECK FOR WHITE NOISE

\begin{tabular}{|c|c|c|c|c|c|c|}
\hline $\begin{array}{r}\text { To } \\
\text { Lag }\end{array}$ & $\begin{array}{l}\text { Chi- } \\
\text { Square }\end{array}$ & $\mathrm{df}$ & $\begin{array}{l}\text { P(White- } \\
\text { Noise) }\end{array}$ & Autocorrelations & & \\
\hline $\begin{array}{r}6 \\
12\end{array}$ & $\begin{array}{l}2.91 \\
4.65\end{array}$ & $\begin{array}{r}6 \\
12\end{array}$ & $\begin{array}{ll}0.820 & -0.074 \\
0.969 & -0.055\end{array}$ & $\begin{array}{rrr}-0.099 & -0.071 & 0.198 \\
-0.013 & -0.077 & -0.022\end{array}$ & $\begin{array}{l}-0.071 \\
-0.003\end{array}$ & $\begin{array}{l}0.036 \\
0.146\end{array}$ \\
\hline
\end{tabular}

(b) PARTIAL AUTOCORRELATIONS

Partial

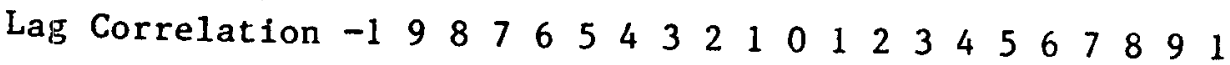

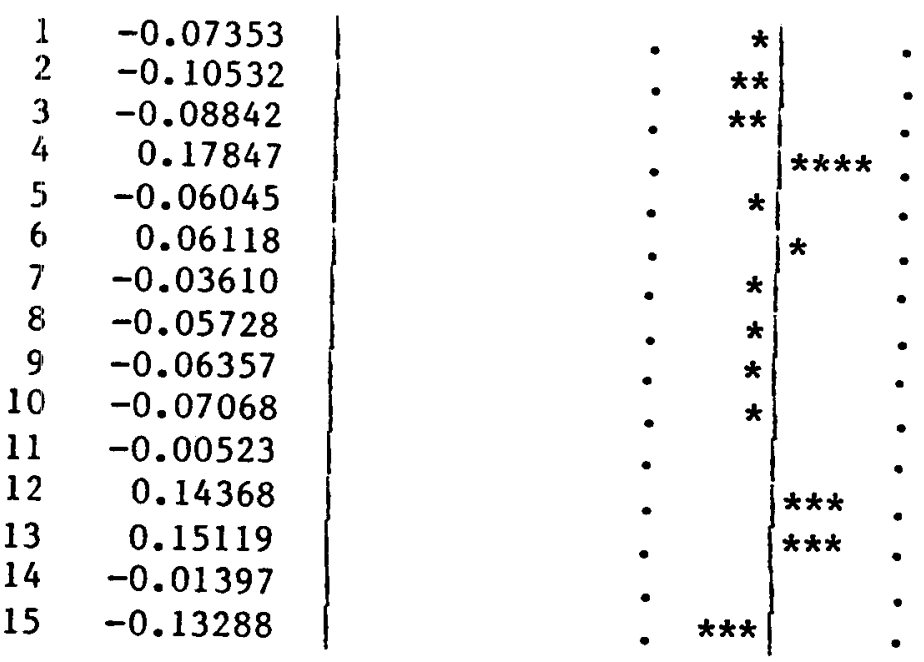

Note: - Marks of Approx. two Standard Errors

Figure 9.11: (a) Q-statistic and (b) Partial Autocorrelation of First Difference Series for Franklin County Vehicle Registration 
Tippecanoe County Vehicle Registration Series: First Difference

(a) Box-Pierce Q-statistic:

AUTOCORRELATION CHECK FOR WHITE NOISE

\begin{tabular}{|c|c|c|c|c|c|c|c|}
\hline $\begin{array}{r}\text { To } \\
\text { Lag }\end{array}$ & $\begin{array}{l}\text { Chi- } \\
\text { Square }\end{array}$ & df & $\begin{array}{l}\text { P(White- } \\
\text { Noise) }\end{array}$ & & Autocorrelations & & \\
\hline $\begin{array}{r}6 \\
12\end{array}$ & $\begin{array}{r}8.39 \\
10.73\end{array}$ & $\begin{array}{r}6 \\
12\end{array}$ & $\begin{array}{l}0.211 \\
0.552\end{array}$ & $\begin{array}{l}0.382 \\
0.009\end{array}$ & $\begin{array}{rrr}0.142 & 0.072 & 0.166 \\
0.001 & -0.126 & -0.104\end{array}$ & $\begin{array}{r}0.028 \\
-0.123\end{array}$ & $\begin{array}{l}0.033 \\
0.029\end{array}$ \\
\hline
\end{tabular}

(b) PARTIAL AUTOCORRELATIONS

Partial

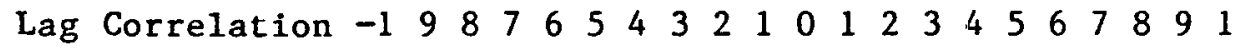

\begin{tabular}{rr|}
1 & 0.38190 \\
2 & -0.00450 \\
3 & 0.02299 \\
4 & 0.15407 \\
5 & -0.10612 \\
6 & 0.04932 \\
7 & -0.01619 \\
8 & -0.03067 \\
9 & -0.12357 \\
10 & -0.02562 \\
11 & -0.07277 \\
12 & 0.12749 \\
13 & 0.02686 \\
14 & -0.28412 \\
15 & 0.06480
\end{tabular}

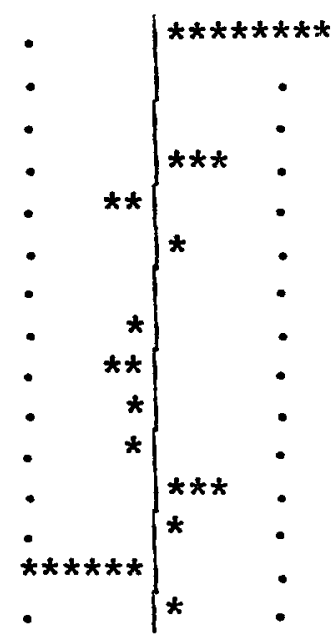

Note: - Marks of Approx. two Standard Errors

Figure 9.12: (a) Q-statistic and (b) Partial Autocorrelation of First Difference Series for Tippecanoe County Vehicle Registration 


\subsubsection{Model Estimation}

The ESTIMATE statement of the SAS ARIMA procedure [SAS/ETS 1984] is used to estimate the parameters and to diagnosis the fit. The maxImum likelihood technique is used in this estimation.

The following diagnostic information and statistics are used to check the model or to compare the alternative models produced in the identification stage.

1. Chi-Square statistics and/or Probability of White Noise - The description of these statistics was provided in Section 9.2.2.3.

2. Root Mean Square Error (RMSE) -- Section 5.4 has a concise description of this statistic.

3. Akaike's information criterion (AIC) [Akaike 1981] -- Section 5.3.5 has a succinct description of this information.

4. t-statistic and significance of parameters of model - Section 6.4.2 has a brief description of these statistics.

5. Correlation between parameters - A description can be found in Section 6.4.2.

The above statistics and information on the different ARIMA models produced in the identification stage for the few selected counties are presented in Tables 9.3 and 9.4. The models with nonsignificant parameters and high correlation between parameters (if applicable) have been dropped in making the final model selections. Table 9.4 shows that some or all parameters of any overfitted model are nonsig- 
Table 9.3: Statistics of ARIMA Models for Few Selected Counties

\begin{tabular}{|c|c|c|c|c|c|c|}
\hline \multirow[b]{2}{*}{$\begin{array}{l}\text { County } \\
\text { No. Name } \\
= \pm x====\neq=== \pm==\end{array}$} & \multirow{2}{*}{$\mid \begin{array}{c}\text { Mode 1: } \\
\operatorname{ARIMA}(p, d, q) \\
==x===== \pm====== \pm=\end{array}$} & \multicolumn{3}{|c|}{ Model Characteristics } & \multicolumn{2}{|c|}{ Normality } \\
\hline & & $\mid \begin{array}{l}\text { P(Wite- } \\
\text { Noise }[* *] \\
========= \pm=*\end{array}$ & $\begin{array}{c}\text { RMSE } \\
=======\end{array}$ & $\mid \begin{array}{c}\text { AIC } \\
=== \pm====\end{array}$ & $\begin{array}{l}\text { W-Value } \\
=======\end{array}$ & $\mid \begin{array}{l}\text { PROB }<\text { W } \\
======\end{array}$ \\
\hline 24 Franklin & $\begin{array}{l}\operatorname{ARIMA}(1,1,0) \\
\operatorname{ARIMA}(0,1,1) \\
\operatorname{ARIMA}(0,1,0)[*]\end{array}$ & $\mid \begin{array}{l}0.653-0.928 \\
0.678-0.933 \\
0.714-0.963\end{array}$ & $\begin{array}{l}357.0 \\
356.7 \\
353.2\end{array}$ & $\begin{array}{l}556.50 \\
556.44 \\
554.71\end{array}$ & $\mid \begin{array}{l}0.9421 \\
0.9470 \\
0.9208\end{array}$ & $\mid \begin{array}{l}0.073 \\
0.100 \\
0.015\end{array}$ \\
\hline 79 Tippecanoe & $\begin{array}{l}\operatorname{ARIMA}(1,1,0) \\
\operatorname{ARIMA}(0,1,1) \\
\operatorname{ARIMA}(1,1,1) \\
\operatorname{ARIMA}(0,1,0)\end{array}$ & $\mid \begin{array}{l}0.592-0.924 \\
0.557-0.917 \\
0.531-0.879 \\
0.123-0.466\end{array}$ & $\mid \begin{array}{r}995.5 \\
1009.8 \\
1009.6 \\
1074.4\end{array}$ & $\begin{array}{l}634.62 \\
635.65 \\
636.62 \\
639.27\end{array}$ & $\mid \begin{array}{l}0.9587 \\
0.9464 \\
0.9589 \\
0.9450\end{array}$ & $\mid \begin{array}{l}0.295 \\
0.097 \\
0.298 \\
0.089\end{array}$ \\
\hline 91 White & $\begin{array}{l}\operatorname{ARIMA}(1,1,0) \\
\operatorname{ARIMA}(0,1,1) \\
\operatorname{ARIMA}(1,1,1) \\
\operatorname{ARIMA}(0,1,0)\end{array}$ & $\mid \begin{array}{l}0.771-0.921 \\
0.546-0.970 \\
0.539-0.909 \\
0.066-0.336\end{array}$ & $\begin{array}{l}314.7 \\
288.4 \\
291.9 \\
347.4\end{array}$ & $\begin{array}{l}547.15 \\
543.93 \\
545.24 \\
553.47\end{array}$ & $\mid \begin{array}{l}0.9773 \\
0.9602 \\
0.9841 \\
0.9903\end{array}$ & $\left|\begin{array}{l}0.694 \\
0.320 \\
0.901 \\
1.000\end{array}\right|$ \\
\hline
\end{tabular}

[*] Model Selected.

[**] Range of values with lags $6,12,18$ and 24 . 
Table 9.4: Significance and Correlation of Parameters of ARIMA Models for Few Selected Counties

\begin{tabular}{|c|c|c|c|c|c|c|c|}
\hline \multirow{2}{*}{$\begin{array}{c}\text { County } \\
\text { No. Name: } \\
==x==m==s:==\end{array}$} & \multirow{2}{*}{$\mid \begin{array}{c}\text { ARIMA } \\
\text { Model: } \\
(p, d, q) \\
======x=\end{array}$} & \multicolumn{3}{|c|}{ Parameter- } & \multicolumn{3}{|c|}{ Correlation } \\
\hline & & $\begin{array}{l}\text { Name } \\
==x==\end{array}$ & $\left\{\begin{array}{l}\text { Estimate } \\
=======\end{array}\right.$ & $\mid \begin{array}{c}t \text {-value } \\
========\end{array}$ & $\left\{\begin{array}{l}\text { Parameter } \\
=== \pm=====\end{array}\right.$ & $\left.\right|_{=== \pm= \pm=} ^{A R} 1$ & $\begin{array}{c}\text { MA } 1 \\
:======\end{array}$ \\
\hline \multirow{3}{*}{$\begin{array}{l}4 \text { Frank- } \\
\text { lin }\end{array}$} & $(1,1,0)$ & $\begin{array}{l}\text { MU } \\
\text { AR1 }\end{array}$ & $\begin{array}{r}302.77 \\
-0.0756\end{array}$ & $\left|\begin{array}{r}5.61 \\
-0.45(*)\end{array}\right|$ & $\mathrm{MU}$ & 0.020 & $\mathrm{NA}(* *)$ \\
\hline & $(0,1,1)$ & $\begin{array}{l}\text { MU } \\
\text { MA1 }\end{array}$ & $\begin{array}{l}302.47 \\
0.0961\end{array}$ & $\begin{array}{l}5.77 \\
0.58 \text { (*) }\end{array}$ & MU & $\mathrm{NA}$ & -0.019 \\
\hline & $(0,1,0)$ & MU & 304.03 & 5.31 & - & NA & $\mathrm{NA}$ \\
\hline \multirow{4}{*}{$\begin{array}{l}79 \begin{array}{l}\text { Tippe- } \\
\text { canoe }\end{array}\end{array}$} & $(1,1,0)$ & $\begin{array}{l}\text { MU } \\
\text { AR } 1\end{array}$ & $\begin{array}{r}1579.00 \\
0.4190\end{array}$ & $\begin{array}{l}5.80 \\
2.78\end{array}$ & $M U$ & 0.023 & NA \\
\hline & $(0,1,1)$ & $\begin{array}{l}\text { MU } \\
\text { MA1 }\end{array}$ & $\begin{array}{l}1606.59 \\
-0.3556\end{array}$ & $\begin{array}{r}7.29 \\
-2.12\end{array}$ & MU & $\mathrm{NA}$ & -0.041 \\
\hline & $(1,1,1)$ & $\begin{array}{l}\text { MU } \\
\text { AR } 1 \\
\text { MAI }\end{array}$ & $\begin{array}{r}1578.40 \\
0.4243 \\
0.0063\end{array}$ & $\begin{array}{l}5.69 \\
1.08(*) \\
0.011^{(*)}\end{array}$ & $\begin{array}{l}\text { MU } \\
\text { AR } 1\end{array}$ & -0.043 & $\begin{array}{r}-0.056 \\
0.922\end{array}$ \\
\hline & $(0,1,0)$ & MU & 1608.79 & 9.23 & - & NA & $\mathrm{NA}$ \\
\hline \multirow{4}{*}{91 White } & $(1,1,0)$ & $\begin{array}{l}\text { MU } \\
\text { ARI }\end{array}$ & $\begin{array}{l}343.07 \\
0.4546\end{array}$ & $\begin{array}{l}3.75 \\
3.03\end{array}$ & MU & 0.052 & NA \\
\hline & $(0,1,1)$ & $\begin{array}{l}\text { MU } \\
\text { MA1 }\end{array}$ & $\begin{array}{r}366.35 \\
-0.9993\end{array}$ & $\left|\begin{array}{r}4.15 \\
-0.07 \text { (*) }\end{array}\right|$ & MU & NA & -0.060 \\
\hline & $(1,1,1)$ & $\begin{array}{l}\text { MU } \\
\text { AR1 } \\
\text { MA1 }\end{array}$ & $\begin{array}{r}356.48 \\
-0.9995 \\
-0.2737\end{array}$ & $\mid \begin{array}{r}5.01 \\
-0.04 \\
-1.06\end{array}$ & $\begin{array}{l}\text { MU } \\
\text { ARI }\end{array}$ & -0.057 & $\begin{array}{r}-0.018 \\
0.646\end{array}$ \\
\hline & $(0,1,0)$ & MU & 345.00 & 6.12 & - & NA & $\mathrm{NA}$ \\
\hline
\end{tabular}

(*) Not Significant at $\alpha=0.05$.

(**) NA $=$ Not Applicable. 
nificant. For Franklin County, $\operatorname{ARIMA}(0,1,0)$ - identified in Section 9.2.2.3 - is the only model with no significant parameter. $\operatorname{ARIMA}(0,1,0)$ is only a difference model with no autoregressive or movIng average parameters. Table 9.3 shows that ARIMA $(0,1,0)$ has the lowest values of RMSE and AIC among the alternative models for FrankIin County. Thus, $\operatorname{ARIMA}(0,1,0)$ has been finally selected to forecast vehicle registrations for Franklin County.

For 79-Tippecanoe County; the parameters of $\operatorname{ARIMA}(1,1,0)$, $\operatorname{ArimA}(0,1,1)$ and $\operatorname{ARIMA}(1,1,1)$ are found to be significant. $\operatorname{ARIMA}(1,1,0)$ is the most warranted model in the identification stage. The overfitted ARIMA $(1,1,1)$ model has nonsignificant AR and MA parameters with high correlation between these parameters. The RMSE and AIC values of $\operatorname{ARIMA}(1,1,0)$ are lowest among all the candidate models, and the probability of white noise of $\operatorname{ARIMA}(1,1,0)$ is high. Thus, $\operatorname{ARIMA}(1,1,0)$ is selected to forecast Tippecanoe County vehicle registrations.

For 91-White County, the overfitted $\operatorname{ARIMA}(1,1,1)$ and $\operatorname{ARIMA}(0,1,1)$ have nonsignificant $A R$ or $M A$ parameters, and a high correlation exists between $A R$ and $M A$ parameters for the $\operatorname{ARIMA}(1,1,1)$ model. Unlike 24Franklin and 79-Tippecanoe, the RMSE and AIC values of the overfitted $\operatorname{ARIMA}(1,1,1)$ and unwarranted $\operatorname{ARIMA}(0,1,1)$ are smaller than those for $\operatorname{ArIma}(1,1,0)$ and $\operatorname{ARIma}(0,1,0)$. But the $\operatorname{ARIMA}(1,1,0)$ has lower $\operatorname{RMSE}$ and $\operatorname{AIC}$ values than the $\operatorname{ARIMA}(0,1,0)$ model. The significance of the parameters in the model is a primary consideration in model selection. Thus, $\operatorname{ARIMA}(1,1,0)$ has been selected to forecast white County vehicle registrations. 
The final models from the Box-Jenkins procedure and their key statistics for each of the 93 series (92 counties and the entire state) are presented in Appendix Table Fl. The estimates of the parameters and their t-values for the ARIMA models are presented in Table F2.

\subsubsection{Model Forecast and Validation}

The FORECAST statement of the SAS ARIMA procedure [SAS/ETS 1984] was used to forecast future values at years 1989 to 2015 , using estimates of the model. The same maximum-likelihood method as used in model estimation is used in model forecasts. The results of these forecasts from ARIMA models for all 92 counties and state vehicle registrations are shown in Appendix Table F3. The forecasts at years 1989-2015 are generated from the last observation (Year 1988) of the series. In addition to these forecasts, one-step ahead forecasts [Abraham \& Leodolter 1983, Box \& Jenkins 1976] are generated for each series at years 1951 to 1988. These one-step ahead forecasts are used to compute the residuals. Normality of these residuals are tested by using the SAS univariate procedure [SAS/Basics 1985]. A detailed discussion of this normality test can be found in Section 6.3 .4 of Chapter 6. The Shapiro-Wilk $W$-value and the P-value are for each model are presented in Appendix Table F1. Table 9.3 also shows these normality statistics for all candidate models of the three selected representative counties. The normality assumption of residuals is satisfied for most of the serles. There are a few series having very low $W$ and probability value. The normality assumption has been 
violated in those cases. The normal probability plots of the three selected counties are presented in Appendix Figures F1.1-F1.3. These plots depict the presence of one or a few outliers (if any) from the expected straight line pattern and thus identify the cause of violation of normality with a low P-value.

By analogy to the plots of residuals against predictor variables in earlier chapters, the plots of residuals are generated against the time variable, Year. Those plots for the three selected counties are presented in Appendix Figures F2.1-F2.3. In spite of the presence of a few outliers (1ying more than 2-3 times STD DEV from the zero residual line), the randomness of the residual plots is apparent in these plots. Normality statistics can be improved by deleting the suspected outliers from the series (See Chapter 6). Because of the problem of estimation with missing data in a series and overall high quality of other results of the models, model refitting by deleting the suspected outliers (if any) has not been tried.

\subsubsection{Autoregression Procedure}

The AUTOREGression (AUTOREG) procedure [SAS/ETS 1984] fits a multiple linear regression model to a selected response time series, with corrections for serial correlation. Unlike the ARIMA procedure, this procedure works even if some values of the independent or dependent variables are missing. The AUTOREG procedure has been used to alleviate the shortcomings of the Ordinary Least Squares regression estimates (see Section 5.2) for the effects of serial correlation. It has also been used to generate forecasts. 


\subsubsection{First Order Autocorrelation Check of Residuals}

Most regression procedures can print a Durbin-Watson statistic that tests for the presence of first-order autocorrelation. When autocorrelation is present, the ordinary least-squares (OLS) parameter estimates are not efficient and the standard error estimates are biased. A common way of detecting a certain type of serial correlation is the Durbin-Watson test [Draper \& Smith 1981]. The alternate hypothesis to detect departures from the null hypothesis (true errors are uncorrelated) is

$$
\mathrm{H}_{\mathrm{a}}: \varepsilon_{t}=\rho \varepsilon_{t-1}+e_{t}
$$

where $|\rho|<1$ and $e_{t}$ is an uncorrelated series, and the errors $e_{t}$ are assumed to be $\mathrm{N}\left(0, \sigma^{2}\right)$ variables. This type of error term, in which $\varepsilon_{t}$ is related to $\varepsilon_{t-1}$, is called an $A R$ error of the first order. The Durbin-Watson statistic (d) is given by

$$
d=\frac{\sum_{t=2}^{n}\left(\hat{\varepsilon}_{t}-\hat{\varepsilon}_{t-1}\right)^{2}}{\sum_{t=1}^{n} \hat{\varepsilon}_{t}^{2}}
$$

where $\hat{\varepsilon}_{t}=Y_{t}-\hat{Y}_{t}$. If the actual errors $\varepsilon_{t}$ are uncorrelated, the numerator of d in Equation (9.2) has an expected value of about 2(n1) $\sigma^{2}$ and the denominator has an expected value of about $n \sigma^{2}$. Thus, if the errors $\varepsilon_{t}$ are uncorrelated, the ratio d should be about 2 . Positive autocorrelation means that $\varepsilon_{t}$ is closer to $\varepsilon_{t-1}$ than in the 
Independent case, so $\left|\varepsilon_{t}-\varepsilon_{t-1}\right|$ should be smaller. It follows that $d$ should also be less. The smallest possible value for $d$ is zero. If $d$ is significantly less than 2, positive autocorrelation is present. When is a Durbin-Watson statistic significant? The answer depends on the number of independent variables in the regression and the number of observations. The critical boundary values of the Durbin-Watson test have been taken from the tables in Draper and Smith [1981]. The tables use $k^{-}=k^{-1}$, where $k^{-}$is the number of explanatory variables, and $n$ (number of observations) to obtain the bounds $d_{L}$ (lower bound) and $d_{U}$ (upper bound) for any given regression [Draper \& Smith 1981]. A Durbin-Watson statistic $d>2$ is evidence of negative autocorrelation. To see that the evidence is true, one needs to compute $d^{-}=4-d$ and use the standard Durbin-Watson test on $d^{-}$. In Section 9.2.3.3, the Durbin-Watson statistic will be used to compare the OLS and AUTOREG procedures for forecasting vehicle registrations.

Three caveats apply to the Durbin-Watson test. First, it is designed to detect first-order AR errors. Although this type of autocorrelation is only one possibility, it seems to be the most common. The test has some power against other types of autocorrelation. Second, the Durbin-Watson bounds are not valid when the lagged values of the dependent variable appear on the right side of the regression. Third, the Durbin-Watson statistic often lies in the critical region, $d_{L}<d<d_{U}$, even though no real autocorrelation is present. 


\subsubsection{Model Description}

Parameter estimates produced by the AUTOREG procedure are usually similar to ordinary least-square estimates, but the standard errors can be very different, affecting significance tests. AUTOREG is a useful tool for forecasting. It uses the time series part of the model as well as the systematic part in generating predicted values. The AUTOREG procedure estimates the parameters of a linear model whose error term is assumed to be an autoregressive process of a given order $p$, denoted $\operatorname{AR}(p)$. The model for time $t$ is as follows:

$$
y_{t}=x_{t}^{-\beta}+\nu_{t}
$$

where $\nu_{t}$ is assumed to be generated by an autoregressive process of order $p(\operatorname{AR}(p))$. That is

$$
\nu_{t}=\varepsilon_{t}-\alpha_{1} \nu_{t-1}-\ldots \ldots-\alpha_{p} \nu_{t-p}
$$

where

$y_{t}$ are the dependent values,

$x_{t}$ is a vector of regression values,

$\beta$ is a vector of structural parameters, and

$\alpha_{1}$ 's $(1=1, \ldots, p)$ are the autoregressive parameters.

The $\varepsilon_{t}$ are normally and independently distributed with a mean of zero and a variance of $\sigma^{2}$. 
An autoregressive model can be specified for the error term. Its parameters can be estimated in one of four ways, depending on what method is used in estimates. As mentioned in Section 9.2.1, the maxImum likelihood method usually provides the most accurate estimates of model error autocorrelation parameters. After an ols fit of the dependent series, the residuals are used to estimate the error autocorrelation parameters. These parameters are then used to reestimate the regression parameters by generalized least squares. After the regression parameters and the autoregression parameters are estimated, the autoregressive model has been used to forecast future observations. A Durbin-Watson statistic is computed from the oLS residuals. AUTOREG procedure also computes Akaike's information criterion (AIC) [Akaike 1981].

\subsubsection{Model Estimation}

To determine the number of autoregressive parameters of residuals after the OLS fit, a number of trial runs with a large number of autocorrelation lags in the models for different series have been made. The results of these trial runs show that only the first lag autoregressive parameter (AR1) is significant with a constant term and time variable in the model. For 24-Franklin County, Table 9.5 shows the significance of parameters for an overfitted autoregressive model with 8 lags. 
Table 9.5: Nonsignificance of Overfitted Autoregressive Lags for Franklin County Vehicle Registrations

\begin{tabular}{|c|c|c|c|}
\hline $\begin{array}{l}\text { Variable } \\
===x====\end{array}$ & $\begin{array}{l}\text { Estimate } \\
=========\end{array}$ & $\left.\right|_{x=======} ^{t}$ & $\begin{array}{l}\text { Prob }>t \\
===x===\end{array}$ \\
\hline Constant & -597216 & -13.460 & 0.0001 \\
\hline Year & 308.88 & 13.740 & 0.0001 \\
\hline AR 1 & -0.8298 & -4.313 & 0.0002 \\
\hline AR2 & 0.0403 & 0.164 & 0.8709 \\
\hline AR 3 & -0.0197 & -0.082 & 0.9352 \\
\hline AR4 & -0.2289 & -0.948 & 0.3508 \\
\hline AR 5 & 0.2396 & 0.990 & 0.3302 \\
\hline AR6 & -0.2351 & -0.955 & 0.3476 \\
\hline AR7 & 0.2374 & 0.949 & 0.3502 \\
\hline AR8 & 0.0351 & 0.177 & 0.8605 \\
\hline
\end{tabular}

This table shows that only ARl with a constant term and Year is significant (i.e., Prob>t or P-value is very low, usually less than 0.05).

The following statistics and diagnostic measures are obtained for each Autoregressive model.

1. Durbin-Watson d statistic for OLS and AUTOREG procedures. A description of this statistic was provided in Section 9.2.3.1.

2. Coefficient of Multiple Determination $\left(R^{2}\right)$. Section 5.3 has the definition of $R^{2}$.

3. RMSE or sqrt(MSE). A brief description of this statistic can be found in Section 5.4 .

4. Akaike's Information Criterion (AIC) [Akaike 1981]. Section 5.3.5 has described this statistic.

5. t-value of the parameters and their significance. Section 6.4 .2 has a concise description. 
The results of items 1 to 4 in the above listing are shown in Appendix Table F4 for all 93 series. The estimation of the model parameters and their $t$-values are presented in Appendix Table F5. The computed $t$-values of the parameters of the models indicate that almost all parameters are significant at an $\alpha$-level as low as 0.0001 . Table F4 shows very high $\mathrm{R}^{2}$ values (about 99 percent) for all AUTOREG models. A significant increase in the d-statistic is noticed in the AUTOREG model with respect to that in the OLS model. Usually, the problem of first-order autocorrelation associated with oLS model has been removed in AUTOREG mode1. The control statistics have been provided in the footnote to Table F4. Even when autocorrelation has not been removed completely, a significant improvement in the d-statistic can be found in the AUTOREG mode1 over the OLS model.

Table 9.6 has compared the $D-W d, R^{2}, \sqrt{M S E}$, and AIC between the OLS with time, AUTOREG with time and ARl, and the resulting ARIMA mode1 (see Section 9.2.2.4) for the three selected counties. In spite of close and high $\mathrm{R}^{2}$ values, a significant improvement has achieved in $D-W^{-} s, \sqrt{M S E}$ and AIC in the AUTOREG model with respect to OLS model. Although $R^{2}$ is not possible for the ARIMA model, the $\sqrt{\text { MSE }}$ and AIC statistics imply that there is no significant difference in the quality of results between the AUTOREG and ARIMA models. 
Table 9.6: Key Statistics of OLS, AUTOREG and ARIMA Model for Few Selected Counties Vehicle Registrations

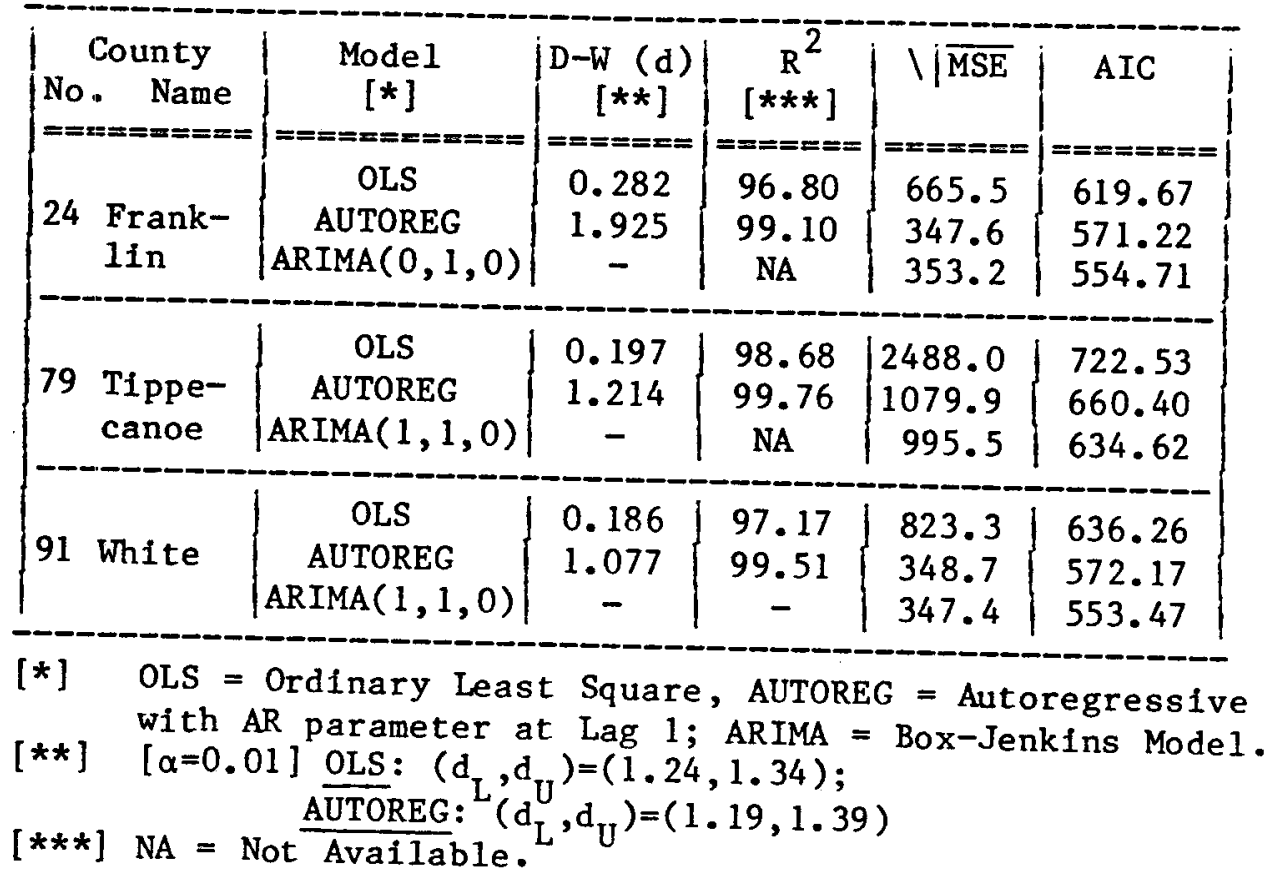

\subsubsection{Model Forecast and Validation}

The AUTOREG models from Section 9.2.3.3 are used to forecast the future values of county and state vehicle registrations for years 1989 to 2015. The results of these forecasts are shown in Appendix Table F6. These forecasts consist of both the structural (part 1 of Equation 9.6) and autoregressive (part 2 of Equation 9.6) parts of the autoregressive mode1. Forecasts are also made for the time series at years 1951 to 1988. These forecasts were used to evaluate the performance of the models. The normality assumption of these residuals is tested by using the SAS UNIVARIATE procedure [SAS/Basics 1985]. Appendix Table $F 4$ has presented the results of the normality statistics - Shapiro-Wilk W-value and the associated P-value. The quality 
of the results of the normality of these AUTOREG models and the ARIMA models in Section 9.2.2.5 is similar. For those series with very low probability results, the normality assumption has been violated for the presence of at least one outlier. The normal probability plots of the three selected counties are shown in Appendix Figures F1.4-F1.6. Once again, these plots are similar to those shown for ARIMA models in Figures F1.1-F1.3. However, a slight improvement in the P-value has been achieved for the three selected AUTOREG models. The plots of residuals against time, presented in Appendix Figures F2.4-F2.6, show the evidence of presence outliers. Randomness in the residuals can be noted in these plots.

\subsubsection{Combination of Forecasts and Comparison of Models}

The combination of forecasts is thought to be a practical and simple way to produce better forecasts. An extensive literature review supports the combination of forecasts. Although there is currently general agreement that combination can increase accuracy, there is still concern about the preferred method of combination.

Armstrong [1984] recommended developing several simple methods and combining the forecasts rather than developing one complex model. He said "If one does not know which of a set of forecasts is most accurate, then combining forecasts produces dramatic gain. Given that we know little about the best way to welght the components of a combined forecast, use methods that are as different as possible, and simply weight each forecast equally. One should not introduce complexities unless absolutely necessary." 
A number of researchers have found advantages in combining models. The combination could be based on any criterion - for example, Mean Absolute Percentage Error (MAPE), Root Mean Square Percen-

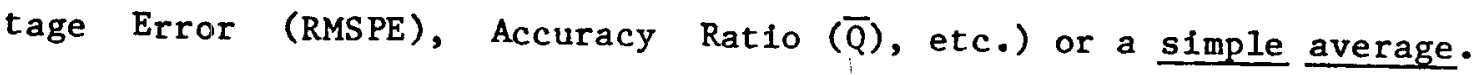
Granger and Ramanathan [1984] said, "In theory, a combined forecast will usually be superior to its constituent models and never be worse than the worse of the constituents". This means that one should never lose by combination and will usually gain.

Both academics and practitioners often recommend combining forecasts. This is considered especially useful if the methods are different. Combinations of forecasts are intuitively appealing. They guard against mistakes and unreasonable assumptions, and thus help to avold large errors. They also use more information. A statistical summary [Table 2, p. 96 of Armstrong 1986] shows that the combination of forecasts from two different methods yielded a 6.6 percent reduction in error compared to the average component. The error reduction ranged from 0 to 23 percent across the eight studies. Combinations of forecasts from three methods are expected to yield further improvements, but the two studies avallable on combining three methods found no gain and an 18 percent error reduction. Combinations of forecasts derived by simflar methods also help. Armstrong [1984] found an average error reduction of 42 percent when two methods were combined (averaged over four studies). Makridakis and Winkler [1983], in their study of 111 time series, found a 7.2 percent reduction for two methods, with continuing gains as methods were added (for example, a 16.3 percent reduction with five methods). Research to date suggests 
that equal weighting of the various forecasts is sufficient.

Both the Box-Jenkins and Autoregressive procedures in this study have been found to deliver quality forecasts in this study. The procedures can hardly blamed for failing to deliver more than is possible from a scanty data base. The optimality of these forecasts rests on two assumptions: that the model is correct and the model structure does not change over time (particularly in the future).

Based on the quality of the statistics of both Box-Jenkins and Autoregressive procedures, and lessons from the literature on combining forecasts, the combination of forecasts in this study has been done with a simple average of the individual forecasts. Appendix Tables F7 shows the results of this combination. It is highly recommended that combined forecasts, rather than a single forecast of vehicle registration (if required) be used by the traffic model.

The performance measures -- ME, MPE, MAE, MAPE, RMSPE, and $\bar{Q}-$ of the forecasts from Box-Jenkins, Autoregressive and their combination for the two selected representative counties are shown in Table 9.7. Some of these forecast measures have been introduced in earlier chapters and their full description is provided in next chapter. Despite the merits and demerits of these forecast measures, all the measures suggest that both Box-Jenkins and Autoregressive have produced forecasts similar in quality. The high quality of their forecasts is verified by (1) very low values of MPE, MAPE, RMSPE, and (2) an accuracy ratio $(\bar{Q})$ close to 1 - a value that reflects good forecasts. 
Table 9.7: Forecast Measures of Time Serles Models for Two Selected Counties Vehicle Registrations

\begin{tabular}{|c|c|c|c|c|c|c|c|}
\hline \multirow{2}{*}{ County } & \multirow{2}{*}{ Mode1 } & \multicolumn{6}{|c|}{ Forecast Measures } \\
\hline & & ME & MPE & MAE & MAPE & jRMSPE & $\bar{Q}$ \\
\hline $\begin{array}{l}\text { Frank- } \\
\text { lin }\end{array}$ & $\mid \begin{array}{c}\text { Box-Jenkin } \\
\text { Autoreg. } \\
\text { Combined }\end{array}$ & $\begin{array}{r}-1.74 \\
21.05 \\
9.66\end{array}$ & $\mid \begin{array}{l}0.216 \\
0.434 \\
0.325\end{array}$ & {$\left[\begin{array}{l}244.7 \\
245.5 \\
242.2\end{array}\right.$} & $\mid \begin{array}{l}2.602 \\
2.602 \\
2.575\end{array}$ & $\begin{array}{l}4.154 \\
3.960 \\
14.023\end{array}$ & $\left\{\begin{array}{l}1.027 \\
1.027 \\
1.027\end{array}\right.$ \\
\hline $\begin{array}{l}\text { Tippe- } \\
\text { canoe }\end{array}$ & $\mid \begin{array}{c}\text { Box-Jenkin } \\
\text { Autoreg. } \\
\text { Combined }\end{array}$ & $\begin{array}{r}-23.55 \\
56.82 \\
16.58\end{array}$ & $\mid \begin{array}{l}0.049 \\
0.387 \\
0.218\end{array}$ & $\mid \begin{array}{l}779.0 \\
809.7 \\
762.3\end{array}$ & $\mid \begin{array}{l}1.430 \\
1.562 \\
1.428\end{array}$ & $\mid \begin{array}{l}1.951 \\
2.202 \\
2.001\end{array}$ & $\mid \begin{array}{l}1.014 \\
1.016 \\
1.014\end{array}$ \\
\hline
\end{tabular}

The forecast measures of combined forecasts also illustrate the high quality forecasts of vehicle registration. It is also proved from Table 9.7 that there is no absolute superiority of the autoregressive or the Box-Jenkins procedure over the other.

It is expected that a combination of forecasts will continue to produce good forecasts, even if one constituent forecast is faulty. Appendix Figures F3.1-F3.3 and F3.4-F3.6 present the plots of observed values of vehicle registration vs. their forecasts from Autoregressive, Box-Jenkins and their combination for the two selected counties 24-Franklin and 79-Tippecanoe, respectively. The points in these plots lie very. close to the $45^{\circ}$ line. Appendix Figures F4.1-F4.3 and F4.4-F4.6 show the plots of observed values of vehicle registration and forecasts from Autoregressive, Box-Jenkins and their combination for the two selected counties 24-Franklin and 79-Tippecanoe, respectively. Although different forecasts for each of these counties are drawn on separate plots, use of the same scale allows one to see that 
the forecasts from different procedures are almost same or close to each other.

\subsection{City Population Forecasts}

Forecasts of city population, like vehicle registration in Section 9.2, are not avallable from any source, to date. This study has used a very simple but commonly-used method to forecast city population. The method uses the "ratio" of city population to county population to forecast the city population from the forecasts of county population available from other sources. Forecasts of city population will be used mainly to forecast traffic by the elasticity model (if required) of Chapter 6. No model in Chapter 6 has both county and city population in its equation at the same time. Thus, the ratio method of forecasting city population will not violate the independence of the variables in the model.

\subsubsection{Ratio Method of City Population Forecasts}

The underlying idea of the "ratio" technique [Krueckeberg \& Silvers 1974] can be summarized as follows: The population growth of a city (CTY) is highly dependent on what happens to the population in its surrounding region, e.g., a township or county (CNTY/TWN). The "ratio" technique assumes that the nature of this interdependence is likely to persist in the near future. If a surrounding region's population projections are available, then one can estimate the city's share of the region's projected total. Algebraically, the "ratio" technique can be represented as: 


$$
\frac{\mathrm{POP}_{\mathrm{CTY}}^{t+n}}{\mathrm{POP}_{\mathrm{CNTY} / \mathrm{TWN}}^{\mathrm{t}+\mathrm{n}}}=\frac{\mathrm{POP}_{\mathrm{CTY}}^{\mathrm{t}}}{\mathrm{POP}_{\mathrm{CNTY} / \mathrm{TWN}}^{t}}
$$

where,

$$
\begin{aligned}
\text { POP }_{\text {CTY }}^{j} & =\text { City Population at year } j \\
\text { POP }_{\text {CNTY } / \text { TWN }}^{j} & =\text { Township(s) or County(ies) Population at year } j
\end{aligned}
$$

For projection purposes, the terms can be rearranged as follows:

$$
\mathrm{POP}_{\mathrm{CTY}}^{t+n}=\left|\frac{\mathrm{POP}_{\mathrm{CTY}}^{t}}{\mathrm{POP}_{\mathrm{CNTY} / \mathrm{TWN}}^{t}}\right| \mathrm{POP}_{\mathrm{CNTY} / \mathrm{TWN}}^{\mathrm{t}+\mathrm{n}}
$$

Equation (9.9) simply says that the future city population will be some fraction ( ${ }^{2} P_{\mathrm{CTY}}^{\mathrm{t}} / \mathrm{POP}_{\mathrm{CNTY} / \mathrm{TWN}}^{\mathrm{t}}$ ) of the future population of the region $\left(\mathrm{POP}_{\mathrm{CNTY} / \mathrm{TWN}}^{\mathrm{t}+\mathrm{n}}\right)$. Equation $(9.9)$ appears similar to a linear model of general form $Y=a+b X$; where a (the intercept) is assumed to be zero, and the independent variable is the population size of the surrounding township or county, and the b coefficient is the ratio of population sizes. The only historical data required in this "ratio" method is the population of the city and its surrounding township or county at one recent point in time. One may think that this "ratio" technique is inferior to the linear model based on a series of historical data. To guard against that possibility, the method can be improved by looking at several points in time and take an average or trend of the ratios. County or township population projections are often based on "cohort survival technique" [Krueckeberg \& Silvers 
1974]. Using that sophisticated technique's population projection as input to the "ratio" method, one can easily see the efficiency of this simple but popular method.

In this study, only one point (Year 1980) has been used to determine the ratio for Equation (9.9). This ratio can be modified and/or calibrated as soon as 1990 census population data at the city and county levels become available, by determining the trend of ratio or average ratio. The 1970 census year data are thought to be distant in time and have not been considered in determining the trend of the ratio or the average ratio. This study has projected the city population for all the cities in the State of Indiana with a 1980 population of 5000 or more. In the ratio technique, the appropriate surrounding area to serve as a defining context for a city is based on the following considerations:

1. the size of the city - the bigger the city, the bigger the surrounding region, and

2. the location of the city within a township and/or county. For example, if a city is located very close to the boundary of another county/township, then the adjacent county/township is also included in the surrounding region.

The surrounding townships or counties used in this study are given in Appendix Table F8. The cities in Table F8 have been listed alphabet1cally, where rank of a city is based on the city's 1980 population size. Table F8 also shows the county name and its number for each city. Finally, Equation (9.9) has used to forecast the city popula- 
tion. The results for these forecasts at years 1985-2020 at every 5 years are shown in Table F9. The forecasts from Table F9 will be used in any traffic model that uses city population as a predictor variable.

\subsection{Chapter Summary}

The main purpose of this chapter is to produce reliable forecasts of input variables for the traffic models when no forecasts are available from outside sources. This chapter has described the techniques used to forecast those input variables - vehicle registration and city population. To forecast vehicle registrations, the popular BoxJenkins time-series analysis and a comparatively simple Autoregressive time serles procedure have been used. The Autoregressive method was found to be as accurate as the Box-Jenkins method. The results of these two methods have been compared and combined to produce better forecasts. The performance measures of the methods have been evaluated and the results show the high quality of the forecasts for county and state vehicle registration. City population has been predicted by using a ratio technique, which is very simple but uses the input of more sophisticated methods. The results of all forecasts have been shown in tabular form. The model building process has been demonstrated with tables and figures. 
CHAPTER 10

MODEL EVALUATION, COMBINATION AND CALIBRATION

\subsection{Introduction}

The most important rule for choosing a forecasting method is its accuracy, or how well the forecast matches the actual future value. In this chapter, several measures to determine the accuracy of forecasting models are summarized from the forecasting literature. Some of those measures are used to evaluate the performance of the models developed in this study. Based on the performance of individual models, forecasts from different models have been combined. Finally, a method to determine when there will be a need to change or recalibrate a forecasting model or its parameters is presented.

\subsection{Performance Measures Criteria}

The performance of a forecast can be checked against its actual value it becomes known. The selection of forecast criteria is usually arbitrary, but has for the most part been limited to those that are frequently seen in statistics and econometrics: coefficient of multiple determination $\left(R^{2}\right)$, standard error of estimate, mean absolute error, range, and mean error. The major difficulty is the absence of 
a single universally accepted measure of forecasting accuracy.

This section describes some measures of forecast accuracy [Ah1burg 1989, Armstrong 1978, Chisholm \& Whitaker 1971, Meyer \& Glauber 1964, and Montgomery \& Johnson 1976], discusses their advantages and disadvantages, then decides on their uses and roles in this study. The following notation has been adapted for this section:

- $A_{i}$ is the 1 th actual value.

- $\mathrm{F}_{i}$ is the ith forecast.

- $\mathrm{n}$ is the number of forecasts made in the forecast horizon.

Mean Error (ME)

The Mean Error (ME) and Mean Percentage Error (MPE) are calculated from

$$
\begin{array}{r}
M E=\frac{\sum_{i=1}^{n}\left(F_{i}-A_{i}\right)}{n} \\
M P E=\frac{\sum_{i=1}^{n}\left(F_{i}-A_{i}\right)}{n}(100)
\end{array}
$$

They should not be used alone because one could have a ME or MPE equal to zero when large errors are present if the large errors had different sigris and canceled one another. This study has used these measures primarily to prove this weakness. 
Mean Absolute Deviation (MAD or MAE)

The mean absolute deviation (MAD or MAE) is calculated from

$$
\text { MAD or MAE }=\frac{\sum_{i=1}^{n}\left|F_{i}-A_{i}\right|}{n}
$$

MAD is appropriate when the cost function is linear and symmetric. An example is when the cost of an error of 10 units is twice that of an error of 5 units, regardless of the sign of $F_{i}-A_{1}$. It does not have the "cancellation" problem associated with ME and MPE. Because of its simplicity, this study has used MAE as a measure of forecast accuracy.

Mean Squared Error (MSE)

Mean Squared Error (MSE) is commonly used in many statistical methods of forecasting. MSE is defined as

$$
\text { MSE }=\frac{\sum_{i=1}^{n}\left(F_{1}-A_{i}\right)^{2}}{n}
$$

This measure has two major drawbacks. First, it refers to fitting a model to historical data. An MSE of zero can always be obtained in the fitting phase by using a polynomial of sufficiently high order. The MSE calculated during the fitting phase of forecasting may give little indication of the accuracy of the model in forecasting. A second drawback of the MSE as a measure of forecasting accuracy is that different methods often use MSE in the different procedures in 
their model fitting phase. Thus, comparison of the methods that use MSE in their fitting procedure in different ways is of limited value.

In the forecasting phase, the use of MSE as a measure of accuracy can also create problems. MSE does not represent absolute measure and is not particularly appropriate for making comparisons among formal forecasting methods (for example, methods based on statistical theory) and naive methods (for example, ad hoc methods). Thus, one should look for alternate measures of accuracy that seek to: (a) express accuracy in relative terms and (b) allow comparison among formal methods and naive methods of forecasting [Armstrong 1978].

\section{Root Mean Square Error (RMSE)}

The Root Mean Square Error (RMSE) is calculated from

$$
\operatorname{RMSE}=\left[\frac{\sum_{i=1}^{n}\left(F_{i}-A_{i}\right)^{2}}{n}\right]^{1 / 2}
$$

RMSE is similar to MAD. A good approximate relationship is: RMSE $=0.8 \mathrm{MAD}:$ It is difficult to visualize what the RMSE means in practical terms, but all one need to do is multiply the RMSE by 1.25 to get an approximation of the MAD. One should avoid the RMSE when there are outliers, because the outliers will have a excessively strong effect on the measure. One legitimate reason for using the RMSE arises when the cost associated with an error increases as the square of the error. 
Mean Absolute Percentage Error (MAPE)

The mean absolute percentage error (MAPE) is calculated from

$$
\text { MAPE }=\left[\begin{array}{c|c|}
n & F_{i}-A_{i} \\
A_{i} & \frac{A_{i}}{n}
\end{array}\right](100)
$$

The MAPE is similar to the MAD except that it is dimensionless. This is helpful in making comparisons among forecasts from different situations. The MAPE is appropriate when the cost of errors is more closely related to the percentage error than to units of error. This assumption is often reasonable. Problems may arise if $A_{i}$ values are close to zero, but this problem does not arise in this study. Consequently, this study uses MAPE as one measure of forecasting model accuracy.

Root Mean Square Percentage Error (RMSPE)

The Root Mean Square Percentage Error (RMSPE) is calculated from

$$
\operatorname{RMSPE}=\left[\frac{\sum_{i=1}^{n}\left[\frac{F_{i}-A_{i}}{A_{i}}\right]^{2}}{n}\right]^{1 / 2}
$$

The RMSPE is similar to MAPE, except it uses the square of the error in its cost function instead of a linear error term. Use of RMSPE 
arises may be appropriate when the cost associated with an error Increases as the square of the error. Because this type of error function seems applicable in this study, RMSPE is used as a measure of accuracy.

Theil's measure of inequality (U)

The 1i.terature reflects some confusion regarding the mathematical representation of Theil's U statistic. There are actually two measures (U1 and U2). Theil proposed both, but at different times and under the same symbol. This section will present the different forms of the $U$ statistic and determine its applicability to this study.

Theil's U1 statistic [Armstrong 1978, Meyer \& Glauber 1964, Chisholm \& Whitkar 1971] is based on a comparison of the predicted change with the observed change. It is a measure of the degree to which the forecasted value approximates the actual one, or more precisely, the amount by which $\alpha$ and $\beta$ differ from 1 and 0 , respectively, in the equation $F_{i}=\alpha A_{i}+\beta$. The Theil Ul coefficient, originally proposed by Theil in 1958, is estimated by

$$
U 1=\frac{\sqrt{\frac{1}{n} \sum_{i=1}^{n}\left(F_{i}-A_{i}\right)^{2}}}{\sqrt{\frac{1}{n_{i=1}^{n} \sum_{i}^{2}}+\sqrt{\frac{1}{n_{i=1}^{n} \sum_{i}^{2}}}}}
$$

The denominator of this statistic normalizes it so that its domain is the closed interval of zero to one. Obviously, $U 1=0$ is a perfect 
forecast, since $F_{i}-A_{i}=0$ for all $i$. At the other extreme, $U 1=1$ is the case of the worst possible forecasts. Ahlburg [1989] dropped the first term of the denominator in Equation (10.8).

The definition of the U2 statistic developed by Thei1 [1966] and presented by Makridakis and Wheelwright [1978] is different from Equation (10.8). Their definition of the $U 2$ statistic is as follows:

$$
U 2=\frac{\sqrt{\frac{1}{n-1} \sum_{i=1}^{n-1}\left(F P E_{i+1}-A P E_{i+1}\right)^{2}}}{\sqrt{\frac{1}{\frac{1}{n-1}_{i=1}^{n-1}\left(A_{i+1}\right)^{2}}}}
$$

where,

$$
\begin{aligned}
& F P E_{i+1}=\frac{F_{i+1}-A_{1}}{A_{i}} \text {, the predictive relative change), and } \\
& \mathrm{APE}_{i+1}=\frac{A_{i+1}-A_{i}}{A_{i}} \text { the actual relative change. }
\end{aligned}
$$

The Equation (10.9) can be simplified to the form shown in Equation (10.10), by substituting the values of $F P E_{i+1}$ and $A P E_{i+1}$, as

$$
\mathrm{U} 2=\frac{\sqrt{\sum_{i=1}^{n-1}\left[\frac{F_{i+1}-A_{i+1}}{A_{i}}\right]^{2}}}{\sqrt{\sum_{i=1}^{n-1}\left[\frac{F_{i+1}-A_{i+1}}{A_{i}}\right]^{2}}}
$$

A major difference between Equation (10.10) and the U2 statistic [Theil 1966] is that Theil mentioned predicted and observed changes, 
while Equation (10.10) [Makridakis \& Wheelwright 1978] shows relative changes. The definition of U2 presented by Armstrong [1978] is same as that of Theil [1966]. There are also differences between the Equations $(10.8)$ and $(10.10)$. In Equation (10.8), the denominator represents the sum of the squares of predicted and actual values over the number of forecasts $n$. In Equation (10.10), the denominator represents sum of the squares of the relative change in actual values. Comparing the numerator of Equation (10.10) with Equation (10.8) shows that the numerator of Equation (10.8) is the sum of change of predicted and actual values (after, $1 / n$ is being factored out from numerator and denominator) and that of Equation (10.10) is the sum of relative changes.

Ahlburg's definition of the second Theil inequality statistic, U2', is completely different from Equation (10.9) or (10.10). The U2statistic has been defined as

$$
\mathrm{U}^{-}=\left[\frac{\sum_{i=1}^{n}\left(F_{i}^{-}-A_{i}^{-}\right)^{2}}{\sum_{i=1}^{n}\left(\bar{A}^{-}-A_{i}^{-}\right)^{2}}\right]^{1 / 2}
$$

where, $\bar{A}^{-}$is the sample mean of the ${A^{-}}_{i}^{-} s$ and

$$
\begin{aligned}
& F_{1}^{-}=\ln \left(F_{i}\right)-\ln \left(F_{i-1}\right) \\
& A_{i}^{-}=\ln \left(A_{1}\right)-\ln \left(A_{i-1}\right)
\end{aligned}
$$


$\mathrm{U} 2$ seems reasonable and is easier to interpret because the $\mathrm{U} 2=$ 1.0 benchmark is based on a no-change model. However, U2 does not pick up errors in forecasting levels, but measures only errors in changes. Despite the advantages of $\mathrm{U} 2$ over $\mathrm{Ul}, \mathrm{U} 1$ has been more widely advocated. The confusion of $U 1$ and $U 2$ implies that these measures are not well understood. Armstrong [1978] drew the conclusion that there is little need for Theil's U, when other measures can do the job and do it more simply.

Coefficient of Variation (CV)

The coefficient of variation (CV) is calculated from

$$
\mathrm{CV}=\frac{\mathrm{RMSE}}{\sum_{i=1}^{n} \mathrm{~A}_{i} / \mathrm{n}}
$$

The definition of RMSE (Root Mean Square Error) appeared in Equation (10.5). The $\mathrm{CV}$ relates the RMSE to the average value of the actual data. This unitless measure is similar to the MAPE, but the MAPE is easier to interpret.

Coefficient of determination $\left(R^{2}\right)$

The coefficient of determination $\left(R^{2}\right)$ is calculated from 


$$
R^{2}=\frac{\sum_{i=1}^{n}\left[\left(F_{i}-\bar{F}\right)\left(A_{i}-\bar{A}\right)\right]^{2}}{\left[\sum_{i=1}^{n}\left(F_{i}-\bar{F}\right)^{2}\right]\left[\sum_{i=1}^{n}\left(A_{i}-\bar{A}\right)^{2}\right]}
$$

where $\bar{A}$ is the average of $A_{i}$ and $\bar{F}$ is the average of $F_{i} \cdot R^{2}$ is the most popular measure used by researchers in forecasting problems. $R^{2}$ is interpreted as the "proportion of variance" explained. An $\mathrm{R}^{2}$ of zero means that the model is not useful in explaining fluctuations, but it does not reflect its ability to forecast levels; thus, $R^{2}=0$ may not be completely bad forecast. Similarly, an $\mathrm{R}^{2}$ of 1.0 does not represent a perfectly good forecast.

Sometimes, $R_{a}^{2}$ (the adjusted $R^{2}$ ) is used instead of $R^{2} \cdot R_{a}^{2}$ compensates for the loss in degrees of freedom in the model. $R_{a}^{2}$ can be negative [Saha 1986]. For more than one variable, $R_{a}^{2}$ is preferable to $\mathrm{R}^{2}$. $\mathrm{R}^{2}$ has meaning primarily when comparing different models in a given situation. Because of its popularity, the simple correlation coefficient ( $r$ ) - represented as the square root of $R^{2}$ in Equation (10.13) - is used in this study.

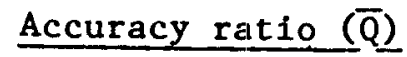

The accuracy ratio $(\bar{Q})$ is calculated from

$$
\bar{Q}=\frac{\sum_{i=1}^{n} Q_{i}}{n}
$$


where

$$
Q_{i}=\left[\begin{array}{ll}
A_{i} / F_{1} & \text { if } A_{i}>F_{i} \\
F_{i} / A_{i} & \text { if } F_{i}>A_{i}
\end{array}\right.
$$

$\bar{Q}$ is similar to the MAPE. One key difference is that it is not bounded on both sides; it can range from 1 to infinity. Therefore, the accuracy ratio is preferable to the $\overline{\mathrm{MAPE}}$ when dealing with large errors. Because of $t$ is simple, easy-to-understand, and free of units, this study has used $\bar{Q}$ as a measure of accuracy.

\subsection{Evaluation and Combination}

The elasticity models developed in this study (see Tables 6.266.28 ), by their nature, use growth of areawide variables to determine the traffic growth. Because of this, they do not account for localized impacts, such as land use changes, that affect traffic. The various computer-based travel demand forecasting procedures (Section 2.3) also depend on forecasts of socioeconomic varfables to forecast traffic. Therefore, no matter which traffic forecasting method is used, results should be interpreted cautiously, and should possibly consider the use of several methods to determine the range of a reasonable forecast.

Although there are other measures of accuracy than those presented in Section 10.2, one can argue that the 11st of measures presented in the previous section should be enough for most forecast- 
ing problems. The typical procedure is to compare the various forecasting models using each of the selected measures [Armstrong 1978]. The selection of a measure of accuracy depends to a great extent on the situation. The MAPE provides a good measure for most situations. $\mathrm{R}^{2}$ is a popular measure, but with all its problems, using it alone to evaluate a forecasting method may be misleading. It appears, from the discussion in Section 10.2, that many measures could be redundant to each other. In the absence of any universally accepted criteria, this study has considered the following list of measures to evaluate and compare the traffic models developed in this study.

1. Mean Error (ME)

2. Mean Percentage Error (MPE)

3. Mean Absolute Error (MAE)

4. Mean Absolute Percentage Error (MAPE)

5. Correlation Coefficient ( $r$ )

6. Root Mean Square Percentage Error (RMSPE)

7. Accuracy Ratio $(\bar{Q})$

The results of these forecasting measures for Interstate, Urban Principal Arterial and Urban Minor Arterial \& Collector are presented in Tables 10.1, 10.2 and 10.3, respectively. The Principal Component Analysis (PCA) based models presented in Chapter 7 have not been evaluated or compared in this chapter because of their complexity. The large number of original variables in PCA based models has not improved the model statistics in PCA based regression models significantly as compared to the model statistics in elasticity models (see 
Table 10.1: Forecast Measures from Regression, E1asticity and Lag-AADT Models of Interstate Highways

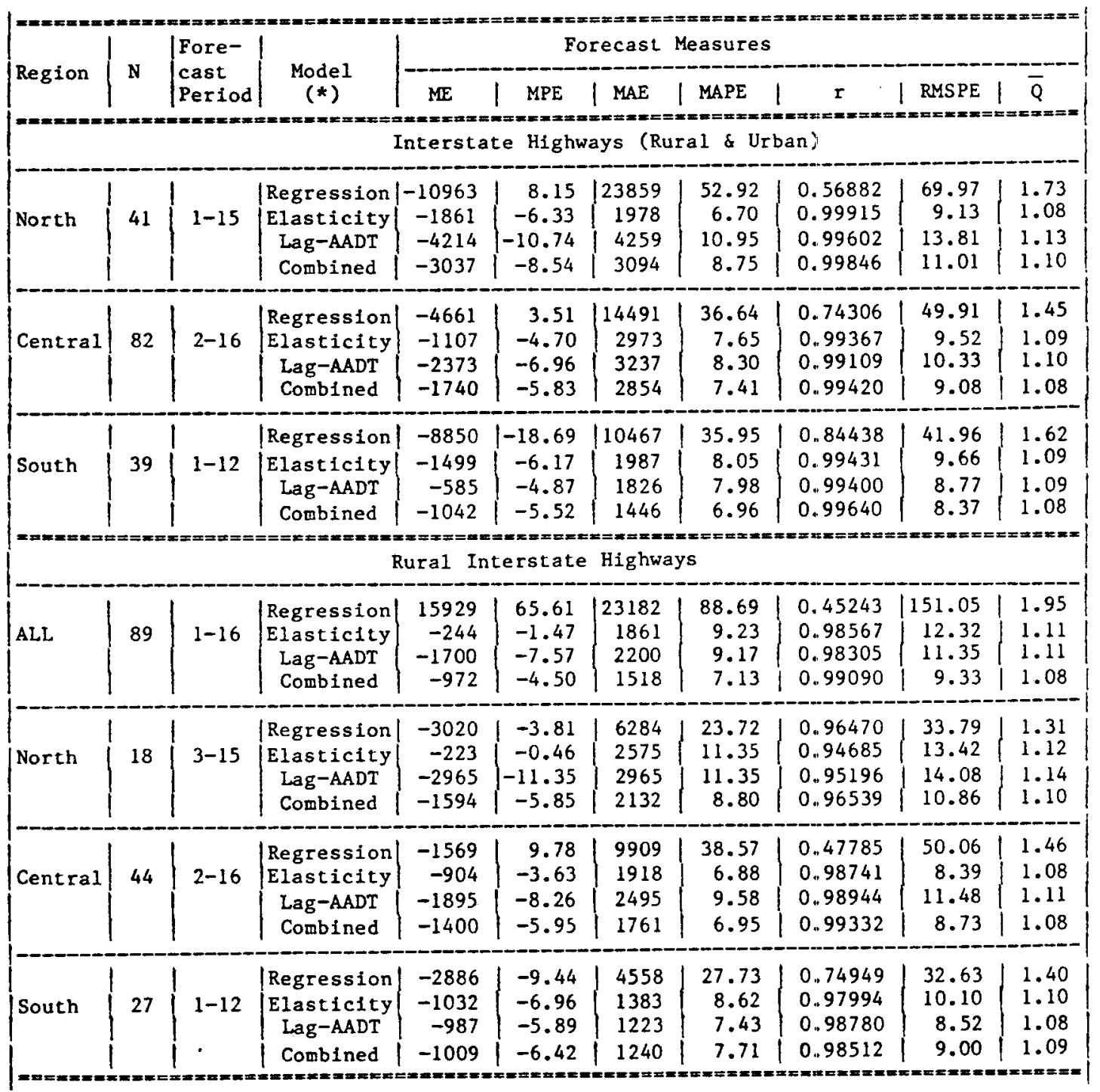


Table 10.1, continued

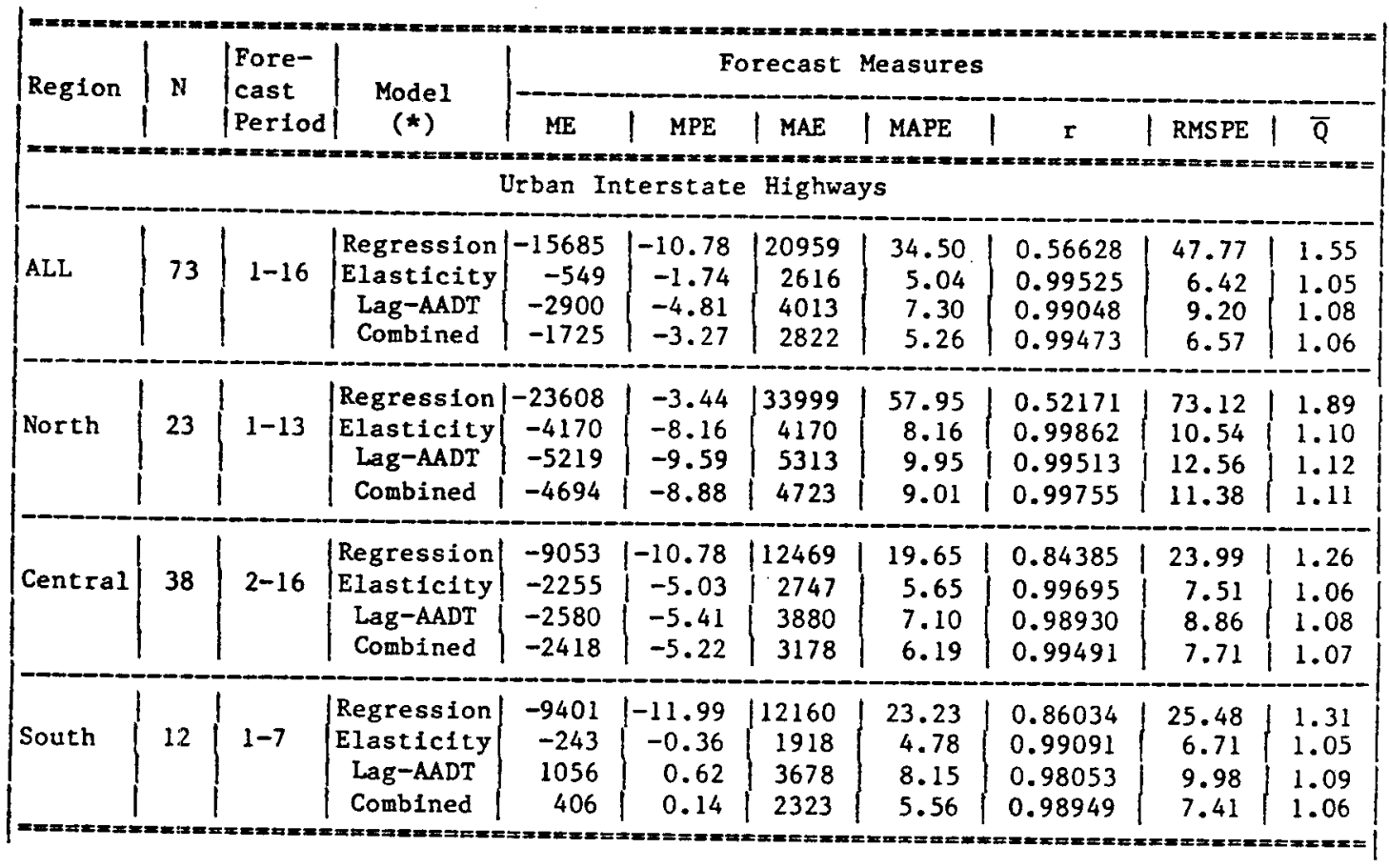

(*) i. See Tables $6.23,6.26$ and 8.4 for Elasticity, Regression and Lag--AADT models, respectively.

ii. Combined model forecasts are equal to average forecasts of Elasticity and Lag-AADT models. 
Table 10.2: Forecast Measures from Regression, Elasticity and Lag-AADT Models of Urban Principal Arterial

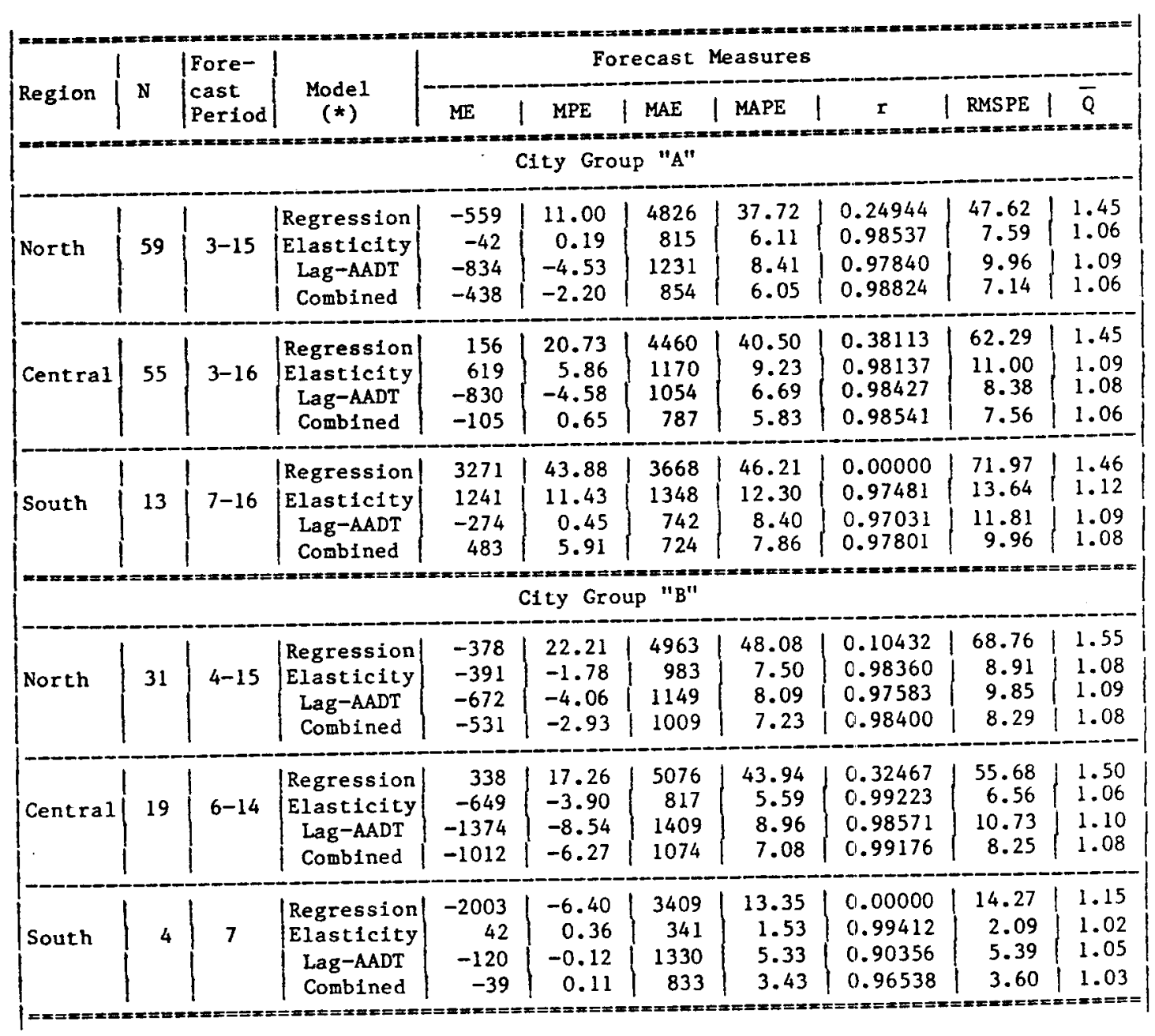


Table 10.2, continued

\begin{tabular}{|c|c|c|c|c|c|c|c|c|c|c|}
\hline \multirow{2}{*}{ Region } & \multirow{2}{*}{$\mathbf{N}$} & \multirow{2}{*}{$\left|\begin{array}{l}\text { Fore- } \\
\text { cast } \\
\text { Period }\end{array}\right|$} & \multirow{2}{*}{$\begin{array}{l}\text { Model } \\
(*)\end{array}$} & \multicolumn{7}{|c|}{ Forecast Measures } \\
\hline & & & & ME & | MPE & MAE & MAPE & $\mathbf{r}$ & RMSPE & $\bar{Q}$ \\
\hline & & & & & 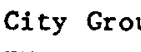 & 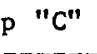 & & & & \\
\hline North & 51 & $3-17$ & $\left|\begin{array}{c}\text { Regression } \\
\text { Elasticity } \\
\text { Lag-AADI } \\
\text { Combined }\end{array}\right|$ & $\begin{array}{r}86 \\
310 \\
-645 \\
-168 \\
\end{array}$ & $\begin{array}{r}12.83 \\
2.10 \\
-4.80 \\
-1.32\end{array}$ & $\begin{array}{r}2624 \\
963 \\
1086 \\
800 \\
\end{array}$ & $\begin{array}{r}27.80 \\
7.90 \\
9.27 \\
6.74 \\
\end{array}$ & $\begin{array}{l}0.62233 \\
0.97384 \\
0.95862 \\
0.97155\end{array}$ & $\begin{array}{r}45.52 \\
8.99 \\
11.10 \\
8.33 \\
\end{array}$ & $\begin{array}{l}1.30 \\
1.08 \\
1.10 \\
1.07\end{array}$ \\
\hline Central & 61 & $3-14$ & $\left|\begin{array}{c}\text { Regression } \\
\text { Elasticity } \\
\text { Lag-AADT } \\
\text { Combined }\end{array}\right|$ & $\begin{array}{r}-123 \\
50 \\
-1145 \\
-547\end{array}$ & $\begin{array}{r}14.04 \\
0.10 \\
-7.63 \\
-3.73\end{array}$ & $\begin{array}{r}3582 \\
1053 \\
1342 \\
939\end{array}$ & $\begin{array}{r}33.97 \\
8.41 \\
9.43 \\
7.11\end{array}$ & $\begin{array}{l}0.67284 \\
0.98054 \\
0.97315 \\
0.98205\end{array}$ & $\begin{array}{r}47.46 \\
9.89 \\
11.54 \\
9.06\end{array}$ & $\begin{array}{l}1.38 \\
1.09 \\
1.11 \\
1.08\end{array}$ \\
\hline South & 8 & $3-17$ & $\left|\begin{array}{c}\text { Regression } \\
\text { Elasticity } \\
\text { Lag-AADT } \\
\text { Combined }\end{array}\right|$ & $\begin{array}{r}-3624 \\
-220 \\
-220 \\
-220\end{array}$ & $\left\{\begin{array}{l}-12.81 \\
-0.08 \\
-0.55 \\
-0.31\end{array}\right.$ & $\begin{array}{r}5009 \\
870 \\
1021 \\
848\end{array}$ & $\begin{array}{r}34.68 \\
6.68 \\
6.67 \\
5.53\end{array}$ & $\begin{array}{l}0.00000 \\
0.98437 \\
0.96953 \\
0.97914\end{array}$ & $\begin{array}{r}40.32 \\
8.15 \\
8.08 \\
7.38\end{array}$ & $\begin{array}{l}1.60 \\
1.07 \\
1.07 \\
1.06\end{array}$ \\
\hline & & & & & City Gr & "D" & & & & \\
\hline North & 20 & $4-14$ & $\left|\begin{array}{c}\text { Regression } \\
\text { Elasticity } \\
\text { Lag-AADT } \\
\text { Combined }\end{array}\right|$ & $\begin{array}{r}-1044 \\
-1455 \\
-679 \\
-1067\end{array}$ & $\mid \begin{array}{r}5.65 \\
-13.98 \\
-0.21 \\
-7.10\end{array}$ & $\begin{array}{l}3209 \\
1455 \\
1861 \\
1472\end{array}$ & $\begin{array}{l}37.74 \\
13.98 \\
20.97 \\
14.33\end{array}$ & $\begin{array}{l}0.31932 \\
0.95211 \\
0.76915 \\
0.92081\end{array}$ & $\begin{array}{l}54.61 \\
16.91 \\
34.31 \\
17.99\end{array}$ & $\begin{array}{l}1.44 \\
1.18 \\
1.23 \\
1.17\end{array}$ \\
\hline Central & 20 & $4-16$ & $\left|\begin{array}{c}\text { Regression } \\
\text { Elasticity } \\
\text { Lag-AADT } \\
\text { Combined }\end{array}\right|$ & $\begin{array}{r}742 \\
191 \\
-373 \\
-91\end{array}$ & $\begin{array}{r}19.36 \\
2.80 \\
-2.96 \\
-0.08 \\
\end{array}$ & $\begin{array}{r}2216 \\
750 \\
665 \\
510\end{array}$ & $\begin{array}{r}32.92 \\
9.01 \\
7.03 \\
5.78 \\
\end{array}$ & $\begin{array}{l}0.12065 \\
0.93910 \\
0.95824 \\
0.95593\end{array}$ & $\begin{array}{r}51.71 \\
10.62 \\
8.37 \\
7.56\end{array}$ & $\begin{array}{l}1.35 \\
1.09 \\
1.08 \\
1.06\end{array}$ \\
\hline South & 3,8 & $4-12$ & $\left|\begin{array}{c}\text { Regression } \\
\text { Elasticity } \\
\text { Lag-AADT } \\
\text { Combined }\end{array}\right|$ & $\begin{array}{r}-292 \\
-623 \\
-1421 \\
-1022\end{array}$ & $\begin{array}{r}8.94 \\
-4.86 \\
-11.29 \\
-8.07\end{array}$ & $\begin{array}{r}3138 \\
932 \\
1421 \\
1091\end{array}$ & $\begin{array}{r}30.05 \\
8.13 \\
11.29 \\
8.80\end{array}$ & $\mid \begin{array}{r}-0.01143 \\
0.97483 \\
0.96646 \\
0.97719\end{array}$ & $\begin{array}{r}38.59 \\
9.60 \\
13.57 \\
10.57\end{array}$ & $\begin{array}{l}1.35 \\
1.09 \\
1.14 \\
1.10\end{array}$ \\
\hline
\end{tabular}

(*) i. See Tables $6.24,6.27$ and 8.5 for Elasticity, Regression and Lag-AADT models, respectively.

1i. Combined model forecasts are equal to average forecasts of Elasticity and Lag-AADT models. 
Table 10.3: Forecast Measures from Regression, Elasticity and Lag-AADT Models of Urban Minor Arterial \& Collector

\begin{tabular}{|c|c|c|c|c|c|c|c|c|c|c|}
\hline \multirow{2}{*}{$\begin{array}{l}\text { City } \\
\text { Group }\end{array}$} & \multirow{2}{*}{$\mathbf{N}$} & \multirow{2}{*}{$\begin{array}{l}\text { Fore- } \\
\text { cast } \\
\text { Period }\end{array}$} & \multirow{2}{*}{$\begin{array}{c}\text { Model } \\
(*)\end{array}$} & \multicolumn{7}{|c|}{ Forecast Measures } \\
\hline & & & & ME & MPE & M & TP & 1 & RMSPE & $\bar{Q}$ \\
\hline A & 12 & $3-16$ & $\left|\begin{array}{c}\text { Regression } \\
\text { Elasticity } \\
\text { Lag-AADT } \\
\text { Combined }\end{array}\right|$ & $\begin{array}{r}-468 \\
2092 \\
125 \\
1109\end{array}$ & $\begin{array}{r}6.92 \\
15.00 \\
1.49 \\
8.25\end{array}$ & $\begin{array}{l}3203 \\
2675 \\
1286 \\
1458\end{array}$ & $\begin{array}{l}29.12 \\
20.20 \\
11.47 \\
11.15\end{array}$ & $\begin{array}{l}0.37517 \\
0.93524 \\
0.93675 \\
0.96852\end{array}$ & $\begin{array}{l}39.70 \\
24.81 \\
14.46 \\
14.01\end{array}$ & $\begin{array}{l}1.34 \\
1.20 \\
1.12 \\
1.11\end{array}$ \\
\hline B & 14 & $3-14$ & $\left|\begin{array}{c}\text { Regression } \\
\text { Elasticity } \\
\text { Lag-AADT } \\
\text { Combined }\end{array}\right|$ & $\begin{array}{r}823 \\
180 \\
-564 \\
-192\end{array}$ & $\begin{array}{r}85.72 \\
15.79 \\
-5.25 \\
5.27\end{array}$ & $\begin{array}{r}3508 \\
1315 \\
632 \\
886\end{array}$ & $\mid \begin{array}{r}109.01 \\
25.66 \\
7.44 \\
14.91\end{array}$ & $\begin{array}{l}0.56514 \\
0.93452 \\
0.97563 \\
0.96471\end{array}$ & $\begin{array}{r}170.24 \\
33.85 \\
11.14 \\
18.95\end{array}$ & $\begin{array}{l}2.14 \\
1.27 \\
1.09 \\
1.16\end{array}$ \\
\hline C & 18 & $3-17$ & $\left|\begin{array}{c}\text { Regression } \\
\text { Elasticity } \\
\text { Lag-AADT } \\
\text { Combined }\end{array}\right|$ & $\begin{array}{r}-1121 \\
-310 \\
-282 \\
-296\end{array}$ & $\begin{array}{r}7.17 \\
-1.87 \\
-1.53 \\
-1.70\end{array}$ & $\begin{array}{r}3107 \\
755 \\
759 \\
754\end{array}$ & $\begin{array}{r}40.85 \\
9.44 \\
9.35 \\
9.34\end{array}$ & $\begin{array}{l}0.28198 \\
0.96558 \\
0.96519 \\
0.96556\end{array}$ & $\begin{array}{l}58.44 \\
15.40 \\
15.23 \\
15.30\end{array}$ & $\begin{array}{l}1.51 \\
1.10 \\
1.10 \\
1.10\end{array}$ \\
\hline D & 33 & $3-16$ & $\mid \begin{array}{c}\text { Regression } \\
\text { Elasticity } \\
\text { Lag-AADT } \\
\text { Combined }\end{array}$ & $\begin{array}{r}-4167 \\
830 \\
-536 \\
147\end{array}$ & $\mid \begin{array}{r}-16.11 \\
9.58 \\
-5.58 \\
1.92\end{array}$ & $\begin{array}{r}5283 \\
1013 \\
714 \\
545\end{array}$ & $\begin{array}{r}65.20 \\
12.21 \\
8.77 \\
6.99\end{array}$ & $\begin{array}{l}0.00932 \\
0.98426 \\
0.98688 \\
0.98949\end{array}$ & $\begin{array}{r}75.73 \\
15.22 \\
10.84 \\
9.04\end{array}$ & $\begin{array}{l}2.66 \\
1.13 \\
1.10 \\
1.07\end{array}$ \\
\hline
\end{tabular}

(*) 1. See Tables $6.25,6.28$ and 8.6 for Elasticity, Regression and Lag-AADT models, respectively.

ii. Combined model forecasts are equal to average forecasts of Elasticity and Lag-AADT models. 
Chapter 6). The second and third columns of Tables 10.1-10.3 show the number off forecasts and the range of the forecast period - the time difference between the year of forecast and the base period (if any). Base period information (as required) has been used by all models except the regression model. For elasticity and lag-AADT models, each forecast has been made for a roadway segment at its last year of traffic data with an earlier year AADT for that segment as its base year AADT. Also, the base and forecast years (and hence the forecast period) for a forecast are kept same for all the models. Different forecast periods have resulted for the following reasons:

a. The traffic volume data are not available at an equal spacing over time.

b. Different forecast periods allow testing the model performance over a short term (say, < 5 years), medium term (say, 5-15 years), and long term (say, $\geq 15$ years).

With enough forecasts for each case, a separate analysis at different forecast ranges could be done to see the model's performance over the short, medium and long term separately. However, for comparing different forecasting models, using a wide range of forecast periods is not expected to change the conclusions, as long as the same base and future years are used among the competing models.

The forecast measures MAE, MAPE, RMSPE and $\bar{Q}$ have been primarily used in this study for model evaluation and comparison. In addition, the error function of each of these measures is different. By comparing all these measures in Tables 10.1 to 10.3 , one can see that the 
regression model does not provide good forecasts. The elasticity model (the variant of the regression model) and the lag-AADT model are clearly superior to the regression model because of the significant Improvement seen in

$$
\begin{aligned}
& \text { 1. low values of MAE, MAPE and RMSPE, } \\
& \text { 2. high values of } r \text {, and } \\
& \text { 3. accuracy ratio }(\bar{Q}) \text { of close to unity. }
\end{aligned}
$$

The ranges of forecast measures for models in the three major highway categories are shown in Table 10.4. This summary table also shows the clear margin of improvement in the elasticity and the lag-AADT models over the regression model. The main reason for this improvement is that the elasticity and lag-AADT models recognize the impacts of nonresident travel in the form of base year and lag year AADT. Based on performance, this study does not recommend the use of regression models to forecast traffic.

The results of $M E, M P E$ and $r$ show that they are either ineffective or redundant to other measures. The use of ME and MPE can be misleading. For example, the following results show that, although the regression models are as good as the other models based on ME or MPE, the other measures (MAE, MAPE, RMSPE and $\bar{Q}$ ) show that the regression models are much inferior to the other models.

1. MPE for the north and central regions of Interstate Highways, and for the north region of both rural and urban interstate highways. (See Table 10.1)

1i. ME for the central region of city groups " $A$ " and " $B$ ", the north 
Table 10.4: Ranges of Forecast Measures of Models for Major
Highway Categories

\begin{tabular}{|c|c|c|c|c|c|c|c|c|c|}
\hline \multirow{2}{*}{$\begin{array}{l}\text { Highway } \\
\text { Category } \\
== \pm==x= \pm= \pm\end{array}$} & \multirow{2}{*}{ Models } & \multicolumn{2}{|c|}{ MAE } & \multicolumn{2}{|c|}{ MAPE } & \multicolumn{2}{|c|}{ RMSPE } & \multicolumn{2}{|l|}{$\bar{Q}$} \\
\hline & & $\begin{array}{c}\text { Lower } \\
=x=x=x\end{array}$ & $\begin{array}{c}\text { Upper } \\
\text { U=x=mm=m }\end{array}$ & $\begin{array}{c}\text { Lower } \\
=\approx= \pm m=m\end{array}$ & $\begin{array}{c}\text { Upper } \\
=x=x==x=x\end{array}$ & Lower & 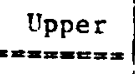 & 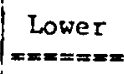 & Upper \\
\hline $\begin{array}{l}\text { Inter- } \\
\text { state } \\
\text { Highway: }\end{array}$ & $\begin{array}{r}\text { Regression } \\
\text { Elastlcity } \\
\text { Lag-AADT } \\
\text { Combined }\end{array}$ & $\begin{array}{l}4558 \\
1383 \\
1223 \\
1240\end{array}$ & $\begin{array}{r}33999 \\
4170 \\
5313 \\
4713\end{array}$ & $\begin{array}{r}19.65 \\
4.78 \\
7.10 \\
5.26\end{array}$ & $\begin{array}{r}88.69 \\
11.35 \\
11.35 \\
9.01\end{array}$ & $\begin{array}{r}23.99 \\
6.42 \\
8.52 \\
6.57\end{array}$ & $\begin{array}{r}151.05 \\
13.42 \\
14.08 \\
11.38\end{array}$ & $\begin{array}{l}1.26 \\
1.05 \\
1.08 \\
1.06\end{array}$ & $\begin{array}{l}1.95 \\
1.12 \\
1.14 \\
1.11\end{array}$ \\
\hline $\begin{array}{l}\text { Urban } \\
\text { Principal } \\
\text { Arterla]. }\end{array}$ & $\left\{\begin{array}{r}\text { Regression } \\
\text { Elasticity } \\
\text { Lag-AADT } \\
\text { Combined }\end{array}\right.$ & $\begin{array}{r}2216 \\
341 \\
665 \\
510\end{array}$ & $\begin{array}{l}5076 \\
1455 \\
1861 \\
1472\end{array}$ & $\begin{array}{r}13.35 \\
1.53 \\
5.33 \\
3.43\end{array}$ & $\begin{array}{l}48.08 \\
13.98 \\
20.97 \\
14.33\end{array}$ & $\begin{array}{r}14.27 \\
2.09 \\
5.39 \\
3.60\end{array}$ & $\begin{array}{l}71.97 \\
16.91 \\
34.31 \\
17.99\end{array}$ & $\begin{array}{l}1.15 \\
1.02 \\
1.05 \\
1.03\end{array}$ & $\begin{array}{l}1.60 \\
1.18 \\
1.23 \\
1.17\end{array}$ \\
\hline $\begin{array}{l}\text { Urban } \\
\text { Minor } \\
\text { Art.\& } \\
\text { Collector } \\
\text { r=m=x }==x=x\end{array}$ & $\mid \begin{array}{r}\text { Regression } \\
\text { Elasticity } \\
\text { Lag-AADT } \\
\text { Combined }\end{array}$ & $\begin{array}{r}3107 \\
755 \\
632 \\
545\end{array}$ & $\begin{array}{l}5283 \\
2675 \\
1286 \\
1458\end{array}$ & $\begin{array}{r}29.12 \\
9.44 \\
7.44 \\
6.99\end{array}$ & $\begin{array}{r}109.01 \\
25.66 \\
11.47 \\
14.91\end{array}$ & $\begin{array}{r}39.70 \\
15.21 \\
10.84 \\
9.04\end{array}$ & $\begin{array}{r}170.24 \\
33.85 \\
15.23 \\
18.95\end{array}$ & $\begin{array}{l}1.34 \\
1.10 \\
1.09 \\
1.07\end{array}$ & $\begin{array}{l}2.66 \\
1.27 \\
1.12 \\
1.16\end{array}$ \\
\hline
\end{tabular}

Note: Lower $=$ Lower value of the forecast measure, and

Upper = Upper value of the forecast measure. 
and central regions of city group " $\mathrm{C}$ ", and the south region of city group "D" of urban principal arterial. (See Table 10.2)

iii. MPE for north region of city group " $D$ " of urban principal arterial. (See Table 10.2)

iv. ME and MPE of city group " $\mathrm{A}$ " of urban minor arterial \& collector. (See Table 10.3)

Although the correlation coefficient $(r)$ is redundant to other measures (MAE, MAPE, RMSPE and $\bar{Q}$ ), the values of $r$ show a very high degree of correlation between the predicted and observed AADT for both elasticity and lag-AADT models. The inferiority of regression models is also reflected in the low values of $r$ for most of the cases of Tables 10.1 to 10.3 .

By comparing the values of MAE, MAPE, RMSPE, and $\bar{Q}$ from Tables 10.1-10.3 and their ranges in Table 10.4, one can see that both elasticity and lag-AADT models have forecasted traffic equally well. Recall that the lag-AADT model was built by using the traffic data only, while the elasticity model was built based on regression analysis using economic and demographic variables and AADT in the model formulation. The MAE measures in Tables 10.1-10.3 show the expected value of error in absolute terms. Table 10.4 has shown the ranges of these values. Although some high values of MAE are noted for Interstate highways, the percentage error in absolute values (MAPE) are in most of those cases less than 10 percent. On the other hand, despite the high value of MAPE for some cases of noninterstate highways, a low value of MAE has resulted from forecasts. The RMSPE, 
which uses square of the error in the cost function, shows that the values of RMSPE are usually below 10 percent, but some high values are noticed in noninterstate cases with low traffic volumes. The accuracy ratio $(\bar{Q})$ in Tables $10.1-10.4$ shows that expected values of prediction are usually within 10 percent of the observed AADT values.

"One can never lose by combining the forecasts". This statement has first mentioned in Section 9.2.4. Based on the comparable performance of elasticity and lag-AADT models, this study has combined the forecasts from these constituent models by applying equal weights to each. The following cases show an improvement in forecast measures (MAE, MAPE, RMSPE and $\bar{Q}$ ) for combined forecasts versus any of their constituent models' forecasts:

A. Interstate Highways (See Table 10.1):

- Central and south regional model of Interstate highways.

- North and statewide (the "ALL" case) of rural Interstate highways.

B. Noninterstate Highways (See Tables $10.2 \& 10.3$ ):

- Central and south regional model for city group " $A$ " of urban principal arterial.

- North, central and south regional model for city group "C" of urban principal arterial.

- Central region of city group "D" of urban principal arterial. 
- City group "D" model of urban minor arterial \& collector.

The above list indicates a clear margin of improvement in all four forecast measures for 11 out of 27 models in the three highway categories. Moreover, an improvement in some of the forecast measures was noticed in the following cases:

- $M A E$ and $\bar{Q}$ for the central region and MAE of the south region of rural interstate (See Table 10.1).

- MAPE, RMSPE and $\bar{Q}$ for the north region of city groups " $A$ " and " $B$ " of urban principal arterial (See Table 10.2).

For the rest of the cases, the performance measures of combined forecasts are between the performance measures of the constituent models. In other words, the combined forecasts can never be worse than the worst forecast of the constituent models. In fact the combined forecast often better than the forecast of any of the constituent models. Based on the study results in this section, the combined forecasts that resulted from a simple average of the elasticity and lag-AADT model forecasts are highly recommended to be used to forecast traffic on state highways.

\subsection{Calibration and Tracking Signal}

In most forecasting systems, it is important to monitor the forecast errors to ensure that the system remains valid. The accuracy of a forecasting method is determined by analyzing forecasting errors experienced. A combination of forecast models may have been used to generate forecasts. The accuracy of the forecasts is measured at 
every period, when the actual data are available, by a tracking signal test. For unbiased forecasts, the tracking signal will lie within certain limits with a preset probability; otherwise (for biased forecasts), the signal will fall outside the limits. If the forecasting system yields unbiased estimates, the tracking signal should be within the preset limit. When the tracking signal deviates from the limit by more than a prescribed amount, an investigation is made to determine if the forecasting model should be modified by recalibrating the mode1(s).

The first tracking signal used in forecasting was the simple cumulative sum (cumsum) of the errors [Thomopoulos 1980]. The simple cumsum is defined by the ratio of the sum of errors at the end of each period to the mean absolute deviation (MAD) of the errors. The ratio should fluctuate around zero if the errors are unbiased. Users should take note if the ratio exceeds a control limit. Control limits for tracking signal test can be set according to the probability of getting a false report, defined as a Type I error - a case where the control limit is exceeded due to chance.

This study has used the confidence interval of four forecast measures (MAE, MAPE, RMSPE, and $\bar{Q}$ ) from Section 10.4 to Install a tracking signal test and thus determine the need for changes in the forecasting model(s) and/or parameter(s). The description follows. The standard deviation of the errors, measured from the performance analysis of the individual forecast methods and the combined forecast method has been used to determine the 90- and 95-percent probability limits of the errors. The use of 90-percent probability limits 
accepts a risk of error of 10 percent (i.e., 10 out of 100 forecast results may lie outside the boundary set by a 90-percent limit). Confidence limits of 90-percent and 95-percent have been proposed because different users may be willing to accept different risks of error. Even the same user can take different risks for different cases, for example, a lower level of risk for a higher level of highway. Also, the risk taking behavior may change with time for an individual. However, the 5 percent and 10 percent risks of error (or 95-percent and 90-percent) are believed to be the most common level of risk that a user will choose, and are commonly used in statistical control. However, if the errors of the combined forecasts were found exceed the 90- or 95-percent 1imit, errors of the individual forecasts will be checked to determine whether the component models need to be modified.

Appendix Tables G1-G6 present the 90-percent and 95-percent confidence limits of the four forecast measures (MAE, MAPE, RMSPE and $\bar{Q}$ ) for the combined and constituent models of all groups of highways. The following list shows the description of all those tables of confidence limits for the forecast measures.

1. Table Gl: 90-percent confidence 1imits for models of Interstate Highways.

2. Table G2: 95-percent confidence 1imits for models of Interstate Highways.

3. Table G3: 90-percent confidence limits for models of Urban Principal Arterial.

4. Table G4: 95-percent confidence limits for models of Urban Principal Arterial.

5. Table G5: 90-percent confidence limits for models of Urban Minor Arterial \& Collector.

6. Table G6: 95-percent confidence limits for models of Urban Minor Arterial \& Collector. 
The minimum possible value of $\bar{Q}$ is 1.0 . For MAE, MAPE and RMSPE, it is zero. The lower limits (L90 or L95) in Tables Gl-G6 are not controlling factor, but the upper limits (U90 or U95) will be used as control points for the monitoring procedure or tracking signal. It may possible that one or two of the forecast measures may exceed the control limit for a forecast made from a model, while the others are not. In that case, the user or modeler needs to apply his (her) judgment to decide whether that particular forecast will be counted to see if the the risk level has exceeded. But, if 3 out of 4 forecast measures exceed the control limit, then it is probably necessary to change or to calibrate the involved model, if the user risk level is also exceeded. For example, with a 10 percent level of risk, if at least three forecast measures exceed their control limits in more than 10 percent of the forecasts, then it has to taken as a signal to change or calibrate the models. This test should be applied primarily to the combined forecasts. When a necessity to calibrate or to change the combined forecast appears, the proposed tracking signal test will be applied to the errors of the component models to see whether one or both component models need to be fixed.

\subsection{Chapter Summary}

The performances of all the traffic forecasting models developed in earlier chapters, except the PCR models, have been tested in this chapter. Because no criteria are universally accepted, several performance measuring criteria have been checked. Comparison of these criteria among the models has concluded that the forecasts from the 
regression model are consistently inferior to the forecasts of the elasticity model (the variant of regression model) and the lag-AADT mode1. On the other hand, both elasticity and the lag-AADT models have showed almost equal performance. Combined forecasts - the simple average of the elasticity and lag-AADT model forecasts - usually have demonstrated better performance than any of the constituent models. Thus, combined forecasts are highly recommended to forecast traffic on the state highway system. Finally, a procedure is suggested to monitor the forecasts and to see if there is a need to change or to recalibrate the models or their parameters based on model performance measures and the user's (or modelers) willingness to accept a certain level of risk. 
CHAPTER 11

SUMMARY AND CONCLUSIONS

\subsection{Introduction}

The primary purpose of this research was to develop a procedure to forecast traffic volumes on urban segments of the state and interstate highway systems for the Indiana Department of Transportation. In this study, a detailed statistical analysis was conducted to develop alternative models to forecast traffic. The models and the procedures described in this study are intended to provide highway planners with a tool for simple, fast and inexpensive estimation of traffic projections. Emphasis was also placed on achieving reliable input information for the forecasts of any background factors (independent variables) used in the models. This study improves on previous efforts to build simple and rellable procedures to forecast traffic. Among the several models developed in this study, elasticity and lag-AADT models are finally selected because of their simplicity and better performance. Accurate forecasts of the input variables are ensured for the success of the elasticity models. In the absence of any universally-accepted forecasting measuring criteria, this study has employed several measures to evaluate the forecasting models. 
often, the combined forecasts developed in this study were found to perform better than the forecasts from any of the constituent models. This chapter summarizes the study's analysis and conclusions. Some problems and limitations of the models -- and suggestions to overcome them - are discussed. This chapter also presents guidelines on how to implement the models and makes recommendations for further studies.

\subsection{Summary of Findings}

The analyses conducted in this study were performed with traffic data gathered from the paper files of INDOT. Other input data were obtained from a variety of sources, for example, the libraries at Purdue, Census Publications, IU Business Center, and W\&P Economics. The nationwide survey on "Forecasting Traffic on State Highways" (ITE Technical Council Committee 6F-41, informational report) showed the lack of any universally accepted method to address the issue of traffic forecasts in small- to medium-sized urban areas. This study has developed an efficient, inexpensive and easy-to-use method to forecast the traffic on state highways. The greatest strength of this study is the application and description of different statistics, ranging from simple to sophisticated. This report contains a full description of the entire model building process. The major findings of the research are summarized in the following sections. 


\subsubsection{Data Evaluation and Candidate Elasticity Model}

The variables and the data bases used in this study have been described in Chapter 4. Table 4.7 contains a summary of the quality (cost, avallability and reliability) of the variables. The details of the analyses and their results for the evaluation of data and for selection of the candidate regression/elasticity models are presented in Chapter 5. The framework of the analyses for Interstate and noninterstate highways are presented in Figures 5.1 and 5.2 , respectively. Several variable selection criteria $\left(R_{p}^{2}, R_{a}^{2}, C_{p}, P C, A I C\right)$ have been employed to select the most appropriate model. Despite the merits and demerits of using the $R^{2}\left(R_{p}^{2}\right)$ criterion, it has been adopted by the researchers as the single most useful criterion to gauge the "goodness" of a statistical model. However, analyses in this research were aided by other statistics. The data structure of this study includes the two data bases (segment and county/city average) and the four formats (raw, raw E\&L, density, and density E\&L). A sensitivity analysis of the "maximum" $R^{2}$ value among the competing models that are without any inconsistency in the sign of regression coefficients identifies the combination of county/city average data base and raw format as the best combination from all possible combination of the data structure. A significant improvement in model statistics was found in county/city average data bases over segment data bases. Because all the predictor variables used in this study are totals at either the city, county, or state level, it was not surprising to have traffic data on a county/city average basis perform better than traffic data on a more localized basis. 
A similar sensitivity analysis among the data groups revealed the following:

- For Interstate Highways: (1) For separate analysis of rural and urban interstate highways, both statewide and regional (north, central and south regions) models are found to be suitable.

A statewide model for the entire interstate network in Indiana was not satisfactory. (3) The improvement of the model statistics is better in case of analysis by geographical region than by the extent of roadway mileage and city group.

- For Urban Principal Arterial: (1) The combination of city group and geographic region performs better than any other possible combination. This second-level stratification by geographical region under each city group has been established as superior to grouping by city group alone. (2) Results improved when the data of city groups $A, B$ and $C$ in the south region were pooled. The alternate was having unstable results with too few observations in each city group.

- For Minor Arterial \& Collector: The second-level stratification by geographic region and extent of highway mileage resulted in poor results and groups with too few observations. However, the analysis at this level of stratification identified areas of weakness in the data. After pooling data by city group, the quality of model statistics improved.

The candidate models resulted from narrowing down a list of possible candidate models by comparing the model statistics $\left(R^{2}, R_{a}^{2}, P C, R M S E\right.$, 
AIC and $\mathrm{C}_{\mathrm{p}}$ ) and by applying judgment on the quality (availability, cost and reliability) of the data for the predictor variables in the model. The list of those candidate models are shown in Chapter 5.

\subsubsection{Elasticity Based Traffic Mode1}

The final regression equations and the elasticity models developed in this study were shown in Chapter 6. The influential analysis identified the individual outliers and the outlier groups and their effect on model statistics. The diagnostic statistics employed to do influential analysis were shown in Section 6.2.1. Modification of the data (if necessary) or deletion of outliers (if justified) was found to inprove the quality of model statistics significantly. For example, fit improved the statistics for the normality assumption of errors. Validity of the regression assumptions (1inearity, constant variance, and normality of errors) were checked through formal tests and graphical plots - residual and normal probability plots. Results of significance tests showed that the model parameters are highly significant. A significance of relation also resulted for all the models. The collinearity test found that no significant relationship existed between the variables of each model. The final regression equations of each group, shown in Tables 6.23-6.25, were produced by comparing the results of the above tests and some model statistics $\left(R^{2}, R_{a}^{2}\right.$, RMSE, and $C V$ ) of the candidate models, and by applying judgment regarding the quality of predictor variables. The $R^{2}$ values for the regression models of interstate highways, urban principal arterial, and urban minor arterial \& collector have ranges 50.35- 
88.86, 51.81-94.14 and 62.52-87.18 percent, respectively, with one or two variables in each equation. Each of these models is simple and does not contain more than two predictor variables. The elasticity models developed from the regression models are shown in Tables 6.266.28. These models are statistically sound and simple. They require the base year traffic data and base and future year data for the predictor variable(s).

\subsubsection{Principal Component Based Traffic Mode1}

The procedure and results for Principal Component Analysis (PCA) were shown in Chapter 7. The PCA was carried out for each group of filtered data which resulted from the influential analysis of Chapter 6. The PCA used 11 predictor variables, but had eliminated the problem of multicollinearity by computing the artficial variables (principal components) which are independent to each other. Some key results of the Principal Component Regression (PSR) model with PCs in the equation are shown in Tables 7.2-7.4. Appendix Tables D1-D3 show the relationship between the PCs entered in the equation and the original variables. The PCR equations for interstate highways, urban principal arterial and urban minor arterial \& collector are shown in Tables $7.5,7.6$ and 7.7 , respectively. These tables show a one-to-one comparison of $\mathrm{R}^{2}$ values between the PCR models and the regression or elasticity models from Chapter 6. The $\mathrm{R}^{2}$ values for the PCR models had ranges $61.46-90.26,52.87-97.57$ and $66.87-89.31$ percent for the three major highway categorles in this study. The $\mathrm{R}^{2}$ ranges for the regression/elasticity models are $50.35-88.86,51.81-94.14$ and $62.52-$ 
87.18 percent. This range comparison, plus one-to-one comparisons for each model shown in Tables $7.5-7.7$, do not show any significant improvement from the complex PCR model over the simple regression or elasticity model developed in Chapter 6. However, the complex PCR models, which need all variables to compute PCs in the model equation, certified the goodness of the model statistics for the regression or elasticity models that have fewer variables in their equations.

\subsubsection{Lagged Traffic (Lag-AADT) Mode1}

The information of traffic data itself and the rate at which traffic had changed were used to develop the inexpensive and simple lagged traffic mode1. The segment data base of Chapter 4 with a "raw" format (see Chapter 5) was used to build this model. Although Equation (8.2) had used "rate of AADT change", in its rearranged form, Equation (8.3) needs only one AADT value to forecast traffic on a highway segment. Because the data bases used to build lag-AADT models are different from those used in in Chapters 6 and 7, the model statistics of the lag models are not directly compared to the other models ${ }^{-}$. However, the traffic forecasting performance of the lag-AADT models were compared with the others in Chapter 10. The lag traffic models for interstate, urban principal arterial and urban minor arterial and collector were presented in Tables 8.1, 8.2 and 8.3, respectively. These tables show, as expected, a very $h i g h R^{2}$ values (about 99 percent). The parameters of the models and the regression relationships were found highly significant at very low $\alpha$ levels. The nonnormality of the error terms were because of the presence of 
outliers. The forecasting performance of these models was tested by skipping the last year of AADT values from each segment, reformulating the models, and comparing the resulting forecasts against the skipped values. Very high forecasting quality performance measures and model statistics were found for the models (shown in Appendix E) built with partial data.

\subsubsection{Time Series and Ratio Method Forecasts}

No forecasts of vehicle registrations and city population are available from outside sources. The structure and results of procedures developed to make these forecasts are shown in Chapter 9 and Appendix F. A ratio method of forecasting city population was described in Section 9.3.1. Its forecasts for the cities of Indiana were shown in Appendix F. Forecasts of vehicle registration were made through two time series analysis techniques - the Box-Jenkins ARIMA model and the autoregressive procedure. Parameters of the models were found to be significant at 5 percent level of significance. Both autoregressive and ARIMA procedures produce high quality forecasts in this study. Use of these two different types of models also increased confidence in the forecasts. A combined forecast of vehicle registrations was made by simple averaging of the forecasts from the BoxJenkins and the autoregressive procedures. The forecast measures of the combined forecasts and the forecasts from the constituent models for the two selected counties were shown in Table 9.7. The values of the performance criteria verified the reliability of the vehicle registration forecasts. 


\subsubsection{Traffic Mode1 Evaluation}

Level of accuracy is a primary concern for the users of traffic forecasts. Inaccurate forecasts can lead to under or over-designed facilities. To determine the tolerable errors in forecast, one needs to know how much error a traffic forecast can have without causing major design changes. However, boundary cases must be examined more carefully. In that case, it will be wise to develop forecasts from several methods to arrive at a "consensus range" of the traffic estimate.

Usually, the error in various travel forecasting methods can be estimated by testing the methods against the actual counts and comparing the results. An extensive study has determined that errors from mainframe traffic simulation are about 10 percent [Trombly \& Ouderkirk $1986]$.

This study used several forecasting measuring criteria (Section 10.2) to evaluate the performance of the traffic models. The results, shown in Tables 10.1-10.3, show that the mean absolute percentage errors, defined in Section 10.2, are usually less than 10 percent. Regression models developed in Chapter 6 were found to be ineffective in forecasting project site traffic. Despite the difference in model statistics and procedures, both elasticity and lag-AADT models were found to be highly efficient models for forecasting traffic. They were found to work equally well. The combined forecasts, formed by simple averaging of the forecasts from the elasticity and lag-AADT models, did better than efther the constituent model in many cases. 
Combined forecasts are recommended to forecast traffic on the state highway segments. Forecasts from several models provides the opportunity to assess the sensitivity of the forecasts to different model forms. Finally, a procedure, shown in Section 10.4, was developed to monitor the forecasts and to see if there will be any need in the future to recalibrate the models and their parameters.

\subsection{Implementation Guidelines}

One purpose of this research was to prepare guidelines for the use of the models developed in this study. The work elements undertaken to accomplish that purpose included review of the related 1iterature, preparation of the data bases, testing different data bases and their formats and stratification, analysis and development of different types of traffic models, forecasts of input variables for which no outside source is available, evaluation of different models, and development of a monitoring procedure to determine the need for model recalibration. Figures $11.1,11.2$ and 11.3 present the process for implementation of the models to forecast traffic on interstate highways, urban principal arterial, and urban minor arterial \& collector, respectively.

The steps that are recommended for the implementation of the elasticity and lag-AADT models to predict the future traffic for urban segment of the state highways and interstate highways of Indiana are listed below.

1. Find the exact location (i.e., city \& county) of the roadway for 


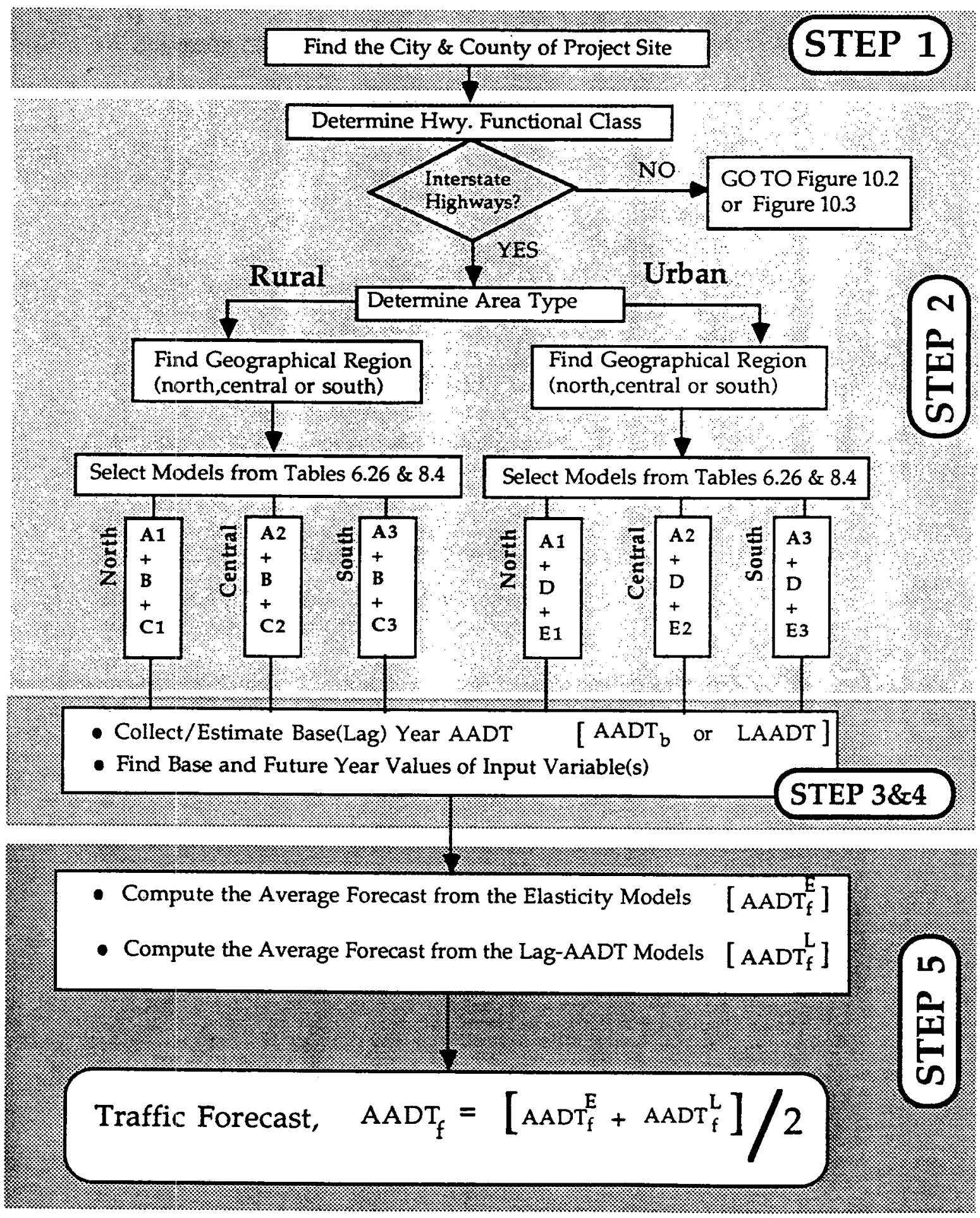

Figure 11.1: Process for Implementation of the Model on Interstate Highways 


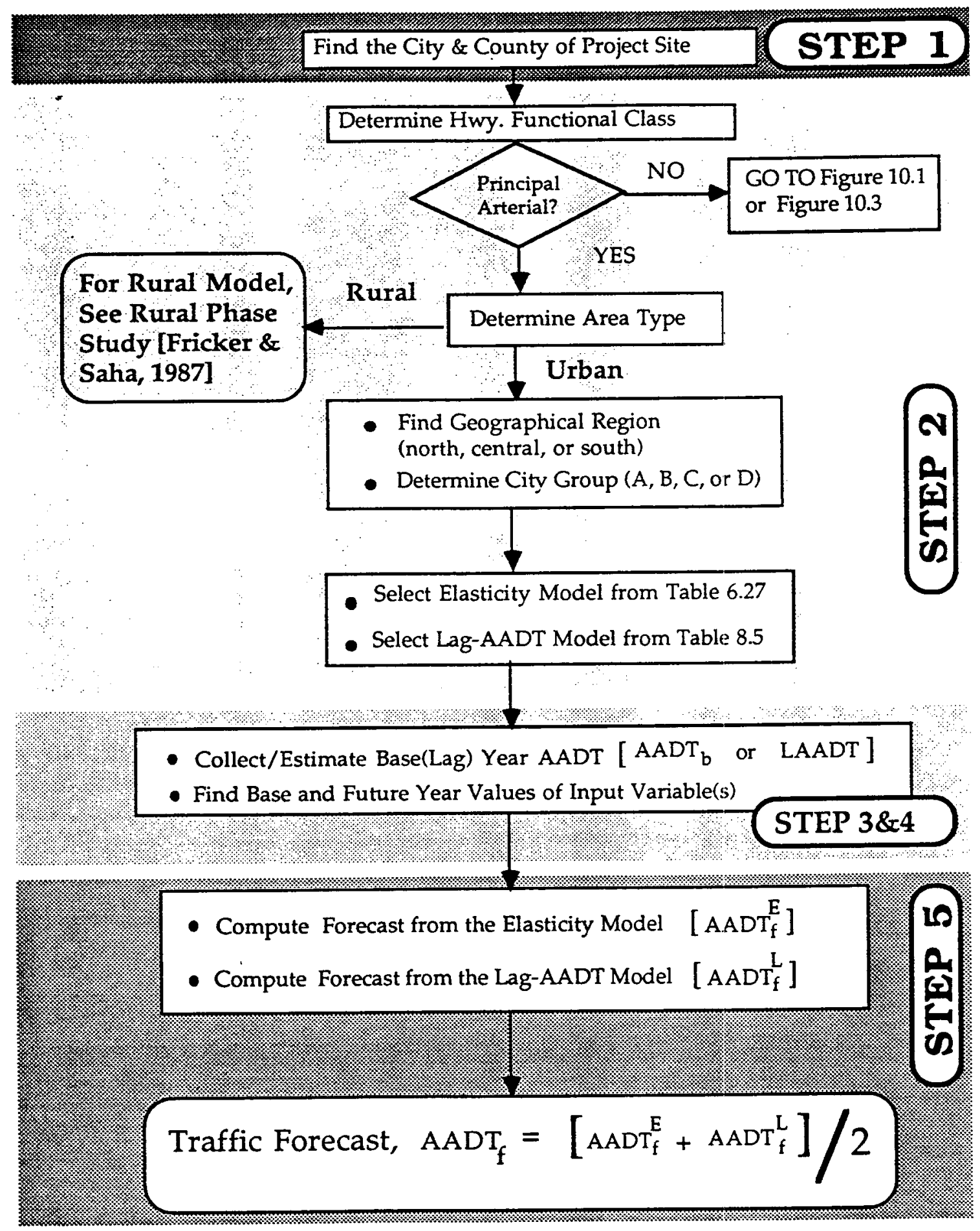

Figure 11.2: Process for Implementation of the Model on Urban Principal Arterial 


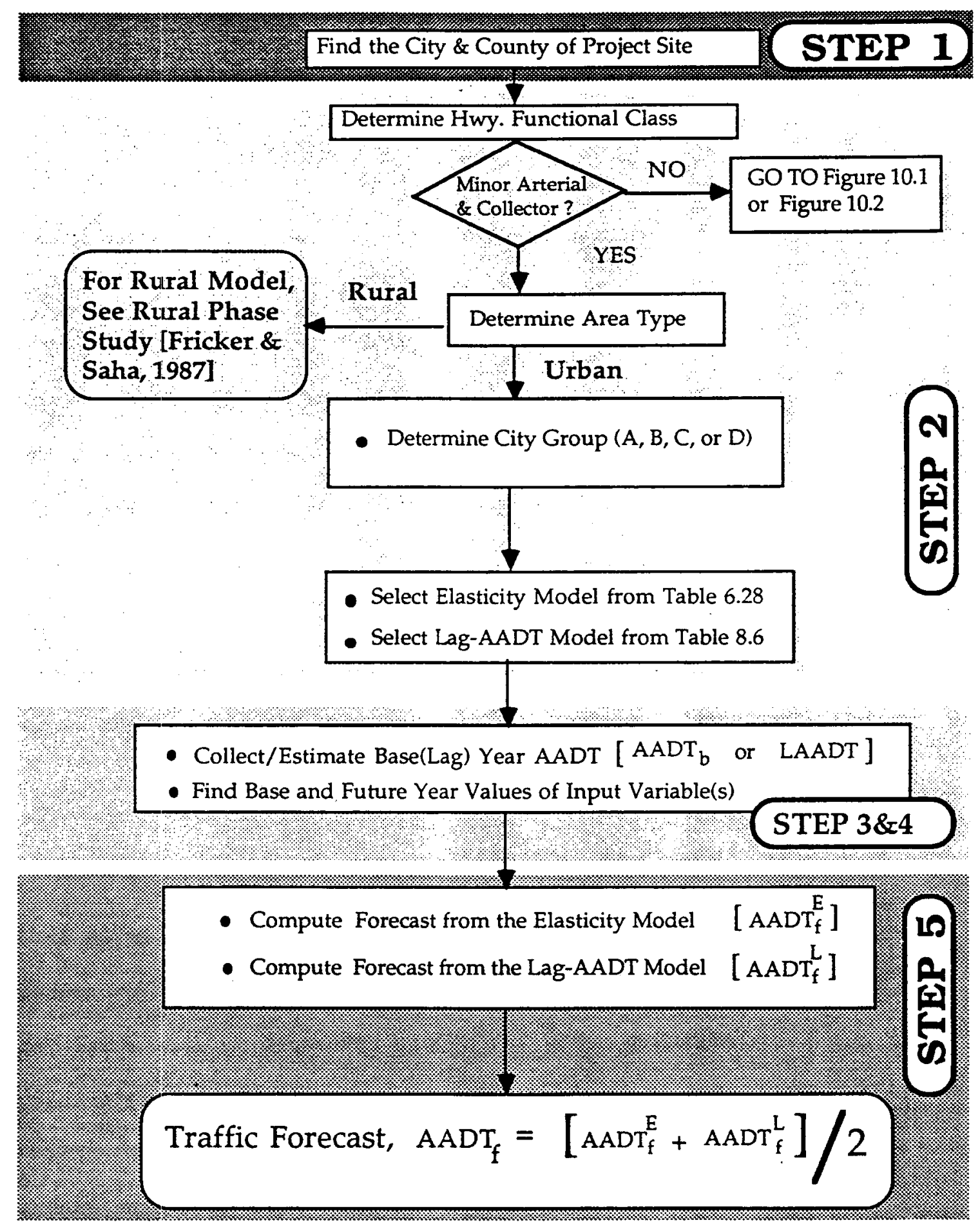

Figure 11.3: Process for Implementation of the Model on Urban Minor Arterial \& Collector 
which the forecast is needed.

2. Select the traffic models that will be used to predict traffic.

a. Determine the functional class of roadway.

This will determine which models are applicable to the project site. To determine the functional class, the functional classification system map - prepared by Program Development Division of INDOT - and the road inventory file of INDOT, will be the two best guides. Moreover, the definitions provided in Table 4.1 of this study would be helpful to find the appropriate highway class. The project site will be classified into one of the four categories of highways provided listed Table 4.1. If the classification is not clear-cut, then personal judgment should be used, and documentation provided.

b. Examine the area type.

Check if the project site is rural or urban in nature. If the project site is in a rural area for noninterstate highways, then the models developed in the rural phase of this study (Fricker \& Saha, 1987) will be applicable to that site. Otherwise, the procedures described in this section will be applied. Suburban segments can be identified as urban segments, because data from suburban segments were included with urban segments in the model buflding process. 
c. Find the geographical region.

Consult Figure 4.2 of this dissertation to determine the geographical region (north, central and south) of the project site. This finding will be used to select the appropriate model for urban principal arterial models and the regional model of interstate highways.

d. Determine the city group.

Find the city group (A, B, C or D) based on the size of the city population. Section 4.6 .1 of this study introduced the city group based on 1980 city population. In using the forecasting models, population in the base year can be used to determine the city group. If the population is not known, a population estimate from the closest census year population to the base year either by ratio method in Section 9.3 .1 or by linear interpolation or extrapolation from city population forecasts in Table F9. The city group information will be used to select models for noninterstate urban highways.

e. Select appropriate models.

The information from steps $2 \mathrm{a}$ to $2 \mathrm{~d}$ are used to find the appropriate mode1(s). Steps $2 \mathrm{a}$ and $2 \mathrm{~b}$ are used to determine highway class and its area type.

1. Interstate Highways: Find the appropriate elasticity models from Table 6.26 , by using the the information 
obtained on area type (step 2b) and geographical region (2c). For each project site, three alternative models - regional interstate model, statewide rural or urban interstate model, and regional rural or urban interstate model -- will be identified in this table. Figure 11.1 shows those appropriate models for each case. For instance, if the project site is identified as being in the northern region and in a rural area, then the models $\mathrm{Al}, \mathrm{B}$ and $\mathrm{Cl}$ from Table 6.26 will be the selected for that project site. Similarly, three alternative lag-AADT models will be selected from Table 8.4 .

ii. Urban Principal Arterial: The information on geographical region (step 2c) and on city group (step 2d) of the project site will be used to find the appropriate elasticity model from Table 6.27. Similarly, the lagAADT will be used identified from Table 8.5 .

iii. Urban Minor Arterial \& Collector: The city group (step 2d) is the only basis for selecting the appropriate model for this highway category, if the area type (step 2b) and the functional class (step 2a) had identified the project site as "Urban Minor Arterial \& Collector". The appropriate elasticity and lag-AADT models will be identified from Tables 6.28 and 8.6 , respectively.

3. Collect the base year $\mathrm{AADT}\left(\mathrm{AADT}_{\mathrm{b}}\right)$ and the lag year AADT (LAADT). 
The base or lag year may be different from the present/current year, but it is the year for which most recent data needed by the are available. $\mathrm{AADT}_{\mathrm{b}}$ (required by the elasticity mode1) or LAADT (required by the lag-AADT mode1) for a project site can be one value for a small project (e.g., intersections) or a series of estimates for roadway sections for a larger project (e.g., lane widening). The primary source of data is the Highway Department's Traffic Volume Book. If this source fails to provide such information, then it could be determined from short-term counts at the project site, using the procedures described in section 2.7 .

4. Collect the base and future year data for the predictor variables used in the elasticity models.

The description of the predictor variables in Section 4.6.3 is a guide to the sources of the required predictor variables. Except vehicle registration and city population forecasts, the forecasts of the predictor variables used in the models are available from outside sources. The combined vehicle registration forecasts developed by time series analysis and presented in Appendix Table F7 will serve as future year values for vehicle registration at the county and state level. The ratio method of making city population forecasts presented in Appendix Table F9 can be used as required by any mode1. The 1ag-AADT models need only the estimate of city population in the base year (if required) to select the appropriate model for noninterstate high- 
ways. They do not need any other information for the predictor variables, to forecast traffic.

5. Estimate the future year AADT.

a. Calculate the future year AADT by using the appropriate elasticity model(s) (Tables 6.26-6.28), as determined in step $2 e$, with the values found in steps 3 and 4 . Denote the future ("f"-year) AADT estimate from the elasticity (E) model as $\mathrm{AADT}_{\mathrm{f}}^{\mathrm{E}}$. For each interstate highway segment, the average forecasts from the three alternative models will be designated as $\operatorname{AADT}_{\mathrm{f}}^{\mathrm{E}}$. Only one forecast will be computed for each noninterstate highway segment.

b. Calculate the future year AADT by using appropriate lag-AADT model(s) (Tables 8.4-8.6), as determined in step $2 \mathrm{e}$ with the values found in steps 3 and 4 . Denote the AADT estimate from the lag-AADT ( or "L") model as $\mathrm{AADT}_{\mathrm{f}}^{\mathrm{L}}$

c. Find the average of the two AADT estimates found in steps $5 \mathrm{a}$ and $5 b$.

$$
\mathrm{AADT}_{f}=\left[\mathrm{AADT}_{\mathrm{f}}^{\mathrm{E}}+\mathrm{AADT}_{\mathrm{f}}^{\mathrm{L}}\right] * 0.5
$$

where,

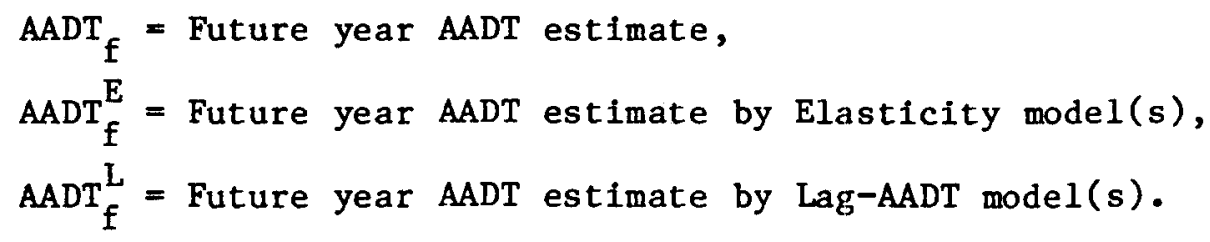


The AADT forecast ( AADT $_{f}$ ) from Equation (11.1) will be used as the "future year AADT" for the project site. The user may give different weight to the AADT found in step $5 \mathrm{a}$ or step 5b, based on model performance. The Equation (11.1) would then be modified to be

$$
\operatorname{AADT}_{\mathrm{f}}^{\mathrm{W}}=\mathrm{w} * \operatorname{AADT}_{\mathrm{f}}^{\mathrm{E}}+(1-\mathrm{w}) * \operatorname{AADT}_{\mathrm{f}}^{\mathrm{L}}
$$

where,

$$
\begin{aligned}
& \operatorname{AADT}_{\mathbf{f}}^{\mathrm{W}}=\text { Weighted AADT estimate, } \\
& w=\text { Weight given to AADT estimate from } \\
& \text { Elasticity mode1, } 0 \leqslant w \leqslant 1.00 \text {, } \\
& \text { other terms are as defined in Equation (11.1). }
\end{aligned}
$$

In the future, the performance of the models can be used to determine the weight "w" in Equation (11.2). But, based on comparable values of the forecasting measuring criteria (MAE, MAPE, RMSPE and $\bar{Q}$ ) for the elasticity and lag-AADT models, and improved performance of the combined forecasts, a simple average, shown in Equation (11.1), is recommended at present.

6. Adjust the estimated future year AADT.

If necessary, the forecast from step 5 can be adjusted by using information on changes in fuel price, fuel supply (energy crisis), fleet fuel efficiency (miles per gallon), household 
size, inflation rate, etc., which have not been included in model formulation because of insufficient information and future uncertainty about those factors. Empirical results [see Chapter 9 of Trombly \& Ouderkirk 1986] can be useful in incorporating such adjustments, if the users have a "feel" for any of these factors. This step is completely optional and it is left to the user to decide whether to modify the forecast values obtained in step 5. Because of its optional nature, this step was not shown in the process for implementation in Figures 11.1 to 11.3 .

\subsection{Problems, Limitations and Suggestions}

The results from this research should be generalized very cautiously outside the bounds of the data and analysis methods described in this report. An important limitation is that the traffic data are from Indiana highways and streets.

A few problems may appear as soon as users begin to use the models to predict traffic. The most serious problem is one that is common to all forecasting processes: the accuracy of the model is determined to a large extent by the accuracy of the input, especially the future values of the predictor variables, as required by the elasticity models. However, this study has focussed on obtaining reliable data as much as possible, efther from outside sources or by developing its own forecast of the input variables.

Applicability of the models to some locations may also cause problems. How can a user decide whether the project area, for which 
future forecast of traffic is needed, is "rural" enough for the model(s)? It is difficult to provide guidelines to assist in this issue, but suggestions [TRB/Special Report 209 1985] exist. Judgment is required in making this determination. In approximate terms, highways with more than 10 uncontrolled access points per mile (on one side) would be considered to be "suburban". Also, any highway on which left or right turns cause appreciable delay to through vehicles would also be classified as "suburban". Multilane suburban highways and rural roads differ from suburban arterials in the following features: (1) their roadside development is not as intense, (2) the density of traffic access points is not as high, and (3) signalized intersections are more than 2 miles apart. In fact, highways with signal spacing of 2 miles or more could be treated as "rural" highways. Increased use of the developed models will lessen this problem. In the meantime, and in the spirit of this study, it may be wise in such borderline cases generate both a rural and an urban forecast.

The elasticity model formulation in Chapter 3 assumes that elasticities are constant over time. A similar linear assumption has been made in the formulation of the lag-AADT model. Historically, travel has been growing at a fairly constant rate for many years. Although fuel shortages interrupted this rate of increase for a while, it has resumed. Therefore, any assumption of constant elasticities would not introduce substantial errors. On the other hand, variable elasticities are not very common in traffic forecasting. They involve more sophisticated and expensive analysis [Kanafani 1983]. This sophistication and expense are against this study's philosophy that the models 
should be easy to understand and inexpensive to use. Elasticities could be recalculated periodically as new information become available, and the appropriateness of earlier values could be checked. If the elasticities seem to change significantly (for example, more than 10 percent), then the new set of elasticities should be used in the model. A similar check could be made on the coefficients of the lagAADT models.

Users are expected to weigh the results of the forecasting models in terms of the local situation, and adjust them according to their professional judgment.

\subsection{Future Research Direction}

The methodologies presented in this report were based on and tested against coverage station traffic counts. The aggregate and disaggregate traffic forecasting models for rural roads of Indiana were developed using only the elasticity method, by using the traffic data from continuous count stations [Fricker \& Saha 1987]. Continuous count stations are the only locations where "true" historic AADT counts are available. Because of the few useful data points from continuous stations data, this study was carried out using traffic data from coverage count stations. The success of this study can be attributed to the determination of data format, data bases and their stratification scheme, and the filtering of traffic data through influential diagnostics. However, further traffic forecasting studies will be helped by the availability of more traffic data, because of the installation of more continuous count stations at locations 
representing a variety of highway categories and traffic characteristics. It is expected that traffic data from the increased number of continuous count stations and from the coverage count stations will provide better statistical results and model performance by using the methodologies of this study.

The data bases and models developed in this study can be updated as new counts become available. Efforts to convert the raw information electronically (automatically) to the form needed by the data base of this study are needed to reduce the bulk of amount of time spent preparing data manually.

Another useful extension of this study would be the use of the methodologiles in the rural area by using the traffic count from coverage count stations as well as from continuous count stations and providing more weight on data from continuous stations. The earlier rural study [Fricker \& Saha 1987], which was based on only continuous stations at rural area, suffered from inadequate traffic data. The preliminary analysis, presented in Chapter 5 and early parts of Chapter 6 of this report regarding data format, data bases and their stratification scheme and data filtering, were impossible with the scanty rural, data base. The time constraints of that study did not allow a test of alternative models (for example, lag-AADT, PSR) and the exhaustive performance evaluation that was done in this study. Similarly, this study's methodologies can be tested on the local roads as well as on the noninterstate highways of urban areas with populations more than 50,000. And, if possible, the results from such a study could be compared to the results of any sophisticated 4-step 
sequential modeling process.

An area that needs special attention is the development of the formal procedure to incorporate the factors which can not be addressed in the model formulation, either because of incompatibility in the model formulation or the lack of enough data. A few of these factors were mentioned in the last (optional) step of the implementation guidelines in Section 11.3.

A possible application as well as an useful extension of this study is to use the results of this study to estimate and forecast of Vehicle Miles of Travel (VMT). Among other studies, the highway cost-allocation study is very much dependent on VMT. The Appendix B of the 1988 Update of the Indiana Highway Cost-Allocation Study [Sinha et al. 1989] showed an disaggregate approach to compute and forecast VMT based on AADT estimate and forecast. An implementation of that approach can be undertaken to estimate and forecast of VMT by using the results of the present study.

Another future effort should be to determine the best weights to use in combining forecasts, as discussed in Chapter 10 of this study, by automatically updating the weights as the actual data for the forecast years become avallable. Also, policies and sensitivity levels with which to define acceptable forecast accuracy need to be established. Perhaps a nationwide survey of experts and an analysis the performance of different forecasting studies could be undertaken. This study has identified a policy to gauge or monitor forecast accuracy for its own forecast measures. 
Time series analysis could be used to forecast future traffic, given the avallability of enough data as required by the procedure. According to Armstrong [1984 and 1986], the time series approach could be combined with the present approaches to obtain reliable traffic forecasts. Time series analysis treats traffic volume as a function of time and uses land use development as the starting point to formulate the traffic growth - as time passes, more land is developed and traffic Increases proportionally. Time series analysis is also a way to introduce time lags, especially with respect to economic predictor variables, to see if better $\mathrm{ADT}$ forecasting models are possible.

The area that the researchers feel will be useful to investigate is the model transferability - the applicability of the models developed in this study to similar states like Indiana. Hartgen [1990] has identified a procedure to determine the similarities among the financial, infrastructure, and organizational structures of the state highway systems, and to group states according to these similaritles. His study has identified states that are based on similar systems, rather than classifying them by closeness and geography. If the performance of the traffic models developed in this study and tested in a "similar" state have been found to be similar to the current study, then the models in this study can be applied directly to that "simflar" state without going through the modeling process. Although, the resulting parameters of the models reflect the validity of the model in the State of Indiana, the procedures discussed in this study were not specific to Indiana. The transferability of the model can be tested in states not similar to Indiana, either directly or by 
developing models using the methodologies of this study on the data of that other state. This consideration may help to fill the gap in the unification of the modeling process, especially to rural and small- to medium-sized urban area, where enough funds are not usually available to do extensive studies.

\subsection{Conclusions}

The principal objective of this study was to develop simple, fast and inexpensive traffic forecasting models for the urban segments of the state and interstate highway network in Indiana. This study has developed two different types of data bases and then tested four forms of data and different data groupings to select the best suitable data structure. The study has identified and developed different suitable methodologies and then applied various statistical analyses to find the best models. Alternative models have been developed in this study to use them at the project level. The choice of predictor variables for the models was based on a combination of various statistical analyses and subjective judgment regarding quality of those variables.

The analyses done to develop the elasticity-based as well as the lag-AADT models are as reliable as possible within the limitations of the data. The developed models could be updated as new data becomes available. The developed elasticity models provide better statistical results than those found in a previous, similar study [Trombly \& Ouderkirk 1986]. Although the varlable selection criterla used in this study are not based solely on the statistical results, this study is based on extensive statistical analysis. The study serve be as a 
stand-alone guide to building and testing the various statististical models to forecast traffic. The variables used in the models were found to be statistically significant. It was found that no other variables will provide additional significant predictive power in the mode1s.

The step-by-step instructions in Section 11.3 are provided to give a structured approach to implementing the models. The developed models are expected to provide highway planners with a simple, fast, and inexpensive estimation procedure to predict future traffic.

In most states, the task of traffic forecasting is heavily dependent on AADT counts from continuous count stations. This study has shown that reliable traffic forecasting models are possible even with traffic data from coverage counts. Much of the credit can be attributed to the data sufficiency, and improved model specification and structure. The reliability of the models is reflected in the values of the selected forecasting measures in Chapter 10. Any state with adequate historical traffic data from coverage and continuous count stations can use this model building approach to determine future year AADT on state highway and interstate segments of sma11- to medium-size urban areas.

Many of the results summarized elsewhere in this study are mutually supportive. For instance, different forecasting measures (see Chapter 10) showed that quality of the forecast measures remained unchanged in spite of different error functions implicit in the measures. Other examples of this "mutual support" were the variable 
selection criteria in Chapters 5 and 6 and the model statistics of Chapters 5 through 9. Such results increased confidence in the procedures and analysis methods employed to build models throughout the study.

Some of the results of this research did not apply directly to forecasting traffic but were found useful in selecting efficient models and in gauging model quality. For instance, the principal component models of Chapter 7 with eleven predictor variables validated the simple elasticity model with for fewer (viz. $\leqslant 2)$ variables without any major change in model statistics. This study has also developed forecasts for input variables that required by the elasticity model, but for which no forecasts are available from outside sources.

The most obvious and direct use of a traffic forecasting model is for the estimation of the benefits from alternate highway system improvement projects. A second application would be as an aid to the appropriate design of a project (for example, number of lanes or type of traffic control). The identification of potential problem segments in the state highway system could be accomplished by using the models to identify rapid traffic growth areas. Undoubtedly, more work must be done in this area to improve the accuracy and reliability of a traffic projection model. It is important to note that the developed models in this report are not purported to be perfect forecasting tools, if such a model could ever exist. Users are expected to weigh the results in terms of the local situation, and make adjustments using their professional judgment. 
The use of different models to forecast traffic has provided an opportunity to search for some degree of consensus. The results of different models developed in this study were compared by observing their performance as well as their model statistics (if applicable). The efficient traffic models (elasticity and lag-AADT) forecasts are combined based on the performance of the individual models. The results of computations will not be more accurate than the input data on which they were based. In the absence of any predictor variables other than AADT itself, the lag-AADT model with lower data requirements, can be expected to perform better than the other models developed in this study. Travel demand can increased rapidly in some part because of steady growth in employment, population, etc. In such cases, it is expected that the elasticity model will outperform the lag-AADT model, which does not account for such explosions in past traffic volumes. This study has verified that combined forecasts from the lagged and elasticity models models outperform the forecasts from the best individual model alone, in many cases. Finally, it is expected that the suggested procedure of combining the forecasts from the elasticity and the lag-AADT models will provide more reliable traffic forecasts to highway planners. 
LIST OF PEFERENCES 
LIST OF REFERENCES

Abraham B. and Ledolter J. [1983], Statistical Methods for Forecasting, John Wiley \& Sons, Inc.

Ahlburg D.A. [1989], "How Accurate are the U.S. Bureau of the Census Projections of Total Live Births?", Journal of Forecasting, Vol.1, pp. 365-374.

Ahmed S.A. [1989], "Operational Filtering of Traffic Data", Journal of Forecasting, Vol.8, pp. 19-32.

Akaike H. [1981], Modern Development of Statistical Methods (Chapter 6), "Trends and Progress in System Identification" Edited by: Eykhoff, Pieter, Pergamon Press.

Albright D. [1985], "Abstract on Trend Line Forecasting Heavy Commercial Traffic as a Percent of Average Daily Traffic", New Mexico State Highway Department.

Amemiya T. [1980], "Selection of Regressors", International Economic Review, 21:2, pp. 331-353.

AASHTO [1984], A Policy on Geometric Design of Highways and Streets, AAs̄HTo, 444 North Capitol Street, N.W. Washington,

American Association of State Highway and Transportation Officials [1986], AASHTO Guide for Design of Pavement

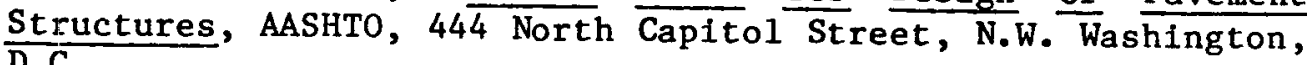
D.C.

Armstrong J.S. [1978], Long-Range Forecasting: From Crystal Bal1 to Computer, New York: John Willey \& Sons, Inc.

Armstrong J.S. [1984], "Forecasting by extrapolation: Conclusion from 25 years Research", Interfaces, 14:6(Nov.-Dec.), pp.5256.

Armstrong J.S. [1986], "The Ombudsman: Research on Forecasting: A Quarter-Century Review (1960-1984)", Interfaces, 16:1 (Jan.Feb.), pp.89-109.

Belsley D.A., Kuh E., and Welsch R.E. [1980], Regression Diagnostics: $\frac{\text { Identifying }}{\text { Collinearity, Influential Data }}$, and 
Box G.E.P. and Jenkins G.M. [1976], Time Series Analysis: Forecasting and Control (Revised edition), San Francisco: Holden-Day.

Box G.E.P. and Pierce D.A. [1970], "Distribution of the Residual Autocorrelations in Autoregressive-Integrated Moving-Average Time Series Models", Journal of the American Statistical Association, Vol. 65, pp. 1509-26.

Brocklebank J.C. and Dickey D.A. [1986], SAS system for forecasting time series, SAS Institute $\overline{\text { Inc., }}$ Cary, North Carolina, USA.

Bureau of Labor Statistics, "Handbook of Labor Statistics", Bulletin 2217, U.S. Department of Labor, June 1984.

Bureau of the Census, "County and City Data Book (1956, 1962, 1967, 1972, 1977, 1983): A Statistical Abstract Supplement", U.S. Department of Commerce.

Bureau of the Census, "County Business Patterns - Indiana, 1970 1988", U.S. Department of Commerce.

Bureau of the Census, "Characteristics of the Population, Number of Inhabitants, Indiana, 1980 Census of Population", U.S. Department of Commerce.

Chan Yupo [1985], "Highway Travel and Fuel Consumption from 1970-1980", Transportation Quarterly, Vo1.39, No. 1, January 1985, pp 39-57.

Chisholm R.K. and Whitaker G.R. (Jr.) [1971], Forecasting Methods, Richard D. Irwin, Inc.

COMSIS Corp. [1985], "Simplified Project Forecasting (SPF) Users Guide", Prepared for Office of Planning FHWA.

Covalt O.D. [1958], "Indiana Highway Needs Study", HRB Bulletin 194, Jan. 1958, pp 14-27, JHRP, Purdue Unfversity.

Deacon J.A., Pigman J.G. and Mohsenzadeh A. [1987], "Traffic Volume Estimates and Growth Trends", Kentucky Transportation Research Program, University of Kentucky, UKTRP-87-32, Nov. 1987.

Draper N.R. and Smith H. [1981], Applied Regression Analysis, John Wiley and Sons, New York.

Economic Statistics Bureau of Washington, D.C., The Handbook of Basic economic Statistics - Labor, Industry, Commerce and Agriculture; April 1984, Vol. XXXXIX, No. 4, pp. 99-101. 
Federal Highway Administration, "Guide for Traffic Volume Counting Manual", Bureau of Public Roads, U.S. Department of Transportation, March 1970.

Freund R.J. and Minton P.D. [1979], Regression Methods - $\underline{\text { Tool }}$ for Data Analysis, Marcel Dekker, Inc., New York.

Fricker Jon D. and Saha S.K. [1987], "Traffic Volume Forecasting Methods for Rural State Highways - Final Report", FHWA/IN/JHRP-86/20, Joint Highway Research Project, School of Civil Engineering, Purdue University.

Granger C.W.J. and Ramanathan R. [1984], "Improved Methods of Combining Forecasts", Journal of Forecasting, Vo1.3, pp 197204.

Harr M.E. [1987], Reliability-Based Design in Civil Engineering, McGraw-Hi11, Inc.

Hartgen. D.T. [1981], "What will happen to travel in the next 20 years?", Transportation Research Record 807, TRB, Dec. edition, pp. 21-27.

Hartgen D.T. [1990], "Similarities among the State Highway Systems", - a paper submitted to the annual meeting of Transportation Research Board, No. 89059, January 1990.

Hazen P.I. [1982], "Summary of Recent National and State Highway Travel Forecast Studies", U.S. Department of Transportation, Program Management division, Federal Highway Administration.

Hutchinson B.G. [1974], Principles of Urban Transport Systems Planning, McGraw-Hil1, Inc.

Indiana Bureau of Motor Vehicles, "Motor Vehicle Registration in Indiana By County $(1940,41,1950$ - 1988)".

Indiana Department of Transportation (INDOT), "Traffic Statistics - 1981,1982,1983,1984,1985,1986 and 1987", Division of Program Development, INDOT.

INDOT, "Division of Program Development Mileage Report -Functional Class/Federal Aid", 1988, INDOT.

INDOT, "Road Inventory File", Research and Training Center, West Lafayette, Indiana.

Indiana Employment Security Division (IESD), "County Employment Patterns, 1970 - 1983", Research and Statistics Section, IESD.

Indiana University, "Indiana County Population Projections 19852020", School of Business, Indiana Business Research Center, Indiana University, 1986, 1988. 
Indiana University, "Indiana Township Population Projections 1985-2020", School of Business, Indiana Business Research Center, Indiana University, 1986.

Indiana University, "Preliminary Estimates of the Intercensal Population of Counties in Indiana", School of Business, Indiana Business Research Center, Indiana University.

Indiana University, Statistical Information System (STATIS), The Economic Development Information Network (EDIN), School of Business, Indiana Business Research Center, Indiana University, 1987.

ITE (Institute of Transportation Engineers) Technical Council Committee 6F-41 [1990], "Traffic Forecasting on State Highway Segments", A Draft Final Report (unpublished as of July 30, 1990), March 1990.

Ismart D.R. and Schoener G.E. [1987], "Urban Arterial Planning and Analysis", Journal of Transportation Engineering, ASCE, Vo1.113, No.3, May 1987.

Jenkins G.M. [1979], Practical Experiences with Modelling and Forecasting Time Series, Time Series Library, Gwilym Jenkins \& Partners (Overseas) Ltd.

Jenkins G.M. [1982], "Some Practical Aspects of Forecasting in Organizations", Journal of Forecasting, Vo1.1, pp. 3-21.

Judge G.G., Hill R.C., Griffiths W.E., Lu kepohl H. and Lee T. [1980], Introduction to the Theory and Practice of Econometrics, Second Edition, John wiley \& Sons, Inc.

Johnson R.A. and Wichern D.W. [1982], Applied Multivariate Statistical Analysis, Englewood Cliffs, NJ, Printice Ha11, Inc.

Kanafani A. [1983], Transportation Demand Analysis, McGraw-Hil1 Book Company.

Krueckeberg D.A. and Silvers A.L. [1974], Urban Planning Analysis: Methods and Models, John Wiley \& Sons, Inc.

Mahmassani H.S. and Sinha K.C. [1978], "Framework for the Transferability of Trip Generation Parameters of Small Urban Areas" - Interim Report, Joint Highway Research Project (JHRP), Report No. JHRP-78-13, Purdue University, Indiana.

Mahmoud E. [1984], "Accuracy in Forecasting: A Survey", Journal in Forecasting, Vol.3, pp. 139-159. 
Makridakis S. [1986], "The Art and Science of Forecasting - An Assessment and Future Directions", International Journal of Forecasting, Vol.2, pp. 15-39.

Makridakis S. and Wheelwright S.C. [1978], Forecasting: Methods and Applications, John Wiley \& Sons, Inc.

Makridakis S. and Winkler R.L. [1983], "Average of Forecasts: Some Empirical Results", Management Science, Vol.29, No.9, pp. 987-996.

Memmott J.L. and Buffington J.L. [1981], "Predicting Traffic Volume Growth Rates Resulting from Changes in Highway Capacity and Land Development", Texas Transportation Institute, Texas A\&M University.

Memmott J.L. [1983], "Factors that Affect Growth Rates and Projections of Traffic Volumes for Use in Highway Economic Models", Transportation Research Record 912.

Meyer J.R. and Glauber R.R. [1964], "Investment Decisions, Economic Forecasting, and Public Policy", Division of Research, Graduate School of Business Administration, Harvard University, Boston.

Meyer M.D. and Miller E.J. [1984], Urban Transportation Planning: A Decision - Oriented Approach, McGraw-Hill Book Company.

Miller D.M. [1985], "The Anatomy of a Successful Forecasting Implementation", International Journal of Forecasting, Vol.1,
pp. 69-78.

Magridge M.J.H. [1977], Traffic Forecasting, Highways and Road Construction International, Embankment Press Ltd., London, pp.
5-10.

Montgomery D.C. and Johnson L.A. [1976], Forecasting and Time Series Analysis, McGraw-Hill Book Company.

Moore D.S. and McCabe G.P. [1989], Introduction to the Practice of Statistics, W.H. Freeman and Company.

Moorthy C.K. and Ratcliffe B.G. [1988], Short Term Traffic Forecasting Using Time Series Methods, Transportation Planning and Technology, Vol. 12, pp. 45-56.

Ne1son C.R. [1973], Applied Time Series Analysis for Managerial Forecasting, San Francisco: Holden-Day, Inc.

Neter J., Wasserman W. and Kutner M.H. [1985], Applied Linear Statistical Models, Second Edition, Richard D. Irwin, Inc., 
Neveu A.J. [1982], "Quick Response Procedures to Forecast Rural Traffic", Transportation Research Record 944.

Prem C.E. [1987], "Streamling the Technical Planning Process", ITE Journal, October 1987, pp. 33-38.

Reeves G.R. and Lawrence K.D. [1982], "Combining Multiple Forecasts Given Multiple Objectives", Journal of Forecasting, Vol.1, pp. 271-279.

Richards M.G. and Ben-akiva M.E. [1975], A Disaggregate Travel Demand Mode1, Saxson House, Ltd and Lexington Books, Inc. Ritchie S.G. [1986], "A Statistical Approach to Statewide Traffic Counting", Transportation Research Board Annual Meeting, Washington DC, January 1986.

Roberts Thomas H., "Forecasting Growth Parameters in Smaller Urban Areas", NCHRP 187, pp. 71-72.

Saha S.K. [1986], "Traffic Volume Forecasting Methods for Rural State Highways", MSCE Thesis, School of Civil Engineering, Purdue University.

Saha, S.K. and Fricker J.D. [1988], "Traffic Volume Forecasting Methods for Rural State Highways", Transportation Research Record 1203, pp. 10-26.

SAS Institute Inc. [1985], SAS User' ${ }^{\prime}$ suide: Basics, Version 5 Edition, SAS Institute Inc., Cary, North Carolina.

SAS Institute Inc. [1985], SAS User ${ }^{-}$s Guide: Statistics, Version 5 Edition, SAS Institute Inc., Cary, North Carolina.

SAS Institute Inc. [1984], SAS/ETS User's Guide, Version 5 Edition, SAS Institute Inc., Cary, North Carolina, USA.

Schultz R.L. [1984], "The Implementation of Forecasting Mode1s", Journal of Forecasting, Vol.3, pp. 43-45.

Sinha K.C., Saha S.R., Fwa T.F., Tee T.F. and Michael H.L. [1989]," "1988 Update of the Indiana Highway Cost-Allocation Study: Draft Fina1 Report", FHWA/In/JHRP-89/4, Joint Highway Research Project, School of Civil Engineering, Purdue University.

Stopher P.R. and Meyburg A.H. [1975], Urban Transportation Modeling and Planning, Lexington Books, Inc.

Theil H. [1965], Economic Forecasts and Policy, Amsterdam: North Holand Publishing Co., pp. 32-38.

Theil H. [1966], "Applied Economic Forecasting", Amsterdam: North-Holland Publishing Company. 
Thomopoulos N.T. [1980], Applied Forecasting Methods, New Jersey: Prentice-Ha11, Inc.

Transportation Research Board (TRB) [1980], Transportation Planning for Small and Medium Sized Communities, Special Report 187, TRB, Washington, D.C.

Transportation Research Board (TRB) [1985], "Highway Capacity Manual", Special Report 209, TRB, National Research Council, Washington, D.C.

Trombly J.W. and Ouderkirk [1986], "Highway Traffic Forecasting Guide", Department of Transportation, State of New York.

Woh1 M. [1970], "A Methodology for Forecasting Peak and Off-Peak Travel Volumes", Highway Research Record No. 322, p. 183-219.

Woods \& Poole Economics (W\&P), Inc. [1988], 1794 Columbia Road NW, Washington, DC 20009, "1987 Indiana' State Profile". 
APPENDICES 
Appendix A 
Table Al: Segment AADT Raw Data Base for Urban Minor Arterial \& Collector

\begin{tabular}{|c|c|c|c|c|c|c|c|c|c|c|c|}
\hline (1) & (2) & (3) & (4) & (5) & (6) & $(7)$ & 1) (8) & (9) & $(10)$ & (11) & (12) \\
\hline OBS & $\begin{array}{l}\text { CT } \\
\text { YN }\end{array}$ & CTYNM & $\begin{array}{l}\text { CTY } \\
\text { AR }\end{array}$ & $\begin{array}{l}\text { PCTY } \\
80\end{array}$ & $\begin{array}{l}\text { CTY } \\
\text { GR }\end{array}$ & $\begin{array}{l}\mathrm{Y} \\
\mathrm{TYN}\end{array}$ & CNTYNM & CNTYAR & $\begin{array}{l}\text { GRE } \\
\text { GION }\end{array}$ & $\begin{array}{l}\text { PCNT } \\
\text { Y } 80\end{array}$ & FUNC \\
\hline $\begin{array}{r}1 \\
1 \\
1 \\
1 \\
-1\end{array}$ & $\begin{array}{l}13 \\
13 \\
13 \\
13 \\
13\end{array}$ & $\begin{array}{l}\text { richmond } \\
\text { richmond } \\
\text { richmond } \\
\text { richmond } \\
\text { richmond }\end{array}$ & $\begin{array}{l}16.3 \\
16.3 \\
16.3 \\
16.3 \\
16.3\end{array}$ & $\begin{array}{l}41349 \\
41349 \\
41349 \\
41349 \\
41349\end{array}$ & $\begin{array}{l}\mathbf{a} \\
\mathbf{a} \\
\mathbf{a} \\
\mathbf{a} \\
\mathbf{a}\end{array}$ & $\begin{array}{l}89 \\
89 \\
89 \\
89 \\
89\end{array}$ & $\begin{array}{l}\text { wayne } \\
\text { wayne } \\
\text { wayne } \\
\text { wayne } \\
\text { wayne }\end{array}$ & $\begin{array}{l}404.25 \\
404.25 \\
404.25 \\
404.25 \\
404.25\end{array}$ & $\begin{array}{l}c \\
c \\
c \\
c \\
c\end{array}$ & $\begin{array}{l}76058 \\
76058 \\
76058 \\
76058 \\
76058\end{array}$ & $\begin{array}{l}\text { uma } \\
\text { uma } \\
\text { uma } \\
\text { uma } \\
\text { uma }\end{array}$ \\
\hline $\begin{array}{r}42 \\
42 \\
42 \\
-2\end{array}$ & $\begin{array}{l}87 \\
87 \\
87 \\
87\end{array}$ & $\begin{array}{l}\text { tipton } \\
\text { tipton } \\
\text { tipton } \\
\text { tipton }\end{array}$ & $\begin{array}{l}1.6 \\
1.6 \\
1.6 \\
1.6\end{array}$ & $\begin{array}{l}5004 \\
5004 \\
5004 \\
5004\end{array}$ & $\begin{array}{l}d \\
d \\
d \\
d\end{array}$ & $\begin{array}{l}80 \\
80 \\
80 \\
80\end{array}$ & $\begin{array}{l}\text { tipton } \\
\text { tipton } \\
\text { tipton } \\
\text { tipton }\end{array}$ & $\begin{array}{l}260.75 \\
260.75 \\
260.75 \\
260.75\end{array}$ & $\begin{array}{l}c \\
c \\
c \\
c\end{array}$ & $\begin{array}{l}16819 \\
16819 \\
16819 \\
16819\end{array}$ & $\begin{array}{l}\text { uma } \\
\text { uma } \\
\text { uma } \\
\text { uma }\end{array}$ \\
\hline
\end{tabular}

\begin{tabular}{|c|c|c|c|c|c|c|c|c|c|c|c|c|c|}
\hline$(1)$ & (2) & (7) & (13) & (14) & (15) & (16) & $(17)$ & (18) & (19) & $(20)$ & (21) & (22) & (23) \\
\hline OBS & $\begin{array}{l}\mathrm{CT} \\
\mathrm{YN}\end{array}$ & $\begin{array}{l}\mathrm{CN} \\
\mathrm{IYN}\end{array}$ & M88TOT & $\begin{array}{l}\text { M88 } \\
\text { FUNC }\end{array}$ & $\begin{array}{l}\text { PM88 } \\
\text { FUNC }\end{array}$ & FAS & HWY & $\mathrm{SEC}$ & YEAR & AADT & CTYPOP & $\begin{array}{l}\text { CNIY } \\
\text { POP }\end{array}$ & $\begin{array}{l}\text { CNTY } \\
\mathrm{HH}\end{array}$ \\
\hline 1 & 13 & 89 & 235.29 & 58.54 & 24.88 & fau & s. 121 & $1 \mathrm{ln}$ & 70 & 7111 & 43999 & 79109 & 25243 \\
\hline 1 & 13 & 89 & 235.29 & 58.54 & 24.88 & fau & $\operatorname{sr} 121$ & $1 \ln$ & 75 & 5658 & 43898 & 77351 & 26191 \\
\hline 1 & 13 & 89 & 235.29 & 58.54 & 24.88 & fau & B 5121 & $1 \ln$ & 79 & 8291 & 42395 & 76300 & 27164 \\
\hline 1 & 13 & 89 & 235.29 & 58.54 & 24.88 & fau & $\operatorname{sr} 121$ & $1 \ln$ & 82 & 8820 & 40752 & 75000 & 27172 \\
\hline-1 & 13 & 89 & 235.29 & 58.54 & 24.88 & fau : & sr 121 & $\ln$ & 85 & 10020 & 39030 & 72200 & 26418 \\
\hline 42 & 87 & 80 & 40.17 & 10.47 & 26.06 & fau & sr19 & $7 b$ & 73 & 3254 & 5028 & 16700 & 5529 \\
\hline 42 & 87 & 80 & 40.17 & 10.47 & 26.06 & fau $s$ & sr19 & $7 b$ & 77 & 3728 & 4756 & 16900 & 5831 \\
\hline 42 & 87 & 80 & 40.17 & 10.47 & 26.06 & fau & st 19 & $7 b$ & 80 & 3754 & 5004 & 16819 & 5993 \\
\hline-2 & 87 & 80 & 40.17 & 10.47 & 26.06 & fau s & $\operatorname{sr} 19$ & $7 b$ & 84 & 4090 & 4694 & 16200 & 5700 \\
\hline
\end{tabular}


Table Al, continued

\begin{tabular}{|c|c|c|c|c|c|c|c|c|c|c|c|c|}
\hline$(1)$ & (2) & $(7)$ & (19) & (24) & (25) & (26) & (27) & (28) & (29) & $(30)$ & (31) & (32) \\
\hline OBS & $\begin{array}{l}\text { CT } \\
\text { YN }\end{array}$ & $\begin{array}{l}\mathrm{CN} \\
\mathrm{TYN}\end{array}$ & YEAR & CNTYVR & $\begin{array}{l}\text { CNTY } \\
\text { EMP }\end{array}$ & $\begin{array}{l}\text { CNTY } \\
\text { INC }\end{array}$ & STPOP & STHH & STVR & STEMP & STINC & GAS \\
\hline $\begin{array}{r}1 \\
1 \\
1 \\
1 \\
-1\end{array}$ & $\begin{array}{l}13 \\
13 \\
13 \\
13 \\
13\end{array}$ & $\begin{array}{l}89 \\
89 \\
89 \\
89 \\
89\end{array}$ & $\begin{array}{l}70 \\
75 \\
79 \\
82 \\
85\end{array}$ & $\begin{array}{l}46259 \\
52634 \\
57978 \\
57679 \\
57560\end{array}$ & $\begin{array}{l}26456 \\
26412 \\
27575 \\
24275 \\
24895\end{array}$ & $\begin{array}{r}9381 \\
9539 \\
10716 \\
9543 \\
10743\end{array}$ & $\begin{array}{l}5195392 \\
5309147 \\
5475000 \\
5485000 \\
5503000\end{array}$ & $\begin{array}{l}1609443 \\
1747281 \\
1896477 \\
1946384 \\
1990746\end{array}$ & $\begin{array}{l}3134244 \\
3772173 \\
4319035 \\
4342071 \\
4533362\end{array}$ & $\begin{array}{l}1580100 \\
1603117 \\
1929580 \\
1719673 \\
1892613\end{array}$ & $\begin{array}{r}9374 \\
10344 \\
11556 \\
10292 \\
11562\end{array}$ & $\begin{array}{l}2366 \\
2644 \\
2757 \\
2336 \\
2424\end{array}$ \\
\hline $\begin{array}{l}42 \\
42 \\
42 \\
-2\end{array}$ & $\begin{array}{l}87 \\
87 \\
87 \\
87\end{array}$ & $\begin{array}{l}80 \\
80 \\
80 \\
80\end{array}$ & $\begin{array}{l}73 \\
77 \\
80 \\
84\end{array}$ & $\begin{array}{l}14114 \\
15615 \\
16151 \\
16424\end{array}$ & $\begin{array}{l}2020 \\
2355 \\
2677 \\
2278\end{array}$ & $\begin{array}{l}12022 \\
11830 \\
11423 \\
12336\end{array}$ & $\begin{array}{l}5329000 \\
5405000 \\
5490224 \\
5493000\end{array}$ & $\begin{array}{l}1711108 \\
1824325 \\
1927052 \\
1970890\end{array}$ & $\begin{array}{l}3652567 \\
4071356 \\
4362862 \\
4388881\end{array}$ & $\begin{array}{l}1702849 \\
1727408 \\
1844200 \\
1795825\end{array}$ & $\begin{array}{l}10978 \\
11311 \\
10830 \\
11000\end{array}$ & $\begin{array}{l}2732 \\
2795 \\
2538 \\
2405\end{array}$ \\
\hline
\end{tabular}

Note: See Table 4.3 for description of columns. 
Table A2: Segment AADT Density Data Base for Urban Minor Arterial \& Collector

\begin{tabular}{|c|c|c|c|c|c|c|c|c|c|c|c|}
\hline (1) & (2) & (3) & (4) & (5) & (6) & $(7)$ & 7) (8) & (9) & $(10)$ & (11) & (12) \\
\hline OBS & $\begin{array}{l}\text { CT } \\
\text { YN }\end{array}$ & CTYNM & $\begin{array}{l}\text { CTY } \\
\text { AR }\end{array}$ & $\begin{array}{l}\text { PCTY } \\
80\end{array}$ & $\begin{array}{l}\text { CTY } \\
\text { GR }\end{array}$ & $\begin{array}{l}\mathrm{Y} \\
\mathrm{TYN}\end{array}$ & IN CNTYNM & CNTYAR & $\begin{array}{l}\text { GRE } \\
\text { GION }\end{array}$ & $\begin{array}{l}\text { PCNT } \\
\text { Y80 }\end{array}$ & FUNC \\
\hline $\begin{array}{r}1 \\
1 \\
1 \\
1 \\
-1\end{array}$ & $\begin{array}{l}13 \\
13 \\
13 \\
13 \\
13\end{array}$ & $\begin{array}{l}\text { richmond } \\
\text { richmond } \\
\text { richmond } \\
\text { richmond } \\
\text { richmond }\end{array}$ & $\begin{array}{l}16.3 \\
16.3 \\
16.3 \\
16.3 \\
16.3\end{array}$ & $\begin{array}{l}41349 \\
41349 \\
41349 \\
41349 \\
41349\end{array}$ & $\begin{array}{l}\text { a } \\
\text { a } \\
\text { a } \\
\text { a } \\
\text { a }\end{array}$ & $\begin{array}{l}89 \\
89 \\
89 \\
89 \\
89\end{array}$ & $\begin{array}{l}\text { wayne } \\
\text { wayne } \\
\text { wayne } \\
\text { wayne } \\
\text { wayne }\end{array}$ & $\begin{array}{l}404.25 \\
404.25 \\
404.25 \\
404.25 \\
404.25\end{array}$ & $\begin{array}{l}c \\
c \\
c \\
c \\
c\end{array}$ & $\begin{array}{l}76058 \\
76058 \\
76058 \\
76058 \\
76058\end{array}$ & $\begin{array}{l}\text { uma } \\
\text { uma } \\
\text { uma } \\
\text { uma } \\
\text { uma }\end{array}$ \\
\hline $\begin{array}{l}42 \\
42 \\
42 \\
-2\end{array}$ & $\begin{array}{l}87 \\
87 \\
87 \\
87\end{array}$ & $\begin{array}{l}\text { tipton } \\
\text { tipton } \\
\text { tipton } \\
\text { tipton }\end{array}$ & $\begin{array}{l}1.6 \\
1.6 \\
1.6 \\
1.6\end{array}$ & $\begin{array}{l}5004 \\
5004 \\
5004 \\
5004\end{array}$ & $\begin{array}{l}\text { d } \\
\text { d } \\
\text { d } \\
\text { d }\end{array}$ & $\begin{array}{l}80 \\
80 \\
80 \\
80\end{array}$ & $\begin{array}{l}\text { tipton } \\
\text { tipton } \\
\text { tipton } \\
\text { tipton }\end{array}$ & $\begin{array}{l}260.75 \\
260.75 \\
260.75 \\
260.75\end{array}$ & $\begin{array}{l}c \\
c \\
c \\
c\end{array}$ & $\begin{array}{l}16819 \\
16819 \\
16819 \\
16819\end{array}$ & $\begin{array}{l}\text { uma } \\
\text { uma } \\
\text { uma } \\
\text { uma }\end{array}$ \\
\hline
\end{tabular}

\begin{tabular}{|c|c|c|c|c|c|c|c|c|c|c|c|c|c|}
\hline (1) & (2) & $(7)$ & (13) & $(14)$ & (15) & (16) & $(17)($ & $(18)$ & (19) & (20) & (21) & (22) & (23) \\
\hline OBS & $\begin{array}{l}\text { CT } \\
\text { YN }\end{array}$ & $\begin{array}{l}\text { CN } \\
\text { TYN }\end{array}$ & M88ToT & $\begin{array}{l}\text { M88 } \\
\text { FUNC }\end{array}$ & $\begin{array}{l}\text { PM88 } \\
\text { FUNC }\end{array}$ & FAS & HWY S & SEC & YEAR & AADT & CTYPOP & $\begin{array}{l}\text { CNTY } \\
\text { POP }\end{array}$ & $\begin{array}{l}\text { CNTY } \\
\mathrm{HH}\end{array}$ \\
\hline $\begin{array}{r}1 \\
1 \\
1 \\
1 \\
-1\end{array}$ & $\begin{array}{l}13 \\
13 \\
13 \\
13 \\
13\end{array}$ & $\begin{array}{l}89 \\
89 \\
89 \\
89 \\
89\end{array}$ & $\begin{array}{l}235.29 \\
235.29 \\
235.29 \\
235.29 \\
235.29\end{array}$ & $\begin{array}{l}58.54 \\
58.54 \\
58.54 \\
58.54 \\
58.54\end{array}$ & $\begin{array}{l}24.88 \\
24.88 \\
24.88 \\
24.88 \\
24.88\end{array}$ & $\begin{array}{l}\text { fau } \\
\text { fau } \\
\text { fau } \\
\text { fau } \\
\text { fau }\end{array}$ & $\begin{array}{l}\operatorname{sr} 121 \\
s r 121 \\
s+121 \\
s r 121 \\
s r 121\end{array}$ & $\begin{array}{l}\ln \\
\ln \\
\ln \\
\ln \\
\ln \end{array}$ & $\begin{array}{l}70 . \\
75 . \\
79 . \\
82 . \\
85.1\end{array}$ & $\begin{array}{r}7111.0 \\
5658.0 \\
8291.0 \\
8820.0 \\
10020.0\end{array}$ & $\begin{array}{l}2699.3 \\
2693.1 \\
2600.9 \\
2500.1 \\
2394.5\end{array}$ & $\begin{array}{l}195.7 \\
191.3 \\
188.7 \\
185.5 \\
178.6\end{array}$ & $\begin{array}{l}62.4 \\
64.8 \\
67.2 \\
67.2 \\
65.4\end{array}$ \\
\hline $\begin{array}{r}42 \\
42 \\
42 \\
-2\end{array}$ & $\begin{array}{l}87 \\
87 \\
87 \\
87\end{array}$ & $\begin{array}{l}80 \\
80 \\
80 \\
80\end{array}$ & $\begin{array}{l}40.17 \\
40.17 \\
40.17 \\
40.17\end{array}$ & $\begin{array}{l}10.47 \\
10.47 \\
10.47 \\
10.47\end{array}$ & $\begin{array}{l}26.06 \\
26.06 \\
26.06 \\
26.06\end{array}$ & $\begin{array}{l}\text { fau } \\
\text { fau } \\
\text { fau } \\
\text { fau }\end{array}$ & $\begin{array}{l}\text { sr } 19 \\
\text { sr } 19 \\
\text { sr19 } \\
\text { sr } 19\end{array}$ & $\begin{array}{l}7 \mathrm{~b} \\
7 \mathrm{~b} \\
7 \mathrm{~b} \\
7 \mathrm{~b}\end{array}$ & $\begin{array}{l}73 . \\
77 . \\
80 . \\
84 .\end{array}$ & $\begin{array}{l}3254.0 \\
3728.0 \\
3754.0 \\
4090.0\end{array}$ & $\begin{array}{l}3142.5 \\
2972.5 \\
3127.5 \\
2933.7\end{array}$ & $\begin{array}{l}64.0 \\
64.8 \\
64.5 \\
62.1\end{array}$ & $\begin{array}{l}21.2 \\
22.4 \\
23.0 \\
21.9\end{array}$ \\
\hline
\end{tabular}


Table A2, continued

\begin{tabular}{|c|c|c|c|c|c|c|c|c|c|c|c|c|}
\hline (1) & (2) & $(7)$ & (19) & (24) & (25) & (26) & (27) & (28) & (29) & (30) & (31) & $(32)$ \\
\hline OBS & $\begin{array}{l}\text { CT } \\
\text { YN }\end{array}$ & $\begin{array}{l}\mathrm{CN} \\
\text { TYN }\end{array}$ & YEAR & CNTYUR & $\begin{array}{l}\text { CNTY } \\
\text { EMP }\end{array}$ & $\begin{array}{l}\text { CNTY } \\
\text { INC }\end{array}$ & STPOP & STHH & STVR & STEMP & SIINC & STGAS \\
\hline $\begin{array}{r}1 \\
1 \\
1 \\
1 \\
-1\end{array}$ & $\begin{array}{l}13 \\
13 \\
13 \\
13 \\
13\end{array}$ & $\begin{array}{l}89 \\
89 \\
89 \\
89 \\
89\end{array}$ & $\begin{array}{l}70 . \\
75 . \\
79 . \\
82 . \\
85\end{array}$ & $\begin{array}{l}58.5 \\
68.0 \\
76.0 \\
76.9 \\
79.7\end{array}$ & $\begin{array}{l}65.4 \\
65.3 \\
68.2 \\
60.0 \\
61.6\end{array}$ & $\begin{array}{r}9381.0 \\
9539.0 \\
10716.0 \\
9543.0 \\
10743.0\end{array}$ & $\begin{array}{l}144.4 \\
147.6 \\
152.2 \\
152.5 \\
153.0\end{array}$ & $\begin{array}{l}44.7 \\
48.6 \\
52.7 \\
54.1 \\
55.3\end{array}$ & $\begin{array}{l}60.4 \\
71.1 \\
78.9 \\
79.2 \\
82.4\end{array}$ & $\begin{array}{l}43.9 \\
44.6 \\
53.6 \\
47.8 \\
52.6\end{array}$ & $\begin{array}{r}9374.0 \\
10344.0 \\
11556.0 \\
10292.0 \\
11562.0\end{array}$ & $\begin{array}{l}2366.0 \\
2644.0 \\
2757.0 \\
2336.0 \\
2424.0\end{array}$ \\
\hline $\begin{array}{l}42 \\
42 \\
42 \\
-2\end{array}$ & $\begin{array}{l}87 \\
87 \\
87 \\
87\end{array}$ & $\begin{array}{l}80 \\
80 \\
80 \\
80\end{array}$ & $\begin{array}{l}73 . \\
77 . \\
80 . \\
84 .\end{array}$ & $\begin{array}{r}84.5 \\
92.4 \\
96.0 \\
101.4\end{array}$ & $\begin{array}{r}7.7 \\
9.0 \\
10.3 \\
8.7\end{array}$ & $\begin{array}{l}12022.0 \\
11830.0 \\
11423.0 \\
12336.0\end{array}$ & $\begin{array}{l}148.1 \\
150.2 \\
152.6 \\
152.7\end{array}$ & $\begin{array}{l}47.6 \\
50.7 \\
53.6 \\
54.8\end{array}$ & $\begin{array}{l}68.5 \\
75.3 \\
79.5 \\
79.9\end{array}$ & $\begin{array}{l}47.3 \\
48.0 \\
51.3 \\
49.9\end{array}$ & $\begin{array}{l}10978.0 \\
11311.0 \\
10830.0 \\
11000.0\end{array}$ & $\begin{array}{l}2732.0 \\
2795.0 \\
2538.0 \\
2405.0\end{array}$ \\
\hline
\end{tabular}

Note: See Table 4.3 for description of columns. 
Table A3: Segment AADT, Earlier \& Later Years Density Data Base for Urban Minor Arterial \& Collector

\begin{tabular}{|c|c|c|c|c|c|c|c|c|c|c|c|}
\hline (1) & (2) & (3) & (4) & (5) & (6) & $(7)$ & 1) (8) & (9) & $(10)$ & (11) & (12) \\
\hline OBS & $\begin{array}{l}\text { CT } \\
\text { YN }\end{array}$ & CTYNM & $\begin{array}{l}\text { CTY } \\
\text { AR }\end{array}$ & $\begin{array}{l}\text { PCTY } \\
80\end{array}$ & $\begin{array}{l}\text { CTY } \\
\text { GR }\end{array}$ & $\begin{array}{l}\mathrm{CN} \\
\mathrm{TYN}\end{array}$ & CNTYNM & CNTYAR & $\begin{array}{l}\text { GRE } \\
\text { GION }\end{array}$ & $\begin{array}{l}\text { PCNT } \\
\text { Y80 }\end{array}$ & FUNC \\
\hline $\begin{array}{l}1 \\
1 \\
2 \\
2\end{array}$ & $\begin{array}{l}13 \\
13 \\
13 \\
13\end{array}$ & $\begin{array}{l}\text { richmond } \\
\text { richmond } \\
\text { richmond } \\
\text { richmond }\end{array}$ & $\begin{array}{l}16.3 \\
16.3 \\
16.3 \\
16.3\end{array}$ & $\begin{array}{l}41349 \\
41349 \\
41349 \\
41349\end{array}$ & $\begin{array}{l}a \\
a \\
a \\
a\end{array}$ & $\begin{array}{l}89 \\
89 \\
89 \\
89\end{array}$ & $\begin{array}{l}\text { wayne } \\
\text { wayne } \\
\text { wayne } \\
\text { wayne }\end{array}$ & $\begin{array}{l}404.25 \\
404.25 \\
404.25 \\
404.25\end{array}$ & $\begin{array}{l}c \\
c \\
c \\
c\end{array}$ & $\begin{array}{l}76058 \\
76058 \\
76058 \\
76058\end{array}$ & $\begin{array}{l}\text { uma } \\
\text { uma } \\
\text { uma } \\
\text { uma }\end{array}$ \\
\hline $\begin{array}{l}41 \\
41 \\
42 \\
42\end{array}$ & $\begin{array}{l}87 \\
87 \\
87 \\
87\end{array}$ & $\begin{array}{l}\text { tipton } \\
\text { tipton } \\
\text { tipton } \\
\text { tipton }\end{array}$ & $\begin{array}{l}1.6 \\
1.6 \\
1.6 \\
1.6\end{array}$ & $\begin{array}{l}5004 \\
5004 \\
5004 \\
5004\end{array}$ & $\begin{array}{ll}\mathrm{d} & 8 \\
\mathrm{~d} & 8 \\
\mathrm{~d} & \varepsilon \\
\mathrm{d} & \varepsilon\end{array}$ & $\begin{array}{l}80 t \\
80 t \\
80 t \\
80 t\end{array}$ & $\begin{array}{l}\text { tipton } \\
\text { tipton } \\
\text { tipton } \\
\text { tipton }\end{array}$ & $\begin{array}{l}260.75 \\
260.75 \\
260.75 \\
260.75\end{array}$ & $\begin{array}{l}c \\
c \\
c \\
c\end{array}$ & $\begin{array}{l}16819 \\
16819 \\
16819 \\
16819\end{array}$ & $\begin{array}{l}\text { uma } \\
\text { uma } \\
\text { uma } \\
\text { uma }\end{array}$ \\
\hline
\end{tabular}

\begin{tabular}{|c|c|c|c|c|c|c|c|c|c|c|c|c|c|}
\hline$(1)$ & (2) & (7) & (13) & (14) & (15) & (16) & (17) & $(18)$ & (19) & $(20)$ & (21) & $(22)$ & (23) \\
\hline OBS & $\begin{array}{l}C T \\
Y N\end{array}$ & $\begin{array}{l}\text { CN } \\
\text { TYN }\end{array}$ & M88TOT & $\begin{array}{l}\text { M88 } \\
\text { FUNC }\end{array}$ & $\begin{array}{l}\text { PM88 } \\
\text { FUNC }\end{array}$ & FAS & HWY & SEC & YEAR & $\mathrm{AADT}$ & CTYPOP & $\begin{array}{l}\text { CNTY } \\
\text { POP }\end{array}$ & $\begin{array}{l}\text { CNTY } \\
\mathrm{HH}\end{array}$ \\
\hline $\begin{array}{l}1 \\
1 \\
2 \\
2\end{array}$ & $\begin{array}{l}13 \\
13 \\
13 \\
13\end{array}$ & $\begin{array}{l}89 \\
89 \\
89 \\
89\end{array}$ & $\begin{array}{l}235.29 \\
235.29 \\
235.29 \\
235.29\end{array}$ & $\begin{array}{l}58.54 \\
58.54 \\
58.54 \\
58.54\end{array}$ & $\begin{array}{l}24.88 \\
24.88 \\
24.88 \\
24.88\end{array}$ & $\begin{array}{l}\text { fau } \\
\text { fau } \\
\text { fau } \\
\text { fau }\end{array}$ & $\begin{array}{l}s+121 \\
s r 121 \\
s r 121 \\
s r 121\end{array}$ & $\begin{array}{l}\ln \\
\ln \\
2 n \\
2 n\end{array}$ & $\begin{array}{l}72.50 \\
82.00 \\
72.50 \\
82.00\end{array}$ & $\begin{array}{l}6384.5 \\
9043.7 \\
5529.0 \\
5732.7\end{array}$ & $\begin{array}{l}2696.2 \\
2498.5 \\
2696.2 \\
2498.5\end{array}$ & $\begin{array}{l}193.5 \\
184.3 \\
193.5 \\
184.3\end{array}$ & $\begin{array}{l}63.6 \\
66.6 \\
63.6 \\
66.6\end{array}$ \\
\hline $\begin{array}{l}41 \\
41 \\
42 \\
42\end{array}$ & $\begin{array}{l}87 \\
87 \\
87 \\
87\end{array}$ & $\begin{array}{l}80 \\
80 \\
80 \\
80\end{array}$ & $\begin{array}{l}40.17 \\
40.17 \\
40.17 \\
40.17\end{array}$ & $\begin{array}{l}10.47 \\
10.47 \\
10.47 \\
10.47\end{array}$ & $\begin{array}{l}26.06 \\
26.06 \\
26.06 \\
26.06\end{array}$ & $\begin{array}{l}\text { fau } \\
\text { fau } \\
\text { fau } \\
\text { fau }\end{array}$ & $\begin{array}{l}\operatorname{sr} 19 \\
\operatorname{sr} 19 \\
\operatorname{sr} 19 \\
\operatorname{sr} 19\end{array}$ & $\begin{array}{l}6 b \\
6 b \\
7 b \\
7 b\end{array}$ & $\begin{array}{l}75.00 \\
82.00 \\
75.00 \\
82.00\end{array}$ & $\begin{array}{l}4337.0 \\
5170.5 \\
3491.0 \\
3922.0\end{array}$ & $\begin{array}{l}3057.5 \\
3030.6 \\
3057.5 \\
3030.6\end{array}$ & $\begin{array}{l}64.4 \\
63.3 \\
64.4 \\
63.3\end{array}$ & $\begin{array}{l}21.8 \\
22.4 \\
21.8 \\
22.4\end{array}$ \\
\hline
\end{tabular}


Table A3, continued

\begin{tabular}{|c|c|c|c|c|c|c|c|c|c|c|c|c|}
\hline (1) & (2) & (7) & (19) & (24) & (25) & (26) & (27) & (28) & (29) & $(30)$ & (31) & (32) \\
\hline OBS & $\begin{array}{l}\text { CT } \\
\text { YN }\end{array}$ & $\begin{array}{l}\text { CN } \\
\text { TYN }\end{array}$ & YEAR & CNTYVR & $\begin{array}{l}\text { CNTY } \\
\text { EMP }\end{array}$ & $\begin{array}{l}\text { CNTY } \\
\text { INC }\end{array}$ & STPOP & STHH & STVR & STEMP & STINC & STGAS \\
\hline $\begin{array}{l}1 \\
1 \\
2 \\
2\end{array}$ & $\begin{array}{l}13 \\
13 \\
13 \\
13\end{array}$ & $\begin{array}{l}89 \\
89 \\
89 \\
89\end{array}$ & $\begin{array}{l}72.50 \\
82.00 \\
72.50 \\
82.00\end{array}$ & $\begin{array}{l}63.3 \\
77.5 \\
63.3 \\
77.5\end{array}$ & $\begin{array}{l}65.4 \\
63.3 \\
65.4 \\
63.3\end{array}$ & $\begin{array}{r}9460.0 \\
10334.0 \\
9460.0 \\
10334.0\end{array}$ & $\begin{array}{l}146.0 \\
152.6 \\
146.0 \\
152.6\end{array}$ & $\begin{array}{l}46.7 \\
54.0 \\
46.7 \\
54.0\end{array}$ & $\begin{array}{l}65.8 \\
80.2 \\
65.8 \\
80.2\end{array}$ & $\begin{array}{l}44.3 \\
51.3 \\
44.3 \\
51.3\end{array}$ & $\begin{array}{r}9859.0 \\
11136.7 \\
9859.0 \\
11136.7\end{array}$ & $\begin{array}{l}2505.0 \\
2505.7 \\
2505.0 \\
2505.7\end{array}$ \\
\hline $\begin{array}{l}41 \\
41 \\
42 \\
42\end{array}$ & $\begin{array}{l}87 \\
87 \\
87 \\
87\end{array}$ & $\begin{array}{l}80 \\
80 \\
80 \\
80\end{array}$ & $\begin{array}{l}75.00 \\
82.00 \\
75.00 \\
82.00\end{array}$ & $\begin{array}{l}88.4 \\
98.7 \\
88.4 \\
98.7\end{array}$ & $\begin{array}{l}8.3 \\
9.5 \\
8.3 \\
9.5\end{array}$ & $\begin{array}{l}11926.0 \\
11879.5 \\
11926.0 \\
11879.5\end{array}$ & $\begin{array}{l}149.2 \\
152.7 \\
149.2 \\
152.7\end{array}$ & $\begin{array}{l}49.2 \\
54.2 \\
49.2 \\
54.2\end{array}$ & $\begin{array}{l}71.9 \\
79.7 \\
71.9 \\
79.7\end{array}$ & $\begin{array}{l}47.7 \\
50.6 \\
47.7 \\
50.6\end{array}$ & $\begin{array}{l}11144.5 \\
10915.0 \\
11144.5 \\
10915.0\end{array}$ & $\begin{array}{l}2763.5 \\
2471.5 \\
2763.5 \\
2471.5\end{array}$ \\
\hline
\end{tabular}

Note: See Table 4.3 for description of columns. 
Table A4: County or City Average Raw Data Base for Urban Minor Arterial \& Collector

\begin{tabular}{|c|c|c|c|c|c|c|c|c|c|c|c|}
\hline (1) & (2) & (3) & (4) & (5) & (6) & $(7)$ & ) $(8)$ & (9) & $(10)$ & (11) & (12) \\
\hline OBS & $\begin{array}{l}\text { CT } \\
\text { YN }\end{array}$ & CTYNM & $\begin{array}{l}\text { CTY } \\
\text { AR }\end{array}$ & $\begin{array}{l}\text { PCTY } \\
80\end{array}$ & $\begin{array}{l}\text { CTY } \\
\text { GR }\end{array}$ & ${ }_{\text {TYN }}^{\mathrm{CN}}$ & CNTYNM & CNTYAR & $\begin{array}{l}\text { GRE } \\
\text { GION }\end{array}$ & $\begin{array}{l}\text { PCNT } \\
\text { Y80 }\end{array}$ & FUNC \\
\hline $\begin{array}{r}2 \\
2 \\
2 \\
2 \\
-1\end{array}$ & $\begin{array}{l}13 \\
13 \\
13 \\
13 \\
13\end{array}$ & $\begin{array}{l}\text { richmond } \\
\text { richmond } \\
\text { richmond } \\
\text { richmond } \\
\text { richmond }\end{array}$ & $\begin{array}{l}16.3 \\
16.3 \\
16.3 \\
16.3 \\
16.3\end{array}$ & $\begin{array}{l}41349 \\
41349 \\
41349 \\
41349 \\
41349\end{array}$ & $\begin{array}{ll}\mathrm{a} & \varepsilon \\
\mathrm{a} & \varepsilon \\
\mathrm{a} & \varepsilon \\
\mathrm{a} & \varepsilon \\
\mathrm{a} & \varepsilon\end{array}$ & $\begin{array}{l}89 \\
89 \\
89 \\
89 \\
89\end{array}$ & $\begin{array}{l}\text { wayne } \\
\text { wayne } \\
\text { wayne } \\
\text { wayne } \\
\text { wayne }\end{array}$ & $\begin{array}{l}404.25 \\
404.25 \\
404.25 \\
404.25 \\
404.25\end{array}$ & $\begin{array}{l}\text { c } \\
c \\
c \\
c \\
c\end{array}$ & $\begin{array}{l}76058 \\
76058 \\
76058 \\
76058 \\
76058\end{array}$ & $\begin{array}{l}\text { uma } \\
\text { uma } \\
\text { uma } \\
\text { uma } \\
\text { uma }\end{array}$ \\
\hline $\begin{array}{l}42 \\
42 \\
42 \\
-2\end{array}$ & $\begin{array}{l}87 \\
87 \\
87 \\
87\end{array}$ & $\begin{array}{l}\text { tipton } \\
\text { tipton } \\
\text { tipton } \\
\text { tipton }\end{array}$ & $\begin{array}{l}1.6 \\
1.6 \\
1.6 \\
1.6\end{array}$ & $\begin{array}{l}5004 \\
5004 \\
5004 \\
5004\end{array}$ & $\begin{array}{ll}\mathrm{d} & \varepsilon \\
\mathrm{d} & 8 \\
\mathrm{~d} & 8 \\
\mathrm{~d} & 8\end{array}$ & $\begin{array}{l}80 t \\
80 t \\
80 t \\
80 t\end{array}$ & $\begin{array}{l}\text { tipton } \\
\text { tipton } \\
\text { tipton } \\
\text { tipton }\end{array}$ & $\begin{array}{l}260.75 \\
260.75 \\
260.75 \\
260.75\end{array}$ & $\begin{array}{l}c \\
c \\
c \\
c\end{array}$ & $\begin{array}{l}16819 \\
16819 \\
16819 \\
16819\end{array}$ & $\begin{array}{l}\text { uma } \\
\text { uma } \\
\text { uma } \\
\text { uma }\end{array}$ \\
\hline
\end{tabular}

\begin{tabular}{|c|c|c|c|c|c|c|c|c|c|c|c|c|c|}
\hline (1) & (2) & (7) & (13) & (14) & (15) & (16) & $(17)$ & $(18)$ & (19) & $(20)$ & (21) & $(22)$ & (23) \\
\hline OBS & $\begin{array}{l}\text { CT } \\
\text { YN }\end{array}$ & $\begin{array}{l}\text { CN } \\
\text { TYN }\end{array}$ & M88TOT & $\begin{array}{l}\text { M88 } \\
\text { FUNC }\end{array}$ & $\begin{array}{l}\text { PM88 } \\
\text { FUNC }\end{array}$ & FAS & HWY & SEC & YEAR & AADT & CTYPOP & $\begin{array}{l}\text { CNTY } \\
\text { POP }\end{array}$ & $\begin{array}{l}\text { CNTY } \\
\mathrm{HH}\end{array}$ \\
\hline $\begin{array}{r}2 \\
2 \\
2 \\
2 \\
-1\end{array}$ & $\begin{array}{l}13 \\
13 \\
13 \\
13 \\
13\end{array}$ & $\begin{array}{l}89 \\
89 \\
89 \\
89 \\
89\end{array}$ & $\begin{array}{l}235.29 \\
235.29 \\
235.29 \\
235.29 \\
235.29\end{array}$ & $\begin{array}{l}58.54 \\
58.54 \\
58.54 \\
58.54 \\
58.54\end{array}$ & $\begin{array}{l}24.88 \\
24.88 \\
24.88 \\
24.88 \\
24.88\end{array}$ & $\begin{array}{l}\text { fau } \\
\text { fau } \\
\text { fau } \\
\text { fau } \\
\text { fau }\end{array}$ & $\begin{array}{l}\text { sr } 121 \\
\text { sr } 121 \\
\text { sr } 121 \\
\text { sr } 121 \\
\text { sr } 121\end{array}$ & $\begin{array}{l}2 \\
2 \\
2 \\
2 \\
2\end{array}$ & $\begin{array}{l}70.00 \\
75.00 \\
79.00 \\
82.00 \\
85.00\end{array}$ & $\begin{array}{l}6516 \\
5397 \\
6994 \\
7330 \\
7840\end{array}$ & $\begin{array}{l}43999 \\
43898 \\
42395 \\
40752 \\
39030\end{array}$ & $\begin{array}{l}79109 \\
77351 \\
76300 \\
75000 \\
72200\end{array}$ & $\begin{array}{l}25243 \\
26191 \\
27164 \\
27172 \\
26418\end{array}$ \\
\hline $\begin{array}{l}42 \\
42 \\
42 \\
-2\end{array}$ & $\begin{array}{l}87 \\
87 \\
87 \\
87\end{array}$ & $\begin{array}{l}80 \\
80 \\
80 \\
80\end{array}$ & $\begin{array}{l}40.17 \\
40.17 \\
40.17 \\
40.17\end{array}$ & $\begin{array}{l}10.47 \\
10.47 \\
10.47 \\
10.47\end{array}$ & $\begin{array}{l}26.06 \\
26.06 \\
26.06 \\
26.06\end{array}$ & $\begin{array}{l}\text { fau } \\
\text { fau } \\
\text { fau } \\
\text { fau }\end{array}$ & $\begin{array}{l}\text { sr } 19 \\
\operatorname{sr} 19 \\
\operatorname{sr} 19 \\
\operatorname{sr} 19\end{array}$ & $\begin{array}{l}4 \\
4 \\
4 \\
4\end{array}$ & $\begin{array}{l}73.00 \\
77.00 \\
80.00 \\
84.00\end{array}$ & $\begin{array}{l}6338 \\
7247 \\
7242 \\
7870\end{array}$ & $\begin{array}{l}5028 \\
4756 \\
5004 \\
4694\end{array}$ & $\begin{array}{l}16700 \\
16900 \\
16819 \\
16200\end{array}$ & $\begin{array}{l}5529 \\
5831 \\
5993 \\
5700\end{array}$ \\
\hline
\end{tabular}


Table A4, continued

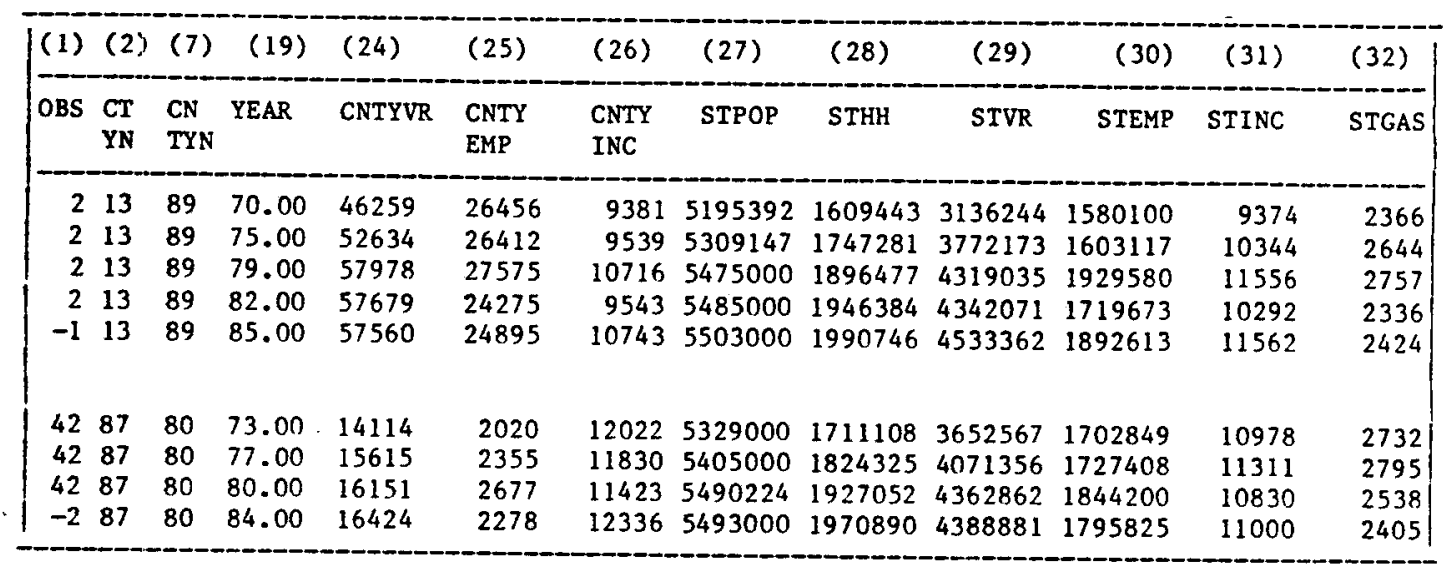

Note: See Table 4.3 for description of columns. 
Table A5: County or City Average Raw Data Base for Urban Principal Arterial

\begin{tabular}{|c|c|c|c|c|c|c|c|c|c|c|c|}
\hline (1) & (2) & (3) & (4) & (5) & (6) & $(7)$ & ) (8) & (9) & $(10)$ & (11) & (12) \\
\hline OBS & $\begin{array}{l}C r \\
\text { YN }\end{array}$ & CTYNM & $\begin{array}{l}\text { CTY } \\
\text { AR }\end{array}$ & $\begin{array}{l}\text { PCTY } \\
80\end{array}$ & $\begin{array}{l}\text { CTY } \\
\text { GR }\end{array}$ & $\begin{array}{l}\mathrm{CN} \\
\mathrm{TYN}\end{array}$ & N CNTYNM & CNTYAR & $\begin{array}{l}\text { GRE } \\
\text { GION }\end{array}$ & $\begin{array}{l}\text { PCNT } \\
\text { Y80 }\end{array}$ & FUNC \\
\hline $\begin{array}{l}11 \\
11 \\
11 \\
11 \\
-1\end{array}$ & $\begin{array}{l:}11 \\
11 \\
11 \\
11 \\
11\end{array}$ & $\begin{array}{l}\text { kokomo } \\
\text { kokomo } \\
\text { kokomo } \\
\text { kokomo } \\
\text { kokomo }\end{array}$ & $\begin{array}{l}14.5 \\
14.5 \\
14.5 \\
14.5 \\
14.5\end{array}$ & $\begin{array}{l}47808 \\
47808 \\
47808 \\
47808 \\
47808\end{array}$ & $\begin{array}{l}a \\
a \\
a \\
a \\
a\end{array}$ & $\begin{array}{ll}34 & h \\
34 & h \\
34 & h \\
34 & h \\
34 & h\end{array}$ & $\begin{array}{l}\text { howard } \\
\text { howard } \\
\text { howard } \\
\text { howard } \\
\text { howard }\end{array}$ & $\begin{array}{l}293.00 \\
293.00 \\
293.00 \\
293.00 \\
293.00\end{array}$ & $\begin{array}{l}\mathbf{n} \\
\mathbf{n} \\
\mathbf{n} \\
\mathbf{n} \\
\mathbf{n}\end{array}$ & $\begin{array}{l}86896 \\
86896 \\
86896 \\
86896 \\
86896\end{array}$ & $\begin{array}{l}\text { upa } \\
\text { upa } \\
\text { upa } \\
\text { upa } \\
\text { upa }\end{array}$ \\
\hline $\begin{array}{l}98 \\
98 \\
98 \\
-2\end{array}$ & $\begin{array}{l}87 \\
87 \\
87 \\
87 \\
t\end{array}$ & $\begin{array}{l}\text { tipton } \\
\text { tipton } \\
\text { tipton } \\
\text { tipton }\end{array}$ & $\begin{array}{l}1.6 \\
1.6 \\
1.6 \\
1.6\end{array}$ & $\begin{array}{l}5004 \\
5004 \\
5004 \\
5004\end{array}$ & $\begin{array}{ll}\mathrm{d} & \varepsilon \\
\mathrm{d} & \varepsilon \\
\mathrm{d} & \varepsilon \\
\mathrm{d} & \varepsilon\end{array}$ & $\begin{array}{ll}80 & t \\
80 & t \\
80 & t \\
80 & t\end{array}$ & $\begin{array}{l}\text { tipton } \\
\text { tipton } \\
\text { tipton } \\
\text { tipton }\end{array}$ & $\begin{array}{l}260.75 \\
260.75 \\
260.75 \\
260.75\end{array}$ & $\begin{array}{l}c \\
c \\
c \\
c\end{array}$ & $\begin{array}{l}16819 \\
16819 \\
16819 \\
16819\end{array}$ & $\begin{array}{l}\text { upa } \\
\text { upa } \\
\text { upa } \\
\text { upa }\end{array}$ \\
\hline
\end{tabular}

\begin{tabular}{|c|c|c|c|c|c|c|c|c|c|c|c|c|c|}
\hline (1) & (2) & (7) & (13) & (14) & (15) & (16) & $(17)$ & $(18)$ & $(19)$ & (20) & (21) & (22) & (23) \\
\hline OBS & $\begin{array}{l}\mathrm{CT} \\
\mathrm{YN}\end{array}$ & $\begin{array}{l}\mathrm{CN} \\
\text { TYN }\end{array}$ & M88TOT & $\begin{array}{l}\text { M88 } \\
\text { FUNC }\end{array}$ & $\begin{array}{l}\text { PM8R } \\
\text { FUNC }\end{array}$ & FAS & HWY & SEC & YEAR & AADT & CTYPOP & $\begin{array}{l}\text { CNTY } \\
\text { POP }\end{array}$ & $\begin{array}{l}\text { CNTY } \\
\mathrm{HH}\end{array}$ \\
\hline $\begin{array}{l}11 \\
11 \\
11 \\
11 \\
-1\end{array}$ & $\begin{array}{l}11 \\
11 \\
11 \\
11 \\
11\end{array}$ & $\begin{array}{l}34 \\
34 \\
34 \\
34 \\
34\end{array}$ & $\begin{array}{l}296.52 \\
296.52 \\
296.52 \\
296.52 \\
296.52\end{array}$ & $\begin{array}{l}17.47 \\
17.47 \\
17.47 \\
17.47 \\
17.47\end{array}$ & $\begin{array}{l}5.89 \\
5.89 \\
5.89 \\
5.89 \\
5.89\end{array}$ & $\begin{array}{l}\text { fap } \\
\text { fap } \\
\text { fap } \\
\text { fap } \\
\text { fap }\end{array}$ & $\begin{array}{l}\text { us } 31 \\
\text { us } 31 \\
\text { us } 31 \\
\text { us } 31 \\
\text { us } 31\end{array}$ & $\begin{array}{l}11 \\
11 \\
11 \\
11 \\
11\end{array}$ & $\begin{array}{l}73.00 \\
77.00 \\
80.00 \\
83.00 \\
87.00\end{array}$ & $\begin{array}{l}17336 \\
18283 \\
17384 \\
19926 \\
20701\end{array}$ & $\begin{array}{l}51915 \\
52109 \\
47808 \\
45714 \\
45723\end{array}$ & $\begin{array}{l}85700 \\
87100 \\
86896 \\
84900 \\
83750\end{array}$ & $\begin{array}{l}27338 \\
29907 \\
31050 \\
30560 \\
30620\end{array}$ \\
\hline $\begin{array}{l}98 \\
98 \\
98 \\
-2\end{array}$ & $\begin{array}{l}87 \\
87 \\
87 \\
87\end{array}$ & $\begin{array}{l}80 \\
80 \\
80 \\
80\end{array}$ & $\begin{array}{l}40.17 \\
40.17 \\
40.17 \\
40.17\end{array}$ & $\begin{array}{l}4.81 \\
4.81 \\
4.81 \\
4.81\end{array}$ & $\begin{array}{l}11.97 \\
11.97 \\
11.97 \\
11.97\end{array}$ & $\begin{array}{l}\text { fap } \\
\text { fap } \\
\text { fap } \\
\text { fap }\end{array}$ & $\begin{array}{l}\text { sr } 28 \\
\text { sr } 28 \\
\text { sr } 28 \\
\text { sr } 28\end{array}$ & $\begin{array}{l}2 \\
2 \\
2 \\
2\end{array}$ & $\begin{array}{l}73.00 \\
77.00 \\
80.00 \\
84.00\end{array}$ & $\begin{array}{l}6714 \\
7416 \\
7587 \\
9815\end{array}$ & $\begin{array}{l}5028 \\
4756 \\
5004 \\
4694\end{array}$ & $\begin{array}{l}16700 \\
16900 \\
16819 \\
16200\end{array}$ & $\begin{array}{l}5529 \\
5831 \\
5993 \\
5700\end{array}$ \\
\hline
\end{tabular}


Table A5, continued

\begin{tabular}{|c|c|c|c|c|c|c|c|c|c|c|c|c|}
\hline$(1)$ & (2) & (7) & (19) & (24) & (25) & (26) & (27) & (28) & (29) & (30) & (31) & $(32)$ \\
\hline OBS & $\begin{array}{l}\text { CT } \\
\text { YN }\end{array}$ & $\begin{array}{l}\text { CN } \\
\text { TYN }\end{array}$ & YEAR & CNTYVR & $\begin{array}{l}\text { CNTY } \\
\text { EMP }\end{array}$ & $\begin{array}{l}\text { CNTY } \\
\text { INC }\end{array}$ & STPOP & STHH & STVR & STEMP & STINC & STGAS \\
\hline 11 & 11 & $\begin{array}{l}34 \\
34\end{array}$ & $\begin{array}{l}73.00 \\
77.00\end{array}$ & $\begin{array}{l}64291 \\
70848\end{array}$ & $\begin{array}{l}39793 \\
38728\end{array}$ & $\begin{array}{l}12622 \\
12359\end{array}$ & $\begin{array}{l}5329000 \\
5405000\end{array}$ & 1711108 & 3652567 & 1702849 & 10978 & 2732 \\
\hline 11 & $\begin{array}{l}11 \\
11\end{array}$ & $\begin{array}{l}34 \\
34\end{array}$ & 80.00 & 73529 & 38128 & $\begin{array}{l}12359 \\
11334\end{array}$ & $\begin{array}{l}5405000 \\
5490224\end{array}$ & $\begin{array}{l}1824325 \\
1927052\end{array}$ & $\begin{array}{l}4071356 \\
4362862\end{array}$ & $\begin{array}{l}1727408 \\
1844200\end{array}$ & 11331 & $\begin{array}{l}2795 \\
2538\end{array}$ \\
\hline 11 & 11 & 34 & 83.00 & 71313 & 32599 & 10811 & 5476000 & 1953931 & 4276264 & $\begin{array}{l}1844200 \\
1679775\end{array}$ & $\begin{array}{l}10830 \\
10445\end{array}$ & $\begin{array}{l}2538 \\
2381\end{array}$ \\
\hline-1 & 11 & 34 & 87.00 & 69622 & 36198 & 13322 & 5532700 & 2009770 & 4451900 & 1945174 & 11593 & 2630 \\
\hline 98 & 87 & 80 & 73.00 & 14114 & 2020 & 12022 & 5329000 & 1711108 & 3652567 & 1702849 & 10978 & 2732 \\
\hline 98 & 87 & 80 & 77.00 & 15615 & 2355 & 11830 & 5405000 & 1824325 & 4071356 & 1727408 & 11311 & 2795 \\
\hline 98 & 87 & 80 & 80.00 & 16151 & 2677 & 11423 & 5490224 & 1927052 & 4362862 & 1844200 & 10830 & 2538 \\
\hline-2 & 87 & 80 & 84.00 & 16424 & 2278 & 12336 & 5493000 & 1970890 & 4388881 & 1795825 & 11000 & 2405 \\
\hline
\end{tabular}

Note: See Table 4.3 for description of columns. 
Table A6: County or City Average Raw Data Base for Interstate Highways

\begin{tabular}{|c|c|c|c|c|c|c|c|c|c|c|c|}
\hline$(1)$ & (2) & (3) & (4) & (5) & (6) & (7) & )$(8)$ & (9) & (10) & (11) & (12) \\
\hline OBS & $\begin{array}{l}\text { CT } \\
\text { YN }\end{array}$ & CTYNM & $\begin{array}{l}\text { CTY } \\
\text { AR }\end{array}$ & $\begin{array}{l}\text { PCTY } \\
80\end{array}$ & $\begin{array}{l}\text { CTY } \\
\text { GR }\end{array}$ & $\begin{array}{l}\mathrm{CN} \\
\mathrm{TYN}\end{array}$ & N CNTYNM & CNTYAR & $\begin{array}{l}\text { GRE } \\
\text { GION }\end{array}$ & $\begin{array}{l}\text { PCNT } \\
Y 80\end{array}$ & FUNC \\
\hline $\begin{array}{r}2 \\
2 \\
2 \\
2 \\
-1\end{array}$ & $\begin{array}{l}12 \\
12 \\
12 \\
12 \\
12\end{array}$ & $\begin{array}{l}\text { lafayette } \\
\text { lafayette } \\
\text { lafayette } \\
\text { lafayette } \\
\text { lafayette }\end{array}$ & $\begin{array}{l}11.5 \\
11.5 \\
11.5 \\
11.5 \\
11.5\end{array}$ & $\begin{array}{l}43011 \\
43011 \\
43011 \\
43011 \\
43011\end{array}$ & $\begin{array}{l}\mathbf{a} \\
\mathbf{a} \\
\mathbf{a} \\
\mathbf{a} \\
\mathbf{a}\end{array}$ & $\begin{array}{ll}79 & t \\
79 & t \\
79 & t \\
79 & t \\
79 & t\end{array}$ & $\begin{array}{l}\text { tippecanoe } \\
\text { tippecanoe } \\
\text { tippecanoe } \\
\text { tippecanoe } \\
\text { tippecanoe }\end{array}$ & $\begin{array}{l}501.50 \\
501.50 \\
501.50 \\
501.50 \\
501.50\end{array}$ & $\begin{array}{l}c \\
c \\
c \\
c \\
c\end{array}$ & $\begin{array}{l}121702 \\
121702 \\
121702 \\
121702 \\
121702\end{array}$ & $\begin{array}{l}\text { ui } \\
\text { ui } \\
\text { ui } \\
\text { ui } \\
\text { ui }\end{array}$ \\
\hline $\begin{array}{r}117 \\
117 \\
117 \\
-2\end{array}$ & $\begin{array}{l}12 \\
12 \\
12 \\
12\end{array}$ & $\begin{array}{l}\text { lafayette } \\
\text { lafayette } \\
\text { lafayette } \\
\text { lafayette }\end{array}$ & $\begin{array}{l}11.5 \\
11.5 \\
11.5 \\
11.5\end{array}$ & $\begin{array}{l}43011 \\
43011 \\
43011 \\
43011\end{array}$ & $\begin{array}{l}\text { a } \\
\text { a } \\
\text { a } \\
\text { a }\end{array}$ & $\begin{array}{ll}91 & w \\
91 & w \\
91 & w \\
91 & w\end{array}$ & $\begin{array}{l}\text { white } \\
\text { white } \\
\text { white } \\
\text { white }\end{array}$ & $\begin{array}{l}503.75 \\
503.75 \\
503.75 \\
503.75\end{array}$ & $\begin{array}{l}\mathbf{n} \\
\mathbf{n} \\
\mathbf{n} \\
\mathbf{n}\end{array}$ & $\begin{array}{l}23867 \\
23867 \\
23867 \\
23867\end{array}$ & $\begin{array}{l}r i \\
r i \\
r i \\
r i\end{array}$ \\
\hline
\end{tabular}

\begin{tabular}{|c|c|c|c|c|c|c|c|c|c|c|c|c|c|}
\hline (1) & (2) & (7) & (13) & (14) & (15) & (16) & $(17)$ & $(18)$ & $(19)$ & $(20)$ & (21) & (22) & (23) \\
\hline OBS & $\begin{array}{l}\text { CT } \\
\text { YN }\end{array}$ & $\begin{array}{l}\text { CN } \\
\text { TYN }\end{array}$ & M88TOT & $\begin{array}{l}\text { M88 } \\
\text { FUNC }\end{array}$ & $\begin{array}{l}\text { PM88 } \\
\text { FUNC }\end{array}$ & FAS & HWY & SEC & YEAR & AADT & CTYPOP & $\begin{array}{l}\text { CNTY } \\
\text { POP }\end{array}$ & $\begin{array}{l}\text { CNTY } \\
\text { HH }\end{array}$ \\
\hline $\begin{array}{r}2 \\
2 \\
2 \\
2 \\
-1\end{array}$ & $\begin{array}{l}12 \\
12 \\
12 \\
12 \\
12\end{array}$ & $\begin{array}{l}79 \\
79 \\
79 \\
79 \\
79\end{array}$ & $\begin{array}{l}1283.95 \\
1283.95 \\
1283.95 \\
1283.95 \\
1283.95\end{array}$ & $\begin{array}{l}23.62 \\
23.62 \\
23.62 \\
23.62 \\
23.62\end{array}$ & $\begin{array}{l}1.84 \\
1.84 \\
1.84 \\
1.84 \\
1.84\end{array}$ & $\begin{array}{l}\text { fai } \\
\text { fai } \\
\text { fai } \\
\text { fai } \\
\text { fai }\end{array}$ & $\begin{array}{l}165 \\
165 \\
165 \\
165 \\
165\end{array}$ & 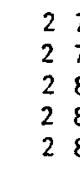 & $\begin{array}{l}75.00 \\
79.00 \\
82.00 \\
85.00 \\
87.00\end{array}$ & $\begin{array}{l}19886 \\
23659 \\
25929 \\
30610 \\
35276\end{array}$ & $\begin{array}{l}48894 \\
46286 \\
43914 \\
44150 \\
44331\end{array}$ & $\begin{array}{l}112408 \\
121600 \\
123900 \\
124500 \\
126800\end{array}$ & $\begin{array}{l}35231 \\
40113 \\
41294 \\
41031 \\
40940\end{array}$ \\
\hline $\begin{array}{r}117 \\
117 \\
117 \\
-2\end{array}$ & $\begin{array}{l}12 \\
12 \\
12 \\
12\end{array}$ & $\begin{array}{l}91 \\
91 \\
91 \\
91\end{array}$ & $\begin{array}{l}1137.14 \\
1137.14 \\
1137.14 \\
1137.14\end{array}$ & $\begin{array}{l}15.22 \\
15.22 \\
15.22 \\
15.22\end{array}$ & $\begin{array}{l}1.34 \\
1.34 \\
1.34 \\
1.34\end{array}$ & $\begin{array}{l}\text { fai } \\
\text { fai } \\
\text { fai } \\
\text { fai }\end{array}$ & $\begin{array}{l}165 \\
165 \\
165 \\
165\end{array}$ & $\begin{array}{ll}2 & 7 \\
2 & 8 \\
2 & 8 \\
2 & 8\end{array}$ & $\begin{array}{l}71.00 \\
80.00 \\
85.00 \\
87.00\end{array}$ & $\begin{array}{r}9866 \\
17915 \\
18855 \\
31126\end{array}$ & $\begin{array}{l}45905 \\
43011 \\
44150 \\
44331\end{array}$ & $\begin{array}{l}21300 \\
23867 \\
23400 \\
23840\end{array}$ & $\begin{array}{l}7051 \\
8798 \\
8817 \\
9050\end{array}$ \\
\hline
\end{tabular}


Table A6, continued

\begin{tabular}{|c|c|c|c|c|c|c|c|c|c|c|c|c|}
\hline$(1)$ & (2) & (7) & (19) & (24) & $(25)$ & $(26)$ & (27) & $(28)$ & (29) & (30) & (31) & (32) \\
\hline OBS & $\begin{array}{l}\text { CI } \\
\text { YN }\end{array}$ & $\begin{array}{l}\mathrm{CN} \\
\mathrm{TYN}\end{array}$ & YEAR & CNTYVR & $\begin{array}{l}\text { CNTY } \\
\text { EMP }\end{array}$ & $\begin{array}{l}\text { CNTY } \\
\text { INC }\end{array}$ & STPOP & STHH & STVR & STEMP & STINC & STGAS \\
\hline $\begin{array}{r}2 \\
2 \\
2 \\
2 \\
-1\end{array}$ & $\begin{array}{l}12 \\
12 \\
12 \\
12 \\
12\end{array}$ & $\begin{array}{l}79 \\
79 \\
79 \\
79 \\
79\end{array}$ & $\begin{array}{l}75.00 \\
79.00 \\
82.00 \\
85.00 \\
87.00\end{array}$ & $\begin{array}{l}70800 \\
81786 \\
84509 \\
87094 \\
87164\end{array}$ & $\begin{array}{l}31945 \\
39198 \\
37998 \\
39237 \\
42395\end{array}$ & $\begin{array}{r}9624 \\
10513 \\
9886 \\
10593 \\
11347\end{array}$ & $\begin{array}{l}5309197 \\
5475000 \\
5485000 \\
5500000 \\
5532700\end{array}$ & $\begin{array}{l}1747281 \\
1896477 \\
1946384 \\
1981498 \\
2009770\end{array}$ & $\begin{array}{l}3772173 \\
4319035 \\
4342071 \\
4460976 \\
4451900\end{array}$ & $\begin{array}{l}1603117 \\
1929580 \\
1719673 \\
1847252 \\
1945174\end{array}$ & $\begin{array}{l}10344 \\
11566 \\
10292 \\
11157 \\
11593\end{array}$ & $\begin{array}{l}2644 \\
2757 \\
2336 \\
2424 \\
2630\end{array}$ \\
\hline $\begin{array}{l}117 \\
117 \\
117 \\
-2\end{array}$ & $\begin{array}{l}12 \\
12 \\
12 \\
12\end{array}$ & $\begin{array}{l}91 \\
91 \\
91 \\
91\end{array}$ & $\begin{array}{l}71.00 \\
80.00 \\
85.00 \\
87.00\end{array}$ & $\begin{array}{l}16164 \\
22321 \\
25368 \\
26078\end{array}$ & $\begin{array}{l}4332 \\
6059 \\
6036 \\
5863\end{array}$ & $\begin{array}{r}9889 \\
10186 \\
10219 \\
12169\end{array}$ & $\begin{array}{l}5250000 \\
5490224 \\
5500000 \\
5532700\end{array}$ & $\begin{array}{l}1645683 \\
1927052 \\
1981498 \\
2009770\end{array}$ & $\begin{array}{l}3210180 \\
4362862 \\
4460976 \\
4451900\end{array}$ & $\begin{array}{l}1531495 \\
1844200 \\
1847252 \\
1945174\end{array}$ & $\begin{array}{r}9669 \\
10830 \\
11157 \\
11593\end{array}$ & $\begin{array}{l}2468 \\
2538 \\
2424 \\
2630\end{array}$ \\
\hline
\end{tabular}

Note: See Table 4.3 for description of columns. 
Appendix B 
Table Bl: City Number, Name and Land Area

\begin{tabular}{|c|c|c|}
\hline $\mid \begin{array}{l}\text { City } \\
\text { No. } \\
== \pm===\end{array}$ & 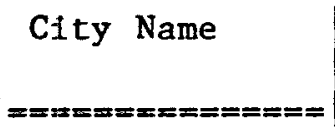 & $\begin{array}{l}\text { Area in } \\
\text { sq. mile } \\
========\end{array}$ \\
\hline 1 & INDIANAPOLIS & 352.0 \\
\hline 2 & FORT WAYNE & 52.6 \\
\hline 3 & GARY & 39.4 \\
\hline 4 & EVANSVILLE & 37.3 \\
\hline 5 & SOUTH BEND & 36.3 \\
\hline 6 & HAMMOND & 23.8 \\
\hline 7 & MUNCIE & 21.9 \\
\hline 8 & ANDERSON & 37.3 \\
\hline 9 & TFRRE HAUTE & 26.3 \\
\hline 10 & BLOOMINGTON & 10.6 \\
\hline 11 & КОКОМО & 14.5 \\
\hline 12 & LAFAYETTE & 11.5 \\
\hline 13 & RICHMOND & 16.3 \\
\hline 14 & ELKHART & 15.9 \\
\hline 15 & MISHAWAKA & 13.2 \\
\hline 16 & EAST CHICAGO & 11.2 \\
\hline 17 & NEW ALBANY & 13.2 \\
\hline 18 & MICHIGAN CITY & 19.6 \\
\hline 19 & MARION & 10.4 \\
\hline 20 & COLUMBUS & 17.1 \\
\hline 21 & PORTAGE & 22.4 \\
\hline 22 & LAWRENCE & 20.9 \\
\hline 23 & HOBART & 14.5 \\
\hline 24 & VALPARAISO & 7.6 \\
\hline 25 & LAPORTE & 11.9 \\
\hline 26 & WEST LAFAYETTE & 4.7 \\
\hline 27 & JEFFERSONVILLE & 6.1 \\
\hline 28 & VINCENNES & 7.4 \\
\hline 29 & NEW CASTLE & 5.9 \\
\hline 30 & GOSHEN & 10.0 \\
\hline 31 & GREENWOOD & 8.4 \\
\hline 32 & CARMEL & 7.8 \\
\hline 33 & LOGANSPORT & 4.9 \\
\hline 34 & CONNERSVILLE & 6.4 \\
\hline 35 & CROWN POINT & 7.0 \\
\hline 36 & HUNTINGTON & 6.6 \\
\hline 37 & FRANKFORT & 4.4 \\
\hline 38 & SEYMOUR & 6.5 \\
\hline 39 & SHELBYVILLE & 7.1 \\
\hline 40 & BEDFORD & 11.9 \\
\hline 41 & LAKE STATION & 7.6 \\
\hline 42 & PERU & 3.6 \\
\hline 43 & CRAWFORDSVILLE & 5.9 \\
\hline
\end{tabular}

\begin{tabular}{|c|c|c|}
\hline $\begin{array}{l}\text { City } \\
\text { No. } \\
======\end{array}$ & City Name & $\begin{array}{l}\text { Area in } \\
\text { sq. mile } \\
========\end{array}$ \\
\hline 44 & BEECH GROVE & 4.1 \\
\hline 45 & WABASH & 5.6 \\
\hline 46 & MADISON & 8.3 \\
\hline 47 & NOBLESVILLE & 5.9 \\
\hline 48 & FRANKLIN & 6.1 \\
\hline 49 & LEBANON & 6.0 \\
\hline 50 & GREENFIELD & 5.1 \\
\hline 51 & WASHINGTON & 4.1 \\
\hline 52 & MARTINSVILLE & 3.2 \\
\hline 53 & ELWOOD & 4.1 \\
\hline 54 & WARSAW & 7.3 \\
\hline 55 & GREENSBURG & 3.0 \\
\hline 56 & JASPER & 5.7 \\
\hline 57 & PRINCETON & 3.5 \\
\hline 58 & BLUFFTON & 5.0 \\
\hline 59 & TELL CITY & 3.8 \\
\hline 60 & DECATUR & 3.1 \\
\hline 61 & GREENCASTLE & 3.7 \\
\hline 62 & AUBURN & 3.8 \\
\hline 63 & BRAZIL & 2.6 \\
\hline 64 & PLYMOUTH & 5.8 \\
\hline 65 & MT. VERNON & 2.2 \\
\hline 66 & HARTFORD CITY & 3.3 \\
\hline 67 & KENDALLVILLE & 4.3 \\
\hline 68 & PORTLAND & 2.4 \\
\hline 69 & NEW HAVEN & 2.7 \\
\hline 70 & GAS CITY & 2.4 \\
\hline 71 & LINTON & 5.0 \\
\hline 72 & BOONVILLE & 2.8 \\
\hline 73 & RUSHVILLE & 1.8 \\
\hline 74 & ALEXANDRIA & 2.4 \\
\hline 75 & NORTH VERNON & 4.1 \\
\hline 76 & WINCHESTER & 2.4 \\
\hline 77 & WHITING & 1.9 \\
\hline 78 & CHARLESTOWN & 2.2 \\
\hline 79 & ANGOLA & 2.1 \\
\hline 80 & HUNTINGBURG & 2.9 \\
\hline 81 & SALEM & 2.9 \\
\hline 82 & CLINTON & 2.0 \\
\hline 83 & MONTICELLO & 2.6 \\
\hline 84 & COLUMBIA CITY & 2.1 \\
\hline 85 & SCOTTSBURG & 3.5 \\
\hline 86 & ROCHESTER & 2.5 \\
\hline 87 & TIPTON & 1.6 \\
\hline
\end{tabular}

Source: (i) County and City Data Book, Bureau of Census.

(ii) Statistical Information system (STATIS), The Economic Development Information Network (EDIN), Indiana University. 
Table B2: County Number, Name and Land Area

\begin{tabular}{|c|c|c|}
\hline \multicolumn{2}{|r|}{ County } & Area in \\
\hline No. & 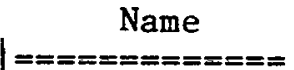 & $\begin{array}{l}\mathrm{sq} \cdot \mathrm{mile} \\
========x\end{array}$ \\
\hline 1 & adams & 341.25 \\
\hline 2 & allen & 662.00 \\
\hline 3 & bartholomew & 407.25 \\
\hline 4 & benton & 407.50 \\
\hline 5 & blackford & 166.25 \\
\hline $\begin{array}{l}6 \\
7\end{array}$ & $\begin{array}{l}\text { boone } \\
\text { brown }\end{array}$ & $\begin{array}{l}424.25 \\
313.75\end{array}$ \\
\hline 8 & carroll & 372.50 \\
\hline 9 & cass & 414.25 \\
\hline 10 & clark & 378.00 \\
\hline 11 & clay & 361.00 \\
\hline 12 & clinton & 405.50 \\
\hline 13 & crawford & 308.25 \\
\hline 14 & daviess & 431.50 \\
\hline 15 & dearborn & 306.75 \\
\hline 16 & decatur & 372.25 \\
\hline 17 & dekalb & 364.50 \\
\hline 18 & delaware & 393.00 \\
\hline 19 & dubois & 430.00 \\
\hline 20 & elkhart & 466.50 \\
\hline 21 & fayette & 215.00 \\
\hline 22 & floyd & 149.75 \\
\hline 23 & fountain & 397.75 \\
\hline 24 & franklin & 387.25 \\
\hline 25 & fulton & 368.75 \\
\hline 26 & gibson & 492.00 \\
\hline 27 & grant & 416.50 \\
\hline 28 & greene & 546.75 \\
\hline 29 & hamilton & 398.75 \\
\hline 30 & hancock & 306.50 \\
\hline 31 & harrison & 484.25 \\
\hline 32 & hendricks & 411.00 \\
\hline 33 & henry & 396.00 \\
\hline 34 & howard & 293.00 \\
\hline 35 & huntington & 366.75 \\
\hline 36 & jackson & 515.25 \\
\hline 37 & jasper & 561.25 \\
\hline 38 & jay & 384.50 \\
\hline 39 & jefferson & 363.75 \\
\hline 40 & jennings & 377.75 \\
\hline 41 & johnson & 319.50 \\
\hline 42 & knox & 519.00 \\
\hline 43 & kosciusko & 540.00 \\
\hline 44 & lagrange & 380.25 \\
\hline 45 & lake & 504.00 \\
\hline 46 & laporte & 601.75 \\
\hline 47 & lawrence & 453.75 \\
\hline
\end{tabular}

\begin{tabular}{|c|c|c|}
\hline \multicolumn{2}{|c|}{ County } & \multirow{2}{*}{$\begin{array}{c}\text { Area in } \\
\mathrm{sq} \cdot \mathrm{mile} \\
========\end{array}$} \\
\hline$===$ & $\begin{array}{c}\text { Name } \\
====:===m====\end{array}$ & \\
\hline 48 & madison & 453.00 \\
\hline 49 & marion & 395.00 \\
\hline 50 & marshall & -443.75 \\
\hline 51 & martin & 340.50 \\
\hline 52 & miami & 371.00 \\
\hline 53 & monroe & 385.25 \\
\hline 54 & montgomery & 505.50 \\
\hline 55 & morgan & 408.25 \\
\hline 56 & newton & 404.00 \\
\hline 57 & noble & 412.75 \\
\hline 58 & ohio & 87.00 \\
\hline 59 & orange & 407.25 \\
\hline 60 & owen & 387.00 \\
\hline 61 & parke & 444.25 \\
\hline 62 & perry & 382.00 \\
\hline 63 & pike & 339.50 \\
\hline 64 & porter & 420.25 \\
\hline 65 & posey & 410.00 \\
\hline 66 & pulaski & 434.50 \\
\hline 67 & putnam & 484.00 \\
\hline 68 & randolph & 454.75 \\
\hline 69 & ripley & 445.75 \\
\hline 70 & rush & 408.25 \\
\hline 71 & st. joseph & 460.75 \\
\hline 72 & $\operatorname{scot} t$ & 192.00 \\
\hline 73 & shelby & 411.50 \\
\hline 74 & spencer & 399.00 \\
\hline 75 & starke & 309.25 \\
\hline 76 & steuben & 308.25 \\
\hline 77 & sullivan & 453.25 \\
\hline 78 & switzerland & 223.00 \\
\hline 79 & tippecanoe & 501.50 \\
\hline 80 & tipton & 260.75 \\
\hline 81 & union & 164.00 \\
\hline 82 & vanderburgh & 237.25 \\
\hline 83 & vermillion & 260.75 \\
\hline 84 & vigo & 407.50 \\
\hline 85 & wabash & 398.00 \\
\hline 86 & warren & 366.50 \\
\hline 87 & warrick & 391.00 \\
\hline 88 & washington & 516.00 \\
\hline 89 & wayne & 404.25 \\
\hline 90 & wells & 369.50 \\
\hline 91 & white & 503.75 \\
\hline 92 & whitley & 336.25 \\
\hline & STAT & 35973.25 \\
\hline
\end{tabular}

Source: (i) County and City Data Book, Bureau of Census.

(ii) Statistical Information system, The Economic Development Information Network (EDIN), Indiana University. 
Table B3: 1988 County Functional Class Mileage of State of Indiana

\begin{tabular}{|c|c|c|c|c|c|c|c|c|c|c|}
\hline No. & $\begin{array}{l}\text { County } \\
\text { - Name }\end{array}$ & Total & $\begin{array}{l}\text { Rural } \\
\text { total }\end{array}$ & $\begin{array}{l}\text { Urban } \\
\text { tota1 }\end{array}$ & UMAC & TUMAC & UPA & ZUPA & INT & 7INT \\
\hline (1) & $\begin{array}{c}(2) \\
x=== \pm=\end{array}$ & (3) & (4) & (5) & (6) & (7) & $(8)$ & (9) & $(10)$ & (11) \\
\hline $\begin{array}{l}1 \\
2 \\
3\end{array}$ & $\begin{array}{l}\text { ADAMS } \\
\text { ALLEN } \\
\text { BARTHOLOMEW }\end{array}$ & $\begin{array}{r}875.86 \\
2458.03 \\
1009.88\end{array}$ & $\begin{array}{r}819.27 \\
1393.66 \\
767.47\end{array}$ & $\begin{array}{r}56.59 \\
1064.37 \\
242.41\end{array}$ & $\begin{array}{r}16.70 \\
237.75 \\
47.86\end{array}$ & $\begin{array}{l}29.51 \\
22.34 \\
19.74\end{array}$ & $\begin{array}{r}5.58 \\
59.02 \\
22.29\end{array}$ & $\begin{array}{l}9.86 \\
5.55 \\
9.20\end{array}$ & $\begin{array}{r}0.00 \\
30.46 \\
22.52\end{array}$ & $\begin{array}{l}0.00 \\
1.24 \\
2.23\end{array}$ \\
\hline $\begin{array}{l}4 \\
5 \\
6\end{array}$ & $\begin{array}{l}\text { BENTON } \\
\text { BLACKFORD } \\
\text { BOONE }\end{array}$ & $\begin{array}{r}833.95 \\
431.63 \\
1077.94\end{array}$ & $\begin{array}{l}833.95 \\
372.65 \\
992.75\end{array}$ & $\begin{array}{r}0.00 \\
58.98 \\
85.19\end{array}$ & $\begin{array}{r}0.00 \\
11.94 \\
19.63\end{array}$ & $\begin{array}{r}0.00 \\
20.24 \\
23.04\end{array}$ & $\begin{array}{l}0.00 \\
6.87 \\
7.76\end{array}$ & $\begin{array}{r}0.00 \\
11.65 \\
9.11\end{array}$ & $\begin{array}{r}0.00 \\
0.00 \\
35.02\end{array}$ & $\begin{array}{l}0.00 \\
0.00 \\
3.25\end{array}$ \\
\hline 7 & BROWN & 453.91 & 453.91 & 0.00 & 0.00 & 0.00 & 0.00 & 0.00 & 0.00 & 0.00 \\
\hline 8 & CARROLL & 915.29 & 915.29 & 0.00 & 0.00 & 0.00 & 0.00 & 0.00 & 0.00 & 0.00 \\
\hline 9 & CASS & 1108.65 & 970.43 & 138.22 & 23.92 & 17.31 & 11.77 & 8.52 & 5.50 & 0.50 \\
\hline $\begin{array}{l}10 \\
11\end{array}$ & $\begin{array}{l}\text { CLARK } \\
\text { CLAY }\end{array}$ & $\begin{array}{l}875.49 \\
870.21\end{array}$ & $\begin{array}{l}587.26 \\
779.74\end{array}$ & $\begin{array}{r}288.23 \\
90.47\end{array}$ & $\begin{array}{l}80.03 \\
18.18\end{array}$ & $\begin{array}{l}27.77 \\
20.10\end{array}$ & $\begin{array}{r}18.49 \\
9.46\end{array}$ & $\begin{array}{r}6.42 \\
10.46\end{array}$ & $\begin{array}{l}28.55 \\
12.53\end{array}$ & $\begin{array}{l}3.26 \\
1.44\end{array}$ \\
\hline 12 & CLINTON & 999.65 & 921.87 & 77.78 & 19.76 & 25.40 & 9.53 & 12.25 & 10.47 & 1.05 \\
\hline 13 & CRAWFORD & 587.81 & 587.81 & 0.00 & 0.00 & 0.00 & 0.00 & 0.00 & 16.98 & 2.89 \\
\hline 14 & DAVIESS & 976.55 & 895.45 & 81.10 & 25.53 & 31.48 & 5.43 & 6.70 & 0.00 & 0.00 \\
\hline 15 & DEARBORN & 678.62 & 678.62 & 0.00 & 0.00 & 0.00 & 0.00 & 0.00 & 17.01 & 2.51 \\
\hline $\begin{array}{l}16 \\
17\end{array}$ & $\begin{array}{l}\text { DECATUR } \\
\text { DERALB }\end{array}$ & $\begin{array}{l}805.81 \\
961.35\end{array}$ & $\begin{array}{l}750.14 \\
903.01\end{array}$ & $\begin{array}{l}55.67 \\
58.34\end{array}$ & $\begin{array}{l}14.36 \\
14.24\end{array}$ & $\begin{array}{l}25.79 \\
24.41\end{array}$ & $\begin{array}{l}5.49 \\
2.99\end{array}$ & $\begin{array}{l}9.86 \\
5.13\end{array}$ & $\begin{array}{l}22.72 \\
18.21\end{array}$ & $\begin{array}{l}2.82 \\
1.89\end{array}$ \\
\hline 18 & DELAWARE & 1346.18 & 794.25 & 551.93 & 152.31 & 27.60 & 41.37 & 7.50 & 32.54 & 2.42 \\
\hline 19 & DUBOIS & 886.12 & 766.06 & 120.06 & 33.29 & 27.73 & 12.56 & 10.46 & 3.32 & 0.37 \\
\hline 20 & ELKHART & .62 & 915.09 & 688.53 & 164.66 & 23.91 & 56.81 & 8.25 & 21.33 & 1.33 \\
\hline 21 & FAYETTE & 482.49 & 399.41 & 83.08 & 16.90 & 20.34 & 12.25 & 14.74 & 0.00 & 0.00 \\
\hline 22 & FLOYD & 518.84 & 309.06 & 209.78 & 49.01 & 23.36 & 12.35 & 5.89 & 16.23 & 3.13 \\
\hline 23 & FOUNTAIN & 878.67 & 878.67 & 0.00 & 0.00 & 0.00 & 0.00 & 0.00 & 16.95 & 1.93 \\
\hline 24 & FRANKLIN & 745.82 & 745.82 & 0.00 & 0.00 & 0.00 & 0.00 & 0.00 & 4.36 & 0.58 \\
\hline 25 & FULTON & 938.69 & 894.40 & 44.29 & 8.19 & 18.49 & 2.90 & 6.55 & 0.00 & 0.00 \\
\hline 26 & GIBSON & .51 & 11 & 63.04 & 14.05 & 22.29 & 4.63 & 7.34 & 4.74 & 0.38 \\
\hline 27 & GRANT & .11 & 970.27 & 286.84 & 78.59 & 27.40 & 22.99 & 8.01 & 19.77 & 1.57 \\
\hline 28 & GREENE & 113 & 108 & 48.04 & 9.86 & 20.52 & 4.11 & 8.56 & 0.00 & 0.00 \\
\hline 29 & HAMILTON & 1281.80 & 1006.28 & 275.52 & 67.53 & 24.51 & 18.32 & 6.65 & 13.43 & 1.05 \\
\hline 30 & HANCOCK & 835.35 & 753.25 & 82.10 & 22.96 & 27.97 & 8.99 & 10.95 & 19.12 & 2.29 \\
\hline 31 & HARRISON & 989.41 & 989.41 & 0.00 & 0.00 & 0.00 & 0.00 & 0.00 & 17.33 & 1.75 \\
\hline 32 & HENDRICKS & 1045.74 & 917.35 & 128.39 & 22.50 & 17.52 & 16.72 & 13.02 & 26.75 & 2.56 \\
\hline 33 & HENRY & 1077.92 & 960.77 & 117.15 & 26.26 & 22.42 & 11.11 & 9.48 & 20.31 & 1.88 \\
\hline 34 & HOWARD & 953.21 & 656.69 & 296.52 & 91.39 & 30.82 & 17.47 & 5.89 & 0.00 & 0.00 \\
\hline 35 & HUNTINGTON & 970.46 & 859.19 & 111.27 & 29.04 & 26.10 & 13.05 & 11.73 & 23.96 & 2.47 \\
\hline 36 & JACKSON & 1030.05 & 915.22 & 114.83 & 31.27 & 27.23 & 5.97 & 5.20 & 22.01 & 2.14 \\
\hline 37 & JASPER & 1155.71 & 1155.71 & 0.00 & 0.00 & 0.00 & 0.00 & 0.00 & 32.01 & 2.77 \\
\hline 38 & JAY & 929.89 & 879.97 & 49.92 & 14.75 & 29.55 & 4.21 & 8.43 & 0.00 & 0.00 \\
\hline 39 & JEFFERSON & 735.91 & 662.28 & 73.63 & 17.33 & 23.54 & 12.59 & 17.10 & 0.00 & 0.00 \\
\hline 40 & JENNINGS & 760.75 & 714.30 & 46.45 & 10.14 & 21.83 & 8.60 & 18.51 & 0.00 & 0.00 \\
\hline 41 & JOHNSON & & & & & & 20.09 & & 17.23 & 1.97 \\
\hline 42 & RNOX & 1200.50 & 106 & 139.9 & 30.58 & 21.84 & 8.24 & 5.89 & 8.71 & 0.73 \\
\hline 43 & KOSCIUSKO & 1481.42 & 1355.91 & 125.51 & 22.58 & 17.99 & 11.12 & 8.86 & 4.22 & 0.28 \\
\hline
\end{tabular}


Table B3, continued

\begin{tabular}{|c|c|c|c|c|c|c|c|c|c|c|}
\hline \multicolumn{2}{|c|}{ County } & \multirow[t]{2}{*}{ Total } & \multirow{2}{*}{$\begin{array}{l}\text { Rural } \\
\text { total }\end{array}$} & \multirow{2}{*}{$\begin{array}{l}\text { Urban } \\
\text { total }\end{array}$} & \multirow[t]{2}{*}{ UMAC } & \multirow[t]{2}{*}{ ZUMAC } & \multirow[t]{2}{*}{ UPA } & \multirow[t]{2}{*}{ ZUPA } & \multirow[t]{2}{*}{ INT } & \multirow[t]{2}{*}{ \%INT } \\
\hline No & Name & & & & & & & & & \\
\hline (1) & (2) & (3) & (4) & (5) & (6) & (7) & (8) & (9) & $(10)$ & (11) \\
\hline \multicolumn{2}{|r|}{$== \pm=-$} & $=$ & & & & & & & & \\
\hline 44 & LAGRANGE & 942.24 & 942.24 & 0.00 & 0.00 & 0.00 & 0.00 & 0.00 & 24.78 & 2.63 \\
\hline 45 & LAKF & 2447.36 & 445.45 & 2001.91 & 428.78 & 21.42 & 134.64 & 6.73 & 79.69 & 3.26 \\
\hline 46 & LAPORTE & 1621.55 & 1219.09 & 402.46 & 91.63 & 22.77 & 40.35 & 10.03 & 37.79 & 2.33 \\
\hline 47 & LAWRENCE & 919.76 & 788.70 & 131.06 & 36.10 & 27.54 & 9.60 & 7.32 & 6.08 & 0.66 \\
\hline 48 & MADISON & 1556 & 980 & 575. & 149.01 & 25 & 42.86 & 7.45 & 17 & 1.15 \\
\hline 49 & MARION & 3336.12 & 0.00 & 3336.12 & 801.86 & 24.04 & 199.77 & 5.99 & 130.83 & 3.92 \\
\hline 50 & MARSHALL & 1180.47 & 1110.86 & 69.61 & 15.99 & 22.97 & 3.17 & 4.55 & 3.80 & 0.32 \\
\hline 51 & MARTIN & 472.51 & 472.51 & 0.00 & 0.00 & 0.00 & 0.00 & 0.00 & 0.00 & 0.00 \\
\hline 52 & MIAMI & 977.14 & 878 & 98.65 & 20.94 & 21.23 & 10.01 & 10.15 & 1.56 & 0.16 \\
\hline 53 & MONROE & 930.62 & 668 & 262 & 71 & 27. & 20.31 & 7.75 & 6.33 & 0.68 \\
\hline 54 & MONTGOMERY & 1095.51 & 1017.67 & 77.84 & 24.86 & 31.94 & 7.18 & 9.22 & 23.20 & 2.12 \\
\hline 55 & MORGAN & 905.00 & 809.24 & 95.76 & 20.96 & 21.89 & 10.60 & 11.07 & 11.34 & 1.25 \\
\hline 56 & NEWTON & 828.71 & 828.71 & 0.00 & 0.00 & 0.00 & 0.00 & 0.00 & 2.88 & 0.35 \\
\hline 57 & NOBLE & 101 & 97 & 48 & 12.83 & 26. & 4.02 & 8.22 & 0.00 & 0.00 \\
\hline 58 & OHIO & 174.91 & 174.91 & 0.00 & 0.00 & 0.00 & 0.00 & 0.00 & 0.00 & 0.00 \\
\hline 59 & ORANGE & 751.39 & 751.39 & 0.00 & 0.00 & 0.00 & 0.00 & 0.00 & 0.00 & 0.00 \\
\hline 60 & OWEN & 733.07 & 733.07 & 0.00 & 0.00 & 0.00 & 0.00 & 0.00 & 0.00 & 0.00 \\
\hline 61 & PARKE & 877.36 & 877 & 0.00 & 0.00 & 0.00 & 0.00 & 0.00 & 0.00 & 0.00 \\
\hline & PERR & 98.77 & 64 & 54. & 9.39 & 17. & 5 & 7 & 12 & 1.81 \\
\hline 63 & PIKE & 658.17 & 658.17 & 0.00 & 0.00 & 0.00 & 0.00 & 0.00 & 0.00 & 0.00 \\
\hline 64 & PORTER & 1308.12 & 789.42 & 518.70 & 132.31 & 25.51 & 54.74 & 10.55 & 32.16 & 2.46 \\
\hline 65 & POSEY & .45 & 99 & 55. & 15.09 & 21 & 6.05 & 10.91 & 17.44 & 2.02 \\
\hline & PUL & 10 & 10 & & & 00 & & 0.00 & 0.00 & .00 \\
\hline 67 & PUTNAM & 962.05 & 904.85 & 57.20 & 12.74 & 22.27 & 6.68 & 11.68 & 20 & 2.11 \\
\hline 68 & RANDOLPH & 1075.72 & 1042.48 & 33.24 & 9.20 & 27.68 & 3.37 & 10.14 & 1.35 & 0.13 \\
\hline 69 & RIPLEY & 930.01 & 930.01 & 0.00 & 0.00 & 0.00 & 0.00 & 0.00 & 8.40 & 0.90 \\
\hline 70 & RUSH & 883.24 & 840.14 & 43.10 & 8.14 & 18.89 & 7.32 & 16.98 & 0.00 & 0.00 \\
\hline 71 & ST. JOSEPH & 1867.02 & 750.97 & 1116.05 & 173.78 & 15.57 & 71.06 & 6.37 & 39.38 & 2.11 \\
\hline 72 & SCOTT & 453.49 & 417.94 & 35.55 & 8.85 & 24.89 & 0.00 & 0.00 & 11.55 & 2.55 \\
\hline 73 & SHELBY & 1018.46 & 936.90 & 81.56 & 23.56 & 28.89 & 8.59 & 10.53 & 27.21 & 2.67 \\
\hline 74 & SPENCER & 935.96 & 935.96 & 0.00 & 0.00 & 0.00 & 0.00 & 0.00 & 9.25 & 0.99 \\
\hline 75 & STARKE & 839.39 & 839.39 & 0.00 & 0.00 & 0.00 & 0.00 & 0.00 & 0.00 & 0.00 \\
\hline 76 & STEUBEN & 838.24 & 79 & 42 . & 7.99 & 18.63 & 4.58 & 10.68 & 41.19 & 4.91 \\
\hline 77 & SULLIVAN & 1072.28 & 1072.28 & 0.00 & 0.00 & 0.00 & 0.00 & 0.00 & 0.00 & 0.00 \\
\hline 78 & SWITZERLAND & 435.43 & 435.43 & 0.00 & 0.00 & 0.00 & 0.00 & 0.00 & 0.00 & 0.00 \\
\hline 79 & TIPPECANOE & 1283.95 & 923.63 & 360.32 & 92.01 & 25.54 & 45.89 & 12.74 & 23.63 & 1.84 \\
\hline 80 & TIPTON & .93 & 24.76 & 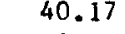 & 47 & & 81 & 11.97 & 0.00 & 00 \\
\hline 81 & UNION & 05 & 32 & 0.00 & 0.00 & 0.00 & 0.00 & 0.00 & 0.00 & 0.00 \\
\hline 82 & VANDERBURGH & 1063.40 & 359.98 & 703.42 & .78 .89 & 25.43 & 37.47 & 5.33 & 7.16 & 0.67 \\
\hline 83 & VERMIZLION & 561.02 & 502.35 & 58.67 & 9.09 & 15.49 & 5.18 & 8.83 & 8.31 & 1.48 \\
\hline 84 & VIGO & 1332.56 & 827.94 & 504.62 & 113.57 & 22.51 & 45.25 & 8.97 & 16.43 & 1.23 \\
\hline
\end{tabular}


Table B3, continued

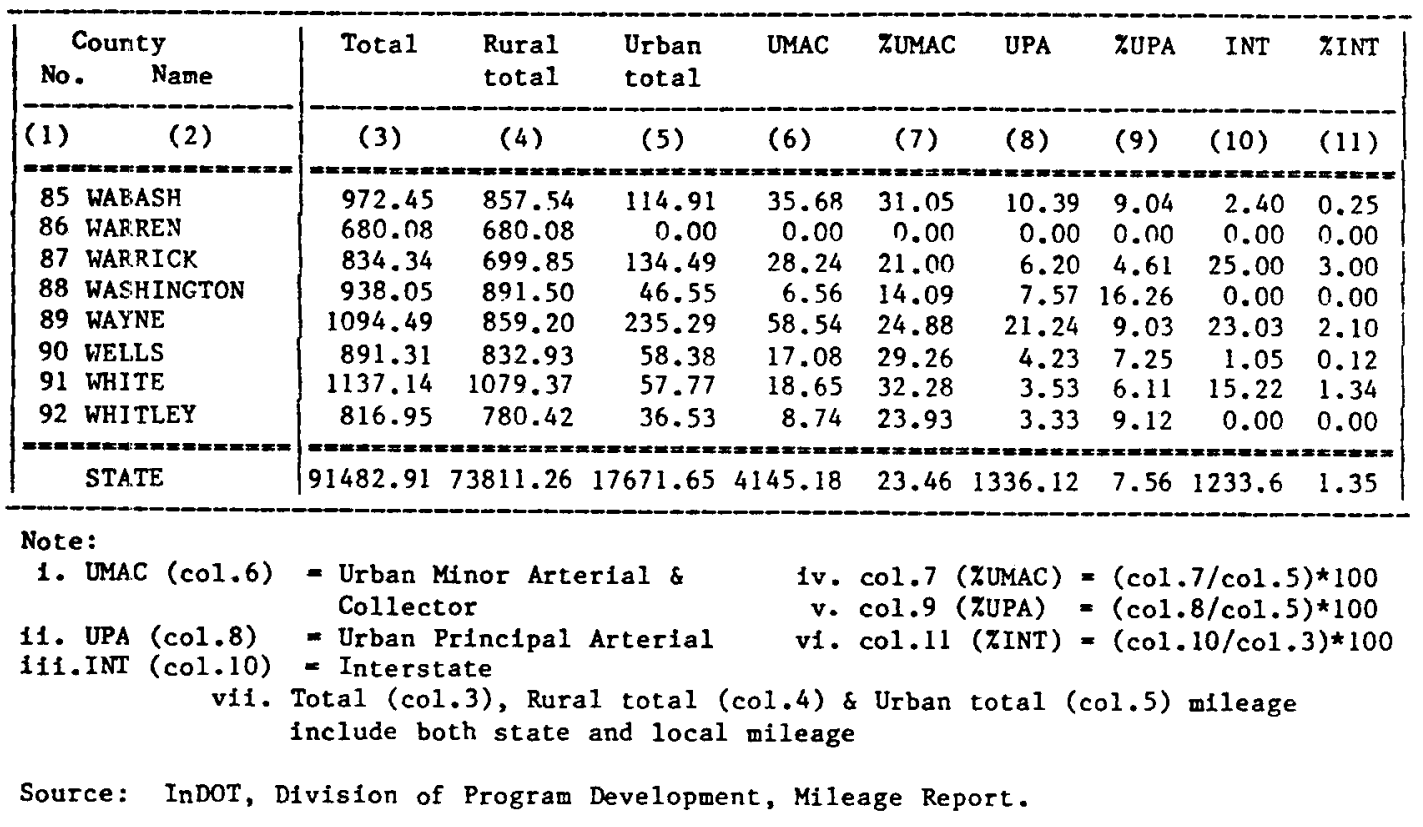


Table B4: Traffic Data Retrieval Form

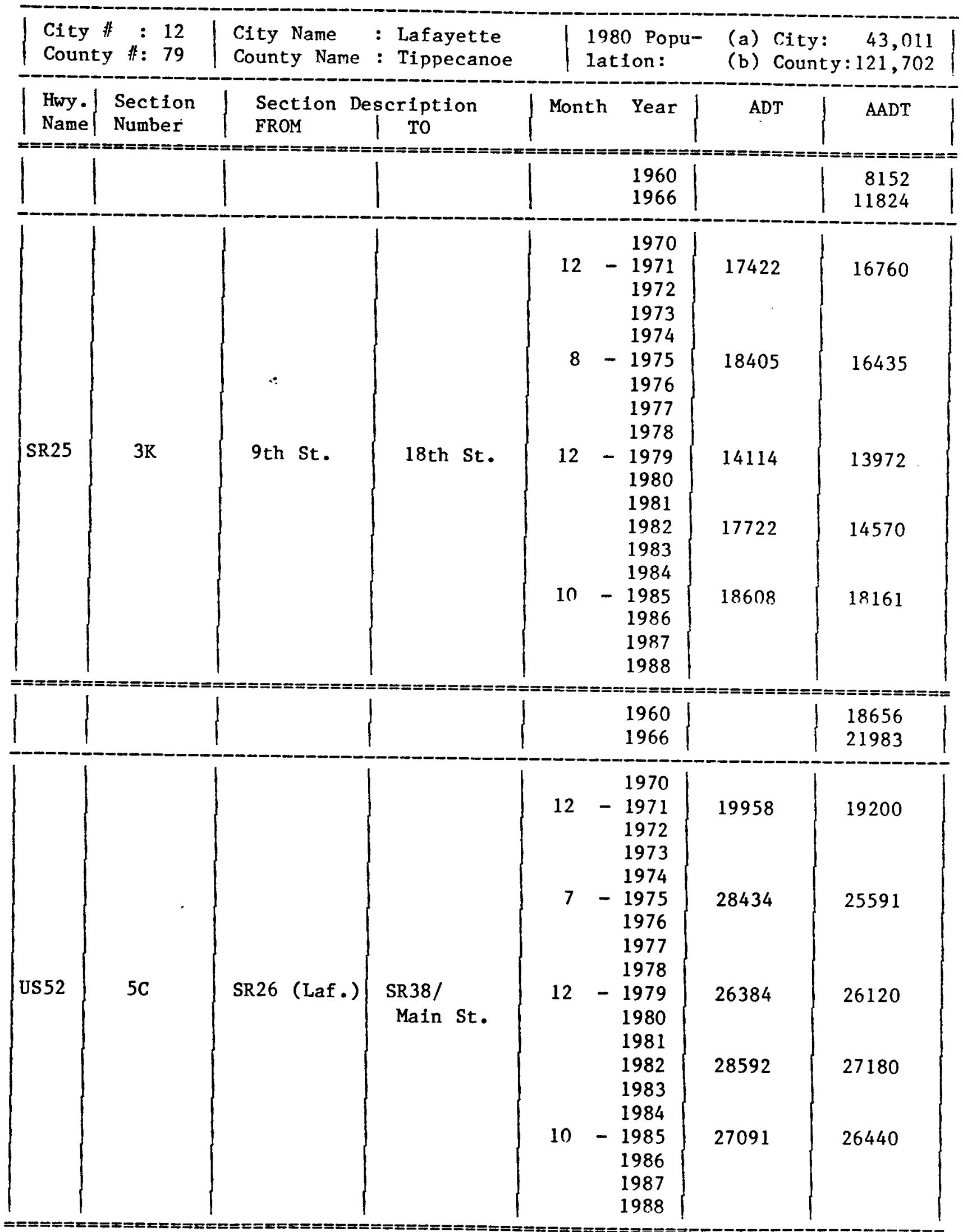


Appendix C 
Table Cl: Best Models for Interstate Highways

[Data Format: Raw and County Avg. by Year]

\begin{tabular}{|c|c|c|c|c|c|c|c|c|c|c|c|c|c|c|}
\hline $\begin{array}{l}\text { Case } \\
N \\
-\end{array}$ & $\left|\begin{array}{l}\text { of } \\
\text { Var } \\
-=\end{array}\right|$ & $R^{2}$ & $\mathrm{R}_{\mathrm{a}}^{2}$ & PC & RMSE & AIC & $c_{p}$ & Var & riables 1 & in Mode 1 & & & $b$-coef & ficients \\
\hline \begin{tabular}{|l|}
$R I+U I$ \\
$A L L$ \\
$N=313$ \\
\end{tabular} & $\begin{array}{r}1 \\
2 \\
3 \\
4 \\
-\end{array}$ & $\left|\begin{array}{l}0.5030 \\
0.5229\end{array}\right|$ & $\begin{array}{l}0.5014 \\
0.5199\end{array}$ & $\begin{array}{l}0.5034 \\
0.4863\end{array}$ & $\begin{array}{l}13714 \\
13457\end{array}$ & $\begin{array}{l}5965.4 \\
5954.5\end{array}$ & $\begin{array}{l}61.64 \\
48.75\end{array}$ & $\begin{array}{l}\text { CNTYPOP } \\
\text { CNTYPOP }\end{array}$ & STEMP & & & $\begin{array}{l}0.0629 \\
0.0631\end{array}$ & 0.0242 & \\
\hline $\begin{array}{l}\mathrm{RI}+\mathrm{UI} \\
\text { CTYGR=A } \\
\mathrm{N}=79\end{array}$ & $\begin{array}{r}1 \\
2 \\
3 \\
4 \\
-\end{array}$ & $\begin{array}{l}0.2376 \\
0.2998\end{array}$ & $\begin{array}{l}0.2277 \\
0.2814\end{array}$ & $\mid \begin{array}{l}0.8020 \\
0.7555\end{array}$ & $\begin{array}{l}8105 \\
7818\end{array}$ & $\begin{array}{l}1424.0 \\
1419.3\end{array}$ & $\begin{array}{l}201.68 \\
181.12\end{array}$ & $\begin{array}{l}\text { CNTYINC } \\
\text { CNTY INC }\end{array}$ & CNTY POP & & & $\begin{array}{l}3.2232 \\
2.2224\end{array}$ & 0.0630 & \\
\hline $\begin{array}{l}R I+U I \\
C T Y G R=A+ \\
N=76\end{array}$ & $\begin{array}{l}1 \\
2 \\
3 \\
4\end{array}$ & $\left|\begin{array}{l}0.3917 \\
0.4028\end{array}\right|$ & $\begin{array}{l}0.3835 \\
0.3864\end{array}$ & $\begin{array}{l}0.6412 \\
0.6463\end{array}$ & $\begin{array}{l}19887 \\
19839\end{array}$ & $\left|\begin{array}{l}1506.4 \\
1507.0\end{array}\right|$ & $\begin{array}{l}40.50 \\
40.44\end{array}$ & $\begin{array}{l}\text { CNTYPOP } \\
\text { CNTYPOP }\end{array}$ & STINC & & & $\begin{array}{l}0.0587 \\
0.0584\end{array}$ & 4.8356 & \\
\hline $\begin{array}{l}\text { RI+UI } \\
\text { CTYGR=B } \\
\text { N=41 }\end{array}$ & $\begin{array}{l}1 \\
2 \\
3 \\
4\end{array}$ & $\left|\begin{array}{l}0.3851 \\
0.6015 \\
0.6295 \\
0.6415\end{array}\right|$ & $\begin{array}{l}0.3693 \\
0.5806 \\
0.5994 \\
0.6017 \\
\end{array}$ & $\begin{array}{l}0.6780 \\
0.4614 \\
0.4506 \\
0.4581\end{array}$ & $\begin{array}{l}15940 \\
12999 \\
12703 \\
12668\end{array}$ & $\begin{array}{l}795.4 \\
779.6 \\
778.7 \\
779.3\end{array}$ & $\begin{array}{r}18.47 \\
0.95 \\
0.43 \\
1.34 \\
\end{array}$ & $\begin{array}{l}\text { CNTYEMP } \\
\text { CNTYHH } \\
\text { CNTYHH } \\
\text { CNTYHH } \\
\end{array}$ & $\begin{array}{l}\text { CTYPOP } \\
\text { CTYPOP } \\
\text { CTYPOP }\end{array}$ & $\begin{array}{l}\text { STHH } \\
\text { STHH }\end{array}$ & CNTYINC & $\begin{array}{l}0.1030 \\
0.1102 \\
0.1079 \\
0.0924 \\
\end{array}$ & $\begin{array}{l}3.5719 \\
3.3942 \\
2.9945 \\
\end{array}$ & $\begin{array}{l}0.0381 \\
0.0419 \quad 2.4143\end{array}$ \\
\hline $\begin{array}{l}R I+U I \\
\text { CTYGR-C } \\
N=61\end{array}$ & $\begin{array}{l}1 \\
2 \\
3 \\
4 \\
\end{array}$ & $\left|\begin{array}{l}0.6404 \\
0.6712 \\
0.6745\end{array}\right|$ & $\begin{array}{l}0.6343 \\
0.6598 \\
0.6574\end{array}$ & $\begin{array}{l}0.3840 \\
0.3628 \\
0.3712\end{array}$ & $\begin{array}{l}11242 \\
10842 \\
10882\end{array}$ & $\begin{array}{l}1139.9 \\
1136.5 \\
1137.8\end{array}$ & $\begin{array}{l}7.79 \\
4.24 \\
5.65\end{array}$ & $\begin{array}{l}\text { CNTYPOP } \\
\text { CNTYPOP } \\
\text { CNTYPOP }\end{array}$ & $\begin{array}{l}\text { CNTYINC } \\
\text { CNTYINC }\end{array}$ & STINC & & $\begin{array}{l}0.0617 \\
0.0578 \\
0.0588\end{array}$ & $\begin{array}{l}3.4664 \\
2.7105\end{array}$ & 2.4688 \\
\hline $\begin{array}{l}\text { RI+UI } \\
\text { CTYGR=D } \\
\mathrm{N}=56 \\
\\
-2=0=-\end{array}$ & \begin{tabular}{c|}
1 \\
2 \\
3 \\
4 \\
$-=-$
\end{tabular} & 0.1290 & 0.1129 & 0.9355 & 4597 & 946.5 & 55.40 & STEMP & & & & 0.0151 & & \\
\hline $\begin{array}{l}\mathrm{RI}+\mathrm{UI} \\
\mathrm{MFUNC}-\mathrm{FI} \\
\mathrm{N}=47\end{array}$ & $\begin{array}{l}1 \\
2 \\
3 \\
4 \\
-\end{array}$ & $\begin{array}{l}0.5186 \\
0.5298\end{array}$ & $\begin{array}{l}0.5079 \\
0.5085\end{array}$ & $\begin{array}{l}0.5242 \\
0.5343\end{array}$ & $\begin{array}{l}5545 \\
5542\end{array}$ & $\begin{array}{l}812.3 \\
813.2\end{array}$ & $\begin{array}{l}40.11 \\
40.17\end{array}$ & $\begin{array}{l}\text { CNTYHH } \\
\text { CNTYPOP }\end{array}$ & STEMP & & & $\begin{array}{l}0.8658 \\
0.2965\end{array}$ & 0.0081 & \\
\hline $\begin{array}{l}\mathrm{RI}+\mathrm{UI} \\
\mathrm{MFUNC}=\mathrm{F} 2 \\
\mathrm{~N}=62\end{array}$ & $\begin{array}{l}1 \\
2 \\
3 \\
4\end{array}$ & $\mid \begin{array}{l}0.6896 \\
0.7281 \\
0.7497 \\
0.7559\end{array}$ & $\begin{array}{l}0.6844 \\
0.7188 \\
0.7387 \\
0.7387\end{array}$ & $\begin{array}{l}0.3311 \\
0.2996 \\
0.2848 \\
0.2870\end{array}$ & $\begin{array}{l}7640 \\
7211 \\
6977 \\
6951\end{array}$ & $\begin{array}{l}1110.7 \\
1104.5 \\
1101.3 \\
1101.8\end{array}$ & $\begin{array}{r}18.67 \\
11.16 \\
7.81 \\
8.30\end{array}$ & $\begin{array}{l}\text { CNTYVR } \\
\text { CNTYEMP } \\
\text { CNTYVR } \\
\text { CNTYVR }\end{array}$ & $\begin{array}{l}\text { CTYPOP } \\
\text { CTYPOP } \\
\text { CTYPOP }\end{array}$ & $\begin{array}{l}\text { CNTYEMP } \\
\text { CNTYEMP }\end{array}$ & STINC & $\begin{array}{l}0.5484 \\
1.4499 \\
0.2870 \\
0.2595\end{array}$ & $\begin{array}{l}0.0230 \\
0.0168 \\
0.0170\end{array}$ & $\begin{array}{l}0.7476 \\
0.7852 \quad 2.0981\end{array}$ \\
\hline
\end{tabular}


Table $\mathrm{Cl}$, continued

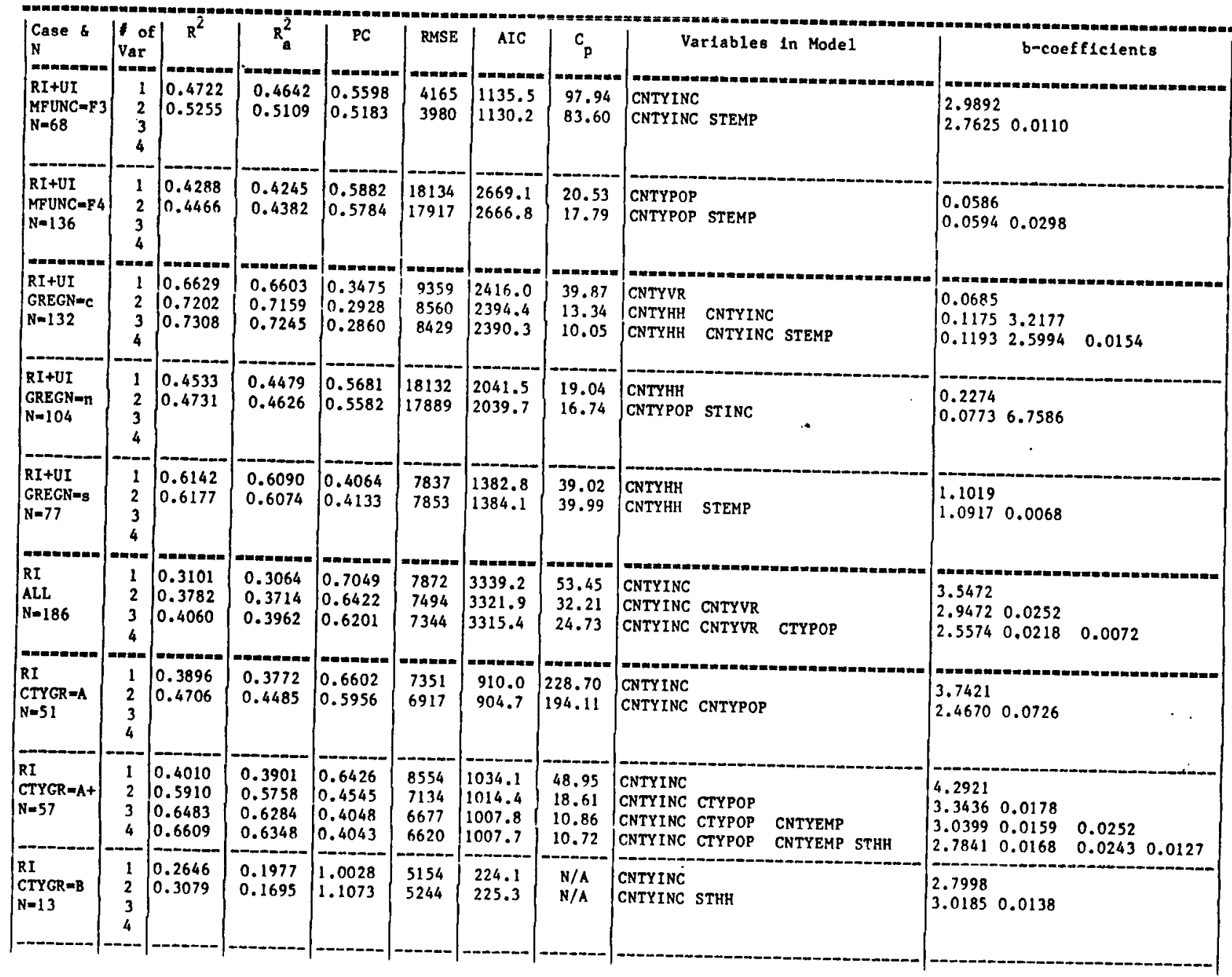


Table $\mathrm{Cl}$, continued

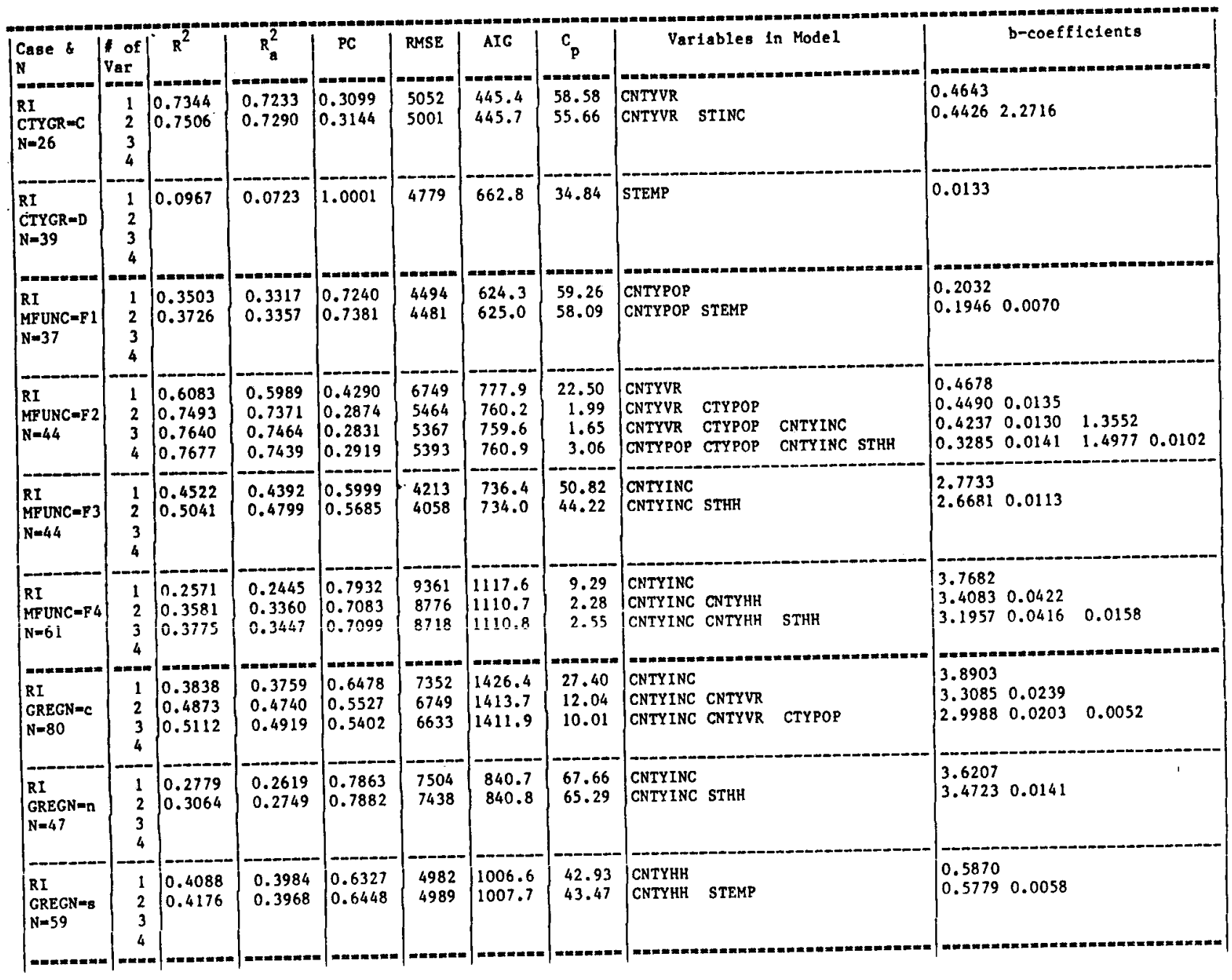


Table $\mathrm{Cl}$, continued

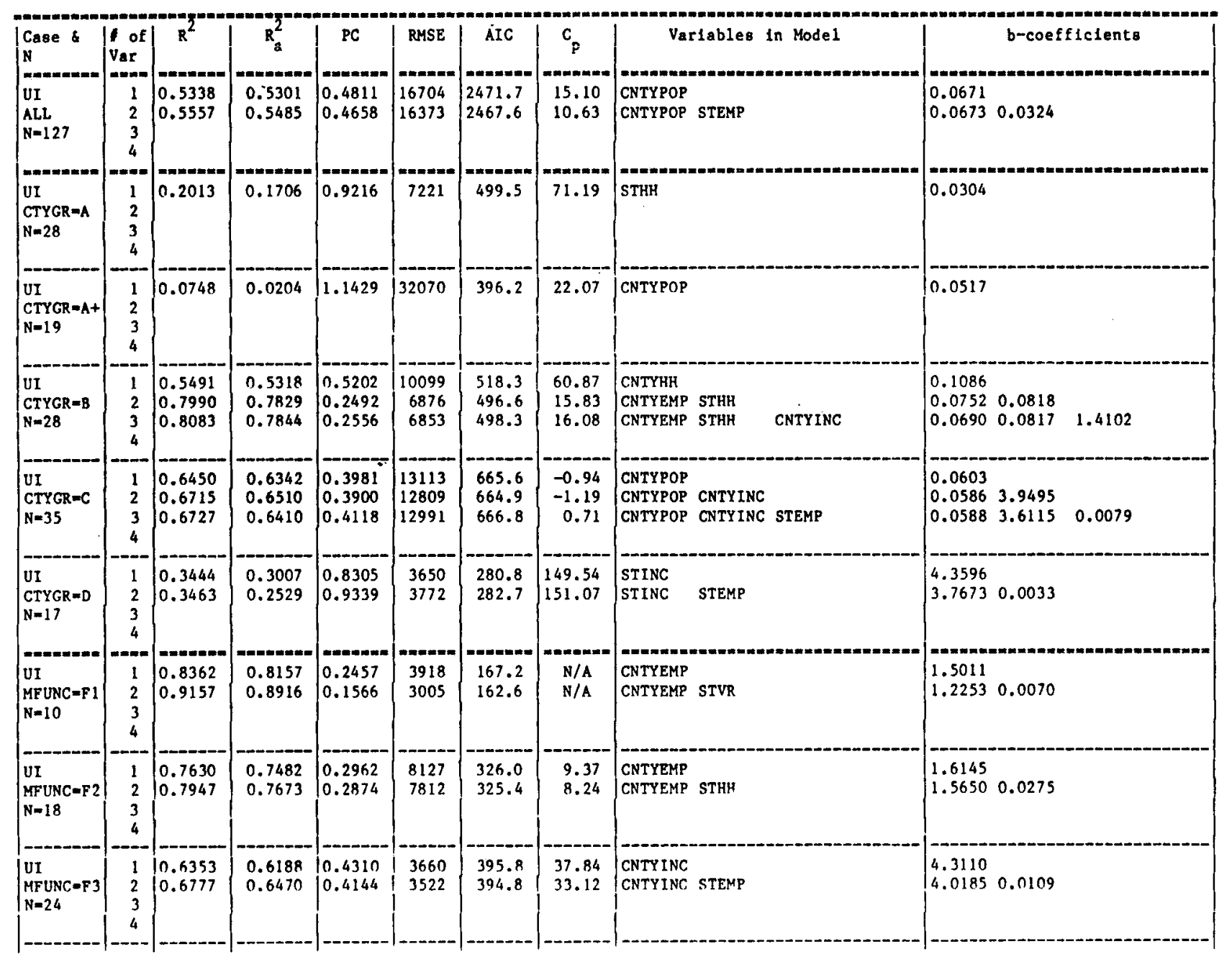


Table C1, continued

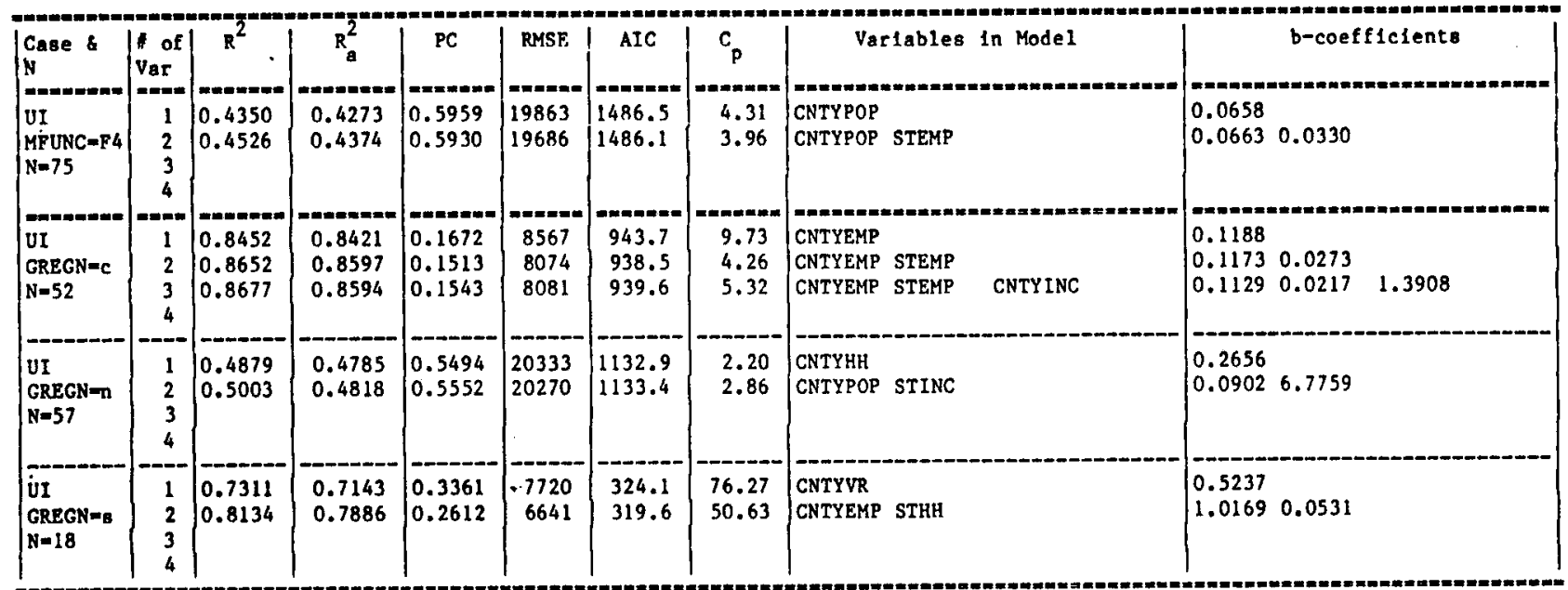

Note: Cases for $\mathrm{N}<5$ have not been recorded 
Table C2: Best Models for Interstate Highways

[Data Format: Raw E\&L and County Avg. by Year]

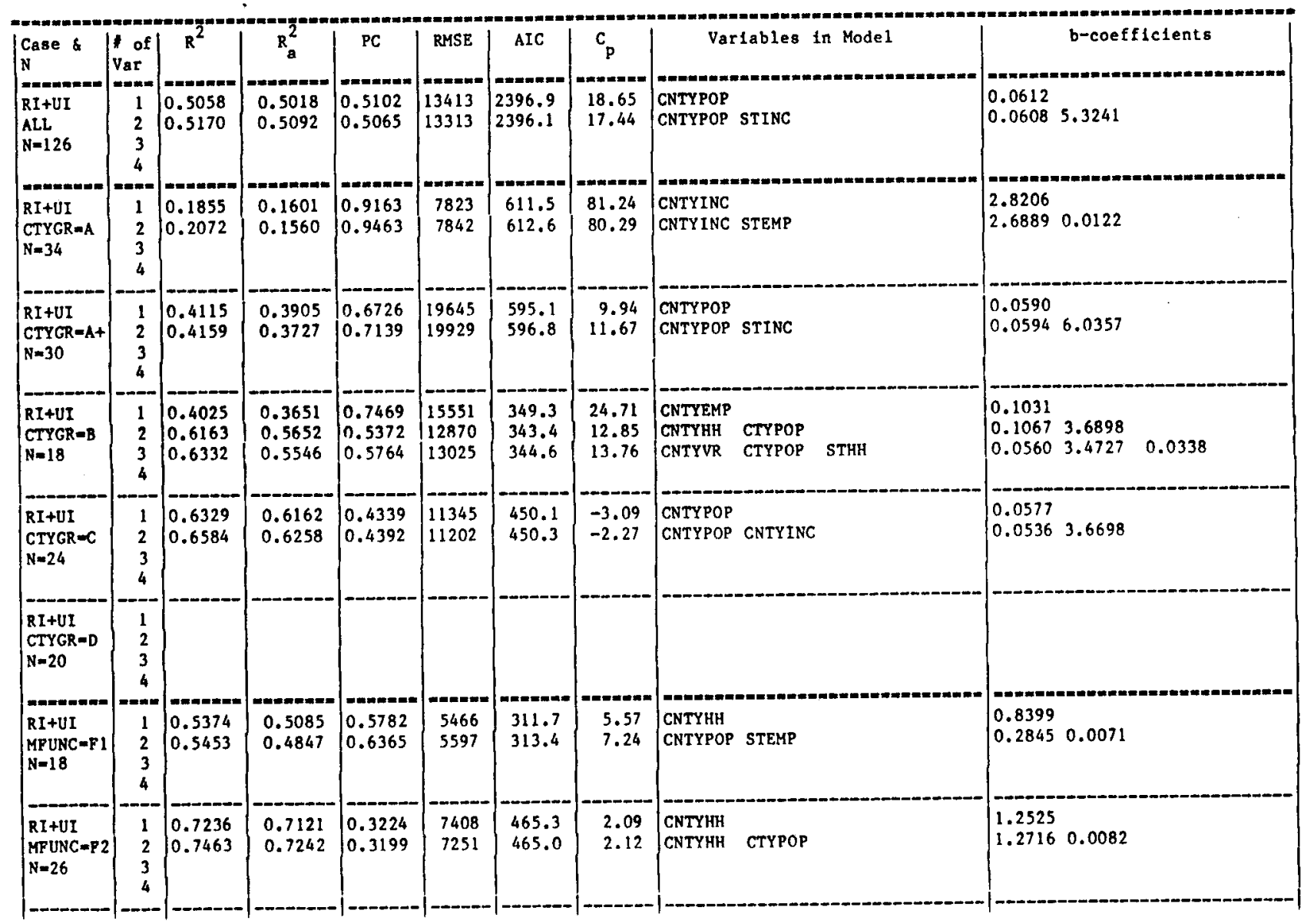


Table C2, continued

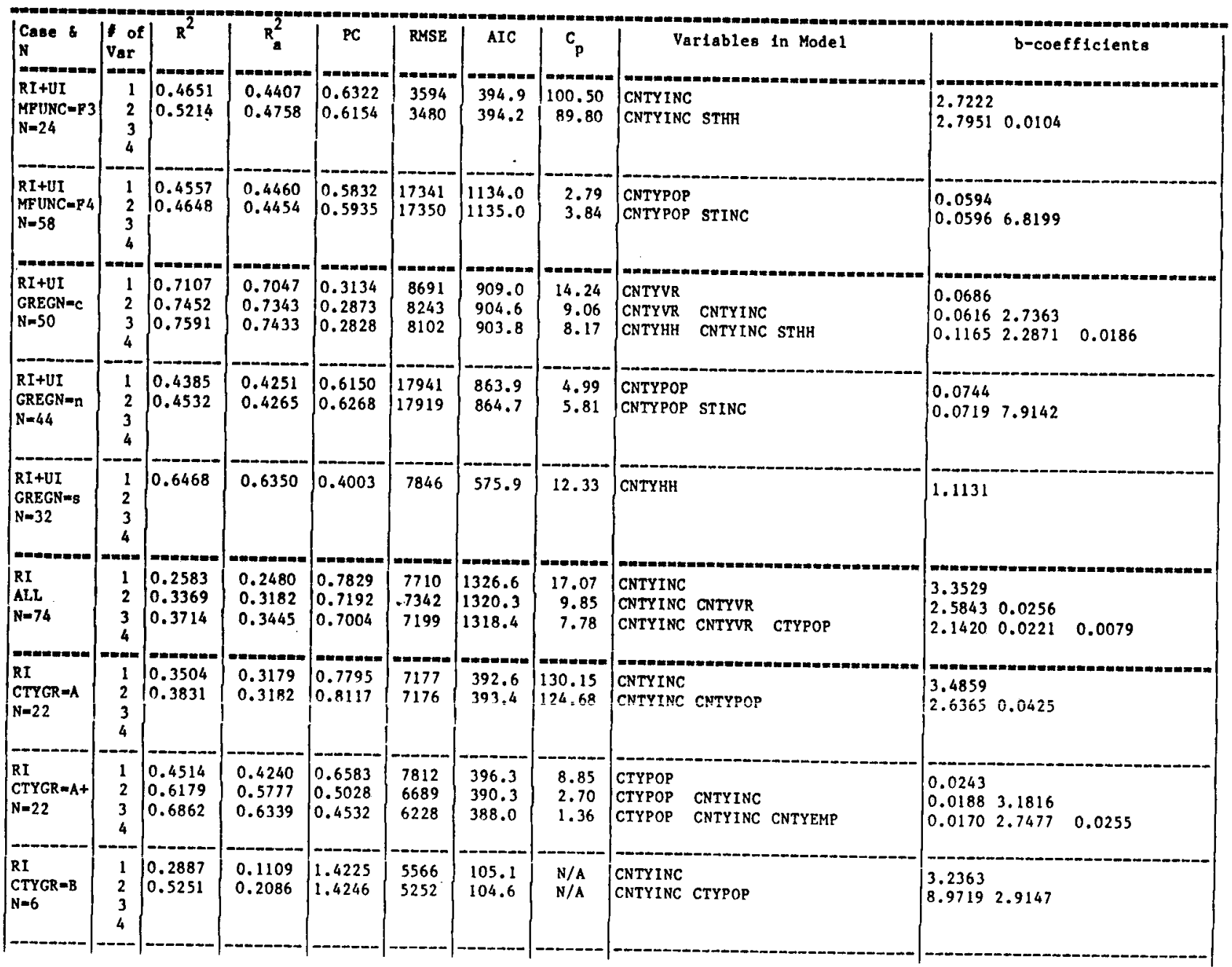


Table C2, continued

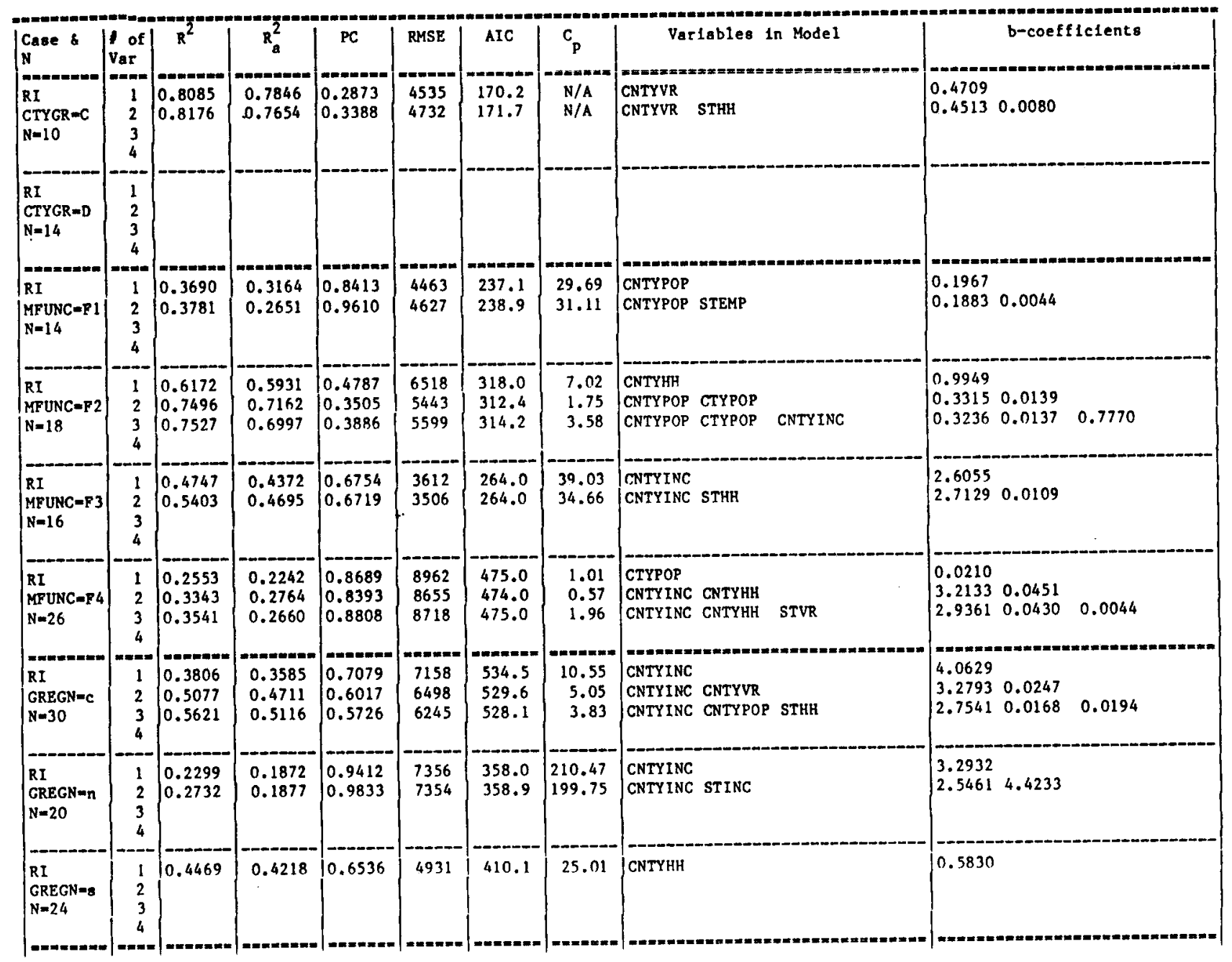


Table C2, continued

\begin{tabular}{|c|c|c|c|c|c|c|c|c|c|}
\hline 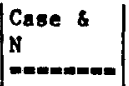 & $\mid \begin{array}{ll}1 & \text { of } \\
\operatorname{Var} \\
-=-\infty\end{array}$ & $\mathrm{R}^{2}$ & $\mathrm{R}_{\mathrm{a}}^{2}$ & $\mid \begin{array}{c}\mathrm{PC} \\
-1-+-\infty\end{array}$ & RMSR & AIC & $C_{p}$ & $\mid \begin{array}{r}\text { Varlables In Mode1 } \\
\text { | }\end{array}$ & $b$-coefficlents \\
\hline $\begin{array}{l}\text { UI } \\
\text { ALL } \\
N=52\end{array}$ & $\begin{array}{l}1 \\
2 \\
3 \\
4\end{array}$ & $\mid \begin{array}{l}0.5197 \\
0.5363\end{array}$ & $\begin{array}{l}0.5101 \\
0.5174\end{array}$ & $\begin{array}{l}0.5187 \\
0.5204\end{array}$ & $\begin{array}{l}16598 \\
16474\end{array}$ & $\begin{array}{l}1012.5 \\
1012.7\end{array}$ & $\begin{array}{l}2.13 \\
2.40\end{array}$ & $\begin{array}{l}\text { CNTYPOP } \\
\text { CNTYPOP STVR }\end{array}$ & $\begin{array}{ll}0.0646 & \\
0.0620 & 0.0099\end{array}$ \\
\hline $\begin{array}{l}\operatorname{man}=-m=\infty \\
\text { UI } \\
\text { CTYGR-A } \\
N=12\end{array}$ & \begin{tabular}{r|}
$-=-\infty$ \\
1 \\
2 \\
3 \\
4
\end{tabular} & $=-$ & $=-m=$ & 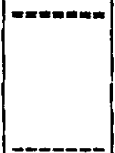 & $-\infty=-\infty$ & |--m-n=- & 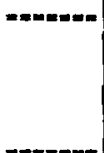 & $=-\infty$ & |=--..- \\
\hline $\begin{array}{l}U I \\
C T Y G R=A+ \\
N=8\end{array}$ & $\begin{array}{l}1 \\
2 \\
3 \\
4\end{array}$ & 0.1293 & -0.0158 & 1.4511 & 33460 & 168.4 & 75.12 & CNTYPOP & 0.0652 \\
\hline $\begin{array}{l}\text { UI } \\
\text { CTYGR }=B \\
N=12\end{array}$ & $\begin{array}{l}1 \\
2 \\
3 \\
4\end{array}$ & $\left|\begin{array}{l}0.6252 \\
0.8512 \\
0.8946\end{array}\right|$ & $\begin{array}{l}0.5877 \\
0.8181 \\
0.8550\end{array}$ & $\left|\begin{array}{l}0.5248 \\
0.2480 \\
0.2108\end{array}\right|$ & $\begin{array}{l}8870 \\
5892 \\
5259\end{array}$ & $\begin{array}{l}219.9 \\
210.9 \\
208.8\end{array}$ & $\begin{array}{l}84.78 \\
30.84 \\
22.09\end{array}$ & $\begin{array}{l}\text { CNTYHH } \\
\text { CNTYEMP STPOP } \\
\text { CNTYEMP STVR STINC }\end{array}$ & $\begin{array}{lll}0.1059 & & \\
0.0738 & 0.1189 & \\
0.0873 & 0.0352 & 32.8320\end{array}$ \\
\hline $\begin{array}{l}\text { UI } \\
\text { CTYGR-C } \\
\mathbb{N}=14\end{array}$ & $\begin{array}{l}1 \\
2 \\
3 \\
4\end{array}$ & $\left|\begin{array}{l}0.6291 \\
0.6548\end{array}\right|$ & $\begin{array}{l}0.5982 \\
0.5921\end{array}$ & $\left|\begin{array}{l}0.4945 \\
0.5334\end{array}\right|$ & $\begin{array}{l}13448 \\
13551\end{array}$ & $\begin{array}{l}268.0 \\
269.0\end{array}$ & $\begin{array}{r}-0.93 \\
0.45\end{array}$ & $\begin{array}{l}\text { CNTYPOP } \\
\text { CNTYPOP CNTYINC }\end{array}$ & $\begin{array}{ll}0.0568 \\
0.0548 & 5.1102\end{array}$ \\
\hline $\begin{array}{l}\text { UI } \\
\text { CTYGR=D } \\
N=6\end{array}$ & $\begin{array}{l}1 \\
2 \\
3 \\
4 \\
\end{array}$ & 0.3118 & 0.1398 & 1.3763 & 3081 & 97.9 & $\mathrm{~N} / \mathrm{A}$ & STHH & 0.0130 \\
\hline $\begin{array}{l}\mathrm{UI} \\
\mathrm{MFUNC}=\mathrm{F} 2 \\
\mathrm{~N}=8\end{array}$ & $\begin{array}{l}1 \\
2 \\
3 \\
4\end{array}$ & $\begin{array}{l}0.7699 \\
0.7965\end{array}$ & $\begin{array}{l}0.7315 \\
0.7151\end{array}$ & $\begin{array}{l}0.3835 \\
0.4477\end{array}$ & $\begin{array}{l}8416 \\
8670\end{array}$ & $\begin{array}{l}146.3 \\
147.3\end{array}$ & $\begin{array}{l}32.84 \\
30.58\end{array}$ & $\begin{array}{l}\text { CNTYEMP } \\
\text { CNTYEMP STHH }\end{array}$ & $\begin{array}{l}1.5084 \\
1.37920 .0263\end{array}$ \\
\hline $\begin{array}{l}\text { UI } \\
\text { MFUNC=P3 } \\
\text { No8 }\end{array}$ & $\begin{array}{l}1 \\
2 \\
3 \\
4\end{array}$ & $\mid \begin{array}{l}0.6911 \\
0.7248\end{array}$ & $\begin{array}{l}0.6397 \\
0.6147\end{array}$ & $\mid \begin{array}{l}0.5148 \\
0.6055\end{array}$ & $\begin{array}{l}3071 \\
3176\end{array}$ & $\begin{array}{l}130.2 \\
131.2\end{array}$ & $\begin{array}{l}\text { N/A } \\
\text { N/A }\end{array}$ & $\begin{array}{l}\text { CNTYINC } \\
\text { CNTYINC STHH }\end{array}$ & $\begin{array}{l}\begin{array}{l}4.8178 \\
4.7991\end{array} \quad 0.0087\end{array}$ \\
\hline $\begin{array}{l}\mathrm{UI} \\
\text { MFUNC-F4 } \\
\mathrm{N}=32\end{array}$ & $\begin{array}{l}1 \\
2 \\
3 \\
4 \\
\end{array}$ & $\begin{array}{l}0.4620 \\
0.4707\end{array}$ & $\begin{array}{l}0.4441 \\
0.4342\end{array}$ & $\begin{array}{l}0.6097 \\
0.6388\end{array}$ & $\begin{array}{l}19363 \\
19534\end{array}$ & $\begin{array}{l}633.7 \\
635.2\end{array}$ & $\begin{array}{l}-3.66 \\
-2.05\end{array}$ & $\begin{array}{l}\text { CNTYPOP } \\
\text { CNTYPOP STINC }\end{array}$ & $\begin{array}{l}0.0674 \\
0.0670 \quad 7.8636\end{array}$ \\
\hline
\end{tabular}


Table C2, continued

\begin{tabular}{|c|c|c|c|c|c|c|c|c|c|}
\hline Case \& & $\mid \begin{array}{l}\text { of } \\
\text { Var }\end{array}$ & $\mathrm{R}^{2}$ & $\mathrm{R}_{\mathrm{a}}^{2}$ & PC & RMSE & AIC & $c_{p}$ & Variables In Model & $b$-coefficlents \\
\hline $\begin{array}{l}U I \\
G R E G N-C \\
N=20\end{array}$ & $\begin{array}{l}1 \\
2 \\
3 \\
4\end{array}$ & $\begin{array}{l}0.8736 \\
0.8834\end{array}$ & $\begin{array}{l}0.8666 \\
0.8697\end{array}$ & $\begin{array}{l}0.1544 \\
0.1577\end{array}$ & $\begin{array}{l}7858 \\
7767\end{array}$ & $\begin{array}{l}360.7 \\
361.1\end{array}$ & $\begin{array}{l}-2.99 \\
-1.99\end{array}$ & $\begin{array}{l}\text { CNTYHH } \\
\text { CNTYHH STHH }\end{array}$ & $\begin{array}{ll}0.1582 & \\
0.1521 & 0.0213\end{array}$ \\
\hline $\begin{array}{l}\text { UI } \\
\text { GREGN=n } \\
\text { N=24 }\end{array}$ & $\begin{array}{l}1 \\
2 \\
3 \\
4\end{array}$ & $\begin{array}{l}0.4563 \\
0.4673\end{array}$ & $\begin{array}{l}0.4316 \\
0.4166\end{array}$ & $\begin{array}{l}0.6425 \\
0.6849\end{array}$ & $\begin{array}{l}20838 \\
21112\end{array}$ & $\begin{array}{l}479.2 \\
480.8\end{array}$ & $\begin{array}{l}-2.78 \\
-1.13\end{array}$ & $\begin{array}{l}\text { CNTYPOP } \\
\text { CNTYPOP STINC }\end{array}$ & $\begin{array}{ll}0.0871 \\
0.0845 & 9.4675\end{array}$ \\
\hline $\begin{array}{l}\text { UI } \\
\text { GREGN=8 } \\
N=8\end{array}$ & $\begin{array}{l}1 \\
2 \\
3 \\
4\end{array}$ & $\begin{array}{l}0.7825 \\
0.8523\end{array}$ & $\begin{array}{l}0.7462 \\
0.7932\end{array}$ & $\mid \begin{array}{l}0.3625 \\
0.3249\end{array}$ & $\begin{array}{l}7332 \\
6618\end{array}$ & $\begin{array}{l}144.1 \\
143.0\end{array}$ & $\begin{array}{l}23.97 \\
16.98\end{array}$ & $\begin{array}{l}\text { CNTYVR } \\
\text { CNTYVR STHH }\end{array}$ & $\begin{array}{ll}0.5316 \\
0.4641 & 0.0384\end{array}$ \\
\hline
\end{tabular}

Note: Cases for $\mathrm{N}<5$ have not been recorded 
Table C3: Best Models for Interstate Highways

[Data Format: Density and County Avg. by Year]

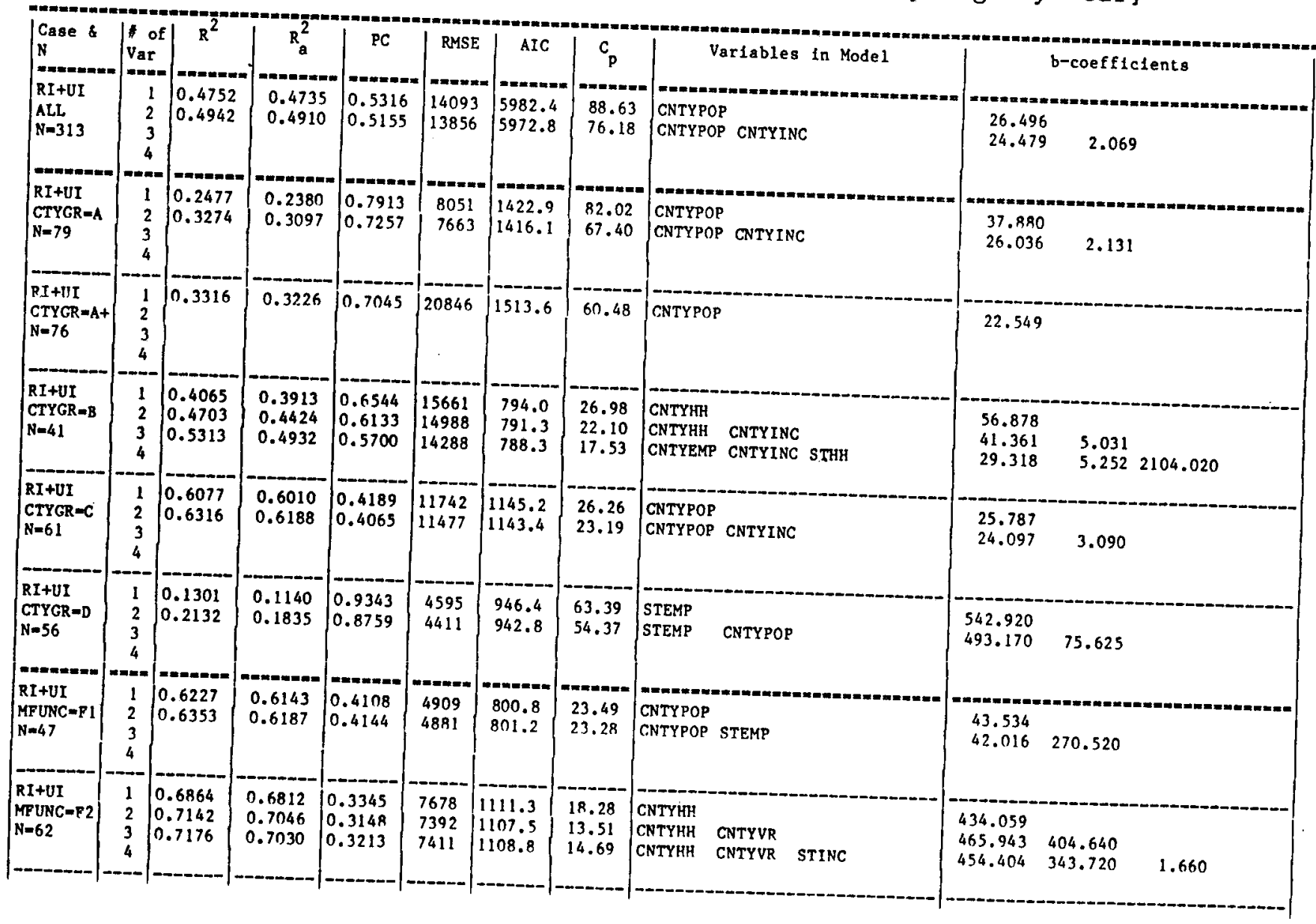


Table $\mathrm{C} 3$, continued

\begin{tabular}{|c|c|c|c|c|c|c|c|c|c|c|c|}
\hline$\left|\begin{array}{|l|}\text { Case } \\
\mathrm{N} \\
-2-1\end{array}\right|$ & $\left|\begin{array}{ll}\text { lof } \\
\mathrm{Var} \\
0 \mathrm{man}\end{array}\right|$ & $R^{2}$ & $R_{a}^{2}$ & PC & RMSE & $\int_{0=0}^{A I C}$ & $c_{p}$ & Variables in Model & 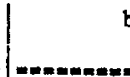 & $-\operatorname{coeff} 1 \mathrm{c} 1$ & lents \\
\hline$\left|\begin{array}{l}R I+U I \\
M F U N C=F 3 \\
N=68\end{array}\right|$ & $\begin{array}{l}1 \\
2 \\
3 \\
4\end{array}$ & $\begin{array}{l}0.4716 \\
0.5259\end{array}$ & $\begin{array}{l}0.4642 \\
0.5113\end{array}$ & $\begin{array}{l}0.5598 \\
0.5178\end{array}$ & $\begin{array}{l}4165 \\
3978\end{array}$ & $\begin{array}{l}1135.5 \\
1130.2\end{array}$ & $\begin{array}{l}97.16 \\
82.76\end{array}$ & $\begin{array}{l}\text { CNTYINC } \\
\text { CNTYINC STEMP }\end{array}$ & $\begin{array}{l}2.989 \\
2.761\end{array}$ & 398.040 & \\
\hline $\mid \begin{array}{l}\mathrm{RI}+\mathrm{UI} \\
\mathrm{MFUNC}=\mathrm{F} 4 \\
\mathrm{~N}=136 \\
-\end{array}$ & $\begin{array}{r}1 \\
2 \\
3 \\
4 \\
-2-\infty\end{array}$ & $\begin{array}{l}0.3903 \\
0.4045\end{array}$ & $\begin{array}{l}0.3858 \\
0.3955\end{array}$ & $\mid \begin{array}{l}0.6279 \\
0.6224\end{array}$ & $\begin{array}{l}18735 \\
18585\end{array}$ & $\begin{array}{l}2677.9 \\
2676.8\end{array}$ & $\begin{array}{l}36.64 \\
34.73\end{array}$ & $\begin{array}{l}\text { CNTYPOP } \\
\text { CNTYPOP STEMP } \\
\end{array}$ & $\begin{array}{l}23.106 \\
23.344\end{array}$ & 956.560 & \\
\hline $\begin{array}{l}\mathrm{RI}+\mathrm{UI} \\
\text { GREGN=C } \\
\mathrm{N}=132\end{array}$ & $\begin{array}{l}1 \\
2 \\
3 \\
4 \\
\end{array}$ & $\left|\begin{array}{l}0.6622 \\
0.7234 \\
0.7347\end{array}\right|$ & $\begin{array}{l}0.6596 \\
0.7192 \\
0.7285\end{array}$ & $\begin{array}{l}0.3482 \\
0.2894 \\
0.2819\end{array}$ & $\begin{array}{l}9369 \\
8510 \\
8368\end{array}$ & $\begin{array}{l}2416.3 \\
2319.9 \\
2388.4\end{array}$ & $\begin{array}{l}59.57 \\
27.57 \\
23.33\end{array}$ & $\begin{array}{ll}\text { CNTYHH } & \\
\text { CNTYHH } & \text { CNTYINC } \\
\text { CNTYHH } & \text { CNTYINC STEMP }\end{array}$ & $\begin{array}{l}52.520 \\
46.573 \\
47.359\end{array}$ & $\begin{array}{l}3.175 \\
2.537\end{array}$ & 568.600 \\
\hline $\begin{array}{l}R I+U I \\
G R E G N=n \\
N=104\end{array}$ & $\begin{array}{l}1 \\
2 \\
3 \\
4\end{array}$ & $\left|\begin{array}{l}0.4728 \\
0.4898\end{array}\right|$ & $\begin{array}{l}0.4677 \\
0.4797\end{array}$ & $\begin{array}{l}0.5478 \\
0.5405\end{array}$ & $\begin{array}{l}17805 \\
17603\end{array}$ & $\begin{array}{l}2038.0 \\
2036.0\end{array}$ & $\begin{array}{r}10.98 \\
9.42\end{array}$ & \begin{tabular}{|l} 
CNTYKH \\
CNTYHH
\end{tabular} & $\begin{array}{l}119.648 \\
118.375\end{array}$ & 5.901 & \\
\hline 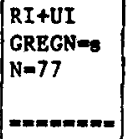 & $\begin{array}{r}1 \\
2 \\
3 \\
4 \\
=-2=\end{array}$ & $\mid \begin{array}{l}0.5645 \\
0.5667\end{array}$ & $\begin{array}{l}0.5587 \\
0.5549\end{array}$ & $\begin{array}{l}0.4587 \\
0.4685\end{array}$ & $\begin{array}{l}8326 \\
8361\end{array}$ & $\begin{array}{l}1392.2 \\
1393.8\end{array}$ & $\begin{array}{l}36.87 \\
38.32\end{array}$ & $\begin{array}{l}\text { CNTYEMP } \\
\text { CNTYEMP STVR }\end{array}$ & $\begin{array}{l}304.974 \\
302.827\end{array}$ & 135.540 & \\
\hline $\begin{array}{l}\mathrm{RI} \\
\text { ALL } \\
\mathrm{N}=186 \\
-\end{array}$ & $\begin{array}{r}1 \\
2 \\
3 \\
4 \\
----\end{array}$ & $\left|\begin{array}{l}0.3101 \\
0.3904 \\
0.4019 \\
-0 .+n=-1\end{array}\right|$ & $\begin{array}{l}0.3064 \\
0.3837 \\
0.3920 \\
-\end{array}$ & $\begin{array}{l}0.7049 \\
0.6296 \\
0.6244\end{array}$ & $\begin{array}{r}7872 \\
7420 \\
7370 \\
\\
-=-1+\end{array}$ & $\begin{array}{l}3339.2 \\
3318.2 \\
3316.7\end{array}$ & $\begin{array}{l}53.45 \\
29.31 \\
27.38\end{array}$ & $\begin{array}{l}\text { CNTYINC } \\
\text { CNTYINC CNTYPOP } \\
\text { CNTYINC CNTYPOP STEMP }\end{array}$ & $\begin{array}{l}3.547 \\
2.966 \\
2.727\end{array}$ & $\begin{array}{l}8.291 \\
8.569\end{array}$ & 333.890 \\
\hline $\begin{array}{l}\text { RI } \\
\text { CTYGR }=A \\
N=51\end{array}$ & $\begin{array}{l}1 \\
2 \\
3 \\
4\end{array}$ & $\begin{array}{l}0.3896 \\
0.4254\end{array}$ & $\begin{array}{l}0.3772 \\
0.4051\end{array}$ & $\begin{array}{l}0.6602 \\
0.6464\end{array} \mid$ & $\begin{array}{l}7351 \\
7206\end{array}$ & $\begin{array}{l}910.0 \\
908.9\end{array}$ & $\begin{array}{r}228.70 \\
70.27\end{array}$ & $\begin{array}{l}\text { CNTYINC } \\
\text { CNTYINC CNTYPOP }\end{array}$ & $\begin{array}{l}3.742 \\
2.996\end{array}$ & 16.761 & \\
\hline $\begin{array}{l}R I \\
\text { CTYGR=A+ } \\
N=57\end{array}$ & $\begin{array}{l}1 \\
2 \\
3 \\
4\end{array}$ & $\mid \begin{array}{l}0.4010 \\
0.5192\end{array}$ & $\begin{array}{l}0.3901 \\
0.5014\end{array}$ & $\begin{array}{l}0.6426 \\
0.5342\end{array}$ & $\begin{array}{l}8554 \\
7734\end{array}$ & $\begin{array}{l}1034.1 \\
1023.6\end{array}$ & $\begin{array}{l}48.95 \\
62.96\end{array}$ & $\begin{array}{l}\text { CNTYINC } \\
\text { CNTYINC CNTYHH }\end{array}$ & $\begin{array}{l}4.292 \\
3.696\end{array}$ & 19.453 & \\
\hline $\begin{array}{l}\text { RI } \\
\text { CTYGR-B } \\
N=13\end{array}$ & $\begin{array}{l}1 \\
2 \\
3 \\
4\end{array}$ & $\mid \begin{array}{l}0.2646 \\
0.5438 \\
0.7061\end{array}$ & $\begin{array}{l}0.1977 \\
0.4526 \\
0.6081\end{array}$ & $\mid \begin{array}{l}1.0028 \\
0.7258 \\
0.5551\end{array}$ & $\begin{array}{l}5154 \\
4257 \\
3602\end{array}$ & $\begin{array}{l}224.1 \\
219.8 \\
216.1\end{array}$ & $\begin{array}{l}N / A \\
N / A \\
N / A\end{array}$ & 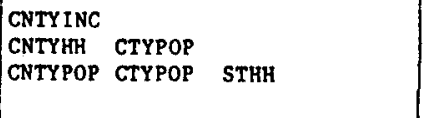 & $\begin{array}{r}2.800 \\
141.278 \\
64.993\end{array}$ & $\begin{array}{l}16.966 \\
23.173\end{array}$ & 1330.820 \\
\hline
\end{tabular}


Table C3, continued

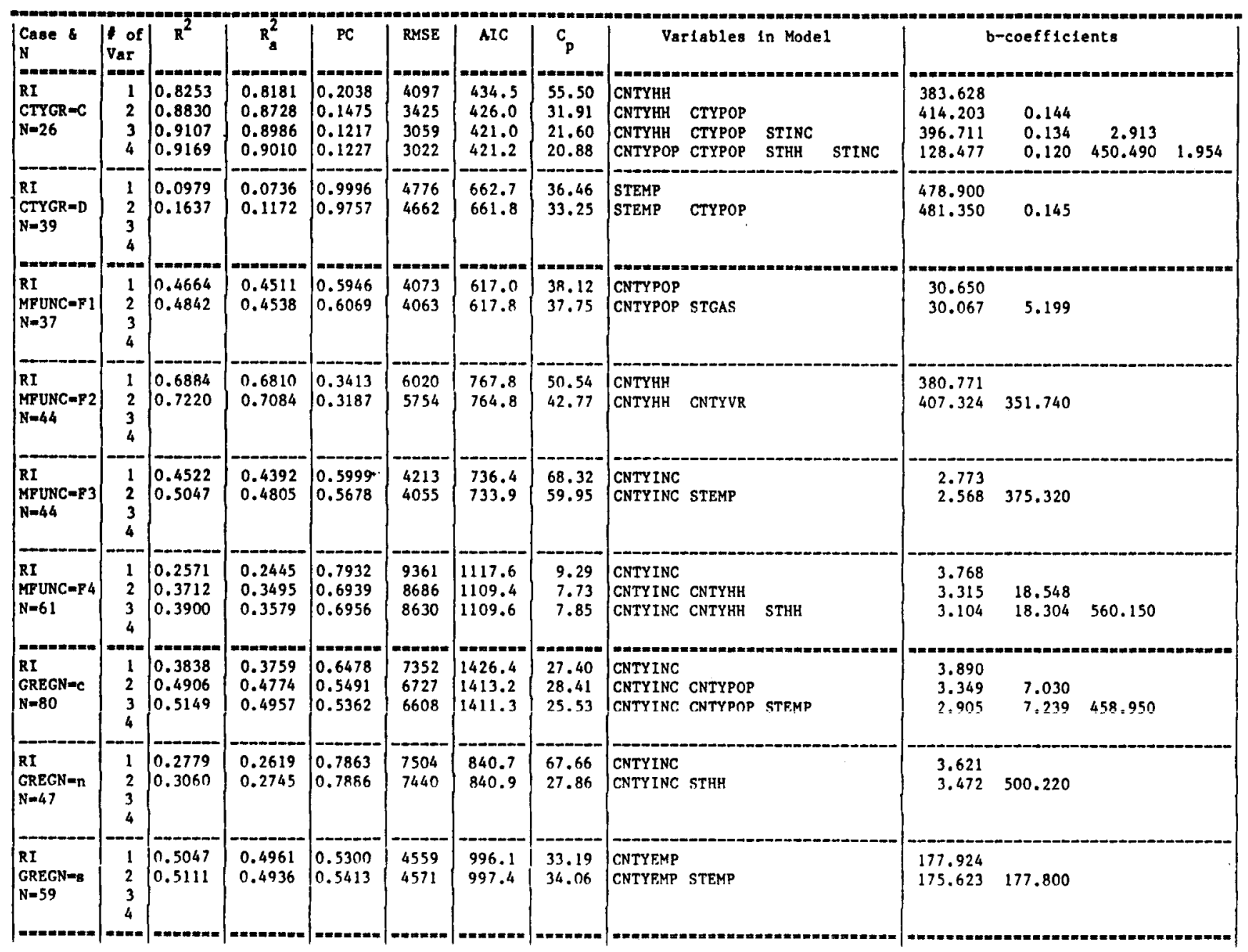


Table C3, continued

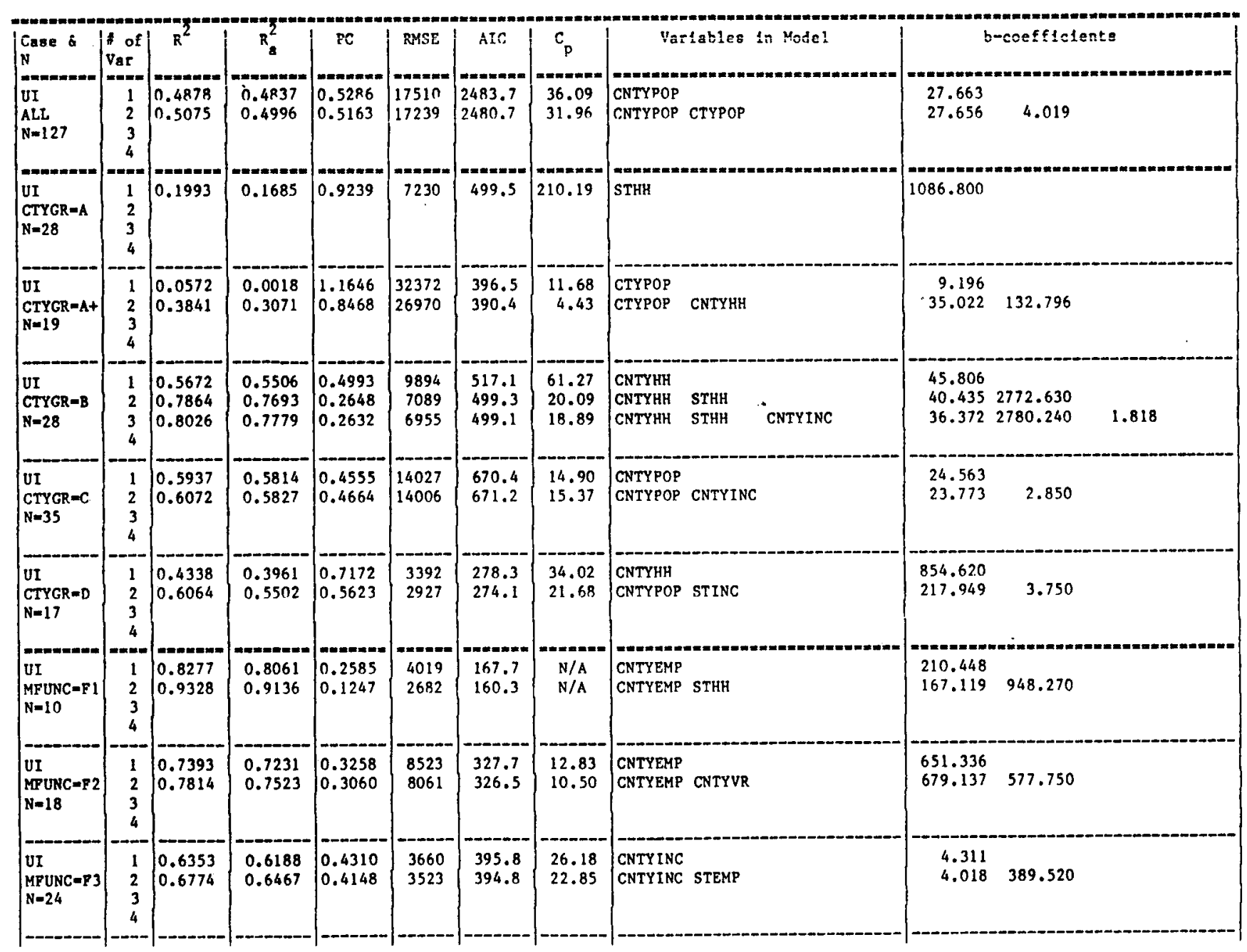


Table C3, continued

\begin{tabular}{|c|c|c|c|c|c|c|c|c|c|c|c|}
\hline $\begin{array}{l}\text { Case } \\
N\end{array}$ & $\left|\begin{array}{l}1 \\
\text { of } \\
\text { Var }\end{array}\right|$ & $\mathbb{R}^{2}$ & $\mathrm{R}_{\mathrm{a}}^{2}$ & PC & RMSE & AIC & $c_{p}$ & Variables in Model & & $-\operatorname{coeff}$ lcle & \\
\hline $\begin{array}{l}\text { UI } \\
\text { MFUNC=F4 } \\
\mathrm{N}=75\end{array}$ & $\begin{array}{l}1 \\
2 \\
3 \\
4\end{array}$ & $\begin{array}{l}0.3742 \\
0.3909\end{array}$ & $\begin{array}{l}0.3657 \\
0.3740\end{array}$ & $\begin{array}{l}0.6600 \\
0.6598\end{array}$ & $\begin{array}{l}20904 \\
20766\end{array}$ & $\begin{array}{l}1494.1 \\
1494.1\end{array}$ & $\begin{array}{l}21.69 \\
21.22\end{array}$ & $\begin{array}{l}\text { CNTYPOP } \\
\text { CNTYPOP STVR }\end{array}$ & $\begin{array}{l}24.513 \\
23.817\end{array}$ & 801.950 & \\
\hline $\begin{array}{l}=-m=-m=-1 \\
U I \\
\text { GREGN=C } \\
N=52\end{array}$ & $\left|\begin{array}{c|}-\infty-\infty \\
1 \\
2 \\
3\end{array}\right|$ & $\mid \begin{array}{l}-. .5-=- \\
0.8457 \\
0.8661 \\
0.8681\end{array}$ & $\begin{array}{l}0.8427 \\
0.8607 \\
0.8599\end{array}$ & $\begin{array}{l}0.1666 \\
0.1503 \\
0.1539\end{array}$ & $\begin{array}{l}=0+0= \\
8550 \\
8046 \\
8069\end{array}$ & $\left\{\begin{array}{l}=-10 * \ldots \\
943.5 \\
938.2 \\
939.4\end{array}\right.$ & $\begin{array}{r}-101.42 \\
83.67 \\
83.75\end{array}$ & $\begin{array}{l}\text { CNTYEMP } \\
\text { CNTYEMP STEMP } \\
\text { CNTYEMP STEMP CNTYINC }\end{array}$ & $\begin{array}{l}46.791 \\
46.204 \\
44.676\end{array}$ & $\begin{array}{l}990.250 \\
810.120\end{array}$ & 1.237 \\
\hline $\begin{array}{l}\text { UI } \\
\text { GREGN-n } \\
\text { N=57 }\end{array}$ & \begin{tabular}{l|}
1 \\
2 \\
3 \\
4
\end{tabular} & $\left|\begin{array}{l}0.5056 \\
0.5163\end{array}\right|$ & $\begin{array}{l}0.4966 \\
0.4984\end{array}$ & $\begin{array}{l}0.5304 \\
0.5374\end{array}$ & $\begin{array}{l}19979 \\
19943\end{array}$ & $\begin{array}{l}1130.8 \\
1131.6\end{array}$ & $\begin{array}{l}8.02 \\
8.69\end{array}$ & $\begin{array}{l}\text { CNTYHH } \\
\text { CNTYHH }\end{array}$ & $\begin{array}{l}138.443 \\
137.266\end{array}$ & 5.493 & \\
\hline $\begin{array}{l}\text { UI } \\
\text { GREGN=8 } \\
N=18\end{array}$ & $\begin{array}{l}1 \\
2 \\
3 \\
4\end{array}$ & $\left|\begin{array}{l}0.5472 \\
0.7705\end{array}\right|$ & $\begin{array}{l}0.5189 \\
0.7399\end{array}$ & $\begin{array}{l}0.5660 \\
0.3213\end{array}$ & $\begin{array}{r}10017 \\
7366\end{array}$ & $\begin{array}{l}333.5 \\
323.3\end{array}$ & $\begin{array}{l}62.23 \\
26.64\end{array}$ & $\begin{array}{l}\text { CTYPOP } \\
\text { CTYPOP CNTYVR }\end{array}$ & $\begin{array}{r}11.803 \\
8.753\end{array}$ & 1255.440 & \\
\hline
\end{tabular}

Note: Cases for $\mathrm{N}<5$ have not been recorded 
Table C4: Best Models for Interstate Highways

[Data Format: Dens1ty E\&L and County Avg: by Year]

\begin{tabular}{|c|c|c|c|c|c|c|c|c|c|}
\hline $\mid \begin{array}{l}\text { Case } \\
N \\
-\end{array}$ & $\left|\begin{array}{c}1 \\
\text { of } \\
\text { Var } \\
=\end{array}\right|$ & $\mathrm{R}^{\mathrm{R}^{2}}$ & $\mathrm{R}_{\mathrm{a}}^{2}$ & PC & RMSE & AIC & $c_{p}$ & Variables in Model & $b$-coefficients \\
\hline $\begin{array}{l}R I+U T \\
A L L \\
\hat{N}=126\end{array}$ & $\begin{array}{r}1 \\
2 \\
3 \\
4 \\
-n=-\end{array}$ & $\begin{array}{l}0.4860 \\
0.4956\end{array}$ & $\begin{array}{l}0.4819 \\
0.4874\end{array}$ & $\begin{array}{l}0.5306 \\
0.5290\end{array}$ & $\begin{array}{l}13678 \\
13605\end{array}$ & $\begin{array}{l}2402.0 \\
2402.0\end{array}$ & $\begin{array}{l}27.55 \\
26.76\end{array}$ & $\begin{array}{l}\text { CNTYPOP } \\
\text { CNTYPOP STINC }\end{array}$ & $\begin{array}{l}26.115 \\
25.912 \quad 4.913\end{array}$ \\
\hline $\begin{array}{l}\mathrm{R} \dot{\mathrm{I}}+\mathrm{UI} \\
\mathrm{CTYGR}=\mathrm{A} \\
\mathrm{N}=34\end{array}$ & $\begin{array}{c}1 \\
1 \\
2 \\
3 \\
4\end{array}$ & $\begin{array}{l}-.2069 \\
0.2578 \\
0.2578\end{array}$ & $\begin{array}{l}0.1821 \\
0.2099\end{array}$ & $\begin{array}{l}0.8922 \\
0.8859\end{array}$ & $\begin{array}{l}7719 \\
7587\end{array}$ & $\begin{array}{r}610.6 \\
610.4\end{array}$ & $\begin{array}{l}45.41 \\
42.58\end{array}$ & $\begin{array}{l}\text { CNTYPOP } \\
\text { CNTYPOP GNTYINC }\end{array}$ & $\begin{array}{l}31.861 \\
22.116\end{array}$ \\
\hline $\mid \begin{array}{l}R I+U I \\
\text { CTYGR }=A+ \\
N=30\end{array}$ & $\begin{array}{l}1 \\
2 \\
3 \\
4\end{array}$ & 0.3546 & 0.3315 & 0.7376 & 20573 & 597.8 & 16.94 & CNTYPOP & 22.857 \\
\hline $\begin{array}{l}R I+U I \\
\text { CTYGR=B } \\
N=18\end{array}$ & $\begin{array}{l}1 \\
2 \\
3 \\
4\end{array}$ & $\begin{array}{l}0.4287 \\
0.4905\end{array}$ & $\begin{array}{l}0.3930 \\
0.4226\end{array}$ & $\begin{array}{l}0.7141 \\
0.7133\end{array}$ & $\begin{array}{l}15206 \\
14831\end{array}$ & $\begin{array}{l}348.5 \\
348.5\end{array}$ & $\begin{array}{l}26.54 \\
24.15\end{array}$ & $\begin{array}{l}\text { CNTYHH } \\
\text { CNTYINC STVR }\end{array}$ & $\begin{array}{l}56.744 \\
12.452 \quad 2810.790\end{array}$ \\
\hline $\begin{array}{l}\text { RI+UI } \\
\text { CTYGR=C } \\
\mathrm{N}=24\end{array}$ & $\begin{array}{l}1 \\
2 \\
3 \\
4\end{array}$ & $\mid \begin{array}{l}0.6129 \\
0.6304\end{array}$ & $\begin{array}{l}0.5953 \\
0.5952\end{array}$ & $\begin{array}{l}0.4575 \\
0.4752\end{array}$ & $\begin{array}{l}11651 \\
11652\end{array}$ & $\begin{array}{l}451.3 \\
452.2\end{array}$ & $\begin{array}{l}0.12 \\
1.21\end{array}$ & $\begin{array}{l}\text { CNTYPOP } \\
\text { CNTYPOP CNTYINC }\end{array}$ & $\begin{array}{l}24.670 \\
22.975\end{array}$ \\
\hline $\begin{array}{l}R T+U I \\
C T Y G R=D \\
N=20 \\
-\end{array}$ & $\begin{array}{r}1 \\
2 \\
3 \\
4\end{array}$ & 0.1269 & 0.0784 & 1.0671 & 4263 & 336.2 & 71.22 & CNTYHH & 239.749 \\
\hline $\begin{array}{l}\mathrm{RI}+\mathrm{UI} \\
\mathrm{MFUNC}=\mathrm{FI} \\
\mathrm{N}=18\end{array}$ & $\begin{array}{l}1 \\
2 \\
3 \\
4\end{array}$ & $\begin{array}{l}0.6466 \\
0.6540\end{array}$ & $\begin{array}{l}0.6245 \\
0.6078\end{array}$ & 0.4418 & $\begin{array}{l}4778 \\
4883\end{array}$ & $\begin{array}{l}306.9 \\
308.5\end{array}$ & $\begin{array}{l}1.30 \\
2.98\end{array}$ & $\begin{array}{l}\text { CNTYPOP } \\
\text { CNTYPOP STHH }\end{array}$ & $\begin{array}{l}42.374 \\
41.172 \quad 188.600\end{array}$ \\
\hline $\begin{array}{l}R I+U I \\
\text { MFUNC }=\mathrm{F} 2 \\
\mathrm{~N}=26\end{array}$ & $\begin{array}{l}1 \\
2 \\
3 \\
4\end{array}$ & $\begin{array}{l}0.7214 \\
0.7326\end{array}$ & $\begin{array}{l}0.7098 \\
0.7093\end{array}$ & $\begin{array}{l}0.3250 \\
0.3372\end{array}$ & $\begin{array}{l}7437 \\
7444\end{array}$ & $\begin{array}{l}465.5 \\
466.4\end{array}$ & $\begin{array}{l}6.35 \\
7.22\end{array}$ & $\begin{array}{l}\text { CNTYHH } \\
\text { CNTYHH }\end{array}$ & $\begin{array}{l}441.771 \\
456.149 \quad 265.440\end{array}$ \\
\hline
\end{tabular}


Table $\mathrm{C4}$, continued

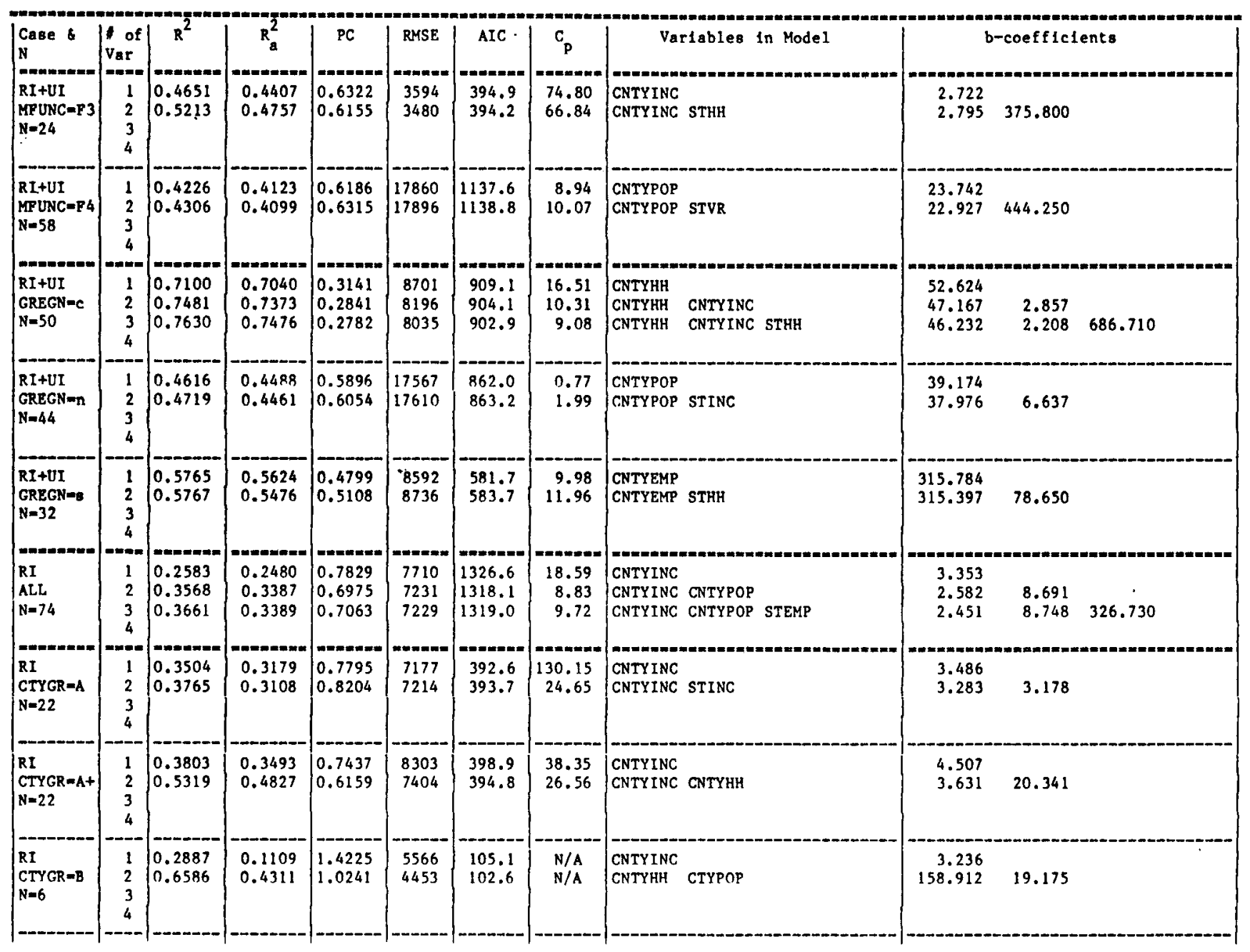


Table C4, continued

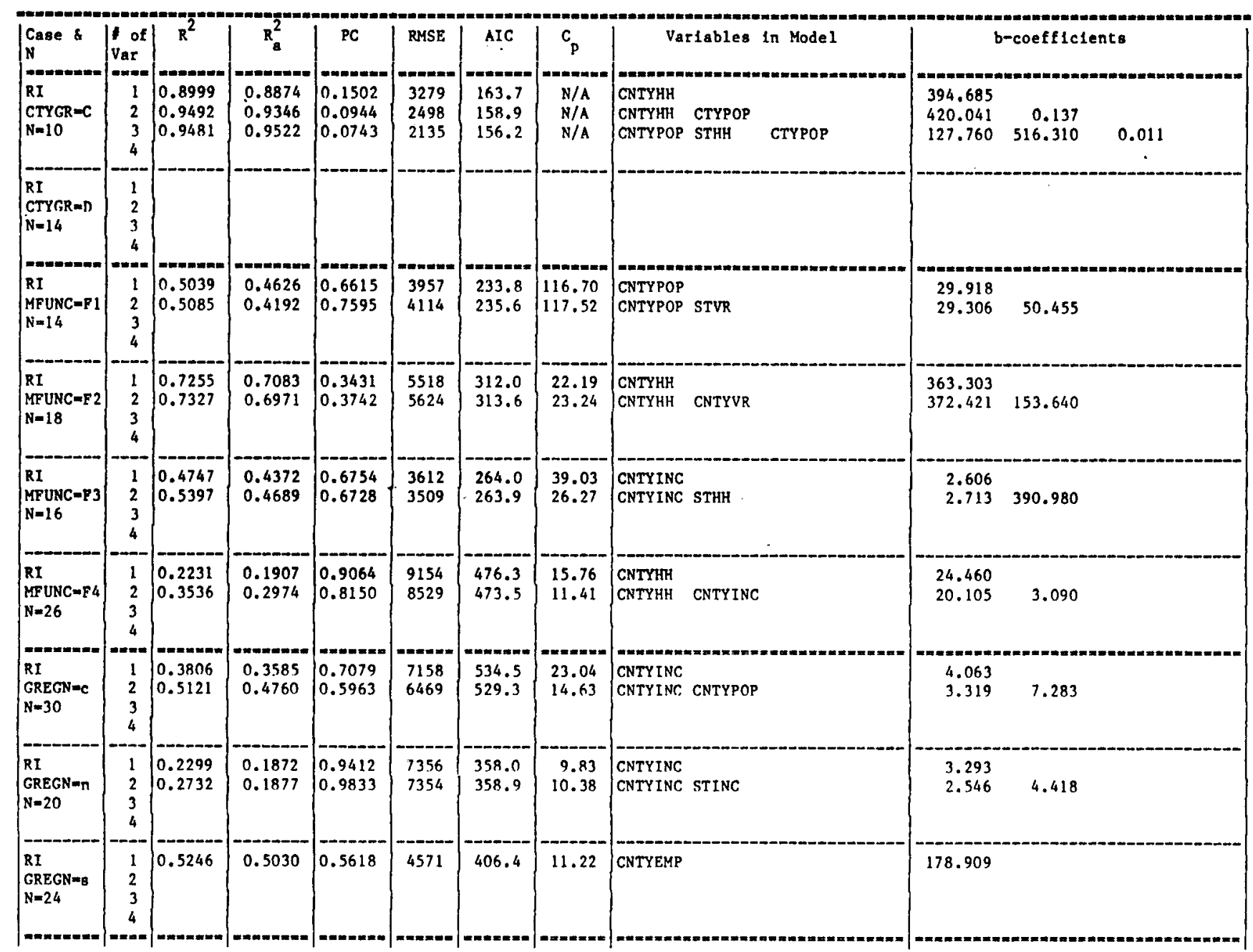


Table C4, continued

\begin{tabular}{|c|c|c|c|c|c|c|c|c|c|c|c|}
\hline 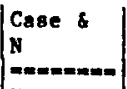 & $\left|\begin{array}{c}\text { of } \\
\operatorname{Var} \\
-=-=\end{array}\right|$ & $\mathrm{R}^{2}$ & $\mathrm{R}_{\mathrm{a}}^{2}$ & $\mid \mathrm{PC}$ & $\mid \begin{array}{l}\text { RMSE } \\
=-=-=\end{array}$ & AIC & $C_{p}$ & Varlables In Model & & $b-c o e f f i c t$ & ents \\
\hline $\begin{array}{l}\mathrm{UI} \\
\mathrm{ALL} \\
\mathrm{N}=52 \\
\mathrm{n}=-+-2=-\end{array}$ & $\begin{array}{l}1 \\
2 \\
3 \\
4 \\
-1\end{array}$ & $\begin{array}{l}0.4855 \\
0.5086\end{array}$ & $\begin{array}{l}0.4753 \\
0.4885\end{array}$ & $\begin{array}{l}0.5556 \\
0.5516\end{array}$ & $\begin{array}{l}17179 \\
16961\end{array}$ & $\begin{array}{l}1016.1 \\
1015.7\end{array}$ & $\begin{array}{l}8.68 \\
8.14\end{array}$ & 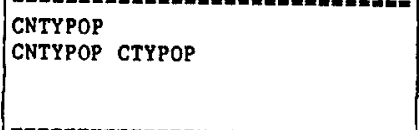 & $\begin{array}{r}27.016 \\
2727.164\end{array}$ & 4.116 & \\
\hline $\begin{array}{l}\mathrm{UI} \\
\mathrm{CTYGR}=\mathrm{A} \\
\mathrm{N}=12\end{array}$ & $\begin{array}{l}1 \\
2 \\
3 \\
4\end{array}$ & 0.1266 & 0.0392 & 1.2228 & 7562 & 216.2 & N/A & STEMP & 878.930 & & \\
\hline $\begin{array}{l}\mathrm{UI} \\
\mathrm{CTYGR}=\mathrm{A}+ \\
\mathrm{N}=8\end{array}$ & $\begin{array}{l}1 \\
2 \\
3 \\
4 \\
-\end{array}$ & 0.1136 & -0.0341 & 1.4773 & 33761 & 168.5 & 8.52 & STVR & 2861.920 & & \\
\hline $\begin{array}{l}\text { UI } \\
\text { CTYGR=B } \\
N=12\end{array}$ & $\begin{array}{l}1 \\
2 \\
3 \\
4\end{array}$ & $\begin{array}{l}0.6495 \\
0.8431 \\
0.8869 \\
0.9074\end{array}$ & $\begin{array}{l}0.6145 \\
0.8083 \\
0.8445 \\
0.8544\end{array}$ & $\left|\begin{array}{l}0.4907 \\
0.2615 \\
0.2262 \\
0.2250\end{array}\right|$ & $\begin{array}{l}8577 \\
6049 \\
5447 \\
5270\end{array}$ & $\begin{array}{l}219.2 \\
211.5 \\
209.6 \\
209.2\end{array}$ & $\begin{array}{l}84.03 \\
35.19 \\
25.69 \\
22.32\end{array}$ & \begin{tabular}{|llll} 
CNTYHH & & & \\
CNTYPOP & STPOP & & \\
CNTYHH & STVR & STINC & \\
CNTYPOP STPOP & STINC & CTYPOP \\
\end{tabular} & $\begin{array}{l}45.072 \\
14.806 \\
47.655 \\
21.897\end{array}$ & $\begin{array}{l}4246.290 \\
2180.100 \\
4667.860\end{array}$ & $\begin{array}{l}35.614 \\
45.963\end{array} \quad 3.640$ \\
\hline $\begin{array}{l}\operatorname{UI} \\
\operatorname{CTYGR}=\mathrm{C} \\
N=14\end{array}$ & $\begin{array}{l}1 \\
2 \\
3 \\
4 \\
-\end{array}$ & $\mid \begin{array}{l}0.5921 \\
0.6006\end{array}$ & $\begin{array}{l}0.5582 \\
0.5280\end{array}$ & $\left|\begin{array}{l}0.5438 \\
0.6173\end{array}\right|$ & $\begin{array}{l}14103 \\
14577\end{array}$ & $\begin{array}{l}269.4 \\
271.1\end{array}$ & $\begin{array}{l}-6.37 \\
-4.45\end{array}$ & $\begin{array}{l}\text { CNTYPOP } \\
\text { CNTYPOP CNTYINC }\end{array}$ & $\begin{array}{l}23.608 \\
22.811\end{array}$ & 2.993 & \\
\hline $\begin{array}{l}U I \\
C T Y G R=D \\
N=6\end{array}$ & \begin{tabular}{r|}
1 \\
2 \\
3 \\
4 \\
$-=-1-$
\end{tabular} & 0.5857 & 0.4821 & 0.8286 & 2391 & 94.9 & N/A & CNTYHH & 586.566 & & \\
\hline $\begin{array}{l}U I \\
M F U N C=F 2 \\
N=8\end{array}$ & \begin{tabular}{l|}
1 \\
2 \\
3 \\
4 \\
-
\end{tabular} & $\mid \begin{array}{l}0.7395 \\
0.7807\end{array}$ & $\begin{array}{l}0.6961 \\
0.6930\end{array}$ & $\begin{array}{l}0.4342 \\
0.4824\end{array}$ & $\begin{array}{l}8954 \\
8939\end{array}$ & $\begin{array}{l}147.3 \\
147.3\end{array}$ & $\begin{array}{l}47.85 \\
41.64\end{array}$ & $\begin{array}{l}\text { CNTYEMP } \\
\text { CNIYEMP GNTYVK }\end{array}$ & $\begin{array}{l}602.260 \\
593.598\end{array}$ & 657.120 & \\
\hline $\begin{array}{l}\mathrm{UI} \\
\mathrm{MFUNC}-\mathrm{F} 3 \\
\mathrm{~N}=8\end{array}$ & $\begin{array}{l}1 \\
2 \\
3 \\
4 \\
\end{array}$ & $\left|\begin{array}{l}0.6911 \\
0.7694\end{array}\right|$ & $\begin{array}{l}0.6397 \\
0.6771\end{array}$ & $\begin{array}{l}0.5148 \\
0.5074\end{array}$ & $\begin{array}{l}3071 \\
2907\end{array}$ & $\begin{array}{l}130.2 \\
129.8\end{array}$ & $\begin{array}{l}N / A \\
N / A\end{array}$ & $\begin{array}{l}\text { CNTYINC } \\
\text { CNTYINC CTYPOP }\end{array}$ & $\begin{array}{l}4.818 \\
5.940\end{array}$ & 7.443 & \\
\hline $\begin{array}{l}\text { UI } \\
\text { MFUNC=F4 } \\
N=32\end{array}$ & $\begin{array}{l}1 \\
2 \\
3 \\
4\end{array}$ & $\begin{array}{l}0.4082 \\
0.4178\end{array}$ & $\begin{array}{l}0.3884 \\
0.3776\end{array}$ & $\mid \begin{array}{l}0.6707 \\
0.7027\end{array}$ & $\begin{array}{l}20309 \\
20487\end{array}$ & $\begin{array}{l}636.7 \\
638.2\end{array}$ & $\begin{array}{l}2.78 \\
4.28\end{array}$ & $\begin{array}{l}\text { CNTYPOP } \\
\text { CNTYPOP STINC }\end{array}$ & $\begin{array}{l}25.555 \\
25.387\end{array}$ & 8.288 & \\
\hline
\end{tabular}


Table C4, continued

\begin{tabular}{|c|c|c|c|c|c|c|c|c|c|}
\hline $\begin{array}{l}\text { Case \& } \\
\text { N }\end{array}$ & of & $\mathbb{R}^{2}$ & $R_{a}^{2}$ & PC & RMSE & AIC & $c_{p}$ & Variables in Model & $b$-coefficients \\
\hline $\begin{array}{l}\text { UI } \\
\text { GRBGW=C } \\
\mathbb{N}=20\end{array}$ & $\begin{array}{l}1 \\
2 \\
3 \\
4\end{array}$ & $\mid \begin{array}{l}0.8753 \\
0.8852\end{array}$ & $\begin{array}{l}0.8684 \\
0.8717\end{array}$ & $\begin{array}{l}0.1524 \\
0.1553\end{array}$ & $\begin{array}{l}7805 \\
7705\end{array}$ & $\begin{array}{l}360.4 \\
360.3\end{array}$ & $\begin{array}{l}32.07 \\
30.25\end{array}$ & $\begin{array}{l}\text { CNTYHH } \\
\text { CNTYHK }\end{array}$ & $\begin{array}{l}62.320 \\
59.901 \quad 776.520\end{array}$ \\
\hline $\begin{array}{l}\text { UI } \\
\text { GREGN=n } \\
N=24\end{array}$ & $\begin{array}{l}1 \\
2 \\
3 \\
4\end{array}$ & 0.4768 & $\begin{array}{l}0.4530 \\
0.4374\end{array}$ & 0.6183 & $\begin{array}{l}20442 \\
20732\end{array}$ & $\begin{array}{l}478.3 \\
479.9\end{array}$ & $\begin{array}{r}-0.57 \\
1.07\end{array}$ & $\begin{array}{l}\text { CNTYPOP } \\
\text { CNTYPOP CTYPOP }\end{array}$ & $\begin{array}{l}45.398 \\
44.361\end{array}$ \\
\hline $\begin{array}{l}\text { UI } \\
\text { GREGN=8 } \\
N=8\end{array}$ & $\begin{array}{l}1 \\
2 \\
3 \\
4\end{array}$ & $\begin{array}{l}0.6504 \\
0.8294\end{array}$ & $\begin{array}{l}0.5921 \\
0.7612\end{array}$ & $\mid \begin{array}{l}0.5827 \\
0.3752\end{array}$ & $\begin{array}{l}9295 \\
7112\end{array}$ & $\begin{array}{l}147.9 \\
144.2\end{array}$ & $\begin{array}{l}51.88 \\
25.26\end{array}$ & \begin{tabular}{|l} 
CNTYVR \\
CNTYVR
\end{tabular} CTYPOP & $\begin{array}{l}2166.470 \\
1528.160\end{array}$ \\
\hline
\end{tabular}

Note: Cases for $\mathrm{N}<5$ have not been recorded 
Table C5: Best Models for Urban Principal Arterial

[Data Format: Raw and City Avg. by Year]

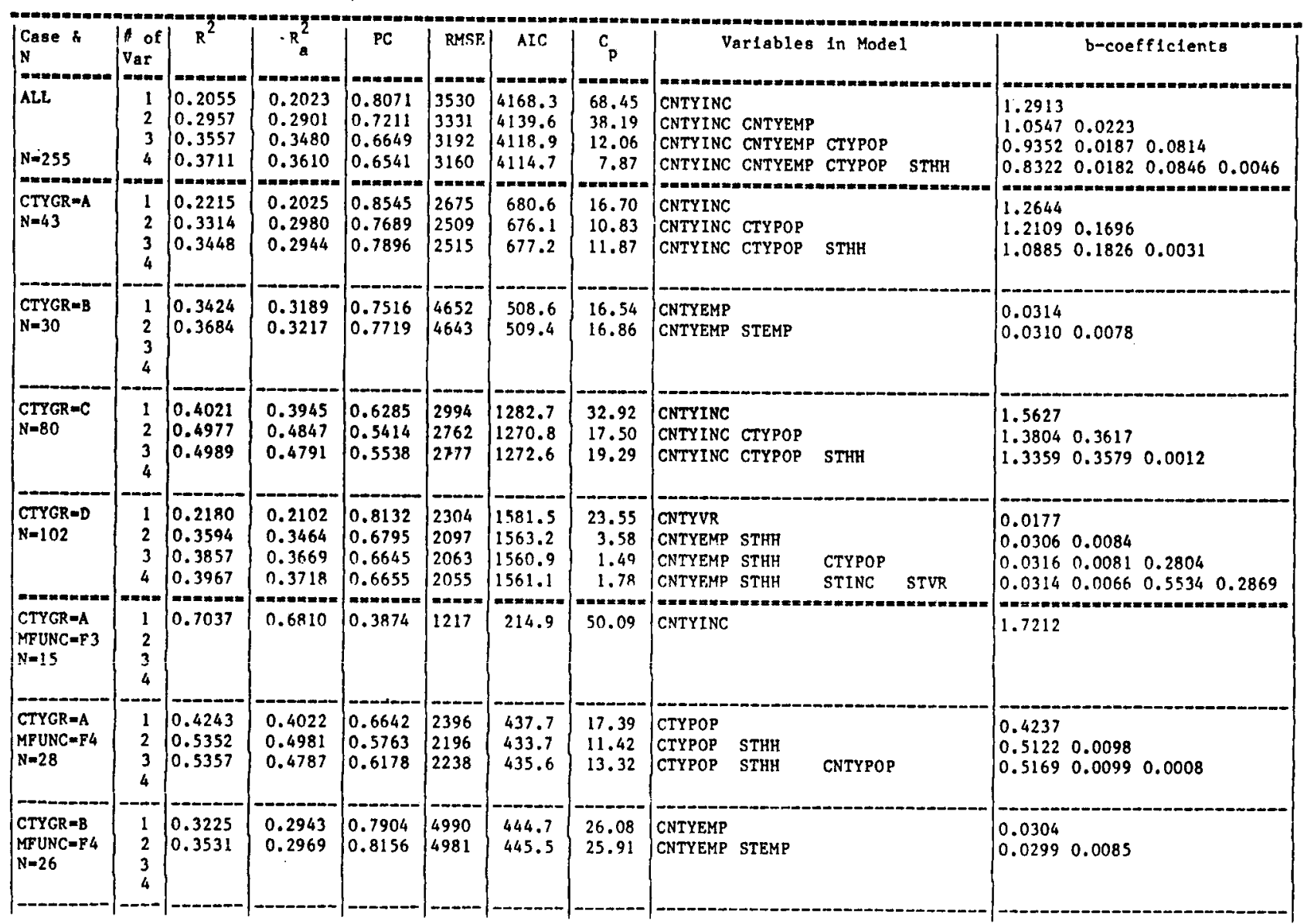


Table C5, continued

\begin{tabular}{|c|c|c|c|c|c|c|c|c|c|c|c|c|}
\hline 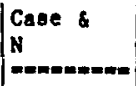 & $\left|\begin{array}{l}\text { of } \\
\operatorname{Var} \\
\operatorname{mon}\end{array}\right|$ & $\left\{\begin{array}{c}R^{2} \\
=-a m m a=\end{array}\right.$ & $\mathrm{R}_{\mathrm{a}}^{2}$ & $\mid \begin{array}{c}\text { PC } \\
-\ldots\end{array}$ & RMSE & AIC & $c_{p}$ & & arlables & in Model & & $b$-coefficients \\
\hline $\begin{array}{l}\text { CTYGR }=C \\
M F U N C=P 2 \\
N=28\end{array}$ & $\begin{array}{l}1 \\
2 \\
3 \\
4 \\
-\end{array}$ & $\left|\begin{array}{l}0.3297 \\
0.4579\end{array}\right|$ & $\begin{array}{l}0.3039 \\
0.4146\end{array}$ & $\mid \begin{array}{l}0.7734 \\
0.6722\end{array}$ & $\begin{array}{l}1189 \\
1191\end{array}$ & $\begin{array}{l}398.5 \\
394.5\end{array}$ & $\begin{array}{l}38.02 \\
28.15\end{array}$ & $\begin{array}{l}\text { STINC } \\
\text { STINC }\end{array}$ & СNTYHH & & $\begin{array}{l}1.4139 \\
1.0418\end{array}$ & 0.3301 \\
\hline $\begin{array}{l}\text { CTYGR }=C \\
\text { MFUNC-F3 } \\
\text { N=26 }\end{array}$ & $\begin{array}{l}1 \\
2 \\
3 \\
4 \\
--\end{array}$ & $\begin{array}{l}0.5089 \\
0.7667 \\
0.8064 \\
0.8114\end{array}$ & $\begin{array}{l}0.4885 \\
0.7464 \\
0.7799 \\
0.7754 \\
\end{array}$ & $\begin{array}{l}0.5728 \\
0.2942 \\
0.2641 \\
0.2785\end{array}$ & $\begin{array}{l}2889 \\
2034 \\
1895 \\
1914\end{array}$ & $\begin{array}{l}416.3 \\
398.9 \\
396.1 \\
397.4 \\
\end{array}$ & $\begin{array}{l}60.51 \\
19.21 \\
14.53 \\
15.70\end{array}$ & $\begin{array}{l}\text { CNTYHY } \\
\text { CNTYR } \\
\text { CNTYPOP } \\
\text { CNTYPOP } \\
\end{array}$ & $\begin{array}{l}\text { CTYPOP } \\
\text { CTYPOP } \\
\text { CTYPOP }\end{array}$ & $\begin{array}{l}\text { CNTYEMP } \\
\text { CNTYEMP STINC }\end{array}$ & $\begin{array}{l}0.5136 \\
0.1913 \\
0.1087 \\
0.1110\end{array}$ & $\begin{array}{lll}0.6040 & & \\
0.6992 & 0.3571 & \\
0.6897 & 0.3100 & 0.4624\end{array}$ \\
\hline $\begin{array}{l}\text { CTYGR }=C \\
\text { MFUNC }=\mathrm{F} 4 \\
\mathrm{~N}=26\end{array}$ & $\begin{array}{l}1 \\
2 \\
3 \\
4 \\
-\end{array}$ & $\begin{array}{l}0.6379 \\
0.6627\end{array}$ & $\begin{array}{l}0.6229 \\
0.6334\end{array}$ & $\begin{array}{l}0.4224 \\
0.4252\end{array}$ & $\begin{array}{l}2749 \\
2710\end{array}$ & $\begin{array}{l}413.7 \\
413.9\end{array}$ & $\begin{array}{l}13.34 \\
12.92\end{array}$ & $\begin{array}{l}\text { CNTYINC } \\
\text { CNTYINC }\end{array}$ & CTYPOP & & $\begin{array}{l}1.9709 \\
1.8779\end{array}$ & 0.1797 \\
\hline $\begin{array}{l}\text { CTYGR =D } \\
\text { MFUNC=F1 } \\
N=41\end{array}$ & $\begin{array}{l}1 \\
2 \\
3 \\
4\end{array}$ & 0.0906 & 0.0673 & 1.0026 & 2299 & 636.6 & 30.66 & STHH & & & 0.0066 & \\
\hline $\begin{array}{l}\text { CTYGR=D } \\
\text { MFUNC=F2 } \\
N=27\end{array}$ & $\begin{array}{l}1 \\
2 \\
3 \\
4\end{array}$ & $\begin{array}{l}0.7961 \\
0.8376 \\
0.8629\end{array}$ & $\begin{array}{l}0.7879 \\
0.8240 \\
0.8450\end{array}$ & $\mid \begin{array}{l}0.2366 \\
0.2030 \\
0.1848\end{array}$ & $\begin{array}{r}1055 \\
961 \\
902\end{array}$ & $\begin{array}{l}377.8 \\
375.7 \\
371.1\end{array}$ & $\begin{array}{l}81.24 \\
62.03 \\
51.10\end{array}$ & $\begin{array}{l}\text { CNTYVR } \\
\text { CNTYVR } \\
\text { CNTYVR }\end{array}$ & $\begin{array}{l}\text { CTYPOP } \\
\text { CTYPOP }\end{array}$ & STINC & $\mid \begin{array}{l}0.3221 \\
0.2679 \\
0.2252\end{array}$ & $\begin{array}{l}0.4764 \\
0.5589\end{array} 0.68803$ \\
\hline $\begin{array}{l}\text { CTYGR }=D \\
\text { MFUNC=F3 } \\
N=15\end{array}$ & $\begin{array}{l}1 \\
2 \\
3 \\
4\end{array}$ & $\begin{array}{l}0.5571 \\
0.8651 \\
0.8971\end{array}$ & $\begin{array}{l}0.5230 \\
0.8426 \\
0.8690\end{array}$ & $\mid \begin{array}{l}0.5792 \\
0.2023 \\
0.1777\end{array}$ & $\begin{array}{r}1777 \\
1021 \\
931\end{array}$ & $\begin{array}{l}226.3 \\
210.5 \\
208.4\end{array}$ & $\begin{array}{r}26.08 \\
2.29 \\
1.61\end{array}$ & $\begin{array}{l}\text { CNTYEMP } \\
\text { CTYPOP } \\
\text { CNTYEMP }\end{array}$ & $\begin{array}{l}\text { STHH } \\
\text { CTYPOP }\end{array}$ & STHH & $\begin{array}{l}0.5384 \\
0.8359 \\
0.1848\end{array}$ & $\begin{array}{ll}0.0122 \\
0.7450 & 0.0089\end{array}$ \\
\hline $\begin{array}{l}\text { CTYGR=D } \\
\text { MPUNC=F4 } \\
N=19\end{array}$ & $\begin{array}{r}1 \\
2 \\
3 \\
4 \\
-\infty\end{array}$ & $\begin{array}{l}0.5644 \\
0.6379 \\
0.6801\end{array}$ & $\begin{array}{l}0.5388 \\
0.5927 \\
0.6161\end{array}$ & $\mid \begin{array}{l}0.5381 \\
0.4978 \\
0.4906\end{array}$ & $\begin{array}{l}1940 \\
1823 \\
1769\end{array}$ & $\begin{array}{l}289.5 \\
288.1 \\
287.7\end{array}$ & $\begin{array}{l}4.19 \\
2.95 \\
3.10\end{array}$ & $\begin{array}{l}\text { CNTYYR } \\
\text { CNTYEMP } \\
\text { CNTYHH } \\
\end{array}$ & $\begin{array}{l}\text { STPOP } \\
\text { CTYPOP }\end{array}$ & STEMP & $\begin{array}{l}0.0200 \\
0.0338 \\
0.0375\end{array}$ & $\begin{array}{l}0.0093 \\
1.4908 \quad 0.0082\end{array}$ \\
\hline $\begin{array}{l}\text { CTYGR }=A \\
\text { GREGN=C } \\
N=15\end{array}$ & $\begin{array}{l}1 \\
2 \\
3 \\
4\end{array}$ & $\begin{array}{l}0.5771 \\
0.8230\end{array}$ & $\begin{array}{l}0.5446 \\
0.7935\end{array}$ & $\begin{array}{l}0.5530 \\
0.2655\end{array}$ & $\begin{array}{l}1760 \\
1185\end{array}$ & $\begin{array}{l}226.0 \\
214.9\end{array}$ & $\begin{array}{r}244.72 \\
98.04\end{array}$ & $\begin{array}{l}\text { CNTYEMP } \\
\text { CNTYPOP }\end{array}$ & CNTYINC & & $\mid \begin{array}{l}0.3753 \\
0.0970\end{array}$ & 2.2656 \\
\hline $\begin{array}{l}\text { CTYGR }=A \\
\text { GREGN=n } \\
N=23\end{array}$ & $\begin{array}{l}1 \\
2 \\
3 \\
4\end{array}$ & $\begin{array}{l}0.4630 \\
0.5284 \\
0.5303\end{array}$ & $\begin{array}{l}0.4385 \\
0.4813 \\
0.4561\end{array}$ & $\mid \begin{array}{l}0.6381 \\
0.6131 \\
0.6675\end{array}$ & $\begin{array}{l}2445 \\
2351 \\
2406\end{array}$ & $\begin{array}{l}360.8 \\
359.9 \\
361.8\end{array}$ & $\begin{array}{l}18.72 \\
16.19 \\
18.06\end{array}$ & $\begin{array}{l}\text { CTYPOP } \\
\text { CTYPOP } \\
\text { CTYPOP }\end{array}$ & $\begin{array}{l}\text { STHH } \\
\text { STHH }\end{array}$ & CNTYPOP & $\mid \begin{array}{l}0.4496 \\
0.5121 \\
0.5163\end{array}$ & $\begin{array}{ll}0.0080 \\
0.0081 & 0.0009\end{array}$ \\
\hline
\end{tabular}


Table C5, continued

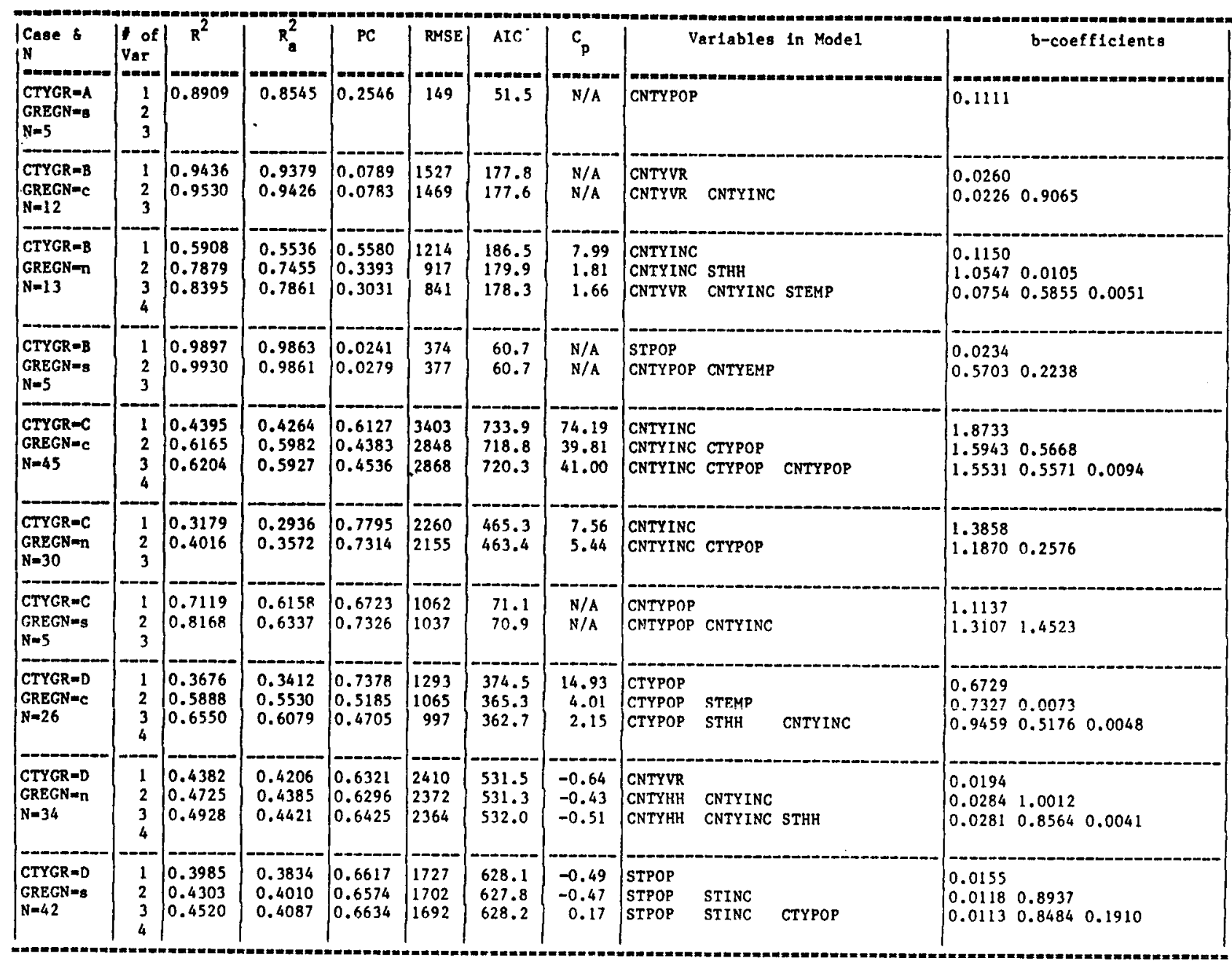

Note: Cases for $\mathrm{N}<5$ have not been recorded 
Table C5.1: Best Models for Urban Principal Arterial by Region [Datà Format: Raw and City Avg. by Year]

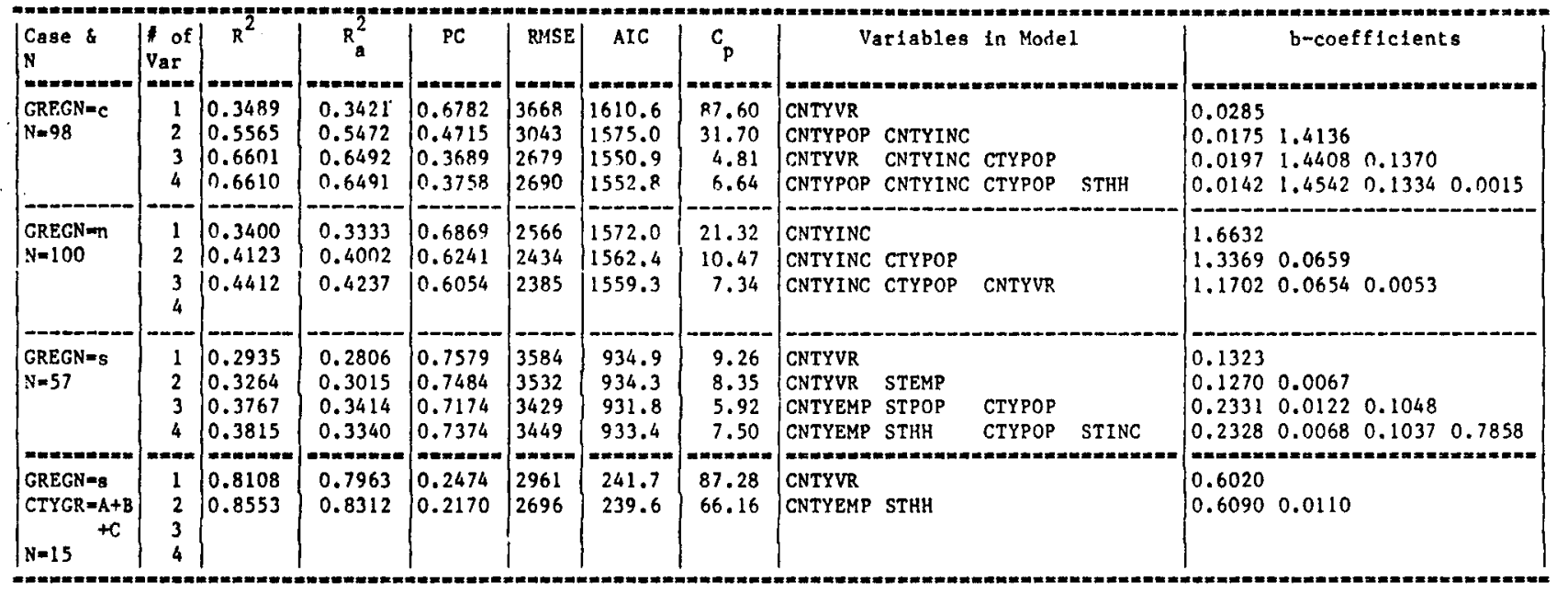


Table C6: Best Models for Urban Principal Arterial

[Data Format: Raw E\&L and City Avg. by Year]

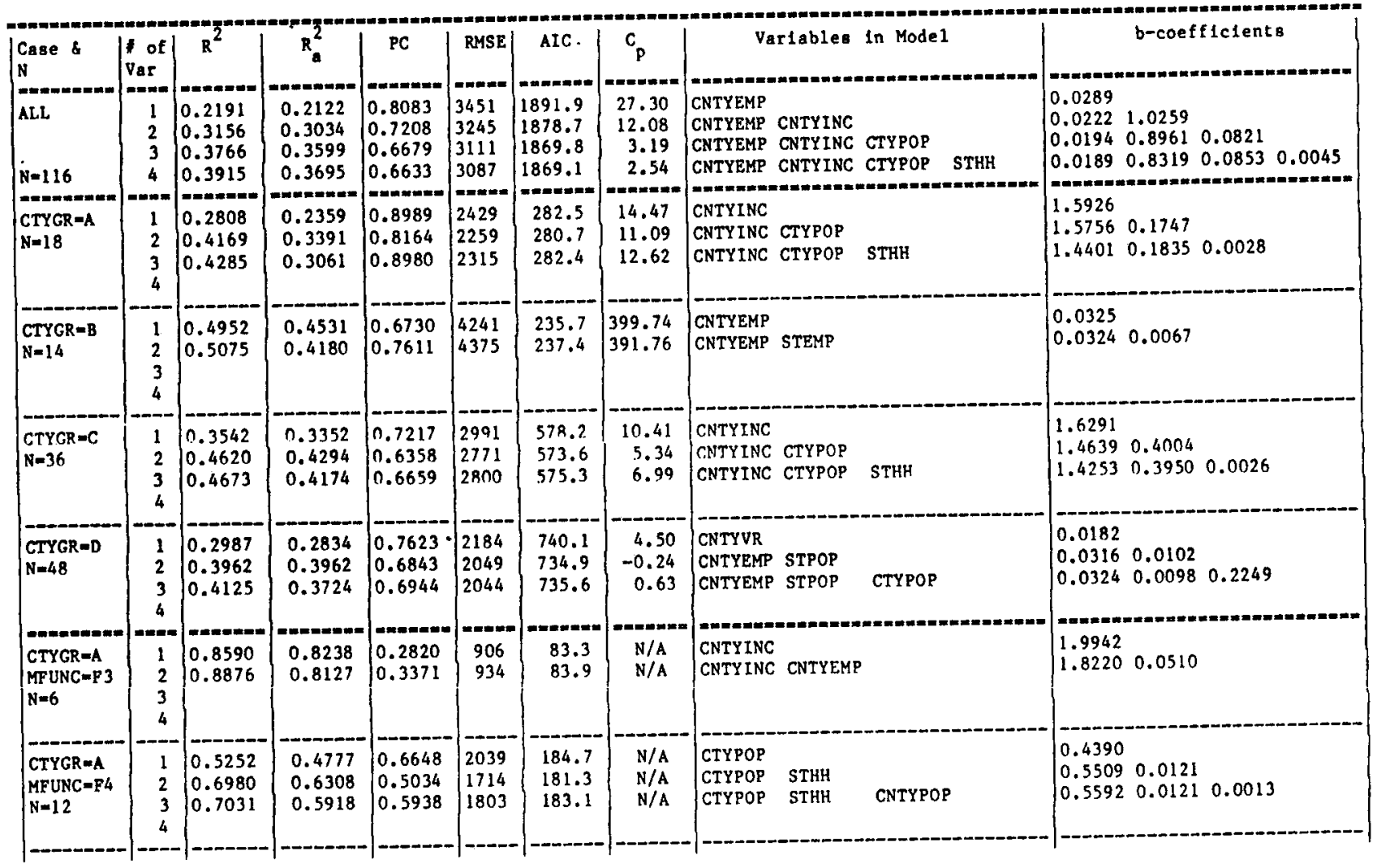


Table C6, continued

\begin{tabular}{|c|c|c|c|c|c|c|c|c|c|c|c|c|}
\hline $\begin{array}{l}\text { Case \& } \\
\text { w }\end{array}$ & $\left|\begin{array}{ll}1 & \text { of } \\
\mathrm{Vaz}\end{array}\right|$ & $\mathbf{R}^{2}$ & $\mathbf{R}_{\mathrm{a}}^{2}$ & $\mathrm{PC}$ & RMSE & AIC & $c_{p}$ & & ariables & In Model & & $b$-coefficients \\
\hline $\begin{array}{l}= \\
\text { CTYGR-B } \\
\text { MPUNC-P4 } \\
\mathrm{N}=12\end{array}$ & \begin{tabular}{|c|}
- \\
1 \\
2 \\
3 \\
4
\end{tabular} & $\begin{array}{l}0.4728 \\
0.4892\end{array}$ & $\begin{array}{l}0.4201 \\
0.3756\end{array}$ & $\begin{array}{l}-0.7381 \\
0.8514\end{array}$ & $\begin{array}{l}-4619 \\
46192 \\
4792\end{array}$ & $\begin{array}{l}204.3 \\
205.9\end{array}$ & $\begin{array}{l}\mathrm{N} / \mathrm{A} \\
\mathrm{N} / \mathrm{A}\end{array}$ & $\begin{array}{l}\text { CNTYEMP } \\
\text { CNTYEMP }\end{array}$ & STEMP & $\operatorname{lng} x=0$ & $\begin{array}{l}0.0317 \\
0.0313\end{array}$ & 0.0079 \\
\hline $\begin{array}{l}\text { CTYGR=C } \\
M P U N C=F 2 \\
N=12\end{array}$ & $\begin{array}{l}1 \\
2 \\
3 \\
4\end{array}$ & $\begin{array}{l}0.4203 \\
0.5132\end{array}$ & $\begin{array}{l}0.3623 \\
0.4050\end{array}$ & $\mid \begin{array}{l}n .8116 \\
0.8114\end{array}$ & $\begin{array}{l}967 \\
934\end{array}$ & $\begin{array}{l}166.8 \\
166.7\end{array}$ & $\begin{array}{l}\mathrm{N} / \mathrm{A} \\
\mathrm{N} / \mathrm{A}\end{array}$ & $\begin{array}{l}\text { CNTYVR } \\
\text { STEMP }\end{array}$ & CNTYPOP & & $\begin{array}{l}0.1392 \\
0.0056\end{array}$ & 0.1037 \\
\hline $\begin{array}{l}\text { CTYGR }=\mathrm{C} \\
\text { MFUNC=F3 } \\
\mathrm{N}=12\end{array}$ & $\begin{array}{l}1 \\
2 \\
3 \\
4\end{array}$ & $\begin{array}{l}0.5167 \\
0.8186 \\
0.8527\end{array}$ & $\begin{array}{l}0.4684 \\
0.7783 \\
0.7975\end{array}$ & $\begin{array}{l}0.6766 \\
0.3023 \\
0.2946\end{array}$ & $\begin{array}{l}3027 \\
1955 \\
1868\end{array}$ & $\begin{array}{l}194.2 \\
184.4 \\
183.9\end{array}$ & $\begin{array}{l}\mathrm{N} / \mathrm{A} \\
\mathrm{N} / \mathrm{A} \\
\mathrm{N} / \mathrm{A}\end{array}$ & \begin{tabular}{|l} 
CNTYPOP \\
CNTYVR \\
CNTYPOP
\end{tabular} & $\begin{array}{l}\text { CTYPOP } \\
\text { CTYPOP }\end{array}$ & CNTYEMP & $\begin{array}{l}0.1637 \\
0.2029 \\
0.1064\end{array}$ & $\begin{array}{l}0.6639 \\
0.77430 .4244\end{array}$ \\
\hline $\begin{array}{l}\text { CTYGR }=C \\
\text { MFUNC }=F 4 \\
N=12\end{array}$ & $\begin{array}{l}1 \\
2 \\
3 \\
4\end{array}$ & $\begin{array}{l}0.5378 \\
0.5607\end{array}$ & $\begin{array}{l}0.4916 \\
0.4631\end{array}$ & $\begin{array}{l}0.6471 \\
0.7321\end{array}$ & $\begin{array}{l}3067 \\
3152\end{array}$ & $\begin{array}{l}194.5 \\
195.9\end{array}$ & $\begin{array}{l}\text { N/A } \\
\text { N/A }\end{array}$ & $\begin{array}{l}\text { CNTYINC } \\
\text { CNTYINC }\end{array}$ & CTYPOP & & $\begin{array}{l}2.0978 \\
2.0443\end{array}$ & 0.2100 \\
\hline $\begin{array}{l}\text { CTYGR-D } \\
\text { MFUNC-F1 } \\
N=20\end{array}$ & $\begin{array}{l}1 \\
2 \\
3 \\
4\end{array}$ & & & & & & & & & & & \\
\hline $\begin{array}{l}\text { CTYGR=D } \\
\text { MFUNC=F } 2 \\
N=12\end{array}$ & $\begin{array}{l}1 \\
2 \\
3\end{array}$ & $\begin{array}{l}0.8592 \\
0.9008 \\
0.9236\end{array}$ & $\begin{array}{l}0.8451 \\
0.8787 \\
0.8949\end{array}$ & $\begin{array}{l}0.1972 \\
0.1654 \\
0.1528\end{array}$ & $\begin{array}{l}867 \\
767 \\
714\end{array}$ & $\begin{array}{l}164.2 \\
161.9 \\
160.8\end{array}$ & $\begin{array}{l}\text { N/A } \\
\text { N/A } \\
\text { N/A }\end{array}$ & $\begin{array}{l}\text { CNTYVR } \\
\text { CNTYVR } \\
\text { CNTYVR }\end{array}$ & $\begin{array}{l}\text { CTYPOP } \\
\text { CTYPOP }\end{array}$ & STEMP & $\begin{array}{l}0.3156 \\
0.2654 \\
0.2270\end{array}$ & $\begin{array}{ll}0.4441 \\
0.4983 & 0.0038\end{array}$ \\
\hline $\begin{array}{l}\text { CTYGR=D } \\
\text { MPUNC=F3 } \\
N=6\end{array}$ & $\begin{array}{l}1 \\
2 \\
3\end{array}$ & $\begin{array}{l}0.5836 \\
0.9418 \\
0.9534\end{array}$ & $\begin{array}{l}0.4795 \\
0.9029 \\
0.9834\end{array}$ & $\begin{array}{l}0.8329 \\
0.1747 \\
0.2332\end{array}$ & $\begin{array}{r}1783 \\
770 \\
844\end{array}$ & $\begin{array}{l}91.4 \\
81.6 \\
82.3\end{array}$ & $\begin{array}{l}\text { N/A } \\
\text { N/A } \\
\text { N/A }\end{array}$ & $\begin{array}{l}\text { CNTYEMP } \\
\text { CTYPOP } \\
\text { CNTYEMP }\end{array}$ & $\begin{array}{l}\text { STHH } \\
\text { CTYPOP }\end{array}$ & CNTYVR & $\begin{array}{l}0.5224 \\
0.7813 \\
0.2958\end{array}$ & $\begin{array}{lll} & & \\
8 & 0.0121 & \\
8 & 0.6958 & 0.1595\end{array}$ \\
\hline $\begin{array}{l}\text { CTYGR }=D \\
\text { MFINC }=F 4 \\
N=10\end{array}$ & $\begin{array}{l}1 \\
2 \\
3 \\
4\end{array}$ & $\begin{array}{l}0.5754 \\
0.6131 \\
0.6601\end{array}$ & $\begin{array}{l}0.5223 \\
0.5026 \\
0.4902\end{array}$ & $\begin{array}{l}0.6369 \\
0.7185 \\
0.7930\end{array}$ & $\begin{array}{l}2036 \\
2077 \\
2103\end{array}$ & $\begin{array}{l}154.1 \\
155.2 \\
155.9\end{array}$ & $\begin{array}{l}N / A \\
N / A \\
N / A\end{array}$ & $\begin{array}{l}\text { CNTYEMP } \\
\text { CNTYEMP } \\
\text { CNTYHH }\end{array}$ & $\begin{array}{l}\text { STINC } \\
\text { STINC }\end{array}$ & CTYPOP & $\begin{array}{l}0.0346 \\
0.0302 \\
0.0329\end{array}$ & $\begin{array}{lll}6 & & \\
2 & 1.4310 \\
9 & 1.8138 & 1.3940\end{array}$ \\
\hline
\end{tabular}


Table C6, continued

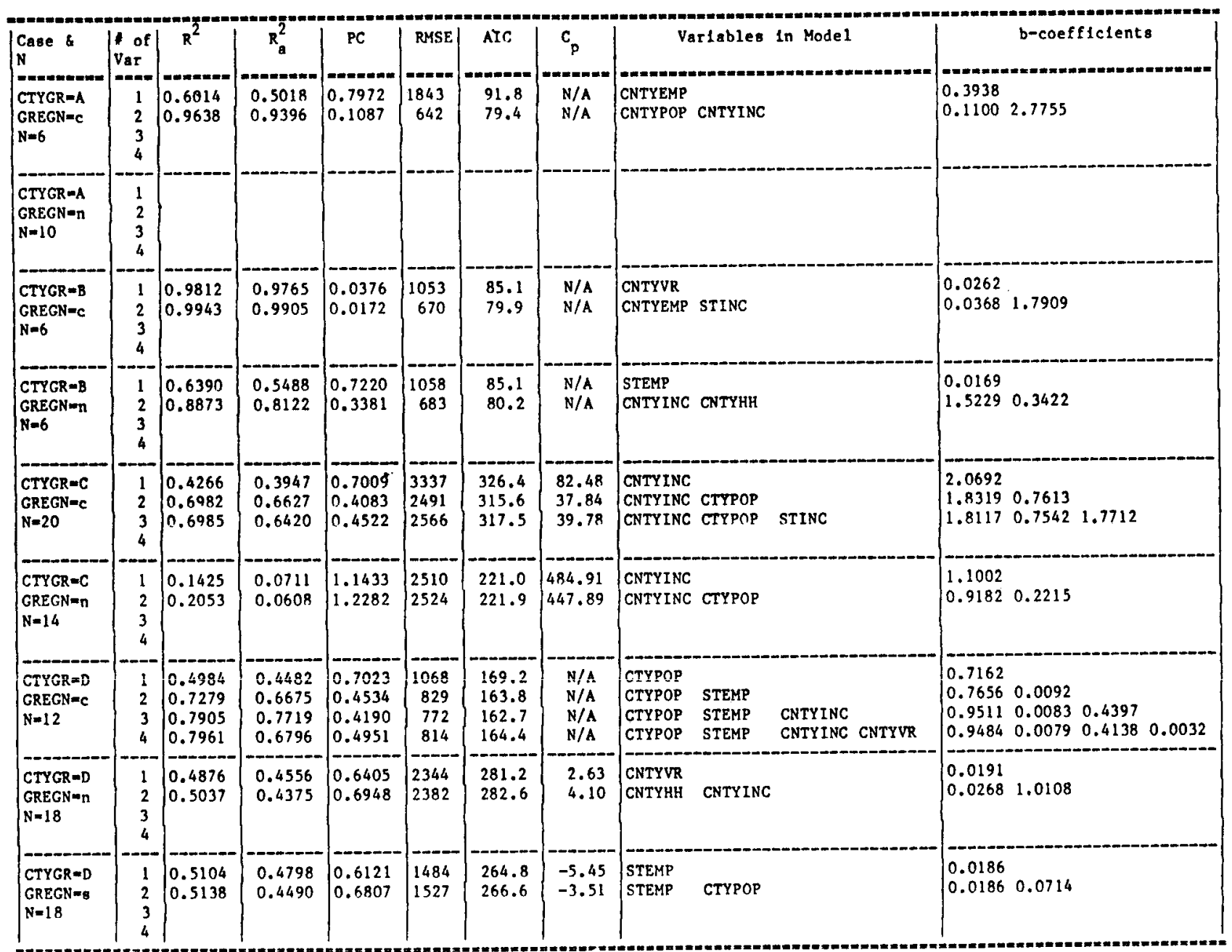

Note: Cases for $\mathrm{N}<5$ have not been recorded 
Table C7: Best Mode1s for Urban Principal Arterial

[Data Format: Density and City Avg. by Year]

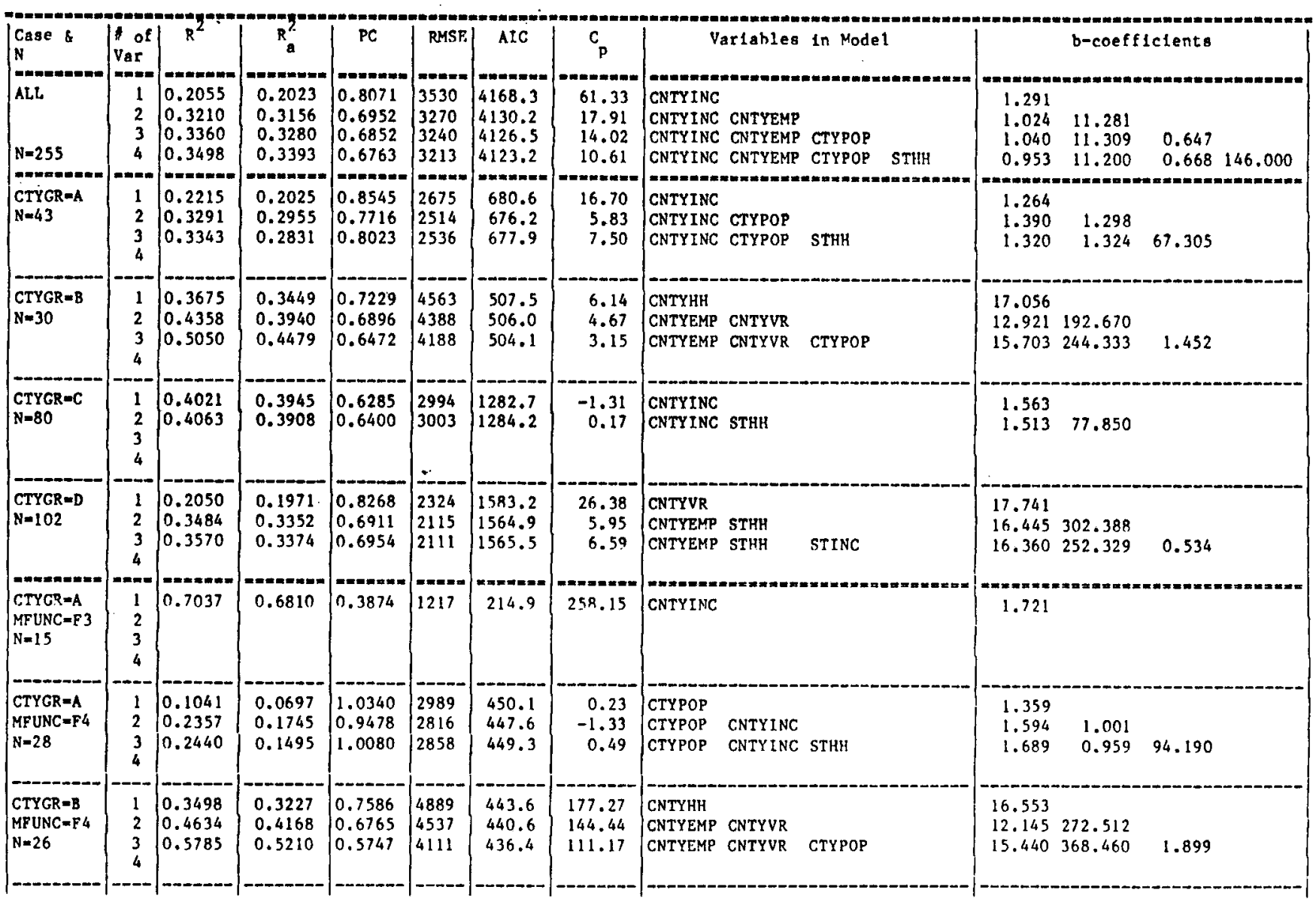


Table C7, continued

\begin{tabular}{|c|c|c|c|c|c|c|c|c|c|c|c|c|c|c|}
\hline 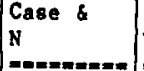 & $\left|\begin{array}{ll}1 & \text { of } \\
\mathrm{Var} \\
-\end{array}\right|$ & $\mathrm{R}^{2}$ & $\mathrm{R}_{\mathrm{a}}^{2}$ & PC & RMSE & $\mid \begin{array}{c}\text { AIC } \\
-=-a\end{array}$ & $c_{p}$ & & Jariables & In Mode? & & $b-\operatorname{coef} f \mathrm{f}$ & icients & \\
\hline $\begin{array}{l}\text { CTYGR=C } \\
\text { MFUNC=F2 } \\
\text { N=28 }\end{array}$ & $\begin{array}{l}1 \\
2 \\
3 \\
4\end{array}$ & $\begin{array}{l}0.3297 \\
0.4616 \\
0.4875\end{array}$ & $\begin{array}{l}0.3039 \\
0.4186 \\
0.4234\end{array}$ & $\begin{array}{l}0.7734 \\
0.6676 \\
0.6834\end{array}$ & $\begin{array}{l}1189 \\
1087 \\
1082\end{array}$ & $\begin{array}{l}398.5 \\
394.3 \\
394.9\end{array}$ & $\begin{array}{l}27.99 \\
19.76 \\
19.76\end{array}$ & $\begin{array}{l}\text { STINC } \\
\text { STINC } \\
\text { STINC }\end{array}$ & $\begin{array}{l}\text { CNTYHH } \\
\text { CNTYHH }\end{array}$ & CNTYEMP & $\begin{array}{l}1.414 \\
1.269 \\
1.152\end{array}$ & $\begin{array}{l}72.590 \\
50.990\end{array}$ & 72.146 & \\
\hline $\begin{array}{l}\text { CTYGR }=C \\
\text { MFUNC }=\text { F3 } \\
N=26\end{array}$ & $\begin{array}{l}1 \\
2 \\
3 \\
4\end{array}$ & $\begin{array}{l}0.5215 \\
0.5878 \\
0.5915 \\
0.5971\end{array}$ & $\begin{array}{l}0.5015 \\
0.5520 \\
0.5357 \\
0.5203\end{array}$ & $\begin{array}{l}0.5583 \\
0.5197 \\
0.5571 \\
0.5948\end{array}$ & $\begin{array}{l}2852 \\
2704 \\
2753 \\
2798\end{array}$ & $\begin{array}{l}415.6 \\
413.7 \\
415.5 \\
417.2\end{array}$ & $\begin{array}{r}-2.23 \\
-2.97 \\
-1.12 \\
0.64\end{array}$ & $\begin{array}{l}\text { CNTYEMP } \\
\text { CNTYEMP } \\
\text { CNTYEMP } \\
\text { CNTYEMP }\end{array}$ & $\begin{array}{l}\text { CTYPOP } \\
\text { CTYPOP } \\
\text { CTYPOP }\end{array}$ & $\begin{array}{l}\text { CNTYPOP } \\
\text { CNTYPOP STINC }\end{array}$ & $\begin{array}{l}280.609 \\
322.355 \\
268.960 \\
236.506\end{array}$ & $\begin{array}{l}1.530 \\
1.435 \\
1.438\end{array}$ & $\begin{array}{r}8.917 \\
12.585\end{array}$ & 0.501 \\
\hline $\begin{array}{l}\text { CTYGR }=C \\
\text { MFUNC }=F 4 \\
N=26\end{array}$ & $\begin{array}{l}1 \\
2 \\
3 \\
4 \\
-\end{array}$ & $\begin{array}{l}0.6379 \\
0.6664\end{array}$ & $\begin{array}{l}0.6229 \\
0.6157\end{array}$ & $\begin{array}{l}0.4224 \\
0.4458\end{array}$ & $\begin{array}{l}2749 \\
2775\end{array}$ & $\begin{array}{l}413.7 \\
415.1\end{array}$ & $\begin{array}{r}9.08 \\
10.36\end{array}$ & $\begin{array}{l}\text { CNTYINC } \\
\text { CNTYINC }\end{array}$ & CNTYUR & & $\begin{array}{l}1.971 \\
1.774\end{array}$ & 55.350 & & \\
\hline $\begin{array}{l}\text { CTYGR=D } \\
\text { MFUNC=F! } \\
\text { N=41 }\end{array}$ & $\begin{array}{l}1 \\
2 \\
3 \\
4 \\
-\end{array}$ & 0.0906 & 0.0673 & 1.0026 & 2299 & 636.6 & 14.28 & STHH & & & 238.133 & & & \\
\hline $\begin{array}{l}\text { CTYGR }=D \\
\text { MFUNC }=\text { F2 } \\
\text { N=27 }\end{array}$ & \begin{tabular}{l|l}
1 \\
2 \\
3 \\
4 \\
-
\end{tabular} & $\begin{array}{l}0.3112 \\
0.3767 \\
0.4212\end{array}$ & $\begin{array}{l}0.2837 \\
0.3248 \\
0.3457\end{array}$ & $\begin{array}{l}0.7990 \\
0.7791 \\
0.7801\end{array}$ & $\begin{array}{l}1939 \\
1883 \\
1853\end{array}$ & $\begin{array}{l}410.7 \\
410.0 \\
410.0\end{array}$ & $\begin{array}{l}44.21 \\
39.82 \\
37.48\end{array}$ & $\begin{array}{l}\text { STEMP } \\
\text { STEMP } \\
\text { STEMP }\end{array}$ & $\begin{array}{l}\text { CNTYINC } \\
\text { CNTYINC }\end{array}$ & CTYPOP & $\begin{array}{l}374.610 \\
249.557 \\
213.999\end{array}$ & $\begin{array}{l}0.833 \\
1.097\end{array}$ & 1.682 & \\
\hline $\begin{array}{l}\text { CTYGR=D } \\
\text { MFUNC }=\text { F3 } \\
N=15\end{array}$ & $\begin{array}{l}1 \\
2 \\
3 \\
4 \\
\end{array}$ & $\begin{array}{l}0.5876 \\
0.6196\end{array}$ & $\begin{array}{l}0.5559 \\
0.5562\end{array}$ & $\begin{array}{l}0.5392 \\
0.5706\end{array}$ & $\begin{array}{l}1715 \\
1714\end{array}$ & $\begin{array}{l}225.3 \\
226.1\end{array}$ & $\begin{array}{l}-2.83 \\
-1.46\end{array}$ & $\begin{array}{l}\text { CNTYEMP } \\
\text { CNTYEMP }\end{array}$ & CNTYHH & & $\begin{array}{l}260.864 \\
261.813\end{array}$ & 79.846 & & \\
\hline 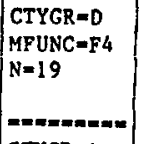 & $\begin{array}{l}1 \\
2 \\
3 \\
4 \\
-=-1\end{array}$ & $\begin{array}{l}0.5319 \\
0.6069 \\
0.6208 \\
-\ldots \ldots\end{array}$ & $\begin{array}{l}0.5043 \\
0.5567 \\
0.5450\end{array}$ & $\begin{array}{l}0.5783 \\
0.5418 \\
0.5814\end{array}$ & $\begin{array}{l}2011 \\
1902 \\
1927 \\
-1\end{array}$ & $\begin{array}{l}290.9 \\
289.7 \\
290.9 \\
\end{array}$ & $\begin{array}{l}26.81 \\
22.19 \\
22.86\end{array}$ & $\begin{array}{l}\text { CNTYEMP } \\
\text { CNTYEMP } \\
\text { CNTYEMP }\end{array}$ & $\begin{array}{l}\text { STỸūP } \\
\text { CNTY INC }\end{array}$ & C CTYPOP & $\begin{array}{r}20.641 \\
19.091 \\
8.978\end{array}$ & $\begin{array}{r}324.480 \\
1.277\end{array}$ & 5.087 & \\
\hline $\begin{array}{l}\text { CTYGR=A } \\
\text { GREGN=C } \\
N=15\end{array}$ & $\begin{array}{r}1 \\
2 \\
3 \\
4 \\
-\end{array}$ & $\begin{array}{l}0.4340 \\
0.7131\end{array}$ & $\begin{array}{l}0.3905 \\
0.6652\end{array}$ & $\begin{array}{l}0.7400 \\
0.4304\end{array}$ & $\begin{array}{l}2035 \\
1509\end{array}$ & $\begin{array}{l}230.4 \\
222.2\end{array}$ & $\begin{array}{l}312.25 \\
154.88\end{array}$ & $\begin{array}{l}\text { CNTYEMP } \\
\text { CNTYINC }\end{array}$ & CTYPOP & & $\begin{array}{r}241.653 \\
2.613\end{array}$ & 2.567 & & \\
\hline $\begin{array}{l}\text { CTYGR }=A \\
\text { GREGN=n } \\
N=23\end{array}$ & $\begin{array}{l}1 \\
2 \\
3 \\
4 \\
\end{array}$ & $\begin{array}{l}0.2181 \\
0.2620\end{array}$ & $\begin{array}{l}0.1809 \\
0.1882\end{array}$ & $\begin{array}{l}0.9308 \\
0.9594\end{array}$ & $\begin{array}{l}2954 \\
2940\end{array}$ & $\begin{array}{l}369.5 \\
37 n .2\end{array}$ & $\begin{array}{l}7.52 \\
8.13\end{array}$ & $\begin{array}{l}\text { CNTYINC } \\
\text { CNTYINC }\end{array}$ & CTYPOP & & $\begin{array}{l}1.430 \\
1.335\end{array}$ & 0.994 & & \\
\hline
\end{tabular}


Table C7, continued

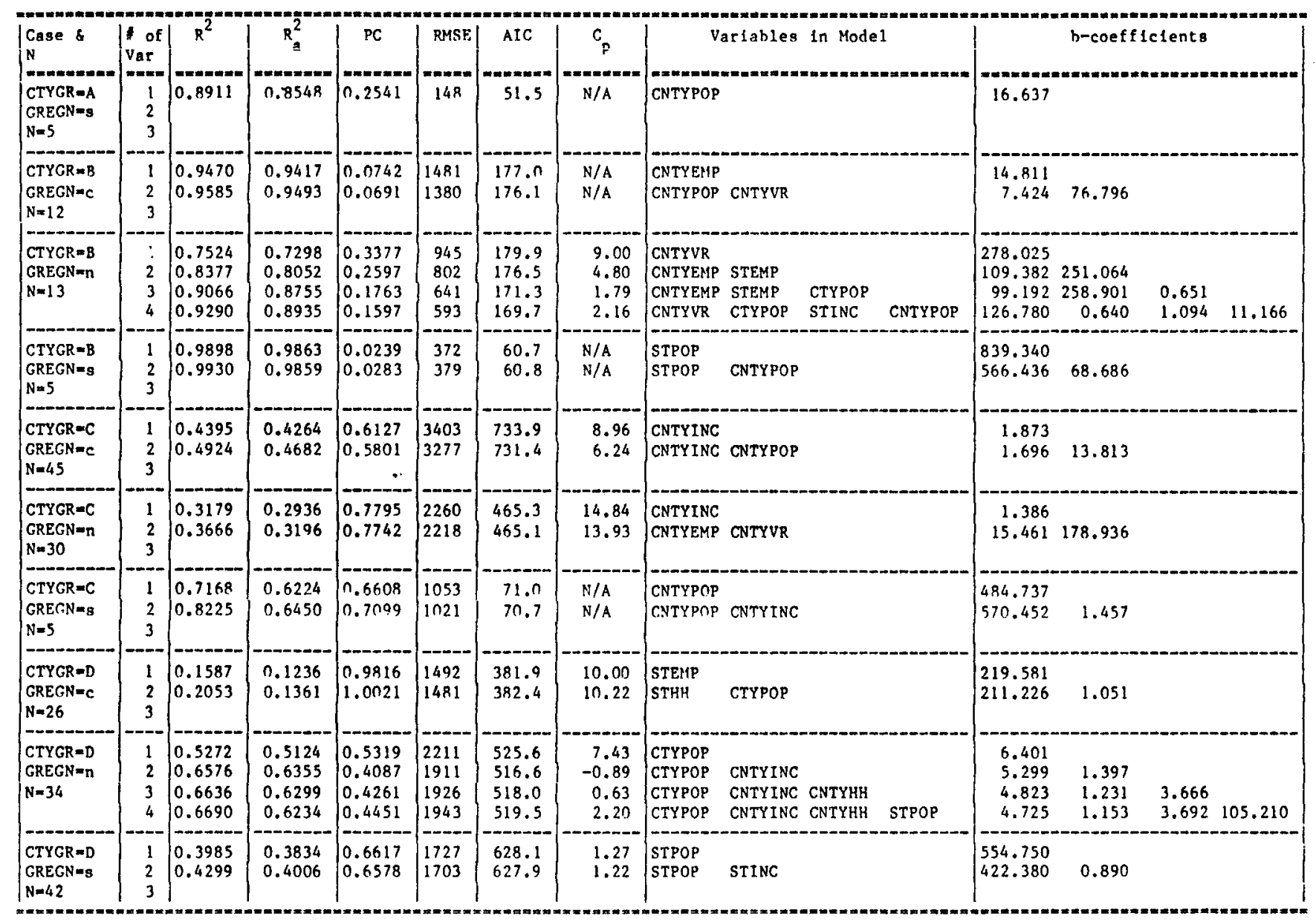

Note: Cases for $\mathrm{N}<5$ have not been recorded 
Table C8: Best Models for Urban Principal Arterlal

[Data Format: Density E\&L and City Avg. by Year]

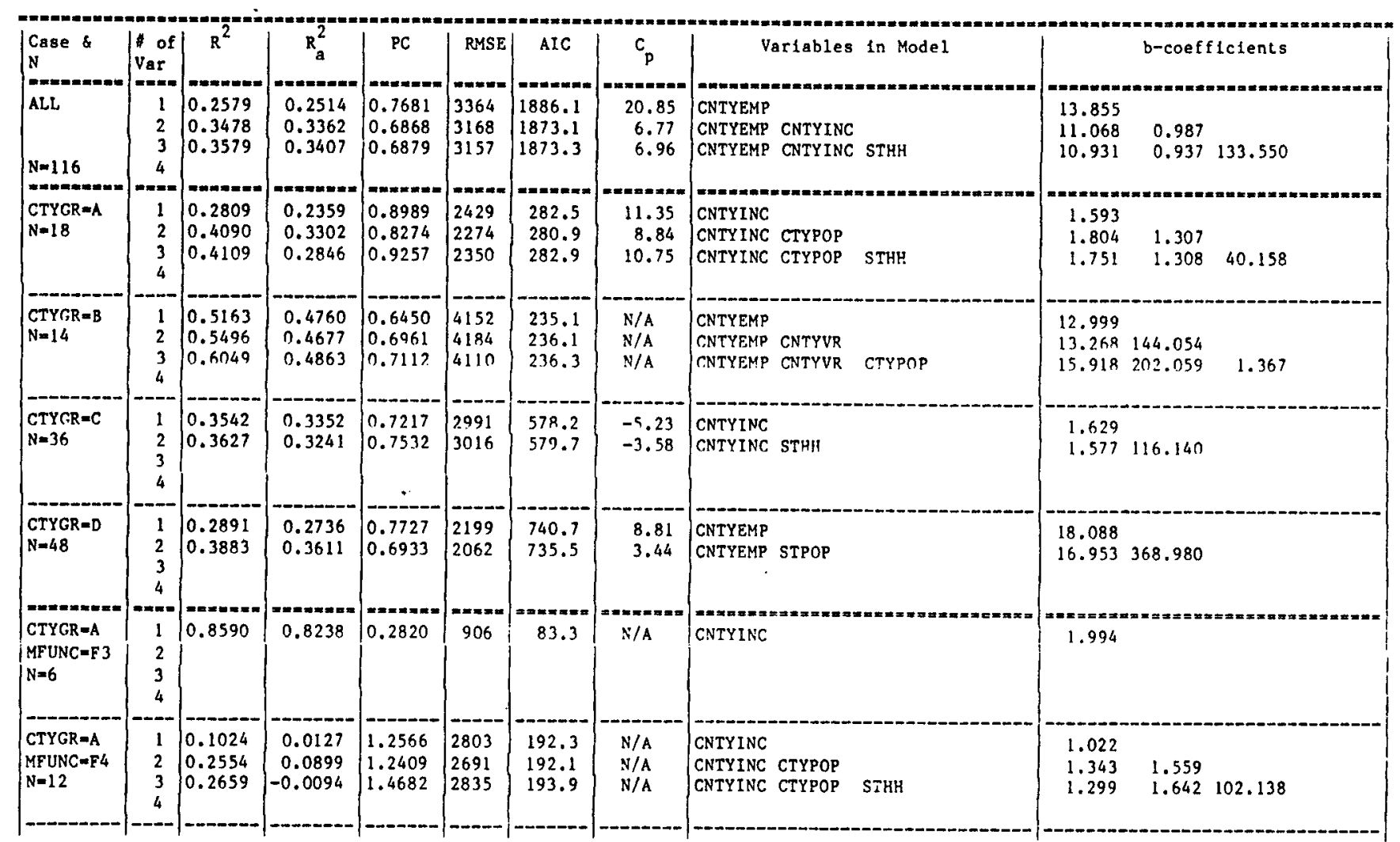


Table C8, continued

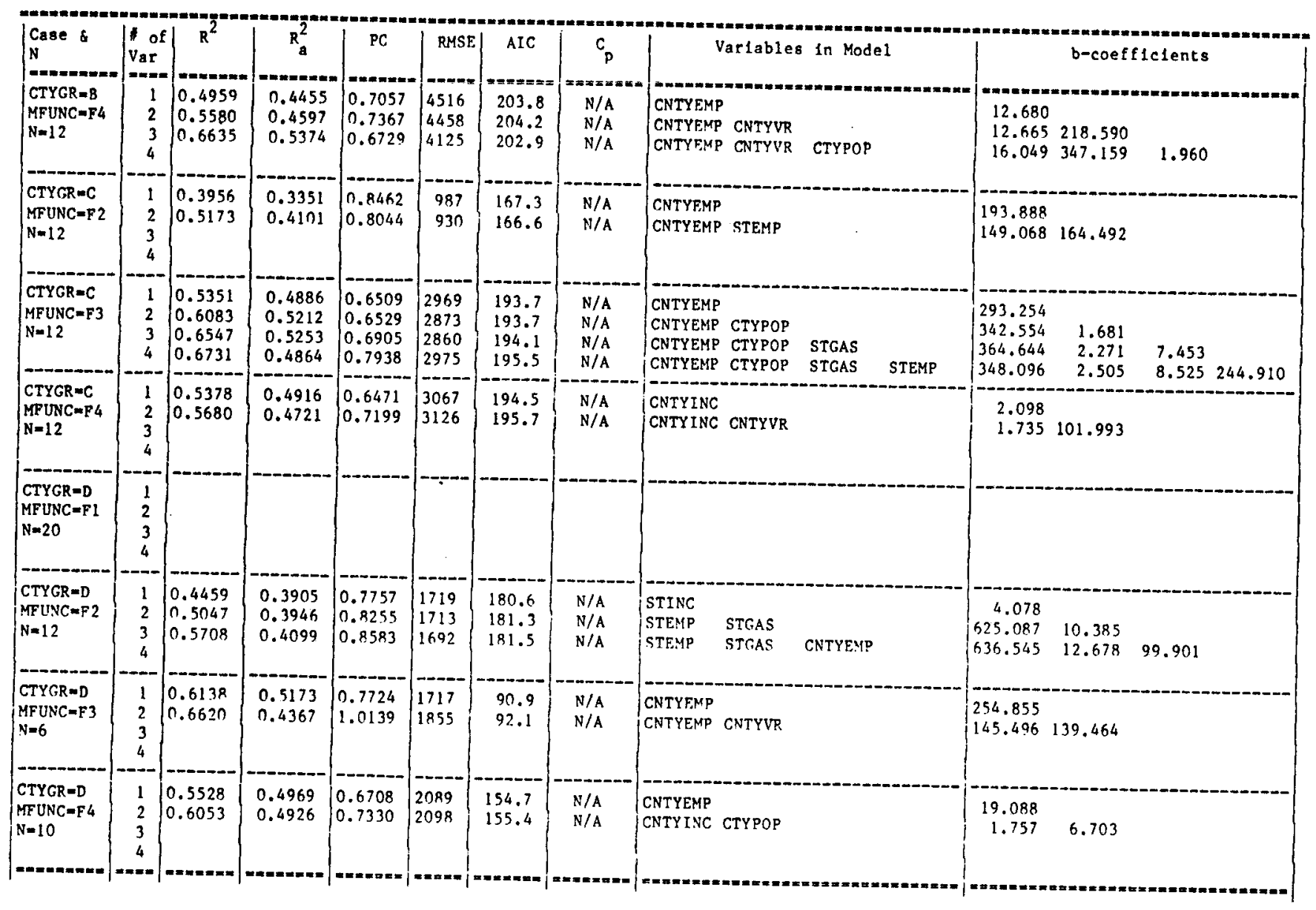


Table C8, continued

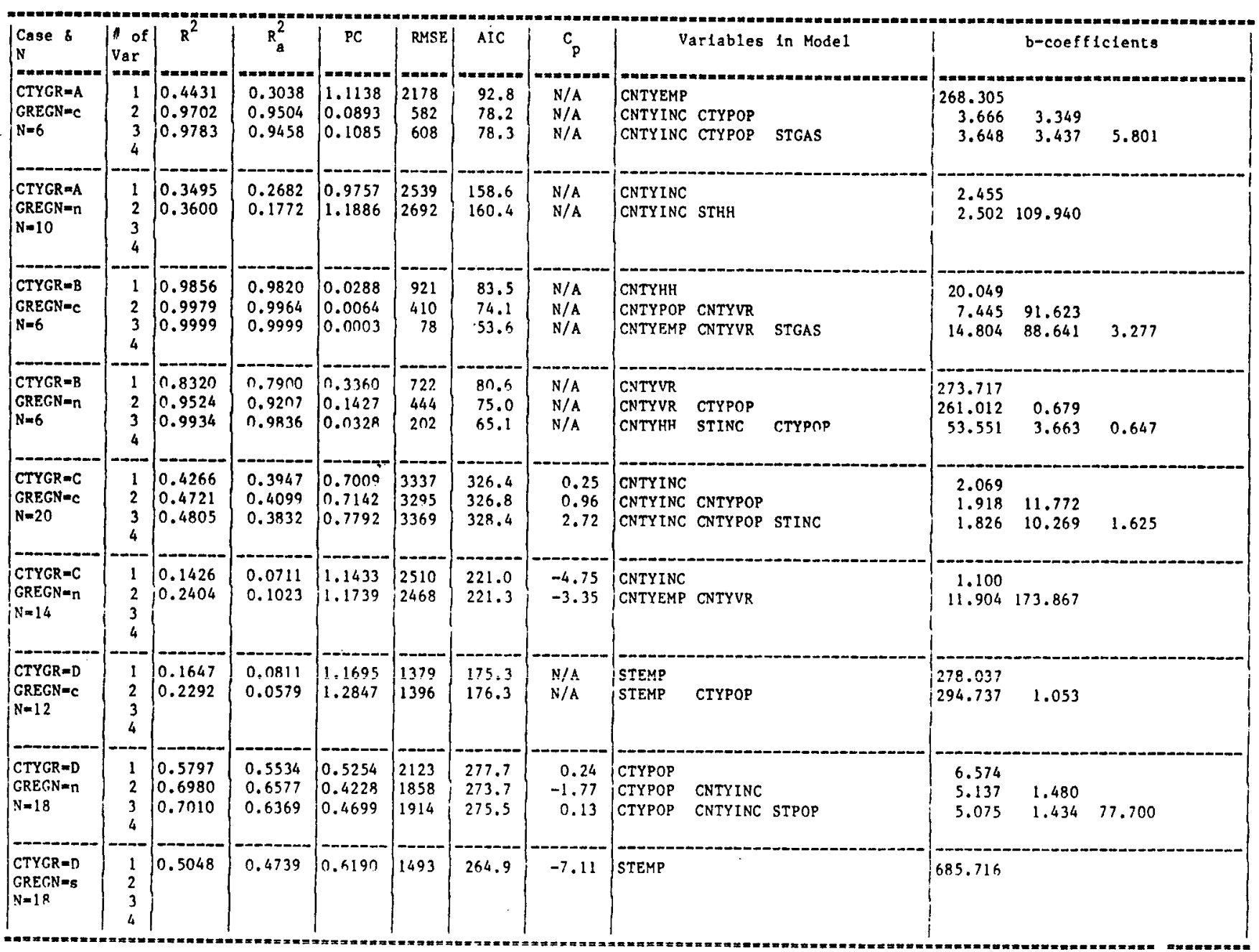

Note: Cases for $\mathrm{N}<5$ have not been recorded 
Table C9: Best Models for Urban Minor Arterial \& Collector [Data Format: Raw and City Avg. by Year]

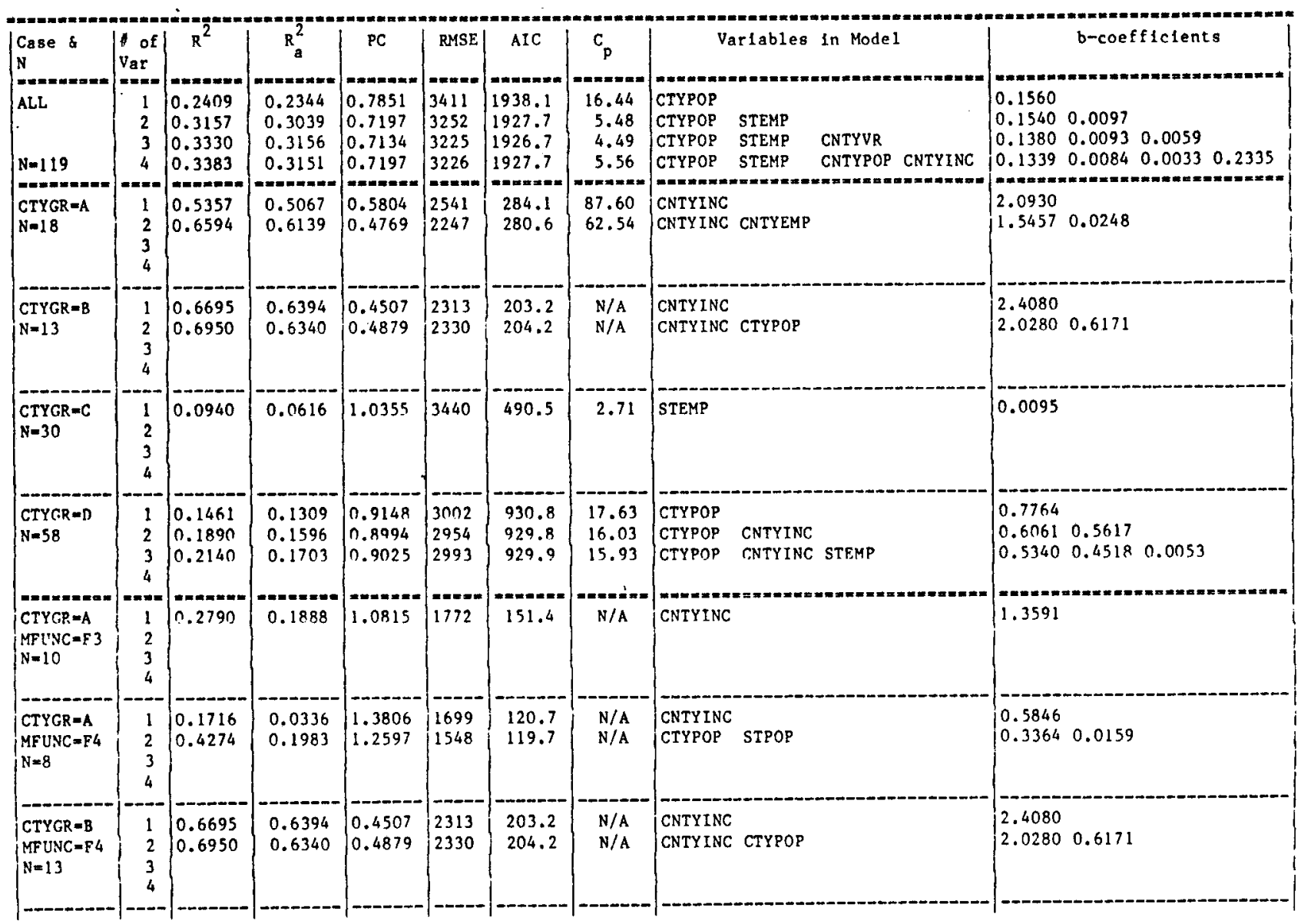


Table C9, continued

\begin{tabular}{|c|c|c|c|c|c|c|c|c|c|}
\hline $\begin{array}{l}\text { Case \& } \\
\mathrm{N}\end{array}$ & $\left|\begin{array}{l}\text { of } \\
\text { var }\end{array}\right|$ & $R^{2}$ & $\mathrm{R}_{\mathrm{a}}^{2}$ & PC & RMSE & AIC & $c_{p}$ & Variables In Model & $b$-coefficients \\
\hline $\begin{array}{l}\text { CTYGR=C } \\
\text { MFUNC=F2 } \\
N=14\end{array}$ & $\begin{array}{c}=- \\
1 \\
2 \\
3 \\
4\end{array}$ & 0.1745 & 0.1057 & $\mid=-1007$ & 4589 & $=-237.9$ & 33.22 & 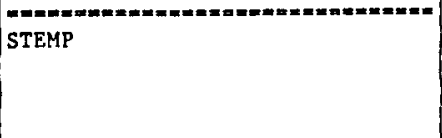 & 0.0169 \\
\hline $\begin{array}{l}\text { CTYGR }=C \\
\text { MFUNC }=F 3 \\
N=5\end{array}$ & $\begin{array}{l}1 \\
2 \\
3 \\
4\end{array}$ & 0.1634 & 0.1155 & 1.9521 & 463 & 62.8 & $\mathrm{~N} / \mathrm{A}$ & STEMP & 0.0010 \\
\hline $\begin{array}{l}\text { CTYGR }=C \\
\text { MFUNC }=F 4\end{array}$ & $\begin{array}{l}1 \\
2\end{array}$ & $\begin{array}{l}0.7830 \\
0.8180\end{array}$ & $\begin{array}{l}0.7588 \\
0.7725\end{array}$ & 0.3135 & $\begin{array}{l}1178 \\
1144\end{array}$ & $\begin{array}{l}157.4 \\
157.4\end{array}$ & $\begin{array}{l}\text { N/A } \\
\text { N/A } \\
N / A\end{array}$ & $\begin{array}{l}\text { CNTYHH } \\
\text { CNTYEMP STPOP }\end{array}$ & $\begin{array}{lll}0.0329 & & \\
0.0331 & 0.0111 & \\
0.0062 & 0.0094 & 0.0172\end{array}$ \\
\hline $\mathrm{N}=11$ & $\begin{array}{l}3 \\
4\end{array}$ & 0.8363 & 0.7662 & 0.3507 & 1160 & & & CNTYHH STPOP CNTYEMP & \\
\hline $\begin{array}{l}\text { CTYGR-D } \\
\text { MFUNC-FI } \\
\mathrm{N}=30\end{array}$ & $\begin{array}{l}1 \\
2 \\
3 \\
4\end{array}$ & & & & & & & & \\
\hline $\begin{array}{l}\text { CTYGR }=D \\
\text { MFUNC=F2 } \\
N=9\end{array}$ & $\begin{array}{l}1 \\
2 \\
3 \\
4\end{array}$ & $\begin{array}{l}0.9379 \\
0.9678\end{array}$ & $\begin{array}{l}0.9291 \\
0.9570\end{array}$ & $\begin{array}{l}0.0974 \\
0.0645\end{array}$ & $\begin{array}{l}686 \\
534\end{array}$ & $\begin{array}{l}119.3 \\
115.4\end{array}$ & $\begin{array}{l}\mathrm{N} / \mathrm{A} \\
\mathrm{N} / \mathrm{A}\end{array}$ & $\begin{array}{l}\text { CNTYEMP } \\
\text { CNTYPOP STGAS }\end{array}$ & $\begin{array}{ll}1.1835 & \\
0.5219 & 2.6181\end{array}$ \\
\hline $\begin{array}{l}\text { CTYGR }=D \\
\text { MFUNC=F } 3\end{array}$ & $\frac{1}{2}$ & 0.6737 & 0.6329 & 0.4895 & 1310 & 145.3 & N/A & STHH & 0.0130 \\
\hline$N=10$ & $\begin{array}{l}3 \\
4\end{array}$ & 0.8171 & 0.7649 & 0.3395 & 1048 & 141.5 & $\mathrm{~N} / \mathrm{A}$ & CNTYEMP & $0.0083 \quad 0.2840$ \\
\hline CTYGR $=D$ & 1 & 0.9484 & 0.9410 & 0.0811 & 475 & 112.7 & $\mathrm{~N} / \mathrm{A}$ & CNTYVR & 0.0218 \\
\hline MFUNC $=F 4$ & 2 & 0.9830 & 0.9774 & 0.0339 & 294 & 104.7 & N/A & CTYYOP CNTYINC & $0.6939 \quad 2.1797$ \\
\hline - & 3 & 0.9840 & 0.9744 & $0.416 n$ & 313 & $10 \% .1$ & $N / A$ & CTYPOP CNTYINC CNTYEMP & 2.01220 .59340 .0040 \\
\hline $\begin{array}{l}\text { CTYGR }=A \\
\text { GREGN }=\mathrm{C} \\
\mathrm{N}=5\end{array}$ & $\begin{array}{l}1 \\
2 \\
3 \\
4\end{array}$ & 0.5165 & 0.3553 & 1.1281 & 745 & 67.5 & N/A & STHY & $\begin{array}{l}=3=\pi=0 \\
0.0042\end{array}$ \\
\hline $\begin{array}{l}\text { CTYGR=A } \\
\text { GRERN=n } \\
\mathrm{N}=8\end{array}$ & $\begin{array}{l}1 \\
2 \\
3 \\
4\end{array}$ & $\begin{array}{l}0.1716 \\
0.4274\end{array}$ & $\begin{array}{l}0.0336 \\
0.1984\end{array}$ & $\begin{array}{l}1.3806 \\
1.2597\end{array}$ & $\begin{array}{l}1700 \\
1548\end{array}$ & $\begin{array}{l}120.7 \\
119.7\end{array}$ & $\begin{array}{l}\mathrm{N} / \mathrm{A} \\
\mathrm{N} / \mathrm{A}\end{array}$ & $\begin{array}{l}\text { CNTYINC } \\
\text { CTYPOP STPOP }\end{array}$ & $\begin{array}{lll}0.5846 & \\
0.3364 & 0.0159\end{array}$ \\
\hline
\end{tabular}


Table C9, continued

\begin{tabular}{|c|c|c|c|c|c|c|c|c|c|}
\hline 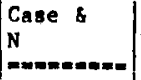 & $\mid \begin{array}{ll}\mid & \text { of } \\
\text { Var } \\
-a-\end{array}$ & $\mid R^{2}$ & $\left.\right|_{-\infty} ^{R_{a}^{2}}$ & $\mid \begin{array}{c}P C \\
= \pm= \pm=x= \pm\end{array}$ & RMSF & AIC & $\mathrm{c}_{\mathrm{p}}$ & Varíables in Model & $b$-coefficients \\
\hline $\begin{array}{l}\text { CTYGR }=A \\
\text { GREGN=8 } \\
N=5\end{array}$ & $\begin{array}{l}1 \\
2 \\
3 \\
4\end{array}$ & 0.7965 & 0.7287 & 0.4747 & 810 & 68.4 & $\mathrm{~N} / \mathrm{A}$ & CNTYMH & 0.6484 \\
\hline $\begin{array}{l}\text { CTYGR =B } \\
\text { GREGN=C } \\
N=5\end{array}$ & $\begin{array}{l}1 \\
2 \\
3 \\
4\end{array}$ & 0.2099 & -0.0534 & 1.8435 & 509 & 63.8 & $N / A$ & CNTYPOP & 0.0351 \\
\hline $\begin{array}{l}\text { CTYGR }=B \\
\text { GREGN=n } \\
N=8\end{array}$ & $\begin{array}{c}1 \\
2 \\
3 \\
4 \\
-\end{array}$ & 0.3389 & 0.2286 & 1.1018 & 2146 & 124.4 & $N / A$ & CNTYINC & 1.2822 \\
\hline $\begin{array}{l}\text { CTYGR }=C \\
\text { GREGN=C } \\
N=12\end{array}$ & $\begin{array}{l}1 \\
2 \\
3 \\
4\end{array}$ & & & & & & & & \\
\hline $\begin{array}{l}\text { CTYGR }=\mathrm{C} \\
\text { GREGN=n } \\
\mathrm{N}=13\end{array}$ & $\begin{array}{l}1 \\
2 \\
3 \\
4\end{array}$ & $\mid \begin{array}{l}0.7574 \\
0.7869\end{array}$ & $\begin{array}{l}0.7353 \\
0.7443\end{array}$ & $\begin{array}{l}0.3309 \\
0.3409\end{array}$ & $\begin{array}{l}975 \\
958\end{array}$ & $\begin{array}{l}180.8 \\
181.1\end{array}$ & $\begin{array}{l}\mathrm{N} / \mathrm{A} \\
\mathrm{N} / \mathrm{A}\end{array}$ & $\begin{array}{l}\text { CNTYHH } \\
\text { CNTYHH STHH }\end{array}$ & $\begin{array}{ll}0.0250 \\
0.0236 & 0.0027\end{array}$ \\
\hline $\begin{array}{l}\text { CTYGR }=C \\
\text { GREGN=s } \\
N=5\end{array}$ & $\begin{array}{l}1 \\
2 \\
3 \\
4\end{array}$ & $\left|\begin{array}{l}n .8893 \\
0.994 n\end{array}\right|$ & $\begin{array}{l}0.8525 \\
0.9880\end{array}$ & $\begin{array}{l}0.2581 \\
0.0239\end{array}$ & $\begin{array}{r}1393 \\
397\end{array}$ & $\begin{array}{l}73.8 \\
61.2\end{array}$ & $\begin{array}{l}N / A \\
\because / A\end{array}$ & $\begin{array}{l}\text { CNTYYH } \\
\text { CNTYEMP STINC }\end{array}$ & $\begin{array}{l}3.6792 \\
6.3572 \quad 2.8209\end{array}$ \\
\hline $\begin{array}{l}\text { CTYGR }=D \\
\text { GREGN-C } \\
N=8\end{array}$ & $\begin{array}{r}1 \\
2 \\
3 \\
4 \\
-\end{array}$ & $0.88 n 6$ & 0.8606 & 0.1991 & 834 & 109.3 & $\mathrm{~N} / \mathrm{A}$ & CNTYINC & 1.5171 \\
\hline $\begin{array}{l}\text { CTYGR=D } \\
\text { GREGN=n } \\
N=16\end{array}$ & $\begin{array}{l}1 \\
2 \\
3 \\
4\end{array}$ & $\begin{array}{l}0.2997 \\
0.3588\end{array}$ & $\begin{array}{l}0.2497 \\
0.2601\end{array}$ & $\begin{array}{l}0.9004 \\
0.9372\end{array}$ & $\begin{array}{r}1097 \\
957\end{array}$ & $\begin{array}{l}225.9 \\
226.5\end{array}$ & $\begin{array}{l}-3.24 \\
-1.97\end{array}$ & $\begin{array}{l}\text { CNTYVR } \\
\text { CNTYVR STVR }\end{array}$ & $\begin{array}{l}0.0074 \\
0.0070 \quad 0.0008\end{array}$ \\
\hline $\begin{array}{l}\text { CTYGR }=D \\
\text { GREGN=8 } \\
N=34\end{array}$ & $\begin{array}{l}1 \\
2 \\
3 \\
4\end{array}$ & 0.1897 & 0.1644 & 0.9115 & 3430 & 555.5 & 14.58 & CTYPOP & 0.9395 \\
\hline
\end{tabular}

Note: Cases for $\mathrm{N}<5$ have not been recorded 
Table c10: Best Models for Urban Minor Arterial \& Collector [Data Format: Raw E\&L and City Avg. by Year]

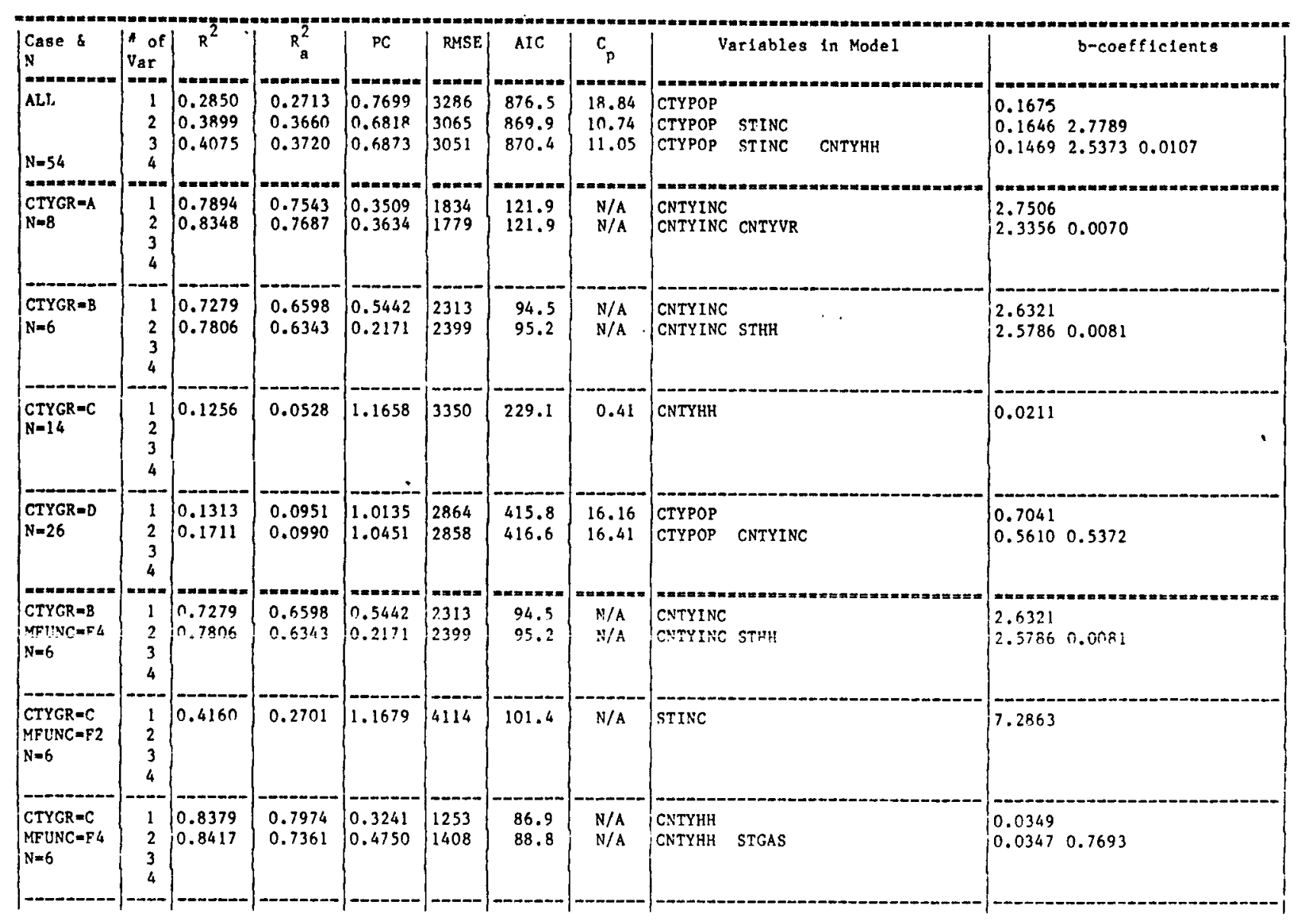


Table C10, continued

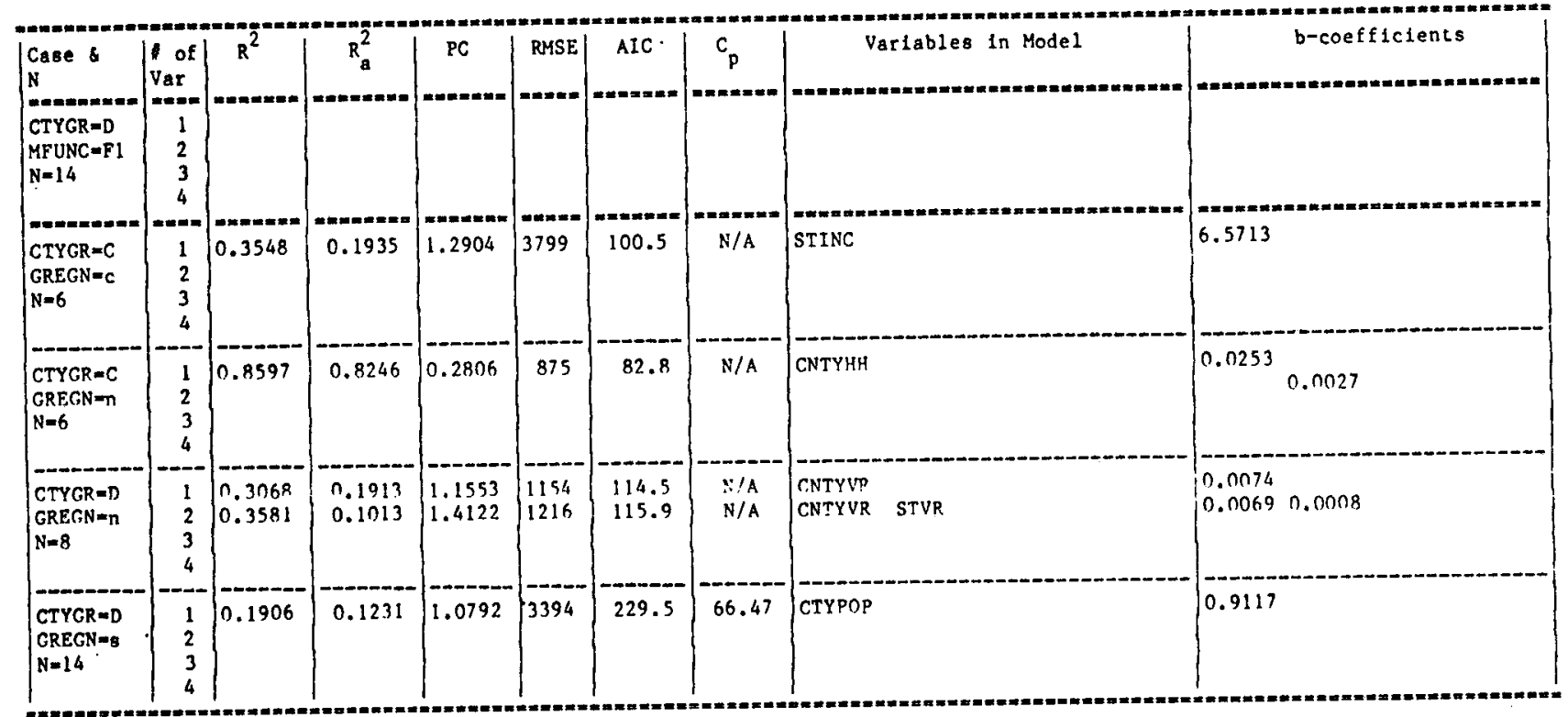

Note: Cases for $\mathrm{N}<5$ have not been recorded 
Table C11: Best Models for Urban Minor Arterial \& Collector [Data Format: Density and City Avg. by Year]

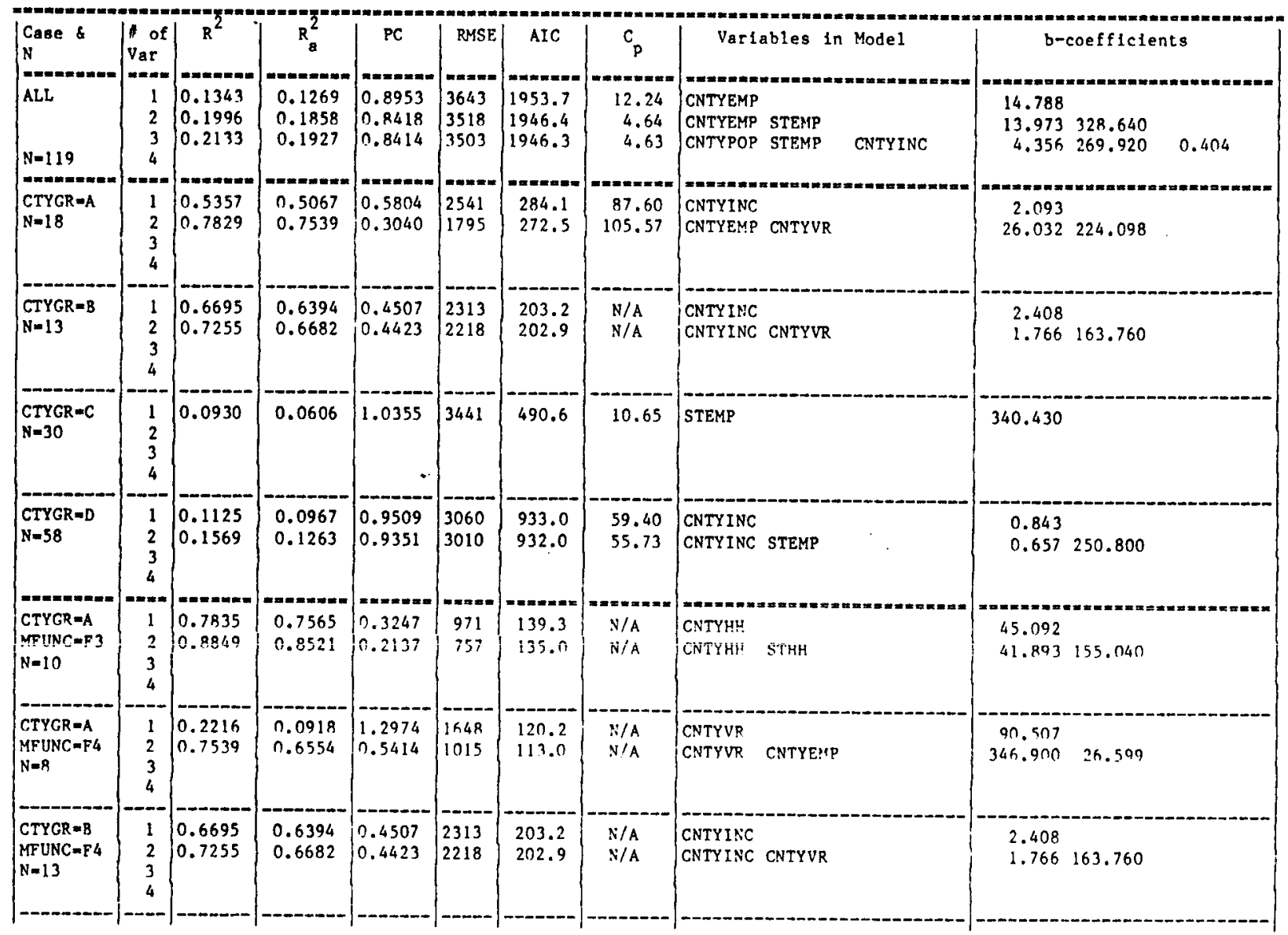


Table Cl1, continued

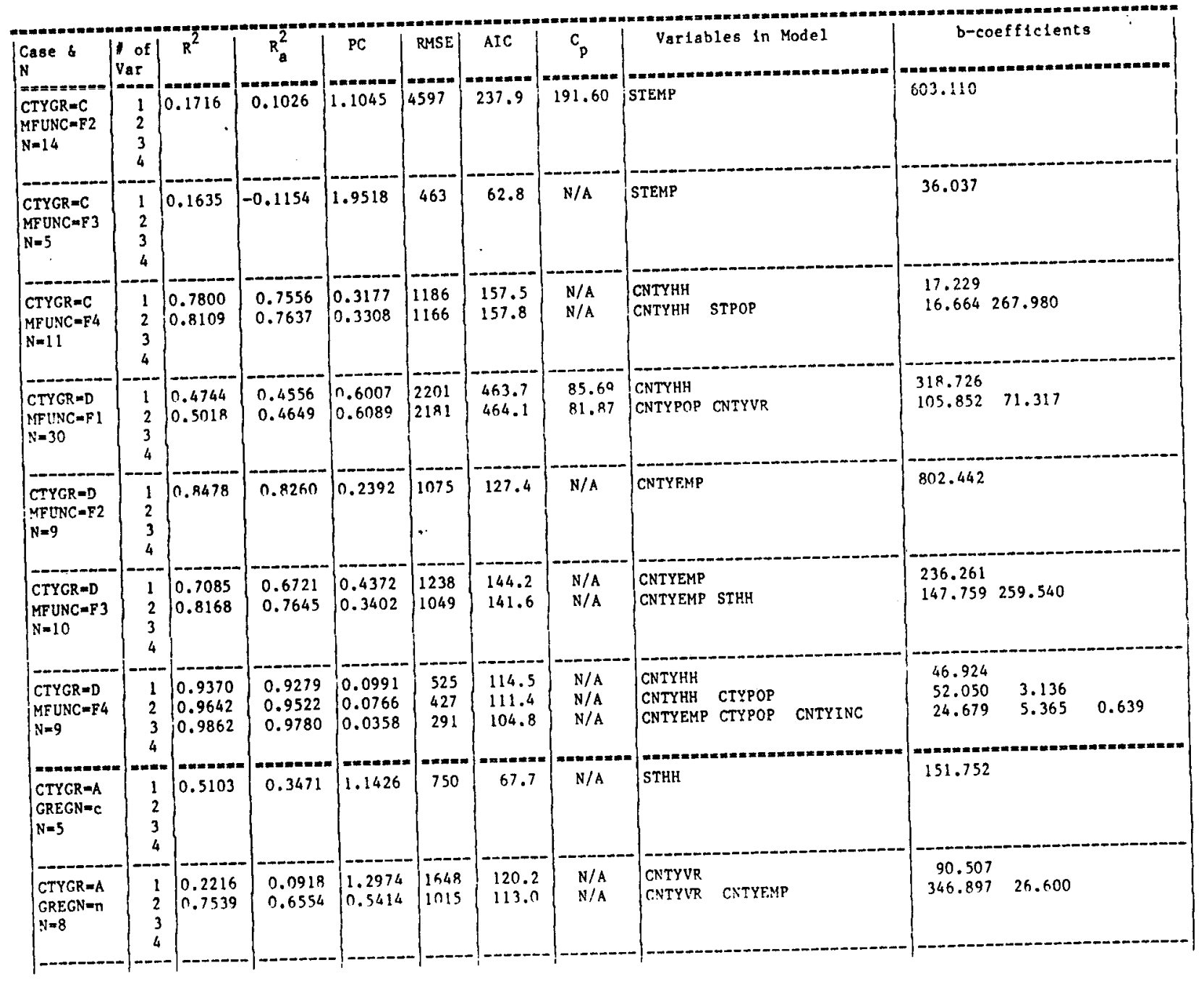


Table C11, continued

\begin{tabular}{|c|c|c|c|c|c|c|c|c|c|}
\hline $\begin{array}{l}\text { Case : } \\
\text { N }\end{array}$ & $\left|\begin{array}{ll}\text { A } & \text { of } \\
\text { Var }\end{array}\right|$ & $\mathrm{R}^{2}$ & $\mathrm{R}_{\mathrm{a}}^{2}$ & $\mathrm{PC}$ & RMSE & AIC & $c_{p}$ & Variables in Model & $b$-coefficlents \\
\hline $\begin{array}{l}\text { CTYGR=A } \\
\text { GREGN=8 } \\
N=5\end{array}$ & $\begin{array}{r}-=- \\
1 \\
2 \\
3 \\
4\end{array}$ & 0.7966 & 0.7288 & 0.4746 & 810 & 68.4 & $\mathrm{~N} / \mathrm{A}$ & CNTYH & 97.047 \\
\hline $\begin{array}{l}\text { CTYGR }=B \\
\text { GREGN=C } \\
N=5\end{array}$ & $\begin{array}{l}1 \\
2 \\
3 \\
4\end{array}$ & 0.2103 & -0.0530 & 1.8427 & 509 & 63.8 & $\mathrm{~N} / \mathrm{A}$ & CNTYPOP & 17.535 \\
\hline $\begin{array}{l}\text { CTYGR }=B \\
\text { GREGN=n } \\
N=8\end{array}$ & $\begin{array}{l}1 \\
2 \\
3 \\
4\end{array}$ & $\begin{array}{l}0.8549 \\
0.8972\end{array}$ & $\begin{array}{l}0.8307 \\
0.8560\end{array}$ & $\left|\begin{array}{l}0.2419 \\
0.2263\end{array}\right|$ & $\begin{array}{l}1005 \\
927\end{array}$ & $\begin{array}{l}112.3 \\
111.3\end{array}$ & $\begin{array}{l}N / A \\
N / A\end{array}$ & $\begin{array}{l}\text { CTYPOP } \\
\text { CTYPOP CNTYVR }\end{array}$ & $\begin{array}{l}3.037 \\
1.615 \quad 155.009\end{array}$ \\
\hline $\begin{array}{l}\text { CTYGR }=C \\
\text { GREGN=C } \\
N=12\end{array}$ & $\begin{array}{l}1 \\
2 \\
3 \\
4\end{array}$ & 0.1425 & 0.0568 & 1.2005 & 4043 & 201.1 & $\mathrm{~N} / \mathrm{A}$ & STINC & 2.720 \\
\hline $\begin{array}{l}\text { CTYGR=C } \\
\text { GREGN=n } \\
\mathrm{N}=\mathrm{I3}\end{array}$ & $\begin{array}{l}1 \\
2 \\
3\end{array}$ & $\mid \begin{array}{l}0.7547 \\
0.8707 \\
0.9325\end{array}$ & $\begin{array}{l}0.7324 \\
0.8448 \\
0.9099\end{array}$ & $\mid \begin{array}{l}0.3345 \\
0.2069 \\
0.1275\end{array}$ & $\begin{array}{l}981 \\
747 \\
568\end{array}$ & $\begin{array}{l}180.8 \\
174.6 \\
168.2\end{array}$ & $\begin{array}{l}N / A \\
N / A \\
N / A\end{array}$ & $\begin{array}{l}\text { CNTYHH } \\
\text { CNTYEMP CTYPOP } \\
\text { CNTYEMP CTYPOP } \\
\text { STHH }\end{array}$ & $\begin{array}{llll}12.870 & & & \\
18.920 & 1.683 & \\
18.184 & 1.752 & 141.189\end{array}$ \\
\hline $\begin{array}{l}\text { CTYGR }=C \\
\text { GREGN=s } \\
N=5\end{array}$ & $\begin{array}{l}1 \\
2 \\
3 \\
4\end{array}$ & $\mid \begin{array}{l}0.8831 \\
0.9926\end{array}$ & $\begin{array}{l}0.8442 \\
0.9851\end{array}$ & $\mid \begin{array}{l}0.2727 \\
0.0297\end{array}$ & $\begin{array}{r}1432 \\
442\end{array}$ & $\begin{array}{l}74.1 \\
62.3\end{array}$ & $\begin{array}{l}\mathrm{N} / \mathrm{A} \\
\mathrm{N} / \mathrm{A}\end{array}$ & $\begin{array}{l}\text { CNTYHH } \\
\text { CNTYEMP STINC }\end{array}$ & $\begin{array}{ll}1577.910 \\
2699.420\end{array} \quad 2.855$ \\
\hline $\begin{array}{l}\text { CTYGR }=D \\
\text { GREGN=C } \\
N=8\end{array}$ & $\begin{array}{l}1 \\
2 \\
3 \\
4\end{array}$ & 0.8806 & 0.8606 & 0.1991 & 834 & 109.3 & $\mathrm{~N} / \mathrm{A}$ & CNTYINC & 1.517 \\
\hline $\begin{array}{l}\text { CTYGR }=\mathrm{D} \\
\text { CREGN=n } \\
N=16\end{array}$ & $\begin{array}{l}1 \\
2 \\
3 \\
4\end{array}$ & $\left\{\begin{array}{l}0.2654 \\
0.549 ?\end{array}\right.$ & $\begin{array}{l}n .2130 \\
0.4799\end{array}$ & $\begin{array}{l}0.9444 \\
0.5589\end{array}$ & $\begin{array}{r}1124 \\
914\end{array}$ & $\begin{array}{l}227.0 \\
221.0\end{array}$ & $\begin{array}{l}32.72 \\
17.44\end{array}$ & $\begin{array}{l}\text { CNTYHH } \\
\text { CNTYHH CNTYVR }\end{array}$ & $\begin{array}{ll}11.351 & \\
16.586 & 91.996\end{array}$ \\
\hline $\begin{array}{l}\text { CTYGR }=D \\
\text { GREGN=s } \\
N=34\end{array}$ & $\begin{array}{l}1 \\
2 \\
3 \\
4\end{array}$ & 0.1778 & $\begin{array}{l}0.1521 \\
0.1539\end{array}$ & $\begin{array}{l}0.9450 \\
0.9486\end{array}$ & $\begin{array}{l}3445 \\
3451\end{array}$ & $\begin{array}{l}555.9 \\
556.8\end{array}$ & $\begin{array}{l}40.07 \\
39.73\end{array}$ & $\begin{array}{l}\text { CNTYINC } \\
\text { CNTYINC STEMP }\end{array}$ & $\begin{array}{l}1.173 \\
0.945220 .820\end{array}$ \\
\hline
\end{tabular}

Note: Cases for $\mathrm{N}<5$ have not been recorded 
Table C12: Best Mode1s for Urban Minor Arterial \& Collector [Data Format: Density E\&L and City Avg. by Year]

\begin{tabular}{|c|c|c|c|c|c|c|c|c|c|c|}
\hline Case \& & $\begin{array}{l}\text { of } \\
\text { Var } \\
\text { Var }\end{array}$ & $\mathrm{R}^{2}$ & $\mathrm{R}_{\mathrm{a}}^{2}$ & PC & RMSE | & AIC & $C_{p}$ & Variables In Model & & b-coefficlents \\
\hline $\begin{array}{l}\text { ALL } \\
N=54\end{array}$ & $\begin{array}{r}1 \\
2 \\
3 \\
4\end{array}$ & 0.1852 & $\begin{array}{l}0.1696 \\
0.2231\end{array}$ & 0.8774 & $\begin{array}{l}3508 \\
3393\end{array}$ & $\begin{array}{l}883.6 \\
880.9\end{array}$ & $\begin{array}{l}17.13 \\
13.59\end{array}$ & $\begin{array}{l}\text { CNTYEMP } \\
\text { CNTYHH STINC }\end{array}$ & $\begin{array}{l}15.323 \\
14.331\end{array}$ & 2.324 \\
\hline $\begin{array}{l}\text { CTYGR }=\mathrm{A} \\
\mathrm{N}=8\end{array}$ & \begin{tabular}{c|}
-1 \\
1 \\
2 \\
3 \\
4
\end{tabular} & $\begin{array}{l}0.7896 \\
0.8735\end{array}$ & $\begin{array}{l}0.7545 \\
0.8230\end{array}$ & $\mid \begin{array}{l}0=1507 \\
0.3507 \\
0.2782\end{array}$ & $\begin{array}{l}1834 \\
1557\end{array}$ & $\begin{array}{l}121.9 \\
119.8\end{array}$ & $\begin{array}{l}\mathrm{N} / \mathrm{A} \\
\mathrm{N} / \mathrm{A}\end{array}$ & $\begin{array}{l}\text { CNTYINC } \\
\text { CTYPOP CNTYVR }\end{array}$ & $\begin{array}{l}2.750 \\
8.242\end{array}$ & 339.141 \\
\hline $\begin{array}{l}\text { CTYGR=B } \\
N=6\end{array}$ & $\begin{array}{l}1 \\
2 \\
3 \\
4 \\
\end{array}$ & $\mid \begin{array}{l}0.7279 \\
0.8312\end{array}$ & $\begin{array}{l}0.6598 \\
0.7187\end{array}$ & $\left\{\begin{array}{l}0.5442 \\
0.5064\end{array}\right.$ & $\begin{array}{l}2313 \\
2104\end{array}$ & $\begin{array}{l}94.5 \\
93.7\end{array}$ & $\begin{array}{l}N / A \\
N / A\end{array}$ & $\begin{array}{l}\text { CNTYINC } \\
\text { CNTYINC CNTYVR }\end{array}$ & $\begin{array}{l}2.632 \\
1.765\end{array}$ & 222.666 \\
\hline $\begin{array}{l}\text { CTYGR }=C \\
N=14\end{array}$ & $\begin{array}{l}1 \\
2 \\
3 \\
4\end{array}$ & 0.1152 & 0.0415 & 1.1797 & 3371 & 229.3 & 1134.28 & STEMP & 454.310 & \\
\hline $\begin{array}{l}\text { CTYGR=D } \\
N=26\end{array}$ & $\begin{array}{l}1 \\
2 \\
3 \\
4 \\
\end{array}$ & 0.0978 & 0.0602 & 1.0526 & 2918 & 416.8 & 38.55 & CNTYINC & 0.790 & \\
\hline $\begin{array}{l}\text { CTYGR }=8 \\
\text { MFUNC=F4 } \\
Y=6\end{array}$ & $\begin{array}{c}-=- \\
1 \\
2 \\
3 \\
4\end{array}$ & $\mid \begin{array}{l}0.7279 \\
0.8312\end{array}$ & $\begin{array}{l}0.6598 \\
0.7187\end{array}$ & $\begin{array}{l}0.5442 \\
0.5064\end{array}$ & $\begin{array}{l}2313 \\
2104\end{array}$ & $\begin{array}{r}94.5 \\
93.7\end{array}$ & N/A & $\begin{array}{l}\text { CNTYINC } \\
\text { CNTYINC CNTYVR }\end{array}$ & $\begin{array}{l}2.632 \\
1.765\end{array}$ & 222.666 \\
\hline $\begin{array}{l}\text { CTYGR=C } \\
M F U N C=F 2 \\
N=6\end{array}$ & $\begin{array}{l}1 \\
2 \\
3 \\
4 \\
-\end{array}$ & 0.4160 & 0.2701 & 1.1679 & 4114 & 101.4 & $\mathrm{~N} / \mathrm{A}$ & STINC & 7.286 & \\
\hline $\begin{array}{l}\text { CTYGR=C } \\
M N U N C=F 4 \\
Y a 6\end{array}$ & $\begin{array}{l}1 \\
2 \\
3 \\
4\end{array}$ & $\left|\begin{array}{l}0.8313 \\
0.8359\end{array}\right|$ & $\begin{array}{l}0.7891 \\
n .7265\end{array}$ & $\begin{array}{l}0.3375 \\
0.4923\end{array}$ & $\begin{array}{l}1259 \\
1434\end{array}$ & $\begin{array}{l}87.2 \\
89.0\end{array}$ & $\begin{array}{l}N / A \\
N / A\end{array}$ & $\begin{array}{l}\text { CNTYHH } \\
\text { CNTYYH CNTYENP }\end{array}$ & $\begin{array}{l}18.291 \\
14.001\end{array}$ & 4.433 \\
\hline
\end{tabular}


Table C12, continued

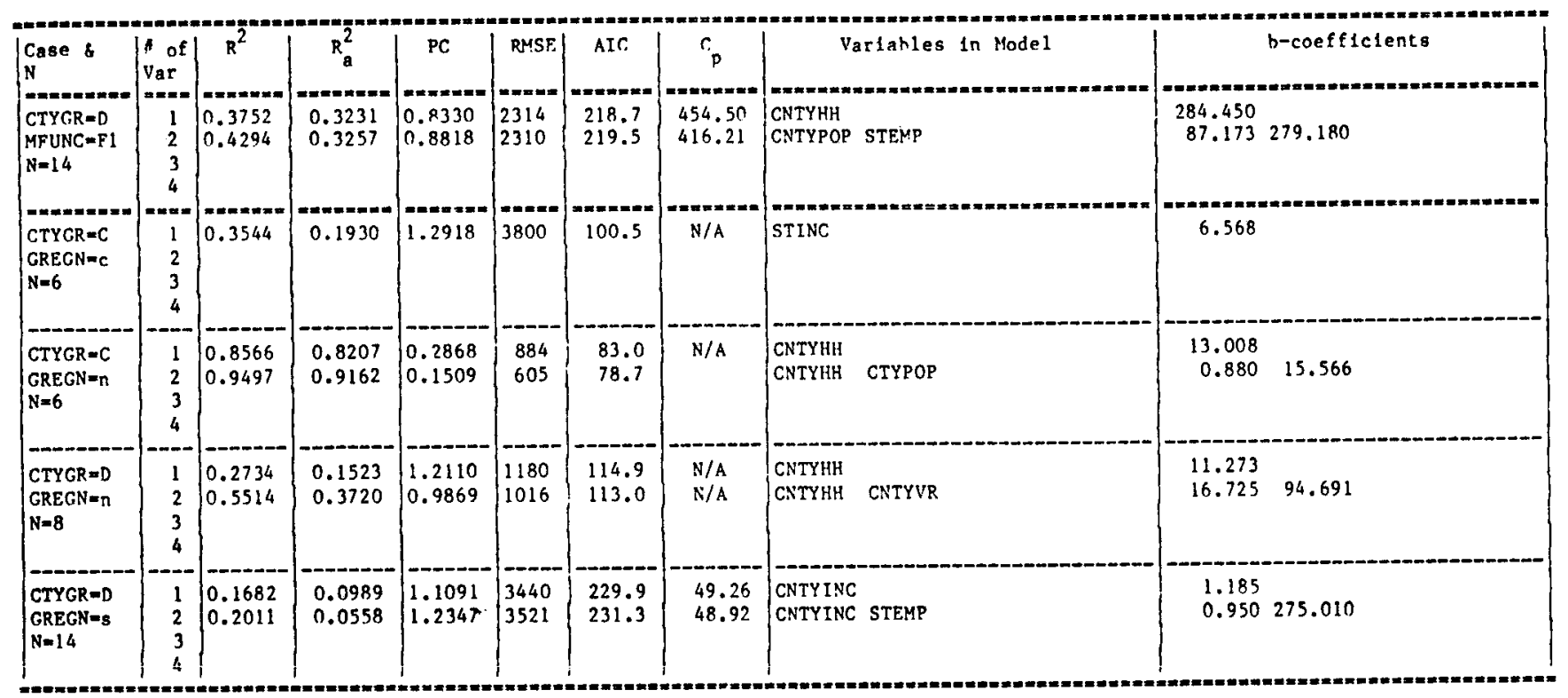

Note: Cases for $\mathrm{N}<5$ have not been recorded 
Appendix D 
Table DI: Elgenvectors or Coeffictents of Principal Components (PCs) of Final Regression Equations for Interstate Highways

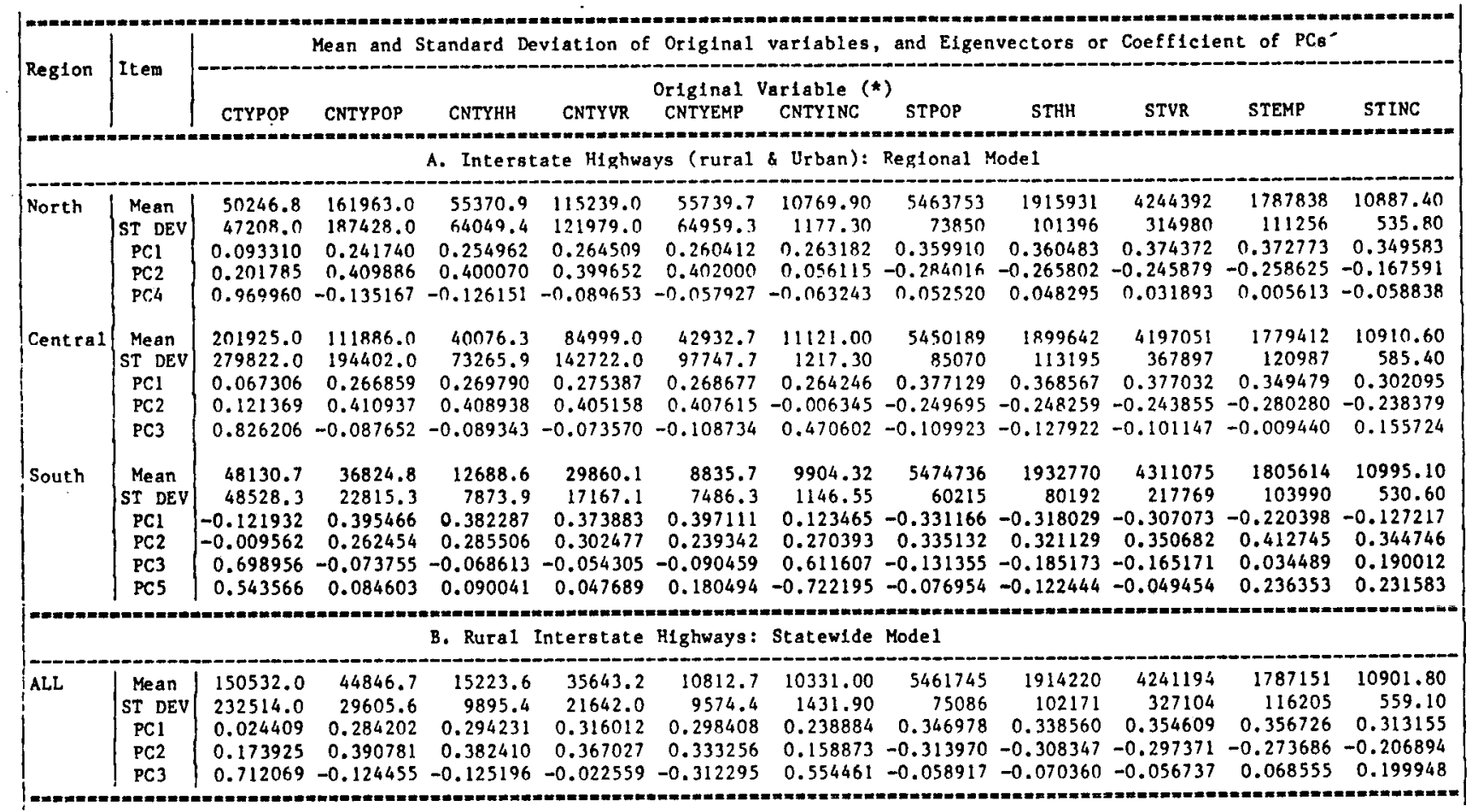


Table D1, continued

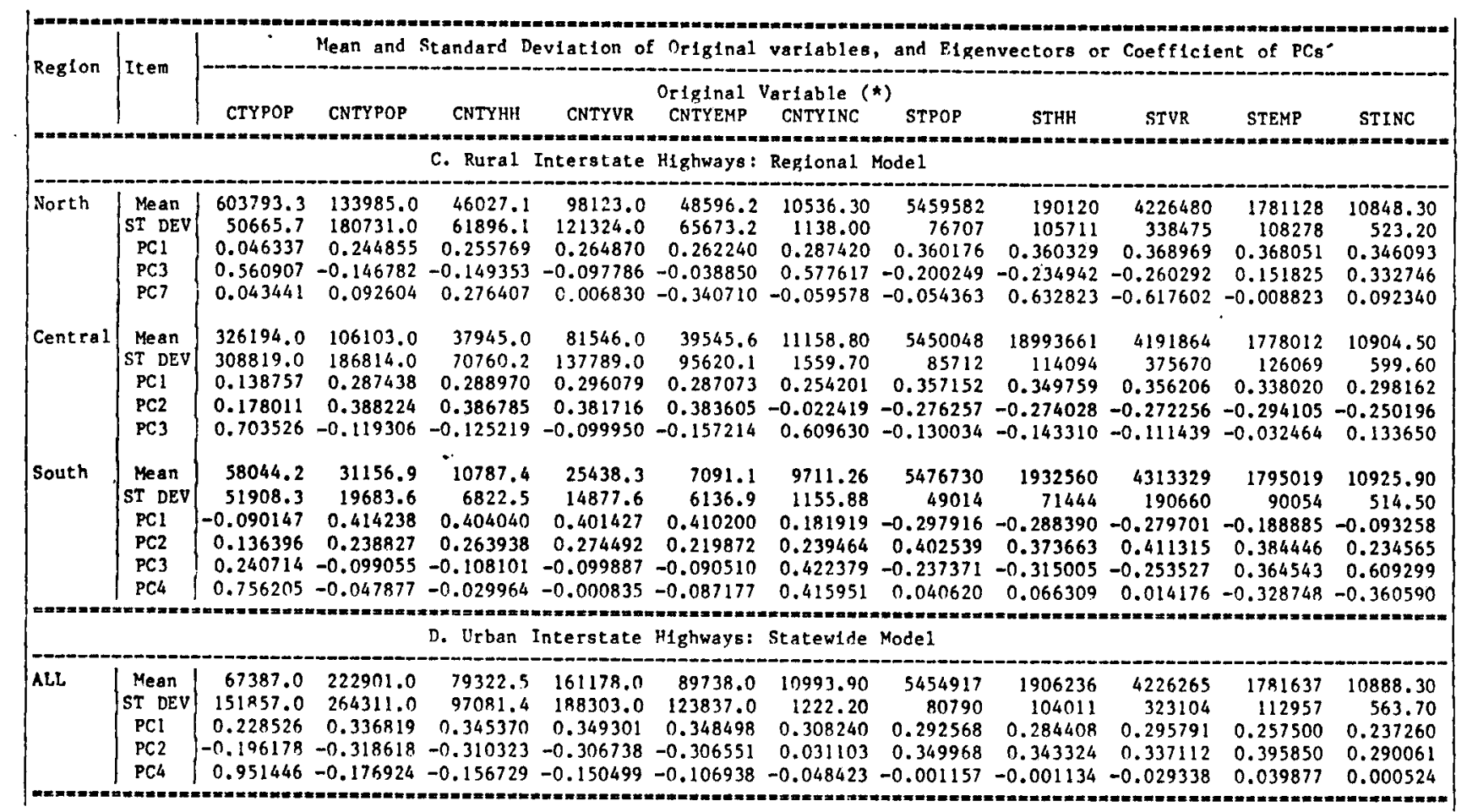


Table D1, continued

\begin{tabular}{|c|c|c|c|c|c|c|c|c|c|c|c|c|}
\hline \multirow{2}{*}{ Region } & \multirow{2}{*}{ Item } & \multicolumn{11}{|c|}{ Mean an } \\
\hline & & CTYPOP & CNTYPOP & CNTYHH & CNTYVR & $\begin{array}{c}\text { Original } \\
\text { CNTYEMP }\end{array}$ & $\begin{array}{c}\text { Variable } \\
\text { CNTYINC }\end{array}$ & STPOP & STHH & STVR & STEMP & STINC \\
\hline & & & & Urban & & & $x_{0}$ & & & & & \\
\hline North & $x$ & 44730.8 & 286608.0 & 98425.9 & 196656.0 & 96940.0 & 11043.90 & 5463905 & 1918376 & 4262 & 1787788 & 10914.80 \\
\hline & ST DEV & $\begin{array}{r}55117.1 \\
-0\end{array}$ & 215583.0 & 74020.7 & 137269.0 & $\begin{array}{r}72302.6 \\
\end{array}$ & $\begin{array}{r}1008.80 \\
0\end{array}$ & $\begin{array}{r}69915 \\
0\end{array}$ & & $\begin{array}{r}275364 \\
0\end{array}$ & $\begin{array}{r}104922 \\
0.352069\end{array}$ & 533.20 \\
\hline & PC2 & $\begin{array}{l}0.140015 \\
0.13015\end{array}$ & $\begin{array}{r}-0.336848 \\
0.347029\end{array}$ & $\begin{array}{r}=0.314178 \\
0.365359\end{array}$ & $\begin{array}{r}-0.316579 \\
0.371566\end{array}$ & $\begin{array}{r}-0.331466 \\
0.357705\end{array}$ & 0.208292 & 0.274856 & & 0.308083 & $\begin{array}{l}0.352069 \\
0.296508\end{array}$ & $\begin{array}{l}0.238534 \\
0.286391\end{array}$ \\
\hline & PC4 & 0.924476 & -0.126039 & -0.110230 & -0.064660 & -0.024383 & -0.030525 & 0.123698 & 0.134290 & 0.088007 & -0.078964 & -0.253543 \\
\hline Centra1 & Mean & 91302.0 & 247273.0 & 91017.0 & 183998.0 & 06.0 & 1144 & 545 & 190 & 42 & 1784388 & 10927.00 \\
\hline & ST DEV & 208998.0 & 315481.0 & 119241.0 & 232426.0 & $15984 \mathrm{~L} .0$ & 1033.00 & 80958 & 106634 & 334551 & 112019 & 3.90 \\
\hline & $\mathrm{PCl}$ & 0.224985 & 0.339951 & 0.344082 & 0.345785 & 0.345985 & 0.306846 & 0.307679 & 0.297693 & 0.303560 & 0.252732 & 0.206605 \\
\hline & PC2 & -0.206394 & 0.315620 & 0.310501 & 0.307767 & 0.307879 & 0.038420 & 0.334817 & 0.333675 & 0.329176 & 0.378647 & 0.308573 \\
\hline & PC4 & 0.944366 & -0.136504 & -0.133591 & -0.136685 & -0.128459 & -0.153183 & -0.022680 & -0.012383 & -0.020290 & 0.075412 & 0.079286 \\
\hline South & Mean & 21399.2 & 58239.3 & 20056.7 & 44765.2 & 15146.2 & 9648.06 & 5440118 & 1890307 & 4186146 & 1776511 & 10791.30 \\
\hline & ST DEV & 13282.0 & 28979.3 & & & & & 99861 & & & 137326 & 697.50 \\
\hline & $\mathrm{PCl}$ & 0.307087 & 0.300205 & 0.314334 & 0.318355 & 0.260426 & 0.359868 & 0.291789 & 0.278049 & 0.303326 & 0.282115 & 0.289904 \\
\hline & PC2 & -0.086342 & -0.359251 & -0.335772 & -0.322627 & -0.387249 & -0.025499 & 0.337306 & 0.344138 & 0.310801 & 0.317924 & 0.257220 \\
\hline & $\mathrm{PC} 3$ & -0.478276 & 0.118278 & 0.122706 & 0.161621 & 0.221958 & -0.455552 & 0.318544 & 0.325524 & 0.320707 & -0.211305 & -0.323035 \\
\hline
\end{tabular}

(*) See Table 4.3 of Chapter 4 for description of Original variables 
Table .D2: Eigenvectors or Coefficients of Principal Components (PCs) of Final Regression Equations for Urban Principal Arterial

\begin{tabular}{|c|c|c|c|c|c|c|c|c|c|c|c|c|}
\hline & & & & $t$ & & & In 3000 & to 50,0001 & & & & \\
\hline North & $\left|\begin{array}{c}\text { Mean } \\
\text { ST DEV } \\
\text { PC3 } \\
\text { PC4 }\end{array}\right|$ & $\begin{array}{r}41985.8 \\
5 n 08.2 \\
0.353476 \\
0.667093\end{array}$ & $\begin{array}{r}184855.0 \\
169470.0 \\
0.008524 \\
-0.037438\end{array}$ & $\begin{array}{r}62634.1 \\
56634.7 \\
0.007572 \\
-0.025738\end{array}$ & $\begin{array}{ll}1 & 132696.0 \\
7 & 105396.0 \\
2 & 0.038664 \\
8 & 0.000509\end{array}$ & $\begin{array}{r}69628.5 \\
55349.5 \\
0.115924 \\
0.052353\end{array}$ & $\begin{array}{rr}5 & 11331.20 \\
5 & 982.80 \\
4 & 0.638445 \\
3 & 0.228652\end{array}$ & $\begin{array}{r}5442145 \\
72169 \\
0.005027 \\
0.231154\end{array}$ & $\begin{array}{r}1882983 \\
102372 \\
-0.045747 \\
0.272747\end{array}$ & $\begin{array}{r}4175026 \\
287127 \\
-0.017592 \\
0.231628\end{array}$ & $\begin{array}{r}1767658 \\
91605 \\
0.337877 \\
-0.197699\end{array}$ & $\begin{array}{r}10979.40 \\
411.00 \\
0.579475 \\
-0.526632\end{array}$ \\
\hline Central & $\begin{array}{c}\text { Mean } \\
\text { ST DEV } \\
\text { PC1 } \\
\text { PC3 } \\
\text { PC4 }\end{array}$ & $\begin{array}{r}38863.4 \\
7706.5 \\
0.054854 \\
-0.391462 \\
0.422657\end{array}$ & $\begin{array}{r}86392.7 \\
25930.1 \\
0.169026 \\
0.160610 \\
-0.040925\end{array}$ & $\begin{array}{r}28798.4 \\
7907.0 \\
0.231217 \\
0.042494 \\
-0.062611\end{array}$ & $\begin{array}{rr}4 & 61157.2 \\
0 & 14637.8 \\
0.321267 \\
4 \\
0.116283 \\
-0.184236\end{array}$ & $\begin{array}{r}29938.4 \\
5440.4 \\
0.294509 \\
0.523122 \\
-0.093580\end{array}$ & $\begin{array}{cc}4 & 10533.40 \\
4 & 1094.10 \\
9 & 0.158099 \\
2 & 0.556841 \\
0 & 0.032814\end{array}$ & $\begin{array}{r}5403535 \\
117344 \\
0.381860 \\
-0.208676 \\
-0.307573\end{array}$ & $\begin{array}{r}1845014 \\
142321 \\
0.372993 \\
-0.295426 \\
-0.284776\end{array}$ & $\begin{array}{r}4015922 \\
513686 \\
0.384625 \\
-0.288704 \\
-0.185369\end{array}$ & $\begin{array}{r}1724947 \\
144671 \\
0.376790 \\
-0.051267 \\
0.398216\end{array}$ & $\begin{array}{r}10603.40 \\
731.90 \\
0.353793 \\
0.071451 \\
0.635100\end{array}$ \\
\hline South & $\mid \begin{array}{c}\text { Mean } \\
\text { ST DEv } \\
\text { PC1 } \\
\text { PC2 } \\
\text { PC3 }\end{array}$ & $\begin{array}{r}23412.1 \\
11297.6 \\
0.110765 \\
0.237269 \\
0.757189\end{array}$ & $\begin{array}{r}57616.8 \\
24427.2 \\
0.250630 \\
0.406293 \\
-0.121656\end{array}$ & $\begin{array}{r}.19726 .2 \\
8339.3 \\
0.290312 \\
0.367958 \\
-0.118935\end{array}$ & $\begin{array}{r}42923.3 \\
19564.0 \\
0.325062 \\
0.318280 \\
-0.158328\end{array}$ & $\begin{array}{r}14895.9 \\
9813.8 \\
0.204427 \\
0.392272 \\
-0.363881\end{array}$ & $\begin{array}{r}9632.67 \\
815.99 \\
0.324942 \\
0.141461 \\
0.478435\end{array}$ & $\begin{array}{r}5403418 \\
126268 \\
0.344784 \\
-0.286155 \\
-0.062623\end{array}$ & $\begin{array}{r}1848838 \\
147759 \\
0.341793 \\
-0.279808 \\
-0.072370\end{array}$ & $\begin{array}{r}4041886 \\
522547 \\
0.358549 \\
-0.263436 \\
-0.035332\end{array}$ & $\begin{array}{r}1752719 \\
141390 \\
0.336851 \\
-0.258473 \\
0.001224\end{array}$ & $\begin{array}{r}10681.60 \\
849.10 \\
0.331463 \\
-0.267696 \\
0.030535\end{array}$ \\
\hline & & & & cy & " & y Popula & on: 20,000 & to 30,000$\}$ & & & & \\
\hline North & $\left|\begin{array}{c}\text { Mean } \\
\text { ST DFV } \\
\text { PC1 } \\
\text { PC2 }\end{array}\right|$ & $\begin{array}{r}23286.3 \\
2555.1 \\
0.215837 \\
-0.025468\end{array}$ & $\begin{array}{r}111175.0 \\
9586.0 \\
0.316054 \\
-0.252397\end{array}$ & $\begin{array}{r}36318.2 \\
4250.6 \\
0.347710 \\
-0.296452\end{array}$ & $\begin{array}{r}93978.9 \\
12150.8 \\
0.356031 \\
-0.031083\end{array}$ & $\begin{array}{r}30383.2 \\
3121.7 \\
0.263403 \\
-0.113722\end{array}$ & $\begin{array}{r}11690.20 \\
1126.80 \\
0.054259 \\
0.630114\end{array}$ & $\begin{array}{r}5441148 \\
74170 \\
0.362274 \\
-0.023661\end{array}$ & $\begin{array}{r}1879797 \\
110411 \\
0.360062 \\
-0.092822\end{array}$ & $\begin{array}{r}4161632 \\
350337 \\
0.374415 \\
0.020379\end{array}$ & $\begin{array}{r}1773192 \\
109362 \\
0.288475 \\
0.395226\end{array}$ & $\begin{array}{r}10931.20 \\
603.40 \\
0.221916 \\
0.520602\end{array}$ \\
\hline Central & \begin{tabular}{|c|} 
Mean \\
ST \\
DEV \\
PC1 \\
PC2
\end{tabular} & $\begin{array}{r}23286.3 \\
2555.1 \\
0.355433 \\
-0.206873\end{array}$ & $\begin{array}{r}111175.0 \\
9586.0 \\
0.357384 \\
-0.228450\end{array}$ & $\begin{array}{r}36318.2 \\
4260.6 \\
0.360982 \\
-0.218044\end{array}$ & $\begin{array}{r}83978.9 \\
12150.8 \\
0.362250 \\
-0.214424\end{array}$ & $\begin{array}{r}30383.2 \\
3121.7 \\
0.360139 \\
-0.221297\end{array}$ & $\begin{array}{r}11690.20 \\
1126.80 \\
0.343566 \\
-0.082748\end{array}$ & $\begin{array}{r}5441148 \\
74170 \\
0.242777 \\
0.393868\end{array}$ & $\begin{array}{r}1879797 \\
110411 \\
0.231910 \\
0.388013\end{array}$ & $\begin{array}{r}4161632 \\
350337 \\
0.238948 \\
0.404086\end{array}$ & $\begin{array}{r}1773192 \\
109362 \\
0.192110 \\
0.436492\end{array}$ & $\begin{array}{r}10931.20 \\
603.40 \\
0.173057 \\
0.310125\end{array}$ \\
\hline South & $\left|\begin{array}{c}\text { Mean } \\
\text { ST DEV } \\
\text { PC1 } \\
\text { PC2 } \\
\text { PC3 }\end{array}\right|$ & $\begin{array}{r}23412.1 \\
11297.6 \\
0.110765 \\
0.237269 \\
0.757189\end{array}$ & $\begin{array}{r}57616.8 \\
24427.2 \\
0.250630 \\
0.406293 \\
-0.121656\end{array}$ & $\begin{array}{r}19726.2 \\
8339.3 \\
0.290312 \\
0.367958 \\
-0.118935\end{array}$ & $\begin{array}{r}42923.3 \\
19564.0 \\
0.325062 \\
0.318280 \\
-0.158328\end{array}$ & $\begin{array}{r}14895.9 \\
9813.8 \\
0.204427 \\
0.392272 \\
-0.363881\end{array}$ & $\begin{array}{r}9632.67 \\
915.99 \\
0.324942 \\
0.141461 \\
0.478435\end{array}$ & $\begin{array}{r}5403418 \\
126268 \\
0.344784 \\
-0.286155 \\
-0.062623\end{array}$ & $\begin{array}{r}1848838 \\
147759 \\
0.341793 \\
-0.279808 \\
-0.072370\end{array}$ & $\begin{array}{r}4041886 \\
522547 \\
0.358549 \\
-0.263436 \\
-0.035332\end{array}$ & $\begin{array}{r}1752719 \\
141390 \\
0.336851 \\
-0.258473 \\
0.001224\end{array}$ & $\begin{array}{r}10681.60 \\
849.10 \\
0.331463 \\
-0.267696 \\
0.030535\end{array}$ \\
\hline
\end{tabular}


Table D2, cont1nued

\begin{tabular}{|c|c|c|c|c|c|c|c|c|c|c|c|c|}
\hline & Item & CT & Mean a & CNTYHH & CNTYVR & $\begin{array}{l}\text { Or1ginal v } \\
\text { CNTYEMP }\end{array}$ & $\begin{array}{l}\text { Varlable (*) } \\
\text { CNTYINC }\end{array}$ & STPOP & STHH & STVR & STEMP & \\
\hline & & & & 1ty Group & "C" ICity & y Populat1o & 0.10000 & to 20,000 ) & & & & \\
\hline North & $\left|\begin{array}{c}\text { Mean } \\
\text { ST DEV } \\
\text { PCI } \\
\text { PC3 } \\
\text { PC5 } \\
\text { PC6 }\end{array}\right|$ & $\begin{array}{r}15561.1 \\
3190.4 \\
0.046423 \\
0.768395 \\
0.204035 \\
0.143988\end{array}$ & $\begin{array}{r}156814.0 \\
190959.0 \\
0.257111 \\
-0.071711 \\
0.078380 \\
0.080193\end{array}$ & $\begin{array}{r}53704.0 \\
65147.2 \\
0.265353 \\
-0.083263 \\
0.118462 \\
0.064821\end{array}$ & $\begin{array}{r}111561.0 \\
122965.0 \\
0.274762 \\
-0.045757 \\
0.046379 \\
0.002746\end{array}$ & $\begin{array}{r}56063.1 \\
64123.3 \\
0.272769 \\
0.053817 \\
-0.058514 \\
-0.096822\end{array}$ & $\begin{array}{r}10882.60 \\
1066.80 \\
0.284369 \\
0.528159 \\
-0.492302 \\
-0.379081\end{array}$ & $\begin{array}{r}5428020 \\
96473 \\
0.374678 \\
-0.165437 \\
-0.176925 \\
-0.067760\end{array}$ & $\begin{array}{r}1870793 \\
123747 \\
0.367707 \\
-0.180529 \\
-0.194633 \\
-0.126593\end{array}$ & $\begin{array}{r}4124551 \\
410463 \\
0.382885 \\
-0.178029 \\
-0.188060 \\
0.194724\end{array}$ & $\begin{array}{r}1767027 \\
118360 \\
0.329240 \\
0.007166 \\
0.752377 \\
-0.451842\end{array}$ & $\begin{array}{r}10864.00 \\
657.20 \\
0.316183 \\
0.147806 \\
0.140125 \\
0.743543\end{array}$ \\
\hline Central & $\mid \begin{array}{c}\text { Mean } \\
\text { ST } \\
\text { DEV } \\
\text { PC1 } \\
\text { PC3 } \\
\text { PCS }\end{array}$ & $\begin{array}{r}13476.5 \\
3040.7 \\
0.124816 \\
0.648420 \\
0.277294\end{array}$ & $\begin{array}{r}58439.0 \\
29493.4 \\
0.287261 \\
0.041413 \\
-0.000082\end{array}$ & $\begin{array}{r}19665.5 \\
10297.4 \\
0.300728 \\
-0.021451 \\
0.018805\end{array}$ & $\begin{array}{r}48101.6 \\
25366.8 \\
0.322389 \\
0.068354 \\
-0.072533\end{array}$ & $\begin{array}{r}13009.4 \\
9700.8 \\
0.304509 \\
-0.085346 \\
0.202654\end{array}$ & $\begin{array}{r}11830.30 \\
1619.60 \\
0.231354 \\
0.631080 \\
-0.544854\end{array}$ & $\begin{array}{r}540180 \\
84042 \\
0.345353 \\
-0.225219 \\
-0.199916\end{array}$ & $\begin{array}{r}1867771 \\
115302 \\
0.335180 \\
-0.248027 \\
-0.275852\end{array}$ & $\begin{array}{r}4123813 \\
399729 \\
0.353954 \\
-0.182862 \\
-0.119473\end{array}$ & $\begin{array}{r}1753963 \\
115814 \\
0.348274 \\
-0.098116 \\
0.189366\end{array}$ & $\begin{array}{r}10889.90 \\
584.60 \\
0.288225 \\
0.108866 \\
0.642934\end{array}$ \\
\hline South & $\mid \begin{array}{c}\text { Mean } \\
\text { ST DEV } \\
\text { PC1 } \\
\text { PC2 } \\
\text { PC3 }\end{array}$ & $\begin{array}{r}23412.1 \\
11297.6 \\
0.110765 \\
0.237269 \\
0.757189\end{array}$ & $\begin{array}{r}57616.8 \\
24427.2 \\
0.250630 \\
0.406293 \\
-0.121656\end{array}$ & $\begin{array}{r}19726.2 \\
8339.3 \\
0.290312 \\
0.367958 \\
-0.118935\end{array}$ & $\begin{array}{r}42923.3 \\
19564.0 \\
0.325062 \\
0.318280 \\
-0.158328\end{array}$ & $\begin{array}{r}14895.9 \\
9813.8 \\
0.204427 \\
0.392272 \\
-0.363881\end{array}$ & $\begin{array}{r}9632.67 \\
915.99 \\
0.324942 \\
0.141461 \\
0.478435\end{array}$ & $\begin{array}{r}5403418 \\
126268 \\
0.344784 \\
-0.286155 \\
-0.062623\end{array}$ & $\begin{array}{r}1848838 \\
147759 \\
0.341793 \\
-0.279808 \\
-0.072370\end{array}$ & $\begin{array}{r}4041886 \\
522547 \\
0.358549 \\
-0.263436 \\
-0.035332\end{array}$ & $\begin{array}{r}1752719 \\
141390 \\
0.336851 \\
-0.258473 \\
0.001224\end{array}$ & $\begin{array}{r}10681.60 \\
849.10 \\
0.331463 \\
-0.267696 \\
0.030535\end{array}$ \\
\hline & & & & & & & & & & & & \\
\hline North & $\left|\begin{array}{c}\text { Mean } \\
\text { ST DEV } \\
\text { PC1 } \\
\text { PC2 }\end{array}\right|$ & $\begin{array}{r}6311.1 \\
1285.5 \\
-0.005251 \\
0.108624\end{array}$ & $\begin{array}{r}106788.0 \\
157128.0 \\
0.326345 \\
-0.337831\end{array}$ & $\begin{array}{r}36689.6 \\
54173.8 \\
0.328878 \\
-0.330767\end{array}$ & $\begin{array}{r}79466.0 \\
109166.0 \\
0.333479 \\
-0.330478\end{array}$ & $\begin{array}{r}38313.1 \\
58836.4 \\
0.327188 \\
-0.341586\end{array}$ & $\begin{array}{r}10401.90 \\
870.80 \\
0.319431 \\
-0.147193\end{array}$ & $\begin{array}{r}5422841 \\
85161 \\
0.316055 \\
0.339313\end{array}$ & $\begin{array}{r}1857278 \\
116190 \\
0.308132 \\
0.344021\end{array}$ & $\begin{array}{r}4092834 \\
386917 \\
0.317404 \\
0.339056\end{array}$ & $\begin{array}{r}1747745 \\
103745 \\
0.311938 \\
0.342199\end{array}$ & $\begin{array}{r}10825.70 \\
464.60 \\
0.268565 \\
0.227064\end{array}$ \\
\hline Centra1 & $\mid \begin{array}{c}\text { Mean } \\
\text { ST DEv } \\
\text { PC1 } \\
\text { PC2 } \\
\text { PC3 } \\
\text { PC4 }\end{array}$ & $\begin{array}{r}6399.7 \\
1424.8 \\
-0.123839 \\
0.062372 \\
0.631416 \\
0.688302\end{array}$ & $\begin{array}{r}42162.2 \\
42568.6 \\
0.300443 \\
-0.388774 \\
0.115786 \\
0.020796\end{array}$ & $\begin{array}{r}14706.7 \\
15010.3 \\
0.306011 \\
-0.382321 \\
0.112301 \\
-0.010271\end{array}$ & $\begin{array}{r}33954.2 \\
33947.1 \\
0.320231 \\
-0.364867 \\
0.105128 \\
-0.001040\end{array}$ & $\begin{array}{r}10893.0 \\
14720.5 \\
0.312760 \\
-0.375642 \\
0.070646 \\
0.030003\end{array}$ & $\begin{array}{r}10011.80 \\
1120.70 \\
0.255366 \\
0.002811 \\
-0.600174 \\
0.263066\end{array}$ & $\begin{array}{r}5422582 \\
90423 \\
0.333554 \\
0.313572 \\
0.181517 \\
-0.205186\end{array}$ & $\begin{array}{r}1855616 \\
121198 \\
0.318448 \\
0.310992 \\
0.243129 \\
-0.267691\end{array}$ & $\begin{array}{r}4086697 \\
415317 \\
0.342760 \\
0.311275 \\
0.147053 \\
-0.168656\end{array}$ & $\begin{array}{r}1759172 \\
105936 \\
0.341591 \\
0.282096 \\
0.014358 \\
-0.173238\end{array}$ & $\begin{array}{r}10876.80 \\
627.40 \\
0.297223 \\
0.230301 \\
-0.291811 \\
0.532337\end{array}$ \\
\hline South & $\left|\begin{array}{c}\text { Mean } \\
\mathrm{ST} \\
\mathrm{DEV} \\
\mathrm{PCl} \\
\mathrm{PC2}\end{array}\right|$ & $\begin{array}{r}6610.9 \\
1681.2 \\
0.078036 \\
-0.092799\end{array}$ & $\begin{array}{r}37053.2 \\
20018.3 \\
0.160098 \\
0.475768\end{array}$ & $\begin{array}{r}12572.9 \\
6952.5 \\
0.192474 \\
0.455093\end{array}$ & $\begin{array}{r}29830.3 \\
15454.9 \\
0.239374 \\
0.433834\end{array}$ & $\begin{array}{r}10528.8 \\
7957.2 \\
0.163180 \\
0.449591\end{array}$ & $\begin{array}{r}9964.64 \\
2 \\
2337.37 \\
0.274203 \\
0.053806\end{array}$ & $\begin{array}{r}5434029 \\
90975 \\
0.414137 \\
-0.179485\end{array}$ & $\begin{array}{r}1876976 \\
118104 \\
0.409120 \\
-0.154179\end{array}$ & $\begin{array}{r}4147053 \\
378395 \\
0.416829 \\
-0.169548\end{array}$ & $\begin{array}{r}1771152 \\
103890 \\
0.394434 \\
-0.173522\end{array}$ & $\begin{array}{r}10884.60 \\
584.70 \\
0.322341 \\
-0.223252\end{array}$ \\
\hline
\end{tabular}

(*) See Table 4.3 of Chapter 4 for description of Original variables 
Table D3: Eigenvectors or Coefficients of Principal Components (PCs) of Final Regression Equations for Urban Minor Arterial \& Collector

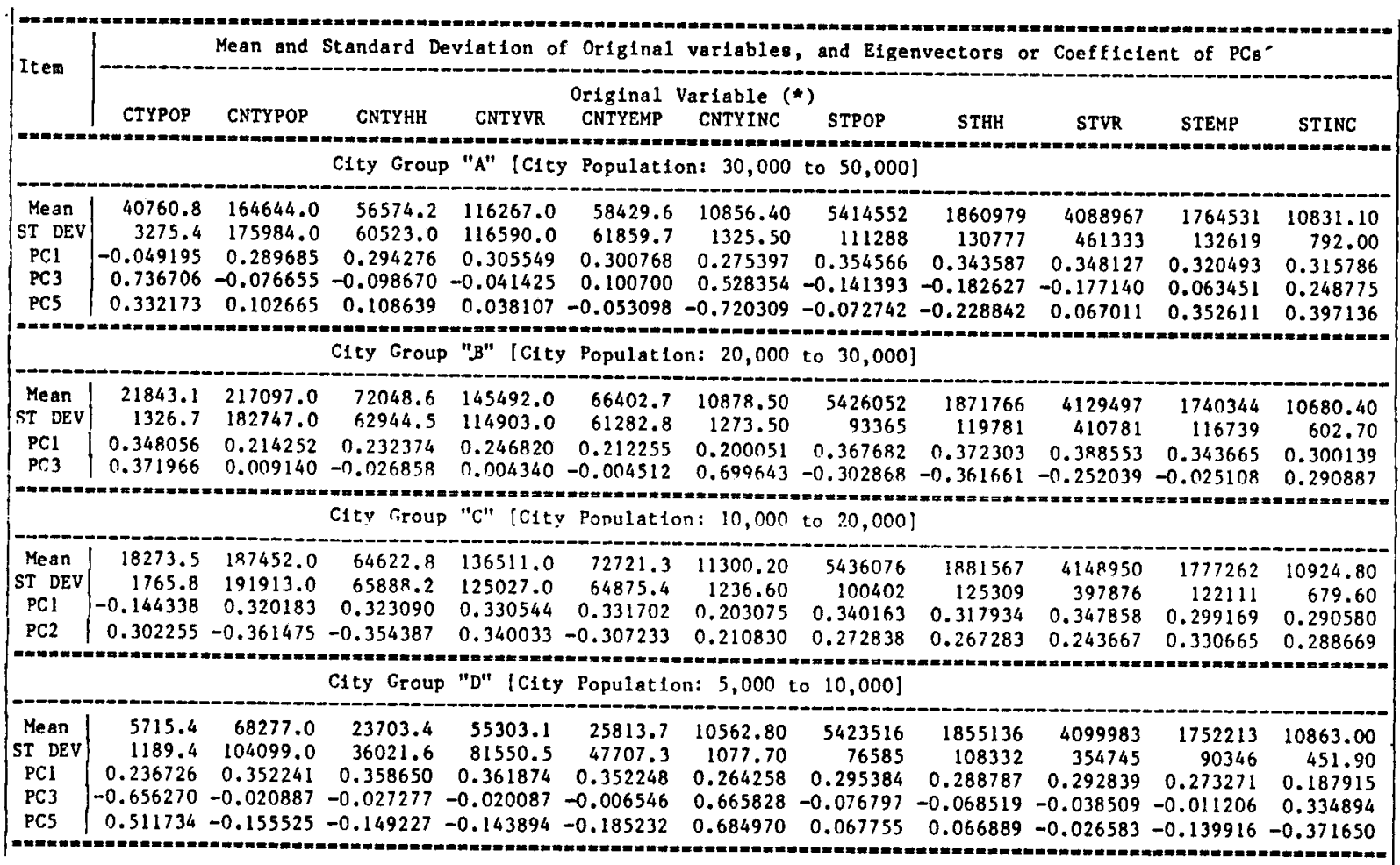

(*) See Table 4.3 of Chapter 4 for description of Original variables 
Slope of the plot: 2515.15

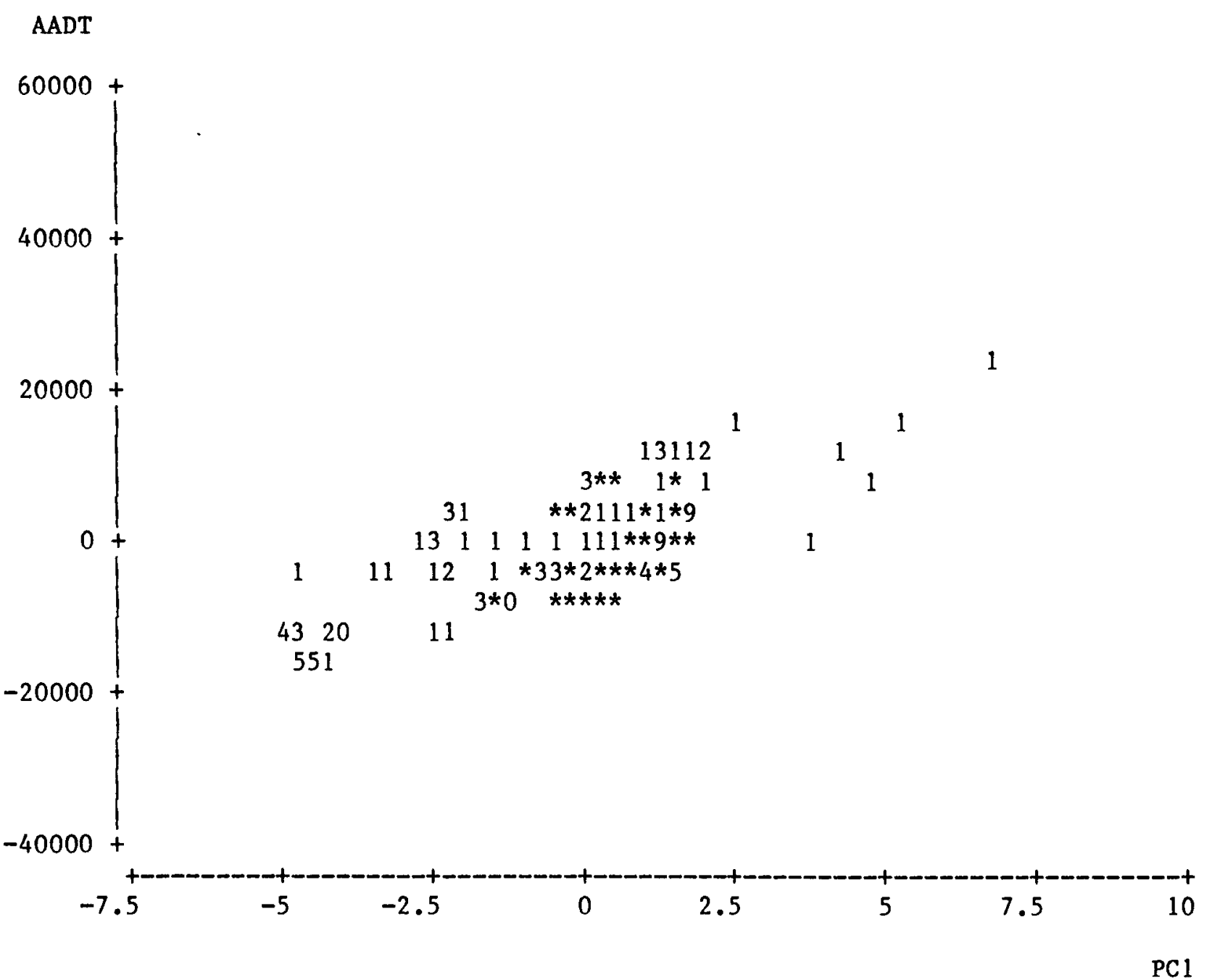

Figure D1.1: Partial Regression Residua1 P1ot of PC1 for Central Region of Rural Interstate Highways 
Slope of the plot : 778.50

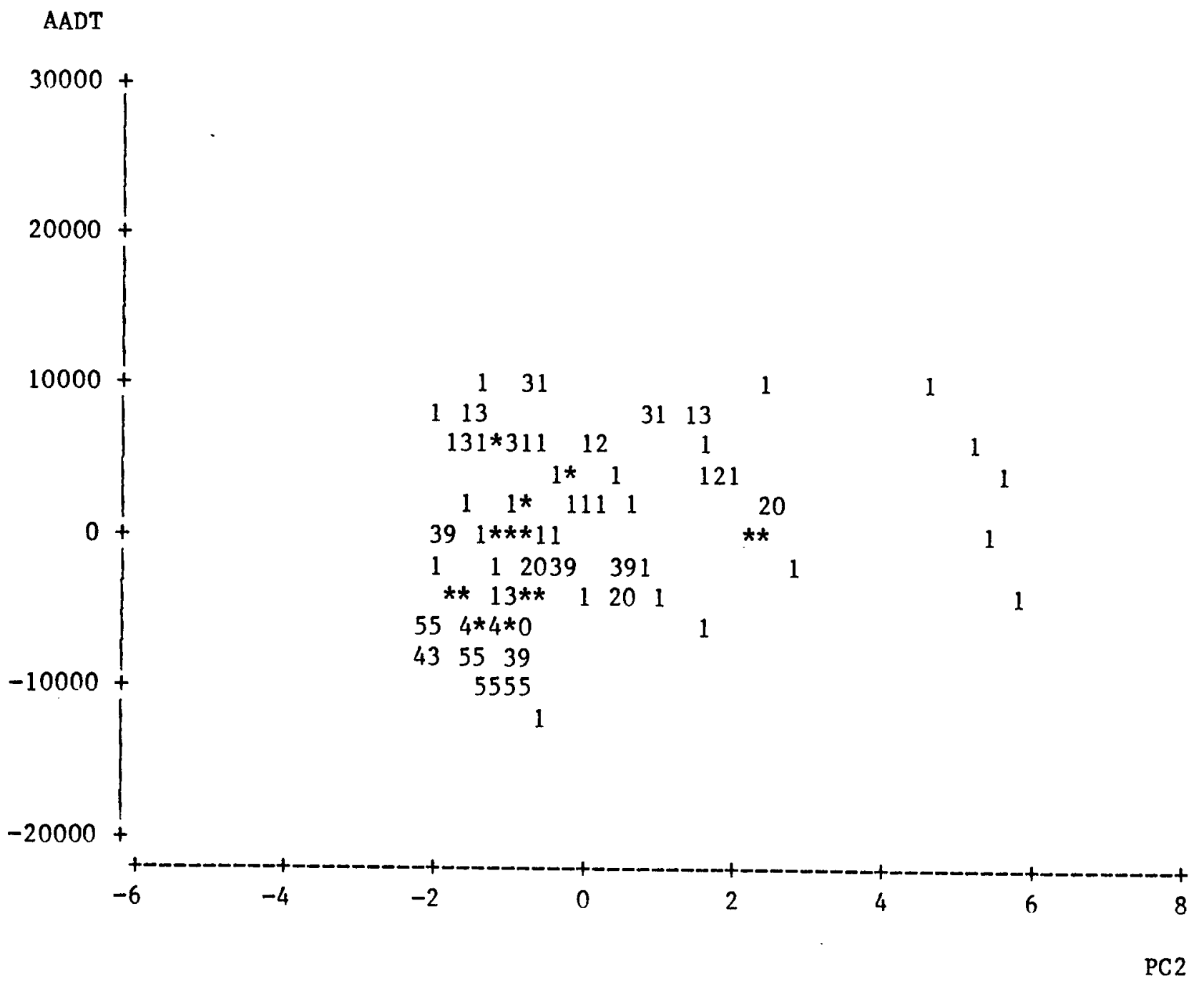

Figure D1.2: Partial Regression Residual P1ot of PC2 for Centra1 Region of Rural Interstate Highways 
Slope of the plot: 2934.67

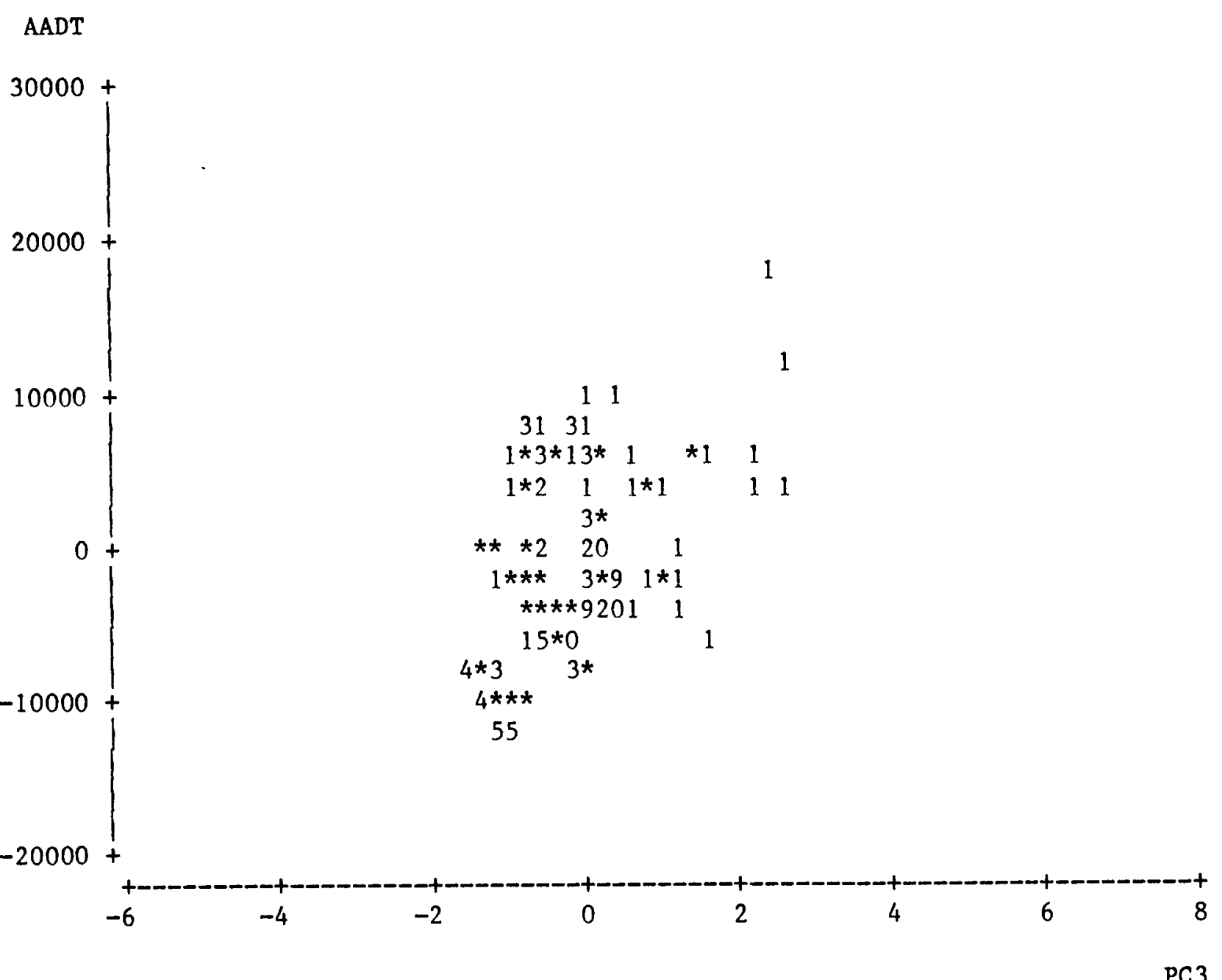

F1gure D1.3: Partial Regression Residual P1ot of PC3 for Central Region of Rural Interstate Highways 


\section{Slope of the plot: 6665.45}

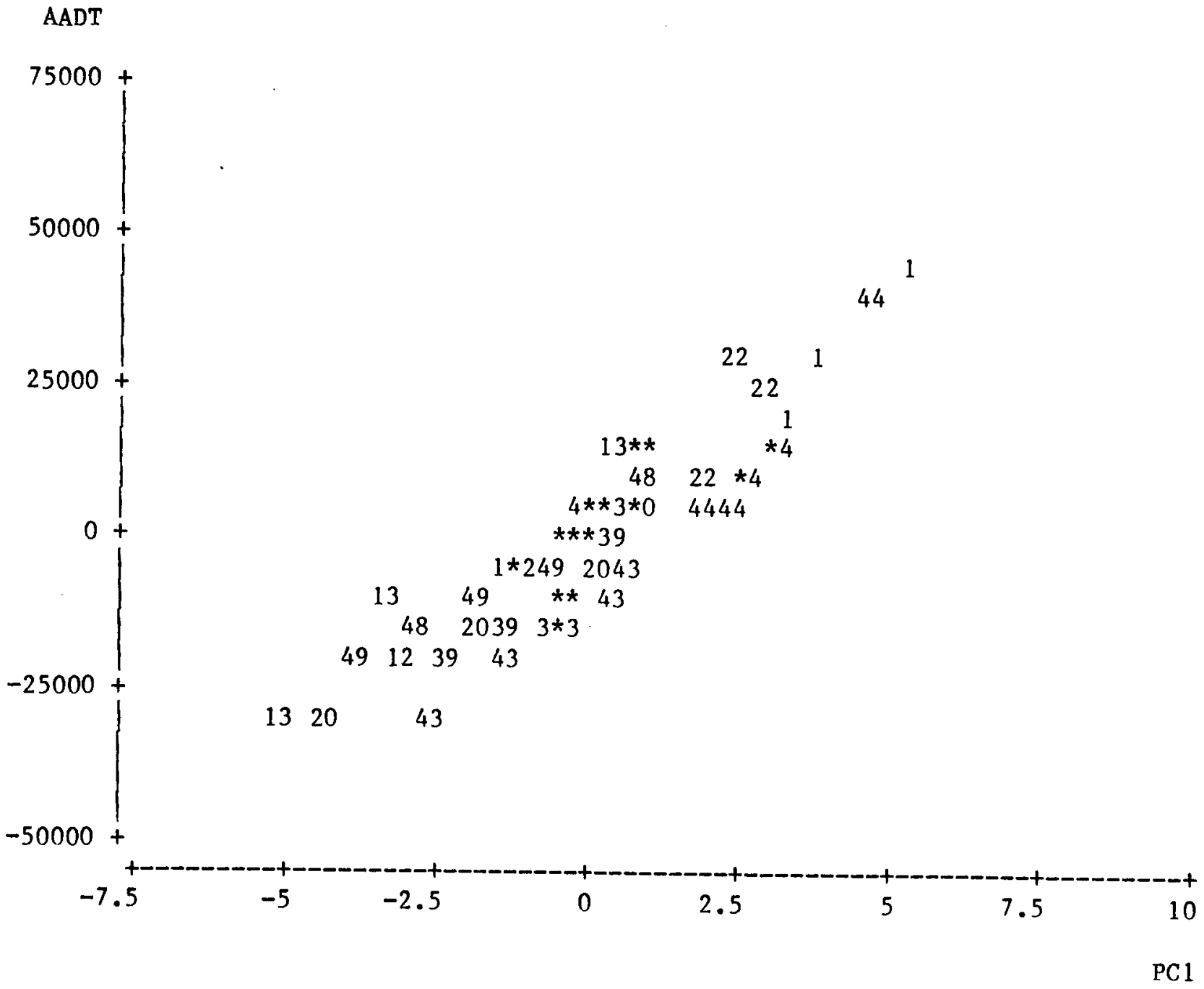

Figure D1.4: Partial Regression Residual Plot of PCl for Central Region of Urban Interstate Highways 
Slope of the plot: -4378.18

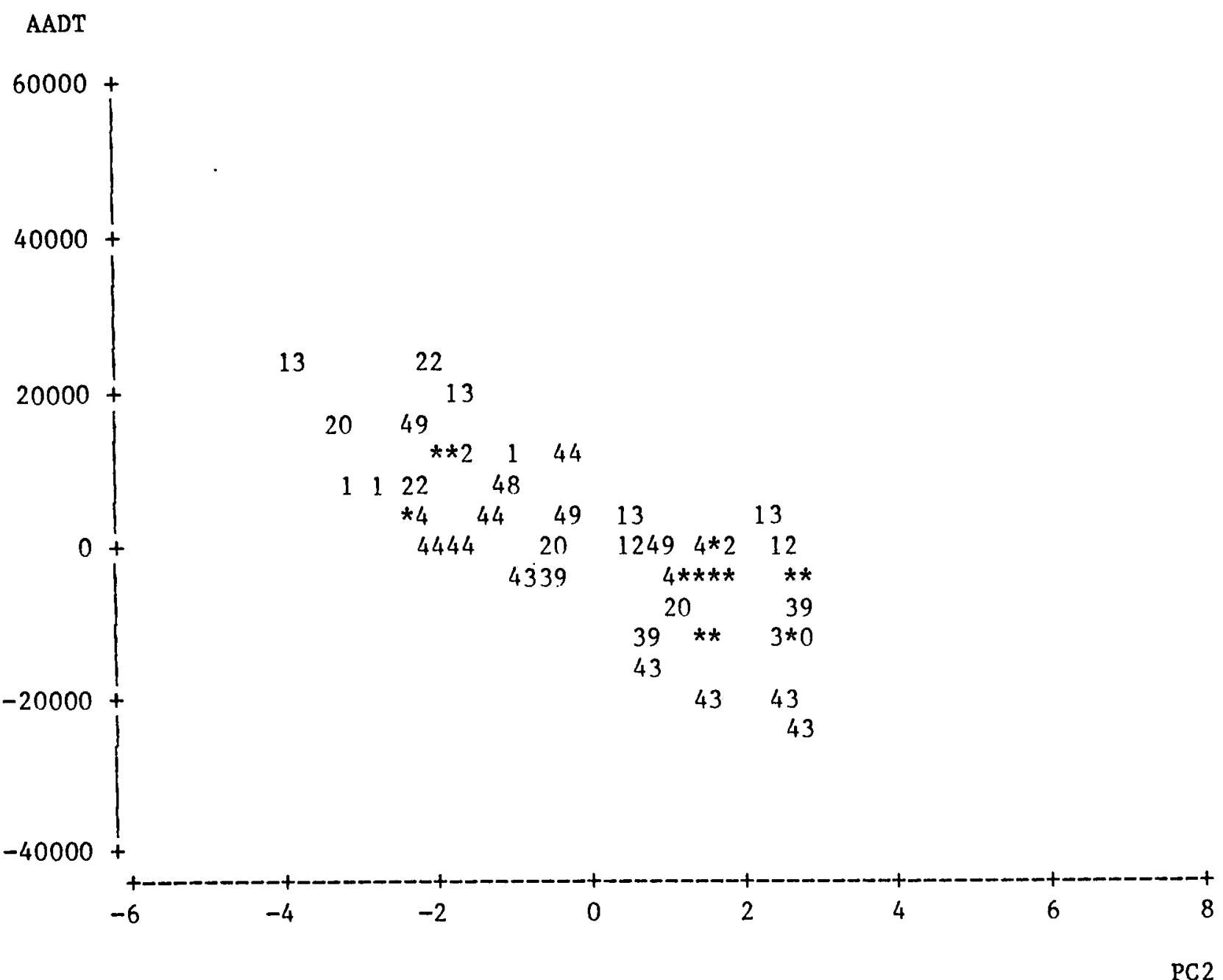

Figure D1.5: Partial Regression Residual P1ot of PC2 for Central Region of Urban Interstate Highways 
Slope of the plot: -2932.51

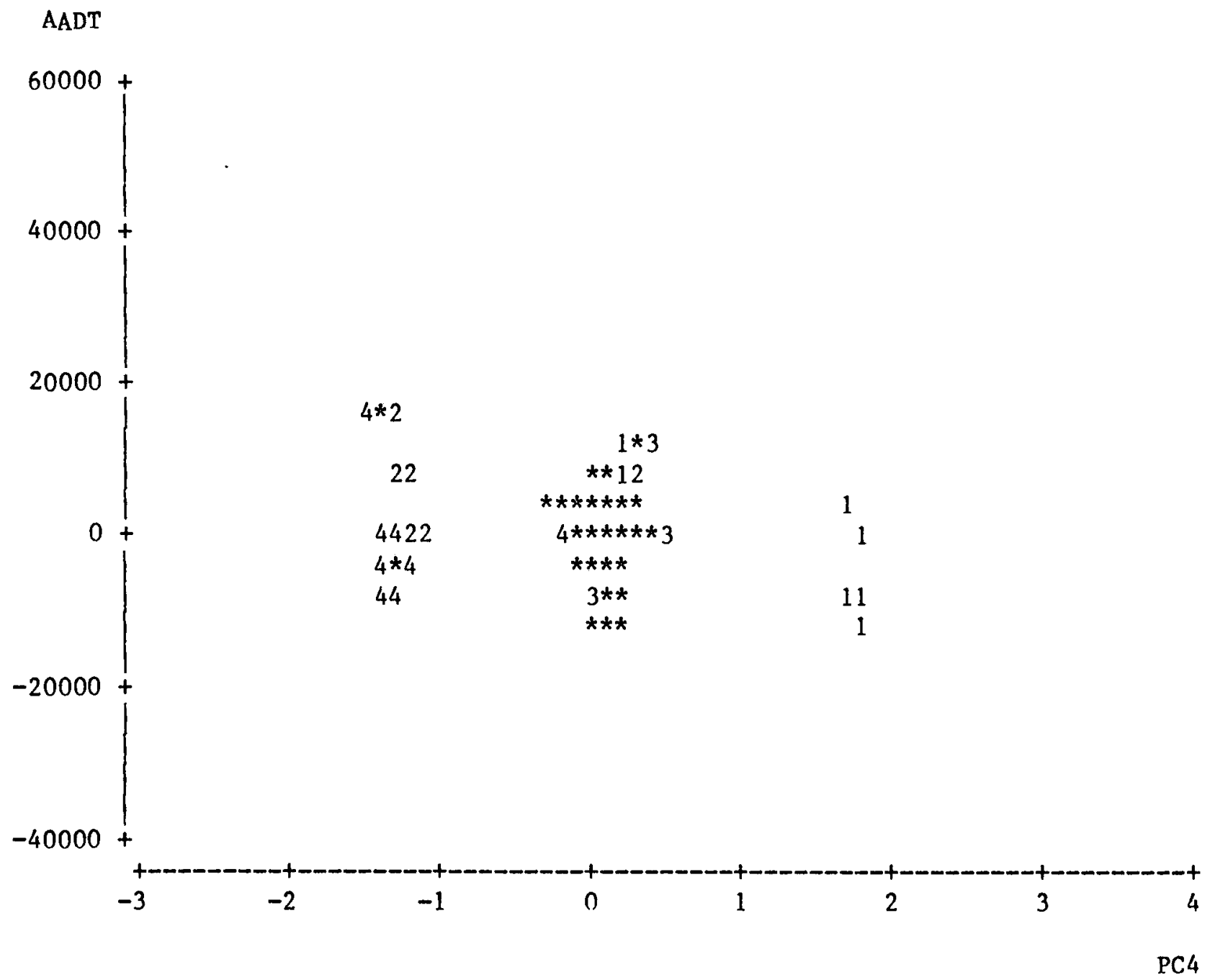

Figure D1.6: Partial Regression Residual P1ot of PC4 for Central Region of Urban Interstate Highways 
Slope of the plot : 798.94

\section{AADT}

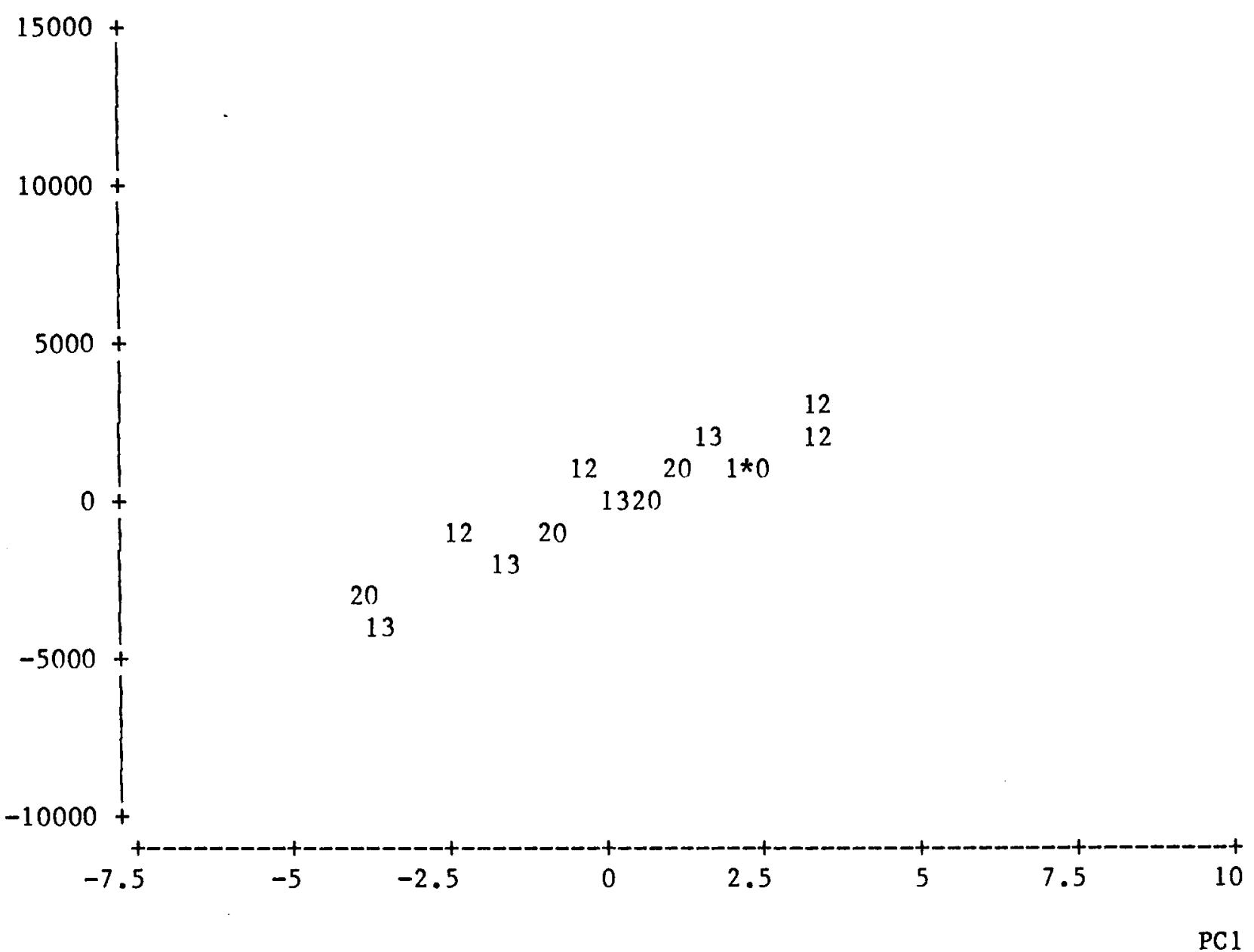

Figure D1.7: Partial Regression Residual Plot of PCl for City Group "A" and Region "c" of Urban Principal Arterial 
Slope of the plot: 1775.23

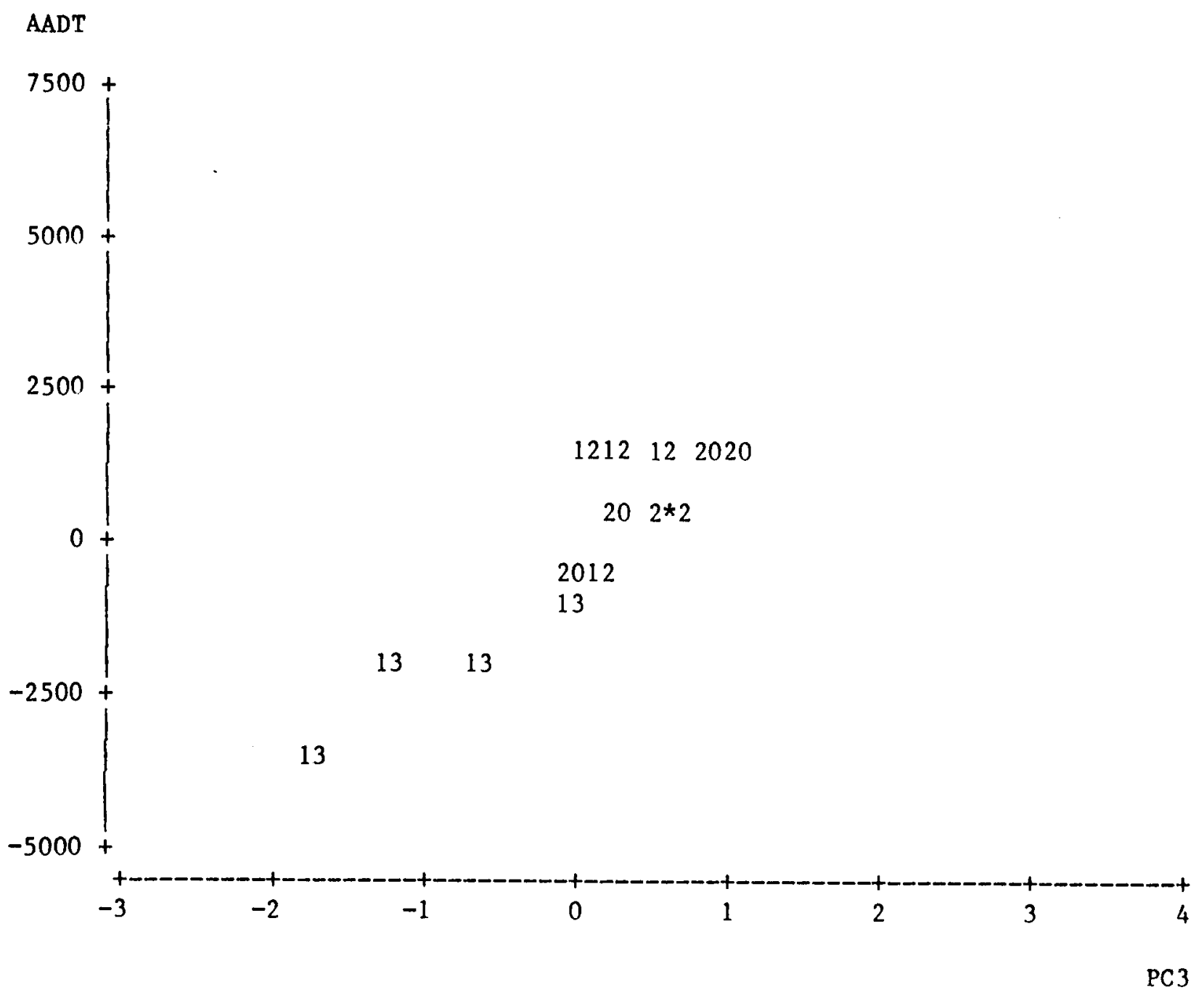

Figure D1.8: Partial Regression Residual Plot of PC3 for City Group 
Slope of the plot : -1192.98

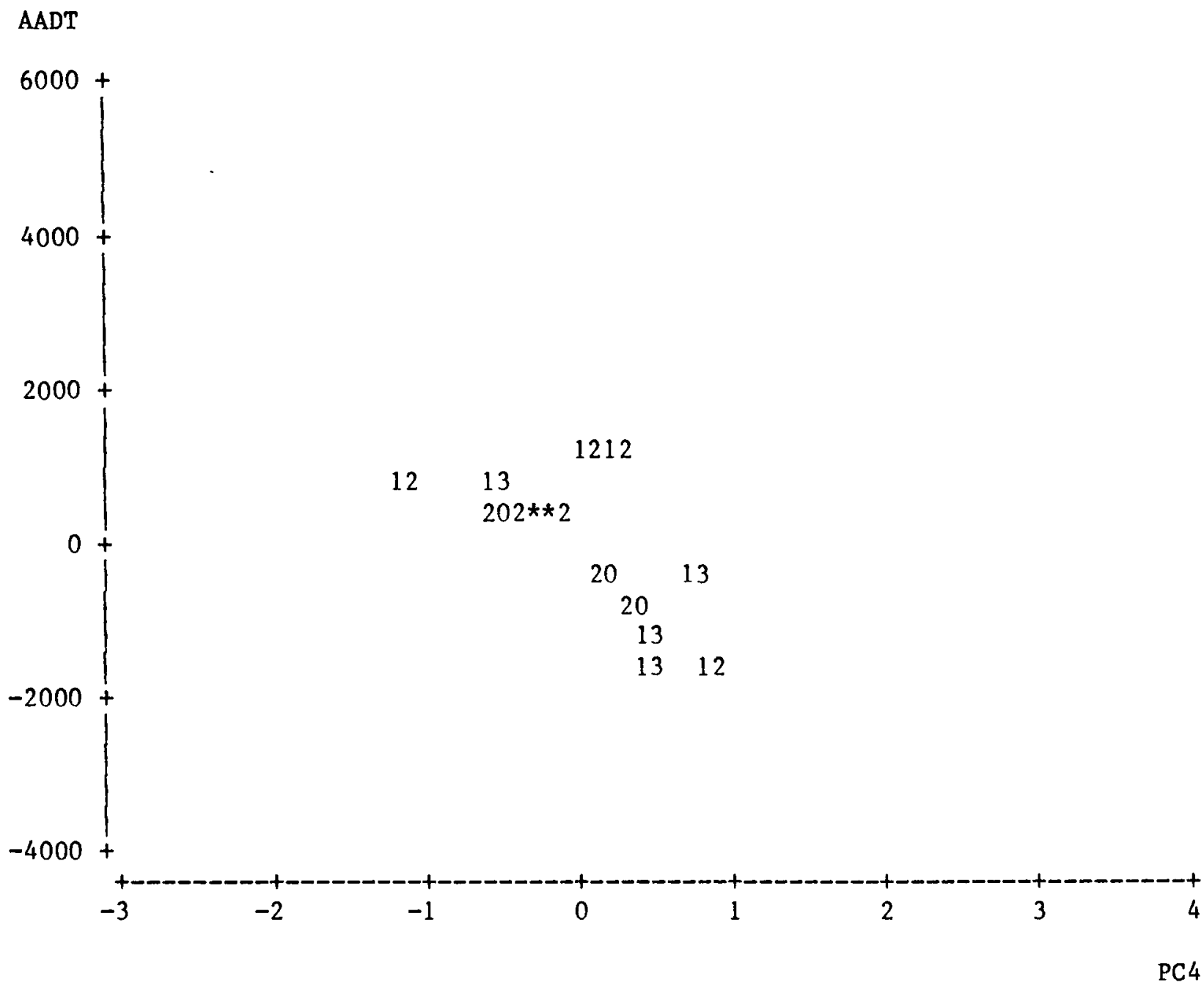

Figure D1.9: Partial Regression Residual Plot of PC4 for City Group "A" and Region "c" of Urban Principal Arterial 
Slope of the plot: 254.15

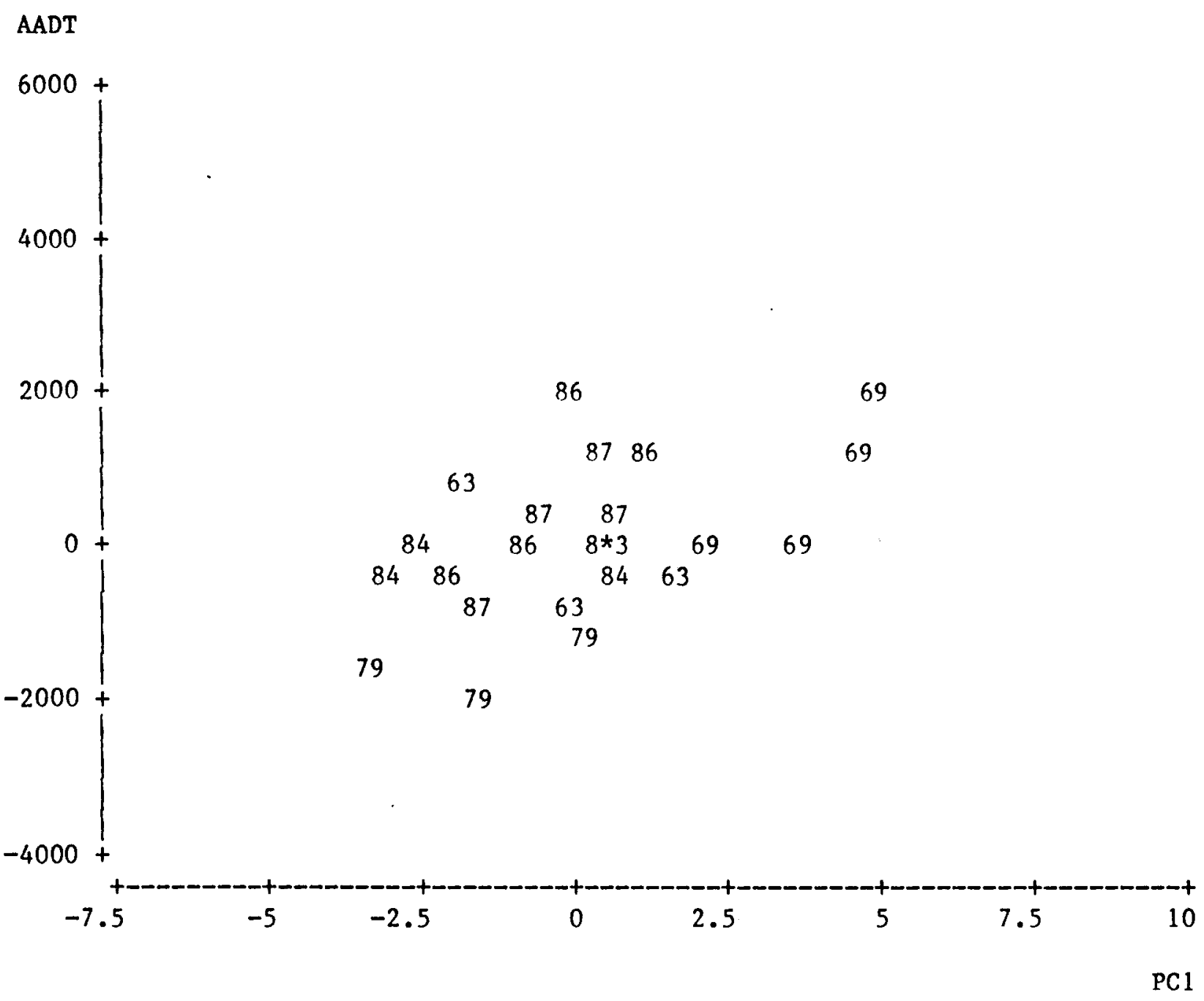

Figure D1.10: Partial Regression Residual Plot of PCl for City Group "D" of Urban Minor Arterial \& Collector 
Slope of the plot : 1033.76

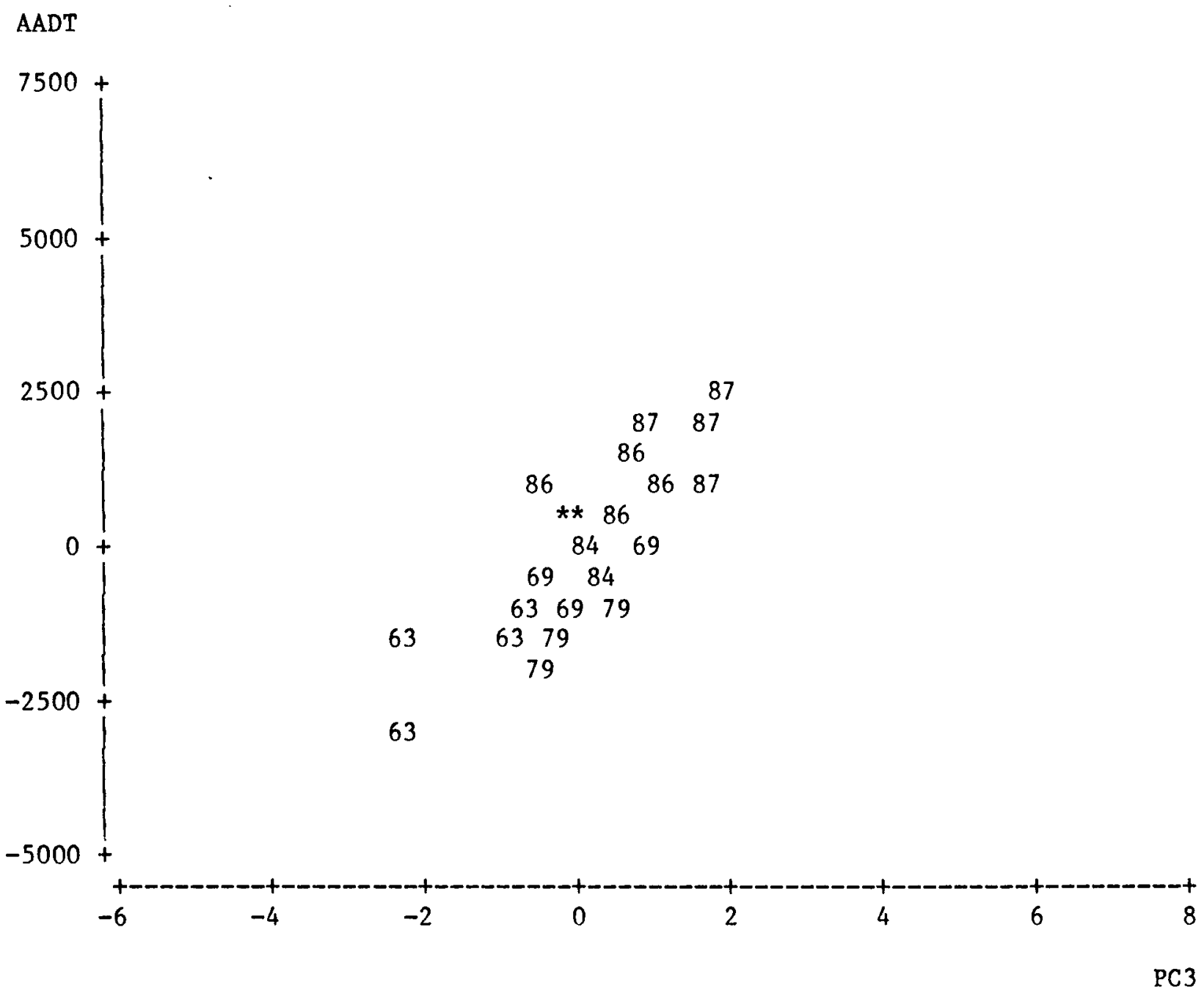

Figure D1.11: Partial Regression Residual P1ot of PC3 for City Group "D" of Urban Minor Arterial \& Collector 
Slope of the plot : 1386.16

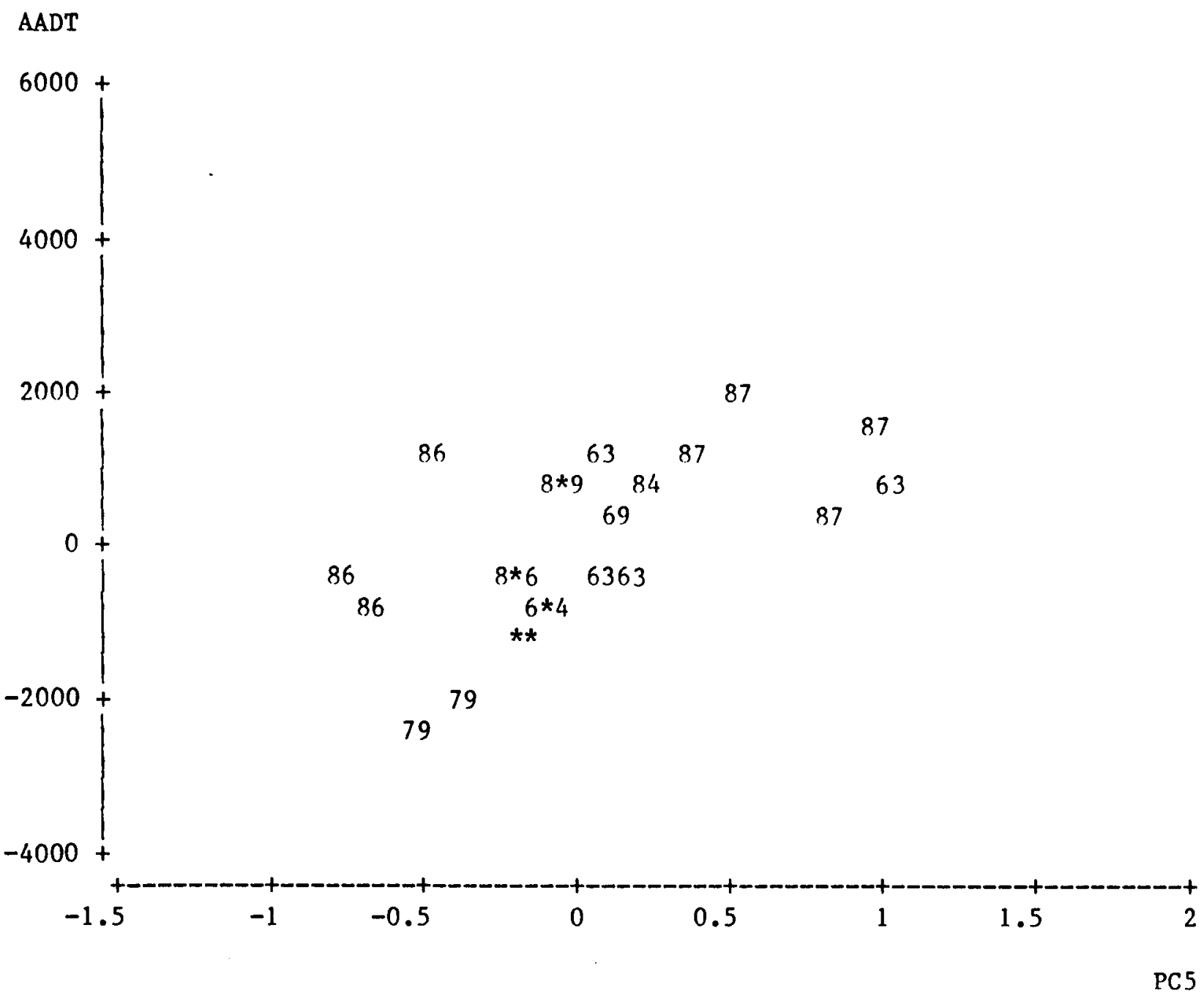

Figure D1.12: Partial Regression Residual Plot of PC5 for City Group "D" of Urban Minor Artertal \& Collector 
LEGEND: $A=1$ OBS, $B=2$ OBS, ETC.

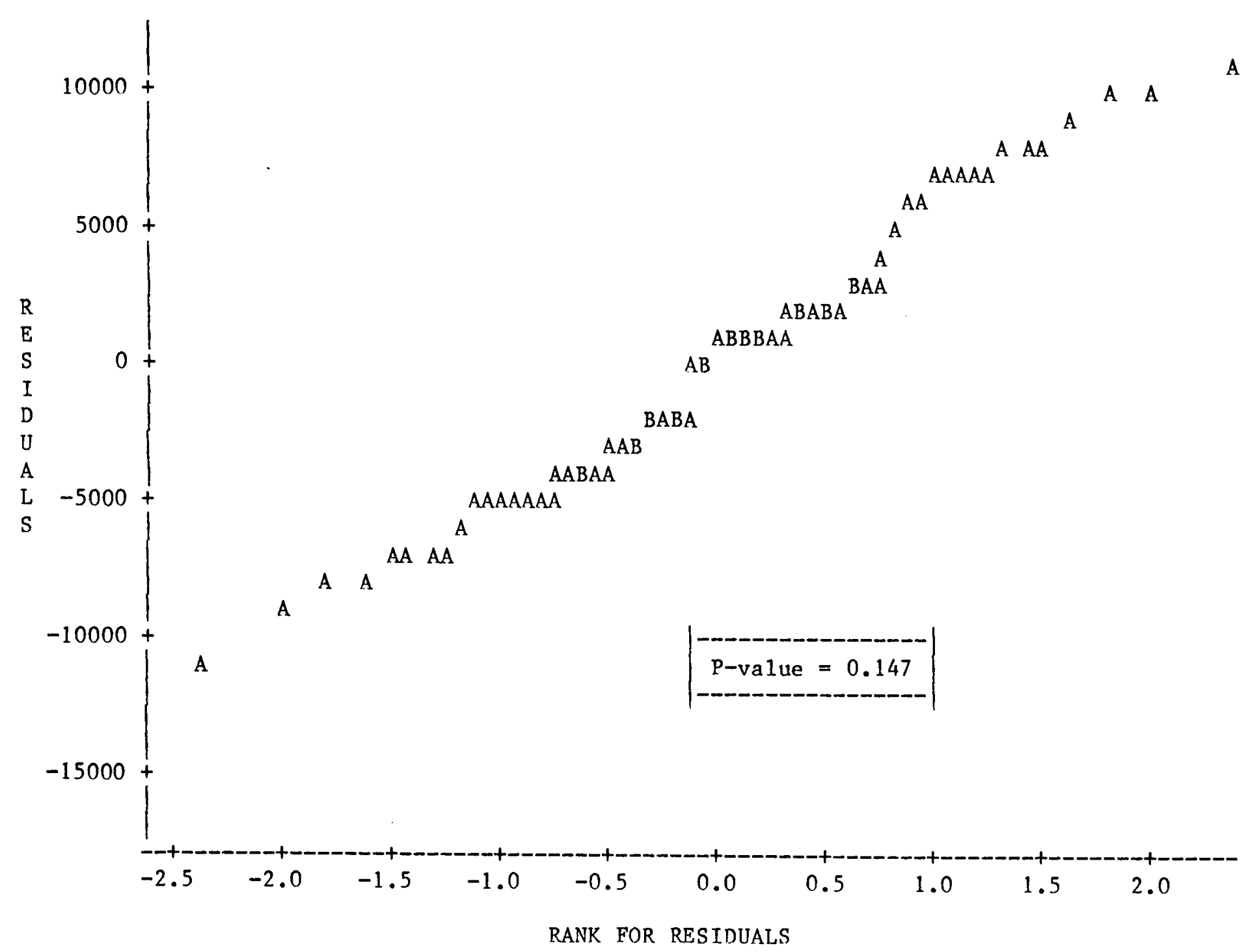

Figure D2.1: Normal Probability P1ot for Central Region of Rural Interstate Highways 
LEGEND: $A=1$ OBS, $B=2$ OBS, ETC.

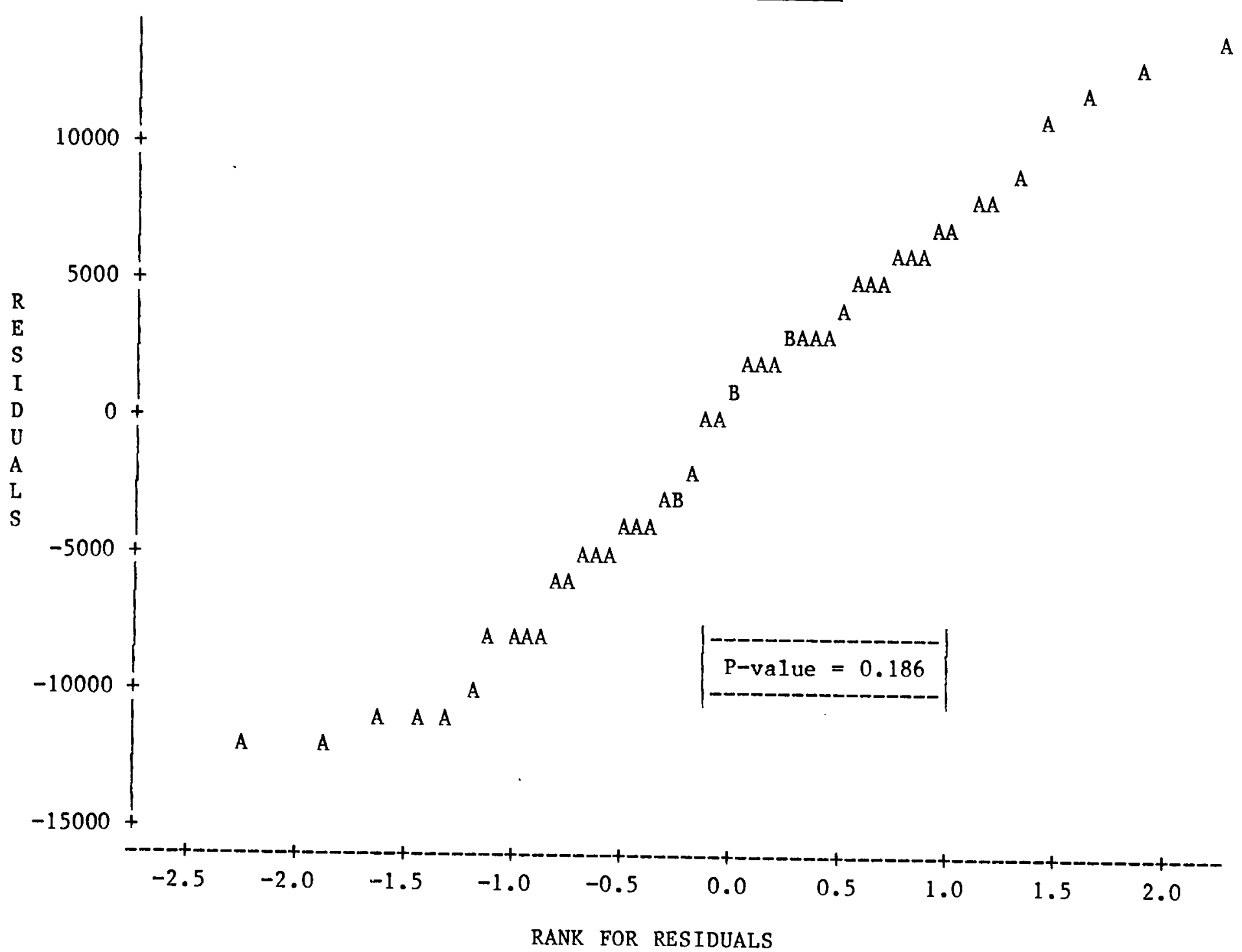

Figure D2.2: Normal Probability P1ot for Central Region of Urban Interstate Highways 
LEGEND: $A=1$ OBS, $B=2$ OBS, ETC.

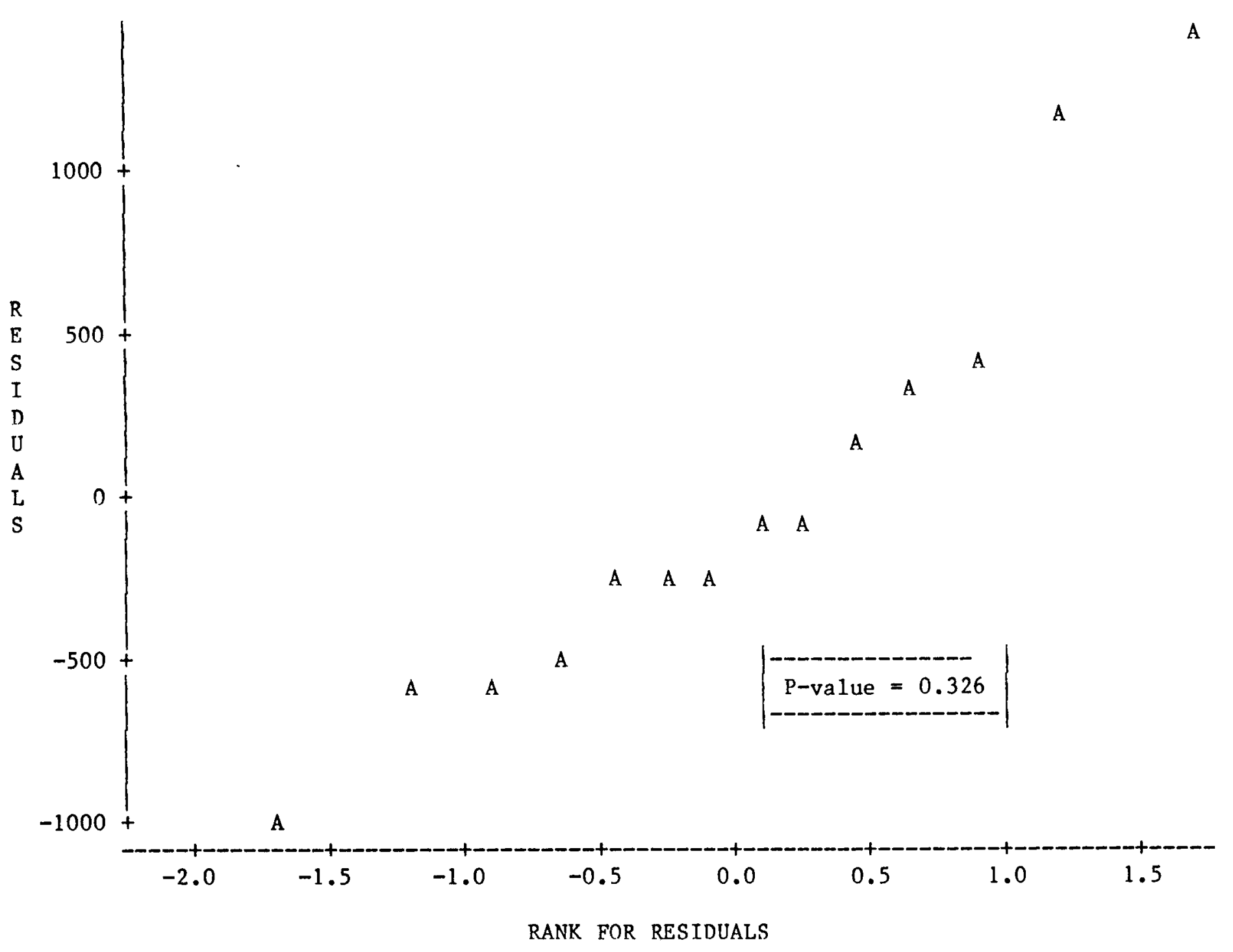

Figure D2.3: Normal Probability P1ot for City Group "A" and Region " $c$ " of Urban Principal Arterial 
LEGEND: $A=1$ OBS, $B=2$ OBS, ETC.

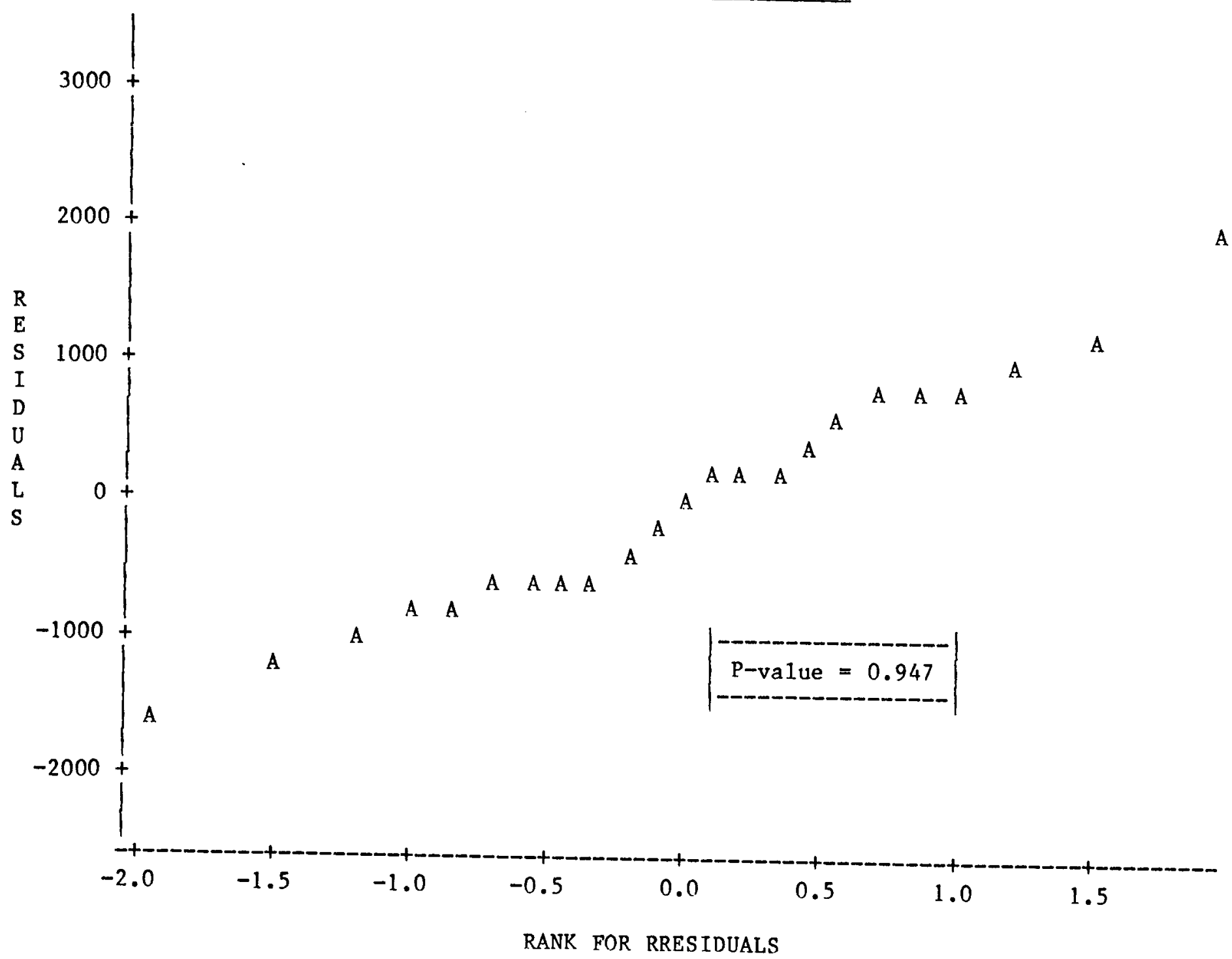

Figure D2.4: Norma1 Probability P1ot for City Group "D" of Urban Minor Arterial \& Collector 
LEGEND: $A=1$ OBS, $B=2$ OBS, ETC.

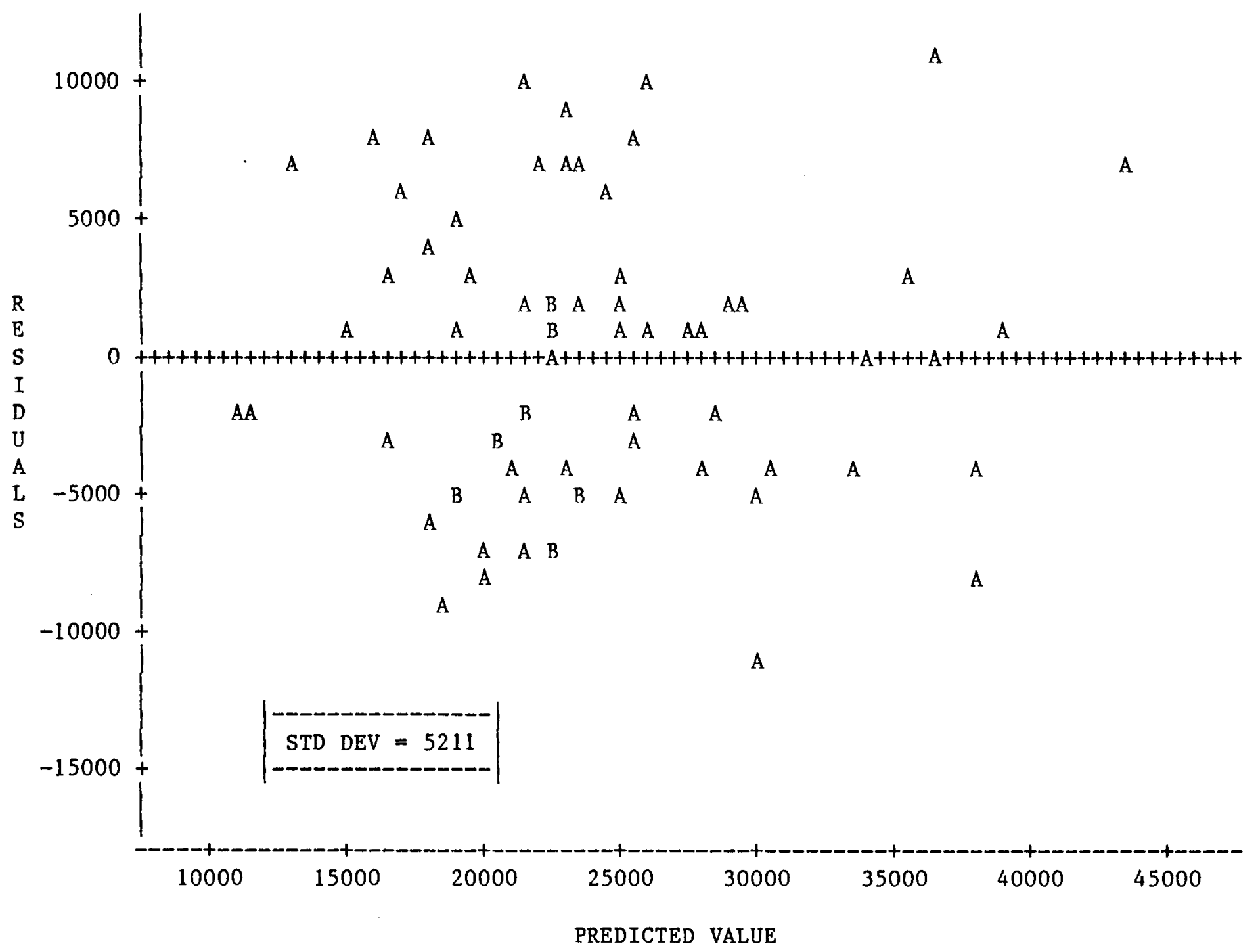

Figure D3.1: Residual Plot against Predicted value for Central Region of Rural Interstate Highways 
LEGEND: $A=1$ OBS, $B=2$ OBS, ETC.

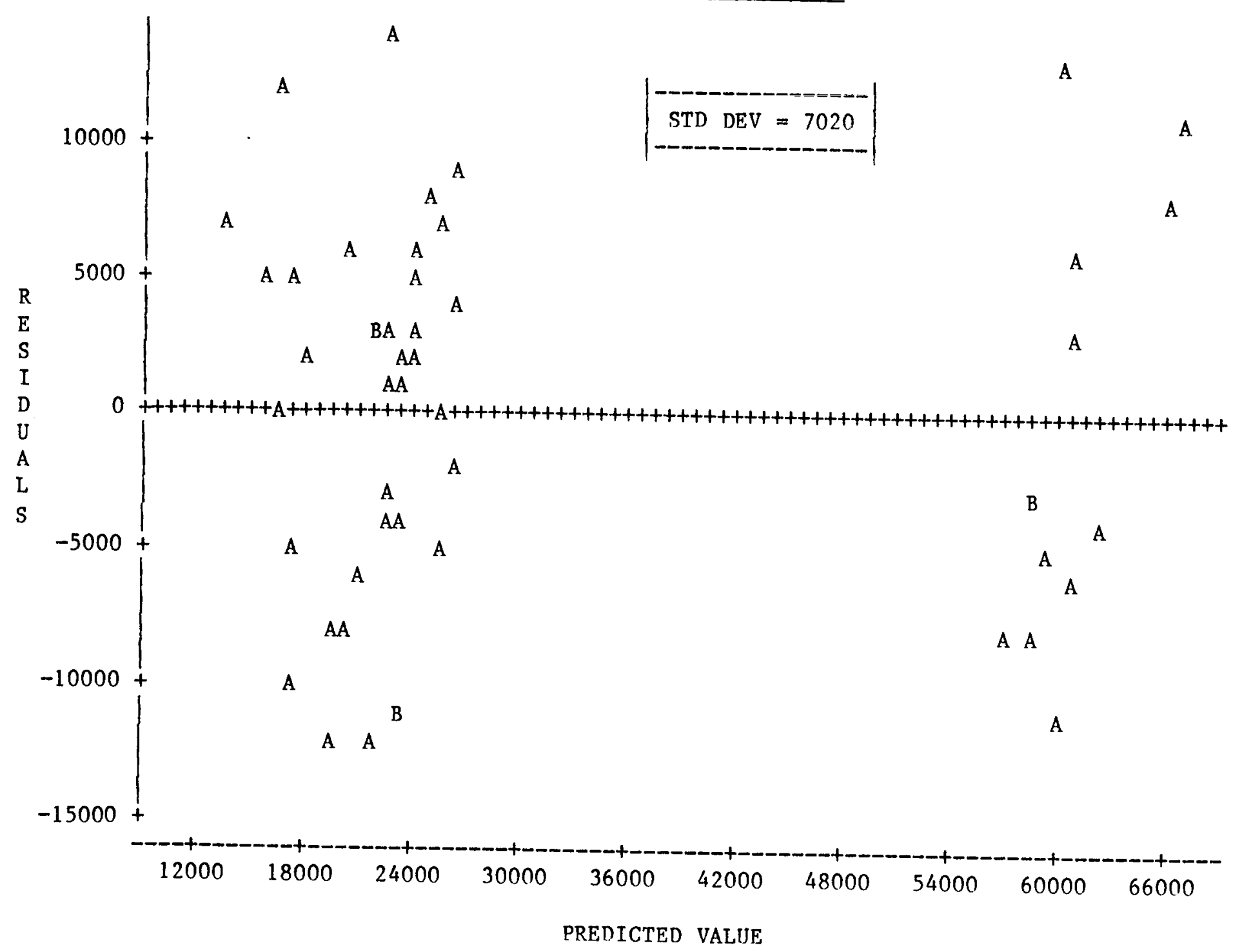

Figure D3.2: Residual Plot against Predicted value for Central Region of Urban Interstate Highwavs 
LEGEND: $A=1$ OBS, $B=2$ OBS, ETC.

A

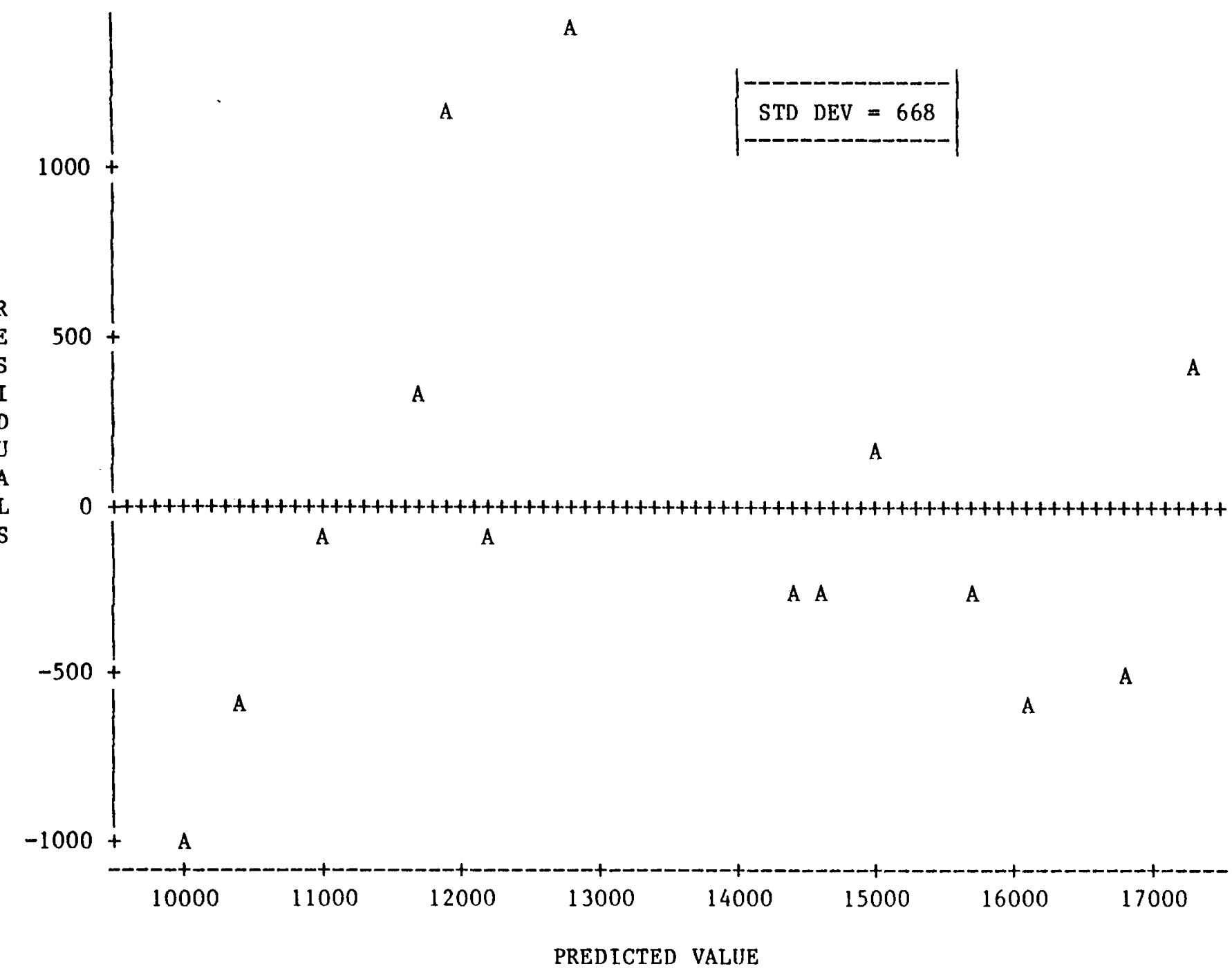

PREDICTED VALUE

Figure D3.3: Residual P1ot agalnst Predicted value for City Group

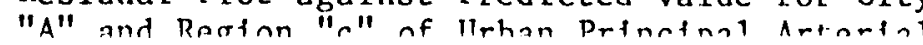


LEGEND: $\mathrm{A}=1 \mathrm{OBS}, \mathrm{B}=2 \mathrm{OBS}$, ETC.

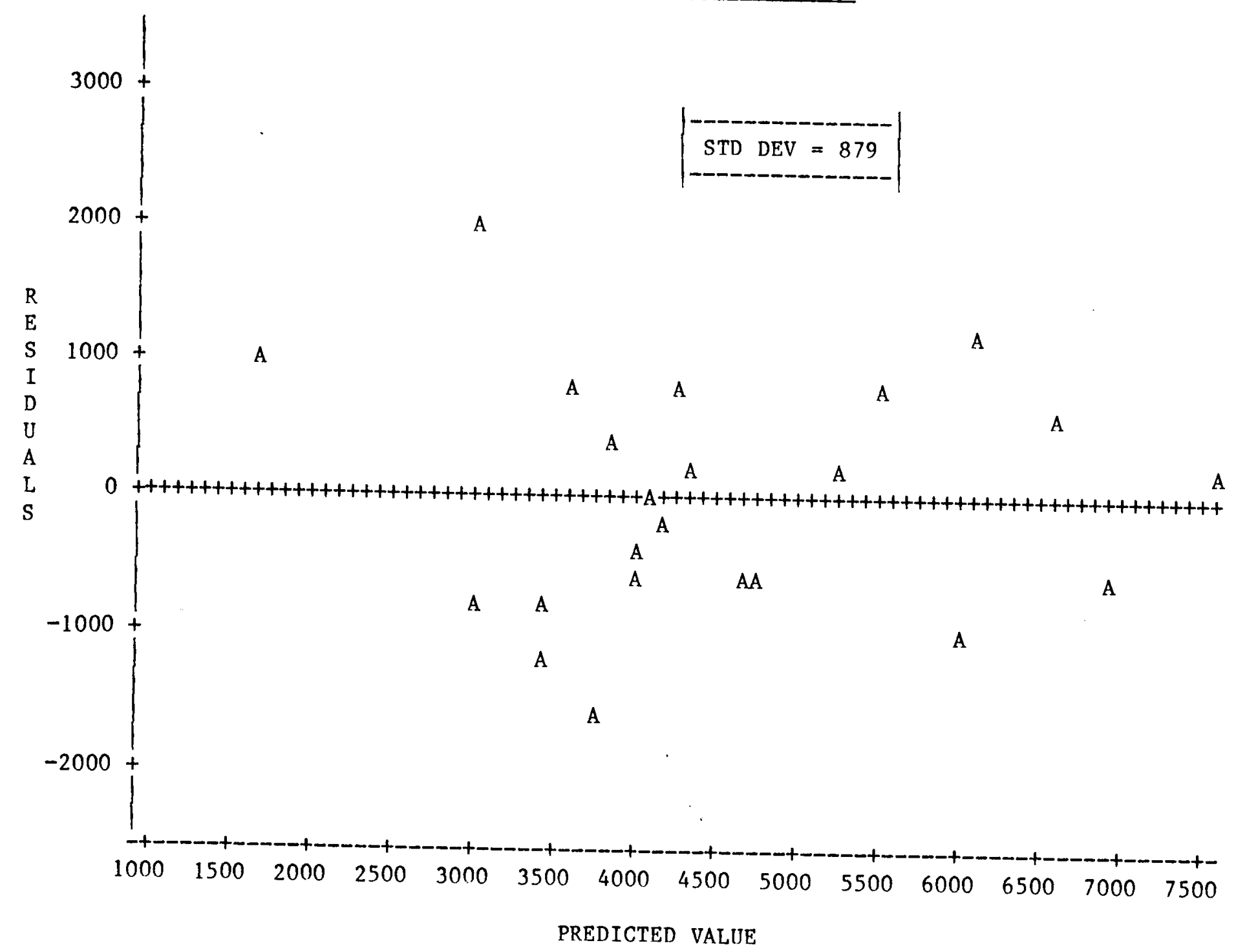

Figure D3.4: Residual P1ot against Predicted value for City Group "D" of Urban Minor Arterial \& Cnllertar 
LEGEND: $\mathrm{A}=1$ OBS, $\mathrm{B}=2 \mathrm{OBS}, \mathrm{ETC}$.

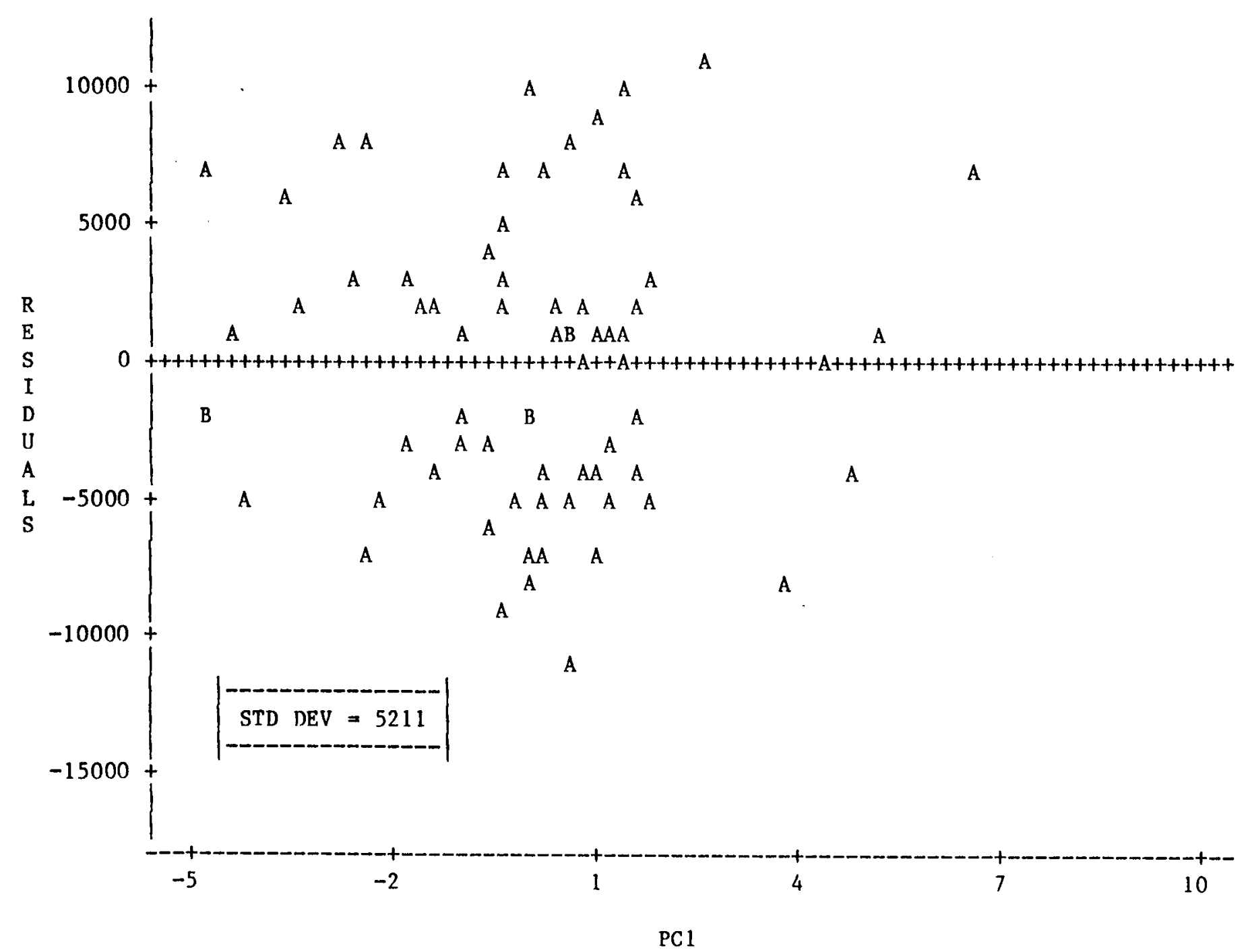


LEGEND: $A=1$ OBS, $B=2$ OBS, ETC.

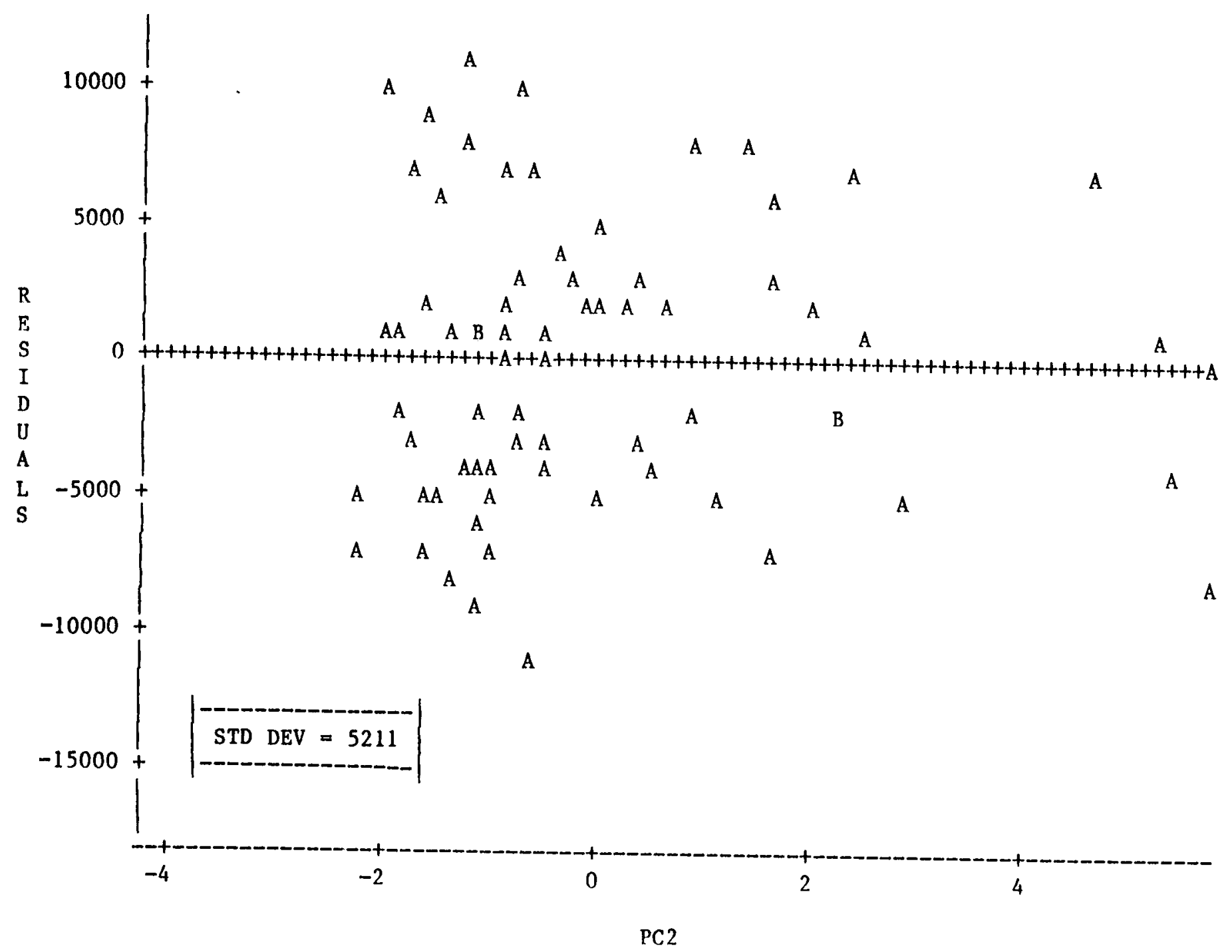


LEGEND: $\mathrm{A}=1$ OBS, $\mathrm{B}=2 \mathrm{OBS}, \mathrm{ETC}$.

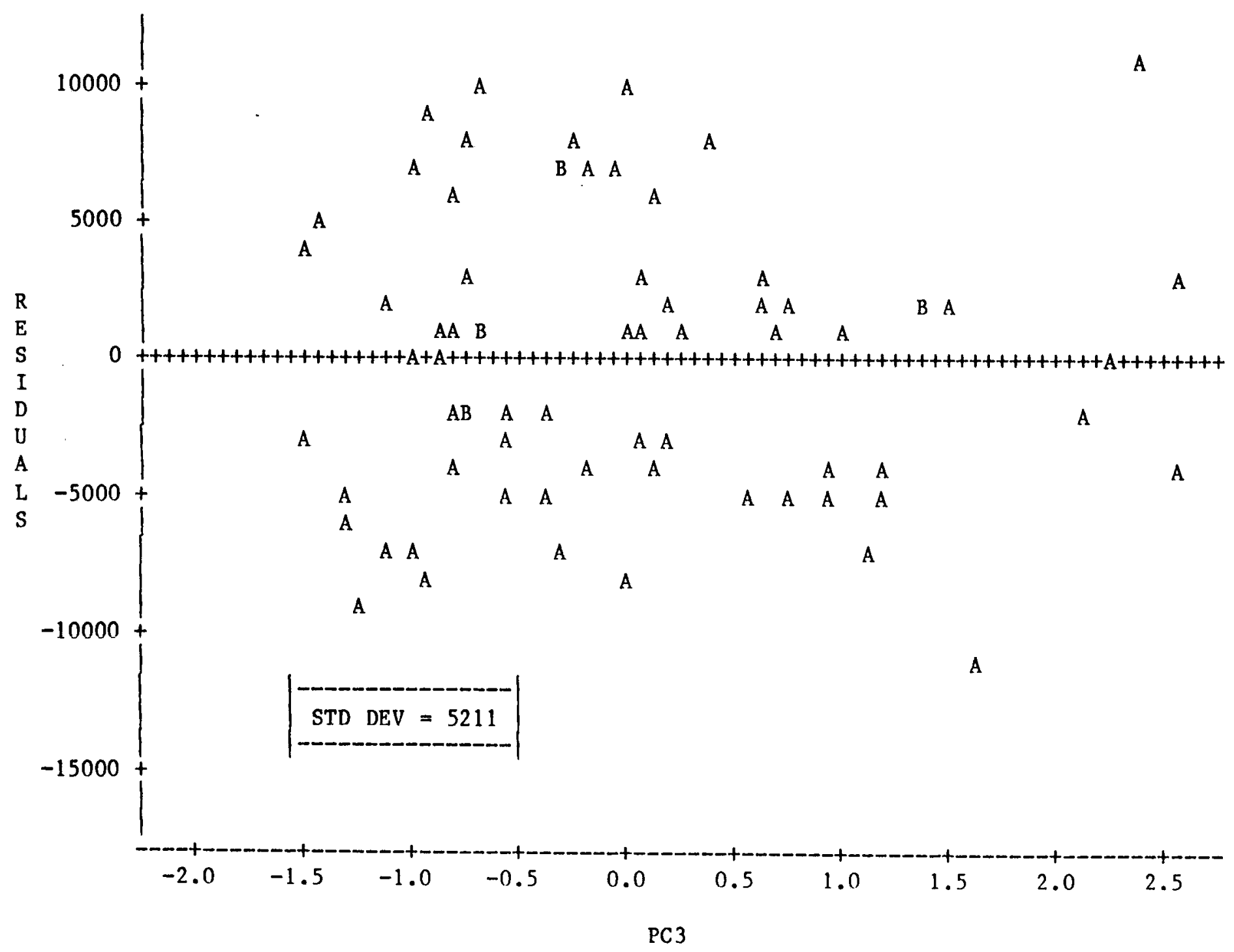


LEGEND: $A=1$ OBS, $B=2$ OBS, ETC.

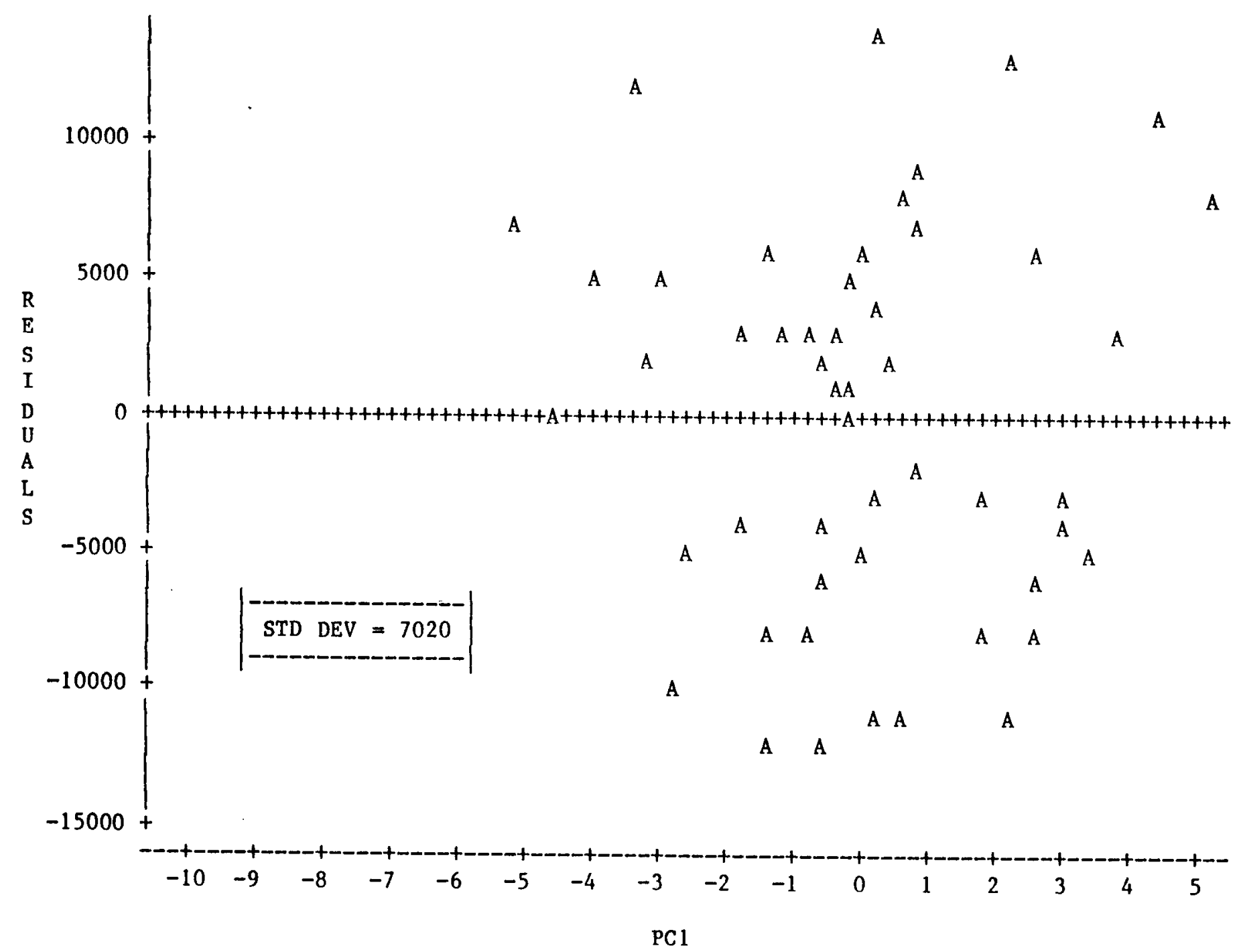

F1gure D4.4: Residua1 Plot against PCl for Central Region of Irhan Tutaretaten Wirhr..nem 
LEGEND: $A=1$ OBS, $B=2$ OBS, ETC.

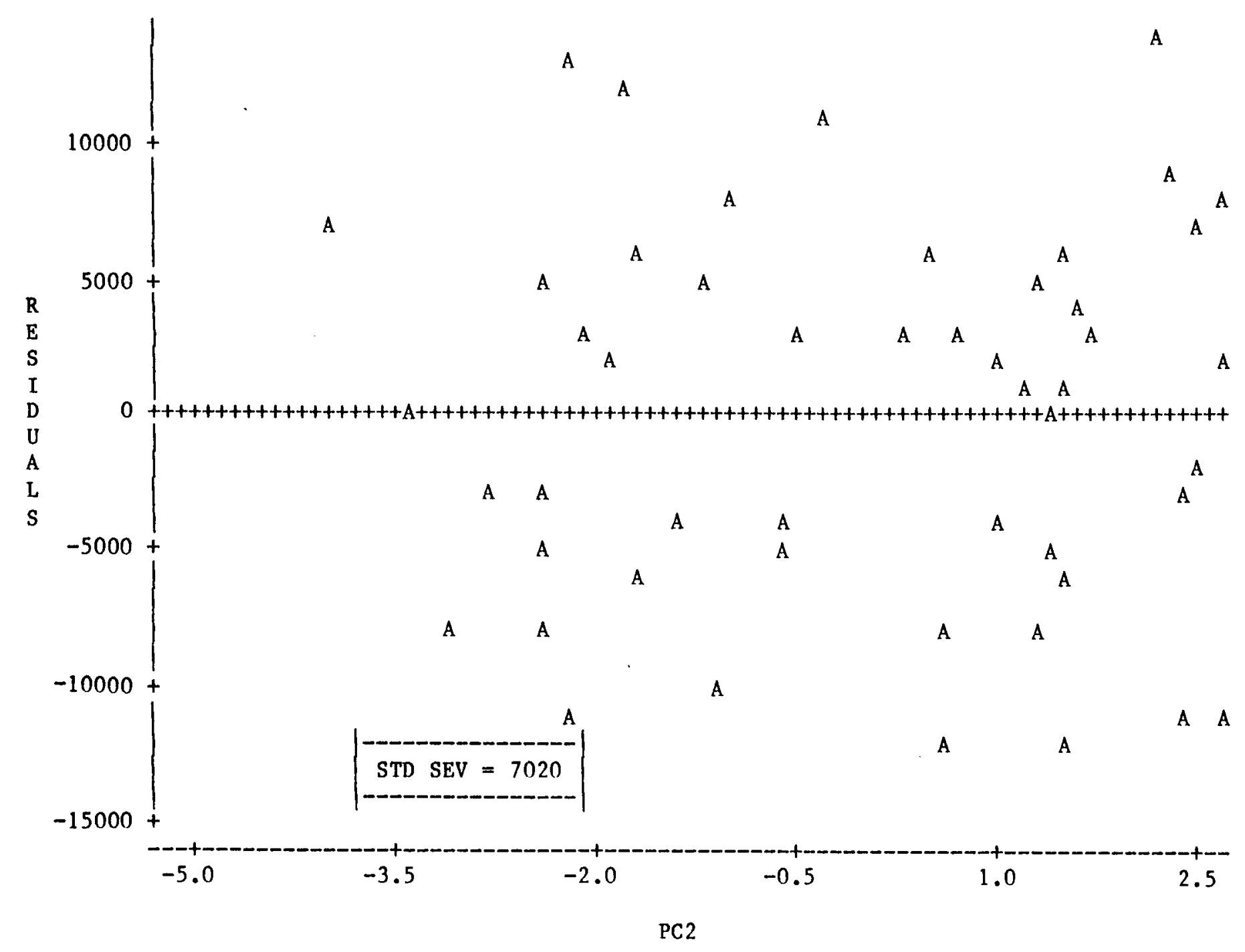

Figure D4.5: Residual P1ot against PC2 for Central Region of Urban Interstate Highwavs 
LEGEND: $A=1$ OBS, $B=2$ OBS, ETC.

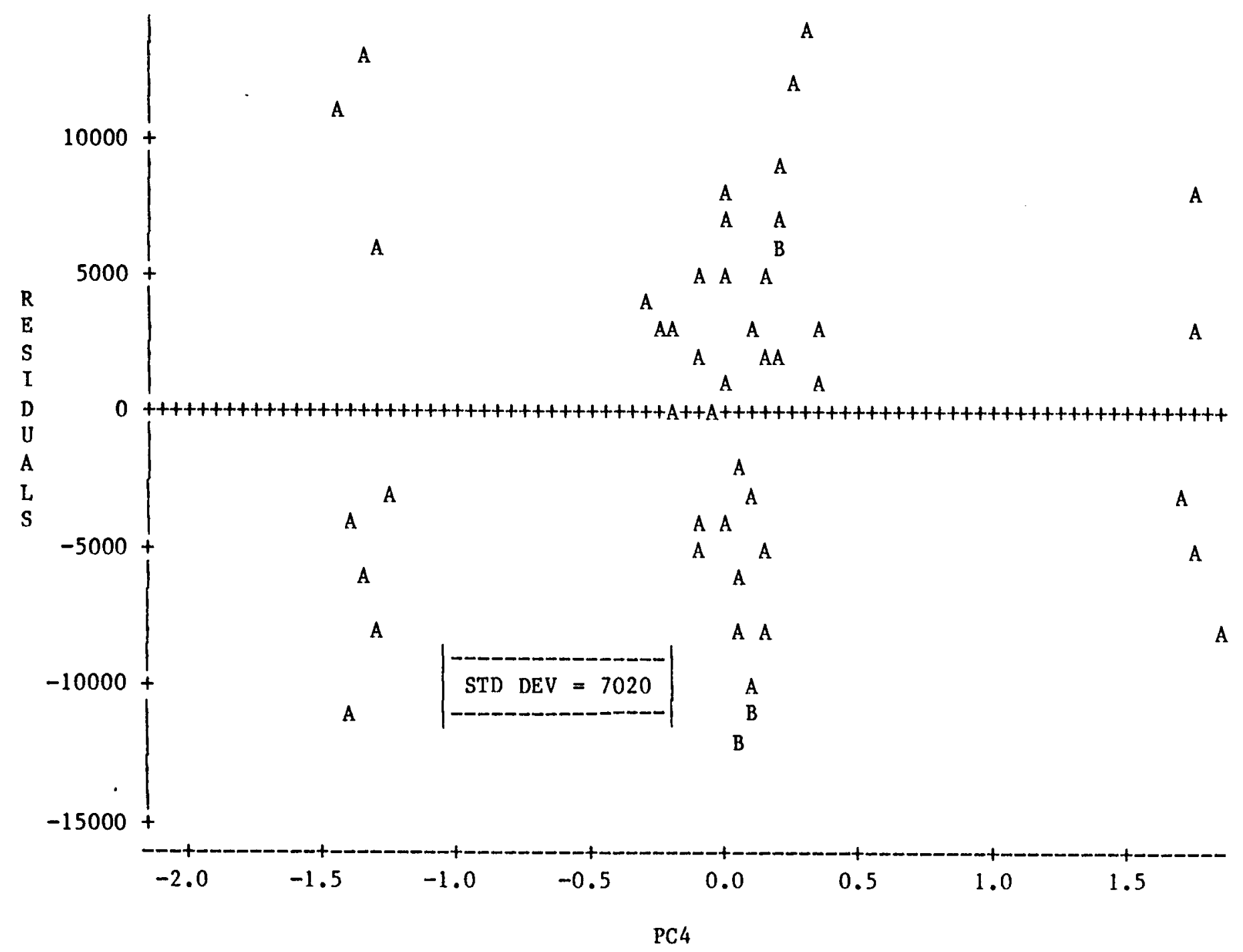

Figure D4.6: Residual Plot against PC4 for Central Region 
LEGEND: $\mathrm{A}=1$ OBS, $\mathrm{B}=2$ OBS, ETC.

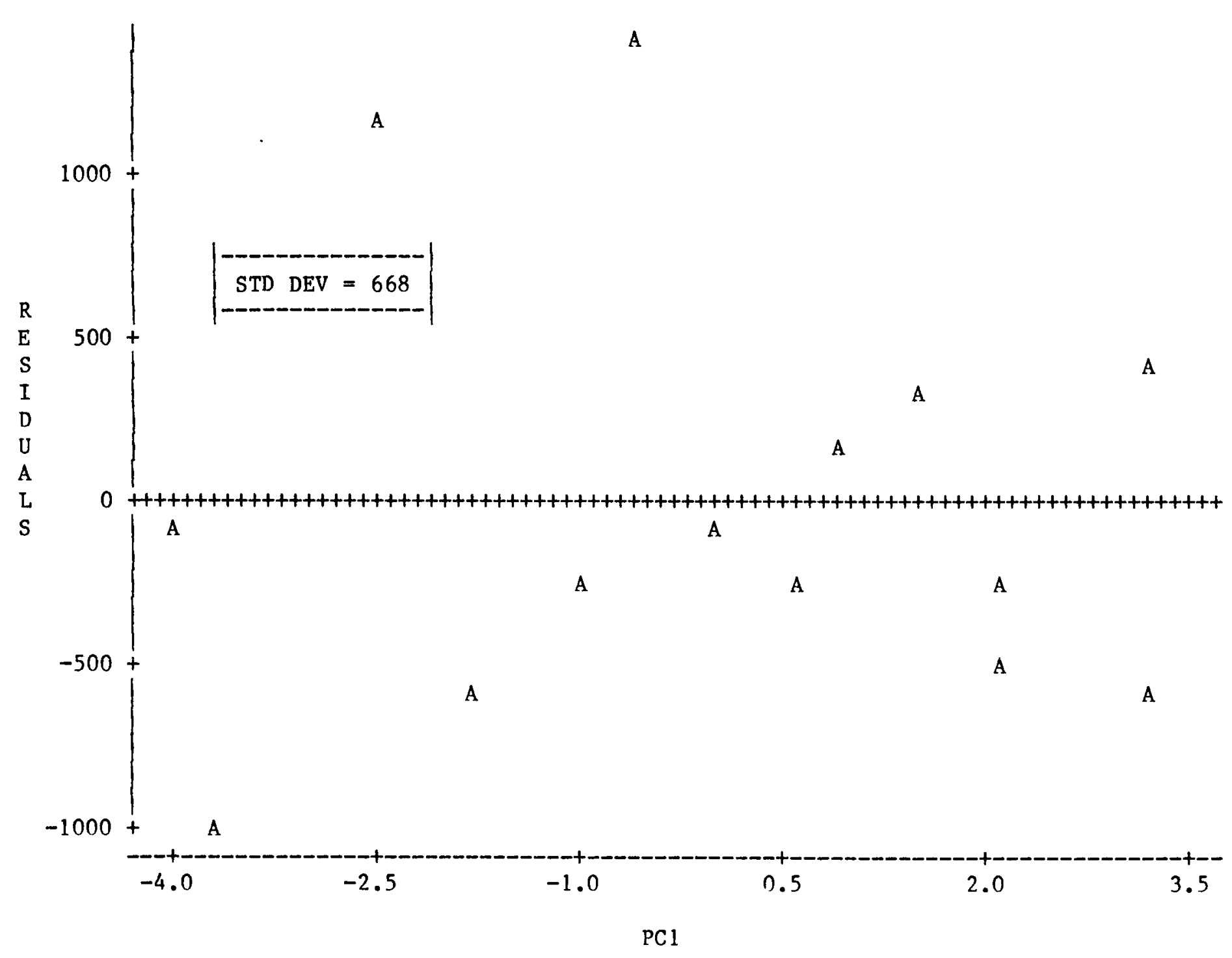

Figure D4.7: Residual Plot against PCl for City Group "A" and Reginn "r" nf Trhan Princinal Artorial 
LEGEND: $A=1$ OBS, $B=2$ OBS, ETC.

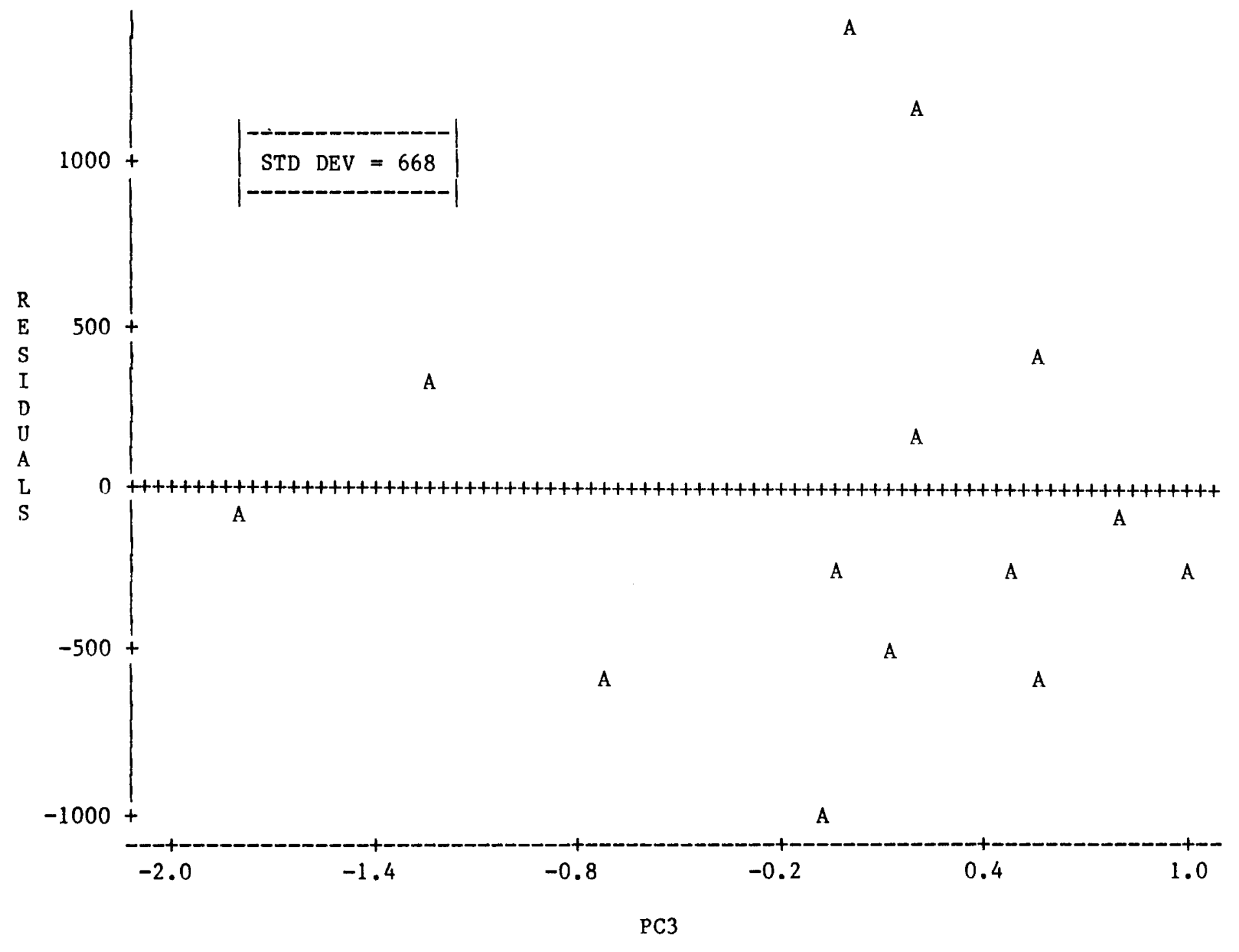

Figure D4.8: Residual Plot against PC3 for City Group "A" and Region "c" of Urban Principal Arterial 
LEGEND: $A=1$ OBS, $B=2$ OBS, ETC.

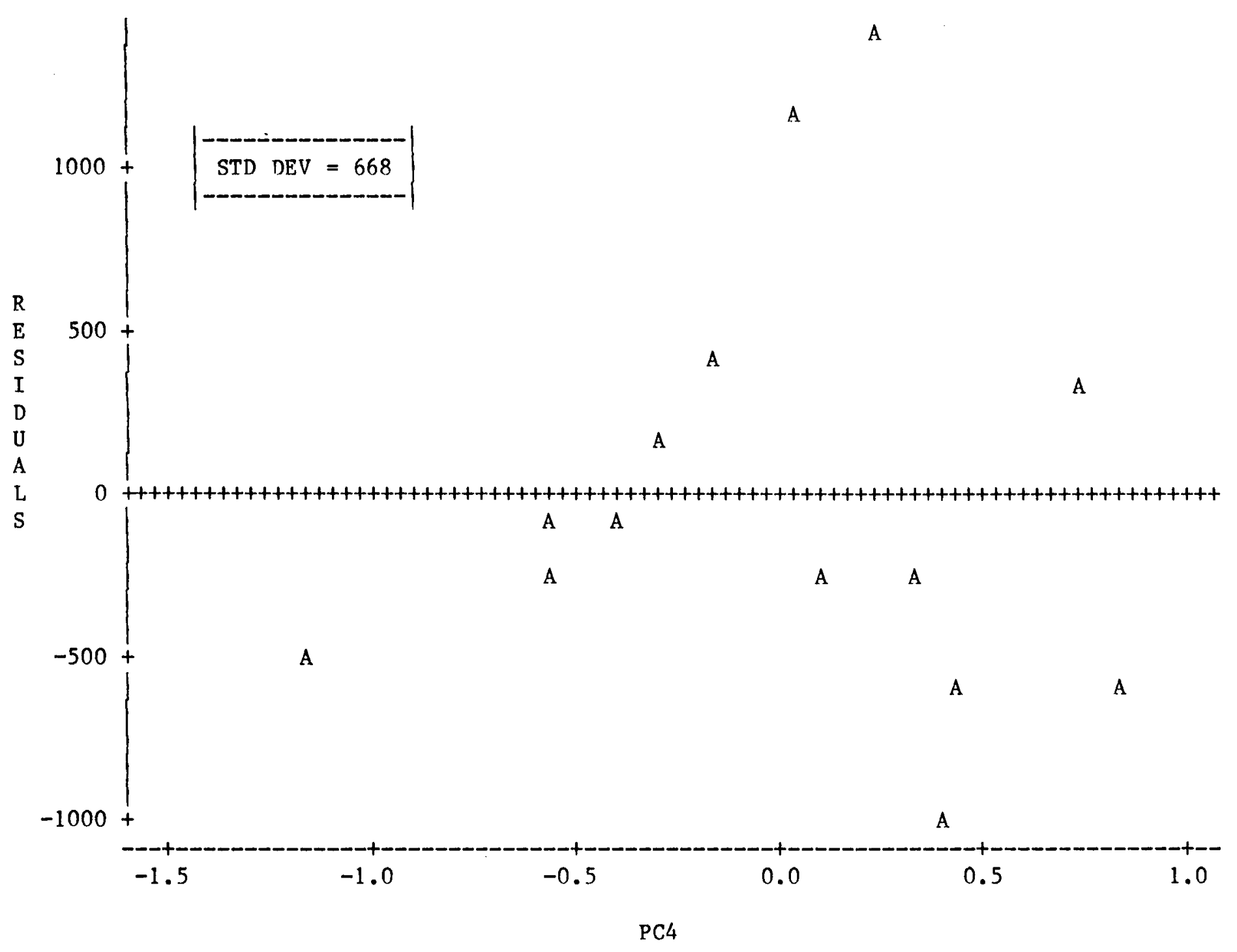
and Region "r" of Virhan Princinal Arterial 
LEGEND: $A=1$ OBS, $B=2$ OBS, ETC.

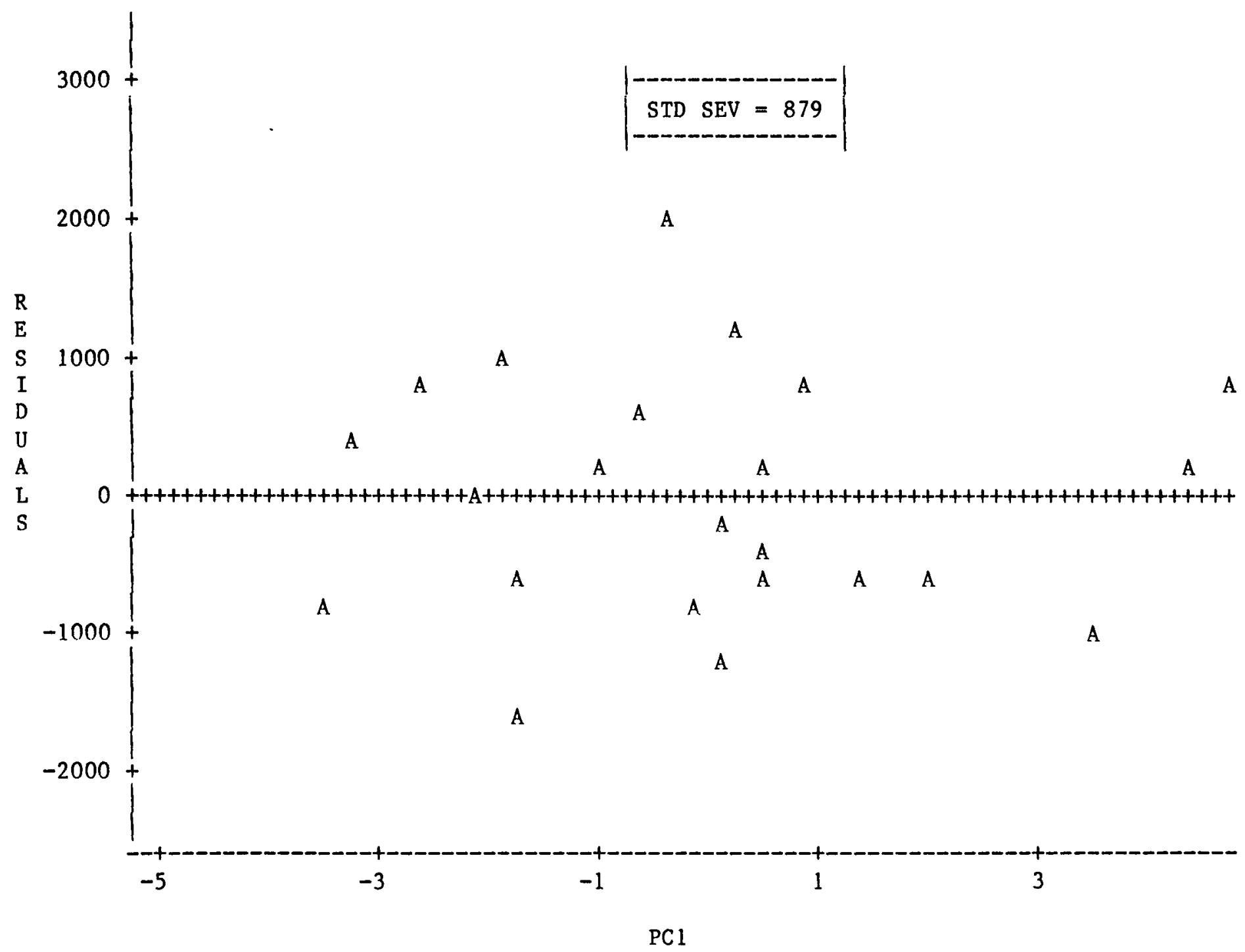

Figure D4.10: Residual P1ot against PCl for City Group "D" of Urban Minor Arterlal \& Collector 
LEGEND: $A=1$ OBS, $B=2$ OBS, ETC.

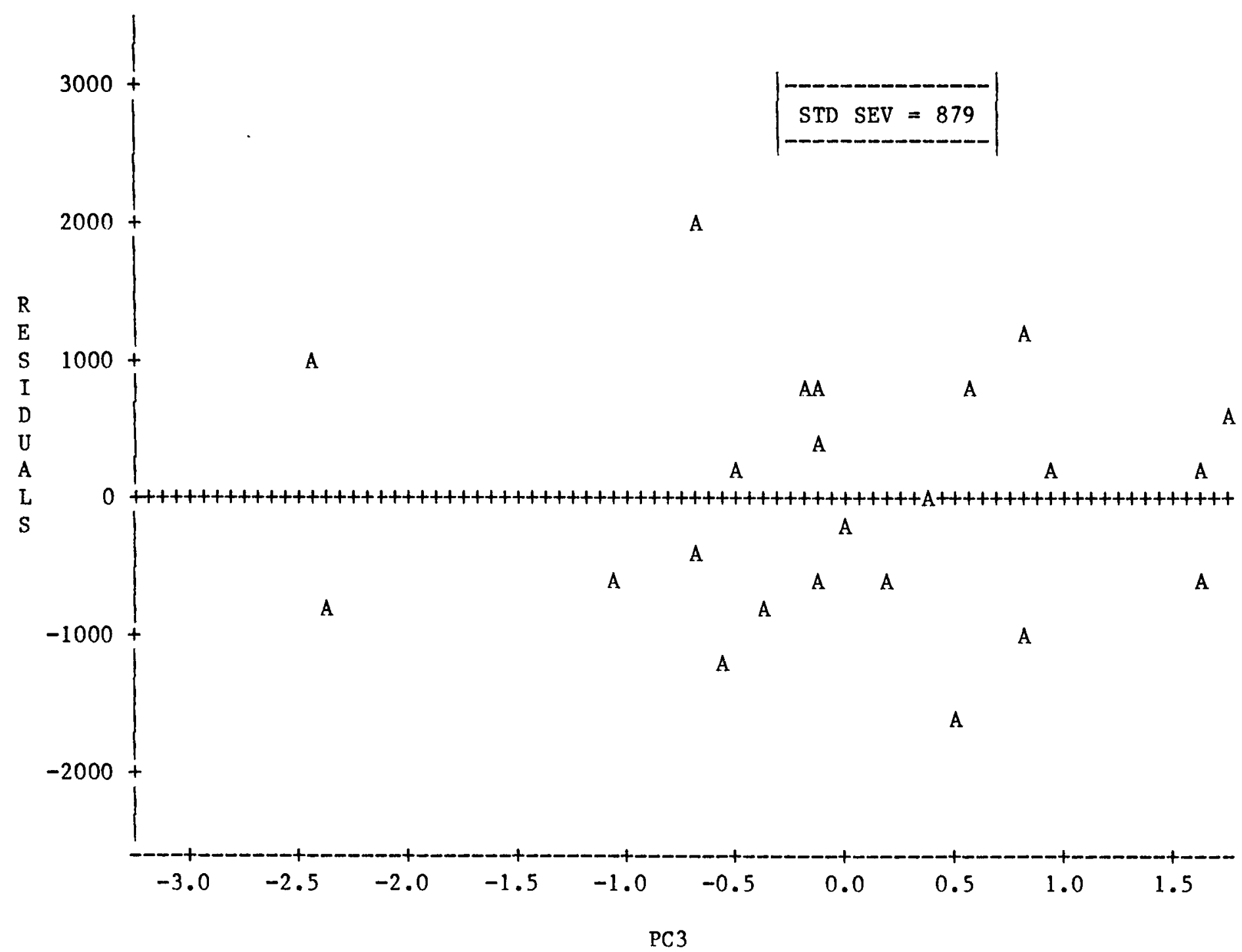

F1gure D4.11: Residua1 P1ot against PC3 for C1ty Group "D" of Urban Minor Arterial \& Collector 
LEGEND: $A=1$ OBS, $B=2$ OBS, ETC.

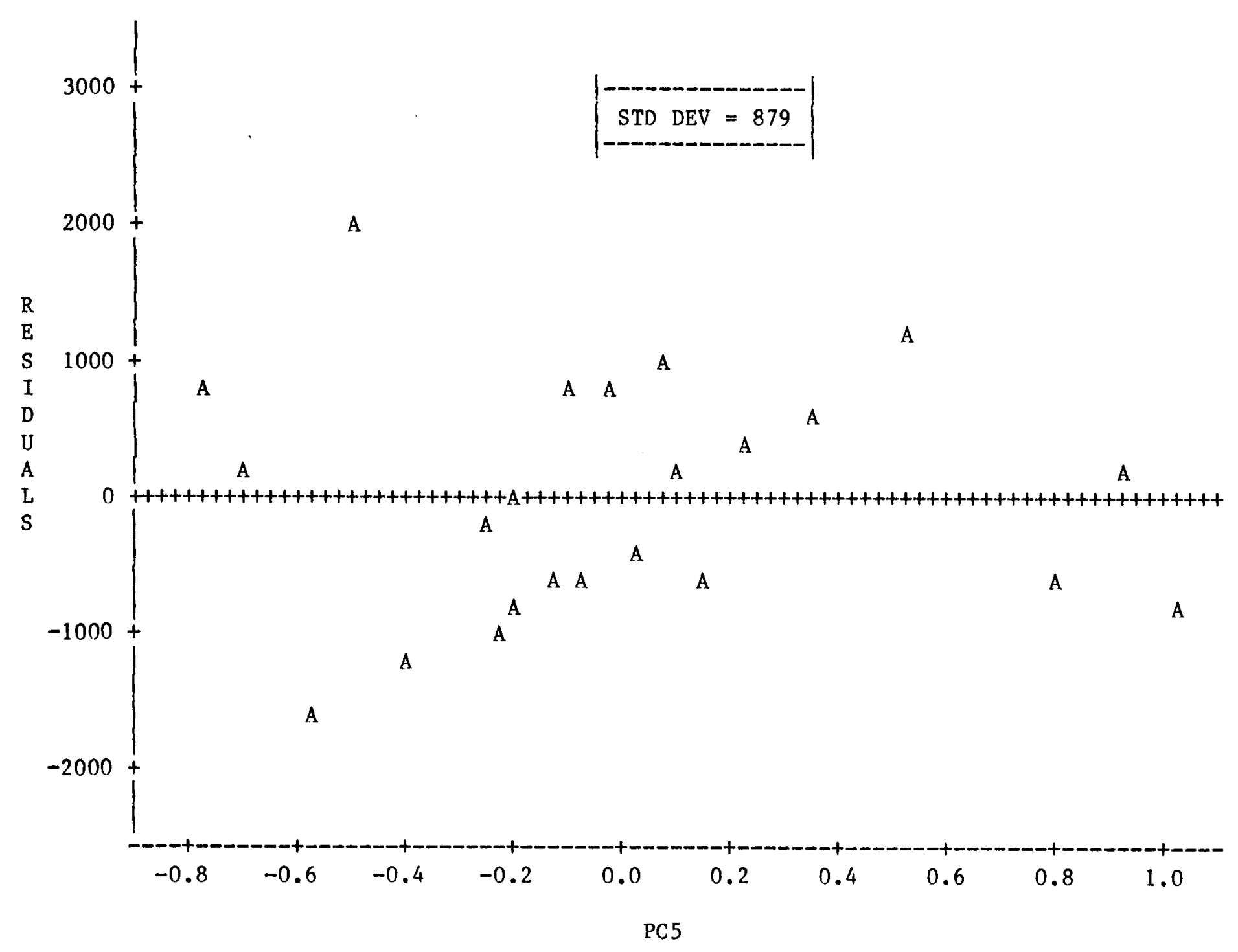

Figure D4.12: Residual Plot against PC5 for City Group "D" of Urban Minor Arterial \& Collector 
LEGEND: $A=1$ OBS, $B=2$ OBS, ETC.

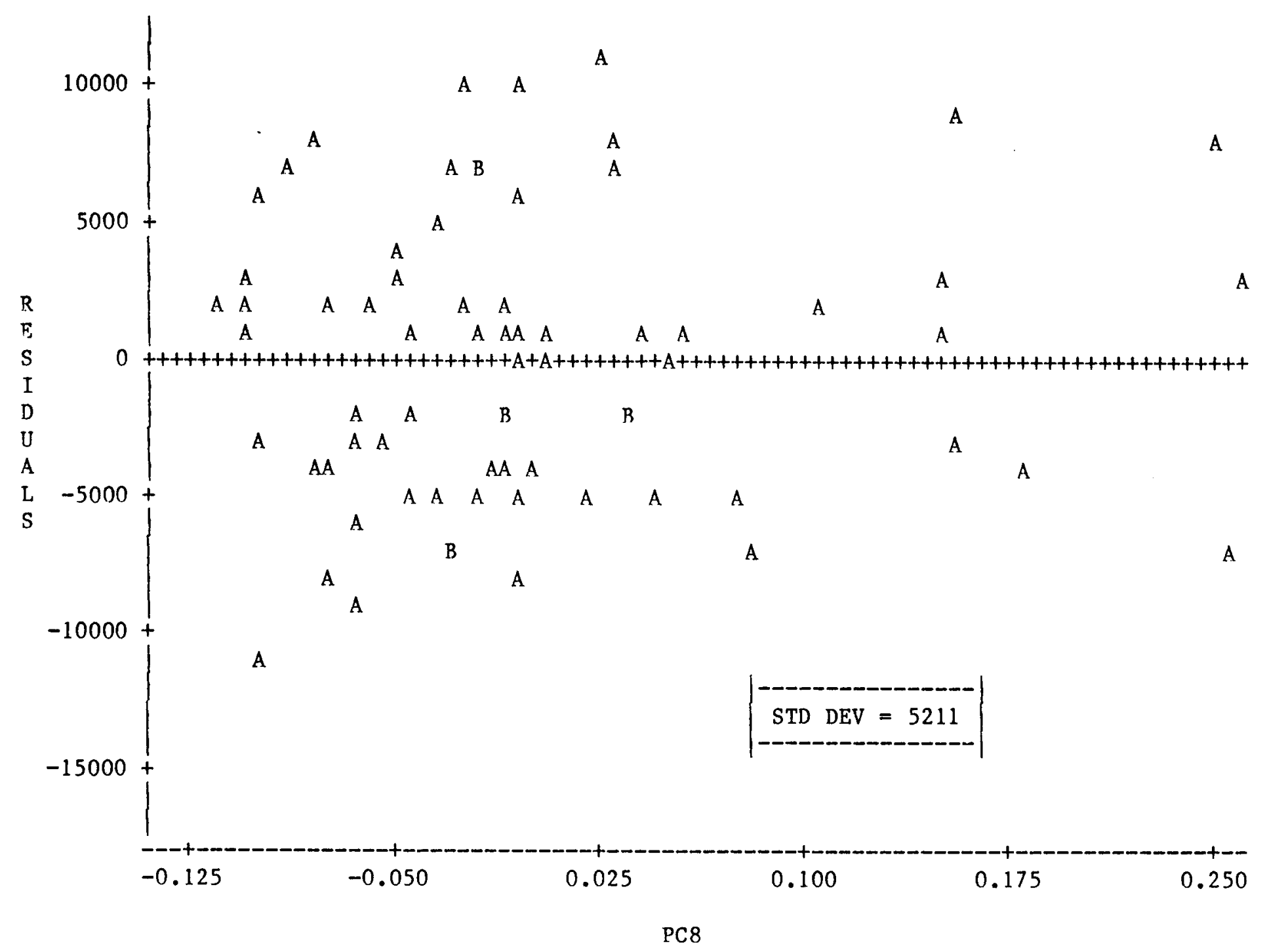

Ff.gure D5.1: Residual P1ot against PC8 (not in mode1) for Central Regton of Rural Tnteratate He ohwava 
LEGEND: $\mathrm{A}=1$ OBS, $\mathrm{B}=2 \mathrm{OBS}, \mathrm{ETC}$.

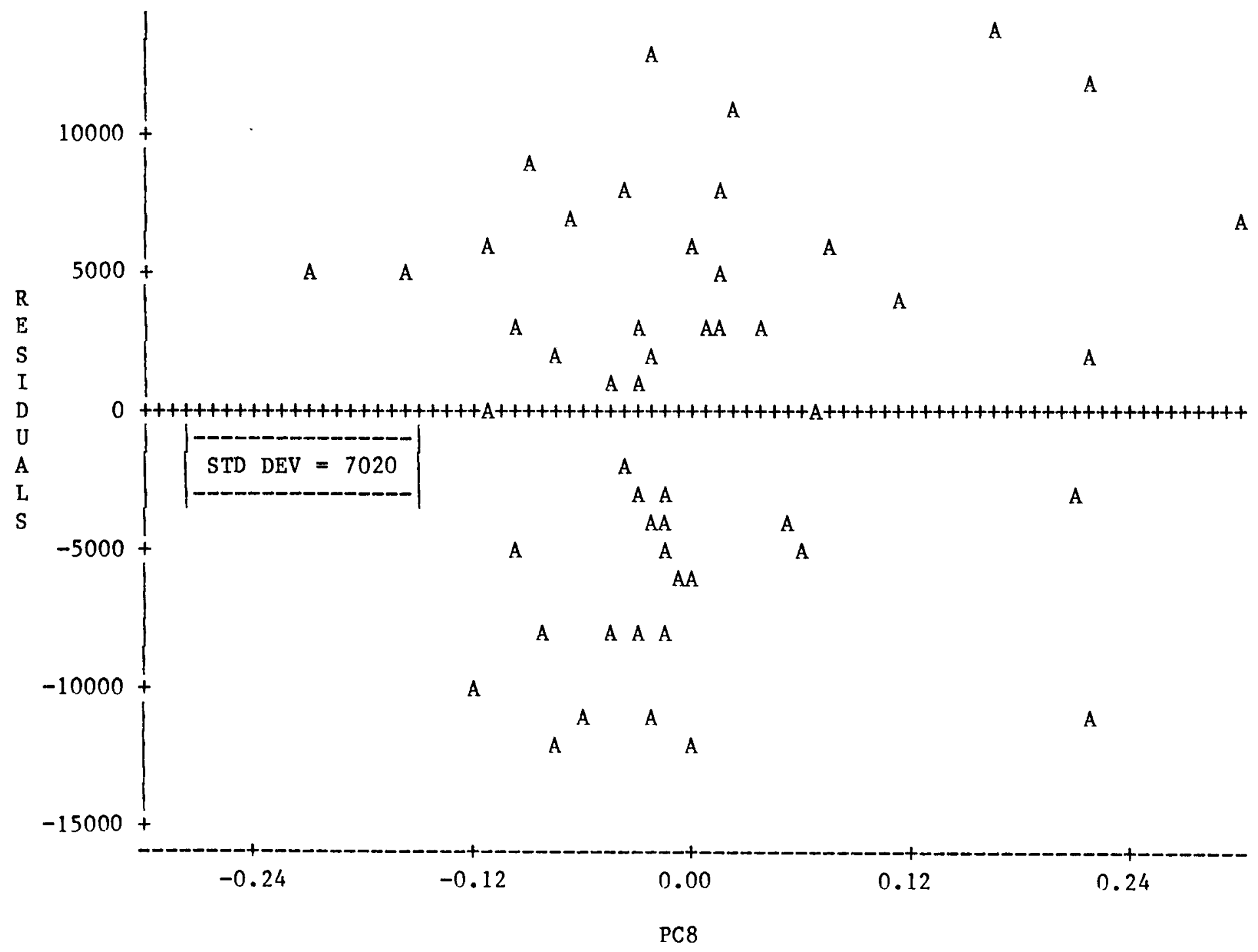

Figure D5.2: Residual P1ot against PC8 (not in mode1) for

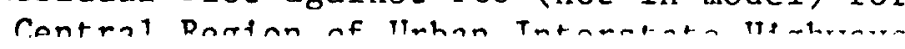


LEGEND: $A=1$ OBS, $B=2$ OBS, ETC.

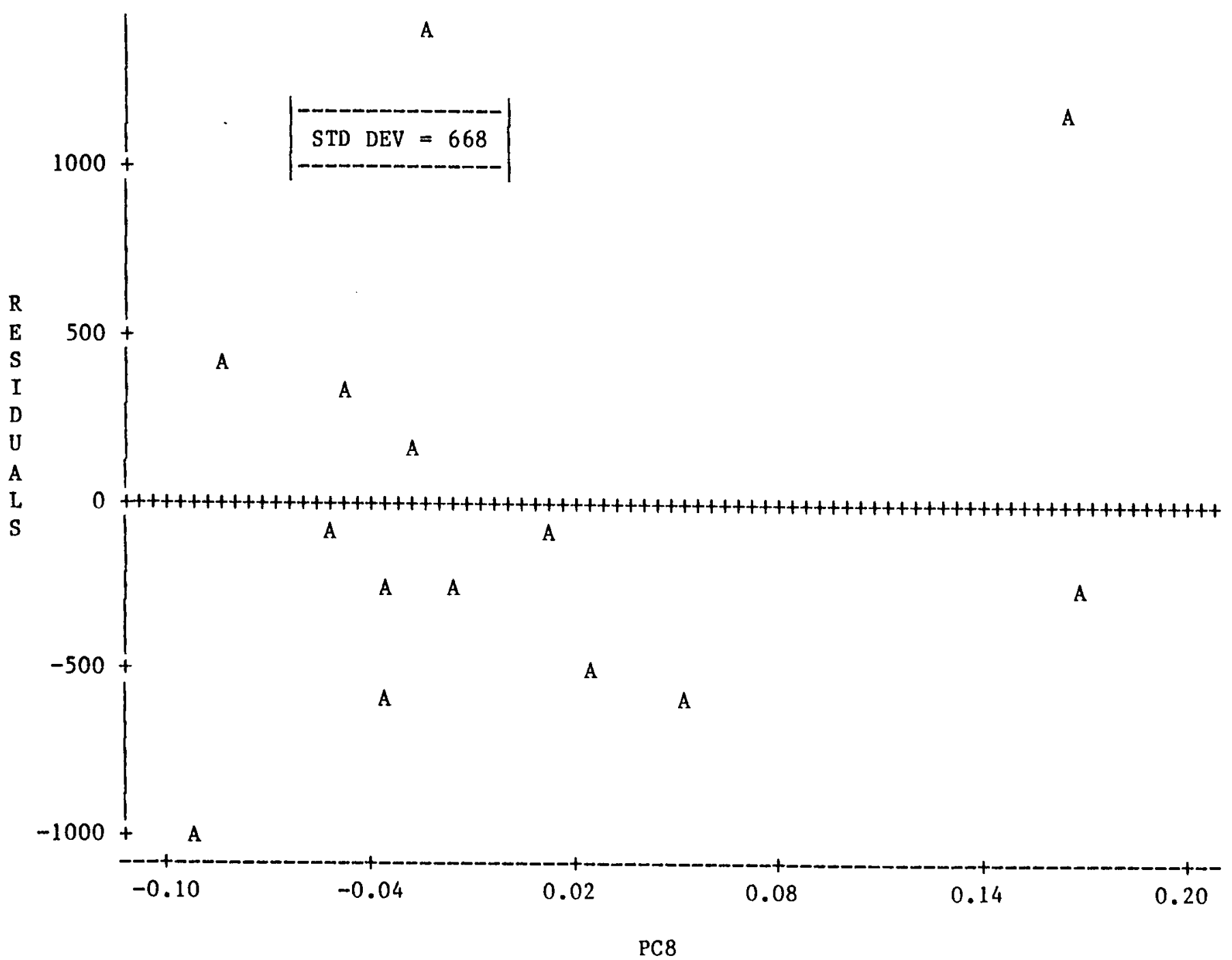


LEGEND: $\mathrm{A}=1$ OBS, $\mathrm{B}=2$ OBS, ETC.

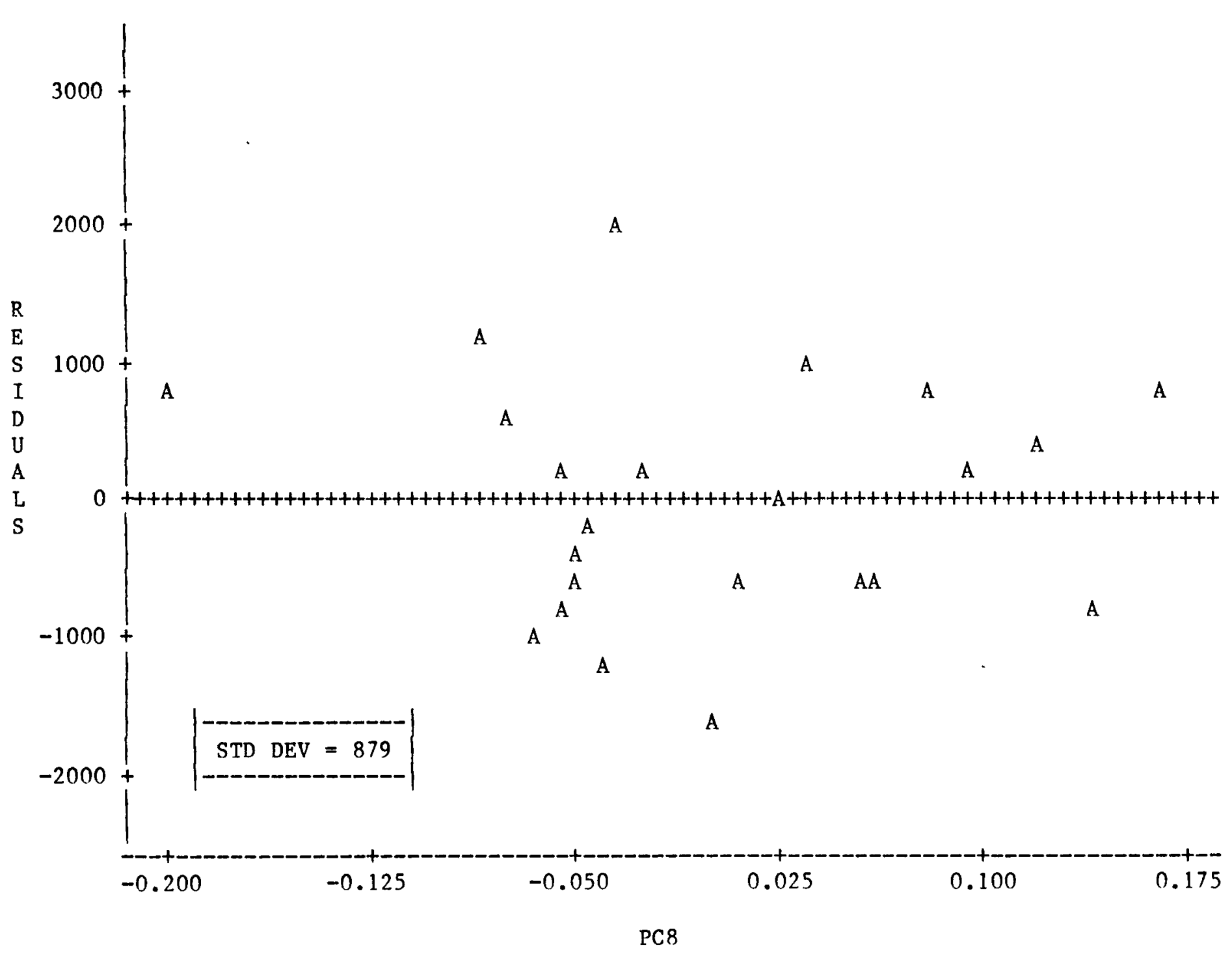

Figure D5.4: Residual P1ot against PC8 (not in mode1) for City Group "D" of Urban Minor Arterial \& Collector 
Appendix E 
Table E1.l: Partial-Lagged Regression Results of Interstate Highways

\begin{tabular}{|c|c|c|c|c|c|c|c|c|c|}
\hline \multirow{3}{*}{ Region } & \multicolumn{2}{|c|}{ Model Statistics } & \multicolumn{5}{|c|}{ Significance Statistics \& Parameter Values } & \multicolumn{2}{|c|}{ Normality Stat. } \\
\hline & $\mathrm{R}^{2} \mathrm{R}^{2}$ & $\begin{array}{l}\text { RMSE } \\
\text { C.V. } \\
\text {. }\end{array}$ & $\begin{array}{c}\text { F-Value } \\
\text { Prob }>F\end{array}$ & $\int_{\text {Name }}^{\text {Para }}$ & $\begin{array}{l}\text { ameter } \\
\text { Estimate }\end{array}$ & $\begin{array}{c}t- \\
\text { value }\end{array}$ & Prob $>t$ & $\mathrm{~N}$ & $\mid \begin{array}{c}W / D(*) \\
(P-v a l \text { ue })\end{array}$ \\
\hline & \multicolumn{9}{|c|}{ A. Interstate Highways (Rural \& Urban): Regional Model } \\
\hline North & $\begin{array}{l}99.79 \\
99.78\end{array}$ & $\begin{array}{r}1605.2 \\
4.143\end{array}$ & $\begin{array}{r}34083.2 \\
0.0001\end{array}$ & $\mid \begin{array}{l}\text { CONST } \\
\text { LAADT } \\
\text { RAADT }\end{array}$ & $\begin{array}{r}-19.49398 \\
1.00458 \\
2.98118\end{array}$ & $\begin{array}{r}-0.098 \\
226.650 \\
40.429\end{array}$ & $\begin{array}{l}0.9219 \\
0.0001 \\
0.0001\end{array}$ & 149 & $\begin{array}{c}0.1268 \\
(<0.01)\end{array}$ \\
\hline Central & $\left\{\begin{array}{l}98.50 \\
98.49\end{array}\right.$ & $\begin{array}{r}2485.7 \\
6.992\end{array}$ & $\left\{\begin{array}{r}10376.6 \\
0.0001\end{array}\right.$ & $\begin{array}{l}\text { CONST } \\
\text { LAADT } \\
\text { RAADT }\end{array}$ & $\begin{array}{r}350.01753 \\
1.02776 \\
1.36517\end{array}$ & $\begin{array}{r}1.238 \\
132.038 \\
27.684\end{array}$ & $\begin{array}{l}0.2166 \\
0.0001 \\
0.0001\end{array}$ & 320 & $\begin{array}{c}0.1112 \\
(<0.01)\end{array}$ \\
\hline \multirow[t]{2}{*}{ South } & $\begin{array}{l}98.81 \\
98.78\end{array}$ & $\begin{array}{r}1343.5 \\
7.474\end{array}$ & $\begin{array}{l}4714.6 \\
0.0001\end{array}$ & $\begin{array}{l}\text { CONST } \\
\text { LAADT } \\
\text { RAADT }\end{array}$ & $\begin{array}{r}-108.99377 \\
1.03591 \\
2.21005\end{array}$ & $\begin{array}{l}-0.468 \\
72.149 \\
18.300\end{array}$ & $\begin{array}{l}0.6404 \\
0.0001 \\
0.0001\end{array}$ & 117 & \multirow[t]{2}{*}{$\begin{array}{r}0.1602 \\
(<0.01)\end{array}$} \\
\hline & B. Rural & Interst & te Highway & ys: Sta & $\begin{array}{l}x=x==x=x=0 \\
\text { atewide Mode }\end{array}$ & 1 & & & \\
\hline \multirow[t]{2}{*}{ ALL } & $\begin{array}{l}97.52 \\
97.50\end{array}$ & $\begin{array}{r}1862.6 \\
8.562\end{array}$ & $\begin{array}{l}7027.6 \\
0.0001\end{array}$ & $\left\{\begin{array}{l}\text { CONST } \\
\text { LAADT } \\
\text { RAADT }\end{array}\right.$ & $\begin{array}{r}640.19078 \\
0.99501 \\
1.68258\end{array}$ & $\left\{\begin{array}{r}3.067 \\
100.818 \\
24.894\end{array}\right.$ & $\begin{array}{l}0.0023 \\
0.0001 \\
0.0001\end{array}$ & \multirow[t]{2}{*}{361} & \multirow[t]{2}{*}{$\begin{array}{c}0.1448 \\
(<0.01)\end{array}$} \\
\hline & C. Rural & st & High & $\begin{array}{l}\text { ys: Reg } \\
\text { ys: Res= }\end{array}$ & $\begin{array}{l}=x=x=x=1 \\
\text { Ional Model }\end{array}$ & & & & \\
\hline North & $\begin{array}{l}95.49 \\
95.36\end{array}$ & $\begin{array}{r}1495.9 \\
7.835\end{array}$ & $\begin{array}{r}740.3 \\
0.0001\end{array}$ & $\mid \begin{array}{l}\text { CONST } \\
\text { LAADT } \\
\text { RAADT }\end{array}$ & $\begin{array}{r}185.11105 \\
1.00078 \\
2.73211\end{array}$ & $\begin{array}{r}0.355 \\
37.594 \\
12.201\end{array}$ & $\begin{array}{l}0.7238 \\
0.0001 \\
0.0001\end{array}$ & 73 & $\begin{array}{r}0.1626 \\
(<0.01)\end{array}$ \\
\hline Central & $\begin{array}{l}96.96 \\
96.93\end{array}$ & $\begin{array}{r}2167.8 \\
8.094\end{array}$ & $\begin{array}{l}3159.3 \\
0.0001\end{array}$ & $\begin{array}{l}\text { CONST } \\
\text { LAADT } \\
\text { RAADT }\end{array}$ & $\begin{array}{r}840.56657 \\
0.99257 \\
1.59519\end{array}$ & $\begin{array}{r}2.270 \\
67.753 \\
18.524\end{array}$ & $\begin{array}{l}0.0243 \\
0.0001 \\
0.0001\end{array}$ & 201 & $\begin{array}{r}0.1424 \\
(<0.01)\end{array}$ \\
\hline South & $\begin{array}{l}96.51 \\
96.42\end{array}$ & $\begin{array}{r}1051.3 \\
8.495\end{array}$ & $\begin{array}{l}1159.8 \\
0.0001\end{array}$ & $\begin{array}{l}\text { CONST } \\
\text { LAADT } \\
\text { RAADT }\end{array}$ & $\begin{array}{r}120.19918 \\
1.02032 \\
1.88571\end{array}$ & $\begin{array}{r}0.431 \\
46.099 \\
12.951\end{array}$ & $\begin{array}{l}0.6678 \\
0.0001 \\
0.0001\end{array}$ & 87 & $\begin{array}{r}0.2074 \\
(<0.01)\end{array}$ \\
\hline
\end{tabular}


Table E1.1, continued

\begin{tabular}{|c|c|c|c|c|c|c|c|c|c|}
\hline \multirow{2}{*}{ Region } & \multicolumn{2}{|c|}{ Model Statistics } & \multicolumn{5}{|c|}{ Significance Statistics \& Parameter Values } & \multicolumn{2}{|c|}{ Normality Stat. } \\
\hline & $\mathrm{R}^{2} \mathrm{R}^{2}$ & $\begin{array}{l}\text { RMSE } \\
\text { C.V. }\end{array}$ & $\mid \begin{array}{r}\text { F-Value } \\
\text { Prob }>\text { F }\end{array}$ & $\begin{array}{c}\text { Para } \\
\text { Name }\end{array}$ & $\begin{array}{l}\text { Est Imate } \\
\text { Ester }\end{array}$ & $\begin{array}{c}t- \\
\text { value }\end{array}$ & Prob>t & $\mathrm{N}$ & $\mid \begin{array}{c}\text { W/D (*) } \\
\text { (P-value) }\end{array}$ \\
\hline & Urba & nterst & te Highwa & s: Sta & atewide Mode & & & & \\
\hline ALL & $\begin{array}{l}98.75 \\
98.73\end{array}$ & $\begin{array}{r}3284.7 \\
6.483\end{array}$ & $\begin{array}{l}8739.6 \\
0.0001\end{array}$ & $\begin{array}{l}\text { CONST } \\
\text { LAADT } \\
\text { RAADT }\end{array}$ & $\begin{array}{r}515.12102 \\
1.02651 \\
1.62377\end{array}$ & $\begin{array}{r}1.176 \\
124.878 \\
24.210\end{array}$ & $\begin{array}{l}0.2409 \\
0.0001 \\
0.0001\end{array}$ & 225 & $\begin{array}{r}0.1345 \\
(<0.01)\end{array}$ \\
\hline & E. Urban & Interst & te Highwa & ys: $\operatorname{Re}$ & gional Model & & & & \\
\hline North & $\begin{array}{l}99.82 \\
99.81\end{array}$ & $\begin{array}{r}1720.9 \\
2.986\end{array}$ & $\mid \begin{array}{r}19758.7 \\
0.0001\end{array}$ & $\begin{array}{l}\text { CONST } \\
\text { LAADT } \\
\text { RAADT }\end{array}$ & $\begin{array}{r}-113.20860 \\
1.00492 \\
3.01027\end{array}$ & $\left\{\begin{array}{r}-0.322 \\
178.844 \\
35.829\end{array}\right.$ & $\begin{array}{l}0.7481 \\
0.0001 \\
0.0001\end{array}$ & 225 & $\begin{array}{r}0.1345 \\
(<0.01)\end{array}$ \\
\hline Central & $\begin{array}{l}98.33 \\
98.30\end{array}$ & $\begin{array}{r}2888.3 \\
5.735\end{array}$ & $\begin{array}{l}3418.4 \\
0.0001\end{array}$ & $\begin{array}{l}\text { CONST } \\
\text { LAADT } \\
\text { RAADT }\end{array}$ & $\begin{array}{r}372.50506 \\
1.03337 \\
1.29483\end{array}$ & $\begin{array}{r}0.558 \\
77.206 \\
19.517\end{array}$ & $\begin{array}{l}0.5777 \\
0.0001 \\
0.0001\end{array}$ & 119 & $\begin{array}{r}0.1046 \\
(<0.01)\end{array}$ \\
\hline South & $\begin{array}{l}97.42 \\
97.22\end{array}$ & $\begin{array}{r}1937.5 \\
5.662\end{array}$ & $\begin{array}{r}509.0 \\
0.0001\end{array}$ & $\begin{array}{l}\text { CONST } \\
\text { LAADT } \\
\text { RAADT }\end{array}$ & $\begin{array}{r}731.22153 \\
1.00116 \\
2.40671\end{array}$ & $\begin{array}{r}0.644 \\
25.395 \\
9.653\end{array}$ & $\begin{array}{l}0.5253 \\
0.0001 \\
0.0001\end{array}$ & 30 & $\begin{array}{r}0.9726 \\
(0.641)\end{array}$ \\
\hline
\end{tabular}

(*) Wilk-Shapiro ' $W$ ' for $N<51$ or Kolmogorov ${ }^{-} \mathrm{D}^{\prime}$ for $\mathrm{N} \geqslant 51$, and $\mathrm{P}$-value $=\mathrm{PROB}>\mathrm{D}$ or $\mathrm{PROB}\langle\mathrm{W}$. 
Table E1.2: Partia1-Lagged Regression Results of Urban Principal Arterial

\begin{tabular}{|c|c|c|c|c|c|c|c|c|c|}
\hline \multirow{3}{*}{ Region } & \multicolumn{2}{|c|}{ Mode1 Statistics } & \multicolumn{5}{|c|}{ Significance Statistics \& Parameter Values } & \multicolumn{2}{|c|}{ Normality Stat. } \\
\hline & $\int_{x=m x=2}^{R^{2}} R^{2}$ & $\begin{array}{l}\text { RMSE } \\
\text { C. V. } \\
==x===0\end{array}$ & $\mid \begin{array}{c}\text { F-Value } \\
\text { Prob }>\text { F }\end{array}$ & Name & $\begin{array}{l}\text { rameter } \\
\text { Estimate }\end{array}$ & $\begin{array}{c}t- \\
\text { value }\end{array}$ & Prob $>t$ & $\mathrm{~N}$ & $\mid \begin{array}{c}\text { W/D (*) } \\
(\mathrm{P} \text {-value })\end{array}$ \\
\hline & City Gro & "A" ICi & ity Popul & lation: & 30,000 to & $50,000]$ & & $F=x$ & \\
\hline North & $\begin{array}{l}99.36 \\
99.35\end{array}$ & $\begin{array}{l}459.8 \\
3.687\end{array}$ & $\mid \begin{array}{r}15249.1 \\
0.0001\end{array}$ & $\left\{\begin{array}{l}\text { CONST } \\
\text { LAADT } \\
\text { RAADT }\end{array}\right.$ & $\begin{array}{r}27.75357 \\
0.98467 \\
3.61682\end{array}$ & $\left\{\begin{array}{r}0.341 \\
167.541 \\
107.280\end{array}\right.$ & $\begin{array}{l}0.7334 \\
0.0001 \\
0.0001\end{array}$ & 200 & $\begin{array}{r}0.1365 \\
(<0.01)\end{array}$ \\
\hline Central & $\begin{array}{l}99.05 \\
99.04\end{array}$ & $\begin{array}{l}537.4 \\
4.204\end{array}$ & $\begin{array}{l}8615.7 \\
0.0001\end{array}$ & $\begin{array}{l}\text { CONST } \\
\text { LAADT } \\
\text { RAADT }\end{array}$ & $\begin{array}{r}-16.36854 \\
1.00096 \\
3.94064\end{array}$ & $\left\{\begin{array}{r}-0.151 \\
121.059 \\
52.842\end{array}\right.$ & $\begin{array}{l}0.8802 \\
0.0001 \\
0.0001\end{array}$ & 168 & $\begin{array}{r}0.1498 \\
(<0.01)\end{array}$ \\
\hline South & $\begin{array}{l}99.11 \\
99.06\end{array}$ & $\begin{array}{l}281.8 \\
2.727\end{array}$ & $\begin{array}{l}2003.1 \\
0.0001\end{array}$ & $\left\{\begin{array}{l}\text { CONST } \\
\text { LAADT } \\
\text { RAADT }\end{array}\right.$ & $\begin{array}{r}322.05516 \\
0.96382 \\
3.92732 \\
==x==x=x=x\end{array}$ & $\begin{array}{c}1.827 \\
57.059 \\
30.153 \\
=======\end{array}$ & $\begin{array}{l}0.0761 \\
0.0001 \\
0.0001\end{array}$ & 39 & $\begin{array}{r}0.9577 \\
(0.269)\end{array}$ \\
\hline & City Gro & "B" [C & ity Popul & $\begin{array}{l}=x=x=x= \\
\text { ation: }\end{array}$ & 20,000 to 3 & $\begin{array}{l}x=x===== \\
30,000]\end{array}$ & & & \\
\hline North & $\begin{array}{l}99.97 \\
99.97\end{array}$ & $\begin{array}{l}101.4 \\
0.864\end{array}$ & $\begin{array}{l}124971 \\
0.0001\end{array}$ & $\left\{\begin{array}{l}\text { CONST } \\
\text { LAADT } \\
\text { RAADT }\end{array}\right.$ & $\begin{array}{r}-6.16070 \\
1.00205 \\
3.95342\end{array}$ & $\left\{\begin{array}{r}-0.237 \\
488.078 \\
176.114\end{array}\right.$ & $\begin{array}{l}0.8132 \\
0.0001 \\
0.0001\end{array}$ & 85 & $\begin{array}{r}0.2414 \\
(<0.01)\end{array}$ \\
\hline Central & $\begin{array}{l}99.87 \\
99.87\end{array}$ & $\begin{array}{l}248.4 \\
2.033\end{array}$ & $\begin{array}{r}18172.8 \\
0.0001\end{array}$ & $\begin{array}{l}\text { CONST } \\
\text { LAADT } \\
\text { RAADT }\end{array}$ & $\begin{array}{r}36.87571 \\
0.99978 \\
3.87413\end{array}$ & $\begin{array}{r}0.491 \\
186.890 \\
76.484\end{array}$ & $\begin{array}{l}0.6240 \\
0.0001 \\
0.0001\end{array}$ & 50 & $\begin{array}{r}0.8329 \\
(<0.01)\end{array}$ \\
\hline South & $\begin{array}{l}99.20 \\
99.20\end{array}$ & $\begin{array}{l}415.6 \\
1.726\end{array}$ & $\begin{array}{r}558.9 \\
0.0001\end{array}$ & $\begin{array}{l}\text { CONST } \\
\text { LAADT } \\
\text { RAADT }\end{array}$ & $\begin{array}{r}2116.79778 \\
0.91194 \\
3.83773\end{array}$ & $\begin{array}{r}3.125 \\
31.102 \\
16.353\end{array}$ & $\begin{array}{l}0.0122 \\
0.0001 \\
0.0001\end{array}$ & 12 & $\begin{array}{r}0.9485 \\
(0.573)\end{array}$ \\
\hline
\end{tabular}


Table E1.2, continued

\begin{tabular}{|c|c|c|c|c|c|c|c|c|c|}
\hline \multirow{3}{*}{ Region } & \multicolumn{2}{|c|}{ Model Statistics } & \multicolumn{5}{|c|}{ Significance Statistics \& Parameter Values } & \multicolumn{2}{|c|}{ Normality Stat. } \\
\hline & 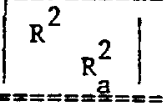 & $\begin{array}{l}\text { RMSE } \\
\text { C.V. } \\
== \pm=x==\end{array}$ & $\begin{array}{r}\text { F-Value } \\
\text { Prob }>\text { F }\end{array}$ & $\begin{array}{l}\text { Para } \\
\text { Name }\end{array}$ & $\begin{array}{l}\text { ameter } \\
\text { Estimate }\end{array}$ & $\begin{array}{c}\mathrm{t}- \\
\text { value }\end{array}$ & Prob>t & $\mathrm{N}$ & $\left\{\begin{array}{c}W / D(*) \\
(P \text {-value })\end{array}\right.$ \\
\hline & City Group & "C" $[\mathrm{C}$ & ty Popul & ation: & 10,000 to 2 & $, 000]$ & & & \\
\hline North & $\begin{array}{l}99.20 \\
99.00\end{array}$ & $\begin{array}{l}366.2 \\
3.348\end{array}$ & $\begin{array}{l}7561.9 \\
0.0001\end{array}$ & $\begin{array}{l}\text { CONST } \\
\text { LAADT } \\
\text { RAADT }\end{array}$ & $\begin{array}{r}292.60243 \\
0.96857 \\
3.70205\end{array}$ & $\begin{array}{r}3.023 \\
111.348 \\
67.347\end{array}$ & $\begin{array}{l}0.0029 \\
0.0001 \\
0.0001\end{array}$ & 153 & $\begin{array}{r}0.1681 \\
(<0.01)\end{array}$ \\
\hline Central & $\begin{array}{l}98.81 \\
98.79\end{array}$ & $\begin{array}{l}568.4 \\
5.070\end{array}$ & $\begin{array}{l}7483.1 \\
0.0001\end{array}$ & $\begin{array}{l}\text { CONST } \\
\text { LAADT } \\
\text { RAADT }\end{array}$ & $\begin{array}{r}107.53486 \\
0.99093 \\
3.67712\end{array}$ & $\begin{array}{r}1.029 \\
109.667 \\
48.916\end{array}$ & $\begin{array}{l}0.3049 \\
0.0001 \\
0.0001\end{array}$ & 184 & $\begin{array}{r}0.1887 \\
(<0.01)\end{array}$ \\
\hline South & $\begin{array}{l}96.53 \\
96.20\end{array}$ & $\begin{array}{l}720.6 \\
6.958\end{array}$ & $\begin{array}{r}292.1 \\
0.0001\end{array}$ & $\begin{array}{l}\text { CONST } \\
\text { LAADT } \\
\text { RAADT }\end{array}$ & $\begin{array}{r}456.57292 \\
0.96474 \\
3.25878\end{array}$ & $\begin{array}{r}0.805 \\
16.026 \\
14.685\end{array}$ & $\begin{array}{l}0.4301 \\
0.0001 \\
0.0001\end{array}$ & 24 & $\begin{array}{r}0.7506 \\
(<0.01)\end{array}$ \\
\hline & Sity Gro & "D" [Ci & ty Popt & ion: & 5,000 to 10 & 0] & & & \\
\hline North & $\begin{array}{l}99.55 \\
99.53\end{array}$ & $\begin{array}{l}246.7 \\
2.727\end{array}$ & $\begin{array}{l}6201.7 \\
0.0001\end{array}$ & $\mid \begin{array}{l}\text { CONST } \\
\text { LAADT } \\
\text { RAADT }\end{array}$ & $\begin{array}{r}78.21203 \\
0.99380 \\
3.43147\end{array}$ & $\left\{\begin{array}{r}0.899 \\
111.342 \\
37.050\end{array}\right.$ & $\begin{array}{l}0.3726 \\
0.0001 \\
0.0001\end{array}$ & 59 & $\begin{array}{r}0.0942 \\
(>0.15)\end{array}$ \\
\hline Central & $\begin{array}{l}99.42 \\
99.40\end{array}$ & $\begin{array}{l}227.3 \\
2.602\end{array}$ & $\begin{array}{l}4964.9 \\
0.0001\end{array}$ & $\begin{array}{l}\text { CONST } \\
\text { LAADT } \\
\text { RAADT }\end{array}$ & $\begin{array}{r}173.05329 \\
0.98695 \\
3.46190\end{array}$ & $\begin{array}{r}1.870 \\
94.978 \\
37.466\end{array}$ & $\begin{array}{l}0.0665 \\
0.0001 \\
0.00 \cap 1\end{array}$ & 61 & $\begin{array}{r}0.1076 \\
(0.079)\end{array}$ \\
\hline South & $\begin{array}{l}98.75 \\
98.73\end{array}$ & $\begin{array}{l}393.9 \\
3.835\end{array}$ & $\begin{array}{l}4385.0 \\
0.0001\end{array}$ & $\mid \begin{array}{l}\text { CONST } \\
\text { LAADT } \\
\text { RAADT }\end{array}$ & $\begin{array}{r}227.37756 \\
0.99171 \\
3.17555\end{array}$ & $\begin{array}{r}1.943 \\
84.986 \\
39.837\end{array}$ & $\begin{array}{l}0.0546 \\
0.0001 \\
0.0001\end{array}$ & 114 & $\begin{array}{r}0.2295 \\
(<0.01)\end{array}$ \\
\hline
\end{tabular}

(*) Wi1k-Shapiro ' $\mathrm{W}^{-}$for $\mathrm{N}<51$ or Kolmogorov ' $\mathrm{D}^{-}$for $\mathrm{N} \geqslant 51$, and $\mathrm{P}$-value $=\mathrm{PROB}>\mathrm{D}$ or $\mathrm{PROB} \angle \mathrm{W}$. 
Table E1.3: Partia1-Lagged Regression Results of Urban Minor Arterial \& Collector

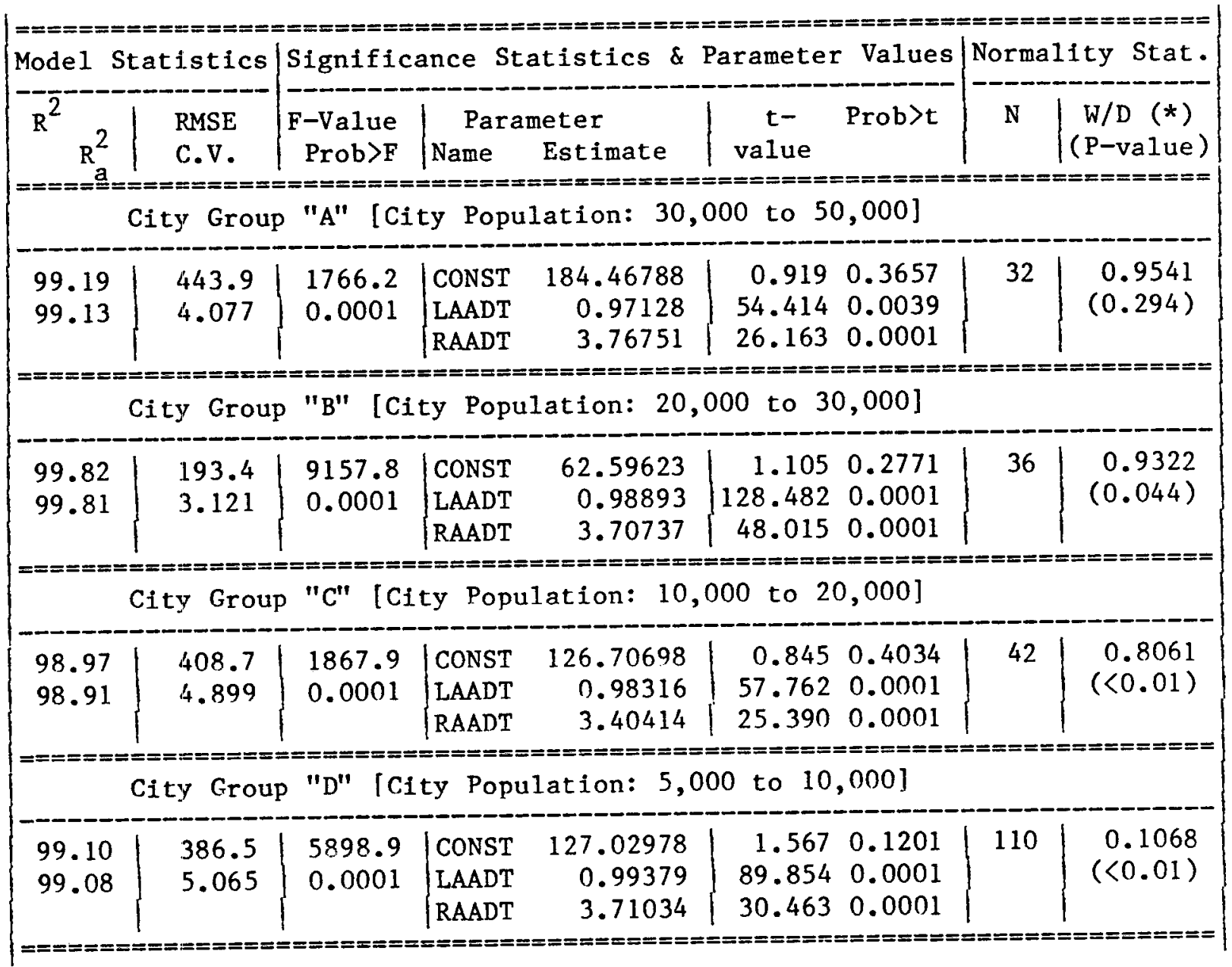

(*) Wilk-Shapiro ' $W$ ' for $N<51$ or Kolmogorov ${ }^{\prime} D^{-}$for $N \geqslant 51$, and $\mathrm{P}$-value $=\mathrm{PROB}>\mathrm{D}$ or $\mathrm{PROB}\langle\mathrm{W}$. 
Table E2.1: Forecast Measures from Partia1-Lagged Model of Interstate Highways

\begin{tabular}{|c|c|c|c|c|c|c|c|}
\hline \multirow{2}{*}{\multicolumn{2}{|c|}{$\begin{array}{l}============== \\
\text { Region }\left|\begin{array}{l}\text { No. of } \\
\text { Fore- } \\
\text { casts }\end{array}\right|\end{array}$}} & \multirow[b]{2}{*}{$\begin{array}{l}\text { Forecast } \\
\text { Period } \\
\text { Range }\end{array}$} & \multicolumn{5}{|c|}{ Forecast Measures } \\
\hline & & & ME & MPE & MAE & MAPE & $\mathbf{r}$ \\
\hline \multirow{2}{*}{\multicolumn{8}{|c|}{ 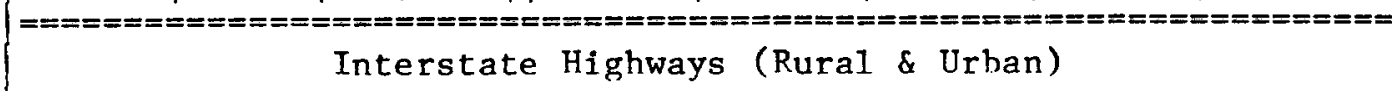 }} \\
\hline & & & & & & & \\
\hline North & 41 & $1-15$ & -2783 & -3.96 & 4673 & 12.43 & 0.99084 \\
\hline Central & 82 & $2-16$ & -3156 & -8.11 & 3509 & 8.68 & 0.99135 \\
\hline South & 39 & $1-12$ & -1414 & -5.62 & 1815 & 7.30 & 0.99326 \\
\hline \multicolumn{8}{|c|}{ 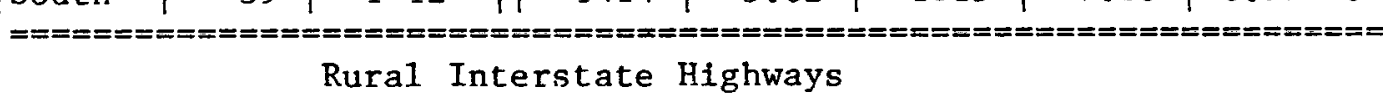 } \\
\hline ALL & 89 & $1-16$ & -2334 & -8.72 & 2469 & 9.69 & 0.98738 \\
\hline North & 18 & $3-15$ & -2649 & -10.31 & 2762 & 10.76 & 0.95204 \\
\hline Central & 44 & $2-16$ & -2774 & -9.38 & 2829 & 9.65 & 0.99134 \\
\hline South & 27 & $1-12$ & -1066 & -6.14 & 1311 & 7.70 & 0.97964 \\
\hline \multicolumn{8}{|c|}{ 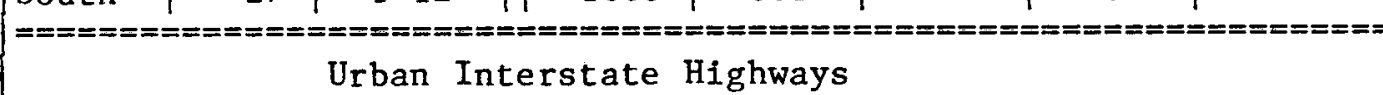 } \\
\hline ALL & 73 & $1-16$ & -3359 & -6.19 & 4255 & 7.72 & 0.99011 \\
\hline North & 23 & $1-13$ & -5424 & -9.96 & 5472 & 10.17 & 0.99461 \\
\hline Central & 38 & $2-16$ & -3915 & -7.52 & 4706 & 8.53 & 0.98473 \\
\hline & 12 & $1-7$ & -3284 & -5.90 & 3966 & 7.64 & 0.97832 \\
\hline
\end{tabular}


Table E2.2: Forecast Measures from Partial-Lagged Model of Urban Principal Arterial

\begin{tabular}{|c|c|c|c|c|c|c|c|}
\hline$==-=$ & $===x===$ & $====$ & & & cast & asures & \\
\hline Region & $\left|\begin{array}{l}\text { No. of } \\
\text { Fore- } \\
\text { casts }\end{array}\right|$ & $\begin{array}{l}\text { Forecast } \\
\text { Period } \\
\text { Range }\end{array}$ & ME & MPE & MAE & MAPE & $r$ \\
\hline$:======$ & $=======$ & $==-======$ & $\begin{aligned}= & ==== \\
& \text { City G }\end{aligned}$ & $\begin{array}{l}\text { oup "A" }===== \\
\text { oup }\end{array}$ & & & \\
\hline North & 59 & $3-15$ & -905 & -4.93 & 1301 & 8.73 & 0.97317 \\
\hline Central & 55 & $3-16$ & -905 & -5.40 & 1053 & 6.97 & 0.98501 \\
\hline South & 13 & $7-16$ & -304 & 0.41 & 778 & 8.86 & 0.97048 \\
\hline$== \pm===$ & $====3$ & $========$ & $===x==0$ & $:======$ & $=====$ & $====0$ & $========$ \\
\hline & & & City G & oup "B" & & & \\
\hline North & 31 & $4-15$ & -731 & -4.38 & 1168 & 8.16 & 0.97710 \\
\hline Central & 19 & $6-14$ & -1205 & -6.98 & 1302 & 8.27 & 0.98618 \\
\hline South & 4 & $7-7$ & -361 & -1.02 & 1326 & 5.32 & 0.90361 \\
\hline$=======$ & $====5$ & $=====$ & $======-$ & $:=======$ & $=====0$ & $======$ & $=======0=$ \\
\hline & & & City G & oup "C" & & & \\
\hline North & 51 & $3-17$ & -663 & -4.93 & 1109 & 9.33 & 0.95686 \\
\hline Central & 61 & $3-14$ & -1281 & -8.01 & 1441 & 9.94 & 0.97099 \\
\hline South & 8 & $3-17$ & -114 & 0.23 & 1028 & 6.92 & 0.96878 \\
\hline$=======$ & $==$ & $=====$ & $\begin{array}{l}====== \\
\text { City G }\end{array}$ & $\begin{array}{l}====== \\
\text { oup "D" }\end{array}$ & & & \\
\hline North & 20 & $4-14$ & -1588 & -14.69 & 1588 & 14.69 & 0.95353 \\
\hline Central & 20 & $4-16$ & -469 & -4.05 & 656 & 6.70 & 0.96045 \\
\hline Sout & 38 & $4-12$ & -1375 & -10.84 & 1377 & 10.86 & 0.96637 \\
\hline
\end{tabular}


Table E2.3: Forecast Measures from Partial-Lagged Mode1 of Urban Minor Arterial \& Collector

\begin{tabular}{|c|c|c|c|c|c|c|c|}
\hline \multirow[b]{2}{*}{$\begin{array}{l}\text { City } \\
\text { Group }\end{array}$} & \multirow[b]{2}{*}{$\begin{array}{l}\text { No. of } \\
\text { Fore- } \\
\text { casts }\end{array}$} & \multirow[b]{2}{*}{$\begin{array}{l}\text { Forecast } \\
\text { Period } \\
\text { Range }\end{array}$} & \multicolumn{5}{|c|}{ Forecast Measures } \\
\hline & & & ME & MPE & MAE & MAPE & $\mathbf{r}$ \\
\hline \multicolumn{8}{|c|}{ 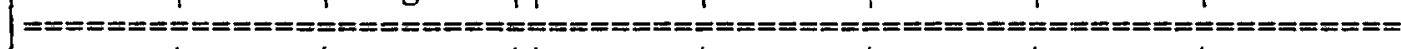 } \\
\hline A & 12 & $3-16$ & -4 & 0.37 & 1309 & 11.20 & 0.94132 \\
\hline B & 14 & $3-14$ & -558 & -4.40 & 663 & 7.95 & 0.97496 \\
\hline $\mathrm{C}$ & 18 & $3-17$ & -221 & -0.86 & 768 & 9.28 & 0.96290 \\
\hline D & 33 & $3-16$ & -426 & -4.05 & 690 & 8.69 & 0.98643 \\
\hline
\end{tabular}




\section{Slope of the plot : 0.99805}

\section{(AADT)}

A $300000+$

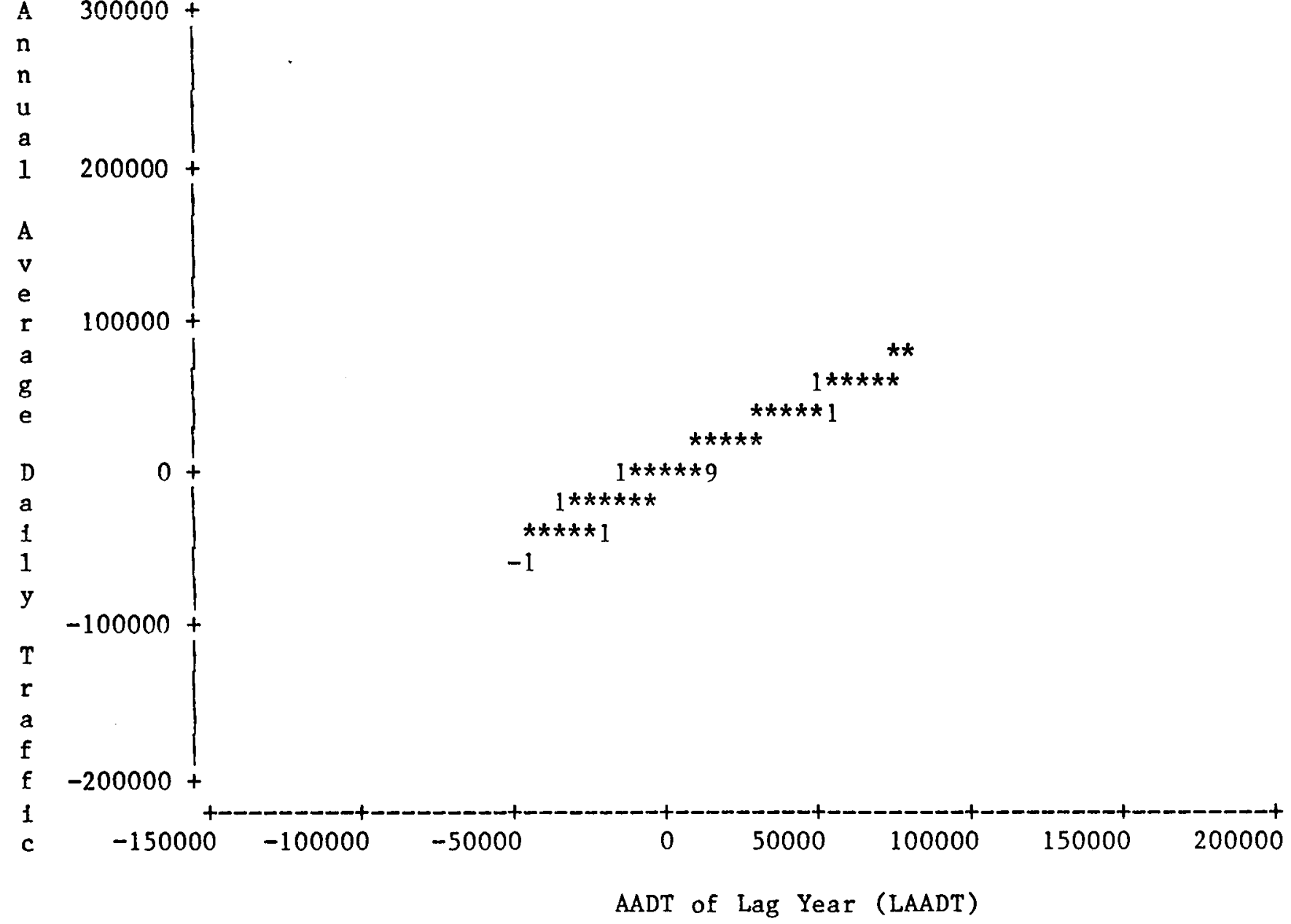

Figure E1.1: Partial. Regression Residual P1ot of LAADT for North Region of Urban Interstate Highways 
Slope of the plot: 2.46689

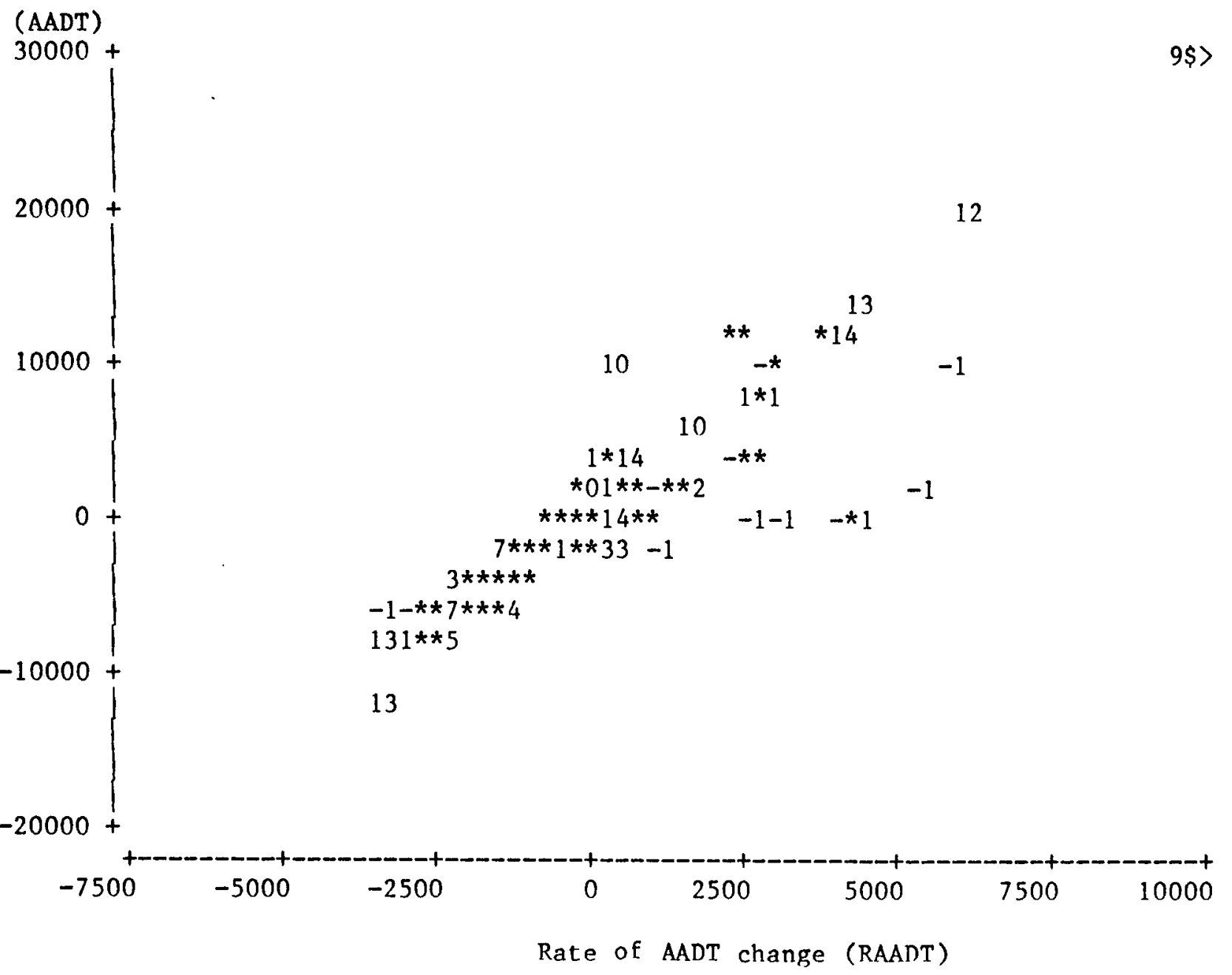

Figure E1.2: Partial Regression Residual Plot of RAADT for North Region of Urban Interstate Highways 
Slope of the plot: 0.98818

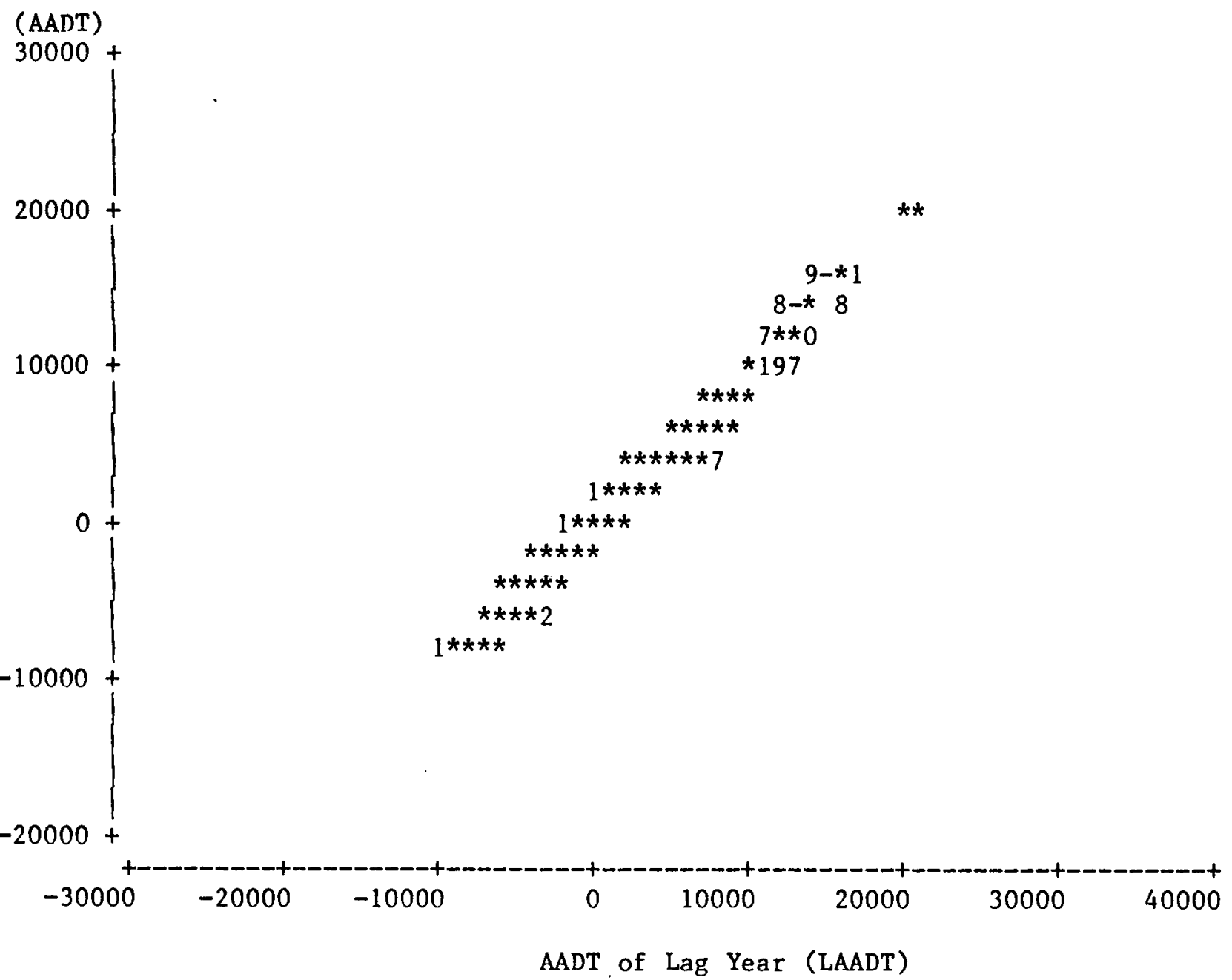

Figure E1.3: Partial Regression Residual P1ot of LAADT for City Group "A" and Region " $n$ " of Urban Principal Arterial 


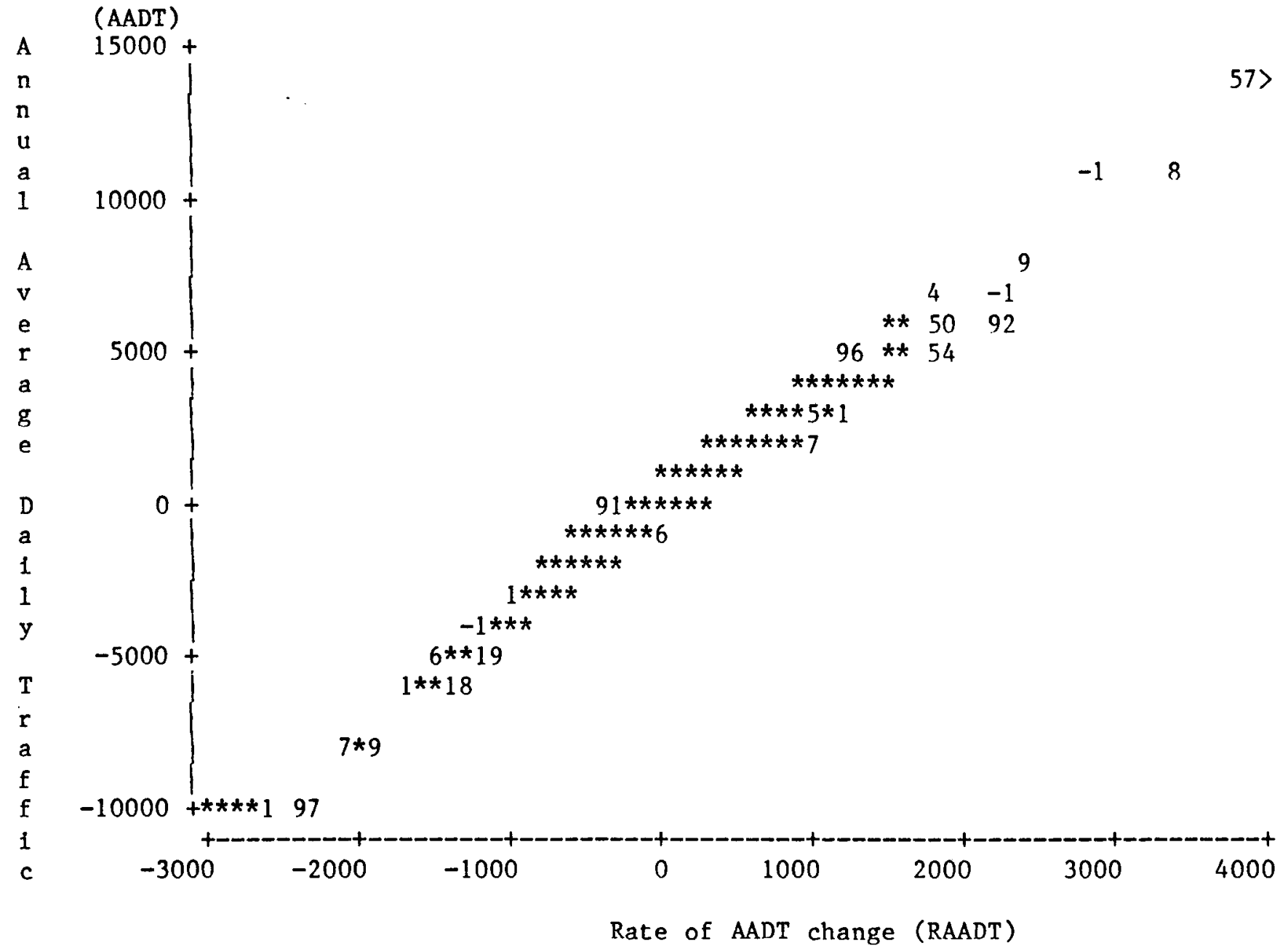




\section{Slope of the plot : 0.99339}

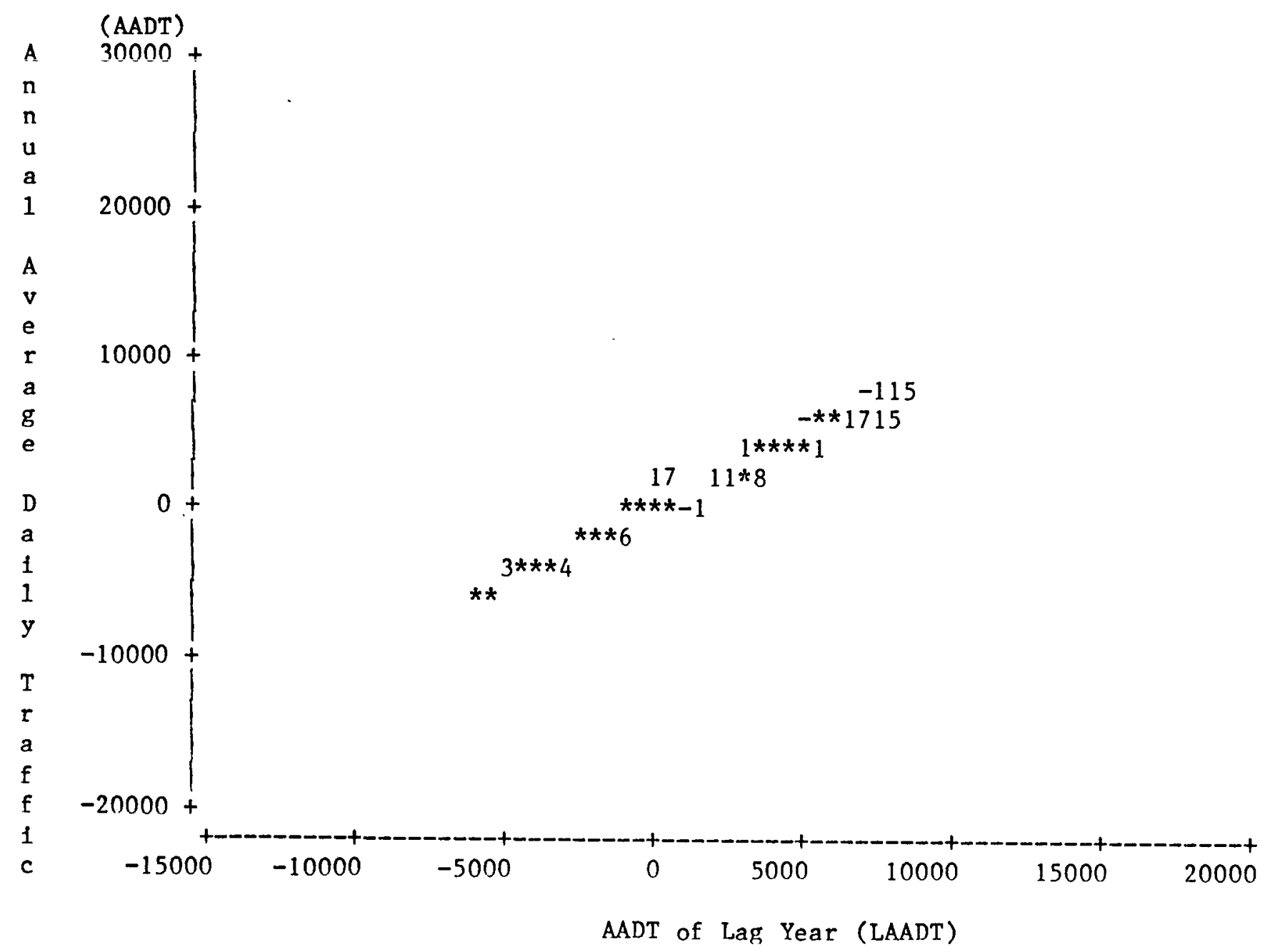

Figure E1.5: Partial Regression Residual P1ot of LAADT for City Group "C" of Urban Minor Arterial \& Collector 
Slope of the plot : 3.37950

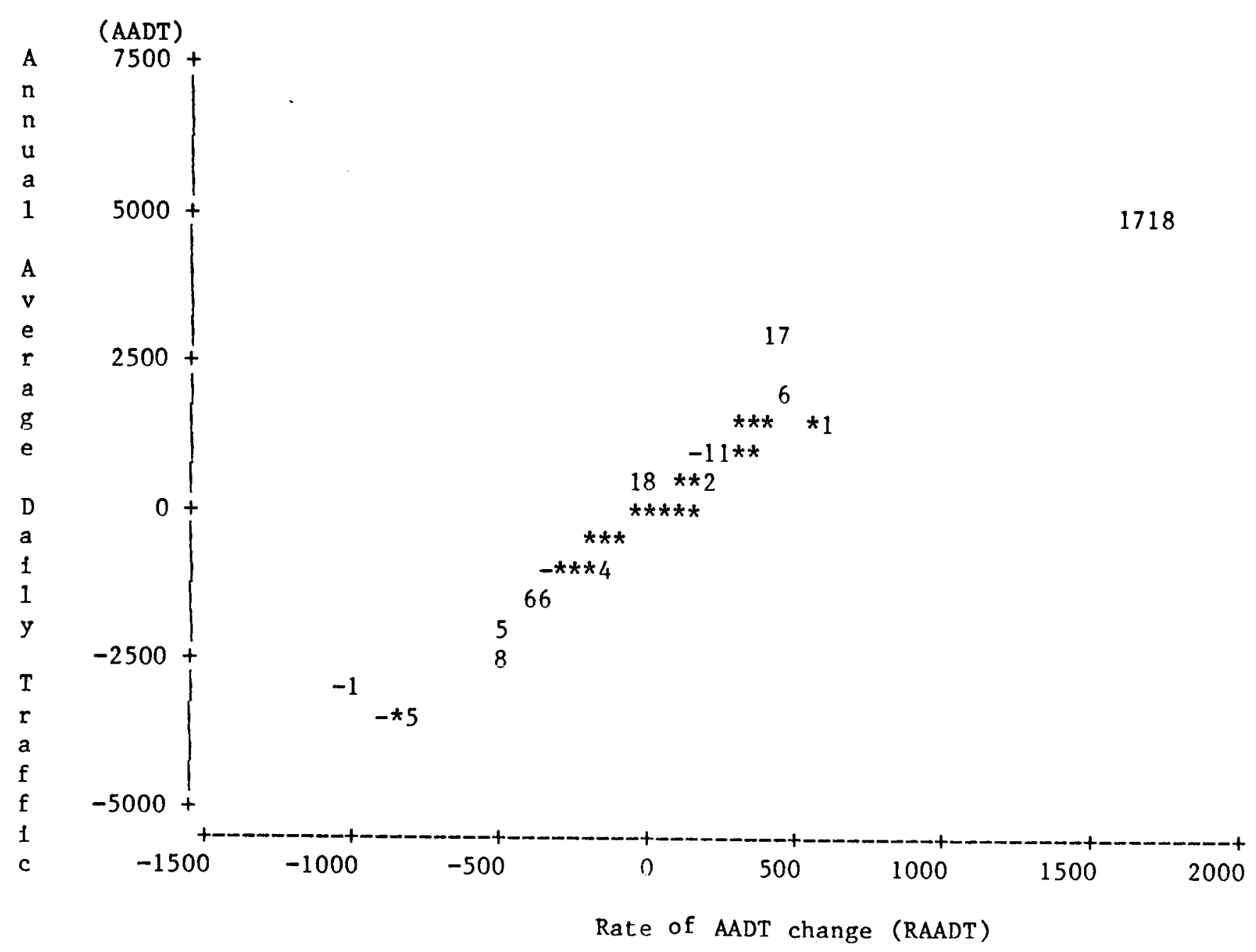

Figure E1.6: Partial Regression Residual Plot of RAADT for City Group "C" of Urban Minor Arterial \& Collector 
$\frac{\text { LEGEND: } A=1 \text { OBS, } B=2 \text { OBS, ETC. }}{\text { P-value: }<0.01}$

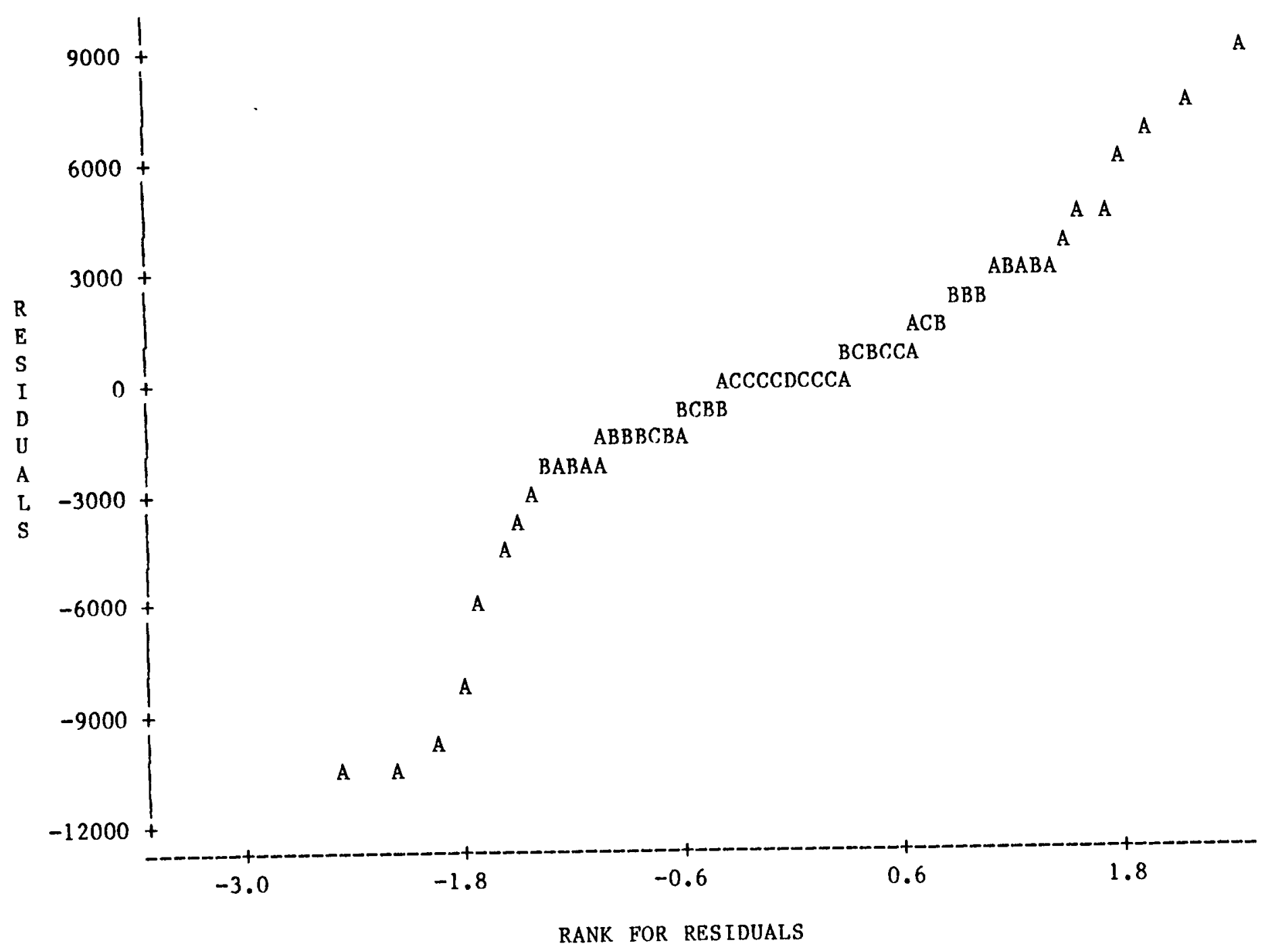

Figure E2.1: Norma1 Probability P1ot for North Region of Urban Interstate Highways 
$\frac{\text { LEGEND: } A=1 \text { OBS, } B=2 \text { OBS, ETC. }}{\text { P-value: } \angle 0.01}$

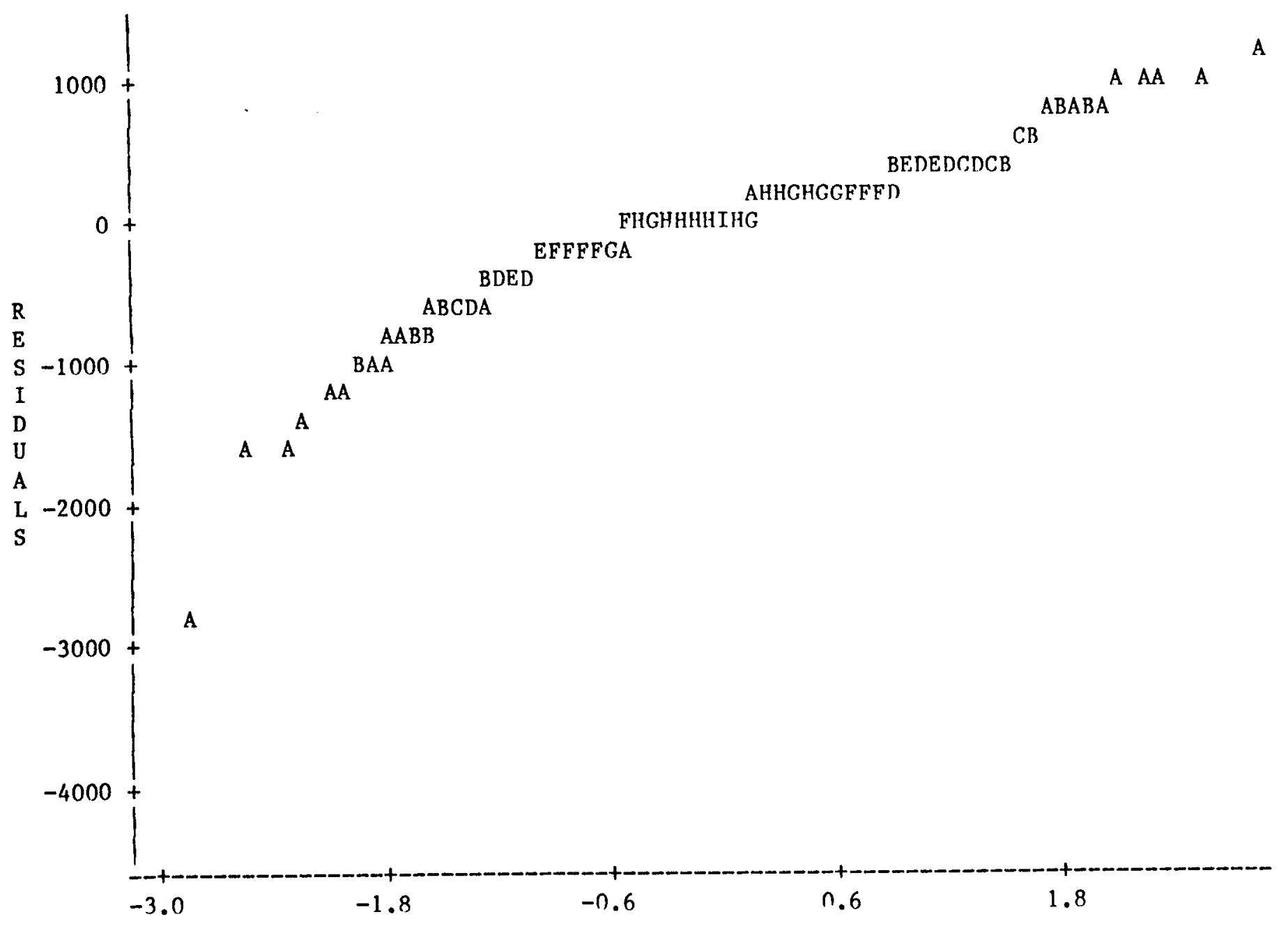

RANK FOR RESIDUALS

Figure E2.2: Normal Probability Plot for City Group "A" and Region " $n$ " of Urban Principal Arterial 
$\frac{\text { LEGEND: } A=1 \text { OBS }, B=2 \text { OBS, ETC. }}{\mathrm{P} \text {-value: }<0.01}$

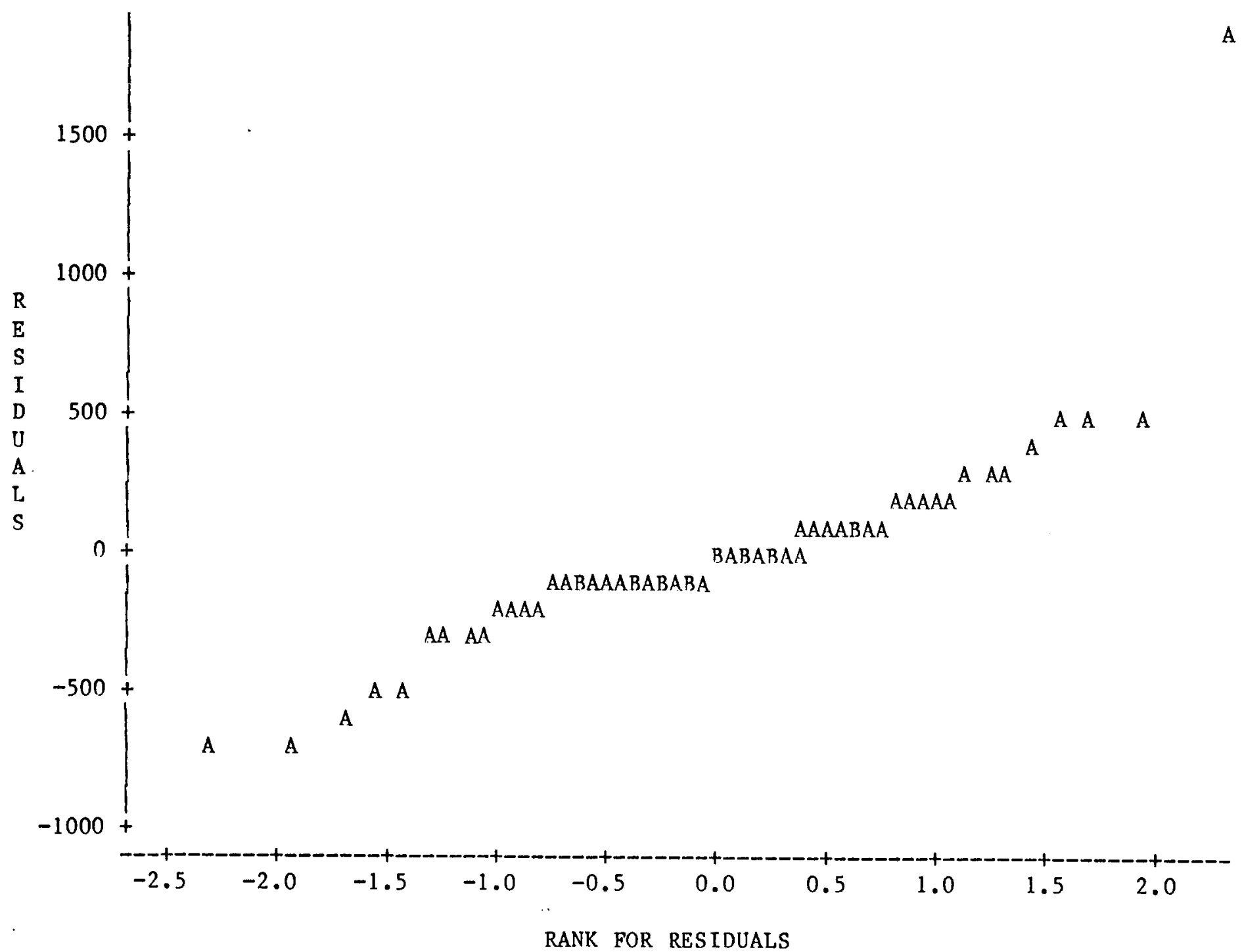

Figure E2.3: Normal Probability Plot for City Group "C" of Irhan Minnr Artortal \& Cnllortar 
$\underbrace{\text { STD DEV }: 2985}_{\text {LEGEND: } A=1 \text { OBS }, B=2 \text { OBS, ETC. }}$

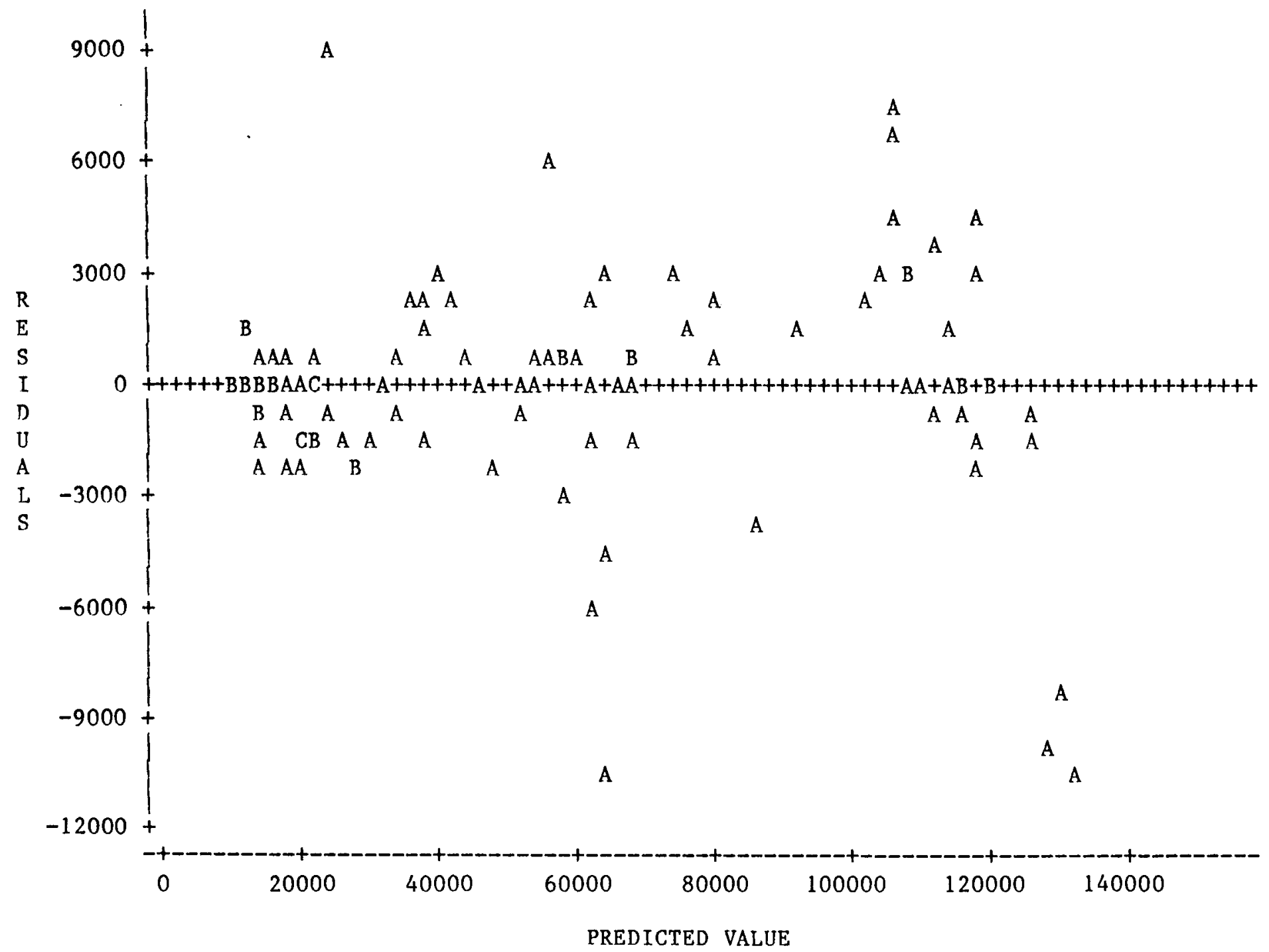

Figure E3.1: Residual Plot against Predicted value for North Region of Urban Interstate Highways 
$\frac{\text { LEGEND: } A=1 \text { OBS, } B=2 \text { OBS, ETC. }}{\text { STD DEV }: 438}$

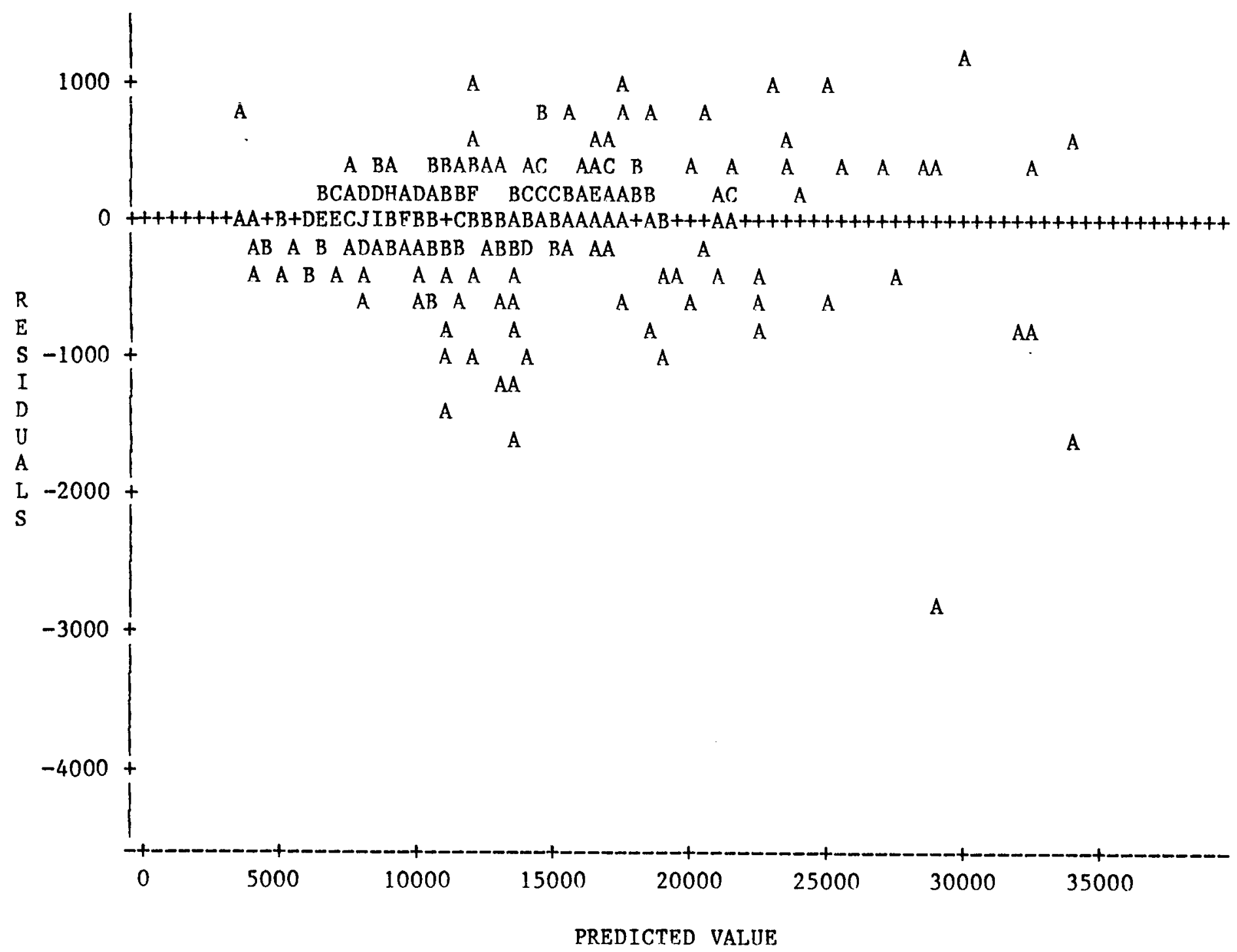


$\frac{\text { LEGEND: } A=1 \text { OBS, } B=2 \text { OBS, ETC. }}{\text { STD DEV }: 352}$

A

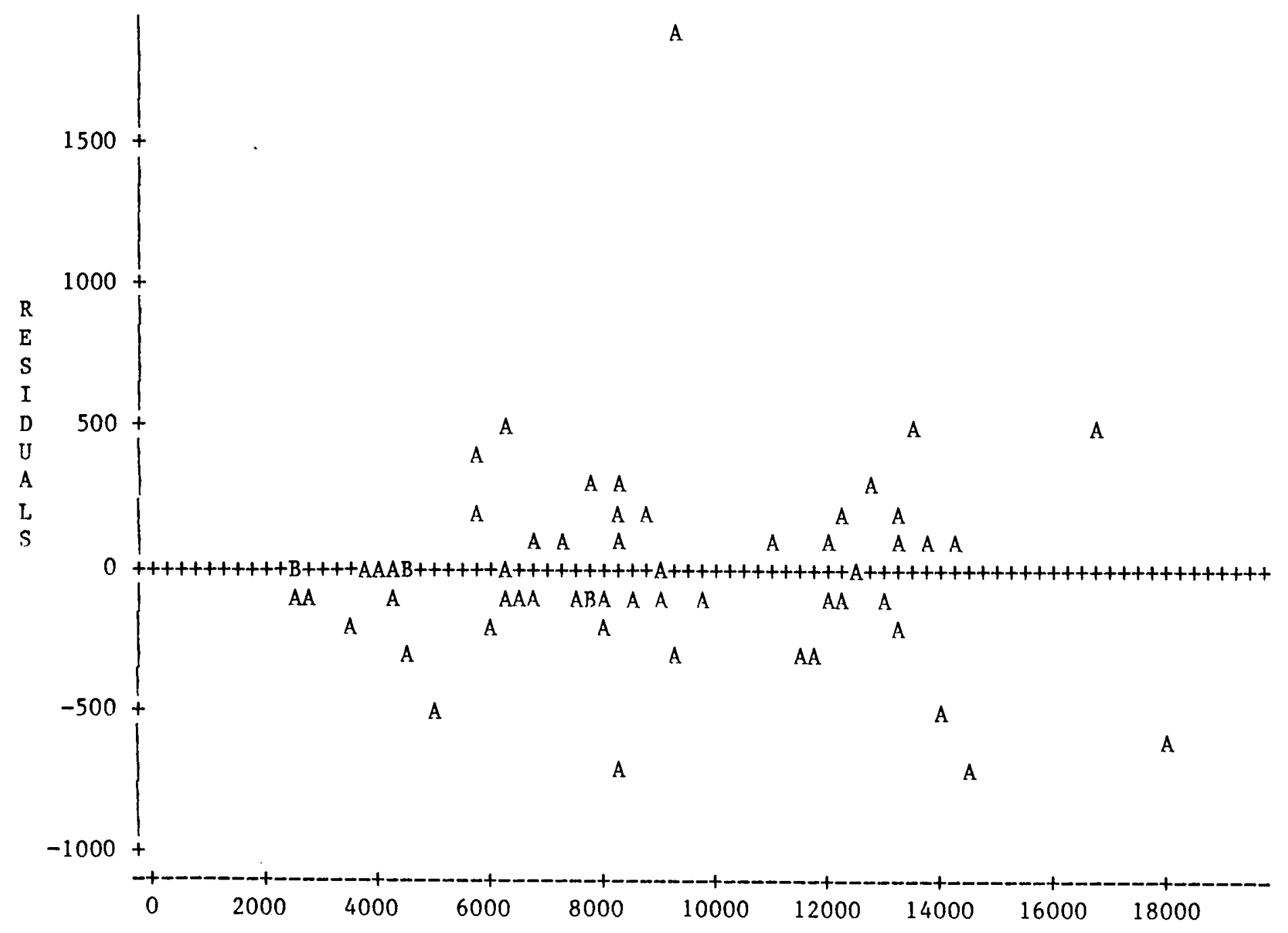

PREDICTED VALUE

Figure E3.3: Residual Plot agalnst Predicted value for City Group "C" of Urban Minor Arterial \& Collector 
$\frac{\text { LEGEND: } A=1 \text { OBS }, B=2 \text { OBS, ETC. }}{\text { STD DEV: } 2985}$

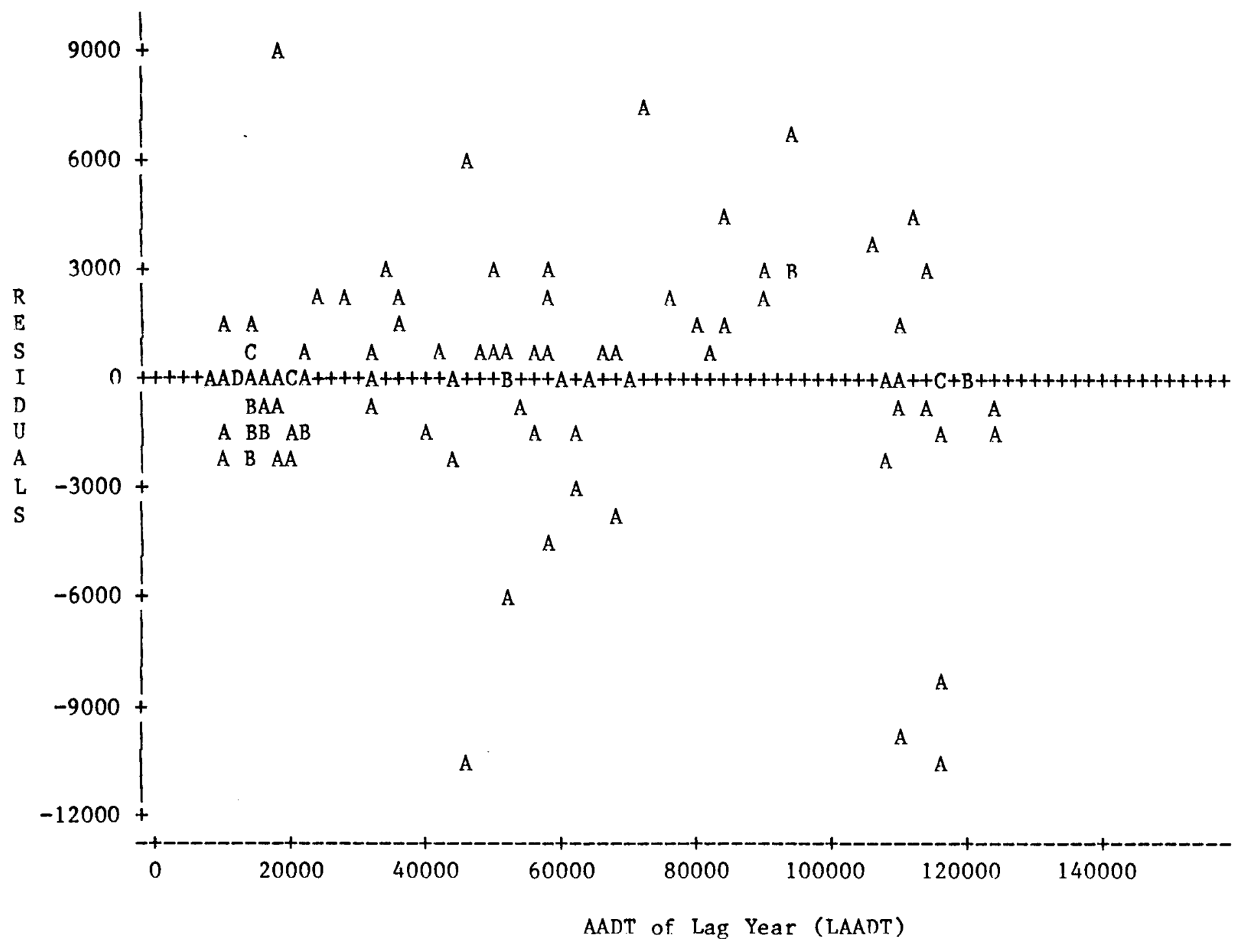

Figure E4.1: Residual Plot against LAADT for North

Region of Urban Tnterstate Highways 
$\frac{\text { LEGEND: } A=1 \text { OBS, } B=2 \text { OBS, ETC. }}{\text { STD DEV: } 2985}$

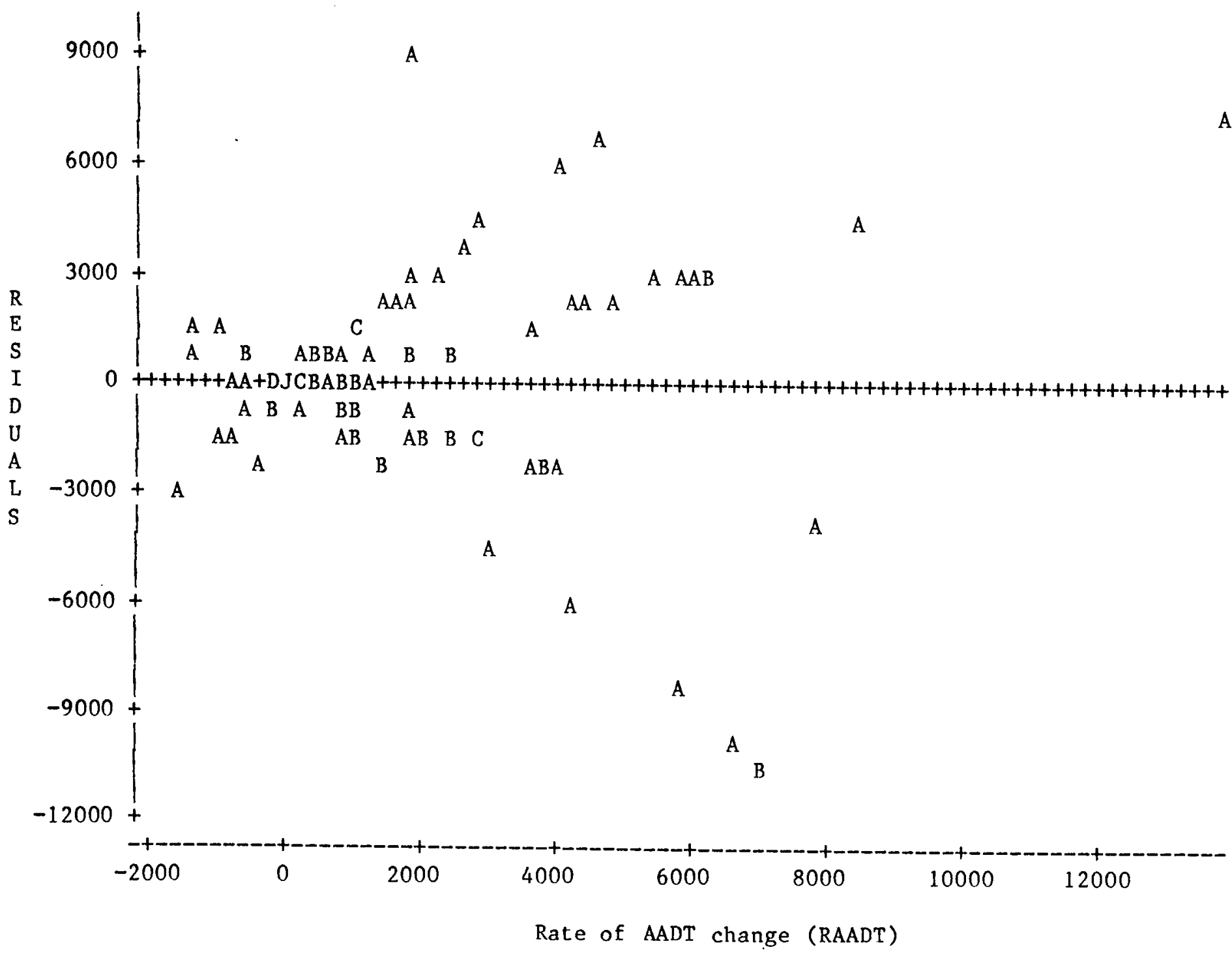

A A


$\frac{\text { LEGEND: } A=1 \text { OBS, } B=2 \text { OBS, ETC. }}{\text { STD DEV: } 438}$

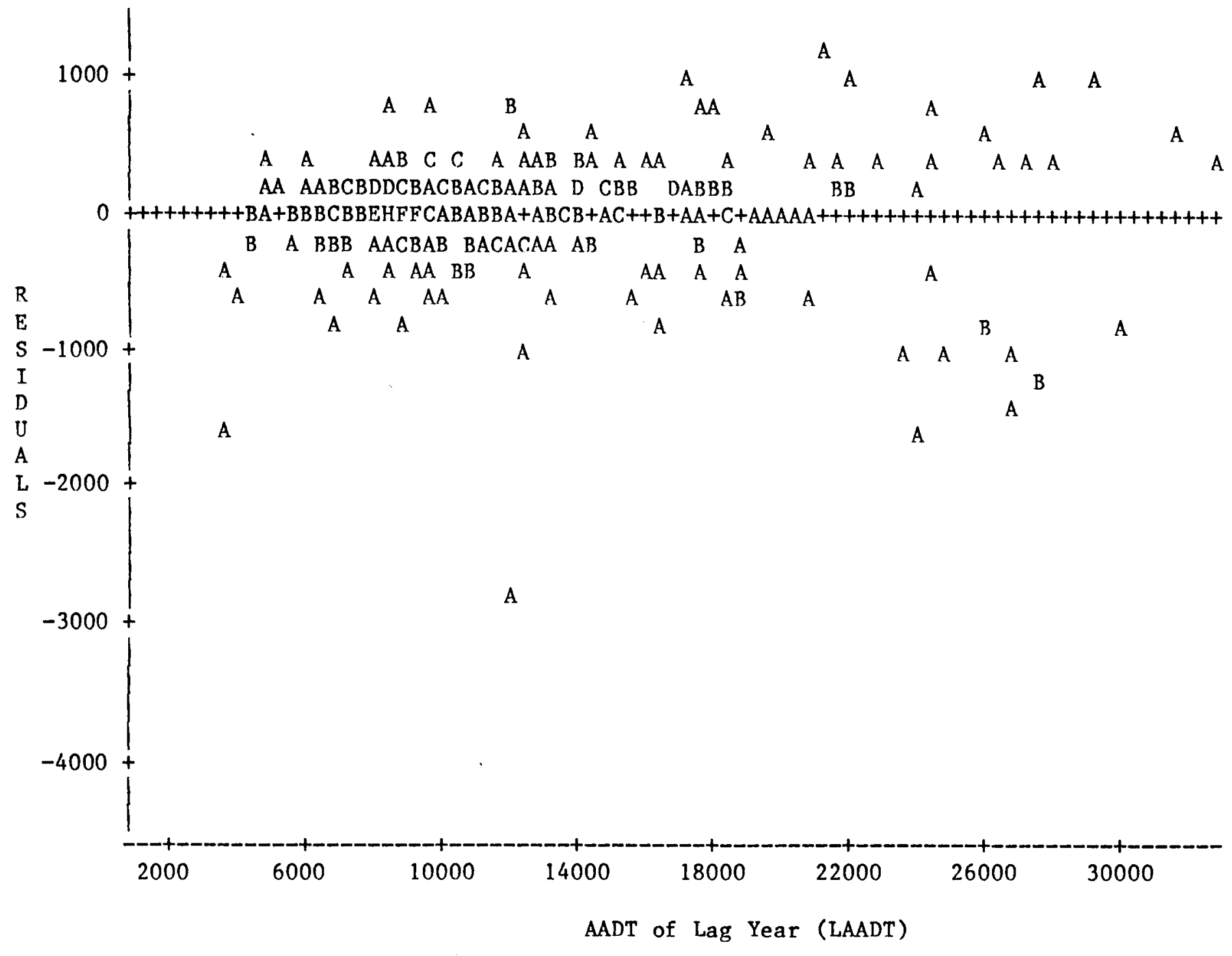

Figure E4.3: Residual Plot against LAADT for City Group "A" and Region " $n$ " of Urban Principal Arterial 
LEGEND: $\mathrm{A}=1$ OBS, $\mathrm{B}=2$ OBS, ETC.

STD DEV: 438

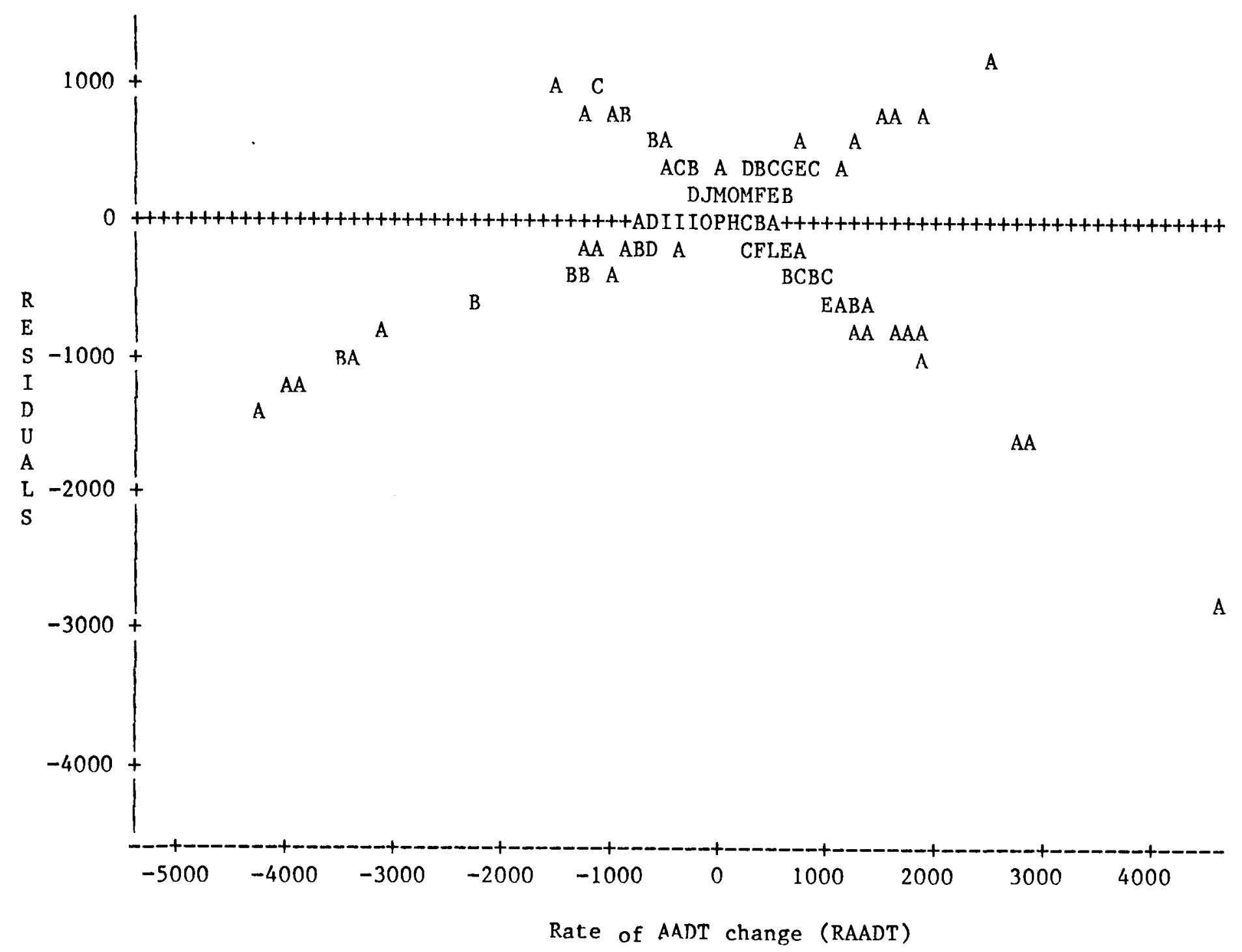

Figure E4.4: Residua1 Plot against RAADT for City Group "A" and Region " $n$ " of Urban Principal Arterial 
LEGEND: $A=1$ OBS, $B=2$ OBS, ETC. STD DEV: 352

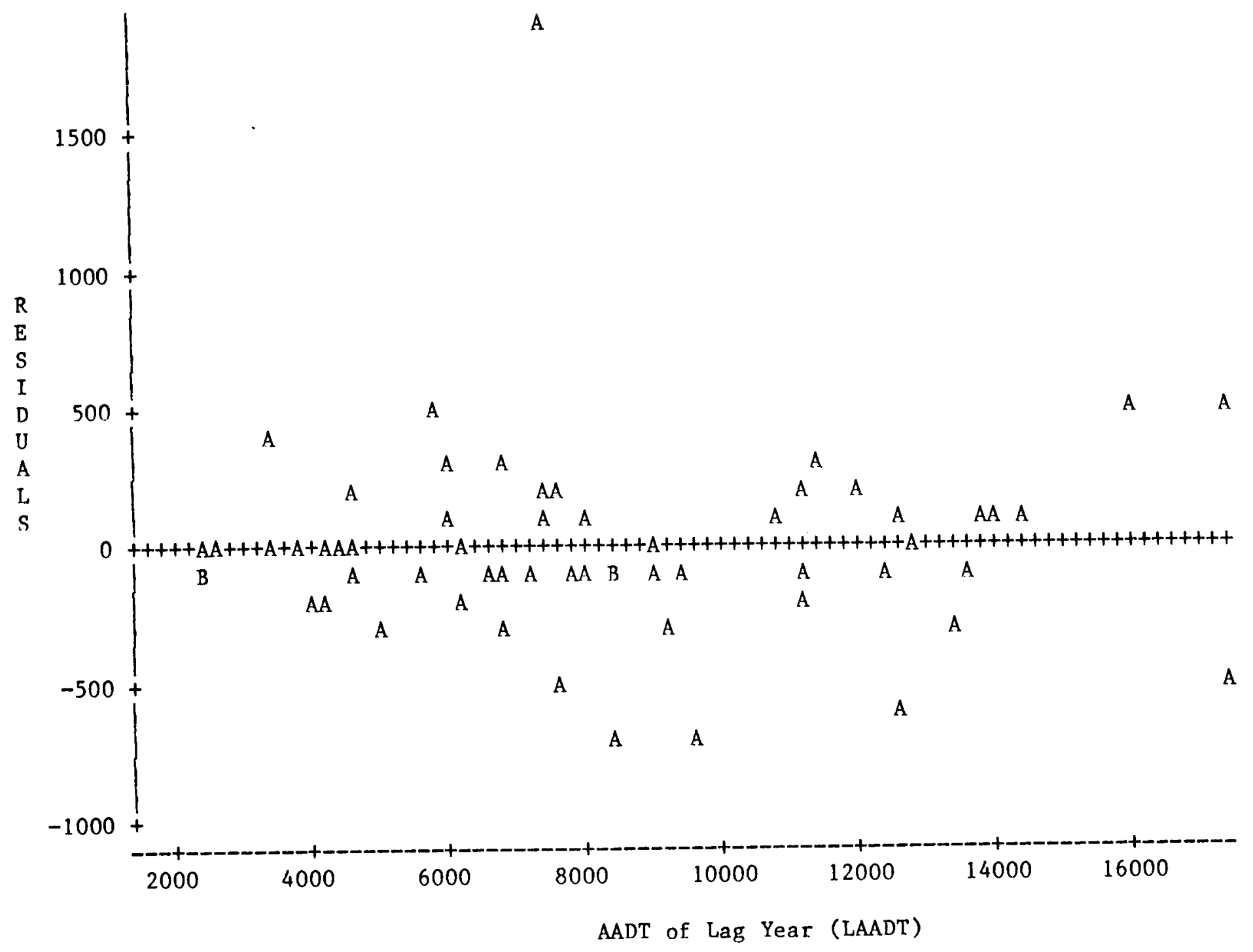

Figure E4.5: Residua1 P1ot against LAADT for City Group "C" of Urhan Minor Arterial \& Collector 

LEGEND: $A=\frac{1}{\mathrm{OBS}, \mathrm{B}=2 \text { OBS, ETC. }}$

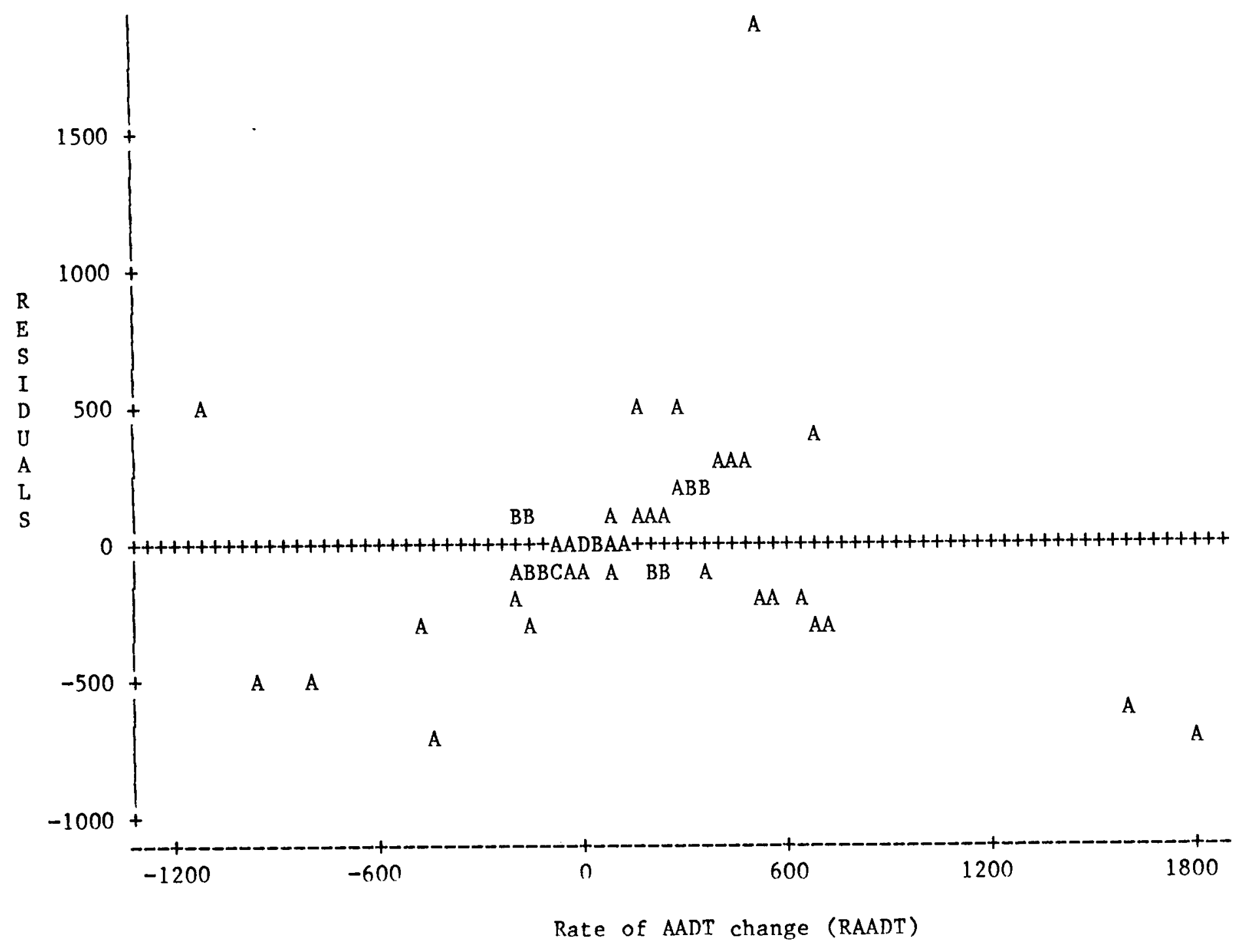

Figure E4.6: Residual Plot against RAADT for City Group "C" of Urban Minor Arterial \& Collector 
LEGEND: $\mathrm{A}=1$ OBS, $\mathrm{B}=2$ OBS, ETC.

Pearson Correlation Coefficient $(r)=0.99461$

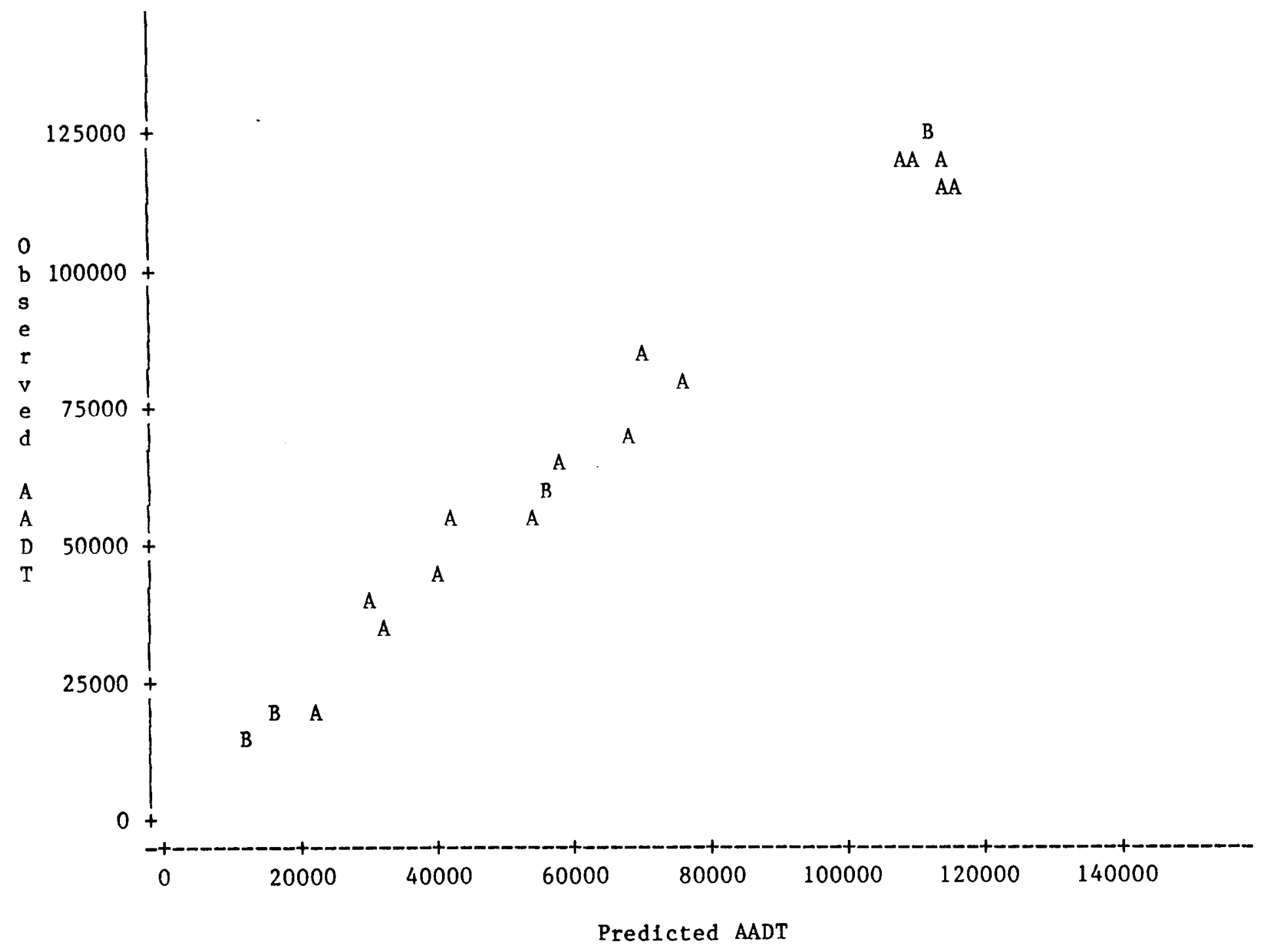

Figure E5.1: Plot of Observed against Predicted AADT from Lagged Regression for North Regional Model of Urban Interstate Highways 
LEGEND: $A=1$ OBS, $B=2$ OBS, ETC.

Pearson Correlation Coefficient $(r)=0.97317$

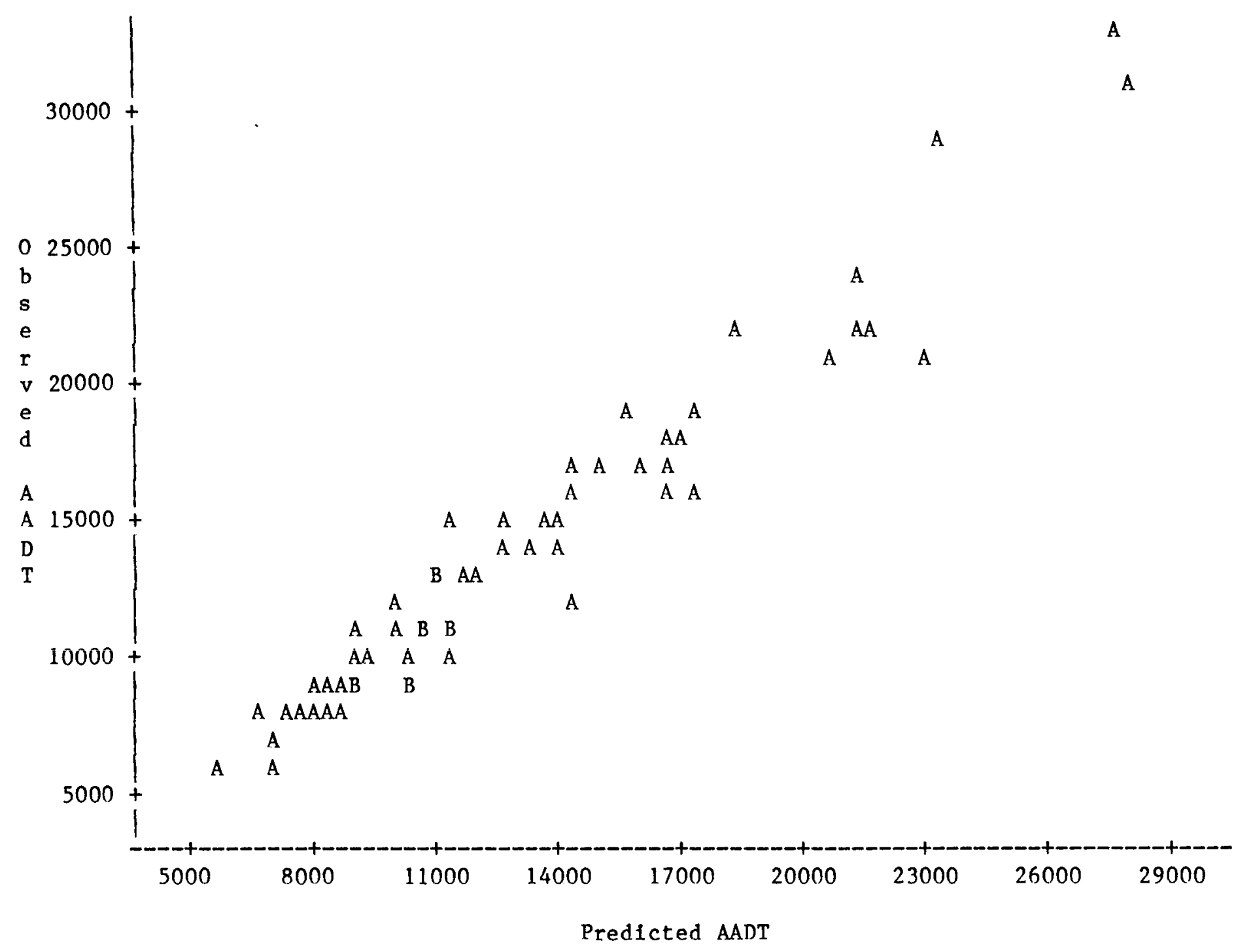

Figure E5.2: P1ot of Observed against Predicted AADT from Lagged Regression for City Group "A" and Region " $n$ " of Urban Principal Arterial 
LEGEND: $\mathrm{A}=1$ OBS, $\mathrm{B}=2$ OBS, ETC.

Pearson Correlation Coefficient $(r)=0.96290$

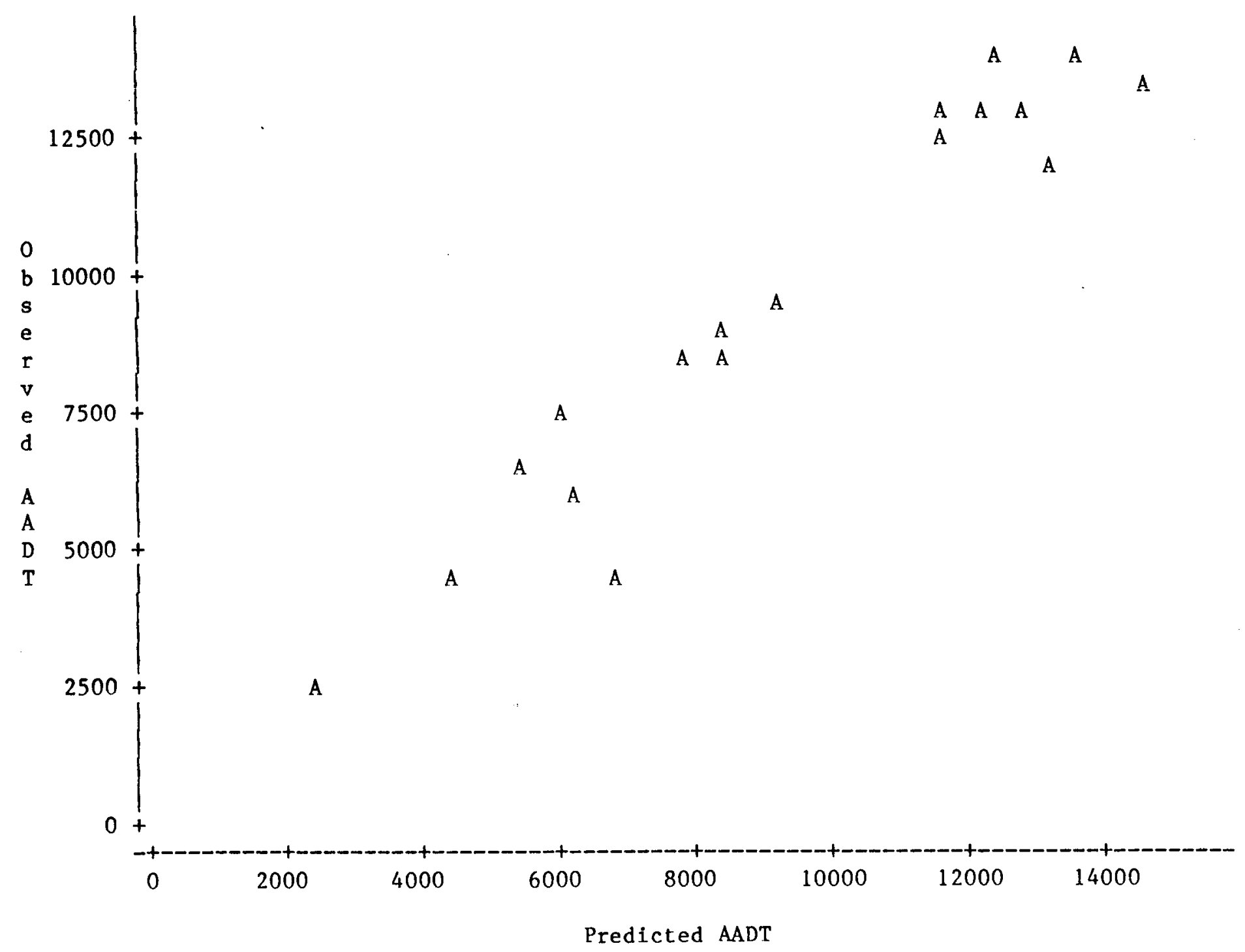

Figure E5.3: Plot of Observed against Predicted AADT from Lagged Regression for City Group "C" of Urban Minor Arterial \& Collector 
LEGEND: $A=1$ OBS, $B=2$ OBS, ETC.

Pearson Correlation Coefficient $(r)=0.66700$

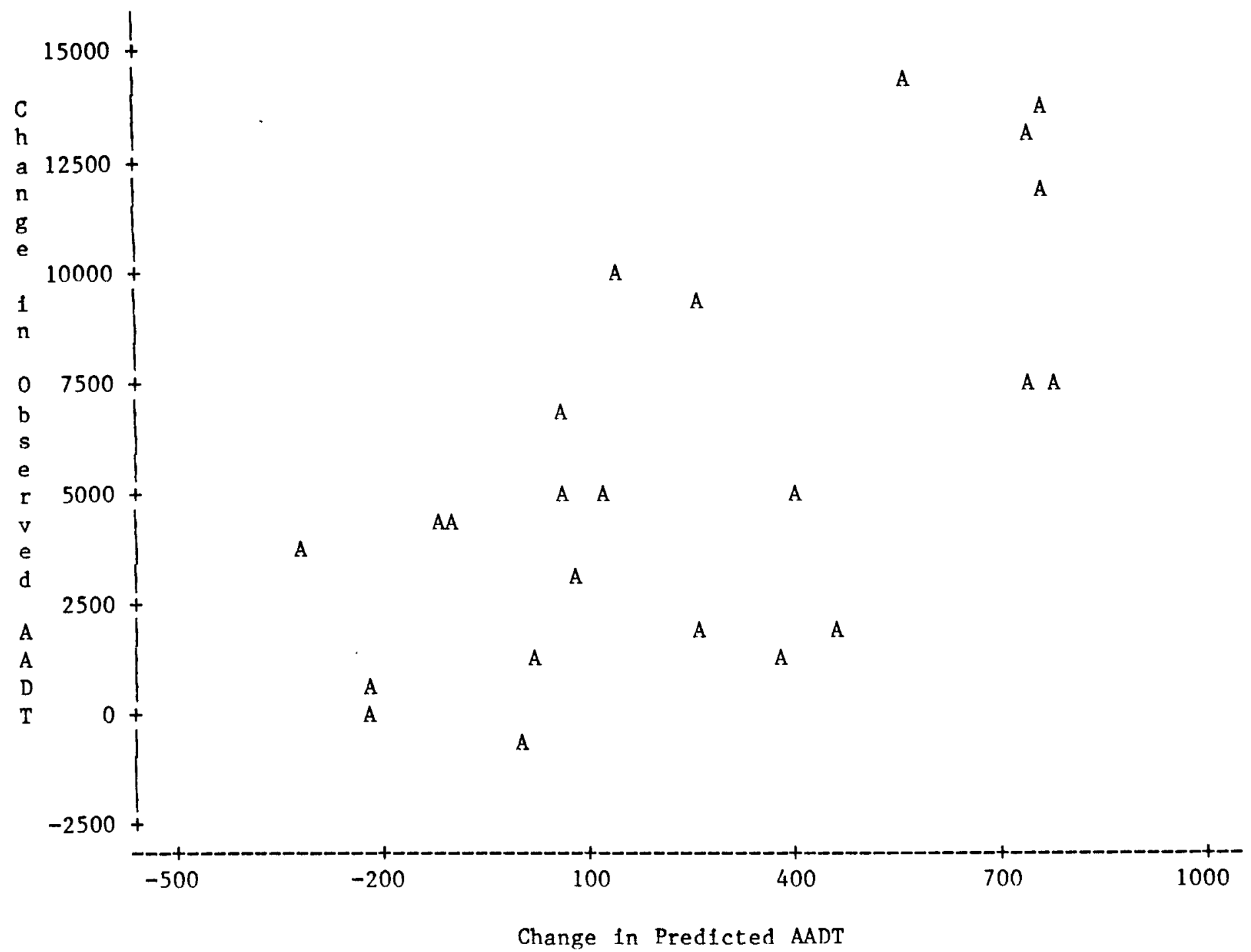

Figure E6.1: Scatterplot of Change in Observed vs. Change in Predicted AADT

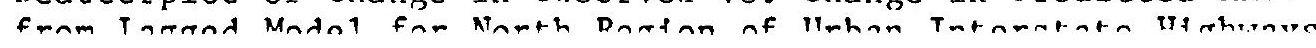


LEGEND: $\mathrm{A}=1 \mathrm{OBS}, \mathrm{B}=2 \mathrm{OBS}$, ETC.

Pearson Correlation Coefficient $(\dot{r})=0.17264$

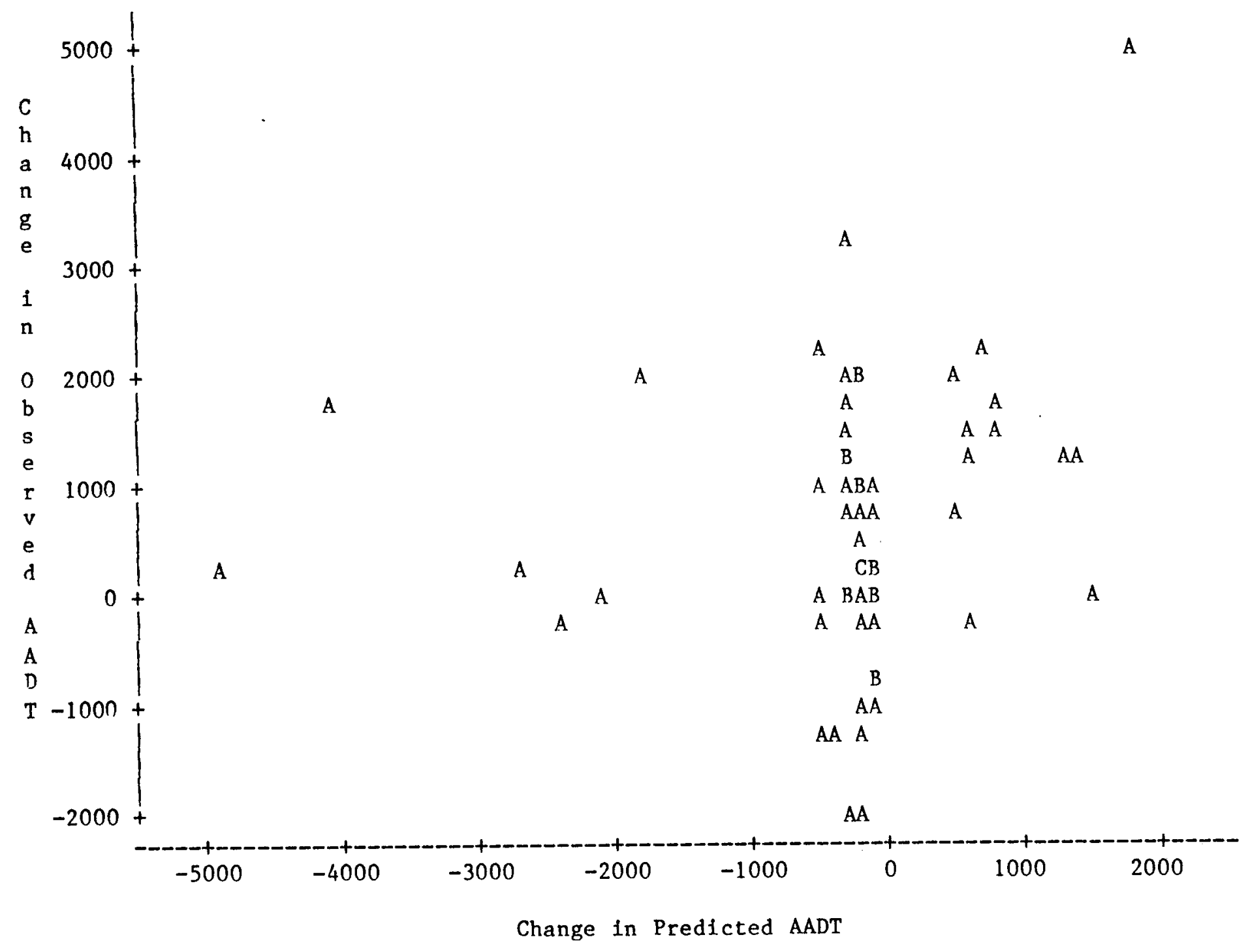

Figure E6.2: Scatterplot of Change in Observed vs. Change in Predicted AADT from 


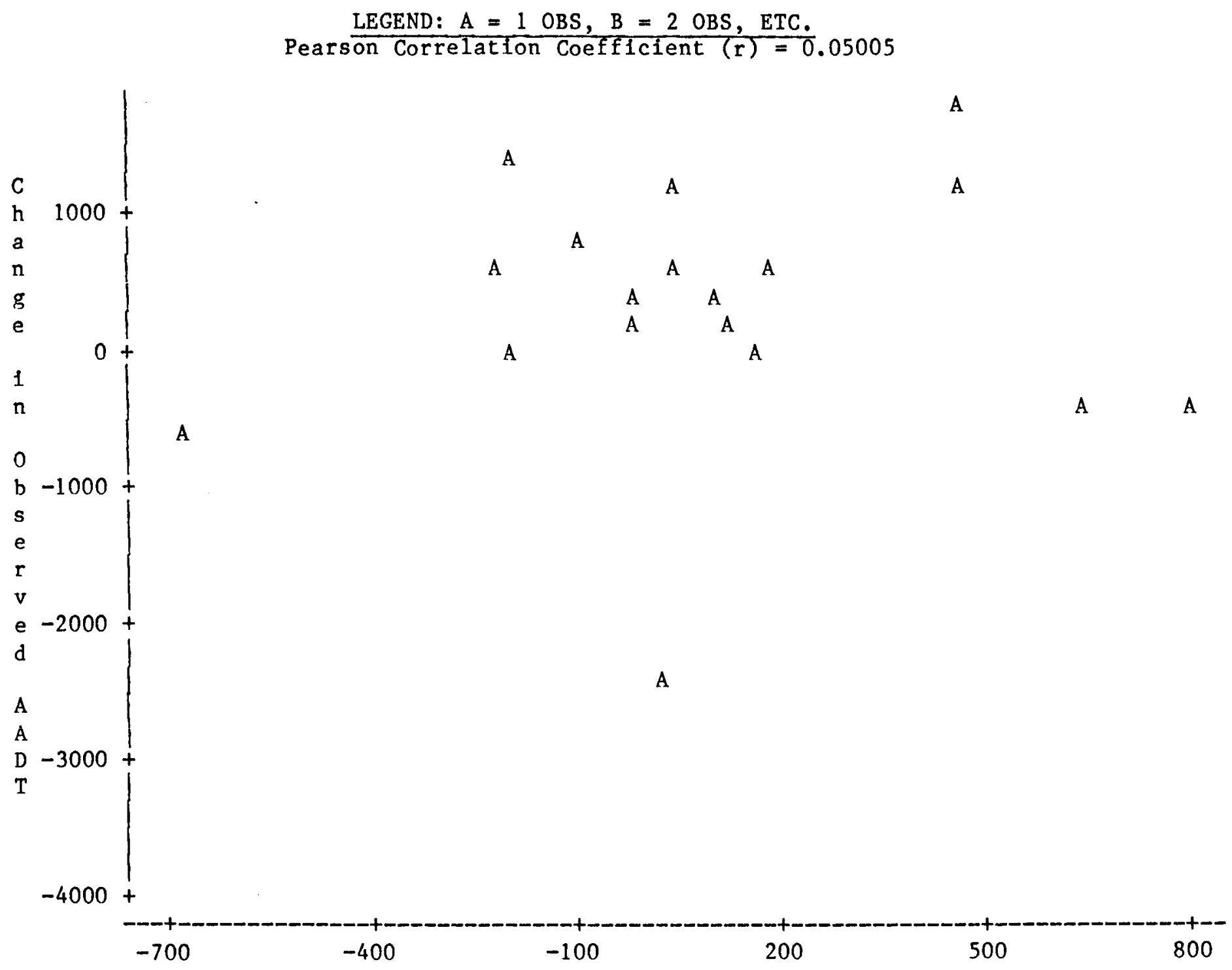

Change in Predicted AADT

Figure E6.3: Scatterplot of Change in Observed vs. Change in Predicted AADT from Tagoed Monel for Citu Groun "C" of Nrhan Minor Artertal \& Collector 
Appendix $F$ 
Table F1: ARIMA Model for Vehicle Registrations

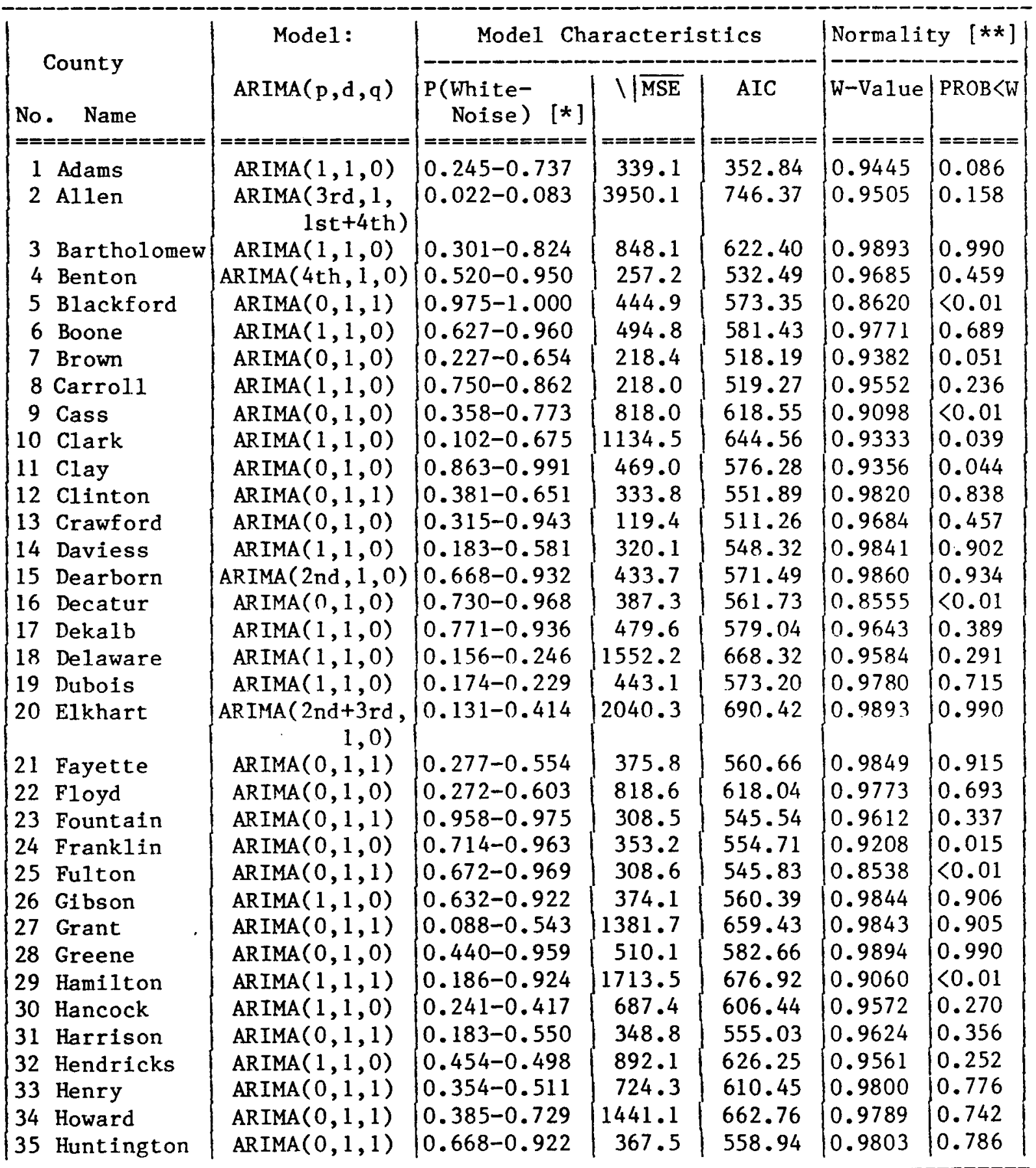


Table Fl, continued

\begin{tabular}{|c|c|c|c|c|c|c|}
\hline \multirow{4}{*}{$\mid$\begin{tabular}{c|} 
County \\
No. $\quad$ Name \\
$=======-==x x==x$
\end{tabular}} & \multirow{4}{*}{$\begin{array}{c}\text { Model: } \\
\operatorname{ARIMA}(p, d, q) \\
=\approx= \pm===== \pm====\end{array}$} & \multicolumn{3}{|c|}{ Model Characteristics } & \multicolumn{2}{|c|}{ Normality $[* *]$} \\
\hline & & & & & & \\
\hline & & $\left\{\begin{array}{l}P(\text { White } \\
\text { Noise })[*]\end{array}\right.$ & $\backslash / \overline{M S E}$ & AIC & alue & $P R O B<W$ \\
\hline & & $==x== \pm x==0=0$ & $== \pm===$ & $=====x=$ & $====0=$ & $======$ \\
\hline 36 Jackson & $\operatorname{ARIMA}(0,1,0)$ & $0.217-0.415$ & 484.2 & 578.70 & 0.9792 & 0.753 \\
\hline 37 Jasper & $A(1,1,0)$ & $0.923-0.986$ & 563.0 & 591.48 & 0.9467 & 0.098 \\
\hline 38 Jay & $\operatorname{ARIMA}(0,1,0)$ & $0.804-0.989$ & 624.7 & 598.06 & 0.8447 & $<0.01$ \\
\hline 39 Jefferson & $\operatorname{ARIMA}(0,1,0)$ & $0.597-0.995$ & 547.0 & 587.96 & 0.9211 & 0.016 \\
\hline 40 Jennings & $\operatorname{ARIMA}(0,1,1)$ & $0.549-0.794$ & 250.9 & 529.97 & 532 & 0.203 \\
\hline 41 Johns on & $A(1,1,0)$ & -0.550 & 947.0 & 630.86 & 27 & 0.553 \\
\hline 42 Knox & $(1,1,0)$ & & 474.1 & & & \\
\hline 43 Kosciusko & ARI & .848 & 746.9 & +.07 & & 0.442 \\
\hline 44 Lagr & ARI & .107 & & & & 0.744 \\
\hline 45 Lake & ARI & .567 & 6399.2 & & & 0.915 \\
\hline 46 Laport & ARI & .902 & 1717.9 & & & 0.435 \\
\hline 47 Lawr & ARI & .732 & 490.4 & & & 0.483 \\
\hline 48 Madison & AR I & .748 & 1793.4 & & 8 & 0.585 \\
\hline 49 Marion & ARI & .370 & 9503.1 & 94 & 8 & 0.831 \\
\hline 50 Mars & AR I & .690 & 532.7 & & & 0.914 \\
\hline 51 Mar & ARIMA & $268-0.805$ & 202.6 & .48 & & 0.036 \\
\hline 52 Miami & ARIMA ( 2nc & $0.091-0.246$ & 678.6 & 605.52 & 0.9 & 0.279 \\
\hline 53 Monr & & $0.898-0.993$ & 1312.2 & 654.46 & 0. & 0.954 \\
\hline 54 Montg & AR IMA $(1$, & $0.540-0.743$ & 418.1 & .61 & 0 . & 10. \\
\hline 55 Morg & $A(0,1,0)$ & $0.599-0.937$ & 791.6 & .05 & 0.9 & 0.850 \\
\hline 56 New & AR & $0.111-0.222$ & 228.8 & .35 & 0. & 0.317 \\
\hline 57 Noble & $A(0,1,0)$ & $0.681-0.912$ & 660.2 & .26 & 0 & 0.839 \\
\hline 58 Ohio & $\operatorname{ARIMA}(0,1$ & $0.227-0.758$ & 76.5 & .46 & & 0.456 \\
\hline 59 Orange & $A(1,1,0)$ & $0.444-0.882$ & 178.5 & .98 & 06 & 0.326 \\
\hline 60 Owen & ARIMAC & $0.834-0.959$ & 345.5 & .04 & 0.9712 & 0.506 \\
\hline 61 Parke & $(2 \mathrm{nd}, 1,0)$ & $0.301-0.748$ & 330.0 & 550.78 & 0.8859 & $<0.01$ \\
\hline 62 Perry & $(1,0)$ & $0.212-0.604$ & 429.0 & 569.50 & & 0.099 \\
\hline 63 Pike & AR & $0.686-0.828$ & 246.8 & 527.48 & 0.9 & 0.486 \\
\hline 64 Porter & $(1,1,0)$ & $0.364-0.909$ & 1438.6 & 662.97 & 0.9783 & 0.726 \\
\hline 65 Pose & $(1,1,0)$ & 0.31 & 354.3 & 26 & & 1.000 \\
\hline $66 \mathrm{Pula}$ & & & & & & \\
\hline 67 Putnam & ARIMA 0 , & -0.946 & 383.2 & 560.92 & 0 . & 0.213 \\
\hline 68 Randolph & AR IMA $(1$, & .951 & 551.3 & & & \\
\hline 69 Ripley & $\operatorname{ARIMA}(0,1,0)$ & 0.65 & 375.7 & 559.41 & 0. & $<0.01$ \\
\hline 70 Rush & $\operatorname{ARIMA}(0,1,0)$ & $0.640-0.955$ & 412.4 & 566.50 & 0.8082 & $k<0.01$ \\
\hline
\end{tabular}


Table Fl, continued

\begin{tabular}{|c|c|c|c|c|c|c|}
\hline \multirow{3}{*}{$\begin{array}{l}\text { County } \\
\text { No. Name } \\
==== \pm== \pm======\end{array}$} & \multirow{3}{*}{$\begin{array}{c}\text { Mode1: } \\
\operatorname{ARIMA}(p, d, q) \\
==============\end{array}$} & \multicolumn{3}{|c|}{ Model Characteristics } & \multicolumn{2}{|c|}{ Normality $[* *]$} \\
\hline & & & & & & \\
\hline & & $\begin{array}{l}P(\text { White- } \\
\text { Noise) [*] } \\
==========\end{array}$ & $==== \pm==$ & AIC & $-\mathrm{V}-1$ & $B \cap R<$ \\
\hline 71 St. Joseph & $\operatorname{ARIMA}(0,1,0)$ & $0.053-0.278$ & 2777.7 & 711.46 & 0.9811 & 0.811 \\
\hline $72 S \cot t$ & $0,1,1)$ & $0.664-$ & 271.5 & & & 628 \\
\hline 73 Shelby & $1,1,0)$ & $0.505-$ & 494.2 & 581 & & 786 \\
\hline 74 Spencer & $A(0,1,0)$ & 0.69 & 367.1 & 55 & & 046 \\
\hline 75 Starke & $, 1,0)$ & 0.36 & 340.8 & & & 227 \\
\hline 76 Steuben & $A R$ & 71 & & & & .01 \\
\hline 77 Sullivan & ARI & & & & & 01 \\
\hline 78 Switzerland & $1,0)$ & & & & & 618 \\
\hline 79 Tippecanoe & $A(1,1,0)$ & & & & & 295 \\
\hline 80 Tipton & ARI & & 279.6 & & & 716 \\
\hline 81 Union & $, 1,0)$ & & & & & 085 \\
\hline 82 Vanderburgh & $\begin{array}{c}\text { ARIMA }(1 \mathrm{st}+3 \mathrm{rd}+ \\
4 \mathrm{th}, 1,0)\end{array}$ & 0.34 & 1883.1 & .97 & & 0.609 \\
\hline 83 Vermil & $\operatorname{ARIMA}(0,1,0)$ & 0.32 & & & & 688 \\
\hline 84 Vigo & $\operatorname{ARIMA}(0,1,1)$ & 0.44 & 10 & & & 85 \\
\hline 85 Wabash & $\operatorname{ARIMA}(0,1,0)$ & 0.74 & & 69 & & 348 \\
\hline 86 Warren & $\operatorname{ARIMA}(0,1,0)$ & 0.70 & & .66 & & .282 \\
\hline 87 Warrick & $(, 1,0)$ & 0.34 & & & & 0.381 \\
\hline 88 Washington & & 0.70 & & & & .208 \\
\hline 89 Wayne & , 1) & 0.62 & 1066.9 & & & .564 \\
\hline 90 Wells & $, 1,1)$ & 0.33 & & & & .569 \\
\hline 91 White & AR & 0.77 & 314.7 & 15 & & 0.694 \\
\hline 92 Whitley & ARII & 0.50 & 378.9 & 561.11 & 710 & 0.500 \\
\hline & ARIMAl & $10.322-0.467$ & 59535 & 945.50 & 0.9841 & 10.902 \\
\hline
\end{tabular}

[*] Range of values with autocorrelation lags of $6,12,18$ and 24 . [**] Number of residuals $=38$. 
Table F2: Parameter of ARIMA Model for Vehicle Registrations

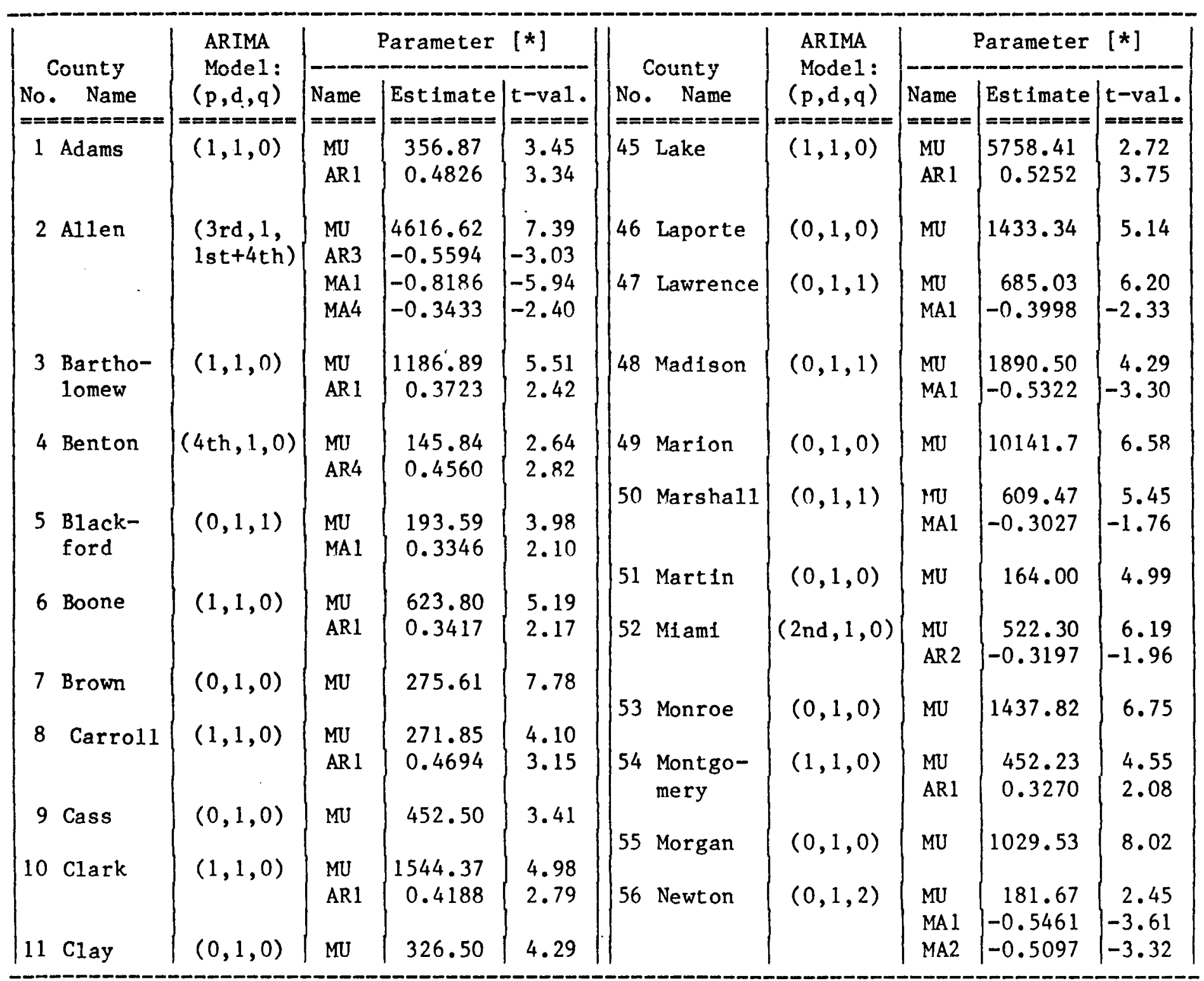


Table F2, continued

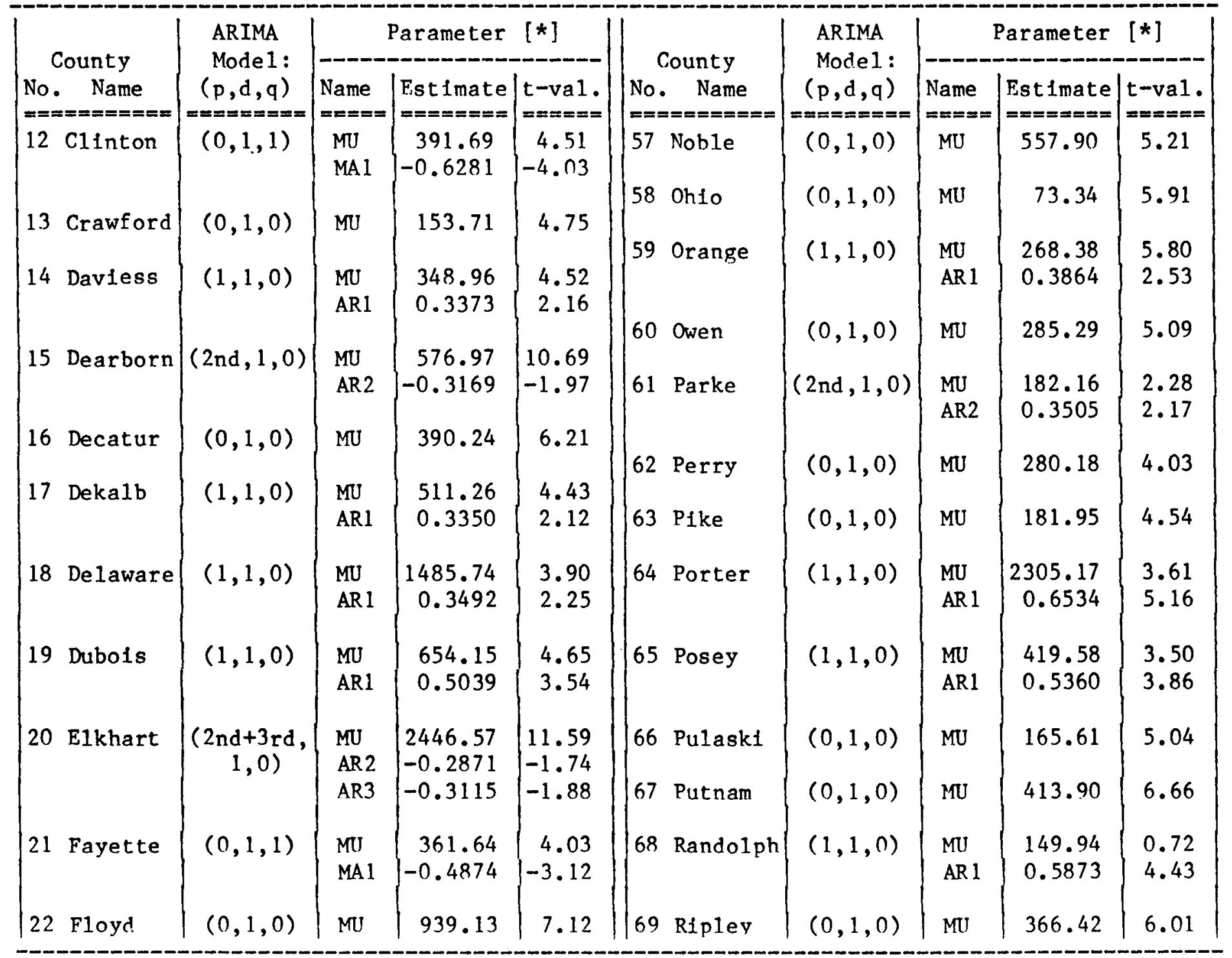


Table F2, continued

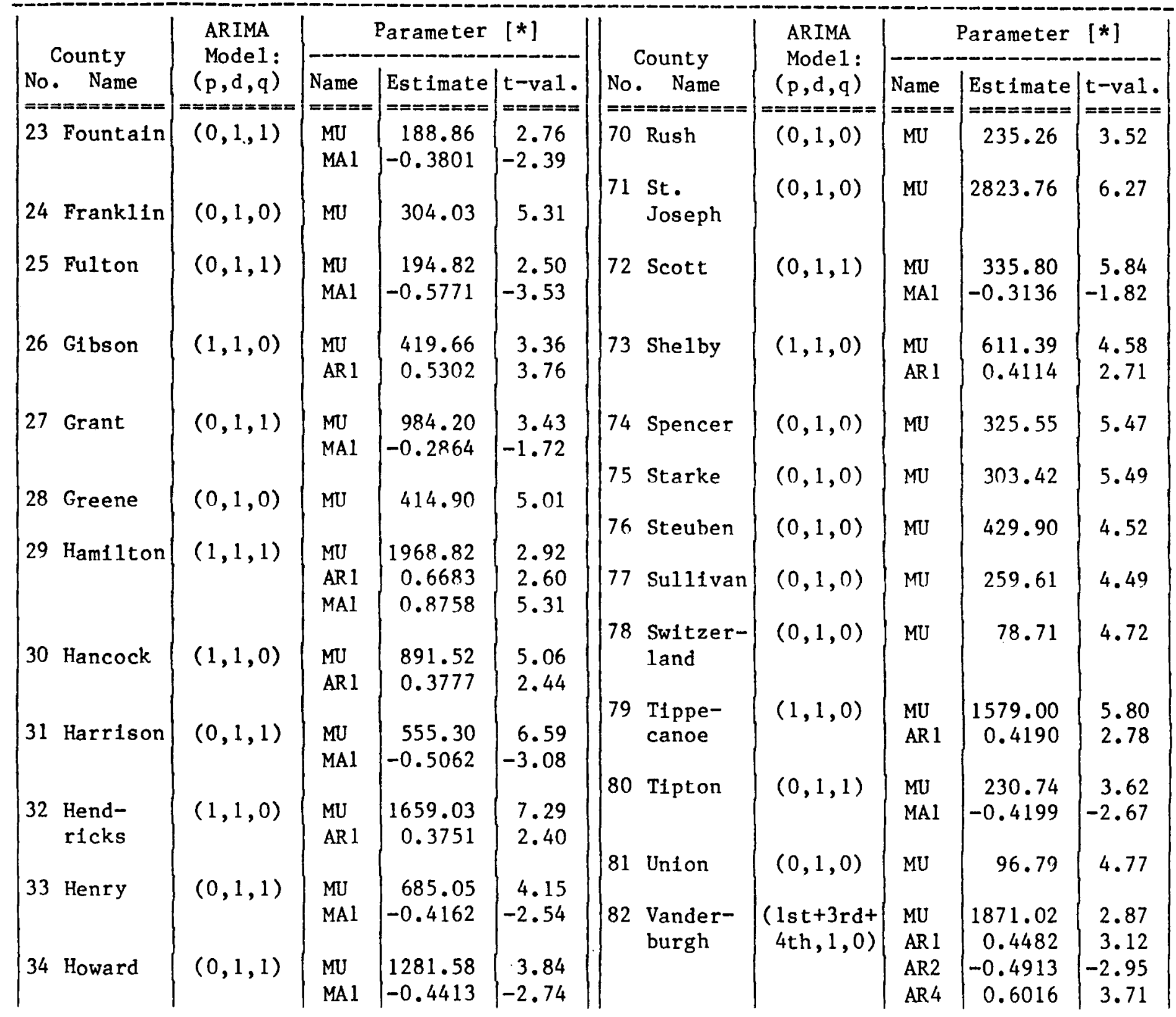


Table F2, continued

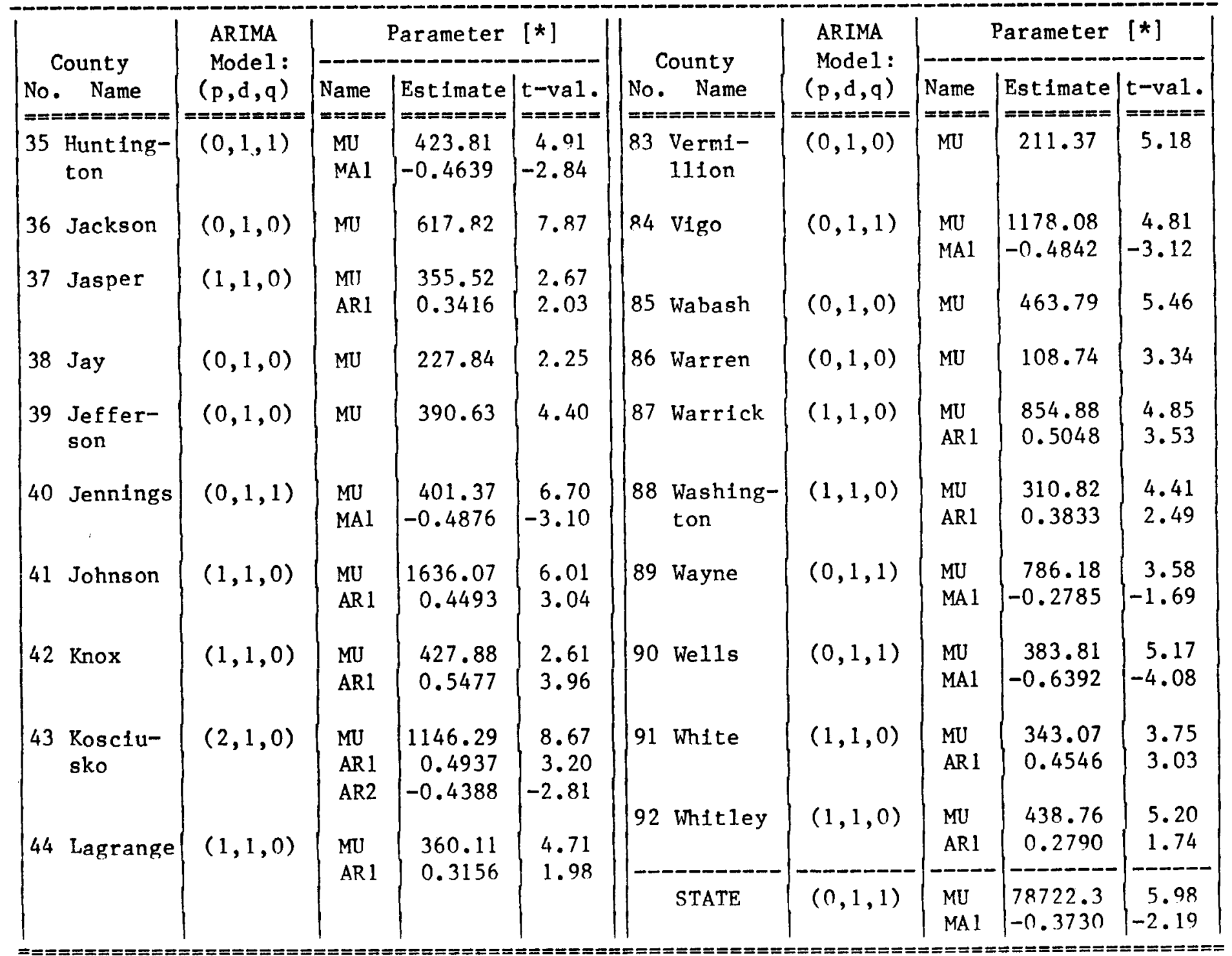


Table F3: 1989-2015 Vehicle Registration Forecasts by ARIMA Mode1

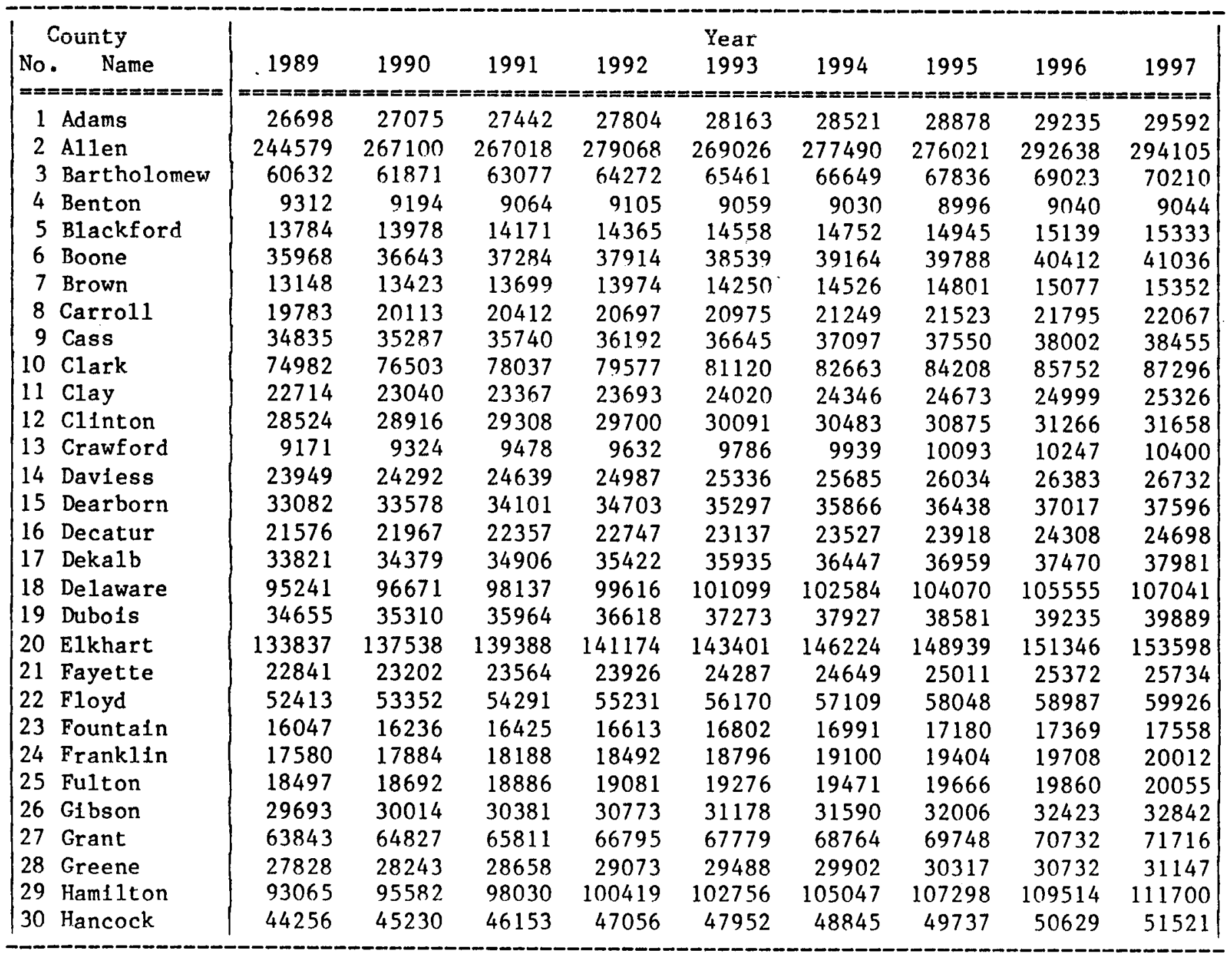


Table F3, continued

\begin{tabular}{|c|c|c|c|c|c|c|c|c|c|}
\hline \multirow{3}{*}{$\begin{array}{l}\text { County } \\
\text { No. Name } \\
==============\end{array}$} & \multicolumn{9}{|c|}{ Year } \\
\hline & 1989 & 19 & 99 & 992 & 1993 & 99 & 1995 & 99 & 1997 \\
\hline & & & & $-5-4$ & & & & & \\
\hline 31 Harrison & 28625 & 29180 & 29735 & 30291 & 30846 & 31401 & 31957 & 32512 & 33067 \\
\hline 32 Hendricks & 74488 & 76287 & 77998 & 79677 & 81343 & 83005 & 84665 & 86325 & 87984 \\
\hline 33 Henry & 45604 & 46289 & 46974 & 47659 & 48344 & 49029 & 49714 & 50399 & 51084 \\
\hline 34 Howard & $\begin{array}{l}72690 \\
31827\end{array}$ & $\begin{array}{l}73971 \\
32251\end{array}$ & & & $\begin{array}{l}77816 \\
33522\end{array}$ & & & & $\begin{array}{l}82942 \\
35217\end{array}$ \\
\hline $\begin{array}{ll}35 & \text { Huntingt } \\
36 & \text { Jackson }\end{array}$ & $\begin{array}{l}318 \\
351\end{array}$ & $\begin{array}{l}32251 \\
35731\end{array}$ & 36348 & $\begin{array}{l}33098 \\
36966\end{array}$ & 4 & $\begin{array}{l}33946 \\
38202\end{array}$ & $\begin{array}{l}343 / 0 \\
38820\end{array}$ & $\begin{array}{l}34 / 94 \\
39438\end{array}$ & 5 \\
\hline 37 Jasper & 23084 & 23468 & 23852 & 24236 & 24620 & 25004 & 25388 & 25772 & 26156 \\
\hline 38 Jay & 20377 & 20605 & 20833 & 21060 & 21288 & 21516 & 21744 & 21972 & 22200 \\
\hline 39 Jefferson & 23805 & 24195 & 24586 & 24977 & 25367 & 25758 & 26148 & 26539 & 26930 \\
\hline 40 Jennings & 20797 & 21198 & 21600 & 22001 & 22402 & 22804 & 23205 & 23607 & 24008 \\
\hline 41 Johnson & 74497 & 76173 & 77827 & 79471 & 81110 & 82748 & 84385 & 86021 & 87657 \\
\hline $42 \mathrm{Knox}$ & 34240 & 34558 & 34925 & 35320 & 35730 & 36148 & 36570 & 36995 & 37422 \\
\hline 43 Kosclusko & 60801 & 62086 & 62976 & 63 & 65 & 66 & 67 & & 69575 \\
\hline 44 Lagrange & 20959 & 213 & 21717 & 22 & 22 & 22801 & 23 & & 23881 \\
\hline 45 Lake & 335361 & 341383 & 347279 & 353110 & 358907 & 364685 & 370454 & 376218 & 381979 \\
\hline 46 Laporte & 84924 & 86358 & 87791 & 89224 & 90658 & 92091 & 93524 & 94958 & 96391 \\
\hline 47 Lawrence & 39190 & 39875 & 40560 & 41245 & 41930 & 42615 & 43300 & 43985 & 44671 \\
\hline 48 Madison & 115980 & 117871 & 119761 & 121652 & 123542 & 125433 & 127323 & 129214 & 131104 \\
\hline 49 Marion & 612979 & 623120 & 633262 & 643404 & 653546 & 663687 & 673829 & 683971 & 694113 \\
\hline 50 Marsha11 & 38221 & 38830 & 39440 & 40049 & 40659 & 41268 & 41878 & 42487 & 43097 \\
\hline 51 Martin & 9880 & 10044 & 10208 & 10372 & 10536 & 10700 & 10864 & 11028 & 11192 \\
\hline 52 Miamt & 34003 & 34568 & 34971 & 35480 & 36040 & 36567 & 37077 & 37598 & 38124 \\
\hline 53 Monroe & 727 & & & & & & 8 & 16 & 84253 \\
\hline 54 Montgomery & 32368 & 32846 & 33307 & 33762 & 34215 & 34 & 35120 & 35572 & 36024 \\
\hline 55 Morgan & 50727 & 51756 & 52786 & 538 & 54845 & 55874 & 56 & 57933 & 58963 \\
\hline 56 Newton & 12729 & 13067 & 13249 & 13430 & 13612 & 13794 & 13975 & 14157 & 14339 \\
\hline 57 Noble & 34307 & 34865 & 35423 & 35981 & 36539 & 37096 & 37654 & 38212 & 38770 \\
\hline 58 Ohio & 4662 & 4736 & 4809 & 4882 & 4956 & 5029 & 5102 & 5176 & 5249 \\
\hline 59 Orange & 16492 & 16761 & 17030 & 17298 & 17566 & 17835 & 18103 & 18372 & 18640 \\
\hline 60 Owen & 15986 & 16272 & 16557 & 16842 & 17127 & 17413 & 17698 & 17983 & 18269 \\
\hline 61 Parke & 14008 & 14213 & 14326 & 14517 & 14675 & 14860 & 15033 & 15217 & 15396 \\
\hline 62 Perry & 16658 & 16938 & 17219 & 17499 & 17779 & 18059 & 18339 & 18620 & 18900 \\
\hline
\end{tabular}


Table F3, continued

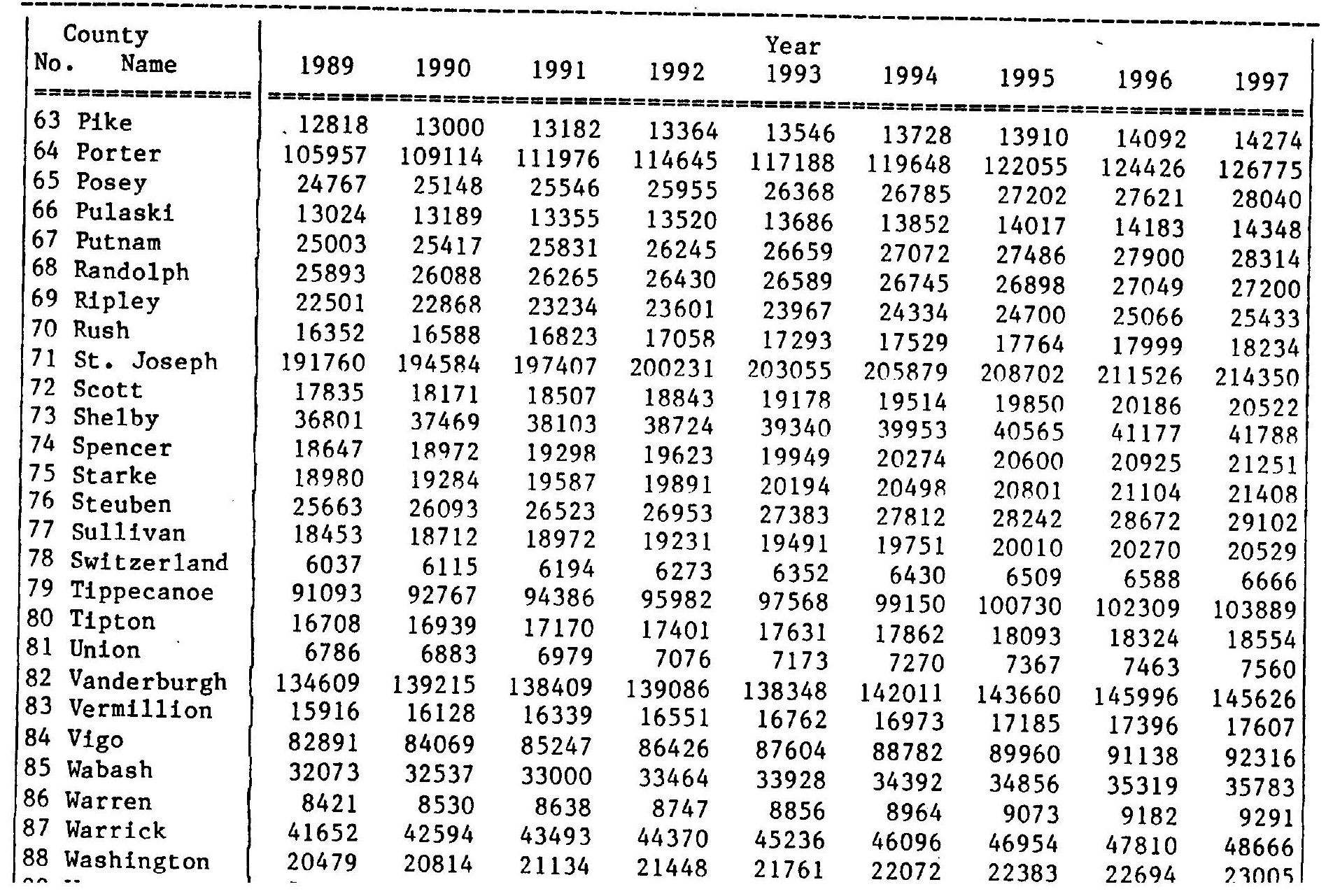


Table F3, continued

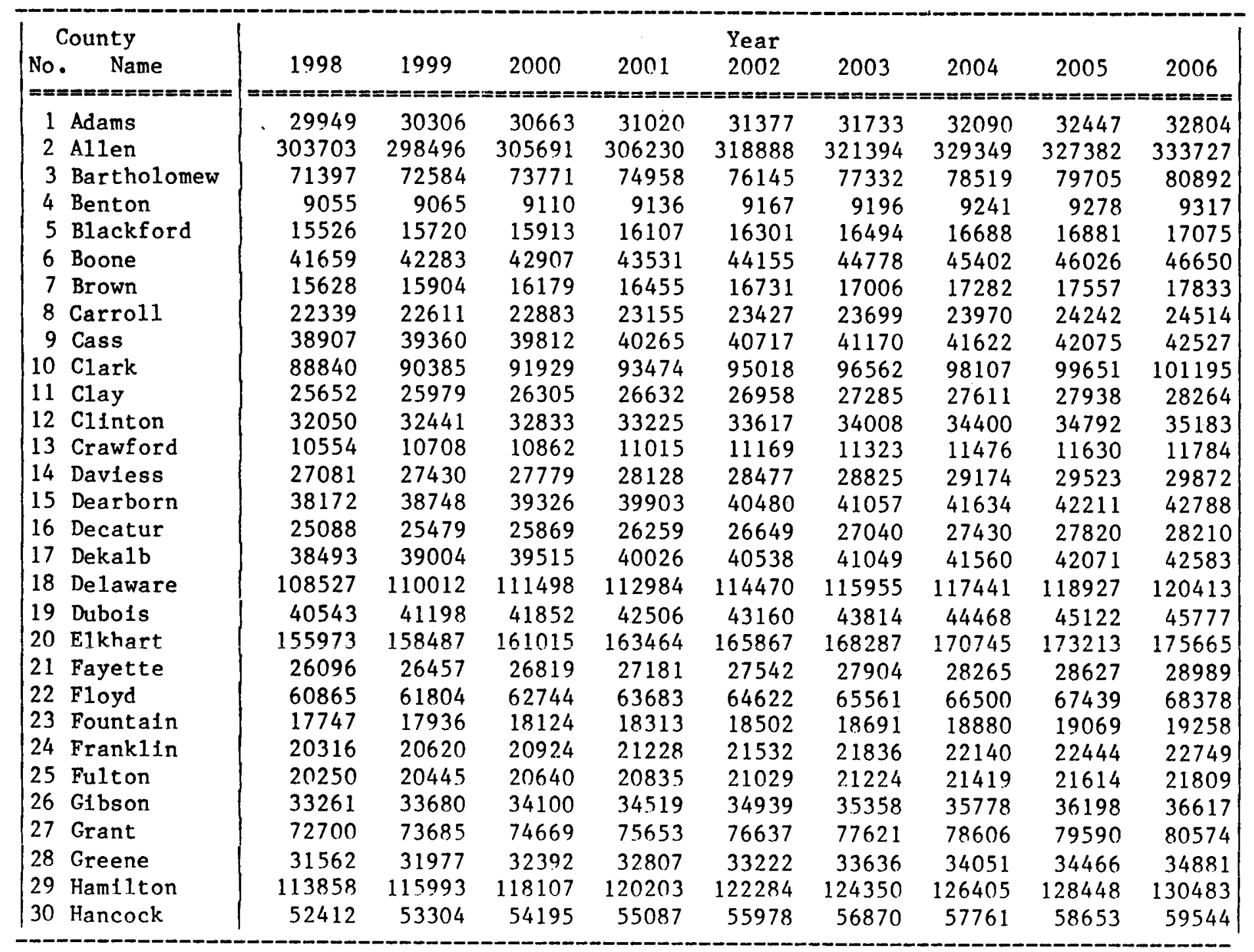


Table F3, continued

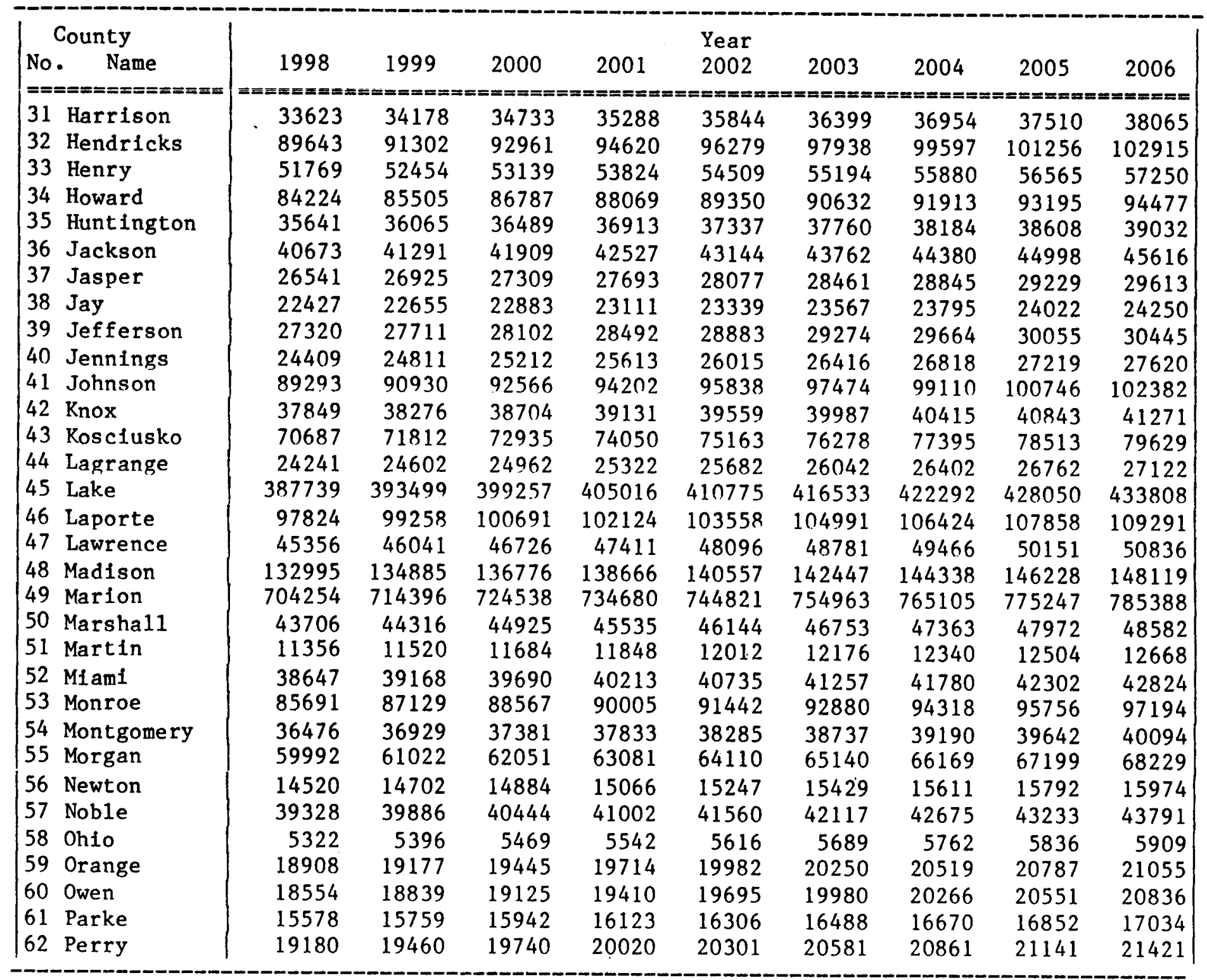


Table F3, continued

\begin{tabular}{|c|c|c|c|c|c|c|c|c|c|}
\hline & \multicolumn{9}{|c|}{ Year } \\
\hline \multirow{2}{*}{$\begin{array}{l}\text { County } \\
\text { No. Name } \\
===============\end{array}$} & 1998 & 1999 & 2000 & 2001 & 2002 & 2003 & 2004 & 2005 & 2006 \\
\hline & $===$ & 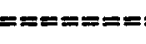 & $====m=s$ & 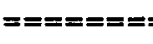 & $===s==$ & $==-=$ & $== \pm=$ & $====$ & $===$ \\
\hline 63 Pike & 14456 & 14637 & 14819 & 15001 & 15183 & 15365 & 15547 & 15729 & 15911 \\
\hline 64 Porter & 129108 & 131432 & 133749 & 136062 & 138373 & 140681 & 142988 & 145295 & 147601 \\
\hline 65 Posey & 28459 & 28879 & 29298 & 29718 & 30137 & 30557 & 30977 & 31396 & 31816 \\
\hline 66 Pulaski & 14514 & 14680 & 14845 & 15011 & 15177 & 15342 & 15508 & 15673 & 15839 \\
\hline 67 Putnam & 28728 & 29142 & 29556 & 29970 & 30384 & 30797 & 31211 & 31625 & 32039 \\
\hline 68 Rando1ph & 27351 & 27501 & 27651 & 27802 & 27952 & 28102 & 282.51 & 28401 & 28551 \\
\hline 69 Ripley & 25799 & 26166 & 26532 & 26899 & 27265 & 27631 & 27998 & 28364 & 28731 \\
\hline 70 Rush & 18470 & 18705 & 18940 & 19175 & 19411 & 19646 & 19881 & 20117 & 20352 \\
\hline 71 St. Joseph & 217174 & 219997 & 222821 & 225645 & 228469 & 231292 & 234116 & 236940 & 239764 \\
\hline $\begin{array}{ll}72 & \text { Scott } \\
73 & \text { Shelby }\end{array}$ & $\begin{array}{l}20857 \\
42399\end{array}$ & $\begin{array}{l}21193 \\
43011\end{array}$ & $\begin{array}{l}21529 \\
43622\end{array}$ & $\begin{array}{l}21865 \\
44234\end{array}$ & $\begin{array}{l}22200 \\
44845\end{array}$ & $\begin{array}{l}22536 \\
45457\end{array}$ & $\begin{array}{l}22872 \\
46068\end{array}$ & $\begin{array}{l}23208 \\
46679\end{array}$ & $\begin{array}{l}23544 \\
47291\end{array}$ \\
\hline $\begin{array}{l}74 \text { Spencer } \\
75 \text { Starke }\end{array}$ & $\begin{array}{l}21577 \\
21711\end{array}$ & $\begin{array}{l}21902 \\
22015\end{array}$ & $\begin{array}{l}22228 \\
22318\end{array}$ & $\begin{array}{l}22553 \\
22622\end{array}$ & & & & & $\begin{array}{l}24181 \\
24139\end{array}$ \\
\hline 76 Steuben & 29532 & 29962 & 30392 & 30822 & 31252 & 31681 & 32111 & 32541 & 32971 \\
\hline 77 Sullivan & 20789 & 21049 & 21308 & 21568 & 21828 & 22087 & 22347 & 22606 & 22866 \\
\hline 78 Switzerland & 6745 & 6824 & 6903 & 6981 & 7060 & 7139 & 7217 & 7296 & 7375 \\
\hline 79 Tippecanoe & 105 & 10 & 108 & 110 & 111 & 113 & 114942 & 116521 & 118100 \\
\hline 80 Tipton & 18785 & 19016 & 19247 & 19477 & 19708 & 19939 & 20170 & 20400 & 20631 \\
\hline 81 Union & 7 & 54 & 30 & 7947 & 8044 & 8141 & 8238 & 8334 & 8431 \\
\hline 82 Vanderburgh & 147679 & 149270 & 152396 & 153392 & 155117 & 156138 & 158813 & 160589 & 162748 \\
\hline 83 Vermillion & 17819 & 18030 & 18241 & 184 & 18664 & 18876 & 19087 & 19298 & 19510 \\
\hline 84 Vigo & 93494 & 94672 & 95850 & 97028 & 98206 & 99384 & 100563 & 101741 & 102919 \\
\hline 85 Wabash & 36247 & 36711 & 37175 & 37638 & 38102 & 38566 & 39030 & 39493 & 39957 \\
\hline 86 Warren & 9399 & 9508 & 9617 & 9726 & 9834 & 9943 & 10052 & 10161 & 10269 \\
\hline 87 Warrick & 49521 & 50376 & 51231 & 52086 & 52941 & 53796 & 54651 & 55506 & 56361 \\
\hline 88 Washington & 23316 & 23626 & 23937 & 24248 & 24559 & 24870 & 25181 & 25491 & 25802 \\
\hline 89 Wayne & 65909 & 66695 & 67481 & 68267 & 69054 & 69840 & 70626 & 71412 & 72198 \\
\hline 90 We11s & 29253 & 29637 & 30021 & 30405 & 30789 & 31172 & 31556 & 31940 & 32324 \\
\hline 91 White & 26 & & & & & & & 28912 & 29255 \\
\hline 92 Whitley & 31263 & 31701 & 32140 & 32579 & 33018 & 33456 & 33895 & 34334 & 34773 \\
\hline STATE & 5367705 & 46427 & 5525149 & 5603872 & 682594 & 5761316 & 5840039 & 5918761 & 5997483 \\
\hline
\end{tabular}


Table F3, continued

\begin{tabular}{|c|c|c|c|c|c|c|c|c|c|}
\hline $\begin{array}{l}\text { County } \\
\text { No. Name } \\
==== \pm== \pm== \pm=====\end{array}$ & $\begin{array}{c}2007 \\
========\end{array}$ & $\begin{array}{l}2008 \\
======0\end{array}$ & $\begin{array}{c}2009 \\
==== \pm==\end{array}$ & $\begin{aligned} & 2010 \\
&======\end{aligned}$ & $\begin{array}{l}\text { Year } \\
2011 \\
== \pm====\end{array}$ & $\begin{array}{l}2012 \\
== \pm===\end{array}$ & $\begin{array}{c}2013 \\
=======\end{array}$ & $\begin{array}{l}2014 \\
======\end{array}$ & 2015 \\
\hline 1 Adams & 33161 & 33518 & 33875 & 34232 & 34588 & 34945 & 35302 & 35659 & 36016 \\
\hline 2 Allen & 335610 & 345617 & 348819 & 355673 & 355877 & 361652 & 364437 & 372665 & 376334 \\
\hline 3 Bartholomew & 82079 & 83266 & 84 & 85640 & 86827 & 88014 & 89200 & 90387 & 91574 \\
\hline 4 Benton & 9355 & 9401 & 9443 & 9485 & 9528 & 9574 & 9618 & 9662 & 9706 \\
\hline 5 Blackford & 17 & 17462 & 17656 & 17849 & 18043 & 18237 & 18430 & 18624 & 18817 \\
\hline 6 Boone & 47274 & 47897 & 48521 & 49145 & 49769 & 50393 & 51016 & 51640 & 52264 \\
\hline 7 Brown & 18109 & 18384 & 18660 & 18935 & 19211 & 19487 & 19762 & 20038 & 20313 \\
\hline 8 Carroll & 24786 & 25058 & 25330 & 25602 & 25873 & 26145 & 26417 & 26689 & 26961 \\
\hline 9 Cass & 42980 & 43432 & 43885 & 44337 & 44790 & 45242 & 45695 & 46147 & 46600 \\
\hline 10 Clark & 102740 & 104284 & 105828 & 107373 & 108917 & 110462 & 112006 & 113550 & 115095 \\
\hline 11 Clay & 28591 & 28917 & 29244 & 29570 & 29897 & 30223 & 30550 & 30876 & 31203 \\
\hline 12 CiInton & 35575 & 35967 & 36358 & 36750 & 37142 & 37533 & 37925 & 38317 & 38709 \\
\hline 13 Crawford & 11938 & 12091 & 12245 & 12399 & 12552 & 12706 & 12860 & 13014 & 13167 \\
\hline 14 Daviess & 30221 & 30570 & 30919 & 31268 & 31617 & 31966 & 32315 & 32664 & 33013 \\
\hline 15 Dearborn & 43365 & 43942 & 44519 & 45095 & 45672 & 46249 & 46826 & 47403 & 47980 \\
\hline 16 Decatur & 28601 & 28991 & 29381 & 29771 & 30161 & 30552 & 30942 & 31332 & 31722 \\
\hline 17 Deka1b & 43094 & 43605 & 44117 & 44628 & 45139 & 45650 & 46162 & 73 & 47184 \\
\hline 18 Delaware & 121898 & 123384 & 124870 & 126356 & 127841 & 129327 & 130813 & 132299 & 133784 \\
\hline 19 Dubois & 46431 & 47085 & 47739 & 48393 & 49047 & 49702 & 50356 & 51010 & 51664 \\
\hline 20 E1khart & 178101 & 180540 & 182988 & 185440 & 187888 & 190333 & 192777 & 195224 & 197672 \\
\hline 21 Fayette & 29350 & 29712 & 30074 & 30435 & 30797 & 31159 & 31520 & 31882 & 32244 \\
\hline 22 Floyd & 69318 & 70257 & 71196 & 72135 & 73074 & 74013 & 74952 & 75891 & 76831 \\
\hline 23 Fountain & 19446 & 19635 & 19824 & 20013 & 20202 & 20391 & 20580 & 20768 & 20957 \\
\hline 24 Frank11n & 23053 & 23357 & 23661 & 23965 & 24269 & 24573 & 24877 & 25181 & 25485 \\
\hline 25 Fult on & 220 & & 93 & 22588 & 22783 & 22978 & 23172 & 23367 & 23562 \\
\hline 26 Gibson & 37037 & 37457 & 37876 & 38296 & 38716 & 39135 & 39555 & 39975 & 40394 \\
\hline $27 \mathrm{Gr}$ & 81558 & 82542 & 83527 & 84511 & 85495 & 86479 & 87463 & 88448 & 89432 \\
\hline 28 Greene & 35296 & 35711 & 36126 & 36541 & 36956 & 37371 & 37785 & 38200 & 38615 \\
\hline 29 Hamilton & 132509 & 134528 & 136541 & 138549 & 140551 & 142550 & 144544 & 146536 & 148525 \\
\hline 30 Hancock & 60436 & 61327 & 62219 & 63110 & 64002 & 64893 & 65785 & 66676 & 67568 \\
\hline
\end{tabular}


Table F3, continued

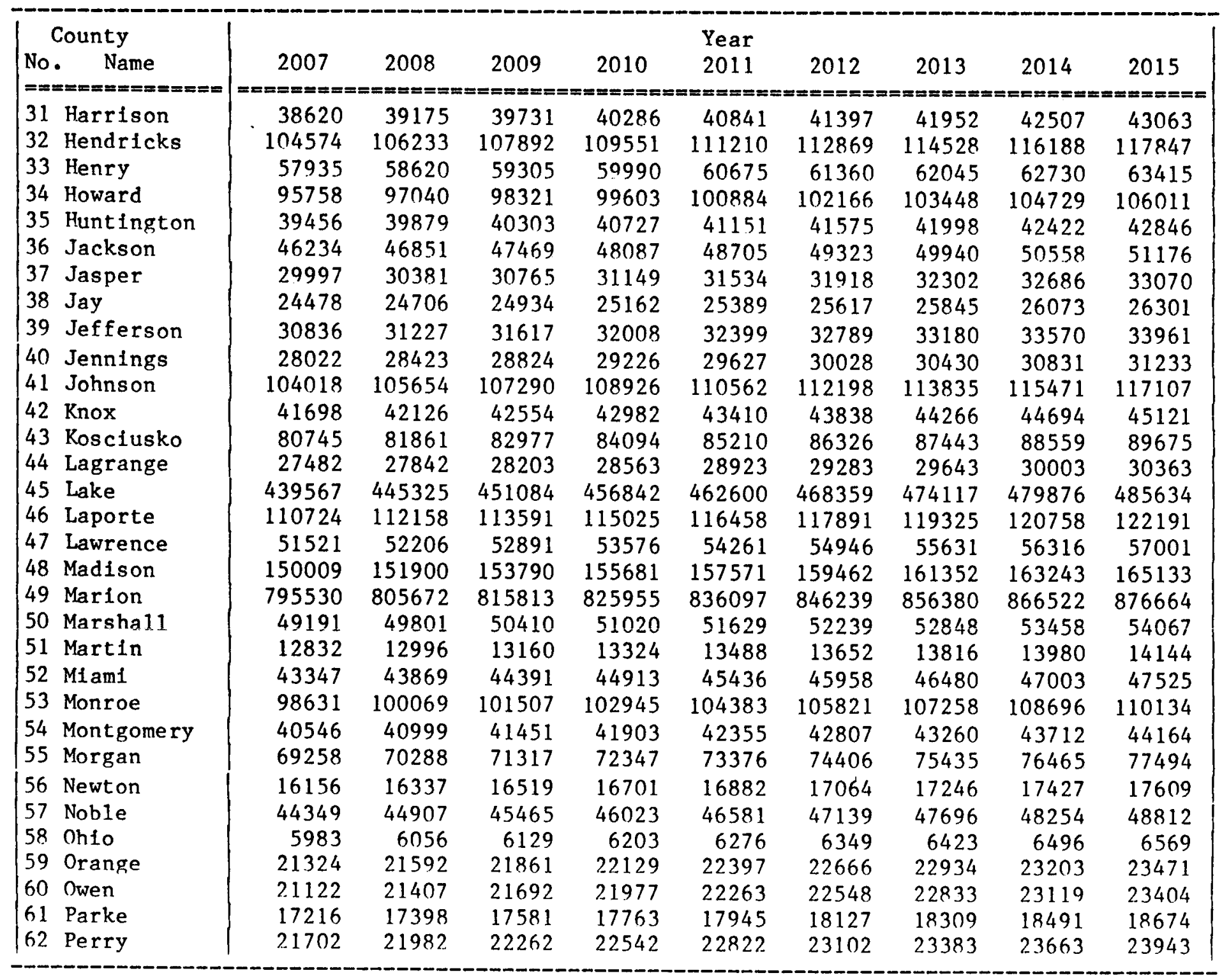


Table F3, continued

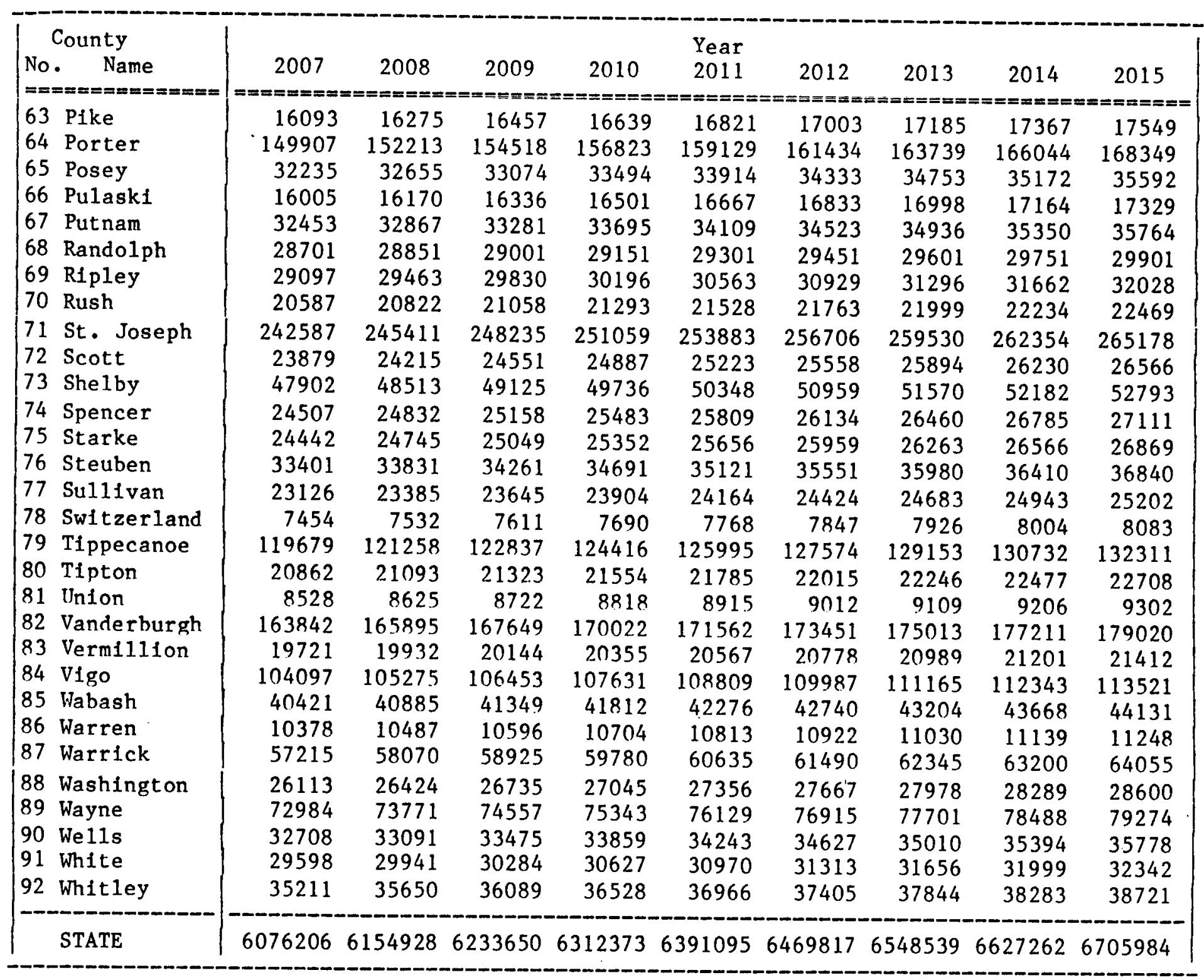


Table F4: Autoregression Model for Vehicle Registrations

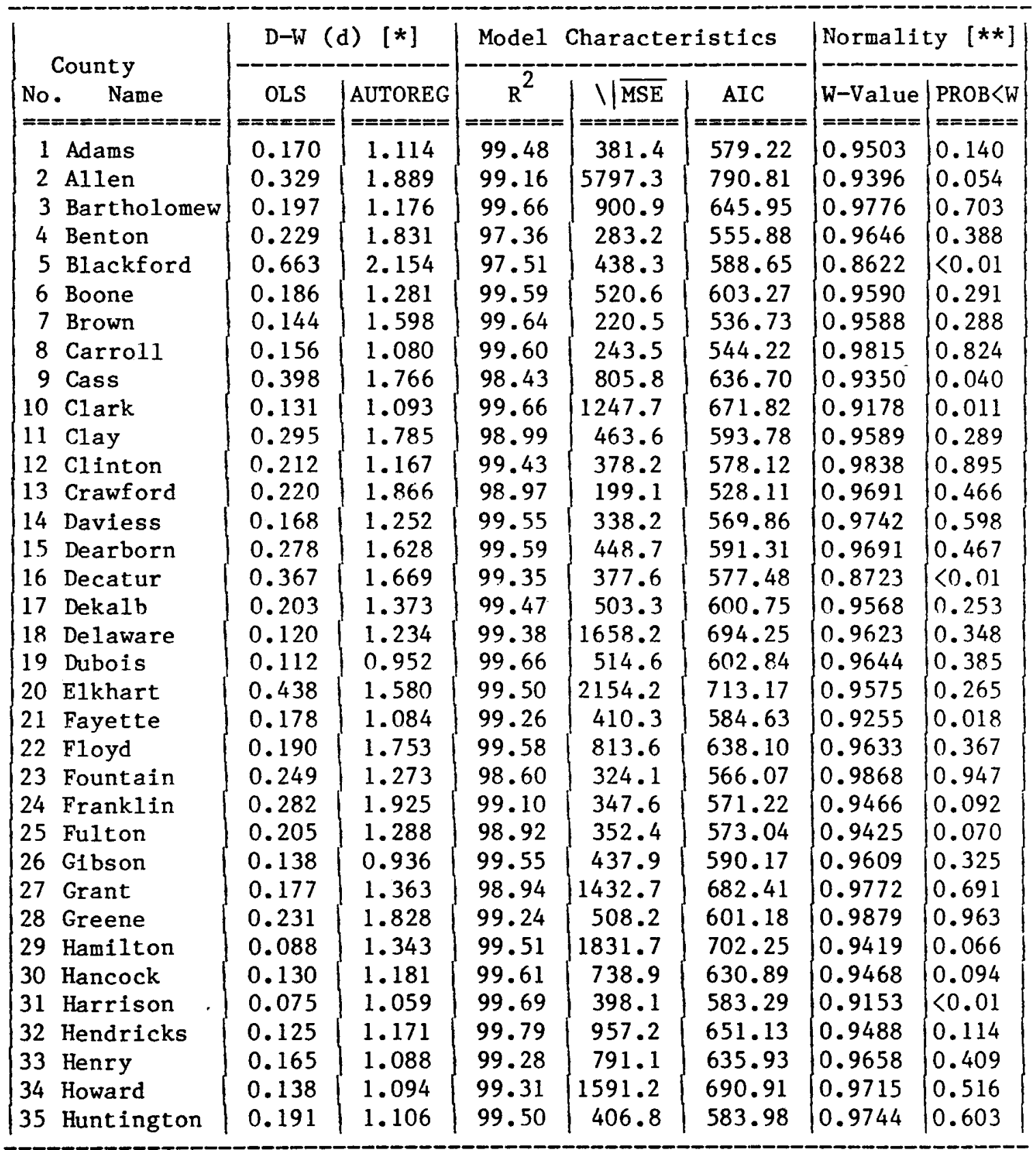


Table F4, continued

\begin{tabular}{|c|c|c|c|c|c|c|c|c|}
\hline \multirow{2}{*}{\multicolumn{2}{|c|}{$\begin{array}{l}\text { County } \\
\text { No. Name } \\
======== \pm====\end{array}$}} & \multicolumn{2}{|c|}{$D-W(d)[*]$} & \multicolumn{3}{|c|}{ Mode1 Characteristics } & \multicolumn{2}{|c|}{ Normality [**] } \\
\hline & & $\begin{array}{c}\text { OLS } \\
=== \pm===\end{array}$ & $\left\{\begin{array}{l}\text { AUTOREG } \\
===\approx===\end{array}\right.$ & $======m$ & $\left\{\begin{array}{l}\backslash \sqrt{\mathrm{MSE}} \\
==== \pm===\end{array}\right.$ & $\begin{array}{c}\mathrm{AIC} \\
========\end{array}$ & $\begin{array}{l}\text { W-Value } \\
=== \pm===\end{array}$ & $\begin{array}{l}\mathrm{PROB} O W \\
======\end{array}$ \\
\hline 36 & Jackson & 0.248 & 1.402 & 99.65 & 482.2 & 597.06 & 0.9922 & 1.000 \\
\hline & Jasper & 0.207 & 1.879 & 98.90 & 585.1 & 612.22 & 0.9656 & 0.406 \\
\hline 38 & Jay & 0.426 & 2.020 & 96.61 & 604.8 & 614.11 & 0.8910 & $<0.01$ \\
\hline 39 & Jeffersion & 0.415 & 1.966 & 99.06 & 538.2 & 605.21 & 0.9616 & 0.337 \\
\hline 40 & Jennings & 0.117 & 1.051 & 99.69 & 283.7 & 556.43 & 0.9394 & 0.052 \\
\hline 41 & Johnson. & 0.095 & 1.039 & 99.74 & 1059.3 & 659.21 & 0.9737 & 0.583 \\
\hline 42 & Knox & 0.156 & 0.846 & 99.30 & 569.9 & 610.94 & 0.9511 & 0.153 \\
\hline 43 & Kosciusko & 0.219 & 1.301 & 99.63 & 860.3 & 642.39 & 0.9241 & 0.017 \\
\hline 44 & Lagrange & 0.139 & 1.355 & 99.51 & 342.5 & 570.95 & 0.9542 & 0.207 \\
\hline 45 & Lake & 0.103 & 0.919 & 99.06 & 7580.1 & 813.01 & 0.9561 & 0.240 \\
\hline 46 & Laporte & 0.251 & 1.823 & 99.42 & 1709.8 & 695.74 & 0.9524 & 0.177 \\
\hline 47 & Lawrence & 0.241 & 1.165 & 99.64 & 519.4 & 602.76 & 0.9876 & 0.959 \\
\hline 48 & Madison & 0.135 & 0.994 & 99.37 & 2078.9 & 711.61 & 0.9834 & 0.882 \\
\hline 49 & Marion & 0.264 & 1.517 & 99.47 & 9466.5 & 829.23 & 0.9802 & 0.782 \\
\hline 50 & Marshall & 0.179 & 1.372 & 99.53 & 550.5 & 607.63 & 0.9637 & 0.372 \\
\hline 51 & Martin & 0.251 & 1.760 & 99.28 & 202.3 & 529.40 & 0.9401 & 0.056 \\
\hline 52 & Miami & 0.370 & 1.464 & 99.03 & 694.7 & 625.19 & 0.9590 & 0.291 \\
\hline 53 & Monroe & 0.321 & 2.113 & 99.54 & 1299.2 & 674.16 & 0.9902 & 0.990 \\
\hline 54 & Montgomery & 0.222 & 1.292 & 99.52 & 436.5 & 589.44 & 0.9676 & 0.441 \\
\hline 55 & Morgan & 0.182 & 1.671 & 99.66 & 794.9 & 636.40 & 0.9812 & 0.813 \\
\hline 56 & Newton & 0.173 & 1.129 & 99.11 & 258.6 & 548.83 & 0.9795 & 0.760 \\
\hline 57 & Noble & 0.535 & 1.891 & 99.17 & 629.4 & 617.00 & 0.9806 & 0.795 \\
\hline 58 & Ohio & 0.177 & 1.655 & 99.36 & 77.1 & 454.54 & 0.9854 & 0.923 \\
\hline 59 & Orange & 0.222 & 1.139 & 99.70 & 190.9 & 524.79 & 0.9801 & 0.780 \\
\hline 60 & Owen & 0.249 & 1.835 & 99.40 & 345.7 & 571.15 & 0.9582 & 0.278 \\
\hline 61 & Parke & 0.238 & 2.076 & 98.54 & 346.9 & 571.39 & 0.9413 & 0.063 \\
\hline 62 & Perry & 0.297 & 1.709 & 98.88 & 424.9 & 587.02 & 0.9395 & 0.053 \\
\hline 63 & Pike & 0.202 & 1.567 & 99.06 & 245.6 & 544.48 & 0.9715 & 0.517 \\
\hline 64 & Porter & 0.104 & 0.684 & 99.65 & 1896.4 & 704.64 & 0.9441 & 0.079 \\
\hline 65 & Posey & 0.114 & 0.868 & 99.52 & 421.3 & 587.23 & 0.9630 & 0.362 \\
\hline 66 & Pulaski & 0.209 & 1.578 & 99.25 & 201.9 & 529.24 & 0.9836 & 0.889 \\
\hline 67 & Putnam & 0.295 & 1.866 & & 380.6 & 578.48 & 0.9589 & 0.290 \\
\hline 68 & Randolph & 0.326 & 1.528 & 98.25 & 602.9 & 614.72 & 0.8547 & $<0.01$ \\
\hline 69 & Ripley & 0.324 & 2.3 & 99.41 & & 576.56 & 0.9578 & 0.271 \\
\hline 70 & Rush & 0.347 & 1.913 & 91 & 411.9 & 584.63 & 0.8764 & $\mid<0.01$ \\
\hline
\end{tabular}


Table F4, continued

\begin{tabular}{|c|c|c|c|c|c|c|c|}
\hline \multirow{3}{*}{$\begin{array}{l}\text { County } \\
\text { No. Name } \\
== \pm==========\end{array}$} & \multirow{2}{*}{\multicolumn{2}{|c|}{$D-W(d)[*]$}} & \multirow{2}{*}{\multicolumn{3}{|c|}{ Mode1 Characteristics }} & \multicolumn{2}{|c|}{ Normality [**] } \\
\hline & & & & & & & \\
\hline & OLS & $\left\{\begin{array}{l}\text { AUTOREG } \\
==x====\end{array}\right.$ & $\begin{array}{c}R^{2} \\
===x==\end{array}$ & $\left\{\begin{array}{l}\backslash \overline{\mathrm{MSE}} \\
======\end{array}\right.$ & $\begin{array}{c}\mathrm{AIC} \\
=======\end{array}$ & $\left\{\begin{array}{l}\text { W-Value } \\
=======\end{array}\right.$ & $\left\{\begin{array}{l}\text { PROB }<\mathrm{W} \\
======\end{array}\right.$ \\
\hline 71 St. Joseph & 0.468 & 1.660 & 99.44 & 2679.7 & 730.15 & 0.9886 & 0.974 \\
\hline $72 S \cot t$ & 0.175 & 1.362 & 99.59 & 279.7 & 554.76 & 0.9739 & 0.589 \\
\hline 73 Shelby & 0.194 & 1.108 & 99.59 & 537.3 & 605.75 & 0.9844 & 0.907 \\
\hline 74 Spencer & 0.207 & 1.757 & 99.34 & 367.0 & 575.92 & 0.9201 & 0.013 \\
\hline 75 Starke & 0.194 & 1.551 & 99.32 & 339.5 & 569.78 & 0.9795 & 0.760 \\
\hline 76 Steuben & 0.249 & 1.884 & 98.84 & 586.0 & 612.30 & 0.8276 & $<0.01$ \\
\hline 77 Sullivan & 0.310 & 1.653 & 98.84 & 355.7 & 573.26 & 0.9404 & 0.058 \\
\hline 78 Switzerland & 0.297 & 1.726 & 99.14 & 101.9 & 475.68 & 0.9700 & 0.483 \\
\hline 79 Tippecanoe & 0.197 & 1.214 & 99.76 & 1079.9 & 660.40 & 0.9661 & 0.415 \\
\hline 80 Tipton & 0.261 & 1.360 & 99.19 & 294.0 & 558.47 & 0.9778 & 0.708 \\
\hline 81 Union & 0.325 & 1.618 & 99.15 & 123.4 & 490.48 & 0.9486 & 0.110 \\
\hline 82 Vanderburgh & 0.204 & 1.434 & 99.40 & 2294.4 & 719.12 & 0.9566 & 0.250 \\
\hline 83 Vermillion & 0.208 & 1.430 & 99.22 & 250.2 & 545.87 & 0.9936 & 1.000 \\
\hline 84 Vigo & 0.146 & 1.158 & 99.49 & 1140.6 & 665.02 & 0.9606 & 0.319 \\
\hline 85 Wabash & 0.263 & 1.492 & 99.29 & 518.5 & 602.56 & 0.9686 & 0.458 \\
\hline 86 Warren & 0.363 & 1.772 & 98.38 & 196.4 & 526.58 & 0.9658 & 0.409 \\
\hline 87 Warrick & 0.068 & 0.965 & 99.68 & 645.8 & 620.95 & 0.9290 & 0.025 \\
\hline 88 Washington & 0.170 & 1.507 & 99.58 & 287.8 & 557.41 & 0.9506 & 0.146 \\
\hline 89 Wayne & 0.223 & 1.398 & 99.01 & 1092.6 & 660.98 & 0.9813 & 0.816 \\
\hline 90 We1ls & 0.131 & 0.861 & 99.55 & 352.4 & 573.14 & 0.9734 & 0.575 \\
\hline 91 White & 0.186 & 1.077 & 99.51 & 348.7 & 572.17 & 0.9799 & 0.775 \\
\hline 92 Whitley & 0.158 & 1.379 & 99.53 & 390.9 & 581.01 & 0.9591 & 0.293 \\
\hline STATE & 0.192 & 1.201 & 99.63 & 63553 & 978.04 & 0.9461 & 0.090 \\
\hline
\end{tabular}

[*] i. $[\alpha=0.01]$ OLS: $\left(d_{L}, d_{U}\right)=(1.24,1.34)$ AUTOREG: $\left(d_{L}, d_{U}\right)=(1.19,1.39)$

ii. $[\alpha=0.05]$ OLS: $\left(d_{L}, d_{U}\right)=(1.43,1.54) ;$ AUTOREG: $\left(d_{L}, d_{U}\right)=(1.38,1.60)$

[**] Number of residuals $=39$ 
Table F5: Parameter of Autoregression Model for Vehicle Registrations

\begin{tabular}{|c|c|c|c|c|c|c|}
\hline County & \multicolumn{3}{|c|}{ Parameter Estimate } & \multicolumn{3}{|c|}{ t-value $[*]$} \\
\hline $\begin{array}{l}\text { No. Name } \\
=============\end{array}$ & $\begin{array}{c}\text { Const } \\
===========\end{array}$ & $\begin{array}{c}\text { Year } \\
== \pm=== \pm=\end{array}$ & $\begin{array}{c}\text { AR } 1 \\
========\end{array}$ & $\begin{array}{l}\text { Const. } \\
=======\end{array}$ & $\begin{array}{l}\text { Year } \\
=====\pi=\end{array}$ & $\begin{array}{c}\mathrm{ARl} \\
=======\end{array}$ \\
\hline 1 Adams & -744337 & 387.62 & -0.9329 & -10.05 & 10.30 & -17.95 \\
\hline 2 Allen & -9441216 & 4879.86 & -0.8637 & -11.09 & 11.28 & -9.55 \\
\hline 3 Bartholomew & -2338426 & 1206.65 & -0.9074 & -14.91 & 15.15 & -13.60 \\
\hline 4 Renton & -186007 & 98.63 & -0.9239 & -3.01 & 3.14 & -12.92 \\
\hline 5 Rlackford & -424032 & 220.58 & -0.6823 & -12.43 & 12.73 & -5.18 \\
\hline 6 Boone & -1240895 & 641.71 & -0.9161 & -13.36 & 13.61 & -15.08 \\
\hline 7 Brown & -545474 & 280.70 & -0.9473 & -10.71 & 10.85 & $-19 \cdot 10$ \\
\hline 8 Carroll & -545635 & 284.26 & -0.9339 & $-11 \cdot 42$ & 11.72 & -18.09 \\
\hline 9 Cass & -950196 & 496.28 & -0.8320 & -8.85 & 9.09 & -7.63 \\
\hline $10 \mathrm{Clark}$ & -3087642 & 1590.91 & -0.9428 & $-11 \cdot 28$ & 11.44 & -18.49 \\
\hline 11 Clay & -677403 & 352.32 & -0.8640 & -10.40 & 10.64 & -10.39 \\
\hline 12 Clinton & -751287 & 392.11 & -0.8929 & $-12 \cdot 34$ & 12.68 & $-12 \cdot 33$ \\
\hline 13 Crawford & -302985 & 156.79 & -0.8981 & -9.04 & 9.21 & $\mid-12 \cdot 40$ \\
\hline 14 Daviess & -703513 & 365.95 & -0.9343 & -10.19 & 10.44 & $-17 \cdot 20$ \\
\hline 15 Dearborn & -1119860 & 579.23 & -0.8750 & -16.68 & 16.99 & -10.81 \\
\hline 16 Decatur & -761928 & 394.20 & -0.8121 & -17.71 & 18.04 & -8.23 \\
\hline 17 Dekalb & -1028573 & 533.78 & -0.9261 & -10.63 & 10.86 & -15.73 \\
\hline 18 Delaware & -2943407 & 1529.71 & -0.9554 & -5.74 & 5.86 & -16.17 \\
\hline 19 Dubois & -1302953 & 672.28 & -0.9487 & -11.46 & 11.64 & -20.91 \\
\hline 20 E1khart & -4870840 & 2516.70 & -0.7827 & -22.38 & 22.76 & -7.68 \\
\hline 21 Fayette & -699004 & 363.05 & -0.9092 & -9.70 & 9.92 & -13.79 \\
\hline 22 Floyd & -1885282 & 974.19 & -0.9163 & $-13 \cdot 14$ & 13.37 & $-15 \cdot 46$ \\
\hline 23 Fountain & -384655 & 201.78 & -0.8938 & -6.69 & 6.90 & -10.67 \\
\hline 24 Franklin & -590108 & 305.33 & -0.8511 & $-12 \cdot 92$ & 13.16 & -9.95 \\
\hline 25 Fulton & -423269 & 222.00 & -0.9321 & -6.22 & 6.42 & -17.82 \\
\hline 26 Gibson & -888260 & 461.79 & -0.9443 & -9.55 & 9.78 & -20.01 \\
\hline 27 Grant & -1951923 & 1014.92 & -0.9303 & -5.70 & 5.83 & -12.83 \\
\hline 28 Greene & -848351 & 440.65 & -0.8962 & -10.49 & 10.73 & -13.21 \\
\hline 29 Hamilton & -3967982 & 2039.70 & -0.9655 & -6.99 & 7.06 & -21.24 \\
\hline 30 Hancock & -1757857 & 905.69 & -0.9387 & -11.65 & 11.82 & -18.70 \\
\hline 31 Harrison & -1073732 & 553.71 & -0.9684 & -8.95 & 9.08 & -24.24 \\
\hline 32 Hendricks & -3231128 & 1661.33 & -0.9419 & -15.96 & 16.15 & -18.98 \\
\hline 33 Henry & -1339820 & 696.89 & -0.9180 & -9.05 & 9.27 & -14.50 \\
\hline 34 Howard & -2541022 & 1315.35 & -0.9496 & -6.04 & 6.15 & -16.88 \\
\hline 35 Huntington & -843817 & $44 \cap .27$ & $\mid-0.9126$ & -11.89 & 12.21 & -14.74 \\
\hline
\end{tabular}


Table F5, continued

\begin{tabular}{|c|c|c|c|c|c|c|}
\hline County & \multicolumn{3}{|c|}{ Parameter Fstimate } & \multicolumn{3}{|c|}{ t-value $[*]$} \\
\hline $\begin{array}{l}\text { No. Name } \\
=======x======\end{array}$ & $\begin{array}{c}\text { Const. } \\
===========\end{array}$ & $\begin{array}{c}\text { Year } \\
=======\end{array}$ & $\begin{array}{c}\text { AR } 1 \\
========\end{array}$ & $\begin{array}{l}\text { Const. } \\
=======\end{array}$ & $\begin{array}{c}\text { Year } \\
== \pm====\end{array}$ & $\begin{array}{c}\text { AR } 1 \\
=======\end{array}$ \\
\hline 36 Jackson & -1246589 & 644.61 & -0.8936 & $-16 \cdot 30$ & 16.60 & -12.77 \\
\hline 37 Jasper & -791974 & 409.91 & -0.9001 & -8.35 & 8.51 & $-13 \cdot 60$ \\
\hline 38 Jay & -479596 & 251.88 & -0.7863 & -7.59 & 7.85 & -7.39 \\
\hline 39 Jefferson & -831320 & 430.13 & -0.8302 & -12.95 & 13.19 & -9.30 \\
\hline 40 Jennings & -776283 & 400.45 & -0.9503 & -11.68 & 11.86 & -20.03 \\
\hline 41 Johnson & -3170493 & 1630.82 & -0.9514 & -12.99 & 13.15 & -21.03 \\
\hline $42 \operatorname{Knox}$ & -873033 & 456.90 & -0.9551 & -5.01 & 5.16 & $-16 \cdot 22$ \\
\hline 43 Kosciusko & -2178096 & 1124.92 & -0.9102 & -14.59 & 14.84 & -14.29 \\
\hline 44 Lagrange & -715954 & 370.26 & -0.9421 & -9.76 & 9.94 & -18.65 \\
\hline 45 Lake & -10961179 & 5688.15 & -0.9645 & -3.84 & 3.92 & $-16 \cdot 67$ \\
\hline 46 Laporte & -2902492 & 1503.78 & -0.8873 & $-10 \cdot 12$ & 10.32 & -10.60 \\
\hline 47 Lawrence & -1342928 & 694.91 & -0.8802 & $-17 \cdot 42$ & 17.74 & $-11 \cdot 77$ \\
\hline 48 Madison & -3686988 & 1913.32 & -0.9422 & -7.46 & 7.61 & -15.98 \\
\hline 49 Marion & -20181695 & 10466.0 & -0.8864 & -12.33 & 12.58 & -9.96 \\
\hline 50 Marsha11 & -1203659 & 624.18 & -0.9165 & -12.37 & 12.63 & -15.41 \\
\hline 51 Martin & -339700 & 175.86 & -0.9031 & -10.02 & 10.21 & $-13 \cdot 48$ \\
\hline 52 Miami & -1053834 & 547.49 & -0.8429 & -11.30 & 11.55 & -8.60 \\
\hline 53 Monroe & -2949154 & 1519.77 & -0.8649 & -16.49 & 16.73 & -10.95 \\
\hline 54 Montgomery & -917211 & 477.57 & -0.9076 & -12.30 & 12.61 & -14.03 \\
\hline 55 Morgan & -2055788 & 1058.82 & -0.9256 & -13.73 & 13.92 & -16.41 \\
\hline 56 Newton & -364943 & 190.05 & -0.9275 & -7.19 & 7.37 & -15.93 \\
\hline 57 Noble & -1112325 & 576.34 & -0.7282 & -20.74 & 21.15 & -6.52 \\
\hline 58 Ohio & -144617 & 74.99 & -0.9355 & -8.58 & 8.75 & -15.95 \\
\hline 59 Orange & -535957 & 277.84 & -0.8946 & -17.56 & 17.92 & -12.79 \\
\hline 60 Owen & -555160 & 286.79 & -0.8991 & -8.93 & 9.07 & $-11 \cdot 14$ \\
\hline 61 Parke & -392342 & 204.70 & -0.8947 & -6.75 & 6.94 & -11.85 \\
\hline 62 Perry & -587331 & 304.06 & -0.8690 & -9.35 & 9.53 & -10.15 \\
\hline 63 Pike & -368800 & 191.98 & -0.8970 & -9.26 & 9.50 & -12.97 \\
\hline 64 Porter & -4533899 & 2331.86 & -0.9520 & -10.87 & 11.00 & -22.71 \\
\hline 65 Posey & -843173 & 436.43 & -0.9476 & -9.39 & 9.57 & $-21 \cdot 36$ \\
\hline 66 Pulaski & -335261 & 175.18 & -0.9023 & -10.05 & 10.34 & $-13 \cdot 63$ \\
\hline 67 Putnam & -845258 & 437.71 & -0.8787 & -15.08 & 15.37 & $-11 \cdot 68$ \\
\hline 68 Randolph & -529541 & 279.38 & -0.9157 & -4.99 & 5.18 & $-15 \cdot 30$ \\
\hline 69 Ripley & -745074 & 385.87 & -0.8605 & $-14 \cdot 92$ & 15.21 & -10.83 \\
\hline 70 Rush & -450685 & 235.33 & -0.8759 & -6.52 & 6.70 & -9.03 \\
\hline
\end{tabular}


Table F5, continued

\begin{tabular}{|c|c|c|c|c|c|c|}
\hline \multirow{2}{*}{$\begin{array}{l}\text { County } \\
\text { No. Name } \\
===============\end{array}$} & \multicolumn{3}{|c|}{ Parameter Estimate } & \multicolumn{3}{|c|}{ t-value $[*]$} \\
\hline & $\begin{array}{c}\text { Const. } \\
==========\end{array}$ & $\begin{array}{c}\text { Year } \\
====-===\end{array}$ & AR 1 & $\begin{array}{l}\text { Const. } \\
=======\end{array}$ & $\begin{array}{c}\text { Year } \\
=======\end{array}$ & $\operatorname{AR}_{======}^{\mathrm{AR}}$ \\
\hline $\begin{array}{ll}71 & \text { St. Joseph } \\
72 & \text { Scott }\end{array}$ & $\begin{array}{r}-5699940 \\
-664563\end{array}$ & $\begin{array}{r}2964.41 \\
343.02\end{array}$ & $\begin{array}{l}-0.7701 \\
-0.9115\end{array}$ & $\begin{array}{l}-21.51 \\
-13.84\end{array}$ & $\begin{array}{l}22.03 \\
14.07\end{array}$ & $\begin{array}{r}-7.01 \\
-14.88\end{array}$ \\
\hline 73 Shelby & -1226955 & 635.57 & -0.9171 & -12.61 & 12.86 & -14.97 \\
\hline 74 Spencer & -660711 & 341.54 & -0.9089 & -10.63 & 10.82 & -14.55 \\
\hline 75 Starke & -611637 & 317.22 & -0.9014 & -10.85 & 11.08 & -13.33 \\
\hline 76 Steuben & -847531 & 438.48 & -0.8988 & -8.42 & 8.58 & -11.68 \\
\hline 77 Su11ivan & -510186 & 266.26 & -0.8833 & -7.94 & 8.15 & -8.94 \\
\hline 78 Switzerland & -16 & 84.32 & -0.8737 & $-11 \cdot 15$ & 11.46 & -11.63 \\
\hline 79 Tippecanoe & -3215587 & 1662.88 & -0.9329 & -14.98 & 15.25 & $-17 \cdot 40$ \\
\hline 80 Tipton & -471442 & 245.67 & -0.8929 & -9.52 & 9.76 & -11.41 \\
\hline 81 Union & $-2 \cap \cap 286$ & 104.16 & -0.8536 & -12.37 & 12.66 & -10.32 \\
\hline 82 Vanderburgh & -4164783 & 2164.85 & -0.9509 & -7.88 & 8.06 & -13.21 \\
\hline 83 Vermillion & -422693 & 220.68 & -0.8928 & -10.49 & 10.78 & -12.28 \\
\hline 84 Vigo & -2265143 & 1181.79 & -0.9530 & -6.11 & 6.27 & -14.51 \\
\hline 85 Wabash & -935622 & 486.93 & -0.8714 & $-12 \cdot 31$ & 12.61 & -10.63 \\
\hline 86 Warren & -228129 & 119.11 & -0.8282 & -9.43 & 9.69 & $-8 \cdot 48$ \\
\hline 87 Warrick & -1680322 & 865.23 & -0.9658 & -9.73 & 9.86 & -25.74 \\
\hline 88 Washington & -640686 & 332.23 & -0.9427 & -10.27 & 10.48 & -18.73 \\
\hline 89 Wayne & -1564114 & 817.00 & -0.9052 & -7.60 & 7.81 & -11.60 \\
\hline 90 Wells & -749552 & 389.68 & -0.9391 & -10.54 & 10.79 & -19.28 \\
\hline 91 White & -696346 & 361.93 & -0.9281 & -10.19 & 10.43 & $-16 \cdot 19$ \\
\hline 92 Whitley & -864279 & 448.04 & -0.9226 & -11.85 & 12.09 & -15.80 \\
\hline STATE & -155807093 & 80712.4 & -0.9169 & -12.94 & 13.20 & -13.93 \\
\hline
\end{tabular}

[*] In most cases, parameters are significant at $\alpha-1$ evel of 0.0001 
Table F6: 1989-2015 Vehicle Registration Forecasts by AutoRegressive Procedure

\begin{tabular}{|c|c|c|c|c|c|c|c|c|c|}
\hline County & \multicolumn{9}{|c|}{ Year } \\
\hline No. Name & 1989 & 1990 & 1991 & 1992 & 1993 & 1994 & 1995 & 1996 & 1997 \\
\hline 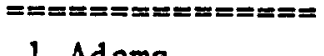 & & $=====8$ & $==x=x=0$ & $====x=$ & $==x===$ & 0 & 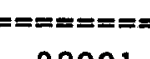 & $==x==x=$ & $======$ \\
\hline 1 Ad ams & 26684 & 27068 & 27452 & 27837 & 28221 & 28606 & 28991 & 29376 & 29762 \\
\hline 2 A1len & 254786 & 261034 & 267095 & 272996 & 278757 & 284399 & 289936 & 295384 & 300755 \\
\hline 3 Bartholomew & 60612 & 61910 & 63200 & 64482 & 65757 & 67025 & 68288 & 69546 & 70799 \\
\hline $\begin{array}{l}4 \text { Benton } \\
5 \text { B1ackford }\end{array}$ & $\begin{array}{r}9612 \\
14080\end{array}$ & $\begin{array}{r}9753 \\
14496\end{array}$ & $\begin{array}{r}9892 \\
\end{array}$ & 10027 & $\begin{array}{l}10159 \\
15445\end{array}$ & $\begin{array}{l}10289 \\
15708\end{array}$ & 10416 & 10542 & 10665 \\
\hline 6 Boone & 35805 & $\begin{array}{l}14446 \\
36418\end{array}$ & $\begin{array}{l}14850 \\
37033\end{array}$ & $\begin{array}{l}1.5162 \\
37651\end{array}$ & $\begin{array}{l}15445 \\
38271\end{array}$ & $\begin{array}{l}15708 \\
38893\end{array}$ & $\begin{array}{l}15958 \\
39516\end{array}$ & $\begin{array}{l}16198 \\
40141\end{array}$ & $\begin{array}{l}16432 \\
40767\end{array}$ \\
\hline 7 Brown & 13136 & 13401 & 13667 & 13934 & 14201 & 14469 & 14738 & 15007 & 15277 \\
\hline 8 Carroll & 19678 & 19968 & 20258 & 20547 & 20836 & 21124 & 21412 & 21700 & 21988 \\
\hline 9 Cass & 35220 & 36000 & 36732 & 37425 & 38085 & 38717 & 39327 & 39917 & 40492 \\
\hline 10 Clark & 75177 & 76853 & 78525 & 80192 & 81855 & 83513 & 85168 & 86819 & 88467 \\
\hline 11 C1ay & 22825 & 23251 & 23667 & 24075 & 24475 & 24868 & 25256 & 25639 & 26018 \\
\hline 12 C1inton & 28196 & 28632 & 29064 & 29492 & 29915 & 30336 & 30753 & 31167 & 31580 \\
\hline $\begin{array}{l}13 \text { Crawford } \\
14 \text { Daviess }\end{array}$ & $\begin{array}{r}9144 \\
24009\end{array}$ & $\begin{array}{r}9273 \\
24398\end{array}$ & $\begin{array}{r}9406 \\
24786\end{array}$ & $\begin{array}{r}9541 \\
25172\end{array}$ & $\begin{array}{r}9678 \\
25557\end{array}$ & $\begin{array}{r}9817 \\
25941\end{array}$ & $\begin{array}{r}9958 \\
26324\end{array}$ & $\begin{array}{l}10101 \\
26705\end{array}$ & $\begin{array}{l}10245 \\
27086\end{array}$ \\
\hline 15 Dearborn & 32827 & 33331 & 33844 & 34365 & 34894 & 35429 & 35969 & 36515 & 37064 \\
\hline 16 Decatur & 21686 & 22166 & 22629 & 23080 & 23520 & 23952 & 24376 & 24795 & 25209 \\
\hline 17 Deka1b & 33660 & 34154 & 34651 & 35150 & 35652 & 36156 & 36662 & 37171 & 37681 \\
\hline 18 Delaware & 95612 & 97301 & 98982 & 100657 & 102325 & 103987 & 105643 & 107294 & 108939 \\
\hline 19 Dubois & 34649 & 35299 & 35950 & 36603 & 37256 & 37910 & 38565 & 39222 & 39878 \\
\hline 20 Elkhart & 133366 & 136212 & 138987 & 141705 & 144380 & 147020 & 149634 & 152226 & 154802 \\
\hline 21 Fayette & 22657 & 23061 & 23461 & 23857 & 24250 & 24641 & 25029 & 25415 & 25799 \\
\hline 22 Floyd & 52443 & 53412 & 54381 & 55351 & 56322 & 57292 & 58263 & 59235 & 60206 \\
\hline 23 Fountain & 16046 & 16315 & 16577 & 16832 & 17082 & 17327 & 17567 & 17803 & 18035 \\
\hline 24 Franklin & 17522 & 17778 & 18040 & 18309 & 18584 & 18863 & 19146 & 19432 & 19721 \\
\hline 25 Fulton & 18293 & 18516 & 18738 & 18960 & 19182 & 19404 & 19627 & 19849 & 20071 \\
\hline 26 Gibson & 29938 & 30417 & 30894 & 31370 & 31846 & 32321 & 32795 & 33268 & 33741 \\
\hline 27 Grant & 63700 & 64928 & 66142 & 67342 & 68529 & 69703 & 70867 & 72021 & 73164 \\
\hline 28 Greene & 27879 & 28343 & 28804 & 29263 & 29720 & 30175 & 30629 & 31082 & 31533 \\
\hline 29 Hamilton & 92389 & 94312 & 96238 & 98.169 & 100103 & 102041 & 103982 & 105927 & 107875 \\
\hline 30 Hancock & 44023 & 44900 & 45780 & 46660 & 47543 & 48427 & 49312 & 50198 & 51086 \\
\hline
\end{tabular}


Table F6, continued

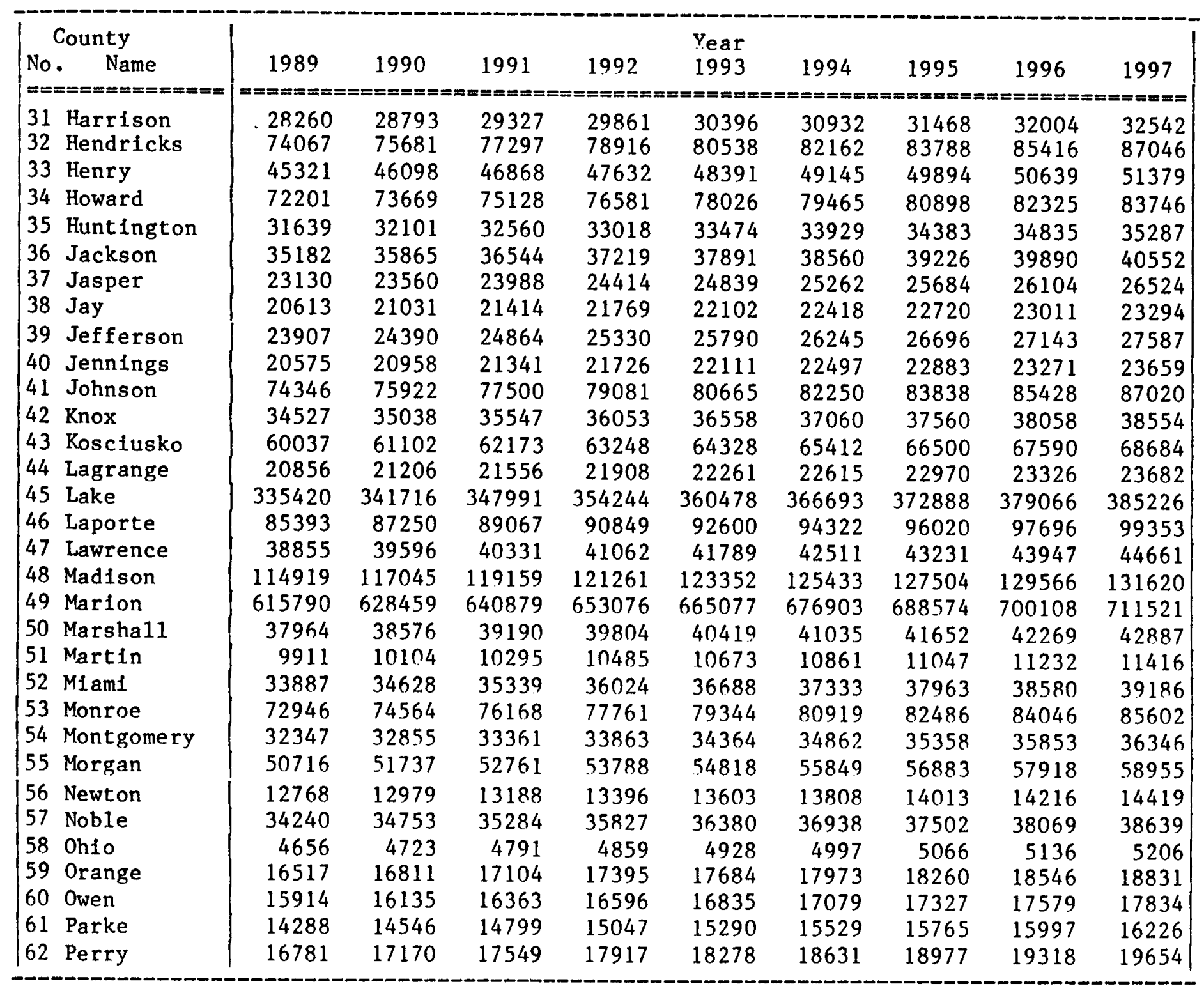


Table F6, continued

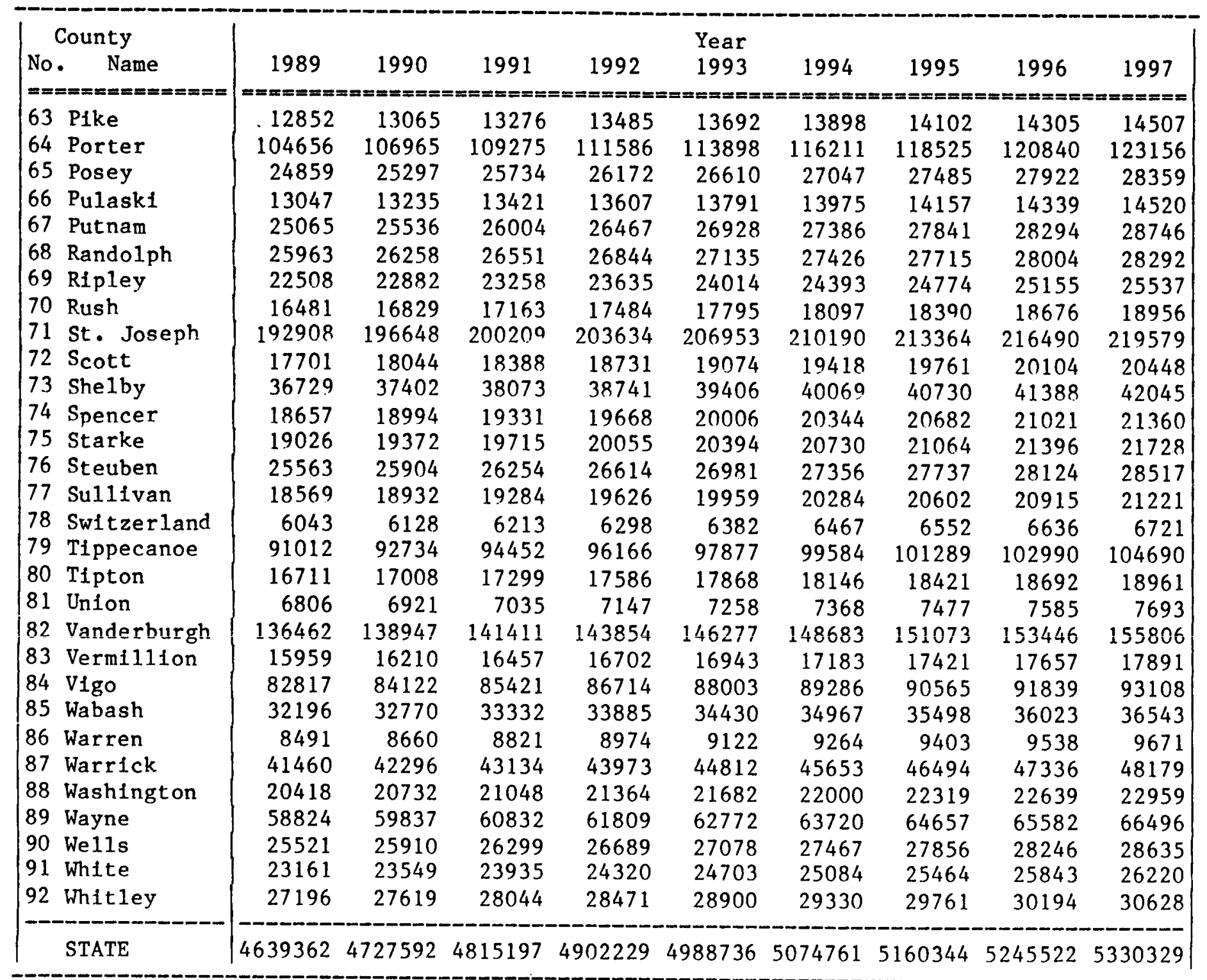


Table F6, continued

\begin{tabular}{|c|c|c|c|c|c|c|c|c|c|c|}
\hline \multirow{2}{*}{\multicolumn{2}{|c|}{$\begin{array}{l}\text { County } \\
\text { No. Name } \\
==============\end{array}$}} & 1998 & 1999 & 2000 & 2001 & $\begin{array}{l}\text { Year } \\
2002\end{array}$ & 2003 & 2004 & 2005 & 2006 \\
\hline & & $======0$ & -4 & 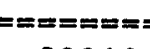 & $==$ & $==x==0=$ & 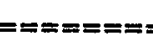 & $===0=$ & & 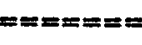 \\
\hline 1 & Adams & 30147 & 30533 & 30919 & 31305 & 31691 & 32077 & 32463 & 32850 & 33236 \\
\hline 2 & A1len & 306058 & 311304 & 316500 & 321653 & 326769 & 331852 & 336908 & 341940 & 346951 \\
\hline 3 & Bartholomew & 72048 & 73293 & 74534 & 72 & 77008 & 78240 & 79470 & 80698 & 81924 \\
\hline 4 & Benton & 10786 & 10906 & 11024 & 11141 & 11256 & 11370 & 11482 & 11594 & 11705 \\
\hline 5 & Blackford & 16662 & 16889 & 17114 & 17337 & 17560 & 17782 & 18003 & 18224 & 18445 \\
\hline 6 & Boone & 41395 & 42023 & 42653 & 43284 & 43916 & 44548 & 45182 & 45816 & 46450 \\
\hline 7 & Brown & 15548 & 15819 & 16091 & 16363 & 1 & 16 & 17182 & 17455 & 17730 \\
\hline 8 & Carroll & 22275 & 22563 & 22850 & 23137 & 2 & 23 & 23996 & 24283 & 24569 \\
\hline 9 & Cass & 41053 & 41604 & 42145 & 42679 & 43207 & 29 & 44247 & 44761 & 45272 \\
\hline 10 & Clark & 90111 & 91753 & 93391 & 95027 & 96660 & 98291 & 99919 & 101545 & 103170 \\
\hline 11 & Clay & 26393 & 26765 & 27135 & 275 & 27867 & 28230 & 28592 & 28 & 29312 \\
\hline 12 & C1inton & 31990 & 32398 & 32804 & 33209 & 33613 & 34015 & 34416 & 34816 & 35216 \\
\hline 13 & Crawford & 10390 & 10537 & 10684 & 10833 & 10 & 11 & 11283 & 11434 & 11586 \\
\hline 14 & Daviess & 27465 & 27844 & 28222 & 28599 & 28975 & 29 & 29726 & 30100 & 30474 \\
\hline 15 & Dearborn & 37617 & 38174 & 38733 & 392 & 39 & 40425 & 40993 & 41562 & 42132 \\
\hline 16 & Decatur & 25619 & 26027 & 26432 & 26835 & 27236 & 27636 & 28035 & 28433 & 28830 \\
\hline 17 & Dekalb & 38193 & 38707 & 39222 & 39738 & 56 & 40 & 41295 & 41816 & 42338 \\
\hline 18 & Delaware & 110579 & 112214 & 113845 & 115471 & 117092 & 118710 & 120324 & 121934 & 123540 \\
\hline 19 & Dubois & 40 & 41195 & 41854 & 42514 & 43 & 5 & 44497 & 45159 & 45822 \\
\hline 20 & E1khart & 157365 & 159919 & 162464 & 165003 & 167537 & 170067 & 172594 & 175120 & 177643 \\
\hline 21 & Fay & 26180 & & 26939 & 27316 & 27692 & 280 & 28441 & 28813 & 29185 \\
\hline 22 & F1oyd & 61178 & 62150 & 63122 & 64094 & 65066 & 66039 & 67012 & 67984 & 68957 \\
\hline 23 & Fountain & 18264 & & & 18936 & 19155 & 19372 & 19588 & 19802 & 20015 \\
\hline 24 & Franklin & 20013 & 20306 & $206 \cap 2$ & 20898 & 21196 & 21496 & 21796 & 22097 & 22398 \\
\hline 25 & Fulton & 20293 & 20515 & 20737 & 20959 & 21181 & 21404 & 2.1626 & 21848 & 22070 \\
\hline 26 & Gtbson & 34213 & 34685 & 35156 & 35627 & 36097 & 36566 & 37036 & 37504 & 37973 \\
\hline 27 & Grant & 74299 & 75425 & 76544 & 77655 & 78760 & 79858 & 80951 & 82038 & 83120 \\
\hline 28 & Greene & 31983 & 32432 & 32880 & 33328 & 33775 & 34221 & 34666 & 35112 & 35556 \\
\hline 29 & Hamilton & 109827 & 111781 & 113738 & 115 & 117661 & 119626 & 121594 & 123565 & 125538 \\
\hline 30 & Hancock & 51975 & 52864 & 53755 & 54647 & 55539 & 56433 & 57327 & 58221 & 59117 \\
\hline
\end{tabular}


Table F6, continued

\begin{tabular}{|c|c|c|c|c|c|c|c|c|c|c|}
\hline \multirow{3}{*}{\multicolumn{2}{|c|}{$\mid$\begin{tabular}{l}
\multicolumn{2}{c}{ County } \\
No. $\quad$ Name \\
$====== \pm=== \pm====$
\end{tabular}}} & \multicolumn{9}{|c|}{ Year } \\
\hline & & 1998 & 1999 & 2000 & 2001 & 2002 & 2003 & 2004 & 200 & 2006 \\
\hline & & & & & & . & $====$ & $====$ & 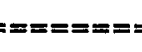 & $==x= \pm=$ \\
\hline 31 & Harrison & 33079 & 33618 & 34156 & 34695 & 35235 & 35775 & 36316 & 36856 & 37398 \\
\hline 32 & Hendricks & 88678 & 903 & 91947 & 93584 & 95222 & 96861 & 98502 & 100144 & 101787 \\
\hline 33 & Henry & 52117 & 52850 & 53581 & 54309 & 55035 & 55758 & 56479 & 57198 & 57915 \\
\hline 34 & Howard & 85162 & 86573 & 87979 & 89380 & 90777 & 92170 & 93559 & 94944 & 96326 \\
\hline 35 & Huntington & 7 & 7 & & & & & 38424 & 38869 & 39314 \\
\hline 36 & Jackson & 41212 & 41871 & 42528 & 43183 & 43838 & 44491 & 45144 & 45795 & 46446 \\
\hline 37 & Jasper & 26942 & 27360 & 27777 & 28193 & 28 & 29024 & 29438 & 298 & 30266 \\
\hline 38 & Jay & 23570 & 23841 & 24108 & 24372 & 24 & 24 & 25 & 25 & 25662 \\
\hline 39 & Jefferson & 28029 & 28469 & 28908 & 29344 & 297 & 30 & 3 & 2 & 15 \\
\hline 40 & Jennings & 24048 & 24437 & 24827 & 25217 & 256 & 25 & & & 76 \\
\hline 41 & Johnson & 88614 & 902 & 918 & 932 & 95 & 96 & 982 & 99 & 101423 \\
\hline 42 & Knox & 39049 & 39542 & 40033 & 40523 & 410 & 414 & 41983 & 42467 & 42950 \\
\hline 43 & Kosciusko & 69781 & 708 & 719 & & 74. & 75 & 76 & $77:$ & 78630 \\
\hline 44 & Lagrange & 24039 & 24398 & 247 & 25 & & 25 & 26 & & 26922 \\
\hline 45 & Lake & 391370 & 397497 & 403609 & 409 & 415 & 421 & 427 & 433 & 439982 \\
\hline 46 & Laporte & 100993 & 102617 & 104227 & 105826 & 107414 & 108 & 110 & 112 & 113680 \\
\hline 47 & Lawrence & 45373 & 46082 & 46 & 96 & 48 & 48 & 49 & 9 & 10 \\
\hline 48 & Madison & 133665 & 135703 & 137734 & 139758 & 141775 & 143787 & 145792 & 147793 & 149788 \\
\hline 49 & Mar & 72 & 7340 & 745162 & 756212 & 767196 & 778121 & 788994 & 799821 & 810606 \\
\hline 50 & Marsha11 & 43506 & 44125 & 44744 & 45364 & 45984 & 46604 & 47225 & 47846 & 48467 \\
\hline 51 & Mar & 11 & 11782 & 11964 & 12146 & 12327 & 12507 & 12687 & 12867 & 13046 \\
\hline 52 & Miami & 397 & 403 & 40 & 415 & 421 & 426 & 432 & 438 & 44360 \\
\hline 53 & Monroe & 87152 & & & & & & & & 99445 \\
\hline 54 & Montgomery & 36838 & 37328 & 37817 & 38305 & 38792 & 39278 & & & 40733 \\
\hline 55 & Morgan & 59994 & 61034 & 62076 & 63119 & 64163 & 65208 & & 67 & 68349 \\
\hline 56 & Newton & 14620 & 14821 & 15021 & 152 & 15 & 156 & 15814 & & 16207 \\
\hline 57 & Nob1e & 39210 & 39783 & 40356 & 40 & 41506 & 420 & 42657 & 43233 & 43808 \\
\hline 58 & Ohio & 5277 & 5348 & 5419 & 5490 & 5561 & 56 & 5705 & 57 & 5850 \\
\hline 59 & Orange & 19116 & 19400 & 196 & 19966 & 20248 & 20530 & 20811 & 21092 & 21372 \\
\hline 60 & Owen & 18093 & 18354 & 18618 & 18885 & 19153 & 19423 & 19695 & 19969 & 20243 \\
\hline 61 & Parke & 16453 & & & & 17339 & 175 & 17773 & & 18201 \\
\hline 62 & Perry & 19986 & 20314 & 20639 & 20962 & 21282 & 21599 & 21915 & 22230 & 22543 \\
\hline
\end{tabular}


Table F6, continued

\begin{tabular}{|c|c|c|c|c|c|c|c|c|c|c|}
\hline No. & $\begin{array}{l}\text { County } \\
\quad \text { Name }\end{array}$ & 1998 & 1999 & 2000 & 2001 & $\begin{array}{l}\text { Year } \\
2002\end{array}$ & 2003 & 2004 & 2005 & 200 \\
\hline & 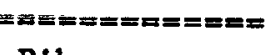 & 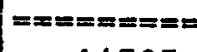 & & & - & $=-=$ & $=== \pm$ & $== \pm====$ & $==x== \pm$ & $==3=0$ \\
\hline $\begin{array}{l}63 \\
64\end{array}$ & $\begin{array}{l}\text { Pike } \\
\text { Porter }\end{array}$ & $\begin{array}{r}14707 \\
125472\end{array}$ & $\begin{array}{r}14907 \\
127789\end{array}$ & $\begin{array}{r}15107 \\
130107\end{array}$ & $\begin{array}{r}15305 \\
132426\end{array}$ & $\begin{array}{r}15503 \\
134745\end{array}$ & $\begin{array}{r}15700 \\
137065\end{array}$ & $\begin{array}{r}15896 \\
139385\end{array}$ & & $\begin{array}{r}16288 \\
144028\end{array}$ \\
\hline 65 & Posey & 28797 & 29234 & 29671 & 30108 & 30546 & 30983 & 31420 & 31857 & 32294 \\
\hline 66 & Pulaski & 14701 & 14881 & 15061 & 15240 & 15419 & 15597 & 15776 & 15953 & 16131 \\
\hline 67 & Putnam & 29195 & 29644 & 30090 & 30536 & 30981 & 31425 & 31868 & 32311 & 32753 \\
\hline 68 & Randolph & 28579 & 28866 & 29152 & 29437 & 29722 & 30006 & 30290 & 30574 & 30857 \\
\hline 69 & Ripley & 25919 & 26302 & 26686 & 27069 & 27453 & 27838 & 28222 & 28607 & 28991 \\
\hline 70 & Rush & 19230 & 19500 & 19765 & 20027 & 20 & 20 & 20 & 21044 & 21293 \\
\hline 71 & St. Joseph & 222640 & 225678 & 228699 & 231708 & 234706 & 237696 & 240681 & 243660 & 246637 \\
\hline 72 & $S \cot t$ & 20791 & 21134 & 21477 & 21821 & 22164 & 22507 & 22850 & 23193 & 23536 \\
\hline 73 & Shelby & 42699 & 43353 & 44004 & 55 & 45 & & 46 & 47 & 47890 \\
\hline 74 & Spencer & 21699 & 22039 & 22378 & 22718 & 23 & 23 & 23 & 24079 & 24419 \\
\hline 75 & Starke & 22057 & 22386 & 22713 & 23039 & 23 & & 24 & 24 & 24659 \\
\hline 76 & Steuben & 28913 & 29315 & 29719 & 30128 & 30539 & 30 & 31 & 38 & 32209 \\
\hline 77 & Sullivan & 21524 & 21822 & 22116 & 22407 & 22695 & 22 & 23 & 45 & 23825 \\
\hline $\begin{array}{l}78 \\
79\end{array}$ & $\begin{array}{l}\text { Switzerland } \\
\text { Tippecanoe }\end{array}$ & $\begin{array}{r}6805 \\
106386\end{array}$ & $\begin{array}{r}6890 \\
108081\end{array}$ & $\begin{array}{r}6974 \\
09773\end{array}$ & $\begin{array}{r}7059 \\
1463\end{array}$ & 7143 & 72 & 7312 & 6 & 7481 \\
\hline 80 & Tipton & 19228 & 19492 & 19754 & $\begin{array}{l}3 \\
4\end{array}$ & $\begin{array}{r}1131 \\
202\end{array}$ & 11 & 20 & $\begin{array}{l}18207 \\
21042\end{array}$ & $\begin{array}{r}119890 \\
21296\end{array}$ \\
\hline 81 & Union & 7800 & 79 & & & $\begin{array}{r}20273 \\
8225\end{array}$ & $\begin{array}{r}20 \\
8\end{array}$ & $\begin{array}{r}207 \\
84\end{array}$ & $\begin{array}{r}21042 \\
8541\end{array}$ & $\begin{array}{r}21296 \\
8646\end{array}$ \\
\hline 82 & Vanderburgh & 158151 & 160485 & 162806 & 165117 & 167418 & $\begin{array}{r}8331 \\
169710\end{array}$ & $\begin{array}{r}171992 \\
17190\end{array}$ & $\begin{array}{r}8541 \\
174267\end{array}$ & $\begin{array}{r}8646 \\
76534\end{array}$ \\
\hline 83 & Vermillion & 18123 & 18355 & 18585 & 18814 & 19043 & 19270 & 19497 & $\begin{array}{r}174267 \\
19723\end{array}$ & $\begin{array}{r}176534 \\
19949\end{array}$ \\
\hline 84 & Vigo & 94374 & 95635 & 96893 & 98147 & 99398 & 100646 & 101890 & 103132 & 104371 \\
\hline 85 & Wabash & 37059 & 37571 & 38080 & 38586 & 39 & 39 & 40090 & 40588 & 41085 \\
\hline 86 & Warren & 9801 & 9929 & 10056 & 10181 & 10 & 10 & 10551 & 10674 & 10795 \\
\hline 87 & Warrick & 49022 & 49867 & 50712 & 51558 & 524 & 53 & & 54947 & 55796 \\
\hline 88 & Washington & 23281 & 23602 & 23925 & 24248 & 24571 & 24895 & 25220 & 25545 & 25870 \\
\hline 89 & Wayne & 67402 & 68299 & 69188 & 70071 & 70947 & 718 & 72683 & 44 & 74401 \\
\hline 90 & Wells & 29024 & 29414 & 29803 & 30192 & 30582 & 305 & 31361 & 31750 & 32140 \\
\hline 91 & White & 265 & & 27346 & 27719 & 28092 & 28464 & & & 29575 \\
\hline 92 & Whitley & 31063 & 31499 & 31936 & 32373 & 32812 & 332.51 & 33691 & 34132 & 34573 \\
\hline & STATE & 4795 & 50 & 2818 & & & 33 & 0 & 33 & 81220 \\
\hline
\end{tabular}


Table F6, continued

\begin{tabular}{|c|c|c|c|c|c|c|c|c|c|c|}
\hline $\begin{array}{l}\text { No. } \\
===\end{array}$ & $\begin{array}{l}\text { County } \\
\text { Name } \\
:== \pm===== \pm===\end{array}$ & $\begin{array}{c}2007 \\
== \pm==\end{array}$ & $\begin{array}{l}2008 \\
z== \pm=x\end{array}$ & 2009 & $\begin{array}{c}2010 \\
=======\end{array}$ & $\begin{array}{l}\text { Year } \\
2011\end{array}$ & 2012 & $\begin{array}{c}2013 \\
=x== \pm=\end{array}$ & $\begin{array}{c}2014 \\
:=== \pm=\end{array}$ & $\begin{array}{l}2015 \\
==:== \pm\end{array}$ \\
\hline 1 & Adams & 33622 & 34009 & 34396 & 34782 & 35169 & 35556 & 35943 & 36330 & 36717 \\
\hline & Allen & & 356 & & 3 & & 37 & & & \\
\hline 3 & Bartholomew & 83149 & 84371 & 8 & 2 & 1 & 89 & 4 & 91680 & 92895 \\
\hline $\begin{array}{l}4 \\
5\end{array}$ & $\begin{array}{l}\text { Benton } \\
\text { Blackford }\end{array}$ & $\begin{array}{l}11815 \\
18666\end{array}$ & $\begin{array}{l}11924 \\
18887\end{array}$ & $\begin{array}{l}12032 \\
19108\end{array}$ & $\begin{array}{l}12139 \\
19328\end{array}$ & $\begin{array}{l}12246 \\
19549\end{array}$ & $\begin{array}{l}12: \\
19\end{array}$ & & & \\
\hline 6 & Boone & 47086 & 47722 & 48358 & 48995 & 49632 & 50269 & 509 & 51546 & \\
\hline 7 & Brown & 180 & 18279 & 185 & 188 & & 193 & & 34 & 10 \\
\hline 8 & Carroll & 24855 & 25141 & 25 & & & & & & 27139 \\
\hline 9 & Cass & & 46 & 46 & & & 48 & & & 99 \\
\hline 10 & Clark & 10 & 106 & 108 & 10 & & 112 & 114 & 116 & 117714 \\
\hline 11 & Clay & 29671 & 30 & 30 & & & & & & \\
\hline 12 & C1in & 35614 & 36012 & 36409 & 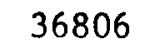 & 2 & & & & 83 \\
\hline 13 & Cra & 11 & 11 & 12 & 1 & 12 & 7 & & & 71 \\
\hline $\begin{array}{l}14 \\
15\end{array}$ & $\begin{array}{l}\text { Davi } \\
\text { Dear }\end{array}$ & & & & & & & & & \\
\hline 16 & Dec & 29227 & 29623 & 300 & 30 & 30 & 31 & 31 & & \\
\hline 17 & Deka & 42861 & 433 & 43 & 44 & & & & & 69 \\
\hline 18 & Delaware & 125143 & 126742 & 128 & 129 & 13. & 133111 & 134 & 136 & 137860 \\
\hline 19 & Dub & 4 & 9 & 4 & 8 & 42 & 98 & & & 06 \\
\hline 20 & Elkhart & 180165 & 182685 & 185205 & 187 & 190 & 192 & 195 & 197 & 14 \\
\hline 21 & Fay & 29556 & 29927 & 30 & 30 & & 12 & & 37 & 03 \\
\hline 22 & Floyd & 699 & 709 & 718 & 728 & 73 & 74797 & 75 & 76 & 77718 \\
\hline 23 & Four & & & 20647 & & & 212 & 21 & 21 & \\
\hline 24 & Franklin & & 23003 & 23306 & 23609 & & 24217 & 24521 & 24825 & 25129 \\
\hline 25 & Fulton & & 22514 & 22736 & & & 23402 & 23624 & 23846 & 24068 \\
\hline 26 & Gibson & 38441 & 38908 & 39376 & 39843 & 40309 & 40776 & 41242 & 41708 & 42174 \\
\hline 27 & Gra & & 85271 & 86340 & 87405 & 88467 & 89525 & 90581 & 91633 & 92683 \\
\hline 28 & Gre & & & & & & 38216 & 38659 & 39101 & 39543 \\
\hline 29 & & & & & & 135435 & 137420 & 139408 & 141397 & 143388 \\
\hline 30 & Hancock & 60013 & 60910 & 61807 & 62705 & 63603 & 64502 & 65401 & 66300 & 67200 \\
\hline
\end{tabular}


Table F6, continued

\begin{tabular}{|c|c|c|c|c|c|c|c|c|c|c|}
\hline $\begin{array}{l}\text { No. } \\
===\end{array}$ & $\begin{array}{l}\text { County } \\
\text { Name }\end{array}$ & 2007 & 2008 & 2009 & 2010 & $\begin{array}{l}\text { Year } \\
2011\end{array}$ & 2012 & 2013 & 2014 & 2015 \\
\hline 31 & Harrison & 37939 & 38482 & 39024 & 39567 & 40110 & 40653 & 41197 & $===== \pm$ & $=====$ \\
\hline 32 & Hendricks & 103431 & 105076 & 106722 & 108369 & 110017 & 111666 & $\begin{array}{r}41197 \\
113315\end{array}$ & $\begin{array}{r}41741 \\
114965\end{array}$ & $\begin{array}{r}42286 \\
116616\end{array}$ \\
\hline 33 & Henry & 58631 & 59345 & 60058 & 60769 & 61479 & 62188 & 62896 & 63603 & 64310 \\
\hline 34 & Howard & 97705 & 99080 & 100452 & 101822 & 103188 & 104552 & 105914 & 107273 & 108630 \\
\hline 35 & Huntington & 39759 & $4 \cap 203$ & 40647 & 41091 & 41534 & 41977 & 42420 & 42863 & 43305 \\
\hline 36 & Jackson & 47097 & 47746 & 48395 & 49044 & 49692 & 50340 & 50987 & 51635 & 52282 \\
\hline 37 & Jasper & 30679 & 31092 & 3150.5 & 31917 & 32 & 32741 & 33153 & 33564 & 33976 \\
\hline 38 & Jay & 25917 & 26171 & 26424 & 26678 & 26930 & 27183 & 27436 & 27688 & 27940 \\
\hline 39 & Jefferson & 31948 & 32380 & 32811 & 33243 & 33674 & 34 & 34536 & 34966 & 35397 \\
\hline 40 & Jennings & 27569 & 27962 & 28356 & 28750 & 29144 & 29539 & 29934 & 30329 & 30724 \\
\hline 41 & Johnson & 103030 & 104639 & 106248 & 107859 & 109470 & 111082 & 112696 & 114 & 115925 \\
\hline 42 & Knox & 43432 & 43913 & 44392 & 44871 & 45348 & 45825 & 46301 & 46776 & 47250 \\
\hline 43 & Kosciusko & 79742 & 80856 & 81 & 83087 & 84204 & 85321 & 86439 & 87558 & 88677 \\
\hline 44 & Lagrange & 27285 & 27648 & 28011 & 28375 & 28740 & 29104 & 29469 & 29835 & 30200 \\
\hline 45 & Lake & 445999 & 452004 & 457998 & 463982 & 469954 & 475917 & 481870 & 487813 & 493748 \\
\hline $\begin{array}{l}46 \\
47\end{array}$ & $\begin{array}{l}\text { Laporte } \\
\text { Lawrence }\end{array}$ & $\begin{array}{r}115230 \\
51710\end{array}$ & $\begin{array}{r}116775 \\
52410\end{array}$ & $\begin{array}{r}118315 \\
53109\end{array}$ & $\begin{array}{r}119852 \\
53807\end{array}$ & $\begin{array}{r}121384 \\
54505\end{array}$ & $\begin{array}{r}122913 \\
55203\end{array}$ & $\begin{array}{r}124439 \\
55900\end{array}$ & $\begin{array}{r}125963 \\
56598\end{array}$ & $\begin{array}{r}127485 \\
57294\end{array}$ \\
\hline 48 & Madison & 151779 & 153765 & 155747 & 157725 & 159700 & 161670 & 163638 & 165602 & 167563 \\
\hline 49 & Marion & 821356 & 832073 & 842761 & 853425 & 864065 & 8746 & 885 & 895 & 906451 \\
\hline 50 & Marshal1 & 49089 & 49711 & 50333 & 50955 & 51577 & 52199 & 52822 & 53445 & 54068 \\
\hline 51 & Martin & 13225 & 13403 & 13582 & 13760 & 13938 & 14115 & 14293 & 14470 & 14647 \\
\hline 52 & Miami & 44918 & 45475 & 46030 & 46583 & 47136 & 47688 & 48240 & 48790 & 49341 \\
\hline 53 & Monroe & 100973 & 102500 & 104026 & 105551 & 107075 & 108599 & 110123 & 111645 & 113168 \\
\hline 54 & Montgomery & 41216 & $\begin{array}{l}41699 \\
70447\end{array}$ & 42181 & 42663 & 43145 & 43626 & 44107 & 44587 & 45068 \\
\hline 55 & Morgan & 69398 & 70447 & & 72549 & 73600 & 74652 & 75705 & 76758 & 77811 \\
\hline 56 & Newton & 16403 & 16598 & 16793 & 16988 & 17182 & 17376 & 17570 & 17764 & 17957 \\
\hline 57 & Nob1e & 44385 & 44961 & 45537 & 46113 & 46689 & 47266 & 47842 & 48418 & 48995 \\
\hline 58 & Oh1o & $\begin{array}{r}5922 \\
\end{array}$ & 5995 & 6068 & 6141 & 6214 & 6287 & 6360 & 6434 & 6507 \\
\hline 59 & Orange & 21653 & 21933 & 22 & 22492 & & 23051 & 23330 & 23609 & 23888 \\
\hline 60 & Owen & 20519 & 20796 & 21074 & 21353 & 21633 & 21914 & 22195 & 22476 & 22758 \\
\hline 61 & Parke & 18414 & 18626 & 18837 & 19048 & 1925 & 19467 & 19676 & 19884 & 20092 \\
\hline 62 & Perry & 22855 & 23166 & 23476 & 23785 & 24094 & 24402 & 24709 & 25016 & 25323 \\
\hline
\end{tabular}


Table F6, continued

\begin{tabular}{|c|c|c|c|c|c|c|c|c|c|c|}
\hline \multirow{3}{*}{\multicolumn{2}{|c|}{$\mid \begin{array}{l}\text { County } \\
\text { No. } \quad \text { Name } \\
==============\end{array}$}} & \multicolumn{9}{|c|}{ Year } \\
\hline & & 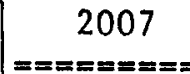 & 2008 & 2009 & 2010 & 2011 & 2012 & 2013 & 2014 & 2015 \\
\hline & & \multicolumn{9}{|c|}{ 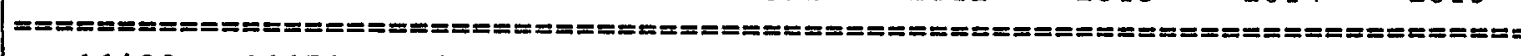 } \\
\hline 63 & Pike & 16483 & 16678 & 16873 & 17068 & 17262 & 17456 & 17649 & 17843 & 18036 \\
\hline 64 & Porter & 146350 & 148672 & 150995 & 153318 & 155642 & 157966 & 160291 & 162615 & 164941 \\
\hline 65 & Posey & 32731 & 33168 & 33605 & 34041 & 34478 & 34915 & 35352 & 35789 & 36226 \\
\hline 66 & Pulask1 & 16308 & 16486 & 16663 & 16839 & 17016 & 17192 & 17369 & 17545 & 17721 \\
\hline 67 & Putnam & 33194 & 33635 & 34076 & 34516 & 34956 & 35396 & 35835 & 36274 & 36713 \\
\hline 68 & Randolph & 31140 & 31422 & 31705 & 31987 & 32269 & 32550 & 32832 & 33113 & 33394 \\
\hline 69 & Ripley & 29376 & 29762 & 30147 & 30532 & 30917 & 31303 & 31688 & 32074 & 32460 \\
\hline 70 & Rush & 21540 & 21786 & 22030 & 22273 & 22516 & 22757 & 22998 & 23238 & 23477 \\
\hline 71 & St. Joseph & 249610 & 252582 & 255552 & 258520 & 261488 & 264455 & 267421 & 270387 & 273352 \\
\hline 72 & $S \cot t$ & 23879 & 24223 & 24566 & 24909 & 25252 & 25595 & 25938 & 26281 & 26624 \\
\hline 73 & Shelby & 48535 & 49178 & 49821 & 50464 & 51105 & 51747 & 52387 & 53028 & 53668 \\
\hline 74 & Spencer & 24759 & 25100 & 25441 & 25782 & 26123 & 26463 & 26804 & 27145 & 27487 \\
\hline 75 & Starke & 24981 & 25303 & 25624 & 25945 & 26266 & 26586 & 26906 & 27225 & 27544 \\
\hline 76 & Steuben & 32632 & 33056 & 33482 & 33908 & 34337 & 34766 & 35196 & 35627 & 36058 \\
\hline 77 & Sul1ivan & 24103 & 24379 & 24655 & 24929 & 25202 & 25475 & 25747 & 26018 & 26289 \\
\hline 78 & Switzerland & 7565 & 7650 & 7734 & 7818 & 7903 & 7987 & 8071 & 8156 & 8240 \\
\hline 79 & Tippecanoe & 121571 & 123250 & 124929 & 126606 & 128283 & 129959 & 131634 & 133308 & 134981 \\
\hline 80 & Tipton & 21549 & 21801 & 22053 & 22304 & 22554 & 22804 & 23054 & 23303 & 23551 \\
\hline 81 & Union & 8751 & 8856 & 8961 & 9065 & 9170 & 9274 & 9379 & 9483 & 9587 \\
\hline 82 & Vanderburgh & 178794 & 181047 & 183294 & 185536 & 187772 & 190003 & 192230 & 194453 & 196671 \\
\hline 83 & Vermillion & 20174 & 20398 & 20623 & 20846 & 21070 & 21293 & 21516 & 21738 & 21961 \\
\hline 84 & Vigo & 105607 & 106840 & 108071 & 109300 & 110527 & 111751 & 112974 & 114194 & 115413 \\
\hline 85 & Wabash & 41580 & 42074 & 42567 & 43060 & 43552 & 44043 & 44533 & 45024 & 45513 \\
\hline 86 & Warren & 10916 & 11037 & 11158 & 11278 & 11398 & 11518 & 11638 & 11757 & 11877 \\
\hline 87 & Warrick & 56645 & 57495 & 58346 & 59197 & 60048 & 60900 & 61753 & 62605 & 63459 \\
\hline 88 & Washington & 26196 & 26522 & 26848 & 27175 & 27502 & 27830 & 28157 & 28485 & 28813 \\
\hline 89 & Wayne & 75254 & 76104 & 76950 & 77794 & 78635 & 79474 & 80311 & 81146 & 81979 \\
\hline 90 & Wells & 32529 & 32919 & 33308 & 33698 & 34087 & 34477 & 34866 & 35256 & 35645 \\
\hline 91 & White & 29944 & 30313 & 30681 & 31049 & 31417 & 31784 & 32150 & 32517 & 32883 \\
\hline 92 & Whitley & 35015 & 35457 & 35900 & 36343 & 36786 & 37230 & 37674 & 38119 & 38563 \\
\hline & STATE & 3651 & 45939 & 3097 & S & & & & 1200 & \\
\hline
\end{tabular}


Table F7: 1989-2015 Vehicle Registration Forecasts (Combined)

\begin{tabular}{|c|c|c|c|c|c|c|c|c|c|c|}
\hline \multirow{3}{*}{\multicolumn{2}{|c|}{$\mid \begin{array}{l}\text { County } \\
\text { No. } \quad \text { Name } \\
=======x====== \pm\end{array}$}} & \multicolumn{9}{|c|}{ Year } \\
\hline & & 1989 & 1990 & 1991 & 1992 & 1993 & 1994 & 1995 & 1996 & 1997 \\
\hline & & \multicolumn{9}{|c|}{ 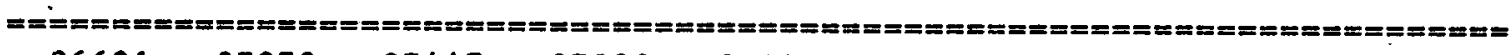 } \\
\hline 1 & Adams & 26691 & 27072 & 27447 & 27820 & 28192 & 28563 & 28935 & 29306 & 29677 \\
\hline 2 & Allen & 249683 & 264067 & 267057 & 276032 & 273892 & 280945 & 282979 & 294011 & 297430 \\
\hline 3 & Bartholomew & 60622 & 61890 & 63139 & 64377 & 65609 & 66837 & 68062 & 69285 & 70505 \\
\hline 4 & Benton & 9462 & 9474 & 9478 & 9566 & 9609 & 9660 & 9706 & 9791 & 9854 \\
\hline 5 & Blackford & 13932 & 14237 & 14511 & 14763 & 15002 & 15230 & 15452 & 15669 & 15882 \\
\hline 6 & Boone & 35 & 36530 & 37159 & 37782 & 38405 & 39028 & 39652 & 40276 & 40901 \\
\hline 7 & Brown & 13142 & 13412 & 13 & 13954 & 14226 & 14497 & 14770 & 15042 & 15315 \\
\hline 8 & Carroll & 19 & 200 & 20 & 20622 & 20905 & 2.1187 & 21467 & 21748 & 22028 \\
\hline 9 & Cass & 35 & 35 & 36236 & 36809 & 37365 & 37 & 38438 & 38960 & 39473 \\
\hline 10 & Clark & 75080 & 766 & 78 & 79885 & 814 & 830 & 84688 & 86 & 87882 \\
\hline 11 & Clay & 22769 & 23146 & 23517 & 23884 & 24 & 7 & 4 & 25 & 25672 \\
\hline 12 & C11nton & 28360 & 28774 & 29186 & 29596 & 30003 & 30409 & 30814 & 31 & 19 \\
\hline 13 & Crawford & 9157 & 9299 & 9442 & 9586 & 9732 & 9878 & 10026 & 74 & 10323 \\
\hline 14 & Daviess & 23979 & 24345 & 24713 & 25080 & 25447 & 25813 & 26179 & 26544 & 26909 \\
\hline 15 & Dearborn & & & 33972 & 34534 & 35096 & 35647 & 36203 & 36766 & 37330 \\
\hline 16 & Decatur & 21631 & 22066 & 22493 & 22913 & 23329 & 23739 & 24147 & 24551 & 24954 \\
\hline 17 & Deka1b & & & 34778 & 35286 & 35794 & 36302 & 36810 & 37320 & 37831 \\
\hline 18 & Delaware & 95427 & 96986 & 98560 & 100137 & 101712 & 103286 & 104857 & 106425 & 107990 \\
\hline 19 & Dubois & & & 57 & 10 & 54 & 37 & 38573 & 39228 & 39884 \\
\hline $\begin{array}{l}20 \\
21\end{array}$ & $\begin{array}{l}\text { E1khart } \\
\text { Fayette }\end{array}$ & $\begin{array}{r}133602 \\
22749\end{array}$ & $\begin{array}{r}136875 \\
23132\end{array}$ & $\begin{array}{r}139188 \\
23512\end{array}$ & $\begin{array}{r}141440 \\
23891\end{array}$ & $\begin{array}{r}143891 \\
24269\end{array}$ & $\begin{array}{r}146622 \\
24645\end{array}$ & $\begin{array}{r}149287 \\
25020\end{array}$ & $\begin{array}{r}151786 \\
25394\end{array}$ & $\begin{array}{r}154200 \\
25766\end{array}$ \\
\hline 22 & Floyd & 52428 & 53382 & 54336 & 55291 & 56246 & 57201 & 58156 & 59111 & 60066 \\
\hline 23 & Fountain & 16046 & 16275 & 16501 & 16723 & 16942 & 17159 & 17373 & 17586 & 17797 \\
\hline 24 & Franklin & 17551 & 17831 & 18114 & 18401 & 18690 & 18981 & 19275 & 19570 & 19867 \\
\hline 25 & Fu1ton & 18395 & 18604 & 18812 & 19021 & 19229 & 19438 & 19646 & 19855 & 20063 \\
\hline 26 & Gibson & 29816 & 30215 & 30638 & 31072 & 31512 & 31955 & 32400 & 32846 & 33291 \\
\hline 27 & Grant & 63771 & 64877 & 65976 & 67068 & 68154 & 69233 & 70307 & 71376 & 72440 \\
\hline 28 & Greene & 27854 & 28293 & 28731 & 29168 & 29604 & 30039 & 30473 & 30907 & 31340 \\
\hline 29 & Hamilton & 92727 & 94947 & 97134 & 99294 & 101430 & 103544 & 105640 & 107721 & 109788 \\
\hline 30 & Hancock & 44140 & 45065 & 45966 & 46858 & 47747 & 48636 & 49525 & 50414 & 51303 \\
\hline
\end{tabular}


Table F7, continued

\begin{tabular}{|c|c|c|c|c|c|c|c|c|c|}
\hline County & \multicolumn{9}{|c|}{ Year } \\
\hline Name & 1989 & 1990 & 1991 & 1992 & 1993 & 1994 & 1995 & 1996 & 1997 \\
\hline 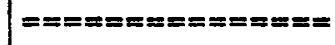 & & & & & & & & & \\
\hline $\begin{array}{l}31 \text { Harrison } \\
32 \text { Hendricks }\end{array}$ & $\begin{array}{l}28443 \\
74278\end{array}$ & $\begin{array}{l}28987 \\
75984\end{array}$ & $\begin{array}{l}29531 \\
77648\end{array}$ & $\begin{array}{l}30076 \\
79297\end{array}$ & $\begin{array}{l}30621 \\
80941\end{array}$ & $\begin{array}{l}31167 \\
82584\end{array}$ & $\begin{array}{l}31712 \\
84227\end{array}$ & $\begin{array}{l}32258 \\
85871\end{array}$ & $\begin{array}{l}32804 \\
87515\end{array}$ \\
\hline 33 Henry & 45462 & 46193 & 46921 & 47646 & 48368 & 49087 & 49804 & 50519 & 51232 \\
\hline 34 Howard & 72446 & 73820 & 75191 & 76558 & 77921 & 79282 & 80639 & 81993 & 83344 \\
\hline 35 Huntington & 31733 & 32176 & 32617 & 33058 & 33498 & 33938 & 34376 & 34814 & 35252 \\
\hline $\begin{array}{l}36 \text { Jackson } \\
37 \text { Jasper }\end{array}$ & $\begin{array}{l}35148 \\
23107\end{array}$ & $\begin{array}{l}35798 \\
23514\end{array}$ & $\begin{array}{l}36446 \\
23920\end{array}$ & $\begin{array}{l}37093 \\
24325\end{array}$ & $\begin{array}{l}37737 \\
24730\end{array}$ & $\begin{array}{l}38381 \\
25133\end{array}$ & $\begin{array}{l}39023 \\
25536\end{array}$ & $\begin{array}{l}39664 \\
25938\end{array}$ & $\begin{array}{l}40304 \\
26340\end{array}$ \\
\hline $38 \mathrm{Jay}$ & 20495 & 20818 & 21123 & 21415 & 21695 & 21967 & 22232 & 22491 & 22747 \\
\hline 39 Jefferson & 23856 & 24293 & 24725 & 25153 & 25579 & 26001 & 26422 & 26841 & 27259 \\
\hline 40 Jennings & 20686 & 21078 & 21470 & 21863 & 22257 & 22650 & 23044 & 23439 & 23833 \\
\hline 41 Johnson & 74422 & 76048 & 77664 & 79276 & 80888 & 82499 & 84112 & 85725 & 87339 \\
\hline 42 Knox & 34383 & 34798 & 35236 & 35687 & 36144 & 36604 & 37065 & 37527 & 37988 \\
\hline 43 Kosciusko & 60419 & 61594 & 62574 & 63577 & 64679 & 65822 & 66943 & 68038 & 69130 \\
\hline 44 Lagrange & 20907 & 21277 & 21636 & 21994 & 22351 & 22708 & 23065 & 23423 & 23782 \\
\hline 45 Lake & 335391 & 341550 & 347635 & 353677 & 359693 & 365689 & 371671 & 377642 & 383603 \\
\hline 46 Laporte & 85159 & 86804 & 88429 & 90037 & 91629 & 93207 & 94772 & 96327 & 97872 \\
\hline 47 Lawrence & & 39735 & 40446 & 41154 & 41859 & 42563 & 43266 & 43966 & 44666 \\
\hline 48 Madison & 115450 & 117458 & 119460 & 121457 & 123447 & 125433 & 127414 & 129390 & 131362 \\
\hline 49 Marion & 6114 & 625790 & 637071 & 648240 & 659312 & 670295 & 681202 & 692040 & 702817 \\
\hline 50 Marsha11 & 38092 & 38703 & 39315 & 39927 & 40539 & 41152 & 41765 & 42378 & 42992 \\
\hline 51 Martin & 9895 & 10074 & 10252 & 10428 & 10605 & 10780 & 10955 & 11130 & 11304 \\
\hline $52 \mathrm{Miami}$ & 33945 & 34598 & 35155 & 35752 & 36364 & 36950 & 37520 & 38089 & 38655 \\
\hline 53 Monroe & 72849 & 74377 & 75897 & 77413 & 78923 & $8043 n$ & 81932 & 83431 & 84928 \\
\hline 54 Montgomery & 32357 & 32851 & 333.34 & 33812 & 34289 & 34765 & 35239 & 35712 & 36185 \\
\hline 55 Morgan & 50721 & 51747 & 52774 & 53802 & 54831 & 55862 & 56893 & 57926 & 58959 \\
\hline 56 Newton & 12748 & 1302.3 & 13218 & 13413 & 13608 & 13801 & 13994 & 14187 & 14379 \\
\hline 57 Noble & 34273 & 34809 & 35353 & 35904 & 36459 & 37017 & 37578 & 38141 & 38704 \\
\hline 58 Ohio & 4659 & 4729 & 4800 & 4871 & 4942 & 5013 & 5084 & 5156 & 5228 \\
\hline 59 Orange & 16504 & 16786 & 17067 & 17346 & 17625 & 17904 & 18182 & 18459 & 18736 \\
\hline 60 Owen & 15950 & 16203 & 16460 & 16719 & 16981 & 17246 & 17512 & 17781 & 18051 \\
\hline 61 Parke & 14148 & 14380 & 14563 & 14782 & 14982 & 15194 & 15399 & 15607 & 15811 \\
\hline 62 Perry & 16719 & 17054 & 17384 & 17708 & 18028 & 18345 & 18658 & 18969 & 19277 \\
\hline
\end{tabular}


Table F7, continued

\begin{tabular}{|c|c|c|c|c|c|c|c|c|c|}
\hline \multirow{3}{*}{ 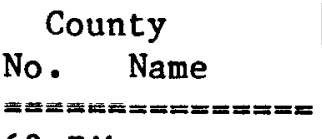 } & \multicolumn{9}{|c|}{ Year } \\
\hline & 989 & 1990 & 1991 & 1992 & 1993 & 1994 & 1995 & 1996 & 1997 \\
\hline & & & angra & 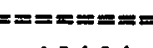 & $== \pm==$ & $==m=x$ & 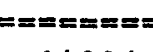 & $:=== \pm$ & $==== \pm$ \\
\hline 63 Pike & 12835 & 13032 & 13229 & 13424 & 13619 & 13813 & 14006 & 14198 & 14390 \\
\hline 64 Porter & 105307 & 108040 & 110626 & 113116 & 115543 & 117930 & 120290 & 122633 & 124966 \\
\hline 65 Posey & 248 & 25222 & 25640 & 26063 & 26489 & 26916 & 27343 & 27771 & 28200 \\
\hline 66 Pulaski & 13035 & 13212 & 13388 & 13564 & 13739 & 13913 & 14087 & 14261 & 14434 \\
\hline 67 Putnam & 25 & 7 & 25917 & 26356 & 26 & 27229 & 27664 & 28097 & 28530 \\
\hline 68 Rando1ph & 25928 & 26173 & 26408 & 26637 & 268 & 27085 & 27307 & 27527 & 27746 \\
\hline 69 Ripley & 22504 & 228 & 23246 & 236 & & 24363 & 24737 & 25111 & 25485 \\
\hline 70 Rush & 16417 & 16708 & 3 & 17 & 4 & 17813 & 18077 & 18 & 18595 \\
\hline 71 St. Joseph & 192334 & 195616 & 198808 & 201 & 205 & 208035 & 211033 & 214008 & 216965 \\
\hline $72 \mathrm{scot} t$ & 17768 & 18108 & 18447 & 18 & 1 & 19 & & & 85 \\
\hline 73 Shelby & 36765 & & 380 & 38 & 39 & 40 & & & 16 \\
\hline 74 Spencer & 18652 & 18983 & 193 & 19 & 19 & 20 & 20 & 20 & 05 \\
\hline 75 Starke & 190 & 19 & 19 & 19 & & & & 21 & 21568 \\
\hline 76 Steuben & 256 & 25 & 26 & 26 & 27. & 27 & 27 & & \\
\hline 77 Sullivan & 18511 & 18822 & 19 & 19 & 19 & 20 & 20 & 20 & 20875 \\
\hline 78 Switzerland & 6040 & 6122 & 6203 & 5 & & 49 & & 2 & 6694 \\
\hline 79 Tippecanoe & 91053 & 92751 & 94419 & 96 & 97 & 99 & 101 & 102 & 104290 \\
\hline 80 Tipton & 16710 & 16974 & 17235 & 174 & 17 & 04 & & & 58 \\
\hline 81 Union & 6796 & 6902 & 7007 & 7111 & 7215 & 19 & 7422 & 7524 & 7626 \\
\hline 82 Vanderburgh & 135536 & 139081 & 139910 & 141470 & 142313 & 145347 & 147367 & 149721 & 150716 \\
\hline 83 Vermillion & 15938 & 16169 & 16398 & 16626 & 16853 & 17078 & 17303 & 17526 & 17749 \\
\hline 84 V1go & 82854 & 840 & 85334 & 86570 & 878 & 89034 & 90263 & 91489 & 92712 \\
\hline 85 Wabash & 32134 & 32653 & 33166 & 33675 & 341 & 34679 & 35177 & 35671 & 36163 \\
\hline 86 Warren & 8456 & 8595 & 8730 & 8861 & 9 & 9114 & 9238 & 9360 & 9481 \\
\hline 87 Warrick & 41556 & 42445 & 43313 & 44171 & 45024 & 45874 & 46724 & 47573 & 48422 \\
\hline 88 Washington & 20448 & 20773 & 21091 & 21406 & 21721 & 22036 & 22351 & 22666 & 22982 \\
\hline 89 Wayne & & & & 61500 & 62375 & 63242 & 64103 & 64959 & 65809 \\
\hline 90 Wells & & 26046 & 26433 & 26819 & 27206 & 27593 & 27979 & 28366 & 28752 \\
\hline 91 White & & & & & & & & 25833 & 26193 \\
\hline 92 Whitley & 27241 & 27682 & 28117 & 28550 & 28984 & 29419 & 29854 & 30289 & 30726 \\
\hline STATE & & & & & & & & & \\
\hline
\end{tabular}


Table F7, continued

\begin{tabular}{|c|c|c|c|c|c|c|c|c|c|c|}
\hline \multirow{2}{*}{\multicolumn{2}{|c|}{$\begin{array}{l}\text { County } \\
\text { No. Name } \\
===== \pm========\end{array}$}} & \multicolumn{9}{|c|}{ Year } \\
\hline & & \multicolumn{9}{|c|}{ 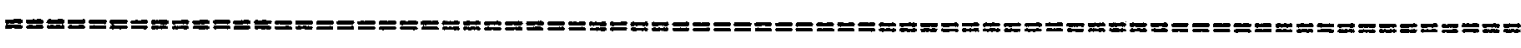 } \\
\hline 1 & Adams & 30048 & 30419 & 30791 & 31162 & 31534 & 31905 & 32277 & 32648 & 33020 \\
\hline 2 & Allen & 304881 & 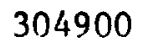 & 311096 & 3139 & & 326623 & 333129 & 334661 & 340339 \\
\hline 3 & Bartholomew & 71723 & 72938 & 74153 & 75365 & & 77786 & 78994 & 80202 & 81408 \\
\hline 4 & Benton & 21 & 998 & 10067 & 10138 & 11 & 10283 & 10362 & 10436 & 10511 \\
\hline 5 & ford & 1 & & 1 & & & & 17 & & 60 \\
\hline 6 & Boone & 415 & 42 & 42 & 7 & & 446 & 45292 & & 50 \\
\hline 7 & Bro & 15588 & 15 & 161 & 16 & & 7 & 17232 & & 81 \\
\hline 8 & Carroll & 22307 & 22587 & 22866 & 23146 & 23 & 23704 & 23983 & 24 & 24541 \\
\hline 9 & Cass & 39980 & 40482 & 40979 & 41472 & 41962 & 42449 & 42934 & 43418 & 43900 \\
\hline 10 & Clark & 89476 & 910 & 92660 & & & 97 & 99013 & 100 & 102183 \\
\hline 11 & $\mathrm{Cl}$ & 26023 & & 267 & & & & & & \\
\hline 12 & $\mathrm{C} 11$ & 32020 & 32 & 32819 & & & & 8 & & 199 \\
\hline 13 & $\mathrm{Cr}$ & 10 & 10 & 107 & & & 7 & 11 & & \\
\hline 14 & Dav & 27273 & 27 & 280 & & & & & & 173 \\
\hline 15 & Dea & 37895 & 384 & 390 & 39 & 40 & 40741 & 413 & & 42460 \\
\hline 16 & Dec & 25354 & 257 & 261 & 265 & 26 & 27338 & 277 & 26 & 28520 \\
\hline 17 & Deka1b & 343 & & 393 & 39 & 4 & 40912 & 41428 & 44 & 42460 \\
\hline 18 & Delaware & 109553 & 1111 & 112672 & 114 & 11 & 117 & 118883 & 120 & 121977 \\
\hline 19 & Dubois & 40540 & 41 & 41853 & 42 & 4 & 43825 & 44483 & 41 & 45799 \\
\hline 20 & E1khart & 156669 & $159 ?$ & 161740 & 164 & 16 & 169 & 171670 & 67 & 176654 \\
\hline 21 & $\mathrm{Fa}$ & & & 26 & & 2 & 27 & 28353 & 20 & 29087 \\
\hline 22 & F1oy & 61021 & & 625 & & & & $66^{\circ}$ & & 68668 \\
\hline 23 & Fo & & & & & & & & & \\
\hline 24 & 1 in & 201 & 20 & 20763 & 210 & & 21666 & 219 & & 22573 \\
\hline 25 & $\mathrm{Fu}$ & & & & & & & & & 21939 \\
\hline 26 & G1 & 3 & 34 & 34628 & & & & 36 & & 37295 \\
\hline 27 & Gri & 7 & & & & & & & & 81847 \\
\hline 28 & Gre & 31772 & 32 & 32636 & 33 & 3 & 29 & & & 35219 \\
\hline 29 & Ham & 111843 & 113887 & 115923 & 117951 & 1190 & $121 \mathrm{c}$ & 1240 & 126 & 128011 \\
\hline 30 & ck & 52193 & 53084 & 75 & 54867 & 55759 & 56651 & 57544 & 58437 & 59331 \\
\hline
\end{tabular}


Table F7, continued

\begin{tabular}{|c|c|c|c|c|c|c|c|c|c|c|}
\hline \multirow{2}{*}{\multicolumn{2}{|c|}{$\begin{array}{l}\text { County } \\
\text { No. } \quad \text { Name } \\
==========\equiv \equiv \equiv \equiv \equiv=0\end{array}$}} & 1998 & 1999 & 2000 & 2001 & $\begin{array}{l}\text { Year } \\
2002\end{array}$ & 2003 & 2004 & 2005 & 2006 \\
\hline & & & & $======0$ & & & $==$ & & & . \\
\hline $\begin{array}{l}31 \\
22\end{array}$ & Harrison & 33351 & 33898 & 34445 & 34992 & 35539 & 36087 & 36635 & 37183 & 37731 \\
\hline 32 & Hendricks & 89161 & 90807 & 92454 & 94102 & 95751 & 97400 & 99050 & 100700 & 102351 \\
\hline 33 & Henry & 51943 & 52652 & 53360 & 54067 & 54772 & 55476 & 56179 & 56881 & 57583 \\
\hline 34 & Howard & 84693 & 86039 & 87383 & 88725 & 90064 & 91401 & 92736 & 94070 & 95402 \\
\hline 35 & Huntington & 35689 & 36126 & 36562 & 36998 & 37434 & 37869 & 38304 & 38739 & 39173 \\
\hline 36 & Jackson & 40943 & 41581 & 42218 & 42855 & 43491 & 44127 & 44762 & 45397 & 46031 \\
\hline 37 & Jasper & 26741 & 27142 & 27543 & 27943 & 28343 & 28742 & 29142 & 29541 & 29940 \\
\hline 38 & Jay & 22999 & 23248 & 23496 & 23741 & 23986 & 24230 & 24472 & 24714 & 24956 \\
\hline 39 & Jefferson & 27675 & 28090 & 28505 & 28918 & 29332 & 29744 & 30157 & 30569 & 30980 \\
\hline 40 & Jennings & 24228 & 24624 & 25019 & 25415 & 25811 & 26208 & 26604 & 27001 & 27398 \\
\hline 41 & Johnson & 88954 & 90570 & 92187 & 93804 & 95423 & 97042 & 98661 & 100282 & 101903 \\
\hline 42 & Knox & 38449 & 38909 & 39368 & 39827 & 40285 & 40742 & 41199 & 41655 & 42110 \\
\hline 43 & Kosciusko & 70234 & 71346 & 72458 & 73568 & 74677 & 75789 & 76902 & 78015 & 79129 \\
\hline 44 & Lagrange & 24140 & 24500 & 24859 & 25219 & 25579 & 25939 & 26300 & 26661 & 27022 \\
\hline 45 & Lake & 389555 & 395498 & 401433 & 407361 & 413282 & 419195 & 425102 & 431002 & 436895 \\
\hline 46 & Laporte & 99409 & 100938 & 102459 & 103975 & 105486 & 106992 & 108493 & 109992 & 111486 \\
\hline 47 & Lawrence & 45364 & 46061 & 46758 & 47453 & 48148 & 48843 & 49537 & 50230 & 50923 \\
\hline 48 & Madison & 133330 & 135294 & 137255 & 139212 & 141166 & 143117 & 145065 & 147011 & 148954 \\
\hline 49 & Marion & 713541 & 724217 & 734850 & 745446 & 756009 & 766542 & 777050 & 787534 & 797997 \\
\hline $\begin{array}{l}50 \\
51\end{array}$ & $\begin{array}{l}\text { Marshall } \\
\text { Martin }\end{array}$ & $\begin{array}{l}43606 \\
11478\end{array}$ & $\begin{array}{l}44220 \\
11651\end{array}$ & $\begin{array}{l}44834 \\
11824\end{array}$ & $\begin{array}{l}45449 \\
11997\end{array}$ & $\begin{array}{l}46064 \\
12169\end{array}$ & $\begin{array}{l}46679 \\
12342\end{array}$ & $\begin{array}{l}47294 \\
12513\end{array}$ & $\begin{array}{l}47909 \\
12685\end{array}$ & $\begin{array}{l}48525 \\
12857\end{array}$ \\
\hline 52 & Miami & 39215 & 39770 & 40322 & 40872 & 41420 & 41965 & 42509 & 43051 & 43592 \\
\hline 53 & Monroe & 86422 & 87914 & 89404 & 90893 & 92380 & 93866 & 95352 & 96836 & 98320 \\
\hline 54 & Montgomery & 36657 & 37128 & 37599 & 38069 & 38539 & 39008 & 39477 & 39945 & 40413 \\
\hline 55 & Morgan & 59993 & 61028 & 62063 & 63100 & 64137 & 65174 & 66212 & 67250 & 68289 \\
\hline $\begin{array}{l}56 \\
57\end{array}$ & $\begin{array}{l}\text { Newton } \\
\text { Noble }\end{array}$ & $\begin{array}{l}14570 \\
39269\end{array}$ & $\begin{array}{l}14761 \\
39834\end{array}$ & $\begin{array}{l}14952 \\
40400\end{array}$ & $\begin{array}{l}15143 \\
40966\end{array}$ & $\begin{array}{l}15333 \\
41533\end{array}$ & $\begin{array}{l}15523 \\
42099\end{array}$ & $\begin{array}{l}15712 \\
42666\end{array}$ & $\begin{array}{l}15901 \\
43233\end{array}$ & $\begin{array}{l}16090 \\
43800\end{array}$ \\
\hline 58 & Ohio & 5300 & 5372 & 5444 & 5516 & 5589 & 5661 & 5734 & 5807 & 5879 \\
\hline 59 & Orange & 19012 & 19288 & 19564 & 19840 & 20115 & 20390 & 20665 & 20939 & 21214 \\
\hline 60 & Owen & 18323 & 18597 & 18871 & 19147 & 19424 & 19702 & 19980 & 20260 & 20540 \\
\hline 61 & Parke & 16016 & 16218 & 16421 & 16622 & 16822 & 17022 & 17221 & 17420 & 17618 \\
\hline 62 & Perry & 19583 & 19887 & 20190 & 20491 & 20791 & 21090 & 21388 & 21686 & 21982 \\
\hline
\end{tabular}


Table F7, continued

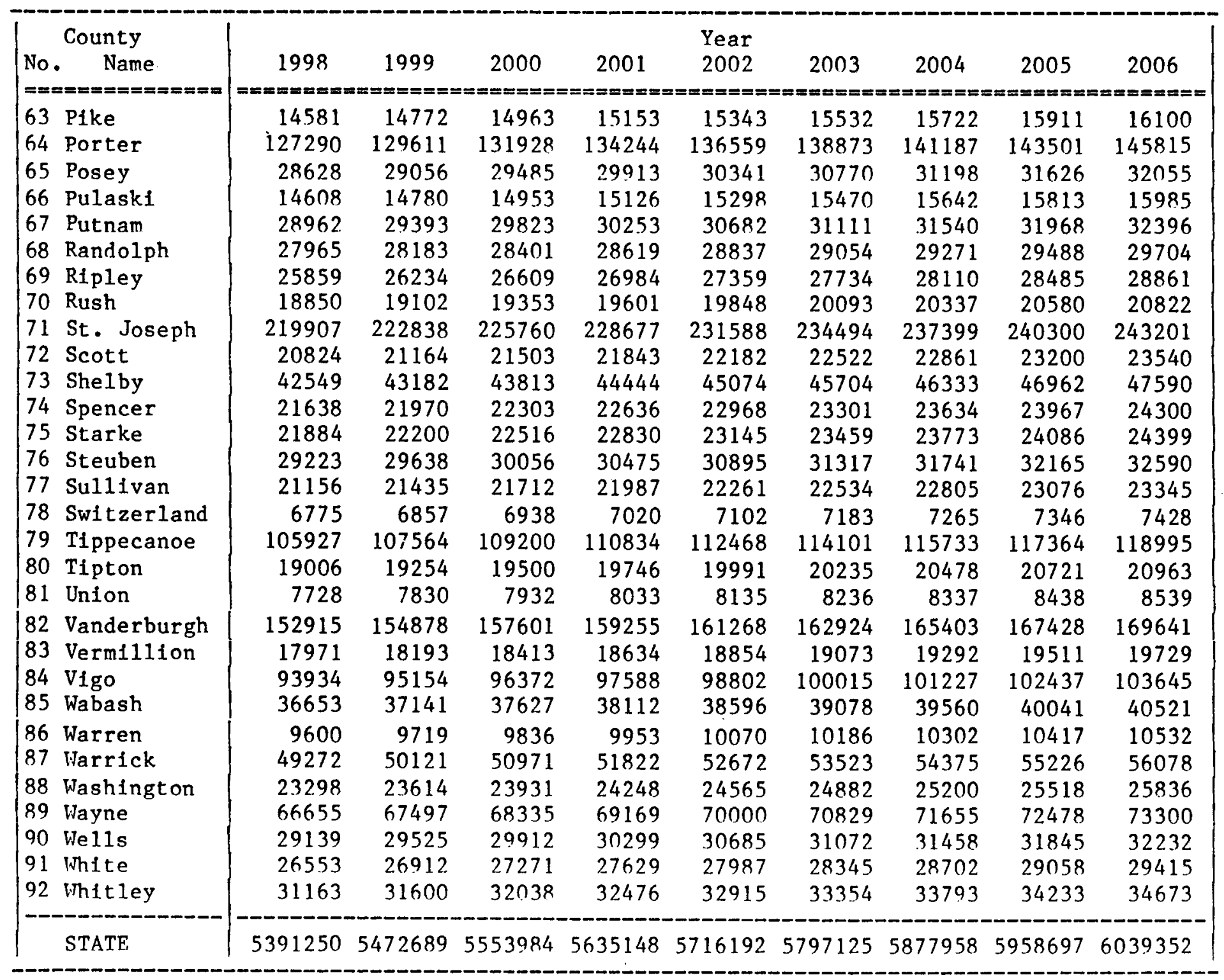


Table F7, continued

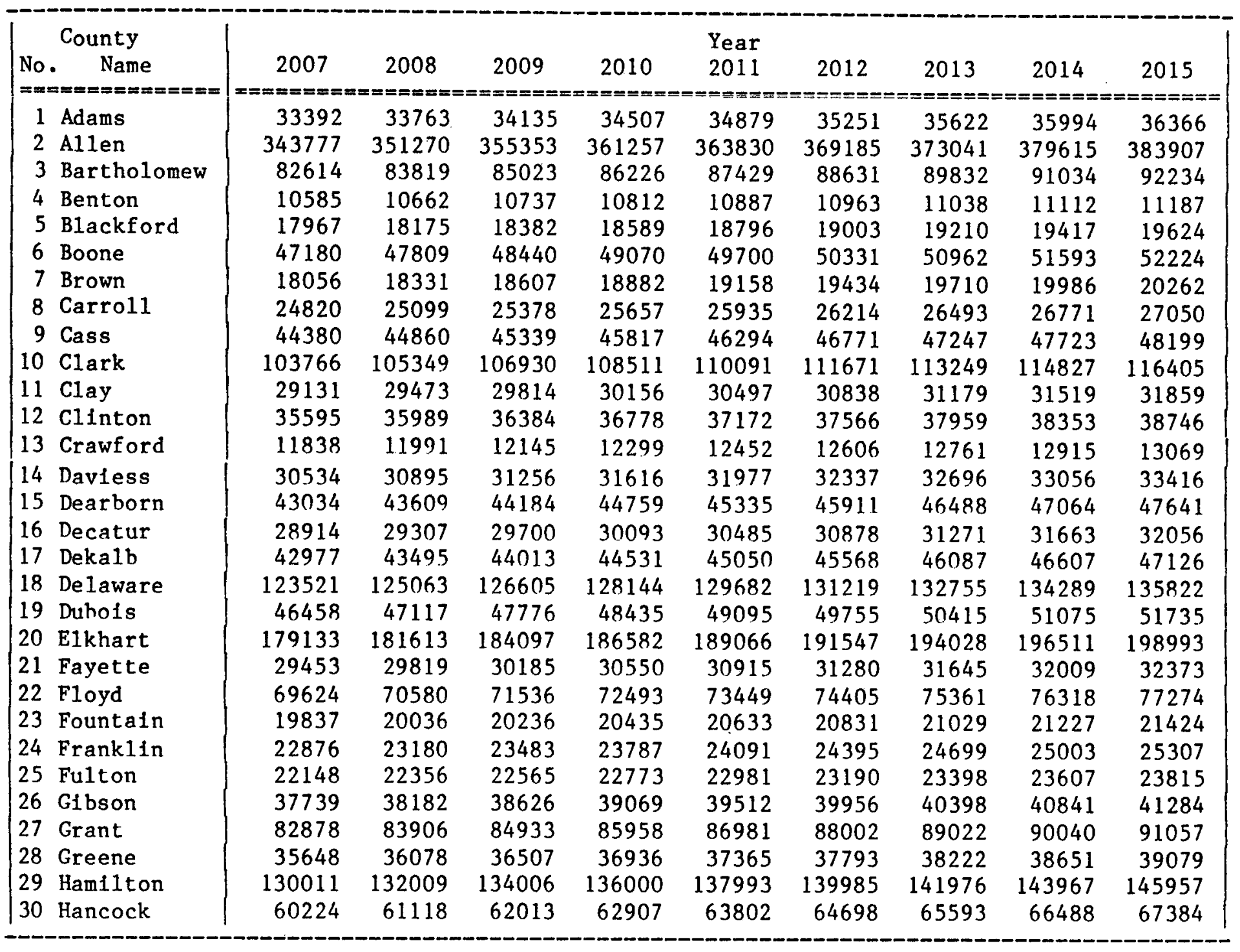


Table F7, continued

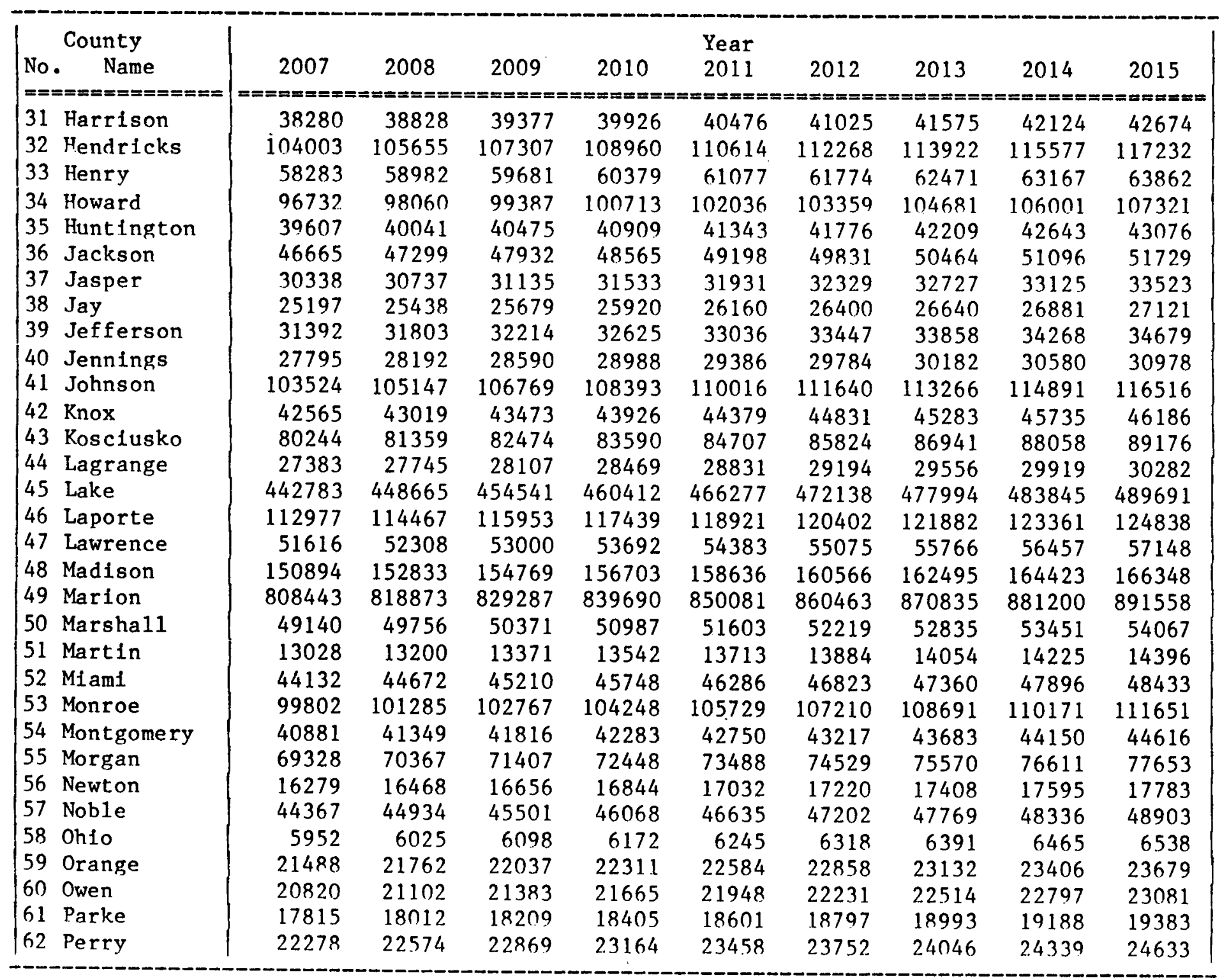


Table F7, continued

\begin{tabular}{|c|c|c|c|c|c|c|c|c|c|c|}
\hline No. & $\begin{array}{l}\text { County } \\
-\quad \text { Name }\end{array}$ & 2007 & 2008 & 2009 & 2010 & $\begin{array}{l}\text { Year } \\
2011\end{array}$ & 2012 & 2013 & 2014 & 2015 \\
\hline$===$ & 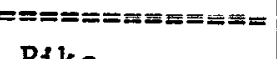 & $=======0$ & $======$ & & $=====0$ & & $== \pm==0$ & $:======$ & $s==s===$ & $==x==x=$ \\
\hline $\begin{array}{l}63 \\
64 \\
65\end{array}$ & $\begin{array}{l}\text { Pike } \\
\text { Porter } \\
\text { Posey }\end{array}$ & $\begin{array}{r}16288 \\
148129 \\
32483\end{array}$ & $\begin{array}{r}16477 \\
150443 \\
32911\end{array}$ & $\begin{array}{r}16665 \\
152757 \\
33340\end{array}$ & $\begin{array}{r}16853 \\
155071 \\
33768\end{array}$ & $\begin{array}{r}17041 \\
157386 \\
34196\end{array}$ & $\begin{array}{r}17229 \\
159700 \\
34624\end{array}$ & $\begin{array}{r}17417 \\
162015 \\
35052\end{array}$ & $\begin{array}{r}17605 \\
164330 \\
35481\end{array}$ & $\begin{array}{r}17792 \\
166645 \\
35909\end{array}$ \\
\hline $\begin{array}{l}66 \\
67\end{array}$ & $\begin{array}{l}\text { Pulaski } \\
\text { Putnam }\end{array}$ & $\begin{array}{l}16156 \\
32824\end{array}$ & $\begin{array}{l}16328 \\
33251\end{array}$ & $\begin{array}{l}16499 \\
33678\end{array}$ & $\begin{array}{l}16670 \\
34105\end{array}$ & $\begin{array}{l}16841 \\
34532\end{array}$ & $\begin{array}{l}17012 \\
34959\end{array}$ & $\begin{array}{l}17183 \\
35386\end{array}$ & $\begin{array}{l}17354 \\
35812\end{array}$ & $\begin{array}{l}17525 \\
36239\end{array}$ \\
\hline $\begin{array}{l}67 \\
68\end{array}$ & $\begin{array}{l}\text { Putnam } \\
\text { Randolph }\end{array}$ & $\begin{array}{l}32824 \\
29921\end{array}$ & $\begin{array}{l}33251 \\
30137\end{array}$ & 30353 & 30569 & 30785 & 31001 & 31216 & 31432 & 31647 \\
\hline 69 & Ripley & 29237 & 29612 & 29988 & 30364 & 30740 & 31116 & 31492 & 31868 & 32244 \\
\hline 70 & Rush & 21064 & 21304 & 21544 & 21783 & 22022 & 22260 & 22498 & 22736 & 22973 \\
\hline 71 & St. Joseph & 246099 & 248997 & 251894 & 254790 & 257686 & 260581 & 263476 & 266371 & 269265 \\
\hline 72 & $S \cot t$ & 23879 & 24219 & 24558 & 24898 & 25237 & 25577 & 25916 & 26256 & 26595 \\
\hline 73 & Shelby & 48218 & 48846 & 49473 & 50100 & 50726 & 51353 & 51979 & 52605 & 53231 \\
\hline 74 & Spencer & 24633 & 24966 & 25299 & 25632 & 25966 & 26299 & 26632 & 26965 & 27299 \\
\hline 75 & Starke & 24712 & 25024 & 25337 & 25649 & 25961 & 26272 & 26584 & 26896 & 27207 \\
\hline 76 & Steuben & 33016 & 33443 & 33871 & 34300 & 34729 & 35158 & 35588 & 36019 & 36449 \\
\hline 77 & Sullivan & 23614 & 23882 & 24150 & 24417 & 24683 & 24949 & 25215 & 25480 & 25745 \\
\hline 78 & Swltzerland & 7509 & 7591 & 7672 & 7754 & 7835 & 7917 & 7999 & 8080 & 8162 \\
\hline 79 & Tippecanoe & 120625 & 122254 & 123883 & 125511 & 127139 & 128767 & 130394 & 132020 & 133646 \\
\hline 80 & Tipton & 21205 & 21447 & 21688 & 21929 & 22169 & 22410 & 22650 & 22890 & 23129 \\
\hline 81 & Union & 8640 & 8740 & 8841 & 8942 & 9042 & 9143 & 9244 & 9344 & 9445 \\
\hline 82 & Vanderburgh & 171318 & 173471 & 175472 & $177779^{\circ}$ & 179667 & 181727 & 183622 & 185832 & 187846 \\
\hline 83 & Vermillion & 19947 & 20165 & 20383 & 20601 & 20818 & 21035 & 21252 & 21470 & 21686 \\
\hline 84 & V1go & 104852 & 106058 & 107262 & 108466 & 109668 & 110869 & 112070 & 113269 & 114467 \\
\hline 85 & Wabash & 41000 & 41480 & 41958 & 42436 & 42914 & 43391 & 43869 & 44346 & 44822 \\
\hline 86 & Warren & 10647 & 10762 & 10877 & 10991 & 11105 & 11220 & 11334 & 11448 & 11562 \\
\hline 87 & Warrick & 56930 & 57783 & 58635 & 59488 & 60342 & 61195 & 62049 & 62903 & 63757 \\
\hline 88 & Washington & 26154 & 26473 & 26792 & 27110 & 27429 & 27748 & 28068 & 28387 & 28706 \\
\hline 89 & Wayne & 74119 & 74937 & 75753 & 76568 & 77382 & 78195 & 79006 & 79817 & 80626 \\
\hline 90 & We1ls & 32618 & 33005 & 33392 & 33778 & 34165 & 34552 & 34938 & 35325 & 35712 \\
\hline 91 & White & 29771 & 30127 & 30483 & 30838 & 31193 & 31548 & 31903 & 32258 & 32612 \\
\hline 92 & Whitley & 35113 & 35554 & 35994 & 36435 & 36876 & 37318 & 37759 & 38201 & 38642 \\
\hline & STATE & 6119929 & 434 & 0874 & 1254 & 41578 & 32 & 080 & 2266 & 6762412 \\
\hline
\end{tabular}


Table F8: Sourrounding County and Township for Cities of Indiana

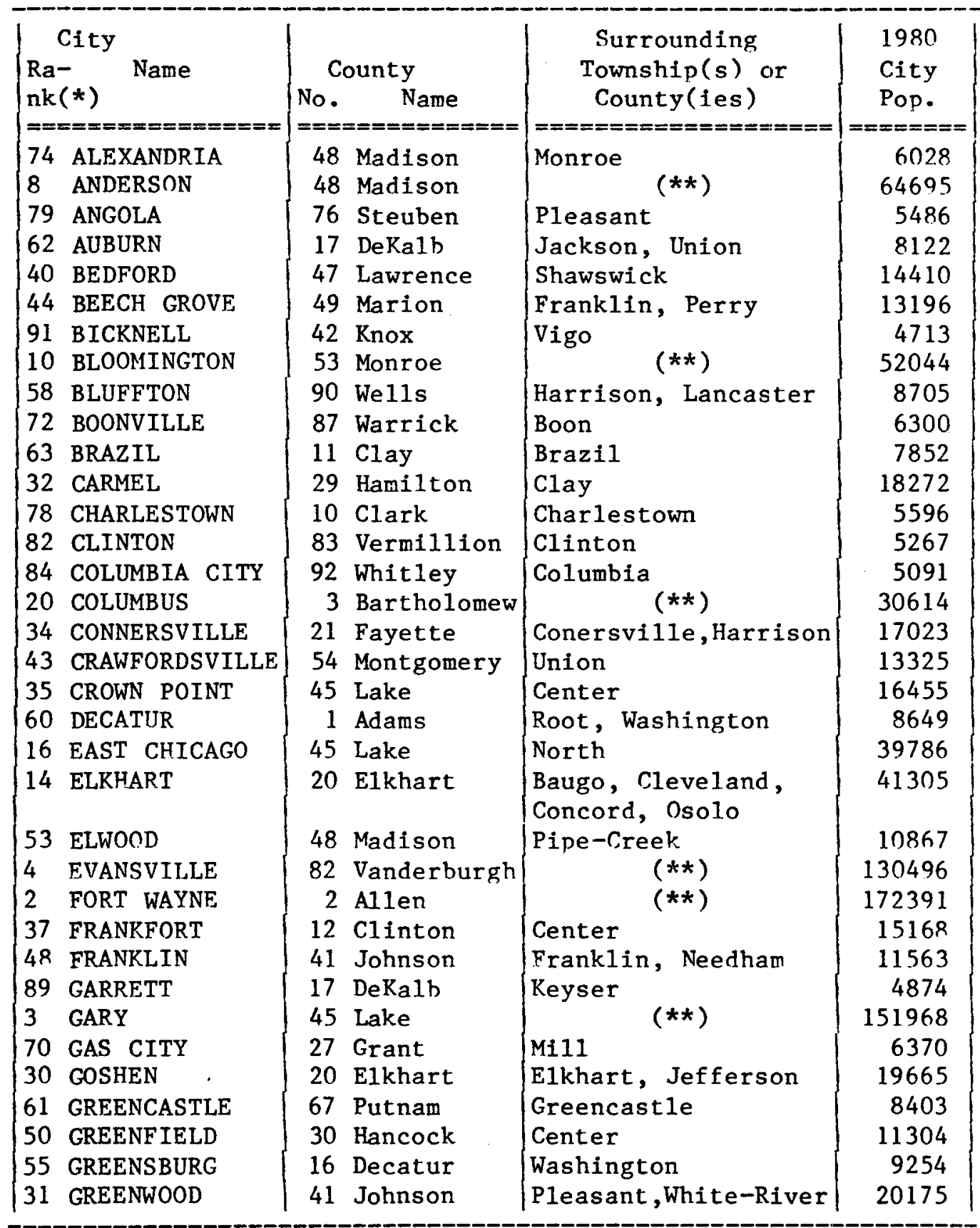


Table F8, continued

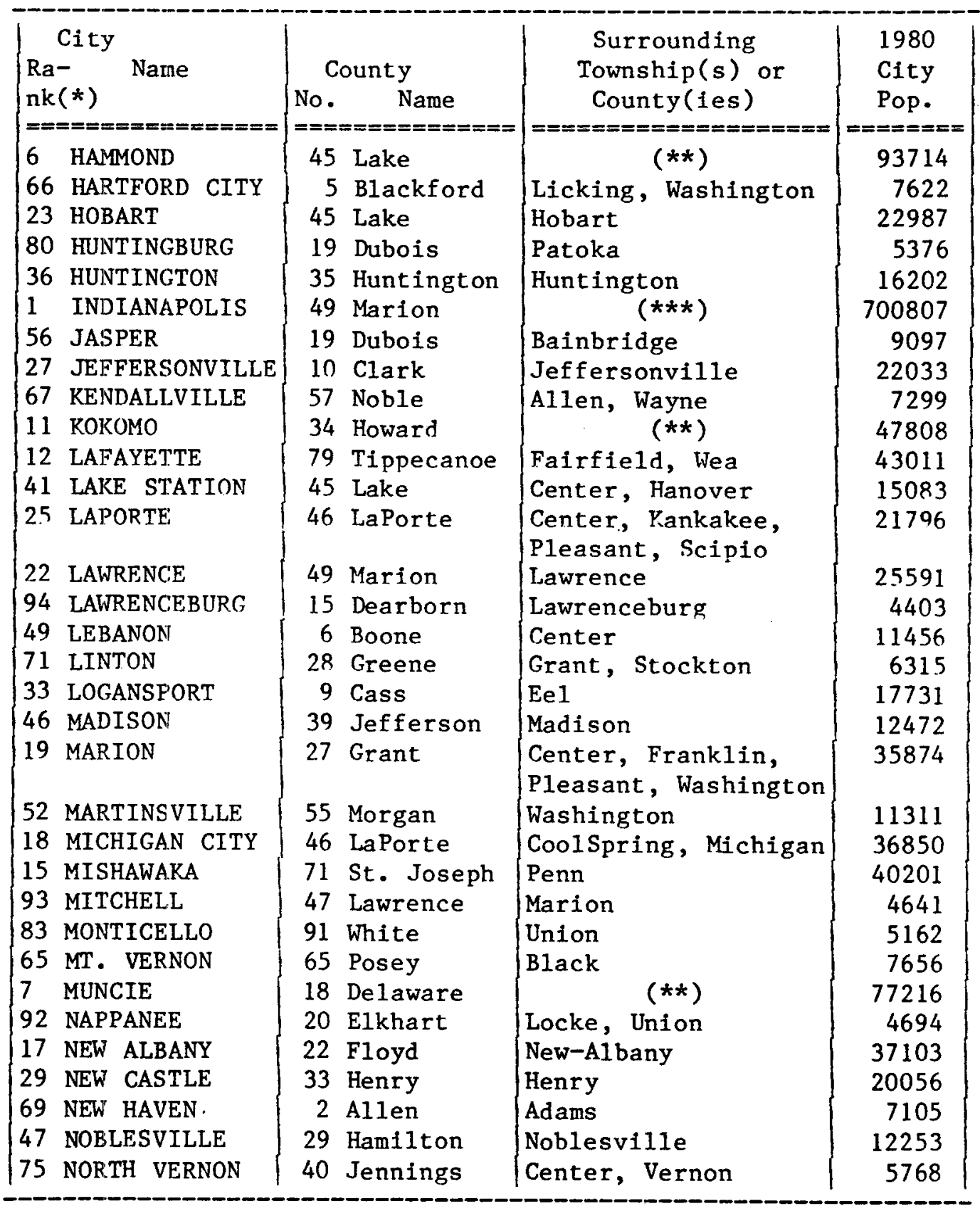


Table F8, continued

\begin{tabular}{|c|c|c|c|}
\hline \multirow{3}{*}{$\begin{array}{l}\text { City } \\
\mathrm{Ra} \\
\mathrm{nk}(*) \\
========x========\end{array}$} & \multirow{3}{*}{$\begin{array}{l}\text { County } \\
0 . \quad \text { Name } \\
============\end{array}$} & Surrounding & 1980 \\
\hline & & Township(s) or & City \\
\hline & & $\begin{array}{c}\text { County(ies) } \\
==========-x=======\end{array}$ & $\begin{array}{l}\text { Pop. } \\
=======\end{array}$ \\
\hline 42 PERU & 52 Miami & Peru, Washington & 13764 \\
\hline 64 PLYMOUTH. & 50 Marshall & Center & 7693 \\
\hline 21 PORTAGE & 64 Porter & Portage & 27409 \\
\hline 68 PORTLAND & 38 Jay & Wayne & 7074 \\
\hline 57 PRINCETON & 26 Gibson & Patoka & 8976 \\
\hline 88 RENSSELAER & 37 Jasper & Marion & 4994 \\
\hline 13 RICHMOND & 89 Wayne & Center, Wayne & 41349 \\
\hline 86 ROCHESTER & 25 Fulton & Rochester & 5050 \\
\hline 73 RUSHVILLE & 70 Rush & Rushville & 6113 \\
\hline 81 SALEM & 88 Washington & Washington & 5290 \\
\hline 85 SCOTTSBURG & $72 S \cot t$ & Vienna & 5068 \\
\hline 38 SEYMOUR & 36 Jackson & Jackson, Redding & 15050 \\
\hline 39 SHELBYVILLE & 73 Shelby & Addison & 15003 \\
\hline 5 SOUTH BEND & 71 St. Joseph & $(* *)$ & 109727 \\
\hline 90 SULLIVAN & 77 Sullivan & Hamilton & 4774 \\
\hline 59 TELL CITY & 62 Perry & Troy & 8704 \\
\hline 9 TERRE HAUTE & 84 Vigo & $(* *)$ & 61125 \\
\hline 87 TIPTON & 80 Tipton & Cicero & 5004 \\
\hline 24 VALPARAISO & 64 Porter & Center, Washington & 22247 \\
\hline 28 VINCENNES & $42 \mathrm{Knox}$ & Vincennes & 20857 \\
\hline 45 WABASH & 85 Wabash & Noble & 12985 \\
\hline 54 WARSAW & 43 Kosciusko & Wayne & 10647 \\
\hline 51 WASHINGTON & 14 Daviess & Washington & 11325 \\
\hline 26 WEST LAFAYETTE & 79 Tippecanoe & Wabash & 21247 \\
\hline 77 WHITING & 45 Lake & North & 5630 \\
\hline 76 WINCHESTEP & 68 Randolph & White-River & 5659 \\
\hline
\end{tabular}

(*) Based on 1980 City Population

(**) County in Second Column

(***) 49-Marion, 29-Hami1ton, 32-Hendricks, 55-Morgan, 41-Johnson, 73-She1by, 30-Hancock, 6-Boone 
Table F9: City Population Forecasts by Ratio Method

\begin{tabular}{|c|c|c|c|c|c|c|c|c|c|}
\hline${ \hline \multirow { 3 } { * }\multicolumn{2}{|c|}{\begin{array}{l}\text { City } \\
\mathrm{Ra}- \\
\mathrm{nk}(*) \\
================\end{array}}}$ & \multicolumn{8}{|c|}{ YEAR } \\
\hline & & 1985 & 1990 & 1995 & 2000 & 2005 & 2010 & 2015 & 2020 \\
\hline & & \multicolumn{8}{|c|}{ 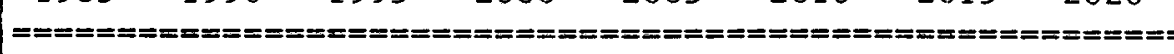 } \\
\hline 74 & ALEXANDRIA & 6145 & 6262 & 6351 & 6390 & 6406 & 6412 & 6412 & 6390 \\
\hline 8 & ANDERSON & 64147 & 64003 & 63934 & 63827 & 63729 & 63613 & 63479 & 63232 \\
\hline 79 & ANGOLA & 5825 & 6111 & 6327 & 6479 & 6584 & 6660 & 6719 & 6725 \\
\hline 62 & AUBURN & 8427 & 8724 & 8993 & 9254 & 9523 & 9741 & 9937 & 10096 \\
\hline 40 & BEDFORD & 14592 & 14767 & 14900 & 14984 & 14991 & 14956 & 14907 & 14816 \\
\hline 44 & BEECH GROVE & 13521 & 13905 & 14158 & 14269 & 14320 & 14363 & 14427 & 14455 \\
\hline 91 & BICKNELL & 4627 & 4619 & 4550 & 4465 & 4422 & 4397 & 4431 & 4439 \\
\hline 10 & BLOOMINGTON & 52297 & 53651 & 55568 & 57186 & 58682 & 60352 & 62559 & 65336 \\
\hline 58 & BLUFFTON & 8988 & 9284 & 9554 & 9811 & 10055 & 10266 & 10443 & 10568 \\
\hline 72 & BOONVILLE & 6603 & 6901 & 7188 & 7447 & 7663 & 7894 & 8109 & 8280 \\
\hline 63 & BRAZIL & 7815 & 7777 & 7749 & 7721 & 7759 & 7815 & 7871 & 7936 \\
\hline 32 & CARMEL & 21471 & 24379 & 26968 & 29232 & 31042 & 32045 & 32342 & 32202 \\
\hline 78 & CHARLESTOWN & 5888 & 6162 & 6387 & 6551 & 6667 & 6721 & 6709 & 6642 \\
\hline 82 & CLINTON & 5402 & 5504 & 5585 & 5633 & 5660 & 5666 & 5622 & 5563 \\
\hline 84 & COLUMBIA CITY & 5263 & 5442 & 5607 & 5760 & 5892 & 5991 & 6058 & 6124 \\
\hline 20 & COLUMBUS & 31621 & 32585 & 33365 & 33939 & 34240 & 34217 & 33944 & 33469 \\
\hline 34 & CONNERSVILLE & 17245 & 17512 & 17772 & 18024 & 18291 & 18482 & 18628 & 18734 \\
\hline 43 & CRAWFORDSVILLE & 13531 & 13664 & 13749 & 13761 & 13755 & 13701 & 13610 & 13446 \\
\hline 35 & CROWN POINT & 16791 & 17167 & 17435 & 17578 & 17709 & 17942 & 18284 & 18702 \\
\hline 60 & DECATUR. & 9096 & 9569 & 10047 & 10558 & 11093 & 11635 & 12208 & 12800 \\
\hline 16 & EAST CEICAGO & 37695 & 36181 & 35104 & 34495 & 34380 & 34662 & 35217 & 35845 \\
\hline 14 & ELKHART & 42042 & 42905 & 43794 & 44626 & 45442 & 46216 & 46932 & 47523 \\
\hline 53 & ELWOOD & 10662 & 10557 & 10465 & 10387 & 10324 & 10282 & 10275 & 10261 \\
\hline 4 & EVANSVILLE & 130262 & 130099 & 129226 & 127879 & 126585 & 125705 & 125004 & 124202 \\
\hline 2 & FORT WAYNE & 176204 & 180696 & 184790 & 188228 & 191473 & 194712 & 197898 & 200527 \\
\hline 37 & FRANKFORT & 14964 & 14880 & 14862 & 14899 & 14992 & 15103 & 15205 & 15307 \\
\hline 48 & FRANRLIN & 11620 & 11825 & 12017 & 12173 & 12378 & 12619 & 12832 & 12995 \\
\hline 89 & GARRETT & 4858 & 4890 & 4937 & 5017 & 5104 & 5191 & 5278 & 5342 \\
\hline 3 & GARY & 147391 & 145052 & 143733 & 143445 & 144578 & 146746 & 149518 & 152215 \\
\hline 70 & GAS CITY & 6306 & 6280 & 6242 & 6184 & 6109 & 6056 & 6019 & 5961 \\
\hline 30 & GOSHEN & 20645 & 21610 & 22459 & 23226 & 23936 & 24536 & 25033 & 25435 \\
\hline 61 & GREENCASTLE & 8365 & 8143 & 8013 & 7921 & 7944 & 7944 & 7860 & 7776 \\
\hline 50 & GREENF IELD & 11843 & 12411 & 12915 & 13314 & 13594 & 13755 & 13874 & 13895 \\
\hline 55 & GREENSBURG & 9303 & 9409 & 9515 & 9629 & 9784 & 9931 & 10077 & 10208 \\
\hline 31 & GREENWOOD & 22593 & 24899 & 26867 & 28454 & 29553 & 30007 & 30124 & 29990 \\
\hline
\end{tabular}


Table F9, continued

\begin{tabular}{|c|c|c|c|c|c|c|c|c|c|}
\hline \multirow{2}{*}{\multicolumn{2}{|c|}{$\begin{array}{l}\text { City } \\
\operatorname{Ra}-\quad \text { Name } \\
\mathrm{nk}(*) \\
==x============x=\end{array}$}} & \multicolumn{8}{|c|}{ YEAR } \\
\hline & & 1985 & 1990 & 1995 & 2000 & 2005 & & 2015 & 2020 \\
\hline 6 & HAMMOND & 90892 & 89449 & 88636 & 88458 & 9157 & 494 & & \\
\hline 66 & HARTFORD CITY & 7352 & & & & 6820 & 6777 & 6728 & 6671 \\
\hline 23 & HOBART & 23030 & 23041 & 22998 & 23009 & 23160 & 23495 & 23911 & 24305 \\
\hline 80 & HUNTINGBURG & 5559 & 5774 & 5981 & 6149 & 6.276 & 6387 & 6491 & 6579 \\
\hline 36 & HUNTINGTON & 16251 & 16348 & 16461 & 16631 & 16873 & 17189 & 17496 & 17715 \\
\hline 1 & INDIANAPOLIS & 711536 & 726248 & 738125 & 747442 & 756.381 & 763025 & 767152 & 767849 \\
\hline 56 & JASPER & 9384 & 9724 & 10019 & 10261 & 10434 & 10623 & 10820 & 10971 \\
\hline 27 & JEFFERSONVILLE & 23217 & 24263 & 25111 & & 39 & 26382 & 26339 & 141 \\
\hline 67 & KENDALLVILLE & 7662 & 8031 & 8371 & 8678 & & 81 & 9354 & 9499 \\
\hline 11 & KOKOMO & 48034 & 48567 & 491 & 496 & 50 & 50 & 37 & 30 \\
\hline 12 & LAFAYETTE & 425 & 42748 & & & 45 & 33 & 30 & 93 \\
\hline 41 & LAKE STATION & 15388 & 15708 & 159 & 16033 & & 16353 & 16668 & 17046 \\
\hline 25 & LAPORTE & 21724 & 21822 & 21966 & 22090 & 22 & 22378 & 22528 & 22639 \\
\hline 22 & LAWRENCE & 26920 & 28020 & & 28981 & $29:$ & 29302 & 29241 & 28975 \\
\hline 94 & - LAWRENCEBURG & 4549 & 4695 & 48 & 4951 & 9 & 5183 & 5293 & 5384 \\
\hline 49 & LEBANON & 12077 & 12683 & 132 & 13 & 14244 & 14643 & 14945 & 15145 \\
\hline 71 & LINTON & 6483 & & & & 6905 & 6938 & 6952 & 6965 \\
\hline 33 & LOGANSPORT & 17065 & 16642 & 16 & 16 & 16145 & 16 & 16 & 16530 \\
\hline 46 & MADISON & 12749 & 130 & 132 & 13 & 13794 & & 13 & 47 \\
\hline 19 & MARION & 34212 & 331 & 323 & & 313 & & 30 & 22 \\
\hline 52 & MARTINSVILLE & 11882 & 124 & 130 & 13 & 138 & & 13 & 14432 \\
\hline 18 & MICHIGAN CITY & 36865 & 37022 & & & & & 4 & \\
\hline 15 & MISHAWAKA & 40906 & & & & & & 42401 & \\
\hline 93 & MITCHELL & 4855 & 5032 & 5162 & 525 & 5297 & 5292 & 5276 & 5224 \\
\hline 83 & MONTICELLO & 5383 & 5581 & 5725 & 58 & 5890 & 5956 & 5973 & 5995 \\
\hline 65 & MT. VERNON & 7884 & 81 & 8317 & & 8603 & & & \\
\hline 7 & MUNCIE & 75102 & 74592 & 74778 & 74970 & 75246 & 75727 & 76543 & 77768 \\
\hline 92 & NAPPANEE & 4893 & 5087 & 5257 & 5415 & & & 5807 & \\
\hline 17 & NEW ALBANY & 36773 & 366 & & & & & 37069 & 37171 \\
\hline 29 & NEW CASTLE & 19373 & 18953 & 18645 & 18390 & 18203 & 18075 & 17948 & 17798 \\
\hline 69 & NEW HAVEN & 7101 & 7158 & 7230 & 7290 & 7328 & 7377 & 7444 & 7515 \\
\hline 47 & NOBLESVILLE & 13648 & 14932 & 16099 & 17131 & 18000 & 18584 & 18941 & 19116 \\
\hline 75 & NORTH VERNON & 6025 & 6294 & 6516 & 6674 & 6797 & 6878 & 6902 & 6931 \\
\hline
\end{tabular}


Table F9, continued

\begin{tabular}{|c|c|c|c|c|c|c|c|c|c|}
\hline \multirow{3}{*}{\multicolumn{2}{|c|}{$\mid \begin{array}{l}\text { City } \\
\mathrm{Ra}- \\
\operatorname{nk}(*) \\
=================\end{array}$}} & \multicolumn{8}{|c|}{ YEAR } \\
\hline & & 5 & 1990 & 1 & 2000 & & & & \\
\hline & & & & & & & $==m===$ & $x===$ & - \\
\hline & $\begin{array}{l}\text { PERU } \\
\text { PLYMOUTH }\end{array}$ & $\begin{array}{r}13592 \\
7943\end{array}$ & $\begin{array}{r}13639 \\
8193\end{array}$ & $\begin{array}{r}13733 \\
8423\end{array}$ & $\begin{array}{r}13897 \\
8619\end{array}$ & $\begin{array}{r}14123 \\
8815\end{array}$ & $\begin{array}{r}14419 \\
8991\end{array}$ & $\begin{array}{r}14771 \\
9167\end{array}$ & $\begin{array}{r}15106 \\
9302\end{array}$ \\
\hline 21 & PORTAGE & 30641 & 33701 & 36306 & 38346 & 39821 & 40621 & 40938 & 40855 \\
\hline 68 & PORTLAND & 7082 & 7090 & 7156 & & 0 & 7 & 6 & 08 \\
\hline 57 & PRINCE'TON & 9 & 5 & & & 9769 & & & 26 \\
\hline 88 & RENSSELAER & & 3 & & & 1 & & & 6 \\
\hline 13 & RICHMOND & 400 & 391 & 38 & 38 & 37886 & 37 & 37 & \\
\hline 86 & ROCHESTER & 52 & 54 & 1 & 5 & 8 & 5 & & \\
\hline 73 & RUSHVILLE & 58 & 5665 & 3 & 6 & 4 & & & \\
\hline 81 & SALEM & 5463 & 5647 & 5820 & 5 & 2 & 1 & & 22 \\
\hline 85 & SCOTTSBURG & 52 & 55 & 57 & 2 & 0 & 3 & 2 & \\
\hline 38 & SEYMOUR & 1 & 162 & 16719 & 17142 & 17457 & 17 & 7 & 03 \\
\hline 39 & SHELBYVILLE & 6 & 146 & 145 & 14579 & 14648 & 00 & 52 & 95 \\
\hline 5 & SOUTH BEND & 08 & 107 & 107 & 961 & 106707 & 106780 & 107 & 3 \\
\hline 90 & SULLIVAN & 4829 & 4884 & 4918 & 4966 & 5001 & 5021 & 1 & 28 \\
\hline 59 & TELL CITY & 8641 & 86 & 9 & 8648 & 8691 & 8773 & $8 \varepsilon$ & 54 \\
\hline 9 & TERRE HAUTE & 59412 & 58210 & 57 & 567 & 56323 & 55969 & 55 & 35 \\
\hline 87 & TIPTON & 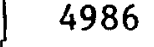 & 49 & & & 5016 & 5022 & & 22 \\
\hline 24 & VALPARAISO & 23912 & 25513 & 270 & 28 & 29478 & 30 & & \\
\hline 28 & VINCENNES & 2 & 20314 & & & 20 & & & \\
\hline 45 & WABASH & 128 & 12812 & 12 & 12 & 12745 & 12 & & 82 \\
\hline 54 & WARSAW & 11683 & 12657 & 13495 & 14213 & 14822 & & & \\
\hline 51 & WASH INGTO & 1 & & & & 12092 & & & \\
\hline 26 & WEST LAFAYETTE & 21 & & & & & & & \\
\hline 77 & WHITING & & & & & & 49 & & 072 \\
\hline 76 & WINCHESTER & 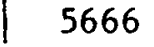 & $3 / 1$ & 578 & & 2001 & 5928 & 5968 & 5961 \\
\hline
\end{tabular}


$\frac{\text { LEGEND: } A=1 \text { OBS, } B=2 \text { OBS, ETC. }}{\text { P-value: } 0.015}$

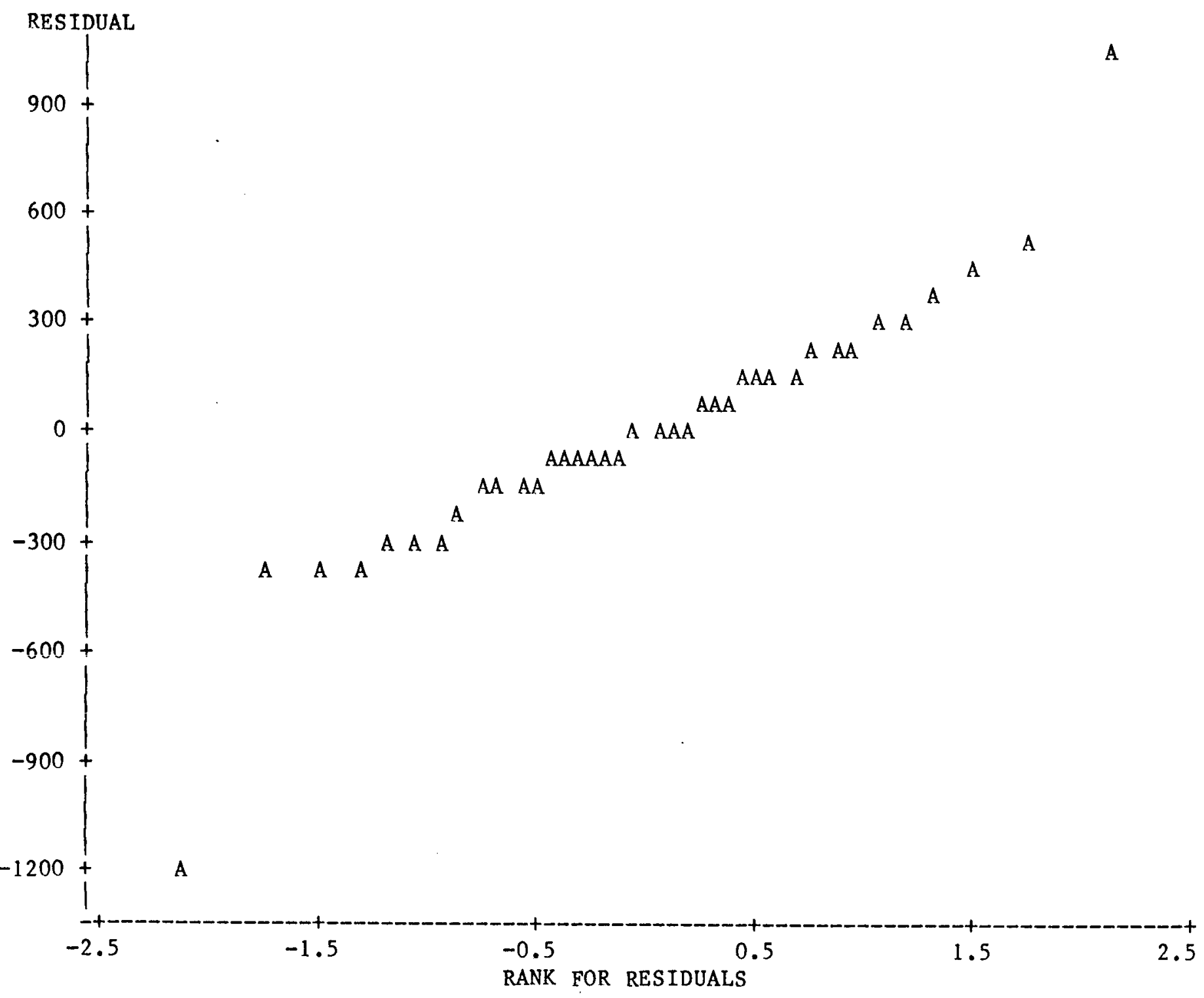

Figure F1.1: Norma1 Probability Plot of Residuals from $\operatorname{ARIMA}(0,1,0)$ 
LEGEND: $A=1$ OBS, $B=2$ OBS, ETC.

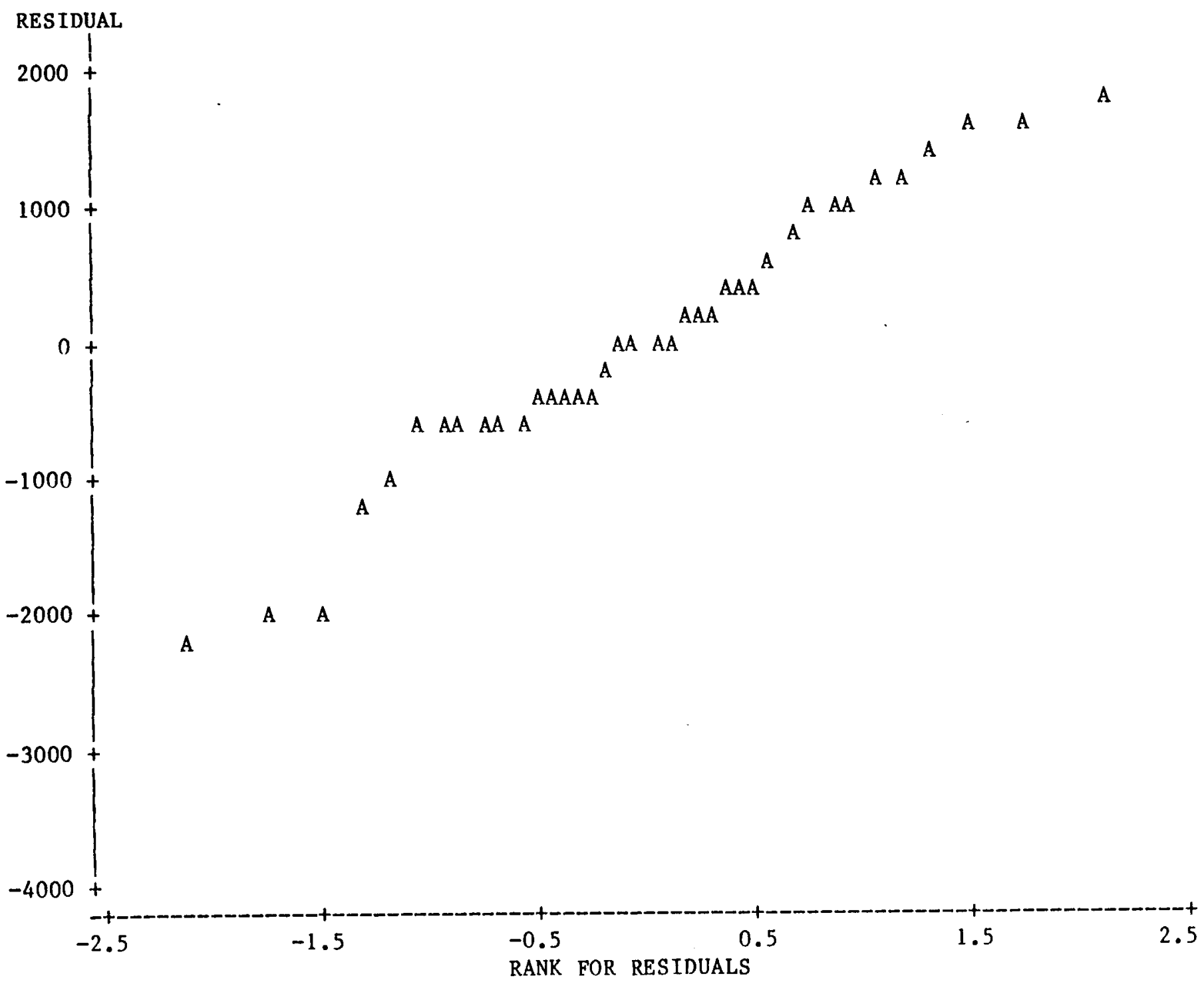

Figure F1.2: Norma1 Probability Plot of Residuals from $\operatorname{ARIMA}(1,1,0)$ 
$\frac{\text { LEGEND: } A=1 \text { OBS, } \mathrm{B}=2 \text { OBS, ETC. }}{\text { P-value: } 0.694}$

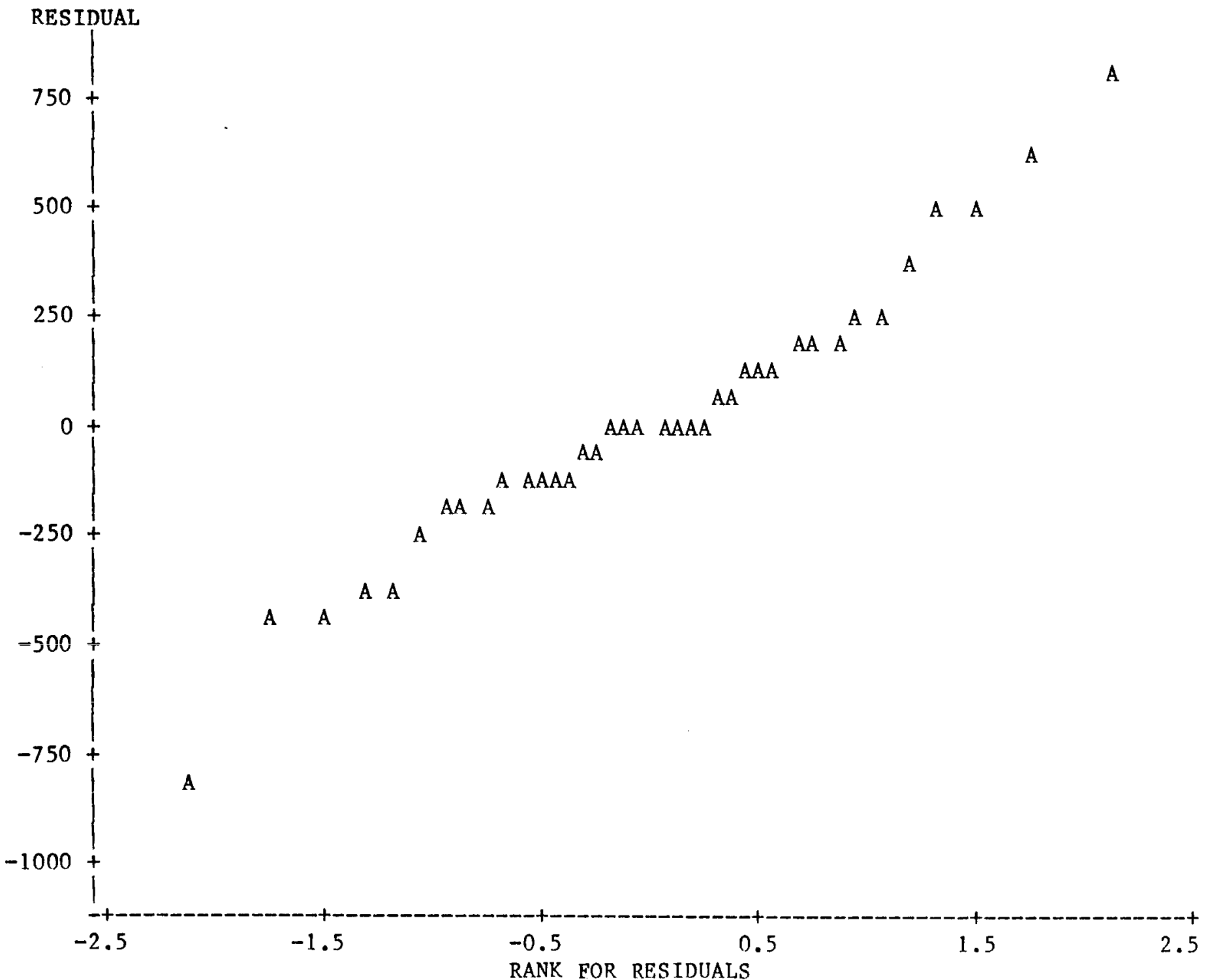

Figure F1.3: Normal Probability Plot of Residuals from $\operatorname{ARIMA}(1,1,0)$ 
$\frac{\text { LEGEND: } A=1 \text { OBS, } \mathrm{B}=2 \text { OBS, ETC. }}{\text { P-value: } 0.092}$

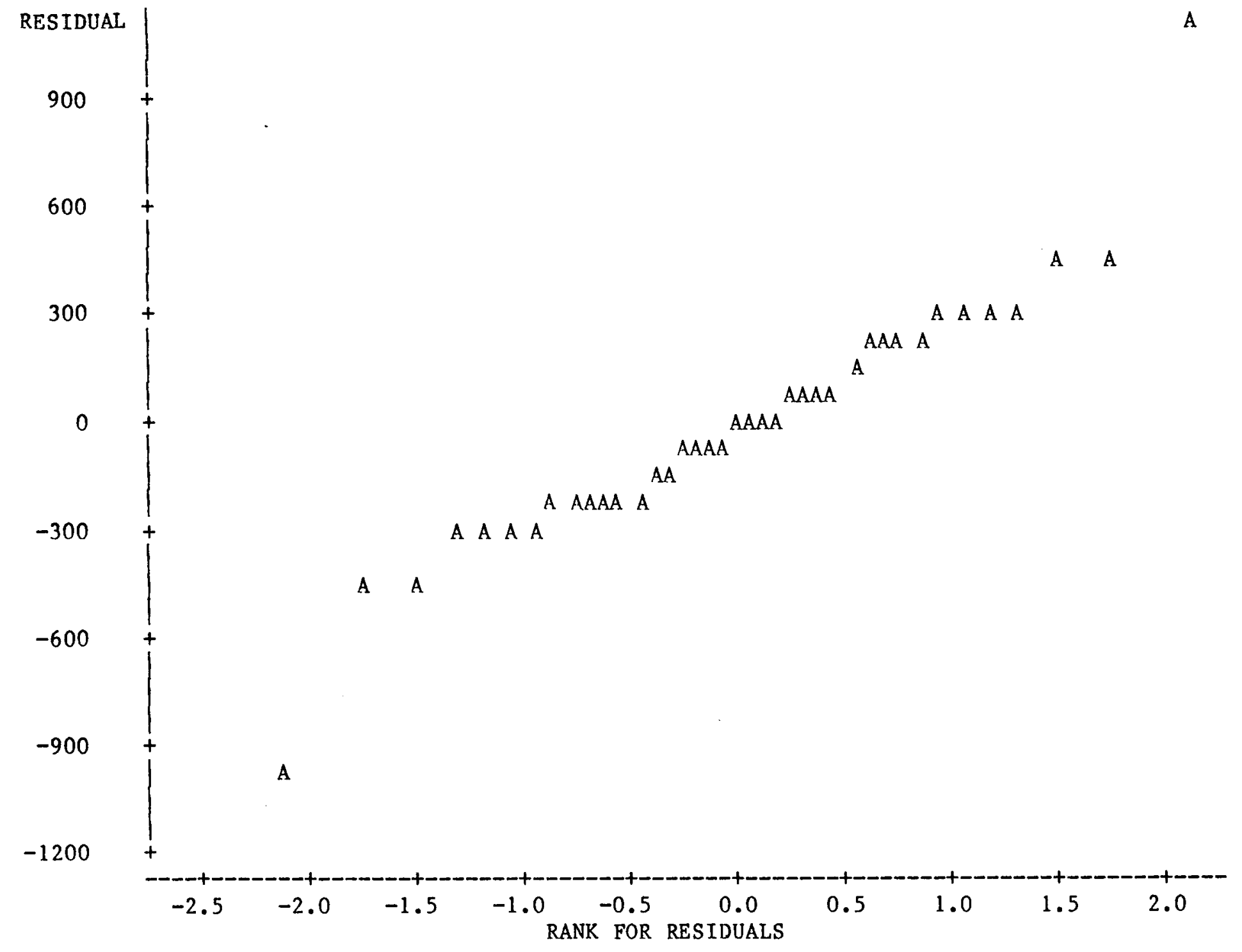

Figure F1.4: Normal Probability Plot of Residuals from Autoregressive Forecasts of Frank1in County Vehicle Registration 
$\frac{\text { LEGEND: } \mathrm{A}=1 \text { OBS, } \mathrm{B}=2 \text { OBS, ETC. }}{\text { P-value: } 0.415}$

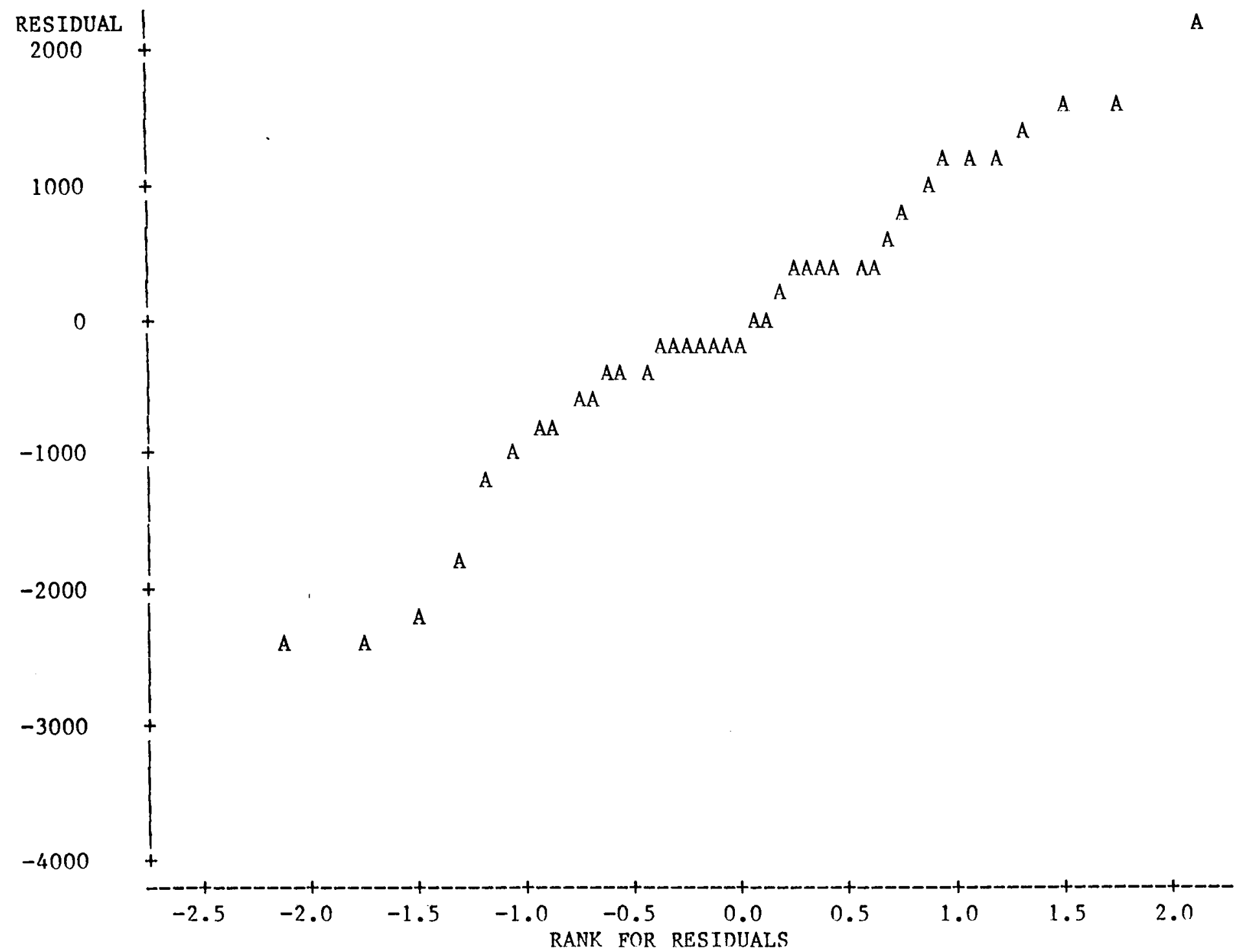

Figure F1.5: Normal Probability P1ot of Residuals from Autoregressive Forecasts of Tippecanoe County Vehicle Registration 
$\frac{\text { LEGEND: } A=1 \text { OBS, } B=2 \text { OBS, ETC. }}{\text { P-value: } 0.775}$

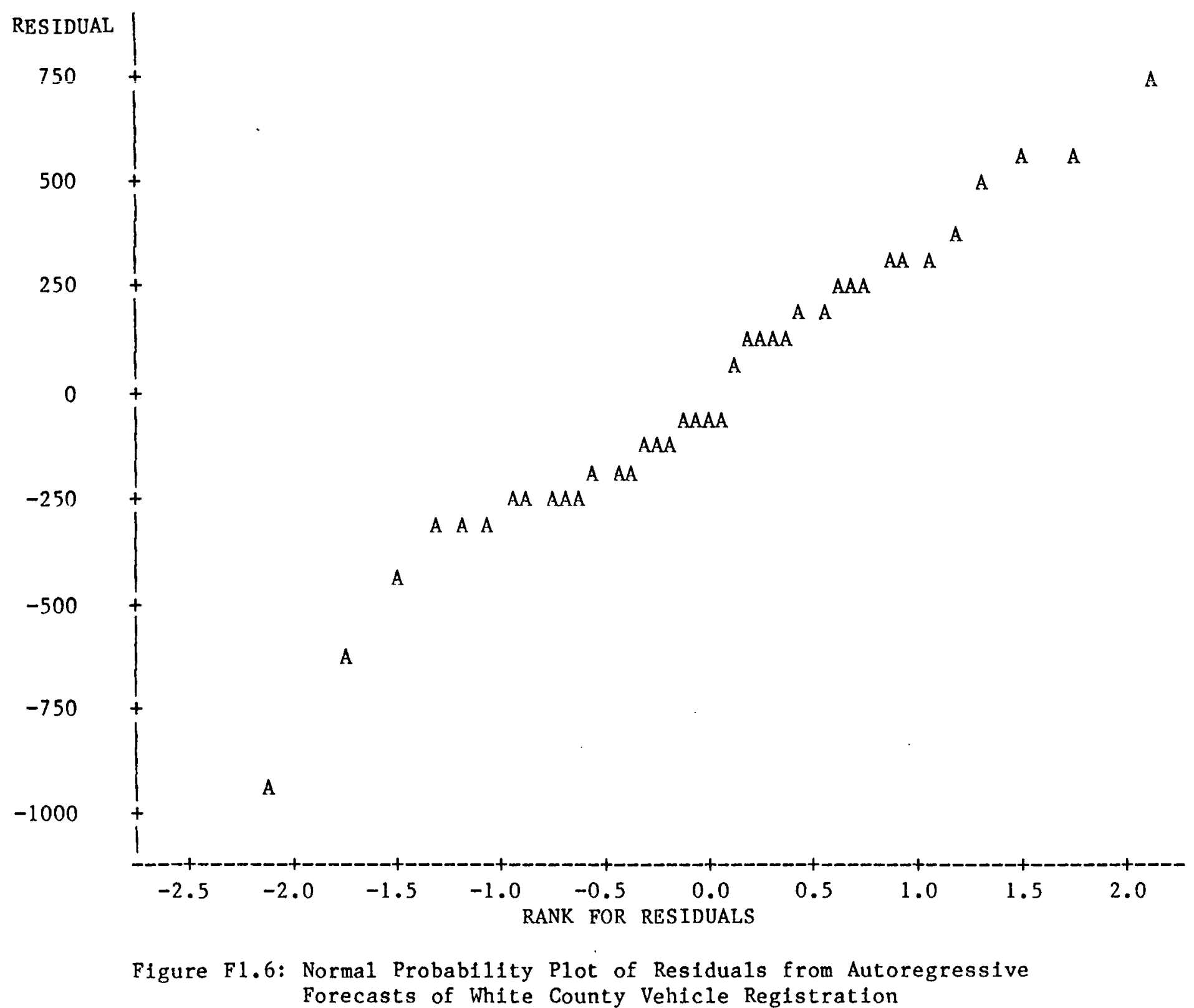


LEGEND: $\mathrm{A}=1$ OBS, $\mathrm{B}=2$ OBS, ETC.

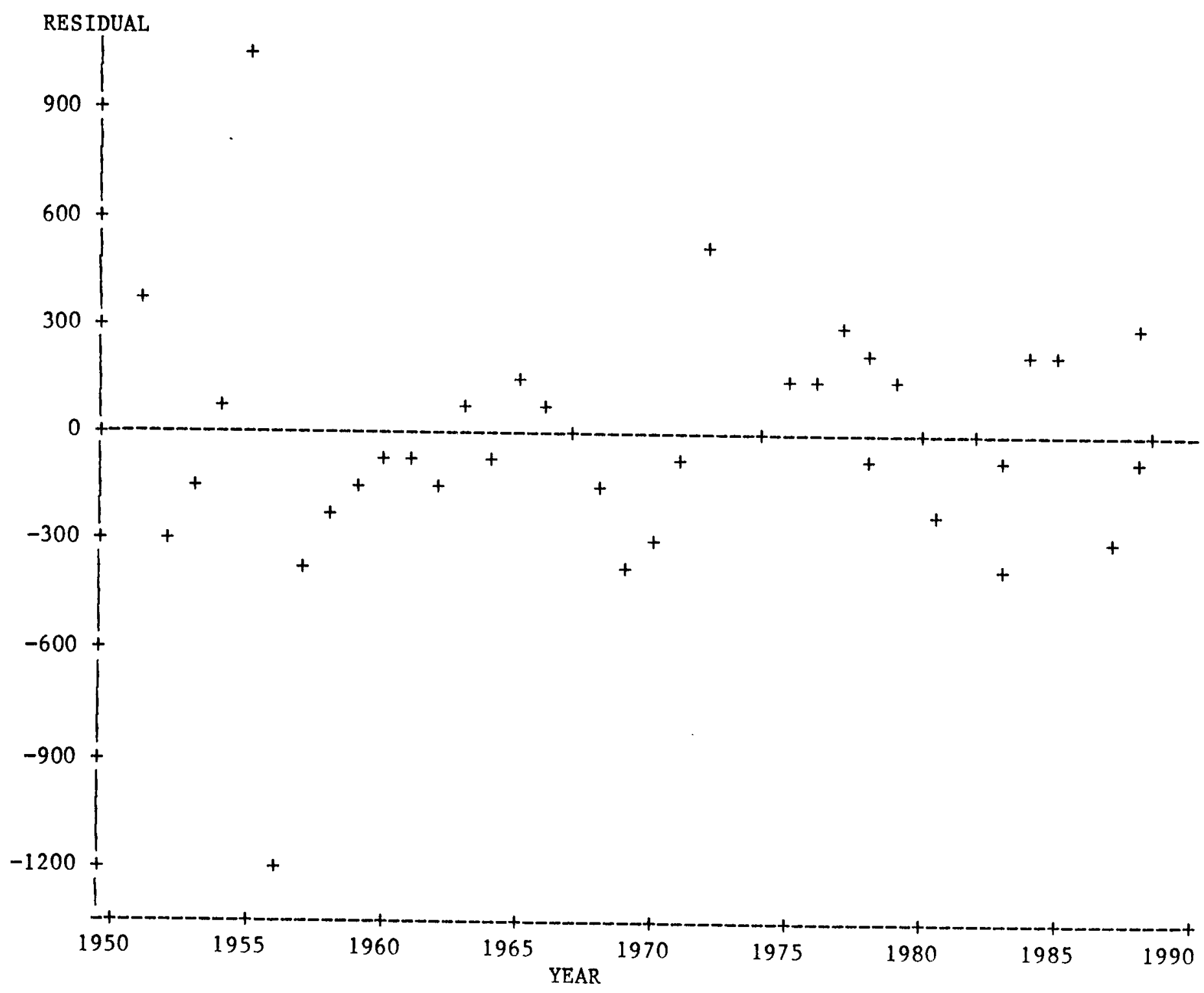

Figure F2.1: Residual P1ot against Time from $\operatorname{ARIMA}(0,1,0)$ Forecasts 
LEGEND: $\mathrm{A}=1 \mathrm{OBS}, \mathrm{B}=2 \mathrm{OBS}, \mathrm{ETC}$.

STD DEV: 992

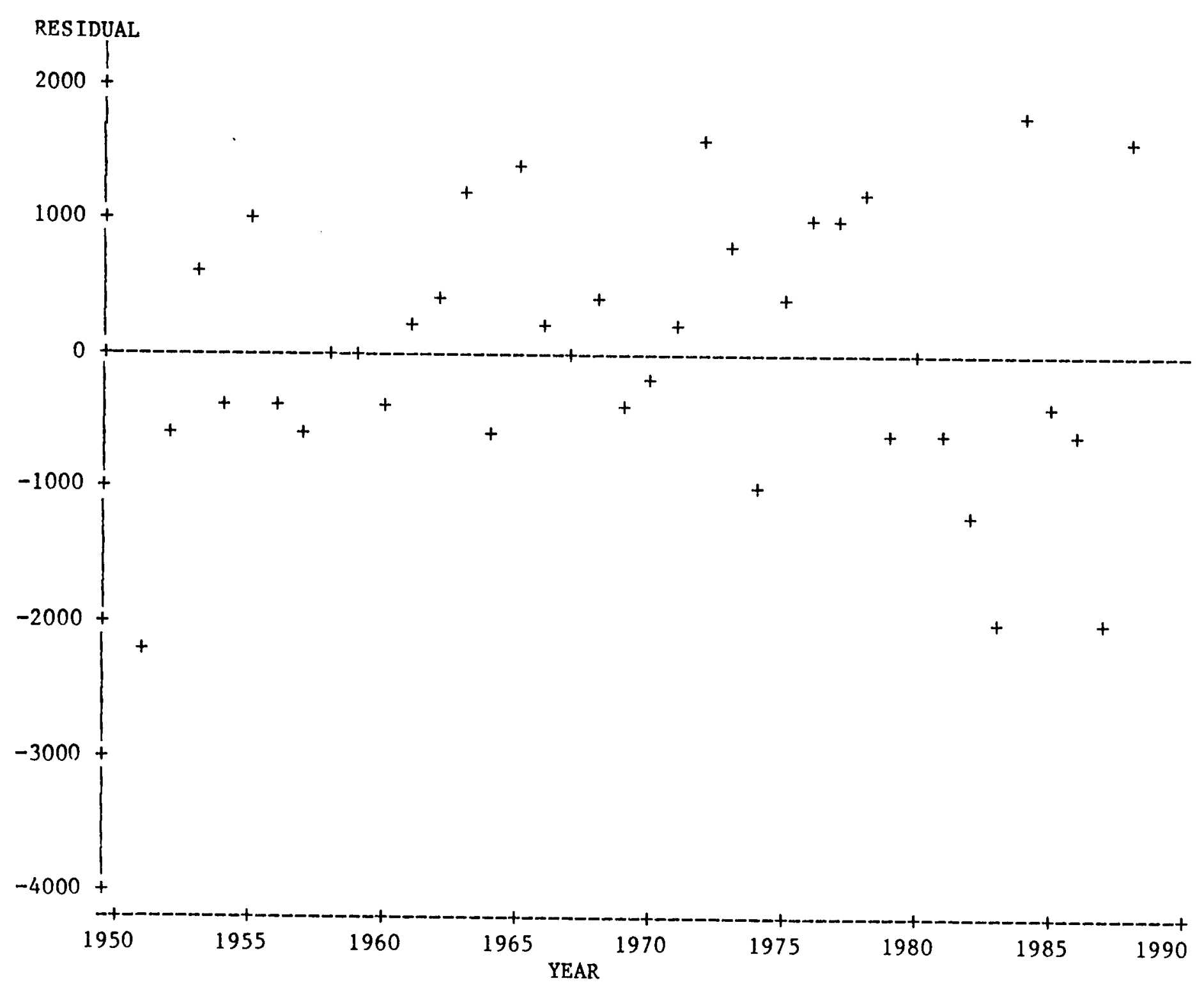

Figure F2.2: Residual Plot against Time from $\operatorname{ARIMA}(1,1,0)$ Forecasts 


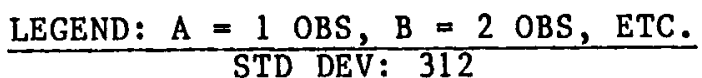

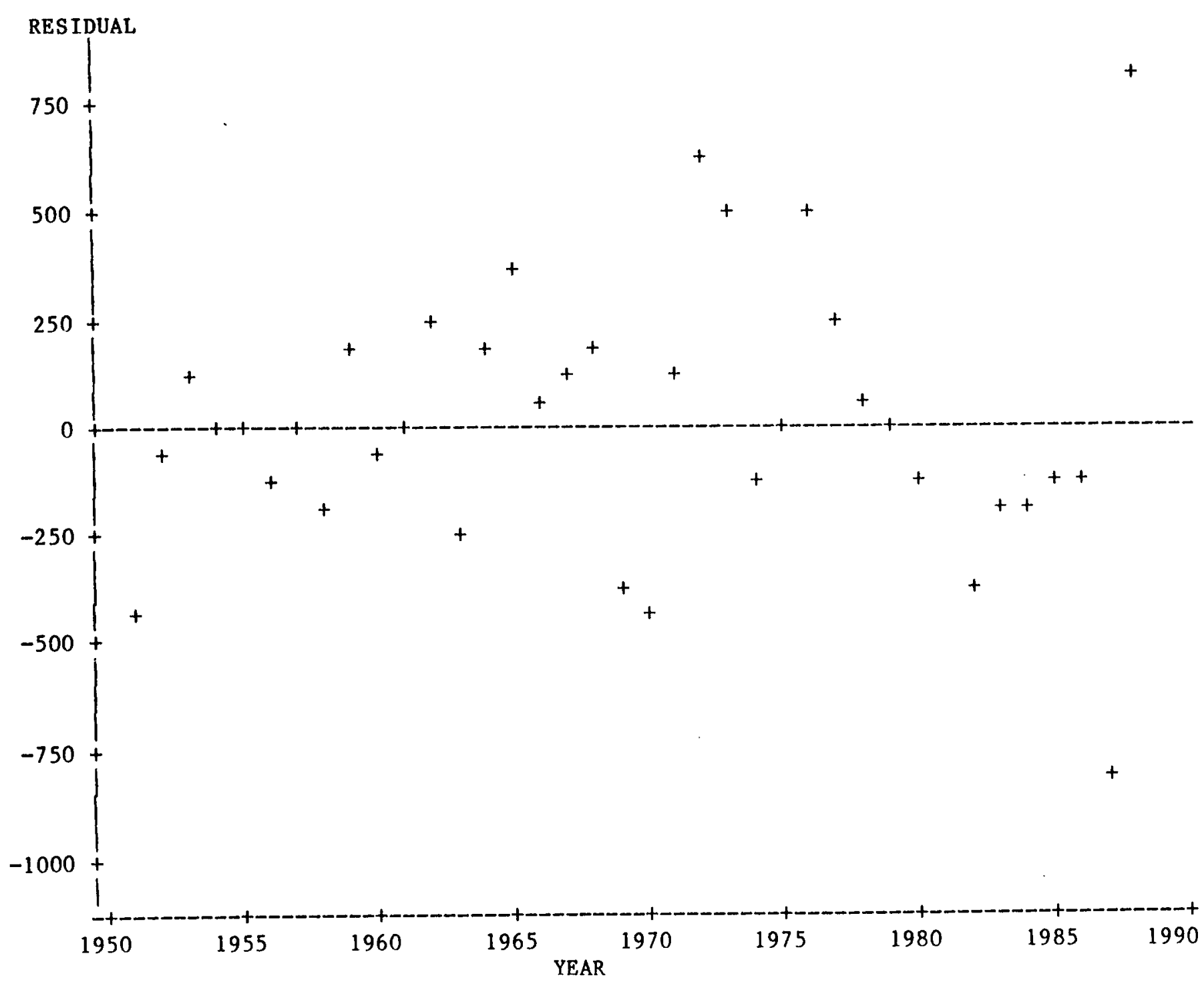

F1gure F2.3: Residual P1ot against Time from $\operatorname{ARIMA}(1,1,0)$ Forecasts of White County Vehicle Registration 
$\frac{\text { LEGEND: } A=1 \text { OBS, } \mathrm{B}=2 \text { OBS, ETC. }}{\text { STD DEV: } 344}$

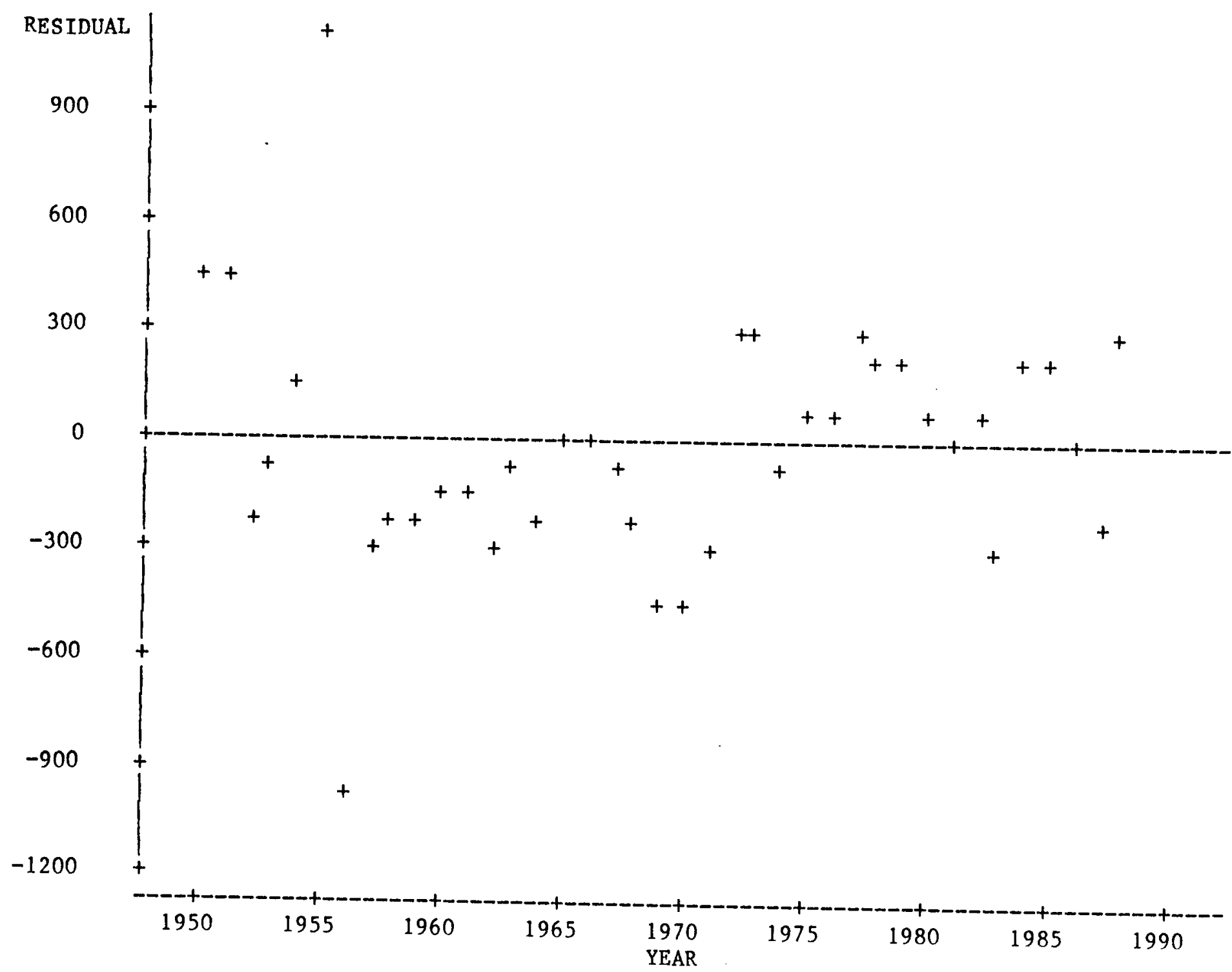

Figure F2.4: Residual P1ot against Time from Autoregressive Forecasts of Frank1in County Vehicle Registration 
$\frac{\text { LEGEND: } A=1 \text { OBS, } B=2 \text { OBS, ETC. }}{\text { STD DEV: } 1064}$

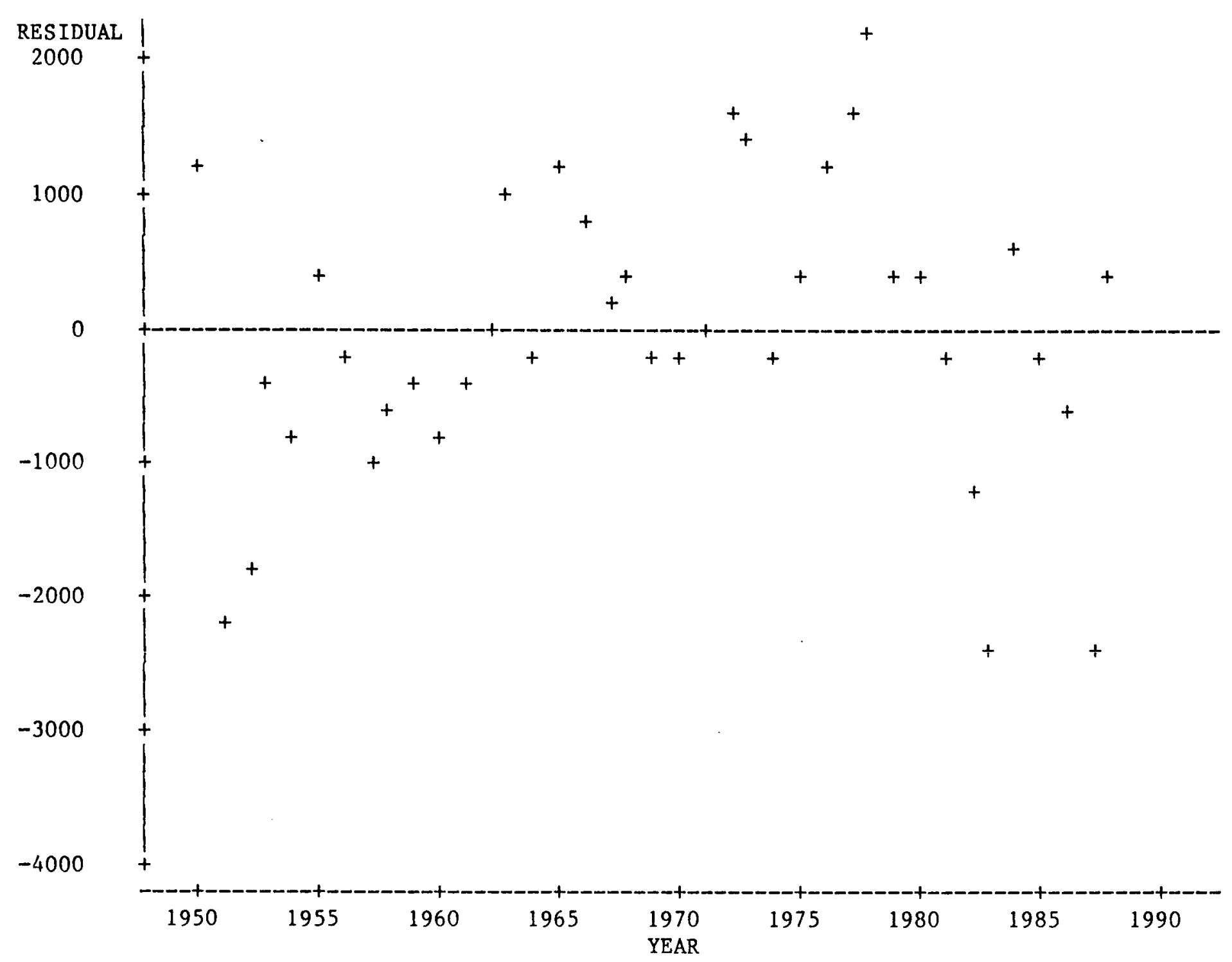

Figure F2.5: Residual P1ot against Time from Autoregressive Forecasts of Tippecanoe County Vehicle Registration 
LEGEND: $A=1$ OBS, $B=2$ OBS, ETC.

STD DEV: 341

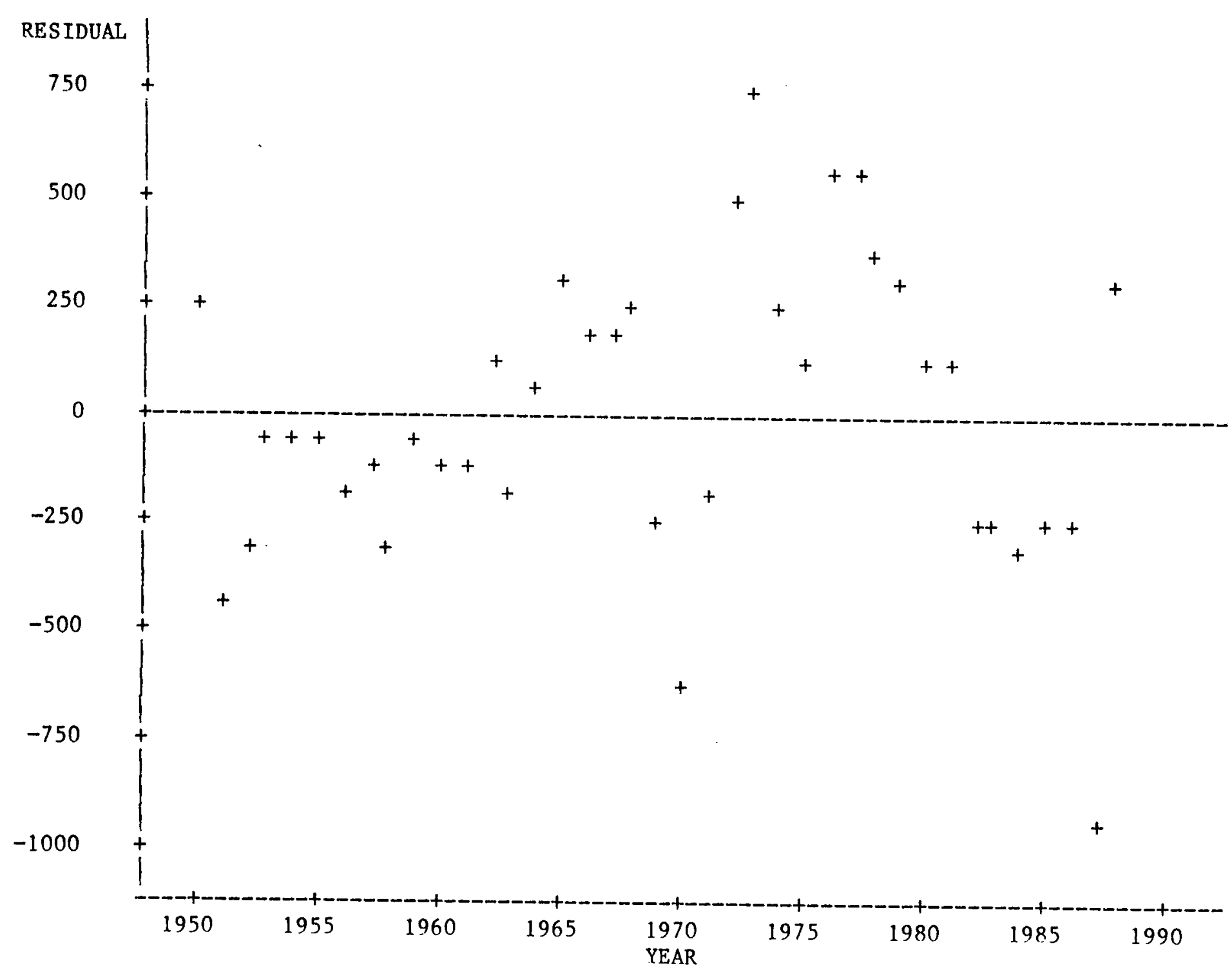

Figure F2.6: Residual P1ot against Time from Autoregressive Forecasts of White County Vehicle Registration 
LEGEND: $A=1$ OBS, $B=2$ OBS, ETC.

Pearson Correlation Coefficient $(r)=0.99537$

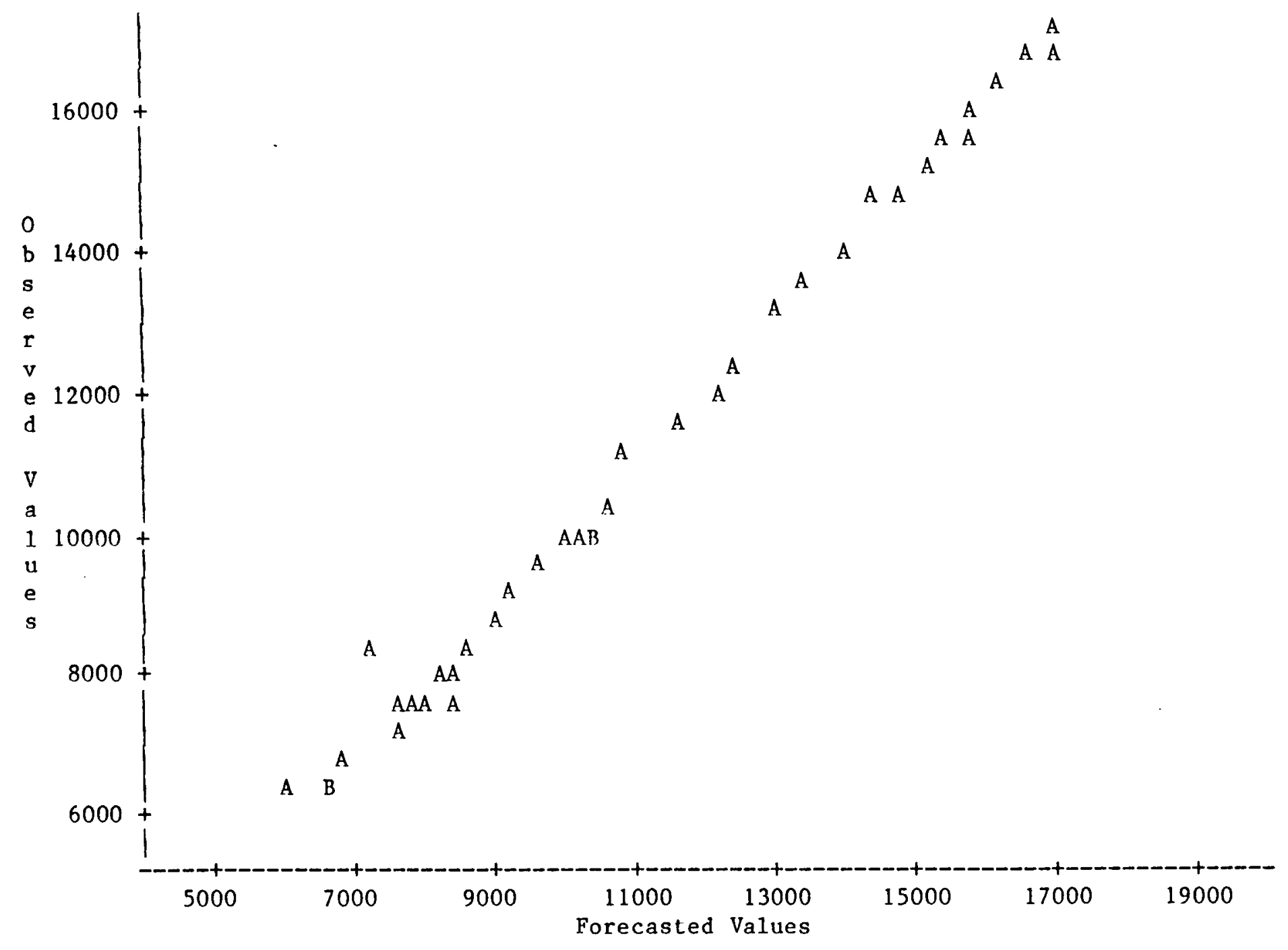

Figure F3.1: P1ot of Observed values against Autoregressive Forecasts of Franklin County Vehicle Registration 
LEGEND: $A=1$ OBS, $B=2$ OBS, ETC.

Pearson Correlation Coefficient (r) $=0.99492$

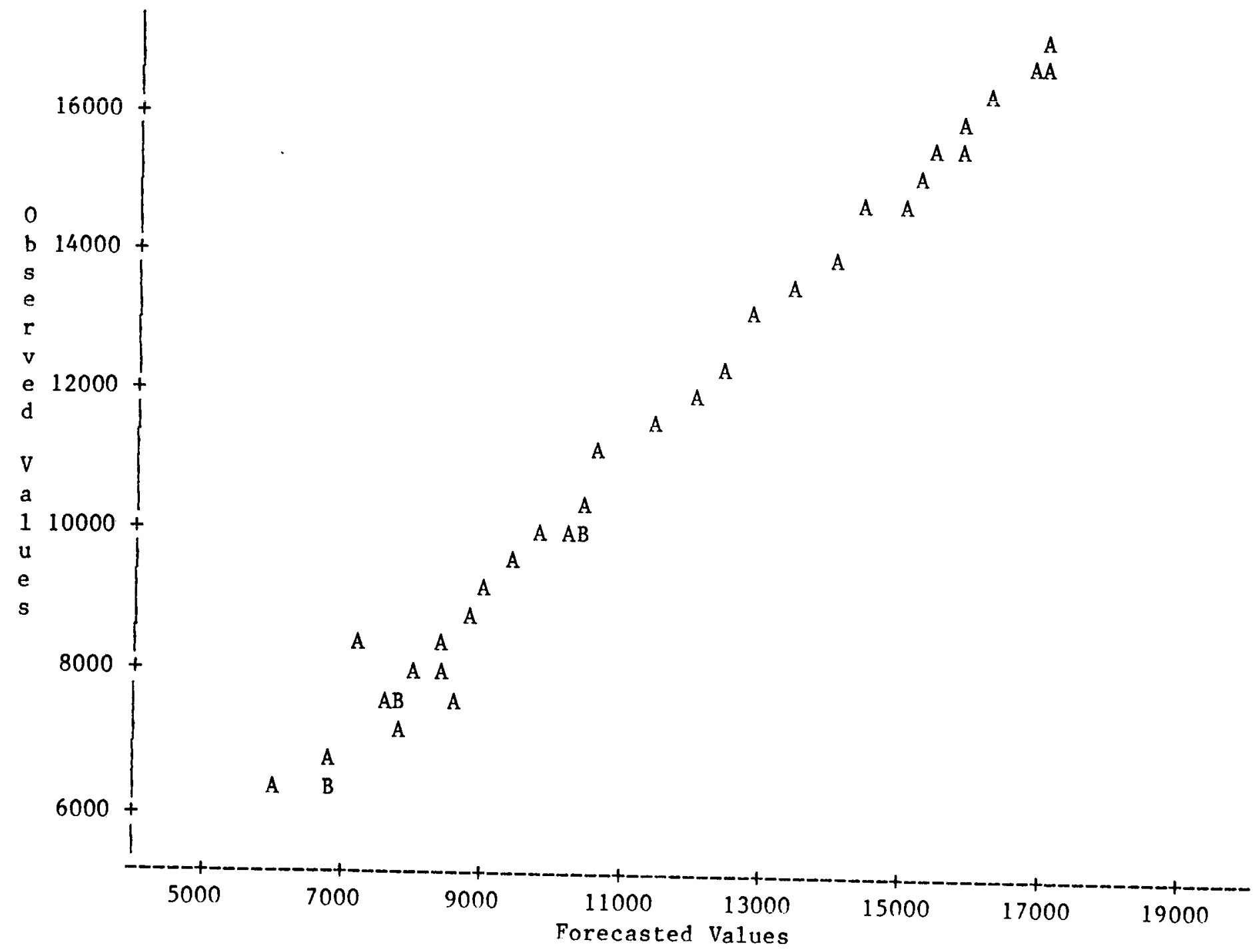

Figure F3.2: P1ot of Observed values against Box-Jenkin Forecasts of Franklin County Vehicle Registration 
LEGEND: $A=1$ OBS, $B=2$ OBS, ETC.

Pearson Correlation Coefficient $(r)=0.99524$

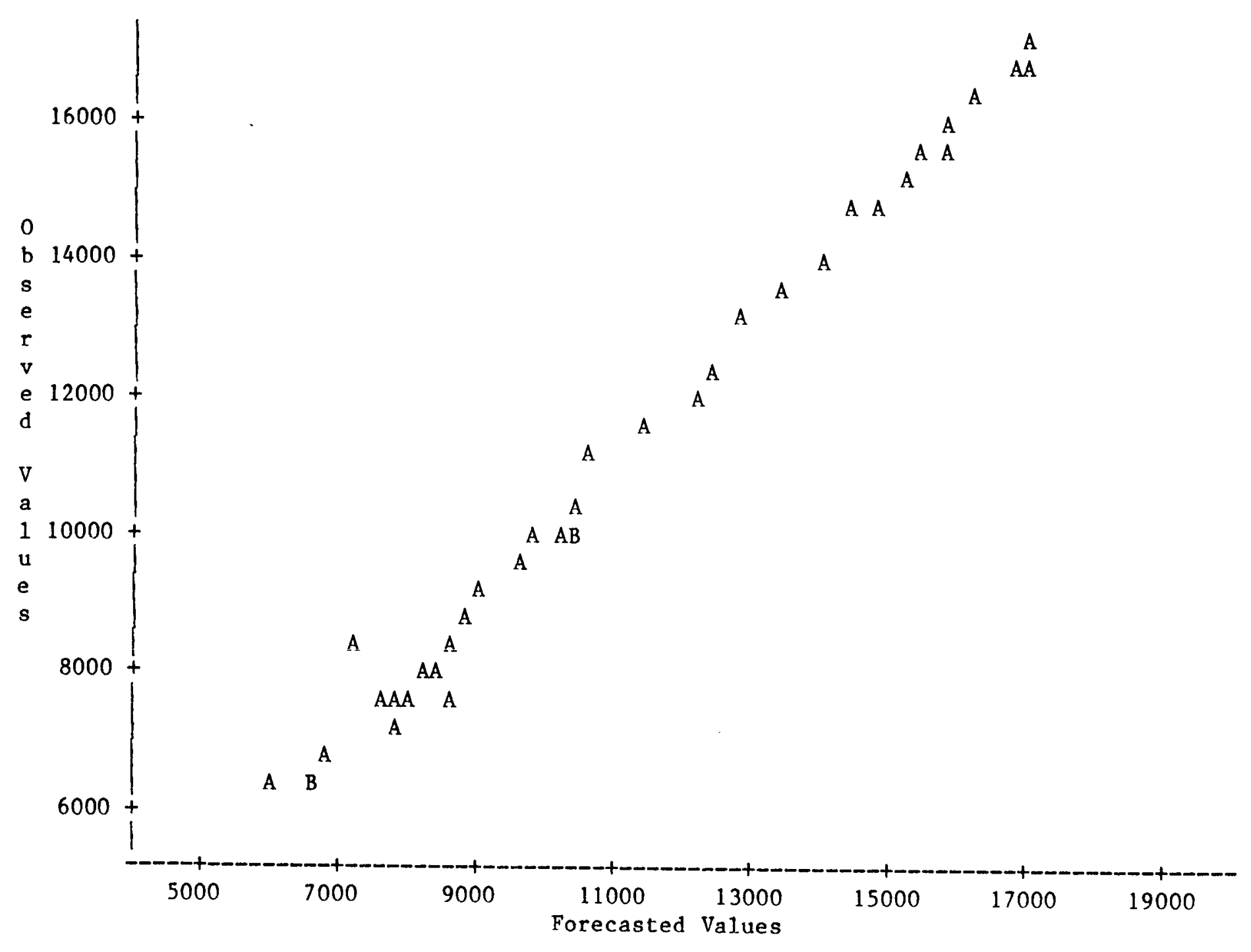

F1gure F3.3: Plot of Observed values against Combined Forecasts of Franklin County Vehicle Registration 
LEGEND: $A=1$ OBS, $B=2$ OBS, ETC.

Pearson Correlation Coefficlent $(r)=0.99876$

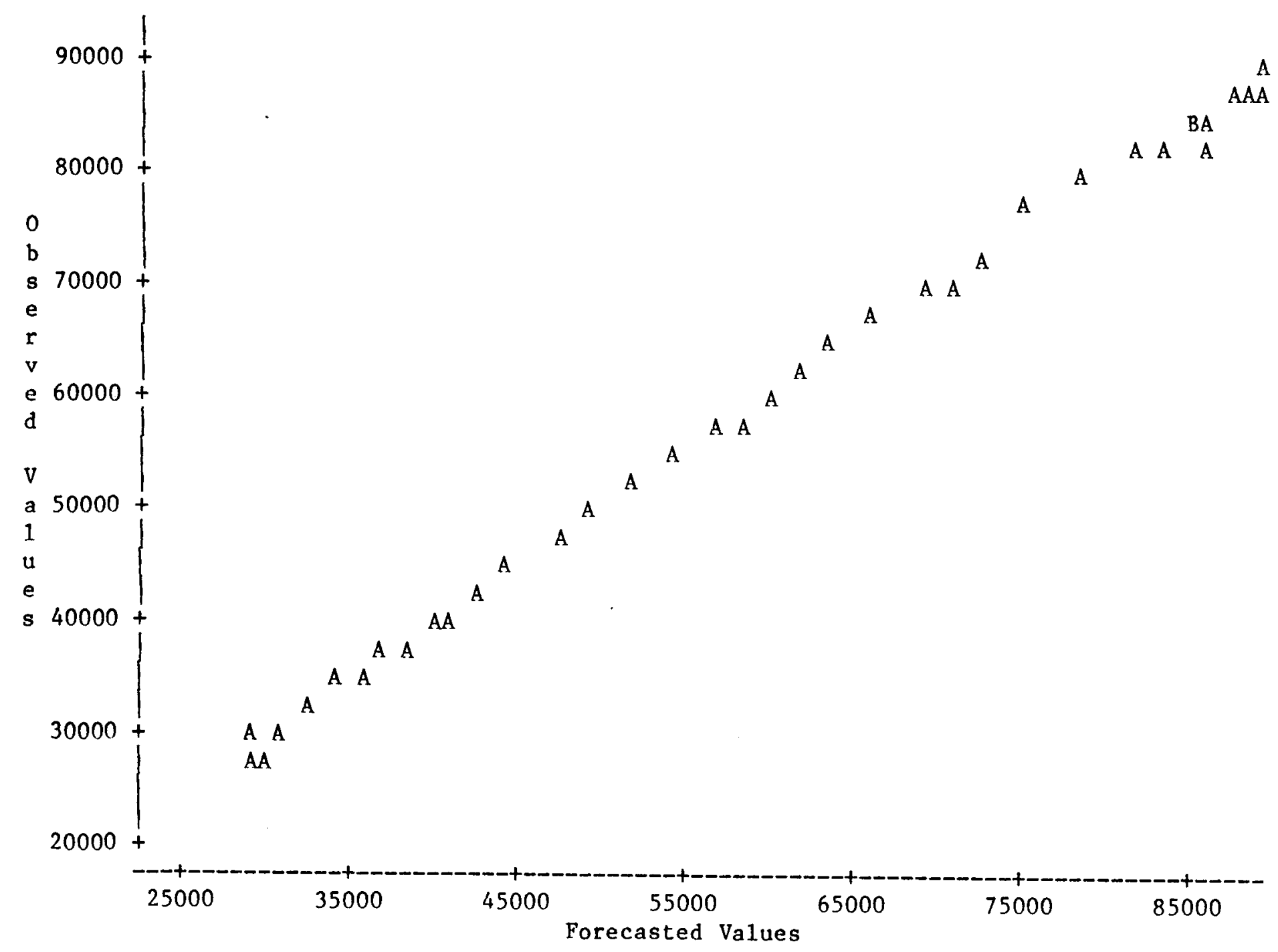

Figure F3.4: P1ot of Observed values against Autoregressive Forecasts of Tippecanoe County Vehicle Registration 
LEGEND: $\mathrm{A}=1$ OBS, $\mathrm{B}=2$ OBS, ETC.

Pearson Correlation Coefficient $(r)=0.99889$

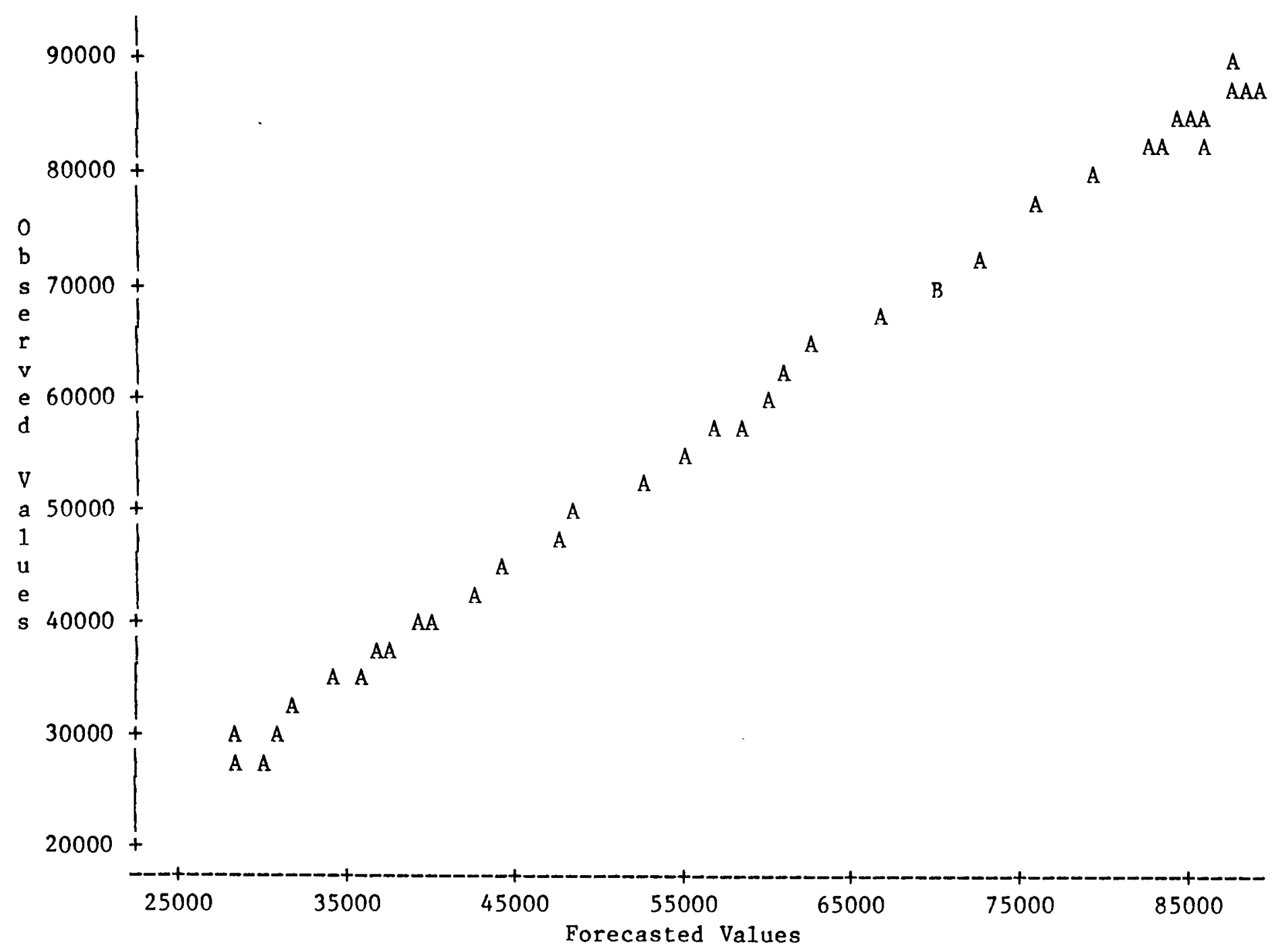

Figure F3.5: P1ot of Observed values against Box-Jenkin Forecasts of Tippecanoe County Vehicle Registration 
LEGEND: $\mathrm{A}=1$ OBS, $\mathrm{B}=2$ OBS, ETC.

Pearson Correlation Coefficient $(\mathbf{r})=0.99889$

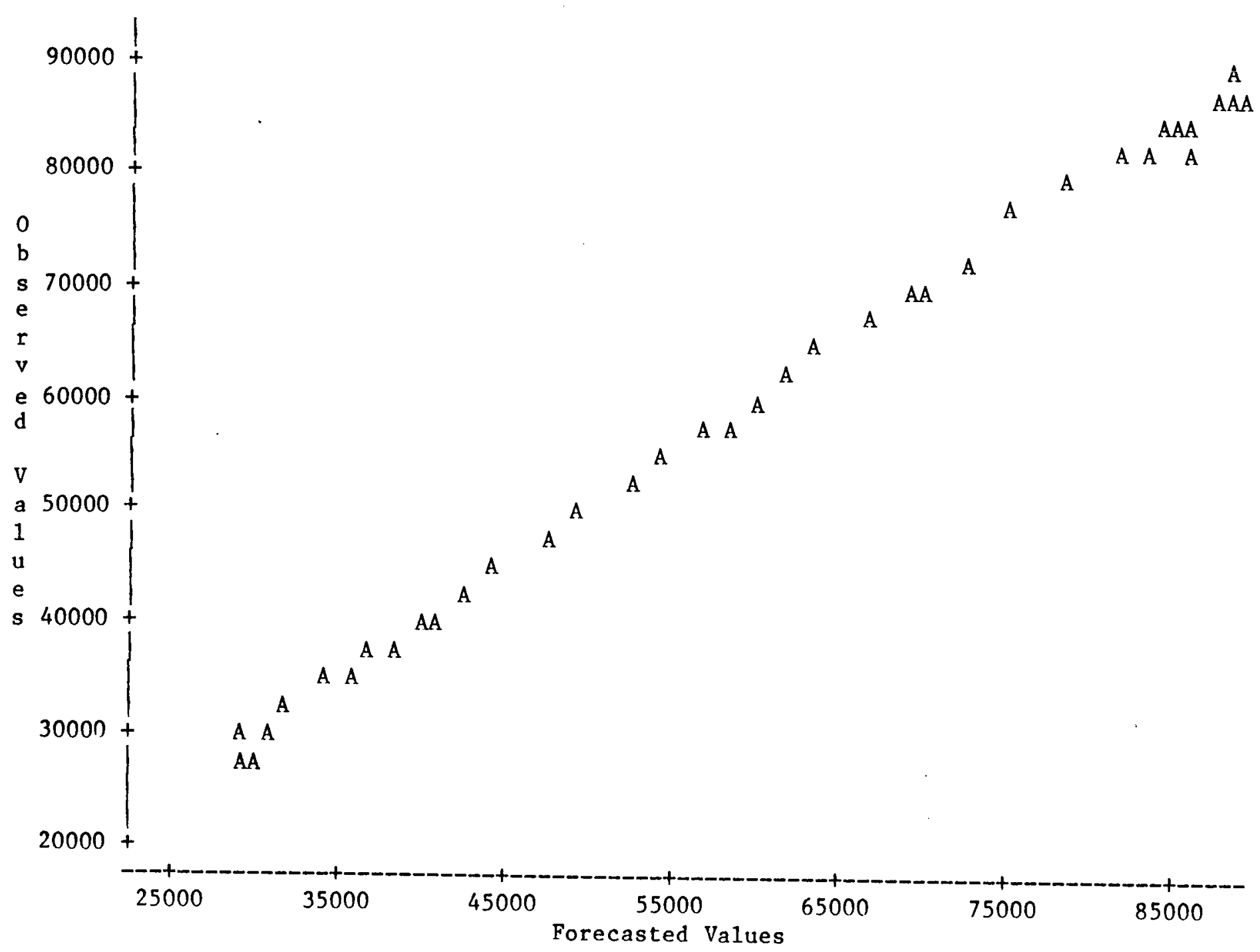

Figure F3.6: P1ot of Observed values against Combined Forecasts of Tippecanoe County Vehicle Registration 
SYMBOL: $r=$ Observed Value; $a=$ Autoregressive Forecast

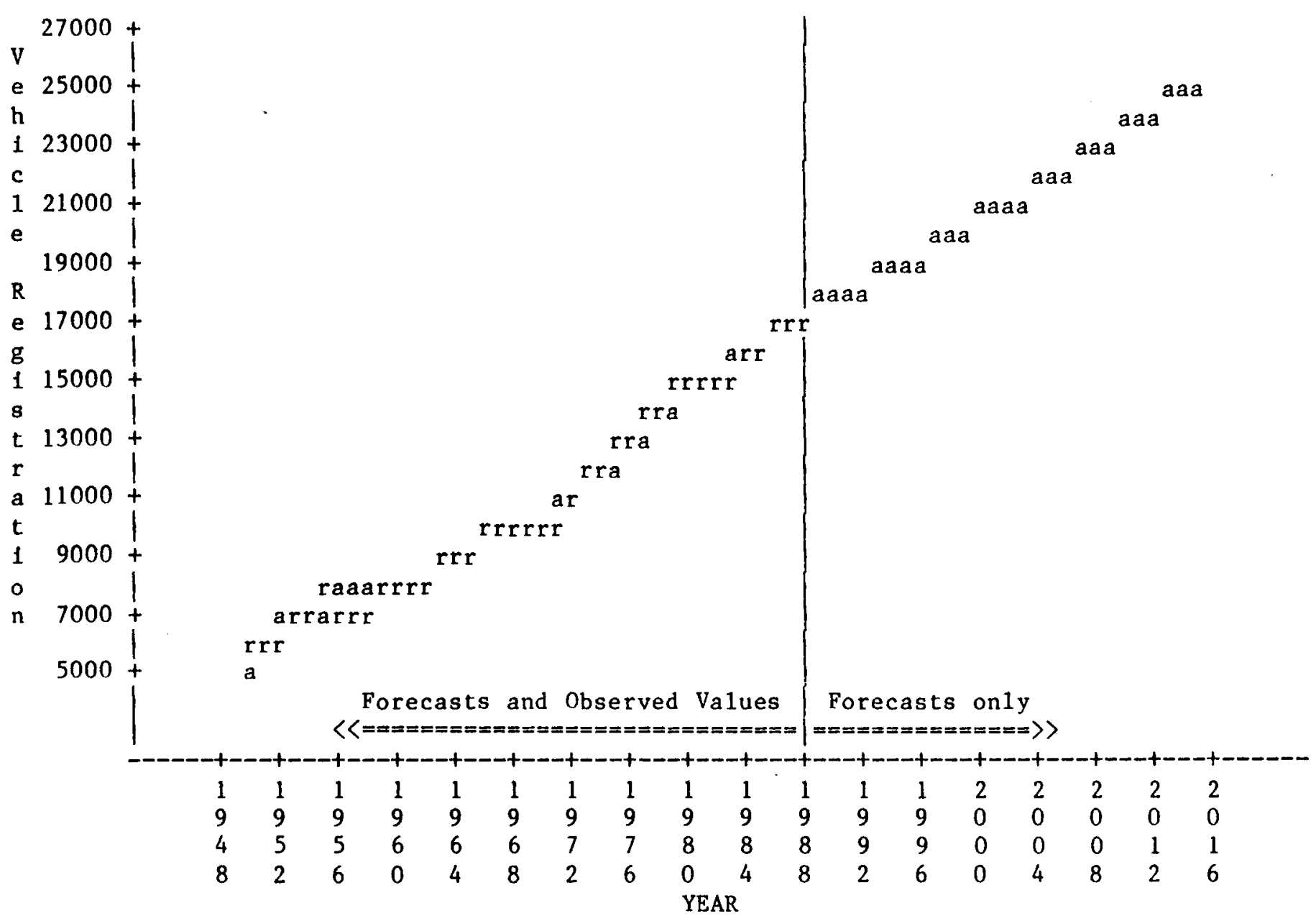

F1gure F4.1: Observed values and Autoregressive Forecasts

Note: One symbol before 1988 means that both forecast and observed value are slinerimnosed in nlot. 
SYMBOL: $r=$ Observed Value; $a=$ Autoregressive Forecast

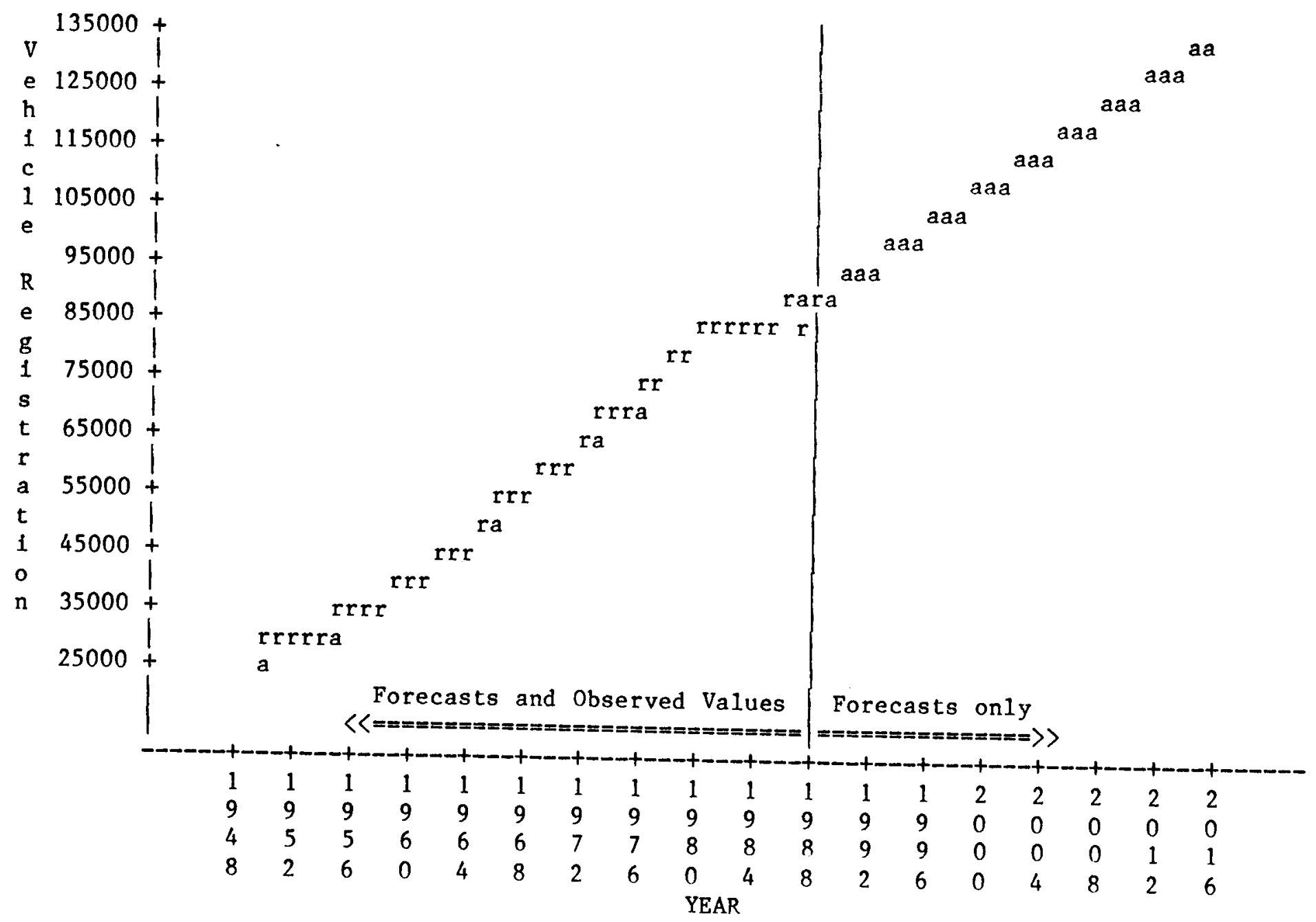

Figure F4.2: Observed values and Autoregressive Forecasts

Note: One symbol before 1988 means that both forecast and observed value are superimposed in plot. 
SYMBOL: $r=$ Observed Value; $b=$ Box-Jenkin Forecast

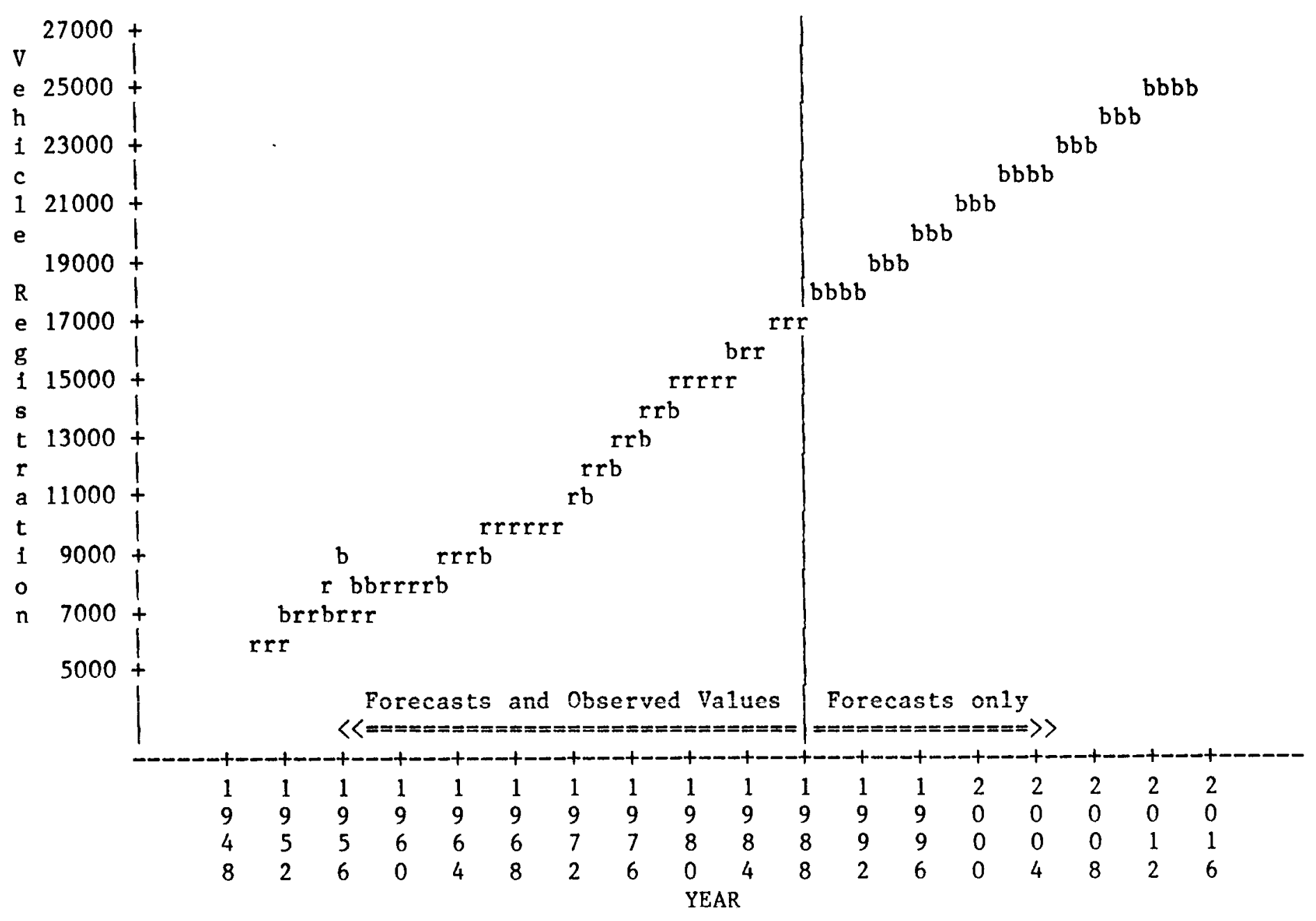

Figure F4.3: Observed values and Box-Jenkin Forecasts are superimposed in plot. 
SYMBOL: $r=$ Observed Value; $b=$ Box-Jenkin Forecast

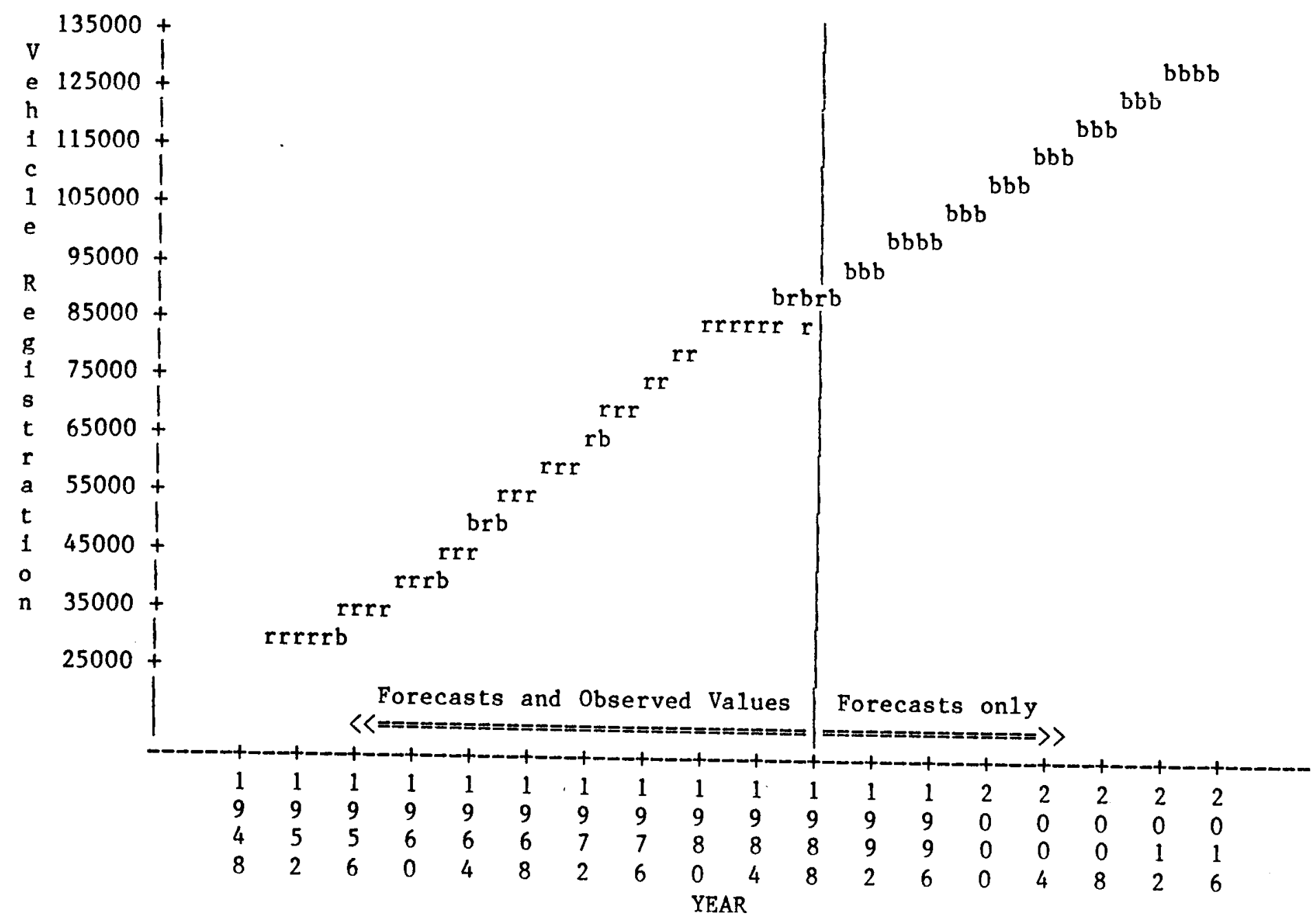

Figure F4.4: Observed values and Box-Jenkin Forecasts

Note: One symbol before 1988 means that both forecast and observed value are superimposed in plot. 
SYMBOL: $r=$ Observed Value: $c=$ Combined Forecast

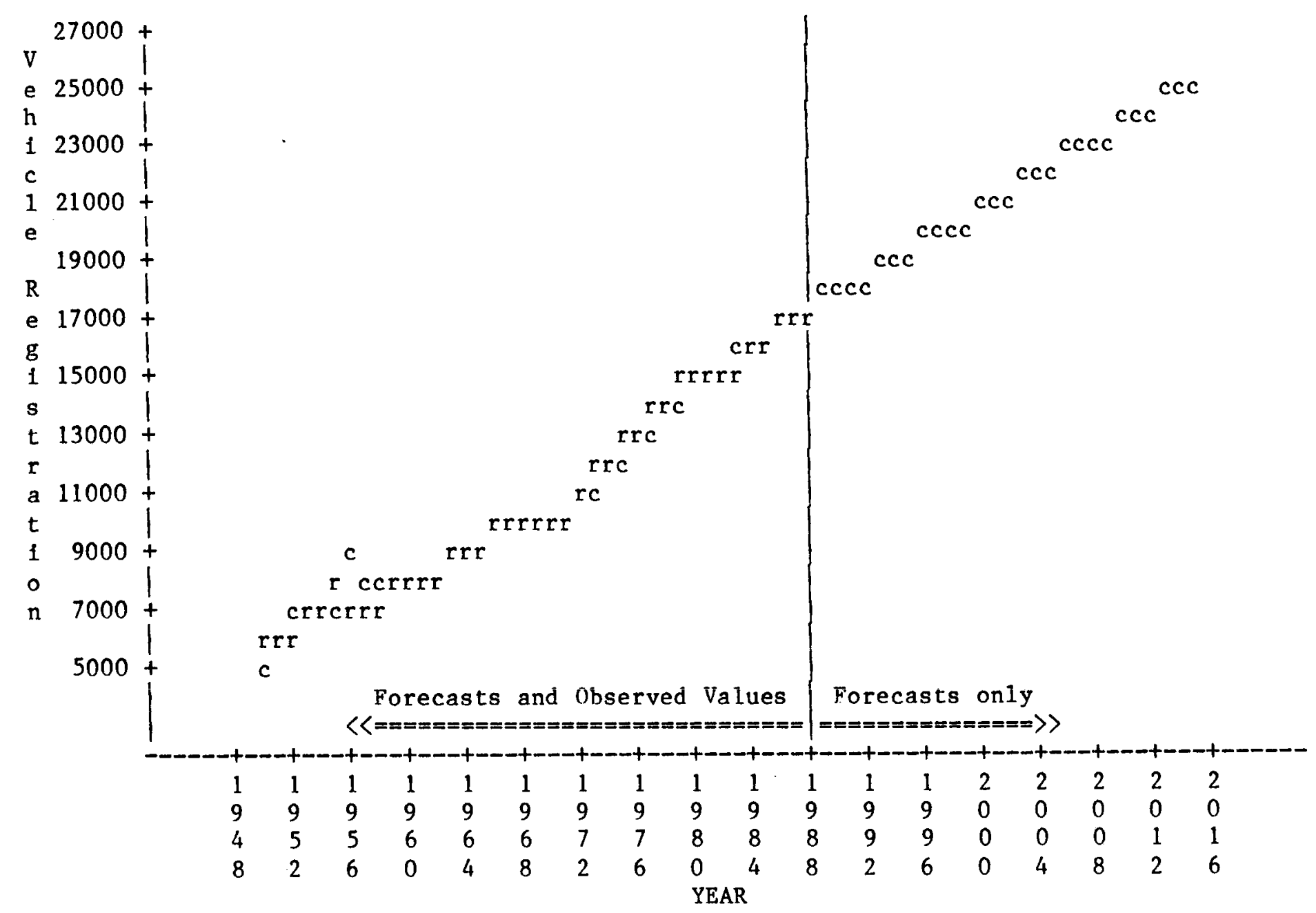

Figure F4.5: Observed values and Combined Forecasts

Note: One symbol before 1988 means that both forecast and observed value are superimposed in plot. 
SYMBOL: $r=$ Observed Value; $c=$ Combined Forecast

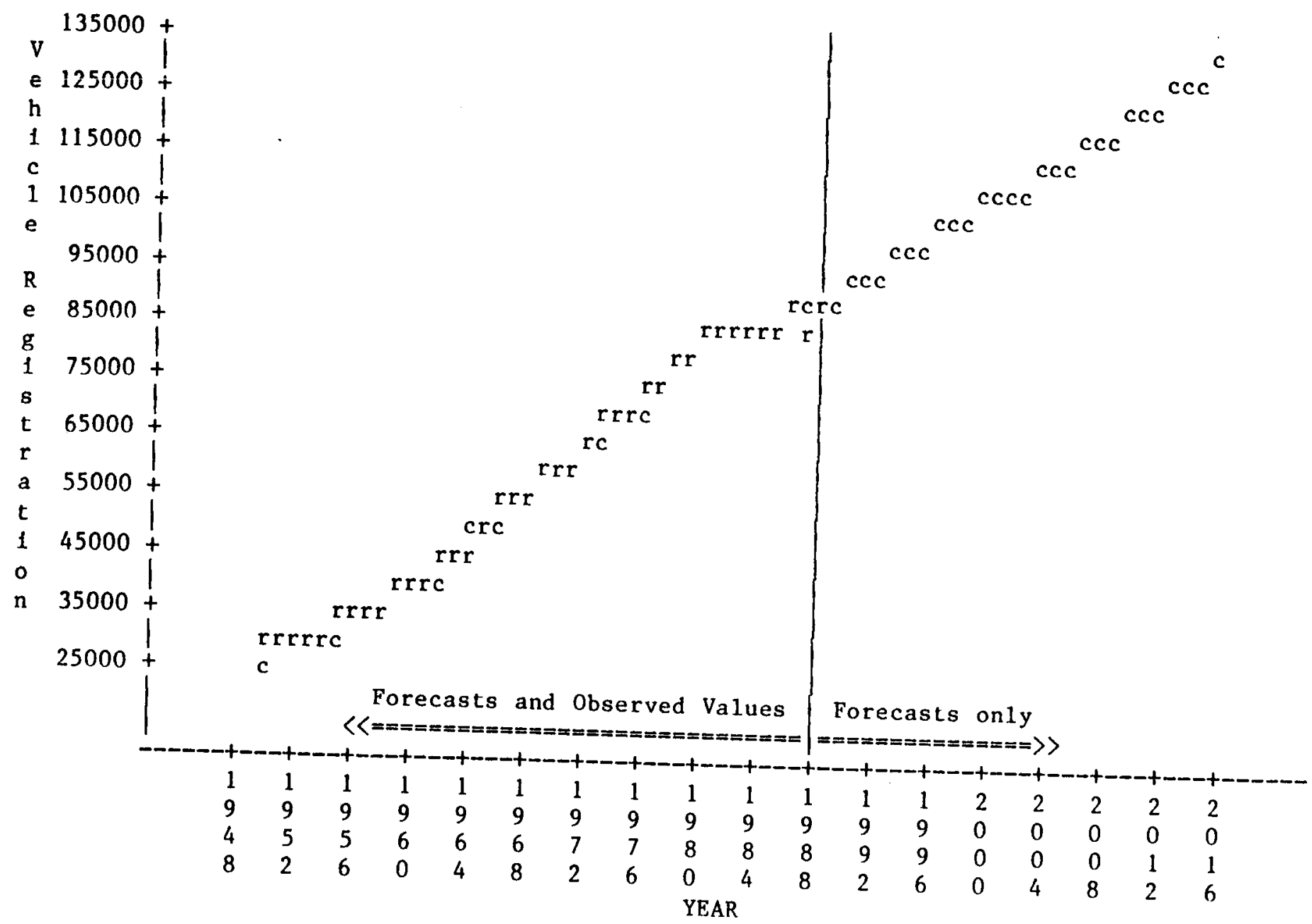

Figure F4.6: Observed values and Combined Forecasts of Tippecanoe County Vehicle Registration 
Appendix G 
Table G1: 90-Percent Confidence Limits of Forecast Measures for Models of Interstate Highways

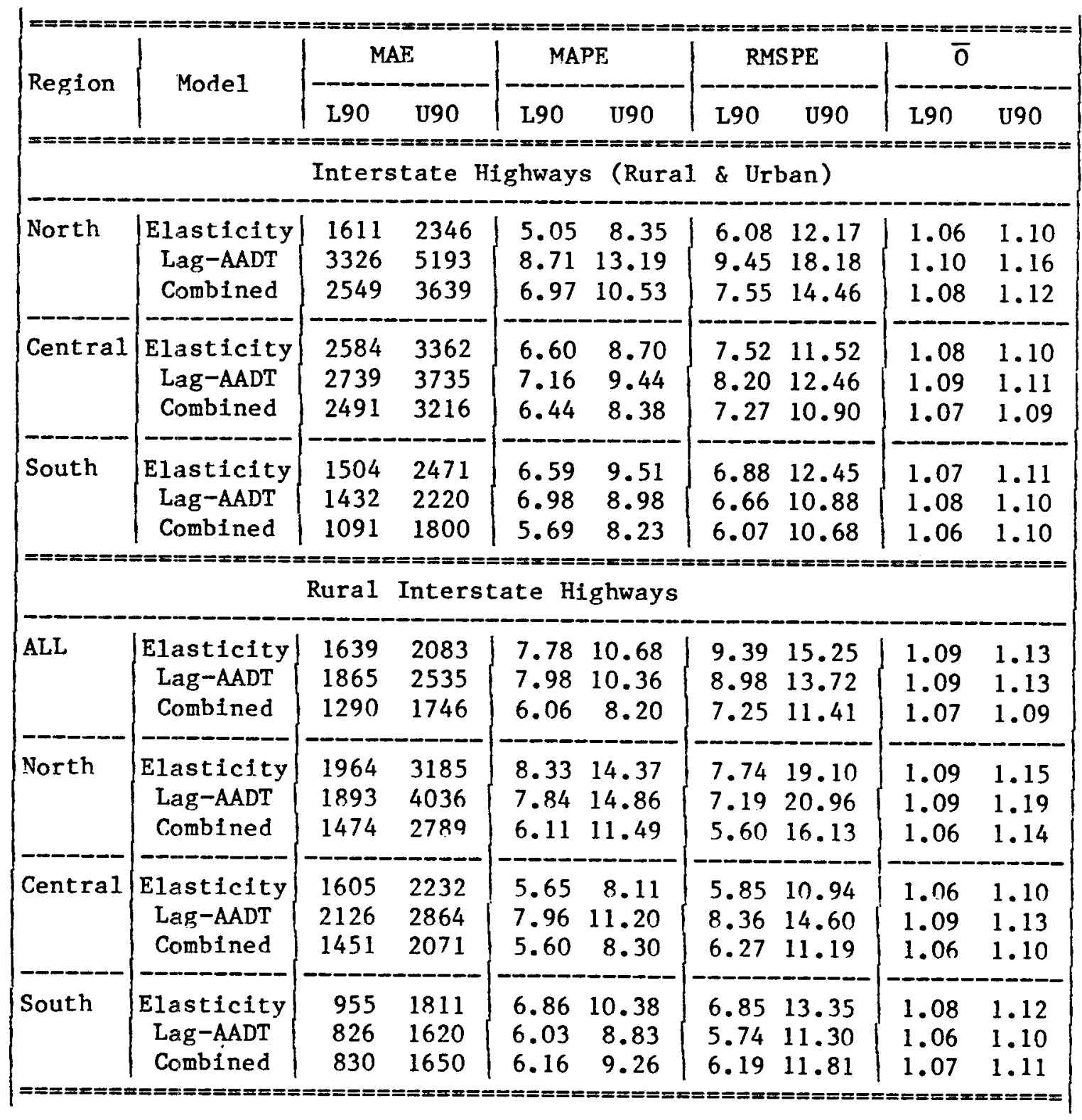


Table Gl, continued

\begin{tabular}{|c|c|c|c|c|c|c|c|c|c|}
\hline \multirow{2}{*}{ Region } & \multirow[b]{2}{*}{ Mode1 } & \multicolumn{2}{|c|}{ MAE } & \multicolumn{2}{|c|}{ MAPE } & \multicolumn{2}{|c|}{ RMSPE } & \multicolumn{2}{|l|}{$\bar{Q}$} \\
\hline & & L90 & U90 & L90 & U90 & L90 & U90 & L90 & U90 \\
\hline$== \pm==x$ & $=$ & $\begin{array}{l}===x= \\
\text { Urban }\end{array}$ & $\begin{array}{l}=== \\
\text { Inter }\end{array}$ & $\begin{array}{l}==== \\
\text { ate H }\end{array}$ & $\begin{array}{l}== \pm=x= \\
\text { lghways }\end{array}$ & $=x=0$ & $==:===x$ & $:=== \pm= \pm$ & $==$ \\
\hline ALL & $\left|\begin{array}{c}\text { Elasticity } \\
\text { Lag-AADT } \\
\text { Combined }\end{array}\right|$ & $\begin{array}{l}2315 \\
3408 \\
2415\end{array}$ & $\begin{array}{l}2918 \\
4618 \\
3229\end{array}$ & $\begin{array}{l}4.26 \\
6.20 \\
4.49\end{array}$ & $\begin{array}{l}5.82 \\
8.40 \\
6.03\end{array}$ & $\begin{array}{l}4.83 \\
7.14 \\
5.07\end{array}$ & $\begin{array}{r}8.01 \\
11.25 \\
8.06\end{array}$ & $\begin{array}{l}1.04 \\
1.07 \\
1.05\end{array}$ & $\begin{array}{l}1.06 \\
1.09 \\
1.07\end{array}$ \\
\hline North & $\begin{array}{c}\text { Elasticity } \\
\text { Lag-AADT } \\
\text { Combined }\end{array}$ & $\begin{array}{l}3345 \\
3864 \\
3647\end{array}$ & $\begin{array}{l}4994 \\
6762 \\
5800\end{array}$ & $\begin{array}{l}5.72 \\
7.15 \\
6.46\end{array}$ & $\begin{array}{l}10.60 \\
12.75 \\
11.56\end{array}$ & $\begin{array}{l}5.60 \\
7.24 \\
6.30\end{array}$ & $\begin{array}{l}15.49 \\
17.88 \\
16.46\end{array}$ & $\begin{array}{l}1.07 \\
1.08 \\
1.07\end{array}$ & $\begin{array}{l}1.13 \\
1.16 \\
1.15\end{array}$ \\
\hline Central & $\begin{array}{c}\text { Elasticity } \\
\text { Lag-AADT } \\
\text { Combined }\end{array}$ & $\begin{array}{l}2390 \\
3207 \\
2729\end{array}$ & $\begin{array}{l}3104 \\
4553 \\
3627\end{array}$ & $\begin{array}{l}4.28 \\
5.63 \\
4.91\end{array}$ & $\begin{array}{l}7.02 \\
8.57 \\
7.47\end{array}$ & $\begin{array}{l}4.70 \\
6.01 \\
5.26\end{array}$ & $\begin{array}{l}10.32 \\
11.71 \\
10.17\end{array}$ & $\begin{array}{l}1.04 \\
1.06 \\
1.05\end{array}$ & $\begin{array}{l}1.08 \\
1.10 \\
1.09\end{array}$ \\
\hline South & $\begin{array}{c}\text { Elasticity } \\
\text { Lag-AADT } \\
\text { Combined }\end{array}$ & $\begin{array}{l}1147 \\
2332 \\
1495\end{array}$ & $\begin{array}{l}2689 \\
5025 \\
3150\end{array}$ & $\begin{array}{l}2.22 \\
5.04 \\
2.90\end{array}$ & $\begin{array}{r}7.34 \\
11.26 \\
8.22\end{array}$ & $\begin{array}{l}1.96 \\
4.15 \\
2.21\end{array}$ & $\begin{array}{l}11.47 \\
15.80 \\
12.61\end{array}$ & $\begin{array}{l}1.02 \\
1.05 \\
1.03\end{array}$ & $\begin{array}{l}1.08 \\
1.13 \\
1.09\end{array}$ \\
\hline
\end{tabular}

Note: $L 90=$ Lower 1 imit of $90 \%$ confidence 1 imit, and U90 = Upper limit of $90 \%$ confidence limit. 
Tab1e G2: 95-Percent Confidence Limits of Forecast Measures for Models of Interstate Highways

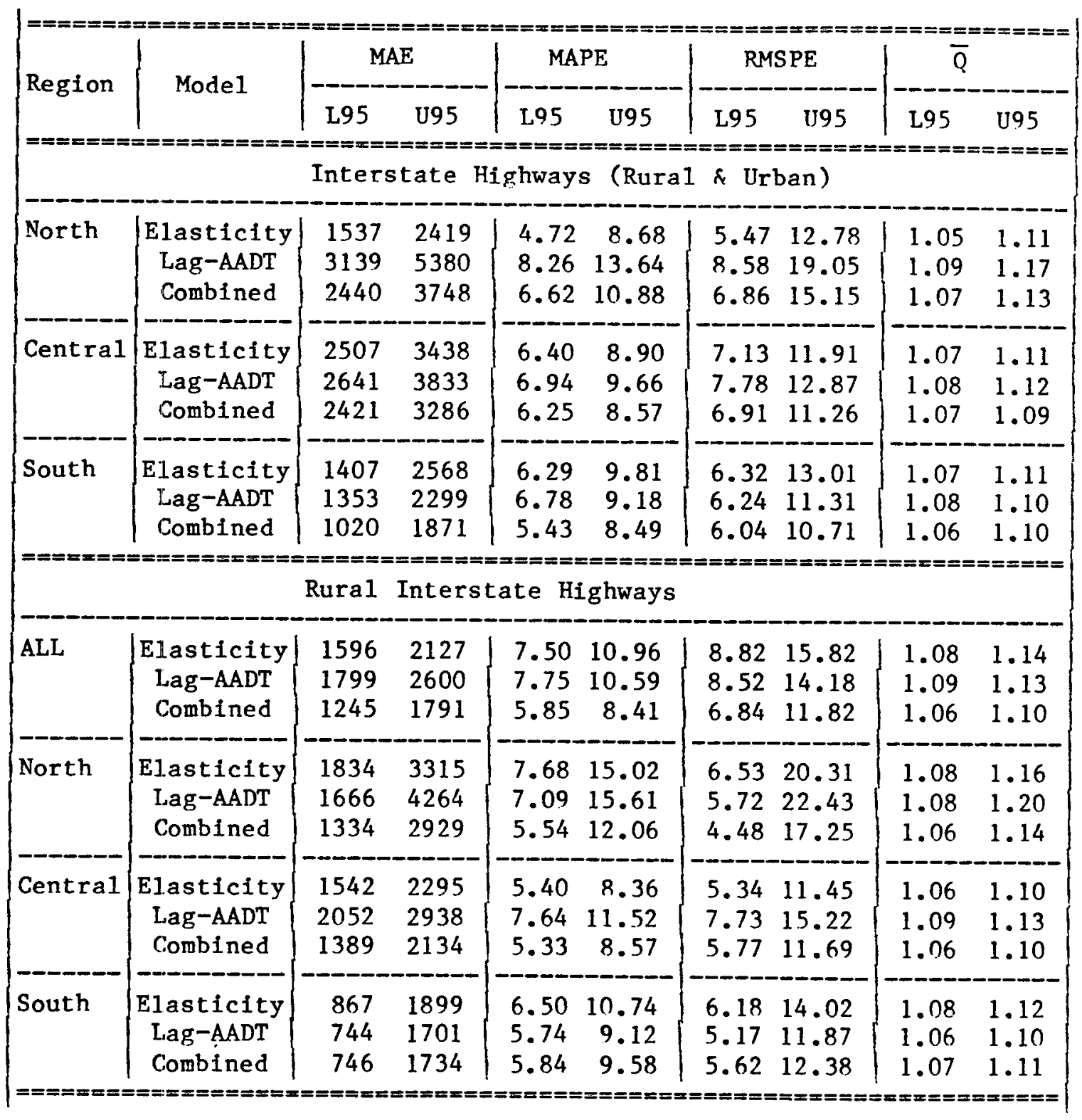


Table G2, continued

\begin{tabular}{|c|c|c|c|c|c|c|c|c|c|}
\hline \multirow{2}{*}{ Region } & \multirow{2}{*}{ Mode1 } & \multicolumn{2}{|c|}{ MAE } & \multicolumn{2}{|c|}{ MAPE } & \multicolumn{2}{|c|}{ RMSPE } & \multicolumn{2}{|l|}{$\bar{Q}$} \\
\hline & & L95 & U95 & L95 & U95 & L95 & u95 & L95 & U95 \\
\hline$===x==$ & $===x=x====$ & $\begin{array}{l}===== \\
\text { Urban }\end{array}$ & $\begin{array}{l}===== \\
\text { Inters }\end{array}$ & $\begin{array}{l}===== \\
\text { ate Hi }\end{array}$ & $\begin{array}{l}===== \\
\text { ghways }\end{array}$ & $====x$ & $== \pm==x$ & $==== \pm$ & \\
\hline ALL & $\mid \begin{array}{c}\text { Elasticity } \\
\text { Lag-AADT } \\
\text { Combined }\end{array}$ & $\begin{array}{l}2255 \\
3289 \\
2335\end{array}$ & $\begin{array}{l}2977 \\
4736 \\
3308\end{array}$ & $\begin{array}{l}4.11 \\
5.98 \\
4.33\end{array}$ & $\begin{array}{l}5.97 \\
8.62 \\
6.19\end{array}$ & $\begin{array}{l}4.52 \\
6.73 \\
4.78\end{array}$ & $\begin{array}{r}8.32 \\
11.66 \\
8.36\end{array}$ & $\begin{array}{l}1.04 \\
1.06 \\
1.05\end{array}$ & $\begin{array}{l}1.06 \\
1.10 \\
1.07\end{array}$ \\
\hline North & $\mid \begin{array}{c}\text { Elasticity } \\
\text { Lag-AADT } \\
\text { Combined }\end{array}$ & $\begin{array}{l}3173 \\
3563 \\
3423\end{array}$ & $\begin{array}{l}5166 \\
7064 \\
6024\end{array}$ & $\begin{array}{l}5.21 \\
6.56 \\
5.94\end{array}$ & $\begin{array}{l}11.11 \\
13.34 \\
12.08\end{array}$ & $\begin{array}{l}4.57 \\
6.14 \\
5.24\end{array}$ & $\begin{array}{l}16.52 \\
18.98 \\
17.51\end{array}$ & $\begin{array}{l}1.06 \\
1.07 \\
1.07\end{array}$ & $\begin{array}{l}1.14 \\
1.17 \\
1.15\end{array}$ \\
\hline Central & $\begin{array}{c}\text { Elasticity } \\
\text { Lag-AADT } \\
\text { Combined }\end{array}$ & $\begin{array}{l}2318 \\
3072 \\
2640\end{array}$ & $\begin{array}{l}3175 \\
4688 \\
3716\end{array}$ & $\begin{array}{l}4.00 \\
5.33 \\
4.66\end{array}$ & $\begin{array}{l}7.30 \\
8.87 \\
7.72\end{array}$ & $\begin{array}{l}4.14 \\
5.44 \\
4.77\end{array}$ & $\begin{array}{l}10.88 \\
12.28 \\
10.66\end{array}$ & $\begin{array}{l}1.04 \\
1.06 \\
1.05\end{array}$ & $\begin{array}{l}1.08 \\
1.10 \\
1.09\end{array}$ \\
\hline South & $\mid \begin{array}{c}\text { Elasticity } \\
\text { Lag-AADT } \\
\text { Combined }\end{array}$ & $\begin{array}{r}974 \\
2028 \\
1308\end{array}$ & $\begin{array}{l}2862 \\
5328 \\
3337\end{array}$ & $\begin{array}{l}1.65 \\
4.34 \\
2.30\end{array}$ & $\begin{array}{r}7.91 \\
11.96 \\
8.82\end{array}$ & $\begin{array}{l}0.89 \\
2.84 \\
1.04\end{array}$ & $\begin{array}{l}12.54 \\
17.11 \\
13.79\end{array}$ & $\begin{array}{l}1.01 \\
1.05 \\
1.02\end{array}$ & $\begin{array}{l}1.09 \\
1.13 \\
1.10\end{array}$ \\
\hline
\end{tabular}

Note: $\mathrm{L} 95=$ Lower 1 imit of $95 \%$ confidence limit, and U95 = Upper Iimit of $95 \%$ confidence limit. 
Table G3: 90-Percent Confidence Limits of Forecast Measures for Models of Urban Principal Arterial

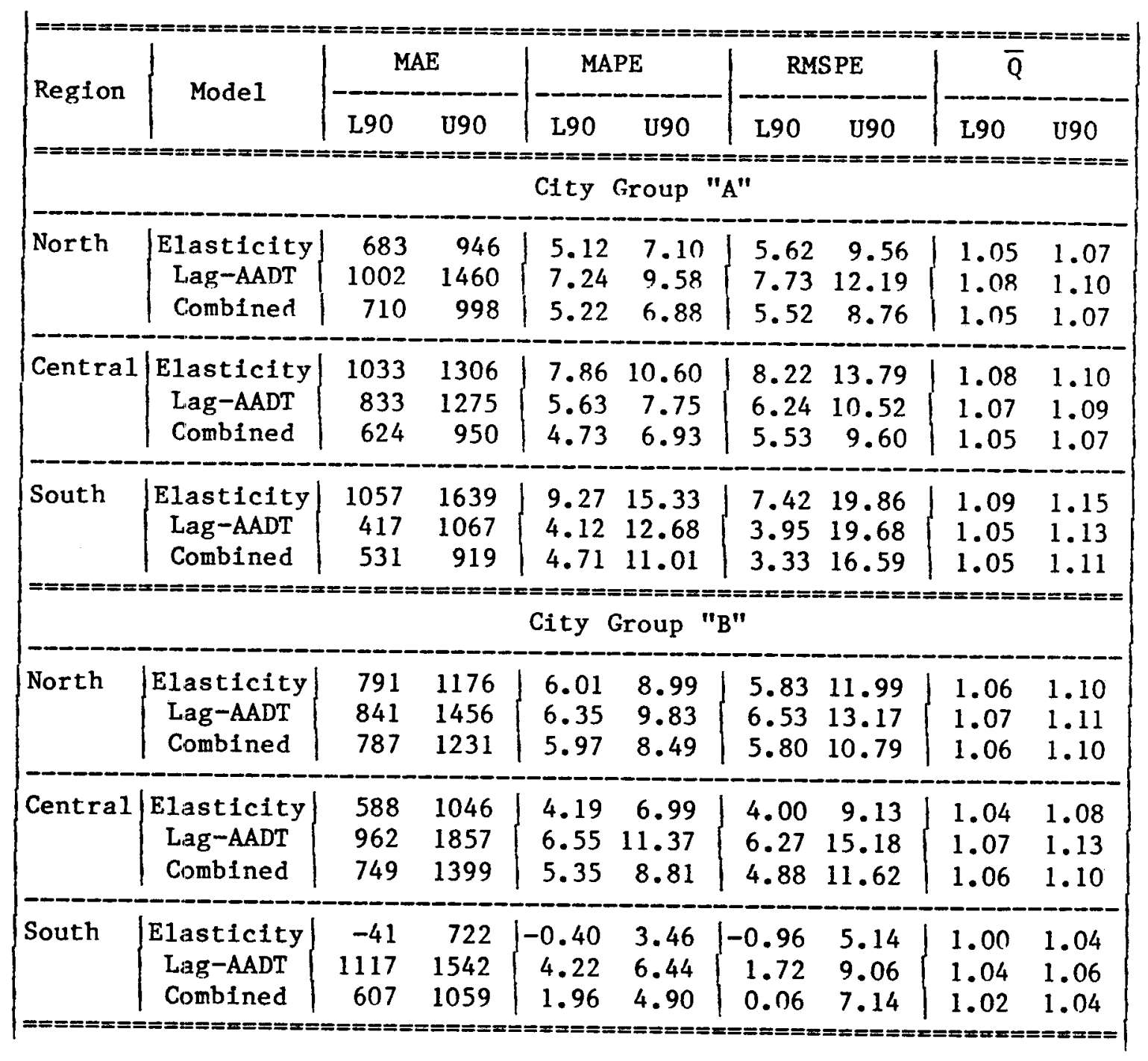


Table G3, continued

\begin{tabular}{|c|c|c|c|c|c|c|c|c|}
\hline \multirow{3}{*}{ Region } & \multirow[b]{2}{*}{ Mode1 } & \multicolumn{2}{|c|}{ MAE } & MAPE & \multicolumn{2}{|c|}{ RMSPE } & \multicolumn{2}{|l|}{$\bar{Q}$} \\
\hline & & L90 & U90 & L90 U90 & L90 & U90 & L90 & U90 \\
\hline & \multicolumn{8}{|c|}{ City Group "C" } \\
\hline North & $\left|\begin{array}{c}\text { Elasticity } \\
\text { Lag-AADT } \\
\text { Combined }\end{array}\right|$ & $\begin{array}{l}811 \\
889 \\
642\end{array}$ & $\begin{array}{r}1116 \\
1282 \\
957\end{array}$ & $\begin{array}{rr}6.88 & 8.92 \\
7.82 & 10.72 \\
5.58 & 7.90\end{array}$ & $\begin{array}{l}6.97 \\
8.23 \\
6.16\end{array}$ & $\begin{array}{l}11.02 \\
13.96 \\
10.50\end{array}$ & $\begin{array}{l}1.07 \\
1.08 \\
1.06\end{array}$ & $\begin{array}{l}1.09 \\
1.12 \\
1.08\end{array}$ \\
\hline Central & $\left|\begin{array}{c}\text { Elasticity } \\
\text { Lag-AADT } \\
\text { Combined }\end{array}\right|$ & $\begin{array}{r}908 \\
1075 \\
771\end{array}$ & $\begin{array}{l}1199 \\
1609 \\
1106\end{array}$ & $\begin{array}{rr}7.29 & 9.53 \\
8.00 & 10.86 \\
5.90 & 8.32\end{array}$ & $\begin{array}{l}7.52 \\
8.67 \\
6.56\end{array}$ & $\begin{array}{l}12.26 \\
14.40 \\
11.55\end{array}$ & $\begin{array}{l}1.08 \\
1.09 \\
1.07\end{array}$ & $\begin{array}{l}1.10 \\
1.13 \\
1.09\end{array}$ \\
\hline South & $\left|\begin{array}{c}\text { Elasticity } \\
\text { Lag-AADT } \\
\text { Combined }\end{array}\right|$ & $\begin{array}{l}368 \\
343 \\
239\end{array}$ & $\begin{array}{l}1373 \\
1700 \\
1457\end{array}$ & $\begin{array}{rr}3.33 & 10.03 \\
3.40 & 9.94 \\
2.03 & 9.03\end{array}$ & $\begin{array}{l}1.94 \\
2.79 \\
2.10\end{array}$ & $\begin{array}{l}14.36 \\
13.36 \\
12.65\end{array}$ & $\begin{array}{l}1.04 \\
1.07 \\
1.02\end{array}$ & $\begin{array}{l}1.10 \\
1.59 \\
1.10\end{array}$ \\
\hline $\mathrm{I}===$ & & & & $\begin{array}{l}=x======== \\
\text { City Group " }\end{array}$ & & & & \\
\hline North & $\left|\begin{array}{c}\text { Elasticity } \\
\text { Lag-AADT } \\
\text { Combined }\end{array}\right|$ & $\begin{array}{l}1020 \\
1271 \\
1050\end{array}$ & $\begin{array}{l}1889 \\
2451 \\
1893\end{array}$ & $\mid \begin{array}{ll}10.20 & 17.76 \\
10.20 & 31.74 \\
10.02 & 18.64\end{array}$ & $\mid \begin{array}{r}10.03 \\
11.28 \\
8.86\end{array}$ & $\begin{array}{l}23.80 \\
57.34 \\
27.11\end{array}$ & $\begin{array}{l}1.13 \\
1.12 \\
1.12\end{array}$ & $\begin{array}{l}1.23 \\
1.34 \\
1.22\end{array}$ \\
\hline Central & $\left|\begin{array}{c}\text { Elasticity } \\
\text { Lag-AADT } \\
\text { Combined }\end{array}\right|$ & $\begin{array}{l}566 \\
450 \\
312\end{array}$ & $\begin{array}{l}934 \\
879 \\
709\end{array}$ & $\begin{array}{rr}6.78 & 11.24 \\
5.23 & 8.83 \\
3.85 & 7.71\end{array}$ & $\begin{array}{l}6.08 \\
4.88 \\
4.15\end{array}$ & $\begin{array}{l}15.16 \\
11.87 \\
10.96\end{array}$ & $\begin{array}{l}1.07 \\
1.06 \\
1.04\end{array}$ & $\begin{array}{l}1.11 \\
1.10 \\
1.08\end{array}$ \\
\hline South & $\left|\begin{array}{c}\text { Elasticity } \\
\text { Lag-AADT } \\
\text { Combined }\end{array}\right|$ & $\begin{array}{r}764 \\
1107 \\
854\end{array}$ & $\begin{array}{l}1100 \\
1736 \\
1327\end{array}$ & $\begin{array}{rr}6.71 & 9.55 \\
9.20 & 13.38 \\
7.17 & 10.43\end{array}$ & $\begin{array}{l}6.91 \\
9.53 \\
7.58\end{array}$ & $\begin{array}{l}12.30 \\
17.60 \\
13.57\end{array}$ & $\begin{array}{l}1.07 \\
1.11 \\
1.08\end{array}$ & $\begin{array}{l}1.11 \\
1.17 \\
1.12\end{array}$ \\
\hline
\end{tabular}

Note: $\mathrm{L} 90=$ Lower 1 imit of $90 \%$ confidence 1 imit, and U90 $=$ Upper limit of $90 \%$ confidence limit. 
Table G4: 95-Percent Confidence Limits of Forecast Measures for Models of Urban Principal Arterial

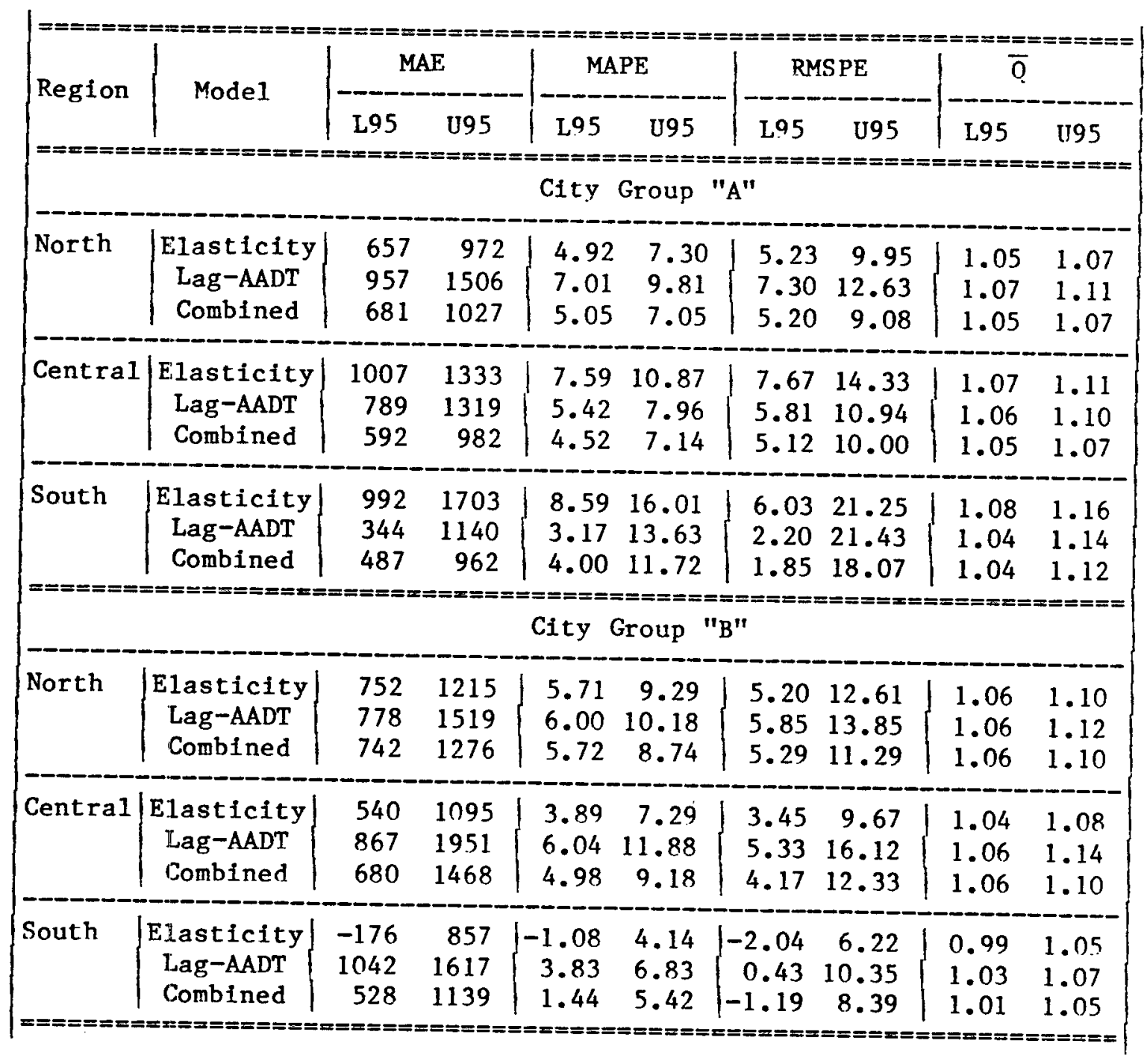


Table G4, continued

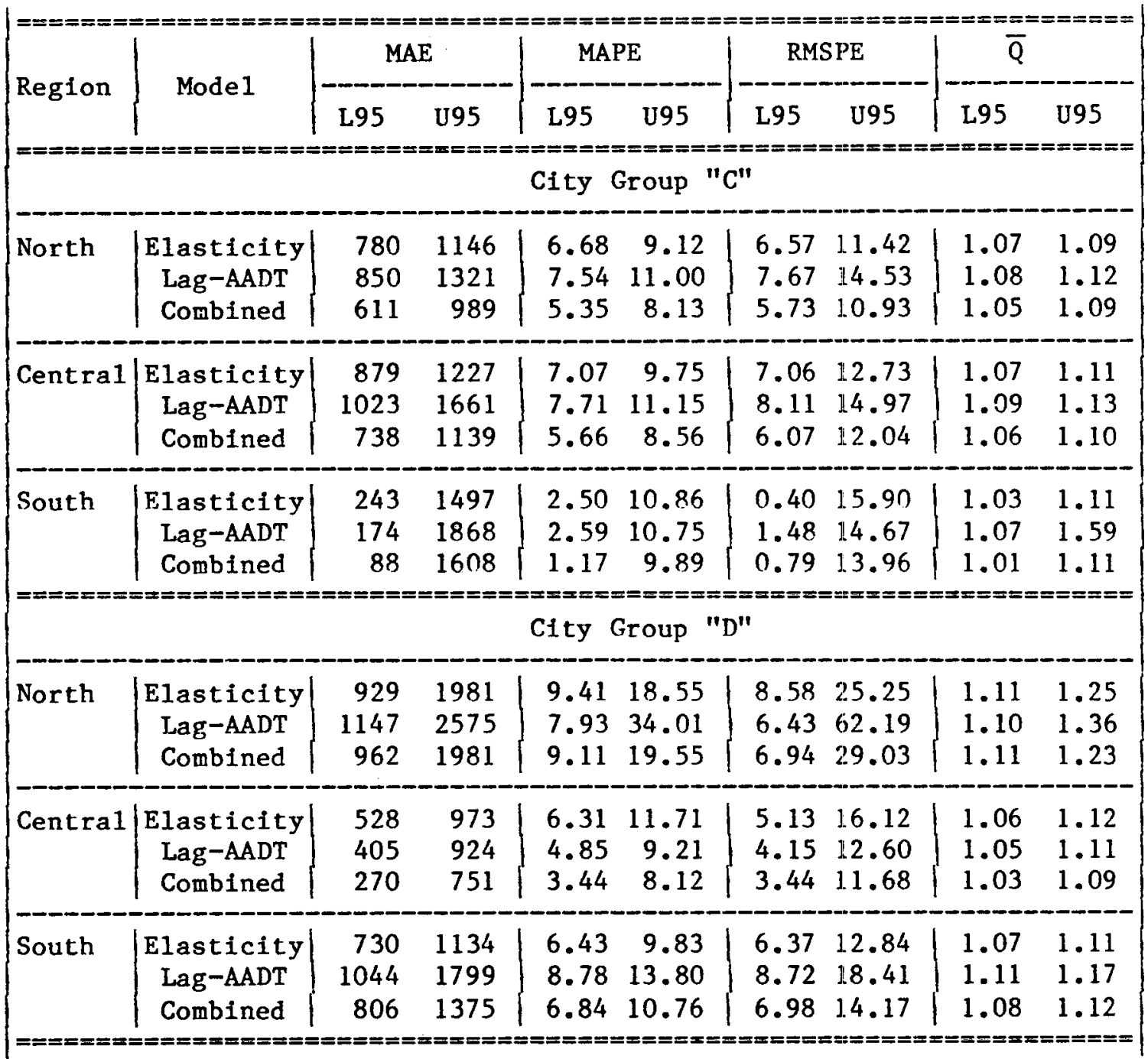

Note: $\mathrm{L} 95=$ Lower 1 imit of $95 \%$ confidence 1 imit, and U95 = Upper 1imit of $95 \%$ confidence 1imit. 
Table G5: 90-Percent Confidence Limits of Forecast Measures for Models of Urban Minor Arterial \& Collector

\begin{tabular}{|c|c|c|c|c|c|c|c|c|c|}
\hline \multirow{2}{*}{$\begin{array}{l}\text { City } \\
\text { Group } \\
=====\end{array}$} & \multirow{2}{*}{$\begin{array}{c}\text { Model } \\
===x====x==\end{array}$} & \multicolumn{2}{|c|}{ MAE } & \multicolumn{2}{|c|}{ MAPE } & \multicolumn{2}{|c|}{ RMSPE } & \multicolumn{2}{|l|}{$\bar{Q}$} \\
\hline & & $\begin{array}{c}\mathrm{L} 90 \\
======\end{array}$ & $\begin{array}{c}\text { U90 } \\
==== \pm=\end{array}$ & $\begin{array}{l}\mathrm{L} 90 \\
=====\end{array}$ & $\begin{array}{c}\text { U90 } \\
=======\end{array}$ & $\begin{array}{l}L 90 \\
======\end{array}$ & $\begin{array}{c}\text { U90 } \\
=======\end{array}$ & $\begin{array}{l}\mathrm{L} 90 \\
=====\end{array}$ & $\begin{array}{c}\text { U90 } \\
======\end{array}$ \\
\hline A & $\begin{array}{l}\text { Elasticity } \\
\text { Lag-AADT } \\
\text { Combined }\end{array}$ & $\begin{array}{r}1370 \\
746 \\
764\end{array}$ & $\begin{array}{l}3979 \\
1826 \\
2152\end{array}$ & $\begin{array}{r}12.41 \\
6.71 \\
5.02\end{array}$ & $\begin{array}{l}27.99 \\
16.23 \\
17.28\end{array}$ & $\begin{array}{r}10.93 \\
7.37 \\
5.63\end{array}$ & $\begin{array}{l}38.69 \\
21.54 \\
22.38\end{array}$ & $\begin{array}{l}1.12 \\
1.07 \\
1.06\end{array}$ & $\begin{array}{l}1.28 \\
1.17 \\
1.16\end{array}$ \\
\hline B & $\begin{array}{l}\text { Elasticity } \\
\text { Lag-AADT } \\
\text { Combined }\end{array}$ & $\begin{array}{l}849 \\
168 \\
461\end{array}$ & $\begin{array}{l}1781 \\
1095 \\
1310\end{array}$ & $\begin{array}{r}14.82 \\
3.36 \\
9.17\end{array}$ & $\begin{array}{l}36.50 \\
11.52 \\
20.65\end{array}$ & $\begin{array}{r}14.94 \\
3.46 \\
8.83\end{array}$ & $\begin{array}{l}52.75 \\
18.82 \\
29.06\end{array}$ & $\begin{array}{l}1.16 \\
1.03 \\
1.09\end{array}$ & $\begin{array}{l}1.38 \\
1.15 \\
1.23\end{array}$ \\
\hline C & $\begin{array}{l}\text { Elasticity } \\
\text { Lag-AADT } \\
\text { Combined }\end{array}$ & $\begin{array}{l}486 \\
494 \\
487\end{array}$ & $\begin{array}{l}1024 \\
1024 \\
1021\end{array}$ & $\begin{array}{l}4.31 \\
4.28 \\
4.23\end{array}$ & $\begin{array}{l}14.57 \\
14.42 \\
14.45\end{array}$ & $\begin{array}{l}4.29 \\
4.27 \\
4.26\end{array}$ & $\begin{array}{l}26.51 \\
26.19 \\
26.34\end{array}$ & $\begin{array}{l}1.05 \\
1.05 \\
1.05\end{array}$ & $\begin{array}{l}1.15 \\
1.15 \\
1.15\end{array}$ \\
\hline D & $\begin{array}{c}\text { Elasticity } \\
\text { Lag-AADT } \\
\text { Combined }\end{array}$ & $\begin{array}{l}743 \\
500 \\
401\end{array}$ & $\begin{array}{r}1284 \\
928 \\
688\end{array}$ & $\begin{array}{l}9.49 \\
6.86 \\
5.27\end{array}$ & $\begin{array}{r}14.93 \\
10.68 \\
8.71\end{array}$ & $\begin{array}{r}10.45 \\
7.16 \\
5.73\end{array}$ & $\begin{array}{l}19.99 \\
14.51 \\
12.35\end{array}$ & $\begin{array}{l}1.10 \\
1.08 \\
1.05\end{array}$ & $\begin{array}{l}1.16 \\
1.12 \\
1.09\end{array}$ \\
\hline
\end{tabular}

Note: $\mathrm{L} 90=$ Lower 1 imit of $90 \%$ confidence limit, and U90 $=$ Upper limit of $90 \%$ confidence limit. 
Table G6: 95-Percent Confidence Limits of Forecast Measures for Models of Urban Minor Arterial \& Collector

\begin{tabular}{|c|c|c|c|c|c|c|c|c|c|}
\hline \multirow{2}{*}{$\begin{array}{l}\text { City } \\
\text { Group } \\
=====\end{array}$} & \multirow[b]{2}{*}{ Mode1 } & \multicolumn{2}{|c|}{ MAE } & \multicolumn{2}{|c|}{ MAPE } & \multicolumn{2}{|c|}{ RMSPE } & \multicolumn{2}{|l|}{$\bar{Q}$} \\
\hline & & $\begin{array}{c}\text { L95 } \\
=====\end{array}$ & $\underbrace{\text { U95 }}_{== \pm x==}$ & $L_{== \pm==}$ & $\begin{array}{c}\text { U95 } \\
==x= \pm==\end{array}$ & $\operatorname{La5}_{==== \pm}$ & $\begin{array}{c}\text { U95 } \\
======\end{array}$ & $\begin{array}{l}\text { L95 } \\
======\end{array}$ & $\begin{array}{l}\text { U95 } \\
=====\end{array}$ \\
\hline A & $\left\{\begin{array}{c}\text { Elasticity } \\
\text { Lag-AADT } \\
\text { Combined }\end{array}\right.$ & $\begin{array}{r}1076 \\
624 \\
607\end{array}$ & $\begin{array}{l}4274 \\
1948 \\
2309\end{array}$ & $\begin{array}{r}10.65 \\
5.63 \\
3.64\end{array}$ & $\begin{array}{l}29.75 \\
17.31 \\
18.66\end{array}$ & $\begin{array}{l}7.80 \\
5.77 \\
3.74\end{array}$ & $\begin{array}{l}41.82 \\
23.14 \\
24.27\end{array}$ & $\begin{array}{l}1.10 \\
1.06 \\
1.05\end{array}$ & $\begin{array}{l}1.30 \\
1.18 \\
1.17\end{array}$ \\
\hline B & $\left\{\begin{array}{c}\text { Elasticity } \\
\text { Lag-AADT } \\
\text { Combined }\end{array}\right.$ & $\begin{array}{r}746 \\
66 \\
368\end{array}$ & $\begin{array}{l}1884 \\
1197 \\
1404\end{array}$ & $\mid \begin{array}{r}12.43 \\
2.47 \\
7.91\end{array}$ & $\begin{array}{l}38.89 \\
12.41 \\
21.91\end{array}$ & $\begin{array}{r}10.79 \\
1.78 \\
6.61\end{array}$ & $\begin{array}{l}56.90 \\
20.51 \\
31.28\end{array}$ & $\begin{array}{l}1.14 \\
1.02 \\
1.08\end{array}$ & $\begin{array}{l}1.40 \\
1.16 \\
1.24\end{array}$ \\
\hline C & $\left\{\begin{array}{c}\text { Elasticity } \\
\text { Lag-AADT } \\
\text { Combined }\end{array}\right.$ & $\begin{array}{l}429 \\
438 \\
430\end{array}$ & $\begin{array}{l}1082 \\
1080 \\
1078\end{array}$ & $\begin{array}{l}3.21 \\
3.20 \\
3.14\end{array}$ & $\begin{array}{l}15.67 \\
15.50 \\
15.54\end{array}$ & $\begin{array}{l}1.92 \\
1.94 \\
1.91\end{array}$ & $\begin{array}{l}28.88 \\
28.52 \\
28.68\end{array}$ & $\begin{array}{l}1.04 \\
1.04 \\
1.04\end{array}$ & $\begin{array}{l}1.16 \\
1.16 \\
1.16\end{array}$ \\
\hline D & $\left\{\begin{array}{c}\text { Elasticity } \\
\text { Lag-AADT } \\
\text { Combined }\end{array}\right.$ & $\begin{array}{l}689 \\
457 \\
373\end{array}$ & $\begin{array}{r}1338 \\
972 \\
716\end{array}$ & $\begin{array}{l}8.94 \\
6.48 \\
4.93\end{array}$ & $\begin{array}{r}15.48 \\
11.06 \\
9.05\end{array}$ & $\begin{array}{l}9.48 \\
6.42 \\
5.06\end{array}$ & $\begin{array}{l}20.95 \\
15.25 \\
13.01\end{array}$ & $\begin{array}{l}1.09 \\
1.07 \\
1.05\end{array}$ & $\begin{array}{l}1.17 \\
1.13 \\
1.09\end{array}$ \\
\hline
\end{tabular}

Note: $\mathrm{L} 95=$ Lower 1 imft of $95 \%$ confidence limit, and U95 = Upper limit of $95 \%$ confidence limit. 
VITA 
VITA

Sunil K. Saha was born on June 30, 1957 in Bangladesh. He received his Bachelor of Science in Civil Engineering from the Bangladesh University of Engineering and Technology (BUET), Dhaka in March 1980. He served as a Lecturer in Civil Engineering from April 1980 to June 1983 and as an Assistant Professor of Civil Engineering from July 1983 to August 1984 at BUET, Dhaka, Bangladesh. He came to Purdue University, Indiana in August 1984 for graduate study. He received his Master of Science in Civil Engineering in August 1986, with an emphasis in Transportation Engineering. Since then, he has been working in the Ph.D. program in the area of Transportation Engineering at Purdue University. He expects to receive his Doctor of Philosophy in Civil Engineering in December 1990. Since August 1984, he has worked as a Graduate Instructor in the School of Civil Engineering at Purdue University. He was a teaching assistant for several courses, including highway transportation characteristics and traffic engineering.

He is a member of the Institute of Transportation Engineers, Transportation Research Board, American Society of Civil Engineers, and Omega Rho honor society. Sunil is married to Puspa R. Saha and they have a son, Sudip K. Saha (Mital), who was born in 1984 . 


\title{
THE DEVELOPMENT OF A PROCEDURE TO FORECAST TRAFFIC VOLUMES ON URBAN SEGMENTS OF THE STATE AND INTERSTATE HIGHWAY SYSTEMS
}

\author{
by \\ Sunil K. Saha \\ Graduate Research Assistant \\ and \\ Jon D. Fricker \\ Research Engineer \\ Joint Highway Research Project \\ Project No.: HPR-0313-(028) \\ File No.: $3-3-41$ \\ Prepared as Part of an Investigation \\ conducted by the \\ Joint Highway Research Project \\ Engineering Experiment Station \\ Purdue University \\ In Cooperation with the \\ Indiana Department of Transportation \\ and the \\ U.S. Department of Transportation \\ Federal Highway Administration
}

The contents of this report reflect the views of the author who is responsible for the facts and accuracy of the data presented herein. The contents do not necessarily reflect the official views or policies of the Federal Highway Administration. This report does not constitute a standard, specifications or regulations.

\section{Department of Civil Engineering \\ Purdue University \\ West Lafayette, Indiana 47907}

August 1990 
IMPLEMENTATION REPORT

The implementation of the results of this study will be accomplished in several ways:

1. By following the step-by-step procedure outlined in this Implementation Report.

2. By using a computer program specially developed to carry out the calculations that are part of that step-by-step procedure.

3. By applying the forecast monitoring procedure outlined in Section 10.4 of the Final Report.

\section{Implementation Guidelines}

One purpose of this research was to prepare guidelines for the use of the models developed in this study. The work elements undertaken to accomplish that purpose included review of the related literature, preparation of the data bases, testing different data bases and their formats and stratification, analysis and development of different types of traffic models, forecasts of input variables for which no outside source is available, 
evaluation of different models, and development of a monitoring procedure to determine the need for model recalibration. Figures $11.1,11.2$ and 11.3 (extracted from the Final Report) present the process for implementation of the models to forecast traffic on interstate highways, urban principal arterials, and urban minor arterials \& collectors, respectively.

The steps that are recommended for the implementation of the elasticity and lag-AADT models to predict the future traffic for urban segment of the state highways and interstate highways of Indiana are listed below.

1. Find the exact location (i.e., city and county) of the roadway for which the forecast is needed.

2. Select the traffic models that will be used to predict trafic.

a. Determine the functional class of roadway.

This will determine which models are applicable to the project site. To determine the functional class, the functional classification system map -prepared by Program Development Division of INDOT -and the road inventory file of INDOT, will be the two best guides. Moreover, the definitions provided in Table 4.1 of the Final Report would be helpful to find the appropriate highway class. The project site 


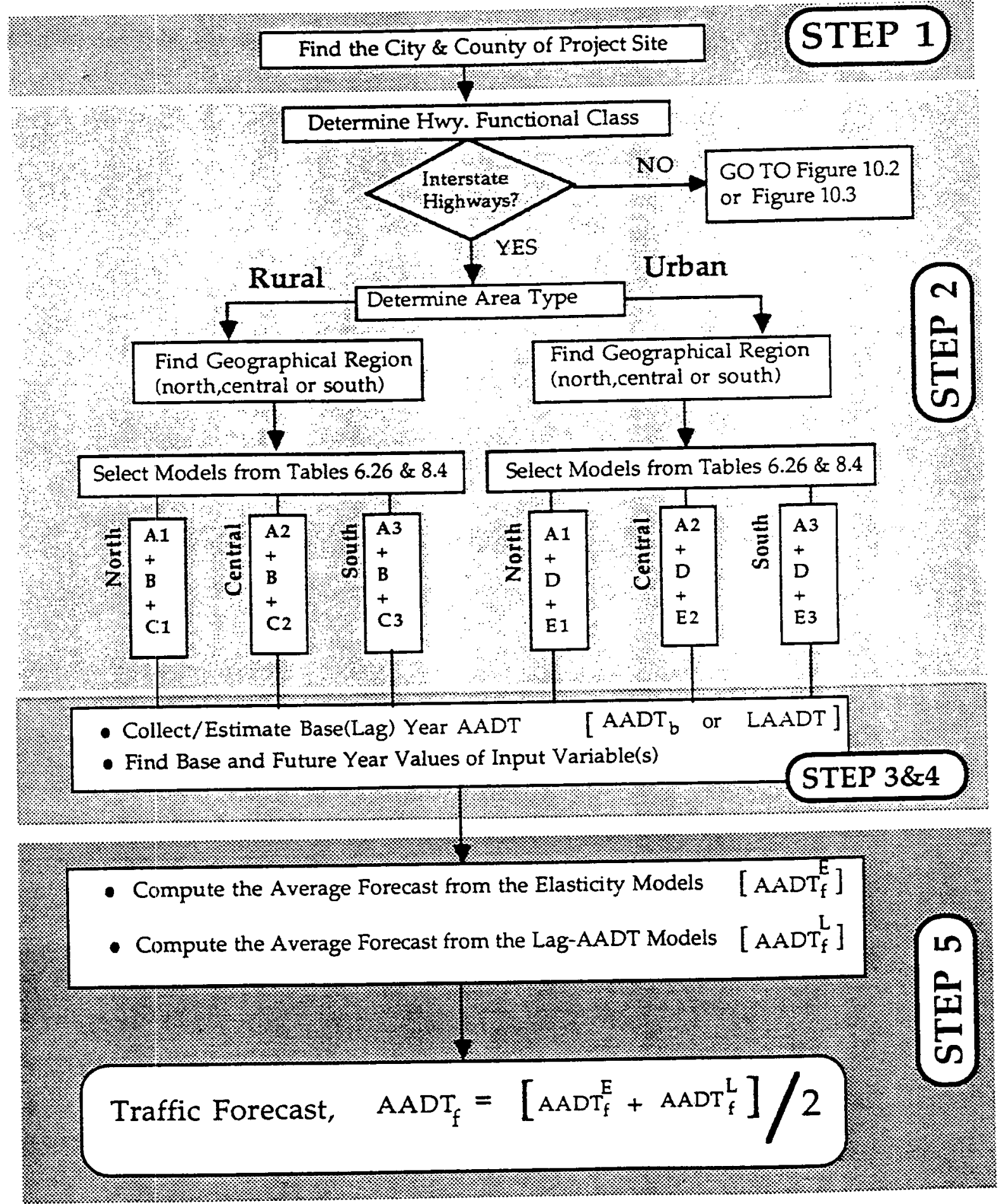

Figure 11.1: Process for Implementation of the Model on Interstate Highways 


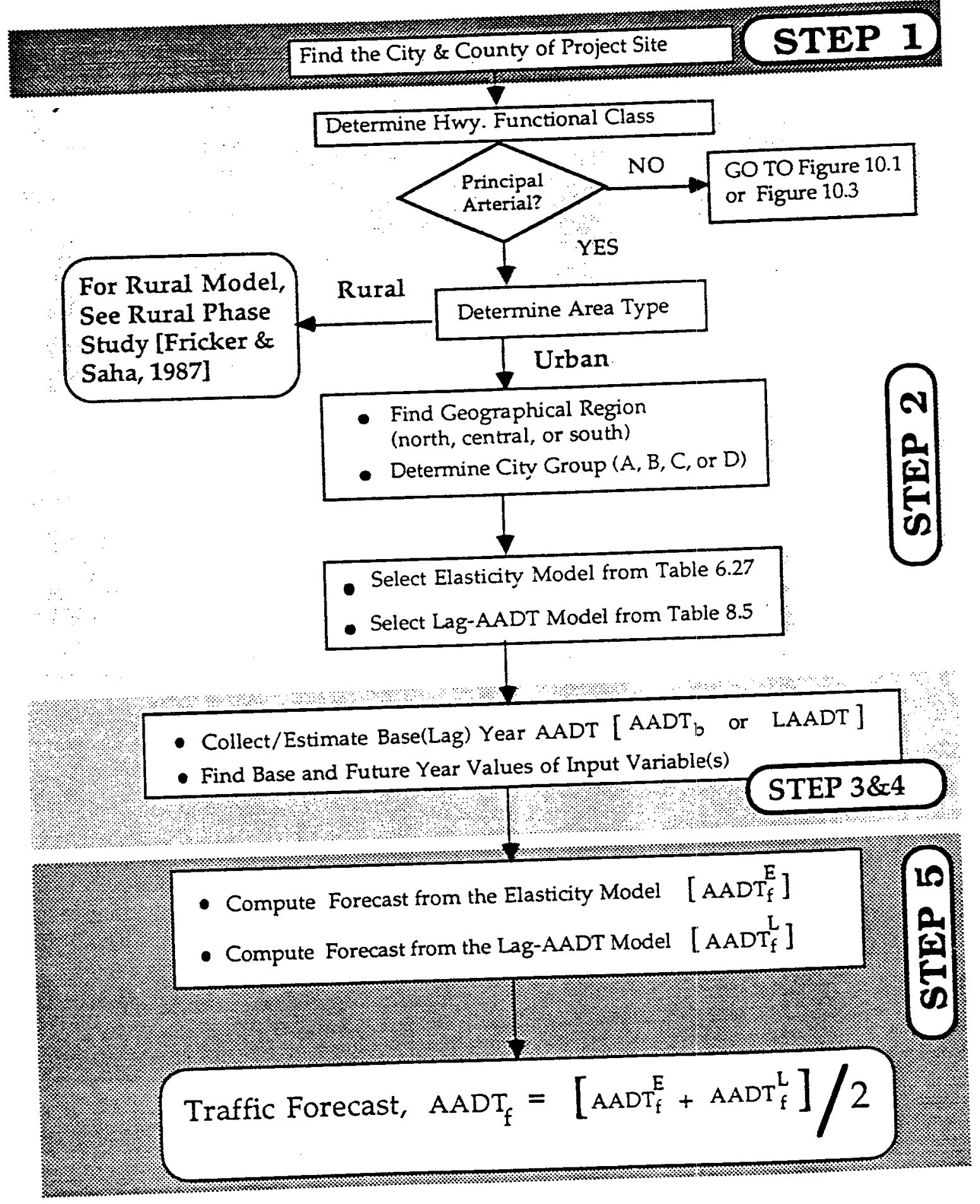

Figure 11.2: Process for Implementation of the Model on Urban Principal Arterial 


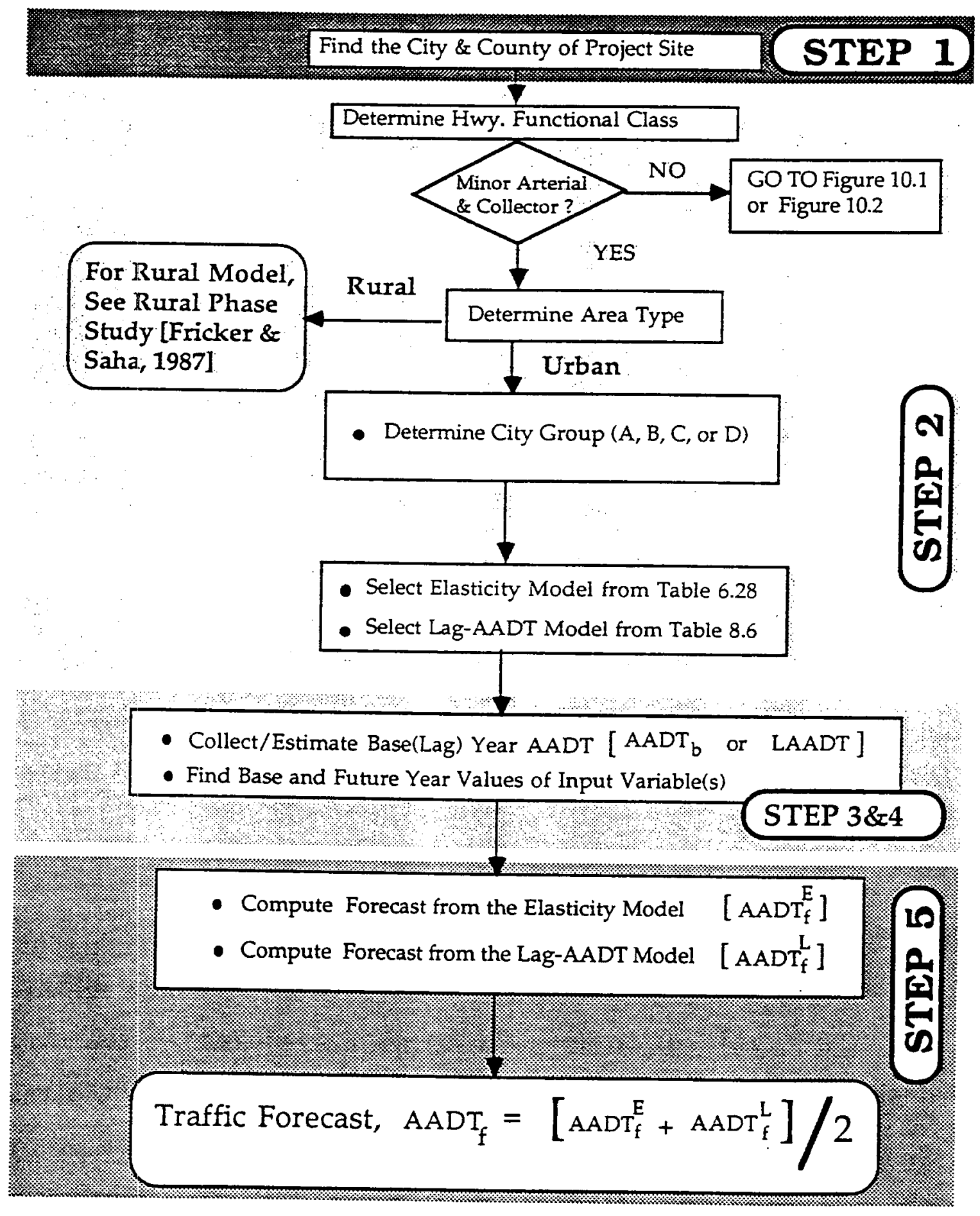

Figure 11.3: Process for Implementation of the Model on Urban Minor Arterial \& Collector 
will be classified into one of the four categories of highways listed in Table 4.1. If the classification is not clear-cut, then personal judgment should be used, and documentation provided.

b. Examine the area type.

Check if the project site is rural or urban in nature. If the project site is in a rural area for noninterstate highways, then the models developed in the rural phase of this study (Fricker and Saha, 1987) will be applicable to that site. Otherwise, the procedures described in this section will be applied. Suburban segments can be identified as urban segments, because data from suburban segments were included with urban segments in the model building process.

c. Find the geographical region.

Consult Figure 4.2 (taken from the Final Report) to determine the geographical region (north, central and south) of the project site. This finding will be used to select the appropriate model for urban principal arterial models and the regional model of interstate highways.

d. Determine the city group. 


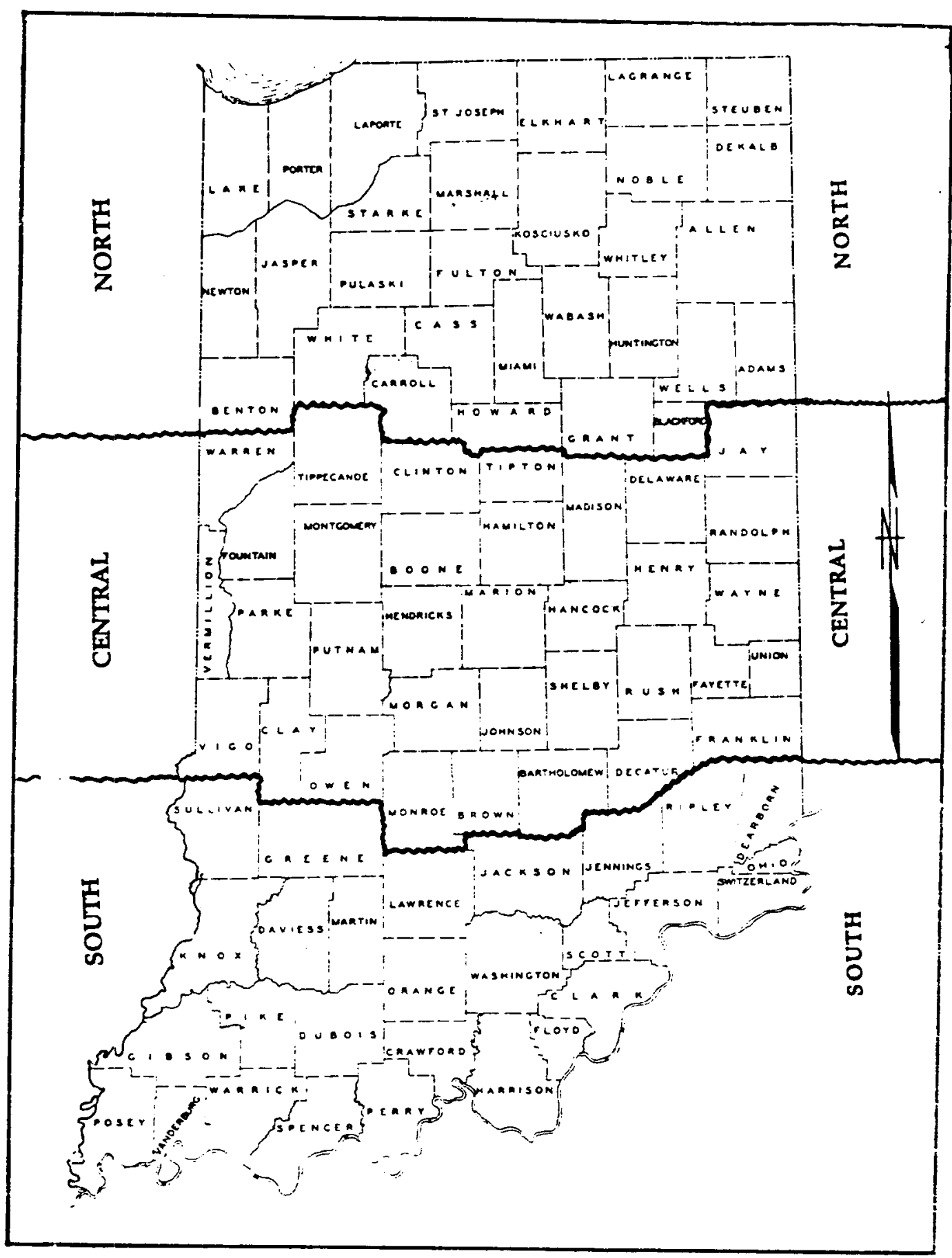

Figure 4.2: Suggested Geographical Boundaries of State of Indiana 
Find the city group ( $A, B, C$ or $D$ ) based on the size of the city population. Section 4.6.1 of the Final Report introduced the city group based on 1980 city population, as summarized below.

\begin{tabular}{|c|c|}
\hline CTYGR & 1980 City Population (1000) \\
\hline A & $30-50$ \\
B & $20-30$ \\
C & $10-20$ \\
D & $5-10$ \\
\hline
\end{tabular}

In using the forecasting models, population in the base year can be used to determine the city group. For interstate highways, an additional group, A+, was designated for cities with more than 50,000 population in 1980. If the population is not known, a population estimate from the closest census year population to the base year either by ratio method (Section 9.3.1 of the Final Report) or by linear interpolation or extrapolation from city population forecasts in Table F9 in Appendix F. The city group information will be used by the computer program to select models for noninterstate urban highways.

e. Select appropriate models.

The information from steps 2 a to $2 d$ are used by the computer program to find the appropriate model(s). Steps $2 a$ and $2 b$ are used to determine 
highway class and its area type.

i. Interstate Highways: The computer program will find the appropriate elasticity models from Final Report Table 6.26, by using the the information obtained on area type (step 2b) and geographical region (2c). For each project site, three alternative models -- regional interstate model, statewide rural or urban interstate model, and regional rural or urban interstate mode1 -- will be identified in this table. Figure 11.1 shows those appropriate models for each case. For instance, if the project site is identified as being in the northern region and in a rural area, then the models $A 1, B$ and $C l$ from Table 6.26 will be the selected for that project site. Similarly, three alternative lag-AADT models will be selected from Final Report Table 8.4 .

ii. Urban Principal Arterial: The information on geographical region (step 2c) and on city group (step 2d) of the project site will be used to find the appropriate elasticity model from Final Report Table 6.27. Similarly, the lagAADT will be used identified from Final Report Table 8.5 . 
iii. Urban Minor Arterial \& Collector: The city group (step 2d) is the only basis for selecting the appropriate model for this highway category, if the area type (step $2 b$ ) and the functional class (step 2a) had identified the project site as "Urban Minor Arterial \& Collector". The appropriate elasticity and lag-AADT models will be identified from Final Report Tables 6.28 and 8.6 , respectively.

3. Collect the base year AADT $\left(\mathrm{AADT}_{\mathrm{b}}\right)$ and the 1 ag year AADT ( LAADT).

The base or lag year may be different from the present/current year, but it is the year for which most recent data needed by the are available. $\operatorname{AADT}_{b}$ (required by the elasticity mode1) or LAADT (required by the 1ag-AADT model) for a project site can be one value for a small project (e.g., intersections) or a series of estimates for roadway sections for a larger project (e.g., lane widening). The primary source of data is the Highway Department's Traffic Volume Book. If this source fails to provide such information, then it could be determined from short-term counts at the project site, using the procedures described in Final Report Section 2.7 . 
4. Collect the base and future year data for the predictor variables used in the elasticity models.

The description of the predictor variables in section 4.6.3 of the Final Report is a guide to the sources of the required predictor variables. Except vehicle registration and city population forecasts, the forecasts of the predictor variables used in the models are available from outside sources. The combined vehicle registration forecasts developed by time series analysis and presented in Final Report Appendix Table F7 will serve as future year values for vehicle registration at the county and state 1 evel. The ratio method of making city population forecasts presented in Appendix Table F9 can be used as required by any mode1. The lag-AADT models need only the estimate of city population in the base year (if required) to select the appropriate model for noninterstate highways. They do not need any other information for the predictor variables, to forecast traffic.

5. Estimate the future year AADT.

a Calculate the future year AADT by using the appropriate elasticity model(s) (Final Report Tables 6.266.28), as determined in step $2 \mathrm{e}$, with the values found in steps 3 and 4 . Denote the future ("f"-year) AADT estimate from the elasticity (E) model as $\operatorname{AADT}_{\mathrm{f}}^{\mathrm{E}}$. 
For each interstate highway segment, the average forecasts from the three alternative models will be designated as $\operatorname{AADT}_{\mathrm{f}}^{\mathrm{E}}$. Only one forecast will be computed for each noninterstate highway segment.

b. Calculate the future year AADT by using appropriate lag-AADT model(s) (Final Report Tables 8.4-8.6), as determined in step $2 e$ with the values found in steps 3 and 4. Denote the AADT estimate from the lag-AADT (or "L") model as $\operatorname{AADT}_{\mathrm{f}}^{\mathrm{L}}$.

c. Find the average of the two AADT estimates found in steps $5 \mathrm{a}$ and $5 \mathrm{~b}$.

$$
\operatorname{AADT}_{f}=\left|\operatorname{AADT}_{\mathrm{f}}^{\mathrm{E}}+\operatorname{AADT}_{\mathrm{f}}^{\mathrm{L}}\right| * 0.5
$$

where,

$\operatorname{AADT}_{f}=$ Future year AADT estimate,

$\operatorname{AADT}_{\mathrm{f}}^{\mathrm{E}}=$ Future year AADT estimate by Elasticity mode1(s), $\operatorname{AADT}_{f}^{L}=$ Future year AADT estimate by Lag-AADT model(s).

The AADT forecast ( $\operatorname{AADT}_{\mathrm{f}}$ ) from Equation (11.1) will be used as the "future year AADT" for the project site. The user may give different weight to the AADT values found in step $5 a$ or step $5 b$, based on model performance. Equation (11.1) would then be modified to be 


$$
\operatorname{AADT}_{\mathrm{f}}^{\mathrm{W}}=\mathrm{w} * \operatorname{AADT}_{\mathrm{f}}^{\mathrm{E}}+(1-\mathrm{w}) * \operatorname{AADT}_{\mathrm{f}}^{\mathrm{L}}
$$

where,

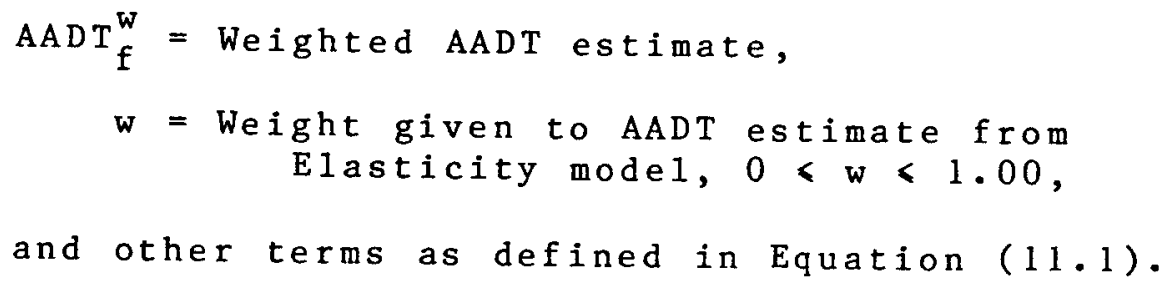

In the future, the performance of the models can be used to determine the weight "w" in Equation (11.2). But, based on comparable values of the forecasting measuring criteria (MAE, MAPE, RMSPE and $\bar{Q}$ ) for the elasticity and lag-AADT models, and improved performance of the combined forecasts, a simple average, shown in Equation (11.1), is recommended at present.

6. Adjust the estimated future year AADT.

If necessary, the forecast from step 5 can be adjusted by using information on changes in fuel price, fuel supply (energy crisis), fleet fuel efficiency (miles per gallon), household size, inflation rate, etc., which have not been included in model formulation because of insufficient information and future uncertainty about those factors. Empirical results can be useful in incorporating such adjustments, if the user has a "feel" for any of these factors. This step is completely optional and it is left to the user to decide whether to modify the forecast values 
obtained in step 5. Because of its optional nature, this step was not shown in the process for implementation in Figures 11.1 to 11.3 . 